\title{
Final Program \\ Forty Fifth Annual Meeting \\ International Neuropsychological Society
}

February 1-4, 2017

New Orleans, Louisiana, USA

9:00 АM-12:00 РМ

1. BELLINGER, DC

9:00 AM-12:00 РМ

1. BIKSON, M

9:00 AM-12:00 PМ

1. DRONKERS, NF

12:00-1:00 PM

1:00-4:00 PM

1. BUSH, S

1:00-4:00 PM

1. CASEY, B

\section{WEDNESDAY, FEBRUARY 1, 2017}

CE Workshop 1. Environmental Chemicals and Children's Brains: How Big a Problem?

Presenter: David C. Bellinger

Bissonet

Environmental Chemicals and Children's Brains: How Big a Problem?

CE Workshop 2. Best-Practices of Transcranial Direct Current Stimulation (tDCS) for Effective and Reliable Outcomes

Presenter: Marom Bikson

Salon D

Best-Practices of Transcranial Direct Current Stimulation (tDCS) for Effective and Reliable

Outcomes

CE Workshop 3. Adult Aphasia: Classifications, Localization, and Neuroimaging

Presenter: Nina F. Dronkers

Salon E

Adult Aphasia: Classifications, Localization, and Neuroimaging

Lunch (On Own)

Conference-Wide

CE Workshop 4. Identifying Ethical Issues in Neuropsychological Subspecialties: Concepts, Cases, and Controversies

Presenter: Shane Bush

Bissonet

Identifying Ethical Issues in Neuropsychological Subspecialties: Concepts, Cases, and Controversies

CE Workshop 5. The Adolescent Brain: Arrested or Adaptive

Development?

Presenter: BJ Casey

Salon D

The Adolescent Brain: Arrested or Adaptive Development? 
1:00-4:00 PM

1. STUSS. DT

\section{2:45-4:00 PM}

1. AGARUNOV E

2. BEDARD, $\mathrm{M}$

3. FIGUEROA, CM

4. KAEMMERER, TD

5. KITCHENER, E

6. MERCER, EN

7. OH. C

8. RYMAN, S

9. SMITH, CT

10. SMITH, HA

11. TURNER. JA

12. VINGERHOETS, G
13. CARBINE, KA
14. KEITH. C
15. LITHFOUS, S
16. MANGAL, P
17. MCKINNEY, TL
18. STEPHENSON, AJ
19. WANG, Y
20. BERMUDEZ, CI
21. BERMUDEZ, CI
22. BERMUDEZ, CI
23. BERMUDEZ, CI
24. CAVACO, S
25. CONSIDINE, CM
26. CORNWELL, M
27. DICKSON, DM
28. KORTHAUER, LE
29. LAFLEUR, J
30. LEE, Y
31. LOCKE, D

\section{CE Workshop 6. Clinical Assessment of Frontal Lobe Functions: A Historical Perspective of the Application of the Boston VA Jamaica Plans VA Process Approach Presenter: Donald T. Stuss Salon E}

Clinical Assessment of Frontal Lobe Functions: A Historical Perspective of the Application of the Boston VA Jamaica Plans VA Process Approach

\section{Poster Session 1. Epilepsy \& Neuroscience Acadia}

\section{Cognitive Neuroscience}

Processing Speed and White Matter Integrity Across Young Adulthood to Late-mid Life The Moderating Influence of the Val66Met Polymorphism of the Brain Derived Neurotrophic Factor Gene in the Relation Between Cognitive Flexibility and Perceived Loneliness Relevant to Depressive Symptoms

Reinforcement Learning and Error-Related Negativity Distinguish Healthy Elders at Genetic Risk for Alzheimer's Disease

Implicit Motor Sequence Learning among Healthy Older Adults and Older Adults with Mild

Neurocognitive Disorder: Examining the Impact of Embedded Associative Structure

Category Specificity in Right Temporal Variant Semantic Dementia

Cognitive Development Trajectories of Planned Cesarean Sections and Vaginal Births

The Effects of Cognitive Loading on Parameters of Gait and Possible Risk of Injurious Falls

Multimodal Cognitive Control: an fMRI study of the AX-CPT

The Neural Basis of Emotional Working Memory and Its Relation to Adaptive Emotional Functioning Does Positive Affect Enhance or Disrupt Cognitive Control Processes? An Event-Related Potential (ERP) Study

Neural Circuits of Source Memory and Imagination

Effective Connectivity Underlying the Production of Speech and Hand Gestures

\section{Electrophysiology/EEG/ERP}

The Relationship Between Food-Related Inhibitory Control, Weight, and BMI Status: Implications for Calorie and Carbohydrate Intake

Effects of Acute Psychosocial Stress on Attentional Resources and Response Inhibition: An Electrophysiological and Behavioral Study

Investigating Face identity Discrimination using Fast Periodic Visual Stimulation (FPVS)

Modulation of Electrocortical Responses to Emotional Pictures in Parkinson's Disease

Medial Frontal Theta Dynamics Predict Temperamental Self-Regulation but Not Executive Response Inhibition

Behavioral Inhibition and Baseline Frontal Asymmetry are Associated with Sleep Quality Components Narcissistic Personality Disorder Modulates the Outcome Evaluation of Trusting Strangers: An Eventrelated Brain Potentials Study

\section{Epilepsy/Seizures}

Delayed Verbal Memory Decline Associated with Left Stereotactic Laser Ablation for the Treatment of Temporal Lobe Epilepsy

How to Measure the Quality of Life in Hispanics with Epilepsy? A Spanish Version of the Personal Impact of Epilepsy Scale in a Multicultural Treatment and Research Setting.

Improvement in Non-Memory Neuropsychological Test Performance Following Stereotactic Laser Ablation for the Treatment of Temporal Lobe Epilepsies.

Visual and Verbal Memory Changes Associated with Left vs. Right Stereotactic Laser Ablation for the Treatment of Temporal Lobe Epilepsies.

Auditory Verbal Learning Test in unilateral MTLE with hippocampal sclerosis

The Clinical and Neuropsychological Characteristics of a Nocturnal-seizure Sample, with Casematched Comparison to a Diurnal-seizure Contrast Sample

The moderating role of seizure variables on social communication difficulties in children with epilepsy

Expressive Language Measures in Focal Epilepsy: How Do They Relate and Do They Help with Lateralization?

Regional Subcortical Volumes are Associated with Pre-Operative Memory Performance in Left Anterior Temporal Lobectomy (ATL) Patients

Impact of neuropsychological consultation in a multidisciplinary pediatric epilepsy clinic

Psychosocial Outcomes 10-years After Diagnosis of Childhood Onset Epilepsy

Ability of the Brown Location Test to Differentiate Right and Left Mesial Temporal Lobe Epilepsy:

Replication in an Epilepsy Monitoring Unit Sample 


\author{
32. LOCKE, D \\ 33. MAGNUSON, S \\ 34. MAIMAN, M \\ 35. MCLAUGHLIN, R \\ 36. NAKHUTINA, L \\ 37. PRITCHETT, A \\ 38. PUENTE, AN \\ 39. RESCH, Z \\ 40. REYES, A \\ 41. RUM, RS \\ 42. SABAT, C \\ 43. STEFANATOS, AK \\ 44. STEFANATOS, G \\ 45. SUDIKOFF, EL \\ 46. WADSWORTH, H \\ 47. ZACHERY, A
}

48. BERNIER, RA

49. BRENNER, E

50. DUDA. B

51. HOSKINSON, KR

52. KRISHNAMURTHY, LC

53. LEE, BG

54. LENGU, K

55. LIGHT, SN

56. MÖLLER, MC

57. MILLER, E

58. NGUYEN-LOUIE, T

59. PETERS, AT

60. ROWLAND. J

61. SPIROU, A

62. TART-ZELVIN, A

63. TRACY, JI

64. TRACY, JI

65. TRAN, S

66. WOODARD, JL

67. WOODBURN, MA

68. WRIGHT, I

69. ZLATAR, ZZ

70. ALEKSONIS, HA

71. ARRINGTON, C

72. CastagNa, PJ

73. COBIA, DJ
Predictive Factors of Alexithymia in Epilepsy and PNES

Construct Validity of NAB Shape Learning in Predicting Mesial Temporal Lobe Seizure Lateralization

Subjective cognitive complaints on the MMPI-2-RF are not associated with cognitive performance on neuropsychological measures among a sample of patients with epilepsy

Parental Helplessness and Executive Functioning in Pediatric Epilepsy

The Relationship Between Prospective Memory Performance, Memory Complaints, and Depression in Ethnically Diverse Patients with Epilepsy

Differences in the Performance of ADHD and Epileptic Patients on Tasks of Attention and Executive Function

Can neuropsychological test scores predict Wada laterality?

Neuropsychological Changes Following Frontal Lobe Resections in Patients with Epilepsy

Reduced frontal lobe neuronal activity at rest contributes to executive function decrements in patients with temporal lobe epilepsy

Cognitive Function, Depression, and Quality of Life in Postoperative Epilepsy Patients

Differential Patterns of Memory Impairment in Temporal Lobe Epilepsy Subtypes

Cognitive and Adaptive Functioning Following Pediatric Hemispherectomy

Preservation of Neurocognitive Function in Continuous Spike and Wave During Sleep (CSWS)

Surgical Resection Effects on Quality of Life in Pediatric Epilepsy

Gender Differences in Neuropsychological Lateralization Accuracy in Temporal-Lobe Epilepsy

Epilepsy Surgery and Language Performance: The Effects of Resection Overlap with Functional Activation

\section{Imaging (Functional)}

Examining Neural Network Representation of Task and Rest Following Moderate and Severe Traumatic Brain Injury

Examining Neural Network Connectivity After Memory Training in Healthy Older Adults

Dual Compensatory Processes of Brain Activations and Deactivations Support Older Adults

Maintenance of Cognitive Function

Functional Magnetic Resonance Imaging of Social Cognition Following Pediatric Traumatic Brain Injury

Test-Retest Reliability of Seed-Based Resting State Reading Networks Differentiate Typical and Poor Readers

[F-18]FDDNP uptake, neurocognition, and number of fights in professional boxers and MMA fighters

Neural Correlates of Gaze Processing Explored with Functional Near-Infrared Spectroscopy (fNIRS) at 9 Months: Relationship to Social and Cognitive Development at 2.5 Years

Fronto-Striatal Correlates of Empathy Subtypes in Healthy Adults

Neural Activity During Vigilance Test Performance - Relation to Cognitive Fatigability in Mild

Traumatic Brain Injury.

Links Between Intrinsic Amygdala Activity and Experiences of Discrimination

fMRI-Based Prediction of Level of Response to Alcohol and Heavier Drinking in Adolescents

Neuroendocrine Modulation of Limbic Activation During Semantic List Learning in Depression Observing Dynamic Networks During the Completion of a Complex Cognitive Task

Exploration of Neuro-Corralates Between Healthy and TBI Individuals as Modulated by Depression An fMRI Study: Neural Mechanisms Associated with Rehearsal Strategy and a Working Memory Task Functional Connectivity Superior to Task FMRI Activation at Predicting Language Performance The Effects of Aging on the Lateralization of Visual-Spatial Semantic Memory in Temporal Lobe Epilepsy

Task-Residual Functional Connectivity In Language and Attention Networks

Resting State Connectivity in Brain Regions Associated with Semantic Familiarity and Knowledge:

Relationships with Neuropsychological Performance

Motor Network Connectivity Differentially Supports Fine and Gross Motor Skills During Childhood Clinical Utility of a Functional Imaging Approach to Visuospatial Memory Lateralization in Epilepsy Objectively-Measured Physical Activity and Sedentary Time and Their Aassociations With Cerebral Blood Flow and Cognition in Older Adults

\section{Imaging (Structural)}

Diffusion Tensor Imaging of the Genu of the Corpus Callosum: Associations with Day-to-Day Executive Function in Pediatric Brain Tumor Survivors

Residualized Tract and Whole Network White Matter Microstructural Integrity Negatively Correlates with Reading Measures

Parsing The Neural Correlates Of Anxious Apprehension And Anxious Arousal In Development: An Examination Of Cortical Thickness In Youth

Hippocampal Shape Features Relate to Limbic Integrity and Episodic Memory Function in

Neuropsychologically Near-Normal Schizophrenia 
74. EDWARDS, M

75. GARCIA-EGAN. PM

76. GOODRICH-HUNSAKER, NJ

77. HORTMAN, K

78. KASSEL, MT

79. MCLAREN, ME

80. PRESSON, N

81. REITER, K

82. RIZVI, B

83. ROCHETTE, AD

84. ROYE, S

85. SEMMEL, E

86. SHAKED, D

87. VELEZ LOPEZ, A

88. WHITNEY, N

89. WIER, R

90. WILLIAMS, VJ

91. CALHOUN, OC

92. CURY, MG

93. DEMETER, G

94. GARCIA, S

95. SEAGLY, KS

96. WILSON, K

4:15-4:30 PM

4:30-5:30 PM

1. HAALAND, KY
Association between network connectivity of select brain regions and cognitive abilities based on deterministic single tensor and multi-fiber diffusion MR tractography

Genetic risk alleles for inflammation and white matter integrity in older healthy adults

Developmental and sex-related changes in white matter integrity in children: tract-based spatial statistics versus deterministic and probabilistic tractography

Volume of the thalamus relates to verbal fluency performance following a major depressive episode Radial Diffusivity of Cingulo-Opercular Network Predicts Attentional Switching Performance in Healthy Aging

Association of Subthreshold Depressive Symptoms with Cortical Thickness and Surface Area of the Insula

Quantification of Diffusion Tractography for Research Application: The Control Group Matters

Cognitive reserve protects against cortical atrophy in those at genetic risk for AD

The Relationship Between White Matter Hyperintensities and Cognition and the Mediating Role of Cortical Thickness

High-Frequency Heart Rate Variability and Structural Brain Integrity in Heart Failure

Dimensions of Executive Functioning and Cortical Thickness in Younger and Older Adults

Diffusion Tensor Imaging of the Inferior Fronto-Occipital Fasciculus: White Matter Integrity and Associations with Visual-Motor Coordination in Pediatric Brain Tumor Survivors

The Relation of Socioeconomic Status and Executive Function: Is Prefrontal Cortex Volume a

Mediator?

Cardiovascular Fitness is Positively Correlated with Left Entorhinal Cortical Thickness in Healthy Young Adults

White Matter Correlates of Early Academic Proficiency in Young School-Age Children

Role of the Cingulum Bundle in Emotional and Behavioral Concerns in Pediatric Brain Tumor Survivors

Age-Dependent Associations Between Cortical Thickness and Cardiorespiratory Fitness

\section{Neurostimulation/Neuromodulation}

Impedance levels and tolerability of $2 \mathrm{~mA} H \mathrm{HD}$ tDCS in older adults

The Effects of Noninvasive Brain Stimulation on Executive Function in Binge Eating Disorder: A Pilot Study

The Effect of Subthalamic Nucleus Deep Brain Stimulation on Memory and Executive Functions in Parkinson's Disease

HD-tDCS as a Neurorehabilitation Technique for a Case of Post-Anoxic Leukoencephalopathy Pairing Brain Stimulation with Psychotherapy: A TMS Pilot Study Treating Combat Related PTSD and Co-Occurring Cognitive Symptoms

Transcranial Direct Current Stimulation (tDCS) Over Left Prefrontal Cortex Improves Visual

Detection of Words

Program Welcome

Program Committee Chair: Benjamin M. Hampstead

Carondelet

Plenary A. The Impact of the Past on Current and Future Views of Limb Apraxia INS President: Kathleen Y. Haaland

\section{Carondelet}

The Impact of the Past on Current and Future Views of Limb Apraxia 
5:30-6:30 PM

6:30-7:30 PM

7:20-8:50 AM

1. HAN. D

7:20-8:50 AM

1. CASSIDY, AR

8:00-9:15 AM

1. ALIOTO, A

2. BAILEY, KC

3. BASHEM, J

4. BEACH, J

5. BERG, J

6. BOETTCHER, A

7. BRITT, JY

8. BUPP, LL

9. BUPP, LL

10. COLVIN, MK

11. COOK, SE

12. CORRERO, AN

13. DE JONGHE, JF

14. DHILLON, S

15. DIXON, A

16. GERSHON, R

17. GODFREY, M

18. GOLDSWORTHY, R

19. GUALTIERI, A

20. GUZMAN, D

21. HALE, C
INS Awards Ceremony

Awards Committee Chair: Roy P.C. Kessels

Carondelet

Welcome Reception

Bissonet

\section{THURSDAY, FEBRUARY 2, 2017}

\section{CE Workshop 7. Financial and Health Decision Making in Old Age: Neuropsychology, Neuroimaging, and Race Considerations Presenter: Duke Han Bissonet}

Financial and Health Decision Making in Old Age: Neuropsychology, Neuroimaging, and Race Considerations

CE Workshop 8. Hearts and Minds: Recent Advances in the Neuropsychology of Pediatric Critical Congenital Heart Disease Presenter: Adam R. Cassidy Salon D

Hearts and Minds: Recent Advances in the Neuropsychology of Pediatric Critical Congenital Heart Disease

\section{Poster Session 2. Adult 1 \& Historical} Acadia

\section{Assessment/Psychometrics/Methods (Adult)}

Neuroanatomical Correlates of the TabCAT Number-Picture Match

Getting the Most out of Your Performance Validity Investment: An Examination of Five Tests among a Mixed Clinical Sample of Veterans

Performance Validity Assessment of Bona Fide and Malingered Traumatic Brain Injury Using Novel Eye-Tracking Systems

Inconsistency in Responding is Associated with Greater Self-Reported Executive Dysfunction Comparing the Electronic and Standard Versions of the Montreal Cognitive Assessment in an Outpatient Memory Disorders Clinic

The relation of self-report pain scales to neuropsychological performance using structural equation modeling

Performance on Benton Facial Recognition Test and Ekman 60 Faces Test in Veterans

Classification Accuracy of Reliable Digit Span and Reliable Digit Span Revised

Classification Accuracy of the Word Choice Test

Using the Addenbrooke's Cognitive Examination - Revised (ACE-R) to Identify the Type and Severity of Cognitive Impairment in Older Adults

Test of Premorbid Functioning Predicts Markers of Intellect in an Outpatient Clinical Sample The Brief Estimate of Seconds Test (BEST): Piloting a New Measure of Chronognosis with ADHD and Memory Disorder Samples from a VA Clinic

Does Depression Explain Poor Effort on Symptom Validity Tests (SVT)?

Predictive Capacity of Symptom Validity Tests for the Detection of Feigned Cognitive Impairment Associated with mild Traumatic Brain Injury (mTBI)

Variation in Alabama Brief Cognitive Screener Score in Amnestic and Non-Amnestic Mild Cognitive Impairment with Level of Education

Equivalence of the NIH Toolbox for Assessment of Neurological and Behavioral Function iPad version

An evidence-based evaluation of neuropsychological tests used to assess dementia among older adults with Down Syndrome

The value of future rewards: Factors that influence discounting rates on a human operant delay discounting task

Influence of Test-Retest Interval on Stability of Neuropsychological Tests in College Athletes

Construct Validity of the Tinker Toy Test

Introducing the ModRey: An Episodic Memory Test for Non-Clinical and Preclinical Populations 
22. HENDRIKS, M

23. HERSHAW. J

24. HOYMAN, LC

25. IBANEZ-CASAS. I

26. IBANEZ-CASAS, I

27. ISAAC. L

28. GOLLA, I

29. KRIVENKO, A

30. LARA-RUIZ. J

31. LAU, I

32. LAU, I

33. MANDERINO, L

34. MARRA, DE

35. MCKAY, D

36. MEAD, C

37. MERRITT, VC

38. MILLOY. A

39. NGUYEN, CM

40. OSWALD, TM

41. OTRUBA. B

42. OZINGA. G

43. PARKER, AF

44. PARKS, A

45. PATT, VM

46. PERSINGER, VC

47. POSSIN, KI

48. QUINN, C

49. RITCHIE, KA

50. SAAD, I

51. SACCÀ, F

52. SCHAEFER, LA

53. SCHREYER-HOFFMAN, G

54. SHAAFI KABIRI, N

55. SHARMA, M

56. SICARD, V

57. SLYNE, KE

58. STAFFARONI, AM

59. STEGMAN, RL

60. STUBBS, WJ

61. TAM, DM

62. TANG, R

63. THOMPSON, LI
A Causal Modeling Approach of WAIS-IV Profiles of Patients With Temporal Lobe Epilepsy, Psychiatric Patients and Matched Controles

Predicting Invalid Responses Using Pupil Diameter During a Cued Attention Task

The Impact of PTSD on Verbal Learning and Memory using the CVLT-II

Protocol for the Development of a Domain Specific Computerized Battery for Cross-Cultural Neurocognitive Assessment: The EMBRACED Project

Word Lists Development for Cross-Cultural Verbal Memory Assessment in a Computerized Neuropsychological Battery: The EMBRACED Project

An Evaluation of Premorbid Intelligence Estimates: BEST and WIAT in a Young, Diverse Population Predictive Value of ECog Total Score and Informant Characteristics on Cognitive Impairment Relationship between the five-point test and a self-report measure of executive functioning in a random sample of English speaking adults

The impact of PTSD symptoms and cognitive performance on student veterans' academic achievement

Association between Performance Validity and Symptom Validity Tests with the MMPI-2-RF among Simulators Feigning Neuropsychological Dysfunction

Classification Accuracy of Performance Validity and Symptom Validity Tests among Examinees Feigning Neuropsychological Dysfunction

Immediate Post-Concussion Assessment and Cognitive Testing Validity Indices Are Correlated with External Performance Validity Measures

An Updated Exploration of the Frequency of Invalid Performance in an Undergraduate Sample

The Relationship between Performance Validity Tests and Diagnoses among Veterans with History of mTBI

Validation of the TabCAT Favorites Memory Test: Neuroanatomical and Memory Correlates in Neurologically Healthy Older Adults

Evaluating the Test-Retest Reliability of Symptom Indices Associated with the Post-Concussion Symptom Scale

Incremental Validity of MoCA Subscale Scores in Amnestic MCI and Alzheimer's Disease

Vascular medical factors associated with health and safety behaviors among older male Veterans residents admitted to an inpatient rehabilitation unit

Embedded Performance Validity Index within the Memory Module of the Neuropsvchological Assessment Battery (NAB): A Pilot Study

The Predictive Value of Verbal Fluency and Story Memory upon Story Recall Using the Repeatable Battery for Assessment of Neuropsychological Status in an Inpatient Setting

Statistical Adaptation of the Boston Naming Test Short Form for Hebrew Speaking Older Adults The New Normal: Creating Updated Norms for Neuropsychological Measures

Clinical Utility of the Visual Object and Spatial Perception Incomplete Letters Test

Digit Vigilance Test: Speed versus Accuracy Tradeoff Revealed

California Verbal Learning Test-II Total Hits and Total False Positives as Embedded Performance

Validity Measures in Mood Disorders

The TabCAT Brain Health Assessment for Detecting Mild Cognitive Impairment and Evaluating Domain-Specific Deficits

Association of Judgment Ability and Functional Status in a Sample of Mixed Rehabilitation Inpatients

An empirical examination of impaired baseline performances on the Standardized Assessment of Concussion

An Update on Normative Data for Neuropsychological Performance on Memory and Language

Measures in a Racially Diverse Older Adult Longitudinal Cohort

Normalization of Timed Neuropsychological Tests With the use of the PATA Rate and Nine-Hole Pegboard Tests

Is the Montreal Cognitive Assessment (MoCA) Related to Functional Outcome and Discharge Destination in a Geriatric Stroke Rehabilitation Population?

Effects of RBANS Demographic Adjustments with a Culturally Diverse Urban Elderly Sample Continuous Performance Test-Identical Pairs (CPT-IP): Characterization and Relation to Other Attention and Working Memory Tasks

Engagement and Motivation: Conventional Paper and Pen Neuropsychological Testing and Virtual Reality

Construct Validity of a Color-Shape Switch Task

Four Year Test-Retest Reliability of the RBANS and Executive Functioning Measures in Healthy Elderly

Four- and Five-Factor Models of WAIS-IV Interpretation in a Clinical Sample

Percentage of Low Scores is a Useful Embedded Measure

Concurrent Validity of the ADHD Symptom Questionnaire Inattention Subscale (ASQ-I): A Pilot Study of Performance on CPT-II and D-KEFS TMT Indices

Survey of Neuropsychologists' Practices and Perspectives Regarding the Utilization of Technicians The Impact of Language and Education on Non-verbal Neuropsychological Measures Cards, Balloons, or Dice? A Comparison of Risky Decision-Making Tasks and Their Associations With Working Memory, Anxiety, Depression, and Risk Taking 


\author{
64. TRIFILIO, E \\ 65. UPSHAW, J \\ 66. VAUGHN, DW \\ 67. VO. TT \\ 68. WALLS, BD \\ 69. WHEELER, JM \\ 70. YOCHIM, B \\ 71. ZAHEED, AB
}

72. BALL, $\mathrm{K}$

73. FORD, AI

74. HAHN-KETTER, AE

75. KIRMSE, R

76. MAIMAN, M

77. MAIMAN, M

78. VARGO, TL

79. AGHVINIAN, M

80. ARCE RENTERIA. M

81. BELTRAN, J

82. FAYTELL, MP

83. FERNANDEZ-GONZALO, S

84. HARDCASTLE, C

85. HUNTER, SJ

86. HUNTER, SJ

87. IUDICELLO, JE

88. JÓDAR, M

89. JONES, J

90. HESTAD, K

91. KEUTMANN, M

92. KORDOVSKI, VM

93. LEVINE, A

94. POSADA-SHEA. C

95. TIERNEY, SM

96. TRAINO, KA

9:00-10:30 АM

1. BIKSON, M
A Tale of Two Stories: Comparing Logical Memory and Newcomer Stories

Allocentric vs. Egocentric Neglect in Stroke Patients: Assessment Through Eye-tracking and Impacts on Functional Outcomes

Blunt(ed) performance: Examiner judgments of cannabis user status predicts neuropsychological outcomes

Promoting Healthy Cognitive Aging: Development and Psychometric Properties of the Healthy Brain Aging Activity Engagement Questionnaire

Utility of the CAARS Validity Scales in Identifying Feigned ADHD, Random Responding, and Genuine ADHD in a College Sample

The Role of Spoken Language and Verbal Mediation in Performance on the Serial Digit Learning-8 Test

Validity of the Verbal Naming Test using a 5-second response time limit

The Chicago Alternative Stroop Test: Developing a Clinical Measure of Incidental Learning

\section{Historical}

The Evolution of Autism Spectrum Disorder: From Severe Psychopathology to the Executive Dysfunction Hypothesis

From Morosis to Neurocognitive Disorders: A History of Dementia and its Neuropsychological Assessment

Future Directions of Neuropsychology from a Training Perspective: Factors Affecting Training Satisfaction from the AACN Student Affairs Committee Survey of Neuropsychology Trainees

Historical Approaches to Neuropsychological Assessment, Theoretical Models, Current Practices and Clinical Applications: An Exploration of Luria and Halstead Theories

Exploring The Relationship between EF Performance Based-Measures and Questionnaires in Neurodevelopmental Disorders (NDDs) Associated with Intellectual Disability (ID)

The History of Assessment in Individuals with Intellectual Disability: Problems. Solutions. Future Directions

Byron P. Rourke: Contributions to the Field of Pediatric Neuropsychology

\section{HIV/AIDS/Infectious Disease}

Quality of healthcare is associated with antiretroviral regimen neuropenetrance and neuropsychological outcomes among HIV+ adults

Characterization of Neurocognitive Intra-Individual Variability Among HIV+ Adults With and

Without Current Cocaine Use

Time-Based Prospective Memory Deficits Are Uniquely Associated with Medication Management Errors in Older Adults Living with HIV

Investigation of the Interrelationships Between Fatigue, Memory Impairment, and Antiretroviral Adherence in HIV Disease

Quality of life and emotional state improve after hepatitis C curative therapy with direct antiviral agents.

Cognitive and Motor Functioning in Older Adults with HIV : A Comparison with Parkinson's Disease Confirmatory Factor Analysis of the Behavioral Rating Inventory of Executive Functioning (BRIEF) in Young Black Men who have Sex with Men (YBMSM)

EF, Frequency of Marijuana Use, and HIV Risk Reduction in Young Black Men who have Sex with Men (YBMSM)

Profiles of HIV-Associated Neurocognitive Impairment in the Context of Low and High Blood-Brain Barrier (BBB) Permeability

Cognitive function improvement in HIV-HCV co-infected Patients after treatment for HCV with direct antiviral agents (DAA).

Apathy is Related to Quality of Life in HIV-infected Adults

HIV and AIDS Associated Neurocognitive Functioning In Zambia- A Gender Perspective Sex and HIV Serostatus Differences in Executive Mechanisms of Verb Fluency Among Drug Users Frequency and Correlates of Low Health Literacy in HIV-Associated Neurocognitive Disorder Suboptimal effort in HIV neuropsychological research studies: does it influence estimated prevalence rates of $\mathrm{HIV}$-associated neurocognitive disorders (HAND)?

Learning and recall of emotionally-laden words among individuals living with HIV and bipolar disorder

Semantic Memory in HIV-Associated Neurocognitive Disorders: An Evaluation of the 'Cortical' vs. 'Subcortical' Hypothesis

Neuropsychological functioning of HIV-negative Cryptococcus meningoencephalitis survivors

\section{Invited Symposium 1. Electrical Brain Stimulation and Cognitive Disorders Chair: Marom Bikson Carondelet}

Electrical Brain Stimulation and Cognitive Disorders 
2. BIKSON, M

3. WOODS, AJ

4. CHARVET, LE

9:00-10:30 AM

1. FERNANDEZ, AL

2. FERRERES, A

3. JUDD, T

4. GROTE, C

5. MANLY, JJ

9:00-10:30 AM

1. LAMAR, M

2. LIBON, DJ

3. $\mathrm{AU}, \mathrm{R}$

4. LAMAR, M

5. KARSTENS, A

6. DIAZ-ORUETA, U

9:00-10:30 AM

1. BLANKEN, AE

2. NATION, DA

3. LOBUE, $\mathrm{C}$

4. YEW, B

5. GLENN, MA

6. MCINTOSH, EC

9:00-10:30 AM

1. TRITTSCHUH, E

2. MISKEY, HM

3. JAK, AJ

4. SCOTT. J

5. WILLIAMSON, JB

6. LERITZ, E
The Basics of tDCS: Technology and Mechanisms

Combating Cognitive Aging and Dementia with Transcranial Direct Current Stimulation (tDCS) At-Home Access: Extending Clinical Trials of Cognitive Remediation and tDCS through Remote Supervision

\section{Symposium 1. Neuropsychology in the Americas Chair: Alberto L. Fernandez \\ Bissonet}

Neuropsychology in the Americas

Neuropsychology in South America

The Past, Present, and Future Development of Neuropsychology in Central America

Neuropsychology in the United States and Canada

Collaboration of Neuropsychologists in the Americas can Accelerate Progress in Research and

Practice

Symposium 2. An integrative look at the Boston Process Approach to Neuropsychology: A review of the history, current research and future directions of error analysis

Chair: Melissa Lamar

Salon D

An integrative look at the Boston Process Approach to Neuropsychology: A review of the history, current research and future directions of error analysis

The history of the Boston Process Approach and the role of Edith Kaplan

Incorporating the Boston Process Approach into Cognitive Assessment in the Digital Era:

Framingham e-Cognitive Health Initiative

Multi-method integration of human connectomics with the Boston Process Approach to neuropsychological assessment

Adapting Boston Process Approach algorithms used in dementia research to a normal aging population

The E-SPACE Project: An international expansion of the Boston Process Approach to incorporate error analysis of behaviour

\section{Paper Session 1. Risk \& Alzheimer's Moderator: Felicia C. Goldstein Salon E}

Longitudinal neurocognitive profiling of empirically-derived Alzheimer's disease variants Independent and Interactive Effects of Cerebral Amyloid and Tau on Neuropsychological Decline and Structural Brain Changes in Older Adults

Traumatic Brain Injury History and Progression from Mild Cognitive Impairment to Alzheimer Disease

Elevated Cerebrovascular Resistance Predicts Increased Amyloidosis, Cerebral Atrophy, and Cognitive Decline

Primacy Effects in Cognitively Normal Older Adults with Alzheimer's Disease Pathology

Untreated Diabetes Associated with Tau Pathology and Worse Cognitive Performance in Older Adults

\section{Paper Session 2. Veterans' Health \\ Moderator: Amy J. Jak \\ Salon F-H}

PTSD + Aging: Psychoeducational Intervention to Promote Self-Efficacy and Healthy Brain Behaviors in Older Veterans with Cognitive Concerns

Cognitive Performance of Veterans in an Inpatient Posttraumatic Stress Disorder Program Pre- and Post-Treatment

Neuropsychological outcomes following hybrid treatment for Veterans with comorbid TBI and PTSD Verbal Memory Functioning Moderates Psychotherapy Treatment Response in PTSD

Lateralized limbic white matter integrity is associated with PTSD symptoms

TBI and PTSD Moderate the Relationship Between Blood Pressure and Cortical Thickness in OEF/

OIF/OND Veterans 
9:30-10:45 AM

1. ALVAREZ, G

2. ANTONINI. T

3. ARCHAMBAULT, $\mathbb{W}$

4. BAILEY, CM

5. BAUGHMAN, BC

6. BIEKMAN, B

7. BURNS, AR

8. COHEN, ML

9. COHEN, P

10. DANGUECAN, AN

11. DUNCAN, B

12. FABER, J

13. FAY-MCCLYMONT, TB

14. HIRST, E

15. HOLLAND, A

16. HOWARTH, RA

17. IWAMOTO, BK

18. JASHAR, D

19. KONTOS, AP

20. KOWALSKI, K

21. KOWALSKI, K

22. KWAN, V

23. LALIBERTE DURISH, C

24. LALIBERTE DURISH, C

25. LALONDE, G

26. LALONDE, G

27. LEBLOND, E

28. LENIHAN, J

29. LINDSEY, HM

30. LIU, JR

31. LORTIE, J

32. LOVE, CE

33. MARINO, C

34. MAXWELL, EC

35. MIETCHEN, JJ

36. MORRIS, S

37. NARAD, M

38. RAMIREZ FLORES, MJ

39. SADY, MD

\section{Poster Session 3. Peds 1 Acadia}

Acquired Brain Injury (TBI/Cerebrovascular Injury \& Disease - Child)

Clinical Features of Young Children with Disorders of Consciousness

Changes in Parenting Skills Following Participation in Two Versions of an Online Parenting Program Designed for Families of Young Children with TBI

Differences in the Psycho-Affective Profile and ADHD Index Scales in Adolescent Hockey Players with a History of Concussions

Pre-Injury Child-SCAT3 Performance in a Youth Sports Sample

The Relationship Between Multi-modal Neuropsychological Measures and Head Impact Telemetry Over the Course of a Single High School Soccer Season

Frontal Lobe Volume and Thickness Differences Between Children With Traumatic Brain Injury and Children With Orthopedic Injury

Changes in Executive Functioning Following Concussion: Comparisons Between ADHD, Learning

Disability, and Typically Developing Children and Adolescents

Parent Ratings of Language Problems After Pediatric Traumatic Brain Injury

Do Initial Symptom Factor Scores Predict Subsequent Impairment following Concussion?

Stability of overall intellectual functioning into early school-age for children with neonatal arterial ischemic stroke

A Comparison of Performance Validity on the CNS Vital Signs (CNSVS) Validity Indicators and

Green's Medical Symptom Validity Test (MSVT) in a Sample of Pediatric Concussion Patients

Executive Functioning and Diffusion Tensor Imaging in TBI

Neuropsychological, behavioural, and quality of life outcomes in a cohort of children with autoimmune encephalopathies

Epidemiology of Sport and Non-Sport-Related Concussion in Adolescents

Predicting Long-Term Cognitive Outcomes of Pediatric Stroke According to Stroke Severity Classification

Functional Outcomes for Pediatric Patients Diagnosed with Anti-NMDA Receptor Encephalitis during Inpatient Rehabilitation

Recovery of Memory Following Pediatric TBI: The Impact of Coma

Retrieval Differences Following Concussion

Comparison of Adolescents with Vestibular and Anxiety Clinical Profiles following Concussion

Are Pre-Injury Sleep Behavior and Acute Post-Concussive Symptoms Predictive of Post-Injury Sleep

Behavior in Children with Mild Traumatic Brain Injury or Orthopedic Injury?

The Relationship Between Pre-Injury Sleep and Acute Post-Concussive Symptoms in Children with

Mild Traumatic Brain Injury and Orthopedic Injury

The Relationship Between Pain and Post-Concussive Symptoms: Comparing Children with Mild

Traumatic Brain Injury to Orthopedic Injury

Persistent Effects of Pediatric Mild Traumatic Brain Injury: The Role of Resilience

The Role of Psychological Resilience in Children with Poor Recovery Following Mild Traumatic Brain Injury

Executive Functions After Mild Traumatic Brain Injury in Preschool Children

Should children with TBI be compared to orthopedic or healthy controls?

Developing an Intervention to Promote Quality of Life in Pediatric Brain Tumor Survivors

Recovery of Attention Deficits following Pediatric TBI: The Impact of Premorbid School Performance

Effects of Injury Severity on White Matter Tract Integrity in Relation to Verbal Memory in Chronic

Pediatric Traumatic Brain Injury

Age-Dependent Association Between Post-Concussional Children and Adolescents and Balance

Performance in Neuropsychological Testing

Assessment Of Executive Functions Using A Colour-Shape Switch-Task In Elite Adolescent Hockey

Players With And Without A History Of Concussion

Parent and Self-Report of Executive Functioning After Moderate to Severe TBI

Self-Awareness of Psychosocial Functioning and Executive Functions Following Moderate and Severe

Pediatric Traumatic Brain Injury

Predictors of Adaptive Functioning Following Childhood Arterial Ischemic Stroke

White Matter Integrity and Neuroanatomical Correlates of CVLT-C Factors in Children with

Traumatic Brain Injury

Effects of Developmental Age on Symptom Reporting and Neurocognitive Performance in Youth after Sports-Related Concussion

Examination of the Impact of a Web-Based Counselor Assisted Problem Solving Intervention (CAPS) on Teen Self-Report Behavior Rating Inventory of Executive Function (BRIEF) Following Pediatric TBI

Theory of mind in children with mild and moderate traumatic brain injury during acute stage Comparison of Symptom Severity and Parent-Child Agreement between Three Causes of Adolescent Concussion: Sports, Motor Vehicle Collision, and Assault 


\section{SANDEL, N \\ 41. SCHMID, AD \\ 42. SCHMID, A \\ 43. SHISHIDO, Y \\ 44. SMITH-PAINE, J \\ 45. SUFRINKO, AM \\ 46. SVINGOS, AM \\ 47. VAUGHAN, CG \\ 48. WILMOTH, K \\ 49. WRIGHT, KL}

50. ABRAMS D

51. AMMONS, C

52. BEDNARZ, H

53. BERGER, NI

54. BERTOLIN, M

55. BRADBURY, KR

56. CASTELLUCGIO, B

57. CHEN, J

58. CHO, I

59. DOLAN, B

60. GAO, Y

61. GREEN, R

62. HERRINGSHAW, AJ

63. JENNINGS, K

64. LANDRY, A

65. LANDRY, A

66. LEE, CM

67. LESSER. R

68. MASSA. J

69. MOULTON, E

70. NAIR, A

71. NAKAGAWA, Y

72. PETERS, AT

73. SHADA, K

74. SHIELDS, BJ

\section{BEARDEN, DJ \\ 76. BRENNER, LA \\ 77. CLEM, M \\ 78. COLVIN, MK \\ 79. GIOIA, A \\ 80. GLASS, L}

Discrimination of Concussed Athletes from Healthy Controls Using a Multimodal Diagnostic Approach

Acceptability of a Return-to-Learn Program for Concussed High School Students

Validation of a Teacher Questionnaire to Assess Concussed Students' Recovery

Cognitive Recovery of Pediatric Patients With Moderate to Severe TBI During Inpatient

Rehabilitation Using the Cognitive \& Linguistic Scale (CALS)

The Moderating Effect of Dopamine Genes on the Association of Family Environment with Longitudinal Executive Function Following Traumatic Brain Injury in Early Childhood: A

Preliminary Study.

Additional Sport Exposure Following Concussion has Dose Response Effect on Recovery Time

Parent-Child Interaction Therapy (PCIT) Reduces Sleep Problems Following Pediatric Traumatic

Brain Injury

The Relation Between Multiple Prior Concussions and Injury Severity

Delayed Clinical Evaluation Following Concussion Predicts Prolonged Return to Play in Female Student-Athletes

Relationship Between Processing Speed with White Matter Hyperintensity Volumes and Injury Severity

\section{Autism Spectrum Disorders}

Early ASD Symptoms, Demographic Characteristics, and Adaptive Skills Predict Change in Cognitive and Language Abilities in Toddlers with ASD

Age and Diagnosis Effects on Mid Fusiform Sulcus Anatomy in Autism Spectrum Disorder Changes in Reading Comprehension as a Result of Language Intervention in Children with Autism Spectrum Disorders

Evaluating the Neural Correlates of Intention Understanding in Autism Spectrum Disorder

Effects of Social Skills Training on Emotional Face Processing in Adolescents With Autism Spectrum Disorder: Behavioral and Electrophysiological Correlates

M-CHAT-R/F Performance in High-Risk Infant Siblings

Verbal Inferential Reasoning in Autism Spectrum Disorder

Differences in Early Temperament Between High Risk Baby Siblings With and Without Autism:

Analyses by Gender

Frontostriatal Structural Connectivity in Autism Spectrum Disorder

Examining the Durability of PEERS for Adolescents With ASD: Maintenance of Neurological and Behavioral Effects

Behavioral and Neural Substrates of Language Delay in Children with Autism Spectrum Disorder

Beery VMI and Structural Volume Correlates in Autism Spectrum Disorder

Neural Correlates of Social Perception in Children with Autism: Local versus Global Preferences

Conversational Speech in Autism: Behavioral, Emotional, and Neuropsychological Viewpoints

Neurocognitive and Adaptive Functioning in Children with Autism Spectrum Disorder and Comorbid Attention Problems

Virtual Reality as an Assessment Modality with Pediatric ASD populations: A Systematic Review Inter-limb Transfer of Kinematic Adaptation in Children with Autism Spectrum Disorder Evolutions in Diagnostic Criteria and Implications: A case study

The Effect of Social Story Training on Social Skills of Cypriot Children with Autism Spectrum Disorder

Change in ASD Symptom Severity Between Ages Two and Four

Altered thalamocortical connectivity in the first-year of life correlates with early social difficulties in high-risk siblings of children with autism

Grammatical Difficulties for Adults with ASD and ADHD

Giant Congenital Melanocytic Nevus in a Two-Year-Old Female with Autism Spectrum Disorder: A

Case Report of Neurocognitive Functioning and Review of Neurological Implications

Examining executive functioning in ASD with or without comorbid ADHD, using the BRIEF

The Relation Between Executive Functioning and Adaptive Skills in Youth with Autism Spectrum Disorder, Level 1

\section{Medical/Neurological Disorders/Other (Child)}

Pain and Cognitive and School Function in Children and Adolescents with Functional Abdominal Pain

Is There a Broader Dyspraxia Phenotype? A Clinical Case Series Analysis

Genotype, Auditory Attention, and Educational Outcomes in Pediatric Acute Lymphoblastic Leukemia

Cognitive and Socio-Emotional Functioning in Children and Adolescents with PANDAS (Pediatric Autoimmune Neuropsychiatric Disorders Associated with Streptococcal Infections)

Feasibility and Validity of a Computerized Cognitive Assessment in Pediatric Patients with Sickle Cell Disease

Characterizing Reading Performance in Elementary School-Age Children with Prenatal Alcohol Exposure 


\author{
81. HAWKS, Z \\ 82. HORTON, DK \\ 83. KENNEDY, T \\ 84. KLIPFEL, K \\ 85. LEPPO, RH \\ 86. MACMULLEN FREEMAN, L \\ 87. OLSON, K \\ 88. POMMY, J \\ 89. RAZ. S \\ 90. RAZ, S \\ 91. RAZ, S \\ 92. RAZ S \\ 93. RIEGER, RE \\ 94. SALAMA. CH \\ 95. BALL, K \\ 96. SCHREIBER, JE \\ 97. SIEBENMORGEN. M \\ 98. TARAZI, R \\ 99. WEGELE, A
}

10:30-10:45 АM

10:45-11:45 АM

1. KNIGHT, RT

11:45 АM-12:45 РМ

12:45-1:45 PM

1. ROSENBAUM, $\mathrm{R}$

12:45-2:15 PM

1. HERMANN, B

2. OJEMANN. J

3. HAMBERGER, MJ

4. DRANE, DL

5. MCDONALD, C
Baseline Cognitive and Neural Differences Distinguish $\mathrm{BH}_{4}$ Responders from Non-Responders Associations Between Age at Diagnosis and Adaptive Skills in Children with Anti-N-Methyl-DAspartate (NMDA) Receptor Encephalitis

Validity of the Kiddie-Sluggish Cognitive Tempo Measure in Children with Sickle Cell Disease Neuropsychological Sequelae of an Internationally Adopted Child

Connecting The Dots: Fine Motor Skills, Executive Functioning Skills, and Math Achievement in Patients with Hearing Loss

Cognitive Functioning in Children with Hypothalamic Hamartoma

Parent-Reported Social and Executive Functions in Children and Adolescents with NF1

Smaller Cortical Volumes in 3.5-to-5 Year-Old Children Born Preterm

Associations of Maternal Intellectual and Socioeconomic Factors with Neuropsychological Outcome of Preterm-Born Preschoolers

Does Motor Development Explain a Unique Portion of the Variance in Cognitive or Language Abilities in Preterm-Born Preschoolers?

Hypoglycemia and Language, Motor, and Cognitive Abilities in Preterm-Born Preschoolers Maternal Hypothyroidism and Neuropsychological Functioning of Preschool Age Children Born Prematurely

Smaller Subcortical Volumes in Preschoolers Born Preterm: A Voxel-Based Morphometry (VBM) Study

The Relationship between the Neurological Predictor Scale and Functional Outcomes of Children with Brain Tumor following Inpatient Rehabilitation

Comprehensive Neuropsychological Testing in a Young Child with Neurofibromatosis Type 1: A Case Study

Cognitive function in preschool-age children with sickle cell disease: associations with home environment and disease-severity

Congenital Heart Disease among Adolescents and Young Adults: Executive Control and Adaptive Functioning

Hydroxyurea Status is Associated with Cognitive Function in Young Children with Sickle Cell Disease Cognition in Children and Adolescents with Myotonic Dystrophy Type 1

AM Coffee Break

Acadia Ballroom

Plenary B. Frontal Cortex and Human Behavior: Evidence from

Intracranial Recording

Presenter: Robert T. Knight

Carondelet

Frontal Cortex and Human Behavior: Evidence from Intracranial Recording

\section{Lunch (On Own)}

Conference-Wide

Early Career Awardee Presentation: A Case Study Approach to Understanding Memory

Award Recipient: R. Shayna Rosenbaum

Salon F-H

A Case Study Approach to Understanding Memory

\section{Invited Symposium 2. Evolution of the Neuropsychology of Epilepsy Surgery \\ Chair: Bruce Hermann \\ Carondelet}

Evolution of the Neuropsychology of Epilepsy Surgery

On the Evolution of Neurosurgery and Neuropsychology in Epilepsy: Epilepsy Surgery

On the Evolution of Neurosurgery and Neuropsychology in Epilepsy: Language

On the Evolution of Neurosurgery and Neuropsychology in Epilepsy: Memory

On the Evolution of Neurosurgery and Neuropsychology in Epilepsy: Executive Functions 
12:45-2:15 PM

12:45-2:15 PM

1. NOSARTI, C

2. COUNSELL, $\mathrm{S}$

3. BOARDMAN, JP

4. GEVA, R

5. CHRISTENSEN LØHAUGEN, C

6. NOSARTI, C

INS Student Liaison Committee Workshop: The Neuropsychologist in the Public Domain: Kids, Academia, and the Law Presenters: Robert T. Knight, Kathleen Y. Haaland, Erin D. Bigler, Donna J. Sorensen

Salon A-C

\section{Symposium 3. Brain and Cognition Following Very Preterm Birth} Chair: Chiara Nosarti

Bissonet

Brain and Cognition Following Very Preterm Birth

Preterm Birth is Associated with Impaired Development of Brain Structural Connectivity Relevant to High Order Cognitive Functions

A Latent Measure Explains Substantial Variance in White Matter Microstructure Across the Newborn Human Brain

Electrophysiological Changes and Attention Correlates Following Preterm Birth

G Born Preterm with Very Low Birth Weight (VLBW) - Never Ending Cognitive Consequences?

Dysconnectivity of Visuospatial Attention Network at Rest and Emotion Recognition in Very-Preterm Born Adults

12:45-2:15 PM

1. DE HAAN, E

2. KARR, JE

3. BOTT, NT

4. HAYES, SM

5. WRIGHT, MJ

6. OBERLIN, LE

12:45-2:15 PM

1. CHEUNG, Y

2. LIU, W

3. BANERJEE, P

4. BRINKMAN, TM

5. FOX, ME

6. WEFEL, JS

1:15-2:30 PM

1. ALIOTO, A

2. BELSER-EHRLICH, J

3. BERNSTEIN, J

4. CAMPBELL, LM

5. CASALETTO, KB

6. CHEN, M

\section{Paper Session 3. Cognitive Neuroscience \\ Moderator: Bruce Crosson Salon D}

Returning into the Footsteps of Berlucchi \& Aglioti: Many Bodies in the Brain. A Review The Dimensionality of Executive Function throughout Adulthood: A Systematic Review and Reanalysis of Latent Variable Studies

Allocentric and Egocentric-based Navigation Learning: Neuroanatomical Correlates fMRI activity during associative encoding is correlated with cardiorespiratory fitness and source memory performance among older adults

The Crucial Role of Cognitive Reserve in the Effect of tDCS on Memory for Individuals With and Without Traumatic Brain Injury in a Simulated Work Environment

The Neural Correlates of Cognitive Dysfunction in Obesity: an fMRI Study

\section{Paper Session 4. Oncology \\ Moderator: Jeffrey S. Wefei \\ Salon E}

Chronic Pulmonary Conditions and Neurocognitive Function in Long-Term Survivors of Childhood Hodgkin Lymphoma

Evolution of Neurocognitive Function in Long-term Survivors of Childhood Acute Lymphoblastic Leukemia Treated with Chemotherapy Only

Visuoconstruction Organizational Strategy and Neuroimaging Outcomes in Long-Term Survivors of Childhood Acute Lymphoblastic Leukemia Treated with Chemotherapy

Genome-Wide Association Study of Attention Problems and Executive Dysfunction in Sdult Survivors of Childhood Leukemia

Attention and Functional Connectivity in Survivors of Childhood Brain Tumors

Validity and Diagnostic Accuracy of the Clinical Trial Battery in Patients with Primary Brain Tumor

\section{Poster Session 4. Aging \& Dementia 1 Acadia}

\section{Aging}

A Longitudinal Study of Cardiorespiratory Fitness, White Matter Integrity, and Cognitive Function in Healthy Older Adults

Exploring Health-Related Quality of Life and Cognitive Functioning in Aging: A Formal Test of the Wilson \& Cleary Model

Examining Cognitive Correlates of Sleep Quality, Daytime Sleepiness, and Insomnia in a Cognitively Healthy Older Adult Sample

Relationship between Cerebral Blood Flow and Famous Face Naming in Cognitively Normal Older Adults

Leisure Activity Participation is Associated with Neuroanatomic Structure in Healthy Aging Adults Neural Correlates of Obstacle Negotiation in Older Adults: An fNIRS Study 
7. CHEY, J

8. CHEY, J

9. CHEY, J

10. CHEY, J

11. CHOI, A

12. COHEN, J

13. DASH, T

14. DEKHTYAR, M

15. DENNY, K

16. DION. C

17. DIVERS, R

18. DUGGER, AJ

19. GARCIA, A

20. GARCIA, A

21. GOGNIAT, M

22. GONZALEZ. I

23. GRACIAN, EI

24. GRAVES, L

25. GRIFFIN, JW

26. GROSS, EZ

27. HAYS, CC

28. HAYS, CC

29. HO, JK

30. JONES, R

31. KAUP, AR

32. KAUR, S

33. KIM. Y

34. KINSELLA, GJ

35. LEAL. C

36. LEAL, G

37. LEVY, S

38. LIEBEL, SW

39. MACE, LC

40. MAYE, JE

41. MELTZER, EP

42. MORIN, R

43. MOSELEY, SA

44. PILLEMER, $\mathrm{S}$

45. POTTER, G

46. RHODES, E

47. RITCHIE, H

48. ROBBINS, R

49. ROGERS, S

50. ROTBLATT, LJ

51. RYCROFT, SS

52. SANDERS, CL

53. SCHMITTER-EDGECOMBE, M
Aging-Associated Reduction in Regional Brain Metabolism, Memory Decline and Education in a 4-year Follow-up Study of Community-Residing Elderly People

Association between the Thickness of the Left Entorhinal Cortex and the Trend in Cohesiveness of Individual's Social Network in a Longitudinal Study of Community-residing Elderly People In-bound but not Out-bound Social Connection Moderated Age-related Brain Aging in Communityresiding Elderly People

Social Network Size Moderates the Relationship between Age and Long-term Memory Score in Community-residing Elderly People over 80 - WITHDRAWN

Preoperative Cognitive Contributions to Cerebral Oximetry Change in Older Adults During Total Knee Arthroplasty

Physical Activity and Processing Speed Across the Lifespan

Dual control mechanism in conflict management for the monolinguals and bilinguals: An fMRI study Imaging, Lifestyle and Demographic Differences in Optimal Executive Function Performers

Long-term change associated with a multi-modal intervention to enhance cognitive compensation strategies and promote brain health activities

Associations of Sedentary Behavior as well as Physical Activity with Learning, Memory, and Hippocampal Volume in a Diverse Sample of Older Adults

When and How Did You Go Wrong? Characterizing Micro-errors in Older Adults

Manual and Computerized Trail Making Test Performance across the Lifespan

Abstract and Concrete Word Processing in the Aging Brain

The Relationship between MoCA Cut-Scores and Brain Volume

The Relationship Between BMI and White Matter Volume in Older Adults

Acculturation as an Important Factor in Neuropsychological Performance in patients with

Alzheimer's Disease among Hispanic Elderly

Transverse Patterning Performance is Not Uniform in Cognitively Normal Older Adults

Modifications to the CVLT-II Novel Recognition Discriminability Measure to Enhance the Detection of Memory Decline in Normal Aging

The Effects of Age on Clustering Strategy During List Acquisition

Poor Financial Decisional Ability is Associated with Elder Financial Exploitation and is Exacerbated among Older Adults with Higher Frequencies of Low Neuropsychological Test Scores

Relationship Between Pulse Wave Velocity, Cerebral Blood Flow, and Memory in Cognitively Normal Older Adults

Subjective Cognitive Decline Modifies the Relationship Between Cerebral Blood Flow and Memory

Function in Cognitively Normal Older Adults

Memory is Preserved in Older Adults Taking AT1-Receptor Blockers

Predictors and the Moderating Effect of General Health and Neuropsychological Performance

Occupational Cognitive Complexity is Associated with Brain Structure and Cognitive Health in MidLife: The CARDIA Study

Inflammation mediates the relationship between metabolic (cholesterol) risk and neuronal viability in middle aged adults

Emotional Information Processing in Older Adults with Depression: Attention and Memory Biases

Memory Groups for Older People: Who Gains? - WITHDRAWN

The Relationship Between Brain Atrophy and Cognitive Performance in Dementia Populations

The Relationship Between White Matter Hyperintensities and Cognitive Performance in Dementia Populations

Mechanisms of Racial Disparities in Cognitive Aging: Use of Causal Mediation in Neuropsychology Research

Executive Functioning Ability is Better Predicted by Cognitive Processing Speed than by White

Matter Hyperintensities or Age

The Serial Position Effect and Hippocampal Asymmetry in Cognitively Normal Older Adults Dispositional Mindfulness as a Predictor of Verbal Memory Performance in Older Adulthood

Emotion Regulation in Relation to Aging and the Preclinical Stages of Dementia

Depression and Cognitive Functioning Among Older Adults with Cancer

Cognitive and Psychosocial Associations of Hearing Loss in Older Adults

The Effect of Perceived Social Support on Cognitive Function Among Older Adults

Physical Frailty and Cognitive Impairment in Late-Life Depression

Grit and Successful Aging in Older Adults

Cognitive and Emotional Associations of Dispositional Mindfulness in Older Adults

Relation Between Social Interaction and Cognitive Functioning in Older Adults: A Feasibility Study Using the EAR Technology

Cognition and Older Adults' Agreeableness: Is There a Relationship?

Effects of Hypertension and its Pharmacological Treatment in the ACTIVE Study

Effects of Age and Task Goal on Naturalistic Visual Behaviors

Risk of Cardiovascular Disease and Cognitive Status in Middle-aged Adults: The Gray Matters Study

Multiple Types of Memory and Everyday Functional Assessment in Community Dwelling Older Adults 


\section{SCHOEN, C \\ 55. SEIDER. T \\ 56. SOTO-AÑARI, M \\ 57. STABLER, AR \\ 58. STRAINGE, L \\ 59. VERNON, EK \\ 60. WALZAK, LC \\ 61. WASSERMAN, VJ \\ 62. WASSERMAN, VJ \\ 63. WEAKLEY, A}
64. ALVAREZ, E
65. CHANEY, G
66. CHERAN, G
67. FOSS, MP
68. PUTCHA, D
69. WYMAN-CHICK, KA

70. BEREZUK. C

71. BEREZUK, C

72. CAMBRONERO, FE

73. CROOK, CL

74. DAVIS, K

75. DENNEY, DA

76. DEVLIN, KN

77. DIAZ SANTOS, AL

78. EMRANI, S

79. EMRANI, S

80. EPPIG. J

81. FIELDS, L

82. GARCIA, S

83. GONZALES, M

84. GUINEA, SF

85. GUINEA, SF

86. HAGERTY, A

87. JANG, JY

88. KIRKLAND CALDWELL, JZ

89. LESNOVSKAYA, A

90. REALE-CALDWELL, A

91. REYNOLDS. GO
Impact of Fall-Related Psychological and Physiological Factors on Dual-Task Performance in Older Adults

Age-Related Changes in Visual Discrimination

The Influence of Cognitive Reserve, Reading Level and Processing Speed on Executive Control Ability in Peruvian Healthy Older Adults

Sleep Disturbance Severity is Associated with Earlier Self-Reported Onset of Cognitive Decline Among Older Adults

Body Mass Index and Executive Functioning in a Longitudinal Study of Healthy Elderly

Sleep Disturbance and its Association with Cognitive Status in a Population Based Sample of Older Adults: The Cache County Memory Study

Vascular Illness Burden Predicts Theory of Mind Performance in Older Adults

Cognitive Markers of Brain Aging: How Young Can We Go?

The Association of Neuropsychological Test Error Responses to Neuroimaging Biomarkers in Young and Middle-Aged Adults

Effectiveness of a Video-based Aging Services Technology Education Program for Clinical Care Professionals

\section{Dementia (Non-AD)}

Differences Between Monolingual and Bilingual Individuals With Mild Cognitive Impairment on Memory Screening

A Meta-analysis of Neuropsychological Functioning, Social Cognition, and Olfaction in the Frontotemporal Dementias

Cognitive and Behavioral Measures in Early bv-FTD

Revision of the Northwestern University Famous Faces Test (NUFFACE-R): Face Naming and Knowledge in Primary Progressive Aphasia (PPA)

Characterization of Cognitive Impairment in Posterior Cortical Atrophy

The Impact of a Previous Diagnosis of Mild Cognitive Impairment on Mood and Quality of Life in Caregivers and Patients Recently Diagnosed With Dementia

\section{MCI (Mild Cognitive Impairment)}

Managing Money Matters: Financial Management is Associated with Increased "Functional Reserve" in Mild Cognitive Impairment

Sex Differences in "Functional Reserve" and Decline in Mild Cognitive Impairment from the Alzheimer's Disease Neuroimaging Initiative: A Longitudinal Analysis

APOE genotype modifies the association between central arterial stiffening and neuropsychological functioning in mild cognitive impairment: The Vanderbilt Memory \& Aging Project

Refinement of a Telephone Screening for Mild Cognitive Impairment

Characterizing Omission Errors in Everyday Task Completion and Cognitive Correlates in Individuals with Mild Cognitive Impairment and Dementia

Differences in Awareness of Memory Function Among Persons with Amnestic MCI vs Subjective Memory Complaints but Normal Memory Function

Diagnosing Mild Cognitive Impairment: Comparison of Conventional, Actuarial, and Statistical Methods

The Lowenstein-Acevedo Scales of Semantic Interference and Learning (LASSI-L), and The ShortTerm Memory Binding Task (STMBT) as Predictors of Mild Cognitive Impairment (MCI)

Visual Versus Verbal Working Memory - I: Differing Between Subtle and Mild Cognitive Impairment Visual Versus Verbal Working Memory - II: Serial Order Position Effects in Subtle and Mild Cognitive Impairment

APOE-E4 Moderates the Relationship between Lobar Microbleeds and a Diagnosis of Mild Cognitive Impairment

Cognitive Functioning in MCI Patients with and without a History of Sports-Related Concussion Preliminary Investigation of Gaze Pattern Differences in MCI and Healthy Older Adults

Cortical Atrophy is Associated with Accelerated Cognitive Decline in Mild Cognitive Impairment with Subsyndromal Depression

Can Subtle ADL Impairments be Traced Along the Continuum of MCI?

Short-Term Memory Binding Deficits Across Subtypes of MCI And Memory Load

Do as I Do, Not as I Say: Relations between Narrative Script Production and Everyday Action Performance

Affective Neuropsychiatric Symptoms and Alzheimer's Disease Biomarkers in Non-demented Older Adults

Sex Differences in Verbal Memory and Hippocampal Volume: The Impact of Amyloid Imaging Measures

Gaze Fixations are Associated With Object Location Memory in Older Controls and Patients With

Mild Cognitive Impairment

Comparison of Performance Validity Tests for the RBANS

Neuropsychiatric Symptoms and Awareness of Cognitive Deficits in Mild Cognitive Impairment 
92. SANTORELLI, GD
93. SOFKO, CA
94. SUHRIE, KR
95. SUHRIE, KR
96. SUMIDA, C
97. THOMAS, KR
98. TIMPANO SPORTIELLO, MR
99. TREMONT, G

2:15-2:45 PM

2:45-3:45 PM

1. VARGHA-KHADEM, F

4:00-5:00 PM

1. ANDERSEN, RA

5:00-6:00 PM

1. PUENTE, AE

5:00-6:30 PM

1. HILLARY, FG

2. LEVIN, H

3. BIGLER, ED

4. HILLARY, FG

\section{5:00-6:30 PM}

1. PILLAY, SB

2. MIOTTO, EC

3. BANGEN, KJ

4. JEFFERSON, AL
Executive Function and Emotion Reactivity to Negative Mood Induction in Older Adults with Mild Cognitive Impairment/Mild Alzheimer's Disease

APOE genotype may modify the association between inflammatory biomarkers and neuropsychological functioning in older adults: The Vanderbilt Memory \& Aging Project Association Between a Brief Telephone Screening Measure and the Repeatable Battery for the Assessment of Neuropsychological Status (RBANS)

Does Performance on the Modified Telephone Interview for Cognitive Status (mTICS) Tell Us Anything About Functional Abilities?

Differences Between Healthy Older Adults and Individuals with Mild Cognitive Impairment on the Medication Management Abilities Assessment: Overdose and Underdose

Longitudinal Trajectories of Informant-Reported Daily Functioning in Empirically-Defined Subtypes of Mild Cognitive Impairment (MCI)

MCI: A Study on Progression to Dementia in High-Risk Individuals

Recognition Memory Heterogeneity in Amnestic Mild Cognitive Impairment

PM Coffee Break

Acadia Ballroom

Plenary C. Developmental Amnesia: Memory Formation in the Absence of Remembering Presenter: Faraneh Vargha-Khadem Carondelet

Developmental Amnesia: Memory Formation in the Absence of Remembering

Plenary D (Birch Memorial Lecture). Cognitive Neural Prosthetics to Overcome Brain and Spinal Cord Injury

Presenter: Richard A. Andersen

Carondelet

Cognitive Neural Prosthetics to Overcome Brain and Spinal Cord Injury

Invited Presentation. From the Laboratory to the Clinic and Back: The Role of Science and Policy Development in Shaping Clinical Neuropsychology

Presenter: Antonio E. Puente

Salon A-C

From the Laboratory to the Clinic and Back: The Role of Science and Policy Development in Shaping Clinical Neuropsychology

Symposium 4. Historical Perspectives in the Study of Neurotrauma: Progress and Pitfalls Over 40 Years of Research Chair: Frank G. Hillary Carondelet

Historical perspectives in the study of neurotrauma: progress and pitfalls over 40 years of research Milestones in Traumatic Brain Injury Research: A Neuropsychological Perspective From Volumetrics to Brain Function, Lessons from TBI Neuroimaging TBI in the age of the human connectome: a critique for a brave new small world

Paper Session 5. Vascular Disease and Injury Moderator: Angela L. Jefferson Bissonet

Where is Wernicke's area? A voxel-based lesion-symptom mapping study of spoken language comprehension in chronic aphasia

Resting State Functional Connectivity and Neural Correlates of Face-Name Encoding in Patients with Left Frontoparietal Stroke

Cerebral Blood Flow and Amyloid-B Interact to Affect Memory Performance in Cognitively Normal Older Adults

Arterial stiffness is related to decreased cerebral blood flow and increased cerebrovascular reactivity in cognitively normal older adults: The Vanderbilt Memory \& Aging Project 
5. WERHANE, ML

6. SCHNEIDER, BC

\section{5:00-6:30 PM}

1. FAMA, $\mathrm{R}$

2. FRAZER, KM

3. REYNOLDS, BW

4. HUNTER, SJ

5. MIGLIORINI, R

6. SCOTT, J

\section{5:00-6:30 PM}

1. TSAPANOU, A

2. FISCHER, JT

3. BEEBE, DW

4. VIQAR, F

5. GOSSELIN, A

6. MARTINDALE, SL
1. NA, S
2. SHAIR, S
3. PONSFORD, J
4. WERRY, AE
5. FELIX, G
6. MEDINA, LD

\section{5:15-6:30 PM}

1. ARMENGOL, CG

2. FRIEDRICH. T

3. HOLLAND, AK

4. AVILA, J

5. BANUELOS, D
Elevated pulse pressure and apolipoprotein-E genotype interact to affect functional decline in cognitively normal older adults

Enlarged perivascular spaces and white matter hyperintensities more strongly relate to neuropsychological functioning than other neuroimaging markers of small vessel disease: The Vanderbilt Memory \& Aging Project

\section{Paper Session 6. Substance Abuse Moderator: Rosemary Fama Salon D}

Neurological and Nutritional Biomarkers of Cognitive Impairment in Alcoholics Assessing cognitive functioning in long-term cocaine users

Ideational Fluency, Sensation Seeking, and Anxiety: A Recipe for Binge Drinking Evidence of increased reward sensitivity in Young Black Men Who Have Sex With Men (YBMSM) who are heavy cannabis users

Exaggerated Aversive Interoceptive Processing in Adolescent Substance Users: An Early Risk Factor for Addiction?

Adolescent and Young Adult Cannabis Users Do Not Show Declines in Neuropsychological

Performance: A Two-Year Longitudinal Study

\section{Paper Session 7. Sleep \\ Moderator: Melissa Lamar \\ Salon E}

Memory and Sleep Problems in the Elderly

Sleep Disturbances and Internalizing Behavior Problems After Pediatric Traumatic Brain Injury Multi-night Sleep Restriction Increases Total Symptom Score on a Concussion Screener in Healthy Adolescents

Sleep Quality Negatively Impacts Cognition in Older Adults with Temporal Lobe Epilepsy Impact of Obstructive Sleep Apnea on Executive Functioning in Forensic Patients with Schizophrenia-Spectrum Disorders

Sleep Quality Affects Cognitive Functioning in Returning Combat Veterans beyond Combat Exposure, PTSD, and Mild TBI History

\section{Paper Session 8. Cross Cultural Moderator: To Be Announced Salon F-H}

Disentangling Race-Related Differences on the Boston Naming Test: Contributions of Socioeconomic Status, Reading and Vocabulary on the Boston Naming Test in a Healthy College Sample Reliability of the CogState Brief and NIH Toolbox Cognition Batteries in African American Elders with Subjective Memory Complaints

The influence of cultural factors on outcome following traumatic brain injury Influence of Demographic Variables on Measures of Attention and Working Memory in Older Adults of Different Races

Illiteracy and Disparities in Cognitive Decline and Alzheimer's Disease among Spanish-Speaking Older Adults

Hippocampal Volume is Related to Cognitive Function in Non-Hispanics but not in Hispanics in a Case-Control Matched Sample

\section{Poster Session 5. Poster Symposia, Genetics, Cross Cultural Acadia}

\section{Behavioral Neurology/Cerebral Lateralization/Callosal Studies}

Challenges in the Development of Lateralization: Implications for the Acquisition of Reading and Writing Skills

The Impact of Age on Navigation Asymmetry in Naturalistic Settings

Support for the Capacity Model of Hostility Using a Dichotic Listening Paradigm: Reductions in Cerebral Laterality for Phoneme Detection Indicate Compromised Cognitive Control in High Hostile Men

\section{Cross Cultural}

Socio-Cultural Impact on Stroop-Interference Performance in Spanish-English Bilinguals The relationship between verbal and nonverbal neuropsychological tests and aspects of English fluency in ethnically diverse individuals 
6. FLOWERS, AT

7. KAUZOR, K

8. LEONG, S

9. PUENTE, AE

10. SEKHON, A

11. STRUTT, AM

12. STUART, JZ

13. THOMAS, S

14. TORRES, VL

15. VELEZ URIBE, I

16. VINCK, K

17. ALEXANDER, CJ

18. CASNAR, C

19. COX, SM

20. FEE, RJ

21. FRANK. J

22. HINTON, VJ

23. LEAFFER, EB

24. LERNER, AL

25. LUCCHETTI. A

26. MCCABE, KL

27. MCKNIGHT, SE

28. OLIVIER, TW

29. TRAVERS, LV

30. VEGA, C

31. YUND, B

32. ASARNOW, R

33. BABIKIAN, T

34. DENNIS, E

35. WILDE, EA

36. OLSEN, A

37. LEVIN, H

38. SANDROFF, BM

39. TOMPOROWSKI, P

40. SANDROFF, BM

41. BEST, J

\section{THORNTON, AE \\ 43. WILLI, T \\ 44. WACLAWIK, K \\ 45. GICAS, K}

Socioeconomic Status and Neuropsychological Assessment Performance

Hispanic Performance on Verbal and Non-verbal Neuropsychological Tests

Exploring the Mini-Mental Status Exam in an African American Sample in a Primarily Urban Setting Rural Russian and American Populations' Performance on Color Trails Test and Trail Making Test Cross-Cultural Differences and Acculturation Effects on WISC-IV Performance in Punjabi Children: A Pilot Study

Assessment of language fluency and confrontation naming in monolingual Spanish speaking older adults

Racial Differences in the Utility of the TOPF in a Hospital Based Outpatient Clinic

Challenges of Developing a Neurocognitive Test Protocol for a Multilingual International Trial : The SIOP Ependymoma II Program

The Big Five Inventory (BFI) and Emotion Word Valence: Does Personality Influence the Appraisal of Emotion Words in Bilinguals?

Testing a Predictive Model for the Appraisal of Valence of Emotion Words in Spanish-English Bilinguals

Cognitive, Academic, and Behavioral Functioning of First and Second Generation Hispanic Children in the Child Welfare System: Findings using the National Survey of Child and Adolescent Well-Being (NSCAW II)

\section{Genetics/Genetic Disorders}

Using the Vineland Adaptive Behavior Scale to Assess Adpative Functioning Differences Between Subtypes of Mucopolysaccharidosis I

ASD Symptomatology and Related Variables in Children with Neurofibromatosis type 1

Neuropsychological and social functioning in children with Neurofibromatosis Type 1, ADHD, and Autism Spectrum Disorder

Underlying contribution of attention and executive functioning to cognition in individuals with dystrophinopathy

Neuropsychological Functioning of an Adult with Fanconi Anemia

The developmental trajectory associated with Glut 1 Deficiency Syndrome

The relationship between cerebral lactic acidosis and memory performance in Mitochondrial Encephalomyopathy, Lactic Acidosis, and Stroke-like Episodes (MELAS)

Examination of COMT Genotype on Verbal and Visual Memory Following Sports-Related Concussion Neurocognitive Variability in Twin Adolescent Males with Williams Syndrome

Quantifying the Resolution of Spatial and Temporal Representation in Children With 22q11.2

Deletion Syndrome - WITHDRAWN

A Rare Case of Spinocerebellar Ataxia Type 16

Systematic Desensitization in a Young Adult with Pelizaeus-Merzbacher Disease

The Neuropsychological Profile of Girls and Women with Turner Syndrome Across the Lifespan Neuropsychological Comorbidities in Tuberous Sclerosis Complex and Autism Spectrum Disorder Relations Between Parent Report of Attention and Sleep in Children with Neurofibromatosis type 1

\section{Acquired Brain Injury (TBI/Cerebrovascular Injury \& Disease - Child)}

Indicators of Recovery/Repair and Neurodegeneration After Moderate-Severe TBI Pediatric Traumatic Brain Injury:Potential Mechanisms - WITHDRAWN

Whole Brain MR Spectroscopic Correlates of Microstructural Abnormalities and Functional Outcomes in Pediatric Moderate/Severe TBI - WITHDRAWN

Multi-Modal Imaging in Pediatric TBI: A Longitudinal Study - WITHDRAWN

Structural Brain Alteration in Children and Adolescents after Moderate to Severe TBI - WITHDRAWN

BOLD Hyper Activation after Pediatric Moderate-Severe Traumatic Brain Injury (msTBI) is Linked to Slow Inter-Hemispheric Transfer Time as Measured with Scalp Event-Related Potential — WITHDRAWN

Reorganization of Social Information Processing After Moderate to Severe TBI in Adolescents - WITHIDRA WN

\section{Cognitive Intervention/Rehabilitation}

Exercise and Cognition: A Multidisciplinary Approach — WITHDRAWN

Exercise and Children's Cognition: Interpretation of Current Research

Exercise and Cognition in Persons with Neurological Disorders - WITHDRAWN

Exercise to Promote Cognition in Older Adults: Current and Future Research to Define Exercise Type and Target Populations

\section{Drug/Toxin-Related Disorders (Including Alcoholism)}

Neuropsychological Aspects in Marginalized Persons with Multimorbidity Structural Correlates of Substance-Induced Psychosis: a Comparison Between Psychostimulant Dependent Individuals With and Without Psychosis

Predictors of One-Year Cognitive Decline in a Marginally Housed, Multimorbid Sample Structural Brain Markers are Differentially Associated with Neurocognitive Profiles in Socially Marginalized Persons with Multimorbid Illness 
46. O'CONNOR. TA

47. WANG, NY

48. MURRAY, NC

49. MURRAY, NC

50. MCCABE, DL

51. LU, W

52. STATUCKA, M

53. WILLIAMS, J

54. OSIPOWICZ, K

55. OSIPOWICZ, K

56. WILLIAMS, J

57. MCWILLIAMS, K

7:00-9:00 PM

7:20-8:50 AM

1. KANA, R

7:20-8:50 AM

1. THAMES, A

8:00-9:15 AM

1. AILION, A

2. AMEDORO, S

3. CHILD, A

4. CLARK, BE

5. CONKLIN. HM

6. COX, LE

7. DASHER, NA

8. ESTEVIS, E

9. GIOIA, A

10. HENNEGHAN, AM

11. IRISH, J

12. JACOLA, LM
The Impact of Traumatic Brain Injury and Aggregate Comorbidities on Cognition and Functioning in a Marginally Housed Sample

The Role of Neurocognition, Psychiatric Symptoms, and Multimorbid Illness In Predicting Everyday and Social Functioning in Marginally Housed Persons

\section{Historical}

Concussion: Past, Present, and Future

Acute Concussion Assessments: The Role of Neuropsychology

The Role of Psychology in Concussion Management

Assessing and Treating Sleep Disturbance and Fatigue after a Concussion(s)

Concussion: Myths and Misconceptions

\section{Memory Functions}

fMRI of Hippocampal Function: Old insights from New Studies mTLE and Verbal Fluency

Smelly Scenes: Role of the Hippocampus in Multisensory Integration of Memories

fMRI Stimuli and Methods

The Unique Role of Time in Autobiographical Memory Consolidation

\section{Student Social, Hosted by the INS Student Liaison Committee}

To Be Announced

\section{FRIDAY, FEBRUARY 3, 2017}

\author{
CE Workshop 9. Autism: Clinical and Translational Insights from Brain \\ Mapping \\ Presenter: Rajesh Kana \\ Bissonet
}

Autism: Clinical and Translational Insights from Brain Mapping

\section{CE Workshop 10. Not All Aging Processes Are Created Equal: Cognitive Aging Among Culturally Diverse Groups \\ Presenter: April Thames \\ Salon D}

Not All Aging Processes Are Created Equal: Cognitive Aging Among Culturally Diverse Groups

\section{Poster Session 6. Adult 2}

Acadia

\section{Cancer}

Childhood brain tumors: A systematic review of the structural neuroimaging literature

Exploratory Analysis of Inductive Reasoning Abilitiesin a Pediatric Neurofibromatosis Type 1

Population

Significant Reading Difficulties in a Pediatric Patient with Left Thalamic Tumor: A Case Report Cancer Patients' Perceptions Of Their Cognitive Functioning After Treatment Is Impacted By

Comments From Others

Computerized Assessment of Cognitive Impairment among Children Undergoing Treatment for Medulloblastoma

Psychosocial Trajectories Among Survivors of Pediatric Brain Tumors: A Growth Mixture Modeling Approach

Effect of Types of GvHD on Patient Mood and Functional Abilities following Bone Marrow

Transplant

Driving Safety in Patients with Primary Brain Tumors

Associations Between Depressive Symptoms and Neuropsychological Functioning in Pediatric Oncology Patients with Brain Tumors (BT) and Acute Lymphoblastic Leukemia (ALL)

Executive Function and Emotional Distress Prior to Breast Cancer Treatment

The Relationship Between Executive Functioning and Adaptive and Maladaptive Behavior in Childhood Cancer Survivors

The Relationship between Procedural Sedation during Treatment and Neurocognitive Outcomes in Survivors of Pediatric Medulloblastoma 


\author{
13. KENNEDY, T \\ 14. LENIHAN, J \\ 15. OLSEN, E \\ 16. PARSONS, M \\ 17. PETERS, JB \\ 18. RICHARD, AE \\ 19. ROSEBERRY, JE \\ 20. SHARKEY, CM \\ 21. TARKENTON, T \\ 22. VERHAAK, A \\ 23. VICKERS, KL \\ 24. WALSH, KS \\ 25. WHITAKER, AM \\ 26. WITHROW, S \\ 27. ZEAL, J
}

28. BONO, AD

29. BONO, AD

30. BRYANT, AM

31. BURNS, CM

32. DEZHKAM, N

33. DOROCIAK, KE

34. DOROCIAK, KE

35. FELLOWS, RP

36. GARCIA, NE

37. GHAZI SAIDI, L

38. GHAZI SAIDI, L

39. GURNANI, A

40. HARCIAREK, M

41. HARCIAREK, M

42. HERNAIZ ALONSO, C

43. HIZEL, L

44. HORTON, DK

45. KEATING, L

46. KURNIADI, N

47. MAHONEY, JJ

48. MANKOWSKA, A

49. PATERSON, TS

50. PIERS, RJ

51. QUASNEY, EE

52. SEMERJIAN, C

53. STEED, D

54. STELMOKAS, J

55. VENEZIA, R

56. WALKER, KA
The Relationship Between Processing Speed and Working Memory in Pediatric Brain Tumor and Acute Lymphoblastic Leukemia

Utility of the BRIEF parent report as a screener for executive dysfunction in patients with pediatric brain tumors

Academic Performance as Predicted by Working Memory, Processing Speed and Radiation Therapy in Pediatric Brain Tumor Survivors

Cognitive Deficits in Older Adults with Glioblastoma (GBM)

The Impact of Sedation for Radiation Therapy on Performance Measures and Caregiver Ratings of

Attention in Survivors of Pediatric Medulloblastoma

Attentional Control and Math Performance in Pediatric Cancer Survivors

Lateralized Cognitive Functioning in the Frontal Lobes: A Brain Tumor Lesion Study

Suicidal Ideation and Executive Functioning in Children with Pediatric Cancer

School Performance in Pediatric Medulloblastoma Survivors

Prediction of Subjective Memory Ability and Patient Self-Report Accuracy Before Resection of High Grade Glioma

The Neuropsychological Impact of Androgen Deprivation Therapy: A Meta-Analytic Review

Neuropsychological Functioning in Children with Medulloblastoma: The Impact of Post-Operative Pediatric Cerebellar Mutism Syndrome Within the First Year Following Diagnosis

Bilingualism as a Potential Protective Factor Against Cognitive Late-Effects following Treatment for Childhood Acute Lymphoblastic Leukemia (ALL)

Verbal Learning and Memory Among Prostate Cancer Patients Undergoing Androgen Deprivation Therapy

Impact of Medical and Treatment Variables on Adaptive Functioning within Eighteen Months of Pediatric Brain Tumor Diagnosis

\section{Medical/Neurological Disorders/Other (Adult)}

Facial Expressivity and Depression in Parkinson's Disease (PD) with Lateralized Motor Impairment Onset

Outcomes and Predictors of the Lee Silverman Voice Treatment (LSVT LOUD) on Facial Mobility and Emotional Expressivity in Parkinson's Disease (PD)

Visuospatial Memory Performance in Parkinson's Disease and Essential Tremor

Cognitive Impairment in Advanced Chronic Kidney Disease

A Review and Neuropsychological Profile of Anti-NMDA-Receptor Encephalitis: A Case Study

Executive Function Moderates the Relationship between Pain Severity and Physical Quality of Life in an Outpatient Sample of Adults with Sickle Cell Disease

Neuropsychological Profile in an Outpatient Sample of Adults with Sickle Cell Disease

Independent and Differential Effects of Cardiometabolic Variables on Executive and Physical

Functioning in Older Adults

Wisconsin Card Sorting Test subscales in Parkinson's disease and Amyotrophic Lateral Sclerosis

Biomarkers of AD, MCI and NCS: MMSE Cortical Thickness, Volumetric and CSF

Striatal Binding Ratios and CSF Biomarkers of Cognition in Parkinson's disease

Adult Man Presenting with Acute Disseminated Encephalomyelitis (ADEM): A Case Study

Attentional-Intentional Brain Networks of Dialyzed Patients With End-Stage Renal Disease Are Not

Entirely Normalized Following Kidney Transplant: Evidence From Event Related Potentials

Disorders of The Attentional-Intentional System in Dialyzed Patients With End-Stage Renal Disease:

Should We Blame Kidney Disease, Dialysis or Both?

Memory Ability Predicts Anesthesia Response in Older Adults During Total Knee Arthroplasty

Working Memory and Recall Domains of the Mini-Mental Status Examination Predict Postoperative

Delirium

Utility of Cognitive and Balance Measures in Predicting Ventriculoperitoneal Shunting

Recommendation in Normal Pressure Hydrocephalus

The Effect of Pre-ICU Depression on Cognition and Emotional Functioning After Critical Illness

Neurocognitive Profiles of Individuals Remitted from Eating Disorders

Referring Providers' Preferences and Satisfaction with Neuropsychological Services

Leftward Bias of Visual Attention in Patients With End-Stage Renal Disease Receiving Dialysis: a Neglected Phenomenon

Modelling Medication Adherence in Renal Transplant Recipients: Cognitive and Psychosocial Impacts Atrial Fibrillation and Cognitive Decline: the Framingham Heart Study

The Impact of Balance Disturbance on an Auditory Sustained Attention Task

Repeatable Battery for the Assessment of Neuropsychological Status (RBANS): Cognitive Profile of Adult Patients with Chiari Malformation Type 1

Cognitive Improvements Persist Post Left Ventricular Assist Device Placement

The Influence of Cognitive Status and Depression on Duration of Hospital Stay in Post-Acute Rehabilitation

Opioid Dosage in Critically III ICU Patients is Associated with Attention Deficits, but not Memory Deficits, at Follow-up

Neuroimaging findings and cognitive functioning in patients with sepsis-associated encephalopathy 
57. WARNER, E

58. YEE, MK

59. CADDEN, M

60. CALVO, D

61. CARLEW, AR

62. COSTA. SL

63. DUNGANSON, H

64. GENOVA, HM

65. GOVEROVER, Y

66. LENGENFELDER, J

67. MORDECAI, K

68. NICCOLAI, L

69. NUNAN-SAAH, J

70. PITTERI, M

71. RAPHAIL, A

72. SACCì, F

73. SANDRY, J

74. STROBER. L

75. ARMSTRONG, G

76. CHAPMAN, S

77. DEV, SI

78. DILORENZO, MG

79. DULAY, M

80. FEDOR, A

81. FERLAND, T

82. FONG. MW

83. K THIRUSELVAM. I

84. LEITNER, D

85. MOORE, MJ

9:00-10:30 AM

1. YEATES, KO

2. PTITO, A

3. YEATES, KO

4. ANDERSON, VA

5. KIRKWOOD, M

6. TAYLOR. H

9:00-10:30 AM

1. PRICE, CG

2. GIOVANNETTI, T
Speed of Clock Drawing is Reduced After Orthopaedic Surgery in Older Adults Diagnostic Criteria for Gulf War Veterans Illness: CDC Versus Kansas Criteria

\section{Multiple Sclerosis/ALS/Demyelinating Disorders}

Acute and Chronic Pain and Cognitive Functioning in Multiple Sclerosis

Can one week of moderate intensity aquatic exercise improve cognition and fitness in MS?

Occupational Attainment as a Proxy of Cognitive Reserve in Patients with Multiple Sclerosis

Understanding difference between the California Verbal Learning Test (CVLT) and Selective

Reminding Test (SRT)

Pilot study of an internet based self-guided mindfulness program for individuals with Multiple

Sclerosis

Cognitive Reserve Protects Against Social Cognition Impairments in Multiple Sclerosis

Exploring Money Management in Persons with MS: A Pilot Study

Remediation of Facial Affect Deficits in Multiple Sclerosis: A Pilot Study

Computerized Cognitive Training for Veterans with Multiple Sclerosis

Medical Decision-Making Capacity and its Cognitive Predictors in Multiple Sclerosis

The Impact of Emotional and Psychosocial Factors on Executive Functioning in Pediatric Multiple Sclerosis

Facial Affect Recognition Deficits In Early Multiple Sclerosis Patients Without Cognitive Dysfunction Cognitive Correlates of Driving Using a Virtual Reality Driving Simulator in Individuals With Multiple Sclerosis

The EDSS integration with the Brief International Cognitive Assessment for Multiple Sclerosis and Orientation Tests

Evaluating a Consolidation and Interference Hypothesis in Multiple Sclerosis

Cognitive health in multiple sclerosis (MS): Impact on fatigue, sleep, well-being, and overall quality of life

\section{Stroke/Vascular Cognitive Impairment}

Quick and Easy: Confirming the Utility of the PHQ-9 in a Stroke Population

Reality monitoring in unawareness of memory deficits

Intra-individual variability in processing speed is related to systolic blood pressure in bipolar disorder Preschool Executive Functioning Abilities Predict Later Academic Achievement in Children with Arterial Ischemic Stroke

Predictors of Cognitive Impairment After Cerebrovascular Accident (CVA)

Is step-count in exoskeleton-assisted locomotor training associated with cognitive functioning after stroke?

Association of Metabolic Syndrome with Cognitive Function in Adults

Neuroanatomic and Neuropsychological Correlates of Post Stroke Functional Status

Anterograde Amnesia for Explicit and Implicit Information in a Case of Bilateral Hippocampal Stroke

Neuropsychological Evaluation Following Subsequent Bilateral Thalamic Infarct - A Case Study

Dissociations Between Visual Neglect and Neglect Dyslexia

\section{Invited Symposium 3. Advances in Pediatric Mild TBI: Toward a Neurobiopsychosocial Model \\ Chair: Keith O. Yeates \\ Discussant: H. Gerry Taylor \\ Carondelet}

Advances in Pediatric Mild TBI: Toward a Neurobiopsychosocial Model

Neuroimaging as a Diagnostic and Prognostic Tool in Pediatric Concussion

Neuropsychological testing as an outcome and predictor in pediatric mild traumatic brain injury

Psychosocial Predictors of and Influences on Outcomes of Pediatric Concussion

Neuropsychological Assessment as an Intervention Model in Pediatric Concussion

Discussion of Issues and Future Directions

\section{Symposium 5. Interdisciplinary Approaches to Understanding Post- Operative Cognitive Complications in Older Adults Chair: Catherine C. Price \\ Discussant: Steve DeKosky Bissonet}

Interdisciplinary Approaches for Understanding Post Operative Cognitive Complications in Older Adults

Baseline Cognitive Function is Significantly Associated with Postoperative Death and Stroke in Aged Adults Following Aortic Valve Replacement 
3. BROWNDYKE, J

4. PRICE, CC

5. FLOYD, TF

9:00-10:30 AM

1. SCHULTHEIS, MT

2. VICKERS, KL

3. TESSIER, J

4. VAKIL, E

5. YUAN, J

9:00-10:30 АM

1. TRACY. JI

2. MARRA, DE

3. OSIPOWICZ, K

4. SCHRAEGLE, $\mathbb{W}$

5. BREWSTER, RC

6. SPAT, J

9:00-10:30 AM

1. SHEPPARD, DP

2. KUHN, T

3. LOJEK, E

4. HARCIAREK, M

5. EVANS, J

6. BOWLER, RM

\section{9:30-10:45 AM}

1. COLEMAN, L

2. GILBART, E

3. JENNETTE, $\mathrm{K}$

4. KIM, M

5. KOHEN, C

6. MAPLE, KE

7. MCDONNELL, M

8. MORGAN, EE

9. MULHAUSER, $\mathrm{K}$

10. PACHECO-COLON, IM
Postoperative Resting-state and Task-based Functional Connectivity Changes and Cognition Following Cardiac Surgery

The Challenge and Promise of Pre-Surgical Cognitive Profiles

The Hypoxia Inducible Factor and Aging-Related Postoperative Cognitive Dysfunction

Symposium 6. Neuropsychology and Technologies: Taking the lead on new opportunities for understanding brain-behavior relationships Chair: Maria T. Schultheis

\section{Salon D}

Neuropsychology and Technologies: Taking the lead on new opportunities for understanding brainbehavior relationships

Testing the Limits: Using VR to Quantify the Impact of ABI on Driving

Subjective and Objective Measurement of Distress During VR Driving in Veterans with PTSD

Direct and indirect measures of context in older versus young adult: The additive contribution of eye tracking

$\mathrm{HbO}_{2}$ Variability During Single- and Dual-Task Gait in Older Adults

\section{Paper Session 9. Epilepsy \\ Moderator: Joseph I. Tracy \\ Salon E}

Functional Connectivity in Epilepsy

Cognitive Reserve Predicts Post-Operative Cognitive Outcomes in an Epilepsy Population

Normative Anticorrelation Inhibits Seizure Generalization in Mesial Temporal Lobe Epilepsy

Hippocampal Sclerosis is a Risk Factor for Depression Features in Youth with Temporal Lobe Epilepsy

White Matter Correlates of Verbal Memory in Left Temporal Lobe Epilepsy: A Study of Structural Connectivity

Healthcare Disparities and Cognitive Performance Among Minority Patients with Epilepsy

\section{Paper Session 10. Medical / Infectious Disease}

Moderator: Marc Norman

Salon F-H

A Comparison of the Sensitivity, Reliability, and Stability of Three Diagnostic Criteria for HIV-

Associated Neurocognitive Disorders

Accelerated Brain Aging and Cognitive Decline in HIV

Neurocognitive and Brain Functions in Highly Functioning and Successfully Treated Young HIV

Seropositive Men

Allocation of Spatially Directed Focal Attention in Patients With End-Stage Renal Disease Receiving

Dialysis: Attentive But Too Engaged

Balancing the Demands of Two Tasks: An Investigation of Cognitive-Motor Dual-Tasking in

Relapsing Remitting Multiple Sclerosis.

Neuropsychological Test Performance in Relation to MRI Manganese Deposition in the Brain

\section{Poster Session 7. Neuropsychiatry}

Acadia

\section{Drug/Toxin-Related Disorders (Including Alcoholism)}

Prenatal Opioid Abuse (POA) Causes Children's Learning/Attentional Problems

Marijuana Use, Aerobic Fitness, Mood, and Disinhibition in Emerging Adults

The Relationship Between Marijuana Use, Inhibitory Control, and Learning Strategy in Adolescents and Young Adults

Deficits of decision-making in college students who participate in binge drinking

The Neurocognitive Effects of Changing Hazardous Drinking Behaviors in Adolescents and Young

Adults

Anterior Cingulate Volume Reductions in Adolescent and Emerging Adult Cannabis Users:

Association with Affective Processing Deficits

Changes in Cognitive Functioning in Patients Receiving Intensive Outpatient Treatment for Alcohol

Abuse

Higher Levels of Emotion Dysregulation in Methamphetamine Users Compared to Non-Users Relates to Neurobehavioral Deficits and Craving

Changes in Cognition Over the Course of Residential Treatment for Alcohol Use Disorder

The Association Between Cannabis Use and Motivation Among Adolescents 
11. PRITCHETT, A

12. SULLIVAN, E

13. ALKOZEI, A

14. ANDERSON, S

15. BEZDEK, M

16. DULAY, M

17. FIGUEROA, P

18. HALLOWELL, ES

19. KAIS, LA

20. KARSTENS, A

21. LIU, H

22. MAHMOOD, $\mathrm{Z}$

23. MATHER, M

24. NG, R

25. REIFE, I

26. RENSBERGER. J

27. SCAVONE, A

28. TAIWO, Z

29. TOCCHINI, S

30. TWAITE, JT

31. VAN MEURS, B

32. ZUCCATO, BG

33. BENDER, S

34. BUESO-IZQUTERDO, N

35. DOMBROWSKI, GV

36. ERDODI, LA

37. ERDODI, LA

38. FARRER, TJ

39. FIELDS, KN

40. FOX. J

41. LADUKE, C

42. MARTIN, P

43. SWIFT, TJ

44. TRAHAN, DE

45. TRAHAN, DE

46. WHITESIDE, D

47. AASE, DM

48. ANGERS, K

49. BABIONE, JM

50. BABU, P

51. BASSO, MR

52. BESSETTE, KL
Effects of Prenatal Cannabis and Tobacco Exposure on Birth Outcome and Temperament Assessing Impulsivity in Cocaine Users Utilizing Hot and Cool Measures of Executive Functioning

\section{Emotional Processes}

Emotional Intelligence Can Be trained via an Online Training Program and is Associated with Better Performance on the IGT

Depression Influence on Executive Functioning and Processing Speed after accounting for Cerebrovascular Risk in Non-Demented Older Adults

Does Emotion Recognition Ability Predict Neural Responses of Empathic Happiness?

Emotion Affects Decision Making and Reasoning After Cerebrovascular Accident (CVA)

Self-Perception of Compassion in Individuals with Agenesis of the Corpus Callosum

The Effect of Severity of Cigarette Smoking and Early Life Adversity on Current Affect

Inhibition and Shifting Processes Influence the Relation Between Savoring Beliefs and Positive Affect

Brain-Behavior Profiles Distinguishing Psychological Resiliencefrom Depression After Trauma in an

Urban Dwelling Sample of Adults: The Possible Role of Rumination

Relationships Between Emotion Regulation and Executive Functions

Neural Correlates of Coping and Perseverance

Associations Between Alexithymia and Emotion Dysregulation

Associations Between Memory Functioning and Internalizing Symptomatology in Children Exposed to Chronic Maternal Depression

Self-Efficacy as a Moderator for the Relationship Between Theory of Mind and Social Problem Solving - WITHDRAWN

Cortisol, DHEA, and Heart Rate Variability in Army National Guard Special Forces

The Influence of Alexithymia and Mindfulness on Perceived Social Support

The Role of Executive Function in Empathic Processing of Positive versus Negative Emotions

Emotional recognition: which are differences in the same perceptual modality?

Examining Relationships between Basic Emotion Perception and Musical Training in the Prosodic,

Facial, and Lexical Channels of Communication and in Music

Sex Differences in Avoidance Behavior and Associated Neural Correlates

The Role of Emotion Regulation in the Relation Between Social Integration and Stress: A Pilot Study

\section{Forensic Neuropsychology}

A Forensic Case Study Involving Unequivocal Severe Brain Injury and Unequivocal Response Bias Impulsivity and external versus internal attributions in male batterers of intimate partner violence Validity Performance in an Anxious Undergraduate Sample

Gender and Lateral Dominance Influences Likelihood of Failure on Performance Validity Tests

The Stroop Test as a Measure of Performance Validity in Adults Clinically Referred for

Neuropsychological Assessment

Fail Rate of Performance Validity Tests in Academic Accommodation Seeking College Students: The Role of Diagnosis on Effort Measurement

Predicting Juvenile Recidivism with the Wechsler Index Scores and Personality Assessment Inventory Scores: The Role of Intraindividual Variability

Neuropsychological Profile and Descriptive Classifications of Mass Murderers

The Neuropsychological Assessment of Justice-Involved Men: A Case for Group-Specific Norms Excessive Decline from Premorbid Functioning (EDPF): Assessing Performance Validity with the WAIS-IV and TOPF

Detecting Simulated Memory Malingering with Eye-Tracking technology

False Positive Rates for Reliable Digit Span in Individuals with Alzheimer's Disease or Other

Dementias

Specificity of the CVMT Symptom Validity Scale in Adults with Alzheimer's Disease or Other Dementias

Logistically Derived Embedded Performance Validity Measures Using Tests of Executive Functioning in a Mild Traumatic Brain Injury Sample

\section{Psychopathology/Neuropsychiatry (Including Schizophrenia)}

PTSD Severity Predicts Working Memory Performance in OEF/OIF/OND Veterans Cognitive performance over five yearsamong individuals with bipolar disorder and unaffected controls using latent growth modeling

PTSD Symptom Severity Predicts Verbal Encoding and Retrieval in Combat-Exposed OEF/OIF/ OND Veterans

Impact of gender and history of childhood trauma on cognitive functioning in patients with bipolar disorder

Inpatient Depressives' Responses to Reward and Punishment Correlate with Distinct Facets of Executive Function

Comorbid Depression and Anxiety Has Greater Top-Down and Bottom-Up Neural Emotional Processing than Depression Alone in the Remitted State 
53. BOBHOLZ, SA

54. BOUCHARD, A

55. BURTON, CZ

56. CAIRNCROSS, M

57. CERNY, BM

58. CLARK, SV

59. COMBS, T

60. CRAWFORD, JL

61. DELDONNO. SR

62. EASTER, RE

63. EYLER, LT

64. FORTE, M

65. GRIMES, KM

66. HAISLEY, LD

67. HUBER. RS

68. HUNT, IJ

69. HWANG, S

70. KARPOUZIAN, T

71. KEILP, JG

72. KLING, LR

73. LAI, JK

74. LUU, H

75. MILLER, M

76. MILLER. ML

77. NGUYEN. TT

78. OSBORN, A

79. PARK, M

80. SHURA, RD

81. SILVA, GR

82. SWIFT, A

83. TORRES, I

84. WILSON. C

85. ZOLLIECOFFER, C.J

10:30-10:45 AM

11:00 AM-12:00 PM

1. CORBETTA, M
Pallidal hypertrophy as a putative neural compensatory response to schizophrenia: Findings in firstepisode schizophrenia

Anticholinergic Drug Burden Predicts Levels of Community Functioning in Outpatients With Serious Mental Illness

Cognition in Bipolar Disorder With and Without Psychosis

Insight Into Neurocognitive Functioning in Psychiatric Illness: A Comparison of Self-Report, Case

Manager Report, and Objective Neuropsychological Test Data

Convergence between scores on the BIS-11 and measures of executive function in individuals with remitted major depression

Clinical and Cognitive Insight in Youth at Ultra High-Risk of Psychosis: Relationships with Cognition, Symptoms, and Default Mode Connectivity

Working Memory Impairments in Schizophrenia: Single Item Maintenance is Compromised Following Intact Updating

Effects of Demographic Characteristics on Cognitive Test Performance in Schizophrenia versus Bipolar Disorder

The Influence of Childhood Trauma and Affective Personality Traits on Neural Correlates of Reward Anticipation

Clinical predictors of decline in cognitive functioning in bipolar disorder

Accelerated Brain Aging in Bipolar Disorder

Mechanisms of Cognitive Disturbance in Schizophrenia

The Development of Subsyndromal Positive Symptomatology: Neurocognitive Performance and Cognitive Biases in Young Adults with Schizotypal Traits

Neuropsychological Assessment to Predict Risk for Physical Restraint and Seclusion on Children's

Psychiatric Inpatient Unit

Relationship of Executive Functioning Deficits to N-acetyl Aspartate (NAA) and Gammaaminobutyric Acid (GABA) in Youth with Bipolar Disorder

Cognitive control disruption and quality of life in individuals with obsessive-compulsive disorder Associations between depression severity and neurocognitive functions in adult patients with major depressive disorder

Eye Movement Biomarkers of High vs. Low dose Lurasidone on Prefrontal Abilities in TreatmentResistant Schizophrenia

Independent Contribution of Neurocognitive Dysfunction to the Risk for Suicidal Behavior in the Context of Other Clinical Predictors

Cluster Analysis-Defined Symptom Subtypes in remitted Major Depressive Disorder

Subjective and Objective Measures of Impulsivity: Relations with Clinical Symptom Severity and Psychosocial Functioning Among Military Veterans with Alcohol Use Disorder and Posttraumatic Stress Disorder

Long-Term Cognitive Functioning in Post-Electroconvulsive Therapy (ECT) Patients

Optical Coherence Tomography of the Retina in Schizophrenia: Relationships with Perceptual Function

Depression. Anxiety, and Quality of Life in Individuals who have undergone Electroconvulsive Therapy

Relationship Between Short-term Intra-individual Variability in Affective Symptoms and Cognitive Performance in Bipolar Disorder

An Exploratory Analysis of PTSD Symptomatology, Gender, Working Memory, and Attention in OIF/ $\mathrm{OEF} / \mathrm{OND}$ Veterans

Cut-off scores of the Schizotypal Personality Questionnaire for screening of high-risk psychosis Evaluating the Motor Slowing Hypothesis of Depression

Verbal Executive Dysfunction in Posttraumatic Stress Disorder: The Role of Clinical Symptoms in Process-Specific Executive Deficits

How Time Flies: The Perception, Perspective and Experience of Time in Bipolar Affective Disorder Metamemory Monitoring in Bipolar Disorder

Association of Neurocognition and Psychosis-Risk Symptoms

The Impact of Disease on the Contribution of Demographic Characteristics to Cognitive Test Performance

\section{AM Coffee Break} Acadia Ballroom

\section{Plenary E. Behavioral Clusters and Brain Network Mechanisms of Impairment and Recovery Presenter: Maurizio Corbetta Carondelet}

Behavioral Clusters and Brain Network Mechanisms of Impairment and Recovery 
12:00-1:00 PM

1:00-2:00 PM

1. LAMAR, M

1:00-2:30 PM

1. STRINGER, AY

2. WILSON, BA

3. CICERONE, KD

4. STRINGER, AY

1:00-2:30 PM

1:00-2:30 PM

1. KREMEN, WS

2. KREMEN, WS

3. SANDERSON-CIMINO, M

4. JEREMY, E

5. MATHER, M

1:00-2:30 PM

1. HOLDER, CM

2. SHAY, N

3. REZAIE, $\mathrm{R}$

4. HOLDER, CM

1:00-2:30 PM

1. FORD, $\mathrm{AI}$

2. WEBER, E

3. KEILP. JG
Lunch (On Own)

Conference-Wide

Benton / Mid-Career Awardee Presentation: Subtle Brain-Behavior Biomarkers of Modifiable Cardiovascular Disease Risk Factors: Implications for Minority Health Disparities, Aging and Dementia Award Recipient: Melissa Lamar Salon F-H

Subtle Brain-Behavior Biomarkers of Modifiable Cardiovascular Disease Risk Factors: Implications for Minority Health Disparities, Aging and Dementia

Invited Symposium 4. A Summit on Cognitive Rehabilitation: Mapping the Past, Defining the Present and Imagining the Future

Chair: Anthony Y. Stringer Carondelet

A Summit on Cognitive Rehabilitation: Mapping the Past, Defining the Present and Imagining the Future

The History of Cognitive Rehabilitation

The Present Status of Cognitive Rehabilitation

The Future of Cognitive Rehabilitation

Panel Discussion, Presented by the INS Student Liaison Committee: International Cross-Cultural Considerations in Research Presenters: Anita Sim, Jonathan Evans, Tedd Judd, Robert K. Heaton Salon A-C

Symposium 7. Locus Coeruleus-Norepinephrine System, Cognitive Effort, and Early Risk for Alzheimer's Disease

Chair: William S. Kremen

Discussant: Mark W. Bondi

Bissonet

Locus Coeruleus-Norepinephrine System, Cognitive Effort, and Early Risk for Alzheimer's Disease Task-Evoked Pupil Response: A Novel Biomarker of Locus Coeruleus Dysfunction Indicating Early Risk for MCI and Alzheimer's Disease

The Relationship Between Locus Coeruleus Integrity and Biomarkers of Alzheimer's Disease: A Magnetic Resonance Imaging Pilot Study

Convergent Evidence of Pupillary Response as an Early Indicator of Locus Coeruleus Dysfunction and Risk for Mild Cognitive Impairment

Locus Coeruleus Neuromelanin MRI Contrast Correlates With Cognitive and Cardiovascular Factors

Symposium 8. Clinical Applications of Functional Neuroimaging for Presurgical Functional Mapping: The Past, Present, and Future Roles for Neuropsychologists

Chair: Christen M. Holder

Discussant: Andrew Papanicolaou Salon D

Clinical Applications of Functional Neuroimaging for Presurgical Functional Mapping: The Past, Present, and Future Roles for Neuropsychologists

The Traditional Use of Invasive Procedures for Determining Localization and Lateralization

Non-Invasive Procedures as the Future of Functional Mapping

Outcomes of Non-Invasive Presurgical Planning

Paper Session 11. Mental IIlness

Moderator: Derin J. Cobia

Salon E

History of Depression: From Possession to Organic Brain Disorder

The Relationship between Depression and Executive Functioning in Child Inpatient Outcomes and Neuropsychological Deficits

Familial Transmission of Neurocognitive Deficits Associated with Suicidal Behavior Risk 
4. STEFFEN-ALLEN, F

1:30-2:45 PM

1. AGHJAYAN, SL

2. ALLISON, S

3. ALVERSON, WA

4. AZAR, M

5. BAENA, AY

6. BAGGER, JE

7. CAHN-WEINER, D

8. CERBONE, B

9. CHILDS, KN

10. CLARK, LR

11. CLEM, M

12. COLVIN. LE

13. CONTRASTANO, CM

14. DALCHAND, E

15. DEFEIS, BL

16. EMMERT, NA

17. FARIAS, S

18. GIGUÈRE-RANCOURT, A

19. HACKETT, K

20. HAMMERS, DB

21. HARMELL, AL

22. JACOBSON, AJ

23. KAY, CD

24. KEMP, E

25. KIELY, T

26. LAST, BS

27. LEE, Y

28. LEE, Y

29. LIU, S

30. LOWE, DA

31. LOWE, DA

32. MAPSTONE, M

33. MARTINEZ, MN

34. MASEY, A

35. MEINERDING, ME

36. MILLER, JB

37. MINOR, A

38. MOORE, $\mathrm{C}$

39. NELSON, NW
Increased Delta as a Compensatory Mechanism During Working Memory in High-Performing Patients with Schizophrenia

\section{Poster Session 8. Aging \& Dementia 2 Acadia}

Dementia (Alzheimer's Disease)

Consistent Report of Subjective Cognitive Decline Longitudinally is Associated With Amyloid Burden Alzheimer Disease Biomarkers and Driving Space in Clinically Normal Older Adults: Role of Spatial Navigation Abilities

Longitudinal Cognitive Asymmetry and Decline in Alzheimer's Disease Patients

Quality of Life in an Ethnically Heterogeneous Community-Based Cohort of Patients with AD

Neuroticism is Associated With Tau Accumulation in Preclinical Autosomal Dominant Alzheimer's

Disease

The Effect of Generation Gap on Informant Ratings using the IQCODE

Cognitive Components of Everyday Functioning in Alzheimer's disease and Lewy Body Dementia The Benefits of Phonemic Cueing in Alzheimer's Disease Patients' Naming Performance

Verbal Fluency Discrepancies: Are They Pathognomonic Indicators of Alzheimer's Type Dementia?

Relationship Between MRI Measures of Cerebral Arterial Flow and Perfusion in Asymptomatic Adults At-Risk for Alzheimer's Disease and Older Adults with Cognitive Impairment

Factor Structure of the 15-Item Geriatric Depression Scale and Predicting Progression to Alzheimer's Disease

Mood and Personality Characteristics Influence Metamemory Accuracy in Healthy Older Adults

The Relationship Among the Judgment of Line Orientation Test, Spatial Abilities, and Executive Functioning

Early Parental Death and Sibship Size: Investigating the Causal Relationship to Alzheimer's Disease in a Multiethnic Cohort

Neurocognitive correlates of psychotic symptoms in a community-based cohort of Mild Cognitive Impairment and Alzheimer's Disease

Using the RBANS, Premorbid Intelligence, and Educational Attainment to Classify Dementia Patients as Normal or Impaired on the ILS Health and Safety Scale

Compensation Strategy Use Among Older Adults: Association with Diagnostic Status,

Neuropsychological Function, and Everyday Function

Nutritional Supplementation in Prevention and Treatment of Cognitive Impairments Associated with Alzheimer's disease

NIH Toolbox Cognition Battery and PROMIS Measures for Detecting Preclinical Alzheimer's Disease Amyloid Positivity using [18F]Flutemetamol-PET and Cognitive Deficits in Non-Demented Community-Dwelling Older Adults

Neuropsychiatric and Cardiovascular Predictors of Alzheimer's disease

Deep Gray Matter Correlates of Symptoms on the Frontal System Behavior Scale in Behavioral Variant Dementia and Early-Onset Alzheimer's Disease

Are Measures of Intraindividual Variability Sensitive to the Preclinical Stage of Alzheimer's Disease in Elders at Genetic Risk?

Sleep Disturbance and Risk of Cognitive Decline in the ADNI Cohort

The Contribution of Neuropsychological Performance, Behavioral and Functional Measures in High\& Low-likelihood Alzheimer's Disease Profiles

Sibling history of dementia is associated with cortical thickness in older adults

Neuropsychological Correlates of Apathy in Cognitively Healthy Middle-Aged Individuals at Risk for Alzheimer's Disease

The Relationship Between Apathy and MMSE in Cognitively Healthy Middle-Aged Individuals at Risk for Alzheimer's Disease

Cognition, Neuropsychiatric Symptoms and Everyday Functioning in Latino Older Adults

Are Changes in Metacognition the First Sign of Prodromal Alzheimer's?

Sex Differences in Cognitive Dysfunction Due to Alzheimer's Disease

Non Standardized Plasma Collection and Handling Procedures Represent Significant Challenges for Blood Based Metabolomic Biomarkers of Alzheimer's Disease

Marital Status and Dementia Risk among Ethnically Diverse Older Adults

Moderating Effects of Cognitive Reserve on Primacy Recall and Alzheimer's Disease Pathology

Correlates of Dependency in a Community Cohort of Individuals with Alzheimer's Disease

Sensitivity of the Montreal Cognitive Assessment Memory Scores to Hippocampal Volume in a Neurodegenerative Disease Sample

Family history and subjective cognitive decline in non-demented older adults

Diagnostic Accuracy of Pathologically Confirmed Alzheimer's Disease in the NACC UDS:

Computational Classification Using Psychometric Measures

Relative predictive values of the MMSE and ADAS-Cog for dementia stage and daily function in Alzheimer's disease 


\author{
40. OLESON, S \\ 41. OLIVEIRA, AA \\ 42. OSBORN, KE \\ 43. PASE, MP \\ 44. PIERS, RJ \\ 45. PILLEMER, S \\ 46. RAHMAN-FILIPIAK, AA \\ 47. ROBBINS, J \\ 48. RODRIGUEZ, IG \\ 49. ROLL, E \\ 50. SHOUEL. HL \\ 51. SUNDERMANN, EE \\ 52. VENKATESAN, UM \\ 53. VILA-CASTELAR, C \\ 54. WAGNER, G \\ 55. WATSON, CW \\ 56. WEISSBERGER, G \\ 57. CAVACO, S \\ 58. ROGERS, S
}

59. ROGERS, S

60. CAVACO, S

61. ALAMEDDINE, LR

62. APPLEMAN, ER

63. CROWLEY, SJ

64. DEVITO, AN

65. DHIMA, K

66. ELLIS, S

$\begin{array}{ll}\text { 67. } & \text { FEE, RJ } \\ \text { 68. } & \text { HARRISON, CE } \\ \text { 69. } & \text { HENDERSHOTT, T } \\ \text { 70. } & \text { HERGERT, DC } \\ \text { 71. } & \text { LAFO, JA } \\ \text { 72. } & \text { LOPEZ, FV } \\ \text { 73. } & \text { MCINERNEY, KF } \\ \text { 74. } & \text { MOFFETT, K } \\ \text { 75. } & \text { PLUIM, CF } \\ \text { 76. } & \text { ROHL, B } \\ \text { 77. } & \text { ROTHLIND, JC } \\ \text { 78. } & \text { SALAZAR, R } \\ \text { 79. } & \text { SALAZAR, R } \\ \text { 80. } & \text { SALAZAR, R } \\ \text { 81. } & \text { SCOTT, BM }\end{array}$

Dietary polyunsaturated fat and cerebral glutamate: interaction with Apolipoprotein E genotype Neuropsychological performance differences between two groups of probable-AD patients from different areas of Brazil

Adverse Vascular Risk Relates to CSF Biomarker Evidence of Axonal Injury Among Amyloid Positive Older Adults

Sugary Beverage Intake and Preclinical Alzheimer's Disease in the Community

How Much Risk is Genetic Risk for Dementia? Framingham Offspring Study

Gender Differences in Factors of Burden and Depression Among Dementia Caregivers

The Anticipatory Dementia Inventory (ADI): An application of the Health Belief Model to fear of dementia in middle- and older-aged adults

The Clinical Utility of the Neurobehavioral Examination in Alzheimer's Disease

The Impact of Cardiovascular Risk Factors (CVRF's) on Neurocognitive Performance in Mild Cognitive Impairment (MCI) and Alzheimer's Disease (AD)

Semantic Knowledge and Everyday Function in People with Dementia

Independent Associations Between Objective Versus Subjective Social Support and Cognition in a Racially Diverse Cohort

Does the Female Advantage in Verbal Memory Mask Alzheimer's Disease Pathology?

What Drives Driving: Differences in the Relationship of Visual Search and Sensory Binding to Driving Performance between Healthy Aging and Alzheimer's Disease

Long-term cognitive effects of Donepezil treatment in patients with Alzheimer's disease: The role of attention.

Pilot Study Examining the Neuropsychological Profiles of Dementia with Lewy Body and Alzheimer's Disease

Racial Discrimination is Associated with Cortical Thinning in Alzheimer's Disease Signature Regions in African American Older Adults

Diagnostic accuracy of memory measures in Alzheimer's dementia and Mild Cognitive Impairment: A systematic review

Higher levels of CSF phosphorylated tau correlate with younger age and poorer memory in

Alzheimer's disease

Clarifying the Types of Memory Deficits in Alzheimer's Disease

\section{Movement and Movement Disorders}

Examining the Effects of Anxiety on Cognition Among Those with Parkinson's Disease Criterion validity of UPDRS-I in the detection of cognitive impairmentin Parkinson's disease Depression Does Not Impact Verbal Fluency in Individuals with Early-Stage Tremor-Dominant Parkinson's Disease

Using Multigenerational Longitudinal Research to Examine for Cognitive Differences Pre-Diagnosis in Parkinson's Disease

Contribution of Cortical White Matter to Motor Sequence Learning in Parkinson's Disease

Neuropsychological Outcomes of a Combined CBT and Executive Skills Training Intervention for Anxious Parkinson's Patients

Hyperlipidemia in Parkinson's Disease: Protective Against Cognitive Decline?

A Study Designed to Examine the Feasibility of a Randomized Single-Blind Cross-Over Trial That

Will Assess the Effects of the Second Generation Dopamine Agonists, Pramipexole Prolonged Release and Ropinirole Modified Release, on Cued Recall Memory in Idiopathic Mild or Moderate Parkinson's Disease Without Cognitive Impairment.

Poor recognition memory in Parkinson's disease may indicate the comorbidity of Alzheimer's disease Specifying the Nonverbal Memory Impairments that Characterize Parkinson's Disease

Predictive Validity of the Mini Mental State Examination, Montreal Cognitive Assessment, and Mattis Dementia Rating Scale-2 in Parkinson's Disease

Awareness in Huntington's Disease

Construct Validity of the University of Florida DBS Cognitive Rating Scale: What cues neuropsychology to raise a red flag for DBS candidacy?

Does the Geriatric Depression Scale Measure Depression in Parkinson's Disease?

Does Exposure to Toxins Influence Cognition in Parkinson's Disease

Corticobasal Syndrome: A Unique Neuropsychological Profile Involving Unilateral Left Ideomotor Apraxia

Changes in Self- and Caregiver-Reported Frontal Behaviors in Parkinson's Disease: A Longitudinal

Study

Sleepiness Across the Cognitive Spectrum in Essential Tremor

Predictors of Multi-Domain Cognitive Decline following Deep Brain Stimulation Surgery for

Treatment of Parkinson's Disease

Parkinson's Disease Affects Category Switching

Predictors of Self-Perceived Stigma in Parkinson's Disease

Social Support Mediates the Relation between Depression and Motor Limitations of Parkinson's Disease

Emotion-semantic priming and electrocortical reactivity in Parkinson's disease 


\author{
82. SEGALÀ, L \\ 83. SPAT, J \\ 84. SPLIT, M \\ 85. STIVER, J \\ 86. SUN-SUSLOW, N \\ 87. SZYMKOWICZ, SM \\ 88. TAYLOR, BP \\ 89. TRAN, B \\ 90. VAN ETTEN. EJ \\ 91. VANDEBUNTE, AM \\ 92. WERTHEIMER, J \\ 93. WIGGINS, ME \\ 94. WYMAN-CHICK, KA \\ 95. YAFFE, B \\ 96. ZANE, KL
}

2:30-3:00 PM

3:00-4:00 PM

1. SULLIVAN, EV

4:00-5:30 PM

1. JOHNSON, SC

2. BARRY-TANNER, T

3. JOHNSON, SC

4. HASSENSTAB, J

5. SOLDAN, A

6. MANLY, JJ

7. JEFFERSON AL

4:00-5:30 PM

1. DOBRYAKOVA, E

2. DOBRYAKOVA, E

3. KAMAT, $\mathrm{R}$

4. BIJANKI, KR

5. GOVEROVER, Y

6. STROBER, L
The Role of Hypertension on Cognition in Parkinson's Disease

Neuropsychological Assessment Evaluating Candidacy for Deep Brain Stimulation in Children with Pharmaco-Resistant Movement Disorders: A Case Series

Rapid Eye Movement Sleep Behavior Disorder and Daytime Sleepiness are related to Poor Attention and Executive Function in Parkinson's disease

Cognitive and Motor Correlates of Depressive Symptoms in Parkinson's Disease

The Role of Social Support on Cognitive Functioning after Deep Brain Stimulation in Parkinson 's disease

Symptom Dimensions of Depression and Apathy and their Relationship with Cognition in Parkinson's Disease

CVLT-II Performance in Huntington's Disease and Parkinson's Disease

Neuropsychological Predictors of Functional Decline in Non-demented Parkinson's Disease Recall and Recognition Discriminability in Parkinson's Disease and Huntington's Disease

The Effect of Depression on the Cognition of those with Parkinson's Disease

Cognition and Parkinson's Disease: The Patient's Perspective

Category Fluency Association Index is Sensitive to Temporal Lobe Atrophy in Parkinson's Disease Neuropsychological Test Performance in Parkinsonism Without Dopaminergic Deficiency on [123I]FP-CIT SPECT Imaging

The Relationship of Motor Dysfunction and Memory Recognition Deficit in Parkinson's Disease Cognitive Slowing and Motor Slowing in Parkinson's Disease

\section{PM Coffee Break} Acadia Ballroom

\section{Plenary F. Contributions to Understanding the Dynamic Course of Alcoholism: An INS Legacy Presenter: Edith V. Sullivan Carondelet}

Contributions to Understanding the Dynamic Course of Alcoholism: An INS Legacy

\section{Invited Symposium 5. The Next Generation: A Look at Cohort Studies of People at Risk for Alzheimer's Disease \\ Chair: Sterling C. Johnson Carondelet}

The Next Generation: A Look at Cohort Studies of People at Risk for Alzheimer's Disease Will I Be Next?

The Wisconsin Registry for Alzheimer's Prevention (WRAP)

Correlating Rates of Change Between Cognition and Biomarkers in Middle Aged Adults at Risk for Alzheimer's Disease: The Adult Children Study

Hypothetical Preclinical Alzheimer Disease Groups and Longitudinal Cognitive Change

Offspring Study of Mechanisms for Racial Disparities in Alzheimer's Disease

The Vanderbilt Memory \& Aging Project: Study Design, Findings, and Future Directions

\section{Symposium 9. Depression in Clinical Conditions: Impact on Behavior, Neural Mechanisms and Quality of Life Chair: Ekaterina Dobryakova Bissonet}

Depression in Clinical Conditions: Assessment, Impact on Behavior, Neural Mechanisms and Quality of Life

Depressive Symptomology Modulates Cortico-Strtiatal Activation During Feedback Presentation in Individuals with Traumatic Brain Injury

Depression and its Correlates in the Context of HIV Infection

Stimulation of the Dorsal Cingulum Produces Euphoria and Positive Emotional Bias in an Epilepsy Patient Undergoing Invasive Presurgical Evaluation

Is Being Younger Better? Age, Depression And QOL in MS

Doctor, Am I Depressed? How to Appreciate the Intricacies of Depression in Neurological Populations and a Guide to Aide in Its Assessment 
4:00-5:30 PM

\author{
1. ARNETT, P \\ 2. GUTY, E \\ 3. GRIMA, NA \\ 4. ASKEN, BM \\ 5. ZAMZOW, J \\ 6. ROMAN, CA
}

4:00-5:30 PM

1. OSBORN, KE

2. GRILLI, MD

3. CASALETTO, KB

4. SALONER, R

5. BERTRAND, E

6. VOGEL, S

4:00-5:30 PM

1. BREARLY, TW

2. ELBIN, R

3. FEENSTRA, HE

4. GIOVANNETTI, T

5. STELMOKAS, J

6. WONG, CG

\section{4:15-5:30 PM}

1. BENNETT, L

2. BERGQUIST, TF

3. BINEY, F

4. CAMPBELL, ME

5. CHAPLIN, AP

6. CHIOU, KS

7. CLARK, AL

\section{Symposium 10. Comorbidities Associated with Neurocognitive Performance in Sports Concussion and MS Chair: Peter Arnett Salon D}

Comorbidities Associated with Neurocognitive Performance in Sports Concussion and MS The Relationship between Headache and Cognitive Impairment following Sports-Related Concussion Melatonin Supplementation Improves Sleep Disturbance Following Traumatic Brain Injury: Preliminary Results from a Randomized Controlled Trial

Physiological Effects of Delayed Removal from Activity Following Sport-Related Concussion as Evidenced in Serum Biomarkers

Sleep and cognitive function in relapsing-remitting multiple sclerosis

Structural Neural Correlates of Cognitive Functioning and Depression in Multiple Sclerosis:

Examining Similarities Across Primary and Secondary Factors

\section{Paper Session 12. Memory \\ Moderator: Roy P.C. Kessels \\ Salon E}

Cognitive Diagnosis Modifies the Effect of Cerebrospinal Fluid Biomarkers of Alzheimer's Disease, Neurodegeneration, and Axonal Injury on Episodic Memory Performance: The Vanderbilt Memory \& Aging Project

The Life Stories of Adults with Amnesia: Insights into the Contribution of the MTL to the HigherOrder Organization of Autobiographical Knowledge

Is "Learning" Episodic Memory? Distinct Cognitive and Neuroanatomic Correlates of Immediate Recall During Learning Trials Among Healthy Aging and Neurodegenerative Cohorts

Worth the Wait: Performance on a One-Week Delayed Recall Task is Associated With Medial Temporal Lobe Structures and Subjective Memory Complaints in Normal Adults

Cortical thickness and metamemory in cognitively diverse older adults

The Relationship Among Memory Performance and Hippocampal Subregion Volumes in a Memory Clinic Population

\section{Paper Session 13. Updating Neuropsychological Practice Moderator: Adam M. Brickman Salon F-H}

Neuropsychological Test Administration by Videoconference: A Systematic Review and Meta-Analysis Comparison of Patient Satisfaction following Face-to-Face and Telehealth Clinical Visits for SportRelated Concussion

Reliability and Validity of an Online Tool for Self-Administered Cognitive Assessment: the Amsterdam Cognition Scan

The Virtual Kitchen: Preliminary Data from A Novel Virtual Reality Test of Mild Difficulties in Everyday Function

Quantitative and Normative Volumetry Using Neuroquant: Association With Memory Performance in Healthy Older Adults and Mild Cognitive Impairment

Age-Related Hearing Loss and Verbal Memory Assessment

\section{Poster Session 9. ABI \& Intervention Acadia}

\section{Acquired Brain Injury (TBI/Cerebrovascular Injury \& Disease - Adult)}

The Role of Athletic Exposure on Neurocognitive Performance in Disability-Seeking, Retired National Football League Players

Relationship of Clinical Characteristics to Functioning in a Sample of Person with Mild Traumatic Brain Injury (TBI) Seeking Treatment

A Case of Significant Retrograde Amnesia and Loss of Autobiographical Memories Following Traumatic Brain Injury

A Comparison of the Buschke Selective Reminding Test and the California Verbal Learning TestSecond Edition in a Stroke Population

Comparing Neuropsychological Outcome in Active Duty Soldiers Following Complicated,

Uncomplicated, and Equivocal Mild Traumatic Brain Injury

Investigation of Response Time as a Process Variable in Metacognitive Functioning After Traumatic Brain Injury

Repetitive Mild Traumatic Brain Injury Moderates the Association Between Age and Cerebral Blood Flow of Medial Temporal Lobe Structures 


\section{ETTENHOFER, M}

9. EVANGELISTA, ND

10. FATOORECHI, S

11. FEDIO, AA

12. FEDIO, AA

13. FONG, AK

14. GAYNOR, LS

15. GENOVA, HM

16. GREIF, SM

17. GROSSNER, EC

18. HAMMOND, J

19. HARIK, L

20. HOFFMAN, SN

21. JURICK, SM

22. KAPLAN, E

23. KAUP, AR

24. KEATLEY, E

25. KEELAN, RE

26. KELLER, AV

27. KILLGORE, W

28. KIM, RT

29. KIRTON, JW

30. KORNBLITH, E

31. LARA-RUIZ, J

32. LEVITCH, C

33. LIPPA, SM

34. LIPPA, SM

35. LIPPA. SM

36. LIPPA, SM

37. LIPPA, SM

38. LIPPA. SM

39. LOPEZ, W

40. LOYNING, A

41. MALLECK. M

42. MASSA, J

43. MCCLINTOCK, KL
Neurocognitive Eye Tracking in Moderate-to-Severe Traumatic Brain Injury: Evidence for Enhanced Sensitivity to Impairment

Brain Derived Neurotropic Factor (BDNF) Val66Met Moderates the Association Between PTSD and Cortical Thickness in Veterans with History of Traumatic Brain Injury

Perceived Workload on Performance Validity Tests in Persons with and without Traumatic Brain Injury

Bringing the Real World Into the Classroom: Individuals With Brain Injury Educate Clinical Psychology Graduate Students

Functional Correlates of Patients' Current and Predicted Future Satisfaction With Their Aggression Following Traumatic Brain Injury

Functional NeuroCognitive Imaging: Rehabilitation of Neurovascular Uncoupling in Concussion Treatment

Alteration and/or loss of consciousness is associated with increased symptom report of PTSD, depression, sleep-related anxiety, and larger amygdala volume in OIF/OEF/OND Veterans Longitudinal White Matter Changes Associated with Executive Functioning Outcome in Chronic Moderate to Severe TBI

Acute Symptom Recall and Sub-Acute Sleep and Mood Dysfunction in Mild Traumatic Brain Injury Examining Metacognitive Accuracy in Moderate and Severe TBI

Trends in Helmet Use and Concussion Among Skiers and Snowboarders: Using an Innovative Data Collection Technique for Injury Prevention

Comparison of the Neurobehavioral Symptom Inventory and the Rivermead Postconcussion Questionnaire

Patterns of Performance and Symptom Validity Tests in Iraq and Afghanistan Veterans with Mild

Traumatic Brain Injury History

Contributions to Executive Dysfunction in Operation Enduring Freedom/Operation Iraqi Freedom (OEF/OIF) Veterans with Post-Traumatic Stress Disorder and Traumatic Brain Injury History Self-Discrepancy and Perfectionism: Two Factors Associated with Depression in Individuals with Traumatic Brain Injury

Lifetime Traumatic Brain Injury with Current Psychiatric Symptomatology Impacts Cognition among Late Middle-Aged Men: Findings from the Vietnam Era Twin Study of Aging (VETSA)

Use of the Depression Anxiety and Stress Scale (DASS) in Varsity Athletes for Baseline Concussion Assessment: Psychometric Properties, Comparison with Non-Athletes, and Frequency of Item Endorsement

The Role of Experienced Affect on Facial Emotion Perception Accuracy in Moderate to Severe Traumatic Brain Injury

The Role of Cognitive Functioning in Suicidal Ideation in Veterans with a History of Mild Traumatic Brain Injury and Comorbid Psychiatric Conditions

Blue Wavelength Light Therapy Increases Thalamic Grey Matter Volume Following Mild Traumatic Brain Injury

Brain Function and Task Performance Predict Self-Reported Disinhibition and Executive Function in Veterans with Mild-Moderate Traumatic Brain Injury

Differential diagnosis of repetitive mild traumatic brain injury and posttraumatic stress disorder:

Three illustrative cases

Neurocognitive Profiles Among Veterans with History of Traumatic Brain Injury

Anxious and Depressive Symptoms in Retired Professional Football Players: A Contrast with Persons

With and Without Moderate-to-Severe Traumatic Brain Injury

Recent and Long-Term Soccer Heading Exposure is Differentially Associated with Cognitive

Impairment in Adult Amateur Players

A Case Series Analysis of the Natural History of Neurocognitive Performance following Moderate,

Severe, and Penetrating Traumatic Brain Injury in U.S. Military Service Members

A Cross-sectional Perspective of Neurocognitive Outcome from Military-related Mild-moderate TBI in the Sub-acute Recovery Period and 1-year post-injury

Neuropsychological Outcome Following Moderate, Severe, and Penetrating Traumatic Brain Injury in U.S. Military Service Members

Predictors of Neurobehavioral Outcome 2-Years Following Mild-Moderate Traumatic Brain Injury

The Natural History of Psychological Symptoms following Moderate, Severe, and Penetrating

Traumatic Brain Injury in Military Service Members: A Case Series Analysis

The relationship between Self-reported Postconcussion Symptoms with Tau and Amyloid-beta 42

Levels following Military-related Mild-Moderate Traumatic Brain Injury

Role of Encoding and Retrieval in Activity Memory Following Traumatic Brain Injury

Comparing Artificial Grammar Learning and Natural Language Learning in Adults with Agrammatic Aphasia

Baseline Postconcussive Symptom Patterns in Athletes and Non-Athletes

The Utility of Guided Relaxation Meditation in Stroke Rehabilitation

Cognitive Effects of Traumatic Brain Injury Assessed with the Digital Clock Drawing Test at the Framingham Heart Study 


\section{MERZ, Z \\ 45. MEYER, J \\ 46. MOORE, RD}

47. MOORE, RD

48. MORENO, CC

49. MOSTI. C

50. PARIKH. SA

51. POLEJAEVA, E

52. RAD, H

53. RASKIN, S

54. RASKIN, S

55. REYNOLDS, M

56. ROY, AA

57. SALINAS, SL

58. SAUVE, W

59. SCHILLING, SL

60. SEAMAN, B

61. SICARD, V

62. SNYDER, AR

63. SORG. SF

64. SULLAN, M

65. TERWILLIGER, V

66. TROYANSKAYA. M

67. TROYANSKAYA, M

68. UKUEBERUWA, DM

69. VOELBEL, G

70. WALKER, A

71. WEBER, E

72. WILLIAMSON. E

73. ALI, S

74. SHEEHAN, JC

75. BALDO, J

76. BROWN, KD

77. CIABATTONI. R

78. HUSSEY, J

79. IMBEAULT. H

80. KIM, K

81. LEON-CARRION, J

82. LEON-CARRION. J

83. MACOUN, S

84. MARTON, K

85. MCGARTHY, J

86. MCFARLAND, CP
Current Public Knowledge Pertaining to Traumatic Brain Injury (TBI): Influence of Demographic Factors, Social Trends, and Sport Concussion Experience on the Understanding of TBI Sequelae Post-Concussion Depression and Cognitive Functioning in Collegiate Athletes

Slow-to-recover athletes exhibit worse emotional status, reduced cognitive performance, and decreased neurophysiological function relative to asymptomatic athletes with and without a history of concussion

The Moderating Influence of Learning Disabilities on the Neuropsychological and Neurophysiological Health of Athletes with a History of Concussion

Effects of Sport Type and Concussion History on Baseline Serum Biomarker Concentrations in Collegiate Athletes

Does the NCAA Concussion Management Plan Matter? How Concussion Education Source Influences Symptom Knowledge in College Athletes

Pre-surgical Executive Functioning in Adult Chiari Malformation Type 1

Task-Irrelevant Interference Mimics Effects of Traumatic Brain Injury on Cognitive Control

The Role of Cognitive Speed and Control in Encoding Deficits Following Traumatic Brain Injury

A Systematic Approach to Prospective Memory Treatment

The Effect of the Cue-Intention Relationship on Prospective Memory Performance in Individuals with Traumatic Brain Injury

Personal Experience with mTBI on Symptom and Recovery Expectations

Characterization of concussion: Injury, symptoms, and reporting behaviors in professional rodeo athletes

Patients with Post-Concussion Syndrome: Impact of Duration of Cognitive Complaints on

Neuropsychological Testing

Sex Differences in Psycho-affective Outcome following Concussion in University Athletes

Suicidality in Military Veterans with Traumatic Brain Injuries

The Effects of Comorbid Mild Traumatic Brain Injury and Alcohol Use Disorder

Persistent Executive Dysfunction Following a Sport-Related Concussion Assessed with a Task-

Switching Paradigm

Symptom Tolerability of Brief Aerobic Exercise After Mild Traumatic Brain Injury

Elevated Intra-individual Variability on Tests of Executive Functions in Veterans with Mild

Traumatic Brain Injury

The relationship between loss of consciousness, sleep-related disorders, and volumetric brain changes in veterans with mild traumatic brain injury

The Role of Oral Contraception on Outcomes Following Concussion in Female Collegiate Athletes: A Pilot Study

Community Functioning, Emotional Status, and Social Cognition Following Combat

Deployment - WITHDRAWN

Post-Deployment Community Integration and Participation - WITHDRAWN

Persistent Symptom Clusters and Objective Cognitive Functioning among Veterans with TBI History Prospective Longitudinal Investigation of White Matter Integrity in Mild Traumatic Brain Injury The Dunning-Kruger Effect and Traumatic Brain Injury

Frontal Systems Behavioral Dysfunction Predicts Employment Status in Moderate-to-Severe TBI

Premorbid IQ Predicts Persistent Postconcussive Self-Reported Symptoms Among Returning

Veterans

\section{Cognitive Intervention/Rehabilitation}

Investigating the Impact of a Computer-Based Cognitive Intervention on Children with Attention/ Executive Function (EF) Problems

Caribbean Quest: Investigating the Impact of a Computerized Cognitive Training Intervention for Children on Cognitive and Academic Outcomes

Feasibility of a Mindfulness-based Stress Reduction Program for Stroke Patients

Development of a User-Friendly Digital Memory Notebook: An Iterative User-Centered Development Process

Impact of Strategy Type on Adherence to a Behavioral Regimen

Meaningful recovery via long-term integrated care: A case study on West Nile Encephalitis

Impacts of a Multi-Domain Cognitive Training in Geriatric Patients Diagnosed with Mild

Neurocognitive Disorder

The efficacy of the Cogmed Working Memory Training ${ }^{\circledR}$ depending on the level of sluggish cognitive tempo (SCT)

Neuropsychological markers for safe driving

Neuropsychological rehabilitation of dysfunctional sphincter control

Cognitive rehabilitation in schools: training the paraprofessional trainer

Relationship Between Baseline Functional Status and Executive Function Training Outcome in

Veterans with Chronic TBI

The Use of Prospective Memory Training for Improving Academic Self-Efficacy in an Undergraduate Population: A Pilot Study

Enhancing Memory and Imagination Improves Problem-Solving Among Individuals with Depression 


\author{
87. MULLEN, C \\ 88. MURDAUGH, D \\ 89. NOVAKOVIC-AGOPIAN, T \\ 90. O'CONNELL, ME \\ 91. PATEL, S \\ 92. PERNA, R \\ 93. POUTIAINEN, E \\ 94. PYNE, S \\ 95. RAMIREZ, FE \\ 96. RAPHAIL, A \\ 97. ROSSI, C \\ 98. VICKERS, KL
}

1. LORING, D

7:20-8:50 AM

1. MARK, VW

9:00-9:30 AM

9:00-10:15 AM

1. ANDERSON JR

2. BEDARD, A

3. CASEY, JE

4. CASSILL, C

5. CRAUN, E

6. DEVITO, AN

7. DEWEY, D

8. DUDA. TA

9. FEDER, A

10. FEIRSEN, N

11. FERENC, L

12. GAU, S

13. GRAVES, B

14. JORGENSON, M

15. KANDASAMY, A
Strategy Training Improves Route Recall: Preliminary Report from a Randomized Controlled Trial of Cognitive Rehabilitation

Efficacy of an Individualized, Manualized Cognitive Remediation Program to Improve Adaptive

Functioning in Pre-Adolescents with Neurological Impairments

Goal-Oriented Attentional Self-Regulation Training in Veterans with PTSD and mTBI

Remote Neuropsychology for Rural Dementia Care: Feasibility of, and Adaptations Required for,

Videoconferenced Support Group and Cognitive Rehabiliation

Simulator Discomfort Across Neurological Populations: Who is Most Affected?

Memory Dysfunction Due to Hemolytic Anemia and Hemochromatosis: A Case Study

Technology Assisted Rehabilitation for Persons With Dementia and Their Family Members - A

Controlled Intervention Study - WITHDRAWN

Effectiveness of a Cognitive Intervention on Metacognitive Awareness in Children with Attention, Self-Regulation, and Executive Function Difficulties

Violation Conscience is Related to Worse Mental Health Among Geriatrics

Eye-Tracking in Neuropsychology: Using the Eyes as the Window to the Mind

The use of b ioengineering techniques in cognitive rehabilitation: a pilot study

Defining the Relationship Between Executive Functioning and Consistency in Regimen Adherence

\section{SATURDAY, FEBRUARY 4, 2017}

\section{CE Workshop 11. How Does Evidence-Based Practice Address the 'Replication Crisis' in Clinical Neuropsychology? \\ Presenters: David Loring, Stephen C. Bowden \\ Bissonet}

How Does Evidence-Based Practice Address the 'Replication Crisis' in Clinical Neuropsychology?

CE Workshop 12. Constraint-Induced Therapies for Neurological Disorders: Contemporary Findings, Application to Disorders of Movement, Aphasia, and Visual Perception, and Increased CNS Neuroplasticity Presenter: Victor W. Mark

Salon D

Constraint-Induced Therapies for Neurological Disorders: Contemporary Findings, Application to Disorders of Movement, Aphasia, and Visual Perception, and Increased CNS Neuroplasticity

\section{INS Business Meeting (Business \& Beignets) Carondelet}

\section{Poster Session 10. Peds 2 Acadia}

\section{ADHD/Attentional Functions}

Differential effects of coffee on sustained attention for good and poor sleepers: Is coffee truly the solution?

Cognitive and Emotional Control in Youth With ADHD, and the Impact of Stimulant and NonStimulant Treatment

Preliminary Validation of the BASC-2 in a Canadian Pediatric Sample with and without ADHD Differences of Attentional Impairment in Obstructive Sleep Apnea and ADHD

Differential working memory abilities for youth with attention deficits

Do Anxiety Symptoms Moderate Working Memory Performance in Underserved Children?

Quality of Life in Adolescents with Developmental Coordination Disorder and Attention Deficit/ Hyperactivity Disorder - WITHDRAWN

Attenuated Graphomotor Procedural Learning in Children and Adolescents with ADHD

Examination of Revisions to a Measure Designed to Detect ADHD Simulators

Executive Functioning and Hyperactivity in Youth with ADHD

Parent-Reported Adaptive Functioning in Preschoolers With or Without ADHD

Comparison of neuropsychological functioning between adults with early- and late-onset DSM-5

Attention-Deficit/Hyperactivity Disorder

Predicting Academic Achievement Using Intelligence, Executive Functioning, and Socioeconomic Status in Children With and Without ADHD

Impact of ADHD on Receptive Language in Children With and Without Language Impairment

The Influence of Impulsivity on Standardized Digital Test Performance 


\section{KINGERY, K \\ 17. KIVISTO, LR \\ 18. LEVITCH. C \\ 19. LONGORIA, J \\ 20. LOTT, M \\ 21. LUNDERVOLD, AJ \\ 22. LUNDERVOLD, AJ \\ 23. MARTINELLI, M \\ 24. MARTINELLI. M \\ 25. MCGLADE, E \\ 26. MLODNICKA, A \\ 27. NELSON. JM}

28. PHILLIPS, AM

29. ROSCH, KS

30. ROSCH, KS

31. SIMONE, AN

32. SWEENEY, KL

33. TADROUS-FURNANZ, SK

34. VOGT, E

35. WOOTEN, K

36. KIRSCH, A

37. KIRSCH, A

38. AUSTIN, CA

39. CANNON, A

40. DEASLEY, S

41. GAUDET, CE

42. GAUDET, CE

43. HEARPS, S

44. HENNRICK, H

45. JOELSON, S

46. ZABEL. TA

47. LALIBERTE DURISH. C

48. LEAFFER, EB

49. LUCCHETTI, A

50. MERCER, EN

51. O'BRIEN, AM

52. O'DESKY. I

53. RACH, A

54. RAO, R

55. RYAN, M
Brain Activity During Periods of Longer Reaction Times: Event-Related Potential Comparisons of Children With and Without ADHD

Sleep Problems in Children With and Without ADHD Using the BEARS Sleep Disorder Screening Tool

Emotion Attribution Impairments, Biases, and Development in Children with Attention Deficit/ Hyperactivity Disorder and Severe Emotion Dysregulation

A revised model of attentional constructs within the Test of Everyday Attention for Children: Results from exploratory factor analysis with a mixed clinical sample

The impact of sleep restriction on sustained attention among healthy adolescents.

Early inattentive behavior predicts high school academic achievement across two culturally and diagnostically diverse samples - WITHDRAWN

Prediction of academic achievement in adolescents from teacher reports of inattention in childhood a classification study - WITHDRAWN

Binge eating is associated with delay discounting and BMI in children with ADHD

Investigating the Impact of Cognitive Load and Motivation on Response Control in Relation to Delay Discounting in Children with ADHD

Speed and Attention in Veterans with and without a History of Suicide Attempts

Impact of Neurodevelopmental Genes on the Trajectory of ADHD severity: A Pilot Study

Comparison of Adolescents and Adults With Purely Inattentive Type ADHD and Combined Type

ADHD on Speeded Cognitive and Academic Measures: Implications for the Sluggish Cognitive Tempo Construct

Symptoms of Hyperactivity/Impulsivity and Increased Error Rates on Graphomotor Tasks Using a Digitizing Tablet

Differential correlations between subcortical volumes and delay discounting in children with and without ADHD

Neuropsychological Correlates of Delay Discounting in Girls and Bovs with ADHD

Persistence of ADHD Symptoms, not Remission, is Associated with Working Memory Difficulties at 8 -vears-old

Developmental Trajectory of Motor Deficits in Preschool Children with and without ADHD

Does processing speed contribute to the far transfer effects of Cogmed Working Memory Training on Reading Comprehension?

Convergence of ADHD Symptom Report and Neuropsychological Tests

Addressing the Sustained Attentional Problems of ADHD Ultra-rapid Metabolizers

Discriminative Power of Rey-Osterrieth Complex Figure Test for Attention-Deficit/Hyperactivity

Disorder Compared to Other Attention and Executive Functioning Measures

\section{Assessment/Psychometrics/Methods (Child)}

Correlates of Rey-Osterrieth Performance Across Memory, Attention, and Visual-Spatial Functioning in a Referred Pediatric Sample

Parent Expectations and Change in Parent Efficacy in Pediatric Neuropsychological Evaluations Improvement or Inflation? Analysis of the Adaptive Behavior Assessment System (ABAS-II Versus ABAS-3) in a Clinical Sample

Concurrent Validity of the BDEFS-CA in a Canadian Sample of Children with and without ADHD

Appraising Neuropsychological Impairment in Children with Severe Psychiatric Disorders: Applying Predicted Base Rates

Rates of Neuropsychological Test Completion Within a Pediatric Inpatient Sample

Pediatric Evaluation of Emotions Relationships and Socialization (PEERS): Interim Analysis of Clinical Data

Informant Reports of Children's Cognitive Abilities Affected by Relationship Factors

Effects of Infant Social-Emotional Problems on Cognitive Performance

Patient Experience of Neuropsychological Assessment: Preliminary Findings from a Pediatric (Ages 5-18) Patient Sample

Convergent and Divergent Validity for a Measure of Psychological Resilience in Children with Mild

Traumatic Brain Injury

Is the WISC-V Yielding Lower Scores? Comparisons of the WISC-IV and WISC-V in Pediatric Epilepsy

A Pilot Study of Critical Flicker Frequency in a Clinical Pediatric Sample

Mullen Early Learning Composite: A Predictor of Later Intelligence Quotient Scores

Confirmatory Factor Analysis of the BDEFS-CA in a Canadian Sample of Children with and without ADHD

WISC Disparity Can Rule In NLD Not Rule Out

Age-Based Child-SCAT3 and SCAT3 Normative Values Using a Youth Football Population

The Relationship of Executive Function and Attention with Sleep Duration in Children with

Obstructive Sleep Apnea

Predictive Utility of Performance-Based and Rating Scale Measures of Inhibition in Preschool Children 


\section{SCHELLER, AD \\ 57. SCHNEIDER, H \\ 58. SHIPLEY, EM \\ 59. STEPHAN, C \\ 60. STEPHAN, C \\ 61. STEPHAN, C \\ 62. WEBER, E \\ 63. WRIGHT, I \\ 64. HOLDNACK, JA}

65. HOLDNACK, JA

66. ALBERT, P

67. AMARAL, JL

68. ARMENGOL, CG

69. ARRINGTON, C

70. CHILD, A

71. CIRINO, PT

72. DAVIS, K

73. GERST, EH

74. HARDY, LM

75. HOKKANEN, L

76. HUSTON-WARREN, EA

77. MANO, Q

78. MARGOLIS, AE

79. REESE, K

80. RIGGALL, E

81. ROBERTS, A

82. SALVADOR-CRUZ, J

83. SALVADOR-CRUZ, J

84. SEESE, S

85. TANAKA, H

86. TIMPANO SPORTIELLO, M

87. WESONGA. EM

88. WINTER, R

89. SRNKA, KD

\section{0:00-11:30 AM}

1. BRICKMAN, AM

2. BRICKMAN, AM

3. BARCH, DM

4. BILDER, RM

5. AU, R
Examination of Automatized Sequences Task as an Index of Performance Validity in General Pediatric Neuropsychological Evaluations

Contrasting Parent vs. Teacher Ratings on the BRIEF-P in Preschool Children with and without ADHD

The Test of Memory Malingering (TOMM): Use with Deaf or Hard of Hearing Children Referred for Neuropsychological Assessment

Cross-setting rater agreement on ADHD symptomatology: What's a clinician to do?

Does adaptive competence rely on better language-based or visually-based reasoning skills?

Parent satisfaction ratings of pediatric neuropsychological assessment services

Assessing effort with the TOMM and Automatized Sequences in a child inpatient setting

A Robust Statistical Approach to Classification of Developmental Change in Cognitive Test Scores

Does Cognitive Variability Differentiate Children with Clinical Disorders from Controls

\section{Learning Disabilities/Academic Skills}

Application of WISC-V Multivariate Base Rate Data to Children with Learning Disorders

Receptive Language Moderates the Effects of AAC Intervention on Communication Skills in Children with Developmental Delays

Service Delivery Outcomes after Assessment in Children with Learning Disorders

Neurological soft signs (NSS) as potential risk indicators of disrupted neurodevelopment in a Mexican sample of elementary school children

Pre-Intervention White Matter Microstructural Integrity Predicts Improvements in Reading Scores in Developmental Dyslexia

Shared and Unique Predictors of Math, Reading, and Attention Skills in Ninth Grade Children

Longitudinal Algebra Prediction for Early versus Later Takers

Disrupted Amygdalar Subregion Functional Connectivity As A Biomarker For Anxiety Symptoms

Associated With Reading Disorder

Processing Speed in Late-Elementary School Children: Examination of the Structure and Its Relation to Reading

Foundational Literacy Skills Among Diverse Children in a Clinic Sample

Midlife Cognition in a Birth Risk Cohort - 40 Year Follow-Up

Executive Functions and Self-Regulated Learning as Predictors of Math Achievement: A Path

Analytic Framework

Attentional control associated with sensitivity to the statistical regularity of subword orthography

Intrinsic Functional Connectivity Within The Left Hemisphere Reading Network And Associations

With Phonological Ability In Children With And Without Reading Disorder

As Easy as ABC? Deaf or Hard-of-Hearing Students' Alphabetic Knowledge and Recitation

Modeling Implicit Sequence Learning in Developmental Dyslexia with and without Specific Language Impairment

Cognitive Correlates of Academic Learning in Fetal Alcohol Spectrum Disorders

Executive Function and Reading Comprehension: Performance of Mexican 9-year-olds on a Non-

Linguistic measure of inhibition.

Neurological Soft Signs (NSS) as Potential Risk Indicators of Disrupted Neurodevelopment in a

Mexican Sample of Elementary School Children

What Do Children with ADHD and ASD Look Like in the Mainstream Classroom? Examining

Executive Functions in School

Slow Reading: A New Neurobiological Phenotype

Developmental Dyslexia and Working Memory

Comorbidity of Learning Disabilities: Prevalence Rates of Co-occuring Reading Disability, Writing

Disability, Math Disability, ADHD, and Other Psychiatric Disorders in a School Sample

Functional MRI Overlap Between ADHD and Developmental Dyslexia on Alternative Force Choice

Tasks: A Meta-Analytic Study

\section{Epilepsy/Seizures}

Intraindividual Variability in Sustained Attention Mediates the Relationship between Age of Epilepsy Onset and IQ and Academic Achievement

Invited Symposium 6. Translational Neuropsychology: Contemplating the Past and Looking Toward the Future

Chair: Adam M. Brickman Carondelet

Translational Neuropsychology: Contemplating the Past and Looking Toward the Future Dietary Flavanols, Hippocampal Subfields, and Cognitive Aging: A Translational Neuropsychology Story

Connectomics and the Brain: Past, Present and Future

Neuropsychological Models: Past, Present and Future

Next Generation Neuropsychology: Digital Biomarkers and Big Data 
6. BAUER, RM

10:00-11:30 AM

1. DROZDICK, L

2. DROZDICK, L

3. NAKONECHNY, A

4. KEMP, S

5. SHAFER, D

10:00-11:30 AM

1. GERNER, GJ

2. NORTHINGTON, F

3. GRAHAM, E

4. PORETTI, A

5. GERNER, GJ

6. BURTON, J

10:00-11:30 AM

1. MEWBORN C

2. SIMON, SS

3. BRENNER, E

4. BELLEVILLE, S

5. KESSELS, RP

6. POLSINELLI, A

10:00-11:30 AM

1. RYAN, NP

2. PULSIPHER, DT

3. BELLEROSE, J

4. MCDONALD, S

5. PRESSON, N

6. TREBLE-BARNA, A
An Interactive Translational Platform for Investigating Age-Related Memory Decline

Symposium 11. Development and Adaptation of Assessment Instruments: Best Practices, Legal Issues, Training, and Lessons Learned

Chair: Lisa Drozdick

Bissonet

Development and Adaptation of Assessment Instruments: Best Practices, Legal Issues, Training, and Lessons Learned

Developing Tests for Global Use

Translation of Assessments: Best Practices and Lessons Learned

Developing Neuropsychology Training Programs in Colombia

Legal Requirements and Recommendations Around Test Adaptation and Translation

Symposium 12. Neonatal Hypoxic-ischemic Encephalopathy in the Posttherapeutic Hypothermia Era: How does a Multi-disciplinary Approach from Bench to Bedside Help Us Understand a Shift in Brain Injury

Patterns and Neurobehavioral Outcomes?

Chair: Gwendolyn J. Gerner

Discussant: Martha Denckla

Salon D

Neonatal Hypoxic-ischemic Encephalopathy in the Post-therapeutic Hypothermia Era: How does a Multi-disciplinary Approach from Bench to Bedside Help Us Understand a Shift in Brain Injury Patterns and Neurobehavioral Outcomes?

Imaging the Spatiotemporal Progression of White Matter Injury after Neonatal Hypoxia Ischemia Blood Biomarkers for Evaluation of Perinatal Encephalopathy

White Matter Injury in Neonatal Hypoxic-ischemic Injury After Therapeutic Hypothermia: Qualitative and Quantitative Analysis of Conventional and Advanced Neuroimaging Techniques Patterns of White Matter Injury and Neurobehavioral Outcomes Following Neonatal Asphyxia and Treatment with Therapeutic Hypothermia

Language Development Following Neonatal Hypoxic-Ischemic Encephalopathy Treated with

Therapeutic Hypothermia

\section{Paper Session 14. Cognitively Based Interventions in Aging Moderator: Sylvie Belleville Salon E}

Cognitive interventions for older adults: A systematic review and meta-analysis of randomized controlled trials

Cognitive and Neuroimaging Changes After Mnemonic Strategy Training in Amnestic Mild Cognitive Impairment: a Randomized, Single-Blind Study

Changes in Resting-State Neural Networks After Memory Training in Amnestic Mild Cognitive Impairment

Cognitive training in persons with MCI has durable effect on memory and generalizes to daily life: Results from the MEMO+ randomized controlled trial

Structured Relearning of Everyday Tasks in Dementia: The Randomized Controlled REDALI-DEM

Trial on Errorless Learning

Mindfulness Training For Improving Cognitive And Emotional Functioning In Healthy, Non-

Meditating Older Adults

Paper Session 15. TBI Across the Lifespan

Moderator: Suzanne Penna

Salon F-H

Uncovering the Neural Correlates of Cognitive, Affective, and Conative Theory of Mind in Paediatric Acquired Brain Disorder: Evidence from Traumatic Brain Injury - WITHDRAWN

Postconcussive Symptoms in Children and Adolescents Are as Common in Other Neurologic/

Neurodevelopmental Disorders as They Are in Concussion

Persistent socio-cognitive clouding following preschool mild TBI

Social Cognition After TBI: Its All About Connections

Quantitative High Definition Fiber Tracking Metrics Differentiate Healthy Control and Chronic TBI Groups

Influence of Dopamine-Related Genes on Neurobehavioral Recovery following Traumatic Brain Injury During Early Childhood 
10:00-11:30 AM
1. SIKKES, S
2. HESSEN, E
3. BUCKLEY, R
4. GIFFORD, $\mathrm{K}$
5. COSENTINO, S
6. APPLE, A

\section{0:30-11:45 AM}

1. WERRY, AE

2. ABRAHAM, N

3. BAILEY, BA

4. BARBOZA, M

5. BERL, M

6. CARMASIN, IS

7. CARVALHO, J

8. COMBS, HL

9. DUGGER, AJ

10. DUVALL, SW

11. EULER, M

12. FALLOWS, R

13. FASANO, ME

14. FRANCHOW, EI

15. HARTLEY, N

16. HAZLETT ELVERMAN, K

17. HOLCOMBE, JS

18. HOLLAND, AK

19. JOHN, SE

20. JOHNSON, N

21. KARR, JE

22. KNEPP, MM

23. KURNIADI, N

24. LE, T

25. MACKIE, M

26. MAYNARD, T

27. MCCUDDY, WT

28. MCLEAN, E

29. MORAN, EE

\section{Paper Session 16. Subjective Cognitive Complaints Moderator: Sietske Sikkes Salon A-C}

Subjective Cognitive Decline and Preclinical Alzheimer's Disease: Harmonization of Measurement Instruments

Subjective cognitive impairment is a predominantly benign condition in memory clinic patients followed for 6 years. The Gothenburg-Oslo MCI study.

Region-specific tau and B-amyloid effects on subjective cognitive concerns in the Harvard Aging Brain Study

Relation of cerebrospinal fluid markers of Alzheimer's disease pathology and subjective cognitive decline; the Vanderbilt Memory and Aging Project

The role of domain-independent health perceptions in Subjective Cognitive Decline

Elevated hippocampal functional connectivity related to memory in breast cancer survivors with selfreported cognitive concerns

\section{Poster Session 11. Cognition Acadia}

\section{Cross Cultural}

Influence of Demographic Variables on Measures of Memory and Language in Older Adults of Different Races

\section{Executive Functions/Frontal Lobes}

Examination of Cognitive Functioning Among Holocaust Survivors Parent-Reported and Performance-Based Changes in Inhibitory Control Following Parent-Child Interaction Therapy (PCIT) in a Pediatric TBI Sample

Preliminary data for comparison of written verbal and non-verbal fluency

Executive Functioning Profiles in Children with Intellectual Disability

Stability of Self-Rated Executive Dysfunction in MCI and Older Adults With Subjective Cognitive Dysfunction

Anxiety and Sleep Dysfunction Predict Executive but not Memory Dysfunction in Healthy Adults Deep Brain Stimulation for Parkinson's disease: An Investigation of Post-surgical Self-regulation and Executive Functioning

Development of Executive Functions: From 5 to 24 Years of Age

Relationship Between Parent Report of Executive Function and Naturalistic Observational Coding in Preterm and Full Term Preschoolers

The Effect of Novelty on Motor Control in Healthy Participants

The Relationship between Interference and Inhibition in a Mixed Clinical Sample

Concurrent Validity of the Behavior Rating Inventory of Executive Function in Clinically-referred Children who are Deaf or Hard-of-Hearing

Naturally-Occurring Expressive Suppression in Older Adulthood: Beyond Executive Functioning

Risky Sexual Behaviors in Homeless Youth: The Influence of Executive Functioning and Depression on the Condom Use of Homeless Youth in Chicago

The Importance of Heart Rate Variability to Executive Functioning Across the Lifespan

Need for Cognition and its Relation to Self-Reported Executive Dysfunction

Assessing Diminishment of Frontal Lobe Function in Trait Aggressive, Violent Prone Men:

Examining Changes in Performance on a Cognitive Estimation Task and Grip Strength Measures

Using a Dual Task Approach

The Unity and Diversity of Neuropsychological Tasks of Executive Functioning: Construct and Ecological Validity of Common Assessment Measures

Developmental Trajectories of Strategic Processing in Children with Phenylketonuria

Multivariate Assessment of Executive Functions: Frequency of Low Scores on the Delis-Kaplan Executive Function System

Rey-Osterrieth Complex Figure Task Performance and Negative Affect Predict Emotion Regulation and Health Behavior

Specificity of Disinhibition in Overweight and Obese Children

Executive Function is Associated with Sleep Disruptions in Young Adults with Depression

Common Deficits of The Cognitive Control Network in Three Neuropsychiatric Disorders

Objective Measurement of Sleep by Smartphone Application - Comparison with Actigraphy and

Relation to Cognition, Mood, and Self-Reported Sleep

The Role of Resting Versus Stress-Induced Autonomic Regulation on Inhibitory Control Performance Across the Lifespan

Depression Explains the Relationship Between Cognition and Social Adjustment in Adults with Cognitive Complaints

Highly Variable Blue Light Exposure is Related to Poor Sleep and Cognition in Young Adults 


\section{NIERMEYER, MA}

31. NIXON, KH

32. NYENHUTS, $R$

33. OBERMEIT, LC

34. OWENS, TE

35. PENNINGTON, N

36. PERSAUD. UD

37. REYNOLDS, BW

38. RIVERA. A

39. SALVADOR-CRUZ, J

40. SANTOS, OA

41. SELEME, ME

42. SO, RP

43. STUDENY, J

44. SULLIVAN, E

45. TAYLOR, S

46. TOLFO, SE

47. TRIPP, J

48. WEILHAMMER, J

49. WELSH, M

50. ZIEMNIK, RE

51. MEMEL. MB

$\begin{array}{ll}\text { 52. } & \text { DUTTA, M } \\ 53 . & \text { FIELDS, L } \\ 54 . & \text { FONG, MW } \\ \text { 55. } & \text { KEY-DELYRIA, S } \\ \text { 56. } & \text { MCAULEY, TL } \\ \text { 57. } & \text { OWENS, TE } \\ \text { 58. } & \text { PILLAY, SB } \\ \text { 59. } & \text { SAADATPOUR, L } \\ & \\ \text { 60. } & \text { ANDERSON, DM } \\ \text { 61. } & \text { BRUNET, HE } \\ \text { 62. } & \text { COOK, A } \\ \text { 63. } & \text { DE WIT, L } \\ \text { 64. } & \text { DULAY, M } \\ 65 . & \text { ESTEVIS, E } \\ \text { 66. } & \text { GAASEDELEN, O } \\ \text { 67. } & \text { KIM, H } \\ \text { 68. } & \text { LABELLE, DR } \\ \text { 69. } & \text { LAJEUNESSE, A } \\ \text { 70. } & \text { NESTER, CO } \\ \text { 71. } & \text { O SHEA, DM }\end{array}$

Slower Action Planning is Associated with More Obstacle Contacts During an Obstructed Walking Task

Can Executive Functioning Bolster Learning During Times of Overwhelming Stress?

Measuring patient self-awareness deficit (PSAD)

The Cognitive Profile of Sexual Risk-Taking Intentions

Effect of Mood Induction and Trait Anxiety on Delay Discounting Performance in fMRI

Frustration Impairs Performance on a Task of Executive Function in Females Only

Cognitive Flexibility and Coping in Adults with Cognitive Complaints

Associations of Decision Making and Concept Formation with Risky Choices in Healthy Adults Competing conflict leads to bilingual disadvantage: Performance on an explicit and implicit Simon task

Executive Function and Reading Comprehension: Performance of Mexican 9-year-olds on a NonLinguistic Measure of Inhibition.

Predicting Performance on a Functional Executive Measure via a Brief Cognitive Screening Test

Executive functioning and academic achievement in a group of elementary school students in Havana

The utility of neuropsychological assessment in detecting adolescents with a history of prior psychiatric hospitalizations

The Executive and Non-Executive Demands of Constructional Measures within a Children's

Psychiatric Inpatient Setting

Trauma and Executive Functioning: An Examination of the Relationship between Trauma and Stroop Task Performance using a Large Web-Based Sample

The Relation Between the Mental Clutter Scale and Self-Reported Executive Dysfunction

The Geriatric Complex Figure: A test for the Assessment of Planning, Visuospatial Ability, and

Memory in Older Adult Populations

The Additive Effect of Low Birth Weight and Seizures on Executive Functioning

The relationship between executive behavioral control and emotional distress in urban homeless youth

College Students with a History of Child Maltreatment and Academic Outcomes: The Mediating Role of Executive Functions

Predictors of Medication Management: Contrasting Verisimilitude and Veridicality

\section{Imaging (Structural)}

Contributions of Visual Integration and Frontotemporal White Matter Integrity on Associative Memory in Older Adults

\section{Language and Speech Functions/Aphasia}

Language Functions in Adults with Epilepsy: A Scoping Review and Data Mining Study

Verbal Fluency and Word Retrieval Difficulties in Healthy Older Adults

Factor Structure of the Boston Diagnostic Aphasia Examination-Third Edition

Ambiguous Sentence Comprehension and Cognitive Control in Adults after TBI

Dissociation Between Implicit and Explicit Access: The Failure of Inhibition Theory

Word and Nonword Reading Differences in Primary Progressive Aphasia Variants and Primary Progressive Apraxia of Speech

Examining the relationship between motor system function and verb processing in aphasia using voxel-based symptom-lesion mapping (VLSM)

Primary Progressive Mixed Transcortical Aphasia, a Case Report

\section{Memory Functions}

Systematic Review of the Relationship Between Hippocampal Volume and Memory Performance in Preterm-Born Individuals

Differences in Learning and Recall Strategies in Older Adults Based on IQ

Is Unusually High Working Memory Performance Associated with SuperAger's Superior Episodic

Memory Performance?

The Effects of Depressive Symptom Dimensions and Education on Verbal Memory

Rates of Memory Loss After Stroke in Areas of the Brain Not Typically Associated with Forgetfulness Performance on the Rey Auditory Verbal Learning Test (RAVLT) in neurologically intact Spanishspeaking older adults

Preliminary Validation of the Subjective Memory Complaint Scale (SMS) for Individuals who have Underwent Electroconvulsive Therapy (ECT)

Effects of Perceptual and Semantic Encoding on Recall and Recognition Memory in Subjective Cognitive Decline

Curiosity is associated with spatial working memory and right mesial temporal volumes

Semi-Naturalistic Prospective Memory Assessment in Mild Cognitive Impairment

Differential Semantic and Episodic Memory for September 11, 2001 in Cognitively Impaired and Healthy Adults

Prospective memory strategy use as a predictor of cognitive status in older adults: the role of subjective memory 


\author{
72. PRESTON, T \\ 73. ROSENBAUM, R \\ 74. ROSENBAUM. $\mathrm{R}$ \\ 75. ROSSETTI, M \\ 76. SEKHON, A \\ 77. STEED, D \\ 78. TALBOT, KS \\ 79. VAKIL, E \\ 80. WONG GONZALEZ, D \\ 81. YANDALL DEJESUS, S
}

82. ALTOMARE, LG

83. BOUKRINA. O

84. GOOD, AJ

85. MANKOWSKA, A

86. SEDGEWICK, JR

87. ZINK, DN

11:30-11:45 AM

12:00-1:00 PM

1. DRONKERS, NF

1:00-2:00 PM
Clinical Evaluation of Prospective Memory in Children: Effect of Distractor Task

Impaired face discrimination following early mediodorsal thalamic damage

Not all types of future thinking are affected in episodic amnesia

Should Male and Female Normative Data be Separate for Verbal Memory Tests? An In-Depth Look at the CVLT-II versus RVLT

Visual Memory Differences Between Older Veterans with PTSD/OSA and OSA Alone

Left Ventricular Assist Device Placement Significantly Improves Memory for Patients with Advanced Heart Failure

Prospective Memory in Childhood: Cognitive and Behavioral Differences Among Subtypes and Experimental Methods

Distinct eve movements for different cognitive processes as expressed in the face recognition task The Effects of Encoding Strategies in Associative Recognition Memory

Spatial Recognition Memory Across the Adult Lifespan: Evidence for Age-Related Deficits in Spatial Pattern Separation in Middle and Old Age

\section{Visuopatial Functions/Neglect/Agnosia}

The Influence of Traumatic Brain Injury on the Allocation of Vertical Spatial Attention Linguistic Processing of Single Words in Spatial Neglect

A 34-Year Follow-Up Study on a Patient with Callosal Disconnection Neglect

Right Hemispheric Dominance for the Allocation of Spatially Directed Focal Attention

Native Reading Direction Modulates Lateral Lighting Biases for 3-Dimensional Stimuli

The Relationship Between Parietal Lobe Integrity and Neuropsychological Tests of Visuospatial

Function

\section{AM Coffee Break}

Acadia Ballroom

Plenary G (Kaplan Memorial Lecture). Language and the Brain: From Past Studies to Future Aspirations

Presenter: Nina F. Dronkers

Carondelet

Language and the Brain: From Past Studies to Future Aspirations

\author{
Kaplan Lecture Luncheon: A Taste of New Orleans \\ Bissonet
}




\title{
Abstracts Presented at the Forty Fifth Annual Meeting International Neuropsychological Society
}

\author{
February 1-4, 2017 \\ New Orleans, Louisiana, USA
}

WEDNESDAY MORNING, FEBRUARY 1, 2017

\section{CE Workshop 1. Environmental Chemicals and Children's Brains: How Big a Problem?}

\section{Presenter: David C. Bellinger}

9:00 a.m.-12:00 p.m.

D.C. BELLINGER. Environmental Chemicals and Children's Brains: How Big a Problem?

The recent lead contamination of the water supply of Flint, MI, was a particularly egregious example of the way in which our health is threatened by exposure to environmental chemicals. Of the tens of thousands chemicals in use, extensive data on toxicity is available for only a small fraction. We are essentially conducting a natural experiment on the population, and exposure standards are established only after epidemiological studies provide unequivocal evidence of danger. Children are the population subgroup that is most vulnerable to environmental chemicals, and the brain the most sensitive organ. This workshop will survey the field of pediatric neurotoxicology, covering the prevalence of children's exposures to different environmental chemicals, the mechanisms of neurotoxicity, the neuropsychological effects, the bases of individual differences in vulnerability, and the contrast between individual and population approaches to estimating the burden of chemical-related morbidities.

Following this lecture, participants will (1) be able to identify the chemicals of greatest concern, (2) understand how chemical exposures impair brain development, (3) understand how early-life exposures to chemicals can cause life-long morbidities in cognition and behavior, and (4) understand how neuropsychologists can contribute to protecting children from environmental chemicals.

Correspondence: David C. Bellinger, PhD, Harvard Medical School and Boston Children's Hospital, 300 Longwood Ave, Boston, MA 02115, United States.E-mail: David.Bellinger@childrens.harvard.edu
CE Workshop 2. Best-Practices of Transcranial Direct Current Stimulation (tDCS) for Effective and Reliable Outcomes

\author{
Presenter: Marom Bikson
}

9:00 a.m.-12:00 p.m.

M. BIKSON. Best-Practices of Transcranial Direct Current Stimulation (tDCS) for Effective and Reliable Outcomes.

Transcranial Direct Current Stimulation (tDCS) is investigated to treat a broad range of neuropsychiatric disorders, to accelerate neurorehabilitation, and to change cognition and behavior in healthy individuals. The tolerability, low-cost, and apparent simplicity of the technique has driven rapid and broad adoption. But safe, effective, and reliable application of tDCS critically depends on the use of proper techniques. This course will first review major findings on tDCS in treatment with a special focus on how tDCS is customized for specific applications. Second, detailed protocols for device, electrode, and subject preparation will be demonstrated. Third, state-of-the-art development including used of concurrent EEG, working with susceptible populations, and High-Definition tDCS (HD-tDCS) will be explained.

As a result of participation in this course, the learner will achieve the following objectives: 1) have a deeper understanding of how tDCS is customized and optimized to specific indications; 2) be familiar with best practices in tDCS preparation and protocol; and, 3) be exposed to the leading edge of technical advancements in tDCS.

Correspondence: Marom Bikson, PhD, Biomedical Engineering, The City College of New York, Steinman Hall T-403B, 160 Convent Avenue, New York, NY 10031,United States.E-mail: bikson@ccny.cuny.edu

\section{CE Workshop 3. Adult Aphasia: Classifications,} Localization, and Neuroimaging

$$
\begin{gathered}
\text { Presenter: Nina F. Dronkers } \\
\text { 9:00 a.m.-12:00 p.m. }
\end{gathered}
$$

N.F. DRONKERS. Adult Aphasia: Classifications, Localization, and Neuroimaging.

Aphasia is a disorder of core language functions that occurs after an injury to the brain. Most of what we have learned about how the brain processes language has come from the study of individuals with aphasia. In this introductory course, we will discuss the different types of aphasia, the parts of the brain that are affected in aphasia, and how to view these 
anatomical structures with neuroimaging. Videos will accompany the lectures to best illustrate the deficits we will be discussing. Participants in this course will (1) become familiar with the different patterns of language disorders that can occur in adults after sudden injury to the brain, (2) become oriented to the anatomical structures of the adult human brain as imaged with Magnetic Resonance Imaging (MRI), and (3) be able to discuss the regions of the brain -- beyond Broca's and Wernicke's areas -- that support the different components of language.
Correspondence: Nina F. Dronkers, PhD, University of California, Davis / VA Northern California Health Care System, 150 Muir Rd (126s), Martinez,CA 94553,United States.E-mail:dronkers@ucdavis.edu

\section{WEDNESDAY AFTERNOON, FEBRUARY 1, 2017}

\section{CE Workshop 4. Identifying Ethical Issues in Neuropsychological Subspecialties: Concepts, Cases, and Controversies}

\author{
Presenter: Shane Bush \\ 1:00-4:00 p.m.
}

S. BUSH. Identifying Ethical Issues in Neuropsychological Subspecialties: Concepts, Cases, and Controversies.

The ability to identify ethical issues is necessary for maintaining high standards of ethical practice, addressing ethical challenges, and avoiding ethical misconduct. Although some ethical requirements, such as professional competence, are consistent across neuropsychological subspecialties, differences in the relative importance of ethical principles and standards are encountered in different practice contexts and with different patient populations. Once ethical issues are identified, use of an ethical decision-making model can assist practitioners in determining how best to approach an issue or resolve a dilemma. Such a model provides a structured means of organizing and considering the various resources that are often necessary for making sound ethical decisions. This workshop will describe ethical issues commonly encountered with different patient populations, such as pediatrics, geriatrics, and military personnel/veterans, and in various practice contexts, including rehabilitation, forensics, and sports. Some general ethical and legal issues, such as test security, will also be covered. The workshop will include audience participation in identifying ethical issues from clinical vignettes. Questions and discussion will be encouraged.

Following this lecture: 1) Participants will be able to describe ethical issues encountered in common neuropsychology subspecialties; 2) Participants will be able to identify ethical issues in clinical vignettes: 3) Participants will be able to describe a decision-making model for addressing ethical challenges in clinical neuropsychology. Correspondence: Shane Bush, PhD, ABPP, Long Island Neuropsychology, P.C., 290 Hawkins Ave, Ste B, Lake Ronkonkoma, NY 11779, United States.E-mail:neuropsych@shanebush.com

\section{CE Workshop 5. The Adolescent Brain: Arrested or Adaptive Development?}

\section{Presenter: BJ Casey}

$$
\text { 1:00-4:00 p.m. }
$$

\section{B. CASEY. The Adolescent Brain: Arrested or Adaptive Development?}

Adolescence is the transition from childhood to adulthood that typically begins with onset of puberty and ends with relative independence from the parent. The adolescent is probably stronger, of higher reasoning capacity, and more resistant to disease than ever before, yet mortality rates during this period increases by $200 \%$. These untimely deaths are not due to disease but to preventable deaths associated with adolescents putting themselves in harm's way. Evidence will be presented that suggests these health statistics are in part due to diminished self-control the ability to inhibit inappropriate desires, emotions, and actions in favor of appropriate ones. Findings of adolescent-specific changes in self-control and underlying brain circuitry are considered in terms of how evolutionarily based biological constraints and experiences shape the brain to adapt to the unique intellectual, physical, sexual, and social challenges of adolescence.

Participation in this course will lead to the learner to: 1) understand what situations may lead to a break down in self-control in adolescents; 2) describe how changes in brain circuitry help to explain these changes; and 3) explain when the capacity for self-control reaches adult like ability.

Correspondence: BJ Casey, PhD, Psychology, Yale University, Kirtland Hall 211, 2 Hillhouse Ave, New Haven, CT 06511, United States. E-mail:bj.casey@yale.edu

\section{CE Workshop 6. Clinical Assessment of Frontal Lobe Functions: A Historical Perspective of the Application of the Boston VA Jamaica Plans VA Process Approach}

Presenter: Donald T. Stuss

$$
\text { 1:00-4:00 p.m. }
$$

D.T. STUSS. Clinical Assessment of Frontal Lobe Functions: A Historical Perspective of the Application of the Boston VA Jamaica Plans VA Process Approach.

The lens of this workshop is the clinical assessment of frontal lobe functioning from a personal historical perspective. The first part of the course emphasizes operational definitions, theoretical and anatomical. Second, results of early studies using commonly used tests of "executive" functioning (e.g., WCST, Stroop) will be highlighted. Third, research based on an approach that emphasizes a process approach will demonstrate that different frontal regions are related to very specific domain general attentional functions. Against this background, we will return to a potential re-interpretation of the standard FL tests. The fifth section briefly summarizes the role of the FL in processes such as theory of mind, and behavioural/emotional self-regulation; together with the attentional studies, this leads to a revised model of FL functioning. Finally, the potential application to rehabilitation will be discussed. As a result of participation in this course, the learner will achieve: (1) a deeper understanding of the complexity of anatomy and functions relationships of the FL: (2) the ability to apply knowledge of FL functions to the interpretation of clinically used FL tests; (3) the ability to assess innovative research techniques for investigating frontal disorders; and (4) the ability to apply this knowledge to neurorehabilitation approaches. 
Correspondence: Donald T. Stuss, PhD, Psychology/Neurology, University of Toronto, 135 George St. South, Suite 805, Toronto, ONM5A 4E8, Canada.E-mail: donaldt@stussassoc.ca

\section{Poster Session 1. Epilepsy \& Neuroscience 2:45-4:00 p.m.}

\section{Cognitive Neuroscience}

E. AGARUNOV, B.L. DEFEIS, Y. GAZES, R.P. SLOAN \& Y. STERN. Processing Speed and White Matter Integrity Across Young Adulthood to Late-mid Life.

Objective: A decline in processing speed across the adult lifespan has been shown to diminish cognitive performance. White matter integrity, measured with diffusion tensor imaging (DTI), declines with aging. We aimed to examine the contribution of white matter integrity, as measured by global mean fractional anisotropy (FA), on processing speed in a sample of healthy adults ranging from 20 to 64 years old. Participants and Methods: 118 sedentary but otherwise cognitively healthy adults were evaluated. Processing speed was measured using the WAIS-R Digit Symbol task. DTI data were acquired with a b value of $800 \mathrm{~s} / \mathrm{mm} 2$ and 55 directions, and processed in FSL with the eddy correct module, and FA was calculated for each voxel using the FDT module in FSL. Mean FA was then calculated as the overall mean across all voxels. Mean FA and demographic variables were used in linear regression models to predict performance on the digit symbol task.

Results: Our sample had a mean age of $39.9( \pm 13.41)$ years, was 68.1/31.9 Female to Male, and had an average education of 16.14 years $( \pm 2.65)$. Controlling for age, gender, education, and ethnicity, mean FA significantly predicted processing speed $(\beta=.213 ; \mathrm{p}=.032)$, with higher mean FA associated with faster processing speed. When VO2max, a measure of aerobic fitness, was also included in the analyses, it did not correlate with processing speed.

Conclusions: Higher mean FA, i.e. more intact white matter integrity, significantly predicted faster processing speed, even when controlling for age. This is notable because higher age correlates strongly with reduced FA. Aerobic capacity was not related to processing speed, but all subjects in this sample were below median VO2max so this may be a function of restricted range. These findings provides some insight into the basis for diminished processing speed in healthy aging.

Correspondence: Emil Agarunov, BS, Columbia Medical Center, 631 West 168th st., P\&S box 16, New York, NY 10032, United States. E-mail: ea2715@columbia.edu

M. BEDARD, R. WOODS, C. CRUMP, J. WILEY, A. MORTON \& H. ANISMAN. The Moderating Influence of the Val66Met Polymorphism of the Brain Derived Neurotrophic Factor Gene in the Relation Between Cognitive Flexibility and Perceived Loneliness Relevant to Depressive Symptoms.

Objective: Brain derived neurotrophic factor (BDNF) is involved in neurogenesis and neuroplasticity in the support of neurons (Binder \&. Scharfman, 2004). A single nucleotide polymorphism of the Val66Met BDNF gene, comprising a substitution of the amino acid valine $(\mathrm{Val})$ to methionine (Met), has been associated with reduced BDNF secretions (Egan et al., 2003), and decreased functional and structural connectivity of the frontal lobe (Kim et al., 2006). Cognitive flexibility, which refers to the ability to shift cognitive sets according to changing environmental demands, may allow for greater affective outcomes during stressor experiences, and may be subserved by prefrontal neurocognitive correlates (Eslinger \& Grattan, 1993). Interestingly, individuals that perceive greater social isolation may also exhibit affective dysfunction, as well as alterations in the functioning of frontal cortical areas (Nakagawa et al., 2015).
Participants and Methods: Undergraduate students $(N=428)$ provided self-report data on cognitive flexibility, perceived loneliness, depressive symptoms, and gave saliva samples for DNA genotyping. Results: Depressive scores increased with greater feelings of loneliness and lower levels of cognitive flexibility. An additional moderation analysis to examine the influence of BDNF genotype on these relations revealed a three-way interaction, which indicated that at lower levels of cognitive flexibility, Met carriers exhibited lower depressive symptoms in the presence of low perceived loneliness. However, with high levels of cognitive flexibility, the $\mathrm{Val} / \mathrm{Val}$ carriers displayed higher levels of depression than did the Met carriers when loneliness was high.

Conclusions: These findings suggest that those with the Val/Val genotype, associated with greater neuroplasticity, may be more sensitive to loneliness experiences, despite reports of being cognitively flexible, and consequently may have adverse neurocognitive vulnerability to depression.

Correspondence: Marc Bedard, Master of Science, Department of Neuroscience, Carleton University, 1125 Colonel By Drive, Ottawa, ON K1S5B6, Canada.E-mail:marc.bedard@carleton.ca

C.M. FIGUEROA, K. HAZLETT ELVERMAN, R.J. MCKINDLES, R.E. MARINELLI, B.D. SCHMIT \& K.A. NIELSON. Reinforcement Learning and Error-Related Negativity Distinguish Healthy Elders at Genetic Risk for Alzheimer's Disease.

Objective: The neural networks underlying reinforcement learning (RL) involve many of the same structures detrimentally affected by Alzheimer's disease (AD), such as the hippocampus. Yet, RL and noninvasive evaluation of its neural underpinnings have been underutilized as a framework for understanding $\mathrm{AD}$ pathology and its pre-clinical states. This study aimed to provide a novel approach for assessing subtle changes in asymptomatic apolipoprotein-E (APOE) $\varepsilon 4$ carriers and non-carriers.

Participants and Methods: Electroencephalography was collected from 40 healthy APOE genotyped older adults (Male $\mathrm{n}=11 ; \mathrm{M}_{\text {age }}=79.30$; $\mathrm{M}_{\text {edu }}=14.88$ years) during an RL task comprised of RL and implicit learning phases. Neural components associated with the error detection system underlying RL, specifically the response error-related negativity (ERN) and the feedback error-related negativity (FRN), were examined for individuals at low risk (APOE $\varepsilon 4-; n=20)$ and high risk for AD (APOE $\varepsilon 4+; n=20)$.

Results: RL task performance did not differ between risk groups. Yet, the high-risk group consistently elicited greater ERN peak amplitudes than the low-risk group. Examination of the FRN peak amplitude showed that the high-risk group did not differ between early and late learning, indicating a deviation from typical RL processes. Implicit learning results revealed that the risk groups differed on intermediate conflict trials, with a trending difference for low conflict trials.

Conclusions: Despite intact learning, the neural correlates of RL are believed to provide evidence that hippocampal dysfuntion disrupts the transfer and use of learned information among high-risk individuals, altering the hippocampal-frontostriatal circuit responsible for adaptive behavior. These results indicate that disease-related changes can be identified prior to clinical diagnosis or functional decline using an RL paradigm and a non-invasive assessment of neural function, which may inform clinical conceptualization, assessment, and treatment.

Correspondence: Christina M. Figueroa, Ph.D., Psychology, Marquette University, 600 N. Wolfe St., Meyer 218, Baltimore, MD 21287-7218, United States. E-mail: christina.figueroa@marquette.edu

T.D. KAEMMERER \& L.H. LU. Implicit Motor Sequence Learning among Healthy Older Adults and Older Adults with Mild Neurocognitive Disorder: Examining the Impact of Embedded Associative Structure.

Objective: Explicit memory decline is a well documented feature of older adulthood and late-life neurocognitive disorders. To date, it remains unclear whether older adult populations experience parallel 
declines in implicit learning and memory, though previous research has suggested age-related deficits in implicit learning when the information being acquired contains higher-order associative structure. We evaluated implicit motor sequence learning among healthy older adults and older adults with mild neurocognitive disorder of probable Alzheimer's or cerebrovascular etiology, with an eve toward the impact of embedded associative structure. Given previous literature implicating medial temporal lobe structures in the implicit learning of higher-order structure, we assessed the extent to which performance on another medial temporal lobe-dependent task (a verbal list-learning measure) predicted higher-order motor sequence learning among older adults.

Participants and Methods: Participants were healthy, communitydwelling older adults $(n=23)$ and older adults with mild neurocognitive disorder $(n=8)$ presenting for neuropsychological evaluation at a university-based outpatient senior health clinic. T-tests and regression analyses were used to analyze between-group learning differences and to explore the extent to which explicit verbal learning predicted secondorder motor sequence learning.

Results: Older adults with mild neurocognitive disorder displayed impaired learning of second-order associations relative to healthy older adults. Across the study, explicit verbal learning performance predicted motor sequence learning of second-order associations above and beyond the effects of age and estimated premorbid intellect.

Conclusions: These preliminary findings provide a meaningful contribution to the learning literature, especially with regard to implicit learning capacity among both healthy and clnical older adult populations.

Correspondence: Tobias D. Kaemmerer, Roosevelt University, 2017 Washington Avenue, Unit 415, St Louis, MO 63103, United States. E-mail:botias108@gmail.com

\section{E. KITCHENER, R. MCCARTHY \& C. KIPPS. Category Specificity in Right Temporal Variant Semantic Dementia.}

Objective: Semantic Dementia (SD) most frequently begins in the left temporal lobe before spreading bilaterally, and predominantly rightsided cases are relatively rare. SD is generally considered to result in generalized semantic loss and category-specificity has only rarely been reported. We have had the opportunity to compare the pattern of semantic memory loss in two cases of right-onset SD with that of another case having left temporal onset.

Participants and Methods: Participants were three SD patients, two right-onset (SW, 54-year-old male; PT, 68-year-old female) and one left-onset (LC, 56-year-old female). We investigated patients' abilities to name and comprehend pictures and words in the categories of people, living things and inanimate objects.

Results: Both right-onset cases showed loss of familiarity for famous people from pictures and also loss of knowledge of famous names, although ability to match faces across viewpoints was within normal range. Patient SW showed greater difficulty with naming objects than living things; however, patient PT did not demonstrate any category effects (simply showing focal prosopagnosia). Left-onset case LC showed a pattern of object naming impairment comparable to SW but with no prosopagnosia. For both SW and LC, object knowledge was significantly more impaired when tested by words rather than through pictures.

Conclusions: Right-onset SD cases showed impairment of person knowledge across verbal and visual domains. Patients LC (left-onset) and SW (right-onset) demonstrated focal progressive aphasia differentially affecting knowledge of objects; however, LC was not prosopagnosic. These findings, taken in conjunction with evidence from the literature of cases of selective loss of animals and living things, raise the possibility of a degree of independence between post-perceptual representations of people, objects and living things. Implications of these findings for theoretical models of semantic organization are discussed.
Correspondence: Erin Kitchener, PhD, Psychology, William James College, Beth Israel Deaconess Medical Centre, Div. Cognitive Neurology, 330 Brookline Ave, Boston, MA 02115. United States. E-mail: ekitchener@gmail.com

E.N. MERCER, J. BECK, J. REMER, T. HARRISON GOLDMAN, V. D'SA \& S. DEONI. Cognitive Development Trajectories of Planned Cesarean Sections and Vaginal Births.

Objective: Prior cross-sectional studies comparing cognitive development in children born via planned Cesarean section (PCS) vs. vaginal birth $(\mathrm{VB})$ present conflicting reports. We sought to further examine the longitudinal cognitive development of PCS and VB children from birth to 68 months.

Participants and Methods: The Mullen Scales of Early Learning (MSEL) was used to assess cognitive development in 52 PCS children ( 23 female) with a mean age of $2.2 \pm 1$.6yrs and $222 \mathrm{VB}$ children ( 68 female) with a mean age of $2 \pm 1.5 y r s$. Children were assessed up to 5 times with an inter-assessment period of 6 or 12 months. The groups did not differ significantly in socio-economic status, SES ( $p=0.24)$, but maternal age of the PCS delivery group was significantly older $(28.5 \pm 5.7$ vs. $32.8 \pm 5.9, \mathrm{p}<0.001)$. The Early Learning Composite (ELC) from the MSEL was used to quantify cognitive ability. A linear mixed-effects model $($ ELC $($ Age $)=\alpha$ Age $+\beta)$ was used to examine the group-wise trend in ELC in each group. An unpaired t-test was used to compare slope $(\alpha)$ and intercept $(\beta)$ values between the groups.

Results: Our results showed no significant difference in intercepts between the groups $(p=0.197)$, suggesting that both groups did not differ at birth. There was a significant difference in the rate (slope) of development $(p<.001)$; the PCS group's ELC scores improved with age while the VB group did not. Despite this, the mean difference at 5 years was 7.05 , within one standard deviation of the test's mean implying both groups are considered normally developing.

Conclusions: Our results do not indicate that PCS children showed a cognitive disadvantage compared to VB children. While we did observe a trend of increasing ability with age in the PCS group, which future research should investigate, the mean of each group was within the 85-115 score range of neurotypical development. The maternal age of the PCS group was slightly older, but prior studies have not revealed significant differences in child cognitive outcomes across this age range of the mothers.

Correspondence: Emily N. Mercer, B.A. in Psychology from Providence College, Engineering (Advanced Baby Imaging Lab), Brown University, 194 First Street, Pawtucket, RI 02861, United States. E-mail: emily_ mercer@brown.edu

L.L. LAPOINTE, C. OH, J.A.G. STIERWALT \& C.G. MAITLAND. The Effects of Cognitive Loading on Parameters of Gait and Possible Risk of Injurious Falls.

Objective: Injurious falls are an intense concern for society and healthcare organizations. The aggregate U.S. national cost of hospital care for adults who came in through emergency departments for an injurious fall was $\$ 6.8$ billion. In Parkinson disease (PD) studies report that nearly $70 \%$ have been hospitalized for injurious falls within the past six months. Many of the causes of injurious falls are obvious: neuromuscular compromise, medications, and motor neuron disease. One less obvious cause is cognitive resource allocation and for the past eight years we have investigated the effects of simultaneous cognitive loading while walking across a variety of participant samples and cognitive load conditions.

Participants and Methods: 112 young and older healthy adults and 20 persons with Parkinson disease were measured under conditions of simultaneous walking and talking during control (just walking), low loads (e.g. counting by ones) and high cognitive-linguistic loads (e.g. subtracting from 95 by 3s). The GAITRite walkway system with 16,000 embedded sensors was used to derive performance across selected spatial and temporal gait parameters. 
Results: Cognitive loading during simultaneous walking was found to be a significant disrupter of gait and a predictor of future falls. Significant differences were found across conditions, especially on measures that are highly correlated with future injurious falls.

Conclusions: Implications for brain-based disorders and the prevention of costly injurious falls were drawn from this aggregate of subjects.

Correspondence: Chorong Oh, School of Communication Science and Disorders, Florida State University, 1121 Martin St, Tallahassee, FL32303, United States.E-mail:co14e@my:fsu.edu

S. RYMAN, A.B. DODD, J. LING, N.A. SHAFF, C.J. WERTZ \& A.R. MAYER. Multimodal Cognitive Control: an fMRI study of the AX-CPT.

Objective: Cognitive control is broadly defined as the set of mechanisms required to pursue a goal, especially when strong competing responses must be overcome. Although the functions of the frontal lobe regions involved in cognitive control are well documented, their role in multisensory contexts are less well understood. The current study examines behavioral and neural measures of cross-modal cognitive control and how they relate to established measures of neurocognitive function.

Participants and Methods: Forty-six healthy adult volunteers $(28$ males; mean age $=31.8 \pm 7.4$ years; mean education $=15.2 \pm 1.9$ years) underwent fMRI while performing a multimodal cognitive control task, the AX Continuous Performance Test (AX-CPT). Additionally, participants completed two valid and reliable measures of neurocognitive function, the EXAMINER and the MCCB. Within subjects t-tests and Pearson zero order correlations were used to examine hypotheses. Results: RT data indicated A cues had significantly faster reaction times than B cues and AX trials had significantly faster reaction times than both $\mathrm{AX}$ and BY trials. Working memory in addition to other measures from both the EXAMINER battery and MCCB were inversely related to RT across several trial types. Preliminary fMRI results indicate activation of the anterior cingulate cortex, ventral and dorsal lateral frontal cortex, superior parietal and temporal cortices, striatum, subthalamic nucleus (STN), thalamus and cerebellum during conditions that required individuals to utilize cognitive control to overcome a pre-potent response.

Conclusions: Behavioral and fMRI data suggest that reaction time, accuracy, and PSC during a cross-modal cognitive control task function similarly to a unimodal task. Cross-modal cognitive control activated widespread regions of the cognitive control network including those implicated in proactive cognitive control as well as response inhibition. Correspondence: Sephira Ryman, University of New Mexico, 800 Madison St. NE, Albuquerque, NM 87110, United States. E-mail: sephira.ryman@gmail.com

C.T. SMITH, R. SMITH, A. SANOVA \& W.D. KILLGORE. The Neural Basis of Emotional Working Memory and Its Relation to Adaptive Emotional Functioning.

Objective: While the neural correlates of exteroceptive working memory (WM) have received considerable empirical analysis, few studies to date focus on WM for emotional information and its relation to emotionfocused attention. Given the known mechanistic overlap of attention and WM, we predicted a similar overlap for the emotional domain. As previous findings link emotion-focused attention to medial prefrontal cortex (MPFC) activation, we hypothesized that MPFC would also be associated with emotional WM (EWM), and examined how individual differences relate to emotional awareness (EA) and emotional intelligence (EI).

Participants and Methods: During fMRI, 26 participants (13 female) completed an EWM task that displayed emotion-provoking and neutral images. We compared a condition where participants held their emotional response to the images in WM $(\mathrm{EWM})$, to a control condition where they held the visual content in WM instead (VWM). They completed measures of EA and EI, including the Levels of Emotional Awareness Scale (LEAS), the Trait Emotional Intelligence Questionnaire (TEIQue), and an EA assessment of emotional perception within animations of moving shapes (Animations Task; AT).

Results: EWM was associated with clusters of activation in left and right MPFC, and a posterior cluster spanning the dorsomedial frontal cortex (DMFC) bilaterally. DMFC activation was associated with LEAS total $(\mathrm{r}=.401)$, LEAS other $(\mathrm{r}=.470)$, and AT $(\mathrm{r}=.53)$. Right MPFC activation correlated positively with AT $(r=.403)$. Left MPFC activation was associated with the emotion regulation, impulse control, stress management, adaptability, and self control subscales of the TEIQue.

Conclusions: This suggests that medial frontal regions may be uniquely engaged during EWM (i.e., relative to VWM), supporting our hypothesis that EWM and emotion-focused attention share overlapping mechanisms. Our results also suggest that medial frontal regions engaged by EWM play a role in cognitive-emotional functions that require recognizing and regulating emotional states.

Correspondence: Courtney T. Smith, University of Arizona, $2875 \mathrm{E} 6 \mathrm{th}$ Street, Unit 101, Tucson, AZ 85716,United States.E-mail: courtneys@ email.arizona.edu

H.A. SMITH, B.S. ADAMS, K.A. CARBINE \& M. LARSON. Does Positive Affect Enhance or Disrupt Cognitive Control Processes? An Event-Related Potential (ERP) Study.

Objective: Previous research indicates positive affect facilitates many cognitive processes; however, recent studies suggest positive affect may be associated with increased distractibility decreasing regulative cognitive control processes. We tested the hypothesis that positive affect is associated with altered context maintenance processes as measured by the cue-related slow wave of the event-related potentials (ERP) relative to evaluative conflict-related neural processes measured by the N450 and conflict slow potential ERPs.

Participants and Methods: ERP data were collected while 44 healthy undergraduate participants completed a modified single-trial cued version of the Stroop task. On each trial, participants viewed a pleasant, neutral or unpleasant International Affective System picture followed by an instruction cue to name the color or read the word of the subsequent congruent or incongruent Stroop stimulus.

Results: Behaviorally, accuracy was decreased following presentation of pleasant or neutral stimuli relative to unpleasant stimuli. The cuerelated slow wave ERP associated with context maintenance did not significantly differentiate color from word instructions cues and did not differ as a function of picture valence (all ps $>.05$ ). Similarly, the N450 ERP associated with conflict detection did not differ by valence. The post-conflict slow potential (conflict SP), however, differed by Stroop congruency and was more positive on incongruent trials during unpleasant and pleasant conditions relative to neutral.

Conclusions: Findings indicate unpleasant IAPS stimuli decrease accuracy on a cued-Stroop paradigm but that ERPs associated with context maintenance and conflict detection are not differentially impacted by picture valence. Post-conflict processing (incongruent conflict SP amplitude) was impacted by the more arousing pleasant and unpleasant conditions-suggesting trial-by-trial arousal levels influence conflict processing more so than context maintenance.

Correspondence: Hilary A. Smith, Clinical Psychology, Brigham Young University, 12973 S. Harvest Field Cir., Riverton, UT 84065, United States.E-mail: hilary.anne.smith@gmail.com

J.A. TURNER, D. BURNET, A. AHMADI, S. HEGERTY \& H. OFFUTT. Neural Circuits of Source Memory and Imagination. Objective: Reality monitoring is the ability to differentiate between actual experiences and self-generated or imagined experiences. The relationship between imagination and memory, and thus reality monitoring, in healthy individuals has been identified in several studies; people considered to be "high imagers" are more susceptible to false memories. We examine the brain networks active during visual and auditory imagination, and relate those to as imagery vividness reports. 
Participants and Methods: We recruited 19 students from the GSU Psychology Research Pool, and acquired fMRI scans on a 3T Siemens scanner while they heard or imagined hearing a set of simple sentences, or saw or imagined seeing a set of pictures. We examined signal changes in the superior temporal gyrus, visual areas, Brodmann areas 46/47, hippocampus and BA 7. Source monitoring was assessed following the scan by testing their ability to remember which sentences or pictures they actually experienced, using a remember/know paradigm. We also collected measures of auditory and visual imagery vividness, and the experience of unusual subclinical experiences.

Results: Participants maintained an average post-scan memory performance $>60 \%$, indicating they were able generally to monitor the source of their memory (real or imagined). In our regions of interest, the experienced stimuli resulted in robust activation, while the imagined stimuli also elicited weak but significant activation. The act of imagining a sentence or picture did not activate any of the regions more strongly than did experiencing the stimuli. We saw no differences in brain responses based on imagination vividness.

Conclusions: As has been found previously, we show that imagining a stimulus weakly activates a similar set of networks as the original stimulus. We have not found cortical regions differentially activated in those with more vivid imaginations. Future analysis will examine differences in encoding stimuli later mis-remembered as experienced when they were actually imagined.

Correspondence: Jessica A. Turner, Psychology, Georgia State University, 140 Decatur St., Atlanta, GA 30303, United States. E-mail: jturner63@gsu.edu

\section{T.R. BAL, R. SIUGZDAITE, D. MARINAZZO \& G. VINGERHOETS. Effective Connectivity Underlying the Production of Speech and Hand Gestures.}

Objective: To investigate the effective connectivity between parietal and frontal regions during the production of two types of complex movement (speech and praxis) in left and right language dominant participants. Participants and Methods: We recruited healthy subjects with typical $(\mathrm{n}=10)$ or atypical $(\mathrm{n}=10)$ language dominance and explored correlations of lateralization indices between activated regions during word generation and tool pantomime tasks using fMRI. In all participants language and praxis shared the same hemisphere and revealed a network of overlapping frontal and parietal regions (Vingerhoets et al., 2013). We re-analyzed this data-set using structural equation modeling (SEM) to explore causal relations between target areas including the dorsolateral prefrontal cortex (dIPFC), ventral and dorsal premotor cortex (vPMC, dPMC), superior and inferior parietal lobe (SPL, IPL).

Results: The resulting model fits both groups of subjects (albeit in their left and right hemispheres respectively) and both tasks (tool use and word generation). The model revealed that SPL feeds to APMC and IPL to vPMC, while both parietal regions project to dIPFC. A strong reciprocal communication exists between $\mathrm{dPMC}$ and $\mathrm{vPMC}$. dIPFC receives parietal input and appears to feed input to both vPMC and dPMC.

Conclusions: With only minor differences between the volunteers with typical and atypical functional asymmetry, the suggested SE model appears to accommodate the neural circuits underlying learned speech and gesture production. The production of complex movements, whether it be speech or tool use, relies on a common, co-lateralized, and perhaps evolutionary related neural connectome.

Correspondence: Guy Vingerhoets, Ghent University, Henri Dunantlaan 2, Ghent B-9000,Belgium.E-mail: guy.vingerhoets@ugent.be

\section{Electrophysiology/EEG/ERP}

K.A. CARBINE, H.A. SMITH, E. MODERSITZKI, J. LECHEMINANT \& M. LARSON. The Relationship Between Food-Related Inhibitory Control, Weight, and BMI Status: Implications for Calorie and Carbohydrate Intake.

Objective: The cognitive control process of food-related inhibition may relate to obesity and diet, which have significant implications for optimal health. Examining neural correlates of cognitive control that influence diet and obesity may provide insight into what neural processes affect health outcomes. We assessed how inhibitory control for high- and low-calorie food cues, measured by the N2 component of the scalprecorded event related potential (ERP), differed by weight $(\mathrm{kg})$, BMI, and related to food intake.

Participants and Methods: 24 lean (BMI $\left.<25_{\mathrm{kg} / \mathrm{m}}{ }^{2}\right), 15$ overweight $\left(25_{\mathrm{kg} / \mathrm{m}}{ }^{2}<\mathrm{BMI}<30_{\mathrm{kg} / \mathrm{m}}{ }^{2}\right)$, and 13 obese $\left(\mathrm{BMI}>30_{\mathrm{kg} / \mathrm{m}}{ }^{2}\right)$ participants completed two food go/no-go tasks, one with high- and one with low-calorie foods as no-go stimuli. Participants were then presented with a weighed ad libitum snack-food buffet to measure immediate food intake. Participants also recorded their food intake across four randomly assigned days using a multiple-pass recall.

Results: A repeated measures ANOVA showed N2 amplitude was larger for no-go than go trials and a Task $\mathrm{x}$ Trial interaction showed no-go was larger than go amplitude on the high-calorie task but did not differ on the low-calorie task $(p s<.01)$. N2 difference amplitude (no-go minus go) on either task did not relate to BMI or weight ( $p \mathrm{~s}>.05$ ). Regressions controlling for sex, age, and weight or BMI showed high-calorie N2 difference amplitude predicted real-world calorie and carbohydrate intake recorded via the recall system. Participants with larger highcalorie N2 difference amplitudes consumed less calories and carbohydrates $(p<<.05)$. High-calorie N2 difference amplitude did not predict calorie or carbohydrate intake from the ad libitum buffet ( $p s>.05)$.

Conclusions: Regardless of BMI and weight, recruiting more cognitive resources to withhold urges to eat high-calorie foods may aid in consuming less food in everyday life. Data suggest N2 ERPs to go/no-go tasks provide valid information about real-world eating behavior and may be used to improve interventions and health outcomes.

Correspondence: Kaylie A. Carbine, B.S., Psychology, Brigham Young University, 6605 Amber Lane, Pleasanton, CA 94566, United States. E-mail: kaylie.carbine@gmail.com

C. KEITH, M. BERTOLIN, R. VAN PATTEN, D.A.S. KAUFMAN \& T.W. BUCHANAN. Effects of Acute Psychosocial Stress on Attentional Resources and Response Inhibition: An Electrophysiological and Behavioral Study.

Objective: A growing body of research has demonstrated the detrimental effects of chronic stress on physical health, cognition, and emotional well-being. Additionally, acute stress has also been associated with decrements in neurocognitive performance. Although a number of studies suggest deleterious effects of acute stress on working memory function, few have examined its influence on attention and response inhibition.

Participants and Methods: In this study, 25 healthy undergraduate males were randomly assigned to perform either the Trier Social Stress Task (TSST; $n=15)$ or a control "placebo TSST" $(n=10)$, followed by a computerized Go-Nogo task during which high density event-related potentials (ERPs) were recorded. Heart rate and cortisol changes were measured as a manipulation check and demonstrated that the TSST did indeed produce a physiological stress response.

Results: Significantly more omission errors were recorded in the stress group relative to the control group. Corresponding electrophysiological analyses demonstrated attenuated P3 amplitudes in the stress group during omission errors. In contrast, no significant group differences were observed for commission errors or $\mathrm{N} 2$ amplitudes.

Conclusions: Our findings suggest that although response inhibition is left intact, acute psychosocial stress may be associated with lapses in attention, resulting in decreased processing of information and increased 
omission errors. Given the prevalence of acute psychosocial stressors in clinical populations, and the fact that attention serves as a gateway to all other cognitive functions, our results suggest that acute stress may have a significant deleterious impact on neuropsychological test performance, and should be appropriately accounted for by researchers and clinicians alike.

Correspondence: Cierra Keith, M.S., Saint Louis University, 4467 Castleman Ave, Apt 2W, St Louis, MO 63110, United States. E-mail: keithc@slu.edu

\section{S. LITHFOUS, A. DUFOUR, O. DESPRES \& B. ROSSION. Investigating Face identity Discrimination using Fast Periodic Visual Stimulation (FPVS).}

Objective: In this study, we investigated face identity discrimination processes using a novel approach in EEG: the Fast Periodic Visual Stimulation (FPVS). This approach consists in the exact synchronization of the brain response to the stimulation frequency and has been validated to study low and high level visual processes.

Participants and Methods: Twenty-eight young adults participated in the study (mean age $=22.8 ; \mathrm{SD}=2.4$ ). Face stimuli were presented using FPVS at a fixed rate of $6 \mathrm{~Hz}$. Adaptation to one face identity was induced by repeated presentation of that identity during a $60 \mathrm{sec}$ adaptation sequence. After a short delay (i.e. between 9 and 15 s), the adapted face was presented in alternation with a novel face identity in a 20 sec testing sequence, resulting in a face identity repetition rate of $3 \mathrm{~Hz}$ (i.e. $6 \mathrm{~Hz} / 2$ ). The magnitude of the $3 \mathrm{~Hz}$ response therefore reflects the spectral signature of adaptation to face identity. A control group was presented only testing sequences, to control for the influence of low-level processes in the $3 \mathrm{~Hz}$ response.

Results: We found a selective electrophysiological response at $3 \mathrm{~Hz}$ distributed in the right occipito-temporal cortex in the adapted, but not in the control group, reflecting the spectral signature of adaptation to face identity. The $6 \mathrm{~Hz}$ presentation frequency elicited a strong response at the exact same frequency, distributed over the medial occipital cortex in both implicit recognition and control groups, reflecting general visual processes.

Conclusions: These results indicate that visual adaptation to one particular face identity produces an objective electrophysiological index of face discrimination between two face identities and provides an objective quantification of visual adaptation effect.

Correspondence: Segolene Lithfous, PhD, Psychological Research Science Institute, University of Louvain, Place du Cardinal Mercier 10, Louvain-la-Neuve 1348, Belgium. E-mail: segolene. lithfous@uclouvain.be

P. MANGAL, J.A. LAFO, J. RENFROE, B.M. SCOTT, M. BRADLEY, M.S. OKUN \& D. BOWERS. Modulation of Electrocortical Responses to Emotional Pictures in Parkinson's Disease.

Objective: Previous research has reported that non-demented Parkinson (PD) patients exhibit blunted physiologic reactivity to emotional stimuli as indexed by startle eyeblink and electrophysiologic responses. This blunted reactivity is associated with apathy and worsening disease severity. The aim of this study was to learn whether PD patients could modulate emotional arousal (late positive potential, LPP) using reappraisal cognitive regulation strategies. We hypothesized that PD patients would have reduced abilities to increase and decrease LPP amplitudes compared to healthy controls (HC).

Participants and Methods: Participants included 27 PD and 18 ageand education-matched HCs, who were not demented, depressed, or apathetic based on standard screening indices. Event related potentials were recorded while participants viewed standard sets of unpleasant and neutral pictures (IAPS) for $3 \mathrm{sec}$ each. Each picture was preceded by a 3 sec cue to "look", "increase", or "decrease" emotional reactivity to the picture. The LPP was averaged over $400-700 \mathrm{~ms}$ post-picture over centroparietal sensors.
Results: Results of a mixed model Group (PD, HC) X Condition (look, increase) ANOVA showed greater LPP amplitude during the increase compared to the look condition in the PD group, but not the HC group. Neither group could decrease physiological reactivity. Cognitive tests of executive function (Trails, Letter Number Sequencing, Stroop) predicted the LPP amplitude for increase and look conditions in the PD group, but not for HCs, or LPP during the decrease condition.

Conclusions: The ability of PD patients to increase their electrocortical responses when cued compared to HCs may be due to differing levels of task motivation. These findings will be discussed in terms of psychological mechanisms and the utility of cognitive regulation strategies in patients with elevated apathy and/or executive deficits. Supported by T32NS082168 (JL,BS), 1R21NS079767 (DB), T32AG020499 (PM), UF National Parkinson Disease COE

Correspondence: Paul Mangal, B.S., Clinical Health Psychology, University of Florida, P.O. Box 100165, Gainesville, FL 32610-0165, United States. E-mail: pmangal@phhp.ufl.edu

T.L. MCKINNEY, E. VACCARIELLO, M. EULER \& Y. SUCHY. Medial Frontal Theta Dynamics Predict Temperamental SelfRegulation but Not Executive Response Inhibition.

Objective: EEG studies suggest that theta-band (4-8 Hz) neural oscillatory processes index medial frontal contributions to cognitive and affective aspects of behavioral self-regulation. However, it is unclear how these experimental findings relate to neuropsychological measures of self-regulation, such as executive response inhibition. This study examined theta-band activity from error trials during complex motor sequencing performance, and tested its differential contribution to executive versus temperamental aspects of behavioral self-regulation.

Participants and Methods: Thirty four healthy undergraduates underwent EEG recording while performing a complex motor sequencing task from which error trials were examined. Response inhibition was operationalized from a composite index of repetition errors obtained from verbal and design fluency tasks. Aspects of temperamental behavioral regulation were examined via NEO-PI-R Conscientiousness and Neuroticism measures. Theta band maximum inter-trial phase clustering (ITPC) was measured from middle-frontal electrodes $(\mathrm{Fz}$ and $\mathrm{FCz}$ ) on error trials time-locked to sequence presentation.

Results: Theta ITPC was positively associated with Conscientiousness $(r=.420)$, including the facets Competence $(r=.466)$ and Order $(r=.451 ;$ all $p$ s $<.013)$. Theta ITPC was only marginally negatively related to Neuroticism $(r=-.312, p=.073)$, although did predict the Impulsiveness facet $(r=-.388, p=.023)$. Despite an acceptable internal consistency $(\alpha=.672)$, the response inhibition composite was not associated with ITPC or any personality measures.

Conclusions: Results suggest that error-related theta oscillations more closely reflect affective regulatory processes than those indexed by executive response inhibition. The pattern of relations suggests that medial frontal theta is associated with greater affective stability in the face of performance lapses. Negative findings for response inhibition suggest a distinction between commission of errors and affective reactions to them. Correspondence: Ty L. McKinney, PhD of Clinical Psychology, Psychology, Univeristy of Utah, 975e 400s \#10, Salt Lake City, UT 84102, United States. E-mail: ty.mckinney.412@gmail.com

A.J. STEPHENSON, A.T. CORSON, E. WATSON \& D.E. EVERHART. Behavioral Inhibition and Baseline Frontal Asymmetry are Associated with Sleep Quality Components.

Objective: Poor sleep has been linked to neurocognitive dysfunction. increased healthcare costs, and morbidity. The Reinforcement Sensitivity Theory (RST) has previously been employed to explore the individual differences associated with sleep-related disorders. RST consists of distinct neurophysiological systems that relate to approach and withdrawal behaviors, including Behavioral Activation (BAS), and Behavioral Inhibition (BIS) Systems. BAS is associated with left frontal alpha activity (measured through EEG) and approach behavior, while 
BIS is associated with right frontal activity and withdrawal behavior. Previous research has linked elevated BIS with emotional dysregulation and poor sleep. In this study it was hypothesized that BIS, and greater right than left anterior activation at baseline would be significantly related to individual components of the PSQI.

Participants and Methods: The relationships between BIS, BAS, frontal baseline EEG asymmetry, and the individual components of sleep quality using the Pittsburgh Sleep Quality Index (PSQI) were examined in a population of 75 university students.

Results: The hypotheses were partially supported. BIS was associated with daytime dysfunction of the PSQI, $r(75)=.337, p<.01)$. Further, greater right activity was weakly associated with sleep duration for $\mathrm{F} 4, r(52)=-.279, p<.05)$, while greater left activity was associated with latency for F3, $r(52)=.333, p<.05)$, and FC3, $r(50)=.310$, $p<.05)$, and efficiency for F7, $r(53)=.453, p<.01), \mathrm{F} 3, r(52)=.308$, $p<.05), \mathrm{FT} 7, r(52)=.354, p<.01)$, and FC3, $r(50)=.300, p<.05)$. Conclusions: These results suggest that the individual components of the PSQI, including daytime dysfunction, sleep duration, latency, and efficiency are related to self-reported behavioral inhibition and frontal EEG asymmetry. Implications for these findings are discussed.

Correspondence: Alexandra J. Stephenson, BS, Psychology, East Carolina University, 104 Rawl Building, Dept. of Psychology, East Carolina University, 1001 East Fifth Street, Greenville, NC 27858, United States.E-mail: stephensona04@students.ecu.edu

\section{Y. WANG, F. GUO \& L. BAI. Narcissistic Personality Disorder Modulates the Outcome Evaluation of Trusting Strangers: An Event-related Brain Potentials Study.}

Objective: Narcissism Personality is often described as a personality characteristic involving arrogance, a feeling of entitlement, and the willingness to exploit others. It's an important factor which can influence the outcome evaluation of trusting strangers. In this study, we aimed to investigate that how narcissistic personality modulated outcome evaluation during the trust game.

Participants and Methods: In the current study, 38 healthy undergraduate participated in the experiment. Participants completed narcissistic personality inventory(NPI) and Beck depression inventory(BDI-II). We employed the event-related potentials (ERPs) while they playing the role of trustor in the one-shot trust game. During this game, they should decide whether to trust or not with different partners over 150 trials. The partners' reciprocation strategy was designed by the experimenter (the reciprocate rate was $50 \%$ ). All the participants were provided with post-decision feedback about the outcome of their decisions in each trail. Results: According to the average score of NPI, 19 participants scored below the 50th percentile of the scores were regarded as relatively low narcissistic, while the other 19 participants scored above the 50th percentile of the scores were regarded as relatively high narcissistic personality. There were significant differences between the two groups on the score of NPI, but not on BDI-II. The dFRN in the relatively high narcissistic group is larger than that in the relatively low narcissistic group. As well, the P300 amplitude elicited by the feedback in the relatively high narcissistic group was larger than that in the relatively low narcissistic group, and this difference was significant at the frontalcentral area $(\mathrm{FCz})$.

Conclusions: These result indicated that narcissistic personality trait influenced the evaluation during trust game, and might lead to less trust others in interpersonal exchange. The present study provides preliminary evidence that the narcissistic personality may modulate the outcome evaluation.

Correspondence: Yiwen Wang, Department of Psychology, Fuzhou University, 2 Xueyuan Rd, Fuzhou 350116, China. E-mail: wangeven@126.com

\section{Epilepsy/Seizures}

C.I. BERMUDEZ, W. JERMAKOWICZ, C. MILLAN, J. KOLCUN, K. MCINERNEY, N. SUN-SUSLOW, L. SEGALA, S. ANDERSON, S. DE LA GUARDA, J.R. JAGID, M. LOWE, M.E. PALOMEQUE, R. RIBOT, E. SERRANO, L. TORNES, N. VELEZ-RUIZ, A. KANNER \& G.J. REY. Delayed Verbal Memory Decline Associated with Left Stereotactic Laser Ablation for the Treatment of Temporal Lobe Epilepsy.

Objective: This study examines whether pre-operative hippocampal volume, ablation size, and post-operative volume loss correlate with percent retention in verbal memory testing following Stereotactic Laser Ablation (SLA) for the treatment of temporal lobe epilepsy.

Participants and Methods: 10 patients underwent left SLA, 7 diagnosed with mesial temporal sclerosis. Mean duration of seizures was 24.3 years $(S D=11.2)$, age at time of surgery $\mathrm{M}=39$ years $(S D=14.1) .60 \%$ males, $80 \%$ White-Hispanics, and 20\% African Americans, average education $\mathrm{M}=11.60$ years $(S D=2.6)$. Neuropsychological evaluations were completed 6-12 months pre and repeated at 6-12 months following SAL. Volumetric analyses were completed by two independent experts using manual tracing with $>90 \%$ inter-rater reliability.

Results: Prior to surgery, standard scores on immediate and delayed verbal memory did not correlate with hippocampal volume, but the ability to retain information from immediate to delayed testing (Immediate recall $\%$ - delayed recall \%) was significantly correlated with hippocampal size $\left(\mathrm{r}=.715, \mathrm{p}<0.05, \mathrm{r}^{2}=0.51\right)$. Patients received $4-6$ ablations $(M=5, S D=.47)$, with total volume loss ranging from $1292-2312 \mathrm{~mm}^{3}$ $(M=1710, S D=389)$, and $74-89 \%$ of the hippocampal structure ablated. Repeated measures ANOVA comparing pre to post test performance on a story memory measure, revealed a significant difference in the percent of information lost from immediate to delayed testing from $-9 \%$ pre to $-21 \%$ following SLA $\left(F(1,4)=7.36, \mathrm{p}<0.05, \mathrm{r}^{2}=0.65\right)$ after controlling for pre-hippocampal volume as it related to \% retention.

Conclusions: Hippocampal size was significantly correlated with ability to retain information over time. Better preserved mesial temporal structures and memory performance increases risk of decline following surgery. However, decline in \% retention was still seen in all patients regardless of poor pre-surgical performance or volume.

Correspondence: Christina I. Bermudez, M.Ed., M.A., M.S., Psychology, Nova Southeastern University, 6510 Main St Apt 312, Miami Lakes, FL 33014, United States. E-mail: christina.bermudez1@gmail.com

C.I. BERMUDEZ, S. DE LA GUARDA, K. MCINERNEY, C. MILLAN, R. FISHER, J. CRAMER \& G.J. REY. How to Measure the Quality of Life in Hispanics with Epilepsy? A Spanish Version of the Personal Impact of Epilepsy Scale in a Multicultural Treatment and Research Setting.

Objective: The Personal Impact of Epilepsy Scale (PIES) is a selfadministered questionnaire originally developed in English (2014). Given the high number of Spanish speaking individuals seeking treatment for epilepsy in the US as well as a higher prevalence and incidence rates of epilepsy in Latin America, our aim was to create a Spanish translation and adaptation of the PIES that could be used in a multicultural treatment and research setting among Hispanics worldwide. Participants and Methods: 48 Patients referred for a neuropsychological evaluation with a diagnosis of epilepsy (English N=36, Spanish $\mathrm{N}=12$ ). Referrals included pre-surgical evaluations ( $\mathrm{N}=23)$, postsurgical follow-up $(\mathrm{N}=6)$, co-occurring psychogenic non-epileptic seizures $(\mathrm{N}=3)$, cognitive complaints $(\mathrm{N}=5)$, and mood disorders $(\mathrm{N}=11)$. Sample was $52 \%$ female, mean age of $37.9(\mathrm{SD}=14.2)$, with $\mathrm{M}=13.9$ years of education ( $\mathrm{SD}=2.3)$, FSIQ $\mathrm{M}=95.4(\mathrm{SD}=13.7)$, Beck Depression Inventory (BDI) M=12.3 (SD=10.23), and Beck Anxiety Inventory $\mathrm{M}=11.7(\mathrm{SD}=10)$. Translation of the measures included independent translation by two native Spanish speakers, back-translation by a native English speaker, expert feedback from neurologist specializing in epilepsy, and community feedback from patients with epilepsy not involved in the study. 
Results: T-test and chi square analysis revealed no significant group differences in age, gender, education, FSIQ, BDI, or BIA scores. A MANCOVA indicated no significant differences between English and Spanish-speaking participants in the PIES total score or sub-scale scores, when controlling for FSIQ, BDI, or BAI scores.

The Total PIES score for the sample $\mathrm{M}=61.5$ ( $\mathrm{SD}=20.4$ ), with the following subscale scores; Seizure Effects $\mathrm{M}=61.2(\mathrm{SD}=26.5)$, Adverse Medication Effects $\mathrm{M}=66.77$ ( $\mathrm{SD}=21.8$ ), Mood and Social Functioning Effects $\mathrm{M}=57$ ( $\mathrm{SD}=25.1)$.

Conclusions: The Spanish translation of the PIES has the potential to be a quick and effective quality of life measure for patients diagnosed with epilepsy. Comprehensive normative data will published when available.

Correspondence: Christina I. Bermudez, M.Ed., M.A., M.S., Psychology, Nova Southeastern University, 6510 Main St Apt 312, Miami Lakes, FL 33014, United States. E-mail: christina.bermudez1@gmail.com

C.I. BERMUDEZ, W. JERMAKOWICZ, C. MILLAN, J. KOLCUN, K. MCINERNEY, N. SUN-SUSLOW, S. DE LA GUARDA, L. SEGALA, S. ANDERSON, J.R. JAGID, M. LOWE, M.E. PALOMEQUE, R. RIBOT, E. SERRANO, L. TORNES, N. VELEZ-RUIZ, A. KANNER \& G.J. REY. Improvement in Non-Memory Neuropsychological Test Performance Following Stereotactic Laser Ablation for the Treatment of Temporal Lobe Epilepsies.

Objective: To examine non-memory cognitive outcome following stereotactic laser ablation (SLA) in refractory temporal lobe epilepsy (TLE). Participants and Methods: 18 patients underwent SLA at the University of Miami. Mean duration of seizures was 29.1 years $(S D=11.3)$, age at time of surgery $\mathrm{M}=43$ years $(S D=13.4), 55 \%$ of the subjects were male, $78 \%$ White (78\% Hispanic) and 22\% African American, with an average education level of 11.39 years $(S D=2.6)$. Neuropsychological evaluations were completed 6-12 months pre and post-SLA. Volumetric analyses were completed by two independent experts using manual tracing with $>90 \%$ inter-rater reliability.

Results: Patients had 3-6 ablations $(M=4.7, S D=.75)$, with a total brain volume ablated ranging from $984-2312 \mathrm{~mm}^{3}$. Percentage of hippocampus ablated from $63-91 \%(M=81.59, S D=7.8)$. There was a significant negative correlation with total ablation size and postoperative Engel classification $(r=-.59, \mathrm{p}<0.05)$. There was a significant positive correlation between number of ablations and post-operative graphomotor processing speed $\left(\mathrm{r}=.468, \mathrm{p}<0.05, \mathrm{r}^{2}=0.22\right)$. No significant correlations were found between ablation size, post hippocampal volume, post-operative seizure frequency, or psychopathology and post-operative test performance.

Statistically significant improvement was observed in overall intellectual capacities (FSIQ, $p<0.05, M=5.33, S E=2.3$ ) and mental set-shifting skills (Trail B, $p<0.05, M=7.55, S E=3.3$ ). Improvement was also noted in dominant hand fine motor dexterity $(M=2.9, \mathrm{~S} E=4.11)$, graphomotor processing speed $(M=3.0, S E=1.85)$, and auditory attention $(M=6.0$, $\mathrm{SE}=5.03)$, but these did not reach statistical significance, possibly due to limited sample size.

Conclusions: The current data suggest that there is an overall improvement in cognitive functioning following SLA, regardless of ablation size, duration of seizure disorder, or post-operative seizure recurrence. Further research includes examining post-operative medications, longterm seizure outcomes, and EEG abnormalities.

Correspondence: Christina I. Bermudez, M.Ed., M.A., M.S., Psychology, Nova Southeastern University, 6510 Main St Apt 312, Miami Lakes, FL 33014, United States. E-mail: christina.bermudez1@gmail.com
C.I. BERMUDEZ, W. JERMAKOWICZ, C. MILLAN, J. KOLCUN, K. MCINERNEY, N. SUN-SUSLOW, S. DE LA GUARDA, L. SEGALA, S. ANDERSON, J.R. JAGID, M. LOWE, M.E. PALOMEQUE, R. RIBOT, E. SERRANO, L. TORNES, N. VELEZ-RUIZ, A. KANNER \&. G.J. REY. Visual and Verbal Memory Changes Associated with Left vs. Right Stereotactic Laser Ablation for the Treatment of Temporal Lobe Epilepsies.

Objective: To examine memory outcome following left vs. right stereotactic laser ablation in patients with intractable temporal lobe epilepsy. Participants and Methods: Of 18 patients who underwent SLA $($ left $=10$, right $=8)$ at the University of Miami, 14 had a diagnosis of lateralized mesial temporal sclerosis (MTS) (left $=7$, right $=7$, language dominance (left=16, right=1, bilateral=1). Age at time of surgery was $\mathrm{M}=43$ years $(\mathrm{SD}=13.4)$. Duration of seizures disorder was $\mathrm{M}=29.1$ years $(\mathrm{SD}=11.3) .55 \%$ males, $78 \%$ White $(78 \%$ Hispanic) and $22 \%$ African American, average education level of $\mathrm{M}=12.4$ years $(\mathrm{SD}=2.6)$. Volumetric analyses were completed by two independent experts using manual tracing with $>90 \%$ inter-rater reliability. Neuropsychological evaluations were completed 6-12 months pre and repeated at 6-12 months post SAL.

Results: Prior to surgery, differences were only present in visual memory $(\mathrm{F}=.355$ (1, 14), $\mathrm{p}<.03$; Left $\mathrm{M}=39.78, \mathrm{SD}=12.13$, right $\mathrm{M}=26.86$, $\mathrm{SD}=8.90)$. Following surgery lateralized analysis showed a significant decline in delayed verbal memory for left ablation only $(\mathrm{F}=1(1,14)$, $\mathrm{p}<0.05$, (Pre $\dot{\mathrm{M}}=42.25 \mathrm{SD}=13.80$ vs. post $\mathrm{M}=37.86, \dot{\mathrm{SD}}=16.16)$. Left ablation patients also had a decline visual memory, but this did not reach statistical significance. In contrast, right ablation patients demonstrated mean improvement in all verbal and visual memory measures, but not a significant change from pre to post scores. Ablation size and hippocampal volume loss was not significantly correlated with performance in either visual or verbal memory measures for both right and left SLA patients.

Conclusions: Findings suggest greater risk for memory morbidity following left SLA surgery. In contrast, right SLA patients, showed mean improvement in all memory scores. Next steps include further analyses of pre-surgical atrophy and lateralization memory index.

Correspondence: Christina I. Bermudez, M.Ed., M.A., M.S., Psychology, Nova Southeastern University, 6510 Main St Apt 312, Miami Lakes, FL 33014, United States. E-mail: christina.bermudez1@gmail.com

S. CAVACO, B. MOREIRA, D. DIAS, A. MARTINS DA SILVA, J. LOPES, J. RAMALHEIRA, J. FREITAS \& J. CHAVES. Auditory Verbal Learning Test in unilateral MTLE with hippocampal sclerosis.

Objective: To assess the lateralizing value of a verbal memory test in unilateral mesial temporal lobe epilepsy with hippocampal sclerosis (MTLE-HS).

Participants and Methods: Twenty-three right MTLE-HS and 26 left MTLE-HS with similar demographic characteristics performed the Portuguese version of the Rey Auditory Verbal Learning Test (AVLT). None of the participants had epilepsy surgery prior to the assessment. Two neurorradiologists, blinded to the neuropsychological test results, identified by consensus the side of sclerosis based on visual inspection of 1.5 or 3.0 Tesla MRI scans. Chi-square test and t-test for independent samples were used for group comparisons. ROC curves were calculated to identify the best cut-off scores to discriminate right and left MTLE-HS.

Results: Regarding ROC curves, the AUC for AVLT Delayed Recall measure (mean scores: right MTLE-HS=8.1 \pm 2 , left MTLE-HS=5.5 \pm 3 ) was 0.799 and for Long-Term Percent Retention index (LTPR; mean scores: right MTLE-HS=81.5 \pm 17.8 , left MTLE-HS=58.2 \pm 30.1 ) was 0.787. A Delayed Recall $<7$ and a LTPR $<72$ were found respectively in $73 \%$ and $77 \%$ of patients with left MTLE-HS, whereas $78 \%$ of right MTLE-HS patients scored 7 or above on the Delayed Recall and $74 \%$ scored 72 or above on the LTPR index. However, none of the AVLT Immediate Recall trials (including the derived indexes: Total Learning 
and Learning Over Trials) or the Delayed Recognition trial discriminated right from left MTLE-HS ( $p>0.05)$.

Conclusions: Structural asymmetry of the hippocampus was strongly associated with a verbal memory test. Impaired delayed recall of verbal information had high concordance with unilateral left MTLE-HS. This cognitive indicator can be useful in determining the lateralization of MTLE-HS before surgery. The study findings are consistent with recent clinical-pathological studies performed in resected hippocampal specimens of MTLE-HS patients.

Correspondence: Sara Cavaco, Neurology, Centro Hospitalar do Porto Portugal, Largo Prof. Abel Salazar, Porto 4099-001, Portugal. E-mail: smcavaco@icbas.up.pt

C.M. CONSIDINE, J.E. ROSEBERRY, D. SABSEVITZ, L. GLASS, J. BINDER \& S. SWANSON. The Clinical and Neuropsychological Characteristics of a Nocturnal-seizure Sample, with Case-matched Comparison to a Diurnal-seizure Contrast Sample.

Objective: Seizure characteristics and neuropsychological dysfunction is better documented within diurnal than nocturnal epilepsy syndromes. It was expected that after controlling for demographic and seizure characteristics, a nocturnal-only seizure (NS) group would perform more poorly than a case-matched diurnal-only seizure group (DS) on select neuropsychological measures, presumably due to disturbed sleep physiology.

Participants and Methods: Clinical and normed neuropsychological data from 31 (women=10; $\mathrm{M}_{\text {age }}=36.65, \mathrm{SD}=13.24 ; \mathrm{M}_{\text {educ }}=13.26$, $\mathrm{SD}=0.65) \mathrm{NS}$ patients was extracted from an epilepsy database $(\mathrm{N}=525$; $5.57 \%$ nocturnal-only seizures). A case-matched (demographics \& seizure characteristics) DS contrast group was created. Correlation analyses between NS seizure-related variables and neuropsychological performances were conducted. The neuropsychological profiles of the NS, DS, and entire database were overlaid for comparison. NS and DS patients were compared using paired t-tests on select neuropsychological measures.

Results: Compared to the entire database sample, the NS subgroup presented with significantly different seizure-foci (more bifrontal, less left temporal; $p=0.048$ ). Reported number of seizures in the past month, lifetime tonic-clonic or status epilepticus episodes, and seizuremedications did not significantly differ between the NS subgroup and the overall database sample. The NS neuropsychological profile revealed weaknesses ( 0.75 SD) in psychomotor-speed and executive functioning relative to norms. Psychomotor speed was significantly weaker in the DS-matched sample $(p=0.003)$.

Conclusions: Nocturnal-only seizure presentation is relatively rare and more likely associated with bifrontal and right temporal seizure foci. NS neuropsychological performance weaknesses are similar to that of DS performances, with the exception of potentially better preserved psychomotor-speed. Potential sleep disturbance due to nocturnal seizure activity does not appear to add to the cognitive morbidity found in epilepsy syndromes.

Correspondence: Ciaran M. Considine, Ph.D., Neuropsychology, Milwaukee VAMC, 5000 W. National Ave., Building 111 - Suite 3304, Milwaukee, WI 53295, United States. E-mail: considine.ciaran@gmail. com

M. CORNWELL, J. SPAT, E. SMITH-HYLE, J. SUZUKI, S. MANDELBAUM, M. BEDERSON, L. LANGAN, A. DESOLLAR, C. STEINBERG \& H.A. BENDER. The moderating role of seizure variables on social communication difficulties in children with epilepsy.

Objective: It has been widely reported throughout the pediatric epilepsy literature that this population is at increased risk of sociallyand developmentally-atypical behaviors (i.e., stereotypies, restricted range of interests and negative reaction to change) and social communication disorders (e.g., uncommon linguistic and conversational characteristics). Both decreased opportunity for socialization and underlying neuropathology have been implicated as etiologies. The present study investigated the "competence correlates" associated with deficits in social interaction in children with different seizure types, varying age at onset, seizure frequencies and treatment regimens.

Participants and Methods: Sixty participants (22 male and 38 female), ranging in age from 6-17 years $(M=11.91$ years, $S D=3.01)$, comprised the sample. All participants had a confirmed diagnosis of epilepsy; children with FSIQs $<70$ were excluded. In addition to a comprehensive neuropsychological test battery, two parent-report behavior rating scales, the Child Behavior Checklist (CBCL) and Behavior Assessment System for Children-2 (BASC-2) were also completed.

Results: Chi-square analyses failed to indicate a relationship between age at seizure onset and frequency on social skills and behavioral function. Of the children receiving AEDs ( $70 \%$ of sample), scales reflecting "thought problems" (CBCL) and "atypicality" (BASC-2) reached statistical significance $(\mathrm{P}<0.05)$. In treatment naïve participants $(30 \%$ of sample), there was an association between seizure type and thought problems, atypical behaviors and a global measure of psychiatric and behavioral symptomatology (BASC Behavioral Symptom Index $\mathrm{p}=0.005)$.

Conclusions: This investigation identified several subgroups of children with epilepsy at increased risk for social skills and social communication deficits. Frequent assessment, psychoeducation, psychotherapy and/or cognitive remediation may be particularly critical for long-term outcomes in this population.

Correspondence: Melinda Cornwell, 1468 Madison Avenue, New York, NY 10029, United States. E-mail: melinda.cornwell@mssm.edu

D.M. DICKSON, V.M. CUELLAR, N. NUSSBAUM \& J.B. TITUS. Expressive Language Measures in Focal Epilepsy: How Do They Relate and Do They Help with Lateralization?

Objective: Research on adult populations with temporal lobe epilepsy suggests that performance on the Boston Naming Test (BNT) has utility in predicting side of seizure focus. Despite common use of the BNT with pediatric epilepsy populations, literature regarding its utility in predicting lateralization of seizure focus is lacking. The present study offers a preliminary exploration of the performance of a pediatric sample with focal epilepsy on measures of expressive language.

Participants and Methods: The expressive language of 30 children with focal epilepsy was evaluated on measures of confrontational naming (BNT), expressive verbal knowledge (Expressive Vocabulary Test-Second Edition; EVT-2), and verbal fluency (Delis-Kaplan Executive Function System; A Developmental NEuroPSYchological Assessment-Second Edition). Age ranged from 6-18 years (mean age $=11.86$; $\mathrm{SD}=3.84)$, and the majority had a right-sided seizure focus $(n=20)$ as confirmed with EEG. Correlational analyses were conducted between measures to consider construct commonality. Independent t-tests were performed to assess the utility of these measures in lateralizing seizure focus.

Results: Performance on all measures was higher for subjects with a right-sided seizure focus, but these differences did not achieve significance; variability was more pronounced amongst subjects with a leftsided focus. The correlation between BNT and EVT-2 standard scores was found to be strongly positive $(r=.85, p<.001)$, providing evidence of construct commonality between these two measures.

Conclusions: These findings suggest that the clinical utility of expressive language measures for lateralizing seizure foci may not apply to pediatric samples. While expressive verbal knowledge may be too broad of a construct to lateralize dysfunction, the lack of group differences in verbal fluency speaks to the importance of using a multifactorial approach when predicting lateralization. Implications, strengths, and limitations of the current study will be discussed.

Correspondence: Duncan M. Dickson, MA, Educational Psychology, University of Texas at Austin, 213 Wisteria Way, San Marcos, TX 78666, United States. E-mail: duncandickson@utexas.edu 
L.E. KORTHAUER, D. SABSEVITZ, E.J. MAHONEY, P. BAUER, E.E. QUASNEY, L. GLASS, S. SWANSON \& J. BINDER. Regional Subcortical Volumes are Associated with Pre-Operative Memory Performance in Left Anterior Temporal Lobectomy (ATL) Patients. Objective: Hippocampal sclerosis and atrophy are common in temporal lobe epilepsy (TLE) patients. Structural hippocampal changes have been associated with pre-operative memory performance and memory outcome following anterior temporal lobectomy (ATL). Additionally, prior research has shown wide extratemporal effects of TLE, including smaller volumes of subcortical structures such as the thalamus, putamen and caudate nuclei. However, little is known about the contribution of extratemporal structures to neuropsychological performance in TLE patients. The objective of this study was to characterize associations between memory performance and regional subcortical volumes in TLE patients.

Participants and Methods: Fifty-eight left hemisphere language dominant TLE patients ( $M$ age $=35.4$ years; $43 \%$ men) underwent neuropsychological testing and MRI prior to left ATL. Memory measures included the Wechsler Memory Scale Logical Memory (LM), Visual Reproduction (VR), 7/24 Spatial Recall Test, and the Selective Reminding Test (SRT). Subcortical volume segmentation was performed using Freesurfer. For each structure of interest, asymmetry scores were calculated by taking the difference between right and left volume and dividing by total bilateral volume of that structure.

Results: Greater rightward hippocampal asymmetry was associated with lower delayed recall on LM $(r=-.45, p=.001)$ and the SRT $(r=-.39, p=.005)$. After controlling for hippocampal asymmetry, greater rightward thalamus asymmetry was associated with lower delayed recall on $7 / 24$ immediate $(r=-.29, p=.05)$ and delayed $(r=-.40, p=.005)$ recall. The association between putamen asymmetry and lower 7/24 immediate recall was marginally significant.

Conclusions: Collectively, findings suggest that the integrity of subcortical structures may contribute to memory performance in TLE patients, even after considering hippocampal volume asymmetries. Subcortical structures may be an important part of a broader network that affects executive control of memory retrieval in TLE.

Correspondence: Laura E. Korthauer, M.A., Psychology, University of Wisconsin-Milwaukee, 2441 E. Hartford Ave., Milwaukee, WI 53212, United States.E-mail: korthau2@uwm.edu

J. LAFLEUR, K.E. PATRICK, J.B. DYKSTRA, J. CASS \& K. MCNALLY. Impact of neuropsychological consultation in a multidisciplinary pediatric epilepsy clinic.

Objective: Neuropsychologists are increasingly expanding their roles within multidisciplinary medical teams and are being tasked with developing innovative models of care that expand beyond traditional outpatient assessment. The current study seeks to investigate the feasibility and utility of a mental health screening process and neuropsychological consultation within a multidisciplinary pediatric epilepsy clinic.

Participants and Methods: During outpatient clinic visits, parents completed the Strengths and Difficulties Questionnaire (SDQ) to screen for emotional, behavioral, and attention problems. Parents also answered questions about their children's academic needs. Based on screening cut-offs, neuropsychology staff then completed brief consultations during the medical appointment. Consultations involved assessing needs for a comprehensive neuropsychological evaluation, placing referrals, and/or providing behavioral recommendations. Follow-up questionnaires were obtained at subsequent clinic visits (number of visits ranged from 2-6). Mixed effects models were conducted to investigate change in SDQ scores over time.

Results: Consults were completed on 224 patients, ages 3-19. Controlling for effects of time, overall SDQ scores significantly declined in patients seen for a neuropsychology consultation $(M$ before $=26.06, S D=7.01$; $M$ after=24.37, $S D=7.25 p<.005)$. Additionally, the proportion of children with elevated scores on the emotional, conduct, or hyperactive SDQ subscales decreased significantly from $70 \%$ to $59 \%(p=.002)$. The proportion of parents who indicated concerns that their child was not receiving adequate services in school also decreased from $48 \%$ to $42 \%(p=.03)$.

Conclusions: Findings suggest that brief consultation during outpatient medical clinic visits may be a valuable service that neuropsychologists can provide as part of a multidisciplinary medical team. Initial data suggest that consultation may lead to improvement in mental health problems and may help link children with appropriate school services. Correspondence: Jacob LaFleur, B.A. Psychology, Behavioral Health, Nationwide Childrens Hospital, $482 \mathrm{~W}$ 2nd Avenue, Columbus, OH 43021, United States.E-mail: jacob.lafleur@ nationwidechildrens.org.

Y. LEE, T.M. MCMILLAN, D. ALMANE, M. HANSON, B. HERMANN \&. J. JONES. Psychosocial Outcomes 10-years After Diagnosis of Childhood Onset Epilepsy.

Objective: Long-term effects psychosocial effects are linked to childhood-onset epilepsy. The present study examines the long-term effects of childhood-onset epilepsy on education, employment, quality of life and disability.

Participants and Methods: Children with epilepsy were followed at baseline within 12 months of epilepsy diagnosis and 10 years later into adulthood. The sample consisted of 55 healthy controls $\left(M_{\text {age }}=12.36\right.$, $S D=2.97)$ and 53 individuals $\left(M_{\text {age }}=12.23, S D=3.23\right)$ with epilepsy (29 localization-related, 24 generalized). Individuals with epilepsy were categorized by remission status $($ remit $=$ no antiepileptic drugs or seizures in the past 12 months, $N=25)$. Participants completed a demographic questionnaire, WHO Disability Assessment Schedule 2.0 (WHODAS), and WHO Quality of Life-BREF (WHOQOL-BREF) at follow-up. Independent samples t-test and chi-square were conducted for data analysis.

Results: Mean age of epilepsy onset was 11.96 years with a duration of 11.36 years. Compared to controls at follow-up, there were significant differences in WHODAS-Cognitive function subscale, $p<.01$; WHODAS-Participation in society subscale, $p=.04$; the WHODAS total score, $p=.03$. However, there were no significant differences between controls and individuals with epilepsy in employment status, current marital status, earnings, and WHOQOL at follow-up. There were no significant differences between remission status groups based on psychosocial outcomes.

Conclusions: At 10-year follow-up significant group differences between the control and epilepsy groups occurred for measures of disability. Notably, there were no significant differences in psychosocial outcomes in the remitted vs. the non-remitted epilepsy groups.

Correspondence: Yun-Pai (Beatrice) Lee, University of WisconsinMadison, 2924 Harvey Street, APT 7H, Madison, WI 53715, United States.E-mail:lee@neurology.wisc.edu

D.K. PARKHURST, C.M. REYNOLDS, K. KIRLIN, S. SHAHID, C.J. DUNCAN, D. LOCKE \& N.A. ROBERTS. Ability of the Brown Location Test to Differentiate Right and Left Mesial Temporal Lobe Epilepsy: Replication in an Epilepsy Monitoring Unit Sample.

Objective: The Brown Location Test (BLT) measures visual memory while minimizing reliance on verbal and writing abilities. Brown and colleagues investigated the BLT's ability to differentiate right and left mesial temporal lobe epilepsy (RTLE and LTLE) among 16 RTLE and 13 LTLE patients. They found both BLT learning and long delay trials predicted RTLE diagnosis with good sensitivity and specificity, with model fit improvement from tests of verbal memory. Patients included in their sample had left hemisphere language lateralization confirmed by Wada testing. Due to the potential diagnostic utility of this measure and yet limited additional research on its ability to differentiate RTLE and LTLE, we sought to replicate Brown's analyses.

Participants and Methods: Participants included 12 RTLE and 19 LTLE patients from the Epilepsy Monitoring Unit (EMU) at Mavo Clinic in Arizona. The BLT consists of learning and recalling locations of shaded circles. We included the Rey Auditory Verbal Learning Test 
(RAVLT) total and long delay scores to test improved categorization by a verbal memory task.

Results: Using bootstrapped hierarchical logistic regressions, diagnosis was not significantly predicted by learning or long delay trials of the BLT. Adding RAVLT scores in the second step did not lead to significance for the predictors or overall models.

Conclusions: Though a small sample size limits the interpretability of null findings, our lack of replication in a similarly-sized sample without strict language lateralization may suggest that Brown and colleagues' large effects may not necessarily translate to a more typical EMU clinical sample. Data collection is ongoing.

Correspondence: Dona Locke, AZ, United States.E-mail:Locke.Dona@ mayo.edu

C.M. REYNOLDS, D.K. PARKHURST, C.J. DUNCAN, S. SHAHID, D. LOCKE, K. KIRLIN \& N.A. ROBERTS. Predictive Factors of Alexithymia in Epilepsy and PNES.

Objective: Alexithymia is a personality trait characterized by a diminished ability to identify and describe feelings, as well as an inability to distinguish physical symptoms associated with emotional arousal. Alexithymia is elevated in both patients with epilepsy and psychogenic nonepileptic seizures (PNES). To expand on previous research considering factors contributing to alexithymia in this population, we examined the extent to which scores on the Toronto Alexithymia Scale (TAS-20) were predicted by performance on measures of executive functioning, language functioning, and depression.

Participants and Methods: We studied 154 PNES and 161 epilepsy patients with video-EEG confirmed diagnoses. Composite scores were created to assess executive functioning (interference scores of the Stroop Color-Word Test and Part B of the Trail Making Test) and language functioning (Animals, Controlled Oral Word Association Test, and Boston Naming Test). Depressive symptoms were measured with the Personality Assessment Inventory.

Results: Hierarchical linear regressions revealed that lower executive function, lower language performance, and higher depression predicted TAS-20 scores, with particularly the latter two predictors. These relationships were not moderated by diagnosis of PNES or epilepsy.

Conclusions: Although alexithymia is prevalent in both epilepsy and PNES, different neuropsychological processes may underlie this deficit in the two groups. Previously we found that relationships with language were stronger with epilepsy, but formal tests of moderation showed that language and depression were significant predictors, above and beyond executive control, in both groups. Thus, alexithymia may reflect impairments in language and psychiatric contributors in both PNES and epilepsy.

Correspondence: Dona Locke, AZ, United States.E-mail:Locke.Dona@ mayo.edu

S. MAGNUSON, J.S. HUNDAL \& B. FUNK. Construct Validity of NAB Shape Learning in Predicting Mesial Temporal Lobe Seizure Lateralization.

Objective: The purpose of this study was to investigate the convergent and discriminant validity of the NAB Shape Learning subtest in a population of epilepsy patients with epileptogenesis in either the left or right mesial temporal lobe.

Participants and Methods: Seventeen adults previously diagnosed with localization-related (focal) epilepsy were interviewed and administered a comprehensive neuropsychological assessment during an inpatient stay on an Epilepsy Monitoring Unit. Each participant was tested individually and the neuropsychological data were collected between January 2015 and June 2016. Ten individuals had epileptogenic foci within the left mesial temporal lobe and seven individuals had epileptogenic foci within the right mesial temporal lobe.

Results: Significant findings emerged from Pearson correlational analysis suggesting that the NAB Shape Learning subtest has adequate convergent validity and discriminant validity. Additionally, a correlational analysis between the NAB Shape Learning subtest and both the WAIS-IV PRI and RCFT-Copy performances was analyzed and show no significant relationship, indicating that visual spatial skills are not a confounding variable effecting NAB Shape Learning performance. Conclusions: The results suggest that the NAB Shape Learning subtest holds promise as a valid measure of nonverbal/visual learning and memory within a seizure disorder patient population. Furthermore, the findings also suggest that the NAB Shape Learning subtest appears to measure nonverbal memory independently of visuospatial and visuoperceptual abilities. Although there was not a statistically significant difference between seizure lateralization and performance on the NAB Shape Learning, which may have been chiefly due to the limited sample size, the preliminary findings are promising. We feel that the NAB Shape Learning subtest warrants additional exploration regarding its ability to accurately differentiate and predict seizure foci lateralization.

Correspondence: Scott Magnuson, Psy.D., Ohio Health Riverside Methodist Hospital, 1200 Darcann Dr, Columbus, OH 43220, United States.E-mail: ScottAMagnuson@Gmail.com

M. MAIMAN, V. DEL BENE, W.S. MACALLISTER, E. FARRELL, L. VAURIO, M. ARCE RENTERIA, M. SLUGS, S. SHELDON $\&$ W. BARR. Subjective cognitive complaints on the MMPI-2-RF are not associated with cognitive performance on neuropsychological measures among a sample of patients with epilepsy.

Objective: Adults with epilepsy frequently exhibit deficits on neuropsychological testing. Fewer studies have examined the assessment and validity of self-reported cognitive complaints. This study sought to evaluate the relationship between subjective cognitive complaints in adults with epilepsy, as measured by the COG scale from MMPI-2-RF, and other measures included in a neuropsychological battery.

Participants and Methods: Twenty-three clinically referred adults (Age: $M=42.67 ; S D=16.18$; IQ: $M=102.00 ; S D=17.52 ;$ ) with a diagnosis of epilepsy or suspected of seizures underwent neuropsychological assessment, which included the Wechsler Abbreviated Scale of Intelligence- $2^{\text {nd }}$ Edition (WASI-2), Repeatable Battery for the Assessment of Neuropsychological Status (RBANS), and Minnesota Multiphasic Personality Inventory-2-Restructured Form (MMPI-2-RF). Subsequently, correlational analyses were used to examine the relationship between subjective cognitive complaints and performance on cognitive testing and other validity scales from the MMPI-2-RF.

Results: Correlational analyses revealed that participants' scores on the Cog scale did not correlate significantly with their cognitive performance on the WASI-2 (Full Scale IQ: $r=-.21$; Verbal IQ: $r=-.06$; Performance IQ: $r=-.27$ ) or RBANS (Immediate Memory: $r<.01$; Visuospatial: $r=-.21$; Language: $r=$. 03; Attention Scale: $r=-.12$; Delayed Memory: $r<$. 01; RBANS Total: $r=-.08)$. Interestingly, their Cog scores did correlate significantly with several validity (RBS: $r=.70$; FBS: $r=.52$ ) scales from the MMPI-2-RF.

Conclusions: This pilot study compared self-reported cognitive complaints with performance on cognitive testing in adults with epilepsy. Consistent with results reported elsewhere, self-report cognitive complaints did not correlate significantly with performance on objective cognitive measures, highlighting the importance of considering both self-report and objective measures when evaluating cognitive skills in this population.

Correspondence: Moshe Maiman, B.A., Psychology, Drexel University, 4700 City Ave. Apt 6307, Philadelphia, PA 19131, United States. E-mail:mainman89@gmail.com

R. MCLAUGHLIN, W.A. SCHRAEgLE, N. NUSSBAUM \& J.B. TITUS. Parental Helplessness and Executive Functioning in Pediatric Epilepsy.

Objective: Quality of life (QOL) in pediatric epilepsy is influenced by many factors, and research suggests that parental coping plays a role. Little is known about the types of variables that impact parental coping. 
This study examines the relationship of coping effectiveness with various epilepsy and cognitive variables.

Participants and Methods: The parents of 39 patients were administered the Illness Cognitions Questionnaire (ICQ) and the Behavior Rating Inventory of Executive Function (BRIEF). Demographic, epilepsy, and cognitive data were obtained from medical records. Correlation analyses were conducted between these variables, and a two-block hierarchical regression was performed to predict parental helplessness.

Results: Correlational analyses revealed significant associations between parental helplessness and number of antiepileptic drugs ( $p=0.018)$, seizure frequency $(p=0.046)$, and the Behavioral Regulation Index (BRI) of the BRIEF $(p=0.025)$. The first stage of the hierarchical regression model revealed no significance with epilepsy, gender, and intelligence as predictors of parental helplessness. The addition of the BRI in the second stage resulted in a significant $\operatorname{model}(p=0.022)$. BRI was the only significant predictor of helplessness (partial $r=0.348$, $\mathrm{p}=0.04)$.

Conclusions: Variance in parental helplessness ratings appeared to be uniquely accounted for by poor behavioral regulation even when accounting for intelligence and epilepsy variables. These findings suggest that executive functioning may play a significant role in a parent's ability to cope with their child's epilepsy. Implications of these findings for developing interventions to improve parental coping and quality of life in epilepsy will be discussed.

Correspondence: Rachael McLaughlin, MA, Neuropsychology, Dell Children's Medical Center, 800 W 38th St, Apt 1110, Austin, TX 78705, United States.E-mail: rdayton88@gmail.com

L. NAKHUTINA, H. PARK, T. MOUNSEY, S.A. MARgOLIS \& A.C. GRANT. The Relationship Between Prospective Memory Performance, Memory Complaints, and Depression in Ethnically Diverse Patients with Epilepsy.

Objective: In people with epilepsy (PWE) there is a poor association between episodic memory complaints and test performance. Rather, episodic memory complaints are often related to depressive symptoms. PWE also have deficits in prospective memory (ProM), but studies of ProM in epilepsy are scarce. We investigated the relationship between ProM complaints, ProM performance, and depression in PWE.

Participants and Methods: Ethnically diverse PWE ( $N=56 ; 59 \%$ Female; 51.8\% African-American, 25\% Caribbean-American, 19.6\% Latino; mean age $40.2 ; \mathrm{SD}=12.5$, mean years of education 12.7 ; $\mathrm{SD}=2.6$ ) completed a ProM measure (Memory for Intentions Screening Test; MIST), the Prospective and Retrospective Memory Questionnaire (PRMQ), and the Neurological Disorder Depression Inventory for Epilepsy (NDDIE). MIST and PRMQ results were characterized, and their relationship with depression on NDDIE was examined.

Results: Mean ProM performance on the MIST was borderline impaired (ProM Total: $\mathrm{z}=-1.8$; 24-hour delay: $73.2 \%$ non-responders). ProM complaints on PRMQ were correlated with MIST ProM Total $(r=-0.26$, $p<0.05)$ but not with 24-hour delay $(r=-0.08, p=0.58)$. After controlling for age and education, ProM complaints did not significantly predict MIST performance (ProM Total: $\beta=-0.152, p=0.22 ; 24$-hour delay: $\mathrm{p}=0.94)$. NDDIE scores were correlated with ProM complaints $(\mathrm{r}=0.29, \mathrm{p}=0.03)$, but not with MIST scores (ProM Total: $\mathrm{r}=0.05$, $p=0.74 ; 24$-hour delay: $r=-0.04, p=0.78)$. In addition, depression was not a significant moderator of the relationship between ProM complaints and MIST performance (ProM Total: $\beta=-0.075, p=0.52$; 24-hour delay: $\beta=-0.194, p=0.15)$.

Conclusions: ProM complaints were not predictive of objective ProM functioning. Consistent with studies of episodic memory, ProM complaints were significantly associated with depressive symptoms. However, depression did not moderate the relationship between ProM complaints and ProM performance.

Correspondence: Luba Nakhutina, Ph.D., Neurology, SUNY Downstate Medical Center, 880 W181st St, Apt. 5A, New York, NY 10033, United States.E-mail: luba.nakhutina@gmail.com
J. SPAT, A. PRITCHETT, S. MANDELBAUM, M. BEDERSON, E. SMITH-HYLE, A. DE-SOLLAR HALE, A.D. BONO, L. MARCUSE, M. FIELDS, J. YOO \& H.A. BENDER. Differences in the Performance of ADHD and Epileptic Patients on Tasks of Attention and Executive Function.

Objective: It has been well established that children with epilepsy have a higher incidence of Attention-Deficit/Hyperactivity Disorder (ADHD) than neurologically healthy controls. However, few studies have established the unique presentation of attentional deficit between these two disorders. An improved understanding of behavioral presentation and neuropsychological endophenotypes would be helpful in order to refine diagnostic decision making and provide more directed treatment interventions.

Participants and Methods: 21 children, mean age $10.1 \pm 2.6$ (11 boys), were administered a comprehensive neuropsychological battery including commonly-administered measures of attention and executive functioning. Of those, 12 had a diagnosis of epilepsy based on the International League Against Epilepsy and 9 had a diagnosis of ADHD (based on parent symptom questionnaire).

Results: No significant differences in inattention and hyperactivity symptoms emerged between the epilepsy and ADHD groups (SNAP-IV Total $p=0.20$ ). A measure of set-shifting and concept formation also failed to yield significant effects. In contrast, on the Connor's Continuous Performance Task, children with epilepsy displayed significantly less response variability than those with ADHD $(p=0.05)$. Also, those with ADHD showed less consistency in reaction times at the different Inter-Stimulus Intervals (i.e., higher standard error by Inter Stimulus Interval $\mathrm{p}=0.05)$.

Conclusions: While many significant cognitive endophenotypes did not emerge between groups of children with epilepsy and ADHD, consistency in reaction time appears to be spared in children with epilepsy, as compared to their peers with ADHD only, thus representing a unique and distinguishing feature. Doing so can help increase diagnostic accuracy and a establish a potential treatment target.

Correspondence: Alexandra Pritchett, B.S., Psychology, Queens College, 57 Hausman St, Brooklyn, NY 11222, United States. E-mail: lexi. pritchett@gmail.com

J. MCNABB, A.N. PUENTE, C. SULLIVAN, M. TESTA \& J. BRANDT. Can neuropsychological test scores predict Wada laterality?

Objective: Concerns about the clinical utility of the Wada test are well documented. Many clinicians now use noninvasive neuroimaging alternatives, but most have not considered neuropsychological test scores as a possibility. This investigation examined if neuropsychological test scores could predict Wada laterality scores.

Participants and Methods: 116 adult temporal lobe epilepsy (TLE) surgical candidates completed neuropsychological evaluations and Wada tests. Seizure laterality was determined by video-EEG monitoring, brain MRI scans, and PET scans. Wada laterality scores were calculated as the difference between scores after right and left injection divided by the sum of both scores, yielding a possible range of -1 (lefthemisphere dominant) to +1 (right-hemisphere dominant). 50 patients also completed post-surgical telephone evaluations.

Results: Two-way between-groups ANOVA (handedness x seizure laterality) revealed right and left TLE groups had left lateralized language, but non-right handers were less lateralized. Both TLE groups had memory laterality contralateral to their seizure focus, and the non-right handers were more lateralized. Hierarchical linear regression revealed seizure laterality and handedness accounted for $8 \%$ of language laterality variance and $31 \%$ of memory laterality variance. Neuropsychological test scores explained incremental variance in predicting language laterality, but not memory laterality. Finally, language laterality was not predictive of any cognitive outcome, and memory laterality only predicted category fluency performance.

Conclusions: This investigation replicates the well-documented finding that seizure laterality and handedness predict Wada laterality. It also shows that neuropsychological test scores provide improved prediction 
of language laterality, but not memory laterality. This suggests that the Wada test provides unique information for surgical determinations. Nonetheless, the utility of the Wada test remains in question as laterality scores had limited prediction of outcomes.

Correspondence: Antonio N. Puente, University of Georgia, Psychology Building, Athens, GA 30601, United States.E-mail:APuente22@gmail. com

Z. RESCH, E. GRACIAN, E. QUASNEY, D. SABSEVITZ, J. BINDER, W. MUELLER \& S. SWANSON. Neuropsychological Changes Following Frontal Lobe Resections in Patients with Epilepsy.

Objective: The purpose of this investigation was to examine neuropsychological functioning after frontal lobe resection in patients with frontal lobe (FL) epilepsy. Change from pre- to post FL resection was examined in the following domains: executive functioning [Trails A \& B, Controlled Oral Word Association Test, Wisconsin Card Sorting Test], memory [Selective Reminding Test (SRT), Wechsler's Memory Scale Logical Memory (LM) and Visual Reproduction (VR)], language [Boston Naming Test], and motor functioning [Finger Tapping, Grooved Pegboard]. Further, we compared the post-operative change scores between groups who underwent either dominant or non-dominant FL resections.

Participants and Methods: Participants included 26 epilepsy patients who completed neuropsychological testing prior to and six months following FL resection (Men=13; Right Resection = 15; Right Handed = 21; Left Dominant for Language $=21$ ) .

Results: Our findings revealed statistically significant post-surgical improvements in LM $(p=.05)$ and VR $(p=.02)$ scores across all FL resection patients. No statistically significant changes were observed for the remaining neuropsychological measures, nor were differences found in the outcomes of those who were left dominant for language and underwent a left vs right FL resection. However, while statistically nonsignificant, the left FL group had slight declines on the SRT while the right FL group improved.

Conclusions: The current study suggests that, overall, FL resections are not associated with cognitive morbidity. While our findings are not consistent with previous investigations that found significant declines in executive functioning and psychomotor abilities following FL surgery, variations in surgical parameters such as size and location of resections are likely also factors. Our findings suggest that there is little risk for decline in executive functions, and that there are improvements in memory following FL resections. Cognitive improvement may be related to seizure remission and/or practice effects.

Correspondence: Zachary Resch, Neuropsychology, Medical College of Wisconsin, 1538 S 4th Street, Milwaukee, WI 53204, United States. E-mail: zachary.resch@my.rfums.org

\section{A. REYES, T. THESESN, W. BARR, C. MORRISON, C. MCDONALD, R. KUZNIECKY, O. DEVINSKY \& K. BLACKMON. Reduced frontal lobe neuronal activity at rest contributes to executive function decrements in patients with temporal lobe epilepsy.}

Objective: Patients with temporal lobe epilepsy (TLE) show reduced bilateral frontal lobe activity at rest, as assessed by the fractional amplitude of low-frequency fluctuations (fALFFs) in the resting-state fMRI BOLD signal. Bilateral cortical thinning has also been observed within the frontal lobes of patients with TLE. We investigate whether reduced fALFF and cortical thinning are associated with performance decrements in set-shifting, an aspect of executive functioning that is commonly impaired in TLE.

Participants and Methods: Resting-state fMRI, volumetric MRI and neuropsychological data were obtained from 22 patients with TLE. fALFF and cortical thickness were calculated at seven gyralbased regions of interest (ROIs) within the frontal lobes. Measures of set-shifting included the number of perseverative errors on the Wisconsin Card Sorting test (WCST-PE) and time to completion of the Trail Making Test-B (TMT-B[M1]). Spearman rho correlations were used to assess the relationship between these measures, fALFF, and cortical thickness.

Results: Lower fALFF in the left rostral middle frontal gyrus $\left(R_{\mathrm{s}}(22)=-0.509, \mathrm{p}=0.015\right)$, right superior frontal gyrus $\left(\mathbf{R}_{\mathrm{s}}(22)=-0.501, \mathrm{p}=0.017\right)$, left pars opercularis $\left(\mathrm{R}_{\mathrm{s}}(22)=-0.440\right.$, $\mathrm{p}=0.04)$, left pars orbitalis $\left(\mathrm{R}_{\mathrm{s}}(22)=-0.568, \mathrm{p}=0.006\right)$, and right medial orbitofrontal gyri $\left(R_{s}(22)=-0.423, p=0.05\right)$ was associated with greater time to completion on the TMT-B. Lower fALFF in the right medial orbitofrontal gyrus was associated with a greater number of WCST-PE $\left(R_{s}(15)=-0.640, p=0.01\right)$. There were no associations between set-shifting measures and cortical thickness in the frontal lobe ROIs.

Conclusions: Patients with TLE show both structural and functional pathology within the frontal lobes. However, a measure of neuronal activity (fALFF) appears to be more sensitive to executive dysfunction in TLE than a measure of [k1] cortical thickness. Thus, reduced fALFF may serve as a regional marker of cognitive dysfunction in patients with epilepsy and other neurological disorders.

Correspondence: Anny Reyes, Neurology, New York University School of Medicine, 605W 177th st apt 41, New York, NY 10033, United States. E-mail:ar4240@nyu.edu

R.S. RUM, A. REALE-CALDWELL, R. SEVER \& M. SCHOENBERG. Cognitive Function, Depression, and Quality of Life in Postoperative Epilepsy Patients.

Objective: The primary goal of this study was to examine the correlation between cognitive functioning, mood, and quality of life (QOL) in postoperative temporal lobe epilepsy patients. Given the high economic and psychosocial burden that accompanies epilepsy, our purpose is to better understand variables that impact post-surgical outcomes. Though epilepsy surgery is considered safe and effective, it is associated with cognitive risks that have been poorly studied areas in epilepsy management.

Participants and Methods: Postoperative (Engel class I-III) epilepsy patients $(\mathrm{N}=63)$ who underwent comprehensive neuropsychological evaluations including the Quality of Life in Epilepsy (QOLIE-31) and the Beck Depression Inventory (BDI) were utilized for this study. Participants were examined at least 6 months after surgery. Correlational analyses were run between QOLIE-31 scores, postoperative neuropsychological measures, and BDI scores.

Results: Age, gender, education, race, condition duration, and condition onset were found to have no significant association with QOL. We found a significant negative correlation between QOLIE-31 total scores and BDI scores $(\mathrm{r}(63)=-.773, \mathrm{p}<.001)$. QOLIE-31 total scores were significantly correlated with better performances on WMS-IV Visual Immediate Index $(\mathrm{r}(46)=.385, \mathrm{p}<.008)$, WMS-IV Visual Delayed Index $(\mathrm{r}(46)=.348, \mathrm{p}<.018)$, WMS-IV Visual Reproduction $(\mathrm{r}(60)=.307, \mathrm{p}<.05)$, and animal naming fluency $(\mathrm{r}(63)=.312$, $\mathrm{p}<.05)$. No significant correlations were found in the areas of executive function, processing speed, and attention.

Conclusions: Results confirm earlier studies indicating lower depression scores are associated with improved quality of life and also highlight the association between objective cognitive functioning and quality of life among postoperative epilepsy patients, particularly in the area of visual memory. Results only apply to patients who have seen some level of improvement in seizure frequency after surgery.

Correspondence: Ruba S. Rum, BA, BS, Department of Psychiatry and Behavioral Neurosciences, University of South Florida, $13303 \mathrm{~N} 53 \mathrm{st}$, Tampa, FL 33617, United States. E-mail: rrum@mail.usf.edu

C. SABAT, W. BARR, C. MORRISON, O. DEVINSKY \& K. BLACKMON. Differential Patterns of Memory Impairment in Temporal Lobe Epilepsy Subtypes.

Objective: To compare the different patterns of episodic and semantic verbal memory performance and impairments, in patients with mesial temporal lobe epilepsy (MTLE) and lateral temporal lobe epilepsy (LTLE). We tested the hypothesis that patients with pathology in 
the mesial temporal lobe will be more impaired in episodic memory, whereas patients with pathology in the lateral temporal lobe will be more impaired in semantic memory.

Participants and Methods: The participants were 26 patients (10 MTLE, 16 LTLE) with refractory temporal lobe epilepsy that had comprehensive neuropsychological assessments as part of the evaluation for posible epilepsy surgery. We compared episodic memory performance, as assessed by the long-delay free recall $z$-score of the California Verbal Learning Test-second edition (CVLT-II) or the Rey Auditory Verbal Learning Test (RAVLT) (whichever was available), and semantic memory performance, as assessed by the total correct $z$-score of the Boston Naming Test (BNT).

Results: Although our findings did not support our hypothesis, we found a significant difference in memory performance between the two temporal lobe epilepsy groups. Patients with MTLE showed significantly lower scores in both episodic and semantic memory than patients with TLE. Furthermore, the MTLE group $z$-scores are more than one standard deviation lower than what is expected for age, showing impairments in episodic and semantic memory. In contrast, the LTLE group showed age-expected performance in both memory systems.

Conclusions: Our results are concordant with findings in previous studies that reported that patients with MTLE have general impairments in verbal declarative memory (Hermann et al, 1997; Hermann et al., 2007; Stravino Messas et al., 2008). We hypothesize that these findings could be related to the presence of mesial temporal sclerosis in participants with MTLE, a developmental lesion that has been previously related to generalized verbal declarative memory impairments. Correspondence: Camila Sabat, New York University, 696 Sackett St Apt 3R, Brooklyn, NY 11217, United States.E-mail: csp380@nyu.edu

\section{A.K. STEFANATOS, K. SARILL, J.R. MADSEN, D.P. WABER, C. VEGA, J. BOLTON \& K. BOYER. Cognitive and Adaptive Functioning Following Pediatric Hemispherectomy.}

Objective: Hemispherectomy has long since been recognized as a successful treatment approach for individuals with medication-resistant multi-lobe seizure activity. While improvements have been documented in seizure control and global parameters such as overall quality of life, relatively few studies have examined the impact of hemispherectomy on cognitive and adaptive functioning in children.

Participants and Methods: Eleven children $(54 \%$ male, mean age $=6$ years, range 1-13) were administered comprehensive neuropsychological assessments before and approximately 8 months following surgical intervention (6 right, 5 left). Assessments included age-appropriate measures of intellectual and adaptive functioning, from which three composite index scores were derived: Verbal Cognition (VC), Nonverbal Cognition (NC), and Adaptive Behavior (AB).

Results: Following surgery, complete seizure freedom was attained in the majority of patients $(n=10)$. Patients fell significantly below the normative means on $\mathrm{VC}, \mathrm{NC}$ and $\mathrm{AB}$ during both the pre- and post-surgical evaluations ( $p=.00$, one-sample t-test). Higher $\mathrm{VC}$ scores relative to $\mathrm{NC}$ scores were evident at pre-surgery $(p=.01)$. However, no significant difference between scores was observed at follow-up ( $p=.33)$. A closer examination of the data revealed that this convergence was largely attributable to decreases in VC in several patients who had undergone right hemispherectomy.

Conclusions: These findings confirm other reports in the literature that there is considerable variability in cognitive outcomes following hemispherectomy in children. They also reveal a trend for substantially lower performance in the nonverbal domain, irrespective of hemispheric localization of seizures. The relationships of these findings to various clinical factors, including change in seizure frequency, age of seizure onset and age at surgery will be discussed.

Correspondence: Arianna K. Stefanatos, Ph.D., Boston Children's Hospital, 300 Longwood Avenue, BCH 3021, Boston, MA 02115, United States.E-mail: arianna.stefanatos@childrens.harvard.edu
G. STEFANATOS, H. ZOBEL, A.K. STEFANATOS \& J. RIVIELLO. Preservation of Neurocognitive Function in Continuous Spike and Wave During Sleep (CSWS).

Objective: Continuous spike-waves during sleep (CSWS) is associated with severe forms of epileptic encephalopathy in which language, cognitive and behavioral disturbances emerge secondary to frequent diffuse spike-waves and slow waves on the EEG during sleep. Although the neuropsychological problems can resolve with the disappearance of CSWS, the effects can sometimes persist. Here, we describe a case where CSWS was evident in a child with remarkably preserved language and general cognitive function.

Participants and Methods: Patient was administered developmental and neurological evaluations following onset of staring spells and unresponsiveness at 3.5 years. Prior developmental history was unremarkable: language and motor milestones emerged in a timely fashion. Comprehensive electrophysiological and neuropsychological evaluations were administered at follow-up at 5-6 years of age.

Results: An MRI revealed scar tissue in posterior periventricular regions of the left cerebral hemisphere. Repeated EEGs have identified awake epileptiform abnormalities and CSWS. CSWS has been resistant to anticonvulsant treatment. Behavioral problems related to poor impulse control and hyperactivity emerged at 3 years and remain evident at 6 years. Otherwise, language, psychosocial and adaptive function are age-appropriate, aside from frequent episodes of nocturnal enuresis. Anomalies of steady-state auditory evoked responses, soft signs on assessment of motor function and left ear advantage on measures of central auditory processing were suggestive of left hemisphere dysfunction.

Conclusions: The overall picture suggests that neural plasticity resulted in hemispheric reorganization of language. Mechanisms for the preservation of cognition in this case and their implications for our understanding of pathogenetic mechanisms in epileptic encephalopathy will be discussed.

Correspondence: Gerry Stefanatos, D.Phil., Communication Sciences \& Disorders, Temple University, 1701 N. 13th St., Philadelphia, PA 19122, United States. E-mail: stefang@temple.edu

E.L. SUDIKOFF, D.N. LORDO, L.A. HARKER \& D.A.S. KAUFMAN. Surgical Resection Effects on Quality of Life in Pediatric Epilepsy. Objective: Surgical resection has been shown to alter outcomes in pediatric epilepsy, including Health-Related Quality of Life (HRQOL). Few studies have used the Reliable Change Index (RCI) as a method of assessing surgical change, and lateralization effects on post-surgical HRQOL outcomes are not clear. This study examined whether surgical resection on pediatric epilepsy patients is associated with improvement in HRQOL, whether changes differ based on surgical side, and whether post-surgical change in HRQOL is associated with post-surgical change in broader socioemotional functioning.

Participants and Methods: Archival pre- and post-surgical data for twenty-four pediatric epilepsy patients (ages 4-19) were reviewed. Preand post-surgical scores from the BASC-2 PRS and Quality of Life in Childhood Epilepsy Questionnaire (QOLCE) were subjected to repeated measures ANOVA. Changes in scores were measured by calculating the Reliable Change Index (RCI) for each child, and then subjected correlational analysis to examine relationships between post-surgical change on the BASC-2 and QOLCE.

Results: Strong main effects of time and laterality were seen in the data, indicating broad improvements post-surgery. Right hemisphere patients scored higher on their ratings of HRQOL, and their scores tended to improve more following surgery than left hemisphere patients. Atypical behavior tended to improve following surgery for left hemisphere patients, while right hemisphere patients showed the opposite trend. Strong correlations were seen between RCI values on the BASC-2 and QOLCE subscales.

Conclusions: Quality of life improved post-surgery, but side of surgery influenced the directionality and type of post-surgical change. A greater patient sample may strengthen current patterns and illuminate 
additional relationships, providing further information for parents and medical treatment teams regarding the nature of post-surgical quality of life change in pediatric epilepsy.

Correspondence: Eliana L. Sudikoff, M.S., 2122 Massachusetts Ave NW Apt 829, Washington, DC 20008, United States.E-mail: esudikoff@ gmail.com

H. WADSWORTH, K. DHIMA, M. OGDEN-EPKER, L. LACRITZ \& M. CULLUM. Gender Differences in Neuropsychological Lateralization Accuracy in Temporal-Lobe Epilepsy.

Objective: Examine gender differences in neuropsychological lateralization accuracy in temporal-lobe epilepsy (TLE).

Participants and Methods: 66 individuals diagnosed with TLE $\left(\mathrm{M}_{\mathrm{Age}}=34.71 ; \mathrm{M}_{\mathrm{Edu}}=12\right.$ years $)$ underwent neuropsychological assessment, MRI, inpatient video-electroencephalography (in an epilepsy-monitoring unit), and in some cases, ictal and interictal SPECT imaging and Wada procedure. Results from each clinical evaluation were presented at a multidisciplinary epilepsy case conference that included Neurology, Neurosurgery, Radiology, and Neuropsychology, with the goal of reaching a consensus on seizure localization and lateralization for surgical treatment planning. Chi-square analysis was used to determine rates of agreement between neuropsychological and consensus lateralization in females $(n=41)$ and males $(n=25)$. Additionally, presurgical agreement rates were examined among males $(n=10)$ and females $(n=18)$ who were seizure-free post-surgery.

Results: Clinician determined neuropsychological lateralization (through blind review by two neuropsychologists) agreed with the case conference consensus in $72 \%$ of males and $59 \%$ of females, for an overall agreement rate of $62 \%$. Despite this difference, chi-square analysis comparing lateralization agreement between males and females was not significant $(p=.20)$. Furthermore, $70 \%$ of males were ultimately seizure-free post-surgery in comparison to $56 \%$ of females

Conclusions: Although chi-square analysis was non-significant, the rates of agreement between neuropsychological and diagnostic case conference lateralization in males vs females is interesting. These findings suggest that neuropsychological techniques may lateralize epilepsy somewhat more accurately in males than in females. Moreover, pilot data suggest that males may be more likely to be seizure-free postsurgery relative to females, which may indicate that case conference lateralization was also more accurate in men. These apparent differences in neurodiagnostic lateralization by gender merit further investigation. Correspondence: Hannah Wadsworth, BA, University of Texas Southwestern Medical Center, 6333 Forest Park Rd, 1st floor BLA 100, Dallas, TX 75390, United States. E-mail: hannah.wadsworth@ utsouthwestern.edu

A. ZACHERY, X. YOU, E. EMERY, S. GERMEYAN, L. SEPETA, M. BERL, E. WIGGS, A. MARTIN, W. GAILLARD \& W. THEODORE. Epilepsy Surgery and Language Performance: The Effects of Resection Overlap with Functional Activation.

Objective: Epilepsy surgery is an effective method for seizure control, but post-surgical language deficits can occur. FMRI can be used to identify brain regions that process language but few studies have used fMRI to investigate post-operative language outcomes in epilepsy patients. We sought to determine whether pre-surgical fMRI activation and resection overlap affected post-surgical language performance.

Participants and Methods: Twenty-four patients (mean age 38 +/- 11 years) referred to the NINDS Epilepsy Section for evaluation of intractable epilepsy completed fMRI language mapping pre-surgery, and sixteen healthy volunteers (mean age $22.1+/-3.1$ years) completed fMRI using an auditory description decision task (ADDT). Patients and controls completed Boston Naming (BN) and Controlled Oral Word Association (COWA) tests with repeated tests after surgery or after a year (controls). Regional laterality indices (LI) for each task were calculated for three language areas: inferior frontal gyrus (IFG), midfrontal gyrus and Wernicke's area. Tissue map differences between pre- and post-surgical anatomy scans were used to create a resection mask and calculate resection volume.

Results: There was no difference between repeated BN or COWA tests for controls. 22 patients had temporal lobe resections (10 left, 12 right), one had a right parietal, and one a right frontal resection. Mean resection volume was $17.4+/-10.5 \mathrm{CC}$ and of the top 10 percent of functional activation, a mean of $0.29+/-0.53 \mathrm{CC}$ was within resection volume. No resection volume was detected for controls. There was no significant correlation $(p=0.44)$ between language lateralization in the IFG or resection volume $(\mathrm{p}=0.12)$ and language performance; however, a negative correlation was found between resection activation and change in BN scores ( $\mathrm{p}=0.001)$.

Conclusions: Changes in language performance after surgery may be due to the overlap of pre-surgical functional activation with resection, rather than resection volume itself, and warrants further study.

Correspondence: Ashley Zachery, Children's National Health System, 111 Michigan Ave NW, DC 20010, United States. E-mail: azachery@ childrensnational.org.

\section{Imaging (Functional)}

R.A. BERNIER, A. ROY, U.M. VENKATESAN, E.C. GROSSNER, E. BRENNER \& F.G. HILLARY. Examining Neural Network Representation of Task and Rest Following Moderate and Severe Traumatic Brain Injury.

Objective: Changes in functional network connectivity following TBI have received increasing attention in recent neuroimaging literature. This study sought to understand how disrupted systems adapt to injury both during resting and goal-directed brain states.

Participants and Methods: Graph theory was implemented to examine functional connectivity in 20 individuals with moderate/severe TBI and 14 healthy controls (HC). Adjacency matrices were created across 264 functionally defined regions of interest (ROIs), and the weights of surviving edges were compared within group between rest and task and between groups during each cognitive state. Relationships between these metrics were then compared with performance on neuropsychological tests.

Results: Neither group showed differences in weighted degree between rest and task at the global level, but local analyses revealed differences at the subnetwork level. During rest, weighted degree was significantly greater in individuals with TBI within the default mode network (DMN) compared to HC, and within the task-positive subnetwork. During task, weighted degree was significantly greater within the DMN in TBI compared to HC. Connectivity between ROIs of different subnetworks did not differ between groups. During rest, greater degree within the DMN was associated with worse performance on Trails B. During task, there was a trending relationship between greater-within DMN degree and better Digit Span Backward performance.

Conclusions: Differences in connectivity between TBI and HC are most salient at the subnetwork level, which may be most relevant to specific cognitive changes after injury. Results suggest that the relation between connectivity and cognitive functioning depends on which state and within which subnetwork the hyperconnectivity occurs, with evidence for connectivity increases within the DMN being associated with poorer performance during rest, but better performance during task. These findings offer important insights into the role of connectivity in recovery after TBI.

Correspondence: Rachel A. Bernier, B.A., Psychology, The Pennsylvania State University, 111 Bruce V. Moore Building, University Park, PA 16802, United States.E-mail: rub221@psu.edu 
E. BRENNER, F.G. HILLARY, A. ROY, R.A. BERNIER, E.C. GROSSNER, K. SATHIAN \& B.M. HAMPSTEAD. Examining Neural Network Connectivity After Memory Training in Healthy Older Adults.

Objective: We investigated functional connectivity using graph theory analyses of resting-state fMRI data in healthy older adults (HOA) who were randomized to mnemonic strategy training (MST) or subtracting cue training (SCT) for face-name associations.

Participants and Methods: Thirty HOAs between 55 and 88 years of age were randomized to equal groups, MST or SCT, and underwent 3 training sessions as well as pre- and post-training fMRI, during which resting-state data were collected. Regions of interest were examined using Power's 264 functionally defined regions (Power et al., 2011). Graph theory was used to analyze connectivity in large-scale networks. Results: MST resulted in greater behavioral improvement than SCT. Decreased connectivity was evident after SCT in the left temporal default mode network (DMN) $(d=0.88)$, left frontal DMN ( $d=0.73)$ right salience network (SN) $(d=0.70)$, cerebellar network, $(d=1.26)$, and fronto-parietal network (FPN) $(d=0.83)$ relative to pre-training. The MST group exhibited increased connectivity in the temporal-parietal network $(d=1.73)$ and left dorsal attention network $(d=1.02)$ relative to pre-training. Comparing the groups, MST significantly increased connectivity in the left frontal DMN $(d=0.73)$, right fronto-parietal SN $(d=0.70)$, left FPN $(d=0.83)$, and cerebellar network $(d=1.26)$.

Conclusions: Findings indicate that there exist changes in connectivity that are specific to intervention. Results suggest that greater behavioral gains following MST are accompanied by increased connectivity in multiple large-scale neural networks in HOAs. Overall, this study provides critical implications concerning the possible long-term neural network changes following treatment.

Correspondence: Einat Brenner, Psychology, Pennsylvania State University, 419 Bruce V. Moore Building, University Park, PA 16802, United States. E-mail: ebb5161@psu.edu

B. DUDA, M. OWENS, C. LINDBERGH, E.S. HALLOWELL, A. MISTRETTA, A. BROWN, X. XU, U.S. CLARK, J. GUNSTAD \& L.H. SWEET. Dual Compensatory Processes of Brain Activations and Deactivations Support Older Adults' Maintenance of Cognitive Function.

Objective: Examine two neurocognitive models of aging to determine if multiple neurocompensatory processes support cognition during working memory in older adults (OAs). Neural activations and deactivations were summarized by brain quadrant and compensatory models were assessed via left-right $(\mathrm{L} / \mathrm{R})$ frontal ratios of volumetric activity. Consistent with the hemispheric asymmetry reduction in older adults (HAROLD) model, it was hypothesized that age would negatively correlate with $\mathrm{L} / \mathrm{R}$ ratios. Consistent with HAROLD, cognitive performance was expected to moderate (i.e., strengthen) the relation between age and $\mathrm{L} / \mathrm{R}$.

Participants and Methods: 45 right-handed, healthy $\mathrm{OAs}(\mathrm{Mage}=63.78)$ underwent an N-Back FMRI paradigm. Neural correlates of 2-Back were compared to resting fixation with a false discovery rate of $p<.01$. Bivariate correlations were conducted to examine age-related alterations in 2-Back activity. Moderation analyses were then performed to determine if the relation between age and $\mathrm{L} / \mathrm{R}$ is influenced by cognition. Results: 2-Back associated brain activity was left-lateralized for activations and deactivations. Age was negatively correlated with $L / R$ for deactivations $(\mathrm{r}=-0.31 ; \mathrm{p}=.04)$ and positively correlated with $\mathrm{L} / \mathrm{R}$ for activations $(r=0.34 ; p=.02)$. N-Back accuracy significantly moderated the relation between age and $L / R$ for deactivations $(\Delta R 2=.13 ; p=.02)$, but not activations. Results revealed a stronger relation between age and $\mathrm{L} / \mathrm{R}$ at higher levels of cognitive performance $(B=-.02 ; \mathrm{p}=.00)$ than lower levels of cognitive performance $(B=-.00 ; p=.89)$.

Conclusions: Results suggest that OAs may employ different neurocompensatory processes that are specific to deactivations (i.e, HAROLD) and activations (i.e. increased left-frontal activity). Reduced asymmetry of deactivations was associated with increased N-Back accuracy. indicating that deactivations may be particularly sensitive to age-related compensation.

Correspondence: Bryant Duda, BA, Clinical Psychology, University of Georgia, 1197 Cedar Shoals Drive, Apt 109, Athens, GA 30605, United States.E-mail:bduda@uga.edu

K.R. HOSKINSON, N. SIMIC, R. WIER, A. ROMYN, M. DENNIS, W.A. CUNNINGHAM \& K.O. YEATES. Functional Magnetic Resonance Imaging of Social Cognition Following Pediatric Traumatic Brain Injury.

Objective: Children are at risk for social-cognitive difficulties after traumatic brain injury (TBI), perhaps due to diminished integrity of brain systems that support such skills, particularly the mentalizing network. The goal of this pilot study was to examine functional brain activity during a test of theory of mind using task-based fMRI, in children with moderate to severe TBI and a comparison group of children with orthopedic injury (OI).

Participants and Methods: Seven children with TBI ( 5 boys; Mean age $=11.69 ;$ Mean time since injury $=2.29)$ and 7 children with OI ( 4 boys; Mean age $=10.52$ ) completed the Jack and Jill (JnJ) Task, which assesses cognitive theory of mind, during fMRI. Parents rated children's executive (BRIEF), psychosocial (CBC), and adaptive (ABAS-3) functioning.

Results: Relative to children with OI, parents rated children with TBI as having more difficulties with metacognition $(d=0.57)$ and behavioral regulation $(d=0.52 ; \mathrm{BRIEF})$, and greater total problems $(d=0.78$; $\mathrm{CBC})$. Groups did not differ in JnJ performance; both groups performed more poorly on theory of mind $(66 \%)$ vs control trials $(82 \%)$. Better JnJ performance was associated with better metacognition $(r=-.72)$ and behavioral regulation $(r=-.65)$, fewer total problems $(r=-.67)$, and better adaptive functioning $(r=.52$; ABAS-3). During fMRI, across groups, children showed increased activation in the mentalizing network (i.e., posterior cingulate, temporal pole, superior temporal, and medial frontal regions). Children with TBI showed relatively greater activation in several regions, particularly a) while making errors, and b) on trials requiring theory of mind.

Conclusions: Task-based fMRI both is feasible and appears sensitive to deficits in social cognition after TBI. Research using task-based fMRI may improve understanding of complex behavioral challenges children face after TBI. Integration with additional imaging metrics (e.g., DTI) may prove especially useful in triaging children at greatest risk, and allocating intervention resources accordingly.

Correspondence: Kristen R. Hoskinson, Ph.D., Center for Biobehavioral Health, The Research Institute at Nationwide Children's Hospital, 700 Children's Drive, Columbus, OH 43205, United States. E-mail: kristen. hoskinson@nationwidechildrens.org

L.C. KRISHNAMURTHY, V. KRISHNAMURTHY, A. EALEY, D. SCHWAM, D. GREENBERG \& R. MORRIS. Test-Retest Reliability of Seed-Based Resting State Reading Networks Differentiate Typical and Poor Readers.

Objective: Dyslexia is a learning disability that arises from disrupted language-related brain networks across all age groups. Resting state functional connectivity Magnetic Resonance Imaging (rsFCMRI) provides a paradigm to investigate brain networks without the need to do any task. However, given the variability in task-independent rsFCMRI signals, it is important to objectively explore the reproducibility of rsFC networks in such populations.

Participants and Methods: Sixteen adults (8 poor/ 8 typical readers) were scanned using rsFCMRI for two sessions separated by $2-4$ weeks on a Siemens 3T Tim Trio. In addition to the standard rsFC preprocessing procedures, we also applied physiological noise correction for respiratory and cardiac pulsations. Whole brain cross-correlation (CC) maps were calculated with the eight following reading/language network seeds: L-supramarginal gyrus (SMG), L-superior temporal gyrus (STG), L-fusiform gyrus (FG), L-pars Orbitalis (pOB), L-pars 
Opercularis (pOP), L-pars Triangularis (pTR), L-angular gyrus (AG), L-medial temporal gyrus (MTG). The Intra-Correlation Coefficient (ICC) was calculated on Fisher Z-transformed CC to assess test-retest reliability of rsFC networks across the two sessions.

Results: Poor and typical readers' rsFC networks are highly reproducible for all eight seeds $(I C C>.7)$, though they have different network relationships. Most notably, poor readers have significant connections (cluster-corrected $p<0.001$ ) to default mode network (DMN) areas, which is not observed in typical readers. Poor readers also show inverse correlations that are not observed in typical readers, and the effect size is higher for typical readers compared to poor readers.

Conclusions: In summary, we see highly reproducible reading networks across both typical and poor, although in poor readers we see connections to DMN. This shows that rsFCMRI can be used as a viable tool to differentiate typical and poor readers and to target specific brain areas for subsequent investigation.

Correspondence: Lisa C. Krishnamurthy, PhD, Physics \& Astronomy, Georgia State University, 831 Marietta St NE, Atlanta, GA 30318, United States.E-mail: lkrishnamurthy@gsu.edu

\section{B.G. LEE, C. BERNICK, V. KEPE, F. DIFILIPPO \& S.J. BANKS. [F-18]FDDNP uptake, neurocognition, and number of fights in professional boxers and MMA fighters.}

Objective: Repeated concussive and sub-concussive blows have been linked to cognitive change. Some research suggests that these cognitive changes are pathologically linked to tau deposition, which can be characterized by Positron Emission Tomography (PET) through the use of [F-18]FDDNP radioisotope, which binds to tau among other proteins. The relationship between tau protein deposition, the extent of mechanical injuries to the brain, and ensuing cognitive function remains opaque. We report cognitive test data and PET imaging findings from a sample of professional fighters.

Participants and Methods: Eighteen active and retired male professional boxers and Mixed Martial Arts athletes completed a brief neurocognitive battery assessing memory, processing speed, attention, and executive functioning; and underwent PET imaging to assess for uptake of [F-18]FDDNP. We ran partial correlations accounting age between their number of fights and [F-18]FDDNP uptake for amygdala, striatum, thalamus, orbitofrontal cortex, and dorsolateral prefrontal cortex: regions shown to be targets for tau deposition in American football players. We computed partial correlations between uptake in these regions and performance on neurocognitive testing accounting for age. Results: The relationship between number of fights and uptake of [F-18]FDDNP was non-significant for all neuroanatomic regions examined accounting for age $(p>.1)$. Verbal memory false-positive errors were correlated with number of fights $(r=.49 ; p=.05)$ and uptake in the orbitofrontal cortex $(r=-.52 ; p<.05)$. Nonverbal memory false positive errors were significantly correlated with number of fights $(r=.50 ; p=.05)$, but not with uptake in any neuroanatomic region. Conclusions: These data do not show a relationship between [F-18] FDDNP uptake and extent of head injury exposure as indexed by number of fights. The seemingly paradoxical relationship between age, verbal false-positive errors, and [F-18]FDDNP uptake is discussed. Correspondence: Bern G. Lee, University of Nevada, Las Vegas, 72 Puerto Viejo Trl, Henderson, NV 89074, United States. E-mail: bernglee@gmail.com

K. LENGU, S. TARABEN, A. FLORES, T. ANDERSEN, C. SWICK, N. WAGLEY, T. NYMAN, K. MCFARLANE, S. BISCONTI, I. KOVELMAN \& R. LAJINESS-O'NEILL. Neural Correlates of Gaze Processing Explored with Functional Near-Infrared Spectroscopy (fNIRS) at 9 Months: Relationship to Social and Cognitive Development at 2.5 Years.

Objective: Research is needed to better understand the neural substrates of social communication and how they relate to the maturity of social and cognitive skills. Because eye gaze processing may be the most rudimentary skill in nonverbal social development, the present study used fNIRS to examine the hemodynamic responses of 9-month-olds while processing social and nonsocial gaze stimuli and tested how these neural correlates relate to cognitive and social development measured between 9 -mos and 2.5-yrs.

Participants and Methods: Fourteen typically-developing infants underwent measurement of oxyHb with a continuous wave fNIRS system (TechEn, Inc., MA, USA) at 9 months of age (Mage $=40.86(1.47)$ weeks) while engaged in gaze shifts to social and nonsocial stimuli. Kendall tau was used to examine the relationships between oxyHb, cognitive testing (Mullen Scales of Early Learning), and infant behaviors at 9-mos, 12-mos, and 2.5-yrs (Infant Behavior Questionnaire-Revised; Brief Infant Toddler Social and Emotional Assessment; Child Development Inventory).

Results: Higher right temporo-parietal (TP) activation during social gaze was associated with decreased emotion regulation at 9-mos, lower social competency at $12-\operatorname{mos}(\tau \mathrm{s}=-.57,-.73$, respectively; $p \mathrm{~s}<.05)$, and less mature social and language skills at 2.5-yrs ( $\tau \mathrm{s}=-.71,-.73$, respectively; $p s<.05)$. By contrast, higher left TP activation during nonsocial stimuli was positively correlated with basic self-care abilities at 2.5 -yrs $(\tau=-.85, p<.05)$.

Conclusions: These results implicate infant RTP activity during social gaze as potentially predictive of later cognitive and social-emotional development. As evidenced by its relationship with lower social and language skills at follow-up, enhanced RTP activation during social gaze processing at 9-mos may reflect delayed development and/or compensation by early social communication networks. These findings may provide a point of reference for future research on vulnerable populations.

Correspondence: Ketrin Lengu, M.S., Department of Psychology, Eastern Michigan University, Science Complex 352, Ypsilanti, MI 48197, United States.E-mail:klengu@emich.edu

S.N. LIGHT, Z. TAIWO, G. MIRABITO \& M. BEZDEK. FrontoStriatal Correlates of Empathy Subtypes in Healthy Adults.

Objective: The unique frontal-subcortical correlates of trait empathy (to "share in" the emotion of others) subtypes were investigated using fMRI.

Participants and Methods: "Empathic concern" and "empathic happiness" (negative and positive vicarious emotion, respectively) were elicited in 16 adults $(6 \mathrm{men} / 10$ women; $M$ age $=22$ years, $S D=7.0)$ who watched video clips from the television show "Extreme Makeover: Home Edition" during fMRI. The clips depict a family in despair and in need of a remodeled home-which elicits empathic concern and/or "empathic cheerfulness" (the desire to cheer someone up), followed by the family's happy reactions to tailored renovations made to each room of their home-which elicits empathic happiness. Trait empathy was measured via self-report outside the scanner.

Results: Mid-anterior orbitofrontal $(\mathrm{mOFC})$ and $\left(R^{2}=44 \%, p<.05\right)$ frontopolar $\left(R^{2}=32 \%, p<.05\right)$ activity during empathic happiness eliciting video clips (and at trend level during empathic concern eliciting video clips $-R^{2}=21 \%, p=.07$ ), relative to the control condition, predicted greater trait empathic concern. Nucleus accumbens core (NAc) activity $\left(R^{2}=21 \%, p=.07\right)$ during empathic happiness eliciting video clips, relative to the control condition, marginally predicted trait empathic happiness. Globus pallidus (GP) activity during empathic concern eliciting video clips predicted greater trait empathic cheerfulness $\left(R^{2}=25 \%, p<.05\right)$.

Conclusions: $\mathrm{mOFC}$ and frontopolar activity similarly predict empathic concern, yet positive valence forms of empathy are distinguishable based on separable patterns of subcortical activation. NAc activation predicts trait empathic happiness, whereas GP activation predicts trait empathic cheerfulness.

Correspondence: Sharee N. Light, Ph.D., Psychology, Georgia State University, Urban Life Building, Room 1190, 140 Decatur Street, Atlanta, GA 30303,United States.E-mail: slight@gsu.edu 
M.C. MÖLLER, L. ENGSTRÖM NORDIN, T. LI, P. JULIN \& A. BARTFAI. Neural Activity During Vigilance Test Performance - Relation to Cognitive Fatigability in Mild Traumatic Brain Injury.

Objective: Fatigue is a challenging and frequent symptom following mild traumatic brain injury (mTBI) but the underlying neuronal mechanisms are not clear. This study set out to investigate fatigability and neural activity in mTBI during the performance of a psychomotor vigilance task (PVT) by using pseudo continuous arterial spin labeling (ASL) MRI technique.

Participants and Methods: Ten patients (37.5 +/- 11.2 years) with chronic fatigue (FSS >4.0) after an mTBI and ten healthy controls (36.9 +/- 11.0 years) were recruited to the study. Participants completed a $20 \mathrm{~min}$ long PVT while measuring reaction time (RT) in the MRI scanner during acquisition of perfusion ASL data. Fatigability over time was analyzed by dividing the performance and CBF (Cerebral Blood Flow) data into quintiles.

Results: Mean reaction time was significantly lower for mTBI patients $(p=.006)$ and decreased further during the PVT while controls performed stably $(p=.005)$. Variability in performance was significantly higher for patients $(p=.006)$, indicating monitoring problems due to salience network dysfunctions. Change of CBF in brain regions including left thalamus, left/right middle frontal gyri and right insula during the PVT task showed significant interaction effects between group and time. Reaction time at the end of the task and subsequently self-reported current fatigue among the patients correlated with high CBF in middle frontal gyrus $(\mathrm{p}=.001)$.

The controls, but not the patients, were able to evaluate energy expenditure in relation to performance, indicating lower self-awareness among the patients.

Conclusions: Patients with chronic fatigue after mTBI have abnormal regional CBF compared to healthy controls during demanding endurance attention tasks and showed altered strength in brain connectivity during rest before and after PVT. These networks have been related to energizing functions of the brain in previous studies.

Correspondence: Marika C. Möller, Ph.D., Department of Clinical Sciences, Karolinska Institutet, Danderyd University Hospital, Dep of Rehabilitation Medicine, Stockholm 182 88, Sweden. E-mail: marika. moller@ds.se

E. MILLER, R. HEGDE \& U.S. CLARK. Links Between Intrinsic Amygdala Activity and Experiences of Discrimination.

Objective: The neural correlates of social discrimination remain inadequately understood. For example, while much is known about the contribution of the amygdalae to threat perception and, to a lesser extent, implicit bias, relatively little is known about their role in the experience of discrimination. Here, we examine the relation between intrinsic amygdala activity (using resting-state fMRI) and perceived discrimination in a diverse sample.

Participants and Methods: We included 51 adults enrolled in a study of neuroAIDS (25-70 yrs; $47 \% \mathrm{HIV}+, 88 \%$ non-White, $40 \%$ female, $39 \%$ homo/bisexual). The Everyday Discrimination Scale (EDS), a self-report measure, assessed frequency of unfair treatment during common everyday situations. We calculated the fractional amplitude of low-frequency fluctuations within the right and left amygdala during a 5-min resting-state fMRI scan to quantify intrinsic amygdala activity. Results: Mean EDS scores were higher in those who were HIV+ (vs. HIV-), White (vs. non-White), male (vs. female), or homo/bisexual (vs. hetero.); except for sexual orientation $(p=.05)$, these group differences were non-significant ( $p s>18)$. A significant association was found between left amygdala activity and $\operatorname{EDS}(\beta=.54, p=.02)$, which remained even when controlling for HIV status, gender, race, and sexual orientation $(p=.01)$. Associations between right amygdala activity and EDS were non-significant $(p=.17)$.

Conclusions: We find evidence that individuals who report a higher frequency of discrimination exhibit greater intrinsic amygdala activity. This association was independent of sociodemographic factors (gender, race, HIV status, sexual orientation). Our data support prior evidence that the left amygdala may be preferentially involved in processing negative cues. Future studies should examine whether greater intrinsic amygdala activity is associated with greater detection of, memory for, or sensitivity to discrimination. Studies should also examine whether experiences of discrimination influence intrinsic amygdala activity. Correspondence: Evan Miller, BS, Icahn School of Medicine at Mount Sinai, New York, NY 10029,United States.E-mail:Evan.Miller@icahn. mssm.edu

T. NGUYEN-LOUIE, C. KOHEN, A. SIMMONS, M. SHUCKIT, T. SMITH \& S. TAPERT. fMRI-Based Prediction of Level of Response to Alcohol and Heavier Drinking in Adolescents.

Objective: Research on level of response (LR) to alcohol has primarily focused on pharmacological responses but less is known about the underlying neural processes. This study examined whether LR predicts drinking patterns after onset and used neurocognitive markers prior to alcohol use initiation (baseline task-based blood oxygen level dependent [BOLD] fMRI, context-dependent functional connectivity, and neuropsychological performances) to prospectively predict low- vs. high LR status in adolescents.

Participants and Methods: Subjective response to alcohol during the first five drinking occasions was assessed using the Self-Rating of the Effects of Alcohol (SRE) questionnaire. Poisson regressions examined whether SRE scores significantly predict future alcohol use patterns after transitioning into alcohol use. Prospective prediction of high- vs. low-responder status was examined using baseline neuropsychological and task-based BOLD performances in logistic regressions and using context-dependent functional connectivity in mixed-effects metaanalysis models.

Results: Youth who exhibited greater BOLD activation at baseline in left temporal and bilateral frontal and parietal regions and decreased functional connectivity among these regions were significantly more likely to be low-responders once alcohol consumption initiated. SRE at alcohol use initiation significantly predicted increased frequency and quantity of alcohol use at follow-up up to three years after onset $(p s<.05)$.

Conclusions: Possible pre-existing neural differences may exist between low- and high-responders even before significant alcohol use has commenced. Brain activation differences may serve as a potential marker for better identifying youth who may be more at risk of developing AUDs, with possible implications in the development of treatment strategies.

Correspondence: Tam Nguyen-Louie, M.S., Psychiatry, SDSU/UCSD, 8950 villa la jolla dr., Suite C213, La Jolla, CA 92037, United States. E-mail:ttn045@ucsd.edu

A.T. PETERS, R. SMITH, M.T. KASSEL, A. VAN METER, E. BRICENO, K. RYAN, M. HAGAN, A. WELDON, A. VEDERMAN, J. ZUBIETA, M. MCINNIS, S. WEISENBACH, M. STARKMAN \& S.A. LANGENECKER. Neuroendocrine Modulation of Limbic Activation During Semantic List Learning in Depression.

Objective: Deficits in learning/memory are common among depressed individuals. Decreased volume and disrupted function in neural structures essential for memory formation, including the hippocampus and prefrontal cortex, is also reported among depressed subjects. The neuroendocrine stress response system is theoretically understood to mediate these cognitive and neural disruptions via modulation of central nervous system targets with high densities of glucocorticoid and mineralcorticoid receptors.

Participants and Methods: We measured the effects of cortisol on neural correlates of memory in 62 adults (18-65 vears) with major depression or bipolar disorder $(n=39)$ and healthy controls $(n=23)$. Subjects provided salivary cortisol samples before completing a semantic word list-learning task during 3-Tesla fMRI. The effects of cortisol on memory were evaluated using an event related model for words recognized minus not recognized. 
Results: Pre-scan cortisol was significantly associated with impaired word recognition in depressed ( $r=-.31$ ), but not healthy individuals $(\mathrm{r}=.08)$. During encoding of correctly recognized words versus not recognized words, cortisol was associated with engagement of the right lingual and fusiform gyri. Depressed subjects showed decreased activity of the right medial frontal gyrus, the right anterior and left posterior cingulate, left precuneus, and the right lingual gyrus. Cortisol predicted attenuated activation of the left posterior cingulate and the left parahippocampal gyrus in depressed subjects.

Conclusions: Whereas acute cortisol may globally mobilize regions of the ventral stream from the primary visual cortex critical for storage of long term memory and pattern recognition, it was associated with specific disruption to function of limbic areas involved in retrieval among depressed subjects. These results provide preliminary evidence that HPA-axis reactivity may selectively interfere with the adaptive recruitment of limbic circuits supporting memory in mood disorders. Correspondence: Amy T. Peters, M.A., Psychiatry and Psychology, University of Illinois at Chicago, $1747 \mathrm{~W}$ Roosevelt Road, MC 747, Chicago, IL 60608, United States.E-mail: apeter51@uic.edu

J. ROWLAND, J. STAPLETON-KOTLOSKI, K. TABER \& D. GODWIN. Observing Dynamic Networks During the Completion of a Complex Cognitive Task.

Objective: To examine the change in whole brain networks over time during completion of a complex cognitive task and how symptoms of PTSD may affect the those networks.

Participants and Methods: Participants included eight right-handed male combat exposed postdeployment veterans. PTSD symptoms were measured using the PCLM. Magnetoencephalography was acquired during completion of the Iowa Gambling Task. Task related brain activity was identified separately for Wins and Losses. Time series of that brain activity were extracted and segmented around the card selection $(-500 \mathrm{~ms}$ to $1000 \mathrm{~ms})$. The weighted phase lag index was used to measure pairwise connectivity between active brain regions and calculated within $250 \mathrm{~ms}$ windows at $50 \mathrm{~ms}$ intervals. Networks were calculated for each $250 \mathrm{~ms}$ window $(n=26)$.

Results: Analyses were conducted in SPSS using a Generalized Linear Models approach with network metrics as outcomes. Outcome (Win/Loss), Time, PCL-M, age, and education were included in the model as well as Outcome*Time and Outcome*PCLM interactions. P-values less than .001 were considered significant. Age and Education were consistently associated with improved network metrics while higher PCLM scores were associated with poorer network metrics. Loss networks had significantly stronger connectivity occurring at significantly higher frequencies producing lower clustering coefficients. Networks did not change significantly over time, and there were no Outcome* Time interactions.

Conclusions: This study demonstrates that meaningful changes in functional networks can be observed over small time scales during the completion of complex cognitive tasks using MEG. The expected associations with age and education were observed. Networks differed depending on outcome, suggesting a single network is unlikely to accurately describe the functional systems underlying task performance. PTSD symptoms were consistently negatively associated with network metrics during task completion, consistent with negative effects of PTSD on executive function.

Correspondence: Jared Rowland, Ph.D., W.G. "Bill" Hefner Veterans Affairs Medical Center, 1601 Brenner Ave - 11M-2, Salisbury, NC 28144, United States. E-mail: jared.rowland@va.gov

\section{A. SPIROU. Exploration of Neuro-Corralates Between Healthy and TBI Individuals as Modulated by Depression.}

Objective: Individuals with traumatic brain injury (TBI) have been shown to suffer from depression. Feedback processing deficits have been shown to be associated with depression in non-TBI individuals. Since both of these constructs rely on the fronto-striatal network, we examined depressive symptomology, motivational tendencies and striatal activation during wins and losses in individuals with TBI.

Participants and Methods: Participants (TBI and healthy controls (HC)) completed a gambling task in an MRI scanner. Depressive symptomology was assessed with the Chicago Multiscale Depression Inventory (CMDI), while motivational tendencies were assessed with the Behavioral Inhibition/Behavioral Activation (BIS/BAS) scale.

Results: Significant between-group differences were observed on each subscale of the CMDI (Mood: $\mathrm{p}=0.039$; Evaluative: $\mathrm{p}=0.038$; Vegetative: $p=0.005$ ), with no differences seen on the BIS/BAS scale. A strong negative correlation was found for the TBI group between the CMDI and BAS questionnaires overall $(\mathrm{r}=-.698, \mathrm{p}=.008)$, with no significant correlations between questionnaires found for the HC group. Using a volumetric analysis after segmentation of sub-cortical structures, a significant difference was found between groups in nucleus accumbens (NAcc) volume, bilaterally (right: $p=0.003$; left: $p=0.009$ ). Using a whole brain analysis, a significant difference after positive feedback presentation was found in the NAcc $(p=0.05)$, but after accounting for the NAcc volumetric differences, there was no longer a difference in activation between groups after positive feedback.

Conclusions: The current study provides evidence on the importance of controlling for volumetric differences in functional neuroimaging studies, specifically when there are differences in activation between HCs and TBI.

Correspondence: Angela Spirou, Traumatic Brain Injury Laboratory, Kessler Foundation, 9 Katharina Place, Washington Township, NJ 07676, United States. E-mail: aspirou@kesslerfoundation.org

A. TART-ZELVIN, A.M. KORELL, R. ECHON, A.L. MILLER, D. CORRELL, D. VOORHEIS, K. OSIPOWICZ, K.J. TURLEYAMES \& X. XU. An fMRI Study: Neural Mechanisms Associated with Rehearsal Strategy and a Working Memory Task.

Objective: Strategy training positively influences working memory (WM) processing and enhances the relationship between WM and higher cognitive functioning (Turley-Ames \& Whitfield, 2003). The goal was to examine the neural activity during a WM task with and without the use of rehearsal strategy (RS).

Participants and Methods: Participants included 40 healthy participants who did not significantly differ on age, gender, or years of education. Participants completed a modified operation span task in an MRI scanner (M-OSPAN; Turner \& Engle, 1989). The experimental group (EG; $n=20$ ) completed the M-OSPAN after one session of RS training. Results: Controls (CG; $n=20$ ) displayed diffuse activation spanning most of the frontal lobes, while the EG demonstrated a significantly more parsimonious activation pattern $(p<.001)$. The EG outperformed the CG on free recall $(t(38)=-2.94, p=.006)$, displaying better performance on the WM task. Region of interest analyses based on extant literature revealed that the EG used significantly more of the left medial frontal gyrus and dorsal frontal gyrus. The control group (CG) used significantly more of the opercular part of the inferior frontal gyrus $(p<.001)$. The hippocampus and parahippocampal regions did not display significant activations.

Conclusions: Impressively, the EG completed the task more efficiently by utilizing significantly fewer brain regions than the CG. Based on correlations, strategy usage is responsible for better free recall. Thus, effective cognitive strategies are reflected in neural efficiency. These findings can help to inform early detection of cognitive decline and early cognitive interventions (e.g., retraining/rehabilitation).

Correspondence: Ariana Tart-Zelvin, M.S., Psychology, Idaho State University, 584 Pheasant Ridge Drive, Apt. A, Chubbuck, ID 83202, United States.E-mail: atartz@gmail.com 
L. KOZLOWSKI, X. HE, M. LEESE \& J.I. TRACY. Functional Connectivity Superior to Task FMRI Activation at Predicting Language Performance.

Objective: Language deficits in temporal lobe Epilepsy (TLE) are associated with altered hemisphere dominance determined by task fMRI activation. Here we tested if functional connectivity $(\mathrm{FC})$ of language network is predictive of language performance and superior to task activation.

Participants and Methods: FMRI scans during a verb generation task (interleaved task on, off) and a rest condition were performed on 64 TLE patients ( $\mathrm{L} / \mathrm{R}=33 / 31)$. Bilateral Broca's and Wernicke's areas were defined anatomically. Laterality Indices (LI) of task activation (on-off contrast) and pair-wise FC during 3 distinct conditions (task on, off, rest) were calculated. Stepwise linear regressions were performed on our language measures [verbal IQ (VIQ), Boston naming test (BNT), phonemic fluency (PF) and semantic fluency (SF)] with LI and FC as independent variables. Models of LI and FC were considered alone, followed by a combined model to compare their predictive strength.

Results: In LTLE, a left bias in LI of Broca's activation was associated with better VIQ and BNT. Stronger right Broca's-Wernicke's FC at on condition was associated with better VIQ and SF. In combined model, VIQ and SF were best predicted by the same right-sided FC; however, BNT was predicted by LI of Broca's activation and left-right Broca's FC at rest. In RTLE, LI of task activation showed no significant association with language performance. Stronger left Broca's-Wernicke's FC was associated with better VIQ at on and PF at off condition. In combined model, we found the same results, with no LI of task activation contributing additionally.

Conclusions: FC appear to be better predictors of language performance. LI of activation predicted language only in LTLE and only involved anterior regions ipsilateral to the seizure focus. FC predicted language in both LTLE and RTLE with the best predictors always involving the contralateral healthy side, commonly emerged at task on condition. These results may imply FC is more sensitive to networks underlying compensatory mechanisms.

Correspondence: Joseph I. Tracy, Ph.D./ABPP, Neurology, Thomas Jefferson University/Sidney Kimmel Medical College, 901 Walnut Street, Health Sciences Bldg Ste 447, Philadelphia, PA 19107, United States. E-mail: joseph.tracy@jefferson.edu

\section{N. SIDEMAN, X. HE, N. KIM \& J.I. TRACY. The Effects of Aging on the Lateralization of Visual-Spatial Semantic Memory in Temporal Lobe Epilepsy.}

Objective: To investigate the impact of aging on the strength of hemispheric asymmetry during a visual-spatial semantic memory (VSSM) task in temporal lobe epilepsy (TLE) patients and health controls (HC). Participants and Methods: Fifty-six TLE patients (LTLE:31; RTLE:25) and 34 matched HC's were recruited. All subjects underwent an fMRI scan while performing a VSSM task and the laterality index (LI) of the hippocampal gyrus (HG) and the para-hippocampal gyrus (PHG) was calculated. The associations of aging and LI in each structure was explored as well as the LI patterns of the two structures in all groups. In both analyses, we subdivided patient groups (with/without hippocampal sclerosis-HS) and tested for differences in LI patterns.

Results: In HCs, correlations were found between age and both the HG $(r=.39, p=.02)$ and PHG LI's $(r=.41, p=.02)$. The PHG correlation reached a level of significance in LTLE subjects $(r=.41, p=.02)$ when covarying for age of seizure onset. Upon further analysis of HS vs. non-HS, a correlation with age was revealed in the PHG in LTLE subjects with HS $(r=.56, p=.03)$. A trend towards significance was also seen in the PHG in LTLE subjects without HS $(r=.46, p=.09)$. A main effect for group was shown $(\mathrm{F}=4.80, \mathrm{p}=.01)$, with the LTLE displaying a higher LI than the HC or RTLE. An interaction $(\mathrm{F}=4.05, \mathrm{p}=.02)$ was also found such that the LI difference between HG and PHG was highest in the RTLE patients. In HS patients, an interaction was found between group (LTLE, RTLE) and structure (HG, PHG) ( $\mathrm{F}=6.09$, $\mathrm{p}=.02$ ), with RTLE showing a greater HG-PHG LI difference.
Conclusions: These results suggest that with normal aging important shifts in lateralization may occur for tasks that demand bi-hemispheric activity. This aging effect seems to differ according to patient type (LTLE vs. RTLE) as well as the presence of HS. Furthermore, the lateralization of HG reorganized as a result of TLE pathology, with this pattern stronger in the setting of HS. The PHG does not show the same dramatic shifts in laterality.

Correspondence: Joseph I. Tracy, Ph.D./ABPP, Neurology, Thomas Jefferson University/Sidney Kimmel Medical College, 901 Walnut Street, Health Sciences Bldg Ste 447, Philadelphia, PA 19107, United States. E-mail: joseph.tracy@jefferson.edu

S. TRAN, S. TOWLER, K.M. MCGREGOR, G. FRISHKOFF, G. KAUNDINYA, L.C. KRISHNAMURTHY, V. KRISHNAMURTHY, A. JAMES \& B. CROSSON. Task-Residual Functional Connectivity In Language and Attention Networks.

Objective: Resting state functional connectivity captures phasic fluctuations in brain activity in the absence of cognitive engagement in a task. Task-residual functional connectivity capitalizes on the remaining variance after mean task-related signal is removed from the time series to identify functional coherence. Although resting state analyses are the preferred method for functional interactions that are unrelated to a specific cognitive state, task-residual analyses may be more sensitive to functional interactions of specific, task-relevant network connections. This study compared task-residual and resting state functional connectivity between nodes of established networks.

Participants and Methods: Thirty-four participants completed a verbal fluency task in the scanner and a resting state scan. The time series from both data sets were correlated between intrahemispheric nodes of language and attention networks.

Results: In contrast to resting state, task-residual functional connectivity between intrahemispheric nodes evidenced significantly stronger laterality indices in the attention network and a trend towards stronger laterality in the language network. Follow-up tests of the language network demonstrated that left hemisphere regions were more strongly correlated than right in task-residual data whereas there were no differences in resting state data.

Conclusions: The observed network specificity lends support to our conceptualization that preferentially increased activity in left hemisphere regions during engagement in a language task remain in the residuals and show a correlation with one another above and beyond spontaneous and intrinsic fluctuations. Using task-residual data may be optimal for characterizing the synchronized fluctuations between regions of discrete networks.

Correspondence: Stella Tran, Psychology, Georgia State University, 140 Decatur St, Atlanta, GA 30303, United States.E-mail:stran12@ student.gsu.edu

J.L. WOODARD, L. DRICOT, M.A. SUGARMAN, A. NORMAN, E.Z. GROSS \& R.J. GENIK. Resting State Connectivity in Brain Regions Associated with Semantic Familiarity and Knowledge: Relationships with Neuropsychological Performance.

Objective: Few studies have investigated relationships between resting connectivity within functionally defined networks and neuropsychological test performance. In this study, we investigated whether resting state functional connectivity (FC) in regions of interest (ROIs) defined by task-activated functional magnetic resonance imaging (fMRI) during semantic memory tasks correlated with performance on semantic (SM) and episodic memory (EM) measures.

Participants and Methods: Fifteen healthy older adults (mean age $=70.2$ years, $\mathrm{SD}=5.4$ years, 11 women) underwent neuropsychological assessment, resting state fMRI, and event-related task-activated fMRI using tests of famous name recognition and knowledge of occupational categories and specific attributes associated with famous persons. Neuropsychological measures included: Wechsler Memory Scale-IV Logical Memory (WMS-IV LM), Rey Auditory Verbal Learning Test 
(RAVLT), Animal Fluency (AF), and Boston Naming Test (BNT). Cross-correlations were computed between the average time-course signals extracted from ROIs involved in SM recognition and knowledge tasks in each participant. These cross-correlations were then correlated with performance on neuropsychological measures of EM (WMS-IV LM, RAVLT) and SM (AF, BNT).

Results: EM immediate recall measures (WMS-IV LM 1 and RAVLT Trial 1) correlated significantly ( $\left.p^{\prime} s<.01\right)$ with the magnitude of the resting state relationship between default mode network (rsDMN) FC and left superior temporal gyrus (STG). EM delayed recall measures (WMS-IV LM 2 and RAVLT Delayed Recall) correlated with rsDMN $\mathrm{FC}$ and posterior cingulate, while marginal correlations ( $p$ 's<.05) were seen between rsDMN FC and left STG. SM performance was correlated with anterior rsDMN FC and posterior cingulate $(\mathrm{AF})$ and between left posterior rsDMN FC and left STG (BNT). Several additional taskactivated regions also correlated with $\mathrm{FC}$ in other resting state networks. Conclusions: Episodic and semantic memory performance correlate with rsDMN FC and left STG and posterior cingulate.

Correspondence: John L. Woodard, Ph.D., Psychology, Wayne State University, 5057 Woodward Ave, 7th Floor, Psychology Department, Northville, MI 48202, United States.E-mail:john.woodard@wayne.edu

M.A. WOODBURN, S. JOELSON, T. HARRISON GOLDMAN, V. D'SA \& S. DEONI. Motor Network Connectivity Differentially Supports Fine and Gross Motor Skills During Childhood.

Objective: Motor development during infancy is one of the most salient milestones - gestures, crawling, posture, and eventually walking. The synaptogenesis and synaptic pruning of the sensorimotor regions are one of the first regions to structurally mature and become functionally available within the first year of life to support these developmental markers. Here, we explore how functional connectivity of the motor network supports fine and gross motor skills.

Participants and Methods: Subjects $(n=41)$ were typically developing children between the ages of 1 to 2 years $($ Mean $=510.5$ days; $\mathrm{SD}=127$ ) with no neurological or psychiatric diagnosis. As part of an ongoing study examining the relationship between structural and functional brain maturation during early childhood, resting state functional and high-resolution MRI scans were acquired within a week from a Mullen Early Life Scale assessment, which includes scaled measures of fine and gross motor skills. Neuroimaging was appropriately processed using SPM8 and the CONN toolbox. Motor network regions of interest were adapted from previous neuroimaging studies, and confirmed using an Independent Component Analysis of this dataset. Graph theory was then used to quantify the global efficiency as a measure of withinnetwork functional connectivity.

Results: Corrected for age, fine motor skills were positively correlated $(p=0.043 ; r=0.330)$ with motor network connectivity, whereas gross motor skills were negatively correlated $(\mathrm{p}=0.021 ; \mathrm{r}=-0.375)$.

Conclusions: This fine and gross motor dissociation makes sense in light of the known changes in functional connectivity during the first vears of life (i.e. increased between network connectivity of motor and frontal/ visual/executive networks and decreased within-network connectivity globally). In other words, fine motor skills may rely more heavily on direct activation of motor cortex, whereas gross motor skills may rely more on integrating motor regions with other functional networks for execution.

Correspondence: Mackenzie A. Woodburn, Cognitive Neuroscience, Cognitive, Linguistics, \& Psychological Sciences, Brown University, 69 Brown St. Box 8314, Providence, RI 02912, United States. E-mail: mackenzie_woodburn@brown.edu

I. WRIGHT \& A. MARSH. Clinical Utility of a Functional Imaging Approach to Visuospatial Memory Lateralization in Epilepsy.

Objective: Localisation of brain function is important for predicting outcome following epilepsy surgery. fMRI is now commonly used in the presurgical evaluation of language lateralisation. The corresponding development of memory paradigms has proved challenging, particularly in the visuospatial domain. Task difficulty, left hemisphere processing bias and verbal strategies constrain the development of a purely visual task. This study aimed to determine the clinical utility of a novel visuospatial memory assessment in clinical populations and, in an fMRI context, maximising BOLD asymmetry to the right by placing a preferential load on spatial memory.

Participants and Methods: 68 healthy controls and 12 patients with temporal lobe epilepsy underwent a forced choice visuospatial recognition task that tested memory for orientation. fMRI data was available for 12 controls and 12 patients. The task is explicitly designed with progressively increasing difficulty. Behavioural data were analysed to determine encoding success across the spectrum of difficulty. Region of interest analysis of fMRI data was undertaken and laterality indices calculated. Predictive validity for both fMRI and behavioural data was explored.

Results: In controls, behavioural results show modest levels of recognition accuracy for visual scenes and concurrent validity alongside established behavioural tests of visuospatial memory. ROI analysis demonstrated successful recruitment of right hippocampus in controls. Patients with right hippocampal pathology poorly and with evidence of reorganisation. Clinical fMRI demonstrated high level of accuracy in determining lateralisation of memory function relative to structural pathology (100\% sensitivity, $60 \%$ specificity).

Conclusions: This paradigm is suitable for determination of hippocampal recruitment for visual memory. The fMRI protocol demonstrated good accuracy for neuroplasticity of visual memory function in clinical samples. Implications for prediction of postoperative memory outcome are discussed.

Correspondence: Ingram Wright, MA(Cantab) PhD DClinPsychol, School of Experimental Psychology, University of Bristol, Bristol Children's Hospital, Upper Maudlin Street, Bristol BS2 8BJ, United Kingdom.E-mail: ingram.wright@bristol.ac.uk

Z.Z. ZLATAR, C.C. HAYS, L.M. CAMPBELL, K.J. BANGEN, M. MELOY \& C.E. WIERENGA. Objectively-Measured Physical Activity and Sedentary Time and Their Aassociations With Cerebral Blood Flow and Cognition in Older Adults.

Objective: Moderate to vigorous physical activity (MVPA; $>3$ METs) has been associated with higher cerebral blood flow (CBF) and better cognition in older adults, whereas sedentary time (ST) is an independent risk factor for poor cognition. Research investigating the associations between objectively-measured PA, ST, CBF, and their impact on cognition, is lacking. We examined associations between objectively-measured MVPA, ST, CBF, and cognition in older adults.

Participants and Methods: 52 cognitively normal participants (mean age $=72$ years; 30 women) underwent $P A$ monitoring (tri-axial accelerometry), neuroimaging (arterial spin labeling MRI) to measure CBF, and cognitive testing (subtests from WMS-R, CVLT-II, DKEFS, famous face naming). Voxel-wise robust regressions were conducted to investigate regional associations of CBF with MVPA and ST independently, adjusting for age and sex. Average CBF in significant brain regions was extracted and correlated with neuropsychological tests.

Results: Higher MVPA was associated with greater CBF in medial, temporal, and frontal regions. In contrast, higher ST was associated with lower $\mathrm{CBF}$ in frontal, temporal, and parietal regions. CBF in the left supplementary motor area, which was significantly associated with MVPA, showed a positive association with famous face naming scores. Contrarily, CBF in the medial temporal pole, which was associated with ST, showed negative associations with memory performance (ps $<0.05$, corrected).

Conclusions: Findings suggest that MVPA supports higher CBF and better cognitive function in cognitively normal older adults. Consistent with evidence that ST is a risk factor for poor cognition, higher ST was related to lower $\mathrm{CBF}$ in regions implicated in aging. Moreover, there was evidence of vascular dysregulation in right temporal pole regions associated with ST, whereby CBF was not supportive of memory functions. 
More research is needed to understand the vascular implications of ST and their effects on cognition.

Correspondence: Zvinka Z. Zlatar, PhD, Psychiatry, University of California, San Diego, 9500 Gilman Dr MC 0664, La Jolla, CA 92093,

United States.E-mail: zvinka@gmail.com

\section{Imaging (Structural)}

H.A. ALEKSONIS, R. WIER, M.M. PEARSON, C.J. CANNISTRACI, A.W. ANDERSON, J.F. KUTTESCH, B.E. COMPAS \& K.R. HOSKINSON. Diffusion Tensor Imaging of the Genu of the Corpus Callosum: Associations with Day-to-Day Executive Function in Pediatric Brain Tumor Survivors.

Objective: Pediatric brain tumor survivors (PBTS) are at increased risk for deficits in executive function that may impact daily activities. PBTS can also show decreased white matter integrity in specific brain regions, including the genu of the corpus callosum ( $\mathrm{gCC}$ ), which is of particular interest because of its frontal lobe connections, potentially contributing to executive function deficits (Fleming et al., 2011). Therefore, we sought to evaluate deficits in executive function in PBTS and to assess whether white matter integrity in the $\mathrm{gCC}$ is associated with these outcomes.

Participants and Methods: Eleven PBTS (ages 8-16) who were $\geq 2$ years post-diagnosis and 14 healthy controls (HC) matched for age and sex were included in the study. DTI tractography (DSI Studio) assessed white matter integrity within the gCC. Primary caregivers completed the Behavior Rating Inventory of Executive Function (BRIEF).

Results: Relative to HC, PBTS had significantly lower gCC mean fractional anisotropy $(\mathrm{mFA})(d=-0.95, p<.01)$ and significantly higher ratings of executive function deficits on the BRIEF Global Executive Composite score $(d=1.44, p<.01)$. Both the Metacognition Index $(d=1.15, p=.01)$ and the Behavioral Regulation Index $(d=1.29, p<.01)$ were higher in PBTS than HC. Hierarchical regression showed that group was a significant predictor of Metacognition Index (adjusted $\mathrm{R}^{2}=.22$ ). The addition of $\mathrm{gCC} \mathrm{mFA}$ to the model predicted significant additional variance in outcome (adj $\mathrm{R}^{2}=.38, \Delta \mathrm{R}^{2}=.18$ ). Group predicted Behavioral Regulation Index scores (adj $R^{2}=.26$ ), but the addition of gCC mFA did not predict significant additional variance.

Conclusions: These results suggest a link between reduced gCC mFA and metacognitive deficits, which may illustrate the behavioral implications of reduced white matter integrity on day-to-day functioning. Tractography in other regions, and linkage with cortical functional activation, should be used to determine additional pathways and networks that contribute to metacognitive functioning deficits in PBTS.

Correspondence: Holly A. Aleksonis, B.S., Center for Biobehavioral Health, Research Institute at Nationwide Children's Hospital, 700 Children's Dr, FB Suite 3B.2, Columbus, OH 43205, United States. E-mail:holly.aleksonis@nationwidechildrens.org

C. ARRINGTON, L.C. KRISHNAMURTHY, J. CRUTCHER, A. EALEY, V. KRISHNAMURTHY, S. DINA, D. GREENBERG \&. R. MORRIS. Residualized Tract and Whole Network White Matter Microstructural Integrity Negatively Correlates with Reading Measures.

Objective: Reading is a complex cognitive process which requires the coordination of multiple underlying cortical regions. As such it also requires the successful communication between these regions via connecting white matter tracts. Research has established a network of white matter tracts such as the superior longitudinal fasciculus (SLF), inferior fronto-occipito fasciculus (IFOF), inferior longitudinal fasciculus (ILF), and the uncinate fasciculus (UF) which are associated with reading.

Participants and Methods: The current study examined relations between measures of white matter integrity and reading in 22 poor and typical adult readers. Tract based spatial statistics were used to extrapolate network and individual tract integrity values from DTI data for the ILF, IFOF, SLF, and UF, bilaterally. Integrity values were correlated with scores from the Woodcock-Johnson III Tests of Achievement reading subtests as well as the Test of Word Reading Efficiency - Second edition Sight Word and Phonetic Decoding Efficiency subtests.

Results: Left total reading network, left ILF, right IFOF, bilateral SLF, and right UF integrity values were correlated with real word and pseudo-word reading $(r=-.42$ to $-.60, p<.05)$. Right SLF and UF were correlated with reading fluency $(r=-.44, p<.05$ and $r=-.54, p<.01$, respectively). Right UF was correlated with reading comprehension $(r=-.51, p<.01)$

Conclusions: Measures of white matter integrity were differentially related to measures of single word reading, reading fluency, and comprehension. Measures microstructural integrity for the left lateralized reading network are related to real word and pseudo-word reading in adults.

Correspondence: C. Nikki Arrington, PhD, Psychology, Georgia State University, 831 Marietta St NW, Atlanta, GA 30318, United States. E-mail: carrington3@gsu.edu

P.J. Castagna, S. ROYE, M. CALAMIA \& S.G. GREENING. Parsing The Neural Correlates Of Anxious Apprehension And Anxious Arousal In Development: An Examination Of Cortical Thickness In Youth.

Objective: Converging neuroscience and psychological research points to two transdiagnostic facets of anxiety that share a bidirectional relationship with executive dysfunction: anxious apprehension and anxious arousal (Sharpe et al., 2015). Anxious apprehension refers the tendency to engage in negative, repetitive thinking. In contrast, anxious arousal denotes hyperarousal of the sympathetic nervous system. We were interested in delineating the neural correlates (i.e., cortical thickness) of anxious apprehension and anxious arousal in a sample of youth.

Participants and Methods: 35 healthy youth $\left(M_{a g e}=13, S D=2.5\right)$ from the NKI-Rockland pilot study completed the Multidimensional Anxiety Scale for Children (MASC) and underwent high-resolution MRI at 3T. Cortical surface reconstruction and thickness estimation were performed using Freesurfer. As a surrogate for anxious apprehension and anxious arousal we used the Social Anxiety and Physical Symptoms MASC subscales, respectively.

Results: Covariate analyses between thickness and each facet of anxiety were conducted while controlling for age, gender, and were corrected for multiple comparisons. We observed significant correlations between cortical thickness and anxious apprehension in the superior frontal gyrus stretching into the ventral medial prefrontal cortex $(Z=2.96, p=.003)$ and the anterior interior insula $(\mathrm{Z}=3.28, p=.001)$. In contrast, anxious arousal was significantly correlated with aspects of the dorsal lateral prefrontal cortex and temporal pole; notably including the rostral middle frontal gyrus $(\mathrm{Z}=5.14, p<.00001)$.

Conclusions: These preliminary findings extend previous research that has examined cortical thickness and negative affect (Ducharme et al., 2014) and anxiety (Newman et al., 2016) in healthy youth. We also find novel evidence implicating neural structures involved in executive function, emotional reactivity, and self-relevant processing in these two dimensions of anxiety.

Correspondence: Peter J. Castagna, M.A., Psychology, Louisiana State University, 9271 Pecan Tree Drive, Baton Rouge, LA 70810, United States.E-mail: pcasta1@lsu.edu

D.J. COBIA, M.J. SMITH, J.G. CSERNANSKY \& L. WANG. Hippocampal Shape Features Relate to Limbic Integrity and Episodic Memory Function in Neuropsychologically Near-Normal Schizophrenia.

Objective: Schizophrenia is a severe psychiatric illness with widespread impairments in cognition; however, a certain percentage of subjects are known to perform in the near-normal range. Little is known about the 
underlying neurobiological features that may be sustaining cognitive performance. The aim of this study was to characterize hippocampal and accompanying limbic structures in neuropsychologically-defined schizophrenia subtypes. It was hypothesized that the "near-normal" group would demonstrate structural integrity similar to healthy individuals, which would relate to episodic memory function.

Participants and Methods: Schizophrenia subjects were clustered into impaired $(\mathrm{NPI}=34)$ and near-normal $(\mathrm{NPNN}=45)$ subgroups using a k-means algorithm; matched healthy comparison subjects were also included $(\mathrm{COM}=56)$. Neuropsychological functioning was assessed through a comprehensive battery, and MR scanning included T1-weighted images. Estimates of hippocampal and cortical structure were derived using surface-based processing pipelines. MANOVA and Pearson models tested group differences and relationships with neuropsychological performance.

Results: Results revealed significant left hippocampal shape abnormalities in NPI $(F=5.27, p=0.006)$, but not NPNN, relative to COM. Significant positive correlations between increased hippocampal outward shape with larger entorhinal $(r=0.33, p=0.03)$ and cingulate $(r=0.44$, $p=0.003)$ cortex were observed in NPNN, but not NPI. In addition, a negative correlation between hippocampal shape and episodic memory $(r=-0.31, p=0.040)$ was observed in NPNN only.

Conclusions: Hippocampal morphology in NPNN appears to be a unique characteristic similar in form to healthy subjects, but related to limbic cortex and episodic memory performance. These findings reinforce the importance of mesial-temporal networks in the pathophysiology of schizophrenia, and may help account for the preservation of function in certain subgroups.

Correspondence: Derin J. Cobia, PhD, Psychology, Brigham Young University, 1036 SWKT, Brigham Young University, Provo, UT 84602 ,

United States. E-mail: derin_cobia@byu.edu

M. EDWARDS, E. HARTMAN, R. CABEEN, S. CORREIA, D. LAIDLAW, P. MALLOY, S. SALLOWAY \& S. DEONI. Association between network connectivity of select brain regions and cognitive abilities based on deterministic single tensor and multi-fiber diffusion MR tractography.

Objective: Diffusion-tensor imaging (DTI) tractography models white matter tracts. Multiple fiber approaches (e.g., ball-and-sticks) can track fiber trajectory in regions of high fiber crossing more efficiently than single-fiber methods. Alzheimer's disease (AD) impacts cortical structural connectivity due to changes in white matter integrity. AD impacts the precuneus, entorhinal cortex (EC), and caudal anterior cingulate (CAC). We sought to examine the association between these brain regions and memory and executive function based on graph theory with a comparison of metrics derived from single -fiber and multi-fiber tractography.

Participants and Methods: Graph theory metric of node degree was derived for precuneus, EC, and CAC bilaterally ( $\mathrm{LH}, \mathrm{RH})$ in 42 participants (controls $n=13$; cognitively impaired $n=29$ ) using deterministic and probabilistic tractography. Analyses were run separately by tractography method. Linear regression models were conducted with ROI entered as the independent variables and neuropsychological tests as dependent variables controlling for age and education. Neuropsychological measures (raw and z-scores) were Trail Making Test B (TMTB) and Hopkins Verbal Learning Test Free Recall (HVLT FR).

Results: Single tensor network: HVLT FR correlated significantly with degree in one ROI: LH EC (all p<0.020). There were no significant correlations between degree and TMTB in any ROI. Multi-fiber network: HVLT FR correlated significantly with three ROI: LH EC, LH precuneus, and RH CAC (all $\mathrm{p}<0.03)$. TMTB correlated significantly with LH precuneus $(p=0.042)$.

Conclusions: Multi-fiber tractography may provide a more robust substrate than single tensor for identifying associations between cognitive function and graph theory-derived network connectivity in brain regions sensitive to $\mathrm{AD}$.
Correspondence: Melissa Edwards, MA, 345 Blackstone Blvd, Providence, RI 02906, United States. E-mail: melissa_edwards@brown.edu

P.M. GARCIA-EGAN, J. HUFFMAN, R. CABEEN, D. LAIDLAW, T. CONTURO, L. BAKER, L.E. SALMINEN \& S. PHILLIPS. Genetic risk alleles for inflammation and white matter integrity in older healthy adults.

Objective: Interleukin-1 $\beta$ (IL-1 $\beta$ ) and Tumor Necrosis Factor-a (TNF- $\alpha$ ) exert pro-inflammatory properties and neuromodulatory functions. Risk alleles associated with IL-1 $\beta(\mathrm{A})$ and TNF- $\alpha(\mathrm{A})$ correspond to poor cognitive outcomes in memory, attention and processing speed. However, it is unclear whether variations in the genes coding for IL-1 $\beta$ and TNF- $\alpha$ influence brain white matter independent of hyperintensities in healthy older adults. We compared the white matter microstructure measure of fiber bundle length (FBL) in healthy older individuals according to their genetic risk configurations for IL-1 $\beta$ and TNF- $\alpha$.

Participants and Methods: Fifty-nine healthy adults age 50 and over underwent diffusion tensor imaging (DTI) and genotyping. Participants were grouped according to genotype configuration for IL-1 $\beta$ (AA/AG; $\mathrm{n}=33$ vs $\mathrm{GG} ; \mathrm{n}=26)$ and TNF- $\alpha(\mathrm{AA} / \mathrm{AG} ; \mathrm{n}=16 ;$ vs. $\mathrm{GG} ; \mathrm{n}=43)$. Quantitative diffusion tractography DTI (qtDTI) was utilized to define FBLs in the corpus callosum (CC), fornix, and uncinate fasciculus (UF). Results: Groups were similar in age, education and sex. Multivariate analyses revealed significant differences between IL-1 $1 \beta$ genotype groups, with shorter FBLs in CC $F(1,57)=4.26, p=0.044)$ and left fornix in the GG genotype group. FBLs were also significantly shorter in left UF in TNF- $\alpha$ GG carriers $F(1,57)=8.23, p=0.006)$.

Conclusions: Results indicate that individual variability in inflammatory mechanisms represents an important mechanism in the neuropathogenesis of reduced FBL in older adults. Additional studies are needed that incorporate levels of inflammation from blood and cerebrospinal fluid into longitudinal models of FBLs.

Correspondence: Paola M. Garcia-Egan, PhD, Psychology, University of Missouri Saint Louis, One University Boulevard, 325 Stadler Hall, SAINT LOUIS, MO 63121-4499, United States.E-mail:pmgb7b@umsl. mail.edu

N.J. GOODRICH-HUNSAKER, T.J. ABILDSKOV, G. BLACK, K.O. YEATES, H. TAYLOR, A. BACEVICE, D. COHEN, L. MIHALOV, B. BANGERT, N. ZUMBERGE \& E.D. BIGLER. Developmental and sex-related changes in white matter integrity in children: tractbased spatial statistics versus deterministic and probabilistic tractography.

Objective: Several different techniques can be used to analyze diffusion weighted imaging. Any technique must be sufficiently sensitive to detect clinical abnormalities. This is especially critical in disorders like mild traumatic brain injury (mTBI), where pathology is likely to be subtle. mTBI represents a major public health concern, especially for youth under 15 years of age. However, the developmental period from birth to 18 years is also a time of tremendous developmental brain changes. Before embarking on an examination of effects of injury, it is important to establish the degree of age- and sex-related differences.

Participants and Methods: Participants were children aged 8-15 years with mTBI or mild orthopedic injuries (OI). DTI scans were obtained within 10 days of injury. We performed tract-based spatial statistics (TBSS), deterministic tractography using AFQ, and probabilistic tractography using TRACULA to evaluate whether any method provides improved sensitivity at identifying developmental and/or sex-related differences.

Results: Boys and girls showed minor group differences. The results from AFQ and TRACULA showed that boys had higher FA and lower RD compared to girls within the left cingulum cingulate bundle. No sex differences were found using TBSS. Across all three methods, many of the tracts, but not all, revealed increases of FA and decreases of RD and MD with age. TBSS analyses resulted in age-related changes across the all WM tracts. AFQ and TRACULA revealed age-related changes within 
the corpus callosum, cingulum cingulate, corticospinal tract, inferior and superior longitudinal fasciculus, and uncinate fasciculus.

Conclusions: The results are in many ways consistent across all three methods. However, results from the tractography methods provide improved sensitivity and better tract-specific results for identifying developmental and sex-related differences within the brain, and are a reliable way to investigate subtle changes in WM integrity.

Correspondence: Naomi J. Goodrich-Hunsaker, PhD, Psychology, Brigham Young University, PO Box 971060, Orem, UT 84097, United States.E-mail:njhunsaker@icloud.com

\section{K. HORTMAN, A. AILION \& T.Z. KING. Volume of the thalamus relates to verbal fluency performance following a major depressive episode.}

Objective: Adults with MDE have shown slower verbal fluency, however the structural underpinnings are unknown. Thalamic volume was examined because of its association with processing speed and verbal fluency. In this pilot study, we hypothesized that thalamus volume would be related to phonemic and semantic verbal fluency in adults with history of depression.

Participants and Methods: DKEFs Phonemic $(\mathrm{PhF})$ and Sematic Fluency (SemF) measures of word generation were administered to 9 adults with MDE history and 9 controls. MDE was on average 21.69 (4.14) years and matched with controls on age (21.47 years $(3.71)$ ), education $(14.44(1.59))$ and gender $(78 \% \mathrm{~F})$. Thalamus volume(Thal) was obtained using FLIRT in FMRIB Software Library(FSL).

Results: Negative correlation indicated higher Thal was correlated with lower fluency performance (PhF LThal $\mathrm{r}=-.69, \mathrm{p}<.01$ RThal $\mathrm{r}=-.64, \mathrm{p}<.01$ and SemF LThal $\mathrm{r}=-.52, \mathrm{p}=.03$ RThal $\mathrm{r}=-.61, \mathrm{p}<.01)$. $T$-test did not reveal group differences, but indicated medium to large effects (LThal $\mathrm{t}(16)=-1.61, \mathrm{p}=.13, d=-.76$; RThal $\mathrm{t}(16)=-1.82, \mathrm{p}=.09$, $d=-.86)$. Regression testing an interaction between the MDE group and $R$ Thal volume on DKEFS PhF was significant $(\mathrm{F}(3,14)=5.66, \mathrm{p}<.01$, Adj. $\left.R^{2}=.45\right)$. Individual variables were not significant, but a large effect for the interaction was found (Beta=-.54, $\mathrm{t}(14)=-1.95, \mathrm{p}=.07$ ), which indicated a steeper slope $\mathrm{R}$ Thal volume and $\mathrm{PhF}$ following MDE.

Conclusions: A large effect was found for the negative relationship between bilateral thalamus volume and verbal fluency in adults with a history of MDE. These findings may be related to serotonin projections to the thalamus. Future longitudinal research should explore the role of serotonin and corticothalamic structure and connectivity.

Correspondence: Kyle Hortman, Georgia State University, 110 Fontaine Way, Fayetteville, GA 30215, United States.E-mail: hortman.kyle@ gmail.com

M.T. KASSEL, C. HUMPHRIES, K. ALTONJI, D.C. OSMON \& M. SABRI. Radial Diffusivity of Cingulo-Opercular Network Predicts Attentional Switching Performance in Healthy Aging.

Objective: The present study investigated the impact of age-related decline in white matter integrity on executive control among the goaldirected, trial-by-trial frontoparietal network (FPN) and the cingulo-opercular network (CON) supporting maintenance and implementation of task set across trials.

Participants and Methods: Eighteen younger (M=26.1, SD=5.7) and 18 older $(\mathrm{M}=62.4, \mathrm{SD}=4.9)$ healthy adults underwent structural MRI (T1- and diffusion-weighted scans) and a neuropsychological battery of attention and executive control. Principal Components Analysis revealed two components of selective attention (Fac1) and switching (Fac2). Composite scores were created for each principal component. Fiber tracts connecting the CON and FPN executive control networks were identified using probabilistic white-matter tractography using seed regions defined based on Power et al. (2011) meta-analysis. Mean radial diffusivity (RD), an indicator of myelin degradation, was extracted for right and left CON and FPN white matter tracks.

Results: Between group ANOVA of RD with network and hemisphere as repeated measures revealed a main effect of group $(p<.001)$, illustrating that older adults have decreased integrity (higher RD) compared to younger adults. Within-group comparisons indicated a main effect of network $(p<.0001)$, such that the CON displayed greater overall integrity compared to the FPN. A network by hemisphere interaction was also evident $(p=.003)$, demonstrating that the left FPN has lower integrity than the right FPN, however there was no difference in laterality apparent in the CON. Multiple regression analyses separated by group revealed no relation between Fac1 and RD of the FPN, nor CON; however, $\mathrm{RD}$ of the $\mathrm{CON}$ predicted Fac2 performance in both younger $(p=.032)$ and older $(p=.023)$ adults. No relation was found between Fac2 and RD of the FPN for either group.

Conclusions: Greater demyelination of the CON may contribute to diminished ability to maintain executive control early in the course of healthy aging.

Correspondence: Michelle T. Kassel, Psychology, University of WisconsinMilwaukee, 2441 E. Hartford Avenue, Garland Hall, 338D, Milwaukee, WI 53211, United States.E-mail: mkassel@uwm.edu

M.E. MCLAREN, V.M. DOTSON, S.M. SZYMKOWICZ, D.M. O' SHEA, A. O'SHEA, E. PORGES, R. COHEN \& A.J. WOODS. Association of Subthreshold Depressive Symptoms with Cortical Thickness and Surface Area of the Insula.

Objective: Decreased insula volumes have been consistently reported in clinical depression; however, few studies have examined subthreshold depression or the potential unique association of different subcomponents of depression with the insula. This study examined the associations of subthreshold depressive symptoms with insular cortical thickness and surface area-the two components of volume. Additionally we examined whether specific subcomponents of depression (i.e., cognitive or somatic symptoms) drove any observed association.

Participants and Methods: Sixty-three healthy older adults completed the Beck Depression Inventory, Second Edition (BDI-II) and underwent magnetic resonance imaging at 3T. Cortical thickness and surface area values were extracted using FreeSurfer Imaging Software. Multiple linear regression analyses were conducted in which depressive symptoms predicted cortical thickness and surface area of the left and right insula while controlling for sex and estimated total intracranial volume. Results: We found a significant negative association between total BDI-II scores and surface area in the left insula $(p=0.036)$. In follow-up analyses, the cognitive subscale was significantly negatively associated with left insula surface area $(p=0.033)$.

Conclusions: Results of this study suggest that subthreshold depressive symptoms are associated with decreased surface area of the insula, and that cognitive, rather than somatic, symptoms of depression drove this effect. The insula is important in strong emotions like disgust, and is a key structure in the hate circuit in depression. Disruption in the hate circuit may alter cognitive control of emotions, which may explain the association between cognitive symptoms such as worthlessness and guilt and the insula. This study highlights the importance of examining subthreshold depression and the unique association that subcomponents of depression may have with the brain.

Correspondence: Molly E. McLaren, PhD, Clinical and Health Psychology, University of Florida, Department of Clinical and Health Psychology, P.O. Box 100165, Gainesville, FL 32610-0165, United States.E-mail:memclaren@phhp.ufl.edu

N. PRESSON, G. VAN EMAN, K. EDELMAN, W. BIRD, D.O. OKONKWO, W. SCHNEIDER \& S.R. BEERS. Quantification of Diffusion Tractography for Research Application: The Control Group Matters.

Objective: Using diffusion imaging tractography, geometric properties of brain white matter pathways (i.e., tract spread and homologue symmetry) can differentiate healthy control and chronic TBI samples (Presson et al., 2014). This study examines whether these quantitative metrics, as well as generalized fractional anisotropy (GFA), vary by age and IQ in healthy controls. 
Participants and Methods: 79 healthy adults with no prior history of concussion, TBI, blast exposure, stroke, or other major neurological disorders completed a high-b-value multishell diffusion MR scan and neuropsychological testing. High Definition Fiber Tracking was used to generate tractography for 13 major white matter tracts and to quantify spread, symmetry, and GFA of each tract. These metrics were correlated with age and a valid estimate of IQ (NART).

Results: As expected from previous DTI studies, GFA was negatively correlated with age in several tracts. Geometric metrics showed a more varied pattern. Spread in Corticospinal Tract and External Capsule was lower in older participants, but spread increased with age in SLF and Splenium tracts. Symmetry decreased with age in Corpus Callosum Body, Genu, and Splenium, as well as the Uncinate Fasciculus. Symmetry increased with age in the Cingulum. Estimated IQ did not correlate with GFA or tract spread, but was negatively correlated with symmetry in the Cingulum, Corpus Callosum, and Inferior Longitudinal Fasciculus. Conclusions: The current results provide evidence that age and IQ are relevant covariates that should be accounted for when analyzing geometric properties of brain tractography to differentiate control and clinical samples.

Correspondence: Nora Presson, PhD, Learning Research and Development Center, University of Pittsburgh, 3939 O'Hara Street, University of Pittsburgh, Pittsburgh, PA 15260, United States. E-mail: presson@pitt.edu

K. REITER, S. POTTS, W.T. MCCUDDY, A. PORCELlI \& K.A. NIELSON. Cognitive reserve protects against cortical atrophy in those at genetic risk for $\mathrm{AD}$.

Objective: Cognitive reserve (CR) is a theoretical construct that explores static and modifiable lifestyle factors that influence the trajectory of age-related cognitive decline. Inheritance of the Apolipoprotein-E $\varepsilon 4$ allele, a known genetic risk for $\mathrm{AD}$, is associated with reduced cortical thickness (CT). The goal of the current study is to examine the relationship of CR and $\varepsilon 4$ inheritance on CT in healthy older adults.

Participants and Methods: Thirty-five adults between ages 51 and 84 completed genetic testing, self-report measures to assess CR, and underwent magnetic resonance imaging (MRI). CR was measured using education, occupation, premorbid IQ, and current levels of cognitive, social, and physical activities. Composite scores were created from factor analysis. All T1-weighted high-resolution images were preprocessed and cortical reconstruction was conducted in FreeSurfer (v. 5.3, http://surfer.nmr.mgh.harvard.edu). CT analyses were examined in the Query Design Estimate Contrast application of FreeSurfer. A general linear model was performed at each vertex and all analyses were corrected for multiple comparisons with false discovery rate <.05. Results:

Statistically significant interactions effects were found, covarying age, between $\varepsilon 4$ inheritance and CR in the left superior temporal (STG), inferior parietal (IPG), insula, lateral occipital and postcentral gyri (postG). Post hoc analyses revealed that greater CT was associated with high CR but this occurred only in $\varepsilon 4$ carriers within the left postG, STG, insula and was a trend in the IPG $(p=.08)$ and lateral occipital $(p=.10)$. No statistically significant interactions were observed in the right hemisphere.

Conclusions: CR modulated the influence of $\varepsilon 4$ inheritance on CT in regions known to be influenced in $\mathrm{AD}$ and would otherwise show reduced CT. In $\varepsilon 4$ carriers specifically, high CR offset cortical thinning. This relationship was not observed in non $\varepsilon 4$ carriers. Thus, CR may prevent cortical atrophy associated with genetic risk to AD.

Correspondence: Katherine Reiter, M.S. working toward Ph.D., Psychology, Marquette University, 3909 N. Murray Ave Apt 804, Milwaukee, WI 53211, United States.E-mail:kereiter@gmail.com
B. RIZVI, A. NARKHEDE, B.S. LAST, V.A. GUZMAN, J.J. MANLY, N. SCHUPF, R. MAYEUX \& A.M. BRICKMAN. The Relationship Between White Matter Hyperintensities and Cognition and the Mediating Role of Cortical Thickness.

Objective: White matter hyperintensities (WMH) on T2-weighted MRI reflect severity of small vessel cerebrovascular disease. They have been linked to cognitive dysfunction and dementia, although the pathway through which WMH affect cognition is largely unknown. One possibility is that WMH promote neurodegeneration, which, in turn, affects cognitive functioning. We examined whether cortical thickness, an MRI marker of neuronal integrity and neurodegeneration, mediates the relationship between WMH and cognition.

Participants and Methods: T1- and T2-weighted MRI scans were obtained among 519 community-dwelling older adults (mean age $=73.9 \pm 5.6,56 \%$ women, $12.8 \pm 4.5$ years of education, $33 \%$ White, $67 \%$ non-White) who were evaluated with a comprehensive neuropsychological battery. Total WMH volume and global and regional cortical thickness were derived. Using conditional process analysis modelling techniques, we examined the association between WMH volume and global cognition and tested whether an overall measure of cortical thickness mediates this relationship statistically. We also tested whether entorhinal cortical thickness specifically mediates the relationship between WMH volume and episodic memory.

Results: Increased total WMH volume was associated with poorer global cognition ( $b=-0.0089, p=0.0008$, CI:-0.0141,-0.0037) and episodic memory $(b=-0.0128, p=0.0018, C I=-0.0208,-0.0048)$. Examination of the indirect statistical effects revealed that global cortical $(b=-0.0017$, CI:-0.0031,-0.0007) and entorhinal cortex thickness $(b=-0.0021$, CI:-0.0050,-0.0004) mediate the relationship of WMH volume on global cognition and memory, respectively. The findings remained when we removed participants with dementia $(\mathrm{n}=10)$.

Conclusions: Our findings suggest that WMH burden promotes global and regional cortical thinning, which, in turn, drives cognitive decline. These observations highlight a potential pathway in which small vessel cerebrovascular disease affects cognition by promoting neurodegenerative changes directly

Correspondence: Batool Rizvi, Neurology, Columbia University Medical Center, 630 W 168th Street, PH 18-301, New York, NY 10032, United States.E-mail:br2499@cumc.columbia.edu

A.D. ROCHETTE, E. GATHRIGHT, A.M. BRICKMAN, M. SPITZNAGEL, R. COHEN, L.H. SWEET, R. JOSEPHSON, J. HUGHES \& J. GUNSTAD. High-Frequency Heart Rate Variability and Structural Brain Integrity in Heart Failure.

Objective: Neurocognitive deficits are common in persons with heart failure (HF) and associated with many poor outcomes, including greater risk for mortality and recurrent hospitalization. Heart rate variability (HRV) has been used to predict poor outcomes in HF; however, no study has examined whether HRV is also associated with neuroimaging markers in older adults with $\mathrm{HF}$.

Participants and Methods: A total of 41 older adults with HF (68\% male; average age 69 years; $90 \%$ class II HF) completed brain MRI as part of a larger project. HRV was assessed via impedence cardiography. Brain structure was analyzed using FreeSurfer Version 5.1. A series of hierarchical linear regression models was conducted to test whether high-frequency HRV was associated with volumes of 1) total gray matter, 2) white matter, and 3) white matter hyperintensities. Results: After controlling for demographic (i.e. age, gender) and medical factors (i.e. diabetes, cardiovascular fitness, intracranial volume), results indicated that greater HRV was associated with greater total gray volume $(\beta=.27, p<.05)$, but no other structural brain indices (all $\mathrm{p}>.05$ ).

Conclusions: Results suggest that greater high-frequency HRV is associated with larger total gray matter volume, even after accounting for common demographic and medical factors. Further work is needed to clarify the etiology of this relationship, particularly studies that examine 
the possible association among HRV, neuroimaging indices, and chronic inflammation.

Correspondence: Amber D. Rochette, BA, Psychology, Kent State University, 3494 Bent Tree Lane, \#213, Stow, OH 44224, United States. E-mail:amber.rochette88@gmail.com

S. ROYe, P.J. Castagna, M. Calamia \& S.G. GREening. Dimensions of Executive Functioning and Cortical Thickness in Younger and Older Adults.

Objective: Executive functioning (EF) includes a set of related, yet distinct abilities supporting goal-directed behavior: inhibition, updating, and switching (Miyake et al., 2000). Previous research (Latzman \& Markon, 2010) has shown that this three-factor model of EF underlies performance on a widely used clinical measure of EF, the Delis-Kaplan Executive Function System (D-KEFS). Fewer studies have examined the structural correlates of dimensions of EF (Friedman \& Miyake, 2016). This study explored the relationship between the D-KEFS measures of inhibition, updating, and switching and cortical thickness within younger and older adults.

Participants and Methods: 155 adults from the Nathan Kline Institute pilot study were included in our analyses. Individuals were divided into Younger $\left(n=114,68 \%\right.$ male, $\mathrm{M}_{\mathrm{age}}=32.30$ years, $\left.\mathrm{SD}=9.38\right)$ and Older $\left(n=49,49 \%\right.$ male, $\mathrm{M}_{\text {age }}=65.43$ years, $\left.\mathrm{SD}=10.20\right)$ adults, based upon their age (20-49 years and 50+ years). Cortical thickness was determined using FreeSurfer's automated cortical parcellation software. Each group was examined independent of one another and scaled scores were used to measure D-KEFS performance.

Results: An exploratory, cortex-wide analysis examined the relationship between cortical thickness and performance on the D-KEFS. All analyses were controlled for using age and gender, and multiple comparisons corrections were performed using a Monte Carlo simulation. We observed significant correlations between thickness and inhibition, in regions associated with EF. Positive correlations were found in the left anterior middle frontal gyrus, left inferior parietal lobule and right anterior middle frontal gyrus, only in the younger adult group (all Z's > 4.0, all $p$ 's > .0001).

Conclusions: These findings extend previous research on the relationship between cortical thickness and EF in adults, by examining individual dimensions of $\mathrm{EF}$ and showing significant clusters within frontal and parietal regions (Yuan \& Raz, 2014).

Correspondence: Scott Roye, M.A., Clinical Psychology, Louisiana State University, 10720 Linkwood Ct, Apt \# 1033, Baton Rouge, LA 70810, United States.E-mail: sroye1@lsu.edu

E.SEMMEL, M.M.PEARSON, C.J.CANNISTRACI, A.W. ANDERSON, J.F. KUTTESCH, B.E. COMPAS \& K.R. HOSKINSON. Diffusion Tensor Imaging of the Inferior Fronto-Occipital Fasciculus: White Matter Integrity and Associations with Visual-Motor Coordination in Pediatric Brain Tumor Survivors.

Objective: The inferior fronto-occipital fasciculus (IFOF) is a white matter tract implicated in executive aspects of visual processing as well as the use of visual information for guiding movement. Pediatric brain tumor survivors (PBTS) experience cognitive deficits related to demyelination of this tract associated with diagnosis and/or tumor-directed therapies (Scantlebury et al., 2016). We expected that PBTS would have reduced white matter integrity in the IFOF and that this would be associated with poorer scores on measures of executive visual and motor processing.

Participants and Methods: We assessed white matter integrity in the IFOF using diffusion tensor imaging in a group of 8-16 year-old PBTS $(\mathrm{n}=16$; mean time since treatment $=4.94)$ and matched controls $(\mathrm{n}=16)$. Participants completed the Trail Making and Color-Word Interference (CWI) Tests from the D-KEFS and the Beery Visual Motor Integration scale. Fractional anisotropy (FA), radial (RD), and axial diffusivity (AD) were calculated using DSI Studio.
Results: Relative to controls, PBTS had lower FA ( $d=1.05)$, and higher $\mathrm{RD}(d=1.47)$ in the right IFOF as well as higher $\mathrm{RD}(d=1.07)$ in the left IFOF. PBTS performed worse on all subscales of Trail Making and on the CWI Inhibition/Switching subscale $(p s<.01)$. Higher FA and lower RD in the right IFOF were correlated with better scores on CWI Word Reading and all Trail Making subscales except Motor Speed $(r=.40-.63 ; p \mathrm{~s}<.05)$. Higher left IFOF RD was associated with poorer CWI Inhibition/Switching and all Trail Making subscales except Motor Speed $(r=.41-.61 ; p s<.05)$.

Conclusions: PBTS have reduced white matter integrity in the IFOF as indicated by DTI. These differences were associated with tests of executive visual and visual-motor coordination, suggesting that the IFOF is important for processing visual information for the purpose of movement and the inhibition of responses to visual stimuli. Future work should identify clinically relevant effects of these deficits to understand how they affect functional outcomes in PBTS.

Correspondence: Eric Semmel, B.A., Psychology, Georgia State University, 180 Jackson Street NE Apt 3217, Atlanta, GA 30312, United States.E-mail: esemmel1@student.gsu.edu

D. SHAKED, L. KATZEL, S.L. SELIGER, C. DAVATZIKOS, R. GULlAPALli, T. KOUO, G. ERUS, M.K. EVANS, A.B. ZONDERMAN \& S.R. WALDSTEIN. The Relation of Socioeconomic Status and Executive Function: Is Prefrontal Cortex Volume a Mediator.

Objective: Lower socioeconomic status (SES) has been associated with lesser cognitive performance and smaller brain volumes. Also, smaller brain volumes have been related to poorer cognitive function. The present study examined whether SES-linked decrements in executive function $(\mathrm{EF})$ were mediated by corresponding reductions in dorsolateral prefrontal cortex (DPFC) gray matter volume.

Participants and Methods: Participants were 212 socioeconomically diverse, African American (AA) and White urban-dwelling adults ( $M$ age $=46.8 ; 39 \%$ AA; $59 \%$ female; $32 \%$ below poverty) from the Healthy Aging in Neighborhoods of Diversity across the Life Span (HANDLS) SCAN study. Participants underwent 3.0-T cranial magnetic resonance imaging. T1-weighted MP-RAGE images were used to derive structural volumetric data. Multiple regression analyses, adjusted for age and sex, examined associations of SES with Trails B, Digit Span Backwards, Verbal Fluency, and DPFC, as well as DPFC-EF relations. Mediation analyses were computed to assess whether significant SES-EF associations were explained by DPFC volume.

Results: SES, EF tests, and DPFC were associated significantly, whereby a lower level of SES, poorer EF, and smaller DPFC were all related. DPFC mediated the relation between SES and Trails B, but not Digit Span Backwards or Verbal Fluency. Race-stratified analyses revealed that significant DPFC mediation of SES-Trails B was present in Whites, but not in AAs.

Conclusions: These results suggest that normal inter-individual variability in DPFC volume is important in the association of SES and mental flexibility for White, but not AA adults. The significant mediation may be partially explained by Trails B's strong reliance on the DPFC relative to the other measures examined. To further elucidate the underlying biological mechanisms for the SES-cognition relation in both AAs and Whites, future research should examine additional ROIs and other brain endpoints such as white matter volume and hyperintensities and neural network connectivity.

Correspondence: Danielle Shaked, MA, Psychology, University of Maryland, Baltimore County, 1244 Battery Ave., Baltimore, MD 21230, United States.E-mail: dshaked1@umbc.edu

A. VELEZ LOPEZ, M. ROSARIO \& K. SCHON. Cardiovascular Fitness is Positively Correlated with Left Entorhinal Cortical Thickness in Healthy Young Adults.

Objective: Structures in the medial temporal lobe (MTL) memory system show experience-dependent neuroplasticity. Within the MTL, 
this plasticity has been observed in the hippocampus (HC) in both humans and animal models. Specifically, rodent model studies have shown a positive correlation between voluntary wheel running and adult neurogenesis in the dentate gyrus subregion of the HC. These models have not examined neuroplasticity in the entorhinal cortex (EC), even though the EC serves as a primary input to the HC, and studies on environmental enrichment have reported greater EC volume in enriched environments. Previous work in our lab in healthy young adults showed a positive correlation between right EC volume, and aerobic fitness (VO2 max). Here, we examined whether cardiovascular fitness predicts entorhinal cortical thickness and hippocampal volume in healthy young adults.

Participants and Methods: We used Freesurfer, a surface-based morphometric analysis method to measure cortical thickness and brain volumes in a young adult cohort ( $n=25,20$ to 33 years). Using multiple regression we first examined whether aerobic fitness predicts EC thickness. Second, we correlated VO2 max and cortical thickness and brain volume data with performance on cognitive tasks thought to be dependent on the EC and HC. Age, gender, and intracranial volume were used as covariates.

Results: VO2 max was significantly associated with left EC thickness $(F(4,20)=4.58, p<.01, r 2=.37)$, and left fusiform gyrus volume $(F(4,20)=5.77, p<.01, r 2=.44)$, but not with HC volume, holding age, gender, and intracranial volume constant. No significant relationships were identified for measures of spatial cognition and memory with EC thickness or VO2 max.

Conclusions: Consistent with our predictions and our previous work, greater VO2 max was associated with greater left EC thickness. This data further supports that cardiovascular fitness may be implicated in the experience-dependent plasticity of structures in the MTL, even in healthy young adults.

Correspondence: Andres Velez Lopez, B.S., Anatomy \& Neurobiology, Boston University, 72 East Concord St., R 1003, Boston, MA 02118, United States.E-mail:andresvl@bu.edu

\section{N. WHITNEY, S. DEONI \& A. MIELE. White Matter Correlates of Early Academic Proficiency in Young School-Age Children. \\ Objective: Converging neuroimaging research has found that white matter differences impact academic outcomes. However, prior literature often utilizes diffusion tensor imaging with older children. To our knowl- edge, this is the first investigation of the relationship between an index of white matter maturation (i.e., myelin) and academic performance in early-elementary school children.}

Participants and Methods: Non-sedated mcDESPOT imaging data was successfully acquired from 84 healthy children between the ages of 4-11 years-old on a Siemens Tim Trio scanner. Three-pool postprocessing was used to calculate quantitative $\mathrm{T}_{1}, \mathrm{~T}_{2}$, and $\mathrm{VF}_{\mathrm{M}}$ maps, which were subsequently aligned to a previously acquired longitidinal template. Nonparametric correlation coefficients were calculated between intravoxel myelin and academic performance on the Letter/Word Reading and Mathematical Calculation subtests from the Academic Achievement Battery ( $\mathrm{A} A \mathrm{~B})$, while controlling for overall cognitve ability and age. A cluster-based approach was used to correct for multiple comparisons.

Results: Results revealed positive correlations between myelin content and academic scores. Specifically, higher reading and math scores were associated with increased myelin in orbitofrontal regions including the genu of the corpus callosum and anterior corona radiata bilaterally, as well as the posterior optic radiations and visual cortex. However, higher math scores were also related to increased myelin in the right parietal lobule and right posterior corona radiata.

Conclusions: Increased myelination largely in frontal and parietal structures is associated with improved performance in academics while controlling for general cognitive ability and age. Orbitofrontal regions are among the last to myelinate; thus, these children may have advanced brain development compared to same-aged peers. Strong overlap in brain regions may reflect lack of controlling for opposing scores in each correlation model, but more likely suggest similar functional characteristics in these areas.

Correspondence: Noelle Whitney, School Psychology, University of Northern Colorado, 4622 Cornish Way, Denver, CO 80239, United States.E-mail: ncwhit001@gmail.com

R. WIER, H.A. ALEKSONIS, M.M. PEARSON, C.J. CANNISTRACI, A.W. ANDERSON, J.F. KUTTESCH, B.E. COMPAS \& K.R. HOSKINSON. Role of the Cingulum Bundle in Emotional and Behavioral Concerns in Pediatric Brain Tumor Survivors.

Objective: Pediatric brain tumor survivors (PBTS) are at risk for emotional and behavioral problems, perhaps due to tumor- or treatment-related changes in brain structures involved in emotion regulation. One such region, the cingulum bundle (CB), is a bilateral white matter tract with limbic connections (Schmidt et al., 2013). We hypothesized that PBTS would display more difficulties with behavior and emotional functioning, and reduced mean fractional anisotropy $(\mathrm{mFA})$ of the $\mathrm{CB}$, relative to healthy controls (HC). We also predicted that $\mathrm{mFA}$ of the $\mathrm{CB}$ would account for significant variance in group differences in these domains.

Participants and Methods: Eleven 8-16 year old PBTS and 14 HC underwent diffusion tensor imaging to assess white matter integrity. Tractography (DSI Studio) quantified $\mathrm{mFA}$ of the CB. PBTS were $\geq 2$ years post-treatment and $\mathrm{HC}$ were matched by age and sex. Parents rated children's emotional and behavioral functioning using the Behavior Rating Inventory of Executive Function and Child Behavior Checklist. Results: Compared to HC, PBTS had lower $\mathrm{mFA}$ in the right $(d=-0.99, \mathrm{p}<.05)$ and left $(d=-1.08, \mathrm{p}<.05)$ CB. Parents of PBTS reported poorer behavioral regulation $(d=1.29, \mathrm{p}<.01)$ and greater internalizing $(d=0.92, p<.05)$ and externalizing symptoms $(d=1.28$, $\mathrm{p}<.01)$. Hierarchical regression combining participant group with right $\mathrm{CB}$ mFA better predicted behavioral regulation (adj. $\mathrm{R}^{2}=.40, \Delta \mathrm{R}^{2}=.14$ ) compared to group alone (adj. $\mathrm{R}^{2}=.26$ ). Similarly, right $\mathrm{CB}$ combined with group better predicted internalizing symptoms (adj. $\mathrm{R}^{2}=.27$, $\Delta \mathrm{R}^{2}=.13$ ) compared to group alone (adj. $\left.\mathrm{R}^{2}=.14\right)$. Group, but not $\mathrm{CB}$ $\mathrm{mFA}$, predicted externalizing symptoms (adj. $\left.\mathrm{R}^{2}=.26\right)$.

Conclusions: Diminished white matter integrity in the $\mathrm{CB}$ may contribute to poor behavior and emotional outcomes following pediatric brain tumor. Clinicians should consider that PBTS with low CB $\mathrm{mFA}$ may be at particular risk for these issues. Further research with a larger sample should clarify this relationship and allow consideration of factors like tumor and treatment type.

Correspondence: Ryan Wier, B.S., Biobehavioral Health, Research Institute at Nationwide Children's Hospital, 700 Children's Drive, FB Suite 3B.2, Columbus, OH OH, United States.E-mail: ryan.wier@ nationwidechildrens.org.

V.J. WILliaMS, J. HAYES, D. FORMAN, D.H. SALAT, R. SPERLING, M. VERFAELLIE \& S.M. HAYES. Age-Dependent Associations Between Cortical Thickness and Cardiorespiratory Fitness.

Objective: Gray matter volume loss in aging is well documented, with cortical thickness serving as a sensitive indicator of age-related cerebral atrophy. Cardiorespiratory fitness (CRF) has been positively associated with gray matter volume in prior literature, and thus may aid in mitigating neurostructural decline in late life. Although associations between brain volume and CRF are well documented, the relation between cortical thickness and CRF has yet to be established in healthy older populations. Furthermore, the association between CRF and gray matter integrity in young adults is largely unexplored.

Participants and Methods: In this study, 32 young (aged 18-31) and 29 older adults (aged 52-82) completed a treadmill based progressive maximal exercise test to evaluate $\mathrm{CRF}$ (peak $\mathrm{VO}_{2}$ ) and structural MRI to quantify whole-brain cortical thickness using a surface-based approach. Results: Differential associations between CRF and cortical thickness among young and older adults were observed primarily in multimodal 
association areas. Greater CRF was associated with thicker cortex in older adults, but with thinner cortex in young adults. The majority of brain regions showing a positive association between CRF and cortical thickness in older adults overlapped with brain areas also demonstrating significant age-related cortical thinning. In follow-up analyses in which age-matched older adults were categorized as high or low fit based on normative data, a stepwise pattern emerged such that the cortex was thinnest in low fit older adults, intermediate in high fit older adults, and thickest in young adults.

Conclusions: CRF may differentially benefit cortical integrity in an age-dependent manner. In young adults, CRF may promote typical cortical development by enhancing or promoting age-expected cortical pruning. In older adults, higher CRF may aid in the preservation of neurostructural integrity, particularly within multimodal cortices most vulnerable to late life cortical atrophy.

Correspondence: Victoria J. Williams, Ph.D., NeuroImaging Research for Veterans Center, VA Boston Healthcare System, 150 South Huntington Ave,151A,Boston, MA 02130,United States.E-mail:tori85@gmail.com

\section{Neurostimulation/Neuromodulation}

\section{O.C. CALHOUN, S. GARCIA, A. LESNOVSKAYA, A. BHAUMIK \& B.M. HAMPSTEAD. Impedance levels and tolerability of $2 \mathrm{~mA} \mathrm{HD}$ tDCS in older adults.}

Objective: Impedance levels and common side effects have been evaluated in traditional (pad-based) tDCS research but little has focused on High Definition (HD) tDCS, especially in older adults. We examined 1) initial impedance levels as well as changes following a 10minute saturation period 2) differences in these values across two consecutive daily sessions 3 ) frequency and severity of side effects between sham and active groups, and 4) the efficacy of blinding using a sham protocol.

Participants and Methods: A mixed sample of 57 older adults with and without memory impairment underwent a total of 170 sessions (82 sham, 88 active) at $2 \mathrm{~mA} \mathrm{HD}$-tDCS for 20 minutes per sessions across a series of studies. Tolerability (i.e., side-effects) and blinding (i.e., asking participants whether they received active or sham stimulation) data were acquired following each session. Impedance levels were recorded immediately after electrode placement and again following a 10-minute saturation period in a subgroup of 33 participants who completed one session (D1) and 31 who completed a second session (D2).

Results: Impedance values changed significantly during the 10-minute saturation period $(F(1,22)=20.08, p<0.001)$ but there was no main effect of session $(\mathrm{F}(1,18)=2.83, p=0.11)$. A time by session interaction revealed greater reductions during D1 than D2 $(\mathrm{F}(1,18)=$ 19.16, $p<0.01)$. Consistent with prior pad-based literature, HD-tDCS was well tolerated as the most commonly reported side effects were tingling (sham $57.3 \%$ vs $48.9 \%$ active), itching (sham $35.4 \%$ vs $33 \%$ active) and burning sensation (sham $57.3 \%$ vs 55.7\% active). Most participants believed they received active stimulation ( $\mathrm{sham}=64 \%$, active $=51 \%)$.

Conclusion: These preliminary findings suggest that a brief saturation period reduces electrical impedance. Tolerability data indicate active and sham HD-tDCS have comparable (mild) side effects that are comparable to pad-based tDCS. The efficacy of sham stimulation protocols is further reinforced by participants' inability to correctly determine whether they received "real" or "fake" HD-tDCS. Correspondence: Oliver C. Calhoun, Bachelor of Science, PsychiatryNeuropsychology, University of Michigan, 2101 Commonwealth Blvd, Suite D, Ann Arbor, MI 48105, United States. E-mail: occ@med.umich.edu
M.G. CURY, M. MARANHÃO, N. MENDES ESTELLA, A. CLAUDINO \& A. BERBERIAN. The Effects of Noninvasive Brain Stimulation on Executive Function in Binge Eating Disorder: A Pilot Study.

Objective: Verify, on a preliminary way, the effects of high frequency repetitive transcranial magnetic stimulation (rTMS), delivered to the dorsolateral pre frontal cortex, on different aspects of executive functioning in binge eating disorder (BED).

Participants and Methods: Eight obese women diagnosed with BED, receiving ten sessions of rTMS, completed a neuropsychological evaluation pre and post rTMS treatment. The cognitive domains assessed through testing were decision making, attention, inhibitory control, set shifting and working memory. All individuals were evaluated using the following tests: Iowa Gambling Test (IGT), Continuous Performance Test (CPT), Stroop test, Number Letter and Keep Track. The estimated baseline intelligence quotient (IQ) was calculated, through Matrix Reasoning and Vocabulary (WAIS-III). The data was analyzed with the Wilcoxon Rank-Sum test, and the Bonferroni correction was adopted to adjust $p$ values $(p \leq 0,00625)$.

Results: No differences were found in cognitive performance pre and post rTMS $\{$ IGT, initial mean $=3,35( \pm 9,06)$, final mean $=8,25$ $( \pm 24,45), p=0,484 ;$ Number Letter, initial mean $=12,27$ $( \pm 9,18)$, final mean $=6,61(+6,26), p=0,050$; Keep Track, initial mean $=15,87( \pm 3,56)$, final mean $=17,37( \pm 1,84), p=0,352$; Stroop test, initial mean $=11,50( \pm 8,51)$, final mean $=4,74( \pm 4,71), \mathrm{p}=0,674$; CPT, initial mean $=2,58( \pm 1,22)$, final mean $=1,72( \pm 1,51), p=0,400\}$.

Conclusions: Despite the negative findings, these preliminary results contributed to neuropsychological battery testing and adequation (application time, training of applicator, acceptance of subjects) and to guide future studies, increasing the sample size and including a control group. Correspondence: Maria Elisa G. Cury, Master's Degree student, PSYCHIATRY, UNIVERSIDADE FEDERAL DE SÃO PAULO, Rua Borges Lagoa, nº 570, São Paulo 04038-020, Brazil. E-mail: m.elisa@ clinicapsico.com.br

G. DEMETER, P. PAJKOSSY, Á. SZOLlosi, Á. LUKÁCS, I. VALÁLIK \& M. RACSMÁNY. The Effect of Subthalamic Nucleus Deep Brain Stimulation on Memory and Executive Functions in Parkinson's Disease.

Objective: The aim of the present study was to asses the effect of bilateral deep brain stimulation (DBS) of the subthalamic nucleus (STN) on memory and executive functions in patients with Parkinson's disease (PD) using an unstimulated PD control group matched on motor symptoms, medication and DBS indication.

Participants and Methods: Ten PD patients with DBS implantation (DBS group) and 10 PD wait-listed patients (control group) participated in the study. A neuropsychological battery was used to assess cognitive functions, including general mental ability (Mini Mental State Examination), verbal (digit span) and spatial short-term memory (Corsi block-tapping task), working memory (n-back task), event-based prospective memory and executive functions (phonemic and semantic verbal fluency, Stroop task, Trail Making B task). Each task was administered twice: before and after surgery in the DBS group with the stimulators on and with a similar time interval between the two task-administration points in the control group.

Results: There was no significant difference between the DBS and the control groups' performance in tasks measuring short-term and working memory and main executive functions. The DBS group showed a significant decline only on the semantic verbal fluency task after surgery compared to its own baseline level $(\mathrm{p}<.05)$. Additionally, the DBS group showed a decreased execution cost score at the second assessment point of the prospective memory task, compared to the control group $(\mathrm{p}<.05)$.

Conclusions: Our results provide support for the cognitive safety of the STN DBS using a wait-listed PD control group. The DBS group showed impaired performance after the surgery only on the semantic verbal fluency task which is in line with findings of previous studies. Furthermore, to the best of our knowledge, this is the first study to show that DBS of the STN boosts prospective memory, in particular intention execution functions. roup $(\mathrm{p}<.05)$. 
Correspondence: Gyula Demeter, PhD, Department of Cognitive Science, Budapest University of Technology and Economics, Egry József 1, Budapest 1111, Hungary. E-mail: demetergy@hotmail.com

S. GARCiA, E.o. GARnetT, S. PELTIER, O.C. CALHOUN, A. LESNOVSKYA, A. BHAUMIK \& B.M. HAMPSTEAD. HD-tDCS as a Neurorehabilitation Technique for a Case of Post-Anoxic Leukoencephalopathy.

Objective: Transcranial direct current stimulation (tDCS) has been used for neurorehabilitation of cognitive and motor impairment associated with neurologic injury and disease as well as a range of mental illnesses. While tDCS has often been used in stroke, we report here the first instance of use in a patient with post-anoxic leukoencepalopathy. Neuropsychological testing revealed diffuse cognitive impairment with executive impairment and pressured speech/language being primary concerns of informants. Thus, we targeted the left lateral prefrontal cortex (PFC) using the more focused high definition tDCS (HD-tDCS) over a month of near daily stimulation sessions.

Participants and Methods: A 62-year-old, right handed, Caucasian male, five years post injury secondary to accidental drug overdose. He underwent neuroimaging, neuropsychological testing, and selfreport questionnaires (patient, family, and caregiver) over two baseline sessions and again one week after HD-tDCS ended. Anodal HD-tDCS was administered at $2 \mathrm{~mA}$ for 20 minutes over the left PFC (F5), for a total of 29 (weekday) sessions.

Results: tDCS was well tolerated. Reliable change indices demonstrated improvement on RBANS delayed memory was significant $(\mathrm{RCI}=2.47$ ). Semantic, but not phonemic, fluency tended to improve. Self-report measures suggested enhanced insight into deficits at post-testing. Informants reported improved executive functioning, inhibition, and memory recall after treatment. GABA decreased from pre to post-treatment in the targeted prefrontal region.

Conclusions: This extended administration of HD-tDCS adds to prior evidence of safety and tolerability while also extending the potential neurorehabilitative uses of this methodology. Considerably more information is needed about the efficacy of, and mechanisms underlying, tDCS; areas we are actively exploring in ongoing work.

Correspondence: Sarah Garcia, PhD, Neuropsychology-Psychiatry, University of Michigan, 2100 Commonwealth, Ann Arbor, MI 48105, United States.E-mail:slga@med.umich.edu

K.S. SEAGLY, A. CHAPPELL, M. CREWE, J.H. GARRITY, P. SAYEGH, S. DEUTSCH \& S. NEUMANN. Pairing Brain Stimulation with Psychotherapy: A TMS Pilot Study Treating Combat Related PTSD and Co-Occurring Cognitive Symptoms.

Objective: Posttraumatic Stress Disorder (PTSD) is a commonly occurring diagnosis, and is particularly prevalent in combat veterans. There has been some success treating PTSD with psychotherapy, but many cases remain refractory to the current standard of care. As such, novel treatments, including transcranial magnetic stimulation (TMS), have been examined and preliminarily found to be of use. However, little research has been conducted looking at combining TMS with evidencebased psychotherapy. The present study examined the feasibility, safety and preliminary effectiveness of pairing TMS and prolonged exposure therapy for the treatment of PTSD and co-occurring cognitive symptoms. Participants and Methods: Low frequency TMS to the right dorsolateral prefrontal cortex and the supplementary motor area combined concurrently with a standardized exposure protocol was administered to nine combat veterans with chronic, treatment-resistant PTSD. Psychological symptoms were measured pre- and post-treatment with the PTSD Checklist, Treatment Outcome PTSD Scale, Beck Depression and Anxiety Inventories, and the Hamilton Depression Rating Scale. Cognitive abilities were measured using CNS Vital Signs ${ }^{\circledR}$. Data were analyzed with repeated measures ANOVAS.

Results: The treatment was safe and well tolerated, and improvements were seen across psychological symptoms with large effect sizes.
Cognitively, processing speed, psychomotor speed, cognitive flexibility and executive function were improved.

Conclusions: Statistically significant results should be interpreted with caution due to small sample size and potential confounds. However, clinical results were striking, with seven of the nine participants no longer meeting criteria for PTSD by the end of the study. Eight of the participants achieved clinically significant cognitive improvements in one or more domains. Results were promising and warrant further investigation with larger sample sizes utilizing a randomized controlled model to confirm and expand upon these findings.

Correspondence: Katharine S. Seagly, Ph.D., Neuropsychology, Rehabilitation Institute of Michigan, 1198 Anita Ave, Grosse Pointe Woods, MI 48236, United States. E-mail: kseagly@dmc.org

K. WILSON, J. WILSON, D. QUINN \& V. CLARK. Transcranial Direct Current Stimulation (tDCS) Over Left Prefrontal Cortex Improves Visual Detection of Words.

Objective: Previous studies have demonstrated that transcranial direct current stimulation (tDCS) may increase diverse types of cognitive ability, in both healthy controls and individuals with brain injury. The present study examined the effect of tDCS on visual word detection speed, a critical component of reading efficiency.

Participants and Methods: Typically-functioning adults $(n=28)$ from the university community were randomly assigned to receive "active" tDCS $(2.0 \mathrm{~mA})$ or "sham" tDCS $(0.1 \mathrm{~mA})$ for 40 minutes in a singleblind design. Anode electrode placement was over the left prefrontal cortex and the cathode was placed over the upper, contralateral arm. Participants completed a timed, computer-based task in which difficult-to-spell-words were presented embedded in a "letter grid". The dependent variable was the average time (in seconds) per word found. Scores were recorded at baseline, during tDCS, and immediately posttDCS. Participants were also asked to return 3-7 days later to perform the task again; $50 \%$ returned.

Results: Groups were equivalent at baseline. The active group detected words faster than the sham group during the delivery of tDCS $(n=28$, $p<0.03, d=.68)$ and immediately afterwards $(p<0.03, d=.87)$. Among participants who returned 7-10 days later $(n=14)$, the active group showed a modest decline from prior testing, but remained faster at detecting words than the sham group $(p<0.01, d=1.48)$.

Conclusions: These findings demonstrate that active tDCS over the left prefrontal cortex improves rapid visual detection of words, suggesting the utility of tDCS for improving written word processing.

Correspondence: Kevin Wilson, Psychology, University of New Mexico, 4100 Ridgeley Ave NE, Albuquerque, NM 87108, United States. E-mail:jkwilson@unm.edu

\section{Program Welcome}

Program Committee Chair: Benjamin M. Hampstead 4:15-4:30 p.m.

\section{Plenary A. The Impact of the Past on Current and Future Views of Limb Apraxia}

\section{INS President: Kathleen Y. Haaland}

$$
\text { 4:30-5:30 p.m. }
$$

\section{K.Y. HAALAND. The Impact of the Past on Current and Future} Views of Limb Apraxia.

This presentation will explore the impact of single cases on past and current conceptualizations of limb apraxia including the work of Hugo Liepmann and several previous INS presidents (Geschwind, Kaplan, 
Heilman, and Gonzalez Rothi). Videotapes of classic apraxic syndromes will be presented. A major focus will be on how the views of the cognitive and neuroanatomical correlates of limb apraxia have changed from the $19^{\text {th }}$ to the $21^{\text {st }}$ century leading to the current emphasis on a left hemisphere cortical network with a left parietal node. Unanswered questions, including the differential roles of left parietal, temporal, and frontal regions in limb praxis, will be discussed in the context of future work that utilizes multi-method approaches that integrate lesion studies with functional imaging and stimulation studies.

Following this lecture, the learner should be able to: 1) Delineate one way that Liepmann's cases influenced his theory of limb apraxia;
2) Discuss one major difference between Geschwind's view and Heilman and Gonzalez Rothi's view of the parietal lobe's role in limb apraxia; 3) Specify one function of the left parietal lobe in limb apraxia; 4) List two methods that have informed current understanding of the neuroanatomical substrates of limb praxis to emphasize a broad left hemisphere network with critical node in the left parietal lobe.

Correspondence: Kathleen Y. Haaland, PhD, Psychiatry and Behavioral Sciences \& Neurology, University of New Mexico, MSC 09 5030, 1 University of New Mexico, Albuquerque, NM 87131, United States.

E-mail:khaaland@unm.edu

\section{WEDNESDAY EVENING, FEBRUARY 1, 2017}

\section{INS Awards Ceremony}

Awards Committee Chair: Roy P.C. Kessels

5:30-6:30 p.m.

Welcome Reception

6:30-7:30 p.m.

\section{THURSDAY MORNING, FEBRUARY 2, 2017}

\author{
CE Workshop 7. Financial and Health \\ Decision Making in Old Age: Neuropsychology, \\ Neuroimaging, and Race Considerations \\ Presenter: Duke Han \\ 7:20-8:50 a.m.
}

D. HAN. Financial and Health Decision Making in Old Age: Neuropsychology, Neuroimaging, and Race Considerations.

Decision making refers to the ability to consider competing alternatives and make an optimal choice. Older adults are regularly faced with consequential decisions regarding financial and health matters, and recent work suggests decision making may decline with age. Age-associated pathological changes in the brain are well documented, suggesting the suboptimal functioning of neural systems may contribute to impaired decision making in old age. While neuroimaging has yielded advances in the knowledge of brain systems involved in younger populations, relatively little is known about the neuroimaging correlates of pathological changes associated with decision making in older adults. This presentation will discuss the neuroimaging correlates of impaired financial and health decision making in old age, as well as the cognitive, affective, and contextual factors that are associated with these patterns. Racial differences in decision making will also be considered in view of the potential mediating and moderating factors that drive them.

As a result of participation in this course, the learner will achieve the following objectives: (1) have a deeper understanding of the critical brain structures that support decision making in old age, and (2) be familiar with cutting-edge translational research techniques for investigating financial and health decision making in old age.

Correspondence: Duke Han, PhD, ABPP-CN, Family Medicine, University of Southern California, 1000 S. Fremont Avenue, Unit 22, HSA Building A-6, 4th Floor, Room 6437A, Alhambra, CA 91803 , United States.E-mail:Duke.Han@med.usc.edu

\section{CE Workshop 8. Hearts and Minds: Recent Advances in the Neuropsychology of Pediatric Critical Congenital Heart Disease}

\author{
Presenter: Adam R. Cassidy
}

7:20-8:50 a.m.

A.R. CASSIDY. Hearts and Minds: Recent Advances in the Neuropsychology of Pediatric Critical Congenital Heart Disease. Congenital heart disease (CHD) constitutes a major global health problem affecting 7 to 9 out of every 1,000 live births worldwide, or over 1 million live births every year. Approximately $25-33 \%$ of these children are born with a critical form of CHD requiring intensive surgical palliation during infancy and/or early childhood. Despite excellent rates of survival in the modern era, children and adolescents with critical CHD remain at high risk for a range of neurological, neurobehavioral, and psychosocial challenges that undermine optimal development and pose a threat to short- and longer-term quality of life. Building on a series of groundbreaking investigations that shed early light on these risks, more recent studies have begun to hone in on mechanisms of brain injury and dysmaturation, identify predictors of outcomes, and elucidate nuanced cognitive, behavioral, social, and self regulatory profiles of children and adolescents with CHD. This workshop will survey research in pediatric congenital heart disease with a particular focus on several recent advances in the neuropsychology of pediatric critical CHD.

Upon conclusion of this course, learners will be able to: 1) Describe how critical CHD affects brain development, and 2) Discuss neurobehavioral and psychosocial risks experienced by children and adolescents with critical CHD.

Correspondence: Adam R. Cassidy, PhD, ABPP, Psychiatry, Harvard Medical School, 300 Longwood Avenue (Fegan 8, Room 830), Boston, MA 02115,United States.E-mail: adam.cassidy@childrens.harvard.edu 
Poster Session 2. Adult 1 \& Historical

8:00-9:15 a.m.

\section{Assessment/Psychometrics/Methods (Adult)}

A. ALIOTO, T. MOSKOWITZ, S. ERLHOFF, N.T. BOTT, J.H. KRAMER, K. RANKIN \& K.L. POSSIN. Neuroanatomical Correlates of the TabCAT Number-Picture Match.

Objective: To investigate the neuroanatomical and cognitive correlates of the TabCAT Number Picture Match ('Match'), a 2-minute tabletbased executive processing speed measure.

Participants and Methods: Individuals diagnosed with a neurodegenerative disease $(\mathrm{N}=144$, age $=70+/-9.5)$ or as neurologically healthy $(\mathrm{N}=154$, age $=74+/-7.6)$ completed a cognitive assessment, and 63 patients and 64 controls also completed 3T MRI. On the Match, subjects were shown a fixed legend of numbers (1-7) paired with simple abstract pictures. When a number appeared on the screen, the subject was instructed to tap the corresponding picture as quickly as possible. Accurate responses were totaled and correlated with measures of executive processing speed (Design Fluency, Digit Symbol, Stroop), memory (California Verbal Learning Test, Benson Recall), language (modified Boston Naming Test), and visuospatial skills (Benson copy). Performance was also correlated with brain volumes using voxelbased morphometry, controlling for age, gender, and total intracranial volume. A permutations-based family-wise error (FWE) correction was employed to account for multiple comparisons.

Results: The Match correlated significantly with measures of executive speed (all ps <.01), but did not correlate with measures of memory, language, or visuospatial skills (all ps $>.2$ ). Match performance was highly sensitive to brain volume reductions with 31 clusters surviving FWE correction. The most significant correlations were with the left thalamus, the right precentral gyrus, the left superior and middle temporal gyri, the right supplementary motor cortex, the left angular gyrus, the left accumbens, and the right caudate (all maxTs > 6.67). Conclusions: The TabCAT Match correlates with tests of executive processing speed and with brain regions important for executive and motor control. The Match is an efficient and valid measure of executive processing speed.

Correspondence: Andrea Alioto, M.S., Memory and Aging Center, University of California San Francisco, 3045 Alma Street, Palo Alto, CA 94306, United States. E-mail: andrea.alioto@ucsf.edu

K.C. BAILEY, J. SOBLE \& J. O'ROURKE. Getting the Most out of Your Performance Validity Investment: An Examination of Five Tests among a Mixed Clinical Sample of Veterans.

Objective: Performance validity tests (PVTs) are extensively examined in the context of Traumatic Brain Injury (TBI) among veterans, but limited research exists with the general clinical veteran population. This retrospective study examined several common PVTs to identify those that balance classification accuracy, sensitivity/specificity, and efficiency in a clinically diverse population.

Participants and Methods: A mixed outpatient clinical sample of 74 veterans completed Green's Word Memory Test (WMT), the Test of Memory Malingering (TOMM), Advanced Clinical Solutions Word Choice Test (WCT), Dot Counting Test (DCT), Rey Fifteen Item Test (RFIT)/Recognition, and Reliable Digit Span (RDS) integrated into a larger clinical battery. Logistic regression determined classification accuracy, and receiver operating characteristic (ROC) curves established sensitivity/specificity values for quick PVTs using the WMT as the diagnostic standard of valid performance.

Results: Sixty percent of participants had a valid performance on the WMT. Educational attainment, race, and language preference did not affect PVT performance. TOMM-Trial 1, WCT, RDS, and DCT all significantly predicted group membership, with classification accuracies of $66.7 \%-83.6 \%$, whereas RFIT/Recognition was nonsignificant. ROC curves revealed areas under the curve (AUCs) of .72-.93, sensitivities of .47-.84, and specificities of .43-.93 based on optimal cut scores for each measure.

Conclusions: Of brief PVT measures examined, TOMM-Trial 1 and DCT had the highest classification accuracy and sensitivity/specificity values, whereas the other measures, while significant, had less robust diagnostic accuracy. Current findings demonstrate the need for clinicians to research and utilize PVTs which balance sound psychometric qualities, clinical utility, time burden, and evaluation costs within the general clinical population.

Correspondence: Kenneth C. Bailey, Ph.D., Neuropsychology, South Texas Veterans Health Care System, 5450 Rowley Rd., Apt 920, San Antonio, TX 78240, United States. E-mail: kenneth.bailey2@va.gov

J. BASHEM, L. RAPPORT, R.J. KANSER, N. BILLINGS, B. VERMILION, S. KROHNER, R. HANKS, R.E. KEELAN, R.D. WHITMAN \& P. SIPLE. Performance Validity Assessment of Bona Fide and Malingered Traumatic Brain Injury Using Novel Eye-Tracking Systems.

Objective: Oculomotor behavior during visual tasks may be a promising avenue to enhance assessment of performance validity. This study evaluated contributions of oculomotor patterns to detection of purposeful poor performance using eye tracking, as compared to a widely-used performance validity test.

Participants and Methods: Participants were 39 adults with moderate-to-severe TBI, 50 healthy comparison (HC) adults, and 42 healthy adults coached to simulate TBI. Participants completed a neuropsychological assessment, including a digitized version of the TOMM, during which oculomotor behaviors were recorded.

Results: Oculomotor indices were generally correlated with neuropsychological indices as predicted by theory. The HC and TBI groups generally produced inverse relationships between key cognitive constructs and oculomotor variables, whereas the SIM group typically produced positive correlations. Results indicated that malingering on the TOMM entails increased cognitive processing as compared to $\mathrm{HC}$ and TBI groups. Single and multivariable logistic regression models with ROC curve analyses examined the oculomotor indices as predictors of group membership. Multiple oculomotor indices showed significant discriminatory power, and one index provided a significant increase to the predictive accuracy of TOMM Total Correct in differentiating bona fide TBI from simulated TBI.

Conclusions: Large effects were observed for several oculomotor behaviors, demonstrating that the inclusion of biomarkers in neuropsychological assessments can significantly improve detection of feigned neurocognitive deficits. Moreover, oculomotor behavior can add unique predictive value in identifying clinical status beyond that provided by standard protocol for the TOMM. The findings indicate that TBI simulators expend greater cognitive effort to fail the TOMM than adults with bona fide TBI and healthy adults providing full effort to pass the TOMM. These behaviors can be captured by eyetracking systems.

Correspondence: Jesse Bashem, Ph.D., Psychology, Wayne State University, 207 Calle Serena, San Clemente, CA 92672, United States. E-mail:bashemj@gmail.com

J. BEACH, S.L. AITA, S. TAYLOR, J.S. HOLCOMBE, M. CALAMIA \& B.D. HILL. Inconsistency in Responding is Associated with Greater Self-Reported Executive Dysfunction.

Objective: Intra-individual variability (IIV) can be conceptualized as an index of consistency. An emerging literature supports IIV as a measure of neuropathology. This study evaluated the association between item level IIV and performance outcome on self-report measures of attention/ executive functioning.

Participants and Methods: 130 university participants $(M$ age $=19.44$, $S D=3.15 ; M$ education $=12.34, S D=.69,3.1 \%$ White, $36.9 \%$ African American, 7.7\% Asian; 73.8\% female). Exclusion criteria included self-reported psychiatric diagnosis. Participants completed the Barkley 
Deficits in Executive Functioning Scale (BDEFS) Long Form and Barkley Adult ADHD Rating Scale IV (BAARS) as part of a larger battery of tests. IIV was calculated as the individual's standard deviation around their own mean. IIV was calculated from raw scores for BDEF and BAARS total score and all subscales.

Results: A series of Spearman's rho correlations were conducted to determine if there was a relationship between IIV (both BDEFS and BAARS) and BDEFS total score. BAARS IIV value and BDEFS total score were significantly correlated $r(128)=.54, p<.01$, demonstrating that lower consistency in reporting on the BAARS was associated with higher reported executive dysfunction on the BDEFS. BDEFS IIV value was also highly correlated with the BDEFS total score $r(128)=.78$, $p<.01$. In addition, the IIV of all BAARS individual subscales $(r=.21$ to .49 , all $p$ values $<.01)$ and IIV of all individual BDEFS subscales $(r=.46$ to $.67, p<.01)$ were all significant predictors BDEFS total score. Conclusions: Increasing item level variability was positively associated with self-reported deficits across two different self-report measures of attention/executive dysfunction in a healthy sample of young adults. These results support IIV of self-reported executive symptoms as a potential marker of neuropathology in line with previous research looking at objective measurement of executive functioning.

Correspondence: Jameson Beach, M.A., 75 South University Blvd, Mobile, AL 36688-0002, United States. E-mail: jdb1623@jagmail. southalabama.edu

J. BERG, J. DURANT, G.C. LEGER, Z.S. NASREDDINE \& J.B. MILLER. Comparing the Electronic and Standard Versions of the Montreal Cognitive Assessment in an Outpatient Memory Disorders Clinic.

Objective: Cognitive assessment is a critical component of clinical care for individuals suspected for neurodegenerative disease. The Montreal Cognitive Assessment (MoCA) is a validated brief paper-and-pencil cognitive screening measure that is routinely employed in clinical practice. An electronic version (eMoCA) of the measure, administered on a tablet computer, has recently been developed. The primary aim of the current study is to compare performance on the standard and electronic versions in an outpatient memory disorders clinic.

Participants and Methods: Participants included 29 new adult patient referrals presenting to clinic with primary memory complaints. Participants were randomly assigned to receive either the eMoCA or MoCA at the first visit and the alternate version two weeks later. Discrepancy scores, concordance correlation coefficients (CCC) and root mean squared differences (RMSD) were calculated between total raw scores from each administration method.

Results: Discrepancy scores ranged from -9.0 to $5.0(M=-0.17$, $S D=2.92$ ) with $52 \%$ of the sample having a raw difference score of 1 point or less. The CCG was 0.70 , indicating a low level of agreement using an absolute definition, and the RMSD was 2.87 . The mean total score on the MoCA $(M=23.48, S D=4.34)$ and eMoCA $(M=23.66$, $S D=3.55)$ were not significantly different.

Conclusions: While preliminary results suggest that the level of absolute agreement between total scores on the MoCA and eMoCA is low, the majority of individuals generated a 1-point or less difference on both versions and there was no mean difference between versions. Continued research on a larger sample will be important in order to establish convergent validity between the electronic and standard versions of the MoCA in a memory disordered population.

Correspondence: Jody-Lynn Berg, M.S., Neuropsychology, Cleveland Clinic Lou Ruvo Center for Brain Health, 888 W. Bonneville Ave., Las Vegas, NV 89106, United States. E-mail: bergj@ccf.org.

A. BOETTCHER, B.D. HILL \& J. EPKER. The relation of self-report pain scales to neuropsychological performance using structural equation modeling.

Objective: Only two studies have examined the latent factor structure of chronic pain, both utilizing non-clinical samples. This study examined the latent factor structure of pain (using self-report pain scales) to a latent factor of cognition (using neuropsychological test data) from a sample of chronic pain patients seen for a presurgical pain evaluation. Participants and Methods: Data from 477 archived files were randomly assigned to an exploratory factor analysis (EFA) subsample $(\mathrm{n}=200$; $61 \%$ female, $87 \%$ White, mean age $=53$ years $[\mathrm{SD}=13.11]$, and mean education=13.2 years $[\mathrm{SD}=2.75]$ ) or a structural equation modeling (SEM) subsample $(\mathrm{n}=277 ; 61 \%$ female, $87 \%$ White, mean age $=53.62$ years $[\mathrm{SD}=13.89]$, and mean education $=13.1$ years $[\mathrm{SD}=2.54])$. All chi square analyses were nonsignificant. Data collected from cognitive tests and self-report pain scales included: Wechsler Abbreviated Scale of Intelligence, Repeatable Battery for the Assessment of Neuropsychological Systems, Trail Making Test Part A \& B, The Modified Somatic Perception Questionnaire, Pain Disability Index, Pain Catastrophizing Scale, and Battery for Health Improvement-Second Edition: Pain Complaints Scale.

Results: Using EFA, two latent factors of pain were identified: "pain distress" and "pain dysfunction." Using SEM, both factors were confirmed and examined in relation to an overall latent factor of cognition ("g"). Greater perceived pain distress and pain dysfunction significantly predicted worse cognition and accounted for $12 \%$ of the variance in $g$.

Conclusions: The two latent factors of chronic pain measured a moderate relationship between global cognitive functioning and selfreported pain. These factors were different than those previously found in the literature. Future research should continue exploring the latent factors of chronic pain.

Correspondence: Anneliese Boettcher, Ph.D., Neuropsychology, KU School of Medicine - Wichita, 139 North Oliver, Wichita, KS 67208, United States.E-mail: boettcheranna@gmail.com

\section{J.Y. BRITT, L.C. HOYMAN \& H.K. ZEINER. Performance on Benton Facial Recognition Test and Ekman 60 Faces Test in Veterans.}

Objective: Facial expressions and recognition play a critical role in social functioning. Systematic research examining facial affect recognition has found conflicting results on whether there are significant deficits in affect recognition in individuals with Post-Traumatic Stress Disorder (PTSD). Some studies have found PTSD to impact identification of expressions of fear and sadness (Poljac et al., 2010). While others have found negative correlations between the severity of civilian adults with PTSD and their ability to recognize expressions of both happiness and fear (Armony et al., 2005). In examining veterans with PTSD, one study found PTSD to negatively impact individuals ability to recognize emotional facial expressions (Poljac et al., 2010) while other studies have not been unsuccessful as replicating the results. However, the literature is still sparse in identifying the impact of facial and affect recognition in veterans with PTSD.

Participants and Methods: This study will attempt to add to the current literature by examining veterans with $(n=56)$ and without a current diagnosis of PTSD $(n=37)$ and compare their performance on the Benton Facial Recognition Test and the Ekman 60 Faces Test. Correlations between PTSD and each measure were conducted.

Results: It was expected that veterans with PTSD would have deficits in their ability to recognize faces and affect. However, this study yielded no significant findings. Although there was no relationship between PTSD and these measures, there was a moderate correlation between the measures ( $r=0.490)$ suggesting beneficial use when used together in testing.

Conclusions: More research is needed to establish a relationship between a diagnosis of PTSD in veterans and social functioning measures. Future research directions will include an analysis of the differences between combat and civilian PTSD on facial and affect recognition.

Correspondence: Jessica Y. Britt, Psychology, Palo Alto University, 1791 Arastradero Road, Palo Alto, CA 94304,United States.E-mail:jbritt@ paloaltou.edu 
L.L. BUPP, R.W. SCHROEDER \& R.J. HEINRICHS. Classification Accuracy of Reliable Digit Span and Reliable Digit Span Revised. Objective: Prior research has indicated that both Reliable Digit Span (RDS) and Reliable Digit Span - Revised (RDS-R; Reliable Digit Span with the sequencing trial) can be useful as embedded performance validity tests (PVTs). Relatively few published studies, however, have directly compared the two measures against each other. The purpose of the present study was to examine the classification accuracy of both RDS and RDS-R to determine if one measure outperforms the other in a mixed clinical sample.

Participants and Methods: Participants were patients referred for neuropsychological evaluations who were classified according to PVT results (excluding RDS and RDS-R). One hundred and sixty-four patients who passed all PVTs administered were compared with 45 patients who failed 2+ PVTs. Receiver operating characteristics (ROC) were utilized to compare these groups to determine area under the curve (AUC), sensitivity (SENS) and specificity (SPEC) rates for RDS and RDS-R.

Results: The AUC was .85 for RDS and .87 for RDS-R. Using the recommended cutoff of $\leq 6$ for RDS, SENS was .24 and SPEC was 92. For RDS-R a cutoff of $\leq 10$ had a SENS of .49 and a SPEC of .98 . Conclusions: The ROC/AUC findings indicate that RDS and RDS-R both demonstrate 'good' discrimination of clinical samples, but RDS-R produced higher sensitivity and specificity rates. Continued crossvalidation of RDS- $\mathrm{R}$ is recommended.

Correspondence: Lindsey L. Bupp, M.S., Psychology, WIchita State University, 753 N. Woodrow Avenue, Wichita, KS 67203, United States. E-mail:lleebupp@gmail.com

\section{L.L. BUPP, R.W. SCHROEDER \& R.J. HEINRICHS. Classification} Accuracy of the Word Choice Test.

Objective: To date, research investigating the classification accuracy of the Word Choice Test (WCT) has been limited to that provided in the administrative and technical manual and three other studies (Bashem et al., 2014; Erdodi et al., 2014; Miller et al., 2012). The purpose of the present study was to further examine the classification accuracy of the WCT

Participants and Methods: Participants were patients referred for neuropsychological evaluations who were classified according to performance validity test (PVT) results (excluding WCT). Two sets of analyses were conducted. In the first set of analyses, 120 patients who passed all administered PVTs were compared with 39 patients who failed 2+ PVTs. In the second set of analyses, a more clinically-realistic "pass" group was used; 197 patients who failed 0-1 PVTs were compared to 39 patients who failed 2+ PVTs. Receiver operating characteristics (ROC) were utilized to determine area under the curve (AUC), sensitivity, and specificity rates.

Results: The first ROC was conducted on the stricter "pass" group (No PVTs failed), with the resulting AUC being .89. The second ROC was conducted with the more liberal "pass" group (0-1 PVTs failed), with the resulting AUC being .85. For both "pass" groups, sensitivity was modest to high (at least .67) when maintaining adequate specificity (.90).

Conclusions: The ROC/AUC findings indicate that the WCT demonstrates 'good' discrimination of clinical samples, with sensitivity rates being modest to high. As such, the WCT appears to be an appropriate freestanding validity test for use in clinical settings.

Correspondence: Lindsey L. Bupp, M.S., Psychology, WIchita State University, 753 N. Woodrow Avenue, Wichita, KS 67203, United States. E-mail:lleebupp@gmail.com
M.K. COLVIN, M. DALY, S. MANCUSO, A. LAFFER \& J.C. SHERMAN. Using the Addenbrooke's Cognitive Examination - Revised (ACE-R) to Identify the Type and Severity of Cognitive Impairment in Older Adults.

Objective: The ACE-R is a cognitive screening measure frequently used to identify neurocognitive disorders. Previous research found it comparable in sensitivity and specificity to the briefer Mini-Mental Status Exam (MMSE) in detecting a neurocognitive disorder. We examined whether the MMSE and ACE-R are sensitive to both severity and type of cognitive impairment and whether the ACE-R subscales add additional useful diagnostic information.

Participants and Methods: 179 patients (mean age $=74.8$ ) completed a clinical neuropsychological evaluation that included the ACE-R. The MMSE is embedded within the ACE-R. Based on clinical history and performance on neuropsychological testing, patients were diagnosed with Mild Cognitive Impairment (MCI) or Dementia (D) of either amnestic (A) or non-amnestic (NA) type (56 MCI-A, 24 D-A, 64 MCI-NA, 35 D-NA).

Results: As expected, total ACE-R score strongly correlated with total MMSE score $(\underline{p}=.000)$ and there was no difference in the strength of this correlation between groups (MCI vs. Dementia, amnestic vs. nonamnestic). Both the total ACE-R and MMSE scores differed depending on severity (MCI > Dementia scores) and type (non-amnestic $>$ amnestic scores) of cognitive impairment with no interaction. Of the five ACE-R subscales, attention \& orientation and memory differed by severity (MCI vs. Dementia) and type of cognitive impairment (amnestic vs. non-amnestic). The visuospatial subscale differed by severity (MCI vs. Dementia) but not type of cognitive impairment (amnestic vs. non-amnestic). Severity of cognitive disorder (MCI vs. Dementia) was predicted by scores on the visuospatial subscale. Type of disorder (amnestic vs. non-amnestic) was predicted by scores on the memory and visuospatial subscales.

Conclusions: The MMSE and ACE-R both differ based on severity and type of cognitive disorder. However, the ACE-R subscales add additional clinical information that can be used to predict severity and type of neurocognitive disorder and mav therefore be a more useful clinical screening tool.

Correspondence: Mary K. Colvin, PhD, Psychiatry, Massachusetts General Hospital, One Bowdoin Square, Suite 701, Boston, MA 02114, United States.E-mail:mcolvin@moh.harvard.edu

S.E. COOK, T.J. FARRER \& J.Z. STUART. Test of Premorbid Functioning Predicts Markers of Intellect in an Outpatient Clinical Sample.

Objective: Updated measures of premorbid functioning (TOPF) and intellect (WAIS-IV) have become available in recent years, but there is little known about how well these measures relate beyond the neurologically intact standardization sample. There is one recent study that suggests the TOPF underestimates premorbid functioning in a sample of individuals seen in a neurodegenerative disease clinic (Berg et al.., 2016). The current study sought to understand how well the TOPF predicts various markers of intellect in a general adult outpatient clinical sample in the south Atlantic region.

Participants and Methods: A total of 469 outpatients (age mean $=56.99, \mathrm{SD}=16.63$, range $16-89$ years, $74 \%$ white, $45 \%$ male. education mean 14.70 years, $S D=2.94$, range $4-20$ years) were administered the TOPF and at least part of WAIS-IV from late 2013 through 2015 as part of the flexible neuropsychology battery chosen by their neuropsychologist. Pearson correlations were utilized to examine the relationship of TOPF with WAIS-IV variables.

Results: TOPF standard score $(\mathrm{N}=469$, mean $=99.96, \mathrm{SD}=14.35$, range $=59-131)$ was significantly correlated with all WAIS-IV variables examined, including Full Scale IQ (with all 10 core subtests administered, $\mathrm{N}=207, r=0.79, p<0.001$ ), Verbal Comprehension Index $(\mathrm{N}=397, r=0.77, p<0.001)$, Vocabulary subtest scaled score $(\mathrm{N}=368, r=0.78, p<0.001)$, Similarities subtest scaled score 
$(\mathbf{N}=397, r=0.63, p<0.001)$, and Information subtest scaled score $(\mathbf{N}=336, r=0.70, p<0.001)$.

Conclusions: Findings suggest that TOPF is highly related to markers of verbal and overall intellect. All correlations examined are relatively equivalent to or modestly higher than those stated in the TOPF manual for the standardization sample of neurologically intact individuals (range of correlations for same variables 0.61-0.75), suggesting that the TOPF predicts intellect equally well in a clinical sample at a general neuropsychology service.

Correspondence: Sarah E. Cook, PhD, Psychiatry and Neurology, Duke University, Box 3333 DUMC, 932, Durham, NC 27705, United States. E-mail:sarah.cook@duke.edu

A.N. CORRERO, C.M. CONSIDINE \& C. ABEARE. The Brief Estimate of Seconds Test (BEST): Piloting a New Measure of Chronognosis with ADHD and Memory Disorder Samples from a VA Clinic.

Objective: Distortions in chronognosis (time perception) are found in multiple neuropsychiatric and neurological populations. The Brief Estimate of Seconds Test (BEST), a new measure of chronognosis designed for practical clinical utility, has demonstrated appropriate psychometrics within a healthy young-adult sample but not with clinical samples. Thus, a prospective pilot study was undertaken, in order to explore the potential utility of collecting larger performance norms for different diagnostic groups.

Participants and Methods: The BEST was administered as part of neuropsychological evaluations for adult attention deficit/hyperactivity disorder (ADHD) and geriatric memory impairment referrals to a VA outpatient clinic. The performances of 13 veterans (women=1; $M_{\text {age }}=71.5, S D=10.8$ ) diagnosed with a primary memory-based neurocognitive disorder (MEM) and the performances of seven veterans (women=2; $M_{\text {age }}=43.7, S D=13.1$ ) diagnosed with ADHD were compared to a sample of 35 healthy controls (HC; women $=30 ; M_{\text {age }}=21.7, S D=2.9$ ). Results: Descriptive analyses identified appropriate psychometrics within each clinical group. Correlation of demographic variables with performance identified education as a covariate for subsequent analyses, but surprisingly not age. An exploratory multinomial logistic regression using BEST subtasks to predict diagnostic group (MEM, ADHD, or HC), after covarying for education, was significant $\left(p=.04\right.$, pseudo- $\left.R^{2}=.22\right)$. Specifically, short prospective verbal $(p<.01)$ and short prospective production $(p=.01)$ estimates predicted diagnostic grouping for both ADHD and MEM groups compared to the HC group.

Conclusions: These pilot data support the BEST's psychometrics and ability to detect dyschronognosia in two clinical samples. The exploratory findings are consistent with ADHD and memory disorder findings in the literature. The low clinical $n$ 's and lack of age-matched normative data for the memory disorders group will be addressed by future research using clinic and community samples.

Correspondence: Anthony N. Correro, M.S., Psychology, Marquette University, Cramer Hall 317, Milwaukee, WI 53233, United States.

E-mail: anthony.correro@marquette.edu

\section{J.F. DE JONGHE, T. SCHOEMAKER \& D. LAM. Does Depression} Explain Poor Effort on Symptom Validity Tests (SVT)?

Objective: Valid assessments require sufficient effort from the part of the testee. Motivation may be compromised, particularly in psychiatric conditions. We examined associations between response bias and self-reported symptoms in depressed and PTSD patients.

Participants and Methods: This is a single site, cross-sectional study using a convenience sample of disability claimants in the Netherlands $(\mathrm{n}=132)$. Patients had depression $(\mathrm{n}=48)$, or PTSD or other anxiety disorders ( $n=37)$. A control group $(n=47 \%)$ had chronic pain disorder, fibromyalgia or chronic fatigue. The Green Word Memory Test (GWMT) was administered to all subjects. The Structured Inventory of Malingered. Symptomatology (SIMS), and the Beck Depression Inventory
(BDI-II) were administered in subsamples. Study outcome was selfreported depressive symptoms in SVT negative cases.

Results: Average age of the participants was 45.1 years (SD 9.5), $48.5 \%$ were female, and $57.7 \%$ had a High school/College/Master's degree. GWMT positive scores were found in $52.3 \%$ of all cases, GWMT and SIMS positive in $33.8 \%$, and GWMT and SIMS negative in $37.7 \%$. No significant group effects on GWMT were found. Average BDI-II scores were 32.8 (SD 13.9) for depressed patients, 28.3 (15.5) for those with anxiety disorders, and 27.6 (14.1) for controls. Kruskal-Walis test showed no group differences for the BDI-II total score $(p=.43)$. Seventy-eight percent of depressed GWMT negative cases reported at least moderate depressive symptoms (BDI-II $>18$ ), and $44.4 \%$ severe symptoms (BDI-II > 29). Approximately half of the GWMT negative cases with anxiety disorders and controls scored BDI-II $>18$.

Conclusions: Non credible test performance is prevalent in disability claimants with affective, mood disorders. However, depressive symptoms per se do not explain poor effort on cognitive tasks.

Correspondence: Jos F. de Jonghe, PhD, Geriatric Medicine 170, Medical Center Alkmaar, Wilhelminalaan 12, Alkmaar 1815 JD, Netherlands. E-mail:j.de.jonghe@mca.nl

S. DHILLON, M. SHARMA, K.K. ZAKZANIS \& R. BAGBY. Predictive Capacity of Symptom Validity Tests for the Detection of Feigned Cognitive Impairment Associated with mild Traumatic Brain Injury (mTBI).

Objective: Diagnostic validity and reliability are concerns for the accurate assessment of cognitive impairment associated with mTBI. Psychological tests with include a distinction between self-report based non-cognitive symptom validity testing (SVTs) and performance based validity testing (PVTs). The Minnesota Multiphasic Personality Inventory-2-RF (MMPI-2-RF) and Personality Assessment Inventory (PAI) include scales that can be used as indices of SVTs. This study aims to (a) compare the effectiveness of the MMPI-2-RF and PAI in detecting overreporting of mTBI symptomology and (b) determine the extent to which the MMPI-2-RF and/or PAI SVTs provide incremental validity beyond that of the PVTs in the detection of feigned uncomplicated mTBI symptoms using an experimental analogue design.

Participants and Methods: Subjects ( $N=102)$ were randomly assigned to a standard instruction (SI) or overreporting (OR) group. At time 1 (T1) both groups completed the MMPI-2-RF and PAI; the SI subjects also completed three PVTs. All subjects return for a second administration (T2). At T2, SI subjects completed the SVTs while OR subjects were instructed to complete the MMPI-2-RF and PAI as if they were suffering from a mTBI, as well as the same battery of PVTs. MANOVA assessed the variance between the SI and OR group and hierarchical logistic regression was used to determine if the SVTs added any significant incremental validity to the PVTs.

Results: The results of our MANOVA are significant, indicating that the manipulation was effective. Regression analyses demonstrate that the indexed SVTs are more predictive of group membership than PVTs. In addition, the MMPI SVTs are most predictive of feigned cognitive impairment.

Conclusions: Administering the MMPI-2-RF along with PVTs in a formal neuropsycholgical evaluation can increase the validity of neuropsychological tests results. When SVTs and PVTs are employed together, however, they serve to account for more observed variance than that demonstrated when PVTs are employed alone.

Correspondence: Sonya Dhillon, Psychological Clinical Science, University of Torotno, 306 - 318 king street east, Toronto, ON m5a0c1, Canada.E-mail:sonya.dhillon@mail.utoronto.ca

A. DIXON, G. PILONIETA \& M.C. NATELSON LOVE. Variation in Alabama Brief Cognitive Screener Score in Amnestic and NonAmnestic Mild Cognitive Impairment with Level of Education.

Objective: To ascertain variations in Alabama Brief Cognitive Screener (ABCs) scores, a test developed to screen for cognitive impairment in 
acute and outpatient settings, based on level of education in patients with mild cognitive impairment.

Participants and Methods: Following IRB approval, ABCs scores and demographic information were obtained from the electronic medical record of patients who had a clinician's diagnosis of mild cognitive impairment (MCI) from 4/30/2012 to 4/30/2014 in the UAB Memory Disorders clinic $(n=213)$. Patients were divided into four categories based on level of education (high school diploma or less (62), some college (55), bachelor's degree (59), and graduate/postgraduate degree (37)) and domain of mild cognitive impairment (amnestic (n=141) vs. non-amnestic $(\mathrm{n}=72)$ ) for analysis. There was no significant difference in ABCs means at initial presentation based on level of education for mild cognitive impairment when not divided by domain as analyzed by ANOVA $(p=0.08)$.

Results: In patients with amnestic MCI, those with a level of education less than or equal to a high school diploma, on average, had a significantly lower ABCs score at initial presentation than those who completed some college or more (means were 23.6 and 25.6, respectively $p=0.01$ ). However, there was no significant difference between the mean scores above that level of education. There was no significant difference in ABCs means based on level of education for non-amnestic MCI.

Conclusions: This may represent the heterogeneity of etiologies of non-amnestic MCI. However, the ABCs' score appears to differ based on level of education at presentation of amnestic MCI. This psychometric property aligns with other bedside screening instruments like the MMSE and MoCA

Correspondence: Amber Dixon, University of Alabama School of Medicine, 1406 Azalea Park Ln, Montoomery, AL 36106, United States. E-mail: amberad@uab.edu

\section{R. GERSHON, C. NOWINSKI \& J. SLOTKIN. Equivalence of the NIH Toolbox for Assessment of Neurological and Behavioral Function iPad version.}

Objective: The NIH Toolbox is a comprehensive and nationally normed set of tests assessing Cognition, Motor, Sensation and Emotion from ages 3-85. Publicly released for web-based administration in 2012, the NIH Toolbox has already been accessed by more than 1,000 investigators and clinicians. In 2013, the National Children's Study chose to release the NIH Toolbox instruments in iPad form. The objective of this study was to demonstrate to potential users that the iPad and web-based versions of the Cognition measures are equivalent.

Participants and Methods: Participants were assigned to one of two conditions: the standard NIH Toolbox (web-based) administration or the iPad version ( $\mathrm{N}=300$ per condition). Participants were randomly selected from existing market research databases to fill age, gender, race/ethnicity and education cells. Participants were first stratified by age, such that 20 participants per each of 15 age bins were assigned to each of the 2 conditions. Within the age strata, participants were matched on gender, ethnicity, and education across the two conditions. Key validation measures were also administered: the PPVT- 4 for all participants and the WISC-IV/WAIS-IV Coding and Letter-Number Sequencing subtests for participants 6 years and older, to ensure both modes of testing compare similarly and favorably to these "gold standards."

Results: No significant difference in scores were encountered for any of the cognitive measures for both children and adults. Minor differences were encountered for some of the cognitive measures in narrow age ranges. However, the results of subsequent regression analyses did not prove significantly different when predicting web-based scores from iPad-based scores.

Conclusions: The iPad version of the NIH Toolbox has been shown to generate scores sufficiently similar to the web-based version to allow results from patients collected on either platform to be compared with one another. This session will discuss overall results, as well as any potential areas for age-specific concern.
Correspondence: Richard Gershon, PhD, Medical Social Sciences, Northwestern University, 6545 North LeMai Avenue, Lincolnwood, IL 60712, United States.E-mail: gershon@northwestern.edu

M. GODFREY, N. RAITANO LEE \& J. GALLO. An evidence-based evaluation of neuropsychological tests used to assess dementia among older adults with Down Syndrome.

Objective: Down Syndrome (DS) is the most common form of intellectual disability with a known genetic etiology. DS results in virtually all adults developing Alzheimer's Disease (AD) neuropathology by age 40 , and $67 \%$ expressing symptoms of dementia by age 72 . Due to increased life expectancy rates for adults with DS, prevalence of adults with comorbid DS and AD has substantially increased and underscores the need for measures with high validity and clinical utility for diagnosing dementia in this population. Efforts to establish standardized tests sensitive to signs of $\mathrm{AD}$ among adults with DS have been proposed and typically target cognitive, functional, and motivational/emotional changes. Of these three domains, tests sensitive to cognitive changes in the DS population are the most tenuous. This study aimed to examine the quality and practical relevance of a set of cognitive tests, which are proposed as useful for diagnosing dementia in the DS population.

Participants and Methods: Specifically, we applied the Critically Appraised Topics Worksheet (CAT; derived from Bowden, Harrison, \& Loring, 2014) to tests measuring general ability, language, memory, orientation and motor skills, to determine their utility for the DS population.

Results: Results of our appraisal suggest that, while some cognitive tests have demonstrated sensitivity to AD in adults with DS and utility in the diagnostic process, improvements are needed.

Conclusions: Moreover, this procedure demonstrates that CAT can evaluate the validity and utility of each cognitive measure in a diagnostic battery and ultimately aid in refining a battery to include only the most clinically useful assessments for a specific population.

Correspondence: Mary Godfrey, Psychology, Drexel University, $30 \mathrm{~S}$ 22nd Street, Apt 10, Philadelphia, PA 19103, United States. E-mail: maryelizabethgodfrey@gmail.com

R. GOLDSWORTHY \& R. SKEEL. The value of future rewards: Factors that influence discounting rates on a human operant delay discounting task.

Objective: Delay Discounting is a choice impulsivity technique used to measure the subjective evaluation of reward values. As shown in previous studies, a reward's value will subjectively decrease as the delay to its receipt becomes longer. Many delay discounting tasks use either hypothetical or monetary reinforcements to estimate impulsive behavior. The present study aimed to refine a novel operant delay discounting measure that utilizes YouTube video clips and their subjective ratings as a measure of reward magnitude. Specifically, both the delay times and differences in quality of the video clips were manipulated to examine how these changes affect discounting behavior.

Participants and Methods: Sixty participants completed the delay discounting task, in which they chose between watching relatively neutral-rated videos immediately and watching highly-rated videos after variable delays. Participants were randomly assigned to one of two conditions in which the quality of the neutral-rated video was manipulated to create a large or small discrepancy between the quality of the two video clips. Participants also completed two self-report impulsivity questionnaires.

Results: The paradigm resulted in discounting behavior for both conditions, in that the delays significantly affected participants' video preferences across both conditions. Increasing the delay time did not result in markedly increased discounting behavior. Changes in reward quality discrepancy did not result in significant differences in responding between groups.

Conclusions: Although refinements to the measure did not result in increased discounting, results provided support that the Delay 
Discounting Task reliably assesses the impact of delays on reward choice responding in a college sample. In addition, the delays significantly impacted responses in a manner consistent with existing temporal discounting measures.

Correspondence: Rachael Goldsworthy, Psychology, CMU, $600 \mathrm{~S}$. Washington Street, APT 19, Mt. Pleasant, MI 48858, United States. E-mail:rgoldswo@gmail.com

A. GUALTIERI, T.J. FARRER, R. ZARZOUR, J. BYTOMSKI \& D. ATTIX. Influence of Test-Retest Interval on Stability of Neuropsychological Tests in College Athletes.

Objective: Neuropsychological assessment is a vital tool in tracking cognition among student-athletes at risk of sustaining concussions. Such assessments begin with baseline testing, which is then repeated annually or at pre-determined intervals to establish new baselines. Some have raised concern about repeated testing interfering with accurate determination of change, suggesting that less frequent intervals are better. We explored whether time interval impacts test-retest reliability.

Participants and Methods: The sample consisted of 241 healthy college athletes (mean age=18.89; $\mathrm{SD}=1.15 ; 75.1 \%$ male). Retesting was done as routine follow-up, without any known interval central nervous system injury. Tests included: Rey Auditory Verbal Learning Test (RAVLT; alt. forms), Brief Visuospatial Memory Test-Revised (BVMT-R; alt. forms); Trail Making Test B, Digit Span backward, Symbol Search, Coding, and Cancellation. The median time interval between $\mathrm{T} 1$ and T2 was 376 days. Test-retest reliability, using Pearson's coefficient $(r)$, was compared for each test for those with a short testing interval (STI; $<376$ days) and for those with a long testing interval (LTI; $>376$ days). Results: Overall, executive tests are more stable (mean STI $r=0.674$; LTI $r=0.612$ ) than memory tests (mean STI $r=0.437$, LTI $r=0.400$ ) regardless of length of time interval, possibly due in part to alternate forms. Despite the relative stability in executive tests, correlations attenuate with the use of longer time intervals for select tests with a decline of $>.10$ on four of nine measures with LTIs.

Conclusions: This analysis suggests that longer time intervals between $\mathrm{T} 1$ and T2 yield lower correlation coefficients on a fair number of measures. Importantly, longer test intervals result in less stability, indicating that retesting infrequently for fear of misinterpreting results is misguided. The challenge of interpreting longitudinal data is better handled through the use of standardized regression based normative trajectories than delay of repeat assessment.

Correspondence: Alex Gualtieri, NC, United States. E-mail: alex. heintzelman@duke.edu

D. GUZMAN, H. FOUTY, E. AILES, K. BROWN, S. LUGAR \& M.R. BASSO. Construct Validity of the Tinker Toy Test.

Objective: Lezak (1982) introduced the Tinker Toy Test (TTT) as a non-verbal measure of executive function that assesses complex planning and problem solving. Examinees are provided 50 pieces of a tinker toy set, and are asked to build an item of their own choosing. No further directions are given. Initial research implied the instrument possessed satisfactory criterion validity, but few studies have elaborated the TTT's merits. Accordingly, this investigation aimed to evaluate the construct validity of the TTT.

Participants and Methods: 40 healthy young adults participated (30 females, 10 males). Participants completed the TTT and the Wechsler Abbreviated Scale of Intelligence - II (WASI-II). Administration of these measures was counterbalanced across participants. Convergent validity was assessed by examining the relationship between TTT scores and the Perceptual Reasoning Index from the WASI-II. Divergent validity was evaluated by the relationship between TTT scores and the Verbal Comprehension Index from the WASI-II.

Results: Because the TTT is conceptualized as a non-verbal measure of executive function, it was anticipated to correlate significantly with the non-verbal component of the WASI-II, and it was expected to not correlate with the verbal domain of the WASI-II. As expected, correlations revealed a significant correlation between the TTT and PRI $(r=. .36, p=. .02)$ and a non-significant correlation between the TTT and VCI $(r=.25, p=.12)$.

Conclusions: This study supports the construct validity of the TTT. This measure correlated most strongly with non-verbal measures. This suggests that the TTT is better at examining nonverbal executive functioning and may help to identify right-frontal lesions.

Correspondence: Daniel Guzman, PhD, The University of Tulsa, 800 South Tucker Drive, Tulsa, OK 74104, United States. E-mail: dag773@ utulsa.edu

C. HALE, B.S. LAST, I.B. MEIER, L. YEUNG, R.P. SLOAN, S.A. SMALL \& A.M. BRICKMAN. Introducing the ModRey: An Episodic Memory Test for Non-Clinical and Preclinical Populations.

Objective: List learning tests are used in practice for diagnostic formulation and in research to characterize episodic memory. Such tools were designed for clinical populations and often suffer from ceiling effects in unimpaired individuals. To address the growing need for neuropsychological instruments that can be used in both normal and preclinical populations, we developed the Modified Rey Auditory Verbal Learning Test, or ModRey, an episodic memory scale that includes three learning trials of 20 unrelated nouns, an interference trial, short delay free recall, long delay free recall, a recognition trial, and a source memory trial. We created two alternate forms to address practice effects for repeated administration.

Participants and Methods: We administered one of the two alternate forms to 230 medically, psychiatrically, and neurologically healthy adults (mean age $=40.2 \pm 15.1$ years-old, range $=20-75$ ). In a subset $(n=86)$, we administered the alternate form (counter-balanced) a mean of $102 \pm 20.2$ days after baseline testing. We examined psychometric properties including frequency distributions, differences in alternate forms, and test-retest reliability, and the effects of age, years of education, and sex.

Results: Primary outcome measures were normally distributed. Differences between the alternate forms were of very small magnitude and not statistically significant. Test-retest reliability was good $(r>0.5)$ for most measures. Higher participant age and lower participant education was associated with poorer performance across most outcome measures. Conclusions: Together with our previous observations of a selective relationship between performance on the ModRey and function of the entorhinal cortex, which mediates episodic memory and contains early pathological changes in Alzheimer's disease, this study demonstrates good psychometric properties of the ModRey. The ModRey is appropriate for episodic memory characterization in normal populations and could be used as an outcome measure in studies involving preclinical populations.

Correspondence: Christiane Hale, M.S., Cognitive Neuroscience, Columbia University, 622 W 168th Street, PH18, Room 333, New York, NY 10032, United States.E-mail: ch3190@cumc.columbia.edu

M. HENDRIKS, M. VAN DIJK, P. VAN DER HEIJDEN, C. SUWARTONO \& T. CLAASSEN. A Causal Modeling Approach of WAIS-IV Profiles of Patients With Temporal Lobe Epilepsy, Psychiatric Patients and Matched Controles.

Objective: Since the publication of the WAIS-IV in 2008, efforts have been made to gain insights into its clinical validation, by applying factor analyses to both clinical and non-clinical samples. This study aims to achieve a better understanding of the factor structure of the Dutch WAIS-IV (WAIS-IV-NL) core battery by applying explorative factor analysis (EFA) and the Bayesian Constraint-based Causal Discovery (BCCD). BCCD is an alternative, explorative approach based on causal modeling. With this algorithm dependencies can be found between variables and determines the reliability of their associations.

Participants and Methods: Using the Dutch version of the WAIS-IV core battery subtests we compared the Intelligence profiles of three 
laerge groups. A group of 202 patients with Temporal Lobe Epilepsy (TLE) and a mixed group of 202 psychiatric patients that was machted for age and educational level, were contrasted with a group normal controls from the Dutch standardization sample $(\mathrm{N}=202)$, that also was matched for age and eductation.

We compared their results both by applying a regular explorative factor analysis and the BCCD method.

Results: EFA revealed 2 factors for the two clinical samples with verbal subtests loading on one factor, and 3 factors for the non-clinical control sample concurrent with verbal comprehension, perceptual reasoning and processing speed. BCCD showed three factors with sufficient reliability $(>80 \%)$ between subtests within all samples. However the subtests Matrix Reasoning, Arithmetic and Digit Span show no relation with the three factors at all, or a less reliable (60-80\%) associations with other subtests.

Conclusions: Although intercorrelations are reflected in the reliability of associations retrieved from causal modeling, EFA and BCCD offer different factor-solutions. In this study, BCCD has shown to detect reliable associations between subtests and offers a more robust basis for clinical interpretation.

Correspondence: Marc Hendriks, PhD, Donders Institute for Brain, Cognition and Behaviour, Radboud University Nijmegen, Montessorilaan 3, Nijmegen6500 HE, Netherlands.E-mail:m.hendriks@donders.ru.nl

J. HERSHAW, E. CORDERO, B. GUISE, D. BARRY, J. KEGEL \& M. ETTENHOFER. Predicting Invalid Responses Using Pupil Diameter During a Cued Attention Task.

Objective: Unobtrusive sensor-based measurements of cognitive processing may be valuable as embedded measures of performance validity. The objective of this study was to evaluate the effect of response bias on pupillary metrics.

Participants and Methods: Forty-eight healthy participants completed a neuropsychological test battery; including $n=42$ instructed to give their best effort and $n=23$ instructed to simulate effects of mild TBI ("fake bad"). An additional n=21 participants with remote mild TBI were instructed to provide their best effort on these measures. Pupillary metrics were computed during a computerized cued attention task in which six unique cue-target combinations (e.g., trial types) were randomly presented. Pretrial pupil diameter (PD) and cue-locked change in PD were compared between the mild TBI patients, biased, and unbiased responders and correlated with cognitive performance. Results: The fake bad group had larger pretrial PD than mild TBI, $p<.05$. Analyses revealed a consistent trend in which the cue-locked change in PD appeared smallest for the fake bad group across several trial types. Large differences in cue-locked PD were noted for trial types eliciting attentional disengagement, $p=.064$, and response inhibition, $p<.05$. Pretrial PD and change in PD on trials indexing response inhibition were related to severity of self-report neurobehavioral symptoms, number of failed PVTs, and performance on TMT A, Digit Span, and Conners' CPT-II, $\mathrm{p}<.05$.

Conclusions: Smaller change in PD indicates that the fake bad group did not process task stimuli to the same extent as unbiased responders and individuals with mild TBI. However, they have more preparatory activation as evidenced by larger pretrial PD, suggesting they potentially maintain a cognitively-taxing strategy of responding with less regard to the unique demands of each trial type. Overall, these findings provide evidence that pupillary response to cognitive tasks could provide an objective measure of response validity to augment existing methods. Disclaimer: The opinions and assertions contained herein are those of the authors, and do not necessarily represent the views of the Department of Defense or the Uniformed Services University of the Health Science. Correspondence: Jamie Hershaw, MS, Medical and Clinical Psychology, Uniformed Services University, 4301 Jones Bridge Road, Bethesda, MD 20814,United States. E-mail: jamie.hershaw.ctr@usuhs.edu
L.C. HOYMAN, J.Y. BRITT \& H.K. ZEINER. The Impact of PTSD on Verbal Learning and Memory using the CVLT-II.

Objective: Posttraumatic stress disorder (PTSD) has been correlated with poor performance on tests of verbal learning and memory (Yehuda et al., 2005). Past research utilized measures such as the California Verbal Learning Test (CVLT) to examine learning and memory in combat veterans with PTSD. Previous results demonstrate that PTSD negatively impacts elderly veteran's long-term memory performance compared to elderly veterans without PTSD (Yehuda et al., 2005). To date, no other study has attempted to replication these findings with a larger veteran sample and a new measure, the CVLT-II (Second Edition). This study bridges the gap in the current literature by identifying group differences in verbal learning and memory performance between veterans with and without PTSD.

Participants and Methods: The sample consisted of veterans with $(n=56)$ and without a diagnosis of PTSD $(n=33)$ in an outpatient neuropsychology clinic. All participants were administered the CVLT-II which includes assessment of verbal learning, proactive interference, immediate recall, and delayed recall.

Results: Correlations and linear regressions were analyzed to better understand the relationship between PTSD and cognitive functioning on measures of verbal learning and memory. Moderate negative correlations were found between PTSD diagnosis and verbal learning ( $r=-.323)$, immediate recall $(\mathrm{r}=-.356)$, and delayed recall $(\mathrm{r}=-.367)$ and a weak correlation between PTSD and proactive interference ( $\mathrm{r}=-.123)$. Using linear regression, it was also shown that PTSD significantly predicts performance on verbal learning (.002), immediate recall (.001), and delayed recall (.001).

Conclusions: Results from this study support previous findings that PTSD negatively impacts verbal learning and memory. Clinicians are encouraged to consider an assessment of verbal learning and memory when a patient is diagnosed with PTSD.

Correspondence: Lisa C. Hoyman, M.S., Palo Alto University, 1791 Arastradero Road, Palo Alto, CA 94304, United States. E-mail: lhoyman@paloaltou.edu

I. IBANEZ-CASAS, J.C. DAUGHERTY, B.E. LEONARD, M. PEREZ GARCIA \& A.E. PUENTE. Protocol for the Development of a Domain Specific Computerized Battery for Cross-Cultural Neurocognitive Assessment: The EMBRACED Project.

Objective: Tests should be tailored to the population they are meant to serve prior to making between groups comparisons (ITC, 2010; AERA, APA \& NCME, 2014). The EMBRACED project follows AERA and ITC guidelines and will be the first comprehensive and domain specific computerized neuropsychological battery intended for widespread international application.

Participants and Methods: Bibliometric sources were utilized using a top-down strategy in 3 phases. In phase 1, more than 160 sources were reviewed. From this, commonly used neuropsychological domains were identified and then included in the battery (Table 1). In Phase 2 the relevant constructs from the identified domains were determined (Table 2). In phase 3 the best tests/tasks to assess those constructs were identified. Results: Phase I: Results revealed the critical domains were attention, executive functions, language, memory, visuospatial, orientation, perception, and social cognition. Substantial overlap and discrepancies within domains were found.

Phase II \& III: For each domain, constructs and tests were identified. Tests with the largest effect sizes and most comprehensive inclusion of constructs were chosen. In verbal memory, a similar task to the CVLT was chosen for the EMBRACED battery due to its high effect size and inclusion of all the relevant constructs. Table 2 provides a step-by-step illustration of the procedure used to assess verbal memory.

Conclusions: The development of a domain specific empirically derived computerized neuropsychological battery id described. Not only does the battery meet traditional standards for test development but will also be applied internationally. Finally, this will be a public domain battery 
intended to be shared globally in an effort to better understand universal as well as more localized assessment of neuropsychological function. Correspondence: Inmaculada Ibanez-Casas, PhD, Psychology, University of North Carolina Wilmington, 137 \#A Lullwater Dr, Wilmington, NC28403,United States.E-mail: ibanezcasasi@uncw.edu

I. IBANEZ-CASAS, B.E. LEONARD, J.C. DAUGHERTY, M. PEREZ GARCIA \& A.E. PUENTE. Word Lists Development for CrossCultural Verbal Memory Assessment in a Computerized Neuropsychological Battery: The EMBRACED Project.

Objective: Word lists are the benchmark for verbal memory and learning assessment. Several factors may affect the quality of results when using these lists. Published test manuals and articles did not provide explanations for how word lists were developed. The aim of this study was to develop as well as to explain the theoretical and practical reasons for two lists of words (one for learning, one for interference) both in English and Spanish.

Participants and Methods: Bibliometric sources determined that word list criteria should include frequency, length, semantic categorization, pan-pronunciation, and commonality across cultures (Table 1). The Spanish Corpus (LEXESP) and English Corpus (SUBTLEXus) were used to determine frequency of words for further statistical comparisons. Word frequency per million is a standard measure of word frequency independent of the corpus size. Three bilingual researchers generated the word lists.

Results: Initially 500 words of intermediate frequency in each corpus were used. However, there were not a sufficient number of words meeting all criteria. Programming the corpus using our criteria to generate its own list also yielded poor results. Finally, it was discovered that determining word categories and generating associated lists within categories satisfying the standards, was successful. Both within-subjects ANOVA and between-groups ANOVA yielded statistically insignificant results within and across both languages (Table 2).

Conclusions: Developing testing materials should be exhaustive, systematic, and reliable according to The Standards for Educational and Psychological Testing (ITC, 2010; AERA, APA \& NCME, 2014). The procedure described has met these requirements and our preliminary data collection provides initial support to suggest that this method is reliable.

Correspondence: Inmaculada Ibanez-Casas, PhD, Psychology, University of North Carolina Wilmington, 137 \#A Lullwater Dr, Wilmington, NC28403,United States.E-mail: ibanezcasasi@uncw.edu

\section{ISAAC. An Evaluation of Premorbid Intelligence Estimates:} BEST and WIAT in a Young, Diverse Population.

Objective: Premorbid intelligence estimates provide a baseline to assess possible deficits due to brain injury. Some of the methods, such as the Best Performance (BEST), were developed with the Wechsler Adult Intelligence Scales - Revised (WAIS-R) and need to be validated on the Wechsler Adult Intelligence Scales - IV (WAIS-IV). This study evaluated premorbid intelligence estimates using the BEST method and tests on the Weschler Individual Achievement Test - III (WIAT-III).

Participants and Methods: Assessment data from 70 participants $(71.4 \%$ female, $\mathrm{n}=50)$ were extracted from a larger set of patients referred to the Assessment Team at an urban university. The participant's ages ranged from 16 to $61(\mathrm{M}=26.35, \mathrm{SD}=9.03)$, had a mean education of 14.51 years $(\mathrm{SD}=2.29)$, and were relatively ethnically diverse, such that $22.9 \%$ were African American $(\mathrm{n}=16), 60 \%$ Caucasian $(\mathrm{n}=42), 7.1 \%$ Hispanic $(\mathrm{n}=5), 4.3 \%$ Asian American $(\mathrm{n}=3), 2.9 \%$ Middle Eastern $(\mathrm{n}=2)$, and $2.9 \%$ Biracial $(\mathrm{n}=2)$. Each was administered the WAIS-IV and WIAT-III as a part of a larger battery.

Results: There was no a signifcant correlation between the full scale intelligence quotient (FSIQ) estimated by the BEST and FSIQ on the WAIS-IV $(r=-.029, p=.822)$. Spelling $(r=.581, p<.001)$, Math
Problem Solving $(r=.663, p<.001)$, and Basic Reading $(r=.506$, $p<.001)$ from the WIAT-III were all significantly correlated with FSIQ. Conclusions: These findings demonstrate that the BEST approach may not be appropriate for premorbid intelligence estimation in a young and relatively diverse population. Embedded in this method are calculations of education, SES, and occupation which may be unstable when younger. Therefore, methods that include these variables may not be suitable for younger individuals and other methods should be considered. The WIAT-III has multiple possibilities, inlcuding Spelling, Math Problem Solving, and Basic Reading, to assist in establishing premorbid intelligence, but these will need to be investigated in brain injured populations.

Correspondence: Lee Isaac, M.Ed, Psychology, La Salle University, 8816 Ridge Ave \#22, Philadelphia, PA 19128, United States. E-mail: lee.v.isaac@gmail.com

A. KOSSMAN, J. MARCEAUX, D.A. GONZÁLEZ \& L. GOLLA. Predictive Value of ECog Total Score and Informant Characteristics on Cognitive Impairment.

Objective: Informant-rated questionnaires are established as meaningful tools when assessing for cognitive impairment. One such questionnaire, the Everyday Cognition scale (ECog), has been shown to be a valid measure of general and domain-specific daily functioning with diagnostic utility, including differentiating between mild cognitive impairment (MCI) and dementia (Farias et al., 2008). However, few studies have addressed the degree to which informant characteristics relate to the utility of responses on the ECog. This study investigated the value of the ECog in predicting the presence and severity of cognitive impairment and the extent to which informant characteristics impacted its predictive power.

Participants and Methods: Participants were 177 veterans referred for neuropsychological evaluation, whose informants completed the ECog. Binary logistic regression (LR) analyses were used to predict cognitive impairment across diagnostic groups: no cognitive impairment, MCI, and dementia, using ECog total score and informant characteristics (e.g., relationship, age, hours spent with veteran).

Results: Preliminary analyses found age and hours spent with the veteran as viable informant variables to include in the model. Models predicting the presence of cognitive impairment, using ECog and these informant characteristics, were significant (MCI vs. no cognitive impairment $[\chi 2=13.2, p=.02]$; dementia vs. no cognitive impairment $[\chi 2=12.2$, $p=.03]$ ), with ECog being the only significant predictor. Classification accuracy in these models was $77.4 \%$ and $62.5 \%$, respectively. ECog did not significantly distinguish MCI from dementia $(\chi 2=2.4, p=.8)$.

Conclusions: Results indicate that the ECog serves as a useful tool for identifying the presence of cognitive impairment, regardless of informant characteristics. It was not helpful, however, in distinguishing between MCI and dementia. Limitations included limited variability of informant characteristics within the current sample.

Correspondence: Lauren Golla, TX, United States.E-mail: golla@ hartford.edu

A. KRIVENKO, S.E. TOLFO, H. O'HARA, S. NAPOLITANO, M. BARBOZA, N. ABRAHAM, G. OZINGA, L. ESKEW \& A.M. POREH. Relationship between the five-point test and a selfreport measure of executive functioning in a random sample of English speaking adults.

Objective: Previous studies have attempted to validate various nonverbal fluency tests such as the Design Fluency Test. the Five-Point Test (5PT). Ruff Figural Fluency Test and the Delis Kaplan Figural Fluency Test. None have examined the construct validity of those measures, in particular, the correlation of these measures and self-reported executive functioning deficits.

Participants and Methods: Preliminary data from a large study examined this issue by correlating the 5PT with The Barkley Deficits in Executive Functioning Scale (BDEFS) in 80 adults gathered as volunteers 
from those serving jury duty in a large urban city. The mean age of participants was $48.5 \pm 15.0$ with an average of $16.1 \pm 3.4$ years of education. The majority was female $(65.4 \%)$, Caucasian $(77.9 \%)$ and had a primary language of English $(97.5 \%)$.

Results: The results showed that age is negatively correlated with number of unique designs, subtractions, and additions on the 5PT, and with the self-management and self-motivation scales on the BDEFS. Education is negatively correlated with BDEFS total score, self-management, self-restraint and self-motivation scales. In analyzing the scales of the BDEFS, the study found that the self-management scale positively correlated with unique designs and subtractions on the 5PT, the selforganization scale negatively correlated with rule breaks on the 5PT, the self-restraint scale negatively correlated with repetitions on the 5PT, the self-motivation scale positively correlated with subtractions on the $5 \mathrm{PT}$ and the self-regulation scale negatively correlated with additions on the 5PT.

Conclusions: The small insignificant correlation between the two measures as well as the nonsensical correlations of the scales of the BDEFS with the 5PT suggests that the link between self-report of such deficits and actual deficits is tenuous at best. These findings are consistent with the literature which indicates that self-report measures of cognitive functioning rarely correlate with actual performance on neuropsychological tests.

Correspondence: Anna Krivenko, Psychology, Cleveland State University, 4467 Habersham Ln S, Richmond Hts, OH 44143, United States.E-mail: anna.krivenko@yahoo.com

J. LARA-RUIZ, K. KAUZOR, G.M. CASTILLO, D. BANUELOS, M. NAKHLA \& J. RAZANI. The impact of PTSD symptoms and cognitive performance on student veterans' academic achievement.

Objective: The current study was conducted to examine the relationship between Post-Traumatic Stress Disorder (PTSD) symptoms and academic achievement among college student veterans.

Participants and Methods: Forty-seven student veterans were administered tests of PTSD symptoms (PTSD Checklists for DSM-5; PCL-5), traumatic brain injury (Ohio State University Traumatic Brain Injury Identification Method; OSU TBI-ID), depression (Beck Depression Inventory-II; BDI-II), anxiety (Beck Anxiety Inventory; BAI), substance abuse and cognitive function. Specifically, domains of attention (Symbol Digit Modality Test; SDMT, Stroop, Digit Span, and Trail Making Test (TMT) part $\dot{A}$ ), executive function (Controlled Oral Word Association Test; COWAT, Wisconsin Card Sorting Test, 64-item version; WCST64, and TMT part B), and memory (Rey-Osterrieth a Complex Figure Test; Rey-O) were assessed.

Results: Bivariate correlation revealed that PTSD was not significantly correlated with academic achievement; however memory was inversely correlated with PTSD $(p<.05)$. Hierarchical regressions found that PTSD symptoms significantly $(p<.05)$ predicted poorer attention domain scores after controlling for depression/anxiety (which was the only other factor associated with PTSD symptoms). Finally, simultaneous regression revealed that in the presence of executive functioning and memory domains, the attention domain was the only significant predictor of academic achievement.

Conclusions: These results highlight the complexity of PTSD in student veterans. Although PTSD symptoms may not be directly impacting academic achievement performance, findings suggest that the subsequent cognitive impairments of PTSD contribute to poorer academic achievement.

Correspondence: Jose Lara-Ruiz, B.A., PSYCHOLOGY, CALIFORNIA STATE UNIVERSITY, NORTHRIDGE, 11722 HARTSOOK ST, Valley Village, CA 91607, United States. E-mail: JOSE.LARARUIZ.392@ MY.CSUN.EDU
L. LAU, M.R. BASSO, E. ESTEVIS, D. WHITESIDE, A.K. MILLER \& D. COMBS. Association between Performance Validity and Symptom Validity Tests with the MMPI-2-RF among Simulators Feigning Neuropsychological Dysfunction.

Objective: Performance validity tests (PVTs) and symptom validity tests (SVTs) are used to detect exaggerated cognitive dysfunction and embellished symptom complaints, respectively. Yet little research has evaluated the relationship between PVTs and SVTs, especially SVTs derived from the MMPI-2-RF. Understanding this relationship could potentially discriminate performance and symptom validity as distinct validity indicators. The present experiment evaluated the association between commonly used PVTs/SVT indices and various MMPI-2-RF scales among participants coached to simulate symptoms of traumatic brain injury.

Participants and Methods: Neurologically-normal university students participated. Coached groups included 20 participants who received detailed information concerning symptoms of head injury, 20 received superficial information, and 20 received detailed symptom information and techniques to avoid detection by PVTs. All coached participants attempted to simulate TBI symptoms. 20 control subjects participated, and were asked to provide optimal effort. The TOMM, WMT, and MMPI-2-RF were administered.

Results: Most of the TOMM and WMT indices correlated significantly with the FBS-r, RBS, and HHI indices ( $r=-0.65$ to -0.76$)$. The PVT and SVT indices correlated with several of the Restructured Clinical $(\mathrm{RC})$ and all of the MMPI-2-RF cognitive complaints scales $(r=-0.49$ to 0.91$)$. All correlations achieved $\mathrm{p}<.001$.

Conclusions: Standalone PVTs and the three SVT indices have significant relationships with several of the RC scales and all of the CG scales. Likely due to shared method variance, this association is stronger for the SVT indices than PVTs. Such findings imply that performance and symptom validity characterize overlapping but distinct indicators of validity during neuropsychological testing.

Correspondence: Lily Lau, PhD, Psychology, University of Tulsa, 226 S Richmond Ave., Tulsa, OK 74112, United States. E-mail: lily-lau@ utulsa.edu

L. LAU, M.R. BASSO, E. ESTEVIS, D. WHITESIDE, A.K. MILLER \&. D. COMBS. Classification Accuracy of Performance Validity and Symptom Validity Tests among Examinees Feigning Neuropsychological Dysfunction.

Objective: Performance validity tests (PVTs) and symptom validity tests (SVTs) are used to detect exaggerated complaints. Although examinees may be coached to defeat such tests, little research has evaluated the effects of coaching on classification accuracy of PVTs and SVTs. In this experiment, neurologically normal participants were coached to simulate symptoms of mild head injury. Three groups received different levels of simulation information. Standalone and embedded PVTs, and SVTs from the MMPI-2-RF were administered.

Participants and Methods: Neurologically-normal university students comprised three groups of simulators (each $n=20$ ) which received either superficial information about head injury symptoms, detailed information about head injury symptoms, or detailed symptom information and techniques to elude detection by PVTs. An honest responding group $(n=20)$ provided optimal performance. The TOMM, WMT, CVLT-2, JLO, and MMPI-2-RF were administered.

Results: The WMT achieved the best classification accuracy of the PVTs and SVTs, with 90-100\% of simulators being detected. Embedded PVTs and the MMPI-2-RF SVTs achieved less effective detection. Notably, the test-coached group eluded detection significantly better than those coached to simulate symptoms alone (10\% eluded detection).

Conclusions: The standalone PVTs accurately classified most examinees simulating head injury. Embedded PVTs and SVTs were less sensitive, especially among examinees who were test-coached. Nevertheless, some of the coached participants successfully eluded detection by the validity tests. Such finding raises concerns regarding the effectiveness of 
PVTs and SVTs in patients who have been coached to elude detection, and enhances the need to maintain test security.

Correspondence: Lily Lau, PhD, Psychology, University of Tulsa, 226 S Richmond Ave., Tulsa, OK 74112, United States. E-mail: lily-lau@ utulsa.edu

L. MANDERINO \& J. GUNSTAD. Immediate Post-Concussion Assessment and Cognitive Testing Validity Indices Are Correlated with External Performance Validity Measures.

Objective: Immediate Post-Concussion Assessment and Cognitive Testing (ImPACT) is widely used in managing sport-related concussions. Athletes may attempt to suppress performance at baseline assessment to mask injury-related deficits. ImPACT uses low scores on five subtests to identify poor effort. The present study uses a simulation design to investigate these validity indices and compare them to external performance validity measures.

Participants and Methods: The ImPACT, Word Memory Test (WMT), and Minnesota Multiphasic Personality Inventory-2 Restructured Form (MMPI-2-RF) were administered to 98 undergraduate students. Participants were instructed to take all measures giving good effort $(\mathrm{N}=44)$ or to simulate a head injury $(\mathrm{N}=54)$ for external incentive (entrance to a raffle). A simulation script that included common symptoms associated with concussion was used.

Results: The simulating group demonstrated poorer ImPACT composite scores than the control group, suggesting they were compliant with instructions. MANOVA showed significant differences on all five ImPACT validity indices between control and simulating groups (Pillai's Trace $=0.27, \mathrm{~F}(5,92)=6.65, \mathrm{p}<0.001)$. ImPACT validity indices demonstrated high specificity $(0.87)$ but lower sensitivity $(0.60)$. All five ImPACT validity indices showed moderate to large correlations with immediate and delayed recall scores on the WMT (range: $=-0.48$, $p<0.001$ to $r=0.78, p<0.001)$. ImPACT validity indices also showed small to moderate correlations with all five MMPI-2-RF over-reporting scales (range: $r=0.21, p<0.05$ to $r=-0.47, p<0.001$ ).

Conclusions: ImPACT validity indices show convergent validity with other performance validity measures and high specificity using a simulation design. Lower sensitivity rates raise concern, as unidentified poor-effort baselines may mask post-injury deficits. This may result in premature return-to-play, putting athletes at risk for further injury or lasting impairment.

Correspondence: Lisa Manderino, Kent State University, 2004 Hidden Lake Dr, Apt F, Stow, OH 44224, United States.E-mail: Imanderi@ kent.edu

D.E. MARRA, E. VOGT, K.A. RITCHIE, W.T. MCCUDDY \& J. HOELZLE. An Updated Exploration of the Frequency of Invalid Performance in an Undergraduate Sample.

Objective: Undergraduate research participants have been the cornerstone of psychological research for decades. It is important to assess whether participants are optimally engaged in research tasks by utilizing performance validity tests (PVT) in order to draw accurate conclusions from testing data. Across published studies, approximately 2 to $56 \%$ of non-clinical student samples have failed at least one PVT. The goal of this study was to determine the percentage of a large undergraduate sample who failed one stand-alone PVT.

Participants and Methods: Healthy undergraduate students $(N=501$; $M_{\text {age }}=19.17(1.36) ; M_{\text {education }}=12.77(1.05) ; 71.7 \%$ female $)$ from a private Midwest university underwent a neuropsychological evaluation for course credit. Task engagement was assessed using the Victoria Symptom Validity Test (VSVT), a stand-alone PVT that masquerades as a working memory task. Invalid performance was defined as $<21$ easy or difficult items.

Results: Nineteen $(3.9 \%)$ typically developing students without a self-reported clinical history exhibited failed the VSVT. Two independent sample $t$-tests revealed that students with self-reported diagnoses of $\operatorname{ADHD}(n=22,4.4 \%)$ or learning disorders (LD; $n=15,3.0 \%)$ did not perform more poorly than typically developing students on the $\operatorname{VSVT}(\mathrm{ADHD} t(480)=0.643, p=.520) ; \operatorname{LD}(t(480)=.345, p=.730)$. Conclusions: Approximately $4 \%$ of a typically developing undergraduate sample participating in research failed a stand-alone PVT, the VSVT. This is notable because there was no apparent incentive for obtaining low scores on tests administered. Further, individuals with clinical conditions were not more likely to fail the VSVT. Previous literature which utilized embedded PVTs reported a meaningfully higher rate of failure and thus, may overestimate performance invalidity in research settings. Nonetheless, evaluating performance and symptom validity in research settings is imperative for ensuring accuracy of data. Correspondence: David E. Marra, MS, Psychology, Marquette University, 1616 N. 50 th St, Milwaukee, WI 53208, United States. E-mail: david. marra@marquette.edu

D. MCKAY, T. NGUYEN, A. CARLEW, A.ZARTMAN, J. WESTHAFER \& B. ARDOLF. The Relationship between Performance Validity Tests and Diagnoses among Veterans with History of mTBI.

Objective: Within the VA system, Veterans with a history of mTBI often perform below expectation on Performance Validity Tests (PVTs; Bruback et al., 2015). Some evidence suggests individuals with a primary psychiatric diagnosis are more likely to fail PVTs within mTBI populations (McCormick, 2013); however, this phenomenon has not been directly compared to those diagnosed with a neurocognitive disorder. The purpose of this study was to examine final primary diagnoses in relation to PVT pass/fail rates in Veterans with a history of mild, uncomplicated TBI with and without psychiatric diagnoses.

Participants and Methods: 91 Veterans from multiple war eras (age range: 22-74) were seen by a VA Neuropsychology Consult Team from 2013-2016 for referral of cognitive complaints due to self-reported mTBI. Participants completed a clinical interview, the Test of Memory Malingering (TOMM) and/or Word Memory Test (WMT), and a flexible neuropsychological battery. Diagnoses were determined by a staff neuropsychologist.

Results: $74.7 \%$ of Veterans received a primary psychiatric diagnosis whereas $9.8 \%$ received a neurocognitive diagnosis (75\% of this group also met criteria for a comorbid psychiatric disorder). Overall, $57.3 \%$ of the sample failed the WMT while $35.6 \%$ failed the TOMM. Diagnostic group was unrelated to WMT pass/fail rate $\left(\chi^{2}(1, N=70)=0.85\right.$, $p=.36)$. Conversely, diagnostic group was significantly related to TOMM pass/fail rate $\left(\chi^{2}(1, N=40)=5.39, p=.02\right)$ where the psychiatric diagnosis group was more likely than the neurocognitive diagnosis group to fail the TOMM. Of the 32 Veterans given both PVTs, $93.7 \%$ failed at least one, $50 \%$ failed the WMT yet passed the TOMM $(75 \%$ from the psychiatric diagnosis group) and $43.8 \%$ failed both (all from the psychiatric diagnosis group).

Conclusions: Results of this study highlight the complex interaction between diagnoses and effort in Veterans with self-reported mTBI. Within our mTBI sample, failing PVTs were common, however, those with psychiatric diagnoses performed worse.

Correspondence: Derek McKay, University of Akron, 6260 Melody Lane, Apt 3030, Dallas, TX 75231, United States.E-mail:dmckay729@gmail. com

C. MEAD, T. MOSKOWITZ, A. ALIOTO, R. SALONER, K.P. RANKIN \& K.L. POSSIN. Validation of the TabCAT Favorites Memory Test: Neuroanatomical and Memory Correlates in Neurologically Healthy Older Adults.

Objective: The TabCAT Favorites Memory Test (FMT) is a tablet-based measure of verbal-visual associative memory requiring 3 minutes of testing time. We correlated performance with: 1) gray matter regions important for memory and, 2) widely-used verbal and visual memory tests.

Participants and Methods: Functionally intact older adults ( $\mathrm{N}=98$; mean age $=76$ ) recruited from the UCSF Memory and Aging Center underwent neuropsychological assessment, and 48 also underwent 
MRI. On the FMT, subjects were instructed to learn each person's favorite things. Across 2 learning trials, they were shown each of four different face photographs twice, once with an animal name and once with a food name (totaling 8 face-word pairs). A delayed recall trial was administered after ten minutes. Accuracy was summed across the second learning and delay trial. Brain regions of interest were analyzed using SPM12.

Results: FMT significantly correlated with medial temporal $(p=.026)$ and parietal $(p=.032)$ lobe cortex volumes, but not significantly with lateral prefrontal cortex. Within the medial temporal lobe, performance correlated significantly with left hippocampus $(p=.013)$, and there were trends with right hippocampus $(\mathrm{p}=.056)$, and left $(\mathrm{p}=.055)$ and right $(p=.085)$ entorhinal cortex. Correlations with left and right parahippocampal gyri were not significant. FMT also correlated with verbal (CVLT-II long delay free recall, $\mathrm{r}=.574, \mathrm{p}<.001$ ) and visual (modified Benson recall, $r=.338 ; \mathrm{p}=.001$ ) memory tests.

Conclusions: Performance on the TabCAT FMT correlates with brain regions important for memory, and with widely-used verbal and visual memory tests. Considering the ease and efficiency of administration, scoring, and data storage, and 4 alternate forms, the FMT is a good choice for a quick and valid measurement of hippocampally-dependent memory.

Correspondence: Christie Mead, M.S., Memory and Aging Clinic, UCSF, 222 Escuela Ave, Apt. 138, Mountain View, CA 94040, United States. E-mail: christie.mead@ucsf.edu

V.C. MERRITT, M.L. BRADSON \& P. ARNETT. Evaluating the Test-Retest Reliability of Symptom Indices Associated with the Post-Concussion Symptom Scale.

Objective: The ImPACT test battery is a commonly used tool in sports concussion assessment. While test-retest reliabilities have been established for the ImPACT cognitive composites, few studies have evaluated the psychometric properties of the ImPACT's Post-Concussion Symptom Scale (PCSS). The purpose of this study was to establish the test-retest reliability of symptom indices associated with the PCSS.

Participants and Methods: Participants included 38 undergraduate students $(50.0 \%$ male) who underwent neuropsychological testing as part of their participation in a research subject pool. The majority of the participants were Caucasian $(94.7 \%)$ and had no history of concussion (73.7\%). All participants completed the ImPACT at two time points, approximately 6 weeks apart. The PCSS was the main outcome measure, and seven symptom indices were calculated (three summary indices and four symptom clusters).

Results: Pearson product moment correlations ( $r$ ) and intraclass correlation coefficients (ICCs) were computed as measures of testretest reliability for all symptom indices. Overall, test-retest reliabilities ranged from low to high $(\mathrm{r}=.13$ to $.80 ; \mathrm{ICC}=.14$ to .76$)$. The cognitive symptom cluster exhibited the highest test-retest reliability $(r=.80$, ICC =.76), followed by the positive symptom total (PST) index, an indicator of the total number of symptoms endorsed $(\mathrm{r}=.71, \mathrm{ICC}=.68)$. In contrast, the total symptom score showed lower test-retest reliability $(\mathrm{r}=.67, \mathrm{ICC}=.59)$. Reliable change indices (RCIs) were also computed. When applying an $80 \%$ confidence interval, $7.9-13.2 \%$ of symptom scores showed reliable change upon retest; when applying a $90 \%$ confidence interval, $5.3-10.5 \%$ of symptom scores showed reliable change upon retest.

Conclusions: These results suggest that evaluating additional symptom indices beyond the total symptom score from the PCSS is beneficial. Findings from this study can be applied to athlete samples to assess reliable change in symptoms following concussion.

Correspondence: Victoria C. Merritt, M.S., Psychology, The Pennsylvania State University, 372 Moore Building, University Park, PA 16802, United States. E-mail: vcabarnes@gmail.com
A. MILLOY, F.C. GOLDSTEIN \& D. LORING. Incremental Validity of MoCA Subscale Scores in Amnestic MCI and Alzheimer's Disease. Objective: The Montreal Cognitive Assessment (MoCA) is commonly used for cognitive screening in dementia clinics. Traditional interpretation is based upon the sum of all items, with a maximum of 30 points. A newer scoring approach has recently been developed consisting of subscale scores for the domains of memory, executive function, visuospatial, language, attention, and orientation. The current study investigated the incremental validity of the MoCA subscale scores vs. the MoCA total score in differentiating persons with normal cognition, amnestic mild cognitive impairment (aMCI), and Alzheimer's disease (AD).

Participants and Methods: MoCA scores were analyzed for 1196 participants in the Alzheimer's Disease Neuroimaging Initiative of whom 401 were diagnosed as cognitively normal, 564 as aMCI, and 231 as AD. MoCA scores were converted into subscale scores based on the item combinations used by Julayanont et al. (2014).

Results: Significant $(\mathrm{p}<.05)$ differences were present between all pairwise group comparisons for each new subscale score as well as the MoCA total score (Controls > aMCI > AD). However, none of the subscale scores had an effect size larger than the total score. In addition, the newly derived Memory subscale (0-15 pts) was compared with the traditional Delayed Recall Score (0-5), and the effect sizes only significantly differed between MCI and AD patients (Memory subscale Cohen's $\mathrm{d}=1.3$; Delayed Recall Score Cohen's d=1.1).

Conclusions: The clinical usefulness of MoCA subscale scores is modest compared to traditional scoring, at least in the context of differentiation of aMCI and AD from normal cognitive aging. Longitudinal studies are necessary to evaluate whether any individual subtest score is more effective in detecting interval decline, and it remains unknown to what degree subtest scores may be sensitive to characterizing the cognitive deficits in other clinical populations.

Correspondence: Aaron Milloy, B.A., 12 Executive Park, NE, GA 30329, United States.E-mail: aaron.m.milloy@emory.edu

C.M. NGUYEN, J. STELMOKAS \& L.A. BIELIAUSKAS. Vascular medical factors associated with health and safety behaviors among older male Veterans residents admitted to an inpatient rehabilitation unit.

Objective: The Independent Living Scales, Health and Safety Subscale (ILS-HS) is a self-reported measure of health and safety awareness. A number of demographic and cognitive variables, particularly memory and executive dysfunction, can contribute to performance on the ILS-HS, though less is known about the increased burden of other medical and vascular disease risk factors.

Participants and Methods: Older male residents completed cognitive testing upon admission $(\mathrm{N}=217$; Mean age $=66.2+/-11.0$ years $)$. Demograpic factors (age and education), neuropsychological test performance (Addenbrooke's Cognitive Examination-III subscales), vascular variables (hypertension, hyperlipidemia, diabetes, myocardial infarction, cerebralvascular accident, cardiovascular disease), and depression (DSM-IV depression criteria), were included in hierarchical regression analyses to evaluate the specific impact of these factors on ILS-HS.

Results: : Demographic (Step $1: \mathrm{R}^{2} \Delta=.055 ; p=.001$ ), neuropsychological (Step $2 \mathrm{R}^{2} \Delta=.088 ; p=<.001$ ), medical (Step $3 \mathrm{R}^{2} \Delta=.017$; $p=.155)$, and $\operatorname{mood}\left(\right.$ Step $\left.4 \mathrm{R}^{2} \Delta=.000 ; p=.914\right)$ factors, collectively, accounted for significant adjusted $15.6 \%$ variance in ILS-HS $(p<0.001)$. After accounting for variance predicted by all variables, history of cardiovascular disease $(p=.004)$ and poor verbal fluency $(p=0.03)$ significantly predicted performance on the ILS-HS.

Conclusions: Findings replicate previous research demonstrating the relationship between reported functional ability and executive functioning, specifically verbal fluency. Of the vascular factors, our results also highlight that cardiovascular disease in particular is the strongest predictor of ILS-HS performance in a post-acute rehabilitation sample. 
Correspondence: Christopher M. Nguyen, Ph.D., Psychiatry and Behavioral Sciences, University of Oklahoma Health Sciences Center, 601 Robert S Kerr Ave, Apt 108, Oklahoma City, OK 73102, United States.E-mail: chris.minh@gmail.com

T.M. OSWALD, R. FAZIO \& G. ANDREWS. Embedded Performance Validity Index within the Memory Module of the Neuropsychological Assessment Battery (NAB): A Pilot Study.

Objective: Measures to gauge optimal effort are necessary within neuropsychological evaluations, particularly within the veteran (Armistead-Jehle \& Buicon, 2012) and forensic settings (Ardolf, Denney, \& Houston, 2007). The precedent regarding the utility of existing recognition memory measures is well established. These measures include the California Verbal Learning Test-II (CVLT-II; Delis, Kramer, \& Ober, 2000) and the Rey Auditory Verbal Learning Test (RAVLT; Schmidt, 1996). However, a literature gap exists concerning the more recent Neuropsychological Assessment Battery (NAB; Stern \&. White, 2003) - Memory Module. This study establishes the clinical utility of NAB Memory Module subtests as embedded performance validity tests (PVTs).

Participants and Methods: The pilot sample consisted of 61 veterans seen within a neuropsychological outpatient clinic for a clinical evaluation. The clinical battery included established PVTs (e.g., DCT, RDS, TOMM), the NAB Memory Module, and other clinically appropriate measures. Utilizing the Slick et al. criteria, non-parametric correlations and ROC analyses were conducted.

Results: Among the List Learning (LL) scores, the Long Delayed Forced-Choice Recognition (LLA_dfc) hits provided the best AUC (.744). The best Shape Learning (SHL) score was the Discriminability Index (SHL_dis; .789). Overall, a combination score was generated to provide the best AUC (.799) and a classification cut score was established.

Conclusions: Initial data analyses revealed numerous significant correlations between existing PVTs and recognition memory tasks within the NAB Memory Module. Furthermore, a performance classification cut score utilizing a combination of memory scores was identified for optimal specificity and sensitivity.

Correspondence: Thomas M. Oswald, Psy.D., Mental Health, Central Arkansas Veterans Healthcare System (CAVHS), 2200 Fort Roots Drive, North Little Rock, AR 72114, United States.E-mail: thomas.oswald@ va.gov

B. OTRUBA, L. YOZAWITZ, M. CORNWELL, J.R. WIENER \& P. UY. The Predictive Value of Verbal Fluency and Story Memory upon Story Recall Using the Repeatable Battery for Assessment of Neuropsychological Status in an Inpatient Setting.

Objective: The Repeatable Battery for the Assessment of Neuropsychological Status (RBANS) has been used within inpatient settings as a brief global measure to identify functional impairment. Story recall is often utilized as a proxy for memory; however research suggest that various domains may impact performance on this task. Story memory is less influenced by other cognitive factors compared to list learning therefore it was utilized in the current study. Verbal fluency has also shown to be significantly correlated with memory performance. In the current pilot study, we sought to determine whether RBANS verbal fluency and story memory impacted story recall performance among patients in acute recovery.

Participants and Methods: Archival data was reviewed from 22 patients (17 male, 5 female) who were admitted to an acute rehabilitation unit in a metropolitan medical center. Mean age was 66 $(\mathrm{SD}=13.01)$, with $59 \%$ being Caucasian, 18\% African American. 9.1\% multi-racial, 9.1\% Hispanic and 4.5\% Asian. Mean education was 12 years $(\mathrm{SD}=3.39)$. The majority of medical diagnoses were accounted for by cerebral vascular etiology. Patients with aphasic diagnoses were excluded from the present study. Stepwise hierarchal regression was utilized to determine the differential effect of verbal fluency and story memory upon story recall.

Results: Results of the analysis revealed story memory significantly predicted story recall $(p=<.0001)$. The first step was significant $F(1,20)=18.28(p=<.0001)$. The second step which included verbal fluency as a predictor also was significant $\mathrm{F}(2,19)=15.20$ (story memory $\mathrm{p}=<.0001$; verbal fluency $\mathrm{p}=.017$ ). Story memory explained $73.4 \%$ variance whereas verbal fluency explained $37.4 \%$ variance.

Conclusions: RBANS verbal fluency and story memory were associated with story recall performance within inpatient clinical care. This suggests that story memory is a multifaceted task dependent upon cognitive factors such as language and learning abilities.

Correspondence: Brittney Otruba, MA, Clinical Psychology, Fielding Graduate University, 5 Birchwood Court, 4A, Mineola, NY 11501, United States.E-mail: brittneyotruba@gmail.com

G. OZINGA, S.E. TOLFO, A. KRIVENKO, S. NAPOLITANO, N. ABRAHAM, M. BARBOZA, H. O'HARA \& A.M. POREH. Statistical Adaptation of the Boston Naming Test Short Form for Hebrew Speaking Older Adults.

Objective: The Boston Naming Test (BNT) is the most frequently administered confrontational naming measure. However, when it is administered to non-English speakers, particularly immigrants, the results might not be valid.

Participants and Methods: The present study examined the performance of 194 older adult Hebrew speakers (41 native and 153 immigrants) on the BNT Short Form. The data was initially subjected to Item Response Theory data analysis, then the test items were reorganized by level of difficulty, dependent upon subpopulation.

Results: Follow-up analysis showed that degree of daily language usage and education, but not age of immigration, correlates with performance on the test. Normative data using a regression-based procedure that takes into account gender and age influences is presented.

Conclusions: The results of the study are discussed in light of previous research regarding late life vocabulary funds, bilingualism, and controlled access in object naming.

Correspondence: Grace Ozinga, Cleveland State University, 11723 Colburn Road, Chardon,OH 44024,United States.E-mail: g.ozinga@ csuohio.edu

A.F. PARKER, G. ARMSTRONG, M.E. CAMPBELL, J. UPSHAW, D. LEITNER, M. LIBBEN \& H. MILLER. The New Normal: Creating Updated Norms for Neuropsychological Measures.

Objective: Valid neuropsychological normative data are essential in the clinical diagnosis of cognitive impairment. Currently, many of the most commonly used neuropsychological assessment measures rely on norms that are out of date and/or demographically inappropriate. This study aims to collect up-to-date norms to replace the existing outdated normative data used for assessing the severity of cognitive dysfunction in patients following brain insult.

Participants and Methods: Sixty-four healthy volunteers aged 60-79 (mean age $=69.1, S D=5.1)$ completed a neuropsychological evaluation. This evaluation consisted of Buschke's Selective Reminding Test (SRT), Auditory Consonant Trigrams (CCC). The Controlled Oral Word Association Task (COWAT) and Color Trails 1 and 2 (CCT-1 and CCT-2). The means and standard deviations of participants' performance were calculated for each measure.

Results: On the recall portion of the SRT, participants scored a mean of $100.65(S D=20.12)$. Further, participants scored an overall mean of $47.43(S D=6.43)$ on the CCC. A mean of $39.32(S D=11.05)$ was found on the COWAT. Finally, a mean of 43.35 seconds $(S D=19.10)$ was found for CCT-1 and a mean of 86.65 seconds $(S D=26.97)$ was found on CGT-2.

Conclusions: Discrepancies were found between the current norms and the normative data collected in this study. Further data collection will continue and means will be recalculated. Upon increased sample 
size, means will be stratified by age and education. Providing clinicians with updated norms is fundamental for accurate diagnosis of cognitive impairments through neuropsychological test performance. Correspondence: Ashleigh F. Parker, University of British Columbia Okanagan, 2543 Paramount Drive, West Kelowna, BC V4T 3H5, Canada.E-mail: ashleighparker94@gmail.com

\section{A. PARKS \& O. PEDRAZA. Clinical Utility of the Visual Object and Spatial Perception Incomplete Letters Test.}

Objective: Visuoperceptual deficits are common in neurodegenerative diseases, particularly synuclienopathies such as dementia with Lewy bodies (DLB). The Incomplete Letters Test (ILT) from the Visual Object and Spatial Perception (VOSP) battery is a brief, untimed, non-motor visuoperceptual task that requires identification of 20 letters in varying degrees of visual degradation. The present study investigated the clinical utility of the ILT in DLB and Alzheimer's dementia (AD).

Participants and Methods: A search of the electronic medical record at Mayo Clinic Florida yielded 97 patients (57\% male) referred for neuropsychological evaluation of possible dementia and who were administered the ILT as part of their exam. Based on the available clinical data, subjects were classified as possible DLB $(n=30)$, possible AD $(n=32)$, or normal cognition (NC: $n=35$ ). Scores from the Dementia Rating Scale-2 (DRS-2), Judgment of Line Orientation (JLO), and WAIS-IV Block Design (BD) were also extracted for analyses.

Results: Age, gender, and education did not differ across the groups. The DLB and AD groups did not differ from each other on the DRS-2 $(\mathrm{M}=124.8, \mathrm{SD}=7.9$ vs. $\mathrm{M}=125.2, \mathrm{SD}=6.3)$, but both groups performed significantly worse than NC patients $(\mathrm{M}=136.4, \mathrm{SD}=5.4)$. The DLB group obtained significantly lower ILT scores $(\mathrm{M}=16.4, \mathrm{SD}=2.7$ ) compared with $\mathrm{AD}(\mathrm{M}=19.2, \mathrm{SD}=1.3)$ and $\mathrm{NC}(\mathrm{M}=19.5, \mathrm{SD}=0.8)$. In a hierarchical logistic regression controlling for the demographic variables, a model with ILT demonstrated $86 \%$ classification accuracy in DLB versus NC. A joint model with JLO, BD, and ILT showed BD and ILT as significant predictors, but without an increase in model accuracy. In contrast and as expected, a model including ILT did not improve accuracy in discriminating $\mathrm{AD}$ from $\mathrm{NC}$ when compared to a base model with demographic variables.

Conclusions: The ILT demonstrates clinical utility in classifying patients with DLB and should be considered in the clinical neuropsychologist's toolbox given its brief administration time and lack of motor demands.

Correspondence: Adam Parks, PhD, 4500 San Pablo Road, Jacksonville, FL 32224, United States.E-mail: parks.adam@mayo.edu

\section{V.M. PATT, G.G. BROWN, M.L. THOMAS \& R.K. HEATON. Digit Vigilance Test: Speed versus Accuracy Tradeoff Revealed.}

Objective: The Digit Vigilance Test (DVT), which requires finding and crossing out a digit in a large, dense array, is widely used in clinical and research settings to measure processing speed and sustained attention. Instead of an expected tradeoff between speed and accuracy, studies have shown DVT completion time and number of errors to be essentially unrelated. The present work re-examines this relationship by modeling DVT performance within the broader context of a comprehensive neuropsychological evaluation.

Participants and Methods: Participants were neuromedically-healthy individuals recruited as part of several studies in the context of the expanded Halstead-Reitan Battery (eHRB) normative effort. Exploratory and Confirmatory factor analyses were carried out to uncover the neurocognitive domains underlying the eHRB and model residual correlations between same-test variables. The relation between $D V T$-time and DVT-error was explored by comparing modeling results with simple bivariate correlation.

Results: The bivariate correlation between DVT-time and DVT-error was negligible in effect size $(r=-.09, p=0.033)$, consistent with previous accounts suggesting limited relatedness between these variables. In the factor analyses, DVT-time and DVT-error loaded on two positively correlated but separate factors: Perceptual Speed and Attention/ Working Memory, respectively. After parsing out the variance accounted for by the positively correlated factors, a significant negative residual correlation was revealed $(r=-.27, p<.001)$.

Conclusions: These results suggest a relative tradeoff between speed and accuracy during the DVT that is usually masked by more general cognitive ability. That is, individuals good at visuomotor speed tasks also tend to be good at error-monitoring. However, among individuals good at both, relatively faster performance is associated with relatively more errors. These results underline the inadequacy of solely considering bivariate correlations to capture complex relationships between some same test-measures.

Correspondence: Virginie M. Patt, M.S., SDSU/UCSD Joint Doctoral Program in Clinical Psychology, 628 N. Illinois St., Arlington, VA 22205, United States.E-mail: vpatt@ucsd.edu

V.C. PERSINGER, D. WHITESIDE, L. BOBOVA, M. VANNUCCI, S. SAIGAL \& M.R. BASSO. California Verbal Learning Test-II Total Hits and Total False Positives as Embedded Performance Validity Measures in Mood Disorders.

Objective: Although performance validity tests (PVTs) are widely used in neuropsychological assessment (Meyers \& Volbrecht, 2003), there are few studies on PVTs in mood disorder patients. Based on previous research (Kizilbash et al., 2002; O'Jile et al., 2005; Rund et al., 2006), PVTs should be effective in mood disorders (e.g. depression and anxiety) patients; however, additional evidence on specific PVTs is necessary. In terms of embedded PVTs, which do not add to the length of neuropsychological test batteries, considerable research has been conducted on the Forced Choice (FC) Recognition subtest of the California Verbal Learning Test, Second Edition (CVLT-II, Delis, Kramer, Kaplan, \& Ober, 2000) but relatively little on CVLT-II Total Hits (TH) and Total False Positives (TFP). This study examined the classification accuracy of TH and TFP as PVTs in a non-compensation seeking mood disorders sample.

Participants and Methods: The sample included 59 individuals (mean age $=55.09, S D=16.21 ;$ mean education $=13.14, S D=3.00 ; 45.8 \%$ males; $89.3 \%$ Caucasian; $88.1 \%$ passed PVTs, $11.9 \%$ failed two or more) consecutively referred for neuropsychological evaluation who met study criteria. Many were diagnosed with a depressive disorder (Unspecified Depressive Disorder, $n=20$; Major Depressive Disorder, $n=14)$, with the remaining $(n=25)$ diagnosed with an anxiety disorder, Bipolar Disorder, or Adjustment Disorder.

Results: It was hypothesized that TH and TFP would have acceptable classification accuracy. The hypothesis was partially supported in that TH had excellent classification accuracy (area under the curve [AUC] $=.84)$, but TFP $(\mathrm{AUC}=.42)$ had poor classification accuracy. When TH specificity $=1.00$, sensitivity $=.46$.

Conclusions: These results provide preliminary support for the classification accuracy of TH in mood disorder patients, but not TFP. Future research should be conducted with larger samples and across different neurological and psychological disorders.

Correspondence: Virginia C. Persinger, PsyD, Clinical Psychology, Adler University, 831 Rice Road, 601, Ridgeland, MS 39157, United States. E-mail: gini.persinger@gmail.com

K.L. POSSIN, T. MOSKOWITZ, N. RABINOWITZ, S. ERLHOFF, A. ALIOTO, E. JOHNSON, B. MILLER, J. KRAMER \& K.P. RANKIN. The TabCAT Brain Health Assessment for Detecting Mild Cognitive Impairment and Evaluating Domain-Specific Deficits.

Objective: To evaluate the sensitivity of the TabCAT Brain Health Assessment to mild cognitive impairment (MCI), and the validity of the memory, visuospatial, and executive function subtests.

Participants and Methods: The TabCAT Brain Health Assessment is a 10-minute tablet-based battery comprised of associative verbal-visual memory ("Favorites"), executive speed ("Match"), visuospatial judgment ("Lines"), and language (animal fluency) tests, and an informant 
questionnaire (the "Brain Health Survey"). We administered it along with a comprehensive neuropsychological evaluation that included the Montreal Cognitive Assessment (MoCA) to older adults diagnosed as neurologically healthy $(\mathrm{N}=89)$, or with MCI $(\mathrm{N}=48)$. The MCI sample was diverse with both common (eg, Alzheimer's disease) and less common (eg, frontotemporal lobar degeneration) etiologies. 120 participants also completed 3T MRI. We included the MoCA and the Brain Health Assessment as predictors of MCI v normal diagnosis in separate discriminant function analyses. Favorites, Match, and Lines were correlated with neuropsychological measures and with regional brain volumes using voxel-based morphometry.

Results: The TabCAT Brain Health Assessment correctly classified 75\% of MCI patients and $96 \%$ of controls. In contrast, the MoCA classified $44 \%$ of the patients and $89 \%$ of the controls. Favorites correlated with memory tests and bilateral hippocampal and entorhinal cortex volumes. Match correlated with executive speed and brain regions important for executive and motor control. Lines correlated with spatial measures and right parietal volumes.

Conclusions: The TabCAT Brain Health Assessment efficiently identifies MCI with greater sensitivity and specificity than the MoCA. The new subtests are valid measures of memory, executive speed, and spatial skills. Comprehensive cognitive assessment is possible in brief screens and may be particularly important for identifying less common neurodegenerative phenotypes.

Correspondence: Katherine L. Possin, Ph.D., Neurology, UCSF, 350675 Nelson Rising Lane, Ste 190, San Francisco, CA 94158, United States. E-mail:kpossin@memory.ucsf.edu

\section{QUINN, L. RABIN, H. JACKSON, A. KAPOOR \& G. SPREHN. Association of Judgment Ability and Functional Status in a Sample of Mixed Rehabilitation Inpatients.}

Objective: Everyday judgment, a higher-order cognitive ability essential for independent living, may become compromised for patients exposed to general anesthesia (during surgery) as well as those with acquired brain injuries, i.e., stroke. During the rehabilitation period, it is important to establish the nature and extent of any such impairment for effective treatment and discharge planning. Unfortunately, there is a dearth of measures used to assess judgment during inpatient rehabilitation. Therefore, the current study investigated the utility of the Test of Practical Judgment (TOP-J) in a mixed sample of rehabilitation inpatients.

Participants and Methods: Participants were 28 adults (mean age $=70$, $\mathrm{SD}=11$ ) with brain-related and musculoskeletal diagnoses (requiring major surgery), recruited from an inpatient rehabilitation facility and evaluated just prior to discharge. Functional status was assessed with the Functional Independence Measure (FIM). Bivariate correlations established the association between TOP-J total scores and the three FIM subtests of problem solving, comprehension, and memory. We also investigated differences in TOP-J scores based on level of global cognition (impaired versus intact MMSE scores using a cutoff of $\leq 26 / 30$ ).

Results: For those with brain-related injuries, TOP-J performance fell close to the mean typically observed in individuals with mild Alzheimer's disease. Within the whole sample, results revealed strong positive correlations between TOP-J total scores and problem solving $(r=.507, p=.006)$, comprehension $(r=.584, p=.001)$, and memory $(\mathrm{r}=.563, \mathrm{p}=.002)$ on the FIM. Participants with normal global cognition obtained significantly higher TOP-J scores than those with abnormal global cognition, $\mathrm{t}(26)=3.34, \mathrm{p}=.003, \mathrm{r}=.548$.

Conclusions: Convergent validity of the TOP-J was supported by the observed correlation with real-world outcomes. Findings may aid researchers and clinicians in objectively evaluating judgment skills in rehabilitation settings.

Correspondence: Crystal Quinn, PhD, Psychology, The Graduate Center, CUNY, 271 E Pine St, Long beach, NY 11561, United States. E-mail: cquinn@gradcenter.cuny.edu
K.A. RITCHIE. An empirical examination of impaired baseline performances on the Standardized Assessment of Concussion.

Objective: Healthy individuals routinely obtain impaired scores on neuropsychological tests. Using a Monte Carlo simulation, this study investigates the frequency at which healthy athletes are likely to achieve impaired Index scores on the Standardized Assessment of Concussion (SAC; McCrea et al., 2000).

Participants and Methods: The current study utilized an archival sample of pre-season, baseline test data from healthy amateur athletes ( $N=2,159$; age $M=17.78(1.93) ; 77.0 \%$ male). The data were collected as part of a large-scale study of neuropsychological outcome following concussion. Participants completed a 2-hour test battery that included the SAC. Raw scores were converted to standard z scores and a correlation matrix was generated among SAC Orientation, Immediate Memory, Concentration, and Delayed Memory indices. A Monte Carlo simulation was conducted using the correlation matrix to determine the likelihood of observing abnormally low scores (Crawford, 2007).

Results: When "abnormal" scores were defined as occurring below the $5^{\text {th }}$ percentile, $18.05 \%$ of healthy athletes are expected to obtain one or more abnormal scores and $1.85 \%$ are estimated to obtain two or more abnormal scores. When "abnormal" scores were more liberally defined as the lowest $25^{\text {th }}$ percentile of the distribution, $65.73 \%$ of healthy athletes are expected to obtain one or more "abnormal" scores, and $26.94 \%$ are estimated to obtain two or more abnormal scores.

Conclusions: Though brief concussion measures are designed to detect subtle cognitive changes associated with concussion, a meaningful proportion of healthy athletes are expected to obtain scores below the 5 th percentile. This is relevant to consider when making return to play decisions by considering differences between baseline and post-injury performances. It is essential to recognize that a low SAC score (or low score on any neurocognitive measure) may reflect normal variability in the population.

Correspondence: Kathryn A. Ritchie, B.A., Psychology, Marquette University, 1930 N. Prospect Ave, \#51, Milwaukee, WI 53202, United States.E-mail: kathryn.ritchie@marquette.edu

L. SAAD, D.A. WOLK \& D. MECHANIC-HAMILTON. An Update on Normative Data for Neuropsychological Performance on Memory and Language Measures in a Racially Diverse Older Adult Longitudinal Cohort.

Objective: This study aimed to provide normative data for a racially diverse elderly sample on neuropsychological measures commonly used in longitudinal and intervention studies. Further investigation of the relationships between demographic variables and performance will also be discussed.

Participants and Methods: Participants included 327 adults (66\% females; ages 50-91 years old; 8-21 years of education; 222 white, 99 black/African American) with normal cognition who are included in the Penn Integrated Neurodegenerative Disease Database (INDD). Uni- and multivariate regression-based models were used to predict performance on Consortium to Establish a Registry for Alzheimer's Disease (CERAD) word list learning (Trials 1-3, total), delayed recall, recognition (Y/N, total), verbal fluency (f-words) and the Boston Naming Test (30 item, odd version). Values obtained from these analyses were entered into a normative data calculator modeled after a version created by Shirk and colleagues (2011). We also report z-scores and related percentiles on a sample case study of a normal older adult showcasing the clinical utility of this method, including the impact of demographic variables. Results: Age, sex, race, and years of education were found to significantly predict performance on most assessments with age being the best overall predictor followed by sex, education, and then race. Multivariate analysis combining these factors significantly predicts performance as well. Using an estimate of premorbid IQ accounted for more variance in the model and provided more accurate $\mathrm{z}$-scores relative to test performance on the Boston Naming Test and verbal fluency.

Conclusions: The present study provides normative data on a racially diverse older adult population on assessments of memory and language 
that do not currently exist in the literature. The data presented here will help to improve diagnostic accuracy of neuropsychological assessments in clinical and research settings.

Correspondence: Laura Saad, BS, Neurology, University of Pennsylvania, 3400 Civic Center Blvd, PCAM - 2 South, Philadelphia, PA 19104, United States.E-mail: laura.saad@uphs.upenn.edu

F. SACCÀ, T. COSTABILE, F. ABATE, A. LIGUORI, F. PACIELLO, C. PANE, A. DE ROSA, G. DE MICHELE \& A. FILLA. Normalization of Timed Neuropsychological Tests With the use of the PATA Rate and Nine-Hole Pegboard Tests.

Objective: Despite patients show frequent physical impairment, timed neuropsychological tests do not take this into account during scoring procedures. We propose a normalization method based on the Pata Rate Task (PRT) and on the Nine-Hole Pegboard Test (9HPT) as a measure of dysarthria and upper limbs dysfunction.

Participants and Methods: We defined the time spent on phonation or on hand movement during neuropsychological testing as Verbal Effort Fraction (VEF) and Motor Effort Fraction (MEF) respectively. This was aimed at excluding the time spent on cognition in each test, and to exclusively modify the VEF and MEF during the normalization procedure. VEF and MEF were measured experimentally on 65 healthy controls on timed neuropsychological tests (Attentional Matrices, Trail Making Test, Symbol Digit Modalities Test, Verbal Fluencies). We developed correction formulas that considered the patient's PRT/9HPT, their normality limits, and the test timing. This allowed us to increase the fixed time of tests proportionally to the grade of disability. For tests with score as a result, time was proportionally reduced. We tested the new method on 25 patients with Friedreich Ataxia (FRDA), as a prototype of dysarthria and upper limbs incoordination.

Results: In healthy controls, VEF and MEF ranged between $13.5 \%$ to $61.7 \%$ of total test time. In FRDA patients, the effect of normalization improved all test results (range of amelioration: 0.51-48.4\%; $\mathrm{p}<0.001$ ). FRDA patients had worst scores in all tests when compared to controls, and this remained significant after correction except for the Attentional Matrices that were similar between FRDA and controls. At the individual level, the normalization method improved equivalent scores with fever patients showing impaired scores.

Conclusions: We propose an innovative normalization method to reduce the impact of neurological disability on timed neuropsychological tests. This could be easily integrated in a clinical setting, as it requires a simple preliminary test with the PRT and 9HPT.

Correspondence: Francesco Saccà, MD, Neuroscience, Reproductive and Odontostomatological Sciences, University Federico II, Via Pansini, 5, Edificio 17, piano terra, Napoli 80131, Italy. E-mail: francesco.sacca@ unina.it

\section{L.A. SCHAEFER. Is the Montreal Cognitive Assessment (MoCA) Related to Functional Outcome and Discharge Destination in a Geriatric Stroke Rehabilitation Population?}

Objective: The Montreal Cognitive Assessment (MoCA) was developed as a screening test for cognitive impairment (Nasreddine et al, 2005). Its relationship to functional outcome in acute rehabilitation patients has been examined in stroke (Toglia et al, 2011) and in a geriatric population (Sweet et al., 2011). More recently, its utility in predicting discharge destination was examined in stroke patients aged 41-92, where it was found not to be predictive (Geubbels et al., 2015). This study examined the relationship between cognitive impairment, as measured by the MoCA, discharge functional status, and discharge destination amongst geriatric stroke rehabilitation inpatients.

Participants and Methods: A retrospective analysis of inpatients aged $70+$ on an acute rehabilitation unit of a hospital with primary diagnosis of stroke who were referred for cognitive assessment $(\mathrm{N}=18$; mean age $=80$ years; 10 male, 8 female) was conducted. All patients were administered the MoCA (range 5-23; mean=15) and FIM. Half were discharged home and half to other facilities. The motor subscale of the FIM (mFIM) at discharge, absolute motor functional gain (mAFG), and motor relative functional efficiency (mRFE) were each computed and correlated with the MoCA, length of stay (LOS), and discharge destination using Pearson correlation coefficients.

Results: There were significant associations between MoCA scores and the mAFG (Pearson $r=.499 ; \mathrm{p}=0.035$ ), but not the mFIM or mRFE. There were also significant correlations between discharge destination and $\mathrm{mAFG}$, mRFE, mFIM, and LOS, but not the MoCA.

Conclusions: This study highlights the value of using the MoCA with geriatric stroke rehabilitation patients given its relationship to functional outcome (mAFG). Muir-Hunter et al. (2016) proposed that mAFG, rather than gain relative to the maximal FIM score (mRFE), better determined rehabilitation success amongst those with cognitive impairment. These results are also similar to those of Geubbels et al. (2015) regarding the MoCA and discharge destination.

Correspondence: Lynn A. Schaefer, PhD, Physical Medicine and Rehabilitation, Nassau University Medical Center, 2201 Hempstead Turnpike, Box 31, East Meadow, NY 11554, United States. E-mail: lschaefe@numc.edu

G. SCHREYER-HOFFMAN \& D. ERLANGER. Effects of RBANS Demographic Adjustments with a Culturally Diverse Urban Elderly Sample.

Objective: The Repeatable Battery for Assessment of Neurological Status (RBANS) has been shown to effectively assess dementia in elderly patients. The RBANS manual provides age-adjusted normative. Duff and Ramezani (2015) developed an RBANS regression formula (REGF) that simultaneously adjusts multiple demographic variablesage, gender, education, and race-that are shown to influence performance. The present study examines the accuracy of RBANS manual and the REGF in identifying cortical disease, using cortical-subcortical deviation score (CSDS).

Participants and Methods: An archival record search in a northeast urban practice identified and utilized data from 18 participants who had completed the RBANS, had good effort scores, had clear evidence of cortical dementia, and were over 65 years old. To assess for classification accuracy, the RBANS and REGF CSDS were compared to clinician's diagnosis.

Results: Results of a Paired Samples t-test $(\mathrm{N}=18)$ revealed statistically significant differences between the RBANS and REGF mean scores on Immediate Memory Index, Delayed Memory Index and Total score. The cortical classification for all participants $(\mathrm{N}=18)$ was $83.3 \%(\mathrm{~N}=15)$ on the RBANS and $72.2 \%(\mathrm{~N}=13)$ on the REGF; for white participants $(\mathrm{N}=13)$ was $84 \%(\mathrm{~N}=11)$ on the RBANS and $69 \%(\mathrm{~N}=9)$ on the REGF; and for non-white participants $(\mathrm{N}=5)$ was $80 \%(\mathrm{~N}=1)$ on the RBANS and REGF.

Conclusions: Neither the RBANS nor the REGF approached $100 \%$ accuracy in classifying cortical dementia. In comparing the CSDS classifications, REGF reduced accuracy with this northeast urban sample, particularly among educated white male participants.

Correspondence: Gabrielle Schreyer-Hoffman, Counseling Psychology Ph.D., Graduate School of Education, Fordham University, 60 East End Ave, apt 20c, New York, NY 10028, United States. E-mail: schreyer. gabri@gmail.com

N. SHAAFI KABIRI, B. DUPEE, A. COTE, M. KRENGEL \& K. THOMAS. Continuous Performance Test-Identical Pairs (CPT-IP): Characterization and Relation to Other Attention and Working Memory Tasks.

Objective: The Continuous Performance Test-Identical Pairs (CPTIP) includes 2-digit (2D), 3-digit (3D), and 4-digit (4D) conditions measuring attention and working memory (WM). Study goals were (1) Characterize these CPT-IP conditions in healthy volunteers (HV) and patients with schizophrenia (SCZ) for their test-retest reliability and ceiling effects; (2) Evaluate the relationship of CPT-IP to other tasks measuring attention and WM. 
Participants and Methods: Thirty-one HV and thirty SCZ adults completed CPT-IP, Attention Network Test (ANT), and a visual WM Change Localization (CL) task twice (14 2 davs). Visit two data was only used for test-retest analyses. CPT-IP d-prime per condition and T score, ANT alerting, orienting, conflict (CE), and grand mean effect (GME), and CL accuracy were analyzed. Incomplete data and outliers were excluded.

Results: $\underline{\mathrm{HV}}:$ 3D and 4D displayed fair test-retest reliability (Intraclass Correlation Coefficient (ICC)>.5). There were no significant relationships between any of the tasks.

SCZ: Good test-retest reliability was observed in all conditions of CPT-IP (ICC>.6). CL correlated with CPT-IP performance across all conditions $(r>.5, p<.01)$. The strength of correlation increased as digit number increased, with the strongest correlation between CL and 4D $(\mathrm{r}=.613, \mathrm{p}<.001)$. Moderate correlation was observed between CL and CPT-T score $(r=.594, p=.001)$. There was a weak to moderate correlation between GME and 2D and 3D, respectively. CL showed moderate correlation with CE (low score indicates better performance) $(\mathrm{r}=-.423, \mathrm{p}<.05)$.

Both groups: A high proportion of both groups reached near-maximum to maximum 2D score $(>33 \%)$, indicating possible ceiling effect. 3D displayed the strongest test-retest reliability (ICC >.7).

Conclusions: Due to strong test-retest reliability and low potential for ceiling effect, 3D may be the most appropriate index for analyses in HV and SCZ. Strong correlation with CL and weak to moderate correlation with GME may indicate that CPT-IP employs a stronger WM demand in SCZ.

Correspondence: Nina Shaafi Kabiri, Anatomy and Neurobiology, Boston University School of Medicine, 650 Albany Street, Unit 140, Boston, MA 02118, United States.E-mail:nskabiri@bu.edu

M. SHARMA, E. JEFFAY, G. FOUSSIAS \& K.K. ZAKZANIS, Engagement and Motivation: Conventional Paper and Pen Neuropsychological Testing and Virtual Reality.

Objective: The primary aim of this study was to examine differences in engagement and situational motivation between conventional paper and pen neuropsychological test measures and immersive virtual reality (VR) in a healthy population. Moreover, we sought to examine simulator sickness symptoms resulting from an immersive VR task developed by the investigators.

Participants and Methods: Healthy undergraduate students ( $N=107)$ were randomly assigned to a paper and pen condition or a VR condition. Both conditions completed three effort tests: Test of Memory Malingering, Rey Fifteen Item Test, and the Victoria Symptom Validity Test. Participants in both conditions also completed the Situational Motivation Scale and an engagement questionnaire. The paper and pen condition were given three tests of attention: the Ruff 2 and 7 Selective Attention Test, the Symbol Digit Modalities Test and the Paced Auditory Serial Addition Test. In contrast, participants in the VR condition completed the OcuVeyorTask, an immersive VR attention task developed to be used with the Oculus Rift, a Head Mounted Display (HMD). Independent t-test was used to compare engagement and situational motivation between groups and paired samples t-test was used to compare simulator sickness pre and post exposure. Pearson's correlation was used to examine the relationship between engagement and situational motivation.

Results: Engagement differences between groups had an effect size of $(d=.31)$. Simulator sickness symptoms significantly increased after exposure to immersive VR $(p=.003)$. Higher engagement scores were associated with higher levels of situation motivation $(p<.001)$.

Conclusions: Results from this study suggest that VR may be a useful cognitive assessment tool however more research is needed to elucidate the relationship between situational motivation and engagement and VR. Future VR development should consider ways to reduce simulator sickness.
Correspondence: Manu Sharma, University of Toronto, 1265 military trail, Scarborough, ON M1C 1A4, Canada. E-mail: mitnu.sharma@ mail.utoronto.ca

V. SICARD, R.D. MOORE \& D. ELLEMBERG. Construct Validity of a Color-Shape Switch Task.

Objective: Task-switching (or set-shifting) is one of many cognitive control abilities. We developed a color-shape switch task based on that of Hillman and Pontifex (2012), which is known to be sensitive to various medical conditions, including brain injury. In the colorshape version of the switch task that we developed, participants first performed two task conditions (homogeneous conditions), one in which they respond to the color of the stimuli, and the other in which they respond to the shape. Then, participants performed a heterogeneous condition, which requires that they respond to either the color or the shape rule set depending on the cue provided. Three different costs are computed from the raw scores: global switch cost, which is thought to be a measure of global cognitive control; local switch cost, which is believed to be a measure of cognitive flexibility; and working memory cost. This study aims to determine the construct validity of this version of the color-shape switch task.

Participants and Methods: Thirty healthy undergraduate students performed a battery of well known and validated neuropsychological tests including the Trail-making Test, the Digit Forward and Backward spans (WAIS-III), the Cognitive Flexibility Inventory, the BRIEF for adults, the Color-Word Interference Subscale (D-KEFS), and the switch task.

Results: Correlation analyses revealed moderate correlations between established neuropsychological tests and the different switch costs ( $\mathrm{rs}=-.49$ to $.41, \mathrm{ps}<.02)$. Among others, the global switch cost was significantly correlated with the Global Executive Composite of the BRIEF-A $(r=-0.49, p=.01)$ and the working memory cost was correlated with the Digit span score $(\mathrm{r}=0.41, \mathrm{p}=.02)$.

Conclusions: These results suggest that the switch task can accurately measure global executive functions, cognitive flexibility and working memory. Further research is needed to investigate the task's test-retest reliability and its validity in clinical populations.

Correspondence: Veronik Sicard, Kinesiology, Université de Montréal, 2100 Boulevard Edouard-Montpetit, Laboratoire des Neurosciences du développement, de l'exercice et de la vision, Montréal, QC H3T1J4, Canada.E-mail:veronik.sicard@gmail.com

K.E. SLYNE, K.J. MANNING, D. KAPLAN, R. KAPLAN \& L. WOLFSON. Four Year Test-Retest Reliability of the RBANS and Executive Functioning Measures in Healthy Elderly.

Objective: Understanding "normal" cognitive change in older adults can improve diagnostic precision. The Repeatable Battery for the Assessment of Neuropsychological Status (RBANS) is increasingly used with older adults, although it does not assess executive functioning. Longitudinal normative data for the RBANS administered on more than two time points and co-normed with executive functioning measures is scarce. The current study aims to expand upon the extant aging literature by analyzing the test-retest reliability of the RBANS and executive functioning measures over three time points.

Participants and Methods: Fifty-eight healthy adults from a larger cohort $(\mathrm{n}=107)$ aged 75-89 $(\mathrm{M}=81.22, \mathrm{SD}=4.01)$ with an average of 14.78 years of education $(\mathrm{SD}=2.91)$ were assessed at baseline and then two and four years later. Participants completed the RBANS, Trail Making Test (TMT), Stroop Test, and a computerized measure of sustained attention and working memory, the California Computerized Assessment Package (CalCAP). Test-retest reliability coefficients, means, and standard deviations for test and change scores at all three time points were calculated.

Results: Subjects were in the average range according to RBANS Total Score (mean score at Time $1=104.31, \mathrm{SD}=16.64$; Time $2=100.33$, $\mathrm{SD}=14.51$; Time $3=94.55, \mathrm{SD}=17.29)$. Reliability coefficients from 
baseline to year four for the RBANS subtests ranged from .37 to .80 . The lowest were observed on Picture Naming (.37) and Figure Copy (.41). All others were above .50 , with the highest being Coding (.72) and List Recall (.72). Stability coefficient ranges for the Stroop CW, CalCAP, and TMT A and B are as follows: .70 to $.82, .36$ to $.65, .35$ to .57 , and .54 to .76 .

Conclusions: We strongly suspect that the lower stability correlations in our sample compared to prior research are due to changes in cognition over a four year test period in people at this end of the age spectrum. This is an important consideration in determining reliable change scores over four years in an elderly population.

Correspondence: Kristin E. Slyne, Psy.D., Psychiatry, UConn Health, 263 Farmington Avenue, Farmington, CT 06030, United States. E-mail: kristin.slyne@gmail.com

\section{A.M. STAFFARONI, M.E. ENG, R. WICKHAM, H.K. ZEINER \& J. MOSES. Four- and Five-Factor Models of WAIS-IV Interpretation in a Clinical Sample.}

Objective: Five-factor models of the Wechsler Adult Intelligence Scale $-4^{\text {th }}$ ed. (WAIS-IV) have been validated using the WAIS-IV standardization and clinical samples. This study aimed to extend these analyses to a sample of patients seen at a neuropsychological clinic.

Participants and Methods: Participants were 322 diagnosticallydiverse Veterans (mean age: 53.99, SD=14.55; mean edu: 13.99 , $\mathrm{SD}=2.24$ ) referred for neuropsychological assessment. Four different models were evaluated using confirmatory factor analysis: 1: A fourfactor, 15-subtest model (Wechsler, 2008); 2: A five-factor, 15-subtest model (Benson, Hulac, \& Kranzler, 2010); 3: A five-factor, 15-subtest model that included a Quantitative Reasoning (RQ) factor (Weiss, Keith, Zhu, \& Chen, 2013); and 4: A five-factor, 12-subtest model that is valid across the lifespan (Niileksela, Reynolds, \& Kaufman, 2013). Models 1-3 were compared using AIC statistics. Model 4 was not directly compared to the other models because it differed in the number of observed variables

Results: Four- and five-factor models were all found to fit the data adequately based on several indices of model fit. The 15-subtest, fivefactor models ( $2 \& 3$ ) performed better than the four-factor model (Model 1) when no additional cross-loadings were specified. In contrast, Model 1 outperformed Models 2 and 3 when the Arithmetic subtest was allowed to cross-load on the Verbal Comprehension factor. Specifying the RQ factor and allowing additional cross-loadings (Model 3) improved fit. The present study also found adequate fit on two of three fit indices for the lifespan model (Model 4).

Conclusions: These findings extend the validation of five-factor models of WAIS-IV interpretation, including a lifespan model, to a diagnostically-diverse neuropsychological sample. The results did not provide a clear statistical rational for choosing between four- and five-factor models. Five-factor models require further study to support their clinical utility. Correspondence: Adam M. Staffaroni, Ph.D., University of California, San Francisco, University of California, San Francisco, 675 Nelson Rising Lane, Suite 190, San Francisco, CA 94158, United States. E-mail: adam.staffaroni@ucsf.edu

\section{R.L. STEGMAN \& W.R. COLE. Percentage of Low Scores is a Useful Embedded Measure.}

Objective: Individuals putting forth poor effort on neuropsychological assessments are likely to have an unusually high number of low scores. The current investigation is an exploration of the use of percentage of low scores (PLS) as an embedded measure of participant effort.

Participants and Methods: Data from 172 consecutive comprehensive neurocognitive assessments from a military concussion clinic were used. All patients had sustained a medically documented mild traumatic brain injury and were tested after full recovery was expected: $\geq 6$ months post injury. Each assessment included 8 or more performance validity measures (PVMs).
A "low score" was defined as: $\geq-1.33 \mathrm{z}$-score / sd, 80 standard, $\leq 37$ T-score, $\leq 6$ scaled, or $\leq 9$ th percentile. PLS was calculated by dividing the total number of low scores by the total number of scores obtained. Results: Average PLS, per number of failed PVMs, were: $0=3.4 \%$ $(n=29), 1=4.3 \%(n=31), 2=8.5 \%(n=34), 3=12.7 \% \quad(n=22), 4=23.7 \%$ $(n=17), 5=25.3 \%(n=10), 6=35.7 \%(n=15) .7=37.2 \%(n=6), 8=30.0 \%$ $(n=4), 9=79.7 \%(n=3), \& 10=82.0 \%(n=1)$. Contingency Table analyses revealed PLS of $15 \%$ is optimal for discriminating between credible and non-credible participants (based on 4+ failed PVMs): PPV=94.64\% and NPV $=92.22 \%$. Additional analyses indicated PLS as low as $12 \%$ is cause for caution.

Conclusions: PLS has demonstrated potential as an embedded measure of effort to discriminate between credible and non-credible data and may be especially useful with review of neuropsychological evaluations during which no or only a few PVMs were administered. Affirmed as well is that a few neuropsychological scores below normal limits are to be expected even with valid data.

Correspondence: Robert L. Stegman, Ph.D., Department of Brain Injury Medicine, Womack Army Medical Center, 205 Angel Oak Dr, Unit 3, Fayetteville,NC28314,United States.E-mail:drstegman@hotmail.com

W.J. STUBBS, T.J. ARENTSEN, E. CROUSE, L. HENNESSEY \& B.L. ROPER. Concurrent Validity of the ADHD Symptom Questionnaire Inattention Subscale (ASQ-I): A Pilot Study of Performance on CPT-II and D-KEFS TMT Indices.

Objective: In a recent pilot study, the ADHD Symptom Questionnaire (ASQ), a 60-item, self-report ADHD measure that includes validity indicators, demonstrated adequate concurrent validity with the CAARS: S-L (Duckett et al., 2016). The current study evaluates the validity of the ASQ-Inattention subscale (ASQ-I) by assessing its relationship with performance on two objective neuropsychological measures, the Connor's Continuous Performance Test (CPT-II) and D-KEFS Trail Making Test (D-KEFS TMT).

Participants and Methods: Participants $(N=47)$ were veterans referred for possible ADHD who completed neuropsychological evaluation. including CPT-II, D-KEFS TMT, and ASQ at a large VAMC, without excessive missing data ( $\geq 2$ items missing). The ASQ includes separate scores for each of the DSM-5 inattention (ASQ-I) and hyperactivity symptoms (ASQ-H). ASQ-I classification was determined when at least 5 inattention symptoms were endorsed (mean score $\geq 3.75$ on 1-6 Likert scale; $n=33$ met criteria, 14 did not). Group differences on selected indices of CPT-II and D-KEFS TMT were examined.

Results: Those meeting above criteria on the ASQ-I subscale produced significantly more commission errors on CPT-II $(t=-2.40, p=.02)$, slower overall reaction time on CPT-II $(t=2.12, p=.04)$, and slower divided attention per D-KEFS TMT Trial 4 performance $(t=.21, p=.045)$ than those who did not. Notably, scores did not significantly differ according to CPT-II omission errors, CPT clinical index for ADHD, or D-KEFS TMT Trial 4 raw scores ( $p s>.05)$.

Conclusions: Current results build on previous preliminary findings on the validity of the ASQ and support its potential utility in clinical diagnosis of ADHD, Inattentive Type, per DSM-5 criteria.

Correspondence: WhitneyJ. Stubbs, The University of Memphis/Memphis Veterans Affairs Medical Center, 5900 Winding River Way Apt 102, Memphis, TN 38120, United States.E-mail: wstubbs1@memphis.edu

D.M. TAM, B. LEMONDA, W. BARR \& L. RABIN. Survey of Neuropsychologists' Practices and Perspectives Regarding the Utilization of Technicians.

Objective: Technicians have been utilized in the field of neuropsychology dating back several decades. As there remains no universally adopted credentialing process, variability continues to exist among neuropsychologists in their utilization and practice of technicians. The current analysis examined data from 506 licensed neuropsychologists (INS and NAN members) in North America who responded to a broader 2011 survey of neuropsychology assessment practices. 
Participants and Methods: Descriptive statistics were calculated for all variables. Chi-Square and independent-samples t-tests were used to assess group differences.

Results: Forty-nine percent of respondents reported engaging in at least some use of technicians. Of those individuals, more than half $(54 \%)$ were female, with a mean age of 47 , and 14.2 years of practice experience. Aggregating responses from respondents who endorsed consistent use of technicians (i.e., 'often' or 'always'), $51 \%$ of those employed individuals who had a Bachelor's degree level of education, and $44 \%$ with a Master's degree (5\% other). An overwhelming majority (96\%) reported providing technicians with training, although variability in responses was evident on the areas of training provided (e.g., ethics, test administration, cultural competency). Of respondents endorsing consistent technician use, $58 \%$ engaged in forensic practice, $56 \%$ utilized computerized assessments, and $11 \%$ conducted non-English assessments.

Conclusions: The current results highlight neuropsychologists' practices on utilization of technicians in professional practice, and indicate differences still exist among beliefs regarding recruitment, training, and certification.

Correspondence: Danny M. Tam, Ph.D., Neurology, NYU Comprehensive Epilepsy Center, 43-18 Main Street, Apt 11B, Flushing, NY 11355, United States.E-mail: danny.tam@outlook.com

\section{R. TANG \& L. SILVA. The Impact of Language and Education on Non-verbal Neuropsychological Measures.}

Objective: Language and education are essential in both verbal and non-verbal neuropsychological performances. Current study examines the impact of language and education on performances on commonly used non-verbal neuropsychological tests among patients in an outpatient neuropsychological clinic.

Participants and Methods: Patients referred to the adult neuropsychology outpatient clinic primarily for memory difficulties were examined in different cognitive functioning domains. Patients were excluded from the analysis if they have any focal damage in the brain, including traumatic brain injury, tumor, or any other known neurological disorder or scored below the cutoff on symptom validity tests. Patients ( $\mathrm{N}=56$, $54 \%$ female) reported an average age of 47.09 years $(\mathrm{SD}=15.48)$ and an average of 11.73 years $(\mathrm{SD}=2.99)$ of education. 48 patients were monolingual English speakers. Performances of interest included Symbol Digit Modalities Test (SDMT; Written and Oral), Trail Making Test Part B (TMT B), Rey-Osterrieth Complex Figure Test (RCFT), copy, and Brief Visuospatial Memory Test, Revised (BVMT-R), delayed recall. Five independent sample t-tests were conducted to examine whether there was a significant difference between monolingual and bilingual speakers on non-verbal test performance. In multiple regression, sex and age were entered in step 1, years of education in step 2 and performance on WASI-II Vocabulary in step 3 as predictors.

Results: There was no significant difference between monolingual and bilingual participants' test performances. In multiple regression, with the exception of BVMT-R, WASI-II Vocabulary was a significant predictor of all four non-verbal tests when age, sex and education were controlled for.

Conclusions: These findings are consistent with existing literature suggesting verbal abilities influence visuo-motor integration and non-verbal problem solving skills. Results also suggest vocabulary might be a more sensitive indicator than education level in predicting non-verbal neuropsychological performance.

Correspondence: Rui Tang, PhD, Psychology, Southern Methodist University, 6421 Lontos dr., Dallas, TX 75214, United States. E-mail: rtang@smu.edu
L.I. THOMPSON, A. SURACE \& S.A. GOLUB. Cards, Balloons, or Dice? A Comparison of Risky Decision-Making Tasks and Their Associations With Working Memory, Anxiety, Depression, and Risk Taking.

Objective: The Iowa Gambling Task (IGT), Balloon Analog Risk Task (BART), and Game of Dice Task (GDT) are three computer tasks frequently used in the study of risky decision-making (DM). Despite this, little research has directly compared these measures, making it difficult to reconcile inconsistencies across findings. The present study examined relationships between the IGT, BART, and GDT, and their associations with factors shown to modulate risky DM, toward bringing greater clarity and generalizability to the literature.

Participants and Methods: An ethnically diverse ( $22 \%$ white) sample of 100 college students ( $64 \%$ female) completed the study for course credit. Measures of DM (IGT, Bechara et al., 1994; BART, Lejuez et al., 2002; GDT, Brand et al., 2005), working memory (WM; WAIS-IV, Digit Span Backwards), and self-reported depression (BDI-II; Beck Depression Inventory, Beck et al., 1996), anxiety (BAI; Beck Anxiety Inventory, Beck \& Steer, 1993), and risk taking (RAS; Risk Behavior Scale, Weber et al., 2002) were collected. Multiple regression was used to examine relationships between the DM tasks, WM, and self-report measures.

Results: BART performance negatively predicted IGT performance $(b=-.22, t(81)=-2.2, p<.05)$. GDT performance positively predicted IGT performance $(b=.28, t(81)=2.7, p<.01)$. WM interacted with depression to predict IGT performance $(b=-.55, t(84)=-2.7, p<.01)$, and with anxiety to predict BART performance $(b=.61, t(84)=2.8$, $p<.01)$, such that below average $\mathrm{WM}$ predicted worse performance in those reporting depression or anxiety, respectively. Self reported risktaking did not predict task performance.

Conclusions: The observed task relationships are consistent with research indicating that the IGT and GDT reward risk averse DM, while the BART rewards moderate risk taking. The tasks differed in their associations with WM, anxiety, and depression, suggesting that these are important factors to consider in cross study comparisons.

Correspondence: Louisa I. Thompson, M.Phil., Psychology, Graduate Center of the City University of New York, 12 Willard Place, Hudson, NY 12534, United States. E-mail: Louisa.Isoud@gmail.com

E. TRIFILIO, L.C. BUTTERFIELD, P. MANGAL, J.E. MAYE, G. SELKE, M. MARSISKE \& D. BOWERS. A Tale of Two Stories: Comparing Logical Memory and Newcomer Stories.

Objective: Many neuropsychological patients require repeat evaluations to determine change over time. This can lead to practice effects, which can impact the validity of the assessment. The current study aimed to determine the validity of an alternative set of verbal memory stories, created by Newcomer et al. (1994) to assess recent memory.

Participants and Methods: We administered the WMS-III Logical Memory (LM) stories and two Newcomer stories (NS) to cognitively healthy older adults $(\mathrm{N}=52)$ under immediate and delayed recall conditions. All were screened for cognitive impairment (mean MMSE $=29.2)$ and significant medical/mental health comorbidities. The NS were scored by blinded raters in 2 ways: verbatim (traditional) and with a newly developed thematic based criteria (similar to LM). Data were analyzed using factor analysis (FA), Pearson correlations, and independent sample t-tests.

Results: Inter-rater reliability of the semantic scoring of the NS was acceptable $(\mathrm{r}=0.940-0.984)$. The verbatim and semantic scorings of the NS were highly correlated $(r=0.918-0.923)$. Importantly, both verbatim and thematic scoring methods of the NS were significantly correlated with LM at immediate and delay $(r=0.572-0.790)$. Factor analysis results involving multiple cognitive measures indicated convergent validity of NS with other memory measures and divergent validity compared to measures of processing speed and visuospatial functioning. Conclusions: The NS appear to be a valid alternative to the LM stories. Future studies would benefit from examining possible differences between the verbatim and thematic NS criteria in relation to structural and functional neuroimaging data. For example, verbatim 
scoring might reflect strong 'pure memory' abilities and correlate with entorhinal cortex volume, while semantic scoring might reflect other neurocognitive or compensatory skills and correlate less strongly with hippocampal areas.

Correspondence: Erin Trifilio, BA, Clinical and Health Psychology, University of Florida, $1185 \mathrm{SW} 9$ th Rd, Gainesville, FL 32601, United States.E-mail:etrifo7@ufl.edu

J. UPSHAW, D. LEITNER, L. OHLHAUSER, M. LIBBEN \& H. MILLER. Allocentric vs. Egocentric Neglect in Stroke Patients: Assessment Through Eye-tracking and Impacts on Functional Outcomes.

Objective: Few studies have investigated the assessment, frequency, and functional impact of egocentric and allocentric neglect among stroke patients. This study aimed to determine a) whether allocentric and egocentric neglect could be dissociated among a sample of stroke patients using eye-tracking, b) the frequencies and attentional patterns of each neglect subtype and c) the nature of the relationship between neglect subtype and functional outcome.

Participants and Methods: 16 acute stroke patients completed comprehensive neuropsychological (NP) assessment batteries, a penciland-paper Apples Test, and an eye-tracking measure of neglect subtype. Descriptive analyses were conducted on Apples Test and eye-tracking scores to determine their sensitivities in detecting neglect subtype. Hierarchical regression was used to determine predictive utility of the eye-tracking measure above that of both NP and Apples Test performance. ANOVA was used to investigate relationships between neglect subtype and functional outcome.

Results: Eye-tracking was more sensitive in identifying neglect subtype than the Apples Test. Classification of neglect subtype based on eye-tracking performance was a significant predictor of functional outcome beyond both NP test performance and Apples Test neglect classification. Patients with no neglect symptoms had better functional outcomes than those with either or both types of neglect. Function of patients with either allocentric or egocentric neglect did not differ significantly from each other. Patients with both types of neglect had the poorest functional outcomes of all four groups.

Conclusions: Neglect subtype classification based on eye-tracking contributes independently to the prediction of functional status beyond that of NP test performance, and is a better predictor of function than the Apples Test. The significant relationship between neglect subtype and functional outcome highlights the importance of sensitive assessment and identification of neglect subtype amongst stroke patients. Correspondence: Jennifer Upshaw, Psychology, University of British Columbia Okanagan, 436 McCarren Avenue, Kelowna, BC V1W4T8, Canada.E-mail:jnupshaw@gmail.com

D.W. VAUGHN, J. WATSON \& R. HIRST. Blunt(ed) performance: Examiner judgments of cannabis user status predicts neuropsychological outcomes.

Objective: Previous research suggests examiner beliefs about acute caffeine administration impact examinee cognitive performance and physiological response (Walach et al., 2001). Hirst and colleagues (2016) found neuropsychologists can discriminate cannabis users from non-users based upon photograph appearance alone. Therefore, this population may be vulnerable to expectancy effects in cognitive research if examiners can guess user status. The present study investigated whether examiner beliefs of cannabis use predicted cognitive performance.

Participants and Methods: Participants included 41 users and 20 non-users randomly assigned to receive either a motivational statement or a neutral statement. Examiners who were blind to user status rated whether they believed the examinee was a cannabis user, then administered a battery of neuropsychological tests assessing core neurocognitive domains. A series of regressions compared neuropsychological performance between the two groups.
Results: Examinees who were judged as cannabis users scored lower on four of eight tests, including California Verbal Learning Test-II, Word Memory Test, Wechsler Adult Intelligence Scale-III Coding subtest, and Trail Making Test. There were no differences in cognitive performance between judged users and non-users on the remaining assessments. After controlling for the motivational statement received, there were no differences on WMT scores. Findings remained consistent after controlling for gender and actual user status.

Conclusions: Results of this study suggest that the test examiners' beliefs of an individual's cannabis user status may affect performance on neuropsychological tests - even when blind to actual user status. These findings have important implications for neuropsychological performance on evaluations in both research and clinical settings, as test scores may partially reflect the examiners' beliefs of an individual's marijuana use status and not their true score.

Correspondence: Dylan W. Vaughn, Clinical Psychology, Palo Alto University, 620 Veterans Blvd Apt 514, Redwood City, CA 94063, United States. E-mail: dvaughn@paloaltou.edu

T.T. VO, C. SUMIDA, R. LAMB \& M. SCHMITTER-EDGECOMBE. Promoting Healthy Cognitive Aging: Development and Psychometric Properties of the Healthy Brain Aging Activity Engagement Questionnaire.

Objective: Accumulating research indicates that activities such as exercise, cognitive/social engagement, good sleep hygiene, stress reduction and proper nutrition are associated with healthy cognitive aging. The Healthy Brain Aging Activity Engagement (HBAAE) questionnaire was developed to capture an individual's general level of engagement in healthy brain aging behaviors.

Participants and Methods: Healthy older $(N=57)$ and younger $(N=222)$ adults rated their engagement on 38 health behavior items using a Likert scale from $1 /$ (strongly disagree) to $5 /$ (strongly agree). The older adults also completed cognitive and physical tests, and social/ personality questionnaires.

Results: A Rasch Model Item Reduction Analysis precipitated the removal of 5 items based on infit and outfit statistics and a Principle Components Analysis (PCA) prompted the removal of one item due to cross loading: all remaining items loaded at .40. The three health domain factors identified via the PCA (Eigenvalues > 2.9) were: physiological, cognitive/social, and health risk behaviors (e.g., smoking), each with Rasch reliability $>.94$. Correlations supported the domain structure of the HBAAE ( $p s<.01)$. Higher endorsement on the physiological factor was associated with better physical function on both objective $(r=.35)$ and self-reported measures $(r=.33)$. Higher endorsement on the cognitive/social factor was associated with higher self-reported levels of social wellbeing $(r=.33)$ and higher levels of the personality characteristics of extraversion $(r=.38)$ and openness $(r=.39)$.

Conclusions: Preliminary data suggests that the HBAAE has strong psychometric properties and may be of value for assessing level of engagement in behaviors which promote healthy cognitive aging.

Correspondence: Thao T. Vo, Bachelor of Science, Psychology, Washington State University, $640 \mathrm{SW}$ Golden Hills Drive A-201, Pullman,WA 99163,United States.E-mail: thao.vo@wsu.edu

B.D. WALLS, E.R. WALLACE, S.L. BROTHERS \& D.T.R. BERRY. Utility of the CAARS Validity Scales in Identifying Feigned ADHD, Random Responding, and Genuine ADHD in a College Sample.

Objective: Due to recent concern about malingered self-report of symptoms of attention-deficit/hyperactivity disorder (ADHD) in college students, there is an urgent need for scales that can detect feigning of this disorder. The present study provided further validation data for a recently developed validity scale for the Conners' Adult ADHD Rating Scale (CAARS), the CAARS Infrequency Index (CII), as well as for the Inconsistency Index (INC).

Participants and Methods: A total of 139 undergraduate students completed the CAARS; 21 individuals with diagnoses of ADHD, 
29 individuals responding honestly, 54 individuals responding randomly to either all or half of the CAARS items, and 35 individuals instructed to malinger ADHD while avoiding detection. A financial incentive of $\$ 25$ was offered for successful feigning.

Results: Overall, the INC showed moderate sensitivity to random responding (.44-.63) and fairly high specificity to ADHD (.86-.91). The CII demonstrated modest sensitivity to malingered ADHD (.31-.46) and excellent specificity to genuine ADHD (.91-.95). Sequential application of validity scales had correct classification rates for honest $(93.1 \%)$, ADHD $(81.0 \%)$, malingering $(57.1 \%)$, half random $(42.3 \%)$, and full random $(92.9 \%)$. Of note, the INC and CII flagged more malingerers as invalid when applied in a stepwise manner $(57.1 \%)$, as opposed to when using the CII alone (34.3\%).

Conclusions: Although the present study demonstrated modest sensitivity in the detection of feigning, the fact that $43-69 \%$ of malingerers went undetected suggests the need for more research. This study has added to the literature by demonstrating the utility of the CAARS validity scales working together to distinguish between various response sets. Finally, if using the algorithm clinically, clinicians should have strong specificity and at least modest sensitivity in the detection of feigning on the CAARS, provided results are successfully cross-validated.

Correspondence: Brittany D. Walls, M.S., Psychology, University of Kentucky, 3901 Rapid Run Dr, Apt 122, Lexington, KY 40515, United States.E-mail: brittany.walls18@gmail.com

\section{J.M. WHEELER, J. MOSES \& J.K. LAI. The Role of Spoken Language and Verbal Mediation in Performance on the Serial Digit Learning-8 Test.}

Objective: The role of spoken language on the performance on the Serial Digit Learning 8 (SDL8) test was examined using the subtest from Benton's Multilingual Aphasia Examination (MAE) through principal component analysis.

Participants and Methods: Archival data of two hundred and twenty-two participants with mixed neuropsychiatric diagnoses who completed SDL8 and MAE were analyzed using principal component analysis; there were no exclusion criteria. The twelve trials of SDL8 were factored using principal component analysis to create a three-factor solution characterized by early-, middle-, and late-appearing items. Subtests from MAE were similarly factored, yielding a four-factor model of spoken language characterized by verbal fluency, naming, repetition, and auditory comprehension. This 4-factor model of spoken language was analyzed along with the 3 -factors from SDL8 to examine the role of spoken language on SDL8 performance.

Results: The analysis produced a five-factor solution showing discrete and specific relationships of language variables to the early, middle, and late trial learning processes of the SDL8 test, $(84 \%$ of the variance explained). Both early- and middle-appearing items were related to repetition, while items on later trials were correlated with auditory comprehension and verbal fluency abilities. Visual naming abilities were found to be independent of SDL8 performance.

Conclusions: Performance on the SDL8 appears to be significantly impacted by spoken language abilities, suggesting the role of verbal mediation in the learning processes of SDL8.

Correspondence: Janel M. Wheeler, Ph.D., Psychology, Palo Alto University, 1281 Lawrence Station Rd Apt. 448, Sunnyvale, CA 94089 , United States. E-mail: jwheeler@paloaltou.edu

\section{B. YOCHIM, S. WITHROW \& J. BALDO. Validity of the Verbal Naming Test using a 5-second response time limit.}

Objective: The Verbal Naming Test (VNT; Yochim et al., 2015) assesses word-finding by having patients name 55 words that are verbally defined for them. Patients are given 10 seconds to generate each word. Given that patients with intact word-finding typically name the words within 2 seconds, and a shorter time limit might increase the sensitivity of the measure to word-finding problems, this study investigated the validity of the VNT with a 5-second time limit.
Participants and Methods: The VNT and NAB Naming test were administered to 130 patients receiving neuropsychological evaluations. The total score was the number of correctly named stimuli within 5 seconds.

Results: The VNT correlated with age, $r=-0.28, \mathrm{p}<.01$, but not with education, $r=0.10, p=0.26$, and males and females obtained equivalent total scores. The VNT correlated with the Naming subtest of the Neuropsychological Assessment Battery (NAB), $r=0.85, p<.001$, with the D-KEFS Letter Fluency subtest, $\mathrm{r}=0.49, \mathrm{p}<.001$, and the D-KEFS Category Fluency subtest, $\mathrm{r}=0.54, \mathrm{p}<.001$. ROC analyses found the Area Under the Curve (AUC) to be 0.90 for detecting dysnomia, 0.74 for detecting DSM-5 Mild Neurocognitive Disorder, and 0.87 for detecting Major Neurocognitive Disorder. A cut score of 47.5 out of 55 resulted in $75 \%$ sensitivity and $77 \%$ specificity in detecting dysnomia.

Conclusions: The VNT with a 5 -second time limit is an effective method of detecting word-finding deficits. Future work will generate normative data using this administration protocol.

Correspondence: Brian Yochim, PhD, National Jewish Health, 1400 Jackson St., B134, Denver, CO 80206, United States. E-mail: YochimBrian@gmail.com

A.B. ZAHEED \& F.W. BYLSMA. The Chicago Alternative Stroop Test: Developing a Clinical Measure of Incidental Learning.

Objective: Implicit learning, or learning without conscious effort, occurs when individuals focus their attention on complex information (Reber, 1995). This type of learning has adaptive value and is pervasive in acquisition of language, motor skills, social norms, and pattern recognition. Frequency detection - learning if something is more or less likely to occur - is one type of implicit learning. Here, the Chicago Alternate Stroop Test (CAST) uses a modification of the Stroop Test method to develop a clinical measure of implicit frequency detection. Modelled on the existing Stroop paradigm, the CAST adds two colors, presenting three colors with equal frequency, one color significantly more frequently, and one significantly less frequently than the other three in an effort to assess implicit frequency detection.

Participants and Methods: Non-clinical adult volunteer participants were recruited for this study. Demographic information, estimated current IQ (WTAR), and CAST performance data were determined. Participants read the entire page of color words, named the color X's are printed in, and completed an interference trial; completion times were recorded. Participants were then asked which color was named most and least often.

Results: In normal healthy volunteers, time to complete each condition of the CAST was generally consistent with typical performance on the traditional Stroop task, with participants taking significantly longer to complete the inhibition condition (W: $45.04 \mathrm{sec}$; C: $67.93 \mathrm{sec}$; C-W: $109.36 \mathrm{sec}$ ). Most normal participants (age range 23-71; education range: 12-20; WTAR range 99-118) were able to correctly identify the colors which were presented with higher frequency $(80 \%)$ and lower frequency $(80 \%)$ than the rest, and accuracy was unrelated to age, education, or speed of performance.

Conclusions: The CAST shows promise as a method for clinical assessment of an aspect of implicit learning. Additional normal control data and data from clinical populations are being gathered and will be presented.

Correspondence: Afsara B. Zaheed, BA, Neuropsychological Services, P.C., 180 N. Michigan Ave., Suite 2210, Chicago, IL 60601, United States.E-mail: abzaheed@neuropsych1.com 


\section{Historical}

K. BALL, C. MENDEZ, R. KIRMSE \&. M. RODRIGUEZ. The Evolution of Autism Spectrum Disorder: From Severe Psychopathology to the Executive Dysfunction Hypothesis.

Objective: Zeitgeist refers to the general beliefs, ideas, and spirit of a time and place. Diagnostic classification of Autism Spectrum Disorder (ASD) can be dated back to Dr. Leo Kanner's 1943 seminal description of Autism where he described the symptoms as being an "obsessive insistence on the preservation of sameness." The aim of this review is to explore how the diagnosis and conceptualization of ASD evolved through the years as a result of a change in Zeitgeist.

Participants and Methods: Material from publications, literature, and systematic reviews were gathered and reviewed from ebscohost, Academic OneFile, and PsycInfo.

Results: From 1950-1960 Autism was referred to as "childhood schizophrenia", and was understood to be the result of "cold mothering" by psychoanalysts. In the 1970s there was a shift in thought and autism was understood as biologic in origin. The following twenty years brought changes in the diagnostic criteria of Autism, requiring deficits in social interactions, communication, and restricted interests or activities, and distinctions between varying presentations were created. Currently, ASD is defined in two categories, and subtypes of the disorder have been eliminated. The executive dysfunction hypothesis in ASD refers to a broad continuum of neuropsychological processes that underlie physical, cognitive, and emotional self-control, and it is widely used to understand the deficits in ASD.

Conclusions: The change in the U.S. Zeitgeist, which called for an increase in scientific thought, allows us to utilize our knowledge of the executive dysfunction implicated in ASD to inform treatment.

Correspondence: Kayleen Ball, M.S., Carlos Albizu University, 9623 SW 163 CT, Miami,FL 33196, United States.E-mail: kayly25@yahoo.com

\section{A.I. FORD. From Morosis to Neurocognitive Disorders: A History of Dementia and its Neuropsychological Assessment.}

Objective: Historical overview of the conceptualization of dementia, including milestones in cognitive assessment.

Participants and Methods: Review of historical writings on the diagnostic conceptualization of dementia and historical dates in neuropsychological assessment.

Results: A basic conceptualization that old age or disease could cause memory or other permanent cognitive impairment has been documented since at least ancient Egypt (2000 BC). But our understanding of dementia as a set of disorders with multiple pathophysiologies is a recent leap in understanding, aided by neuropsychological assessment. In regard to diagnostic specificity, we have moved from observation of cases-anchored by Alois Alzheimer's 1906 case report, to diagnosis of advanced-stage disease, to our current ability to identify mild earlystage or precursor changes. This evolution is reflected in our classification systems, from "organic brain syndrome" in DSM-I to our current concept of multiple subtypes of Neurocognitive disorder in DSM-5. Psychological assessment had a boom with the need to evaluate soldiers for WWI and neuropsychological assessment soon proliferated, helping to refine the understanding of many disorders, including dementia. From the Luria and Reitan approaches, to specific screening tests and commercial test batteries for dementia evaluation, to cognitive testing within dementia treatment trials, neuropsychology has progressively helped refine the concept of dementia. As our field continues to advance and incorporate newer methods and constructs, such as neuroimaging and neuroinflammation, neuropsychology will remain a key contributor to the evaluation and remediation of dementia.

Conclusions: Alongside the historical refinement of dementia diagnoses, we can track the development of cognitive assessment procedures and appreciate the influence that neuropsychology has had in-and will continue to have-in understanding the dementia disorders.
Correspondence: Alicia I. Ford, PhD, Psychiatry and Behavioral Sciences, Oklahoma State University Center for Health Sciences, 1111 W.17th St., Tulsa, OK 74107, United States.E-mail: alicia.ford@ okstate.edu

A.E. HAHN-KETTER, D. WHITESIDE, S.J. TOWNS, L.M. GUIDOTTI BRETING, A.M. BUTTS \& O.A. SANTOS. Future Directions of Neuropsychology from a Training Perspective: Factors Affecting Training Satisfaction from the AACN Student Affairs Committee Survey of Neuropsychology Trainees.

Objective: As with any profession, individuals who comprise the current cohort of trainees are the future of neuropsychology. As such, the issues impacting their training affect all aspects of the future of the profession. A recent comprehensive survey of neuropsychology trainees (doctoral students, interns, and postdoctoral fellows) found that most respondents were satisfied with their programs, yet a significant subset endorsed dissatisfaction with training (Whiteside et al., 2016). This study explores the reasons for dissatisfaction in order to suggest areas of improvement. Participants and Methods: A total of 344 trainees completed the online survey distributed via listservs. Chi-square analyses were used to determine which training factors were associated with level of satisfaction at doctoral, internship, and postdoctoral fellowship levels.

Results: Satisfaction differed slightly across training levels. Doctoral trainees' satisfaction with their programs was significantly associated with the experience of bias against their degree program, experience of harassment by a supervisor/mentor, and/or how familiar the trainee was made regarding relevant issues in the field and steps necessary for their career. For intern and postdoctoral trainees, factors associated with satisfaction also included extent their mentor kept them informed of relevant issues and how well-educated they were about the steps toward a clinical career, but unique to this group was that quality of clinical feedback from supervisors also impacted trainee satisfaction. Conclusions: Across training levels, mentors who inform trainees about the path toward their chosen career and relevant issues in the field appeared to be consistently associated with satisfaction. As trainees mature, quality of supervision also impacts satisfaction. Findings emphasize the importance of effective mentoring beyond job responsibilities, workload, setting, etc. Increasing access to high-quality mentoring may greatly enhance training experiences among future neuropsychologists. Correspondence: Amanda E. Hahn-Ketter, Psy.D., Department of Rehabilitation Medicine, Icahn School of Medicine at Mount Sinai, One Gustave Levy Place, Box 1163, New York, NY 10029, United States. E-mail: amanda.hahn-ketter@mountsinai.org

R. KIRMSE \& U. ALFORD. Historical Approaches to Neuropsychological Assessment, Theoretical Models, Current Practices and Clinical Applications: An Exploration of Luria and Halstead Theories.

Objective: The field of neuropsychology is an integration of neurology, anatomy, physiology, chemistry, pharmacology, and psychology. Historical models of neuropsychological abilities can be attributed back to Halstead and Luria models. These models have largely influenced how current neuropsychological practices are approached. The aim of this review is to explore how these models were established, their primary goals, as well as how these models are currently applied. Specifically, the underlying aims of these theoretical models are explored alongside the evolution of their applicability to neuropsychological testing over the past several decades. A humanistic approach is explored where the individual is emphasized in relation to the aforesaid models from a biopsychosocial perspective, which is a reflection of today's emphasis of an integrated behavioral health ideal.

Participants and Methods: Material from publications, literature, and systematic reviews were gathered and reviewed from google scholar, ebscohost, pubmed, and psycinfo.

Results: The expansive history of neuropsychology is continuously influenced by interrelated fields. Studies have demonstrated the efficacy 
of both flexible and fixed approaches. The application of cross-cultural and integrated health perspectives will allow for an emphasis on the individual, in terms of their strengths and weaknesses, as well as gearing treatments towards their rehabilitation.

Conclusions: Rather than viewing brain impairments through only one of the explored models, there is a benefit to integrating both approaches in order to develop a greater understanding of the individual and injury. In unifying the traditional approaches of localizing deficits and identifying individual strengths and weaknesses, practitioners can value the use of both standardized and flexible approaches. This will ensure the efforts of modern demands on the emphasis of integrated behavioral health and promote an individualized biopsychosoical perspective to assessment and rehabilitation efforts.

Correspondence: Raquel Kirmse, MS, Psychology, University of Texas Health Science Center, 1650 S John King blvd \#2504, Rockwall, TX 75032, United States. E-mail: raquel.kirmse@uthct.edu

M. maiman, M. PEREZ, A. CARolin \& N. RAITANo-LEE. Exploring The Relationship between EF Performance BasedMeasures and Questionnaires in Neurodevelopmental Disorders (NDDs) Associated with Intellectual Disability (ID).

Objective: Executive functioning (EF) deficits characterize most neurodevelopmental disorders (NDDs), including intellectual disability (ID). However, valid EF assessment in ID is difficult due to floor effects on performance measures. The advent of parent questionnaires (1990's) as a complementary approach represented a step forward for this group. However, existing research suggests non-significant to low correlations between these methods. This poster aims to (A) provide a historical review of EF assessment in NDDs associated with ID and (B) extend the findings of a recent review (Toplak et al., 2013) on relations between EF performance and questionnaire measures to include NDDs associated with ID.

Participants and Methods: A literature review was conducted on (A) the history of EF assessment in NDDs associated with ID and (B) studies on relations between these assessment methods in NDDs associated with ID.

Results: Historically, neuropsychologists relied almost exclusively on performance-based EF measures. However, concerns about their ecological validity led to questionnaire development. Unfortunately, systematic reviews of the relations between these methods (e.g., Toplak et al., 2013) report non-significant to low correlations. The current poster extends existing reviews by summarizing research for three NDDs associated with ID: Autism, Fetal Alcohol Syndrome, and Down syndrome.

Conclusions: Consistent with Toplak et al (2013), several but not all studies related to the above-mentioned disorders have reported nonsignificant to low correlations between EF performance measures and questionnaires. These findings are discussed within the context of unique challenges of EF assessment in youth with ID and the potential utility of EF questionnaires for this group.

Correspondence: Moshe Maiman, B.A., Psychology, Drexel University, 4700 City Ave. Apt 6307, Philadelphia, PA 19131, United States. E-mail: mainman89@gmail.com

M. maiman, a. CARolin, M. PEREZ \& N. RAitano-LEE. The History of Assessment in Individuals with Intellectual Disability: Problems. Solutions. Future Directions.

Objective: Despite intellectual disability's (ID) prominent role in the history of modern assessment, there is a growing consensus in the field that current neuropsychological measures are not adequate to quantify individual differences among ID groups of varying severity (BerryKravis et al., 2013). This investigation aims to (a) provide a historical review of assessment in ID, (b) describe challenges with standardized testing in ID, and (c) describe areas of need for future research.

Participants and Methods: A literature review was conducted on (a) the history of ID and the assessment of intelligence, (b) current efforts to measure cognitive functioning in ID, with a focus on the limitations of current assessment methods and (c) alternative methods to measure the severity of cognitive impairments across ID groups.

Results: A number of challenges were revealed, most noticeably floor effects on neuropsychological testing (Edgin et al., 2010). This was particularly apparent on EF tasks (Hooper et al., 2008). Solutions in the literature include applying an alternative approach to IQ score estimation (Hessl et al., 2009), (b) comparing neuropsychological performance to mental rather than chronological age (Lee et al., 2011), and (c) developing new instruments and gathering normative data that are specific to individuals with ID.

Conclusions: Strides have been made in ID assessment; however, concerns remain. This is especially problematic for treatment research, given the need to carefully measure change in cognitive abilities in order to evaluate the efficacy of both pharmacological and behavioral interventions.

Correspondence: Moshe Maiman, B.A., Psychology, Drexel University, 4700 City Ave. Apt 6307, Philadelphia, PA 19131, United States. E-mail:mainman89@gmail.com

T.L. VARGO. Byron P. Rourke: Contributions to the Field of Pediatric Neuropsychology.

Objective: Byron P. Rourke, Ph.D. was an eminent clinical neuropsychologist who has contributed immensely to the field of pediatric neuropsychology. He has served as co-founder and co-editor of Journal of Clinical Neuropsychology, The Clinical Neuropsychologist, and Child Neuropsychologist as well as a consulting editor for The Journal of Child Neurology. His research endeavors have greatly increased our understanding of learning disorders and highlighted the underlying differences in brain functioning that impact learning. This biographical review will summarize his numerous accomplishments and contributions to the field of pediatric neuropsychology.

Participants and Methods: The literature will be reviewed to summarize Byron Rourke's main contributions to the field of pediatric neuropsychology and the learning disorder community. The work of his collaborators and mentees will also be reviewed.

Results: Rourke and his colleagues identified the characteristics of Nonverbal Learning Disorder (NLD). Rourke's neurodevelopmental model of NLD seeks to explain the underlying brain differences that may contribute to this syndrome. His efforts to explain the neuropsychological underpinnings of learning disorders have shaped the field by emphasizing the link between brain functioning and behavior rather than purely academic achievement. His work has propelled other researchers to examine the neuropsychological bases of other clinical syndromes in children. This has guided psychologists in differentiating NLD from other distinct syndromes with some similar symptoms, such as Autism Spectrum Disorder.

Conclusions: Rourke's innovative research has been integral in establishing the sub-specialty of pediatric neuropsychology. More recent advances in neuroimaging techniques may further elucidate the neural correlates of learning disorders among other neurodevelopmental disorders. This may aid the development of more effective intervention strategies and cognitive training programs for children with learning disorders.

Correspondence: Tracy L. Vargo, Palo Alto University, 1731 Cleveland Avenue, San Jose, CA 95126, United States. E-mail: tvargo@paloaltou. edu

\section{HIV/AIDS/Infectious Disease}

M. AGHVinian, A. Villegas, C. GONEN, K. ALVAREZ, M. GARCIA, A. THAMES \& A. ARENTOFT. Quality of healthcare is associated with antiretroviral regimen neuropenetrance and neuropsychological outcomes among HIV+ adults.

Objective: Combined antiretroviral (ARV) therapy is now the standard of care in the treatment of HIV. However, not all patients receive 
high-quality care, and significant health disparities exist within HIV (Gebo et al., 2005; Keruly et al., 2002). Yet it is unclear whether this affects the types of medications prescribed, particularly in terms of neuropenetrance. This study explores how healthcare quality is associated with CNS Penetration Effectiveness (CPE; Letendre et al., 2010) and neuropsychological outcomes.

Participants and Methods: $21 \mathrm{HIV}+$ adults (90\% male; 57\% African American, 43\% non-Hispanic white; age $=53.80 \pm 8.96$, education $=14.05 \pm 2.18)$ completed a comprehensive neuropsychological (NP) battery and neuromedical evaluation, including the "Quality of Care Through the Patient's Eyes" questionnaire-HIV version (QUOTE-HIV) as part of a pilot study.

Results: Preliminary results showed that participants on low CPE $(<8)$ regimens reported significantly lower overall healthcare quality based on QUOTE-HIV $(91.00 \pm 5.71)$ compared to those with high CPE regimens $(100.10 \pm 3.57 ; p<.01)$. Among the three QUOTE-HIV dimension scores, lower professional performance (i.e., performance of the HIV healthcare provider) was significantly associated with worse NP deficit scores (DS) in Attention $(r=-.46, p=.04)$ and Memory $(r=-.61$, $p<.01)$, with a trend for Global DS $(r=-.41, p=.07)$. The remaining dimensions, attitude of the professional and organization of care, were not associated with NP deficits scores.

Conclusions: HIV+ individuals receiving lower quality healthcare were prescribed less neuropenetrant ARV medications. Additionally, worse performance of the HIV healthcare provider, specifically, was associated with worse NP functioning. While further research is being conducted to clarify the nature of these relationships, our preliminary results suggest that quality of care may be a significant factor in HIV-treatment-related disparities.

Correspondence: Maral Aghvinian, B.A., Psychology, California State University, Northridge, 18111 Nordhoff St., Northridge, CA 91330, United States. E-mail:maghvinian@gmail.com

M. ARCE RENTERIA, V.A. GUZMAN, E. TELL, V. DEL BENE, U.S. CLARK, D. BYRD, S. MORGELLO \& M. RIVERA MINDT. Characterization of Neurocognitive Intra-Individual Variability Among HIV+ Adults With and Without Current Cocaine Use.

Objective: Intra-Individual Variability (IIV) in neurocognitive (NC) abilities is associated with subtle changes in frontostriatal functioning and can be indicative of subsequent declines in NC functioning. IIV can be measured in various ways, cross-sectionally such as across scores on a NC battery (i.e., NC dispersion) or across time (i.e., Reaction Time IIV). Given that HIV and active cocaine use can independently affect frontostriatal circuits associated with NC dysfunction, this study sought to characterize diverse measures of IIV within a sample of HIV+ adults with and without recent cocaine use. It was hypothesized that greater IIV (NC dispersion; RT IIV) would be associated with recent cocaine use, greater NC dysfunction, and poorer immunological function.

Participants and Methods: Thirty HIV+ adults (67\% male; mean age $=54$ yrs $[S D=7.4]$; mean education $=12$ yrs $[S D=3]$; Cocaine $+n=16$ ) completed comprehensive neuromedical (CD4 count, viral load) NC, and substance use evaluations, as well as several computerized reaction time (RT) tests (Choice RT, Go/NoGo, Stop-Signal). Cocaine+ was defined as cocaine use within the past 30 days or via positive urine toxicology. Intra-individual standard deviations were calculated for the $\mathrm{NC}$ battery and each of the RT tests.

Results: Cocaine+ and Cocaine- groups did not differ significantly on any IIV measure (RT IIV; NC dispersion; all $p$ 's $>.10$; $d$ 's range .04-.31). $\mathrm{NC}$ dispersion was associated with nadir CD4 $(r=-.46, p=.01)$ across the whole sample.

Conclusions: A significant effect of recent cocaine use on IIV was not observed in this sample of HIV+ adults. This finding adds to the literature demonstrating the reduced impact of active drug use on NC functioning among HIV+ adults. However, similar to prior research, greater IIV (NC dispersion) was associated with peak immunological dysfunction. Future studies should examine links between IIV and markers of
HIV disease progression, as they relate to consequent $\mathrm{NC}$ and functional deficits in HIV+ adults.

Correspondence: Miguel Arce Renteria, B.A., Fordham University, 685 Academy St, Apt 27, New York, NY 10034, United States. E-mail: marce3@fordham.edu

J.L. BELTRAN, S.P. WOODS, M. VERDUZCO, S. LOFT, E.E. MORGAN \& T.V. SMITH. Time-Based Prospective Memory Deficits Are Uniquely Associated with Medication Management Errors in Older Adults Living with HIV.

Objective: Prior research suggests that deficits in time-based Prospective Memory (PM), as measured by clinical tasks, are associated with antiretroviral non-adherence in HIV-infected populations. The current study extends that literature by using experimental measures of timebased PM and medication management skills to determine the cognitive and functional mechanisms of this association among older HIV+ adults. Participants and Methods: Forty-five HIV+ older adults completed a computerized time-based PM experiment in which they were asked to press a button at 2,5 , and 9 minutes during an ongoing language task. From this PM measure we derived measures of accuracy and time monitoring. Participants also completed a mock "pill dispensing" task to assess their ability to manage medications.

Results: Multiple regressions controlling for demographics and global cognitive impairment showed that time-based PM accuracy $(p<.01)$, but not time-monitoring ( $p>10)$, was independently associated with omission errors on the medication management task. PM accuracy was not associated with medication errors of commission ( $p>.10)$.

Conclusions: These preliminary findings suggest that time-based PM accuracy, perhaps as a function of cue detection processes, plays a significant role in medication management skills among older adults living with HIV. These data call attention to the influence of PM on everyday functioning, and specifically highlight the applicability of assessing PM impairment when evaluating medication management skills among older HIV+ individuals.

Correspondence: Jessica L. Beltran, Psychiatry, University of California, San Diego, 220 Dickinson St, San Diego, CA 92103, United States. E-mail: jlbeltran@ucsd.edu

M.P. FAYTELL, C. NEIGHBORS, P.J. MASSMAN, R. CRUTCHLEY \& S.P. WOODS. Investigation of the Interrelationships Between Fatigue, Memory Impairment, and Antiretroviral Adherence in HIV Disease.

Objective: Fatigue and memory impairments are each highly prevalent in HIV disease (e.g., Heaton et al., 2011), and can adversely influence adherence to antiretroviral therapy (ART). However, the interrelationships between fatigue, memory impairments, and ART adherence remain unelucidated. To investigate these interrelationships, the present study adopted the Aaronson et al. (1999) model of fatigue, which predicts that fatigue depletes compensatory resources, thus leaving HIV-positive individuals more vulnerable to the impact of memory impairments on adherence.

Participants and Methods: A sample of 177 participants completed a brief clinical neuropsychological and neuropsychiatric assessment including measures of memory, fatigue, and 30-day electronic monitoring of ART adherence. Path analysis via SEM was used to determine whether fatigue conferred a stronger risk of worse adherence through a direct or an indirect path (i.e., via strategy use). Depression was evaluated as a covariate due to well-established associations with both fatigue and ART adherence.

Results: The best-fitting model included direct paths between fatigue, memory, and strategy use with ART adherence, and an indirect path between fatigue and ART adherence via strategy use (RMSEA=.034). Higher fatigue, worse memory, and greater strategy use were strongly associated with poorer ART adherence. Higher fatigue was also associated with greater strategy use. These relationships persisted when depression was included as a covariate. 
Conclusions: The effect of fatigue on ART adherence was independent from memory, and was not better explained by depression. Unexpectedly, higher fatigue also predicted greater strategy use, suggesting that awareness of diminished resources may prompt HIV-positive individuals toward greater reliance on strategy use. Future investigation of strategies employed to bolster adherence in the presence of fatigue is warranted. Correspondence: Marika P. Faytell, B.A., Psychology, University of Houston, 2925 Helena Street, Houston, TX 77006, United States. E-mail:mpfaytell@uh.edu

S. FERNANDEZ-GONZALO, M. SALA RODÓ, M. GARAU ROLANDI, M. NAVARRO \& M. JÓDAR. Quality of life and emotional state improve after hepatitis $\mathrm{C}$ curative therapy with direct antiviral agents.

Objective: To study the emotional state and the auto- perceived quality of life improvement in HIV-HCV co-infected patients after the cure of HCV with direct antiviral agents. Additionally, we explore if this enhancement is related with biological parameters or with cognitive improvement.

Participants and Methods: $16 \mathrm{HIV}-\mathrm{HCV}$ patients were included. Digit forwards, digit backwards and CPT reaction time were used to assess attention and working memory. Learning, short and long term memory were assessed with the Rey Auditory Verbal Learning Test (RAVLT) 5, RAVLT 3' and RAVLT 20'. Executive function were assessed with TMT A \& B, Stroop test and FAS. The SF-36 were used to assess the quality of life, and HADS to assess Anxiety and depression. HIV related variables: CD4 and nadir, CD8, Viral load. HCV-related: genotype, RNA-VHC, fibrosis.

Results: Depression symptoms significantly improved after treatment $(\mathrm{M} 1=5,81, \mathrm{SD} 1=3,17-\mathrm{M} 2=2,75, \mathrm{SD} 2=2,15 ; \mathrm{P}=0.003)$. Also the general score of the SFS-36 significantly enhanced. (M1=52.67, $\mathrm{SD} 1=25.97-\mathrm{M} 2=63.88, \mathrm{SD} 2=25.22 ; \mathrm{P}=0.03)$. A sub analysis of the different SFS-36 indexes showed a significant change on the Vitality section $(\mathrm{M} 1=59.53, \mathrm{SD} 1=14.99-\mathrm{M} 2=67.19, \mathrm{SD} 2=14.76 ; \mathrm{P}=0.04)$ and a trend to signification in Social Role Functioning (M1=66.80, $\mathrm{SD} 1=28.29-\mathrm{M} 2=84.38, \mathrm{SD} 2=20.16 ; \mathrm{P}=0.06)$

No significant relationship were observed between the biological variables and the cognitive improvement with the functional and emotional changes.

Conclusions: HIV-HCV infected patients improve their quality of life and emotional state after hepatitis C cure. Nevertheless, we did not find any relation between this improvement and biological or cognitive variables. The small size of our sample has to be considered.

Correspondence: Sol Fernandez-Gonzalo, PHD, Barcelona, Spain. E-mail:msfernandez@tauli.cat

C. HARDCASTLE, T. HENDERSHOTT, R. FAMA, E. MULLEROEHRING, E.V. SUllivan, A. PFEFFERBAUM, K. POSTON, H. BRONTË-STEWART \& T. SCHULTE. Cognitive and Motor Functioning in Older Adults with HIV: A Comparison with Parkinson's Disease.

Objective: Combination antiretroviral therapy (cART) has extended the life expectancy decades for HIV infection survivors. A new challenge looms with a potential synergy between immunosenescence and HIV-related inflammatory processes that may increase susceptibility to HIV-related degradation in striatocortical pathways, as is observed in Parkinson's disease (PD), with untoward effects in selective cognitive and motor functions.

Participants and Methods: We administered tests of executive functioning (EXF), information processing (IP), episodic memory (MEM), visuospatial (VS), and motor (MOT) processes to compare the pattern and extent of impairments of 15 HIV (47-71 yrs) and 12 PD (49-79 yrs) on dopaminergic medication relative to 15 normal controls (NC; 46-75 yrs). All scores were standardized on $\mathrm{NC}($ mean $=0 \pm 1 \mathrm{SD})$ for composite score construction. A HAND (HIV-Associated Neurocognitive Disorders) score was derived for HIV and PD.
Results: HIV scored lower than NC on EXF, MEM, and VS, whereas PD scored lower than HIV and NC on MOT. The HAND score, based on impairment in $\geq 2$ domains, categorized $9 \mathrm{HIV}$ and 7 PD as impaired. In HIV, 5 were impaired on 4 and 1 was impaired on all 5 composites, whereas 2 of the 12 PD were impaired on 4 composites and none was impaired on all composites. The majority of HIV $(>50 \%)$ scored more than $1 \mathrm{SD}$ below NC on each cognitive composite score, whereas fewer (17\%-42\%) PD scored at this level. MOT impairment occurred in $33 \%$ of HIV and $67 \%$ of PD.

Conclusions: We identified unique patterns of cognitive and motor impairment in aging HIV and PD patients. Although similar striatocortical pathways have been implicated, HIV was more severely compromised in cognitive functions and PD on medication in motor functions. The motor findings in HIV suggest increased susceptibility of motor system involvement with immunosenescence in HIV in the cART era. Support: AA023165, AA017347, AA017168, NS075097, AG047366, Michael J. Fox foundation for Parkinson's research

Correspondence: Cheshire Hardcastle, CA 94306, United States. E-mail: cheshire.hardcastle@sri.com

D. SMITH, J. SCHNEIDER \& S.J. HUNTER. Confirmatory Factor Analysis of the Behavioral Rating Inventory of Executive Functioning (BRIEF) in Young Black Men who have Sex with Men (YBMSM).

Objective: Although the psychometric properties of the BRIEF have been extensively evaluated in healthy and mixed clinical samples, little is known about whether its structure is consistent across all clinical populations, particularly urban African-American emerging adult men. The application of this scale to evaluate EF in clinical populations is believed to benefit from a broader examination of the test structure in a diverse population, in order to confirm its reliability in research and clinical practice.

Participants and Methods: We recruited 618 YBMSM, ages 16-29, through respondent-driven sampling in Chicago and administered the BRIEF as part of a longitudinal survey of HIV risk behavior. The factor structure of the BRIEF was then evaluated using confirmatory factor analyses of subscores, replicating the procedure of Gioia, Isquith, Retzlaff and Espy (2002), and Roth, Lance, Isquith, Fischer and Giancola (2013), by examining one, two, and three-factor models and their fit with this sample.

Results: Results of YBMSM participants on the BRIEF closely aligned with those obtained in prior analyses. Use of a two-factor model, Behavioral Regulation and Metacognition, fit data significantly better than a unity model. Unlike prior research, creation of a three-factor model did not appear to significantly improve model fit.

Conclusions: Results support the multidimensional structure of the BRIEF, and the utility of the current conceptualization of the BRIEF for a clinical population such as YBMSM, who differ in meaningful ways from those with which the test was initially standardized. Findings highlight the reliability of the BRIEF for use in research and clinical practice with a group of urban African-American emerging adult males, and support comparisons across cultural groups when exploring EF development and its role in risk behavior and decision making.

Correspondence: Scott J. Hunter, PhD, Psychiatry \& Behavioral Neuroscience, University of Chicago, UCM PBN, MC 3077, 5841 S Maryland Ave, Chicago, IL 60637, United States. E-mail: shunter@ uchicago.edu

N. FRANCIS, D. SMITH, J. KAYLEGIAN, J. SCHNEIDER \& S.J. HUNTER. EF, Frequency of Marijuana Use, and HIV Risk Reduction in Young Black Men who have Sex with Men (YBMSM). Objective: YBMSM are disproportionately affected by HIV in the US. Marijuana use and executive functioning (EF) have been separately studied as moderators of HIV risk behavior, but limited research has investigated these factors together in YBMSM. We sought to examine 
how marijuana use and EF, both separately and together, influence decision making about HIV risk reduction in high-risk men.

Participants and Methods: 618 YBMSM were recruited through respondent-driven sampling in Chicago. $70.9 \%$ reported intermittent-to-at least daily use of marijuana. Participants were administered the BRIEF and IGT as part of a longitudinal survey of HIV risk behavior. The relationship between EF, frequency of marijuana use, and HIV risk reduction behavior was examined using logistic regression.

Results: BRIEF scores were variable but average range for the sample. No association was found between frequency of marijuana use and HIV risk reduction, while the decision to reduce number of partners was negatively associated with BRIEF BRI (OR 1.04; 95\% CI: 1.01-1.08), MI (OR 1.04; 95\% CI 1.01-1.07), and GEC (OR 1.01; 95\% CI: 1.00-1.04). Conversely, EF was positively associated with the odds of staying with one partner as HIV risk reduction. Higher BRI (OR 1.03; 95\% CI: 1.011.05), MI (R 1.02; 95\% CI: 1.01-1.03), and GEC (OR 1.01; 95\% CI: 1.00-1.02) scores predicted an increased odds of heavier marijuana use. Conclusions: Results suggest that level of EF impacts decision making regarding HIV risk reduction behavior, specifically choices about number of partners and staying with one specific partner. EF is also influenced by the frequency of marijuana use, although further study is required regarding directionality of influence with this population. Based on our results, we argue that further research should explore EF in relationship to developing individualized HIV prevention strategies, within a context of expected marijuana use.

Correspondence: Scott J. Hunter, PhD, Psychiatry \& Behavioral Neuroscience, University of Chicago, UCM PBN, MC 3077, 5841 S Maryland Ave, Chicago, IL 60637, United States.E-mail: shunter@ uchicago.edu

J.E. IUDICELLO, A. GONGVATANA, D. FRANKLINJR, D. ROSARIO, R.J. ELLIS, R.K. HEATON, I. GRANT \& S.L. LETENDRE. Profiles of HIV-Associated Neurocognitive Impairment in the Context of Low and High Blood-Brain Barrier (BBB) Permeability.

Objective: Despite more effective antiretroviral therapy (ART), neurocognitive impairment (NCI) remains highly prevalent in HIV. One explanation is that the effects of ART on neurocognitive (NC) outcomes vary depending on the permeability of the BBB. This study explored this notion by examining the profiles and predictors of HIV-associated NCI in the context of low and high BBB permeability.

Participants and Methods: Participants included169 HIV+ primarily middle-aged individuals without confounding comorbidities who were on ART $\geq 1$ month. All participants were administered a comprehensive neuromedical and neuropsychological assessment. The CSF/ serum albumin ratio (CSAR) was used as an index of BBB permeability (high=more permeable). Clinical ratings were derived to assess global and individual NC domain impairment.

Results: Recursive partitioning revealed that both Low $(<3.5)$ and High $(\geq 6.1)$ CSARs predicted global NCI. Significantly higher proportions of global NCI were observed in the Low and High CSAR groups $(56 \%$ and $65 \%$ ) relative to the Mid CSAR group $(38 \% ; p s<0.05)$. The High CSAR group had higher proportions of NCI relative to the Low and Mid CSAR groups within the motor ( $48 \%$ vs $25 \%$ and $17 \%$; ps $\leq 0.05$ ) and speed of information processing domains (30\% vs 12 and $13 \%$; ps $<0.01$ and 0.08). Both High and Low CSAR groups had higher proportions of NCI in the attention/working memory domain relative to the Mid CSAR group ( $44 \%$ and $41 \%$ vs $23 \%$; ps $=0.06$ and 0.02 ). NCI within the high CSAR group was associated with ARV and vascular risk factors across domains.

Conclusions: These findings suggest that both low and high CSARs are associated with NCI in HIV, and that the underlying etiologies and profiles of NCI differ in the context of low and high BBB permeability. NCI in the context of low BBB permeability may occur due to ART ineffectiveness, whereas NCI in the context of high BBB permeability may be related to vascular disease and/or neurotoxicity. These findings highlight the potential role of the BBB in the persistence of NCI in HIV.
Correspondence: Jennifer E. Iudicello, M.S., Psychiatry, HIV Neurobehavioral Research Center, $150 \mathrm{~W}$ Washington St, 2nd Floor, San Diego, CA 92103, United States.E-mail: jüdicello@ucsd.edu

M. JÓDAR, S. FERNANDEZ-GONZALO, M. GARAU ROLANDI \& M. SALA RODÓ. Cognitive function improvement in HIV-HCV co-infected Patients after treatment for $\mathrm{HCV}$ with direct antiviral agents (DAA).

Objective: To study the cognitive improvement after HCV cure by direct antiviral agents (DAA) in HIV-HCV co-infected patients. As a secondary objective we explore the relationship between biological HIV-HCV variables and the cognitive changes.

Participants and Methods: 16 patients HIV-HCV co-infected were included in the study. Digit forwards, digit backwards and CPT reaction time were used to assess attention and working memory. Learning, short and long term memory were assessed with the Rey Auditory Verbal Learning Test (RAVLT) 5, RAVLT 3' and RAVLT 20'. Finally, executive functions were assessed with TMT A \& B, Stroop test and FAS. We analyzed immunological and HIV-associated parameters (CD4 and CD8 lymphocyte counts and CD4 Nadir, HIV-RNA) and HCV-RNA and genotype. All patients were evaluated 1 week before and 3 months after therapy, when patients were free of HVC virus (Sustained viral response week 12 ).

Results: After treatment patients significantly improve long-term verbal memory $(\mathrm{M} 1=6.69, \mathrm{SD} 1=1.78-\mathrm{M} 2=8.19, \mathrm{SD} 2=2.66 ; \mathrm{P}=0.03)$ Stroop color $(\mathrm{M} 1=69.8, \mathrm{SD} 1=10.92-\mathrm{M} 2=74.31, \mathrm{SD} 2=11.03$; $\mathrm{P}=0.003)$ and verbal fluency $(\mathrm{M} 1=28,06, \mathrm{SD} 1=12.11-\mathrm{M} 2=31.88$, $\mathrm{SD} 2=11.78 ; \mathrm{P}=0.006)$. A trend to signification was observed between the HCV genotype 4 and the improvement in long-term verbal memory $(\mathrm{X} 2=5.66 ; \mathrm{P}=0.06)$.

Conclusions: Cognitive functions improve in patients with HIV co-infected with HCV, after antiviral treatment and complete HCV cure. The HIV genotype 4 could be a variable related with the better cognitive function in this patients.

Correspondence: Merce Jódar, PhD, Heath and Clinical Psychology, Universitat Autònoma de Barcelona, Dpmt. Clinical and Healh Psychology, Campus de Bellaterra, Edifici B. bellaterra, Barcelona 08193, Spain.E-mail:merce.jodar@uab.cat

\section{J. JONES, T. KUHN, Z. MAHMOOD, C. HINKIN \& A. THAMES. Apathy is Related to Quality of Life in HIV-infected Adults.}

Objective: Apathy (a lack of goal-directed motivation and behavior) is a common symptom in many neurological disorders including HIVAssociated Neurocognitive Disorder. Although apathy is associated with meaningful outcomes in HIV, such as cognitive impairment, there has been a dearth of research linking apathy to other relevant outcomes, including health related quality of life (HRQoL). This study examined the relationship between apathy, depression and HRQoL among HIV+ adults.

Participants and Methods: The sample included $43 \mathrm{HIV}+$ participants and 45 HIV negative controls. Participants completed self-report questionnaires assessing apathy (Frontal Systems Behavior Scale- apathy subscale), HRQoL (Quality of Life Enjoyment and Satisfaction Questionnaire) and depression (Beck Depression Inventory II). Hierarchical regression analyses were computed to examine the impact of apathy on HRQoL while controlling for potential confounds such as depression, cognitive impairment, substance use history, HIV disease severity, and demographics.

Results: Pearson's correlations revealed that HRQoL was significantly related to apathy $(\mathrm{r}=-.570, p<.001)$ among individuals with HIV. Regression analyses revealed that HRQoL was only related to apathy $(p<.001)$, and not other possible confounding variables (demographics, disease severity, depression, substance use history and general cognitive functioning). Both apathy and depression were uniquely related to HRQoL among HIV negative controls. 
Conclusions: Findings provide evidence that apathy is an independent contributor to HRQOL among individuals living with HIV. Future interventions should consider focusing on apathy as a meaningful patient-centered target.

Correspondence: Jacob Jones, Ph.D., UCLA, 760 Westwood Plaza, Los Angeles, CA 90024,United States.E-mail:jacobjones@mednet.ucla.edu

N. KABUBA, J. MENON, D. FRANKLIN JR, R.K. HEATON \& K. HESTAD. HIV and AIDS Associated Neurocognitive Functioning In Zambia- A Gender Perspective.

Objective: Human immunodeficiency virus (HIV) infection and acquired immune deficiency syndrome (AIDS) are frequently associated with neurocognitive impairment (NCI). However, few studies have examined the interrelationship between gender and NCI in the HIV and AIDS population.

Participants and Methods: This cross sectional study examined the neurocognitive functioning of HIV infected male and female adults from urban Zambia, a country in sub-Saharan Africa. Participants included 266 HIV seropositive $(\mathrm{HIV}+)$ adults [males $(\mathrm{n}=107)$ and females $(\mathrm{n}=159)$ ]. Participants completed neurocognitive (NC) assessment by means of a comprehensive neuropsychology test battery using normative data from $324 \mathrm{HIV}$ seronegative (HIV-) controls. The norms corrected for effects of age, education and gender in the general population, and the test battery measures domains of: Attention/Working Memory, Executive Function, Verbal Fluency, Processing Speed, Verbal and Visual Episodic Memory, and fine Motor Skills.

Results: An overall comparison of the HIV+ male and female participants yielded no statistically significant differences. ANCOVA results controlling for disease characteristics, showed that HIV+ female participants were more impaired than the males on Recall scores $[\mathrm{F}(1,117)$ $=9.70, \mathrm{p}=.002$, partial $\left.\mathrm{eta}^{2}=.077\right]$. The females also evidenced a trend towards greater impairment on Learning efficiency $(p=.015)$.

Conclusions: The findings suggest there are gender related differences in NCI after controlling for disease characteristics. It was observed that although the HIV+ females enjoyed better health compared to their $\mathrm{HIV}+$ male counterparts, they still had worse performance on the neuropsychological tests. This implies that HIV may have more NC consequences for Zambian females than males.

Correspondence: Knut Hestad, Trondheim, Norway. E-mail: knut. hestad@hihm.no

M. KEUTMANN, R. GONZALEZ, P.M. MAKI, L. RUBIN \& E. MARTIN. Sex and HIV Serostatus Differences in Executive Mechanisms of Verb Fluency Among Drug Users.

Objective: Action (verb) fluency (AF) is sensitive to HIV-associated neurocognitive impairment and is a critical predictor of activities of daily living. AF is strongly correlated with executive function, critically dependent on the integrity of prefrontal-striatal circuitry and predicts CSF astrocytosis. We recently reported sex and HIV serostatus differences in executive function among substance dependent individuals (SDIs). In this study we extended these findings by comparing AF performance among male and female HIV+ and HIV - SDIs and investigating potential sex differences in the executive component of AF.

Participants and Methods: A group of $149 \mathrm{HIV}+$ and 329 EIA-verified HIV-SDIs (primarily cocaine and alcohol dependent), 85\% African American and $70 \%$ female, completed a measure of action fluency requiring speeded retrieval of activity names, and a measure of decision making under risk (Game of Dice Task) as part of a larger study of sex differences in neurocognitive effects of HIV and drugs of abuse. All participants were verified abstinent during testing.

Results: A $2 \times 2$ ANCOVA controlling for estimated Verbal IQ showed a significant Sex $x$ HIV Serostatus interaction, $p=.002$. The HIV + men outperformed HIV+ women and HIV - men $(p=.05)$. Action Fluency scores correlated significantly with GDT net scores among $\mathrm{HIV}+$ men $(r=.48, p<.001)$, but did not correlate significantly for the other groups ( $\mathrm{p}>.05$ for all tests).
Conclusions: Our results suggest that different cognitive mechanisms contribute to action fluency performance among HIV + male and female SDIs, raising the question of sex differences in the pattern of effects of HIV on prefrontal-striatal circuitry. Supported by HHS R01 DA12828 to E.M. Martin

Correspondence: Michael Keutmann, M.A., Dept of Psychology, University of Illinois, Chicago, IL 60612, United States. E-mail: mkeutm2@uic.edu

V.M. KORDOVSKI, S.P. WOODS \& M. VERDUZCO. Frequency and Correlates of Low Health Literacy in HIV-Associated Neurocognitive Disorder.

Objective: Health literacy entails the ability to understand and apply health-related information to make informed medical decisions. Low health literacy is thought to be common among individuals with HIV, but we know little about the contribution of HIV-associated neurocognitive disorders (HAND) to this public health problem.

Participants and Methods: Participants included $43 \mathrm{HIV}+$ adults with HAND, $50 \mathrm{HIV}+$ adults without HAND, and 42 HIV- comparison participants who completed a comprehensive research neuropsychological battery, neuromedical evaluation, and five well-validated measures of health literacy: Test of Functional Health Literacy in Adults (TOFHLA), Newest Vital Sign (NVS), Rapid Estimate of Adult Literacy in Medicine (REALM), Single Item Literacy Screener (SILS), and the Short Assessment of Health Literacy (SAHL). Published raw cutoff scores were used to classify participants on each health literacy measure and participants who scored below these cut-scores on one or more measures were classified as having low health literacy.

Results: Rates of low health literacy were highest in the group of HIV+ adults with HAND (HAND+= $51 \%$, HAND- $=16 \%$, HIV-: $\left.31 \%, \chi^{2}<.01\right)$. A logistical regression controlling for education revealed that HAND+ adults were nearly 5 times more likely to have low health literacy than HAND- adults (Odds Ratio: 4.9 [1.9-13.8]). Among HAND+ adults, low health literacy was associated with lower scores on delayed memory, executive functions, and health-related physical quality of life, as well as the presence of an AIDS diagnosis (ps<.05).

Conclusions: These findings suggest that HAND is an independent contributor to reduced health literacy, particularly on assessments of self-sufficiency in reading health-related material (i.e., SILS) and of the comprehension and integration of health information (i.e., NVS). Future studies are needed to develop targeted literacy interventions in an effort to enhance health outcomes and quality of life for individuals with HAND.

Correspondence: Victoria M. Kordovski, Psychology, University of Houston, 126 Heyne Building, Suite 204, University of Houston, Houston, TX 77021, United States.E-mail:vkordovski@uh.edu

A. LEVINE, E. MARTIN, N. SACKTOR, C. MUNRO \& J. BECKER. Suboptimal effort in HIV neuropsychological research studies: does it influence estimated prevalence rates of $\mathrm{HIV}$-associated neurocognitive disorders (HAND)?

Objective: Prevalence rates of HAND are determined in large cohort studies that rely on volunteers who receive reimbursement in return for their participation. Anecdotal evidence suggests that many cohort participants do not put forth adequate effort on neurocognitive testing, thereby potentially inflating prevalence estimates. We characterized suboptimal effort in the Multicenter AIDS Cohort Study, including reasons, predictors, and effect on HAND prevalence estimates.

Participants and Methods: After completing neurocognitive testing, 995 participants ( $430 \mathrm{HIV}-, 565 \mathrm{HIV}+$ ) completed the Visual Analogue Effort Scale (VAES). Those with $<100 \%$ then indicated the reason(s) for suboptimal effort. K-means cluster produced 3 groups: Full effort $($ mean $=97 \%)$, Moderate Effort $(79 \%)$, and Poor Effort $(51 \%)$. HAND rates and other variables were compared between these groups. Linear regression examined predictors of VAES score. 
Results: The average VAES score was 91.4 ( $\mathrm{sd}=13$; range 20-100), with no difference between HIV - and HIV+ participants. Among individuals who indicated suboptimal effort, the most common reasons were "tired" (42\%) and "distracted" (36\%). Effort groups had similar age and depression severity. The Poor Effort group had greater ANI and MND diagnosis (both $31 \%$ ) as compared to the Full Effort group $(12 \%$ and $13 \%$ ) and the Moderate Effort group (both 19\%). Finally, we determined predictors of Effort level. Of education, employment status, race, HIV status, and depression, higher education and being employed were significant predictors of effort.

Conclusions: A relationship between effort level and HAND severity was found. This information can be used to implement methods for improving effort in cohort studies and reconsidering HAND prevalence rates.

Correspondence: Andrew Levine, Ph.D., Neurology, UCLA, 1050 Roswell Ave, Long Beach, CA 90804, United States.E-mail: ajlevine@ mednet.ucla.edu

\section{POSADA-SHEA. Learning and recall of emotionally-laden} words among individuals living with HIV and bipolar disorder.

Objective: Both HIV infection and bipolar disorder have been shown to affect learning and recall of verbal information without emotional valence. We previously reported that healthy individuals appeared to have difficulties learning negatively-laden information as compared to positively- or neutrally-laden information (Posada et al, 2014); however, they appeared to recall neutral information better than emotionally-laden information.

Participants and Methods: We assessed 140 individuals (HIV-/ BD- = 29; HIV-/BD+ = 28; HIV+/BD- = 27; HIV+/BD+ = 56) using the Emotional Verbal Learning Test (EVLT) to assess whether the emotional valence of words facilitates learning and recall.

The EVLT consists of three groups of four words with different emotional levels ( 4 positive, 4 negative, 4 neutral). We analyzed 3 outcome measures: learning trials, short delayed recall, and long delayed recall. Results: Participants in the HIV+/BD-, HIV-/BD+, and HIV-/BDgroups learned fewer negative words as compared to positive and neutral words $(p<0.01)$, whereas the HIV+/BD+ group learned more neutral words as compared to positive and negative words. After a short delay, $\mathrm{HIV}-/ \mathrm{BD}+$ and $\mathrm{HIV}+/ \mathrm{BD}$ - participants recalled fewer negative words $(p<0.01)$ whereas the HIV+/BD+ group recalled more neutral words $(\mathrm{p}<0.01)$. After a 20 minute delay, HIV-/BD-, HIV+/BD-, and HIV+/ $\mathrm{BD}+$ participants recalled more neutral words as compared to positive and negative words $(p<0.01)$

Conclusions: Healthy comparison participants and individuals living with HIV and bipolar disorder appear to have difficulties initially learning negatively-laden information as compared to positively- or neutrally- laden information. After a delay, however, individuals across all groups (except HIV-/BD+) recall neutral information better than information that is emotionally laden.

Correspondence: Carolina Posada-Shea, PhD, Psychiatry, Institute of Living/Hartford Hospital, 72 Windsor Rd, Brookline, MA 02445, United States.E-mail: carolina.posada@hhchealth.org

S.M. TIERNEY, S.P. WOODS, M. VERDUZCO, J.L. BELTRAN, P.J. MASSMAN \& R. HASBUN. Semantic Memory in HIVAssociated Neurocognitive Disorders: An Evaluation of the 'Cortical' vs. 'Subcortical' Hypothesis.

Objective: While HIV- associated neurocognitive disorders (HAND) have historically been characterized as a subcortical process, there is some evidence to suggest that the cortical regions that support semantic memory may also be affected. The current study examined the effects of HAND on semantic memory.

Participants and Methods: Participants were ( $\mathrm{N}=459)$ separated into three groups based on neurocognitive status defined by the Frascati criteria: HIV+ individuals with HAND $(\mathrm{n}=85)$, HIV+ individuals without HAND ( $\mathrm{n}=191)$, and HIV negative individuals $(\mathrm{n}=183)$.
All participants completed the Boston Naming Test (BNT) and the Famous Faces subtest of the Kauffman Adolescent and Adult Intelligence Test (KAIT). Analyses of errors committed on the BNT were conducted to further characterize the nature of semantic memory differences across groups.

Results: Linear regressions revealed a significant adverse effect of HAND on total scores on the BNT and the KAIT (all $p s<.01$ ). Analyses of BNT errors showed that individuals with HAND committed increased rates of semantically-related errors as compared to the other two groups (all $p$ s < .05). However, there were no group differences in rates of visually based errors, more commonly observed in subcortical diseases (all $p \mathrm{~s}>.10$ ). Findings regarding the cognitive correlates of semantic memory show that executive $\left(r_{s}=-.24, p=.02\right)$, speed of processing $\left(r_{s}=-.31, p=.004\right)$, and learning (at trend level) domains were shown to be associated with BNT performance, while only the learning domain $\left(r_{s}=-.28, \mathrm{p}=.01\right)$ was significantly related to KAIT scores within the $\mathrm{HAND}+$ group.

Conclusions: Results suggest that HAND may impose adverse effects on individuals' object naming and identification abilities and suggest that there are mild semantic deficits in HAND that parallel traditional cortical diseases such as Alzheimer's Disease.

Correspondence: Savanna M. Tierney, B.A., Psychology, University of Houston, 201 Wilcrest Dr. APT\#203, Houston, TX 77042, United States.E-mail: Savanna386@gmail.com

K.A. TRAINO, J. SNOW, A. PANACKAL, N. BIASSOU, J. BENNETT, P. WILLIAMSON, A. SUMMERS \& T. SHIRAZI. Neuropsychological functioning of HIV-negative Cryptococcus meningoencephalitis survivors.

Objective: There are few studies on cognitive functioning in HIVnegative patients who have survived Cryptococcus meningoencephalitis, a potentially fatal fungal infection of the CNS that can affect HIVpositive, but rarely HIV negative, individuals. We report on the neurocognitive functioning of $\mathrm{HIV}$-negative patients and how it relates to other illness indicators and neuroimaging.

Participants and Methods: Twenty-five immunocompetent Cryptococcus survivors that are being followed at NIH were administered a neuropsychological (NP) battery assessing memory, learning, psychomotor function, language, focused attention, executive function, and information processing. Patients also underwent brain MRI and lumbar puncture.

Results: Thirty-two percent of patients obtained a composite (demographically corrected) T-score (across all NP tests) in the bottom $16^{\text {th }}$ percentile and $12 \%$ obtained scores in the bottom $2^{\text {nd }}$ percentile, suggesting a high rate of impairment in this patient population. For individual domains, the percentage of individuals who scored below the $16^{\text {th }}$ percentile ranged from $59 \%$ for psychomotor speed to $9 \%$ for focused attention. The remaining domains showed high levels of impairment, including information processing $(45.5 \%)$, and language, learning, and memory (all 32\%). However, in relation to MRI and CSF data, few significant associations with NP results were found. On the Beck inventories, $28.6 \%$ of individuals endorsed depression and $45 \%$ endorsed anxiety at moderate to severe degrees.

Conclusions: The majority of Cryptococcus meningoencephalitis patients exhibit NP impairments, with the pattern suggestive of a frontal-subcortical syndrome. However, it remains unclear which parameters best explain observed differences in cognition. Future work should examine the relationship between markers of CNS damage and NP impairment.

Correspondence: Katherine A. Traino, B.S. Psychology, Office of the Clinical Director, National Institute of Mental Health, 10201 Grosvenor Pl, Apt 411, Rockville, MD 20852, United States. E-mail: katherine. traino@nih.gov 


\section{Invited Symposium 1. Electrical Brain Stimulation and Cognitive Disorders}

\author{
Chair: Marom Bikson
}

9:00-10:30 a.m.

M. BIKSON. Electrical Brain Stimulation and Cognitive Disorders. Invited Symposium Summary: Non-invasive electrical stimulation is investigated to remedy cognitive decline associated with a wide range of neuropsychiatric disorders and brain injury, as well as cognitive aging and neurodegenerative disease. This session focuses on one brain stimulation approach, transcranial Direct Current Stimulation (tDCS) that has emerged as a promising intervention to accelerate response to other cognitive or behavioral treatments. Applications span cognitive disorders of stroke, TBI, MS, epilepsy, chronic pain, and age related deficits. tDCS is considered sufficiently tolerated that human trials on cognitive function even include healthy volunteers. Indeed, it is work in healthy subjects that established the basic mechanisms and plausibility of applying tDCS as a clinical intervention. This session covers the technical basics, mechanisms, and applications of tDCS. Insight from animal studies and human neurophysiology indicate tDCS has the broad capacity to modulate cortical excitability and enhance ongoing plasticity. In its function as an enhancer of ongoing activity; tDCS is used in clinical trials along with cognitive or behavioral training. For example, in the treatment of age- or MS- related cognitive decline, tDCS is applied with computerized "brain games" or "cognitive training" to facilitate the neuroplastic response of brain tissue engaged by challenging cognitive tasks. In TBI and stroke rehabilitation, tDCS is used to as tool to boost the efficacy of neuro-rehabilitation therapy, again under the principle that brain plasticity activated by training will be enhanced by electrical stimulation. This session also address state-ofthe-art techniques to enhance the efficacy and deployment of tDCS including use of EEG to guide stimulation, home-base therapies, and focal stimulation with High-Definition tDCS.

Correspondence: Marom Bikson, PhD, Biomedical Engineering, The City College of New York, Steinman Hall T-403B, 160 Convent Avenue, New York, NY 10031, United States.E-mail: bikson@ccny.cuny.edu

\section{BIKSON \& D.Q. TRUONG. The Basics of tDCS: Technology and Mechanisms.}

Transcranial Direct Current Stimulation (tDCS) involves application of weak current to electrodes on the scalp for the purpose of neuromodulation. This talk places tDCS in the context of other brain stimulation techniques and then reviews the fundamental of tDCS application and its mechanisms. The technology can be made specific by 1) placing the electrodes in locations on the scalp in relation to targeted brain regions; and 2) combing tDCS with a task. Placement of electrodes to target specific brain regions is guided by computational models of current flow. Conventional tDCS uses two large electrodes to guide current across the brain. High-Definition tDCS (HD-tDCS) uses arrays of small electrodes to focus current to specific brain regions. Animal studies of DCS indicate that stimulation during ongoing synaptic plasticity can make the plasticity more effective. In this way, is a subject is learning or receiving therapy during tDCS, the effectiveness of that intervention may be enhanced. This also makes tDCS specific since only brain networks activated by the training would be enhanced by electrical stimulation.

Correspondence: Marom Bikson, PhD, Biomedical Engineering, The City College of New York, Steinman Hall T-403B, 160 Convent Avenue, New York, NY 10031,United States.E-mail: bikson@ccny.cuny.edu

\footnotetext{
A.J. WOODS. Combating Cognitive Aging and Dementia with Transcranial Direct Current Stimulation (tDCS)

The world population is rapidly aging. In the United States, the number of adults over 65 years of age is expected to double by the year 2050 .
}

Even in the absence of neurodegenerative disease, aging is associated with a trajectory of decline in thinking and memory abilities, which typically accelerates in the seventh and eight decades of life. This decline has significant impact on maintenance of independence and overall realworld functional abilities. In addition, the rate of cognitive aging is one of the best predictors of With a rapidly aging population also comes an increase in overall number of dementia diagnoses. Unfortunately, there is a paucity of interventions for combating cognitive aging and slowing the onset of dementia. Transcranial Direct Current Stimulation (tDCS) may hold particular promise in the domain, particularly when used as an adjunctive intervention. tDCS provides a non-invasive method for altering the neuroplastic response of brain tissue. Cognitive training is a method that has shown strong impact on slowing or reversing symptoms of cognitive aging, as well as some benefit in preventing dementia. By pairing tDCS with cognitive training, there is a strong potential for enhancing the efficacy of cognitive training and improving both cognitive transfer and functional benefits, as well as potential for either slowing or preventing onset of dementia. This talk 1) examines tDCS application in the context of cognitive aging and dementia (e.g., Alzheimer's disease), 2) describes ongoing clinical trial efforts attempting to combat cognitive aging using tDCS as an adjunctive intervention (e.g., the ACT Study), and 3) discusses important future directions for translation of tDCS into a common and effective clinical tool.

Correspondence: Adam J. Woods, PhD, Department of Aging and Geriatric Research, Center for Cognitive Aging and Memory, McKnight Brain Institute, University of Florida, 2004 Mowry Rd, Office 3118, Gainesville, FL 32610,United States.E-mail: ajwoods@ufl.edu

\section{L.E. CHARVET \& M. SHAW. At-Home Access: Extending Clinical} Trials of Cognitive Remediation and tDCS through Remote Supervision.

Pairing tDCS with cognitive remediation holds promise for greater and more enduring benefit. Research is greatly needed to provide guidelines for safe and optimal use. With rapidly evolving technology that is increasingly available to consumers, the possibility of home access is growing. The majority of individuals living with cognitive disorders are not able to repeatedly travel to an outpatient clinic for the multiple treatment sessions required for training. To meet this need, we have developed a protocol for remotely-supervised tDCS (RS-tDCS) that is combined with cognitive remediation and delivered to participants at home while maintaining clinical standards. Initially developed to target cognitive impairment in multiple sclerosis (MS), the protocol is being expanded for use in other conditions and telerehabilitation programs. The methods, challenges and potential of at-home tDCS will be discussed along with initial efficacy data from an ongoing randomized, sham-controlled trial.

Correspondence: Leigh E. Charvet, Department of Neurology, NYU Langone Medical Center, 240 East 38th Street, New York, NY 10016, United States.E-mail: leigh.charvet@nyumc.org

\section{Symposium 1. Neuropsychology in the Americas}

\author{
Chair: Alberto L. Fernandez
}

$$
\text { 9:00-10:30 a.m. }
$$

\section{A.L. FERNANDEZ, A. FERRERES, D. PINEDA, T. JUDD, C. GROTE} \&. J.J. MANLY. Neuropsychology in the Americas.

Neuropsychology has gone through different development processes in the world. Within the American continent (South, Central, North), neuropsychology development took different courses in the different regions. North American neuropsychology has an empiric and quantitative tradition and a faster development. South America, influenced by other psychological and neuropsychological streams, based much of 
its development on qualitative approaches. Neuropsychology in Central America is in an initial stage.

A summary of the current state of neuropsychological development in each region, including the topics of professional and research interest, will be presented. Besides, the factors that have influenced and continue determining neuropsychology development in each region will be discussed, as well as the future perspectives for its development. In addition, possible cooperative actions between the most important professional organizations in the continent (SLAN, ALAN, INS) will be proposed.

Correspondence: Alberto L. Fernandez, PhD, Neuropsychology, Universidad Católica de Córdoba, Chile 279 PB, Cordoba 5000, Argentina.E-mail:neurorehab@onenet.com.ar

\section{A. FERRERES. Neuropsychology in South America.}

Until the 1970s-1980s neuropsychology in South America was mainly the result of pioneering remarkable individuals. Most of them were neurologists. The foundation of Sociedad Latinoamericana de Neuropsicologia (SLAN) in 1989 in Buenos Aires, was the result of these pioneers' will to develop a regional framework for the emerging discipline. Neuropsychology had started to be acknowledged in the region as a science and as a clinical practice. The change of status of neuropsychology was reflected in four areas: 1) the inclusion of neuropsychology as a regular course within the study plans of Psychology and other similar disciplines; 2) the emergence of postgraduate courses in neuropsychology; 3) the establishment of neuropsychology services in different health institutions; 4) more scientific research involved with neuropsychology (neuropsychological subjects in $\mathrm{PhD}$ dissertations, research proposals and scholarship proposals). A survey was conducted in order to characterize the current status of neuropsychology in Argentina, Bolivia, Brasil, Chile, Colombia, Paraguay, Perú, Uruguay y Venezuela. Representative figures of the discipline were surveyed. Besides, an analysis of the research topics in two regional specialized journals ("Neuropsicología Latinoamericana" and "Neuropsicología, Neuropsiquiatría y Neurociencias), was made.

Correspondence: Aldo Ferreres. E-mail: aldoferreres@gmail.com

\section{T. JUDD. The Past, Present, and Future Development of Neuropsychology in Central America.}

Neuropsychology (NP) has been slowly developing as a clinical service in most of Central America (CA) for about 30 years. There has been sporadic NP research during that time. Forensic NP is very limited. Facilitators of development include clinical needs, especially war rehabilitation; government support; private sector demand; university priorities, and individual interest. To date, development is usually by nationals getting trained outside of the region and through self-study, and occasionally by trainers coming in. Barriers and challenges to NP development include poverty; professional rivalries; limited medical/ mental health, educational, cultural, and legal infrastructure; competing public health needs; the cultural adaptation of foreign NP needed to serve the region; and the diversity of languages and cultures within CA. Few individuals or institutions see neuropsychology as a personal professional identity, but rather, as a skill set for professionals with a broader identity. Suggestions for promoting development of NP include fostering of personal professional relationships, empowering and mentoring young professionals, and developing professional dispute resolution or neutralization mechanisms. Development of NP will also require facilitating interdisciplinary understanding and cooperation and developing professional and institutional identities. A pressing need is to develop sustainable international support that serves reciprocal needs, such as sustained NP organizational outreach; sister university or departmental programs; transnational research consortia; distance or hybrid training; and Internet resources. These themes will be developed through a presentation concerning the first Masters degree in NP in $\mathrm{CA}$ at the Universidad del Valle de Guatemala, just completing its first cohort. Correspondence: TeddJudd. E-mail: teddjudd@gmail.com
C. GROTE. Neuropsychology in the United States and Canada.

The science and practice of neuropsychology has flourished in the United States of America and Canada over the last few decades. It appears that these are the only two countries in the world that require practitioners to hold a doctorate degree. Pursuit of board certification is also common. While the length and cost of career entry is greater than in other countries, the salaries of American and Canadian neuropsychologists are relatively high compared to neuropsychologists elsewhere or other workers in these two countries. Job prospects for neuropsychologists are relatively good with common work settings including academic medical centers, community and veterans hospitals and universities. Many neuropsychologists are self-employed in private practice and/or consult on medicolegal cases. There are a number of challenges facing neuropsychologists, however. These include greatly increased competition for funding of scientific investigations, changing and perhaps decreasing funding from payors, and tests that may not always reflect the increasingly diverse populations that American and Canadian neuropsychologists are attempting to evaluate and treat.

Correspondence: Christopher Grote, Behavioral Sciences, Rush University Medical Center, 1645 W Jackson Ste 400, Chicago, IL 60612, United States.E-mail:Christopher_Grote@rush.edu

\section{J.J. MANLY. Collaboration of Neuropsychologists in the Americas} can Accelerate Progress in Research and Practice.

Most of the scientific literature in neuropsychology has a narrow focus on United States-based populations, settings, and problems. A more diverse, global perspective will benefit all aspects of neuropsychology, because including perspectives from all of North America, the Caribbean, and Central and South America will increase the relevance and accuracy of our knowledge of brain-behavior relationships. Approaches to neuropsychological assessment across the Americas reflect the history of neuropsychology, employing both quantitative and process/qualitative methods that when used in concert, increase productivity and broaden the application of results. Cross-national collaboration will enhance our field's preparation for complex yet critical practical issues for clinical neuropsychology. Cross-national collaboration has the potential of moving beyond the mere documentation of disparities in cognitive function, to discovering the causal mechanisms of disparities, thus empowering interventions and intervention. Contributions to knowledge about language use and bilingualism, the influence of socioeconomic status on the brain and cognition, and cultural influences on neuropsychological measurement are all potential areas of emphasis in neuropsychology across the Americas. We can accelerate engagement through formal collaboration of neuropsychological professional associations in each region, so that the strengths of each organization and each setting can be maximally leveraged. Potential cooperative mechanisms include dedicated scientific interest groups, research and practice summits to develop prioritized recommendations, set-aside funds for studies that include cross-national comparative designs, and exchange programs for trainees that incorporate structured plans for continued collaboration after the exchange is over. INS, ALAN, and SLAN have an opportunity to shape the direction and quality of this development, in partnership.

Correspondence: Jennifer J. Manly, PhD, Neurology, Columbia University, 630 West 168th St., P\&S Box 16, New York, NY 10032, United States.E-mail:.jjm71@cumc.columbia.edu 


\section{Symposium 2. An integrative look at the Boston Process Approach to Neuropsychology: A review of the history, current research and future directions of error analysis}

\author{
Chair: Melissa Lamar \\ 9:00-10:30 a.m.
}

\begin{abstract}
M. LAMAR, D.J. LIBON, R. AU, A. KARSTENS \& U. DIAZORUETA. An integrative look at the Boston Process Approach to Neuropsychology: A review of the history, current research and future directions of error analysis.
\end{abstract}

On this, the $50^{\text {th }}$ anniversary of the International Neuropsychological Society (INS), it seems fitting to highlight another anniversary, that of a key philosophical school of neuropsychological assessment - the Boston Process Approach (BPA). Over the past 40 years, the BPA, first advocated by Dr. Edith Kaplan (INS President 1979), has become one of the primary neuropsychological methods used in research and clinical assessment. The BPA suggests that brain-behavior relationships are often revealed in the analysis of errors and the process by which patients reach a correct solution, rather than solely on psychometric scores. This symposium will review the past, present and future of the BPA while simultaneously allowing generations of Dr. Kaplan's students and collaborators to highlight the influence she and the larger INS community has had on their work. Drs. David Libon and Rodney Swenson (former Boston VA Interns under Dr. Kaplan) will discuss the history of the BPA and the role of their mentor in shaping this work. Dr. Rhoda Au (former Boston VA Intern under Dr. Kaplan) will describe cutting edge research to move the BPA into a digital age of cognitive assessment via the Framingham e-Cognitive Health Initiative. Dr. Melissa Lamar (former Drexel University extern under Dr. Libon with adjunct mentorship by Dr. Kaplan) will outline multi-method integration the BPA to neuropsychological assessment with advanced neuroimaging analytics including connectomics. Lastly, Aimee Karstens (current graduate student of Dr. Lamar) and Dr. Unai Diaz-Orueta (international collaborator of Drs. Lamar and Libon) will demonstrate ways to adapt the BPA to normal brain aging and the E-SPACE project (European Standardised Process Approach to Cognitive Evaluation in Older People), respectively. Ultimately, the audience will leave this symposium with a better appreciation for the BPA both past, present and future; a framework for which will be provided by our Discussant, Dr. David Libon.

Correspondence: Melissa Lamar, PhD, Rush Alzheimer's Disease Center, Rush University Medical Center, 600 S Paulina St, Chicago, IL 60612, United States.E-mail:Melissa_Lamar@rush.edu

\section{D.J. LIBON \& R. SWENSON. The history of the Boston Process} Approach and the role of Edith Kaplan.

Edith Kaplan, the originator of The Boston Process Approach, began her work training the next generation of students in this approach in 1976 when she became the director of Clinical Neuropsychological Services at the Boston VA. The historical roots of the Boston Process Approach, however, can be traced to tenets of Gestalt psychology that championed the notion that complex behavior is best understood when its individual components are viewed as a whole. In fact, the interplay between test stimuli and/ or an experimental paradigm and their constituent parts was suggested by Dr. Kaplan's mentor, Heinz Werner who first explained higher-order cognitive processes underlying perceptual reasoning and concept formation in 1937. Recent empirical work incorporating the Boston Process Approach has helped to differentiate performance between Alzheimer's/Vascular spectrum dementia patients on such tests as the Mini-Mental Sate Examination and the WAIS Similarities subtest; performance that was otherwise indistinguishable on the basic psychometric scores associated with these measures. Thus, when an analysis of process and errors is used to operationally-define key cognitive constructs interesting between-group differences emerge in dementia populations. The added value of an analysis of process and errors has been demonstrated in research designed to define subtypes of risk for dementia, a.k.a., Mild Cognitive Impairment (MCI), providing more nuanced information regarding underlying brain-behavior relations prior to the onset of dementia. In sum, the Boston Process Approach is an effective methodology that enhances the neuropsychological analysis of brain aging and cognition.

Correspondence: David J. Libon, PhD, Geriatrics and Gerontology, Rowan University, 42 East Laurel Rd, Stratford, NJ 08084, United States.E-mail: libondj@gmail.com

R. AU, R.J. PIERS \& S. DEVINE. Incorporating the Boston Process Approach into Cognitive Assessment in the Digital Era: Framingham e-Cognitive Health Initiative.

Effective treatment for Alzheimer's disease (AD) remains elusive. Failed clinical trial studies are presumably because interventions are too late in the course of the disease. Just as the introduction of the Boston Process Approach to neuropsychological assessment expanded diagnostic accuracy, the introduction of digital technology into the cognitive assessment process is allowing for the detection of performance variations at unprecedented levels of precision and granularity. Framingham cognitive aging studies have digitally captured responses to neuropsychological tests since 2005, enabling new directions in preclinical AD research. Beginning in 2005, all participant spoken responses have been digitally recorded ( $\mathrm{n}>7500+)$. In 2011, a digital pen was introduced for recording performance on the digital Clock Drawing Test $(\mathrm{n}>2500)$ and in 2013, expanded use included all participant drawn tests $(n>1500)$. The study population included all Framingham Heart Study participants across 2-3 generations who completed neuropsychological testing (age range 25-100+). This presentation will review a series of results from digitally acquired metrics, particularly those obtained from participants asymptomatic for $\mathrm{AD}$, to highlight the capability to detect differences that traditional measures do not. For example, we have examined decision-making latency measures from the digital Clock Drawing Test and found age-related differences among adults who were deemed cognitively intact (e.g., average Mini-Mental score $=29$ ). Just as we were the first to incorporate the Boston Process Approach into our cohort, we are the first to digitally capture traditional neuropsychological tests within a multi-generation community based sample. This study and our work to date have laid the foundation to identify cognitive biomarkers that can serve as surrogate measures to screen for those who are at increased risk but still asymptomatic for $\mathrm{AD}$.

Correspondence: Rhoda Au, PhD, Anatomy \& Neurobiology, Neurology and Epidemiology, Boston University Schools of Medicine and Public Health, 72 E Concord Street, B6, Boston, MA 02118, United States. E-mail:rhodaau@bu.edu

M. LAMAR, O. AJILORE, L. ZHAN, A. KARSTENS, C. DION, M. GONZALES, J. COHEN \& A. LEOW. Multi-method integration of human connectomics with the Boston Process Approach to neuropsychological assessment.

The Boston Process Approach (BPA) to neuropsychology revolutionized the way clinicians and researchers perceived the performance path to a total score. Likewise, advances in neuroimaging and neuroimage analytics have revolutionized the way we conceptualize brain-behavior relationships. Integrating these two approaches stands to significantly increase our clinical understanding and empirical explorations of normal and pathological aging. We present data from two community-based studies that merged a BPA to neuropsychology with a tractographyderived structural brain connectome to elucidate underlying differences in otherwise normal performances in older adults. Both studies collected whole brain T1-weighted images and diffusion tensor images. In the first study, 72 non-demented/non-depressed individuals were categorized based on whether or not they used 'anchor' digits (i.e., 12,3,6,9) before any other digits while completing the Clock Drawing Test. In a separate study, 80 non-demented/non-depressed individuals were administered 
the Judgement of Line Orientation test and evaluated for oblique effect errors, i.e., errors in identifying diagonal (a.k.a., oblique) lines. In the context of grossly intact clock drawings, Anchorers had better regional integration within local networks involving the left medial orbitofrontal and transverse temporal cortices as well as the right rostral anterior cingulate and superior frontal gyrus versus non-anchorers. Anchorers also exhibited a higher degree of modular integration among heteromodal regions of the ventral visual processing stream versus non-anchorers. Oblique errors were associated with network reductions involving the bilateral superior longitudinal fasciculi, but modular integration analyses were not significant. The BPA plus advanced neuroimaging analytics not only revealed subtle distinctions in brain-behavior relationships but may also signal biomarkers for future longitudinal studies of the development of dementia.

Correspondence: Melissa Lamar, PhD, Rush Alzheimer's Disease Center, Rush University Medical Center, 600 S Paulina St, Chicago, IL 60612, United States.E-mail: Melissa_Lamar@rush.edu

\section{A. KARSTENS, O. AJILORE, A. KUMAR \& M. LAMAR. Adapting Boston Process Approach algorithms used in dementia research to a normal aging population.}

Throughout the dementia literature, the Boston Process Approach has been used to identify subtle distinctions in behavior. For example, error analysis of the Wechsler Similarities subtest in dementia has revealed differences in concrete versus more abstract patterns of performance that relate to differences in working memory and semantic retrieval, cardiovascular disease risk factor (CVD-RF) profiles and left prefrontal grey matter volumes. Attempts to expand this work - and other forms of Boston Process Approach error analyses - to non-demented older adults are often stymied by limited errors and/or highly successful performance. We will discuss ways to consider the Boston Process Approach in normal aging with a focus on Similarities response styles. Eighty-five non-demented older adults with either an abstract $(n=26$; age 67 , MMSE $\sim 29)$, concrete $(\mathrm{n}=24$; age $\sim 68$, MMSE $\sim 28)$ or mixed $(\mathrm{n}=35$; age 70, MMSE $\sim 28)$ response style as determined through a Boston Process Approach to the WAIS-III Similarities subtest were compared across working memory and semantic retrieval, CVD-RF profiles, and grey matter volumes. MANOVAs for working memory, $\mathrm{F}(6,152)=2.18, \mathrm{p}=0.04$, and semantic retrieval, $\mathrm{F}(6,158)=2.04, \mathrm{p}=0.06$, revealed significant differences between groups on the Trail Making Test Part B (abstract $>$ concrete $=$ mixed, $p$-values $\leq 0.01)$ and semantic cluster size from Animal fluency (abstract=concrete $>$ mixed, $p$-values $\leq 0.02$ ). Groups did not differ on CVD-RF profiles or prefrontal grey matter volumes. In sum, adapting Boston Process Approach methods from the dementia literature to a normal aging population is possible and results were seen in differential cognitive but not brain structure profiles. Additional ways to tease apart greater distinctions between our groups of relatively health older adults, e.g. intratest scatter, are discussed as it relates to the Boston Process Approach.

Correspondence: Aimee Karstens, Bachelors of Science, University of Illinois, Chicago, 2134 N Maplewood Ave, Apt. 2, Chicago, IL 60647, United States. E-mail: karsten2@uic.edu

U. DIAZ-ORUETA, A. BLANCO-CAMPAL \& T. BURKE. The E-SPACE Project: An international expansion of the Boston Process Approach to incorporate error analysis of behaviour.

Dementia is a clinical syndrome characterised by cognitive and functional decline that may be caused by a wide variety of neurodegenerative diseases, with Alzheimer's disease being the most common. A cognitive evaluation that relies exclusively on summary achievement scores is considered to be incomplete, unless these are complemented by a qualitative, e.g., Boston Process Approach, analysis of the individual's performance. The goals of the E-SPACE project (European Standardised Process Approach to Cognitive Evaluation in Older People) are (1) to identify widely used cognitive screening measures for the detection of mild cognitive impairment and dementia in primary and secondary care; and (2) to improve existing cognitive screening measures, without significantly affecting normative-data-based administration procedures, by means of the inclusion of qualitative indices and error analyses. The ultimate aim of E-SPACE is to uncover cognitive strategies used by older adults and the underlying primary neurocognitive mechanisms of the impairment responsible for performance failure. Suggested test modifications for instruments such as the Montreal Cognitive Assessment (MoCA) and Addenbrooke's Cognitive Examination -Third Edition (ACE-III), among others, will be presented and potential benefits for the adoption of these indices in clinical practice in primary and secondary care will be outlined.

Correspondence: Unai Diaz-Orueta. E-mail: unai.diaz-orueta@dcu.ie

\section{Paper Session 1. Risk \& Alzheimer's}

\author{
Moderator: Felicia C. Goldstein
}

\section{9:00-10:30 a.m.}

\section{A.E. BLANKEN \& D.A. NATION. Longitudinal neurocognitive profiling of empirically-derived Alzheimer's disease variants.}

Objective: Autopsy studies have identified Alzheimer's disease (AD) variants with different cognitive and brain atrophy profiles. The present study sought to identify AD variants through cluster analysis of in vivo AD biomarkers and neuropsychological measures, and to compare variants on longitudinal cognitive decline and brain atrophy.

Participants and Methods: Alzheimer's Disease Neuroimaging Initiative participants ( $N=977$, non-demented subsample) were subjected to hierarchical cluster analysis using baseline cognition and cerebrospinal fluid biomarkers. Longitudinal (baseline, 1, 2 year) cognitive decline ( $\mathrm{n=747)}$ ) and brain atrophy (hippocampal, $\mathrm{n}=497$; ventricular, $\mathrm{n}=566$; and subregional volumes, $\mathrm{n}=510$ ) were compared with repeated measures ANCOVA, controlling for age, gender, education, and apolipoprotein E4 status, and LSD post-hoc tests.

Results: Subgroups included a group $($ NoAD; n=624) with normal cognition and biomarker status, a typical $\mathrm{AD}$ group $(T A D ; \mathrm{n}=216)$ with greater memory decline and biomarker profile consistent with $\mathrm{AD}$, and an atypical $\mathrm{AD}$ group $(A A D ; \mathrm{n}=137)$ with relatively preserved memory, impairment in language and executive functioning, and intermediate biomarker elevation. In two years $A A D$ continued to show greatest impairment in language and executive ability, whereas $T A D$ had greatest memory impairment. AD groups had more hippocampal atrophy and ventricular enlargement than $N o A D$. Enlargement of the left lateral ventricle was greatest for $A A D(A A D>T A D>N o A D)$. $A A D$ had greater atrophy in the right lateral orbitofrontal and supramarginal, and the left pars triangularis $(A A D>T A D>N o A D)$. $T A D$ had greater atrophy in the bilateral putamen and rostral middle frontal, right frontal pole, left pars orbitalis and posterior cingulate $\left(T A D>A A D>N_{o} A D\right)$.

Conclusions: Two AD variants showed distinct patterns of cognitive impairment over 2 years. $A A D$ outperformed $T A D$ in memory, but showed greater language and executive impairment. AD variants had differing patterns of brain atrophy over 1 year consistent with cognitive profiles.

Correspondence: Anna E. Blanken, PhD, Clinical Psychology, USC, 12550 Fielding Circle, \#1, Playa Vista, CA 90094, United States. E-mail:blanken@usc.edu

D.A. NATION \& J.K. HO. Independent and Interactive Effects of Cerebral Amyloid and Tau on Neuropsychological Decline and Structural Brain Changes in Older Adults.

Objective: The present study investigated the independent and synergistic effects of amyloid beta $\left(\mathrm{A} \beta_{1-42}\right)$ and phosphorylated tau ( $\mathrm{p}$-tau) pathologies on neuropsychological and neurostructural trajectories in older adults. 
Participants and Methods: Participants from the Alzheimer's Disease Neuroimaging Initiative underwent longitudinal assessment $(\mathrm{N}=732$; 0, 12, 24 months), including baseline lumbar puncture and follow-up cognitive exams, and a subset underwent follow-up neuroimaging of brain volume (hippocampal and ventricle volumes; $n=185$ ) and white matter lesion (WML) volume $(\mathrm{n}=546)$. Cerebral spinal fluid (CSF) biomarker profiles (A $\beta$-Ptau-, A $\beta+$ Ptau-, A $\beta$-Ptau+, A $\beta+$ Ptau+) were compared on decline trajectories for memory (AVLT delay and recognition), attention/executive function (Trails $\mathrm{A}$ and $\mathrm{B}$ ) and language (Animals and BNT), and brain atrophy and WML volumes using ANCOVA with repeated measures and post-hoc LSD tests.

Results: The A $\beta+P$ tau+ group exhibited significantly greater hippocampal atrophy compared to all other groups, greater decline on all cognitive tests relative to the $A \beta$-Ptau- and A $\beta$-Ptau+ groups, and increased WML burden relative to the A $\beta$-Ptau- group. The A $\beta+P$ taugroup exhibited greater ventricle volume and worse attention/executive function than the A $\beta$-Ptau+ and A $\beta$-Ptau- groups. The A $\beta+P t a u+$ and $A \beta+P$ tau- groups did not differ in WML volumes, ventricle volume or executive functions.

Conclusions: Findings suggest that when both amyloid and tau pathology are present they have synergistic effects on hippocampal atrophy and decline in memory and language abilities. In the absence of tau pathology, cerebral amyloidosis was associated with similar levels of white matter damage, ventricular enlargement and executive decline, suggesting that amyloid may also have tau-independent effects on cognitive decline through increased white matter changes and brain atrophy. Correspondence: Daniel A. Nation, Ph.D., Psychology, University of Southern California, 3620 South McClintock Ave., Los Angeles, CA 92008,United States. E-mail: danation@usc.edu

\section{LOBUE, F. WOON, H.C. ROSSETTI, L.S. HYNAN, J. HART \& M. CULLUM. Traumatic Brain Injury History and Progression from Mild Cognitive Impairment to Alzheimer Disease.}

Objective: The mechanistic link between traumatic brain injury (TBI) and later cognitive decline is unknown. TBI has been hypothesized to activate a progressive neurodegenerative process, accelerate an already present neurodegenerative disorder, or disrupt neuronal reserve and interact with aging. We utilized a large national cohort to examine whether a history of TBI is associated with progression from mild cognitive impairment (MCI) to Alzheimer disease (AD).

Participants and Methods: Data on subjects with MCI $(\mathrm{n}=2,719)$ were obtained from the National Alzheimer's Coordinating Center. TBI was categorized based on presence/absence of reported TBI with loss of consciousness (LOC) without chronic deficit occurring $>1$ year prior to diagnosis of MCI (TBI+/-). Survival analyses were used to determine if a history of TBI predicted progression from MCI to AD over the course of 9 years, adjusted for demographics, apolipoprotein E-e4, a vascular risk score, and history of psychiatric factors. Random regression models were used to examine whether TBI history also predicted rate of decline on the Clinical Dementia Rating scale Sum of Boxes score (CDR-SB) among subjects who progress to $\mathrm{AD}$.

Results: Across 9 years, TBI history was a significant predictor but associated with slower progression from MCI to a diagnosis of AD in an unadjusted model ( $\mathrm{HR}=0.77 ; 95 \% \mathrm{CI}=0.61-0.97 ; p=.02)$. This association, however, diminished $(p=.15)$ after adjustment for an earlier age of MCI diagnosis (2.6 years earlier for TBI+ subjects, $p<.001)$. A history of TBI was a non-significant predictor for rate of decline on CDR-SB among subjects who progressed to $\mathrm{AD}(b=0.15$, $T=0.88, p=.38$ ).

Conclusions: A history of TBI with LOC was associated with an earlier age of diagnosis of MCI, but not progression to AD. These findings suggest TBI might reduce the threshold for onset of MCI, but once the neurodegenerative process for MCI to AD starts, a history of TBI appears unrelated to subsequent decline.
Correspondence: Christian LoBue, UT Southwestern Medical Center, 5953 Lofland Drive, Frisco, TX 75033, United States. E-mail: christian. lobue@utsouthwestern.edu

B. YEW \& D.A. NATION. Elevated Cerebrovascular Resistance Predicts Increased Amyloidosis, Cerebral Atrophy, and Cognitive Decline.

Objective: Cerebral amyloid deposition on positron-emission tomography (PET) is an in vivo biomarker of Alzheimer's disease (AD) that has been linked to both increased blood pressure and decreased cerebral blood flow (CBF). The ratio of blood pressure to CBF is an index of cerebrovascular resistance (CVRi). This study examined CVRi as a predictor of cerebral amyloid burden, cerebral atrophy and neuropsychological decline in a longitudinal study.

Participants and Methods: Non-demented, stroke-free older adults from the Alzheimer's Disease Neuroimaging Initiative study underwent neuroimaging and neuropsychological testing at baseline, 12 and 24 month follow-up. Florbetapir PET was used to identify participants as amyloid-positive $(n=87)$ or amyloid-negative $(n=112)$. Arterial spin labeling MRI indexed rCBF, by which brachial blood pressure was divided to obtain CVRi. Fludeoxyglucose (FDG)-PET measured neuronal metabolism. CVRi prediction of cerebral amyloid, atrophy and cognitive decline was analyzed using linear mixed models and regression, controlling for age, sex, apolipoprotein E4, education, and FDG-PET.

Results: Baseline CVRi was elevated in amyloid-positive cases and predicted increased cerebral amyloid accumulation, as well as declines in global cognition, memory, and executive function, at follow-up. Among amyloid-negative cases, higher CVRi also predicted greater cortical atrophy.

Conclusions: Increased CVRi is associated with higher amyloid load, and is predictive of cortical atrophy and cognitive decline in older adults. Importantly, results were independent of neuronal metabolism, suggesting observed declines are the product of cerebrovascular dysfunction rather than mere consequences of reduced neural activity. CVRi may therefore instigate neurodegeneration prior to amyloid accumulation, drive amyloid accumulation, and/or interact with cerebral amyloidosis to produce cognitive decline in older adults.

Correspondence: Belinda Yew, University of Southern California, Dept. of Psychology, SGM 501, 3620 South McClintock Ave, Los Angeles, CA 90089,United States. E-mail: belinday@usc.edu

M.A. GLENN, S.T. MOELTER, K. GOLDBERG, D. FISHER \& R.S. WILSON. Primacy Effects in Cognitively Normal Older Adults with Alzheimer's Disease Pathology.

Objective: To determine whether reduced immediate and delayed primacy recall on the CERAD word list memory test (CWL) is predictive of postmortem Alzheimer's disease (AD) pathology in cognitively normal older individuals. We hypothesized that lower primacy scores predict $\mathrm{AD}$ pathology.

Participants and Methods: The study sample included age, gender, and education matched, cognitively normal older adults $(\mathrm{N}=56)$ who participated in the Religious Orders Study or Memory and Aging Project at Rush Alzheimer's Disease Center (ADC). Half ( $\mathrm{N}=28$ ) of the subjects met National Institute on Aging (NIA)-Reagan Institute criteria for a postmortem neuropathological diagnosis of AD. Archival data obtained included neuropathological measures of beta amyloid, neurofibrillary tangles, and global AD pathology quantified across 8 brain regions. CWL item-level performance closest in time to autopsy (0.1-2.0 years) was recoded according to the proportion of words recalled from the primacy region, defined as the first 3 out of 10 total words. Multiple regression analyses were performed, correcting for age, gender, and education.

Results: The proportion of words recalled from the primacy region of CWL for trial 1 and trials 1-3 immediate recall, did not predict measures of AD neuropathology $(p>.10)$. The proportion of words recalled from 
the primacy region on delayed recall of CWL was predictive of global tangles $(b=-2.60$ [95\% CI: $-4.92,-0.27], p=.029)$ and medial temporal lobe (MTL) tangles ( $b=-11.04$ [95\% CI: $-19.88,-2.20], p=.015)$, with lower delayed primacy proportion scores predicting greater tangle pathology. The delayed primacy proportion score accounted for $8 \%$ of the variance in global tangles, and $9 \%$ of the variance in MTL tangles. Conclusions: Assessment of primacy recall, especially delayed primacy proportion scores, may be a sensitive marker of neurobiological dysfunction associated with tangle pathology. Future research may test whether this measure improves detection of early cognitive decline associated with preclinical AD.

Correspondence: Megan A. Glenn, M.A., M.S., Institute for Graduate Clinical Psychology, Widener University, 4708 A Dunbarton Road, Mount Laurel, NJ 08054, United States. E-mail:mglenn1550@mail. usciences.edu

\section{E.C. MCINTOSH \& D.A. NATION. Untreated Diabetes Associated with Tau Pathology and Worse Cognitive Performance in Older Adults.}

Objective: Type 2 diabetes mellitus (T2DM) is associated with increased risk for Alzheimer's dementia. Recent research indicates increased cerebral spinal fluid (CSF) phosphorylated tau ( $\mathrm{p}$-tau) concentrations in older adults with T2DM. To date no studies have investigated these associations in treated versus untreated T2DM. The present study investigated cerebrospinal fluid (CSF) p-tau and neuropsychological performance differences between non-diabetic older adults and those with treated versus untreated T2DM

Participants and Methods: Non-demented older adults from the Alzheimer's Disease Neuroimaging Initiative (age 255 ; N=906) were classified as non-diabetic, treated T2DM or untreated T2DM using fasting blood glucose and medication history. Analysis of covariance and post-hoc LSD tests were used to compare non-diabetic older adults to those with treated and untreated T2DM on CSF phosphorylated tau (p-tau) and neuropsychological test scores, after controlling for age, gender, education, and apolipoprotein (APOE)-e4 carrier status.

Results: Findings revealed that older adults with untreated diabetes had higher CSF p-tau and performed worse on category fluency (Animals) and Digit Symbol Substitution tests relative to those who were non-diabetic, after controlling for all covariates ( $p<.05$ for all). There were no differences between treated T2DM and non-diabetic older adults on any measures $(p>.10)$.

Conclusions: The present study findings indicated increased CSF p-tau and worse performance on two timed neuropsychological tests in untreated T2DM, but not treated T2DM, relative to non-diabetic older adults. These results suggest that glucose-lowering agents may influence CSF p-tau and may preserve processing speed abilities previously implicated in cerebrovascular and Alzheimer's disease. Future research is needed to investigate the mechanisms by which glucose-lowering agents attenuate tau pathology and improve cognitive function.

Correspondence: Elissa C. McIntosh, MA, Psychology, University of Southern California, 3620 McClintock Ave, Suite 501, Los Angeles, CA 90089,United States.E-mail: ecmcinto@usc.edu

\section{Paper Session 2. Veterans' Health \\ Moderator: Amy J. Jak \\ 9:00-10:30 a.m.}

\begin{abstract}
E. TRITTSCHUH, J.I. ALVA, D. CHAPMAN, J. KANG \& S. THIELKE. PTSD + Aging: Psychoeducational Intervention to Promote SelfEfficacy and Healthy Brain Behaviors in Older Veterans with Cognitive Concerns.

Objective: Aging Veterans with PTSD diagnoses are at increased risk for dementia and may present for evaluation based on concerns for
\end{abstract}

cognitive decline. Efficient care models are needed for those that fall in the "gray" zone between normal aging and dementia. The "Memory Skills for Older Adults with PTSD" protocol is an outpatient psychoeducational class series developed at the VA PugetSoundHealthCareSystem and conducted from 2012-2016. The intervention goals were to provide patient-centered education within a group setting to promote translation of skills into daily function and to promote healthy brain aging via behavioral change.

Participants and Methods: Veterans 55+ with a diagnosis of PTSD, subjective and/or mild objective cognitive decline, but no dementia. Participants engaged in $8-1 \mathrm{hr}$ group sessions with leaders from a variety of training backgrounds; groups were conducted at urban and rural sites. SMART goals, behavioral activation, and mindfulness training provided a backbone across sessions. Pre- and post-group data were collected in the form of 1) chart review for healthcare utilization patterns and 2) comparison of Veteran-completed symptom and attitude questionnaires.

Results: Participants rated the group highly ( $\mathrm{n}=97$ participants: 93M/4F; mean age 68, from 13 groups, with $>85 \%$ retention). Postgroup, Veterans reported significantly decreased depressive symptoms, increased sense of self-efficacy, but no reductions in PTSD symptoms or memory complaints. Chart review of the 1 st 50 participants revealed a significant decrease in overall healthcare utilization in the 6 months post-group, in the context of re-engagement in specialty services (e.g., psychiatry).

Conclusions: As this project closes in 2016, analyses are planned for the inclusion of the final Fall 2016 participants, comparison of results by group leader specialty, and of rural vs urban offerings. This type of intervention can efficiently manage the cognitive concerns of Veterans with PTSD at risk for cognitive decline and potentially improve outcomes.

Correspondence: Emily Trittschuh, PhD, GRECC - S-182, VAPSHCS/ University of Washington, 1660 S. Columbian Way, Seattle, WA 98108, United States.E-mail: etritt@gmail.com

H.M. MISKEY, R.E. YOASH-GANTZ, R.D. SHURA, S. KULUBEKOVA \& J. ROWLAND. Cognitive Performance of Veterans in an Inpatient Posttraumatic Stress Disorder Program Pre- and Post-Treatment.

Objective: Some research suggests cognitive impairment is associated with posttraumatic stress disorder (PTSD); preliminary studies indicate potential improvement in cognition following treatment (see Aupperle et al., 2012 for a review). We examined functioning pre- and post-treatment to evaluate changes in psychiatric symptoms and cognition.

Participants and Methods: Eighty-nine Iraq and Afghanistan service members with combat-related PTSD who were enrolled in a six week inpatient PTSD treatment program completed a comprehensive neuropsychological evaluation during the first week of admission. Following program completion, 50 participants repeated the battery prior to discharge and provided data for analysis (Average: age $=39,98 \%$ male, $49 \%$ Caucasian). Paired t-tests using selected scores from the Hamilton Rating Scale (HamD), PCL-Military, Posttraumatic Growth Inventory, Connor Davidson Resilience Scale, PASAT, TMT, COWAT, Fruits and Vegetables, CPT-II, a digit span task, and the CVLT-II were completed to assess for significant change. Correlations using individual change scores (Time 1 minus Time 2 scores) were calculated to determine if change in psychological symptoms was associated with change in cognitive functioning.

Results: Scores significantly improved on PASAT 2.4 second trial $(d=-0.37)$, PASAT total score $(d=-0.40)$, TMT Part B $(d=0.36)$, $\operatorname{HamD}(d=0.87)$, and PCL-M $(d=0.71)$. Improvements on the PASAT and TMT were not associated with decreases in PCL-M or HamD scores. Conclusions: Completion of the PTSD treatment program was associated with decreases in psychological distress and improvements in working memory and divided attention. We were unable to demonstrate a relationship between psychological and cognitive improvements. Implications of these findings will be discussed. 
Correspondence: Holly M. Miskey, PhD, Hefner VA Medical Center, Hefner VA Medical Center, 1601 Brenner Ave, Salisbury, NC 28144, United States.E-mail: Holly.Miskey@va.gov

A.J. JAK, L.D. CROCKER, S.M. JURICK, B. BOYD, M. SANDERSONCIMINO, A.V. KELLER, L. TRENOVA, R. AUPPERLE, C. RODGERS, A. LANG, D. SCHIEHSER, S. NORMAN \& E.W. TWAMLEY. Neuropsychological outcomes following hybrid treatment for Veterans with comorbid TBI and PTSD.

Objective: Comorbidity of PTSD and history of TBI is high among Iraq and Afghanistan-era Veterans (OIF/OEF/OND). Cognitive processing therapy (CPT) is empirically supported for treatment of PTSD, but is not designed to accommodate memory, attention, or problem solving deficits experienced by many Veterans. Compensatory cognitive rehabilitation (CogSMART) is effective for cognitive deficits stemming from a variety of etiologies. We created a hybrid treatment that integrates components of CogSMART into CPT to concurrently address cognitive and mental health challenges in Veterans with PTSD, history of TBI, and cognitive complaints.

Participants and Methods: 107 OIF/OEF/OND Veterans with PTSD and mild-moderate TBI history enrolled in a randomized controlled trial investigating efficacy of our novel hybrid treatment, SMART-CPT, as compared to standard CPT. Veterans were randomized to SMART-CPT $(\mathrm{n}=54)$ or CPT $(\mathrm{n}=53)$, and underwent neuropsychological assessment at baseline, completion of the 12 week intervention, and three months post-treatment. Repeated measures ANOVA was used to compare groups.

Results: Groups were equivalent on demographic and injury variables. Both treatment groups exhibited clinically significant but not statistically different reductions in PTSD, neurobehavioral, and depressive symptoms. The SMART-CPT group completed significantly more treatment sessions (8.5 sessions) than the CPT group (6.6 sessions). The SMART-CPT group showed significantly more improvement than the CPT group in attention and executive functioning.

Conclusions: Individuals with PTSD, TBI, and cognitive complaints can successfully complete structured/empirically supported mental health therapy with or without modifications. Reducing emotional distress in Veterans can also reduce neurobehavioral symptom reporting and improve cognitive functioning. Augmentation of therapy with cognitive compensatory strategies may improve treatment adherence and attention/executive functioning symptoms in this population. Correspondence: Amy J. Jak, Ph.D., VA Healthcare System, San Diego/ UCSD, 3350 La Jolla Village Dr., 151B, San Diego, CA 92161, United States.E-mail:ajak@ucsd.edu

J. SCOTT, G. HARB, J. BROWNLOW, J. GREENE, R.C. GUR \& R.J. ROSS. Verbal Memory Functioning Moderates Psychotherapy Treatment Response in PTSD.

Objective: Posttraumatic stress disorder (PTSD) is associated with cognitive deficits in attention, executive control, and episodic memory, although few studies have investigated the relevance of these cognitive difficulties for treatment outcomes. Here, we examined whether 1) neurocognitive performance, and 2) history of traumatic brain injury (TBI) were associated with response to cognitive-behavioral psychotherapy for PTSD-related sleep problems.

Participants and Methods: In a randomized controlled trial comparing Imagery Rehearsal (IR) to components of CBT for insomnia (cCBT-I) for PTSD-related recurrent nightmares, 94 U.S. Veterans completed tests from the Penn Computerized Neurocognitive Battery (CNB) assessing attention, executive control, episodic memory, and spatial processing. TBI was assessed with a structured clinical interview. Mixed-effects models examined main effects of cognitive functioning and interactions with time for sleep and nightmare outcomes.

Results: Significant verbal immediate memory by time interactions were found for nightmare distress $(p<.001)$, nightmare frequency $(p<.001)$, and sleep quality $(p=0.02)$, even after controlling for overall level of cognitive performance and depression. TBI exhibited main effects on all outcomes $(p<.05)$ but no interactions with time $(p s>10)$.

Conclusions: Verbal memory moderated primary outcomes across two short-term psychotherapy sleep interventions, such that individuals with lower verbal immediate memory performance were less likely to respond to both treatments. Veterans with TBI displayed greater overall symptom severity at all timepoints but did not show altered trajectories of treatment response. Our findings suggest that verbal memory functioning may be an important factor to consider in PTSD treatment implementation and outcomes.

Correspondence: J. Cobb Scott, Ph.D., Psychiatry, Perelman School of Medicine at the University of Pennsylvania, 3400 Spruce Street, 10th Floor, Gates Building, Philadelphia, PA 19104, United States. E-mail: scott1@upenn.edu

\section{J.B. WILLIAMSON, D. LAMB, M. HARCIAREK \& K.M. HEILMAN. Lateralized limbic white matter integrity is associated with PTSD symptoms.}

Objective: Mild traumatic brain injury (mTBI) is linked to post traumatic stress disorder (PTSD). mTBI may damage white matter connection between frontal cortex and amygdala, a mediator of emotions such as fear and anger. Further, based on right-left hemispheric differences in emotional processing, there may be laterality effects of injury.

Participants and Methods: 59 veterans including 23 with mTBI and PTSD, 14 without history of mTBI or PTSD, 7 with history of PTSD. and 15 with only mTBI were assessed. Fractional Anisotropy (FA) was derived from a 64 direction HARDI sequence acquired at 3T using TBSS and then a white matter atlas (Johns Hopkins) was used to identify white matter pathways. Self-report assessments including the PTSD Checklist - Military version and Symptom Checklist 90 Revised were administered. Based on a priori hypotheses, we analyzed the relationship between right and left uncinate fasciculus (UF) DTI-FA and emotional dysregulation.

Results: FA of right UF, a critical limbic-prefrontal pathway, was correlated with symptoms of emotional dysregulation. Lower DTI-FA in this region was associated with symptoms of disturbing memories and dreams, reminders of military experience, avoidance behavior, angry outbursts, and jumpiness/ease of startle. Further, symptoms of hostility and anxiety were also associated. Subjects without history of PTSD had higher DTI-FA in this region.

Conclusions: As predicted, a reduction of right UF connectivity between prefrontal and limbic areas is related to symptoms of emotional dysregulation in a sample of combat veterans with and without TBI and PTSD. This may represent a neurological vulnerability to symptoms of emotional dysregulation in this population.

Correspondence: John B. Williamson, PhD, Neurology, University of Florida, 2775 NW 106 Way, Gainesville, FL 32606, United States. E-mail: john.williamson@neurology.ufl.edu

E. LERITZ, R. MCGLINCHEY, W.P. MILBERG \& R. JORGE. TBI and PTSD Moderate the Relationship Between Blood Pressure and Cortical Thickness in OEF/OIF/OND Veterans.

Objective: It is becoming clear that elevated cardiovascular (CV) risk factors, such as high blood pressure (BP), are prevalent in Veterans of $\mathrm{OEF} / \mathrm{OIF} / \mathrm{OND}$. High BP, especially in mid-life, is associated with serious vascular disease, and higher risk for the development of vascular-related cognitive impairment and dementia. We have demonstrated that even sub-clinically elevated levels of BP can negatively affect aspects of neural integrity, including cortical thickness (CT). PTSD, TBI and blast exposures are prevalent in the OIF/OEF/OND cohort, and also have negative effects on brain structure, but there is little information regarding how these factors interact with $\mathrm{CV}$ risk.

Participants and Methods: 239 OEF/OIF/OND Veterans (mean age 32.5 ) underwent comprehensive physiological/medical, psychiatric, and neuroimaging assessment at the TRACTS National Research Center. Free Surfer morphometric GLM procedures evaluated the association 
between BP and CT along every vertex on the cortical mantle. Hierarchical regression analyses examined the contribution of PTSD, TBI and blast exposure to clusters in which higher BP was associated with reduced CT.

Results: Higher BP was associated with reduced CT in several bilateral regions, including frontal and temporal brain areas. This was seen even for BP values that fell below clinical threshold for hypertension diagnosis. For the frontal cluster, number of lifetime TBIs and a PTSD diagnosis were most predictive of CT, while for the temporal cluster, total number of blast exposures predicted CT.

Conclusions: BP is negatively associated with CT in younger veterans in regions that we have reported are affected in older non-veterans. A history of TBI, blast exposures and PTSD increased the negative effect of BP on CT.

Correspondence: Elizabeth Leritz, PhD, Harvard Medical School, VA Boston Healthcare System, 150 South Huntington Ave, GRECC 182 (JP), Boston, MA 02130,United States.E-mail: elizabeth_leritz@hms. harvard.edu

\section{Poster Session 3. Peds 1 9:30-10:45 a.m.}

\section{Acquired Brain Injury (TBI/ Cerebrovascular Injury \& Disease - Child)}

\section{G. ALVAREZ, S. SUSKAUER \& B. SLOMINE. Clinical Features of Young Children with Disorders of Consciousness.}

Objective: Disorders of Consciousness (DOC), including vegetative (VS) and minimally conscious states (MCS), can occur following severe brain injury. Assessment of DOC is challenging in all patients due to questionable and inconsistent responses; however, evaluation is particularly challenging in young children because of their limited repertoire of developmentally-expected behaviors. The purpose of this study was to describe clinical features in a convenience sample of young children with DOC, including exam findings of those in MCS and those who emerged into a conscious state (CS).

Participants and Methods: Chart review was conducted for 25 children admitted to a pediatric inpatient rehabilitation facility directly from an acute care hospital following a new neurologic injury or illness $(\mathrm{TBI}=5$, Acquired $=20)$. Age ranged from 6 months through 5 years $(M=2.61)$. All children were identified to have DOC upon admission $(\mathrm{VS}=14, \mathrm{MCS}=11)$.

Results: Five children (ages 1-5 years) emerged into CS by discharge. All 5 of these children demonstrated features consistent with MCS at admission. The 11 children in MCS at admission displayed visual fixation and/or contingent affective responding; none showed clear command following. Of those in $\mathrm{VS}$ at admission, $36 \%$ progressed to MCS. At discharge, only 3 children clearly followed commands (ages 2 , 4 , and 5), each of whom were in CS.

Conclusions: These results contribute to the small literature on DOC in children. Consistent with previous studies of older children, higher level of consciousness at admission appears associated with better outcomes at discharge. The vast majority of the sample was not observed to follow simple motor commands. Overall, future research is needed to establish more distinctive and developmentally relevant behaviors suggestive of MCS in young children, as well as identify factors associated with emergence into $\mathrm{CS}$ in this population.

Correspondence: Gabrielle Alvarez, M.S., Neuropsychology, Kennedy Krieger Institute, 2102 Bank St, Baltimore, MD 21231, United States. E-mail: alvarezg@kennedykrieger.org
T. ANTONINI, A. CASSEDY, H. TAYLOR, T. STANCIN, M. KIRKWOOD, K.O. YEATES \& S.L. WADE. Changes in Parenting Skills Following Participation in Two Versions of an Online Parenting Program Designed for Families of Young Children with TBI.

Objective: Traumatic brain injury (TBI) renders young children vulnerable to behavioral difficulties. Positive parent-child interactions may mitigate these difficulties. Parent-skills training programs that focus on improving parental warm responsiveness and consistent discipline may improve parent-child interactions and reduce problem behavior. This study examined changes in observed parenting behaviors following participation in an online parent-skills program designed for families of young children with TBI.

Participants and Methods: Participants included 113 families with a 3-10-year-old child who had sustained a complicated mild to severe TBI. They were randomly assigned to 1 of 3 groups: a 10-14-session online parenting skills program that included didactic information about TBI and stress management $(\mathrm{n}=39)$, an 'express' 7 -session parenting skills program $(n=36)$, or an Internet resource comparison group that offered online TBI information $(\mathrm{n}=38)$. Parent-child interactions were recorded pre- and post-intervention and the number of positive and negative behaviors exhibited by parents was coded. Generalized estimating equations were used to examine post-intervention parenting behaviors across the groups, controlling for site, child's current age, age at injury, time since injury, TBI severity, parent marital status, family income, and observed parenting behaviors at baseline.

Results: Both parenting skills groups exhibited significantly higher numbers of positive behaviors and significantly lower numbers of negative behaviors compared with the Internet resources group. The two parenting skills groups did not differ from each other in positive or negative parenting behaviors.

Conclusions: Both parent-skills programs successfully promoted positive and decreased negative parenting behaviors. However, parents did not show additional benefit from the longer version of the program. Thus, a brief, targeted 'express' parenting program may be the most practical intervention option to improve parent-child interactions post TBI.

Correspondence: Tanya Antonini, Texas Children's Hospital, 4055 South Braeswood Blvd., Apt 212, Houston, TX 77025, United States. E-mail:tanya.antonini@gmail.com

W. ARCHAMBAULT, J. LÉPINE, R.D. MOORE, D. BELLEMARE \& D. ELLEMBERG. Differences in the Psycho-Affective Profile and ADHD Index Scales in Adolescent Hockey Players with a History of Concussions.

Objective: To determine differences in psycho-affective health, executive functions and ADHD symptoms between adolescent hockey players who sustained a concussive injuries and their control teammates.

Participants and Methods: Eighty-four hockey players (54 with a history of concussions, 30 without) from Québec minor hockey Pee-Wee, Bantam and Midget categories (ages 11-17) participated in the current study. Athletes completed a psycho-affective assessment consisting of the Depression (BDI) and Anxiety Inventories (BAI) of the Beck Youth Inventory (BYI). Furthermore, parents filled out the BRIEF and Conners-3 questionnaires evaluating their child's executive functions and ADHD behavioral expression, respectively. Athletes with a diagnosis of ADHD or any other developmental disorder were excluded from analyses. Independent $\mathrm{t}$-tests were used to analyze the mean $t$-score between concussed and control athletes for the BAI, BDI and every scale of the BRIEF and Conners questionnaires.

Results: $\mathrm{BAI}$ and BDI $t$-scores were significantly higher for teenagers with a history of concussion $(\mathrm{BAI}$ means $=47.83$ vs. $44.50 ; \mathrm{p}=.013$; BDI means $=46.65$ vs. $41.70 ; p<.001)$ as was the ADHD Index from the Conners -3 (mean $=0.94$ vs. $0.37 ; p=.043)$. Furthermore, there was a group trend for the ADHD probability factor of the Conners-3 (mean $=29.98$ vs. $24.43 ; p=.078)$. Group means for all the other scales were not significantly different ( $p s \geq .124$ for all). 
Conclusions: Compared to adolescents without a history of concussion, those reporting concussive injuries present some alterations in their psycho-affective health. In addition, even when controlling for ADHD diagnoses, a relation between history of concussion and the degree of ADHD behaviors was observed. Additional longitudinal research is necessary to better understand the nature, duration, and causality of alterations observed in adolescent hockey players with a history of concussion.

Correspondence: William Archambault, Kinesiology, Université de Montréal, 2250 rue Guy, Apt. 103, Montréal, QC H3H 2M3, Canada. E-mail:will_a11@hotmail.com

C.M. BAILEY, J. GAITLEY, S. BRISKIN, S. MADERCIC \& P. FASTENAU. Pre-Injury Child-SCAT3 Performance in a Youth Sports Sample.

Objective: In 2012, the Standardized Concussion Assessment Tool $3^{\text {rd }}$ Edition (SCAT3) was adapted and endorsed for use with pediatric populations (Child-SCAT3) by the most recent international consensus statement on concussion in sport (McCrory et al., 2012). Though widely used, limited information is available regarding its utility in children. The current study examined the performance of healthy youth athletes on Child-SCAT3.

Participants and Methods: 86 healthy youth hockey athletes $(92 \%$ male, $\mathrm{M}$ age=10.19; Age range=5-15) completed a baseline concussion battery that included the Child-SCAT3, concussion symptom questionnaires, and a battery of commonly administered neurocognitive testing. Performance on the cognitive testing from the Child-SCAT3 was examined to determine if age of youth athletes was related to ChildSCAT3 performance and if significant differences existed between older $(13+: n=17)$ versus elementary-age athletes $(5-12: n=69)$. A zeroorder pearson correlation was completed examining the relationship between age and Child-SCAT3 performance. An independent t-test was completed to examine differences in performance by age group.

Results: Age showed a large correlation to Child-SCAT3 performance $(\mathrm{r}=.58, \mathrm{p}<.001)$. Also, mean performance on the cognitive portion of the Child-SCAT3 in middle-school age athletes $(\mathrm{M}=26.41, \mathrm{SD}=1.62)$ was significantly $(\mathrm{p}<.001)$ higher than elementary-age athletes $(\mathrm{M}=23.58$, $\mathrm{SD}=3.33)$.

Conclusions: Findings showed a large effect of age on Child-SCAT3 cognitive performance, with middle-school age athletes showing levels of performance similar to high-school and college-age athletes, while elementary-age athletes (the targeted population of the measure) performed meaningfully below older athletes. The findings suggest that different normative data is required for use of the Child-SCAT3 with younger athletes and may raise questions regarding the overall utility of this measure with elementary-age athletes.

Correspondence: Christopher M. Bailey, Ph.D., Neurology, University Hospitals Case Medical Center, 11100 Euclid Ave, Mail Stop HAN 5040, Cleveland, OH 44106, United States.E-mail: christopher.bailey3@ uhhospitals.org

B.C. BAUGHMAN, B.L. BRETT, R. REZAIE \& S. NARAYANA. The Relationship Between Multi-modal Neuropsychological Measures and Head Impact Telemetry Over the Course of a Single High School Soccer Season.

Objective: Increased attention to the long-term effects of subconcussive head impacts has led to attempts to better quantify these blows and to sensitively measure clinical outcomes. Due to inconsistencies in accelerometer data, clinical utility has yet to be established (Breedlove et al., 2012; Nauheim et al., 2003). Furthermore, debate continues regarding the necessity of neuropsychological testing in athletes with mTBI (Randolph, McCrea, \& Barr, 2003; Van Kampen et al., 2006). We investigated the relationship between these two methodologies in a sample of high school soccer players.

Participants and Methods: A homogenous sample of healthy, adolescent females $(\mathrm{N}=11)$ completed a pre and post season, multimodal neurocognitive battery. Head impacts were quantified using accelerometers (X-Patch, X2 Biosystems). Although not reported here, participants also completed multi-modal neuroimaging (i.e., fMRI, diffusion tensor imaging, magnetoencephalography, transcranial magnetic stimulation). Linear and angular accelerometer metrics were calculated across multiple force thresholds. Reliable change indices (RCIs; Iverson, 2001) were calculated. Correlations were explored between neuropsychological tests and between NP and impact telemetry.

Results: Neuropsychological performance was variable, although no meaningful deficits were noted at any time point. Mean RCIs were not significant $(-.23-.70)$ and not correlated to accelerometers, with the exception of phonemic fluency $(r=.85, \mathrm{p}<.01)$. Correlations between impact telemetry and NP testing were generally non-significant, save for select processing speed, executive function, and delayed memory tasks $(.59-.78)$.

Conclusions: Despite significant head impact telemetry over the course of a single soccer season (e.g., max linear acceleration $>150 \mathrm{~g} ;>150+$, $20 \mathrm{~g}$ blows), there was no consistent evidence to suggest alterations in neuropsychological test performance. Implications for clinical management and future studies will be addressed.

Correspondence: Brandon C. Baughman, Phd, Semmes-Murphey Clinic, 6325 Humphreys Blvd., Memphis, TN 38120, United States. E-mail: bbaughman@semmes-murphey.com

B. BIEKMAN, E.A. WILDE, X. LI, J. HUNTER, G. HANTEN, A.C. VASQUEZ, A. SCHMIDT, Z.D. CHU \& H. LEVIN. Frontal Lobe Volume and Thickness Differences Between Children With Traumatic Brain Injury and Children With Orthopedic Injury.

Objective: Traumatic brain injury (TBI) is one of the most common causes of pathology and the most common cause of death in children. Neuroimaging, specifically volumetric analysis of cortical regions, has been an increasingly significant area of interest. This study contributes to the ongoing research into volumetric differences following pediatric TBI.

Participants and Methods: 16 children with complicated-mild or moderate TBI, 18 children with severe TBI, and 23 children with orthopedic injury (OI), matched on demographic variables such as sex, race, and SES, were recruited. All participants underwent a T1-weighted MRI sequence at 12 months post-injury. The T1 images were processed through the FreeSurfer pipeline, which created cortical thickness and volume measurements using regions of interest based on the Desikan-Killiany atlas. Thickness and volume of the left and right hemispheres were averaged to create a composite.

Results: ANOVA found a significant main effect of injury group for the thickness of the caudal middle frontal cortex $(p=.002)$, lateral orbitofrontal cortex $(p=.006)$, medial orbitofrontal cortex $(p=.02)$, rostral middle frontal cortex $(p=.0004)$, and superior frontal cortex $(p=.0003)$. Additionally, ANOVA found a significant main effect of injury group for the volume of the lateral orbitofrontal cortex $(\mathrm{p}=.002)$, and superior frontal cortex $(p=.03)$.

Conclusions: Consistent with previous findings, children with TBI have cortical thickness and cortical volume differences with OI controls. More research is needed to know whether cortical volume and thickness differences precede or succeed TBI.

Correspondence: Brian Biekman, Physical Medicine \& Rehabilitation, Baylor College of Medicine, 9707 Rathbone, Houston, TX 77031, United States.E-mail:Brian.Biekman@bcm.edu

A.R. BURNS, C. AHRABI-NEJAD, E. DUGI, I. HEATON, J.B. NEWMAN \& G.A. GIOIA. Changes in Executive Functioning Following Concussion: Comparisons Between ADHD, Learning Disability, and Typically Developing Children and Adolescents.

Objective: To examine changes in executive functioning (EF) postconcussion between children with Attention-Deficit/Hyperactivity Disorder (ADHD), Learning Disabilities (LD), and typically developing (TD) children. 
Participants and Methods: Children seen in a specialty concussion clinic within 1 month of injury were classified on premorbid diagnosis by a clinician (according to clinical interview) and matched on gender, age, and days since injury, yielding 38 of each ADHD, LD, and TD with concussion $(68 \%$ male; mean age $=13.79$; mean days since injury $=14.28)$. Patients and parents completed a 30 -item monitoring version of the BRIEF during initial and follow-up clinic visits; scores were adjusted for retrospective pre-injury ratings. One way and Repeated Measures ANOVAs were conducted.

Results: Self-reported change in EF deficits following concussion did not significantly differ across groups. Parents reported significantly greater increases in EF deficits following a concussion when their child had a LD compared to those with ADHD ( $p=0.012)$. Specifically, Working Memory $(\mathrm{p}=0.004)$ and Emotional Control $(\mathrm{p}=0.039)$ deficits were reported to increase more significantly in children with LD than those with ADHD. However, parent report did not indicate a significant difference between TD children and those with either ADHD or LD. All groups reported significant improvements in EF from their initial visit to their follow-up visit, (parent-report $\mathrm{F}(1,64)=15.598, \mathrm{p}=0.000$ and self-report $\mathrm{F}(1,58)=25.785, \mathrm{p}=0.000)$. There were no significant differences in the rate of change in EF across groups.

Conclusions: Following a concussion, ADHD, LD, and TD individuals experience an increase in executive functioning deficits. Based on parent report, those with a $\mathrm{LD}$ experience more significant increases compared to ADHD individuals. Despite this finding, EF functioning difficulties following concussion were found to improve at similar rates over time in TD children and those with ADHD or LD.

Correspondence: Alison R. Burns, PhD, Neuropsychology, Children's National Health System, 15245 Shady Grove Road, Suite 350, Rockville, MD 20850,United States. E-mail: alburns@childrensnational.org.

M.L. COHEN, D. TULSKY, J. WANG, H. BERTISCH, P. KISALA, K.O. YEATES \& F.P. RIVARA. Parent Ratings of Language Problems After Pediatric Traumatic Brain Injury.

Objective: To identify longitudinal trends in parent-reported language problems in children with traumatic brain injury (TBI).

Participants and Methods: Parents of 170 children with TBI completed a battery of outcome measures soon after injury (reporting on premorbid functioning), and again 6 and 12 months post-injury. Of the total sample, $123(72.4 \%)$ children had mild TBI and $47(27.6 \%)$ had moderate or severe (mod/sev) TBI. Language abilities were measured with the TBI-QOL Communication item bank (CIB), a version of the Neuro-QOL Communication item pool that has been optimized and calibrated for individuals with TBI. Items from the short form of the TBI-QOL CIB formed two composite scores - receptive language and expressive language. Analyses targeted both within-group (over time) and between-group (mild versus mod/sev) differences, adjusting for age, sex, and when appropriate, baseline score and TBI severity.

Results: Six months after injury, both groups were rated to have lower receptive language skills than before injury ( $\mathrm{mod} / \mathrm{sev}$ worse than mild). The $\mathrm{mod} / \mathrm{sev}$ group was also rated to have reduced expressive language skills. Twelve months after injury, these problems persisted and were not significantly better than at 6 months.

Conclusions: Parents reported that their children displayed at least subtle language problems 6 and 12 months after TBI, even mild TBI. Further research is needed on how well these perceptions cohere with objective measures of language and with measures of language pragmatics.

Correspondence: Mathew L. Cohen, University of Delaware, $540 \mathrm{~S}$. College Ave., Room 210CC, Newark, DE 19713, United States. E-mail: mlcohen@udel.edu
P. COHEN, A. SUFRINKO, R. ELBIN, M.W. COLLINS \& A.P. KONTOS. Do Initial Symptom Factor Scores Predict Subsequent Impairment following Concussion?

Objective: High symptom scores at initial visit (Meehan et al., 2013) and at baseline (Custer et al., 2016) are predictive of impairment and protracted recovery following concussion. However, the predictive value of symptom factors (e.g., Kontos et al., 2012) on subsequent impairment is unknown. The purpose of this study was to examine the ability of patients' symptom factor scores at their initial clinic visit to predict neurocognitive and vestibular/oculomotor impairment at their second clinic visit.

Participants and Methods: Participants included 72 athletes aged 13-22 with a sport-related concussion. Participants completed the Post-Concussion Symptom Scale (PCSS), Immediate Post-concussion Assessment and Cognitive Testing (ImPACT), and Vestibular/Ocular Motor Screening (VOMS) at 1 week post-injury and again approximately 2-4 weeks post-injury. PCSS scores were aggregated into symptom factors per Kontos et al. (2012). Multiple regressions were conducted with symptom factors at $<1$ week as predictors of ImPACT and VOMS, scores at 2-4 weeks and recovery time.

Results: The cognitive fatigue/migraine symptom factor predicted impairment on visual memory $(\beta=-.348, p=.033)$ and reaction time $(\beta=.003, p=.029)$. The affective symptom factor predicted higher scores on the horizontal $(\beta=.398, p=.019)$ and vertical saccades $(\beta=.390$, $\mathrm{p}=.031)$, and vertical $(\beta=.571, p=.013)$, and horizontal vestibular ocular reflex (VOR) $(\beta=.471, p=.030)$. Symptom factor scores did not significantly predict recovery time.

Conclusions: Clinicians can utilize patient's initial symptom factor scores to better predict subsequent impairment and identify patients for earlier, targeted treatment. Patients with anxiety/mood symptoms following concussion may experience increased vestibular impairment following concussion.

Correspondence: Paul Cohen, PsyD, Orthopedic Surgery, University of Pittsburgh Medical Center, 3200 South Water Street, UPMC Sports Medicine Concussion Program, Pittsburgh, PA 15203, United States. E-mail:cohenpe@upmc.edu

A.N. DANGUECAN, T. WILLIAMS \& R. WESTMACOTT. Stability of overall intellectual functioning into early school-age for children with neonatal arterial ischemic stroke.

Objective: Children with neonatal arterial ischemic stroke (AIS) are at risk for long-term cognitive deficits (Westmacott et al., 2009). Others have studied overall intellectual functioning (full scale IQ; FSIQ) over time in this population, but have not considered stability within or decline from the Average range. The latter can have important implications for prognosis and treatment planning. We examined the stability of Average and below Average FSIQ scores longitudinally (between pre-school and school-age) in children with neonatal AIS, while considering several neurological variables (e.g., seizures, lesion size).

Participants and Methods: Our sample consisted of 24 children with neonatal AIS who underwent neuropsychological testing between 2005 and 2015 at SickKids Hospital in Toronto. All children had both WPPSI (III or IV) and WISC (III, IV, or V) testing with valid FSIQ at an average of 3 years apart. Exclusion criteria included non-neonatal AIS, cerebral sinovenous thrombosis (CSVT), and mova mova disease.

Results: Only lesion size predicted WPPSI and WISC FSIQ. For subsequent analyses, FSIQ was analyzed as a dichotomous variable reflecting Average (standard score $\geq 90$ ) or below Average $(<90)$ functioning. WPPSI and WISC FSIQ scores were highly stable over time $\left(\chi^{2}=10.97\right.$, $p<.01) .10$ patients had below Average FSIQ on the WPPSI, of which 9 remained below Average on the WISC. 14 patients had at least Average FSIQ on the WPPSI, of which 11 remained Average; 3 declined by 15 or 16 standard score points to below Average on the WISC. All 3 patients who showed FSIQ decline had combined cortical/subcortical lesions and hemiparesis.

Conclusions: Pre-school intellectual functioning remains stable into the school-age years for most children with neonatal AIS; thus, clinicians 
may consider using low pre-school FSIQ to advocate for early school/ community interventions. Close monitoring may be warranted for children with cortical/subcortical lesions and hemiparesis, as they may be at increased risk for cognitive decline.

Correspondence: Ashley N. Danguecan, Ph.D., Psychology, Hospital for Sick Children, Hospital for Sick Children, 555 University Avenue, Toronto, ONM5G1X8, Canada.E-mail: ashley.danguecan@sickkids.ca

\section{B. DUNCAN, J. KIEFEL \& K. ONO. A Comparison of Performance Validity on the CNS Vital Signs (CNSVS) Validity Indicators and Green's Medical Symptom Validity Test (MSVT) in a Sample of} Pediatric Concussion Patients.

Objective: The purpose of this study is to compare the CNSVS validity indicators to Green's MSVT and to characterize the populations that passed/failed either measure in a sample of children with concussion. Participants and Methods: This study consists of 48 children (8-16 years) who sustained a concussion within $0-3$ months of testing. Participants were administered a standard neuropsychological battery, including the MSVT, a well-validated tool in pediatric concussion (Kirkwood \& Kirk, 2010).

Results: Chi-Square tests of independence were run to examine the relation between the MSVT and each validity indicator for the major domains of the CNSVS, and the overall CNSVS neurocognition index (CNSVS-NI). The MSVT was significantly related to the CNSVS Processing Speed $\left(X^{2}=7.380, p=.007\right.$ Reaction Time $\left(X^{2}=6.146\right.$, $\mathrm{p}=.013)$, and Composite Memory $\left(\mathrm{X}^{2}=5.812, \mathrm{p}=.016\right)$ domains, as well as the CNSVS-NI $\left(X^{2}=4.438, p=.035\right)$. Subjects were then categorized into four groups based on whether they passed/failed either validity measure (i.e., MSVT and the CNSVS-NI). A one-way ANOVA revealed a significant effect for self-report of hyperactivity $[\mathrm{F}(3,37)=2.983$, $\mathrm{p}=.044]$, such that individuals that passed one or the other validity measure were reporting more hyperactivity compared to individuals that failed both or passed both.

Conclusions: Preliminary results suggest that validity indicators on the CNSVS and the MSVT are fairly similar. However, results also indicated that failing one or the other validity measure was indicative of higher levels of hyperactivity, suggesting possible interactions between hyperactivity, attention, and performance on validity measures. These results highlight the importance of assessing performance validity in the context of comorbid cognitive dysfunctions. This research contributes to the growing literature on performance validity testing in children, specifically using the CNSVS (Brooks, Sherman, \& Iverson, 2014). Correspondence: Brittany Duncan, MA, Neuropsychology, Children's Healthcare of Atlanta, 7075 Derby Court, Cumming, GA 30040, United States.E-mail:brit021491@gmail.com

J. FABER, E.A. WILDE, X. LI, G. HANTEN, J. HUNTER, L. EWINGCOBBS, M.E. AITKEN, M. MACLEOD, Z.D. CHU \& H.S. LEVIN. Executive Functioning and Diffusion Tensor Imaging in TBI.

Objective: Executive functioning complaints are common among children with traumatic brain injury (TBI).

Participants and Methods: The Delis-Kaplan Executive Functioning System (D-KEFS) Color Word Interference test (Delis, Kaplan \& Kramer, 2011), a measure of working memory, cognitive flexibility, and inhibitory control was administered to $21(6 \mathrm{~F} / 15 \mathrm{M})$ children aged $10-18$ years, who sustained moderate to severe TBI as toddlers and were scanned 5-15 years later. The comparison group was 17 (6F/11M) typically-developing children, matched on gender, age, SES, and ethnicity. We examined group differences in D-KEFS Color Word Interference test performance and diffusion tensor imaging (DTI)-derived fractional anisotropy (FA) and apparent diffusion coefficient (ADC) of the corpus callosum (CC), frontal white matter (FWM), uncinate fasciculus, cingulate, and ventral striatum (VS). DTI metrics were correlated with D-KEFS scaled scores for word reading, interference, and interference-switching conditions.
Results: D-KEFS scaled scores on word reading $(p=0.043)$, interference $(p=0.013)$, and interference-switching $(p=0.007)$ conditions significantly differed between the TBI and control groups, with better performance in the control group. The TBI group demonstrated significantly lower FA in the CC total $(p=0.013)$, body $(p=0.016)$, and genu $(p=0.027)$. ADC in the TBI group was significantly higher for the CC total $(p=0.001)$, body $(p=0.005)$, genu $(p=0.002)$, CC splenium $(p=0.002)$, left FWM $(p=0.009)$, and left cingulum $(p=0.050)$. In the TBI group, D-KEFS scores significantly correlated with ADC of the left uncinate fasciculus $(r=-0.460, p=.041)$, such that poorer performance was associated with decreased white matter integrity.

Conclusions: Deficits in executive functioning may persist for years following injury and may detrimentally impact academic and social outcomes. These deficits may be related to injury-induced structural changes in white matter integrity.

Correspondence: Jessica Faber, B. A., Baylor College of Medicine, One Baylor Plaza, Houston, TX 77030, United States. E-mail: jesfaber@ gmail.com

T.B. FAY-MCCLYMONT, K.O. YEATES, S. BENSELER \& B. BROOKS. Neuropsychological, behavioural, and quality of life outcomes in a cohort of children with autoimmune encephalopathies.

Objective: Little is known about the impact of autoimmune encephalopathies on functioning in children. The objective is to examine neuropsychological performance in 12 children with autoimmune encephalopathy, their recovery over time, and behavioural and quality of life outcomes.

Participants and Methods: Participants included 12 children with autoimmune encephalopathy (including 5 anti-NMDAR, 3 autoimmune [unspecified], 2 mycoplasma, 1 Rasmussen's encephalitis, and 1 post-infectious cerebellitis) aged 6 to 17 (mean age $=13.13, \mathrm{SD}=3.6$; 8 girls, 4 boys). Children completed neuropsychological testing from 1 month to 1 year after onset (Time 1 mean $=4.9$ months after initial symptoms), and again from 1 month to 2 years later $(\mathrm{N}=7)$, based on clinical need.

Results: Global functioning was relatively preserved (i.e., Neurocognition index or abbreviated full scale intellectual quotient $=88$ $[\mathrm{SD}=20.65])$ at time 1 . Total number of neuropsychological deficits did not differ by diagnosis ( $p>05$ ). Areas of difficulty included motor speed, reaction time, attention, and executive functioning (deficits apparent in $25-30 \%$ of cases). Children tested closer to time of onset or with continuing seizures throughout recovery fared worse than those tested later in time or without seizures. Parent questionnaires did not reveal marked internalizing or externalizing problems (i.e., BASC Behavioral Symptom Index $\mathrm{T}$ score $=51, \mathrm{SD}=10)$. Parent rated quality of life was mildly impacted in most cases (PedsQL mean=69.28, SD=16.4). Repeat assessment showed reliable change over time, but continuing dysexecutive difficulties in some children, especially those with continuing seizures.

Conclusions: Children with autoimmune encephalopathy demonstrate primarily executive difficulties, with some recovery seen over the first year post-injury. Continuing problems are more prevalent in those with ongoing seizures.

Correspondence: Taryn B. Fay-McClymont, Ph.D., Neuropsychology, Alberta Children's Hospital, 4163500 Varsity Drive NW, Calgary, ABT2L 1Y3, Canada.E-mail: tarynfay@gmail.com

\section{E. HIRST, A.E. THORNTON \& D. COX. Epidemiology of Sport and} Non-Sport-Related Concussion in Adolescents.

Objective: To investigate and report on the prevalence and correlates of concussions experienced within the past year in a provincially representative sample of adolescents. In particular, to evaluate the incidence of concussion across gender, age, and activity, and to examine the health and mental health correlates of concussion. 
Participants and Methods: Data for this study were taken from the most recent BC Adolescent Health Survey (BC AHS, 2013) from the McCreary Centre Society. The BC AHS is a repeated cross-sectional survey of youth ages 12-19 in the province of British Columbia, Canada. The target population included all students enrolled in grades 7 to 12 in mainstream public schools. This provided survey data on 29, 832 students, which is weighted to provide an accurate representation of all students in British Columbia in grades 7 through 12.

Results: To accommodate complex survey data, Complex Samples module in SPSS was used to run crosstabs and logistic regression. Prevalence for concussion in the past 12 months was $16 \%$ (19\% of males, $14 \%$ of females). Rates of concussion were higher in individuals involved in sports with or without a coach compared to those individuals who are not involved in sports $(p<0.001)$. Rates of poor mental health were higher in individuals with a history of at least one concussion in the past 12 months $(p<0.001)$. Reporting a greater number of concussions in the past year was also associated with poorer reported mental health $(p<0.001)$.

Conclusions: Rates of concussion in the adolescent population surveyed in this research are higher than most estimates. This study contributes to the understanding of the epidemiology of concussion and can inform prevention and possible treatment. Further research is required to determine the nature of the risks and consequences of concussion in adolescence.

Correspondence: Emily Hirst, Psychology, Simon Fraser Univsersity, 3755 West 6th Ave, Unit 124, Vancouver, BC V6R1V1, Canada. E-mail: ehirst@psych.ubc.ca

\section{A. HOLLAND, P. PLUMB, K. GOODSPEED, P.L. STAVINOHA \& M.M. DOWLING. Predicting Long-Term Cognitive Outcomes of Pediatric Stroke According to Stroke Severity Classification. \\ Objective: The Pediatric Stroke Outcome Measure (PSOM) rates neuro- logical impairment. This study investigated the relationship between initial PSOM score and long-term cognitive outcome, hypothesizing worse initial PSOM score would predict lower IQ at long-term follow-up. Other factors considered were months post stroke and age at stroke. Participants and Methods: PSOM scores at initial visit and IQ scores at long-term follow-up (M=3.77 years) were obtained for 84 survivors of pediatric stroke ages 4:0-25:6 ( $\dot{M}=11: 5$ years; 37 females). Age at stroke ranged $0-18: 5$ years $(M=7: 8)$. Groups were created based on PSOM score: 52 low-severity (0-1.0) and 32 high-severity (1.5+). Groups did not differ on gender ratios, age at stroke, age at testing, months post stroke, or stroke-related medical variables. PSOM, age at stroke, and months post stroke were entered into stepwise regressions separately for each group.}

Results: Mean IQ for the high-severity group was 79.41 (SD=16.92), significantly lower than the low-severity group mean of 88.08 ( $\mathrm{SD}=16.78 ; p=.024)$. For the low-severity group, the regression was significant $(F=3.503 ; p=.022)$ with only months post stroke contributing significantly $(b=-.487 ; p=.004)$. For the high-severity group, the regression was significant $(F=4.079 ; p=.016)$ with only PSOM score contributing significantly $(b=-.420 ; p=.018)$.

Conclusions: PSOM was more relevant than age at stroke in predicting cognitive outcome, and greater stroke severity was associated with lower $\mathrm{IQ}$ at long-term follow-up. Findings suggest that with initial $\operatorname{PSOM}<1.5$, months post stroke may be more important than age at stroke in determining cognitive outcome, with an inverse relationship between months post stroke and IQ. In contrast, with initial PSOM $>1.0$, severity may be the most significant determining factor of long-term cognitive outcome. Improved outcome predictions could improve efforts toward early intervention. Study limitations and future directions will be discussed. Correspondence: Alice Ann Holland, PhD, Children's Medical Center Dallas, 6300 Harry Hines Blvd., Suite 900, Dallas, TX 75235, United States.E-mail:Alice.Ann.Holland@childrens.com
R.A. HOWARTH, M. JAY, A. KRENTZEL, C. GHILAIN \& L.S. BLACKWELL. Functional Outcomes for Pediatric Patients Diagnosed with Anti-NMDA Receptor Encephalitis during Inpatient Rehabilitation.

Objective: Anti-NMDA receptor encephalitis is an auto-immune disorder with a predictable clinical course. Neurocognitive domains often impacted include attention, executive functioning, memory, and language. Little is known about the early functional outcomes and neurocognitive recovery in pediatric patients. Accordingly, the current study examined the functional status of pediatric patients diagnosed with Anti-NMDA encephalitis during inpatient rehabilitation.

Participants and Methods: Participants included 25 patients $(64 \%$ female; $56 \%$ African American) who were admitted for inpatient rehabilitation. On average, patients were 10.34 years of age $(\mathrm{SD}=4.97$ ) at the time of diagnosis and length of stay ranged from 15 - 108 days $(\mathrm{M}=43.77)$. Functional status upon admission and discharge was evaluated using the Functional Independence Measure for Children (WeeFIM) Developmental Functional Quotient (DFQ, \% of age-appropriate function). A subset of patients were also administered the Cognitive and Linguistic Scale (CALS).

Results: Mean WeeFIM Total DFQ score at admission was 27.40 (range: 15.03 - 62.64) and at discharge was 51.97 (range: 15.03 91.87). Average change in WeeFIM Total DFQ scores from admission to discharge was 25.86 (range: $-8.32-75.17, \mathrm{p}<.01$ ). WeeFIM domain scores improved between admission and discharge (Self-Care: $\mathrm{p}<.001$, Cognition: $p<.01$, Mobility: $p<.001)$. For those patients with CALS data $(n=10)$, correlations with WeeFIM DFQ scores ranged from 0.67 to 0.99 . Younger children exhibited worse functional outcomes compared to older children $(\mathrm{p}<.05)$.

Conclusions: Results suggest that children with Anti-NMDA encephalitis often show functional improvements during inpatient rehabilitation, despite ongoing functional deficits at discharge; however, younger patients may be at particular risk for worse outcomes. Further research is necessary to identify predictors of functional and cognitive outcome in this population.

Correspondence: Robyn A. Howarth, Ph.D., Neuropsychology, Children's Healthcare of Atlanta, Children's Healthcare of Atlanta, Neuropsychology, 5455 Meridian Mark Rd. NE Suite 180, Atlanta, GA30342, United States.E-mail: Robyn.Howarth@choa.org

B.K. IWAMOTO, J. LENIHAN, J. NICHOLS, J. PIVONKA-JONES, K. FREIER-RANDALL \& S. ASHWAL. Recovery of Memory Following Pediatric TBI: The Impact of Coma.

Objective: Memory deficits are frequently reported following pediatric traumatic brain injury (TBI), with outcome and recovery a function of numerous moderating variables. The purpose of the current study was to examine coma as a moderating variable in memory recovery. Research has suggested coma duration may impact neurocognitive recovery.

Participants and Methods: Seventy-five children (4-19 years; $72 \%$ male) with moderate/severe TBI were recruited from a Level 1 Trauma Center ED. They were administered the Children's Memory Scale at 3 - and 12-months post-injury as part of a larger institutional study. Coma was documented in 58 patients (1-20 days, $\mathrm{M}=2.95$ days). Data were analyzed using independent-samples t-tests, bivariate correlation, and repeated measures analysis with coma as the grouping variable.

Results: Results showed general memory scores at both 3 - and 12-months were negatively correlated with the number of days spent in a coma $(r=-.38, p<.01$ and $r=-.30 . p<.05)$. No significant differences were found in general memory scores between groups at 3 -months (coma $\mathrm{M}=90.71$, $\mathrm{SD}=20.58$; no coma $\mathrm{M}=100.93, \mathrm{SD}=20.17 ; t(62)=1.69, p=.096)$ and 12-months post-injury (coma $\mathrm{M}=101.83, \mathrm{SD}=23.22$;no coma $\mathrm{M}=109.79, \mathrm{SD}=13.37 ; t(60)=1.62, p=.113)$. Additionally, no significant effect was found between coma status and general memory scores from 3 - to 12 -months $(F(1,58)=0.04, p=.837)$.

Conclusions: This prospective longitudinal study shows that memory improves at similar rates in both the coma group and the no coma group, however, the number of days in a coma is negatively correlated with 
memory recovery, which is consistent with previous research (Sinha et al., 2013). Length of coma does inform post recovery outcomes and intervention needs.

Correspondence: Brooke K. Iwamoto, Pediatric Psychological Services, Loma Linda University, 19362 Pilario, Rowland Heights, CA 91748, United States.E-mail:biwamoto@llu.edu

D. JASHAR, R. DIDIANO, V. CORELLO-SOARES \& M. BEST. Retrieval Differences Following Concussion.

Objective: Individuals complain of retrieval deficits after concussion. It is important to examine deficits using a comparison group. The aim of this study is to compare the neurocognitive profile of individuals who have experienced one or more concussions (Concussion group) to individuals with no concussions (Control Group).

Participants and Methods: Ninety-nine individuals ( mean age $=15.48$, $S D=2.93$; female $=52$, male $=47$ ) were included in the Concussion group. Twenty-one individuals ( mean age $=9.62, S D=1.25$; female $=9$, male $=12$ ) were included in the Control group. Measures: Auditory Naming Test (ANT; Total Correct and "Tip of Tongue" Responses (TOT; responses after 2 seconds), Boston Naming Test (BNT; Total Correct), and selected subscales from the Wechsler Memory Scales (Logical Memory I and II, Paired Verbal Associations I and II).

Results: Independent-samples t-tests were used to examine score differences between the Control and Concussion groups on Total Correct BNT, TOT ANT, Logical Memory I and II, and Verbal Paired Associations I. There was a difference in scores between Control $(M=96.67, S D=8.71)$ and Concussion groups $(M=94.83, S D=17.29 ; t(48)=-.45, p=.01)$ on Logical Memory I. A Mann-Whitney Signed Rank U Test revealed a difference in ANT Response Time between the Control $(M d=94$, $n=21)$ and Concussion ( $M d=115, n=38)$ groups but not on Total Correct ANT and Verbal Paired Associations II.

Conclusions: The findings indicate that individuals who have experienced concussions may have some mild difficulties with retrieval compared to the Control group. Those in the concussion group were slower at naming and had lower scores in immediate retrieval of contextual information.

Correspondence: Dasal Jashar, Doctorate in Clinical Psychology, Psychology, University of Connecticut, 406 Babbidge Road, Unit 1020, Storrs, CT 06269-1020, United States. E-mail: dasal.jashar@uconn. edu

A.P. KONTOS, E. REYNOLDS, A. SUFRINKO, V.L. REEVES, C.L. HOLLAND \& M.W. COLLINS. Comparison of Adolescents with Vestibular and Anxiety Clinical Profiles following Concussion. Objective: Concussed patients present with clinical profiles including vestibular and anxiety (Collins et al., 2014; in press). However, there is no research on outcomes in patients with different clinical profiles. The purpose of this study was to compare clinical outcomes among concussed adolescents with vestibular, anxiety, and neither-vestibular nor anxiety (NVA) clinical profiles.

Participants and Methods: A vestibular group ( $\mathrm{n}=11$; i.e., presence of vestibular dysfunction/symptoms), an anxiety group ( $\mathrm{n}=10$; i.e., presence of anxiety symptoms with or without vestibular dysfunction/ symptoms) and a NVA (neither anxiety nor vestibular) group $(\mathrm{n}=12)$ were derived from a sample of 33 adolescents, aged 12-20 (M=15.0, $\mathrm{SD}=1.9$ ), with a diagnosed concussion. Participants completed the Post-concussion Symptom Scale (PCSS), Immediate Post-concussion Assessment and Cognitive Testing (ImPACT), Vestibular/Ocular Motor Screening (VOMS), Balance Error Scoring System (BESS) 2-10 days post-concussion. Univariate ANOVAs with Bonferroni corrections were used to compare the groups.

Results: The anxiety group took longer to recover $(\mathrm{F}=2.8, \mathrm{p}=0.05$, $\eta 2=.21 ; \mathrm{M}=63.9, \mathrm{SD}=68.6$ days) than both the vestibular group $(\mathrm{M}=36.6, \mathrm{SD}=11.9)$ and the $\mathrm{NVA}$ group $(\mathrm{M}=19.9, \mathrm{SD}=12.3)$. The anxiety group reported higher symptoms $(F=3.2, p=0.05, \eta 2=0.18)$ and lower processing speed scores $(\dot{F}=5.6, p=0.009, \eta 2=0.21)$ than the other groups. The vestibular and anxiety groups scored worse on vestibular $(\mathrm{F}=4.3, \mathrm{p}=0.02, \eta 2=0.23)$ and ocular motor $(\mathrm{F}=43.5, \mathrm{p}=0.04, \eta 2=0.20)$ outcomes than the NVA group.

Conclusions: Clinicians should employ multimodal comprehensive assessments to identify patients with clinical profiles such as anxiety that may be linked to worse clinical outcomes and longer recovery times. Correspondence: Anthony P. Kontos, PhD, Orthopaedic Surgery, University of Pittsburgh, UPMC Rooney Sports Complex, 3200 South Water St, Pittsburgh, PA 15203, United States. E-mail: akontos@pitt. edu

K. KOWALSKI, L. TOMFOHR-MADSEN, E.D. BIGLER, A. BACEVICE, B. BANGERT, D. COHEN, L. MIHALOV, N. ZUMBERGE, H. TAYLOR \& K.O. YEATES. Are Pre-Injury Sleep Behavior and Acute Post-Concussive Symptoms Predictive of Post-Injury Sleep Behavior in Children with Mild Traumatic Brain Injury or Orthopedic Injury?

Objective: The relationship of injury type, pre-injury sleep problems, and acute PCS to post-injury sleep problems was examined in children with mild traumatic brain injury (mTBI) or orthopedic injury (OI).

Participants and Methods: 59 children with mTBI and 35 with OI aged 8 through 15 years of age were recruited from the Emergency Department of two Ohio children's hospitals at the time of injury. Parents rated their children's pre-injury sleep behavior (Sleep Disorders Inventory for Students) retrospectively, post-acute cognitive and somatic PCS (Health Behavior Inventory-20) at approximately 10 days post-injury, and sleep behavior at 3-month post-injury.

Results: Pre-injury sleep problems $(\beta=.557, p<.001)$ and post-acute somatic PCS $(\beta=.361, p<.001)$, but not injury type $(\beta=-.033, p<.711)$, were significant predictors of 3 -month post-injury sleep problems $\left(\mathrm{R}^{2}=.491, p<.001\right)$. Likewise, pre-injury sleep problems $(\beta=.448$, $p<.001)$ and post-acute cognitive PCS $(\beta=.326, p=.001)$, but not injury type $(\beta=.079, p<.325)$, were significant predictors of 3 -month post-injury sleep problems $\left(\mathrm{R}^{2}=.470, \mathrm{p}<.001\right)$.

Conclusions: Having more pre-injury sleep problems or higher parentrated post-acute PCS are predictive of having more sleep problems 3 months post-injury, regardless of the type of injury. These preliminary findings suggest that preexisting sleep difficulties and acute PCS may play a more critical role in the development of post-injury sleep problems than injury type.

Correspondence: Kristina Kowalski, Ph.D., Department of Psychology, University of Calgary, Alberta Children's Hospital Research Institute, 2888 Shaganappi Trail NW, Calgary, AB T3B 6A8, Canada. E-mail: kristina.kowalski@ucalgary.ca

K. KOWALSKI, L. TOMFOHR-MADSEN, E.D. BIGLER, A. BACEVICE, B. BANGERT, D. COHEN, N. ZUMBERGE, L. MIHALOV, H. TAYLOR \& K.O. YEATES. The Relationship Between Pre-Injury Sleep and Acute Post-Concussive Symptoms in Children with Mild Traumatic Brain Injury and Orthopedic Injury.

Objective: The relationship of injury type and pre-injury sleep problems to acute post-concussive symptoms (PCS) was examined in children with mild traumatic brain injury (mTBI) or orthopedic injury (OI).

Participants and Methods: 95 children with mTBI and 55 with OI aged 8 through 15 years of age were recruited from the Emergency Department of two Ohio children's hospitals at the time of injury. Parents rated their children's pre-injury sleep behavior retrospectively (Sleep Disorders Inventory for Students) and post-acute cognitive and somatic PCS at approximately 10 days post-injury (Health Behavior Inventory-20).

Results: Pre-injury sleep problems and injury type both significantly predicted both somatic $\left(\mathrm{R}^{2}=.227, \mathrm{p}<.001\right)$ and cognitive PCS $\left(\mathrm{R}^{2}=.342, p<.001\right)$ at post-acute assessment. The interaction of pre-injury sleep and injury type did not account for significant additional 
variance in either somatic $\left(\mathrm{R}^{2}\right.$ change $\left.=.002, p=.531\right)$ or cognitive PCS $\left(\mathrm{R}^{2}\right.$ change $\left.=.001, p=0.691\right)$.

Conclusions: Children with more pre-injury sleep problems or a mTBI have higher parent-reported PCS at post-acute assessment than those with fewer pre-injury sleep problems or OI. However, pre-injury sleep problems do not appear to moderate the effect of mTBI on parentreported post-acute PCS. Pre-existing sleep difficulties are an important consideration when evaluating and treating acute PCS in children. Correspondence: Kristina Kowalski, Ph.D., Department of Psychology, University of Calgary, Alberta Children's Hospital Research Institute, 2888 Shaganappi Trail NW, Calgary, AB T3B 6A8, Canada. E-mail: kristina.kowalski@ucalgary.ca

V. KWAN, K. KOWALSKI, M. NOEL, H. TAYLOR, E.D. BIGLER, D. COHEN, L. MIHALOV, N. ZUMBERGE, A. BACEVICE, B. BANGERT \& K.O. YEATES. The Relationship Between Pain and Post-Concussive Symptoms: Comparing Children with Mild Traumatic Brain Injury to Orthopedic Injury.

Objective: The relationship of cognitive and somatic post-concussive symptoms (PCS) to pain intensity was examined in a prospective, longitudinal study of children after a mild traumatic brain injury (mild TBI) or orthopedic injury $(\mathrm{OI})$.

Participants and Methods: Participants included 87 children with mild TBI and 48 children with OI, between 8 and 16 years of age, recruited at the Emergency Department of two children's hospitals in Ohio. Children rated their pain intensity (Numerical Rating Scale-11) and post-concussive symptoms (Health and Behavior Inventory-20) each week, from the time of injury up to six months post-injury, through a smartphone app, email or phone call.

Results: Mixed models predicting PCS, with pain as a time-varying covariate, revealed a stronger relationship between children's pain intensity and both cognitive and somatic PCS in children with mild TBI than in those with OI, after controlling for sex and age at the time of injury $(\beta=0.91, p=0.018$ and $\beta=0.80, p=0.002$ for somatic and cognitive PCS, respectively). In addition, girls showed a stronger relationship than boys between pain and cognitive PCS ( $\beta=-1.08$, $p=0.005)$, but not somatic PCS, after controlling for all other variables. Conclusions: Higher ratings of pain are related to greater increases in both cognitive and somatic PCS in children after mild TBI than OI. These findings suggest that pain should be carefully assessed and become a focus of treatment in settings that manage children after a mild TBI.

Correspondence: Vivian Kwan, Masters, Psychology, University of Calgary, 250 Collegiate Blvd NW, Calgary, AB T2N5A6, Canada. E-mail:vivian.kwan2@ucalgary.ca

C. LALIBERTE DURISH, K.O. YEATES \& B. BROOKS. Persistent Effects of Pediatric Mild Traumatic Brain Injury: The Role of Resilience.

Objective: Previous research has indicated a potential dose-response relationship with respect to the number of mild traumatic brain injuries (mTBIs) a child sustains and the presence of persistent post-concussion symptoms (PCS). As well, studies have shown low psychological resilience to be predictive of PCS in adults with mTBI, though this relationship has yet to be studied in children. Therefore, this study examined the relationship of psychological resilience to persistent PCS following single and multiple pediatric mTBI, compared to orthopaedic injury (OI).

Participants and Methods: Participants $(\mathrm{N}=65)$, ages 8-18 years, who sustained multiple mTBIs $(n=24)$, a single mTBI $(n=22)$, or an OI $(n=19)$, were recruited from existing research databases at a children's hospital in Calgary, Alberta. All participants sustained injuries at least 6 months prior to recruitment, with an average time since injury of 2.68 years. Self-reported psychological resilience was measured using the Connor-Davidson Resilience Scale (CD-RISC), and both selfand parent-reported PCS were measured using the Post-Concussion Symptom Inventory (PCSI). Hierarchical regression analyses examined psychological resilience as a predictor of PCS, both as a main effect and as a moderator of group differences.

Results: Multiple mTBIs and low psychological resilience were both significant predictors of persistent PCS, although the absence of significant interactions indicated resilience likely does not act as a moderator of group differences in PCS.

Conclusions: Sustaining multiple mTBIs may increase a child's risk of persistent PCS; however, high psychological resilience may serve as a protective factor with respect to persistent PCS, regardless of the number or type of injuries a child sustains. Our results provide support for developing interventions aimed at increasing psychological resilience to potentially improve outcomes for children suffering from persistent PCS after mTBI.

Correspondence: Christianne Laliberte Durish, M.Sc., Psychology, University of Calgary, 405-188 15 Ave SW, Calgary, AB T2R1S4, Canada.E-mail: christianne.lalibert@ucalgary.ca

C. LALIBERTE DURISH, K.O. YEATES \& B. BROOKS. The Role of Psychological Resilience in Children with Poor Recovery Following Mild Traumatic Brain Injury.

Objective: Poor recovery from mild traumatic brain injury (mTBI) may stem largely from psychosocial factors, which are not well understood. This study examined whether psychological resilience predicts persistent post-concussion symptoms (PCS) following pediatric mTBI.

Participants and Methods: Children ( $\mathrm{N}=66)$, ages 8 to 17 years, who sustained a mTBI were recruited from a neuropsychology screening clinic at a children's hospital in Calgary, Alberta. All participants sustained mTBIs more than 3 months prior to the clinic visit, and were seen on the basis of poor recovery (i.e., presence of persistent PCS and complaints of cognitive problems). Self-reported psychological resilience was measured using the 10 -item version of the ConnorDavidson Resilience Scale (CD-RISC) and PCS were measured using the self- and parent-reported Post-Concussion Symptom Inventory (PCSI). Hierarchical regression analyses examined psychological resilience as a predictor of PCS.

Results: Low psychological resilience was a significant predictor of greater overall child-reported persistent PCS $\left(R^{2}=.10, \beta=-.32\right.$, $p=.021)$, as well as specific physical $\left(R^{2}=.08, p=.047\right)$, cognitive $\left(R^{2}=.11, p=.014\right)$, and emotional $\left(R^{2}=.15, p=.004\right)$ symptom domains, though was not predictive of fatigue-related symptoms. Low psychological resilience was a significant predictor of parent-reported emotional symptoms $\left(R^{2}=.07, p=.038\right)$, though not overall parent-reported PCS.

Conclusions: Psychological resilience likely plays an important role in recovery from $m$ TBI. Children with poor recovery from $\mathrm{mTBI}$ who report low psychological resilience are more likely to report greater PCS than those with high psychological resilience. These results suggest a need to develop and evaluate interventions focused on increasing psychological resilience to improve outcomes for children suffering from persistent PCS following mTBI.

Correspondence: Christianne Laliberte Durish, M.Sc., Psychology, University of Calgary, 405-188 15 Ave SW, Calgary, AB T2R1S4, Canada.E-mail: christianne.lalibert@ucalgary.ca

G. LALONDE, A. BERNIER, C. BEAUDOIN, J. GRAVEL \& M.H. BEAUCHAMP. Executive Functions After Mild Traumatic Brain Injury in Preschool Children.

Objective: During childhood, the incidence of traumatic brain injury (TBI) is especially high in the preschool years (McKinlay et al., 2008). The young brain is particularly vulnerable to head injury due to inherent physiological and developmental factors (Huelke, 1998). Children who sustain severe TBI tend to show impaired cognitive skills such as executive functions (EF; Ganesalingam, et al., 2011; Nadebaum, et al., 2007). However, little is known of the impact of the most common and mildest form of TBI on EF. The aim of this study was to examine EF in preschoolers following mild TBI (mTBI). 
Participants and Methods: This is a sub-analysis of a larger prospective cohort study of preschool TBI. Twenty-eight children (3.5-5 years; $M$ age $=50, S D=5.3$ months, 16 males) with accidental mTBI were recruited at an urban paediatric Emergency Department immediately following trauma. Six months post-injury they completed EF tests assessing inhibition (Shape stroop; Carlson, 2005), working memory (Spin the pots; Hughes \& Ensor, 2005), processing speed (Codes WPPSI-III; Wechsler, 2002), planning (Hanoi tower; Welsh, et al., 1991) and cognitive flexibility (Dimensional Change Card Sort Test; Zelazo, 2006). ANOVA was used to compare their performance to that of 34 typically developing children (TDC; $M$ age $=50, S D=4.4$ months, 15 males).

Results: Children with mTBI performed significantly worse on the inhibition and processing speed tasks compared to TDC (Welch's $F(1,35.35)=4.51, p=.04, d=0.57 ; F(1,57)=5.06, p=.03$, $d=0.57)$. No differences were found on the other EF tests.

Conclusions: In young children, even mild cases of TBI can negatively impact EF, more specifically inhibition and speed processing. These results are consistent with the hypothesis that skills in a rapid stage of development, such as EF in the preschool years, could be particularly vulnerable to brain disruptions (Ewing-Cobbs, et al., 2004), and suggest monitoring of EF may be important after preschool mTBI.

Correspondence: Gabrielle Lalonde, University of Montreal, 2900 Boulevard Edouard-Montpeti, Montreal, QC H3T 1J4, Canada. E-mail: gabrielle.lalonde@hotmail.com

C. LANDRY-ROY, G. LALONDE, A. BERNIER, C. BEAUDOIN, J. GRAVEL \& M.H. BEAUCHAMP. Should children with TBI be compared to orthopedic or healthy controls?

Objective: In TBI research, control groups typically consist of orthopedic controls (OC) or uninjured healthy controls (HC). It has been argued that OC are most similar to individuals with TBI because they share injury-related experiences and pre-morbid characteristics, but OC are more costly and time consuming to recruit. Mathias et al. (2013) found that adult $\mathrm{OC}$ and $\mathrm{HC}$ were similar on a number of demographic, psychosocial and cognitive variables; though, it is not known whether these results apply to children.

Participants and Methods: This is a substudy of a prospective TBI cohort study. We compared 72 OC recruited in the emergency department and $84 \mathrm{HC}$ recruited in daycares aged between 2 and 5 years of age. OC completed pre-injury questionnaires measuring demographics, behavior (CBCL, ABAS) and family functioning (DAS, FAD, PSI). Six months later, parents completed the same questionnaires and children underwent neuropsychological assessment. HC completed the assessment immediately after recruitment and their caregiver completed the questionnaires only once.

Results: Independent sample $t$-tests and Chi-square showed no group differences on any study variables $(p>0.05)$. They were comparable on socioeconomic status, parental education, ethnicity, birth characteristics and developmental history. The two groups had similar behavioral and family characteristics as reported by the parents. These results were observed when analyzing both pre-injury and post-injury characteristics. Moreover, they had equivalent levels of general intellectual functioning and verbal abilities, and they performed equally on tasks of executive functions and theory of mind.

Conclusions: OC and HC were similar on all variables tested, both when considering pre-injury characteristics of OC and their functioning 6-months post-injury. There may be no major demographic, behavioral, familial or cognitive differences between OC and HC, and no clear advantage in recruiting OC in preschool TBI studies, though other variables may be of interest to explore.

Correspondence: Gabrielle Lalonde, University of Montreal, 2900 Boulevard Edouard-Montpeti, Montreal, QC H3T 1J4, Canada. E-mail: gabrielle.lalonde@hotmail.com
S.L. WADE, E. LEBLOND, S. RAJ, K. BAUM, A. THOMPSON, R. SALLOUM, M. NARAD \& A. PLATT. Developing an Intervention to Promote Quality of Life in Pediatric Brain Tumor Survivors.

Objective: To develop an online intervention for adolescent survivors of pediatric brain tumors (PBT), the second most common childhood cancer. PBTs and their treatments are associated with long-term neurocognitive and psychosocial difficulties. Few interventions target these difficulties in this population. Focus groups conducted with PBT survivors, parents, and care providers identified needs and challenges. Findings were used to develop an online cognitive-behavioral intervention, designed to be both accessible and customizable to unique needs of survivors. We report on focus group findings and background for the intervention.

Participants and Methods: Five PBT survivors (3 females, 2 males; ages 14-25) and 6 parents participated in focus groups, discussing needs, challenges, and suggestions for an intervention targeting survivors of PBT. Survivors also participated in individual qualitative interviews and completed questionnaires rating needs and challenges.

Results: Most survivors $(n=4)$ reported problems with memory, mood, and relationships. Other challenges noted included problems with medication management and fatigue. There was variability in concerns both between survivors (e.g., fatigued affected some "not at all" and others "extremely") and between survivors and their parents (e.g., parents reported organization, planning, and problem solving as affecting their child "a little", while the survivor reported it as "extremely"). Survivors and their families noted challenges changed over time. One survivor commented that her challenges changed "from day to day and week to week".

Conclusions: Survivors and their parents reported a wide range of challenges, though difficulties varied, underscoring the need for customizable interventions. Input suggests that interventions for PBT survivors need to be brief and flexible to address social, emotional, and neurocognitive concerns.

Correspondence: Elizabeth LeBlond, Cincinnati Children's Hospital Medical Center, 2926 Vaughn Street, Cincinnati, OH 45219, United States.E-mail: elizabeth.leblond@cchmc.org

J. LENIHAN, B.K. IWAMOTO, J. PIVONKA-JONES, K. FREIERRANDALL, J. NICHOLS \& S. ASHWAL. Recovery of Attention Deficits following Pediatric TBI: The Impact of Premorbid School Performance.

Objective: The objective of the current study is to describe the trajectory of attention following moderate/severe TBI in a pediatric population with consideration of premorbid school performance. As research has demonstrated reduced attentiveness after TBI, the impact of premorbid school performance on attention was explored.

Participants and Methods: 55 children (6-16 years, M=12.12, $\mathrm{SD}=3.65,70 \%$ male) with moderate/severe TBI were recruited from a Level 1 Trauma Center ED. The TEA-CH was administered at 3 and 12 months post-injury as part of a larger study/assessment battery. Premorbid academic functioning was grouped based on parent report (above average, average, and below average). Data was analyzed using a repeated measures ANOVA and independent t-tests.

Results: Results demonstrated a significant effect between premorbid school performance and attention scores over time $(\mathrm{F}(2,52)=4.08$, $p=<0.03)$. Results from the independent t-tests found a significant difference in scores between average $(\mathrm{M}=11.54, \mathrm{SD}=3.50)$ and below average $(\mathrm{M}=7.50, \mathrm{SD}=3.89)$ group on attention measures 12 months after injury $(t(30)=2.13, p=0.04)$. The above average group did not demonstrate any significant changes from 3 months to 12 months ( $\mathrm{T} 1 \mathrm{M}=9.12 ; \mathrm{T} 12 \mathrm{M}=9.85$ ) and their overall scores did not suggest attention impairment.

Conclusions: This prospective longitudinal study shows that children with below average school performance prior to a TBI demonstrate attention deficits that did not significantly improve over time compared to those who had average school performance prior to injury. Previous evidence supports this finding, suggesting that premorbid educational 
abilities can be a good predictor of outcome after TBI in children (Catroppa et al., 2008).

Correspondence: Jessica Lenihan, Department of Psychology, Loma Linda University, 1601 Barton Road Apt 1706, Redlands, CA 92373, United States.E-mail: jlenihan@llu.edu

H.M. LINDSEY, S. JIVANI, J.J. MIETCHEN, S.D. GALE, E.A. WILDE, J. FABER, M. MACLEOD, M.E. AITKEN, L. EWINGCOBBS \& H.S. LEVIN. Effects of Injury Severity on White Matter Tract Integrity in Relation to Verbal Memory in Chronic Pediatric Traumatic Brain Injury.

Objective: There is a paucity of literature investigating the relationships between injury severity, declarative verbal memory, and the integrity of associated white matter (WM) pathways, particularly for chronic pediatric TBI. The aims of the present study are to determine the following: 1) the effect that injury severity may have on the integrity of WM pathways, 2) the impact that reduced WM integrity may have on declarative memory, and 3) whether the integrity of regional WM pathways plays a mediating role between injury severity and declarative verbal memory in children with chronic TBI.

Participants and Methods: Participants included 21 patients (15 males) who sustained early TBIs-mean age at injury of $4.1(\mathrm{SD}=2.0)$ yearsand were between the ages of 11-17 years at the time of the study. DTI analyses were conducted using quantitative tractography via PRIDE software. WM pathways included were the corpus callosum (CC), uncinate fasciculi (UF), perforant pathways (PP), and the cingulum bundles (CB). Glasgow Coma Scale (GCS) scores were used as an index of injury severity. Declarative verbal memory performance was assessed using standard scores from the long-delay free recall (LDFR) trial of the California Verbal Learning Test - Children's Version. Structural equation modeling was used to conduct the mediation path analyses. Results: There was no significant total effect of GCS on LDFR performance. Distal mediation roles between GCS and LDFR were demonstrated through FA of the CC, the left PP, and the left UF. FA of the right $\mathrm{UF}$, right $\mathrm{PP}$, and right and left $\mathrm{CB}$ did not play mediating roles between GCS and LDFR; however, FA of the left CB predicted LDFR performance.

Conclusions: Verbal memory performance was not predicted by injury severity alone, however a mediating role is played between verbal memory and severity by the integrity of WM pathways, specifically the left PP, left UF, and the CG, in chronic pediatric TBI.

Correspondence: Hannah M. Lindsey, MA, Psychology, Brigham Young University, $1200 \mathrm{~N}$ Terrace Dr, Apt 106, Provo, UT 84604, United States.E-mail: lindseyhm.89@gmail.com

J.R.M. LIU, E. LIMA, K. HARTLINE, N.B. PAWLENKO, A. PATEL, A. RIOPELLE \& A. HERRERA-HAMILTON. Age-Dependent Association Between Post-Concussional Children and Adolescents and Balance Performance in Neuropsychological Testing.

Objective: Limited published data exists on the modified Balance Error Scoring System (BESS) for children and adolescents post-concussion. The current study aims to assess the impact of age at two time points post-concussion as a predictor of change in balance.

Participants and Methods: Participants. Children and adolescents $(\mathrm{N}=265)$ (aged 2.86-22.86; $\mathrm{M}=13.61 ; \mathrm{SD}=3.26 ; 64.3 \%$ male; $35.7 \%$ female) diagnosed with concussion and receiving post-concussion treatment at Children's Hospital Los Angeles (CHLA). All patients were examined at an initial exam and for neuropsychological testing due to persistent symptoms.

Method. Initial exam (3 days-3 months post-injury) BESS scores were compared to follow up scores gathered at neuropsychological exam (3-4 weeks post initial admission).

Results: A paired samples t-test was run to assess whether balance following a concussion increased or decreased after admission. Results show that individuals who sustain a concussion have increased balance at follow up $(N=255, M=25.38, S D=4.51)$ than at initial exam $(N=255, M=23.58, S D=5.17)$. Results of a paired samples t-test indicated there was a significant difference between balance at initial exam and balance at follow up $(t(2)=-6.17, p<.001)$. Two Simple Linear Regressions were conducted in SPSS 21.0 (IBM, 2012). Results indicated that age in years explained a significant amount of the variance on the BESS at initial exam and follow up $\left(R^{2}=.077, R^{2}=.119\right.$, respectfully). Specifically, as age increases by one year, scores on the initial BESS score increase by .277, 95\% CI[.277, .688] $(p<.001)$. Additionally, as age increases by one year, scores on the final BESS increase by $.344,95 \%$ CI $[.346, .687)(p<.001)$.

Conclusions: The results of the study support our hypothesis that age predicts scores on the BESS at initial and follow up exams. Findings suggest that age may be a mediating factor in BESS performance and may have implications for BESS scores across the lifespan and in concussion management.

Correspondence: Jessica R.M. Liu, M.A., Neuropsychology, Children's Hospital Los Angeles, 4650 Sunset Blvd., Attn: Anita Herrera Hamilton, Ph.D., ABPP-CN, Los Angeles, CA 90027, United States.E-mail:jrliu@ mednet.ucla.edu

J. LORTIE, V. SICARD, R.D. MOORE \& D. ELLEMBERG. Assessment Of Executive Functions Using A Colour-Shape Switch-Task In Elite Adolescent Hockey Players With And Without A History Of Concussion.

Objective: Previous research indicates that deficits in executive functions can persist several months following a sport-concussion (Ellemberg et al., 2009; Mayr et al., 2014). We used a switch-task to assess executive functions in elite adolescent hockey players with and without a history of concussion.

Participants and Methods: Thirty-three elite adolescent hockey players (19 history of concussion; 14 controls) completed a switch-task before and after a bout of moderate aerobic exercise. Participants with a history of concussion were 6 to 36 months from their last concussion and incurred 1-3 concussions. Participants in the two groups did not differ in terms of age ( $15.52 \pm 0.71$ years), education $(10.15 \pm 1.25$ years $)$, body mass index $\left(24.03 \pm 2.46 \mathrm{~kg} / \mathrm{m}^{2}\right)$, years of sport participation $(10.70 \pm 1.49$ years $)$ or years of body checking $(3.52 \pm 1.18$ years). The aerobic exercise session consisted of a 20 -minute submaximal effort (60-70\% of maximal heart rate) on a cycle ergometer.

Results: Under the present testing conditions, no difference in cognitive performance was observed before or after a bout of moderate aerobic exercise. Specifically, measures of accuracy, reaction time, local switch cost, global switch cost and working memory cost were similar for both groups before and after exercise ( $p s \geq=0.125)$.

Conclusions: Athletes with a history of concussion did not differ from their matched teammates in terms of executive functions using the colour-shape switch task. These findings contradict to our previous research revealing persistent executive dysfunction in adolescents with a history of concussion (Baillargeon et al. 2012). Additional participants are needed to determine if time since injury and the number of concussions influence executive functioning in adolescent athletes with a history of concussion.

Correspondence: Jean-Christophe Lortie, Master, Kinesiology, Université de Montréal, 2100 Boulevard Edouard-Montpetit, Montreal, QCH3T1J4, Canada.E-mail: jc.lortie96@gmail.com

C.E. LOVE, S.J. TLUSTOS-CARTER, M. KIRKWOOD, K.O. YEATES, H. TAYLOR, T. STANCIN \& S.L. WADE. Parent and Self-Report of Executive Functioning After Moderate to Severe TBI.

Objective: Parents of teens often endorse more executive functioning $(\mathrm{EF})$ problems than teens themselves in a variety of populations (e.g., speech language impairment, myelomeningocele and hydrocephalus). We sought to evaluate the relationship between parent- and self-report 
ratings of $\mathrm{EF}$ in adolescents with traumatic brain injury (TBI) and to examine whether discrepancies varied by injury severity.

Participants and Methods: Participants included 140 adolescents (99 males), aged 11-18 years. Injury severity was defined as lowest Glasgow Coma Scale (GCS) score, with a moderate TBI (n=78, GCS below 13 or 13-15 with intracranial imaging findings) and severe TBI $(n=62)$ group. The Behavior Rating Inventory of Executive Function (BRIEF) Parent- and Self-Report Global Executive Composite scores were utilized.

Results: BRIEF parent and self ratings correlated significantly, although parents reported significantly higher executive impairment than adolescents $(\mathrm{t}[139]=5.27, \mathrm{p}<.001)$. Teens with moderate versus severe injuries did not differ on either self $(\mathrm{t}[138]=1.15, \mathrm{p}=.25)$ or parent ratings $(\mathrm{t}[138]=.807, \mathrm{p}=.42)$. Degree of informant discrepancy using age at injury and SES as covariates did not demonstrate significant effects of injury severity (moderate vs. severe; $\mathrm{F}(1,136)=.48$, $\mathrm{p}=.49$ ). Further, select background variables (e.g., premorbid ADHD) did not predict informant discrepancy.

Conclusions: Following adolescent TBI, parents and teens report differing levels of EF problems, and this discrepancy does not appear to be explained by injury severity defined by initial GCS score. This finding is noteworthy, as another injury severity variable, length of coma, has been found to predict differences in parent and self-report $\mathrm{EF}$ in a different population of adolescents with a history of TBI (Wilson et al., 2011). Our results in conjunction with previous literature suggest that discrepancies between parent and self perceptions of EF may not be affected by TBI severity.

Correspondence: Christina E. Love, MS, Psychology, Florida Institute of Technology, $150 \mathrm{~W}$. University Blvd., Melbourne, FL 32901, United States.E-mail: celove182@gmail.com

C. MARINO. Self-Awareness of Psychosocial Functioning and Executive Functions Following Moderate and Severe Pediatric Traumatic Brain Injury.

Objective: Deficits in self-awareness (SA) are common following pediatric traumatic brain injury (TBI). This study examined executive functions as correlates of SA deficits of internalizing and externalizing symptoms.

Participants and Methods: Eleven children and their parents completed parallel forms of the Behavior Assessment System for Children measuring externalizing symptoms (inattentiveness, hyperactivity) and internalizing symptoms (anxiety, depression, somatization). Neuropsychological measures of executive functions included: sustained and auditory attention, cognitive flexibility, and inhibitory control. Degree of concordance between the parent and self reports were computed to indicate SA deficits. Paired samples t-tests were computed to examine agreement between self and parent reports. Independent samples t-tests were conducted to examine the influence of executive functions on SA impairments of internalizing and externalizing symptoms.

Results: Significant discrepancies were found on internalizing symptomatology, $\mathrm{t}(10)=-3.009, \mathrm{p}=.013$ and attention problems, $\mathrm{t}(10)$ $=-2.710, p=.022$, such that children frequently rated themselves more favorably than their parents. SA deficits of internalizing symptoms significantly related to sustained attention, $\mathrm{t}(9)=-.494, \mathrm{p}=.056$. Moderate to large effect sizes were found between SA of internalizing symptoms and executive functions.

Conclusions: Parent and child reports of child's functioning may be strongly disparate at least when measuring symptoms pertaining to sadness, anxiety, somatization, and inattention. These findings highlight the importance of multiple source assessments when evaluating neurobehavioral outcomes. Poorer sustained attention likely contributes to the child's ability to accurately infer sadness, withdrawal, and anxiety. Moderate to large effect sizes between SA deficits of internalizing and externalizing symptoms and attention, set-switching, and inhibition suggest that these relationships may reach statistical significance with a larger sample.
Correspondence: Cherylynn Marino, Ph.D., Research, Kessler Foundation; Children's Specialized Hospital, 971 Bloomfield Ave, Glen Ridge, NJ 07028, United States.E-mail: cmarino@kesslerfoundation.org.

E.C. MAXWELL, K.E. CARLSON, T. BALLANTYNE, A.L. HOLLATZ, T.J. BERNARD \& R. BOADA. Predictors of Adaptive Functioning Following Childhood Arterial Ischemic Stroke.

Objective: The present study evaluated potential predictors of adaptive functioning (AF) after childhood arterial ischemic stroke (AIS). It was hypothesized that language skills, motor functioning, psychological symptoms, and executive functioning would significantly predict $\mathrm{AF}$, with motor and language skills accounting for most of the unique variance in $\mathrm{AF}$.

Participants and Methods: Participants were children $(n=51$, mean age $=12.1$ years) who suffered an AIS during childhood (range $=1$ month to 19.1 years). Children completed the Grooved Pegboard, Boston Naming Test, Sentence Repetition and Token subtests of the Multilingual Aphasia Examination. Parents completed the Scales of Independent Behavior - Revised, Achenbach Child Behavior Checklist (CBCL), and Behavior Rating Inventory of Executive Function (BRIEF). The assessment was completed at least 10 months post-AIS (mean $=3.96$ years $)$. Analyses were conducted using hierarchical regression.

Results: Language and fine motor skills, introduced first into the model to predict $\mathrm{AF}$, resulted in a significant $R^{2}$ of $0.43, F=14.94, p<0.001$. Both variables accounted for significant unique variance. Executive functioning (BRIEF Metacognitive Index) and Total Problems from the CBCL were entered second, yielding a significant $R^{2}$-change of 0.10 , $F$-change $=4.32, p=0.02$. Only executive functioning accounted for significant unique variance.

Conclusions: Language and fine motor skills accounted for $43 \%$ of the variance in AF in children with AIS. When total psychological problems and executive functioning were added, the variance accounted for increased to $53 \%$; however, total psychological problems was not a unique predictor. Follow-up analyses indicated that internalizing and externalizing problems both uniquely predicted variance in AF, but in opposite directions. Possible mechanisms for the latter finding are discussed. Overall, executive and psychological factors add unique variance beyond motor and language skills, indicating the need for intervention post AIS to facilitate AF.

Correspondence: Emily C. Maxwell, Children's Hospital Colorado, 13123 East 16th Avenue, Box 155, Aurora, CO 80045, United States. E-mail:emily.maxwell@childrenscolorado.org

J.J. MIETCHEN, H.M. LINDSEY, S. JIVANI, S.D. GALE, E.A. WILDE, J. FABER, M. MACLEOD, M.E. AITKEN, L. EWINGCOBBS \& H.S. LEVIN. White Matter Integrity and Neuroanatomical Correlates of CVLT-C Factors in Children with Traumatic Brain Injury.

Objective: Examine the white matter neuroanatomical correlates of the various factors of the CVLT-C in children with chronic traumatic brain injury (TBI).

Participants and Methods: Participants were 22 children with complicated-mild to severe TBI (age at injury $=4.09 \pm 1.97$, age at testing $=11.95 \pm 1.78$, GCS $=7.86 \pm 3.73,31 \%$ female). Fractional anisotropy $(F A)$ was acquired for bilateral frontal white matter, uncinate fasciculi, and perforant pathways using PRIDE software. Performance on the CVLT-C was separated into a six factor model, as has been done in previous research: simple attention, total learning, learning efficiency, delayed free recall, delayed cued recall, and recognition. Stepwise regression was used to examine the relationship between the white matter pathways and the six factors of the CVLT-C while controlling for age and GCS.

Results: Right frontal FA $(p=0.002)$ and left uncinate fasciculus FA $(p=0.014)$ were significant predictors of simple attention. The left perforant pathway FA $(p=0.054)$ was approaching significance as a 
predictor of total learning. Left frontal FA was predictive of learning efficiency $(p=0.002)$, as was age at injury $(p=0.047)$. The left perforant pathway was predictive of both the long delay free recall $(p=0.002)$ and long delay cued recall $(p=0.011)$. Lastly, both the left $(p=0.001)$ and right $(p=0.023)$ perforant pathways were predictive of recognition trials. Conclusions: White matter integrity in frontal and temporal structures was predictive of performance on various factors of the CVLT-C in children with a history of TBI. There is also some evidence of lateralization to the left in frontotemporal white matter structures as they relate to CVLT-C performance.

Correspondence: Jonathan J. Mietchen, BS Psychology, Department of Psychology, Brigham Young University, 1001 SWKT, Provo, UT 846025543, United States. E-mail: jonathan.mietchen@gmail.com

S. MORRIS, D. MURDAUGH, K. ONO \& T. BURNS. Effects of Developmental Age on Symptom Reporting and Neurocognitive Performance in Youth after Sports-Related Concussion.

Objective: Recent research has focused on differences in recovery from sports-related concussion (SRC) based on age; however, few studies have assessed SRC in younger ages. The purpose of this study was to assess age differences in symptoms and neurocognitive performance.

Participants and Methods: Sample included 1,345 acutely concussed ( $<7$ days post-concussion) and 3,529 nonconcussed athletes (ages 8-18) who completed the Immediate Post-Concussive Assessment and Cognitive Testing (ImPACT) battery. Participants were divided into developmental age cohorts to assess differences in symptoms and neurocognitive scores.

Results: Across all age groups, the concussed group demonstrated significantly lower performance than the nonconcussed group in verbal memory ( $p<0.0001)$, visual memory, visuomotor, reaction time, and impulse control $(p<0.0001)$. When assessing effect of age, younger ages performed significantly lower across all ImPACT composite scores than older ages, regardless of whether concussed or nonconcussed. Within each age group, athletes in the middle-aged concussed group (ages 13-16) had significantly lower performance scores compared to same-aged nonconcussed across all composite scores $(p<0.0001)$ and total symptom scores $(p<0.001)$. However, composite scores were not statistically different between the concussed and control group in the youngest (ages 8-12) or oldest (ages 17+) cohorts.

Conclusions: These results revealed age differences in performance not only in concussed but also in nonconcussed youth. Specifically, the youngest and oldest developmental age ranges did not differentiate between concussed and nonconcussed youth athletes, suggestive of test sensitivity differences across age groups. This provides important information for providers to consider when assessing SRC at different ages. Correspondence: Sarah Morris, GA, United States. E-mail: sarahmorris713@gmail.com

M. NARAD, C.A. AUSTIN, H. TAYLOR, T. STANCIN, M. KIRKWOOD \& S.L. WADE. Examination of the Impact of a Web-Based Counselor Assisted Problem Solving Intervention (CAPS) on Teen Self-Report Behavior Rating Inventory of Executive Function (BRIEF) Following Pediatric TBI.

Objective: To examine the impact of a web-based Counselor-Assisted Problem Solving (CAPS) intervention on teen self-reported executive functioning after TBI.

Participants and Methods: This multi-site randomized controlled trial included 132 teens between the ages of 12-17, 1-6 months post hospitalization for a moderate to severe TBI. 65 teens received the CAPS online family-based problem solving intervention, and 67 were part of the Internet Resource Comparison (IRC) group. Parents and teens completed measures at baseline, 6-months, 12-months, and 18-months post baseline. Linear mixed models were used to examine teen selfreport GEC scores from the BRIEF as well as the difference between parent-reported and self-reported GEC scores over time. Age, SES, and injury severity were examined as treatment moderators.
Results: Injury severity moderated the effect of CAPS on teen selfreported executive functioning $(\mathrm{EF})$ over time $(\mathrm{F}(1,295)=4.65, \mathrm{p}=$ $.03)$. No significant contrasts were identified but visual inspection of graphs showed teens with severe TBI in the CAPS group appeared to report greater GEC scores over time while those with severe injuries in the IRC group reported a reduction in GEC scores over time. A significant treatment group*injury severity*time interaction revealed that parent-report and self-report became more similar over time for teens with severe TBI in the CAPS group $(\mathrm{F}(1,286)=4.95, \mathrm{p}=.03)$.

Conclusions: While CAPS has previously been associated with improved parent-report EF in older teens, this was not observed in teen self-report. Rather, teens with severe injuries receiving the CAPS intervention reported increasing EF difficulties. Comparison of parent and teen reported GEC reveals that while teens with severe injuries receiving the CAPS intervention reported greater GEC scores, they begin to approach the parent ratings. These findings suggest that the CAPS intervention may be associated with increased teen self-awareness of deficits rather than a worsening of symptoms.

Correspondence: Megan Narad, PhD, Behavioral Medicine Clinical Psychology/Physical Medicine \& Rehab, Cincinnati Children's Hospital Medical Center, 7521 Glenover Drive, Cincinnati, OH 45236, United States.E-mail:megan.narad@cchmc.org.

M.J. RAMIREZ FLORES, M. COLIN CANCINO, G. SALAS MORALES, J. CONTRERAS VACA \& J. SANTOS CORDERO. Theory of mind in children with mild and moderate traumatic brain injury during acute stage.

Objective: Moderate and severe TBI, in a chronical stage, is known to cause difficulties Theory of Mind (ToM), yet little is known about mild TBI. The purpose of this study is to analyze performance in Tom with children who have suffered mild and moderate TBI during the acute stage of the injury.

Participants and Methods: 15 children participated, between 4 and 8 years old $(x=5.53)$ with mild $(n=9)$ and moderate TBI $(n=6)$, matched by age, education and gender, with 15 children with orthopaedic injuries (OI), both groups with the injury less than a month old. The ToM scale was applied, includes 5 tasks which sum a total of 7 points. A memory control task was applied to confirm the understanding of the whole task. Results: Relative to OI, the TBI group had a lower score for the total score of ToM $(x=4.86 \pm 1.32 ; x=6.0 \pm .92 ; U=54.000 ; p<0.05)$; particularly in content false belief test $(\mathrm{U}=58.500 ; p<0.05)$ and real-apparent emotion test $(\mathrm{U}=60.00 ; p<0.05)$. However, TBI group performed worst in the memory tasks (mild $30 \%$; moderate $60 \%$ ). There was no relationship between ToM and memory tasks in TBI group with good comprehension ( $r=0.095 ; p=0.807)$; nor any difference in total score between mild and moderate TBI $(\mathrm{U}=12,500 ; \mathrm{p}=0.129)$.

Conclusions: The ToM low scores may be due to memory problems, mainly in the moderate TBI group. It needs to be considered that this study is conducted during the acute stage of the injury, thus, it is expected that increase differences in the chronic stage.

Correspondence: Maura J. Ramirez Flores, PhD, FACULTY OF PSYCHOLOGY, UNIVERSIDAD NACIONAL AUTONOMA DE MEXICO, AV. UNIVERSIDAD 3004 COL. COPILCO-UNIVERSIDAD, FACULTAD DE PSICOLOGÍA, MEXICO CITY 04510,Mexico.E-mail:mau_ramz@ comunidad.unam.mx

M.D. SADY, S. COLLIER, P. SIRIJUNTANAN, T.D. GRAHAM, C.G. VAUGHAN \& G.A. GIOIA. Comparison of Symptom Severity and Parent-Child Agreement between Three Causes of Adolescent Concussion: Sports, Motor Vehicle Collision, and Assault.

Objective: The current study examined differences in severity of post-concussion symptoms between three causes of injury (sports, motor vehicle collision (MVC), and assault), and differences in degree of parent-child agreement in ratings.

Participants and Methods: 99 youth (age 12-18) with concussion were evaluated in a specialty clinic (1st visit $2-47$ days post-injury), with one 
of three causes of injury: sports injury $(n=50), M V C(n=26)$, or assault $(\mathrm{n}=23)$. Groups were matched on age, gender, and time since injury, and there were no group differences in preinjury or injury characteristics. Patients and parents completed the Post-Concussion Symptom Inventory (PCSI) and a monitoring version of the Behavior Rating Inventory of Executive Function (BRIEF-m). Repeated measures ANOVA was used to examine group differences in post-concussive problems (preinjury-adjusted), and Fisher's z-tests were used to test differences between parent-child correlations on each subscale.

Results: Simple effects indicated no significant differences between groups on parent or self-report PCSI subscales. On the BRIEF-m, one simple effect was significant on parent report only (Initiate subscale), with the assault group having significantly higher ratings than the sports group. There was a pattern of higher parent-child agreement in sports injuries compared to assault and MVC, but the comparison was statistically significant on only one of the nine scales (BRIEF-m inhibition). Effect sizes were small to negligible.

Conclusions: In adolescents with concussion, severity of symptoms and executive functioning problems did not differ significantly between three injury causes. Rates of parent-child agreement may be somewhat higher after sports injury than assault or MVC, but more research is needed. Future studies should increase sample size, capture data closer to the time of injury, explore additional factors (such as circumstances surrounding assault and MVC, legal involvement, etc.), and compare additional outcomes (including time to recovery).

Correspondence: Maegan D. Sady, Ph.D., Pediatric Neuropsychology, Children's National Medical Center, 15245 Shady Grove Road, Suite 350, Rockville,MD 20850,United States.E-mail:maegan.sady@gmail. com

\section{N. SANDEL, A. SUFRINKO, J. HENLEY, M.W. COLLINS \& A.P. KONTOS. Discrimination of Concussed Athletes from Healthy Controls Using a Multimodal Diagnostic Approach.}

Objective: Evaluate the efficacy of using a multimodal approach to discriminate between acutely concussed individuals and healthy controls.

Participants and Methods: Participants included 23 concussed athletes (56.5\% males) and 25 healthy age and sex matched controls ( $68 \%$ males) aged 12 to 20 years old ( $M=15.21, S D=2.03)$. Participants completed a multimodal evaluation that included the Post-Concussion Symptom Scale (PCSS), computerized neurocognitive testing (Immediate Post-concussion Assessment and Cognitive Testing [ImPACT]), the Balance Error Scoring System (BESS), and the Vestibular/Ocular Motor Screening (VOMS) tool. A discriminant function analysis was conducted to evaluate how well the multimodal approach classified concussed participants from healthy controls. Univariate analyses identified measures in the multimodal approach that best differentiated concussed from healthy participants.

Results: The discriminant function yielded a significant model that differentiated concussed from healthy groups (chi-squared $=24.382$, $d f=6, p<.001)$. The model accurately predicted correct outcomes for $81.3 \%$ of cases (73.9\% - concussed; $88.0 \%$ - healthy controls). Univariate ANOVAs revealed that the concussed and healthy control participants differed significantly on all predictor variables in the multimodal assessment: PCSS $(F=30.45, p<.001)$, ImPACT Memory $(F=4.72$, $p=.04), \operatorname{ImPACT}$ Speed $(F=6.78, p=.01)$, vestibular screening $(F=23.62$, $p<.001)$, and near point convergence $(F=7.30, p<.01)$, with the exception of balance testing $(F=0.91, p=.35)$.

Conclusions: Utilization of a multimodal approach to concussion management during the acute phase of the injury correctly discriminated concussed athletes from healthy controls in $81.3 \%$ of cases. A multimodal approach should include measures of symptoms, cognition, and vestibular/ocular motor function. Balance testing did not discriminate concussed from control participants.
Correspondence: Natalie Sandel, PsyD, MBA, Orthopaedic Surgery, UPMC Sports Medicine Concussion Program, 3200 S. Water St., Pittsburgh, PA 15203,United States. E-mail: sandelnk@upmc.edu

\section{A.D. SCHMID, J. LICHTENSTEIN, K. ATWATER \& K. LINNEA. Acceptability of a Return-to-Learn Program for Concussed High School Students.}

Objective: Few models of implementation for return-to-learn (RTL) post-concussion programs have been tested for acceptability. As part of a larger concussion management demonstration project, this study assessed the acceptability of an RTL program at four high schools.

Participants and Methods: This RTL program included oversight by a team of specialists (neuropsychologist, occupational therapist, physical therapist), education to all school personnel, the formation of school-based concussion management teams, in-school neuropsychological consultation, daily email updates from teachers regarding student progress, and a room where concussed students could engage in replacement activities. Educators were asked to complete a survey regarding their satisfaction with this program.

Results: 168 educators rated high satisfaction with this program $(\mathrm{M}=3.31, \mathrm{SD}=.60$ on a $1-4$ scale). Satisfaction did not differ by any demographic factors. Qualitative analysis of teacher comments revealed 133 positive $(68.9 \%), 49$ negative, and 11 neutral statements. Positive themes included, but were not limited to the following: increased communication, more fluid transition into the classroom, clearer understanding of academic restrictions and expectations, and enhanced teacher support. Negative themes included concern about students manipulating the RTL system to avoid work, students taking too long to return to normal activity, and difficulty modifying work to accommodate the needs of concussed students.

Conclusions: Results of this study demonstrated the acceptability of an RTL program. Educator comments were primarily positive, revealing that they felt better supported and that classroom return was better managed with this program. However, their comments also indicated that this type of program can be time-consuming and that some students appeared to exaggerate symptoms in order to avoid schoolwork.

Correspondence: Amy D. Schmid, Ph.D., Psychiatry, Geisel School of Medicine at Dartmouth, HB 7550 Department of Psychiatry, One Medical Center Drive, Lebanon, NH 03756, United States. E-mail: amy.d.schmid@hitchcock.org.

\section{A. SCHMID, J. LICHTENSTEIN, K. ATWATER, K. LINNEA \& A. MAERLENDER. Validation of a Teacher Questionnaire to Assess Concussed Students' Recovery.}

Objective: Schools are encouraged to use a return-to-learn (RTL) program for concussed students. Outside of counting symptoms, however, few tools are available to monitor students' RTL progress. We sought to validate the Better or Worse Index (BoWI), a daily questionnaire for teachers about students' recovery, by assessing convergent validity with the PCSI, construct validity across time, and acceptability among high school teachers.

Participants and Methods: Across 3 high schools, 33 female and 39 male concussed students participated in the program during the 2015-2016 school year (age: $\mathrm{M}=16.19, \mathrm{SD}=1.31$ ). The following measures were administered each day of their recovery: self-reported symptom rating (Post-Concussive Symptom Inventory; PCSI), and teacher BoWI ratings of each student. The BoWI is a short online questionnaire assessing whether a student seemed "worse" $(0)$, "the same" (1), or "better" (2) compared to before the concussion. Teacher ratings were averaged, resulting in a single daily score for each student. Time was measured in days post-concussion (DPC). At the end of the school year, teachers were surveyed to measure their attitudes (1=very negative, $4=$ very positive) and use (1=never, $4=$ always $)$ of the BoWI.

Results: Regarding convergent and construct validity, correlations were significant and in the expected direction between BoWI and PCSI $(r=.31$, $p<.01)$, and BoWI and DPC $(r=.22, p<.01)$. Teachers' use of the BoWI 
was high $(\mathrm{M}=3.71, \mathrm{SD}=.61)$ and they reported positive attitudes toward the instrument $(\mathrm{M}=3.29, \mathrm{SD}=.68)$.

Conclusions: These results support the validity and acceptability of the BoWI as an instrument to measure readiness to return to a full academic workload after a concussion. The BoWI correlated with an established measure of postconcussive symptoms and showed improved functioning over time. Additionally, teachers reported high use of and positive attitudes toward this measure. These findings highlight the utility and feasibility of daily teacher input during the return to learn process. Correspondence: Amy Schmid, PhD, Psychiatry, Dartmouth Hitchcock Medical Center, 75 April Lane, White River.Junction, VT 05001, United States.E-mail: amyschmid@ymail.com

Y. SHISHIDO, L.S. BLACKWELL, R.D. LATZMAN \& R.A. HOWARTH. Cognitive Recovery of Pediatric Patients With Moderate to Severe TBI During Inpatient Rehabilitation Using the Cognitive \& Linguistic Scale (CALS).

Objective: Serial assessment of cognitive recovery during inpatient rehabilitation is critical for monitoring neurobehavioral status following traumatic brain injury (TBI) to guide appropriate treatment. Given the variable recovery course, further investigation of cognitive recovery of pediatric patients is needed in an inpatient setting. The current study examines cognitive recovery of pediatric patients using the CALS and identifies potential demographic and clinical factors contributing to variability in rates of recovery.

Participants and Methods: Participants included 68 patients (ages 3-20) with moderate to severe TBI who received inpatient rehabilitation. Cognitive recovery was assessed at admission and discharge using the CALS, a 20-item measure designed for serial assessment of cognitive recovery following acquired brain injury.

Results: Patients evidenced significant improvements in cognitive functioning between admission and discharge for CALS total scores $(t(67)=-12.99, p<.01)$ and item scores $(t(67)=-5.01--11.68$, $p<.01)$. Effect sizes varied from medium to large and were larger than those reported in the previous pediatric literature $(M d n=.86)$. Factor analytically derived basic- and high-level functioning scales also showed improvements, with a significantly larger effect size in high-level functioning. Preliminary analyses indicated that CALS total and item level scores did not differ by gender, age, or race.

Conclusions: Results suggest significant improvement in cognitive functions for pediatric patients with TBI during inpatient rehabilitation, with potentially more nuanced recovery patterns at an individual item level. Findings support the importance of serial monitoring during inpatient rehabilitation to assist with identifying appropriate treatments and supports for community and school re-entry.

Correspondence: Yuri Shishido, Ph.D., Psychology, Georgia State University, 20 Parkside Ct. NE, Atlanta, GA 30342, United States. E-mail: yurishishido@gmail.com

J. SMITH-PAINE, A. TREBLE-BARNA, N. ZHANG, H. ZANG, L. MARTIN, K.O. YEATES, H. TAYLOR, S.L. WADE \& B. KUROWSKI. The Moderating Effect of Dopamine Genes on the Association of Family Environment with Longitudinal Executive Function Following Traumatic Brain Injury in Early Childhood: A Preliminary Study.

Objective: To examine whether dopamine (DA) genotypes moderate the association of family environment with executive function $(\mathrm{EF})$ over time following early traumatic brain injury (TBI).

Participants and Methods: Caregivers of 65 children with TBI and 70 children with orthopedic injury (OI) completed the Behavior Rating Inventory of Executive Function (BRIEF) shortly after injury (6, 12, and 18 months) and at extended follow-ups an average of 3.5 and 6.8 years post injury. Salivary DNA was collected for genotyping of DA transporter (DAT1), DA receptor-4 (DRD4), and ankyrin repeat and kinase domain containing 1 (ANKK1). Linear mixed models examined moderating effects of risk allele on associations of injury type
(TBI or OI) and family environment (i.e., parenting style and quality of the home environment) with behavioral ratings of child EF over time. Results: Overall, TBI, high levels of maladaptive parenting styles (permissive and authoritarian), and disadvantageous home environment were associated with worse EF over time. However, DA genetics modified some of these risks. Specifically, for the DAT gene, there were significant interactions among genotype, family environment, and injury type, when considering the influence of authoritarian parenting $(p=.002)$; authoritative parenting $(p=.008)$; and permissive parenting $(p=<.001)$; and significant interaction among genotype and injury type in the model considering home environment $(p=.006)$. For the ANKK1 gene, there were significant interactions among genotype, family environment, and injury type when considering the quality of the home environment $(p=.021)$, and authoritative parenting ( $p=.003)$.

Conclusions: The present results demonstrate that for EF over time after injury in early childhood genetics may mediate the effects of injury type and family environment. These results suggest that knowledge of both family environment and DA genetics may be useful in explaining the heterogeneity in TBI recovery.

Correspondence: Julia Smith-Paine, M.A, Psychology, University of Cincinnati, 6961 Murray Ave., Apt. \#3, Cincinnati, OH 45227, United States.E-mail:smith8j2@mail.uc.edu

A.M. SUFRINKO, R. ELBIN, P. SCHATZ, E. REYNOLDS, N. SANDEL, M.W. COLLINS \& A.P. KONTOS. Additional Sport Exposure Following Concussion has Dose Response Effect on Recovery Time.

Objective: Current guidelines suggest the immediate removal of athletes from contest after sustaining a sport-related concussion (SRC). However, some athletes continue to play after sustaining a SRC, due to lack of awareness of signs/symptoms, sport culture, and limited access to medical professionals. Concussed athletes who remain in play demonstrate worse outcomes, including more severe acute cognitive impairment and longer recovery time. The goal of this study was to determine if there was a dose response of post-injury sport exposure (i.e., minutes remained in game/practice play after SRC) on athletes' severity of symptoms, neurocognitive impairment and recovery time. Participants and Methods: Participants included 59 athletes aged 15.3+/-1.9 who reported remaining in play for $22.9+/-26.8$ minutes (range $=3-160)$ immediately following SRC. Participants were grouped by short duration (3-15 minutes $[n=25]$ ) or long duration ( $>15$ minutes $[n=34]$ ) of continuous play following injury. A Mann-Whitney U test compared groups on recovery time and a series of $t$-tests compared groups on the Post-concussion Symptom Scale (PCSS) and Immediate Post-Concussion Assessment and Cognitive Testing (ImPACT) 1-7 days following SRC.

Results: Athletes in the long duration group took longer to recover $(M=43.79$ days, $S D=26.34)$ compared to those in the short duration group $(M=29.08$ days, $S D=12.90, p=.049)$ There were no significant differences on acute outcomes at 1-7 days post injury on ImPACT scores $(p>.10)$ or PCSS score $(p=.40)$.

Conclusions: Additional exposure to sport immediately following SRC has a dose response effect on clinical outcomes. Further aerobic activity and exposure to additional contact are two potential mechanisms that may exacerbate injury.

Correspondence: Alicia M. Sufrinko, PhD, Department of Orthopaedic Surgery, University of Pittsburgh, 3200 S Water st., Pittsburgh, PA 15203, United States. E-mail: asufrinko@gmail.com

A.M. SVINGOS, D. GARCIA, B.A. BAILEY, S. GREIF, D.M. BAGNER \&. S.C. HEATON. Parent-Child Interaction Therapy (PCIT) Reduces Sleep Problems Following Pediatric Traumatic Brain Injury.

Objective: Pediatric traumatic brain injury (TBI) is associated with a variety of neurocognitive, behavioral, and psychological changes. Sleep difficulties after TBI are common, and can negatively impact both cognition and recovery. Behavioral problems after TBI can disrupt family 
functioning and may affect sleep hygiene and/or sleep initiation. Though Parent-Child Interaction Therapy (PCIT) has been shown to effectively reduce behavior problems, it is unclear whether it may also reduce sleep problems after childhood head injury.

Participants and Methods: Participants $(\mathrm{n}=10)$ were between 2 and 5 years of age, had sustained a recent head injury, and displayed elevated behavioral problems. Injury severity and mechanism varied across the sample, though the majority sustained mild head injuries $(90 \%)$ subsequent to falls $(60 \%)$. Study participants received an intensive format of PCIT (twice a week for 5 weeks). Parent-reported child sleep behaviors were assessed over time using the 7-item Sleep Problems subscale of the Child Behavior Checklist (CBCL).

Results: Paired sample t-tests indicated significant reductions in parent-report of child sleep problems following the PCIT intervention $(\mathrm{t}[1,9]=4.975, \mathrm{p}=.001)$ and at 2 -month follow-up $(\mathrm{t}[1,9]=3.511$, $\mathrm{p}=.007)$.

Conclusions: Findings suggest that behavioral parent training interventions may reduce pediatric sleep difficulties after head injury. Future studies should examine the extent to which injury characteristics and/ or socio-demographic factors may modify observed effects. Researchers should also attempt to examine specific components of PCIT in order to gain insight to the relative contributions of specific intervention elements on pediatric sleep functioning.

Correspondence: Adrian M. Svingos, M.S., Clinical and Health Psychology, University of Florida, P.O. Box 100185, Gainesville, FL 32610, United States. E-mail: adrianmchambers@phhp.ufl.edu

C.G. VAUGHAN, J. KEROACK, A. KRAVITZ, J. WALLEY, M. SADY \& G.A. GIOIA. The Relation Between Multiple Prior Concussions and Injury Severity.

Objective: Examine the relation between the number of prior concussions and post-concussion severity and recovery course.

Participants and Methods: 1178 children (ages 8-18; M = 13.43; $\mathrm{SD}=2.52 ; 64.1 \%$ Male) were evaluated within 30 days of concussion. Concussion history was gathered along with self-reported retrospective pre-injury and post-injury symptoms on the Post-Concussion Symptom Inventory (PCSI). A subsample rated symptoms at visit 2. Mean differences in retrospectively reported pre-injury symptoms, total adjusted (current minus pre) post-injury symptom score, symptom factor scores (physical, emotional, sleep, cognitive), visit 2 adjusted total scores, and visit 1 to visit 2 symptom change were examined by concussion history. Results: Within the $8-12$ age group $(n=410), 71 \%$ had no prior concussions, $20.5 \%$ had 1 or 2 , and $8.5 \%$ had 3 or more. Within the $13-18$ age group $(\mathrm{n}=768), 58.3 \%$ had no prior concussions, $26.3 \%$ had 1 , $9.6 \%$ had 2 , and $5.7 \%$ had 3 or more. Concussion history did not relate to gender, history of ADHD, days from injury to visit, or years of age. Concussion history did not relate to retrospectively reported baseline symptom levels $(\mathrm{p}>.05)$. Total post injury scores at visit 1 , visit 2 , or the visit 1 to visit 2 change did not vary by number of prior concussion $(p>.05)$. The symptom factor scores were similar by concussion history in 13-18 year olds $(p>.05)$. Younger children (age 8-12) with more concussions reported greater post-injury emotional symptoms, but not other symptom factor scores, at visit $1(\mathrm{p}<.05)$ than those with fewer or no prior concussions.

Conclusions: Post-concussion symptom severity and trajectory of symptom recovery were not related to concussion history in this sample of youth. Moreover, those with a history of concussion endorsed similar pre-injury symptom levels than those without prior injury. These findings appear to contradict a commonly reported assumption in the literature that multiple concussions are cumulative, at least in this group level analysis.

Correspondence: Christopher G. Vaughan, Psy.D., Pediatric Neuropsychology, Children's National Health System, 15245 Shady Grove Rd, Suite 350, Rockville, MD 20850, United States. E-mail: cvaughan@cnmc.org
K. WILMOTH, J.M. GALUSHA, S.C. BUNT, E.I. GODBEY, N. HOLDER, J. GARNER-ROBERTS, G. BLOOMGARDEN, N. DIDEHBANI \& C.M. CULLUM. Delayed Clinical Evaluation Following Concussion Predicts Prolonged Return to Play in Female Student-Athletes.

Objective: Variability in adolescents' recovery from sports concussion is poorly understood, and lingering symptoms can negatively impact academic, social, and emotional functioning. Some athletes may be at greater risk for such lingering symptoms and delayed recovery based on gender differences, although the literature is mixed. To this end, we examined the relationship between delayed clinical evaluation following concussion and time to recovery in adolescent student-athletes stratified by gender.

Participants and Methods: Participants were 77 full-time students (52\% female, $78 \%$ White, $25 \%$ with previous concussion) ages $9-19$ who sustained a sports-related concussion and were referred for postconcussion evaluation through clinics participating in ConTex, a longitudinal concussion registry in North Texas. As part of the protocol a Concussion Symptom Log ( $\mathrm{S} x \log )$ was administered at initial visit to assess symptom severity, and medical record review at 3-month follow-up confirmed the time to return-to-play (RTP). A Poisson regression for the number of days to RTP was conducted for each gender based on the time to initial clinic visit and $\mathrm{Sx} \log$ total score.

Results: Athletes presented for initial evaluation 0-15 days after injury $(\mathrm{M}=5.3, \mathrm{SD}=4.3)$. For each additional day between injury and clinic evaluation, female athletes' RTP was $82 \%$ longer, $\mathrm{p}<.001,95 \% \mathrm{CI}[58,106]$. Further, a positive interaction of time to clinic evaluation and $\mathrm{Sx}_{\mathrm{x}} \mathrm{Log}$ total score at initial visit was found, $\mathrm{p}<.001$. In contrast, time to evaluation was not a significant predictor of RTP in male athletes.

Conclusions: Our findings demonstrate a link between delayed clinical evaluation following concussion and increased time until clearance to return to play in female but not male student-athletes. This relationship varied based on injury severity. These results may indicate selective benefits of earlier intervention, though gender differences in concussion management and recovery should be explored further in this population before firm conclusions can be drawn.

Correspondence: Kristin Wilmoth, UT Southwestern Medical Center, 5323 Harry Hines Blvd, Dallas, TX 75390-9044, United States. E-mail: Kristin.Wilmoth@UTSouthwestern.edu

F. ROBERTSON, K.L. WRIGHT, E.D. BIGLER \& R. HOPKINS. Relationship Between Processing Speed with White Matter Hyperintensity Volumes and Injury Severity.

Objective: Pediatric traumatic brain injury (TBI) often results in slowed processing speed (PS) which is associated with white matter hyperintensities (WMH) identified in T2-FLAIR MRI. We explored the relations between WMH volumes derived with two methods, the automated Lesion Segmentation Tool (LST) method and manual tracings with PS and injury severity. If the automated method approximates the "gold standard' tracing method, because of its ease in use it would be the preferred method to use in research.

Participants and Methods: 8 TBI and 6 orthopedic injury (OI) participants ( 9 males and 5 females), were included in the study. Participants were between the ages of 8-13 at time of neuropsychological testing. LST WMH volumes were compared to manually traced WMH volumes using an idependent-samples t-test. Regression analysis assessed the relationship between WMH volumes, PS and injury severity controlling for age and sex.

Results: The mean age of the TBI group was 10.31 years $(\mathrm{SD}=1.3)$ and for the OI group was $10.57(\mathrm{SD}=2.0)$. There was no significant differences between the TBI and OI group for age $(p=.77)$ or for sex $(p=0.23)$. Automated WM volume was determined to be $.033 \mathrm{~cm}^{3}$ (s.d. $=0.076$ ), in contrast to the manual tracing $\mathrm{WM}$ volume that was $1.84 \mathrm{~cm}^{3}$ (s.d. =1.33). While both methods identified WM pathology the WMH volumes between the two techniques were significantly different $(p=.002)$. Although limited by the sample size and therefore statisitical 
power, neither WMH metric significantly related to PS although injury severity was positively correlated with PS $\left(\mathrm{R}^{2}=.347, \mathrm{p}=0.025\right)$.

Conclusions: Differences in how WM pathology is identified and quantified between the two techniques relates in part to differences in segmentation and classification of the adnormalities. Differences between the two techniques resulted in substantially different WMH volume estimations. Ways to improve accuracy in WM pathology identification for pediatric TBI will be discussed.

Correspondence: Kacie L. Wright, Psychology, Brigham Young University, 488 E. 100 S., Orem, UT 84097, United States. E-mail: kacie.birtcher@hotmail.com

\section{Autism Spectrum Disorders}

D. ABRAMS, L. ADAMSON, D. FEIN \& D.L. ROBINS. Early ASD Symptoms, Demographic Characteristics, and Adaptive Skills Predict Change in Cognitive and Language Abilities in Toddlers with ASD.

Objective: Children diagnosed with autism spectrum disorder (ASD) at a young age have diverse language and cognitive outcomes which impact their functional abilities. The present study aims to identify predictors of change in these abilities between ages 2 and 4 .

Participants and Methods: Toddlers ( $n=270 ; 79 \%$ male) diagnosed with ASD at age 2 (T1) were re-evaluated at age 4 (T2). Evaluations included Autism Diagnostic Observation Schedule (ADOS[-2]), Mullen Scales of Early Learning (MSEL), and Vineland Adaptive Behavior Scales(-2).

Results: A multiple linear regression model was developed to predict change (age equivalent range: -94.39-130.39) in each MSEL domain using backward selection. T1 predictors of visual reception change include maternal education, $\beta=5.00$, minority race, -8.05 , intervention hours, -.44 (negatives suggest decline), ADOS social affect (SA), -3.23, and other MSEL scores, $p s<.030$. Predictors of expressive language change include those variables and VABS communication subdomain, $.45, p s<.038$. Finally, predictors of receptive language change include minority race, -10.60, ADOS SA, -1.74, other MSEL scores, and VABS communication subdomain, .65, $p s<.049$. We also examined predictors of change in a positive direction only $(\mathrm{N}=156-185)$. Fine motor was a predictor of growth in all 3 domains, and there was generally less change in males and minority children, $p s<.042$.

Conclusions: Initial child characteristics, including demographic factors, symptom severity, and adaptive skills, independently predict change in language and cognitive abilities in children with ASD between ages 2 and 4 . More intervention was provided to lower functioning children. Fine motor skills, female sex, and majority race were specifically predictive of closing the gap with peers. Results highlight factors, such as early skills and access to resources, that support early positive change in cognition and language.

Correspondence: Danielle Abrams, MA, Psychology, Georgia State University, 230 E Ponce de Leon Ave, Unit 223, Decatur, GA 30030, United States. E-mail: dabrams1@student.gsu.edu

C. AMMONS, M. WINSLETT \& R. KANA. Age and Diagnosis Effects on Mid Fusiform Sulcus Anatomy in Autism Spectrum Disorder. Objective: Aberrant activation and connectivity of the fusiform gyrus (FG) during social tasks is often reported in autism spectrum disorder (ASD). A recently characterized minor sulcus bisecting the FG of healthy control (HC) participants predicted the location of face selective visual areas and divisions of cytoarchitecture in this important social brain region (Weiner et al., 2014). Despite this, the mid fusiform sulcus (MFS) has not been studied in ASD. This study establishes the gross anatomical organization of the MFS in ASD and investigates age, diagnosis, and hemispheric effects on the morphometry of this relatively neglected social brain feature.
Participants and Methods: High resolution T1-weighted images from 115 participants (54 ASD, $61 \mathrm{HC}$ ) ages 9-40 years were acquired on a 3T Siemens MRI scanner. MFS identification and classification were performed by three independent raters using Freesurfer 2D rendering. Gray matter volume, surface area, cortical thickness, and thickness standard deviation (SD) were extracted from subject specific MFS labels and entered into a repeated measures general linear model with hemisphere, age, and diagnosis controlling for overall FG size.

Results: MFS was reliably identified in $97 \%$ of hemispheres with similar gross anatomical features in ASD and HC individuals. Morphometric analysis revealed: 1) a main effect of age on MFS thickness with cortical thinning in older individuals and 2) an interaction effect of hemisphere and diagnosis on MFS thickness SD. In ASD, variability in cortical thickness was relatively stable across hemispheres, whereas HC demonstrated more pronounced regional thickness differences in the right hemisphere. Conclusions: Differences in regional thickness specific to the right MFS in the HC population may be related to functional specificity differences given the importance of right FG in face processing and social cognition. This first investigation of the MFS in ASD provides important insights into the anatomy of this region.

Correspondence: Carla Ammons, M.S., Psychology, University of Alabama at Birmingham, 1620 12th St. S - Apt C, Birmingham, AL 35205, United States.E-mail: cjammons@uab.edu

H. BEDNARZ, A. LEMELMAN, D. MURDAUGH \& R. KANA. Changes in Reading Comprehension as a Result of Language Intervention in Children with Autism Spectrum Disorders.

Objective: Language deficits are a core feature of autism spectrum disorders (ASD) and extend to deficits in reading. A common reading profile in high-functioning children with ASD is intact decoding accompanied by poor comprehension. Visuospatial ability, an area of strength in ASD, could prove useful for reading interventions. Our objective was to test the effectiveness of a visual imagery-based intervention to improve reading comprehension in ASD.

Participants and Methods: Children with ASD (ASD-EXP; $N=22$; ages 8-13 years) participated in a reading intervention program (Visualizing and Verbalizing for Language Comprehension and Thinking; $\mathrm{V} / \mathrm{V})$. Participants were tested on neuropsychological measures before and after 200-hours of face-to-face V/V intervention. This group was compared to a waitlist-control group of children with ASD (ASD-WLC; $\mathrm{N}=17$ ) as well as a typically developing group of children (TD; $\mathrm{N}=16$ ). Results: Within-group analyses revealed a significant improvement in reading comprehension in the ASD-EXP group from pre-to-postintervention, an effect not observed in the ASD-WLC group. In addition, baseline reading comprehension scores significantly predicted interventionrelated change in reading comprehension. Relative to ASD-WLC, children in the ASD-EXP group did not show significantly greater oral or reading comprehension post-intervention.

Conclusions: While we found the V/V intervention to be effective in improving reading comprehension in children with ASD from pre-to-post-intervention, it may not be generalizable, as the same effect was not seen when compared to waitlist control group of children. Future research should focus on increased sample sizes to investigate the effectiveness of this reading intervention.

Correspondence: Haley Bednarz, The University of Alabama at Birmingham, 1944 Mayfair Park Dr Apt 202, Homewood, AL 35209 , United States. E-mail: hbednarz@uab.edu

N.I. BERGER, B. INGERSOLL, M. PONTIFEX \& S. LASZLO. Evaluating the Neural Correlates of Intention Understanding in Autism Spectrum Disorder.

Objective: The purpose of this study was to use Event Related Potentials (ERPs) to assess, for the first time, the neural correlates of intention understanding in children with Autism Spectrum Disorder (ASD) and controls. Given behavioral research suggesting that ASD is associated with impaired intention understanding only when cued using social 
stimuli (e.g., facial expressions), this study also compared differences in neural processing between groups across social and non-social stimuli. Participants and Methods: We examined neural indices of social and non-social intention understanding in 22 school-age ASD children and 22 controls (age, gender, and IQ matched). Participants viewed picture sequences depicting either social or non-social intention. The final picture of the sequence varied such that an actor either completed the intended action (expected) or performed an unintended action (unexpected). We evaluated the P600 as it has been linked to expectancy violations in visual scene processing. Participants made button press responses to intended condition only

Results: Social stimuli: Both groups demonstrated similar ERP responses, characterized by a greater (positive) P600 to unintended vs. intended condition. Response accuracy was significantly worse for the ASD group. Non-social stimuli: No group differences were identified in either response accuracy or ERP responses (greater P600 to unintended vs. intended condition).

Conclusions: Accuracy data concord with previous behavioral findings of a dissociation between social and non-social intention understanding, such that social intention alone is functionally impaired in ASD. However, no group differences were observed for either stimuli type at a neurological level. This is a particularly novel finding, as it demonstrates that observed behavioral impairments are independent from the neural processes supporting basic social intention understanding. Additional work is necessary to characterize this newly identified gap between neurological functioning and overt behavior.

Correspondence: Natalie I. Berger, Psychology, Michigan State University, 316 Physics Rd, East Lansing, MI 48824, United States. E-mail: bergerna@msu.edu

\section{BERTOLIN, B. BRADDOCK \& D.A.S. KAUFMAN. Effects of Social Skills Training on Emotional Face Processing in Adolescents With Autism Spectrum Disorder: Behavioral and Electrophysiological Correlates.}

Objective: Chronic social deficits are regarded as the hallmark impairment of Autism Spectrum Disorder (ASD), manifest early in development, and are typically associated with persistent and lifelong impairments in functioning. Social skills interventions such as the Program for the Education and Enrichment of Relational Skills (PEERS) are currently the most widely used and well-supported of all treatment approaches for adolescents with ASD. Moreover, the PEERS program has been shown to produce neurophysiological changes and enhance socio-emotional skills in this population. However, little is known about the specific effects of social skills training on social attention in adolescents with ASD.

Participants and Methods: Using neuropsychological measures and high-density event-related potentials (ERPs) acquired during a computerized emotional face processing task, we examined emotional face processing in sixteen adolescents with ASD ages 12 to17, both before and after participation in the PEERS program and relative to thirteen of their same-aged typically developing peers.

Results: Results revealed that adolescents with ASD exhibited atypical face processing relative to their typically developing peers, including significant group differences in bilateral N170 and EPN latencies suggesting delays in feature-based and evaluative face processing. Moreover, completion of the PEERS program resulted in shorter N170 and EPN latencies, and these changes were related to beneficial changes in self- and other-reported emotional functioning.

Conclusions: These results suggest neural changes in the evaluative processing of human faces post-PEERS that may be meaningful indicators of social and emotional processing in everyday life. Our findings indicate that the neural mechanisms underlying emotional face processing deficits in ASD appear to be susceptible to psychosocial intervention and suggest that social skills training may improve socioemotional skills by enhancing social attention.
Correspondence: Madison Bertolin, MS, Psychology, Saint Louis University, 4501 Lindell Blvd 7H, Saint Louis, MO 63108, United States.E-mail:stroup@slu.edu

K.R. BRADBURY, D.L. ROBINS, M. BARTON, W.L. STONE, Z.E. WARREN \& D. FEIN. M-CHAT-R/F Performance in HighRisk Infant Siblings.

Objective: The M-CHAT-R/F is a parent-completed, screening instrument for ASD in toddlers. Infant siblings are at higher risk for ASD and for sub-clinical ASD symptoms making screening more complicated in this population. The current study explores how the presence of an older affected child impacts parents' responses on the M-CHAT-R/F for later-born children.

Participants and Methods: Toddlers $\left(\mathrm{n}=186 ; \mathrm{M}_{\mathrm{age}}=21.2\right.$ months, $\mathrm{SD}=4.2$ ) with an older ASD-affected sibling were screened using the M-CHAT-R at three sites across the country. Follow-up interviews were conducted to confirm at-risk responses for children who screened positive. Children who continued to screened positive were invited for a comprehensive evaluation to determine diagnostic classification. M-CHAT-R/F psychometric properties were examined and compared to those from screening in a low-risk population (Robins et al., 2014). Results: High-risk infant siblings showed substantially higher screen positive rates at initial screen $(40.3 \%)$ and follow-up (34.9\%) compared to low-risk populations ( $7.2 \%$ and $2.2 \%$, respectively). Follow-up only incrementally improved positive predictive value (PPV) for ASD in the high-risk population (.44 to .55) compared to in the low-risk population (.138 to .475). PPV remained high for other developmental concerns $\left(\mathrm{PPV}_{\mathrm{HR}}=.983\right.$ vs. $\left.\mathrm{PPV}_{\mathrm{LR}}=.946\right)$. Area under the curve (AUC) was .870 indicating the M-CHAT-R/F demonstrated good accuracy in distinguishing between ASD and non-ASD in a high-risk population and was comparable to accuracy seen in low-risk populations $(\mathrm{AUC}=.907)$. Conclusions: Screening measures, such as the M-CHAT-R/F, perform differently in high-risk infant sibling populations. Follow-up was less critical in a high-risk population. High screen positive rates and PPVs, especially for any developmental concern, highlight the importance and efficacy of screening in high-risk populations.

Correspondence: Kathryn R. Bradbury, University of Connecticut, 406 Babbidge Rd, Unit 1020, Storrs, CT 06269-1020, United States. E-mail: kathryn.bradbury@uconn.edu

B. CASTELLUCCIO, A. CANFIELD \& I. EIGSTI. Verbal Inferential Reasoning in Autism Spectrum Disorder.

Objective: Weaknesses in executive functions, especially mental flexibility, are frequent in autism spectrum disorder (ASD). Despite the prominent role of language and communication weaknesses, very little work has investigated verbal executive abilities in ASD. A deficit in verbally-oriented executive functions, such as the deductive reasoning required to infer meanings of novel expressions, could confer risk for language deficits. The current study investigated verbal inferential reasoning in ASD.

Participants and Methods: Adults with ASD $(n=9)$ and typical development (TD; $n=16$ ) participated in a study of influences on communication and language abilities. All participants were monolingual English speakers with full scale IQ > 77 with no history of brain injury. Groups were matched for age, gender, and full scale IQ. ASD diagnoses were confirmed using the Autism Diagnostic Observation Schedule. Participants completed the Word Context Test, a test of verbal deductive reasoning from the Delis-Kaplan Executive Function System that requires participants to determine the meanings of nonsense words using the context provided by progressively more specific clue sentences. Results: The ASD group showed no impairment relative to the TD group on the Word Context Test. Both groups' means were in the average range on scores capturing overall success and mental flexibility. For each group and for the overall sample, mental flexibility on the test was significantly related to nonverbal IQ but not verbal IQ. In the overall sample, success on the test was also significantly related to nonverbal 
IQ. Word Context Test performance was not related to symptom severity or other executive functions (abstraction or inhibition).

Conclusions: Preliminary findings indicate no weakness in verbal inferential reasoning in average-IQ adults with ASD. Findings suggest that, regardless of diagnosis, nonverbal abstract reasoning abilities (IQ) and mental flexibility in the context of verbal inferential reasoning may share prefrontal substrates.

Correspondence: Brian Castelluccio, Psychological Sciences, University of Connecticut, 406 Babbidge Road, Unit 1020, Storrs, CT 06269 1020,United States.E-mail: brian.castelluccio@uconn.edu

\section{J. CHEN, M. BARTON \& D. FEIN. Differences in Early Temperament Between High Risk Baby Siblings With and Without Autism: Analyses by Gender.}

Objective: To assess whether temperamental differences between baby siblings with and without autism spectrum disorders (ASD) at age 2 hold equally for boys and girls.

Participants and Methods: Baby siblings of children with ASD were screened using the M-CHAT(-R). Parents of children who screened positive completed the Toddler Temperament Scale (TTS) when their child received a diagnostic evaluation. Children were grouped by diagnosis: ASD and non-ASD (includes developmental delay, language disorder, and no diagnosis). Mean scores on five subscales of the TTS (previously indicating group differences; Chen, Barton \& Fein, 2016) were compared between groups using t-tests separately for boys and girls (Boys ASD: n=50; Boys non-ASD: n=45; Girls ASD: n=28, Girls non-ASD: $n=21$ ). Moderation analyses (Gender X Diagnosis) were not feasible due to multicollinearity.

Results: Post-hoc power analyses indicate $97 \%$ and $77 \%$ power for boys and girls, respectively, to detect large effects. Boys, but not girls with ASD were significantly less rhythmic (i.e., less consistent in eating, sleeping, and elimination routines; $\mathrm{p}=.004, \mathrm{~d}=.60$ and $\mathrm{p}=.557, \mathrm{~d}=.18$, respectively for boys and girls), displayed more negative $\operatorname{mood}(p=.008$, $\mathrm{d}=.56$ and $\mathrm{p}=.301, \mathrm{~d}=.30$ ) and were less distractible (i.e., less able to remove themselves from negative behavior patterns; $p<.001, d=1.23$ and $p=.054, d=.57$ ) than non-ASD peers. Girls, but not boys with ASD were significantly more withdrawn than non-ASD peers $(p=.018, d=.70$ and $\mathrm{p}=.200, \mathrm{~d}=.26$ for girls and boys, respectively).

Conclusions: The expression of temperament differences between high risk baby siblings with and without ASD at age 2 may vary with gender. These findings may reflect sex differences in the development of temperament dimensions within ASD, as well as parental gender role expectations (e.g. withdrawn or non-approaching behavior is rated as more atypical for girls). There may have been insufficient power to detect small or medium effects.

Correspondence: Julia Chen, B.A., Psychological Sciences, University of Connecticut, 406 Babbidge Rd Unit 1020, Storrs, CT 06269, United States.E-mail:julia.chen@uconn.edu

I. CHO, M. SCHUETZE, S. VINETTE, S. RAHMAN, K. RIVARD, A. MCCRIMMON, D. DEWEY \& S. BRAY. Frontostriatal Structural Connectivity in Autism Spectrum Disorder.

Objective: Abnormalities in the frontostriatal circuit have been suggested as an underlying cause of the social deficits in Autism Spectrum Disorder (ASD). However, findings on white matter (WM) properties have been mixed. The objective of this study was to investigate frontostriatal WM structure in youth with ASD, using multi-shell diffusion tensor imaging (DTI), connectivity-defined regions of interest and probabilistic fiber tracking of limbic, motor and cognitive pathways.

Participants and Methods: DT images were collected from 19 typically developing (TD; 15 male) and 20 ASD (17 male) participants (age: TD 16.55 \pm 2.2 ; ASD 16.55 \pm 2.5 ; IQ: TD 108.95 \pm 15 ; ASD 101.85 \pm 17 ), using two b-values $(900,2000)$ each at 30 directions. Connectivity based parcellation was conducted on a subset of 10 participants (5 TD) to identify regions of the caudate and putamen most highly connected to anatomically defined 1) orbitofrontal cortex (OFC), 2) Brodmann areas 4 and 6 , and 3) rostral middle frontal and superior frontal gyri. Resulting caudate and putamen sub-regions were then used as seed regions. WM properties of the fibers connecting the caudate and putamen to the three prefrontal targets were averaged over the identified tracts. Mean fractional anisotropy (FA) values were compared between groups, controlling for age, sex and IQ.

Results: A hemisphere $\mathrm{x}$ tract $\mathrm{x}$ group interaction effect was found $(p=0.03)$. This effect was driven by the tracts connecting caudate and putamen seeds to the left OFC, with lower FA values in the ASD group relative to the TD group $(\mathrm{p}=0.03)$.

Conclusions: As the tracts connecting the OFC to the striatum are important for goal directed behaviors, decreased FA may be associated with social impairments in ASD. Furthermore, as many ASD behavioural therapies use reinforcement learning approaches reliant on frontostriatal interactions, characterizing differences in this circuitry can allow for improved therapies.

Correspondence: Ivy Cho, University of Calgary, A0-401 Lower Level, 2888 Shaganappi Trail NW, Calgary, AB T3B 6A8, Canada. E-mail: iykcho@ucalgary.ca

B. DOLAN, D. SNYDER, A. BARRINGTON, A. MCVEY, K. WILLAR, S. PLEISS, J. KARST, A. CARSON \& A. VAUGHAN VAN HECKE. Examining the Durability of PEERS for Adolescents With ASD: Maintenance of Neurological and Behavioral Effects.

Objective: To date, there are no known published studies that have assessed the maintenance of treatment effects in the context of neurological changes and their relationship to behavioral outcomes following a social skills intervention for adolescents with Autism Spectrum Disorder (ASD). The few studies that have incorporated long-term assessment into their design have focused exclusively on sustained behavioral responses to treatment. Individuals with ASD across the lifespan exhibit aberrant neural activity, which is thought to underlie social skill deficits noted in persons on the spectrum. Thus, this study sought to examine the impact of a social skills intervention, the Program for the Education and Enrichment of Relational Skills (PEERS: Laugeson, Frankel, Mogil, \& Dillon, 2009), on the maintenance of neural plasticity and treatment gains in social functioning.

Participants and Methods: Thirty-one adolescents with ASD, age 11-16 years, were recruited for participation in this study and completed the PEERS intervention. Neural activity was assessed via electroencephalography (EEG) in terms of spectral power and asymmetry. Additionally, behavioral outcomes, examining a variety of social domains, at pre, post, and 6-month follow-up, were investigated for their relationship to changes in EEG activity.

Results: Results revealed that adolescents with ASD demonstrated a decrease in gamma activity in the right temporal region following PEERS, which was maintained at 6-month follow-up $(F(1.59,47.69)$ $=7.76, p=.002$, partial $\eta^{2}=.21$, observed power $\left.=.89\right)$. This sustained neural change related to fewer problem behaviors $(r(30)=-.40$, $p=.027)$ and improved social cognition $(r(28)=-.40, p=.033)$, as reported by caregivers.

Conclusions: Findings from the present study highlight the role of neural plasticity as a mechanism for maintaining improvements in behavioral presentation following intervention and represent a promising biomarker for neural response to treatment and maintenance of gains.

Correspondence: Bridget Dolan, Psychology, Marquette University, 827 $S$ Bouldin Street, Baltimore, MD 21224, United States. E-mail: bridget. dolan@marquette.edu

Y. GAO, S. PUNYAMURTHULA, A. JAHEDI, N. BAGGETT, W. ZHAO, C. FONG, I. FISHMAN \& R. MÜLLER. Behavioral and Neural Substrates of Language Delay in Children with Autism Spectrum Disorder.

Objective: Autism Spectrum Disorder (ASD) is characterized by sociocommunicative deficits. Language delay (LD) is one of the first 
detectable signs of ASD; however, the functional brain connectivity of the language network as it relates to LD is poorly understood. The present study aims to characterize LD using language assessments and intrinsic functional connectivity in children with ASD.

Participants and Methods: 50 typically developing (TD) and 53 ASD participants, 8-17 years old, were matched on nonverbal IQ, handedness, gender, and head motion ( $p s>.66)$. A 6-minute resting state protocol was used with regions of interest (ROI) selected from a meta-analysis of 54 fMRI language studies (Rodd et al., 2015). Average BOLD time series extracted from each cortical seed were correlated with each other, yielding 91 ROI pairs. Correlations were transformed to z-scores and compared between groups. Neuroimaging results were correlated with scores from the Clinical Evaluation of Language Fundamentals (CELF) and age of first spoken word (FW) derived from the Autism Diagnostic Interview.

Results: Language delay, indicated by FW, was highly related to expressive, receptive, and overall language abilities $(p s<.005)$ in children with ASD. Between-group comparison revealed 12 pairs of ROI correlations that showed higher connectivity between language ROIs in the ASD group when compared to TD peers. Connectivity between right angular gyrus and left precentral sulcus was significantly anticorrelated with $\mathrm{FW}(\mathrm{r}=-.45, p=.02)$. Connectivity of left precentral gyrus to left angular gyrus was marginally anticorrelated with $\mathrm{FW}$ ( $\mathrm{r}=-.36$, $p=.05$ ) and significantly correlated with CELF Receptive Language scores $(\mathrm{r}=.44, p=.02)$.

Conclusions: Our imaging results support previous findings of aberrant connectivity of the language network in ASD. The link of overconnectivity between left precentral gyrus and left angular gyrus to earlier language development and higher language abilities suggests a compensatory mechanism unique to children with ASD

Correspondence: Yangfeifei Gao, Ph.D. in Clinical Psychology, Clinical Psychology, San Diego State University/UC San Diego, 6363 Alvarado Ct., Suite 200, San Diego, CA 92120, United States. E-mail: yangfeifeig@gmail.com

R. GREEN, E.D. BIGLER, M. PRIGGE, A. FROEHLICH, N. LANGE, A. ALEXANDER \& J. LAINHART. Beery VMI and Structural Volume Correlates in Autism Spectrum Disorder.

Objective: Very few studies have examined the association between visual-motor integration (VMI) abilities and neuroanatomical regions of interest (ROI) in individuals with Autism Spectrum Disorder (ASD). Participants and Methods: The current study included an all-male sample of 41 ASD (ages 3-23) and 27 typically developing (TD) participants (ages 5-26) who completed the Beery-Buktenica Developmental Test of Visual-Motor Integration (Beery VMI) as part of a neuropsychological battery. All participants underwent 3.0 Tesla magnetic resonance imaging (MRI) with image quantification by FreeSurfer software v5.1. The groups were statistically matched on age, handedness, and intracranial volume.

Results: As a group, the ASD participants performed significantly lower on VMI and IQ measures compared to the TD group. VMI performance was significantly correlated with FSIQ and PIQ in the TD group only. No pre-defined neuroanatomical ROIs were significantly different between groups. Significant correlations were observed in the TD group between VMI and total precentral gyrus gray matter volume $(r=.51, p=.006)$ and total frontal lobe gray matter volume $(r=.46, p=.017)$. Total parietal lobe gray matter volume trended toward significance.

Conclusions: The positive correlations relating frontal volumetric findings with VMI are consistent with size-function relations that typically exist in brain regions assumed to participate in motor and executive control needed for a VMI task. Although only trending toward significance, the parietal findings in the TD group support that interpretation as parietal cortical areas participate in the perceptual and attentional aspects of visuomotor control. In contrast, these relations were not observed in the ASD participants, suggesting a mismatch in size-function organization within these regions. Given these statistically significant findings, likely differences in frontal and parieto-frontal network organization between TD and ASD in the performance of visuomotor tasks will be discussed.

Correspondence: Ryan Green, Tripler Army Medical Center, 4 Sand Island Access Rd \#36, Honolulu, HI 96819, United States. E-mail: green.r.ryan@gmail.com

\section{A.J. HERRINGSHAW, S. KUMAR \& R. KANA. Neural Correlates of Social Perception in Children with Autism: Local versus Global Preferences.}

Objective: Autism Spectrum Disorder (ASD) is characterized by social interaction deficits. A detail-oriented information processing bias and an overreliance on brain regions associated with perceptual processing are also seen. In social cognition tasks where strong coherence is the norm, this processing style may lead people with ASD to falter, as opposed to visual search tasks where they perform well. This project examines the neural correlates of global versus local information processing in children with ASD in the context of visual and social processing.

Participants and Methods: ASD $(N=17)$ and Typically Developing (TD) $(N=17)$ children/adolescents completed an emotion and shape judgement task in a Siemens 3.0 Tesla fMRI scanner. The task consisted of stick figure human characters made up of geometric shapes displaying various emotions. Participants either indicated if a given geometric shape (shape/local condition) was present in the figure or the emotion conveyed by the figure (emotion/global condition). Data were analyzed using SPM12.

Results: 1) In the ASD group, global bias predicted significant activity in right angular gyrus in the emotion/global condition; local bias did not account for activation variance in either condition. 2) In TD children, local bias predicted significant activity in the local/shape condition in bilateral thalamus, pre- and post-central gyri, and lingual gyri; global bias did not account for activation variance in either condition. 3) Greater ToM abilities predicted increased activity in the ASD group in bilateral precuneus, lingual and cingulate gyri, and calcarine sulci in both conditions.

Conclusions: For the emotion/global condition in the ASD group, additional neural resources associated with spatial attention and integration were required to override a default local bias. Conversely, for local/ shape condition in the TD group required additional neural resources associated with sensory processing and visual attention to override their strong global processing bias.

Correspondence: Abbey J. Herringshaw, B.A., Psychology, University of Alabama at Birmingham, 1720 2nd Ave S, CH 201, Birmingham, AL 35294-1152, United States. E-mail: abbeyjh@uab.edu

\section{WELKOWITZ \& K. JENNINGS. Conversational Speech in Autism: Behavioral, Emotional, and Neuropsychological Viewpoints.}

Objective: We have completed several studies using the "Speechmatch" iPad program in recent years which suggest the program is useful in teaching individuals with Autism Spectrum Disorder (ASD how to match emotional content in language. These studies form an early foundation for building a model for understanding the pathway to ASD.

Participants and Methods: Male and female participants ranging from school-aged children to adults diagnosed with Autism Spectrum Disorder (ASD) engaged in "Speechmatch" iPad program in a pretest - posttest methodology. Speech parameters, neuropsychological indices of attention, patterns of neural connectivity as measured by fMRI, and converstational outputs were dependent measures in these studies. Sample sizes for these studies are small; they range from 1 to 10 participants.

Results: These studies suggest that "Speechmatch" is useful for teaching emotional content of speech with these participants. Variables including social attention appear to be important components in understanding ASD. As a result of this work and our review of extent literature, we are in the early stages of articulating a model for understanding the path of language delay in ASD. This model acknowledges a fuller 
pathophysiology including both neural connectivity and biochemical predispostions that lead to lack of attention to non-content aspects of speech ultimately resulting in ASD children falling out of social developmental sequence. The goal of intervention then does not become one of simply "correcting the path" but instead putting in place a few critical "pivotal" behaviors that will orient the person toward increased matching of a few speech parameters.

Conclusions: The purpose of this paper is to alert professional psychologists to emerging tools in helping individuals with ASD build social conversational skills. These new skills follow a model that incorporates a path of social development along with biological and situational factors that produce chronic social avoidance and escape.

Correspondence: Karen Jennings, Ph.D., Psychology, Keene State College, 229 Main Street, Keene, NH 03435, United States. E-mail: kjennings1@keene.edu

\section{A. LANDRY, S. LI \& D. KRIZ. Neurocognitive and Adaptive Functioning in Children with Autism Spectrum Disorder and Comorbid Attention Problems.}

Objective: Children with autism spectrum disorder (ASD) and children with attention-deficit/hyperactivity disorder (ADHD) have deficits in neurocognitive and adaptive functioning; however, little research has been conducted with children who have both conditions. This study investigated whether children with ASD and comorbid attention problems (ASD + ADHD) have more neurocognitive and adaptive deficits than children with ASD, ADHD, or another mental health disorder (MHD).

Participants and Methods: Participants included 587 children (5-18 years; IQ > 70) who received evaluations through a hospital-based outpatient autism clinic. Data were grouped by child diagnosis and symptom profiles to create four distinct groups (ASD Only, ADHD Only, ASD + ADHD, and MHD). Standardized measures of neurocognitive and behavioral functioning were utilized.

Results: Group differences were examined with Analyses of Covariance. Children with ASD + ADHD were found to have significantly lower communication and social skills $(p=.006)$, overall adaptive skills ( $p=.024)$, and nonverbal abilities $(p=.031)$ than the ASD Only group. Working memory and verbal comprehension differences were not found $(p=.414)$.

Conclusions: The results of this study suggest that children with ASD $+\mathrm{ADHD}$ have greater adaptive and nonverbal deficits compared to the other groups assessed, affecting their communication and social skills. However, they have similar verbal comprehension and working memory abilities. Overall, this study suggests that co-occurring ASD and ADHD diagnoses produce greater challenges for children, and should therefore be considered in combination when planning treatment interventions to improve outcomes.

Correspondence: Allisen Landry, M.A., Psychology, Pacific University, 2190 W. Burnside St. \#108, Portland, OR 97210, United States. E-mail: land5981@pacificu.edu

A. LANDRY, S. KARAM, S. MASTEL, T. HALL \& T. DUFFIELD. Virtual Reality as an Assessment Modality with Pediatric ASD populations: A Systematic Review.

Objective: Virtual reality (VR) has demonstrated increased reliability and validity for assessing cognitive functions. This review sought to evaluate whether VR assessment is equitable or performs better than traditional modalities in pediatric Autism Spectrum Disorder (ASD) populations.

Participants and Methods: A systematic review was conducted based upon methodological steps outlined by Uman (2011). The research question of interest pertained to psychometric comparisons of VR assessment to traditional modalities (i.e., paper-and-pencil and analogous computerized versions). A comprehensive list of search terms was established resulting in seven population (e.g., autism), four age (e.g., child), and eight exposure (e.g., virtual) terms. Inclusion and exclusion criteria were established based upon researcher consensus. Two independent researchers applied said criteria to manuscripts collected and interrater reliability was calculated using Cohen's Kappa. Discrepancies were discussed with the all researchers involved.

Results: Term combinations (e.g., autism + pediatric + virtual) resulted in 2,544 manuscripts. 1,280 duplicates were removed leaving 1,264 manuscripts. 1,259 manuscripts did not meet inclusion criteria and all remaining manuscripts met the exclusion criteria. The interrater reliability for the raters was found to be Kappa $=0.75$, or substantial agreement. A data extraction and design based quality assessment template were not applied as no manuscripts survived inclusion and exclusion criteria.

Conclusions: Few VR assessment paradigms exist. Of those that do exist, either a lack of comparison to traditional measures is being conducted, or psychometric comparisons of assessment modalities has not been applied to pediatric ASD populations.

Correspondence: Allisen Landry, M.A., Psychology, Pacific University, 2190 W. Burnside St. \#108, Portland, OR 97210, United States. E-mail: land5981@pacificu.edu

\section{C.M. LEE \& J. BO. Inter-limb Transfer of Kinematic Adaptation} in Children with Autism Spectrum Disorder.

Objective: Children with Autism Spectrum Disorder (ASD) often present with significant motor dysfunctions and impaired motor learning, though their learning mechanism is still unclear. A previous study revealed that children with ASD have relatively unstable and less efficient learning from motor planning perspective but can perform a similar level of adaptation by using motor control strategies. The current study examined the inter-limb transfer after children with ASD adapted to visuomotor distortions with their dominant hand, aimed to uncover motor learning processes and generalization among ASD.

Participants and Methods: Thirteen children with ASD, age 8 to 12 , and thirteen age-, gender-, and IQ-matched controls completed a center-out pointing task with the visual feedback of the hand movement rotated $30^{\circ}$ counter-clockwise. The after-effect and transfer-effect on variables of motor planning (directional error [DE], the maximum directional deviation of the actual from the ideal movement) and motor control (movement time [MT], the time moving from the home to the target) were used to measure adaptability and inter-limb transfer.

Results: Results revealed that children with ASD demonstrated successful transfer in motor control $(\mathrm{MT}, \mathrm{p}<.01)$ but not in motor planning (DE, $\mathrm{p}=0.089$ ), whereas the controls displayed significant transfer-effect on both motor planning and control (all $p<.01)$. The magnitude of transfer-effect related to overall adaptability of their dominant hand among all participants.

Conclusions: Children with ASD showed less inter-limb transfer than controls from motor planning perspective, but perform a similar level of learning transfer using motor control strategies to successful compensation for their "noisy" visuomotor mapping. A stable and efficient internal model may be essential for inter-limb transfer in kinematic adaptation.

Correspondence: Chimei M. Lee, Ph.D., Psychology, Eastern Michigan University, 2212 Aldrich Ave S, Apt 104, Minneapolis, MN 55405, United States. E-mail:mchimeilee@gmail.com

\section{R. LESSER \& J. WALKOWIAK. Evolutions in Diagnostic Criteria and Implications: A case study.}

Objective: Complex neurodevelopmental profile, including chromosome $15 q$ deletion syndrome, exemplifies treatment implications of diagnostic criteria changes between DSM-IV-TR and DSM-5, particularly in regard to Autism Spectrum Disorders (ASD) and Intellectual Disability (ID) in children.

Participants and Methods: Nine year old boy presented to pediatric clinic for neuropsychological testing to aid in differential diagnosis and determination of appropriate services in the context of a standing diagnosis of chromosome 15q deletion syndrome. Vineland Adaptive 
Behavior Scale indicated functioning from under 1 year old to mid-2 year old level. Two nonverbal measures of IQ indicated ID in the moderate range $(\mathrm{KABC}, \mathrm{NVI}=41$; PPVT $=49)$. On the ADOS-2- Module 1, he demonstrated communication abilities, reciprocal social behavior, and play skills that were consistent with intelligence. As a result, he did not meet criteria for ASD.

Results: Child met criteria for PDD-NOS under DSM-IV-TR criteria but does not meet criteria for ASD under DSM-5 criteria because deficits in social skills and communication are related to ID. The inclusion of adaptive functioning as a qualifier of severity of ID in the DSM-5 indicates that he now meets criteria for ID, Severe, rather than Mental Retardation, Moderate, as he did under DSM-IV-TR criteria. Unique neurodevelopmental sequelae likely related to chromosome 15 q deletion syndrome further complicate diagnosis and contribute to an atypical presentation.

Conclusions: This child, with a mutation on the chromosome 15q15.3, displays a unique neurodevelopmental profile that exemplifies diagnostic and treatment implications of the removal of PDD-NOS from the DSM. Functional impairments related to chromosome abnormalities, under new diagnostic criteria, lead to more severe and potentially more stigmatizing quantifiers of intellectual disability. These diagnostic changes may result in barriers to treatment for children with atypical symptom presentations.

Correspondence: Rebecca Lesser, MA, Psychiatry, Yale School of Medicine, 155 Bradley Street, Apt 3, New Havent, CT 06511, United States.E-mail: rebecca.lesser@yale.edu

H. OZOGLUOZ \& J. MASSA. The Effect of Social Story Training on Social Skills of Cypriot Children with Autism Spectrum Disorder. Objective: Autism Spectrum Disorder (ASD) affects children and families throughout the world. A number of studies support the use of social stories in improving social skills and decreasing inappropriate behaviors in children with ASD. The present study attempted to examine the use of social story training in a group of Cypriot children and adolescents with ASD. This is the first study to date to examine this underrepresented population. It was hypothesized that children in the social skill intervention group would have higher scores on parent and teacher social skills ratings.

Participants and Methods: Ten children (2 females; 8 males) ranging in age from 8 - 17 who were diagnosed with ASD participated in the study.

Parents and teachers completed the Autism Social Skills Profile questionnaire before and after social story training. Children received social story training in the following three areas: ability to say sorry, cope with ridicule, and avoid unnecessarily touching others. Childen in the experimental group were read stories and given lessons about the stories. Children in the control group were only read the stories.

Results: A 2(Rater) x 2(Time) x 2(Group) Mixed Design ANOVA was calculated to determine if there were differences between the groups social skills. No main effects or interactions were found. It is important to note that a trend toward a significance main effect for rater was found suggesting parents ratings tended to be higher.

Conclusions: The present findings are in contrast to previous research which has supported the use of social story training in chldren with ASD. A potential limitation in the present study is cultural differences in how parents view development disorders. Further, as Northern Cyprus is a small and developing country, there are fewer well trained professionals working in special education schools. Therefore, some children may be misdiagnosed. Future studies are needed to clarify these issues.

Correspondence: Jacqueline Massa, Ph.D., Psychology, Middle East Technical University - Northern Cyprus Campus, Kalkanli-Guzelyurt, Psychology Program R-132, Mersin 10 Turkey 99738, Turkey. E-mail: jmassa@metu.edu.tr
E. MOULTON, M. BARTON \& D. FEIN. Change in ASD Symptom Severity Between Ages Two and Four.

Objective: This study investigates changes in ASD symptom severity between ages 2 and 4 years in children with ASD and compares the magnitude of change in children with lower IQ (LIQ) and higher IQ (HIQ)).

Participants and Methods: Children who screened positive on the MCHAT(-R), were evaluated at age 2 (T1) and 4 (T2) using cognitive, adaptive and ASD-specific measures. Children $(n=130)$ were diagnosed with ASD at both timepoints. Symptom severity was assessed using Childhood Autism Rating Scale factor scores: Social Communication (SC), Stereotyped Behaviors and Sensory Sensitivities (SBSS) and Emotional Reactivity (ER) (Moulton et al., under review). Magnitude of change was compared in children with LIQ and HIQ. Groups $(n=65$, each) were established by dividing the sample based on the whole sample's median ratio IQ score on the Visual Reception (VR) Scale of the MSEL $(M d n=65.83)$. Mean VR IQ: LIQ=51.86 $(S D=11.54)$; $\mathrm{HIQ}=81.06(S D=17.50)(p<.001)$. Groups did not differ in age, gender, or race/ethnicity.

Results: SC: Children with LIQ displayed more severe SC scores at T1 $(p<.001)$ and T2 $(p<.001)$. Both groups displayed a significant decrease in SC severity between T1 and T2

SBSS: Children with LIQ displayed more severe SBSS scores at T1 $(p=.032)$ and T2 $(p=.034)$. Both groups showed no significant change in SBSS severity between T1 and T2.

ER: Children with LIQ displayed more severe ER scores at T1 $(p=.026)$; the groups showed similar scores at T2. Both groups showed no significant change in ER between T1 and T2

Conclusions: Our findings may reflect the focus of intervention on social communication, which appeared to improve between 2 and 4 . The apparent stability of SBSS and ER symptoms may reflect a lack of treatment focus or resistance to improvement for these domains. During the toddler years, children with LIQ and HIQ both show a capacity for improvement in social communication.

Correspondence: Emily Moulton, Ph.D., Psychology, University of Connecticut, 406 Babbidge Road, Storrs, CT 06269, United States. E-mail:emily.moulton@uconn.edu

A. NAIR, J. YANG, C. PONTING, T. TSANG, J. LIU, H. BOWMAN, L. JACKSON, S. BOOKHEIMER \& M. DAPRETTO. Altered thalamocortical connectivity in the first-year of life correlates with early social difficulties in high-risk siblings of children with autism.

Objective: In prior studies (Nair et al., 2013; Nair et al., 2015), we demonstrated that children with autism showed mostly reduced connectivity especially for prefrontal-thalamic networks, accompanied by overconnectivity within temporal-thalamic networks. Given the importance of early identification of biomarkers and endophenotypes of autism, it is crucial to understand how early in the developmental process these differences in thalamocortical networks emerge.

Participants and Methods: Resting-state functional connectivity (rs-fcMRI) data were acquired during natural sleep for 22 infant siblings (9 months old) of children with autism (high-risk group; HR) and 16 infants at low risk (LR) for autism. Analyses were undertaken to examine thalamo-cortical connectivity and to relate connectivity strength in thalamocortical networks to scores on the Autism Observation Scale for Infants (AOSI), Social Responsiveness Scale-2 (SRS-2), and Sensory Profile-2 (SP-2).

Results: Results indicate that the HR group showed thalamocortical patterns similar to older ASD children and adolescents in our prior studies. More specifically, as compared to the LR group, the HR group demonstrated marked bilateral underconnectivity within prefrontalthalamic networks, and overconnectivity within right temporal-thalamic networks. Temporo-thalamic overconnectivity in the HR group was correlated with higher scores, indexing early social difficulties, on the $\operatorname{AOSI}(r=.50, p=.02)$ and SRS-2 $(r=.79, p=.002)$. In contrast, prefrontal-thalamic underconnectivity in the HR group was correlated with 
poorer sensory responsivity to visual $(r=-.66, p=.01)$ and auditory $(r=-.62, p=.02)$ stimuli on SP-2.

Conclusions: These findings suggest that subcortical-cortical connectivity may be disrupted as early as the first months of life in HR infants, and that the altered connectivity may be associated with severity of early social difficulties.

Correspondence: Aarti Nair, Ph.D., Semel Institute, UCLA, $5401 \mathrm{~W}$ Olympic Blvd Apt 3, Los Angeles, CA 90036, United States. E-mail: aartinair@gmail.com

\section{Y. NAKAGAWA \& T. KOYAMA. Grammatical Difficulties for Adults with ASD and ADHD.}

Objective: This study examined the recognition and understanding of Japanese grammatical structures in adults with ASD and ADHD to reveal potential comprehension difficulties.

Participants and Methods: The participants were 82 adults with Developmental Disorders (ASD and ADHD) aged between eighteen to fifty-two, and a control group of eight university students average aged twenty. They were given a Japanese grammatical test (J.COSS: Japanese test for comprehension of syntax and semantics) in a group and a individually given a Wechsler Adults Intelligence Test.

Results: Whenever a participant correctly answered the four questions in each grammatical construct, it was assumed that they could understand that item. The number of items were 18.11/20 in ASDs that were evaluated at higher level, and were 15.25/20 in ADHDs that were evaluated at extremely higher or lower level. There were significant difference between the numbers of participants in each developmental level between groups $(X 2(3)=10.66, p<.05)$. Moreover, there was a strong correlation between J.COSS and IQs scores in ADHDs. Although previous studies showed children with ASDs had difficulty comprehending passive sentences, there were no difficulties observed in this area.

Conclusions: This study examined Japanese grammatical difficulties in adults with ASDs and ADHDs. There was strong correlation between J.COSS scores and IQ scores for ADHDs. Moreover, the participants with ADHD showed an overall delay in grammatical competence. However, the participants with ASDs showed limited indication of grammatical difficulties when compared with control group. This suggests communication difficulties in ASDs cause not only grammatical comprehension. Correspondence: Yoshiko Nakagawa, Ph,D, Clinical Psychology, International University of Health and Welfare, Aoyama Ichome Tower, 1-3-3, MinamiAoyama, Minato-ku 107-0062, Japan. E-mail: SNC59810@nifty.com

\section{A.T. PETERS \& V. TUCHSCHERER. Giant Congenital Melanocytic Nevus in a Two-Year-Old Female with Autism Spectrum Disorder: A Case Report of Neurocognitive Functioning and Review of Neurological Implications.}

Objective: Giant congenital melanocytic nevus (GCMN) is a rare congenital cutaneous syndrome characterized by pigment-based birthmarks. The risk of central nervous system (CNS) melanocytic deposits (neurocutanous melanosis, NCM) is approximately $7 \%$ among individuals with large nevi, resulting in increased risk of developmental delays, seizures, gait abnormalities, and other neurological complications. Patients with GCMN of the head and neck may also have associated neurological complications even without identifiable melanocytic deposits in the CNS. There is currently a paucity of research on the neuropsychological outcomes of these patients, with only a handful of case studies available.

Participants and Methods: The authors report the case of a 33-month old female presenting with GCMN of the dorsal spine, scalp, and face and associated history of 12 facial reconstructive surgeries. A recent MRI was normal, but conducted after the 4-month old recommended benchmark (normal brain myelination can partially obscure NCM abnormalities).
Results: The neuropsychological evaluation revealed low average estimated intellectual functioning, low average expressive language skills, borderline fine motor abilities, and symptoms consistent with autism. Gross motor function and receptive language were intact.

Conclusions: This case illustrates a number of delays in social development, motor skills, and language in a child without identifiable melanocytic deposits in the CNS (i.e., normal imaging). Although CNS deposits may have been detected if imaging was conducted at an earlier age, GCMN of the head/neck may lead to neurocognitive sequelae even in the presence of normal imaging. Children with GCMN need early and frequent neuropsychological screens to facilitate diagnostic clarity and appropriate intervention early in development.

Correspondence: Amy T. Peters, M.A., Psychiatry and Psychology, University of Illinois at Chicago, $1747 \mathrm{~W}$ Roosevelt Road, MC 747, Chicago, IL 60608, United States.E-mail: apeter51@uic.edu

\section{K. SHADA, M. CORDOVA, B. LANGHORST, A. GRAHAM \& D. FAIR. Examining executive functioning in ASD with or without comorbid ADHD, using the BRIEF.}

Objective: Children with ASD and ADHD both experience executive functioning $(\mathrm{EF})$ deficits. Differences in the extent and nature of these deficits may aide differential diagnosis and treatment. However, limited research has been conducted using parent report of $\mathrm{EF}$ for children with ASD and/or ADHD despite the clinical utility of such measures. The Behavior Rating Inventory of Executive Function (BRIEF), an empirical measure of $\mathrm{EF}$, was used to examine differences between groups for ASD, comorbid ASD/ADHD, ASD/subthreshold ADHD, and typically developing (TD) participants.

Participants and Methods: BRIEF scores for 131 children were examined. Four diagnostic groups determined by psychologists and psychiatrists using rigorous standards including standardized diagnostic interviews and assessments include: TD ( $\mathrm{N}=68)$, ASD $(\mathrm{N}=10)$. ASD/ subthreshold ADHD ( $\mathrm{N}=15)$, and comorbid ASD/ADHD ( $\mathrm{N}=38)$.

Results: A Kruskal-Wallis $H$ test yielded statistically significant differences on seven scales: Inhibit $\chi^{2}(3)=44.07, p=<.001$; Shift $\chi^{2}(3)=59.67, p=<.001$; Emotional Control $\chi^{2}(3)=42.45, p=<.001$; Initiation $\chi^{2}(3)=51.54, p=<.001$; Working Memory $\chi^{2}(3)=65.16, p=<.001$; Plan/Organize; $\chi^{2}(3)=54.44, p=<.001$; and Monitor $\chi^{2}(3)=62.98$, $p=<.001$. Significant differences were found between the TD group and two or more diagnostic groups on seven of eight scales. Findings on the Initiate, Working Memory, and Plan/Organize scales were significantly different between the comorbid ASD/ADHD group (respective means: 84.40, 88.47, 85.26) and ASD/subthreshold ADHD group (respective means: $53.27,51.47,54.10)$.

Conclusions: Those with ASD and comorbid ADHD show more EF impairment on parent ratings than those with ASD and subthreshold ADHD. Findings for ASD participants without comorbid/subthreshold ADHD were likely limited by the small group size. Additional analyses will add ASD cases and investigate the corroboration of parent reported EF deficits and task-based performance on Digit Span and D-KEFS (Tower, Color Word, and Trails).

Correspondence: Kiryl Shada, MS, School of Graduate Psychology, Pacific University, 18310 NW Cornell Rd, Apt F, Beaverton, OR 97006, United States. E-mail: shad9079@pacificu.edu

B.J. SHIELDS \& M.N. SCOTT. The Relation Between Executive Functioning and Adaptive Skills in Youth with Autism Spectrum Disorder, Level 1.

Objective: Autism spectrum disorder (ASD) puts youth at risk for a number of cognitive, social-behavioral, and adaptive difficulties compared to typically developing youth. Although youth with ASD without intellectual disability are expected to develop appropriate adaptive skills as they age, this has yet to be supported (Pugliese et al., 2015). One proposed explanation for these continued challenges is weaknesses in executive functioning $(\mathrm{EF})$, given recent research explaining the EF deficits experienced in youth with ASD. However, more research is 
needed to elucidate the link between EF and adaptive skills. As such, we examined the link between EF and adaptive skills in a sample of children with ASD, Level 1.

Participants and Methods: Participants were maternal caregivers of 40 children ( $78 \%$ male; $M=10.78 \pm 2.92,73 \%$ Caucasian) with a primary diagnosis of level 1 ASD presenting at the University of Chicago for neuropsychological evaluation. Caregivers reported on (a) youth EF using the Behavior Rating Inventory of Executive Functioning (Gioia et al., 2000), and (b) youth adaptive skills using the Scales of Independent Behavior-Revised (Bruininks et al., 1996). Hierarchical linear regression analyses were used to examine the relation between $\mathrm{EF}$ and adaptive skills, controlling for age, sex, race, and medication use.

Results: Although BRIEF composite scales were non-significant predictors, maternal reports on the Initiate $(\beta=-.55, t=-2.29, p=.04)$, Organization of Materials $(\beta=-.45, t=-2.20, p=.05)$, and Monitor $(\beta=-.69$, $t=-4.19, p=.001)$ subscales significantly predicted reports of youth adaptive skills.

Conclusions: Poor EF may play in important role in youth with ASD developing adaptive skills. However, it will be important to further distinguish the role of specific EF skills as they relate to successful adaptive development, and focus prevention and intervention efforts that focus on developing the skills that most impact development into adolescence and adulthood.

Correspondence: Brian J. Shields, Ph.D., Medical College of Wisconsin, 2556 N. 69th St., Lower, Milwaukee, WI 53213, United States. E-mail: brianjshields@gmail.com

\section{Medical/Neurological Disorders/Other (Child)}

D.J. BEARDEN, D.P. WABER, J.E. SCHREIBER \& C. MRAKOTSKY. Pain and Cognitive and School Function in Children and Adolescents with Functional Abdominal Pain.

Objective: Functional abdominal pain (FAP) is a frequently occurring complaint among pediatric patients and is associated with cognitive and school problems. Typically, research examining cognitive function in individuals with FAP has been adult focused. Because less is known about associations among pain and cognitive and school functioning in children and adolescents with FAP, the current study explored relations among those constructs in a group of young people.

Participants and Methods: Twenty-seven children and adolescents ( $M=12.61$ years, $S D=2.64 ; 33.3 \%$ male $)$ with FAP underwent evaluations that assessed cognitive, behavioral, and emotional functioning, and pain.

Results: Increased pain was associated with more metacognitive problems (BRIEF) $r(17)=.63, p=.007$, slower performance on WISC-IV Coding, $r(27)=-.52, p=.006$, poorer school function (PEDsQL) $r(25)=-.57, p=.022$, and an increased number of school absences $r(27)=.45, p=.017$.

Conclusions: Increased pain in young people with FAP is related to poorer cognitive and school functioning and more school absences. Correspondence: Donald J. Bearden, PhD, Department of Psychiatry, Boston Children's Hospital \& Harvard Medical School, 1106 Parker Place NE, Atlanta, GA 30324, United States.E-mail: dbearden2@gmail. com

\section{L.A. BRENNER, E. HEIDEMAN \& D.P. WABER. Is There a Broader Dyspraxia Phenotype? A Clinical Case Series Analysis.}

Objective: To explore the research question that dyspraxia is associated with distinct cognitive difficulties in addition to the "pure" motor planning deficits required for diagnosis (Steinman et al 2010).

Participants and Methods: Cases were selected from a larger sample of individuals seen for neuropsychological evaluation over a 2 year period in outpatient medical settings. Children were referred due to cognitive/learning concerns; seven children with dyspraxia diagnoses were identified. Three were excluded ( 1 due to age, 2 due to other medical diagnoses), leaving a final sample of 4 children ( 3 male). Three of the 4 children had a prior diagnosis of dyspraxia made by a neurologist and the fourth was diagnosed based on neuropsychological testing.

Results: Age range was 8 to 13. Evaluation clearly documented the expected motor deficits affecting precision, planning and sequencing. Although full scale IQs were Average or above, there was a distinct pattern of cognitive weaknesses. The most notable trend was inefficiency, even when achievement (final score) was Average - this pattern was most evident on measures of visual-spatial planning and narrative recall. The predominant style of processing was highly fragmented, with reduced appreciation for unifying frameworks, suggesting a core cognitive deficit involving planning/framing.

Conclusions: Our results support a broader dyspraxia phenotype extending beyond the motor domain with the potential to affect academic and adaptive functioning. Analysis of process is of particular importance when conceptualizing dyspraxia because it can contribute to a more complete understanding of the child and inform treatment planning. Correspondence: Laurie A. Brenner, PhD, Neurology, University of Virginia, Box 800394, Charlottesville, CA 22902, United States. E-mail: laurie.brenner@gmail.com

M. CLEM, E. LAMPSON, P.L. STAVINOHA \& A.A. HOLLAND. Genotype, Auditory Attention, and Educational Outcomes in Pediatric Acute Lymphoblastic Leukemia.

Objective: Pediatric acute lymphoblastic leukemia (ALL) survivors often demonstrate visual attention deficits (e.g.., Jacola et al., 2016), but their auditory attention has not been well-studied. The present study addressed this gap, hypothesizing similar deficits would be observed in auditory attention. Additionally, emerging research suggests Apolipoprotein E (APOE) e4 and Catechol-O-methyltransferase (COMT) Val polymorphisms may exacerbate neurotoxic effects of chemotherapy (Small et al., 2011; Krull et al., 2015). Thus, carriers of those alleles were expected to demonstrate worse auditory attention and educational performance relative to non-carriers.

Participants and Methods: 83 ALL survivors (45 females, M=12.58 years) completed the Conners Continuous Auditory Test of Attention (CATA) and provided blood samples for genotyping. Four groups were defined relative to carrier and non-carrier status for both APOE and COMT genotype. Parents reported educational performance and services via structured questionnaire.

Results: There were no significant demographic or treatment-related group differences. Relative to norms, the overall sample exhibited deficits in perseverative commission errors, hit reaction time, and hit reaction time variability $(p ’ s<.05)$, while omission errors trended $(p=.05)$. Genotype did not impact CATA performance. COMT-Val carriers utilized more educational services $(p<.05)$ than non-carriers and showed a trend for more grade retentions $(p=.06)$.

Conclusions: ALL survivors demonstrated auditory attention deficits suggestive of impulsivity and inattentiveness. Although CATA performance was similar for COMT-Val carriers and non-carriers, parent-report findings suggest the former may be more susceptible to subtle neurocognitive deficits affecting educational outcomes. Further research should investigate genotype-based differences in other neurocognitive factors relevant to academic functioning in ALL survivors.

Correspondence: Matthew Clem, MEd, Psychiatry, UT Southwestern Medical Center, 11348 Lippitt Ave, Dallas, TX 75218, United States. E-mail:matthew.clem@utsw.edu

M.K. COLVIN, S. MANCUSO, P. AlLURI, A. LAFFER, L. SHORSER-GENTILE \& K. WILLIAMS. Cognitive and SocioEmotional Functioning in Children and Adolescents with PANDAS (Pediatric Autoimmune Neuropsychiatric Disorders Associated with Streptococcal Infections).

Objective: PANDAS is a pediatric-onset neuropsychiatric disorder characterized by rapid onset of obsessive-compulsive disorder (OCD) 
and/or a tic disorder. Symptoms are associated with streptococcal infection and often episodic but chronic. We examined whether skills associated with frontostriatal networks (executive functions and motor skills) are selectively impaired and the degree to which this may be related markers of immune dysfunction and strep exposure.

Participants and Methods: 16 patients (13 male) ages 6-15 (mean $=10$ years) completed neuropsychological testing. One sample t-tests with Bonferroni corrections were conducted to compare scores to the normative mean. Significant results were correlated with patients' values of IgA, ASO titer levels, and Anti-DNase B levels.

Results: Overall cognitive functioning (Full Scale IQ, mean $\mathrm{Z}=.36$ ) was normal. Patients had significant difficulties with error-monitoring (mean $\mathrm{Z}=-.33, \mathrm{p}<.003$ ) and graphomotor control (mean $\mathrm{Z}=-.98$, $\mathrm{p}<.001)$. Performances on these measures did not vary based on markers of immune function and strep exposure. There was a trend for patients to have stronger verbal abilities (mean $Z=.26, \mathrm{p}<.03$ ), including verbal fluency and retrieval (mean $\mathrm{Z}=.23, \mathrm{p}<.02)$. Measures of speeded motor output were normal; dexterity was fast bilaterally (dominant: $\mathrm{p}<.015$, nondominant: $\mathrm{p}<.003)$. On standardized questionnaires (BRIEF, BASC-2, SRS-2), parents endorsed significant difficulties with behavioral regulation, hyperactivity, anxiety, depression, attention, adaptability, and repetitive behaviors.

Conclusions: Overall, there were difficulties with error-monitoring and graphomotor control in the context of normal intellectual functioning. Results also suggest strengths in fluent verbal and motor output. Performances did not vary based on markers of immune function or strep exposure. On standardized questionnaires, parents reported widespread emotional and behavioral concerns.

Correspondence: Mary K. Colvin, PhD, Psychiatry, Massachusetts General Hospital, One Bowdoin Square, Suite 701, Boston, MA 02114, United States.E-mail: mcolvin@mgh.harvard.edu

A. GIOIA, S. BILLS, T. KENNEDY, S. WISE, K.K. HARDY \& S. HARDY. Feasibility and Validity of a Computerized Cognitive Assessment in Pediatric Patients with Sickle Cell Disease.

Objective: Computerized cognitive testing (CCT) is increasingly being used to evaluate neurocognitive functioning in children and adults; yet, literature demonstrating its appropriateness for use with some medical populations is lacking. We assessed the feasibility and validity of a CCT battery, CogState, to evaluate attention, working memory, and processing speed in children with sickle cell disease (SCD).

Participants and Methods: In this prospective study, 59 children with SCD ages 7-16 ( $M=10.44, S D=2.87,42 \%$ Male $)$ were administered the Wechsler Intelligence Scale for Children (WISC-V), a CCT battery (CogState), and other neuropsychological measures and parent surveys assessing executive function, academic achievement, and quality of life. Results: A data integrity check was completed for each participant to assess the feasibility of CogState. Seventy percent of participants passed the integrity check. Regarding validity, all six CogState tasks were significantly correlated to at least one WISC-V subscale. Identification (IDN) speed (a processing speed and sustained attention task), One Card Learning (OCL) accuracy (a learning and memory task), and One Back (ONB) speed (a working memory task), were all significantly correlated with FSIQ (IDN $r=.36, p<.01$; OCL $r=.37, p<.01$; ONB $r=.38$, $p<.01$ ), PSI (IDN $r=.59, p<.01$; OCL $r=.29, p<.05$; ONB $r=.31, p<.05$ ), and WMI (IDN $r=.35, p<.01$; OCL $r=.34, p<.01$; ONB $r=.32, p<.05$ ). Furthermore, the Groton Maze Task (an executive functioning task) was significantly correlated with WMI $(r=.46, p<.01)$ and the Spatial Span Backwards test $(r=.61, p<.01)$, and the Detection speed task was significantly correlated to FSIQ $(r=.31, p<.05)$.

Conclusions: CogState appears to be a feasible and valid neuropsychological assessment for a youth with SCD. Due to the ease of CCT, assessments such as CogState could be easily integrated into prospective clinical trials to monitor disease- and treatment-related neurotoxicities and in clinical settings to screen for neurocognitive deficits.
Correspondence: Anthony Gioia, Psychology (BS), Neurology, Children's National Medical Center, 2804 Deer Trail Court, Ellicott City, MD 21042,United States. E-mail: agioia@childrensnational.org

\section{GLASS \& S.N. MATTSON. Characterizing Reading Performance in Elementary School-Age Children with Prenatal Alcohol Exposure.}

Objective: The underlying mechanisms of reading-related ability in children with histories of heavy prenatal alcohol exposure have not been comprehensively examined, which may hinder the potential for effective intervention. This study assessed whether different factors contribute to reading performance in children with and without heavy prenatal alcohol exposure.

Participants and Methods: Children (6-12y) with histories of heavy prenatal alcohol exposure $(\mathrm{AE}, \mathrm{n}=32)$ and without $(\mathrm{CON}, \mathrm{n}=40)$ were evaluated on multiple standardized measures of reading (decoding, fluency, comprehension), phonological processing (phonological awareness, phonological memory, rapid naming) and other factors related to reading (home literacy environment, caregiver-rated behavior, vocabulary, attitude towards reading). MANOVA and regression techniques were utilized.

Results: $\mathrm{AE}<\mathrm{CON}$ on all measures except rapid naming. AE had relative weaknesses in phonological awareness, decoding, and comprehension. Aspects of phonological processing accounted for significant variance in reading across groups. Alcohol exposure accounted for additional variance in decoding and comprehension. No interaction effects were significant. Taking the other factors into consideration, vocabulary, behavioral concerns, and attitude towards reading were additional significant contributors. Exposure history continued to account for significant variance, with no significant interactions.

Conclusions: Alcohol-exposed children demonstrated difficulties in reading and pre-reading abilities. Cognitive mechanisms known to contribute to reading in other populations also contributed in children with prenatal alcohol exposure, suggesting that effective interventions targeting phonological processing will likely also improve outcomes for alcohol-exposed children. Other aspects of functioning associated with alcohol exposure should also be considered in the development and dissemination of interventions.

Research supported by NIAAA F31 AA022261 (Glass) and U01 AA014834 (Mattson).

Correspondence: Leila Glass, M.S., SDSU/UCSD Joint Doctoral Program, 6330 Alvarado Court, Suite 100, San Diego, CA 92120, United States.E-mail:lglass@mail.sdsu.edu

\section{Z. HAWKS, J. SHIMONY, J. RUTLIN, D. GRANGE, S. CHRIST \& D. WHITE. Baseline Cognitive and Neural Differences Distinguish $\mathrm{BH}_{4}$ Responders from Non-Responders.}

Objective: Phenylketonuria (PKU) is a metabolic disorder characterized by disruption in the metabolism of phenylalanine (Phe). If left untreated, PKU entails profound neurologic problems and intellectual disability. Recently, sapropterin dihydrochloride $\left(\mathrm{BH}_{4}\right)$ has emerged as a promising pharmaceutical treatment for $\mathrm{PKU}$. $\mathrm{BH}_{4}$ reduces Phe levels and improves white matter integrity in a subset of individuals with PKU known as "responders." Although previous research has identified biochemical and genotypic differences that distinguish $\mathrm{BH}_{4}$ responders from non-responders prior to treatment, cognitive and neural differences remain largely unexplored.

Participants and Methods: To this end, we assessed general intellect and white matter integrity at baseline (i.e., prior to treatment with $\mathrm{BH}_{4}$ ) in 17 responders, 12 non-responders, and 12 healthy controls.

Results: Group-wise comparisons and tract based spatial statistics (TBSS) indicated poorer cognitive functioning and reduced white matter integrity in (1) individuals with PKU relative to controls and (2) non-responders relative to responders. TBSS findings also indicated an inverse relationship between white matter integrity and Phe variability (i.e., SD Phe) in non-responders. 
Conclusions: Given baseline differences between responders and non-responders, combining individuals with PKU into a single experimental group may mask the breadth and severity of PKU-related deficits. These results underscore the importance of characterizing PKU as a multi-faceted, multi-dimensional disorder.

Correspondence: Zoe Hawks, PhD, Psychological \& Brain Sciences, Washington University, 5355 Pershing Ave., Apt. 3C, St. Louis, MO 63112,United States.E-mail: hawksz@wustl.edu

D.K. HORTON, A. WILKINSON-SMITH, R.A. HOWARTH, B. GREENBERG, A. AYERS, P. PLUMB \& S. HUGHES. Associations Between Age at Diagnosis and Adaptive Skills in Children with Anti-N-Methyl-D-Aspartate (NMDA) Receptor Encephalitis.

Objective: Anti-N-methyl-D-aspartate (NMDA) receptor encephalitis is a form of autoimmune limbic encephalitis that can occur throughout the lifespan, often resulting in severe neurological and psychiatric symptoms. Outcome studies in adults have shown that over $75 \%$ of these patients make a full recovery. However, literature on functional outcomes in children is limited. The present study aimed to evaluate adaptive functioning in children with anti-NMDA receptor encephalitis based on age at diagnosis.

Participants and Methods: Parents of 15 children diagnosed between 8 and 179 months of age completed the Adaptive Behavior Assessment System, Second or Third Edition (ABAS) as part of a neuropsychological screening evaluation. Average time since diagnosis at the time of evaluation was 17.1 months. Participants were divided into "young" (71 months or younger, mean age $=32$ months) and "old" (72 months or older, mean age $=121$ months) groups based on age at diagnosis, and Mann-Whitney U tests were conducted to compare mean standard scores for ABAS composites between groups. Pearson correlations between age at diagnosis and $\mathrm{ABAS}$ composites scores were also calculated in the total sample.

Results: Mann-Whitney U tests revealed parent-rated adaptive skills in the "young" group to be significantly lower $(p<.05)$ than the "old" group on Conceptual, Practical, and General Adaptive Composites. Results also revealed statistically significant $(p<.05)$ positive correlations between age at diagnosis and all ABAS composites including General Adaptive Composite $(r=.69)$, Conceptual $(r=.70)$, Social $(r=.59)$, and Practical $(r=.67)$.

Conclusions: Results suggest that the functional impact of anti-NMDA receptor encephalitis may be more severe in children diagnosed at younger ages, possibly due to the adverse effects of this illness during a sensitive period of brain development. Findings have implications for the importance of early intervention for children diagnosed at younger ages. Correspondence: Daniel K. Horton, PhD, Psychiatry, Children's Medical Center Dallas, 4533 Cedar Springs Rd., \#425, Dallas, TX 75219, United States.E-mail: daniel.horton@childrens.com

T. KENNEDY, S. BILLS, S. WISE, K.K. HARDY \& S. HARDY. Validity of the Kiddie-Sluggish Cognitive Tempo Measure in Children with Sickle Cell Disease.

Objective: Sluggish cognitive tempo (SCT) is thought to be comprised of shared symptoms of ADHD-predominantly inattentive type as well as distinct symptoms of low energy of attention. Children with sickle cell disease (SCD) often experience fatigue and attention problems. However, SCT symptoms have never been explored in SCD. We describe SCT symptoms in youth with SCD and examine relationships between SCT and validated measures of cognition.

Participants and Methods: Fifty-nine children with SCD, ages 7 to 16 $(\mathrm{M}=10.59, \mathrm{SD}=2.91,43 \%$ male) were recruited as part of a cognitive training intervention study. Youth completed the Wechsler Intelligence Scale for Children, Fifth Edition (WISC-V) and parents completed the Conners-3 and Kiddie-Sluggish Cognitive Tempo Rating Scale (K-SCT). The K-SCT is comprised of a total score (range $=0-3$; higher scores reflect greater impairment) and 3 subdomains: Daydreams, Working Memory Problems, and Sleepy/Tired.
Results: Higher scores on the K-SCT were correlated with greater difficulties on the Inattention ( $\mathrm{r}=.755, \mathrm{p}=.000)$, Hyperactivity/Impulsivity $(r=.413, p=.002)$, Learning Problems $(r=.785, p=.000)$, and Executive Functioning ( $r=.664, \mathrm{p}=.000)$ subscales of the Conners-3. Sleepy/ Tired was the only K-SCT subscale not significantly correlated with Inattention and Hyperactivity/Impulsivity ( $\mathrm{r}=.254, \mathrm{p}=.059 ; \mathrm{r}=.057$, $\mathrm{p}=.675$, respectively). In a regression controlling for age and gender, higher scores on the Sleepy/Tired subscale predicted lower scores on the WISC-V Processing Speed Index $(b=-7.203, p=.032)$.

Conclusions: The K-SCT exhibited convergent validity with Conners-3 ADHD subscales but, in line with reports in the general literature, was more closely related to inattentive than hyperactive/impulsive symptoms. Sleepy/Tired symptoms of the K-SCT were unrelated to ADHD scales, highlighting a symptom presentation distinct from ADHD. Higher SCT symptoms were associated with greater parent-rated learning problems. The K-SCT appears to be a valid tool for measuring SCT in children with SCD.

Correspondence: Tess Kennedy, B.A., Neuropsychology, Children's National Medical Center, Children's National Health System, 111 Michigan Ave. NW, Washington, DC 20010, United States. E-mail: tkennedy2@childrensnational.org.

\section{K. KLIPFEL \& M. SEMRUD-CLIKEMAN. Neuropsychological} Sequelae of an Internationally Adopted Child.

Objective: This case study focuses on the unusual neuropsychological profile of an internationally adopted child with aim to disseminate known information and to seek consultation regarding remaining diagnostic and treatment questions.

Participants and Methods: The patient was internationally adopted at 4 months-of-age. His medical history is significant for low birth weight, dehydration, low respiratory rate, dysentery, pneumonia, Hepatitis A. tuberculosis exposure, and sepsis. Repeated neuropsychological evaluations, educational records, and consultation from neurology, psychiatry, occupational therapy, rheumatology, ophthalmology, and genetics are present.

Results: Within the context of normal development early, he began to show motor, attention, and sensory processing concerns at the age of 3 years. At age 5, patient showed a significant decline in fine motor and reading skills and onset of hand tremor, mood dysregulation, and physical concerns (e.g., eye asymmetry, staring spells, frontal headaches, hypersomnia). An MRI was notable for a single small focus of $\mathrm{T} 2$ hyperintensity in the posterior left cerebellum. Additional medical work-up showed mild astigmatism, rapid medication metabolization. and a genetic anomaly for the R779Q variant in the SMARCA1 gene. The patient has shown intact intellect and language, alongside impaired attention and executive functions, reading accuracy, verbal memory, visual-spatial skills, fine motor skills, and emotional/behavioral functioning. Interventions have included occupational therapy, medications, IEP services, and counseling, with some improvement.

Conclusions: The current case study demonstrates the complexities observed in an internationally adopted child. Findings also provide evidence for the possible significance of SMARCA1 anomaly, which requires broader investigation within the field.

Correspondence: Katherine Klipfel, PhD, Pediatric Neuropsychology, University of Minnesota Masonic Children's Hospital, University of Minnesota Masonic Children's Hospital, Minneapolis, MN 55455, United States.E-mail:klipf002@umn.edu

R.H. LEPPO, J.S. OGHALAI \& S. CAUDLE. Connecting The Dots: Fine Motor Skills, Executive Functioning Skills, and Math Achievement in Patients with Hearing Loss.

Objective: Children with hearing loss, including those with cochlear implants (CIs), are at particular risk for speech and language delays and later difficulties with reading and math. Research also links poor language skills with deficits in executive functioning skills. Research links language and fine motor skills, as well as fine motor skills and 
academic achievement. This study examined whether fine motor skills predicted executive functioning skills and math achievement for pediatric CI candidates.

Participants and Methods: Data were collected via retrospective chart review of children referred for outpatient neuropsychological evaluation in connection with cochlear implantation. Only participants with VMI-6, Purdue Pegboard, Stanford-Binet-IV Bead Memory subtest, and Tower of London, Drexel University, 2nd Edition scores were included in the sample ( $\mathrm{N}=53,55 \%$ male, age range 7 to 17 years). 25 patients received unilateral CI, 10 bilateral CI, and 18 no CI/not reported. Of the patients who received CIs, $43 \%$ were age 2 or younger at the time of implantation. The majority of patients' primary language was Spoken English (N=34).

Results: In linear regression analyses, VMI Motor Coordination predicted TOL-Dx-2 Total Rule Violations $(p=.03)$ and VMI Visual Perception predicted Bead Memory $(p<.01)$. Purdue Dominant Hand predicted Bead Memory $(p=.024)$ and TOL-Dx-2 Total Initiation Time $\left(\mathrm{R}^{2}=.11, \mathrm{p}=.02\right)$., Non-Dominant Hand predicted TOL-Dx-2 Total Moves $(p=.05)$ and Total Rule Violations $(p=.01)$, and Purdue Both Hands predicted TOL-Dx-2 Total Rule Violations $(p=.02)$. The average math achievement score was a standard score of $85(n=10)$ for computation (WJ-IV Calculation; WRAT-4: Math Computation) and 89 for fluency (WJ-IV Math Fact Fluency).

Conclusions: Preliminary results suggest that fine motor skills predict some aspects of executive functioning for pediatric CI candidates. Results regarding any connection between fine motor skills, executive functioning skills, and math achievement will be included in the final presentation.

Correspondence: Rachel H. Leppo, Ph.D., Psychology Service, Texas Children's Hospital / Baylor College of Medicine, 3210 Norfolk Street, Apt.15309, Houston, TX 77098,United States.E-mail: rhtarantolo@ gmail.com

\section{MACMULLEN FREEMAN, J.B. FULTON \& B. BUTCHER. Cognitive Functioning in Children with Hypothalamic Hamartoma.}

Objective: Hypothalamic hamartoma $(\mathrm{HH})$ is characterized by a hamartoma within regions of the third ventricle and tuber cinereum. Clinical presentation is variable, but often associated with precocious puberty and intractable epilepsy. Children with HH demonstrate a wide range of neurodevelopmental and behavioral deficits. Information is lacking regarding the developmental course and extent of these difficulties. Although there is an emerging literature regarding the neurocognitive deficits associated with $\mathrm{HH}$, no studies have examined the cognitive functioning of preschool children with this condition. The study sought to address the following research questions: What is the range in intellectual functioning in preschool children with HH? Is there a clear cognitive profile or discrepancy in skills in this population?

Participants and Methods: Clinical referrals of patients diagnosed with HH at Phoenix Children's Hospital were examined for the study. A total of 11 participants ( 2 female) were assessed, mean age was 41.2 months (SD 9.9 months). Two of the 11 patients were not included in group intellectual data given their inability to successfully complete intellectual testing. All children were administered the Wechsler Preschool and Primary Scales of Intelligence-Third Ed. (WPPSI-III).

Results: Results indicated that overall IQ was within the average range; however, participants demonstrated a significant discrepancy between verbal and perceptual indices.

Conclusions: In this small sample, preschool children with $\mathrm{HH}$ demonstrated intact overall cognitive functioning; however, significant variability between cognitive domains was present in the majority of participants.

Correspondence: Laura MacMullen Freeman, PhD, Phoenix Children's Hospital, 1701 E Colter St Unit 186, Phoenix, AZ 85016, United States. E-mail: lauramacfreeman@gmail.com
K. OLSON, M. KRIJNEN, K.K. HARDY, B. HOOVER \& M.T. ACOSTA. Parent-Reported Social and Executive Functions in Children and Adolescents with NF1.

Objective: Neurofibromatosis type 1 (NF1) is a genetic disorder associated with cognitive and social difficulties. Recent research has examined social difficulties, though the extent to which social problems reflect core social skills deficits versus problems in other cognitive domains critical to social success is unclear. This study sought to elucidate relationships between parent-reported executive functions (EF) and social functioning.

Participants and Methods: Patients referred to NF Clinic for routine medical care completed packets consisting of Behavioral Rating Inventory of Executive Function (BRIEF), Child Behavior Checklist (CBCL), Social Responsiveness Questionnaire (SRQ), and Social Communication Scale (SCS).

Results: Data were obtained from parents of 31 children (14 male, $45.2 \% ; 17$ female, $54.8 \%)$. Mean age was 10.23 years $(\mathrm{SD}=5.95$, range $=2-25)$. Thirty percent or more of patients had clinically significant problems on each of seven BRIEF subscales, as well as all subscales of SRQ. Males had significantly more communication problems on SCS than females $[t(27)=2.077, p=.047]$. Significant correlations were found between BRIEF subscales Inhibit, Shift, Plan/Organize, and Monitor with all five SRQ subscales $(.392<r<.751$.), SCS Total Score Scale $(.402<r<.556)$ and CBCL Social Problems Scale $(.501<r<.889)$. Twenty-three percent of sample had comorbid attention deficit/hyperactivity disorder (ADHD), and those children had significantly more problems on multiple BRIEF scales including Initiate $[\mathrm{t}(18)=-4.578$, $\mathrm{p}=.000]$ and Plan/Organize $[\mathrm{t}(25)=-5.821, \mathrm{p}=.000]$, as well as CBCL Social Problems Scale $[\mathrm{t}(18)=-2.672, \mathrm{p}=.038]$.

Conclusions: Patients experienced challenges in both EF and social functioning. Children and adolescents with ADHD had significantly poorer functioning in both domains regardless of age. Interventions may benefit from targeting particular EF or social difficulties; however, more research is needed to delineate core social difficulties from challenges in EF that contribute to social success.

Correspondence: Katie Olson, Psy.D., Neurology, Children's National Health System, 111 Michigan Ave, Washington, DC 20010, United States.E-mail:kaolson@childrensnational.org

J. POMMY, R. RIEGER, J. PHILlips, A. CAPRIHAN, E.C. GONZALES, R. CAMPBELL, R. YEO \& R. OHLS. Smaller Cortical Volumes in 3.5-to-5 Year-Old Children Born Preterm.

Objective: Premature birth has been associated with smaller gray matter (GM) volumes in the temporal lobes and other regions in adults (Bauml et al., 2015). The impact of premature birth in preschool-age children has not yet been established. We examined cortical volumes in 3.5-to-5 year-old children born prematurely to assess the impact of gestational age (GA).

Participants and Methods: 59 individuals born prematurely $(\mathrm{GA}$ range $=24-31$ weeks, mean $=27.94, \mathrm{SD}=1.82)$ and 21 individuals born full term $($ mean $=39.1$ weeks, $\mathrm{SD}=1.34)$ were recruited from two sites. Cortical structure was assessed using Voxel-Based Morphometry, an unbiased, voxel-wise assessment of gray matter volumes, utilizing a pediatric template generated from the Template-O-Matic toolbox.

Results: Group comparisons controlling for age, sex, and site revealed smaller GM volumes in the premature group relative to the control group, specifically, in right middle and superior temporal gyri, bilateral inferior frontal gyri, and parahippocampal gyri (corrected FWE <0.05). Within the premature group, decreasing GA was correlated with smaller GM volumes in the right middle temporal gyrus and medial temporal pole, right paracentral gyrus, right precuneus, and bilateral cerebellum (uncorrected $\mathrm{p}<0.001$ ). Regression analyses in the total sample indicated that the volume of these identified cortical structures significantly predicted behavioral abnormalities on all BASC-II summary scales ( $\mathrm{r}$ square values ranged from .13 to .22), but not Full Scale IQ. 
Conclusions: Premature birth is associated with smaller gray matter volumes, mostly in temporal regions, and these are related to the behavioral rather than intellectual consequences of prematurity.

Further, within the premature group, GA is correlated with smaller volumes in right temporal cortex and bilateral cerebellum. Based on previous studies in adults born premature (Bauml et al., 2015), these anatomic variations likely underlie widespread cortical connectivity reductions.

Correspondence: Jessica Pommy, Psychology, University of New Mexico, 1101 Yale Blvd NE, Albuquerque, NM 87106, United States. E-mail: jpommy@gmail.com

A. MATTES, A. HEITZER, J. PIERCY, B. PETERS, J. KLARR \& S. RAZ. Associations of Maternal Intellectual and Socioeconomic Factors with Neuropsychological Outcome of Preterm-Born Preschoolers.

Objective: The neuropsychological outcome of children born preterm is strongly influenced by maternal environmental and genetic factors. Yet little is known about the unique contribution of discrete maternal socioeconomic and intellectual variables to explaining developmental outcome variance in this population. We aimed to examine the strength of association between factors related to the environment provided by the mother (education, occupation, and verbal intelligence) and distinct neuropsychological functions.

Participants and Methods: The cognitive, language and math skills of 98 preterm (<34 weeks gestation) preschoolers (3-4 years of age) were assessed with the Wechsler Preschool and Primary Scale of Intelligence (WPPSI-III/IV), Clinical Evaluation of Language Fundamentals (CELF-P2), and Woodcock Johnson Tests of Achievement (W-J III), respectively. The mothers' verbal intelligence was prorated based on three WAIS-IV subtests, whereas occupational values were assigned using the Hollingshead Four Factor Index. Mothers who did not produce an income were excluded.

Results: Simultaneous multiple regression analyses revealed that years of education, but not occupation or Verbal IQ, explained a unique portion of the variance in the prorated WPPSI-III/IV Full Scale IQ $(F[1,90]=10.48 ; p=.002)$. The mother's Verbal IQ explained a significant portion of the variance in the CELF-P2 Core Language Score $(F[1,88]=8.25, p=.005)$, over and above the variance explained by occupation and years of education, as well as birth weight, intrauterine growth, medical complications, age at testing, and sex. None of the three variables of interest were associated with W-J III math performance (Applied Problems and Quantitative Reasoning subtests).

Conclusions: Although maternal education, occupation, and VIQ share variance in common, each variable may also provide a unique contribution to explained variance in separate neuropsychological functions in preterm-born preschoolers.

Correspondence: Sarah Raz, Ph.D., Merrill Palmer Skillman Institute, Wayne State Univ, Merrill Palmer Skillman Institute, 71 E. Ferry St., Detroit, MI 48202, United States. E-mail: sarahraz@wayne.edu

B. PETERS, A. HEITZER, J. PIERCY, A. MATTES, J. KLARR \& S. RAZ. Does Motor Development Explain a Unique Portion of the Variance in Cognitive or Language Abilities in Preterm-Born Preschoolers?

Objective: Children born prematurely are at risk for motor impairments or delays. However, it is unknown whether motor functioning explains variance in cognitive and language performance, over and above the variance accounted for by sociodemographic and medical risk factors. To address this question, we recruited a sample of preschoolers born preterm ( $<34$ weeks gestation).

Participants and Methods: One hundred and one children (age $44.32 \pm 3.39$ months) participated in this study. We excluded cases with congenital anomalies, perinatal intracranial hemorrhage $>$ Grade 2, periventricular leukomalacia, and cerebral palsy. Motor functioning was evaluated using the Peabody Developmental Motor Scales-Second
Edition (PDMS-2). Cognitive functioning was evaluated using the Information and Block Design subtests from the Wechsler Preschool and Primary Scale of Intelligence-Third/-Fourth Edition (WPPSI-III/-IV). Language functioning was evaluated using the Clinical Evaluation of Language Fundamentals-Preschool-2 (CELF-P2).

Results: We used simultaneous multiple regression analyses with the Fine and Gross Motor Quotients (PDMS-2) as predictors of interest. Adjusted age, sex, SES, intrauterine growth $z$-score, total perinatal complications, and gestational age were our covariates. The Fine and Gross Motor Quotients of the PDMS-2 accounted for variance in both Information $(\mathrm{p}<.001 ; \mathrm{p}<.01$, respectively $)$ and Block Design $(p<.001$; $p<.01$, respectively), and in Expressive $(p<.01 ; p<.05$, respectively) and Receptive Language $(p<.001 ; p<.01$, respectively), scores.

Conclusions: Motor skills contributed to explained variance in both cognitive and language outcome of preterm-born preschoolers, over and above the variance accounted for by sociodemographic and perinatal medical risk. Thus, in the aftermath of perinatal adversity, preschool motor abilities may reflect an important aspect of brain integrity that is not captured by variables reflecting medical risk, such as gestational age, growth restriction, or complications summary scores.

Correspondence: Sarah Raz, Ph.D., Merrill Palmer Skillman Institute, Wayne State Univ, Merrill Palmer Skillman Institute, 71 E. Ferry St., Detroit, MI 48202, United States.E-mail:sarahraz@wayne.edu

J. PIERCY, A. Heitzer, B. PETERS, A. MATTES, J. KLARR \& S. RAZ. Hypoglycemia and Language, Motor, and Cognitive Abilities in Preterm-Born Preschoolers.

Objective: Children born prematurely are at risk for neurocognitive deficits associated with perinatal risk; yet the relationship between metabolic complications and developmental outcome in this population is not fully understood. As neonatal hypoglycemia has been reported to be linked to white matter abnormalities in this at-risk population, we aimed to examine the relationship between hypoglycemia and neurodevelopmental outcome at preschool age.

Participants and Methods: Participants were twenty-seven preterm (<34 weeks gestation) preschoolers $(44.8 \pm 3.0$ months of age) with, and 126 preschoolers without, hypoglycemia observed during hospitalization. We examined outcomes before and after excluding children with severe perinatal intracranial hemorrhage or periventricular leukomalacia, and cerebral palsy. Cognitive, language, and motor functioning were measured using the Wechsler Preschool and Primary Scale of Intelligence (WPPSI-III/-IV), Clinical Evaluation of Language Fundamentals (CELF-P2), and Peabody Developmental Motor Scales (PDMS-2), respectively.

Results: We used simultaneous multiple regression analyses with hypoglycemia as the predictor of interest. Adjusted age, sex, SES, intrauterine growth $(z$-score), and birth weight were covariates. Outcome measures were cognitive, language, and motor indices. Hypoglycemia was associated with lower performance on the prorated Full Scale IQ $(F(1,156)=15.76, p<.001)$ and on both Receptive $(F(1,149)=15.90$, $p<.001)$ and Expressive $(F(1,142)=12.02, p<.001)$ Language Index scores, regardless of exclusion of 'neurological' cases. In contrast, hypoglycemia was not associated with motor performance.

Conclusions: In this exploratory study we documented a link between hypoglycemia and both cognitive and language performance in children born preterm. A larger group of preschoolers with history of this metabolic condition is needed to determine whether the observed associations are likely due to hypoglycemia or to associated medical complications not specified in our model.

Correspondence: Sarah Raz, Ph.D., Merrill Palmer Skillman Institute, Wayne State Univ, Merrill Palmer Skillman Institute, 71 E. Ferry St., Detroit, MI 48202, United States. E-mail: sarahraz@wayne.edu 
A. HEITZER, J. PIERCY, A. MATTES, B. PETERS, J. KLARR \& S. RAZ. Maternal Hypothyroidism and Neuropsychological Functioning of Preschool Age Children Born Prematurely.

Objective: Maternal thyroid dysfunction is known to be associated with impaired neurodevelopment in the offspring. Yet a limited body of research is available on the relationship between thyroid dysfunction in pregnancy and neuropsychological development of children born prematurely. Our goal was to examine the associations between treated maternal hypothyroidism and cognitive, language, and motor outcomes of preterm-born preschoolers.

Participants and Methods: We recruited 137 preterm $(<34$ weeks gestation) preschoolers (3-4 years), 21 with, and 116 without, history of maternal hypothyroidism requiring thyroid replacement hormones. We excluded cases with congenital anomalies, moderate to severe intracranial hemorrhage, cerebral palsy, and prenatal substance exposure. Cognitive, language, and motor abilities were assessed with the Wechsler Preschool and Primary Scale of Intelligence (WPPSI-III/IV), Clinical Evaluation of Language Fundamentals (CELF-P2) and Peabody Developmental Motor Scales (PDMS-2), respectively.

Results: We used simultaneous linear regression analyses with diagnosis of maternal hypothyroidism as predictor of interest. We adjusted for demographic (socioeconomic status, sex, multiple gestation, age at testing) and medical (hospitalization days, birth weight standardized by gestational age, and birth weight) factors. Maternal hypothyroidism was found to be linked to lower scores on the CELF-P2 Core Language Index $[F(1,116)=7.748, p=.006]$ and CELF-P2 Pre-Literacy Rating Scale $[F(1,117)=9.093, p=.003]$, but not to performance on intellectual or motor tests.

Conclusions: We found that within the population of preterm-born preschoolers, those whose mothers were treated antenatally for hypothyroidism obtained lower global language and reading readiness scores. A prospective study with a larger subsample of cases with hypothyroid dysfunction is needed to determine whether the findings are attributable to hypothyroidism, its treatment, or to risk factors that were not included in our model.

Correspondence: Sarah Raz, Ph.D., Merrill Palmer Skillman Institute, Wayne State Univ, Merrill Palmer Skillman Institute, 71 E. Ferry St., Detroit, MI 48202, United States. E-mail: sarahraz@wayne.edu

R.E. RIEGER, J. POMMY, M. WHITE, D. GILL, R. CAMPBELL, J. LOWE, J. PHILLIPS, R. YEO \& R. OHLS. Smaller Subcortical Volumes in Preschoolers Born Preterm: A Voxel-Based Morphometry (VBM) Study.

Objective: Premature birth is a risk factor for altered brain development. Reduced volume of subcortical regions, including the hippocampus, caudate nucleus, and thalamus, have been found in children and adolescents born preterm. However, the specific subcortical regions affected are poorly understood, as are the functional consequences. In the current sample of children age 3.5 to 5 years, we utilized a voxel-based analysis to reveal subcortical variations associated with premature birth, and the importance of such variation for cognitive and behavioral outcome.

Participants and Methods: 59 individuals born prematurely (gestational age range $=24-31$ weeks, mean $=27.94, \mathrm{SD}=1.82)$ and 21 individuals born full term $($ mean $=39.1$ weeks, $\mathrm{SD}=1.34)$ were recruited from two sites. Cortical and subcortical (above cerebellar level) gray matter volumes were assessed with VBM utilizing an age-specific template. Cortical volumes were masked so as to specify subcortical regions for group comparison. Intelligence was assessed with the WPPSI 3 and behavior with the BASC 2 .

Results: Group comparisons controlling for age, sex, and site revealed smaller subcortical volumes in the premature group in portions of the the left caudate, left hippocampus, right hippocampus, and right thalamus (corrected FWE <0.05). The thalamic voxels were mostly dorsomedial. Regression analyses in the total sample indicated that the volume of these identified subcortical structures significantly predicted FSIQ ( $\mathrm{r}$ square $=.20)$, but only one of four BASC 2 subscales.
Conclusions: Premature birth was associated with clusters of voxels distributed across diverse subcortical structures, including the caudate, hippocampus, and thalamus. These effects may contribute to variation in intellectual, and to a lesser extent, emotional function among preschool aged children.

Correspondence: Rebecca E. Rieger, PhD, Psychology, University of New Mexico, 1510 A Gold Ave SE, Albuquerque, NM 87106, United States. E-mail: rerieger@unm.edu

C.H. SALAMA, T. NORRIS, B. SLOMINE, S. SUSKAUER \& C.F. SALORIO. The Relationship between the Neurological Predictor Scale and Functional Outcomes of Children with Brain Tumor following Inpatient Rehabilitation.

Objective: After brain tumor resection, some children exhibit functional declines that warrant inpatient rehabilitation. This study examines the impact of neurological risk factors, as measured by the Neurological Predictor Scale (NPS), on functional outcome among children admitted to inpatient rehabilitation following brain tumor resection. It was hypothesized that total neurological risk would predict change in functioning.

Participants and Methods: Forty-one patients (3-21 years) were admitted to inpatient rehabilitation between 2003-2015 after brain tumor resection ( 24 males; $\mathrm{M}$ age $=9.7$ years). Mean length of stay was 35.61 days (range $=5-116$ ). Tumor location was more commonly infratentorial (68.3\% vs $31.7 \%$ supratentorial). Approximately $23 \%$ received chemotherapy or radiation during inpatient rehabilitation. The Functional Independence Measure for Children (WeeFIM) Developmental Functional Quotient (DFQ) assessed functional independence across domains (self-care, mobility, and cognition) at admission and discharge. The NPS provided a cumulative index of a child's risk factors.

Results: Linear regression revealed that total NPS at admission did not predict change in functioning. Among individual risk factors, chemotherapy before and/or during inpatient rehabilitation was associated with less change in self-care $(r=-.39, p<.05)$, mobility $(r=-.39, p<.05)$, and total functioning $(r=-.39, p<.05)$.

Conclusions: In this group, chemotherapy is an important consideration for functional outcome. Chemotherapy likely represents multiple factors that impact rehabilitation (e.g., aggressive malignancy, adverse medication effects, and disruption in rehabilitation for medical reasons). Further work should investigate what benefits inpatient rehabilitation provides children receiving chemotherapy after brain tumor resection; this information will be important for team planning and family education. Future work should also examine specific predictors of functional change among children receiving chemotherapy during inpatient rehabilitation.

Correspondence: Christina H. Salama, Ph.D., Neuropsychology, Kennedy Krieger Institute/Johns Hopkins School of Medicine, $707 \mathrm{~N}$. Broadway, Department of Behavioral Psychology, Baltimore, MD 21205, United States. E-mail: salama@kennedykrieger.org

B. SCHNEIDER, J. GARCIA, K. BALL, I. GONZALEZ, A. DREHER, G. SANTACRUZ, K. LEON \& S. TANNER-WOODWARD. Comprehensive Neuropsychological Testing in a Young Child with Neurofibromatosis Type 1: A Case Study.

Objective: Neurofibromatosis Type 1 (NF1) is a genetic neurological syndrome noted by various sequelae, including dermal neurofibromas, café-au-lait spots, and possible optic/acoustic gliomas. Neurocognitive deficits in children with NF1 are expected, given the role of neurofibromin in regulating GABA release, which is critical to prefrontalstriatal communication and long-term potentiation in the hippocampus, affecting learning, attention, working memory, and processing speed. Behavioral problems are also common, with more internalizing/externalizing and social difficulties when compared to the general population. Still, no distinct cognitive profile has emerged, particularly with very young children, except for increased deficits when NF1 and ADHD are comorbid. Neuropsychological functioning of a 6 year-old 
Hispanic-American male with this syndrome is presented. Findings will add to the limited literature about neuropsychological profiles and behavioral deficits due to NF1 in young children using updated, comprehensive neuropsychological assessments.

Participants and Methods: Neuropsychological testing of intelligence, achievement, language, memory, perceptual/spatial, executive function, auditory processing, attention/concentration, motor, processing speed, and behavioral/emotional functioning was completed to determine cognitive strengths and weaknesses.

Results: Testing revealed Low Average intelligence and academic achievement about one grade below current placement. Weaknesses were found in attention/concentration, processing speed, auditory processing, visual memory, motor precision, unstructured nonverbal conceptual reasoning, and cognitive flexibility, as well as behavioral problems. Attention deficits/hyperactivity were reported by parents and teachers, consistent with diagnosis of ADHD and Major Neurocognitive Disorder due to NF1 with behavioral disturbance.

Conclusions: Results of comprehensive testing are commensurate with behavioral and cognitive deficits associated with NF1 and comorbid ADHD in young children.

Correspondence: Kayleen Ball, M.S., FL, United States. E-mail: kmarquez632@sunmail.albizu.edu

J.E. SCHREIBER, R. BEAULIEU, L. WHITE, L. HALL, J. ESTEPP \& W. WANG. Cognitive function in preschool-age children with sickle cell disease: associations with home environment and disease-severity.

Objective: Children with sickle cell disease (SCD) are at risk for cognitive problems associated with the adverse neurological consequences of the disease. In addition, a disproportionate number of children with SCD live in lower socioeconomic status (SES) households, and low SES has also been associated with poor cognitive development. The present study examined associations among home environment factors associated with SES, disease severity, and cognitive functioning in preschoolers with SCD.

Participants and Methods: Twenty-two children with SCD underwent a cognitive evaluation at 4.1 years $(S D=.3)$ as part of an IRB-approved study. The research team visited the patient's home within 6 months following the evaluation to complete the Home Observation for Measurement of the Environment (HOME) inventory, a measure of the quality and quantity of stimulation and support available to a child in the home environment. Hemoglobin level was abstracted from the medical record as a measure of disease severity. Bivariate Pearson correlations were used to examine association among variables.

Results: The total score from the HOME inventory was positively correlated with SB5 Quantitative Reasoning $[r(22)=.51, p=.02]$, Working Memory $[r(22)=.56, p<.01]$, the FSIQ $[r(22)=.46, p=.04]$, and the Bracken School Readiness Assessment $[r(22)=.72, p<.01]$, but not with caregiver report of child executive function problems on the BRIEF, $p$ 's $>.05$. Hemoglobin was not significantly associated with any cognitive outcomes, $p$ 's $>05$.

Conclusions: Cognitive functioning and school readiness were associated with stimulation in the home environment but not with a medical indicator of disease severity. Results suggest that the home environment has a greater impact on cognitive functioning in preschool age children with SCD than a measure of disease severity. Further work is needed to understand the influence of home environment on cognitive functioning in children with sickle cell disease.

Correspondence: Jane E. Schreiber, PhD, Department of Psychology, St Jude Children's Research Hospital, 262 Danny Thomas Place, Mail Stop 740, Memphis, TN 38105, United States.E-mail:jane.schreiber@ stjude.org
M. SIEBENMORGEN, M. LOMAN, PHD \& L. GLASS. Congenital Heart Disease among Adolescents and Young Adults: Executive Control and Adaptive Functioning.

Objective: Congenital heart defects (CHD) affect about 8 out of every 1000 infants born in the United States (AHA, 2015). Individuals with this chronic health condition are at increased risk for neurodevelopmental deficits, including lower intellectual functioning, slower processing speed, visual-spatial deficits, and attention problems. While these skills have implications for understanding an individuals' daily functioning, research on adaptive functioning in individuals with CHD is limited. Studies involving other medical populations (e.g., TBI) show that executive dysfunction is related to poor adaptive skills, particularly social skills (Heverly-Fitt et al., 2016). This study aimed to investigate whether a similar relationship exists among adolescents and young adults with CHD. Results will have implications for the type of treatment and recommendations that will be the most beneficial for these individuals.

Participants and Methods: Participants included 22 individuals with a range of CHD diagnoses ( 4 females, 18 Males; age $\mathrm{M}=14.93$, $\mathrm{SD}=3.32)$. Correlation and regression analyses were used to examine the hypotheses.

Results: While trends exist for planning $(\mathrm{F}(3,9)=3.65, \mathrm{p}=.06)$ and problem-solving $(\mathrm{F}(3,13)=2.84, \mathrm{p}=.08)$ to predict adaptive functioning in this small sample, each explain a significant portion of the variance in social skills functioning when age and gender are controlled [planning $(\mathrm{F}(3,9)=7.82, \mathrm{p} \leq 0.01)$; problem solving $(\mathrm{F}(3,13)=3.74, \mathrm{p}<0.05)]$. Sequencing and set-switching were not predictive.

Conclusions: Results suggest that executive control may have a key role in understanding adaptive functioning among individuals with CHD and that emphasizing growth in skills related to improving social functioning (e.g., conflict resolution) may be particularly important.

Correspondence: Marsha Siebenmorgen, PhD, Neurology, Medical College of Wisconson, 3470 N 95th St, Milwaukee, WI 53222, United States.E-mail:msiebenmorge@mcw.edu

R. TARAZI, M. IAMPIETRO, K. PATRICK \& N. APOLLONSKY. Hydroxyurea Status is Associated with Cognitive Function in Young Children with Sickle Cell Disease.

Objective: Cognitive impairment in children with sickle cell disease (SCD) is thought to reflect the complex pathophysiology of the disease. Hydroxyurea (HU) is used in children with SCD to increase fetal hemoglobin $(\mathrm{HbF})$, contributing to a decrease in physical symptoms and to potential protection against cerebral microvasculopathy. There has been minimal investigation into the association between HU use and cognition in this population. The purpose of this study is to examine the relationship between hydroxyurea status and cognition in young children with SCD.

Participants and Methods: Neuropsychological data were collected in a prospective study from 38 children with SCD HbSS or HbS/b ${ }^{0}$ thalassaemia ages 4 to 11 years (Mage $=7.19$ years, $\mathrm{SD}=1.61)$ with no history of stroke or chronic transfusion. Ten children $(26 \%)$ were taking $\mathrm{HU}$ at the time of evaluation.

Results: Results were analyzed using ANCOVAs with HU status included as a factor and hemoglobin and age included as covariates. Controlling for hemoglobin (disease severity) and age, children on HU performed significantly better than children who were not on HU on measures of nonverbal reasoning (SB-V Nonverbal Fluid Reasoning; $[F(1,34)=7.31$, $\left.p=.01, \eta_{\mathrm{p}}^{2}=.18\right]$ ), immediate visual memory (NEPSY-II Design Memory Immediate; $\left.\left[F(1,33)=4.51, p=.04, \eta_{\mathrm{p}}^{2}=.12\right]\right)$, delayed visual memory (NEPSY-II Design Memory Delayed; $[F(1,31)=7.66, p=.01$, $\left.\eta_{p}^{2}=.198\right]$ ), and cognitive inhibition (NEPSY-II Inhibition Inhibition; $\left.\left[F(1,30)=4.39, p=.04, \eta_{\mathrm{p}}^{2}=.13\right]\right)$.

Conclusions: These results provide preliminary evidence that treatment with HU, which is typically prescribed for more symptomatic children assumed to be at higher risk for cognitive effects of SCD, may not only reduce physical symptoms, but also be protective against cognitive 
decline or dysfunction in young children with SCD. Further investigation is warranted.

Correspondence: Reem Tarazi, PhD, Psychiatry, Drexel University, Division of Hematology, Nelson Pavilion, SCHC, 160 East Erie Ave., Philadelphia, PA 19134,United States.E-mail: rtarazi@drexelmed.edu

A. WEGELE, R. YEO, R. CAMPBELL \& L. MORRISON. Cognition in Children and Adolescents with Myotonic Dystrophy Type 1.

Objective: Myotonic Dystrophy Type 1 (DM1) is a progressive neuromuscular disease involving multiple systems. While prior studies have noted significant global cognitive impairment and neuroimaging abnormalities in adults with DM1, there is a paucity of studies investigating cognition in younger patients. Our aims were to characterize the neurocognitive pattern of children and adolescents affected with congenital or childhood onset DM1. A secondary aim was to determine whether or not there was a correlation between CTG repeat length and severity of impairment.

Participants and Methods: Patients with DM1 ( $\mathrm{n}=16 ; 5$ female, 11 male, mean age 17) were administered a neuropsychological test battery to assess verbal and nonverbal intelligence, executive visuospatial, and memory functioning. Results were compared to a healthy control group ( $n=9 ; 3$ female, 6 male, mean age 16) and to a group with Charcot-Marie Tooth disease (CMT) $(n=6 ; 2$ female, 4 male, mean age 18), to control for muscle weakness.

Results: Patients with DM1 showed significant impairment in verbal and nonverbal intelligence $(p<.001)$, executive function $(p=.006)$, and memory $(p<0.001)$ compared to healthy controls. When compared to both healthy and CMT controls, patients with DM1 showed significant impairment in visuospatial $(\mathrm{p}<0.001)$ and verbal processing $(p<0.001)$. The correlation between CTG length repeat and FSIQ was not significant $(\mathrm{n}=15, \mathrm{r}=-.471, \mathrm{p}=.076)$.

Conclusions: Children and adolescents diagnosed with congenital or childhood-onset myotonic dystrophy have global cognitive impairment similar to findings in adult populations. Limitations include a small sample size and a referral center site. Future directions include: expanding the sample size; longitudinal follow-up, and investigating potential differences in degree of impairment between congenital-onset and childhood-onset.

Correspondence: Ashley Wegele, BS, Neurology, University of New Mexico Health Sciences Center, MSC10 5620, 1 University of New Mexico, Albuquerque, NM 87131,United States.E-mail: awegele@ salud.unm.edu

\section{AM Coffee Break}

10:30-10:45 a.m.

\section{Plenary B. Frontal Cortex and Human Behavior: Evidence from Intracranial Recording}

\section{Presenter: Robert T. Knight}

\section{0:45-11:45 a.m.}

\section{R.T. KNIGHT. Frontal Cortex and Human Behavior: Evidence from Intracranial Recording.}

Neuropsychological evidence has documented the critical role of prefrontal cortex (PFC) in the control of cognitive and social processing with extensive lateral or orbital PFC damage resulting in a profound disintegration of goal-directed behavior. This lecture will first describe novel neural activity linked to cognition recently unveiled by intracranial recordings in humans. Second, these brain signals will be used to link PFC function to cognitive control across a range of tasks. Direct cortical recording (electrocorticography; ECoG) provides unique insights in the role of PFC in cognition and social interaction. Since the discovery of the EEG in the 1920 's, neurophysiological dogma stated that the human cortex did not generate reliable rhythms above 50-60 Hz. However, findings over the last decade report neural activity up to $250 \mathrm{~Hz}$ in the human cortex. Every cognitive process examined with intracranial recording including language, attention, memory and decision-making generates task-specific high frequency activity in the range of 70-250 $\mathrm{Hz}$ (high frequency band; HFB). Importantly, the HFB band has superb spatial localization and task specificity. HFB recording has provided novel insights into the role of Broca's area in language processing, the hierarchical organization of PFC, and the critical role of PFC in contextual processing, decision making and working memory. Importantly, the HFB is phase locked to the trough of slower cortical oscillations with different PFC dependent tasks eliciting unique spatial patterns of HFB-theta coupling. These results provide evidence that transient coupling between low- and high-frequency brain activity provides a mechanism for effective communication in distributed neural networks engaged during PFC dependent cognitive processing. The results obtained from the study of PFC patients and from intracranial recording support the proposal that the devastating human prefrontal syndrome can be viewed as a failure of PFC control of distributed neural networks subserving human behavior.

Following this lecture, the learner should be able to: 1) Understand the role of high frequency brain activity in cognition; 2) Understand the role of low frequency brain oscillations in establishing networks supporting cognition; 3) Understand the key role of prefrontal cortex in orchestrating neural networks in the service of cognition Correspondence: Robert T. Knight, MD, Helen Wills Neuroscience Institute, University of California, Berkeley, 130 Barker Hall, Berkeley, CA94720,United States.E-mail:rtknight@berkeley.edu 
Lunch (On Own)

11:45 a.m.-12:45 p.m.

THURSDAY AFTERNOON, FEBRUARY 2, 2017

\section{Early Career Awardee Presentation: A Case Study Approach to Understanding Memory}

\section{Award Recipient: R. Shayna Rosenbaum}

\section{2:45-1:45 p.m.}

\section{R. ROSEnBAUM. A Case Study Approach to Understanding Memory.}

Much of what we know about brain-behavior relations is made possible by the study of neuropsychological cases. Given the ubiquity of functional neuroimaging studies, and the importance they have assumed in elucidating brain function, the goal of my talk is to describe how single cases continue to challenge accepted dogma, to lead to new discoveries, and to suggest hypotheses and theories that steer the field in new directions. Using memory as an example, I will discuss recent findings from case studies that specify critical functions of the hippocampus in episodic memory and spatial memory, and clarify its role in nonmnemonic abilities. Together, this work provides novel, theoretical insights on the nature of hippocampal-neocortical interactions and the types of memory they help represent.

Correspondence: R. Shayna Rosenbaum, PhD, Department of Psychology and Centre for Vision Research, York University, 4700 Keele St., Toronto, ON M3J 1P3, Canada.E-mail: shaynar@yorku.ca

\section{Invited Symposium 2. Evolution of the Neuropsychology of Epilepsy Surgery}

\section{Chair: Bruce Hermann}

$$
\text { 12:45-2:15 p.m. }
$$

B. HERMANN, J. OJEMANN, M.J. HAMBERGER, D.L. DRANE \& C. MCDONALD. Evolution of the Neuropsychology of Epilepsy Surgery.

Invited Symposium Summary: Clinical and experimental neuropsychology has had a long and productive relationship with the field of epilepsy surgery beginning with its earliest days at the Montreal Neurological Institute. As the availability of surgical treatment for medication resistant epilepsy spread throughout the world, neuropsychology has remained a standard component in the evaluation of patients for surgical consideration as well as the evaluation of outcomes following surgery. The resulting clinical and experimental work has contributed to new knowledge regarding brain function, served to characterize patient groups at high and low risk for postoperative cognitive and behavioral complications, and helped to inform advances in surgical approaches and techniques. The 50th anniversary of the International Neuropsychological Society is an appropriate time to take stock of the advances in epilepsy surgery and the neuropsychology of epilepsy surgery that have taken place over this interval. The symposium will begin with an overview of epilepsy surgery and the advances in care and technique occurring over time (Jeffrey Ojemann, MD, University of Washington), followed by speakers who will address the evaluation and outcomes across major cognitive domains including language (Marla Hamberger, $\mathrm{PhD}$, Columbia University), memory (Dan Drane, PhD, Emory University), and executive function (Carrie McDonald, PhD, UCSD). Each speaker will provide a historical perspective of the changes in style, approach and outcome that have occurred over time as well as the latest findings with a view to future directions and opportunities.

Correspondence: Bruce Hermann, PhD, Department of Neurology, University of Wisconsin-Madison, 600 N Highland Ave, Madison, WI 53792, United States.E-mail: hermann@neurology.wisc.edu

J. OJEMANN. On the Evolution of Neurosurgery and Neuropsychology in Epilepsy: Epilepsy Surgery.

The history of modern neurosurgical approaches to epilepsy finds its roots in the $19^{\text {th }}$ century, before even the invention of the roentgenogram. Localization of epileptic foci, including through imaging and physiology, led to an evolution in surgical options and efficacy. At the same time, functional localization, especially of motor, language and, later, memory, provided increased opportunity to provide surgical approaches with less morbidity. Many of these mapping tools (especially for speech and language) also revolutionized surgical treatments of other disorders, such as tumors. Different approaches to acute and chronic direct brain recordings have further advanced the clinical and scientific studies of focal epilepsy. The introduction of vagus nerve stimulation and intracranial therapeutic stimulation (e.g., RNS) open new doors for surgical intervention. Laser ablation provides another example of the ongoing effort to find a maximally effective, minimally invasive intervention with the least possible injury to central nervous system function. These and other advances in the surgical treatment of epilepsy will be reviewed.

Correspondence: Jeffrey Ojemann, MD, Neurological Surgery, University of Washington School of Medicine, 325 9th Ave, Seattle, WA 98104, United States. E-mail: jeff.ojemann@seattlechildrens.org

M.J. HAMBERGER. On the Evolution of Neurosurgery and Neuropsychology in Epilepsy: Language.

The inherent challenge of epilepsy surgery is to remove a sufficient volume of epileptogenic brain tissue to eliminate seizures, yet to avoid the removal or disruption of brain areas that are critical for function, particularly language. With a substantial proportion of epilepsy surgery cases involving frontal and temporal lobe regions, this risk-benefit quandary set the stage for the development of techniques to assess, lateralize and localize language function. Pioneering work at the Montreal Neurological Institute in the mid-1900s included the development of cortical stimulation to identify essential language cortex, the application of intracarotid barbiturate injection to determine hemisphere language dominance, and pre and postoperative neuropsychological assessment to characterize cognitive function and elucidate structure-function relations. Over the past 50 years, significant advances in epilepsy treatment have broadened surgical options for refractory epilepsy, with varied associated levels of risk to language. Traditional methods for language lateralization and localization have been refined, and advances in technology and cognitive neuroscience have led to new methods to determine language dominance and identify brain areas that mediate different aspects of language. In this presentation, we will review our progress over the past 50 years in assessing, localizing and protecting language in the context of surgical epilepsy, and consider the language-related issues that have been difficult to resolve. Although the fundamental tension between elimination of seizures and preservation of function remains, with greater knowledge and more sophisticated tools at hand, we are better equipped to further advance the neuropsychology and cognitive 
neuroscience of language, and meet the challenge of improving language outcome following surgical treatment for epilepsy.

Correspondence: Marla J. Hamberger, Ph.D., Neurology, Columbia University, 710 West 168th Street, New York, NY 10032, United States. E-mail:mh61@columbia.edu

D.L. DRANE. On the Evolution of Neurosurgery and Neuropsychology in Epilepsy: Memory.

Epilepsy surgery has provided a rich substrate for some of the greatest insights into memory over the last 75 years, fostering tight collaborations between neuropsychologists, neurosurgeons, and neurologists, while providing unique opportunities to uncover the neural underpinnings of cognition. The tragedy of Henry Molaison ("H.M.") established the essential role of the temporal lobes in declarative memory, after he was rendered amnestic by a bi-temporal resection. It fostered the development of the $\mathrm{W}$ ada procedure, which remains a solid component of the surgical workup despite the emergence of functional neuroimaging. Epilepsy surgery evaluations remain a window into the brain; and most surgical candidates willingly and valiantly contribute to our ever increasing knowledge base, and our refinement of surgical procedures and the evaluative process. Efforts to prevent adverse cognitive outcomes has led to numerous studies of memory change after surgery, and has afforded an exploration of the relative contribution of disease-related (e.g., seizure frequency, onset age) and surgical variables (e.g., resection extent \& location). This presentation highlights some hallmarks of the epilepsy surgery experience, while emphasizing significant findings regarding memory network models (e.g., material specific patterns), and theories of memory reorganization and outcome prediction (e.g., reserve capacity vs. functional reserve). Novel data from patients undergoing minimally invasive procedures, such as stereotactic laser amygdalohippocampotomy and radiofrequency ablation, will be presented, suggesting our current memory models remain crude and imprecise. These findings highlight that the epilepsy surgery center remains the incubator of cutting-edge neuroscience knowledge, holding promise for a new renaissance of memory models that build on the exquisite lesions produced by these novel interventions and technologies and allowing for more precise targeting of seizure onset zones.

Correspondence: Daniel L. Drane, PhD, Neurology and Pediatrics, Emory University School of Medicine, Woodruff Memorial Research Building, 101 Woodruff Circle, Suite 6111, Atlanta, GA 30322, United States.E-mail:ddrane@emory.edu

\section{MCDONALD. On the Evolution of Neurosurgery and Neuropsychology in Epilepsy: Executive Functions.}

The neuropsychology of temporal lobe epilepsy (TLE) has historically focused on language and memory since these domains are the most frequently impaired and are at highest risk for postoperative decline following anterior temporal lobectomy (ATL). Interest in executive dysfunction $(\mathrm{ExD})$ in epilepsy was largely stimulated by the application of the Wisconsin Card Sorting Test to patients with frontal lobe epilepsy by Milner and collaborators at the Montreal Neurological Institute in the early 1960s. However, interest in ExD in TLE began to materialize in the late 1980s when reports began to appear documenting its presence in TLE and introduced the concept of the nociferous cortex. Since that time, there has been increasing interest in further characterizing ExD in TLE, and particularly in understanding how it is affected by surgical intervention. This presentation will address the evolution of three pivotal debates surrounding ExD in TLE: (1) what is the nature of ExD in TLE, (2) what are the underlying causes, and (3) what changes are seen following ATL or other surgical procedures? Early research efforts relied exclusively on neuropsychological testing before and after surgery to answer these questions and revealed that up to half of patients with TLE show measurable changes in working memory, fluency, and/ or set-shifting. Recently, advanced neuroimaging and new analytic approaches have allowed for greater insight into frontotemporal network dysfunction in TLE that may underlie aspects of ExD presurgically and/ or be disrupted by surgery. Despite these advancements, we still lack an appreciation of which patients will decline in executive functioning following surgery and which patients will improve. Thus, there remains a critical need to identify individual neurobiological and clinical risk factors that predict post-surgical changes in executive functioning in TLE, determine the prognostic value of these changes, and assess their impact on quality of life.

Correspondence: Carrie McDonald, PhD, Center for Multimodal Imaging and Genetics (CMIG), Altman Center for Translational Research Institute, UC San Diego, La Jolla, CA 92037, United States.

E-mail: camcdonald@ucsd.edu

\section{INS Student Liaison Committee Workshop: The Neuropsychologist in the Public Domain: Kids, Academia, and the Law}

\section{Presenters: Robert T. Knight, Kathleen Y. Haaland, Erin D. Bigler, Donna J. Sorensen}

12:45-2:15 p.m.

\section{Symposium 3. Brain and Cognition Following Very Preterm Birth}

\author{
Chair: Chiara Nosarti \\ 12:45-2:15 p.m.
}

\section{NOSARTI, S. COUNSELL, J.P. BOARDMAN, R. GEVA \& G. CHRISTENSEN LØHAUGEN. Brain and Cognition Following Very Preterm Birth.}

This symposium will bring together international experts on the study of structural and functional brain alterations following very preterm birth that have been associated with an increased vulnerability to a variety of neurocognitive problems. Such studies contribute to define possible underlying mechanisms and to identify neurodevelopmentally vulnerable individuals, who could be then targeted with specific interventions. The objectives of the symposium are:

1) To appreciate issues relating to neurodevelopmental alterations which have been associated with an increased vulnerability to a variety of neurocognitive problems

2) To increase the audience's understanding of the complex interactions between various phases and aspects of brain development and environmental factors associated with neurocognitive risk.

The five chosen presenters will provide a comprehensive and multidisciplinary approach to our discussion. Prof Counsell will present a study showing that very preterm birth results in impaired development of brain structural connectivity relevant to high order cognitive functions. Dr Boardman will talk about how the foundational white matter architecture for information processing and intelligence is altered in infants who were born very preterm, although this does not appear to be critical to emergent social cognitive function. Prof Geva will then discuss how atypical electorphysiological activity in early maturing brainstem pathways at birth correlates with to long-term attention regulation capacity in childhood. In an adult sample, Dr Løhaugen will present her work on the association between structural brain alterations and cognitive outcomes in individuals who were born preterm with a very low birth weight. Finally, Dr Nosarti will discuss the association between an impaired ability to recognize visual facial expressions and resting state functional connectivity in adults who were born very preterm. indicating a bottom-up processing dysfunction.

Correspondence: Chiara Nosarti, PhD, Psychosis Studies, Institute of Psychiatry, Psychology and Neuroscience, De Crespigny Park, Denmark Hill, London SE5 8AF, United Kingdom. E-mail: chiara.nosarti@kcl. ac.uk 
D. BATALLE, E.J. HUGHES, H. ZHANG, J. TOURNIER, G. BALL, N. TUSOR, P. ALJABAR, L. WALI, D.C. ALEXANDER, J.V. HAJNAL, C. NOSARTI, A.D. EDWARDS \& S. COUNSELL. Preterm Birth is Associated with Impaired Development of Brain Structural Connectivity Relevant to High Order Cognitive Functions.

Objectives: Infants who are born preterm have a high prevalence of cognitive and behavioural deficits which are evident in childhood and an increased risk of developing psychiatric disorders in adulthood. The wide spectrum of disability associated with preterm birth is consistent with pervasive abnormalities in brain growth and connectivity. We aimed to (i) assess structural brain development from 25 to 45 weeks gestational age (GA) using graph theoretical approaches and (ii) test the hypothesis that preterm birth results in altered weighted network topology, informed by measures of white matter microstructure in the early neonatal period.

Participants and Methods: Sixty-five infants underwent MRI between $25^{+3}$ and $45^{+6}$ weeks GA. Structural networks were constructed using constrained spherical deconvolution tractography weighted by fractional anisotropy and neurite density index.

Results: We observed regional differences in brain maturation, with connections to and from deep grey matter showing most rapid developmental changes during this period. Intra-frontal, frontal to cingulate, frontal to caudate and inter-hemispheric connections matured more slowly. We demonstrated a core of key connections that was not affected by GA at birth. However, local connectivity involving thalamus, cerebellum, superior frontal lobe, cingulate gyrus and short range cortico-cortical connections was related to the degree of prematurity and resulted in altered global topology of the structural brain network.

Conclusions: Our data suggests that impaired connectivity in regions associated with important aspects of cognition and behaviour including executive function, memory, attention, information processing, response inhibition, salience processing and emotion regulation is already present in the early neonatal period and may explain, at least in part, neurocognitive and neurobehavioral impairments in this population.

Correspondence: Serena Counsell, PhD, Centre for the Developing Brain, King's College London, St Thomas' Hospital, London SE1 7EH, United Kingdom.E-mail: serena.counsell@kcl.ac.uk

E.J. TELFORD, S. COX, S. FLETCHER-WATSON, D. ANBLAGAN, S. SPARROW, R. PATAKY, S.I. SEMPLE, M.E. BASTIN \& J.P. BOARDMAN. A Latent Measure Explains Substantial Variance in White Matter Microstructure Across the Newborn Human Brain. Objective. A latent measure of white matter microstructure $(g)$ provides a neural basis for information processing speed and intelligence in adults, but the temporal emergence of $g$ during development and environmental factors that modify it are unknown. Social cognition is one of the first cognitive functions to develop, and it can be evaluated using infant eye-tracking. We tested the hypotheses: $1 . g$ is present in the newborn and is modified by environment (preterm birth); and $2, g$ is associated with infant social cognition.

Participants and methods. Based on diffusion MRI scans from 145 neonates (gestational age [GA] at birth range $23^{+2}$ to $41^{+5}$ weeks), the microstructural properties of eight major white matter tracts were calculated using probabilistic neighborhood tractography. Principal component analyses (PCAs) were carried out on the correlations between the eight tracts, separately for four tract-averaged water diffusion parameters (fractional anisotropy $[\mathrm{FA}]$, and mean $[\mathrm{MD}]$, axial $[\lambda \mathrm{ax}]$ and radial $[\lambda \mathrm{rad}]$ diffusivities). A subset of participants had eye-tracking at median age 7 months.

Results. For all four parameters there was a clear one factor solution, which explained $49 \%(\mathrm{FA}), 54 \%(\mathrm{MD}), 59 \%(\lambda \mathrm{rad})$, and $36 \%(\lambda \mathrm{ax})$ of the variance, and all tracts showed positive loadings and comparable factor structures. GA at birth was associated with the general measure for each water diffusion parameter. There were no significant associations between any general diffusion parameter and a sensitive measure of emergent social cognition derived from an eye-tracking at 7 months (all $\beta_{\text {absolute }} \leq 0.123$, all $p$-values $\geq 0.265$ )
Conclusions. The foundational white matter architecture for information processing and intelligence is established by birth, but is not critical to emergent social cognitive function. The environmental stress of preterm birth is associated with substantially global alterations to white matter connectivity.

Correspondence: James P. Boardman, United Kingdom. E-mail: James. Boardman@ed.ac.uk

R. GEVA, A. DITAL \& J. YARMOLOVKY. Electrophysiological Changes and Attention Correlates Following Preterm Birth.

Objective: The effects of prenatal adversity and preterm birth on the integrity of early maturing neural networks are not well understood. We aimed to evaluate brainstem electrophysiological functions in infants born very preterm and explore its cognitive and socio-emotional correlates later on in development.

Participants and Methods: A large sample of low risk infants born preterm ( $\mathrm{N}=183$, gestation age range: $29-36.1$ weeks) and birth weight 830 to 2644 grams) were recruited and evaluated at the neonatal intensive care unit soon after birth (mean gestation week 34.1) using neurobehavioral evaluation and an auditory brainstem evoked response test ( $75 \mathrm{~dB}$ hearing level, at a $10.1 \mathrm{~Hz}$ rate). They were then enrolled in a prospective follow up study. At 9 years of age a subsample $(\mathrm{N}=44)$ was tested using EEG and their attention was evaluated using gaze tracking paradigms.

Results: Data showed that $37.5 \%$ of the evaluated cases had compromised brainstem functions at birth. At 9 years of age changes in EEG were note in alpha and in theta wave spectrums on EEG in the compromised group as compared with the typical group. Specifically higher EEG frontal alpha power $(\mathrm{p}<0.003)$ and lower frontal theta power $(p<0.007)$ was seen along with specific attention regulation differences $(p<0.046)$ in the children that were diagnosed with compromised neonatal brainstem dysfunction.

Conclusions: Prematurity seems to double the risk for atypical electorphysiological activity in early maturing brainstem pathways at birth. These deficits are later related to long-term changes in brain electrical activity along with attention regulation changes, evident throughout the first decade of life and possibly thereafter.

Correspondence: Ronny Geva. E-mail: Ronny.Geva@biu.ac.il

G. CHRISTENSEN LØHAUGEN, H.F. ØSTGÅRD, A.E. SØLSNES, K.J. BJULAND, S. AANES, L.M. RIMOL, M. MARTINUSSEN, A. BRUBAKK, A.K. HÅBERG, K.K. SRIPADA, L. EIKENES, K.A. EVENSEN, M.S. INDREDAVIK \& J. SKRANES. Born Preterm with Very Low Birth Weight (VLBW) - Never Ending Cognitive Consequences?

Objective: To assess cognitive and neuropsychological functions and explore brain structure/functional relationship in young adults born VLBW compared to term born controls.

Participants and Methods: Neonates born preterm with very low birth weight (bw<1500 grams) and term born controls at St. Olav University Hospital in Trondheim, Norway in 1986-1988 were followed with multidisciplinary assessments into young adulthood. At 19-20 years of age, $55 \mathrm{VLBW}(72 \%)$ and 81 controls $(74 \%)$ received cognitive assessments, 51 VLBW and 66 controls also had cerebral MRI. Cognitive function (IQ) was assessed with full WAIS-III. Neuropsychological assessment included attention/executive (Delis-Kaplan), memory (Wechsler Memory Scale-III) and visuo-motor tests (VMI). DTI and morphological images were obtained on a 1.5 Tesla Siemens Magnetom Symphony Sonata system. The FreeSurfer software package was applied for the analysis of cortical thickness and surface area, and brain structural volumes. Tract-based spatial statistics were carried out to test for voxelwise group differences in fractional anisotropy (FA) of white matter tracts.

Results: The VLBW group had lower IQ and reduced scores within the attention/executive, memory and visual-motor domains. Fewer individuals from the VLBW group were still in school or employed compared to 
controls at 19 years of age. Reductions in cortical surface area, cortical thickness, brain volumes and FA-values of major white matter tracts were found in the VLBW group compared to controls. Deviations in brain morphometry and FA were associated with reduced cognitive functioning in the VLBW group but not in controls.

Conclusions: Our study indicates that deviations in cortical surface area, cortical thickness, brain volumes and white matter tracts are still present in adulthood and may explain the increased prevalence of cognitive and neuropsychological deficits in preterm born VLBW individuals. Correspondence: Gro Christine Christensen Løhaugen. E-mail: Gro. Lohaugen@sshf.no

C. TSENG, V. KAROLIS, J. KROLL, S. FROUDIST-WALSH, S. COUNSELL \& C. NOSARTI. Dysconnectivity of Visuospatial Attention Network at Rest and Emotion Recognition in VeryPreterm Born Adults.

Objective: Very preterm (VPT) birth is associated with socio-emotional problems in childhood, which have been studied as antecedents of later psychiatric disorders. Here we aimed to investigate whether VPT adults displayed impairments in recognizing facial emotions compared to term-born controls and whether such possible impairments were associated with altered resting-state functional connectivity (rsfc). We tested the following hypotheses - emotion recognition in VPT adults is characterized by 1) impaired top-down executive control over emotional processing (e.g. alterations in central executive network (CEN)), and 2) impaired bottom-up responses (e.g. enhanced salience network (SN) connectivity associated with emotional processing or decreased engagement of visuospatial attention network (VAN), which aids the allocation of attentional focus).

Participants and Methods: Ninety-seven VPT adults $(<33$ weeks of gestation) who were admitted to the neonatal unit of University College Hospital, London and 87 term-born controls. Participants completed the Emotion Recognition Task (ERT), a computerised assessment that presents morphs of emotional facial expressions. Analysis of rsfe data involved group independent component analysis performed using FSL's MELODIC, which identified the three networks of interest.

Results: VPT adults were less able to recognize specific emotions than controls $(\mathrm{U}=2815.5, \mathrm{p}<0.001)$. Whilst in controls emotion recognition scores were associated with VAN rsfc $(r=0.24, p=0.028)$, no such pattern was observed in VPT adults; with significant between-group differences $(\mathrm{F}=5.30, \mathrm{p}=0.022)$.

Conclusions: The allocation of attentional focus is important for developing the ability to selectively orient to key people and events, and to regulate emotional reactions to incoming sensory information. Emotion recognition impairments in very preterm samples may reflect bottom-up processing dysfunction and need to be investigated together with attention modulation.

Correspondence: Chiara Nosarti, PhD, Psychosis Studies, Institute of Psychiatry, Psychology and Neuroscience, De Crespigny Park, Denmark Hill, London SE5 8AF, United Kingdom.E-mail: chiara.nosarti@kcl. ac.uk

\section{Paper Session 3. Cognitive Neuroscience}

\section{Moderator: Bruce Crosson}

$$
\text { 12:45-2:15 p.m. }
$$

\section{E. DE HAAN \& C. DIJKERMAN. Returning into the Footsteps of} Berlucchi \& Aglioti: Many Bodies in the Brain. A Review.

Objective: In this presentation, I will review the experimental and theoretical developments over the last twenty years on somatosensory processing.

Participants and Methods: In their original paper on corporeal awareness, Berlucchi and Aglioti's (1997) already stressed the complex, multifaceted nature of how the body is represented in the brain. When Chris Dijkerman and I published our model of somatosensory processing ten years later (2007), we were more influenced by the work of Jacques Paillard, and Mel Goodale and David Milner, postulating two separate routes for processing somatosensory information: one for action planning and one for perception and recognition.

Results: These models triggered a large number of studies and somatosensory processing has now become a major field in cognitive neuroscience (e.g. Medina \& Coslett, 2016).

Conclusions: So, again ten years later, it seems appropriate to take stock. In doing so, I will argue for a distributed system, as suggested by Berlucchi and Aglioti (2010), comprising several representations, such as body image and body schema but also the affective body, tactile exploration, and tactile object representation.

References.

Berlucchi, G. \& Aglioti, S. (1997) The body in the brain: Neural bases of corporeal awareness. Trends in Neuroscience 20, 560-64.

Berlucchi, G. \& Aglioti, S. (2010) The body in the brain revisited. Experimental Brain Research, 200, 25-35.

Dijkerman, H.C. and de Haan, E.H.F. (2007) Somatosensory processes subserving perception and action. Behavioral and Brain Sciences, 30, 189-201

Goodale, M. A. \& Milner, A. D. (1992) Separate visual pathways for perception and action. Trends in Neurosciences 15, 20-25.

Medina, J. \& Coslett, B. (2016) Understanding body representations (Special Issue Eds.). Cognitive Neuropsychology, 33, 1-4.

Correspondence: Edward de Haan, PhD, Psychology, University of Amsterdam, Nieuwe Prinsengracht 129B, Amsterdam 1018WS, Netherlands. E-mail: e.h.f.dehaan@uva.nl

J.E. KARR, C.N. ARESHENKOFF, R.E. KILSHAW, R.J. TONKIN, S.M. HOFER, G.L. IVERSON \& M.A. GARCIA-BARRERA. The Dimensionality of Executive Function throughout Adulthood: A Systematic Review and Reanalysis of Latent Variable Studies.

Objective: Many studies have examined the structure of executive function (EF) through Confirmatory Factor Analysis (CFA); however, most CFA studies report inconsistent factor models across adulthood. This review revisited the published CFA research on EF, aiming to identify the number of EF constructs supported by CFA models and determine the best-fitting EF models by reanalyzing summary data.

Participants and Methods: The search protocol yielded 13 eligible articles (i.e., CFAs of healthy adults) with sufficient data to reanalyze 11 samples. For each study, 5,000 bootstrapped samples were simulated from a multivariate normal distribution based on the correlation matrix, fitting four factor models to each sample: a unidimensional model (UDM), multidimensional model (MDM), bifactor model (BFM), and nested factor model (NFM; bifactor without an inhibition factor). For models that converged, fit was assessed with the Comparative Fit Index $(\mathrm{CFI} \geq .90=$ good fit $)$.

Results: Accepted factor models differed substantially across studies. Twenty different constructs were supported, most frequently shifting $(k=7)$, updating $(k=7)$, and inhibition $(k=4)$. Based on the bootstrapped data across samples, the UDM almost always converged (mean $94 \%$ converged across samples), but rarely fit well (mean $8 \%$ with CFI $\geq .90$ ). Three-factor models including shifting, updating, and inhibition showed the best fit most often (mean \% with CFI $\geq .90$ : MDM=75\%, BFM=84\%, and $\mathrm{NFM}=70 \%)$. However, they converged far less frequently than the UDM (mean \% converged: $\mathrm{MDM}=51 \%, \mathrm{BFM}=10 \%, \mathrm{NFM}=50 \%$ ).

Conclusions: The adult CFA literature supports a multidimensional EF structure, with shifting, updating, and inhibition receiving the most empirical support. However, the interfactor relationships remain ambiguous. Studies variably support the MDM, BFM, or NFM, but in many cases these solutions do not converge. The MDM converged most often across studies, and showed comparable fit to the BFM and NFM; however, it converged in just $51 \%$ of samples and cannot be considered a stable EF model. 
Correspondence: Justin E. Karr, M.Sc., Psychology, University of Victoria, P.O. Box 1700 STN CSC, Victoria, BC V8W 2Y2, Canada. E-mail:jkarr@uvic.ca

N.T. BOTT, T. MOSKOWITZ, S. ERLHOFF, A. ALIOTO, K.P. RANKIN, J. KRAMER \& K.L. POSSIN. Allocentric and Egocentric-based Navigation Learning: Neuroanatomical Correlates.

Objective: Allocentric navigation relies on mapping boundaries or major landmarks irrespective of one's position, whereas egocentric route learning relies on linking self-motion cues with local landmarks. We investigated allocentric- and egocentric-specific navigation learning in healthy older adults $(\mathrm{NC})$ and a mixed sample of subjects with mild neurodegenerative disease (ND) to investigate the neuroanatomic basis of spatial navigation.

Participants and Methods: One hundred and twenty-seven adults (mean age: $68.9 \pm 9.2 ; 64 \mathrm{NC}, 63 \mathrm{ND}$ ) learned the location of a buried treasure in a circular virtual field over 10 trials with only allocentric landmarks available to guide learning. A mostly overlapping sample of older adults (mean age: 70.4 $\pm 8.9 ; 63 \mathrm{NC}, 46 \mathrm{ND}$ ) learned a defined route through a virtual neighborhood over 15 trials using only egocentric and local cues. Both tasks were controlled with a driving simulator. Mean ranked learning trials were correlated with gray matter volume using DARTEL-based voxel-based morphometry controlling for age, sex, and total intracranial volume. A permutations-based family-wise error correction was employed to account for multiple comparisons.

Results: Allocentric learning correlated with gray matter volume clusters in bilateral thalamus, left superior frontal gyrus, right precuneus, and right posterior cingulate. Egocentric route learning correlated with clusters in the inferior and middle frontal gyri superior and middle temporal gyri, left inferior occipital gyrus, left precentral gyrus, and left entorhinal cortex and hippocampus.

Conclusions: Allocentric and egocentric navigation strategies have distinct neuroanatomical underpinnings with a medial parietal-thalamic circuit supporting spatial learning based on landmark-based cues, and lateral frontal and temporal regions supporting route learning based on self-based cues. The role of the thalamus in allocentric navigation is consistent with human and rodent literature suggesting these nuclei may integrate visual cues important for spatial maps.

Correspondence: Nicholas T. Bott, Neurology, University of California San Francisco, 401 Quarry Rd., MC 5719, Palo Alto, CA 94305, United States.E-mail:nbott@stanford.edu

S.M. HAYES, V.J. WILLIAMS, H. LIU, M. VERFAELLIE \& J. HAYES. fMRI activity during associative encoding is correlated with cardiorespiratory fitness and source memory performance among older adults.

Objective: Age-related functional deterioration, which refers to both decreases and increases in brain activity, is commonly observed during associative encoding. Yet, there are remarkable individual differences among older adults $(\mathrm{OA})$. The main goal of the current study was to assess the relationship between fMRI activation during face-name associative encoding, subsequent source memory performance, and cardiorespiratory fitness (CRF), an individual difference factor that may attenuate brain aging.

Participants and Methods: Twenty-six OA and 31 young adults (YA) completed a treadmill-based maximal exercise test to evaluate cardiorespiratory fitness (peak $\mathrm{VO}_{2}$ ) and functional Magnetic Resonance Imaging (fMRI) to examine brain activation during a face-name associative encoding task. Memory performance was assessed with a two alternative forced-choice source memory task.

Results: Among $\mathrm{OA}$, peak $\mathrm{VO}_{2}$, the gold standard for the assessment of CRF, was positively associated with fMRI activity during associative encoding in bilateral prefrontal cortex, medial prefrontal cortex, bilateral thalamus and left hippocampus. Our results indicated that in some regions, high fit $\mathrm{OA}$ showed activation more similar to young adults (i.e., brain maintenance), whereas in other regions, high fit OA demonstrated greater activation than YA (i.e., compensation). Further, fMRI activity in these brain regions was positively associated with source memory performance among OA, with a mediation model demonstrating that activity in medial prefrontal cortex indirectly influenced the relationship between peak $\mathrm{VO}_{2}$ and source memory performance. Conclusions: Our results indicate that CRF contributes to neuroplasticity among $\mathrm{OA}$, reducing age-related differences in some regions while accentuating age-differences in other brain regions. Further, the pattern of increased activation in high fit $\mathrm{OA}$ was linked to better subsequent source memory performance, suggesting that brain regions sensitive to CRF may compensate for age-related memory decline.

Correspondence: Scott M. Hayes, PhD, Memory Disorders Research Center and Neuroimaging Research for Veterans Center, VA Boston Healthcare System, 150 S. Huntington Ave, VA Boston Healthcare System, MDRC 151A, Boston, MA 02130, United States. E-mail: smhayes@bu.edu

M.J. WRIGHT, D. HARDY, A. LEUCHTER, I. COOK, P. VESPA, D. MCARTHUR, D. HOVDA, E. WOO, B. BIRATH, W. LOPEZ \& J. FUSTER. The Crucial Role of Cognitive Reserve in the Effect of tDCS on Memory for Individuals With and Without Traumatic Brain Injury in a Simulated Work Environment.

Objective: Research has called into question the cognitive benefits of transcranial direct current stimulation (tDCS; Sellers, 2015). We sought to determine if potential tDCS benefits in persons with and without traumatic brain injury (TBI) might be related to cognitive efficiency (CE) and cognitive reserve (CR).

Participants and Methods: TBI $(n=18)$ and control $(n=19)$ groups consented to voluntary participation in this IRB approved study. The groups were matched on demographic variables. The TBI group suffered moderate-to-severe injuries that were largely due to accelerationdeceleration events. All participants completed neuropsychological tests and a procedure where they underwent anodal $(2 \mathrm{~mA}$ to left prefrontal cortex for 15min) or sham tDCS during learning trials for a list of workrelated tasks. Following learning and a distractor task, they were guided to a work environment where they were to complete the tasks. Next, they were given recall and recognition tests for the to-be-completed tasks. Results: Nonparametric 3-way ANOVAs were computed with Aligned Rank Transformations that included group (TBI, control), tDCS (anodal, sham), and estimated IQ (EIQ; high, low) or CE (high, low). Interactions were broken down by Mann-Whitney U tests. EIQ was derived from the WTAR and used as an indicator of CR. CE was based on a composite of speeded executive tests. Anodal tDCS improved memory in TBI participants with both higher EIQs and CE. However, it reduced performances in control participants with higher EIQs, but not those with higher CE. Regressions revealed that the interaction between EIQ and CE predicted work-related memory performances across anodal groups $\left(R^{2} \mathrm{~s}=.25-.58, p \mathrm{~s}=.000-.041\right)$.

Conclusions: Our data suggest that the effects of anodal tDCS are governed by the interaction of potential and actual CE and that matching these to the level of stimulation may maximize cognitive results. Also, CE may provide a buffer against stimulation levels that might otherwise be deleterious to performances in some people.

Correspondence: Matthew J. Wright, Ph.D., Psychiatry, Harbor-UCLA Medical Center, 1124 W. Carson St., B-4 South, Rm. 111 (Box 490), Torrance, CA 90502, United States. E-mail: mwright@labiomed.org

L.E. OBERLIN, A. WAIWOOD \& K.I. ERICKSON. The Neural Correlates of Cognitive Dysfunction in Obesity: an fMRI Study.

Objective: Obesity confers risk for neurocognitive disorders in late life and is associated with impairments in several cognitive domains among neurologically healthy adults. However, the neural correlates of cognitive deficits in obesity remain poorly understood. Using functional magnetic resonance imaging (fMRI), we sought to identify variations in functional brain activation in obese versus normal-weight individuals during a 
task measuring attentional control and examine whether differences in functional network efficiency predict neurocognitive performance. Participants and Methods: Seventeen obese $(B M I \geq 30)$ and 17 gender and age-matched healthy-weight participants (BMI $\leq 25)$ underwent functional imaging while completing a standard color-word Stroop task. A whole-brain voxelwise approach was used to examine group differences in activation during congruent, neutral, and incongruent trials. Results: Relative to the normal-weight group, obese participants demonstrated slower reaction times on congruent $(p<.01)$, neutral $(p<.01)$ and incongruent $(\mathrm{p}<.01)$ trials. In addition, normal-weight individuals exhibited a greater magnitude of deactivation than obese subjects during incongruent trials relative to baseline fixation, specifically in brain areas composing the default mode network (DMN) including the superior parietal lobule, the ventral medial prefrontal cortex (vmPFC) and the medial temporal lobe $\left(\mathrm{FDR}_{\text {corrected }} \mathrm{p}<.05\right)$. Furthermore, greater blood-oxygen-level dependent (BOLD) signal suppression in the vmPFC during incongruent trials was correlated with better accuracy on the incongruent condition $(\mathrm{r}=-0.34 ; \mathrm{p}<.05)$.

Conclusions: Obese individuals exhibited less deactivation during incongruent trials in brain regions that comprise the DMN. Furthermore, less successful deactivation in DMN regions was associated with poorer Stroop performance. Variations in functional network efficiency, and in particular failure to suppress activity in resting state networks, may contribute to cognitive deficits in obesity.

Correspondence: Lauren E. Oberlin, MS, Department of Psychology, University of Pittsburgh, 210 South Bouquet Street, 3211 Sennott Square, Pittsburgh, PA 15260, United States.E-mail: leo11@pitt.edu

\section{Paper Session 4. Oncology}

\section{Moderator: Jeffrey S. Wefel}

$$
\text { 12:45-2:15 p.m. }
$$

Y. CHEUNG, N.D. SABIN, D.A. MULROONEY, K.K. NESS, M.J. KRASIN, T.M. BRINKMAN, P. BANERJEE, D. SRIVASTAVA, L.L. ROBISON, M.M. HUDSON \& K.R. KRULL. Chronic Pulmonary Conditions and Neurocognitive Function in Long-Term Survivors of Childhood Hodgkin Lymphoma.

Objective: To compare neurocognitive function in long-term survivors of Hodgkin lymphoma (HL) to community controls and examine associations with pulmonary and cardiac morbidity.

Participants and Methods: 158 HL survivors treated with thoracic radiation (mean[SD] age 37.7[7.8] years, 23.4[8.1] years post-diagnosis) and 164 age- and sex-matched controls completed neuropsychological testing. Age-adjusted Z-scores $(\mu=0, \sigma=1.0)$ were compared between groups. Systematic grading of pulmonary (obstructive, restrictive, diffusion deficits) and cardiac (structural, functional defects, arrhythmias) morbidity was conducted and analyzed as predictors of neurocognitive function using generalized linear modeling, adjusting for age. Analyses were stratified to examine the effect of smoking on neurocognitive function in survivors with/without morbidity.

Results: Survivors performed poorer than controls on CVLT short(mean[SD] Z-scores: $-0.24[1.0] v 0.13[1.0] ; P=0.008)$ and long-delay recall $(-0.29[1.1] v 0.05[1.1] ; P=0.02)$, CPT Omissions $(-0.60[3.1]$ $v 0.24[0.8] ; P=0.005)$ and Coding $(0.14[1.0] v 0.39[1.0] ; P=0.05)$. Moderate to disabling pulmonary $(32 \%)$ and cardiac $(22 \%)$ conditions were present in survivors. Survivors with pulmonary conditions performed worse than survivors without morbidity, on CPT Omissions $(-1.47[3.5] v-0.09[2.2] ; P=0.009)$, Symbol Search $(-0.28[1.0]$ $v 0.46[1.0] ; P=0.003)$ and Coding $(-0.23[0.8] v 0.30[1.0] ; P=0.005)$. Cardiac conditions were not associated with neurocognitive function. In survivors without pulmonary conditions, current smokers were more impaired than non-current smokers $\left(P{ }^{\prime}<0.05\right)$ but in survivors with pulmonary conditions, smoking did not contribute to worse performance.
Conclusions: Roughly 20 years post-treatment, survivors of HL have worse neurocognitive outcomes than controls. Pulmonary morbidity and smoking were associated with worse functioning. Future work should evaluate the impact of pulmonary insufficiency on neurocognitive outcomes. HL survivors should be educated on the neurocognitive effects of smoking.

Correspondence: Yin Ting Cheung, Ph.D., Department of Epidemiology \&. Cancer Control, St. Jude Children's Research Hospital, 262 Danny Thomas Place, Memphis, TN 38105, United States. E-mail: VinTing. Cheung@Stjude.org

W. LIU, Y. CHEUNG, H.M. CONKLIN, L.M. JACOLA, D. SRIVASTAVA, V.G. NOLAN, H.ZHANG, J.G. GURNEY, I. HUANG, L.L. ROBISON, C. PUI, M.M. HUDSON \& K.R. KRULL. Evolution of Neurocognitive Function in Long-term Survivors of Childhood Acute Lymphoblastic Leukemia Treated with Chemotherapy Only. Objective: To evaluate the association between attention problems at the end of chemotherapy with executive function at long-term follow-up among childhood ALL survivors treated on a single institution chemotherapy-only protocol.

Participants and Methods: At end of therapy, the Conners CPT and Parent Rating Scale (CPRS) were collected on 158 survivors $(48 \%$ male; mean age 9.2yrs). At long-term follow-up, survivors (age 13.7yrs; 7.6yrs post-diagnosis) completed the Delis-Kaplan Executive Function System and Rey Complex Figure. Impairment on these outcomes was defined at below $10^{\text {th }}$ percentile of normative data. Poisson regression (GENMOD, SAS) was conducted to test associations between neurocognitive outcomes and high dose IV methotrexate (MTX) plasma concentration (area under curve [AUC]), and number of intrathecal therapies (IT's), adjusted for age at diagnosis. Associations between end of therapy and long-term follow-up testing were evaluated. Mediation analysis (STATA) was conducted to assess treatment effect on long-term outcomes, mediated by end of therapy function.

Results: Survivors performed worse than normative data $(10 \%$ expected) on CPT (38-53\%) and CPRS inattention (20\%) at end of therapy (all $\mathrm{p}$ 's $<0.05$ with FDR adjustment). CPT omissions and risk taking were associated with higher MTX AUC ( $p$ 's $<0.05)$. CPRS inattention was associated with more IT's $(p=0.003)$. At long-term follow-up, more impairment was seen on, number-letter switching $(24 \%)$ and Rey copy $(53.5 \%), p$ 's $<0.05$ (FDR), which were associated with treatment exposures. Survivors with high CPT omissions at end of therapy had increased risk for impaired Rey copy at long-term follow-up (RR 1.9, 95\%CI 1.2-2.9). However, indirect mediation of MTX AUC on Rey copy by CPT omissions was not significant ( $p=0.08$, RR $1.05,95 \% \mathrm{CI}$ 1.0-1.1).

Conclusions: Only chemotherapy, and not end of therapy attention problems, accounts for poor executive function in survivors at longterm follow-up. Monitoring for new neurocognitive problems during long-term survival is recommended.

Correspondence: Wei Liu, PhD, St.Jude Children's Research Hospital, 262 Danny Thomas Place, Memphis, TN 38105, United States. E-mail: wei.liu@stjude.org

P. BANERJEE, W.E. REDDICK, Y. CHEUNG, J. GLASS, L.L. ROBISON, M.M. HUDSON \& K.R. KRULL. Visuoconstruction Organizational Strategy and Neuroimaging Outcomes in LongTerm Survivors of Childhood Acute Lymphoblastic Leukemia Treated with Chemotherapy.

Objective: To examine organizational strategy on the Rey-Osterrieth Figure in association with cortical thickness, regional brain volumes, and white matter (WM) integrity in long-term survivors of childhood acute lymphoblastic leukemia (ALL).

Participants and Methods: 193 ALL survivors (mean[SD] age 14.3[4.7] years; 7.6[1.6] years post-diagnosis) treated on a chemotherapy-only protocol completed the Rey-Osterrieth Figure. Copy accuracy, organizational ability, and error performance scores were calculated 
using the Developmental Scoring System. Cortical thickness and regional white and grey matter volumes were obtained from magnetic resonance imaging, and WM integrity of regions and the frontostriatal tract was assessed using diffusion tensor imaging. Spearman partial correlations were used to examine associations between visuoconstruction and neuroimaging variables, adjusting for age and sex.

Results: Better organization was associated with thicker right caudal $(r=0.18, p=0.03)$ and rostral anterior cingulate cortex (ACG; $r_{s}=0.18$, $p=0.02)$. Fewer errors were associated with thicker right rostral ACC $\left(r_{s}=-0.21, p=0.01\right)$, left and right paracentral cortex $\left(r_{s}=-0.17, p=0.03\right.$; $\left.r_{s}=-0.25, p<0.01\right)$, and left posterior cingulate $\left(r_{s}=-0.20, p=0.01\right)$, as well as greater left and right cerebellar $\left(r_{s}=-0.22, p<0.01 ; r_{s}=-0.18\right.$, $p=0.03)$ and subcortical grey matter $\left(r_{s}=-0.17, p=0.03\right)$ volumes. Copy accuracy was associated with thicker left and right paracentral cortex and left and right posterior cingulate $(p$ 's $<0.05)$. No Rey variables were associated with WM integrity or total WM volume ( $p$ 's $>0.05)$.

Conclusions: In long-term ALL survivors treated with chemotherapy, poor visuoconstruction is related to thin cortices and less grey matter volume in brain regions involved in executive function (ACC), attention/ executive motor control (cerebellum, paracentral, posterior cingulate), and error monitoring (rostral ACC). This may suggest disruption of normal development of the default mode network and related executive networks.

Correspondence: Pia Banerjee, PhD, Epidemiology and Cancer Control, St. Jude Children's Research Hospital, 262 Danny Thomas Place, MS 735, Room S6010, Memphis, TN 38105, United States. E-mail: piabanerjee@gmail.com

T.M. BRINKMAN, Y. CHEUNG, C. LI, C.L. WILSON, G. KANG, W. LIU, K.K. NESS, W.E. REDDICK, M. EDELMANN, D. SRIVASTAVA, G. NEALE, M.M. HUDSON, L.L. ROBISON \& K.R. KRULL. Genome-Wide Association Study of Attention Problems and Executive Dysfunction in Sdult Survivors of Childhood Leukemia.

Objective: To examine genetic predictors of attention problems and executive dysfunction in adult survivors of childhood acute lymphoblastic leukemia (ALL).

Participants and Methods: 724 survivors of ALL (mean [SD] current age $=31.6[7.7]$ years, time since diagnosis $=25.0[7.5]$ years) completed standardized assessment of attention (Conners' Continuous Performance Test; Trails A) and executive function (Trails B, Verbal fluency, Digit Span Backwards). Age-adjusted z-scores and impairment frequencies ( $\geq 2 \mathrm{SD}$ below the normative mean) were calculated. Single nucleotide polymorphisms (SNPs) associated with impairment were identified through a genome-wide association study (Affymetrix 6.0 GeneChip with imputation to the 1000 Genomes Project) using a 2-stage resampling approach with a type 1 error rate of $1 \times 10^{-6}$. Clinical and genetic factors associated with neurocognitive impairment were examined in multivariable logistic regression models.

Results: Twenty percent of survivors were impaired on at least one measure of attention; $24 \%$ on at least one measure of executive function. Prevalence of impairment increased by cranial radiation (CRT) dose (e.g., executive function: no CRT $=19.9 \%$; $\leq 20 \mathrm{~Gy}=21.1 \%$; $>20 \mathrm{~Gy}=33.5 \%)$. Three SNPs were internally validated as associated with attention impairment and 3 SNPs with executive dysfunction. In multivariable models adjusted for ancestry and clinical factors, an interaction between CRT dose and SNP rs11200297 (intron region of NSMCE4A, associated with telomere maintenance) was associated with impaired executive function. Survivors treated with $>20 \mathrm{~Gy}$ CRT had $156 \%$ increased risk of executive dysfunction with each minor allele $(\mathrm{RR}=2.56,95 \%$ CI 1.54-2.86), those treated with $\leq 20 \mathrm{~Gy}$ CRT had $34 \%$ increased risk with each minor allele ( $\mathrm{RR}=1.34,95 \%$ CI 1.04 1.73). The SNP was not associated with dysfunction in survivors treated without CRT.

Conclusions: Genetic variants related to telomere maintenance may modify risk of executive dysfunction in aging adult survivors of childhood ALL treated with high dose CRT.
Correspondence: Tara M. Brinkman, PhD, Epidemiology and Cancer Control, St. Jude Children's Research Hospital, 262 Danny Thomas Place, MS 735, Memphis, TN 38105, United States. E-mail: tara. brinkman@stjude.org

\section{M.E. FOX \& T.Z. KING. Attention and Functional Connectivity in} Survivors of Childhood Brain Tumors.

Objective: To study potential hyperactivity and hyperconnectivity, this study assessed functional connectivity in survivors of childhood brain tumors compared to their healthy peers during an attention task using psychophysiological interaction (PPI) analyses and evaluated for a relationship with performance.

Participants and Methods: Twenty-three survivors at least five years past diagnosis and 23 healthy controls completed a letter n-back task in the scanner. BOLD activation analyses and PPI analyses were conducted on a [0-back - crosshair] contrast to assess activation and connectivity during the task, and correlation and interaction analyses were conducted with the 0-back d' measure, a measure of accuracy.

Results: In the [0-back - crosshair] contrast, survivors showed more activity in a cluster in the left parietal lobe $(B A 19, p=0.037)$, which was then used as the seed region for the PPI analyses. In survivors, left BA19 negatively interacted with the right superior parietal lobe (BA7; z=3.588). In controls, left BA19 negatively interacted with the right BA19 ( $\mathrm{z}=3.573)$ and medial prefrontal cortex (BA9; $\mathrm{z}=3.573$ ). Beta weights of the left BA19-right BA19 interaction were correlated with 0 -back d' values for survivors only $(\mathrm{R}=0.454$, $\mathrm{p}=0.030)$, and the group* connectivity interaction predicted d'values $(\mathrm{F}(3,42)=5.261$, $\mathrm{p}=0.004)$.

Conclusions: Hyperactivity in the left parietal lobe in survivors during the attention task supported the latent resource hypothesis. Connectivity findings were more complex; control subjects showed negative interaction between activation of regions of the attention and default mode networks while survivors did not. Different right parietal regions in survivors and controls negatively interacted with the left parietal seed. Strength of interaction with the region bilateral to the seed showed a significant interaction with group to predict performance on the attention task, with survivors showing a positive relationship and controls showing none.

Correspondence: Michelle E. Fox, Psychology, Georgia State University, 475 Buckhead Ave., \#3402, Atlanta, GA 30305, United States. E-mail: mfox9@student.gsu.edu

J.S. WEFEL, S.Y. PATWARDHAN, M.E. BRADSHAW \& P.J. MASSMAN. Validity and Diagnostic Accuracy of the Clinical Trial Battery in Patients with Primary Brain Tumor.

Objective: Cognitive testing with the Clinical Trial Battery ([CTB]: HVLT-R, TMT, COWA) is increasingly incorporated into neurooncology clinical trials to establish the safety and efficacy of therapies. This study determined the relationship between the CTB Composite score (i.e., average standardized score of 3 CTB tests) and clinical characteristics as well as clinician ratings of impairment.

Participants and Methods: Neuropsychological assessments were completed on 260 newly diagnosed adult primary brain tumor patients. Patients completed a battery of 12 cognitive tests. Two board certified neuropsychologists blinded to patient characteristics reviewed all tests and rated global impairment for each patient using a 9-point scale. Validity related evidence was examined with correlations between cognitive tests scores and functional status, and by $t$-tests and effect size estimates evaluating the effects of tumor grade, laterality, and caudality. Diagnostic accuracy (i.e., sensitivity, specificity, PPV, NPV, and ROC curves) of CTB Composite compared to clinician global impairment rating was examined.

Results: CTB Composite correlated with measures of functional status (KPS: $\rho=0.39, p \leq .0001$ FIM: $\rho=0.46, p \leq .0001)$. Patients with lower grade $(p \leq .0001, \mathrm{~d}=0.49)$ and right hemisphere $(p \leq .005$, $\mathrm{d}=0.36)$ tumor performed better than patients with higher grade 
and left hemisphere tumor. There was no difference between patients with anterior versus posterior tumor. Using CTB Composite cutpoint $\leq-0.5$, diagnostic accuracy was: sensitivity $(0.86)$, specificity $(0.78)$, PPV (0.80), and NPV (0.85). ROC curve analyses identified a cutpoint of -0.63 (sensitivity (0.85), specificity (0.83), PPV (0.84), and NPV $(0.84)$ ) with an AUC of $89.5 \%$.

Conclusions: The CTB Composite score demonstrated adequate validity related evidence for use among primary brain tumor patients. Diagnostic accuracy was best at cutpoint of -0.63 .

Correspondence: Jeffrey S. Wefel, PHD, NEURO-ONCOLOGY, MD ANDERSON CANCER CENTER, 1515 HOLCOMBE BLVD, UNIT 431, HOUSTON, TX 77030, United States.E-mail:JWEFEL@ MDANDERSON.ORG

\section{Poster Session 4. Aging \& Dementia 1 1:15-2:30 p.m.}

\section{Aging}

A. ALIOTO, C. MEAD, K.B. CASALETTO, R. SALONER, S. DUTT, G. MARX \& J.H. KRAMER. A Longitudinal Study of Cardiorespiratory Fitness, White Matter Integrity, and Cognitive Function in Healthy Older Adults.

Objective: Increasing evidence suggests that cardiorespiratory fitness (CRF) protects against age-related declines in processing speed (PS), but the underlying biological mechanisms remain unclear. One hypothesis is that white matter integrity (WM), known to correlate with PS performance over time, mediates this relationship. In this longitudinal study, we evaluated the influence of white matter (WM) integrity on the relationship between baseline CRF and longitudinal PS in a population of healthy older adults.

Participants and Methods: 244 functionally intact older adults (mean age $=73$ ) recruited from the UCSF Memory and Aging Center underwent neuropsychological assessment and MRI. CRF was quantified by a formula based on age, gender, BMI, blood pressure, and selfreported physical activity (Jurca et al., 2005). Longitudinal processing speed trajectories were measured using slope variables. White matter hypointensites (WMH) from T1-weighted images was our measure of WM integrity. Regression models were used to evaluate the hypothesis that the relationship between baseline CRF and longitudinal PS is mediated by WMH.

Results: Higher CRF predicted slower decline in PS, and the association remained significant after controlling for education $(p<.001)$. In addition, higher CRF was a significant predictor of WMH ( $p<.001)$, and WM integrity predicted slower decline in PS ( $\mathrm{p}<.001)$. After controlling for WMH, the contribution of CRF on PS was no longer significant ( $b=-.0077 ; 47 \%$ mediation effect). A bootstrap estimation approach using 1000 samples indicated that the mediation effect was significant (95\% CI=-.0082, -.0078).

Conclusions: As expected, higher baseline CRF contributed to less decline in PS over time via better WM integrity in cognitively normal older adults. Findings support prior research suggesting that CRF may help prevent cerebrovascular disease and associated cognitive decline, and amplify the need to consider lifestyle factors that can preserve brain health among older adult populations.

Correspondence: Andrea Alioto, M.S., Memory and Aging Center, University of California San Francisco, 3045 Alma Street, Palo Alto, CA 94306,United States.E-mail: andrea.alioto@ucsf.edu
J. BELSER-EHRLICH, E. SENG, J. MAHONEY \& R. HOLTZER. Exploring Health-Related Quality of Life and Cognitive Functioning in Aging: A Formal Test of the Wilson \& Cleary Model. Objective: A theoretical framework of health-related quality of life (HRQoL) was proposed by Wilson \& Cleary (1995). Whether this model of HRQoL applies to an aging population has not been explored. Furthermore, the relationship between cognitive functioning and HRQoL is not well-described within this model. The current study examined the model in a sample of community-dwelling older adults and explored the contribution of cognitive functioning.

Participants and Methods: Participants were non-demented older adults $(\mathrm{N}=444$, age range $=65-95$, female $=58 \%)$ who completed self-report and objective measures that represented factors of the HRQoL model. These factors were biological functioning, symptom status, functional status, general health perceptions, overall quality of life, cognitive functioning, individual and environmental characteristics. The primary statistical method utilized was structural equation modeling.

Results: Standardized and unstandardized parameter estimates indicated adequate representation of each measurement variable on the corresponding HRQoL factor. Overall model fit was poor for physical HRQoL $\chi^{2}(140)=565.38, p<0.001$; RMSEA $=0.081$ and mental HRQoL $\chi^{2}(140)=543.78, p<0.001$; RMSEA $=0.081$. Incorporating cognitive functioning into the model also yielded poor model fit for physical $\chi^{2}(158)=701.84, p<0.001 ;$ RMSEA $=0.088$ and mental HRQoL $\chi^{2}(158)=678.80, p<0.001 ;$ RMSEA $=0.086$.

Conclusions: The assumption of the model that measured variables represented only one factor of HRQoL, and a linear progression of factors was not supported. Furthermore, inclusion of cognitive functioning did not improve model fit. This is likely due to associations among physical, psychological, social, functional, and cognitive domains in the aging population.

Correspondence: Janna Belser-Ehrlich, PhD student, Ferkauf Graduate School of Psychology, 1165 Morris Park Ave, Bronx, NY 10461, United States.E-mail: jbelsere@gmail.com

\section{J. BERNSTEIN, M. CALAMIA \& J. KELLER. Examining Cognitive Correlates of Sleep Quality, Daytime Sleepiness, and Insomnia in a Cognitively Healthy Older Adult Sample.}

Objective: To assess associations between self-reported sleep functioning with cognitive functioning at baseline and one-year follow-up in a sample of 1,029 cognitively healthy older adults.

Participants and Methods: Participants were recruited from the Louisiana Aging Brain Study (LABrainS), a longitudinal study of cognitive aging conducted by the Institute for Dementia Research and Prevention. Participants were $67.4 \%$ female and $94.9 \%$ white, with an average of $70.3(S D=7.7)$ years of age and an average of $15.9(S D=2.5)$ years of education. Participants completed multiple self-report sleep questionnaires at baseline and a standardized battery of cognitive measures (i.e., the Uniform Dataset Neuropsychological Battery) at baseline and one-year follow-up.

Results: In a multiple regression model examining associations between daytime sleepiness and overall sleep quality with cognition, greater daytime sleepiness $(\beta=-.069, p<.05)$, but not overall sleep quality $(\beta=-.035, p>.05)$, was associated with worse performance on concurrent measures of executive functioning $\left(R^{2}=.32, \mathrm{~F}(12,883)=34.57\right.$, $)$. In a separate model, age moderated the relationship between insomnia symptoms and executive functioning such that greater insomnia severity had a larger negative association with executive functioning as age increased $\left(R^{2}=.33, \mathrm{~F}(13,996)=39.39, \beta=-.059, \mathrm{p}<.05\right)$.

Conclusions: Sleep problems are associated with worse performance on measures of executive functioning in cognitively healthy older adults, and this relationship is stronger with increased age. Prior work has suggested an association between increased daytime sleepiness and impaired executive functioning in older adults with Parkinson's Disease (e.g., Goldman et al., 2013); our results extend these findings to the cognitively healthy population. 
Correspondence: John Bernstein, University of Rochester, 10 Ingleside Road, Lexington, MA 02420, United States. E-mail: jberns16@u. rochester.edu

L.M. CAMPBELL, C.C. HAYS, Z.Z. ZLATAR, M. MELOY \& C.E. WIERENGA. Relationship between Cerebral Blood Flow and Famous Face Naming in Cognitively Normal Older Adults.

Objective: Cerebral blood flow (CBF) has been implicated as a biomarker of Alzheimer's disease (AD), and research suggests those who demonstrate worse recognition of recent famous names, compared to remote, may be at higher risk for $\mathrm{AD}$. The current study aimed to examine CBF differences between those who perform worse on recent, compared to remote, famous face naming among a group of cognitively normal older adults. We hypothesized that those who performed worse on recent famous face naming would demonstrate lower CBF, supporting famous face naming as a sensitive cognitive marker of early AD pathology.

Participants and Methods: Fifty-two cognitively normal older adults $(\mathrm{m}=72.6, \mathrm{SD}=5.164)$ underwent arterial spin labeling (ASL) MRI to measure whole brain resting $\mathrm{CBF}$ and a famous faces picture naming task to assess recent vs. remote face naming. Voxel-wise independent samples T-tests examined CBF differences between those who performed worse on recent face naming (compared to remote) and those who performed equally or better on recent face naming (compared to remote).

Results: Results demonstrated that those who performed worse on recent famous face naming showed lower CBF, compared to those who did not, in the right hippocampus, right fusiform gyrus, right inferior temporal gyrus, right lingual gyrus, bilateral frontal gyrus, and bilateral anterior and posterior cingulate cortex (voxel-wise threshold $p<0.025$, cluster size corrected for multiple comparisons at $p<0.025$ ).

Conclusions: Those who performed worse on recent famous face naming demonstrated lower $\mathrm{CBF}$ in regions associated with famous face processing. Given that both $\mathrm{AD}$ and $\mathrm{AD}$-risk have been associated with lower CBF, the current results suggest worse performance on recent famous face naming, compared to remote naming, may serve as an early cognitive marker of AD risk among cognitively healthy older adults. Future studies should examine the relationship between CBF and recent versus remote famous face naming longitudinally and in coordination with functional tasks.

Correspondence: Laura M. Campbell, B.S., Research Service, VA San Diego Healthcare System, 3350 La Jolla Village Drive, 151B Attn: Laura Campbell, San Diego, CA 92161, United States. E-mail: lauracampbell166@gmail.com

K.B. CASALETTO, S. DUTT, E. FOX, R. SALONER \& J. KRAMER. Leisure Activity Participation is Associated with Neuroanatomic Structure in Healthy Aging Adults.

Objective: Lifestyle impacts brain development and may represent a modifiable point of intervention to improve brain health with age. Yet, the neurobehavioral impact of differential leisure activities is not well characterized. We aimed to examine the relationship of physical, social, and cognitive leisure activities on neuroanatomic structure among healthy older adults.

Participants and Methods: Forty community-dwelling healthy older adults (mean age $=74.9$ ) completed the modified CHAMPS Activities Questionnaire and a structural brain MRI. A priori regions of interest included medial and lateral temporal, middle and inferior frontal, and parietal lobes. Multivariable regression analyses covaried for age and total intracranial volumes.

Results: Greater proportion of hours engaged in physical activities was associated with larger medial (partial $\mathrm{r}(\mathrm{pr})=0.53, \mathrm{p}=0.003$ ) and lateral temporal $(\mathrm{pr}=0.43, \mathrm{p}=0.02)$, and parietal $(\mathrm{pr}=0.40, \mathrm{p}=0.03)$ lobes, with a trend toward middle frontal gyrus integrity ( $\mathrm{pr}=0.31, \mathrm{p}=0.10$ ). Engagement in more cognitively-demanding activities demonstrated a tendency for larger medial temporal $(\mathrm{pr}=0.25)$ and inferior frontal $(\mathrm{pr}=0.24)$ lobe volumes ( $\mathrm{ps}=0.11)$. Participation in social activities was not meaningfully associated with neuroanatomic structure $(\mathrm{ps}>0.60)$. When all types of activities were simultaneously entered, only physical activities were independently associated with medial temporal, parietal, and lateral temporal lobes ( $\mathrm{ps}<0.05)$. After controlling for vascular health markers (cholesterol ratio, HOMA-IR, BMI, heart rate, systolic blood pressure), only the relationship between physical activities and medial temporal lobe structure remained $(\mathrm{pr}=0.52, \mathrm{p}=0.01)$.

Conclusions: Leisure activities are associated with brain structure in otherwise healthy older adults. Physically-demanding activities may be particularly beneficial for structures involved in episodic memory that are susceptible to age-related dysfunction (e.g., Alzheimer's disease). Correspondence: Kaitlin B. Casaletto, PhD, Psychiatry, University of California, San Francisco, 675 Nelson Rising Lane, San Francisco, CA 94115, United States. E-mail: kaitlin.casaletto@gmail.com

M. CHEN, S. PILlEMER, S. ENGLAND, M. IZZETOGLU, J. MAHONEY \& R. HOLTZER. Neural Correlates of Obstacle Negotiation in Older Adults: An fNIRS Study.

Objective: Unsuccessful Obstacle Negotiation $(\mathrm{ON})$ is a cause of falls among seniors. Older adults are less efficient at avoiding obstacles compared to young adults, especially under attention-demanding conditions. Using functional near-infrared-spectroscopy (fNIRS), recent studies implicated the prefrontal cortex (PFC) in cognitive control of locomotion, notably under dual-task walking conditions. The neural substrates underlying $\mathrm{ON}$, however, have not been established. The current study determined the role of the PFC in ON during walking in seniors.

Participants and Methods: 90 non-demented older adults (mean age $=78.1(\mathrm{SD}=5.5)$ years; \%female=51) underwent fNIRS acquisition to assess changes in hemodynamic activity in the PFC during normal walk $[\mathrm{NW}]$ and walk-while-talk $[\mathrm{WWT}]$ conditions with and without obstacles. Obstacles were presented as red elliptical shapes using advanced laser technology, which resemble potholes, providing ecological validity to our task. A linear mixed effects model was used to determine differences in oxygenation levels among the four task conditions. The presence of slow gait, a risk factor for dementia and falls, served as a predictor hypothesized to moderate the effect of obstacles on PFC activation. Results: PFC oxygenation levels were significantly higher in WWT compared to NW $(p<0.001)$ irrespective of ON. Slow gait moderated the effect of obstacles on oxygenation levels across task conditions. Specifically, PFC oxygenation levels were significantly higher in ON-NW compared to NW $(\mathrm{p}=0.017)$ and ON-WWT compared to WWT $(p<0.001)$ but only among individuals with slow gait.

Conclusions: Consistent with Compensatory Reallocation, ON required greater PFC involvement among individuals with mobility limitations. Correspondence: Michelle Chen, Clinical Psychology with Health Emphasis Ph.D., Ferkauf Graduate School of Psychology, Yeshiva University, 41 Marble Hill Ave, 2, Bronx, NY 10463, United States. E-mail:mich.chen48@gmail.com

H. YOON, S. KIM, J. KIM \& J. CHEY. Aging-Associated Reduction in Regional Brain Metabolism, Memory Decline and Education in a 4-year Follow-up Study of Community-Residing Elderly People. Objective: In this longitudinal resting FDG-PET study, we examined the relationship between regional glucose metabolic change at rest and cognitive decline in the context of aging. We further investigated the role of education in moderating the relationship between metabolism decrease and cognitive aging, testing the cognitive reserve hypothesis. Participants and Methods: Forty-nine healthy elders (age 62-81, mean age $=69.4, \mathrm{SD}=4.39,83.7 \%$ women) underwent both neuropsychological tests and FDG-PET scanning and were followed for 4 years. The contrast scores that reflect changes on cognitive ability and metabolism were calculated by measuring the difference between baseline value and 4 -year follow-up value.

Results: Paired T-test on imaging data revealed significant metabolic decrease in the following brain regions: the middle frontal gyrus 
(MFG), the anterior cingulate cortex (ACC), the posterior cingulate cortex (PCC), and the thalamus. The regional metabolism decrease in the MFG, in particular, was found to be positively correlated with the decline in the Delayed Recognition Index, a composite recognition score from the Elderly Verbal Learning Test (EVLT), the Simple Rey Figure Test (SRFT), and the Story Recall Test (SRT) in Elderly Memory Scale (Chey, 2007). The association, however, was modulated by the years of education: i.e., the effect of metabolic deterioration on recognition decline was weakened in the elderly people with more education.

Conclusions: Elderly participants who had larger MFG metabolism reduction exhibited steeper decline in recognition ability over time. However, people with higher education did not show this trend; instead, they preserved or even improved their recognition ability despite metabolic reduction. This result suggests that education provides additional support to resist against metabolic burden, which is consistent with the cognitive reserve hypothesis.

Correspondence: Jeanyung Chey, Ph.D., Psychology, Seoul National University, Gwanakro 1, Seoul 08826, Korea (the Republic of). E-mail: jychey@snu.ac.kr

\section{H. KIM, J. BAEK, S. KWAK, Y. YOUM \& J. CHEY. Association between the Thickness of the Left Entorhinal Cortex and the Trend in Cohesiveness of Individual's Social Network in a Longitudinal Study of Community-residing Elderly People.}

Objective: The links between social network indices and the brain have been reported. Among the brain regions, entorhinal cortex (EC) is known to be correlated with an individual's social network size, indicating that the memory capacity supports individuals to maintain a large network size. However, most studies mainly focused on the size of network, rather than its characteristics. Therefore, we aimed to discover the relationship between the EC thickness and characteristics of social network by focusing on the trend in cohesiveness of individual's social network in a longitudinal study.

Participants and Methods: 814 elderly population in a township were recruited from Korean Social life, Health, Aging, Project. Interview was conducted for the entire population in a community to measure complete social network characteristics, including network size and cohesiveness with 3-year changes. Individual's social network size and cohesiveness (k-core) were calculated by using social network analysis. 68 old adults (mean age $=71.38 \pm 6.40)$ subsampled and underwent MRI protocol that included the T1 image. Surface-based morphometry were used to measure the thickness of EC. Multiple regression analysis was conducted to investigate the relationship between the thickness of EC and social network size and cohesiveness, respectively.

Results: Decreasing trend in cohesiveness was correlated with thinner left EC $(p<0.05)$, while increasing trend or maintained cohesiveness of an individual's social network predicted thicker left EC $(p<0.05)$. The analyses were adjusted for age, sex, education, network size, and depression level in a multiple regression model. Social network size change did not predict left EC thickness.

Conclusions: EC is one of the structures that is vulnerable to the early pathologies of Alzheimer's disease, and thicker EC can be regarded as a brain reserve. Future follow-up studies are necessary to find whether maintaining or increasing cohesive social relationship affects the integrity of the entorhinal cortex or vice versa.

Correspondence: Jeanyung Chey, Ph.D., Psychology, Seoul National University, Gwanakro 1, Seoul 08826, Korea (the Republic of). E-mail: jychey@snu.ac.kr

S. KWAK, W. JOO, Y. YOUM \& J. CHEY. In-bound but not Outbound Social Connection Moderated Age-related Brain Aging in Community-residing Elderly People.

Objective: Socially integrated lifestyle has protective effect in wide range of late-life health outcome especially age-related cognitive decline. Although studies revealed that the size of individual's social network is associated with less cognitive decline, reported effect are not consistent and its neural evidence remains elusive. We aimed to examine how the number of social connection and its direction had affected brain aging. Participants and Methods: Korean Social life, Health, Aging Project recruited 814 elderly dwelling in a Township Y, and administered social network questionnaires, which involved asking the participants to generate the name of people who have regular contact and discuss important concerns with him/her. From this global network, the number of social ties generated by other people and by oneself each represented in-degree centrality and out-degree centrality, respectively. 68 healthy older adults (mean age $=71.38 \pm 6.40$ ) were subsampled and completed a neuroimaging protocol that included T1 and T2 FLAIR. Each image were used to estimate the volume of lateral ventricles and white matter lesion. Bootstrapped Multiple regression model tested whether two age-related neuropathology are moderated by the number of social ties. Results: We found that the elderly who had higher in-degree centrality had less age effect on the ventricular volume enlargement $(b=-235.54$. $95 \%$ CI $[-380.33,-90.75], p=.002)$ and white matter lesion $(b=-0.01$, $95 \%$ CI $[-0.03,-0.01], p<.001)$. In contrast, out-degree centrality did not affect the relationship between age and brain volume, white matter lesion.

Conclusions: The result shows that the older adults who were designated as important discussion member maintained healthier brain as they got older. This discrepant result between in-degree and out-degree social ties may explain inconsistent results from previous studies. More objectively measured social ties from global network could be better indicator of one's social life that are important for healthy brain aging. Correspondence: Jeanyung Chey, Ph.D., Psychology, Seoul National University, Gwanakro 1, Seoul 08826, Korea (the Republic of). E-mail: jychey@snu.ac.kr

\section{WITHDRAWN: H. KIM, S. KWAK, Y. YOUM \& J. CHEY. Social Network Size Moderates the Relationship between Age and Long-term Memory Score in Community-residing Elderly People over 80.}

Objective: Individual difference in the relationship between brain pathology and cognitive function has been well explicated by the cognitive reserve concept. Although education has been repeatedly found to be a meaningful proxy of cognitive reserve, social connectedness as a possible component of cognitive reserve is unclear. We aimed to investigate the effects of individual's social network size and whether it moderates the relationship between age and cognitive performance.

Participants and Methods: 142 healthy elderly participants (mean age $=72.06 \pm 6.62$ ) were administered a neuropsychological battery. Participants' episodic memory was assessed with the Elderly Verbal Learning Test, the Story Recall Test, and the Simple Rey Figure Test. Social network questionnaires quantified the number of people who have regular contact and discuss important concerns with the participants up to 6 people in addition to presence of one's spouse. To examine the buffering effect of social network toward the age-associated cognitive decline, we divided the elderly participants into old-old group $(n=17$, mean age $=82.12)$ and young-old group $(n=125$, mean age $=70.69)$. ANCOVA was used to test the interaction effect between age group and social network size on memory performance.

Results: There was a significant interaction effect between age group and social network size on cognitive performance, $F(2,133=5.833)$, $p=.004$. In old-old group, the larger social network size was correlated with higher long-term memory score. In the young-old group, however, the memory score was not significantly different depending on individuals' network size.

Conclusions: The results showed that social network size mav moderate the effects of cognitive aging in elderly people over 80 ; i.e., larger social network decreased the clinical manifestation of cognitive decline when it is possible that age-related pathology had been accumulated in later years. The possibility of social network size impacting brain health, however, needs to be investigated. 
Correspondence: Jeanyung Chey, Ph.D., Psychology, Seoul National University, Gwanakro 1, Seoul 08826, Korea (the Republic of). E-mail: jychey@snu.ac.kr

A. CHOI, N.A. SCHWAB, J.J. TANNER, L. HIZEL, S.J. CROWLEY, S. LEVY, C. HERNAIZ ALONSO, E. WARNER, H. PARVATANENI, M. RICE \& C.C. PRICE. Preoperative Cognitive Contributions to Cerebral Oximetry Change in Older Adults During Total Knee Arthroplasty.

Objective: Intraoperative cerebral deoxygenation is reported in elderly patients with impaired preoperative cognition before hip fracture surgery and can serve as a potential risk factor for postoperative decline. However, there is little research examining the cause of oxygen desaturation, specifically around the time of tourniquet removal. Tourniquet removal during total knee arthroplasty is relevant to cognitive outcomes, as it is associated with significant biological stress (i.e. release of emboli, altered blood pressure). For this study, we hypothesized that 1) tourniquet removal in older adults would result in a change in cerebral oximetry values and 2 ) preoperative cognitive abilities would predict cerebral oximetry change before and after tourniquet removal.

Participants and Methods: 50 older adults enrolled in an ongoing federally funded investigation of neuroimaging and cognitive predictors of cognitive decline after unilateral knee replacement surgery. All participants completed neuropsychological assessment. All had surgery with the same surgeon and general anesthetic protocol. Cerebral oximetry was measured bilaterally every 30 seconds during surgery.

Results: Around tourniquet release, oximetry values declined by an average of two percent ( 5 min pre $=69.61 \pm 9.89 \% ; 5$ min post $=68.00 \pm 9.86 \%$; change $=-1.53 \pm 1.87 \%$ ). Some individuals, however, showed increased oximetry values. A greater drop in oximetry saturation (pre-post tourniquet removal) was negatively associated with education $(\mathrm{r}=-0.29, \mathrm{p}=0.05)$ and baseline language tests (fluency/confrontation naming; $r=-0.35, p=0.15$ ).

Conclusions: The time of tourniquet removal during knee replacement surgery is an acute biological stressor and a period of vulnerability for the brain in older adults. Baseline cognitive reserve is a predictor for how the brain responds during tourniquet removal. Some older adults showed an adaptive brain response after tourniquet release. R01NR014181 (CP).

Correspondence: Allison Choi, B.S., Clinical and Health Psychology, University of Florida, 2330 SW Williston Rd, \#713, Gainesville, FL 32608, United States. E-mail: allisonchoi@ufl.edu

J. COHEN, G. GROVE, J.C. WATT, M.E. WOLLAM \& K.I. ERICKSON. Physical Activity and Processing Speed Across the Lifespan.

Objective: Physical activity (PA) has beneficial effects on cognitive functioning in older adults; however, less is known about its effects earlier in adulthood. It is unknown whether PA benefits cognitive functioning in younger adults - a group considered in their cognitive prime. Limitations of past research (e.g., self-report PA metrics, limited neuropsychological batteries, using younger participants as cognitive controls without considering PA) motivated the present examination of PA on cognitive functioning in young and older adult samples.

Participants and Methods: In two groups of 171 cognitively healthy adults (younger: 18-40 years; older: 55 + years), we evaluated the effects of PA on processing speed (PS), a cognitive domain particularly susceptible to aging. PA was quantified using accelerometer metrics. PS was quantified with conditions of the Trail Making Test, Stroop Task, and Flanker Test. Ordinary least squares regression models evaluated the relationship between age, PA level, and PS.

Results: After controlling for age, BMI, and education, there was a main effect of age such that older adults were slower than younger adults on the PS tasks. There was also a main effect of PA on several of the PS tasks (all $\mathrm{p}<.05)$, such that individuals with higher levels of PA were faster than those with lower PA. Finally, there were significant Age $\mathbf{x}$ PA interactions for the Stroop Effect, Flanker Interference Score, and
Flanker Congruent condition (all $\mathrm{p} \leq .05$ ), such that the less active, older adults were slower than both their more active counterparts and the younger group.

Conclusions: PA has beneficial effects on PS across adulthood. Although higher levels of PA positively influence performance on PS regardless of age, remaining physically active may benefit older adults more than younger adults.

Correspondence: Jamie Cohen, Psychology, University of Pittsburgh, 210 S. Bouquet Street, 3122 Sennott Square, Pittsburgh, PA 15260, United States.E-mail: jcohe?@gmail.com

T. DASH, L. GHAZI SAIDI, P. BERROIR, D. ADROVER-ROIG \& A.I. ANSALDO. Dual control mechanism in conflict management for the monolinguals and bilinguals: An fMRI study.

Objective: Interference control in the Bilingual population has been studied a lot in the recent years. The Simon task assesses interference control and recruits a combination of brain areas related to the visual and motor processing as well as executive functions. One of the interesting ways to study the interference control is trail-by-trail analysis of the performance. According to Braver (2012), the performance on the slow trials is indicative of the use of reactive control strategy, while performance on faster trials involves proactive control strategy. The present study aims at providing the neural basis of performance for proactive and reactive control strategy in elderly bilinguals and monolinguals.

Participants and Methods: In an event-related fMRI design ( $N=2 * 10$ ), of two groups (monolinguals, bilinguals), three conditions (congruent, incongruent and neutral), two trial type (first 1/3 fastest trials and last $1 / 3$ slowest trials), $t$-test analysis $(K \geq 20, p \leq 0.001)$ was conducted on the contrast images of interest. Image preprocessing was performed using SPM12 following standard procedures.

Results: When comparing slow and fast responses with both groups and all conditions together, the results show that reactive control strategy requires more neural resources for efficient performance as compared to proactive control strategy. Second set of results on the Simon effect $(\mathrm{IC}>\mathrm{N})$ in individual groups for slow and fast trials separately, indicate that Simon effect was present only in fast trials for the bilingual population.

Conclusions: Performance on fast trials is indicative of the use of proactive control strategy (Braver (2012), in elderly bilinguals. This is while; there is evidence that older adults use reactive control strategy for efficient performance (Czernochowski et al, 2010). Thus, life long use of two languages protects the proactive control strategy, which tends to be vulnerable due to cognitive aging.

Correspondence: Tanya Dash, CRIUGM, 5350 Macdonald avenue, Apartment no 1505, Cote St Luc, Montreal, canada, Montreal, QC h3x 3v2, Canada.E-mail: tani.dash@gmail.com

M. DEKHTYAR, R. BUCKLEY, K.V. PAPP, M.R. LAPOINT \& D.M. RENTZ. Imaging, Lifestyle and Demographic Differences in Optimal Executive Function Performers.

Objective: Memory and executive functioning (EF) skills decline with age. What is unclear is whether imaging, lifestyle or demographic factors can distinguish optimal from typical performance in later life and whether these differ by cognitive domain. Our previous findings suggested that optimal memory performers (i.e., those scoring $\geq 0.5 S D$ on a memory composite) exhibited larger hippocampal volumes in comparison with typical performers. Here we investigate differences between optimal and typical performers on EF rather than memory.

Participants and Methods: A total of $n=125$ clinically healthy older adults $\left(M_{\mathrm{axe}}=79.5 \pm 3.5\right)$ from the Harvard Aging Brain study underwent neuropsychological evaluations, neuroimaging, and pedometer-tracked physical activity monitoring. Participants were classified as either optimal $(n=25)$ or typical EF performers $(n=100)$ if scoring above or below the top quintile. Groups were compared using non-parametric Mann-Whitney $U$ independent-group tests. 
Results: Optimal performers exhibited higher IQ $(p<0.001)$, memory (Logical Memory, $p=0.004$ ) and general cognitive performance (MMSE, $p=0.029)$. They had larger total grey matter volumes $(p=0.002)$ and higher levels of aerobic activity $(p=0.024)$. There was a trend toward higher levels of education ( $\mathrm{p}=0.055)$. No group differences occurred in age $(p=0.184)$ or sex $(p=0.210)$.

Conclusions: Optimal EF performers demonstrated both demographic and lifestyle benefits in comparison with their peers. Some of these factors were premorbid (e.g., IQ), suggesting a potential latent advantage but a modifiable marker was also observed (e.g., physical activity). Optimal EF performers may begin with higher cognitive reserve but potentially exceed their typical peers by making an advantageous lifestyle choice.

Correspondence: Maria Dekhtyar, Boston University, 221 Longwood Ave, BL-104, Boston, IL 02115, United States. E-mail: mdekhtyar@ partners.org

K. DENNY, C. BARBA \& S. FARIAS. Long-term change associated with a multi-modal intervention to enhance cognitive compensation strategies and promote brain health activities.

Objective: To evaluate short- and long-term outcomes of an eight-week multi-modal intervention with two target areas aimed at: 1) enhancing cognitive compensation strategies and 2) promoting healthy lifestyles associated with brain health. Three cognitive compensation strategies were designed to promote habitual use of a calendar, use of task lists for achievement of short- and long-term goals, and the development of functional organizational systems at home. Three brain health modules included physical exercise, engagement in cognitive stimulating activities, and enhancing positive outlook (e.g., practicing gratitude).

Participants and Methods: Participants were 33 older adults living independently. They completed a questionnaire that assessed engagement with compensatory skills and brain health activities before and after class completion. A subset of participants $(n=19)$ completed this questionnaire 5-9 months after group completion to assess maintenance of positive changes.

Results: There were significant increases in the use of compensation strategies $(p=0.001)$ and increased brain health activity engagement $(p=0.004)$ following completion of the intervention. Maintenance results indicated a trend $(p=0.06)$ toward continuing to engage in more compensation strategy activities at follow up compared to preintervention levels, but cognitive activity engagement had declined since class completion. Compensation behaviors related to checking calendars (e.g., weekly calendar review, $p=0.007$ ) and utilizing functional organizational zones $(p=0.02)$ were maintained at follow up.

Conclusions: Use of compensation strategies improved in association with a multi-modal intervention and select healthy lifestyle behaviors were maintained at follow up. Results suggest that improving compensation strategy use may be an ideal intervention target to preserve functional independence in older adults.

Correspondence: Katherine Denny, Ph.D., Neurology, University of California at Davis, 4860 Y Street, Suite 3700, Sacramento, CA 95811, United States.E-mail: katie.denny@gmail.com

C. DION, A. KARSTENS, L. ZHAN, J. COHEN, E.A. BOOTS, O. AJILORE, A. LEOW, D.X. MARQUEZ \& M. LAMAR. Associations of Sedentary Behavior as well as Physical Activity with Learning, Memory, and Hippocampal Volume in a Diverse Sample of Older Adults.

Objective: Increases in moderate-to-vigorous physical activity (MVPA) including jogging and swimming, are associated with increases in learning, memory, and related hippocampal volumes. Although $67 \%$ of adults $\geq 60$ years of age average 8.5 waking hours per day of sedentary behavior (SB), less work exists regarding the role of SB on cognition and brain structure.

Participants and Methods: Cross-sectional associations between activity levels, learning, memory, and hippocampal volume were investigated in a diverse sample of non-demented/non-depressed adults ( $\mathrm{N}=70 ; \sim$ age $=67 \pm 6 ; 43 \%$ female; $61 \%$ minority). Self-reported MVPA and moderate-to-vigorous leisure activity (MVLA) including golf and chair exercises were assessed with the CHAMPS Activities Questionnaire for Older Adults. The Sedentary Behavior Questionnaire measured SB. The California Verbal Learning Test-II Trials1-5 total recall measured learning while long delay free recall measured memory. T1-weighted imaging was used to quantify hippocampal volumes adjusted for total intracranial volume via FreeSurfer (QC'd for accuracy). Separate multi-variable linear regressions adjusting for age, sex and education investigated relationships between individual activity levels, learning, memory, and hippocampal volume.

Results: Higher levels of self-reported MVPA were associated with better learning performance $[b=.29, t(65)=2.66, p=.01]$ but not better memory. Neither MVLA nor SB contributed to learning or memory. In contrast, MVPA was not associated with hippocampal volumes but higher levels of self-reported MVLA $[b=-1.72, t(65)=-1.69, p=.09]$ and higher levels of self-reported SB $[b=-.22, t(65)=-2.16, p=.03]$ did associate with lower hippocampal volumes.

Conclusions: This study replicated findings of the positive association between PA and learning in older adults and extended this work to $\mathrm{SB}$, revealing negative associations between SB (as well as MVLA) and hippocampal volumes. Investigating SB in conjunction with PA may provide a more comprehensive view of brain health in older adults. Correspondence: Catherine Dion, BA, Psychiatry, University of Illinois at Chicago, 1601 W. Taylor, MC912, Chicago, IL 60612, United States. E-mail: cdion@psych.uic.edu

R. DIVERS, T. GIOVANNETTI, S.S. RYCROFT, E. ROLL \& J. HULSWIT. When and How Did You Go Wrong? Characterizing Micro-errors in Older Adults.

Objective: Mild functional difficulties arise in normal aging and are present in people with mild cognitive impairment. On performance-based tests, mild difficulties have been reliably measured by coding "micro-errors," operationalized as subtle inefficient actions. The aim of this study was to characterize micro-errors to understand when and why these errors arise in older adults.

Participants and Methods: 48 young adults (ages 18-23), and 25 healthy older adults (ages 60-75) were video recorded while they performed 2 everyday tasks (breakfast; lunch) with target and distractor objects that were presented on a tabletop. Micro-errors were coded from recordings and characterized on three different levels: 1) timing - beginning, middle, or ending segment of the task; between or within sub-steps of the task 2) monitoring - the extent to which the participant made contact with an incorrect object was noted to indicate the severity of a monitoring failure (i.e., reach no touch - mild failure; reach touchmoderate failure; extra action- severe failure) and 3) object properties target object, distractor object). Nonparametric tests were used to examine group differences.

Results: Older adults made significantly more micro-errors during the middle $(Z=2.13, p<.01)$ and ending $(Z-2.90, p<.01)$ task segments and between subtasks $(\mathrm{Z}=3.00, \mathrm{p}<.01)$. Older adults made more micro-errors that reflected egregious monitoring failures (e.g., extra actions; $\mathrm{Z}=3.58, \mathrm{p}<.01)$. Finally, older adults made significantly more micro-errors to both target $(\mathrm{Z}=2.93, \mathrm{p}<.01)$ and distractor objects $(\mathrm{Z}=3.50, \mathrm{p}<.01)$.

Conclusions: Mild functional difficulties in older adults may be due to interference from past steps and from multiple response options that arise between steps as well as poor or slow response monitoring. Interventions (e.g., cues) should be applied toward the end of tasks and at subtask boundaries and should focus on promoting task monitoring. Correspondence: Ross Divers, Psychology, Temple Univeristy, Temple University, Psychology Department, 1701 N 13th St., Philadelphia, PA 19122, United States.E-mail: ross.divers@temple.edu 
K.J. MOORE, A.J. DUGGER, A.J. BOHLEN \& H. DAVIS. Manual and Computerized Trail Making Test Performance across the Lifespan.

Objective: The Trail Making Test (TMT) is one of the most widely used neuropsychological tests and is thought to measure visual search, scanning, mental elasticity, executive functioning/shifting and processing speed. It was originally used as a test of general intelligence and was later used to detect cognitive decline in individuals suffering from traumatic brain injuries. Performance on a computerized and manual version of the TMT will be examined from childhood through old age to determine the validity of a computerized TMT.

Participants and Methods: The TMT consists of two different sections; parts A and B. In part A of the test, the participant must draw a line between a series of numbers in ascending order, and in part B the participant must draw a line alternating between letters and numbers (1-A-2-B-3 etc.). Performance on the TMT is measured by completion time for each trial. Traditionally the TMT is completed using a paperand-pencil measure and is timed with a stopwatch. A paper-and-pencil and computerized TMT were completed with younger participants (YP) ranging in age from $9-24, n=380$ and older participants $(\mathrm{OP})$ ranging in age from 20-89, $n=476$

Results: Computer and paper-and-pencil part A completion times were significantly correlated for both younger and older participants, YP $r=.60, n=380 p<.01$ and OP $r=.56, n=475, p<.01$. Part B completion times were also significantly correlated for both the younger and older participants, YP $r=.66, n=380, p<.01$ and OP $r=.70$, $n=475, p<.01$

Conclusions: These findings provide support for the implementation of a computerized TMT. A computerized TMT has the benefit of ease of administration and increased accuracy of data collection. The results provide the first combined lifespan norms on a computerized and manual TMT. Further, these results provide support the use of a computerized TMT in both older and younger participants.

Correspondence: Amber.J. Dugger, BA, University of Colorado Colorado Springs, Psychology Department, Colorado Springs, CO 80918, United States.E-mail: adugger@uccs.edu

A. GARCIA, E. PORGES, A.J. WOODS, J. REILLY \& R. COHEN. Abstract and Concrete Word Processing in the Aging Brain.

Objective: While the semantic system is well-characterized in younger adults, the effects of healthy aging on the integrity of this system are still unknown. The current study aimed to extend the characterization of the neural network underlying semantic processing to older adults, specifically probing abstract and concrete words.

Participants and Methods: Thirty-eight adults, aged 40-91. completed a semantic association task and a rhyming task while undergoing functional neuroimaging.

Results: Compared to the rhyming task, the semantic task elicited greater activation of the reported semantic system, including hubs of the semantic network (left anterior temporal lobe and bilateral angular gyrus), higher order association cortices (middle temporal gyrus, anterior cingulate cortex, posterior cingulate cortex), and executive control regions (left inferior frontal gyrus [IFG]). As expected, region of interest analyses revealed significantly greater activation of the left IFG during abstract word processing than concrete word processing $(p<.05)$. Age was not a significant covariate for activation during abstract, concrete, or combined abstract + concrete word processing.

Conclusions: We found no evidence that the semantic system is significantly altered in healthy aging. Like younger adults, older adults utilize a distributed set of secondary association cortices and integrative hub regions to process word meaning. Abstract word processing taxed the fronto-executive systems, which is broadly consistent with the notion that abstraction has increased processing demands. The level of activation in these regions was not significantly changed across the age spectrum.
Correspondence: Amanda Garcia, Clinical and Health Psychology, University of Florida, 3461 SW 2nd Ave. \#404, Gainesville, FL 32607, United States.E-mail: amgar@phhp.ufl.edu

A. GARCIA, A. MILLOY, D. LORING \& F.C. GOLDSTEIN. The Relationship between MoCA Cut-Scores and Brain Volume.

Objective: Researchers have attempted to make the Montreal Cognitive Assessment sensitive to conversion from mild cognitive impairment to Alzheimer's disease by introducing memory, executive, visuospatial, and language indices. The current study examined relationships between recommended clinical cutoffs of these index scores and brain volumes of the memory system.

Participants and Methods: The sample included 890 participants (148 AD, 425 amnestic MCI, 317 cognitively normal controls) in ADNI who received the MoCA and MRI brain scans. MoCA scores were converted to index scores (Julayanont et al., 2014). T-tests and Pearson correlations, collapsed across diagnoses, were calculated to determine the relationship between the MoCA index scores and ROIs.

Results: Persons below the clinical cutoff on the memory index (MIS) had significantly $(\mathrm{p}<.05)$ smaller volumes of the hippocampus $(\mathrm{t}=$ -7.1), entorhinal cortex $(t=-6.8)$, fusiform gyrus $(t=-4.9)$, and middle temporal lobe $(t=-6.6)$. This same pattern held for executive, visuospatial, and language indices. When the index scores and brain volumes were correlated separately based upon whether MoCA scores were above or below the recommended cutoff for each index, the MIS positively predicted volumes of all brain regions for participants with scores in the normal range $(p<.05)$; however, the only significant MIS correlation in participants scoring in the impaired range was hippocampal volume $(\mathrm{p}<.05)$. The executive and visuospatial index scores predicted brain volume for all regions in both cutoff groups, while the language score predicted brain volume only in the impaired cutoff group. Findings remained significant when controlling for age.

Conclusions: The index score cutoffs accurately reflect brain volume in memory regions, thus providing support for their clinical application. The MIS is related to volume loss in persons scoring above the cutoff for impairment, highlighting its clinical relevance in at-risk populations. Correspondence: Amanda Garcia, Clinical and Health Psychology, University of Florida, 3461 SW 2nd Ave. \#404, Gainesville, FL 32607, United States.E-mail: amgar@phhp.ufl.edu

M. GOGNIAT, T. ROBINSON, C. MEWBORN, J. DICKENS \& L. MILLER. The Relationship Between BMI and White Matter Volume in Older Adults.

Objective: The current literature is unclear on the relationship between deviations in normal body mass index (BMI) and cognitive function in later life, and there is similarly a need to understand BMI in relation to brain anatomy influences. Thus, the purpose of this study was to determine the relationship between BMI and white matter volume in healthy older adults.

Participants and Methods: Participants were a total of 49 older adults (age 65+) from the surrounding community of a southeastern college town. Membership in BMI classification was conducted as indicated by the standards set by the CDC. Each participant reported height and weight. In this sample, $33 \%$ (16) of participants were considered "normal," 47\% (23) "overweight," 18\% (9) "obese," and 2\% (1) "underweight." Using magnetic resonance imaging (MRI), a priori white matter volume regions of interest (total volume, lateral orbitofrontal, medial orbitofrontal, and parahippocampal) were determined and processed using FreeSurfer. Linear regressions were used to determine the relationship between BMI and each region of interest.

Results: Linear regressions were calculated to predict total white matter volume, lateral orbitofrontal white matter volume, medial orbitofrontal white matter volume, and parahippocampal white matter volume based on BMI. BMI only significantly predicted medial frontotemporal white matter volume $b=70.385, \mathrm{t}=5.902, p=.028$. 
Conclusions: These results suggest that greater BMI in later life may selectively preserve white matter volume in the medial orbitofrontal area, which is important given that decreased volume in medial orbitofrontal white matter in later life has been associated with Alzheimer's disease (Salat et al., 2009). This is a novel finding that will need to be replicated in the future.

Correspondence: Marissa Gogniat, Clinical Psychology, University of Georgia, 120 Mark Twain Cir, Apt J5, Athens, GA 30605-3688, United States.E-mail:mag53440@uga.edu

I. GONZALEZ, G. LEAL, M. RODRIGUEZ, D. LOEWENSTEIN, R. DUARA \& M. GRIEG. Acculturation as an Important Factor in Neuropsychological Performance in patients with Alzheimer's Disease among Hispanic Elderly.

Objective: The purpose of this study was to investigate correlation between acculturation and performance on neuropsychological tests among Hispanic elderly diagnosed with Alzheimer's disease (AD). It was hypothesized that neuropsychological performance would improve, as acculturation levels increase.

Participants and Methods: This study examined archival data. The sample consisted of $161 \mathrm{AD}$ patients with an age mean of 73.17 ( $\mathrm{SD}=6.6)$, and an education mean of 12.37 ( $\mathrm{SD}=3.9)$. Of those subjects, $67 \%$ of the sample was female. Each Hispanic subject was given a comprehensive neuropsychological evaluation that included tests of different domains, such as memory, language, and executive functioning. As part of their assessment, Hispanic subjects completed the Marin Acculturation Scale.

Results: A correlational analysis revealed significant findings between test performance and acculturation level when controlling for age and education. On Logical Memory delayed (LM-II) $(\mathrm{r}=.245, \mathrm{p}<.002)$, Digit Span Backwards $(\mathrm{r}=.282, \mathrm{p}<.000)$, Trails $\mathrm{B}(\mathrm{r}=-.282, \mathrm{p}<.002)$, and Hopkins Delayed Recall (HDR) ( $\mathrm{r}=.222, \mathrm{p}<.005)$.

Conclusions: These results indicate that Hispanic elderly perform higher in neuropsychological assessments as their level of acculturation increases. Interestingly, positive correlations were found between level of acculturation and performance on all measures except Trails B. Trails B measures cognitive switching, something that bilinguals might have an advantage from switching between languages. Overall, results of this study have implications for diagnostic measures in $\mathrm{AD}$. The results caution professionals to consider factors that could affect performance and urge the use of appropriate norms when working with the Hispanic population.

This study was supported by NIA, NIH, Bethesda, MD, Grant \# 1P50 AG025711-05)

Correspondence: Isabel Gonzalez, Doctorate, Psychology, Carlos Albizu University, 901 NE 191 Street, Miami, FL 33179, United States. E-mail: isagongar@gmail.com

E.I. GRACIAN, M.B. AUSTIFF, J.C. CICHON, B. YANG, D.C. OSMON \& K.E. MOSACK. Transverse Patterning Performance is Not Uniform in Cognitively Normal Older Adults.

Objective: Transverse patterning (TP) is a learning and memory adaptation of the 'rock/paper/scissors' problem that depends on the hippocampus, is sensitive to aging, and requires pattern separation (PS) to solve. PS is a mechanism that reduces interference among highly similar memories; age-related impairments in this construct have been linked as possible early indicators of Alzheimer's disease (AD) onset. Previous investigators dichotomized cognitively normal older adults who passed a cognitive screening into impaired-older $(\mathrm{IO})$ and unimpaired-older (UO) subsets, and found that IO adults were disproportionately deficient in PS abilities. Our purpose, then, was to determine if IO adults were inferior on TP than UO adults and young adults.

Participants and Methods: Participants were 30 young adults and 29 older adults who performed within the cognitively normal range on the Montreal Cognitive Assessment (MoCA). All completed a TP task and a standardized neuropsychological battery that included the Hopkins
Verbal Learning Test-Revised (HVLT-R). Older adults were classified as IO $(n=14)$ or $U O(n=15)$ based on performance on the delayed-recall subtest of the HVLT-R; IO adults did not perform within the normal range.

Results: IO adults were inferior on TP compared to both UO adults (Cohen's $d=1.34)$ and young adults $(d=2.53 ; p<.001)$. Also, UO adults were marginally inferior to young adults $(d=.51 ; p=.048)$ on TP. Conclusions: Consistent with the PS literature, and despite passing a cognitive screening, we found TP deficits in IO adults compared to UO adults and young adults. Forthcoming work should determine if TP performance is similar between IO adults and patients with Mild Cognitive Impairment (MCI). Importantly, TP could be a promising tool to detect pathological PS impairment in IO adults that is predictive of impending MCI and $\mathrm{AD}$.

Correspondence: Enrique I. Gracian, MA, Psychology, University of Wisconsin, Milwaukee, 1869 N. Cambridge Ave, Apt 207, Milwaukee, WI 53202, United States.E-mail: gracian2@uwm.edu

L. GRAVES, H.M. HOLDEN, D. DELIS \& P. GILBERT. Modifications to the CVLT-II Novel Recognition Discriminability Measure to Enhance the Detection of Memory Decline in Normal Aging.

Objective: The CVLT-II includes a measure on the yes/no recognition trial called Novel Recognition Discriminability (NRD) that captures the ability to endorse target items and reject novel distractor items (i.e.. items not presented on the List B trial). Novel distractors include: 1) prototypical words semantically related to target items and 2) words semantically unrelated to target items. A potential limitation of this measure is that, for half of the distractors, the novelty of the item is confounded by their strong semantic relationship to the target items. We compared the original NRD formula with two sub-measures (hits vs. prototypical distractors only [NRD Prototypical] and hits vs. unrelated distractors only [NRD Unrelated] in a sample of healthy young and older adults.

Participants and Methods: The CVLT-II was administered to 69 healthy young adults and 40 healthy older adults. Group comparisons on hits, prototypical FPs, unrelated FPs, and original and modified NRD indices were made using independent t-tests. Within-group comparisons on original and modified NRD indices were made using repeated measures ANOVA and post-hoc pairwise comparisons. Bonferroni corrections were applied.

Results: Older adults had significantly lower hits and higher prototypical FPs than young adults $(p s<.01)$, but comparable unrelated FPs $(p=.48)$. Older adults scored significantly lower than young adults on original and modified NRD indices $(p s<.01)$. In older adults, NRD Unrelated scores were significantly higher than original NRD scores $(p<.001)$, which were higher than NRD Prototypical scores $(p<.001)$. In young adults, NRD Unrelated scores were significantly higher than both original NRD and NRD Prototypical scores $(p s<.01)$, which were comparable.

Conclusions: The ability to reject distractors that are novel but share semantic properties with targets may decline with age. The use of modified NRD indices to parse the semantic association of novel distractors with targets may enhance detection of this decline.

Correspondence: Lisa Graves, SDSU/UCSD JDP in Clinical Psychology, 9270 Regents Road, Unit I, La Jolla, CA 92037, United States. E-mail: lvgraves@gmail.com

J.W. GRIFFIN, J. SAURMAN, C. BUSSELl, A. GURNANI \& B.E. GAVETT. The Effects of Age on Clustering Strategy During List Acquisition.

Objective: Semantic and serial clustering are techniques used to aid recall during list-learning tasks. This study's goal was to analyze semantic and serial clustering strategies during the free recall of words among older and younger adults across list acquisition trials. Differences in cluster strategies between older and younger adults may provide insight into normal cognitive aging. 
Participants and Methods: Participants included 79 adults aged 17-36 years and 79 adults aged 54-89 years representing a "younger" and "older" group respectively. Each participant completed four learning trials from the Memory Assessment Scales List Learning test. Semantic and serial (bidirectional) clustering were calculated using chance-adjusted methods, in accordance with the California Verbal Learning Test Second Edition manual for scoring. Mixed effects regression analysis was used to analyze the effects of age, clustering type, and trial (1-4) on number of clusters produced.

Results: The main effect of cluster type revealed serial clustering was used more frequently than semantic clustering regardless of age group $(b=.98, p<.001)$. Moreover, regression analyses indicated that both types of clustering were used more frequently with each successive trial $(b=.54, p<.001)$. Interaction effects revealed a less positive slope for serial compared to semantic clustering across learning trials $(b=-21$, $p<.05)$ for both age groups. No significant age-based interactions were found.

Conclusions: Older and younger adults demonstrated no differences in clustering strategy during list acquisition suggesting that they do not differ in application of such strategies. Although serial clustering was used more frequently, rate of semantic clustering showed a more rapid increase across trials. This latter finding suggests that people may switch from serial to semantic clustering strategies with increasing exposure to the list. Further research should examine these trends in strategy usage among clinical samples, such as those with Alzheimer's disease.

Correspondence: Jason W. Griffin, B.A., Psyhology, University of Colorado, Colorado Springs, 6850 Sproul Ln, Colorado Springs, CO 80918, United States. E-mail: jgriff12@uccs.edu

E.Z. GROSS, A.A.M. RAHMAN-FILIPIAK, L.J. FICKER \& P. LICHTENBERG. Poor Financial Decisional Ability is Associated with Elder Financial Exploitation and is Exacerbated among Older Adults with Higher Frequencies of Low Neuropsychological Test Scores.

Objective: Poor financial decisional abilities (FDA) and declining cognitive ability are known risk factors for FE, but the relative importance of each and their relationship is not well understood. The present study compared rates of FE by FDA and neuropsychological performance. Participants and Methods: Participants $(\mathbf{N}=200$; mean age $=71.5$, $\mathrm{SD}=7.4$ ) completed the Lichtenberg Financial Decision-Making Rating Scale (LFDRS), a clinician-rated measure of FDA for a current or recent personal decision, including self-reported FE, as well as a neuropsychological battery. Two criteria based on SDs away from the sample mean in the impaired direction were used to classify the frequency of low scores (12 possible): Mild Impairment (MI) $=2$ scores $\geq 2$ SD, and Subtle Impairment $(\mathrm{SI})=4$ scores $\geq 1 \mathrm{SD}$.

Results: Poor FDA and low cognitive scores were relatively rare overall $(\mathrm{FDA}=16[8 \%] ; \mathrm{MI}=16[8 \%] ; \mathrm{SI}=37[18.5 \%])$. The rate of $\mathrm{FE}$ was similar to national prevalence rates $(\mathrm{FE}=36[18 \%])$. FE rates were higher among people with poor FDA $(\mathrm{N}=10[62.5 \%])$ and people who scored low on neuropsychological tests using MI criteria ( $\mathrm{N}=6$ $[37.5 \%])$ and SI criteria $(\mathrm{N}=12[32 \%])$. The highest rate of $\mathrm{FE}$ was among people with both poor FDA and SI ( $\mathrm{N}=8[72 \%])$, while the rate of FE among people with FDA and MI was similar to the rate of FDA alone $(\mathrm{N}=3[60 \%])$. Among people with intact FDA, the rate of FE was not elevated for people who met SI criteria $(N=4[15.4 \%])$ and was somewhat higher for people who met MI criteria ( $\mathrm{N}=3[27 \%])$. Conclusions: The interaction between poor FDA and poor cognition is associated with the highest risk for FE. Poor FDA alone is also associated with a greatly increased risk for FE, but poor cognition alone is not. Life experiences that contribute to FDA may protect against FE despite cognitive deficits. Alternatively, the LFDRS may be more sensitive than neuropsychological tests to cognitive deficits associated with FE. Correspondence: Evan Z. Gross, B.A., Psychology, Wayne State University, 21546 Gregory Street, Dearborn, MI 48214, United States. E-mail:evan.gross@wayne.edu
C.C. HAYS, Z.Z. ZLATAR, L.M. CAMPBELL, M. MELOY \& C.E. WIERENGA. Relationship Between Pulse Wave Velocity, Cerebral Blood Flow, and Memory in Cognitively Normal Older Adults.

Objective: Cerebral blood flow (CBF), an indirect measure of neural function, is associated with cognitive performance and has been implicated in age-related cognitive decline. While the underlying mechanisms of these age-related changes are not well-understood, evidence suggests that peripheral vascular changes such as increased arterial stiffness, measured by pulse wave velocity (PWV), may contribute to hypoperfusion. These findings implicate vascular dysfunction in the etiology of age-related cognitive decline and suggest that CBF and/or PWV may serve as underlying mechanisms of this change, yet few studies have examined the association between PWV and CBF and their relationship with cognitive function.

Participants and Methods: A robust regression model was employed to examine the voxel-wise relationship between PWV and arterial spin labeling (ASL) MRI-measured CBF among a group of 22 cognitively normal older adults (mean age $=72$ ). A verbal memory composite score was derived by averaging $z$-scores for subtests of the CVLT-2 and WMS-R Logical Memory. Linear regression models were used to determine if $\mathrm{CBF}$ within regions that were associated with $\mathrm{PWV}$ was related to verbal memory performance.

Results: Preliminary results demonstrate negative associations between PWV and CBF in the left middle frontal gyrus, right inferior temporal gyrus, and the left hippocampus and parahippocampal gyrus (all p's $<0.01$, corrected) and significant associations between CBF in the left middle frontal gyrus $(B=4.7 ; p=.013)$ and the left hippocampus and parahippocampal gyrus $(B=3.9 ; p=.047$, trend level) and memory performance.

Conclusions: Findings suggest that greater arterial stiffness is associated with reduced $\mathrm{CBF}$ in regions implicated in aging, memory, and $\mathrm{AD}$ risk, and that lower CBF in regions showing associations with PWV is related to poorer memory performance. Longitudinal studies are needed to determine whether changes in PWV precede changes in $\mathrm{CBF}$ and whether the relationship between $\mathrm{CBF}$ and cognition might be mediated by PWV

Correspondence: Chelsea C. Hays, M.S., University of California San Diego, 3148 Hawthorn st APT B, San Diego, CA 92104, United States. E-mail: chelseahays@gmail.com

C.C. HAYS, Z.Z. ZLATAR, L.M. CAMPBELL, M. MELOY \& C.E. WIERENGA. Subjective Cognitive Decline Modifies the Relationship Between Cerebral Blood Flow and Memory Function in Cognitively Normal Older Adults.

Objective: Subjective cognitive decline (SCD), or self-reported cognitive decline despite normal neuropsychological test performance, is a risk factor for objective cognitive decline and Alzheimer's disease (AD). While brain mechanisms contributing to SCD are not well defined, studies show associations with vascular risk factors and altered cerebral blood flow (CBF), suggesting that those with SCD might be experiencing vascular dysregulation, or a disruption in the normal relationship between $\mathrm{CBF}$ and cognition. We examined whether the association between $\mathrm{CBF}$ and verbal memory differs between those with SCD (SCD+) and those without SCD (SCD-).

Participants and Methods: SCD+ was defined as at least one endorsement of memory deterioration and no memory improvements using the Subjective Memory Rating Scale. A verbal memory composite score was derived by averaging $z$-scores for subtests of the CVLT-2 and WMS-R Logical Memory. Linear mixed effect models were employed to investigate whether the voxel-wise relationship between arterial spin labeling (ASL) MRI-measured CBF and verbal memory performance is modified by SCD among a group of 70 cognitively normal older adults (35 SCD+, 35 SCD-; mean age=72) matched on age, gender, and depressive symptoms.

Results: Results indicated that the SCD- group exhibited positive associations between verbal memory and CBF in posterior cingulate cortex, 
middle temporal gyrus, and inferior frontal gyrus, whereas the SCD+ group displayed negative associations between verbal memory and CBF in posterior cingulate cortex, middle temporal gyrus, hippocampus, fusiform gyrus, and inferior frontal gyrus.

Conclusions: Findings suggest that while higher CBF is supportive of memory function in those without SCD, higher CBF may no longer support memory function in those presenting with SCD, perhaps reflecting neurovascular dysregulation associated with aging and $\mathrm{AD}$ risk.

Correspondence: Chelsea C. Hays, M.S., University of California San Diego, 3148 Hawthorn st APT B, San Diego, CA 92104, United States. E-mail: chelseahays@gmail.com

\section{J.K. HO \& D.A. NATION. Memory is Preserved in Older Adults Taking AT1-Receptor Blockers.}

Objective: Prior work suggests some but not all antihypertensive treatments may benefit cognition and risk for Alzheimer's disease, independent of stroke. Angiotensin II type 1 receptor blockers (ARBs) have been highlighted as one antihypertensive drug class that may confer greatest benefit.

Participants and Methods: Participants were 1,626 non-demented adults, ages 55-91 recruited from Alzheimer's Disease Neuroimaging Initiative sites. Three groups were compared: ARB-users (HTN-ARBs), other-antihypertensive-drug-users (HTN-Other) and Normotensives. Groups were compared on cognition, and MRI measures of brain volume and white matter lesions, using repeated measures ANCOVA.

Results: At baseline, the HTN-Other group performed worse than Normotensives on Immediate Recall ( $p=.002)$, Delayed Recall $(p<.001)$, and Recognition memory $(p=.001)$, and Trails $\mathrm{A}(p<.001)$ and $\mathrm{B}$ $(p=.01)$. ARB-users performed better than the HTN-Other group on Recognition memory $(p=.04)$ and worse than Normotensives on Trails A $(p=.04)$. The HTN-Other group remained worse than Normotensives on AVLT Delayed Recall over 3-year follow-up ( $p=.01)$. At baseline and 3-year follow-up, the HTN-Other group exhibited greater white matter lesion volume than Normotensives $(p=.031)$ and ARB-users ( $p=.046$, over 3 -year follow-up). There were no group differences in brain volume.

Conclusions: Hypertensive participants demonstrated worse baseline memory and executive function, and greater memory decline over 3 year follow up compared to normotensives, unless they were ARB-users, who showed better memory than those on other drugs. Other antihypertensive drug users showed more white matter disease than normotensives and ARB-users over 3-year follow-up. Findings suggest ARBs may benefit cognition over other antihypertensive medications, possibly due to stymieing white matter disease.

Correspondence: Jean K. Ho, USC, 7428 Hollywood Blvd Apt 307, Los Angeles, CA 90046, United States.E-mail: jeanho@usc.edu

R. JONES, E. LIMA, C. NORSETH, Y. XU, A. ARECHIGA, S. RAJARAM \& J. SABATÉ. Predictors and the Moderating Effect of General Health and Neuropsychological Performance.

Objective: The current study examines predictors and moderators of general health and its effects on neuropsychological performance in healthy older adults.

Participants and Methods: This study included 365 healthy older adults. Variables included the Health SF-12 survey, the Psychosocial Factor survey, Trails A/B, Rey Auditory Verbal Learning Test (RAVLT), Symbol Digits Modality Test (SDMT), and Controlled Oral Word Association Test (COWAT).

Results: Results revealed that general health was significantly predicted by self-perceived memory complaints $(\beta=-.260, p<.001$, CI $[-.254$, $-.101])$ and spiritual well being $(\beta=-.293, p<.001$, CI $[-.046,-.023])$. General Health significantly predicted cognitive performance on Trails B $(\beta=.164, p<.05$, CI $[3.73,16.45])$ and SDMT $(\beta=-.136, p<.05$, CI $[-2.806,-.381])$. Results indicated social support significantly moderated the relationship between general health and neuropsychological performance on the SDMT $\left(F(3,348)=4.38, \mathrm{p}<.05, R^{2}=.03\right)$. Other results indicated that social support did not significantly moderate neuropsychological performance on Trails A/B, RAVLT, and COWAT (ps $>.05)$.

Conclusions: Results suggest that increased general health complaints may be a possible sign of future cognitive decline, specifically in attention, visual scanning, and motor speed. Proper assessment of health complaints in addition to psychosocial factors such as social support may help reduce the negative impact on cognitive functions and possibly assist in the future detection of dementia. However, future research is needed before assessment of these predictors is used in clinical settings. Correspondence: Rochelle Jones, PsyD, Psychology, Loma Linda University, 25590 Prospect Ave 45B, Loma Linda, CA 92354, United States.E-mail: ryjones@llu.edu

A.R. KAUP, F. XIA, L.J. LAUNER, S. SIDNEY, I. NASRALLAH, C. DAVATZIKOS, N. ALLEN \& K. YAFFE. Occupational Cognitive Complexity is Associated with Brain Structure and Cognitive Health in Mid-Life: The CARDIA Study.

Objective: Occupational cognitive complexity may reduce risk for cognitive decline in aging. How it affects brain structure and whether it exerts influence in mid-life is unclear. We investigated whether occupational cognitive complexity in early to mid-adulthood is associated with brain structure and cognitive health in mid-life.

Participants and Methods: Participants were 677 adults from the Coronary Artery Risk Development in Young Adults (CARDIA) cohort study (ages 18-30 at baseline, $52 \%$ female, $39 \%$ black). We calculated Occupational Cognitive Requirements scores (OCRS) using participants' Census Occupation Codes (Years 10 and 15) and Occupational Information Network data; OCRS reflect the cognitive complexity of participants' most demanding occupation. At Year 25, participants had structural brain MRI, diffusion tensor imaging, and cognitive testing [Digit Symbol, Stroop, and Rey Auditory Verbal Learning Test (RAVLT)]. In regression models, we examined the association between OCRS, gray matter volume, fractional anisotropy (FA), and cognition. Adjusted models included demographics, education, site, intracranial volume, hypertension, diabetes, depressive symptoms, alcohol use, and smoking.

Results: Higher OCRS was associated with higher total FA $(\beta=.08$, $p=.045$ in fully adjusted model), with similar results for frontal, temporal, and occipital FA. OCRS was not associated with total or lobar gray matter volume (all $p>.05$ ). Higher OCRS was associated with better cognitive performance (Digit Symbol $\beta=.15, p<.001$; Stroop: $\beta=.09, p=.03$; RAVLT Long Delay Free Recall: $\beta=.07$, $p=.06$ in fully adjusted models).

Conclusions: Occupational cognitive complexity in early to mid-adulthood is associated with better white matter integrity $(\mathrm{FA})$ and cognitive function in mid-life, possibly promoting cognitive reserve. Whether and how these associations help protect the aging brain into late life remains to be determined.

Correspondence: Allison R. Kaup, PhD, Research / Psychiatry, San Francisco VA Healthcare System / University of California San Francisco, 4150 Clement St, 116B, San Francisco, CA 94121, United States.E-mail: allison.kaup@ucsf.edu

S. KAUR, T. TARUMI, M. GONZALES, M. PYRON, S. OLESON, C. CASSILL, H. TANAKA \& A. HALEY. Inflammation mediates the relationship between metabolic (cholesterol) risk and neuronal viability in middle aged adults.

Objective: High cholesterol is associated with elevated cardiovascular risk and increased risk of dementia in older adults. In particular, oxidized low density lipoprotein (oxLDL) has been highlighted as a salient marker of future cognitive decline. However, the mechanisms underlying this relationship are poorly understood. We aimed to determine how oxLDL affects the brain at midlife by examining concentrations of key cerebral metabolites localized in the occiparietal grey 
matter. As oxLDL is also associated with increased neuroinflammation, we further hypothesized that changes in concentrations of cerebral metabolites would be driven by changes in levels of C-Reactive Protein (CRP), a useful peripheral marker of inflammation.

Participants and Methods: Fasting blood samples were obtained from 60 participants aged $40-60$ years (mean $\pm \mathrm{SD}=52.3 \pm 5.6)$. Participants also underwent structural magnetic resonance imaging, ${ }^{1} \mathrm{H}$ Magnetic Resonance Spectroscopy ( ${ }^{1} \mathrm{H}$ MRS) and neuropsychological assessment. CRP and oxLDL levels were assessed with enzyme linked immunosorbent assays (ELISA). Statistical mediation was assessed using traditional causal steps and nonparametric bootstrapping.

Results: Higher oxLDL was significantly associated with lower concentrations of $\mathrm{N}$ Acetyl Aspartate $(\mathrm{NAA})(\mathrm{b}=-0.29, \mathrm{p}=0.03)$ and lower levels of circulating CRP $(b=-0.37, p=0.03)$. Linear regression and bootstrapping methods indicated that CRP fully mediated the relationship between oxLDL and NAA $(b=0.60,95 \% \mathrm{CI}=-3.79--0.26)$.

Conclusions: The relationship between higher oxLDL and NAA was fully statistically mediated by circulating CRP levels. Inflammation is thus a useful probable mechanism through which executive function decline occurs in individuals with high cholesterol. Inflammation reducing interventions (physical exercise, dietary restriction, nonsteroidal anti inflammatory drug treatment) could thus potentially be utilized to reverse changes in NAA in individuals with high metabolic risk.

Correspondence: Sonya Kaur, M.A., Psychology, University of Texas Austin, 1437 Woodward Avenue, Detroit, MI 48226, United States. E-mail:sonya_kaur@utexas.edu

\section{Y. KIM \& Y. KANG. Emotional Information Processing in Older Adults with Depression: Attention and Memory Biases.}

Objective: Depressed young adults (YA) showed the "negative bias" to pay more attention to the negative information and remember it consistent with their current mood. However, the emotional information processing in depressed older adults $(\mathrm{OA})$ is far from clear yet. The $\mathrm{OA}$ generally showed the "positivity effect" which refers the OA is more likely to process the positive information than the negative ones. Then, do the depressed $\mathrm{OA}$ would show the negative bias even with positivity effect? This study was conducted to investigate the effect of depressive mood in the emotional information processing of the Korean OA.

Participants and Methods: A total of 51 YA (age $=21.43 \pm 1.40 ; 26$ normal, 25 depressed) and $51 \mathrm{OA}$ (age $=68.86 \pm 2.22 ; 25$ normal, 26 depressed) participated in the study. The subjects were classified into normal and depression groups based on the CES-D. All the subjects completed the Health Screening Questionnaire and the Korean MiniMental State Examination. To measure attention and memory biases, computer-administered dot probe task and recognition test that consisted of neutral and emotionally charged faces and words were administered. Results: The overall $\mathrm{OA}$ had a significantly greater attention and memory biases for positive stimuli than negative or neutral ones, whereas the YA showed the negative bias in attention and memory. The $\mathrm{OA}$ also were more likely to disengage attention from negative stimuli. However, the depressed $\mathrm{OA}$ as well as depressed YA had a significantly greater attentional bias for negative stimuli compared to positive or neutral ones. The depressed OA also showed a greater memory bias for negative words, although they did not show any memory biases for either neutral or emotionally charged faces.

Conclusions: These findings suggest that like the depressed YA, the depressed OA have negative emotional information processing bias in attention and memory, although affective optimization is a universal characteristic in the overall OA despite sociocultural differences.

Correspondence: Yujin Kim, Department of Psychology, Hallym University, 1 Hallymdaehakgil, Chuncheon, Gangwondo 24252, Korea (the Republic of).E-mail: sapvogue@naver.com

\section{WITHDRAWN: G.J. KINSELLA, B. ONG \& K. PIKE. Memory} Groups for Older People: Who Gains?

Objective: Interventions to moderate memory difficulties experienced by older people, especially for mild cognitive impairment (MCI), continue to be evaluated. In a recent randomised trial (Kinsella et al, 2015), we demonstrated that a memory group (LaTCH) improved prospective memory for healthy older adults and people with MCI. In this study, we aimed to identify baseline characteristics that predict those participants most likely to gain from intervention.

Participants and Methods: 113 healthy older adult (HOA) and 106 adults with MCI were randomly allocated to a memory group or a waitlist control group, and by six-month follow-up all participants received intervention. High Gainers achieved an increase from baseline prospective memory (CAMPROMPT) of at least $0.8 S D$ (i.e., a large effect) by follow-up. Hierarchical logistic regressions evaluated if baseline demographic, cognitive reserve or readiness for change variables predicted High Gainers vs. Low Gainers. We used a bootstrapping approach to estimate effect size for predictors.

Results: $53(47 \%)$ HOA and 45 (42\%) MCI were determined as High Gainers. Using bootstrap sampling, separate logistic regressions for $\mathrm{HOA}$ and MCI groups demonstrated that demographic and readiness for change variables did not contribute to the model. However, the odds of being a High Gainer significantly increased for better baseline working memory (Digit Span backwards - 1.58 times for HOA, 1.62 times for MCI).

Conclusions: Basic demographics (age, gender, education) and readiness for change are no barriers to gaining benefits from cognitive intervention for memory changes in older age. However, better working memory provides an indicator of those who will gain more from memory groups.

Correspondence: Glynda J. Kinsella, PhD, Psychology, La Trobe University, La Trobe University Road, Plenty Road, Melbourne, VIC 3086, Australia. E-mail: g:kinsella@latrobe.edu.au

G. LEAL, I. GONZALEZ, M. RODRIGUEZ, D. LOEWENSTEIN, R. DUARA \& M. GRIEG. The Relationship Between Brain Atrophy and Cognitive Performance in Dementia Populations.

Objective: Relationships between brain atrophy and cognitive performance among individuals with Mild Cognitive Impairment (MCI) and Alzheimer's disease (AD) were examined.

Participants and Methods: Participants included an MCI group ( $\mathrm{n}=42$; mean age $=76.71, \mathrm{SD}=6.34$; mean education $=13.69, \mathrm{SD}=3.36$ ), and an AD group (n=40; mean age $=77.83, \mathrm{SD}=6.24$; mean education=13.17, $\mathrm{SD}=3.93)$. Mini-Mental State Examination (MMSE), Logical Memory II (LMII), Hopkins Verbal Learning Delayed Recall (HVLT-DR), Visual Reproductions II (VRII), Boston Naming Test (BNT), Trail-Making Test B (TMT-B), Digit-Span Backward (DSB), Category Fluency (CF), and volumetric analysis of medial temporal areas were examined.

Results: Partial correlations controlling for age and education revealed significant relationships among MCI between the right entorhinal volume $(\mathrm{REV})$ and CF $[\mathrm{r}(42)=.345, \mathrm{p}<.05]$, HVLT-DR $[\mathrm{r}(42)=.384$, $\mathrm{p}<.05]$, and VRII $[\mathrm{r}(42)=.347, \mathrm{p}<.05]$; right hippocampus $(\mathrm{RH})$ and $\mathrm{CF}[\mathrm{r}(42)=.348, \mathrm{p}<.05]$; right inferior lateral ventricle (RILV) and BNT $[\mathrm{r}(42)=-.407, \mathrm{p}<.05]$; and left inferior lateral ventricle (LILV) and BNT $[\mathrm{r}(42)=-.327, \mathrm{p}<.05]$. Significant relationships in AD included the MMSE and RILV $[\mathrm{r}(40)=.375, \mathrm{p}<.05]$, left lateral ventricle $(\mathrm{LLV})$ $[\mathrm{r}(40)=.415, \mathrm{p}<.05]$, and right lateral ventricle (RLV) $[\mathrm{r}(40)=.388$, $\mathrm{p}<.05]$; left hippocampus (LH) and LMII $[\mathrm{r}(40)=.374, \mathrm{p}<.05]$, and VRII $[\mathrm{r}(40)=.423, \mathrm{p}<.05]$; left entorhinal volume $(\mathrm{LEV})$ and VRII $[\mathrm{r}(40)=.428 ; \mathrm{p}<.05], \mathrm{RH}[\mathrm{r}(40)=.465, \mathrm{p}<.05]$ and VRII; and REV and VRII $[\mathrm{r}(40)=.437, \mathrm{p}<.05]$.

Conclusions: Results indicated that episodic and visual memory performance are sensitive to medial temporal atrophy in both MCI and AD. Measures of confrontational naming, semantic fluency and rote memory appear to be sensitive to brain atrophy in MCI only.

This study was supported by NIA, NIH, Bethesda, MD, Grant \# 1P50 AG025711-05) 
Correspondence: Giselle Leal, PsyD in Clinical Psychology, Psychology, Carlos Albizu University, 3131 ne 188th St., \#2909, Miami, FL 33180, United States. E-mail: giselle_leal@icloud.com

G. LEAL, I. GONZALEZ, M. RODRIGUEZ, D. LOEWENSTEIN, R. DUARA \& M. GRIEG. The Relationship Between White Matter Hyperintensities and Cognitive Performance in Dementia Populations.

Objective: This study examined the relationship between white matter hyperintensities (WMH) and performance on cognitive measures of memory, attention, and language

Participants and Methods: Eighty-two subjects were in included in this study. An MCI group $(n=42)$ had a mean age of 76.71 $(\mathrm{SD}=6.34)$, and mean education of $13.69(\mathrm{SD}=3.36)$. The $\mathrm{AD}$ group $(n=40)$ had a mean age of $77.83(\mathrm{SD}=6.24)$ and mean education of 13.17 (SD= 3.93). Mini-Mental State Examination (MMSE), Logical Memory II (LMII), Hopkins Verbal Learning Delayed Recall (HVLTDR), Visual Reproductions II (VRII), Boston Naming Test (BNT), TrailMaking Test B (TMT-B), Digit-Span Backward (DSB), and Category Fluency (CF) were examined. A measure of left and right total WMH was examined.

Results: A partial correlation analysis was conducted to clarify the relationship between WMH and performance on neuropsychological measures, controlling for age and education. A significant relationship was found among MCI patients between left WMH and LMII $[\mathrm{r}(41)=.382, \mathrm{p}<.05]$, left WMH and VRII $[\mathrm{r}(41)=-.328, \mathrm{p}<.05]$, right WMH and LMII $[\mathrm{r}(41)=.339, \mathrm{p}<.05]$, and right $\mathrm{WMH}$ and VRII $[\mathrm{r}(41)=.-424, \mathrm{p}<.05]$. Among AD, there was a significant relationship between left WMH and MMSE $[\mathrm{r}(41)=.483, \mathrm{p}<.05]$, and right $\mathrm{WMH}$ and MMSE $[\mathrm{r}(41)=.480, \mathrm{p}<.05]$.

Conclusions: Significant findings indicated that measures of episodic memory and visual memory are sensitive to WMH in both right and left hemisphere. Overall cognitive functioning, as measured by a brief cognitive screener, is related to bilateral WMH.

This study was supported by NIA, NIH, Bethesda, MD, Grant \# 1P50 AG025711-05)

Correspondence: Giselle Leal, PsyD in Clinical Psychology, Psychology, Carlos Albizu University, 3131 ne 188th St., \#2909, Miami, FL 33180, United States.E-mail: giselle_leal@icloud.com

S. LEVY, T.G. HILL-JARRETT, C. STRICKLAND-HUGHES, I. SHPITSER, J. WEUVE, J. MARDEN, L.B. ZAHODNE \& J.J. MANLY. Mechanisms of Racial Disparities in Cognitive Aging: Use of Causal Mediation in Neuropsychology Research.

Objective: The mechanisms that contribute to racial disparities in late life cognition remain poorly understood, in part because potential mediators are not measured and confounds are often unspecified. We hypothesized that use of modern causal mediation methods would reveal (1) significant racial disparities in memory mediated through childhood/adult socioeconomic status (SES) and adult cardiovascular disease (CVD) and (2) non-significant direct effects of race (the inequality in cognitive function remaining after accounting for mediators).

Participants and Methods: Participants included 3,255 older adults who self-identified as African American ( $\mathrm{n}=1,825)$ or non-Hispanic white $(\mathrm{n}=1,430)$ from the Washington Heights Inwood and Columbia Aging Project. Neuropsychological tests measured language, visuospatial skills, processing speed, and memory at baseline and 18-24 month follow-ups. We estimated the direct effects of race on memory at baseline and memory trajectory, and the indirect effects of race through childhood SES (composite of parents' education, education quality, occupation, and rural residence), adult SES (composite of education, income, and occupation), and CVD burden (count of hypertension, stroke, diabetes, and heart disease). We controlled for the confounding effects of age and sex.

Results: African Americans had significantly lower memory scores than Whites $(\beta=-5.97, p<.001)$. The significant indirect effects of race through childhood SES $(\beta=.03, p<.001)$ and adult SES $(\beta=.02$, $\mathrm{p}=.008)$, but not CVD burden $(\beta=-.04, \mathrm{p}=.09)$ completely accounted for direct-effect racial disparities.

Conclusions: Results suggest that a complete reduction of racial disparities in cognitive aging would be achieved if African Americans and Whites had equivalent SES across the life course. Although CVD was more prevalent among African Americans and those with low SES, and was associated with lower cognitive function, CVD was not an independent mediator of disparities.

Correspondence: Shellie-Anne Levy, Ph.D., Clinical and Health Psychology, University of Florida, 4872 NW 42nd Rd, Apt. 104, Gainesville, FL 32606, United States. E-mail: stlevy@phhp.ufl.edu

S.W. LIEBEL, E.S. HALLOWELL, A. OSHRI, E.C. JONES, J. GUNSTAD, R. COHEN \& L.H. SWEET. Executive Functioning Ability is Better Predicted by Cognitive Processing Speed than by White Matter Hyperintensities or Age.

Objective: Older adults are expected to experience age-associated neurocognitive decline in the absence of obvious disease or trauma. These neurocognitive impairments have been directly associated with compromised white matter integrity, and they include poorer performance on assessments of cognitive processing speed (CPS) and executive functions (EF). The present study examined age, CPS, and white matter hyperintensities (WMH) to determine which best predicts EF.

Participants and Methods: A community sample of 47 healthy older adults ( $M$ age $=62.30 ; S D=8.22)$ completed a neuropsychological and MRI assessment. A composite score for CPS and EF was formed with unit-weighted z-scores of constituent tests to provide stable measures of ability in these domains. WMH load was quantified with an automated segmentation technique using high-resolution whole-brain T1-weighted and FLAIR images and corrected for intracranial volume. A stepwise multiple regression model was built to evaluate the incremental predictive utility of age, CPS, and WMH load on EF ability.

Results: A significant regression equation was found in which CPS best predicted EF $\left(R^{2}=.42, \mathrm{~F}[1,45]=32.39, p<.001\right)$. After controlling the variance associated with age and $\mathrm{WMH}$ load, the incremental predictive validity of CPS $\left(\Delta R^{2}=.16, p=.001\right)$ was significant. Although all variables were significantly correlated with $\mathrm{EF}$, neither age $(p=.14)$ nor WMH load $(p=.37)$ significantly improved the predictive value of the model after CPS was entered.

Conclusions: CPS is a fundamental neurocognitive function necessary for EF. The accurate assessment of CPS alone may be particularly useful as an efficient, cost effective, and sensitive marker of EF. It is also a potential alternative to neuroimaging assessments to predict EF-related brain dysfunction.

Correspondence: Spencer W. Liebel, Psychology, University of Georgia, 144 Jolly Lane, Athens, GA 30606,United States.E-mail:swliebel@ uga.edu

L.C. MACE, S.T. MOELTER \& D.A. WOLK. The Serial Position Effect and Hippocampal Asymmetry in Cognitively Normal Older Adults.

Objective: To investigate the predictive value of a measure of the serial position effect in Cognitively Normal (CN) older adults with MRI and cerebrospinal fluid (CSF) biomarkers of Alzheimer's disease (AD).

Participants and Methods: Data used in the preparation of this abstract was obtained with permission from the Alzheimer's Disease Neuroimaging Initiative (ADNI) database (adni.loni.usc.edu). Data from $70 \mathrm{CN}$ participants from the ADNI cohort was examined. Word recall across five learning trials of the Rey Auditory Verbal Learning Test (RAVLT) was coded using a primacy-weighted measure of word recall - retention-weighted scoring (RWS). Using this measure, higher scores are associated with greater recall from primacy list regions. We used a dichotomized scoring technique computed by subtracting left from right hippocampal volumes such that a positive score indicated a smaller left hippocampus. Participants were further categorized into 
CSF pathological and non-pathological groups as per (Shaw et al., 2009 ) using $\mathrm{A} \beta_{42}$ CSF concentrations less than $192 \mathrm{pg} / \mathrm{ml}$ as the cut point for inclusion in the pathological CSF group. A binary logistic regression was conducted to assess whether RWS and/or CSF grouping predicted hippocampal asymmetry $(\mathrm{L}</>\mathrm{R})$, when age and intracranial volume were controlled.

Results: A test of the full model against a constant only model was not statistically significant nor was CSF pathology a significant predictor of hippocampal asymmetry. Lower RWS however, did predict hippocampal asymmetry $(\mathrm{R}>\mathrm{L})$ at a trend level, $p=.06$.

Conclusions: Our findings suggest RWS may have merit in detecting subtle cognitive changes related to preclinical stages of AD including hippocampal asymmetry and lateralization of function, which may change dynamically according to disease progression.

Correspondence: Lauren C. Mace, Master's of Science, Behavioral and Social Sciences, University of the Sciences, 2452 tulip street, Philadelphia, PA 19125, United States.E-mail: laurenmace@gmail.com

J.E. MAYE, L.C. BUTTERFIELD, E. TRIFILIO, P. MANGAL, D. BOWERS \& M. MARSISKE. Dispositional Mindfulness as a Predictor of Verbal Memory Performance in Older Adulthood.

Objective: A growing body of research suggests that mindfulness meditation training may strengthen cognitive performance in younger and even older adults. Existing trait mindfulness (TM), or a disposition toward "non-elaborative, nonjudgmental, present-centered awareness" (Bishop et al., 2004), on the other hand, is inconsistently linked with cognitive performance in college-aged adults and has never been evaluated specifically in older adults. Controlling for emotion and mood factors related to TM, the current study examined associations between TM and several age-vulnerable cognitive domains in a sample of older adults.

Participants and Methods: Participants (N=56; Age 77.4 +/- 8.1) were cognitively normal non-meditation practitioners. Standardized neuropsychological assessments and self-reported emotion/mood and mindfulness instruments were administered. Simple and multiple linear regressions examined associations between TM (Cognitive and Affective Mindfulness Scale-Revised) and each of three separate neurocognitive domain composites calculated from individual neuropsychological measures (Sheline et al. 2006). These three domains were chosen based on previous work suggesting their enhancement in long-term meditators (Lykins et al., 2012; Valentine et al., 1999).

Results: Preliminary bivariate analyses showed TM to be negatively correlated with depression, trait anxiety, and apathy $(\mathrm{r}=-.260$ to -.591 ; $\mathrm{p}<.05)$. As a single independent variable, TM positively predicted delayed episodic (verbal) memory $(R=.271 ; p<.05)$, but not basic attention or executive functioning. The relationship between TM and delayed memory remained salient after controlling for demographics (age, sex, education) and the aforementioned emotion/mood factors $(p<.05)$.

Conclusions: Greater TM appears to be associated with better memory performance in older age, even after considering emotion and mood factors. Given that late life is a time of risk for memory decline, future research should consider possible improvement or maintenance of TM in older age.

Correspondence: Jacqueline E. Maye, M.S., Clinical Psychology, University of Florida, 201 SE 2nd Avenue, STE 325, Gainesville, FL 32601, United States.E-mail: jmaye@phhp.ufl.edu

\section{E.P. MELTZER, J.C. BOROD \& L. RABIN. Emotion Regulation in Relation to Aging and the Preclinical Stages of Dementia.}

Objective: Emotion regulation (ER) is essential for effective functioning in daily life. Research suggests that ER improves in older adulthood despite concomitant declines in cognition and the presumed neural substrates of ER. However, the current understanding of relationships among ER, age, and cognitive difficulties in older adulthood is limited. The current study investigated ER strategy use (adaptive vs. maladaptive) in relation to age and clinical status.
Participants and Methods: Participants were 179 community-dwelling, non-demented older adults from the Einstein Aging Study in the Bronx, New York $(M$ age $=81.4 \pm 5.8$, range $=70-98$ years; $69.3 \%$ female), including (healthy controls [HC, 42\%], subjective cognitive decline [SCD, 37\%], and mild cognitive impairment [MCI, 21\%]). Participants completed two self-report measures of ER strategy use, the Emotion Regulation Questionnaire (ERQ; Gross \& John, 2003) and the Cognitive Emotion Regulation Questionnaire (CERQ; Garnefski, Kraaij, \&. Spinhoven, 2001). Pearson product-moment or Spearman rank-order correlations were utilized to examine associations between age and ERQ and CERQ scores, and analyses of variance were conducted to examine differences in ER strategy use as a function of participant group (i.e., HC, SCD, \& MCI).

Results: Age (in years) was significantly and inversely related to use of adaptive ER strategies on the ERQ $(p=0.020)$ and CERQ $(p=0.008)$. HC participants endorsed greater adaptive ER strategy use than did SCD participants on the CERQ ( $p=0.051)$, and MCI participants endorsed significantly greater maladaptive ER strategy use on the CERQ than did HC $(p=0.009)$ and SCD participants $(p=0.001)$.

Conclusions: Our results provide initial evidence that adaptive ER strategy use declines with advancing age, but not markedly so, and in relation to cognitive difficulties. We discuss the clinical implications of our findings in terms of working psychotherapeutically with older adults who have mild cognitive difficulties.

Correspondence: Erica P. Meltzer, CUNY Graduate Center, 86 Larch Drive, Manhasset Hills, NY 11040, United States.E-mail: epm234@ gmail.com

\section{R. MORIN \& E. MIDLARSKY. Depression and Cognitive Functioning Among Older Adults with Cancer.}

Objective: The proportion of the United States population in older adulthood is growing rapidly, and with that growth comes an increase in diseases such as cancer. As rates of illness increase, there is a concomitant increase in cognitive and psychological correlates of cancer, including depressive symptomatology. Depressive symptoms among older adults often have cognitive sequelae. It is therefore vital to disentangle the relationships among these variables.

Participants and Methods: This study sampled 403 older adults from the Health and Retirement Study who had been diagnosed with cancer. Using latent class growth analysis (LCGA), longitudinal data (prediagnosis to 4 years later) were explored. The goals were to investigate possible trajectories of cognitive functioning (immediate and delayed recall for verbal stimuli), as well as to identify whether depressive symptoms and demographic factors predicted membership in these cognitive classes.

Results: Findings indicated that three classes of cognitive functioning best fit the data: these were High Recall, Middle Recall and Low Recall classes, which represented fairly stable trajectories from pre-diagnosis to a period four years later. Further, higher numbers of depressive symptoms after diagnosis (but not prior to diagnosis) were significantly predictive of membership in the Low Recall class. Depressive symptoms did not distinguish membership likelihood between the High and Middle Recall classes at any time point.

Conclusions: Depressive symptomatology is generally thought to affect cognition in late life. We found that depressive symptoms after a cancer diagnosis, but not before, successfully differentiated between those who had low recall cognition from those with middle and high degrees of recall. Clinical and theoretical implications of this finding are discussed. Correspondence: Ruth Morin, PhD, Clinical Psychology, Teachers College, Columbia University, 327 Bedford Avenue Apt. F5, Brooklyn, NY 11211, United States. E-mail: ruth.t.morin@gmail.com 
S.A. MOSELEY, H. RITCHIE, J. SHEHORN, A. ROSADOMUELLER, E.L. GLISKY \& N. MARRONE. Cognitive and Psychosocial Associations of Hearing Loss in Older Adults.

Objective: Cognitive and psychosocial consequences of age-related hearing loss continue to surface in hearing science and psychology literature. The aim of this study was to further explore these relations using a comprehensive battery of hearing and listening, cognitive, and self-report psychosocial measures.

Participants and Methods: Twenty-nine healthy, community-dwelling older adults (age range $=65-87$ ) with a range of hearing ability completed a battery of tests. Hearing and listening abilities were assessed using pure-tone audiometry, word recognition, sentence recognition in quiet and in the presence of background noise, and self-report questionnaires. Cognitive tests included measures of memory, working memory, executive functioning, and language. Self-report measures assessed social engagement, loneliness, health and mood.

Results: The relations between hearing loss and cognition controlling for the effects of age were explored with first-order partial correlations. Measures of hearing and listening were significantly associated with measures of executive functioning and vocabulary $(r$-values ranging from .36 to $.59, p$ 's <.05), with complex listening measures showing the most robust associations with cognition. Hearing and listening measures were also significantly associated with loneliness, disengagement from conversation, depression, and various subscales of a health survey ( $r$-values ranging from .38 to $.68, p$ 's <.05). Self-reported difficulty hearing speech was strongly related to all psychosocial measures $(r$-values ranging from .48 to $.69, p$ 's $<.05)$.

Conclusions: The present study found that hearing loss was associated with poorer performance on various measures of cognition and lower self-reported psychosocial functioning. The consequences of age-related hearing loss extend beyond difficulties in communication and warrant further scientific investigation as our population ages.

Correspondence: Suzanne A. Moseley, MA, Psychology, University of Arizona, 1503 E University Blvd, Tucson, AZ 85721, United States. E-mail: smoseley@email.arizona.edu

\section{S. PILLEMER \& R. HOLTZER. The Effect of Perceived Social Support on Cognitive Function Among Older Adults.}

Objective: The current study examined whether four empirically validated dimensions of perceived social support, emotional/informational, tangible, affectionate, and positive social interaction, were associated with increased risk of incident cognitive impairment and whether this association was moderated by gender.

Participants and Methods: The sample consisted of 493 non-demented older adults (mean age $=76.58 \mathrm{yrs}$; $\%$ female $=57.2$ ) enrolled in a longitudinal cohort study. Support was assessed using the Medical Outcomes Study-Social Support Survey. Cognitive impairment was defined as 1 standard deviation below the mean on the Repeatable Battery for the Assessment of Neuropsychological Status (RBANS) total score. Cox proportional-hazard models were used to assess the risk of incident cognitive impairment.

Results: Higher levels of tangible support (hazard ratio (HR), 1.748) affectionate support (HR, 1.870), positive social interaction (HR, 1.696), and overall support (HR, 2.059) were associated with increased risk of incident cognitive impairment on the RBANS. Further analyses revealed a moderation effect of gender. Specifically higher scores on tangible support (HR, 1.907), positive social interaction (HR, 2.222), and overall support (HR, 2.738) were associated with increased risk of incident cognitive impairment only in men.

Conclusions: Men who seek more support may do it in order to compensate for abilities or skills that are gradually being lost. Hence, greater perceived support may be a risk factor for overall cognitive decline. These findings could potentially be used in assessment and intervention procedures for older men at risk of cognitive decline.
Correspondence: Sarah Pillemer, Ferkauf Graduate School of Psychology, 15 Whiting Street, Apt 3, Providence, RI 02906, United States.E-mail:sarah.pillemer@gmail.com

\section{G. POTTER. Physical Frailty and Cognitive Impairment in Late-} Life Depression.

Objective: Neuropsychological deficits during late-life depression (LLD) often improve with symptom remission; however, some individuals have persistent cognitive impairment (PCI). Because PCI is a risk factor for cognitive and functional decline in LLD, it is important to discover clinical features that predict this condition and serve as a point of intervention. One feature may be physical frailty, defined as decline across multiple physiological systems leading to decreased reserve against stressors and increased vulnerability to adverse outcomes. While physical frailty is an important syndrome in geriatric medicine, it is less recognized in geriatric neuropsychology. Our objective was to examine the association of physical frailty to PCI among individuals with LLD, and test mediation by vascular brain pathology.

Participants and Methods: Participants were in a prospective treatment study of LLD and age 60+ at study enrollment. Participants completed annual neuropsychological assessment and consensus review to assign cognitive diagnoses. MRI was obtained at study entry, as was physical frailty, defined by: 1) fatigue, 2) slowness, 3) appetite/weight loss, 4) weakness, and 5) illness comorbidity. We compared individuals with diagnoses of cognitively normal vs. PCI over 5 years, including only those remitted from depression. Vascular brain pathology was defined by white matter lesion volume on MRI.

Results: Physically frail individuals had worse neuropsychological performance at enrollment than nonfrail, particularly among executive functions. Individuals with depression and physical frailty at enrollment had significantly higher risk of PCI compared to nonfrail, which was partially mediated by white matter lesion volume.

Conclusions: Physical frailty, which can be reliably measured by questionnaire, has value for identifying individuals with LLD at risk of PCI, and may signal underlying vascular brain pathology. Findings suggest poor physical resilience may be linked to poor cognitive resilience in the context of LLD.

Correspondence: Guy Potter, PhD, Psychiatry, Duke University Medical Center, 2200 West Main Street, Suite A-200, Durham, NC 27705, United States. E-mail: guy.potter@duke.edu

\section{E. RHODES \& T. GIOVANNETTI. Grit and Successful Aging in Older Adults.}

Objective: Grit is a noncognitive trait that has been shown to increase monotonically throughout adulthood and predict late-life cognitive performance. Less is known about the relation between grit and successful aging in older adults.

Participants and Methods: Participants over 55-years-old $(\mathrm{n}=98)$ completed a series of self-report surveys assessing demographics, grit (12-item Grit Scale), physical and emotional functioning (SF-36), and age-related changes in cognitive functioning (ECog). Multiple linear regressions were used to assess the relations between grit, age, and measures of successful aging. Bivariate Pearson correlations were used to assess relations between grit and various components of successful aging. Results: Grit significantly predicted emotional quality of life, $\beta=0.35, t(97)=4.46, p>0.001, R^{2}=0.21$, and age-related changes in cognitive functioning, $\beta=-0.33, t(97)=-3.22, p=0.002$. $R^{2}=0.11$. Grit was positively associated with better emotional wellbeing $(r=0.37, p>0.001)$, energy $(r=0.41, p>0.001)$, social functioning $(r=0.29, p=0.007)$, and reduced limitations due to emotional problems $(r=0.36, p>0.001)$. There was no significant association between grit and age or years of education.

Conclusions: Grit is a previously unexplored factor that shows strong relations with well-validated measures of successful aging. Grit may serve as a protective factor that promotes active adaptation to 
the developmental challenges of aging. Interventions to develop grit throughout the lifespan may improve quality of life in the elderly. Correspondence: Emma Rhodes, MA, Psychology, Temple University, 1701 N 13th Street, Department of Psychology - Temple University, Philadelphia, PA 19122, United States. E-mail: emma.rhodes@temple. edu

H. RITCHIE, A. POLSINELLI, S.A. MOSELEY \& E.L. GLISKY. Cognitive and Emotional Associations of Dispositional Mindfulness in Older Adults.

Objective: Mindfulness is a multi-faceted construct that is associated with cognitive and emotional benefits in young adults and long-term meditators but is rarely studied in older adults. Additionally, few studies have attempted to validate its multi-faceted nature by demonstrating the specificity of facets for predicting behavior. The Five Facet Mindfulness Questionnaire (FFMQ) measures these different facets, including Acting with Awareness (AwA), Observe, Describe, Nonjudgment, and Nonreactivity. The aim of this study was to examine the (a) cognitive and emotional associations of dispositional mindfulness in older adults and (b) multi-faceted nature of mindfulness.

Participants and Methods: Sixty healthy non-meditating older adults $\left(\mathrm{M}_{\text {age }}=75.8, \mathrm{SD}_{\text {age }}=5.6\right)$ completed measures of cognition (attention, updating, inhibition, switching, verbal intelligence, everyday cognitive errors), a measure of emotion regulation, and the FFMQ. We used separate hierarchical regressions to examine whether specific subscales of the FFMQ predicted these domains of cognitive and emotional functioning (while controlling for age, education, and other facets of the FFMQ).

Results: Hierarchical regressions showed higher AwA was associated with better updating and attentional performance, and fewer every day cognitive errors. Similarly, higher Nonjudgment was moderately associated with better emotion regulation. In contrast to these positive associations, higher Observe was associated with more everyday cognitive errors and worse switching performance.

Conclusions: Higher dispositional mindfulness was generally associated with better cognitive and emotional functioning in older adults, although higher scores on Observe suggested poorer functioning. Furthermore, we found some evidence suggesting facets of the FFMQ uniquely predict domains of cognitive and emotional functioning, lending support to the hypothesized multi-dimensional nature of mindfulness.

Correspondence: Hannah Ritchie, Psychology, University of Arizona, 6639 E Broadway Blvd. Apt 106, Tucson, AZ 85710, United States. E-mail:hannahritchie@email.arizona.edu

R. ROBBINS, E.L. GLISKY \& M. MEHL. Relation Between Social Interaction and Cognitive Functioning in Older Adults: A Feasibility Study Using the EAR Technology.

Objective: In older adults higher levels of social engagement are associated with better cognitive function as measured by intelligence tests, executive functions and memory functioning. Research suggests that it is the quality of interactions instead of the quantity that impacts cognition. Participants and Methods: This pilot study used the Electronically Activated Recorder technology to explore the relation between frontal/ executive and memory functioning and social interaction in older adults as measured by type of conversation (substantive talk or small talk) spoken by the participant while with others. The EAR technology was used to collect objective measures of social interaction by recording participants' daily conversations. Participants included 7 females and 3 males with a mean age of 74.5 years (Range $=67-80)$; 17 years of education (Range=14-19); 7 lived alone and 3 lived with a spouse. Neuropsychological tests measured executive functions and memory functioning.

Results: Although small numbers precluded significant findings, a Pearson partial correlation coefficient suggested a positive correlation between memory functioning and substantive talk with others after controlling for living situation (living with a spouse or living alone), $r(8)=.56, n . s$. Results suggested a positive correlation between frontal functioning and percentage of substantive talk with others, $r(10)=.61$, n.s., controlled for living situation. Specifically, the executive function of updating in working memory appears to be correlated with percentage of substantive talk with others, $\mathrm{r}(10)=.41$, n.s.

Conclusions: Preliminary findings from this pilot study suggest that social interaction, in the form of substantive talk, is positively associated with frontal functioning and more specifically, updating, in older adults. Correspondence: Ruth Robbins, Psychology, University of Arizona, 3742 East4th Street, Tucson, AZ 85716, United States. E-mail: ruthrobbins@ email.Arizona.edu

\section{S. ROGERS. Cognition and Older Adults' Agreeableness: Is There a Relationship?}

Objective: Only recent research has started exploring the role of personality as a predictive factor for cognitive performance. This study aimed to determine how specific areas of cognitive functioning vary with the degree of agreeableness in older adults.

Participants and Methods: 79 older adults (59 women, $M$ age $=77$ years, $M$ education $=16$ years ) completed a neuropsychological battery that included measures from the WAIS-IV, WMS-IV, CVLT-II, DKEFS, COWAT, Trails A \& B, BNT, and the ROCF. Participants also completed the NEO-FFI to assess their degree of agreeableness.

Results: Bivariate correlation analyses revealed significant positive relationships between older adults' level of agreeableness and their performance on WAIS-IV Coding, Trails A \& B, DKEFS Color Naming, WMS-IV Logical Memory, and WMS-IV Visual Reproductionm, all $p \mathrm{~s}$ $\leq .05$. When divided into classification categories, those with very low levels of agreeableness performed significantly worse than all other levels of agreeableness on Trails A, Trails B, and RCFT 3' and 30' delays, all $p s<.05$. They also scored worse on DKEFS Color Naming than those with average or high levels of agreeableness, and they performed worse on WMS-IV Visual Reproduction than those with average, high, or very high levels of agreeableness, all $p s<.05$.

Conclusions: The cognitive abilities of older adults seem to significantly vary with their level of agreeableness. Those who are disagreeable experience worse visual processing speed, divided attention, verbal and nonverbal learning, and figure copying relative to those who value getting along with others. The disproportionate benefit in nonverbal abilities suggests the possibility of a right hemisphere preference for agreeableness. These findings suggests that developing skills in agreeableness may promote cognitive health, whereas disagreeableness may contribute to reduced cognitive functioning. Those who are more agreeable may also simply try to perform better in testing environments than those who may be disadvantaged by skepticism.

Correspondence: Steve Rogers, Ph.D., Psychology, Westmont College, 955 La Paz Road, Santa Barbara, CA 93108, United States. E-mail: sarogers@westmont.edu

\section{L.J. ROTBLATT, A. HORGAS \& M. MARSISKE. Effects of} Hypertension and its Pharmacological Treatment in the ACTIVE Study.

Objective: While the negative effects of hypertension on cognition have been well documented, the current study aimed to investigate whether pharmacological treatment of hypertension might moderate these effects, and whether such moderation might vary by cognitive domain. Participants and Methods: Participants were 2802 older adults, aged $65-96$ (75.9\% female), free of frank dementia (MMSE $>23)$ who completed the baseline assessment of ACTIVE Clinical Trial. In addition to cognitive data, participants provided medication audit data. The current study coded the number of antihypertensive and non-cardiovascular medications. The four dependent variables were Reasoning (Word Series, Letter Series and Letter Sets tests), Processing Speed (four subtasks of the Useful Field of View test), Immediate Verbal Memory (HVLT, AVLT and RBMT Paragraph Recall) and the Digit Symbol Substitution Test. 
Results: For all outcomes age, gender, and education were statistically controlled in Block One of a four-block hierarchical regression. Block 2 , representing objectively and subjectively measured hypertension, explained poorer functioning $(\mathrm{p}<.001)$ for our Reasoning composite and for the Digit Symbol Substitution Test. Block 3, representing the number of antihypertensive medications (controlling for overall polypharmacy), explained poorer functioning $(\mathrm{p}<.05)$ in all cognitive domains assessed. In Block 4, medications did not moderate the effects of hypertension for any cognitive outcome; multiple Rs for the final four block models ranged from .422 to .522 .

Conclusions: The results replicate negative effects of hypertension on multiple cognitive outcomes. Antihypertensive treatment apparently served as an indicator of either greater disease severity, ineffective disease management, or iatrogenic effects of medication. Future research will examine compounding risk associated with other indicators of cardiovascular risk and metabolic syndrome, and will also examine the effect of hypertension on ten-year cognitive change trajectories in ACTIVE.

Correspondence: Lindsay J. Rotblatt, Clinical and Health Psychology, University of Florida, 321 SE 3rd Street, E14, Gainesville, FL 32601, United States.E-mail:Irotblatt@ufl.edu

S.S. RYCROFT, T. GIOVANNETTI, J. REILLY, T.F. SHIPLEY, J. HULSWIT \& R. DIVERS. Effects of Age and Task Goal on Naturalistic Visual Behaviors.

Objective: Subtle functional changes occur in normal and pathological aging, but little is known about these changes and their predictive value. This study used a novel task to examine eye movements in healthy older and younger adults while viewing everyday objects during passive and goal-driven behavior. We expected more efficient fixation patterns in goal-driven viewing and less efficient patterns in older adults.

Participants and Methods: 26 young adults (ages 18-22) and 24 non-demented older adults (ages 60-75) viewed everyday objects under two instructions manipulating goal-driven visual behaviors: 1) simply view and 2 ) view while verbalizing steps to complete a complex everyday task (pack a lunch). An SMI RED-m remote eye tracker was used and fixations and dwell times on target and irrelevant areas were coded. Repeated-measures ANOVAs were conducted for effects of group and instruction on visual patterns. Correlations between eye-tracking and self-report and neuropsychological measures were examined.

Results: There was a significant effect of instruction on target $(F=116.22$, $p<0.001)$ and distractor $(F=158.04 ; p<0.001)$ fixations, with increased target and decreased distractor fixations during goal-driven viewing. Further, young adults increased in target dwell times more than older adults from the passive to the goal-driven task $(F=10.88, p=0.002)$. Significant relations emerged between eye-tracking variables and a self-report measure of functioning and tests of executive function.

Conclusions: Eye movements became more directed and efficient when viewing objects with a task goal, and time viewing targets in the goal condition may be useful as a sensitive indicator of functional changes with age. Eye-tracking variables were further validated by relations with self-report, and correlations suggest that executive function is related to the complex eye movement patterns that occur with naturalistic vision. Eye tracking is thus a promising tool for detecting and characterizing risk for decline in the earliest possible stage.

Correspondence: Sarah S. Rycroft, Psychology, Temple University, 1701 N. 13th Street, 6th Floor Weiss Hall, Philadelphia, PA 19122, United States.E-mail:sarah.seligman@temple.edu

C.L. SANDERS, S. BEHRENS, J. TSCHANZ, J. MATYI, M. DECKER, R. HOVEY \& M. NORTON. Risk of Cardiovascular Disease and Cognitive Status in Middle-aged Adults: The Gray Matters Study. Objective: To investigate the relationship between cardiovascular disease (CVD) risk and cognitive decline in middle-aged adults.

Participants and Methods: 146 persons (66\% female) who volunteered for a randomized controlled pilot study of a healthy lifestyle application were studied for 6 months. Mean (sd) age was 54.2 (6.9), with $77 \%$ completing a bachelor's degree or higher. All measures were administered at baseline and 6-months. Ten-year risk of experiencing a CVD event was determined using a risk calculator based on demographic and health indicators from the 2013 guidelines of the American College of Cardiology and American Heart Association Task Force. A score $>=7.5 \%$ was considered higher risk. Cognitive status was assessed by the Montreal Cognitive Assessment (MOCA), Rey Auditory Verbal Learning Test (RAVLT), Multilingual Aphasia Examination (MAE), and the following NIH toolbox measures: List Sorting Working Memory, Flanker Inhibitory Control, and Picture Vocabulary. Linear mixed effects models examined the association between CVD score (time-varying) and cognitive measures. Covariates tested were age, sex, education, and BMI.

Results: Baseline CVD score [Mean (sd)] $=3.3 \%$ (3.1); CVD scores increased by an average of . $3 \%(p<.01)$ for all subjects over 6 months. Low CVD score $(<7.5 \%)$ was associated with higher scores on the MOCA $(b=.7, p=.06)$ and RAVLT sum of trials $(b=3.7)$, recognition errors $(\mathrm{b}=-3.0), \&$ interference $(\mathrm{b}=.8)$, all $p<.05$, with the inclusion of covariates. CVD score was not significantly associated with other test scores nor rate of change on any tests.

Conclusions: Individuals at greater risk of cardiovascular disease have, on average, worse scores on tests of global cognitive functioning and episodic (verbal) memory, even in this sample of subjects with relatively low risk scores. Modifiable lifestyle factors that reduce CVD risk may be a target for intervention to promote cognitive functioning in mid-to-late life.

Correspondence: Chelsea L. Sanders, Bachelors, Psychology, Utah State University, 74 West 300 South, Logan, UT 84321, United States. E-mail: chels.laurae@gmail.com

\section{J. BEAVER \& M. SCHMITTER-EDGECOMBE. Multiple Types of Memory and Everyday Functional Assessment in Community Dwelling Older Adults.}

Objective: Current proxy measures for assessing everyday functioning (e.g., questionnaires, performance-based measures, direct observation) show discrepancies in their rating of functional status. To identify potential sources of discrepancy, the present study investigated the predictive role of content memory (i.e., memory for information), temporal order memory, and prospective memory on multiple proxy measures of functional status in an older adult sample.

Participants and Methods: A total of 197 community-dwelling older adults who both met $(n=45)$ and did not meet $(n=152)$ criteria for mild cognitive impairment (MCI), completed six different assessments of functional status (two questionnaires, two performance-based tasks, two direct observation tasks) as well as measures of content memory, prospective memory, and temporal order memory. Hierarchical linear multiple regression analyses were used to determine which measures of memory predicted each of the six outcome measures of functional ability.

Results: Content memory and prospective memory were found to significantly predict all measures of functional status $(p$ 's $<.05)$, while temporal order memory was significantly predictive of questionnaire and direct observation measures ( $p$ 's $<.05)$, but not performance-based measures.

Conclusions: These results suggest that direct observation and questionnaire measures may be able to capture components of everyday functioning that require context and temporal sequencing abilities, such as multi-tasking, not well captured in many current laboratory performance-based measures of functional status. Future research should aim to inform the development and use of maximally effective and valid proxy measures of functional ability.

Correspondence: Maureen Schmitter-Edgecombe, Ph.D., Psychology, Washington State University, Department of Psychology, Washington State University, Pullman, WA 99164-4820, United States. E-mail: schmitter-e@wsu.edu 
C. SCHOEN \& R. HOLTZER. Impact of Fall-Related Psychological and Physiological Factors on Dual-Task Performance in Older Adults.

Objective: The current study examined the moderating effects of fall-related psychological and physiological factors on dual-task (DT) performance and task prioritization strategy in a cohort of non-demented. community-dwelling older adults (OAs).

Participants and Methods: A cross-sectional sample of 512 OAs provided information regarding fall-related psychological factors including fear of falling $(\mathrm{FoF})$ and balance confidence using the Activities-specific Balance Confidence (ABC) Scale. These individuals also completed a mobility questionnaire assessing fall history and a standardized assessment of balance performance. Participants' gait speed was calculated for both normal and walking-while-talking (WWT) conditions on an instrumented walkway. Cognitive performance was measured by accuracy on a Serial 7s subtraction task for both single and WWT conditions.

Results: Adjusted linear mixed effects models revealed that participants with high-risk psychological factors (i.e., FoF, low BC, or combined FoF/low BC) demonstrated smaller DT costs in gait speed compared to those without these risk factors. Similarly, participants with highrisk physiological factors (i.e., falls, low balance, or combined falls/ low balance) demonstrated smaller DT costs in gait speed compared to those without.

Conclusions: Consistent with the posture-first hypothesis, results suggest that $\mathrm{OAs}$ with these specific fall-related risk factors may be more likely to prioritize mobility over a cognitively demanding interference task in order to maintain safety and postural stability under complex DT conditions. With a more complete understanding of the factors influencing attention and mobility functioning in aging, clinicians may be better able to develop effective screening tools and intervention strategies for fall prevention in community and rehabilitation settings. Correspondence: Chelsea Schoen, M.A., Psychology, Ferkauf Graduate School of Psychology, Yeshiva University, 200 Rector Place, Apt 4C, NY, NY 10280,United States.E-mail: chelseaschoen@gmail.com

\section{T. SEIDER, E. PORGES, A.J. WOODS \& R. COHEN. Age-Related Changes in Visual Discrimination.}

Objective: To characterize age-related changes in visual perceptual, spatial, and velocity discrimination using the Visual Assessment Battery (VAB) (Swearer \& Kane 1996). Prior research demonstrated age-related response time increases and, to a lesser extent, accuracy decreases, for a wide variety of visual discrimination tasks. The current study expands upon this research by including more elderly adults and more precisely measuring form and velocity perception with updated tasks that were piloted on the target demographic.

Participants and Methods: The sample consists of forty older adults (age $\geq 50$ years) who did not meet criteria for mild cognitive impairment or dementia. The task includes three simultaneous match-to-sample paradigms that were identical aside from the type of visual skill tested: discrimination of location, form, and velocity. Each task has three systematically varied levels of difficulty. Additional visual tasks include Benton's Judgment of Line Orientation (JOLO) and Facial Recognition Test (FRT) to independently assess perceptual and spatial ability for validation of the $V A B$

Results: Preliminary data indicated age-related slowing across all VAB tasks, with the degree of slowing varying as a function of task difficulty and type (e.g., greater slowing for velocity discrimination, less for location). Age-related decreases in accuracy were less widespread, but existed for the most difficult level of some tasks. The current study provides normative data on the effects of age, task difficulty, and task type on response times and accuracy. Validity estimates compare location discrimination with JOLO and form discrimination with FRT.

Conclusions: With advanced age, processing speed declines across visual functions. Accuracy is more stable with increasing age. The VAB provides a potentially useful clinical tool to comprehensively assess higher visual function.
Correspondence: Talia Seider, University of California, San Francisco, 2004 Mowry Rd., Gainesville, FL 32610,United States.E-mail: tseider@ phhp.ufl.edu

M. SOTO-AÑARI, G. CÁCERES, N. LÓPEZ \& S.F. GUINEA. The Influence of Cognitive Reserve, Reading Level and Processing Speed on Executive Control Ability in Peruvian Healthy Older Adults. Objective: Executive control modulates many of the neuropsychological characteristics observed in aging, but in turn, it is influenced by factors linked to education as reading level, and basic cognitive mechanisms such as processing speed. Reading level has been proposed as a cognitive reserve measure in elderly people without formal education. The aim of this study was to analyze the influence of cognitive reserve, reading level and processing speed, over cognitive performance and executive control components in Peruvian elderly people.

Participants and Methods: 94 elderly participants, 73 women (mean age: 70.86) and 21 males (mean age: 75.05), were assessed by a comprehensive neuropsychology battery including reading level task, speed of processing test, and the domains of cognitive flexibility, working memory, accessing long-term memory and inhibitory control. Results: We used structural equation modeling to obtain latent variables of executive control. Our analysis showed best fitting model with two separate factors: working memory and accessing long-term memory. However, latent variable cognitive flexibility showed best fitting only if inhibitory control was included. Moreover, we note that the level of reading and processing speed have a significant effect on cognitive flexibility, accessing long-term memory and working memory.

Conclusions: These results show that executive control exerts a modulatory effect on cognitive performance operating like a cognitive reserve mechanism. They also demonstrated the importance of formal education and processing speed in order to explain cognitive performance variability observed in Peruvian healthy elderly people. Correspondence: Marcio Soto-Añari, Ph.D., Universidad Católica San Pablo, Arequipa, Peru.E-mail:msoto@ucsp.edu.pe

A.R. STABLER \& J. MORRIS. Sleep Disturbance Severity is Associated with Earlier Self-Reported Onset of Cognitive Decline Among Older Adults.

Objective: Sleep quality impacts cognitive functioning across the lifespan. (Kronholm et al., 2009). Older adults are more likely to experience both cognitive deterioration and sleep difficulties (Keage et al., 2012). The present study seeks to better understand sleep and cognitive functioning among elderly by investigating the extent to which severity of sleep disturbance is associated with age of cognitive decline onset in a large older adult cohort.

Participants and Methods: Archival data from 5,568 older adult (>60 years) participants was selected from the National Alzheimer's Coordinating Center's Uniform Data Set (NACG-UDS; Weintraub et al., $2009)$, with a sample average of $76.9(\mathrm{SD}=8.6)$ years of age, and 14.4 $(\mathrm{SD}=3.8)$ years of education. Gender distribution was $53.5 \%$ male. Participants estimated the age at which they began experiencing cognitive decline. Sleep disturbance severity data was obtained from answers to sleep-related questions on the Neuropsychiatric Inventory Questionnaire. Analyses involved a correlation of sleep disturbance severity and age of decline onset, followed by the same correlation while controlling for years of education.

Results: Average reported age of cognitive decline onset was 70.7 $(\mathrm{SD}=9.2)$ years. Of the full sample, $48.4 \%$ reported mild sleep disturbance, $35.1 \%$ moderate, and $16.5 \%$ severe. Sleep disturbance severity and reported age of cognitive decline onset were significantly negatively correlated, $r(5,586)=-.038, p=.005$. After controlling for years of education, this significant negative correlation remained, $r(5,586)=-.044, p=.001$

Conclusions: Older adults with more severe sleep problems may experience an earlier onset of decline in cognition. Sleep difficulties among elderly may be related to various psychological, medical, or lifestyle 
factors that need further exploration. Investigation of the specific cognitive problems arising among these earlier decliners with increased sleep difficulties is necessary for developing more effective cognitive decline screening methods.

Correspondence: Anthony R. Stabler, Doctoral, Roosevelt University, 1445 W Augusta Blvd., Chicago, IL 60642, United States. E-mail: astabler@mail.roosevelt.edu

L. STRAINGE, K. GETTENS, K.J. MANNING, R.F. KAPLAN, D. WAKEFIELD, W. WHITE \& L. WOLFSON. Body Mass Index and Executive Functioning in a Longitudinal Study of Healthy Elderly.

Objective: Vascular risk factors in middle age, including hypertension and obesity, are associated with impaired executive functioning. However, preliminary evidence suggests the relationship between weight and cognition among older persons (> 65 years old) may be reversed. The current investigation examined baseline body mass index (BMI) as a predictor of executive function (EF) in a 4-year study of healthy elderly. Participants and Methods: 97 participants between the ages of 75 and 90 years were evaluated at baseline and four years. BMI was calculated from participants' baseline height and weight. EF was assessed using the Trail Making Test Part B and the Stroop Color-Word Test. Hierarchical regression analyses predicting follow-up EF scores from baseline BMI controlled for age, education, estimate of IQ, and baseline performance on respective EF tests.

Results: At baseline, $44 \%$ of participants were overweight (BMI between 25 and $30 \mathrm{~kg} / \mathrm{m}^{2}$ ), and an additional $16 \%$ were obese (BMI $>30 \mathrm{~kg} / \mathrm{m}^{2}$ ). The remaining $40 \%$ of participants had BMIs $<25 \mathrm{~kg} / \mathrm{m}^{2}$. Performance on both tests of EF differed significantly by baseline BMI category (Trails B: $F=4.531, p=0.016$; Stroop: $F=5.163$, $\mathrm{p}=0.009$ ), with better performance in the overweight and obese participants compared to normal weight participants. Regression analyses revealed that higher baseline BMI was significantly predictive of better performance on Trails B at 4-year follow-up $(\beta=-0.285 ; p=0.020)$ and better performance on the Stroop task at 4 -year follow-up $(\beta=0.255$; $\mathrm{p}=0.007)$. BMI was not associated with blood pressure or serum lipoproteins at either time point.

Conclusions: In contrast to historical findings in younger people, the current investigation demonstrated that greater baseline BMI was associated with better EF at 4-year follow-up.

Correspondence: Lauren Strainge, Psychiatry, University of Connecticut Health Center, Department of Psychiatry, UConn Health, 263 Farmington Avenue, MC1410, Farmington, CT 06030-1410, United States.E-mail: lauren.strainge@uconn.edu

E.K. VERNON, S. BEHRENS, J. MATYI \& J. TSCHANZ. Sleep Disturbance and its Association with Cognitive Status in a Population Based Sample of Older Adults: The Cache County Memory Study.

Objective: Sleep disturbance increases with aging, with up to $25 \%$ of older adults reporting having had insomnia. Insomnia and other sleep difficulties have been associated with cognitive decline and risk for Alzheimer's disease (AD). We examined whether sleep disturbance was associated with worse cognitive functioning in a population-based sample of older adults.

Participants and Methods: 4736 individuals (57.2 \% female) participated in a longitudinal study of risk factors for $\mathrm{AD}$ and cognitive decline. Sample age and education [mean (SD)] = 74.6 (6.48) and 3.19 (2.89), respectively. Cognitive status was measured using the Modified MiniMental State Exam (3MS) at 3 - 4 year intervals for up to 12 years (4 visits). Sleep disturbance was ascertained through self-report of having had sleep problems or insomnia. Linear mixed models were used to examine the association between sleep disturbance (time-varying) and cognitive status (3MS). Covariates included age, education, APOE genotype, and gender.
Results: Mean (SD) baseline 3MS score was 89.20 (9.62) (3MS maximum score $=100)$. At baseline, $39.1 \%$ of participants reported having had sleep problems. Report of sleep problems was associated with higher $3 \mathrm{MS}$ scores $(\mathrm{b}=-0.283, \mathrm{p}=0.031)$, with the inclusion of covariates (age, gender, education, and APOE status). There were no differences in rate of decline in 3MS by report of sleep disturbance.

Conclusions: Individuals reporting a sleep disturbance were more likely to perform better on the 3MS compared to those who did not report a sleep disturbance. The results were unexpected and potentially reflect beneficial cognitive effects regarding use of sleep medications or other interventions used to treat sleep disturbance. Future work will examine this issue and type of sleep disturbance in this population.

Correspondence: Elizabeth K. Vernon, BA, Psychology, Utah State University, 694 S 600 E, River Heights, UT 84321, United States. E-mail: ekvernon@aggiemail.usu.edu

L.C. WALZAK \& W.J. L. THORNTON. Vascular Illness Burden Predicts Theory of Mind Performance in Older Adults.

Objective: Theory of Mind (ToM) allows us to reason about cognitive and affective states in order to understand and predict behavior. Past research has identified pulse pressure as a risk factor for reduced ToM in older adults and suggests that cognitive ToM is particularly vulnerable. However, it is unclear whether other health risk factors are linked to reduced ToM and what mechanisms underlie this association. We examined the unique contributions of age, neurocognitive performance, and vascular and nonvascular illness burden in ToM in older adults.

Participants and Methods: 86 older adults (59 females; 27 males, $M=72$ years) completed standardized measures of neurocognition, cognitive ToM, and affective ToM. Participants also completed an inventory of health conditions from which we derived illness burden estimates of vascular and nonvascular risk using beta weights. Using a path analysis approach, we identified direct and indirect predictors of ToM and tested the strength and direction of these predictors through mediation modeling.

Results: Greater severity of vascular illness burden was associated with poorer cognitive ToM $(\dot{\beta}=-.51,95 \% \mathrm{CI}=[-.90,-.41], p<.000)$ and affective ToM $(\beta=-.24,95 \% \mathrm{CI}=[-.58,-.03], p=.03)$ after controlling for age and sex. Nonvascular illness burden did not predict cognitive or affective ToM. Vascular illness burden indirectly influenced cognitive ToM through its effect on executive functioning, which accounted for $18 \%$ of the total effect, $P_{\mathrm{M}}=.18$. Vacular illness burden also directly influenced cognitive ToM independent of its effect on executive functioning $\left(c^{\prime}=-0.51, p=.002\right)$.

Conclusions: Our findings underscore the specific importance of vascular health as a risk factor for declines in ToM. Given that older adults are at increased risk for acquiring vascular risk factors that contribute to cognitive dysfunction and social deficits, a deeper understanding of ToM within a multidimensional framework is clinically relevant for developing effective interventions in later life.

Correspondence: Laura C. Walzak, B.A. (Hons), Clinical Psychology, Simon Fraser University, Department of Psychology, 8888 University Drive, Burnaby, BCV5A 1S6, Canada.E-mail: lwalzak@sfu.ca

V.J. WASSERMAN, Y. LIU, T. ANG, S. KIMBERLY, C. DECARLI \& R. AU. Cognitive Markers of Brain Aging: How Young Can We Go? Objective: Neuroimaging biomarkers have been traditionally accepted as early indices of Alzheimer's disease (AD). Cognitive markers are often presumed to appear later in the course. This study examined to what extent cognitive measures correlated with neuroimaging biomarkers in a young to middle-aged community-based sample.

Participants and Methods: Dementia-free participants of the Framingham Heart Study Third Generation cohort were divided into young (age 25-44) and middle-aged (age 45-64) groups. Both groups were administered a comprehensive neuropsychological (NP) test battery and a brain MRI scan. Brain measures obtained from volumetric MRI 
parameters included volumes of total and lobar grey matter and white matter, hippocampal as well as white matter hyperintensity volume (WMH). Linear regression relating NP scores to MRI metrics within age groups were adjusted for sex \& education.

Results: For both age groups, frontal grey matter was positively associated with tests of memory, attention, executive function and language, while temporal white matter correlated positively with visuospatial functioning. In young adults $(\mathrm{n}=738 ; 56 \%$ female, mean age $=38)$ and in the middle-aged group ( $\mathrm{n}=1312 ; 51.5 \%$ female, mean age $=52)$, larger total white matter volume was associated with better performance in immediate visual memory. Larger hippocampal volume was positively associated with visuospatial functioning in young adults, and with tests of memory and language in middle aged adults. Higher WMH was significant only in middle-aged adults and was associated with poorer performance on memory and executive function tests (all $p<0.05$ ).

Conclusions: These results suggest potential for cognitive indices to serve as surrogates to MRI biomarkers in younger populations asymptomatic for AD.

Correspondence: Victor J. Wasserman, Psychological and Brain Sciences, Boston University, 5 Royce Rd, Apt. 26, Allston, MA 02134, United States.E-mail: wasserman.victor@gmail.com

V.J. WASSERMAN, Y. LIU, T. ANG, S. KIMBERLY, C. DECARLI \& R. AU. The Association of Neuropsychological Test Error Responses to Neuroimaging Biomarkers in Young and MiddleAged Adults.

Objective: Research on Alzheimer's disease is increasingly focused on symptom detection well before overt clinical symptoms are apparent. The Boston Process Approach is a cognitive evaluative approach that considers extraneous and error responses. We examined whether neuropsychological test errors were associated with MRI neuroimaging biomarkers among younger adults presumed to be cognitively intact.

Participants and Methods: The study population included young (aged 25-44) and middle-aged (aged 45-64) Framingham Heart Study $3^{\text {rd }}$ Generation participants who completed neuropsychological testing and MRI brain imaging. Conventional neuropsychological tests were coded for commission errors (confabulation, perseveration, interference, loss of set) and summed to create a Sum of Total Errors (STE) score. Brain MRI outcomes included hippocampus volume, total and lobar grey and white matter volume, and white matter hyperintensity volume (WMH). Linear regression was used to estimate the association between STE score and MRI outcomes within age cohorts.

Results: Among young adults $(\mathrm{n}=738 ; 56 \%$ female, mean age $=38$ years), increasing STE was associated with smaller total and frontal grey brain matter volume $(p<0.01)$ and larger occipital white matter volume $(\mathrm{p}<0.05)$. Among middle-aged adults $(\mathrm{n}=1,312 ; 51.5 \%$ female, mean age $=52$ years), increasing STE was associated with smaller temporal white matter volume and hippocampal volume $(\mathrm{p}<0.05)$.

Conclusions: Our study is the first to describe an association between neuropsychological test errors and subtle differences in brain morphology in a young adult sample. Future research will reveal whether measurement of neuropsychological test commission errors may provide an alternative to more intrusive and expensive neuroimaging biomarkers for early detection of AD.

Correspondence: Victor J. Wasserman, Psychological and Brain Sciences, Boston University, 5 Royce Rd, Apt. 26, Allston, MA 02134, United States.E-mail: wasserman.victor@gmail.com

A. WEAKLEY, M. SCHMITTER-EDGECOMBE \& J.W. TAM. Effectiveness of a Video-based Aging Services Technology Education Program for Clinical Care Professionals.

Objective: Physical, medical, and cognitive changes that often co-occur with advancing age can impact a person's ability to function independently. Although a number of aging services technologies (ASTs) have been developed with demonstrated improvement in functional outcome and safety, an underutilization gap remains. To lessen the gap, the study aim was to develop a video-based program to improve awareness of ASTs and recommendation potential among clinical care providers.

Participants and Methods: Sixty-five clinical providers from a range of professions were presented videos covering ASTs related to memory, medication management, and daily living. Participants completed program effectiveness measures before and after the video program assessing objective and perceived AST knowledge, attitude, stigma, self-efficacy, and recommendation potential.

Results: T-tests revealed that participants' objective and perceived AST knowledge improved from pre to post-program, as did self-efficacy. Participants' recommendation potential also increased with $95.2 \%$ stating they are more likely to recommend ASTs. Participants benefitted equally from the program regardless of years of experience or previous AST familiarity with the exception of objective (high experience group had greater gain) and subjective (low familiarity group had greater gain) knowledge. A hierarchical regression with professional factors entered first, followed by program effectiveness measures, demonstrated that change in self-efficacy and perceived knowledge significantly predicted recommendation potential.

Conclusions: The AST education program was effective at improving care providers' awareness of ASTs. Perceived knowledge gain and improved self-efficacy contributed to overall recommendation potential. Notably, the program appeared to benefit all providers regardless of years of experience or prior AST knowledge.

Correspondence: Alyssa Weakley, Clinical Psychology, Psychology, Washington State University, 710 SE Chinook dr Apt K52, Pullman, WA 99163, United States. E-mail: alyweakley@gmail.com

\section{Dementia (Non-AD)}

E. ALVAREZ, M. RODRIGUEZ, R. DUARA, M. GREIG \& D. LOEWENSTEIN. Differences Between Monolingual and Bilingual Individuals With Mild Cognitive Impairment on Memory Screening.

Objective: The aim of this study was to compare differences in cognitive impairment on a memory screening evaluation between groups of monolingual and bilingual older adults with mild cognitive impairment (MCI).

Participants and Methods: The sample included 50 participants divided into: Bilingual $(n=19)$, monolingual English $(n=15)$, and monolingual Spanish $(\mathrm{n}=16)$ groups. The monolingual English group age range was 55-75 $(\mathrm{M}=67.40, \mathrm{SD}=7.670)$, and education range $12-20$ years $(\mathrm{M}=15.13, \mathrm{SD}=2.696)$. The monolingual Spanish group age range was 57-75 $(\mathrm{M}=70.00, \mathrm{SD}=5.922)$ with years of education between 12-16 ( $\mathrm{M}=13.13, \mathrm{SD}=1.455)$. The Bilingual group age range was $61-75(\mathrm{M}=70.37, \mathrm{SD}=4.573)$, and education range $12-19$ years $(\mathrm{M}=14.95, \mathrm{SD}=2.392)$. The memory screening evaluation included the Mini-Mental Status Examination (MMSE), Logical Memory I (LMI) and II (LMII), Loewestein-Acevedo Scale for Semantic Interference (LASSI), and Category Fluency. MANCOVA was used to compare the cognitive performance in the three groups.

Results: Bilingual individuals exhibited better performance on MMSE $[F(2,49)=4.608, p=.015]$. Sidak's post hoc analysis of means revealed a significant difference between the bilingual and monolingual-Spanish group in MMSE performance ( $p=.021)$.

Conclusions: The MMSE was the only test that showed significance which implicates that this may not be a culturally sensitive screening measure. This is consistent with previous findings in the literature. Examination of the means showed that the bilingual group scored significantly higher than the monolingual Spanish group, indicating that speaking two languages may be a protective factor in overall cognitive functioning. This study was supported by State of Florida, Department of Elder Affairs, Memory Disorders Clinic Grant (2015-16). 
Correspondence: Elvin Alvarez, MS, Psychology, Albizu University, $5201 \mathrm{NW}$ 7th ST, 406W, Miami, FL 33126, United States. E-mail: elvinalvarez@gmail.com

G. CHANEY, J. DERIGHT, S.L. AITA, A. SPEARS, C. ONYIKE \& V. KАMАTH. A Meta-analysis of Neuropsychological Functioning, Social Cognition, and Olfaction in the Frontotemporal Dementias. Objective: Frontotemporal dementia (FTD) constitutes abnormalities in social conduct, self-control, and language due to frontal lobe degeneration. The aim of this meta-analysis was to elucidate which measures of neuropsychological, social cognitive, and olfactory functioning best discriminate the diverse clinical phenotypes of FTD.

Participants and Methods: Our search yielded 1,293 publications, with 144 publications meeting inclusion criteria. Patient groups included behavioral variant FTD (bvFTD; $n=410$ ), semantic variant primary progressive aphasia (svPPA; $n=508)$, nonfluent variant PPA (nfvPPA; $n=269$ ), and logopenic variant PPA (lvPPA; $n=109$ ). We calculated effect size with Cohen's $d$ and assessed heterogeneity with Cochran's $Q$-statistic using a random effects model.

Results: The analyses revealed a large overall effect size across all studies $(d=-2.16)$. For the FTD subtypes, the measures with the largest effect sizes were category fluency for bvFTD $(d=-3.59)$, Frontal Assessment Battery for lvPPA $(d=-8.23)$, Stroop Test for nfvPPA $(d=-5)$, and Graded Naming Task for svPPA $(d=-9.19)$. FTD patients performed worse across all domains, with olfactory performance $(d=-1.69)$ distinguishing the groups more than neuropsychological subdomains and social cognitive tests. Conversely, neuropsychological tests $(d=-2.36)$ had the largest effect size for bvFTD compared to social cognitive and olfactory measures.

Conclusions: Overall, executive functioning measures showed the largest difference between controls and the bvFTD and nfvPPA groups. Tests of language and global cognition were most discrepant for svPPA and lvPPA groups. Additionally, olfactory performance may be a useful diagnostic tool in distinguishing FTD patients; however, these latter findings are based on limited studies suggesting the need for improved social cognitive and olfactory studies in bvFTD. These findings indicate that clinical evaluation of FTD must be as nuanced as the phenotypes themselves.

Correspondence: Grace-Anna Chaney, Bachelor of Science, Psychiatry and Behavioral Sciences, The Johns Hopkins University, 600 N. Wolfe Street, Meyer 218, Baltimore, MD 21287-7218, United States. E-mail: gchaney2@jhmi.edu

G. CHERAN, L. WU, M. MANOOCHEHRI, S. CINES, E. FALLON, T. LYNCH, J. HEIDEBRINK, H. PAULSON, S. LEE, E. HUEY \& S. COSENTINO. Cognitive and Behavioral Measures in Early bv-FTD.

Objective: Early diagnosis of frontotemporal lobar dementia is vital to enable early treatment and behavioral interventions before significant disease progression. Examination of pre-symptomatic carriers of a genetic mutation for FTD offers the opportunity to identify the very earliest cognitive and behavioral features of the disease. The current study examined the sensitivity of a range of cognitive tests for detecting differences between pre-symptomatic carriers of the MAPT mutation and non-carrier controls drawn from the same families.

Participants and Methods: 59 individuals from families carrying MAPT mutations completed a comprehensive battery of neuropsychological tests, and were assigned a Clinical Dementia Rating (CDR) scale score. Questionnaires were completed by a family member informant well known to the individual, reporting on social and behavioral measures. The 59 subjects include 48 non-carriers, 5 carriers assigned a $\mathrm{CDR}=0$ and 6 carriers assigned a $\mathrm{CDR}=0.5$, indicating very mild dementia. A Wilcoxon rank test was used to compare 25 social, behavioral, and cognitive measures between the two groups.

Results: No comparisons survived stringent correction for 25 comparisons, but three measures differed between the two groups with a $p<0.01$ :
Boston Naming, the Perspective-taking score of the Interpersonal Reactivity Index, and the Social Norms Questionnaire from the NACC FTLD Module 2.0. Carriers received lower scores on average in 21 out of 25 tests.

Conclusions: While these results should be interpreted with caution as they do not survive correction for multiple comparisons, they provide early evidence that naming, informant-completed behavioral measures, and measures of crystalized social knowledge may be sensitive to detect the earliest symptoms of bv-FTD.

Correspondence: Gayathri Cheran, Bachelor of Science, Neurology, Columbia University Medical Center, 630 West 168th Street P\&S Box 16, New York, NY 10032, United States. E-mail: gc2646@cumc. columbia.edu

M.P. FOSS, T. GEFEN, E. ROGALSKI \& S. WEINTRAUB. Revision of the Northwestern University Famous Faces Test (NUFFACE-R): Face Naming and Knowledge in Primary Progressive Aphasia (PPA).

Objective: Face naming in Primary Progressive Aphasia (PPA) can result from failures of name retrieval, or an inability to link the face to its name or its semantic associations. The Northwestern University Famous Faces Test was revised (NUFFACE-R) to identify the locus of failure in word-object (i.e., name-face) linkages in patients with PPA. Participants and Methods: Stimuli were 16 updated black-and-white photos of famous persons and their 16 names on individual cards. There were four conditions: 1) face naming; 2) verbal description of facts related to the face; 3) verbal description in response to the printed name; and 4 ) face-to-name matching (multiple-choice format, two cards with 8 faces each). Total score for each condition was 32 . Twenty one cognitively-healthy controls (NC) and 10 individuals with PPA participated in this preliminary study.

Results: No differences were found between groups in age (PPA mean $=68 \pm 8.5 ; \mathrm{NC}$ mean $=67 \pm 11.5)$, education, gender, or racial makeup $(\mathrm{p}<0.05$, for all). NC mean scores were near ceiling and significantly higher than scores of the PPA group on all conditions. The PPA group showed greatest difficulty with face naming (mean=8 \pm 7.4). Although face descriptions (mean $=15 \pm 9.4$ ) and printed name descriptions (mean $=17 \pm 9.4$ ) were at $\sim 50 \%$ accuracy, both were superior to face naming $($ all $\mathrm{p}<0.05)$. PPA patients performed significantly better on face-to-name matching (mean score $=29 \pm 5.6$ ) compared to the other tasks (all $\mathrm{p}<0.01$ ).

Conclusions: The primary locus of face naming failure occurred on tasks requiring name retrieval. Verbal descriptions of the face or name were better, indicating access to semantic information via either the face or the name. Scores were highest for face-name matching, indicating preserved linkages between words and visual face stimuli. Overall, naming failures on the NUFFACE-R appear to represent lexical retrieval problems rather than deficits accessing semantic knowledge.

Correspondence: Maria P. Foss, PHD, Neuroscience and Behavioral Science, University of São Paulo, 600 N Mc Clurg CT \# 407, Rua H, 525 Condominio Quinta da Boa Vista A, Chicago, IL 60611, United States.E-mail:mpfoss@gmail.com

D. PUTCHA, C.D. KAY, S. MCGINNIS, B.C. DICKERSON \& J.C. SHERMAN. Characterization of Cognitive Impairment in Posterior Cortical Atrophy.

Objective: Posterior cortical atrophy (PCA) is a progressive neurocognitive disorder characterized by primary visuoperceptual deficits and parieto-occipital atrophy, with relative sparing of other domains. While visuospatial deficits can remain the primary deficit, we sought to determine the extent to which other cognitive domains are impacted throughout disease progression, and to relate cognitive performance to neurological findings.

Participants and Methods: Twenty individuals (mean age $=63.4$ years, 13F) referred for neuropsychological assessment were diagnosed with PCA based on neurological, neuropsychological exams, and 
neuroimaging. To compare performances across disparate clinical test batteries, we calculated a percent impairment score by domain. Statistical analysis was then conducted to determine associations between cognitive performance, and various neurological findings, specifically the presence of Parkinsonian features.

Results: While visuospatial impairment was prominent as expected (78\%), 87\% impairment was also observed in Processing Speed, $42 \%$ impairment in Attention/Working Memory, 61\% impairment in the Memory, 60\% impairment in Executive Functions, and 48\% impairment in Language. There was a significant difference in memory impairment in patients who exhibited Parkinsonism $(\mathrm{P}+)$ compared to those who did not (P-), with the $\mathrm{P}+$ group demonstrating more severe memory impairment compared to the $\mathrm{P}$ - $\operatorname{group}(\mathrm{t}=2.8, \mathrm{df}=9.3, \mathrm{p}=0.02)$.

Conclusions: This characterization of neuropsychological profiles across different forms of PCA, those with and without Parkinsonian features, may offer clues clarifying previously mixed findings on cognitive performance outside of the visuospatial domain as well as underlving etiology. Future work will investigate structural MRI and molecular biomarkers (e.g., Tau) to further elucidate underlying mechanisms of disease progression.

Correspondence: Deepti Putcha, PhD, Psychiatry, Massachusetts General Hospital, Psychological Assessment Center, One Bowdoin Square,Boston, MA 02114,United States.E-mail: deepti.putcha@ gmail.com

K.A. WYMAN-CHICK, G.M.G. WORTHINGTON, T. JONES, S.A. SPERLING \& C.A. MANNING. The Impact of a Previous Diagnosis of Mild Cognitive Impairment on Mood and Quality of Life in Caregivers and Patients Recently Diagnosed With Dementia.

Objective: It is unknown if a diagnosis of Mild Cognitive Impairment (MCI) prior to conversion to dementia impacts mood or quality of life (QoL) when receiving a diagnosis of dementia. It may serve as a protective factor by preparing patients for a dementia diagnosis, or the knowledge of increased risk for dementia could increase anxiety and negatively impact QoL at dementia diagnosis.

Participants and Methods: Participants were recruited from The University of Virginia Memory and Aging Care Clinic where they were followed closely by neuropsychologists. The study included 18 individuals who converted from MCI to dementia within the past 6 months $(38.8 \%$ male, Age $=77.4 \pm 5.8$, Education $=13.4 \pm 4.8)$ and 17 individuals diagnosed with dementia in the past 6 months who were not previously diagnosed with MCI $(29.4 \%$ male, Age $=77.0 \pm 9.29$, Education $=12.7 \pm 4.4)$. Participants and caregivers completed Quality of Life in Alzheimer's disease (QOL-AD), Center for Epidemiologic Studies Depression Scale-Revised (CESD-R), Neuropsychiatric Inventory Questionnaire (NPI-Q), Instrumental Activities of Daily Living Scale (IADL). Caregivers also completed the Zarit Burden Interview-Short (ZBI). Independent samples t-tests were performed to compare demographics and self-report measures across the two groups.

Results: The group who progressed from MCI did not differ from the group who did not have a previous MCI diagnosis on demographics or on QOL-AD, CESD-R, NPI-Q, or IADL. There was no significant difference between the caregivers on QOL-AD, CESD-R, NPI-Q, or ZBI. Conclusions: In this small sample, a previous diagnosis of MCI did not impact QoL, IADLs, mood, or caregiver burden in patients newly diagnosed with dementia. These preliminary findings are the result of a new program designed to coordinate care for individuals with a new diagnosis of dementia in Virginia. The sample size is expected to increase over time. Future studies will examine if patient-centered coordination of services can improve QoL and delay nursing home placement.

Correspondence: Kathryn A. Wyman-Chick, PsyD, Department of Neurology, University of Virginia, PO BOX 800394, Charlottesville, VA 22908-0394,United States.E-mail:kwyman@pacificu.edu

\section{MCI (Mild Cognitive Impairment)}

C. BEREZUK, K.K. ZAKZANIS, J. RAMIREZ \& S.E. BLACK. Managing Money Matters: Financial Management is Associated with Increased "Functional Reserve" in Mild Cognitive Impairment.

Objective: The purpose of this study is to examine whether financial management is associated with greater functional independence in individuals with mild cognitive impairment (MCI). We hypothesized that financial management experience may increase one's "Functional Reserve", thus promoting independence across other, unrelated instrumental activities of daily living (IADLs).

Participants and Methods: Individuals with MCI were taken from the Alzheimer's Disease Neuroimaging Initiative $(n=862)$. IADLs and IADL experience was assessed with the Functional Activities Questionnaire. We conducted ten Chi-square analyses comparing financial management experience (dichotomous) and dependence on each IADL (categorical).

Results: No group differences existed in Mini-Mental State Examination (MMSE) scores, age, and years of education, although women were more likely to have experience managing finances $(p<.001)$. Chi-Square analyses suggest that financial management experience is associated with greater independence in the ability to manage finances $(p<.001)$; assemble tax and business forms $(\mathrm{p}<.001)$; play a game of skill or hobby $(p=.047)$; follow TV, books, or magazines $(p=.009)$; and remember appointments and important dates $(\mathrm{p}=.002)$.

Conclusions: Although it is intuitive that financial management experience is associated with the ability to manage finances in individuals with MCI, it is interesting that this experience is also related to independence in other IADLs. Given that managing finances is highly multifaceted, shared neural networks may exist between managing finances and other IADLs. Therefore, these findings may have implications for identifying those at a higher risk for functional decline. For example, a lack of financial experience may result in a lower "Functional Reserve", increasing the probability of functional dependence overall. Future longitudinal studies are needed. Additionally, it would be of interest to study the impact that experience in other IADLs, such as shopping and cooking, has on functional disability.

Correspondence: Courtney Berezuk, Psychological Clinical Science, University of Toronto Scarborough, 18 Yonge Street, Unit 3104, Toronto, ON M5E 1Z8, Canada.E-mail: courtney.berezuk@gmail.com

C. BEREZUK, K.K. ZAKZANIS, J. RAMIREZ, M. SHARMA \& S.E. BLACK. Sex Differences in "Functional Reserve" and Decline in Mild Cognitive Impairment from the Alzheimer's Disease Neuroimaging Initiative: A Longitudinal Analysis.

Objective: The purpose of this study was to compare decline of instrumental activities of daily living (IADLs) between men and women with mild cognitive impairment (MCI) and the impact of "Functional Reserve".

Participants and Methods: Participants with MCI at baseline (male=498; female=336) were taken from the Alzheimer's Disease Neuroimaging Initiative. The outcome measure was dependence on the Functional Activities Questionnaire (FAQ). IADL experience was also quantified from the FAQ. We ran linear mixed-effects models with time, sex, age, and baseline Mini-Mental State Examination (MMSE) entered as fixed effects and a random intercept adjusting for unaccounted subject variability. Additional models also included a random slope for time and a fixed effect for IADL experience.

Results: In the first model, women had lower FAQ scores at baseline $(p=.013)$ but showed faster decline over time $(p<.001)$. Additionally, time since baseline $(p<.001)$, greater age $(p=.015)$, and lower baseline MMSE $(\mathrm{p}<.001)$ predicted higher FAQ scores. After accounting for subject variability in functional change, women continued to show lower scores at baseline $(p=.009)$, although no sex difference was found in the rate of change. In the final model, no sex differences existed after 
adjusting for IADL experience. However, experience was associated with better functional ability at baseline $(p<.001)$ but not the rate of decline. Conclusions: These results suggest that men may present with greater functional dependence in MCI, although women may decline at a faster rate. However, this initial female advantage may be mediated by greater IADL experience. We hypothesize that performing IADLs may result in a greater "Functional Reserve", protecting against disability. Women no longer showed faster decline once the rate of change was allowed to vary across subjects, suggesting that other factors may intercede the relationship between sex and function. Further research is indicated given mixed findings in the research literature and in keeping with our findings. Correspondence: Courtney Berezuk, Psychological Clinical Science, University of Toronto Scarborough, 18 Yonge Street, Unit 3104, Toronto, ON M5E 1Z8, Canada.E-mail: courtney.berezuk@gmail.com

F.E. CAMBRONERO, J.E. NEAL, D. LIU, K. GIFFORD, J.G. TERRY, S. NAIR, K.R. PECHMAN, K.E. OSBORN, E. MOORE, E. GORDON, M. BABICZ, J.E. BOGNER, R. MARTIN-WILLETT, C. SEABOLT, M. THURSBY, T.J. HOHMAN, S.P. BELL, T.J. WANG, J.J. CARR \& A.L. JEFFERSON. APOE genotype modifies the association between central arterial stiffening and neuropsychological functioning in mild cognitive impairment: The Vanderbilt Memory \& Aging Project.

Objective: Arterial stiffening increases the risk of incident Alzheimer's disease (AD). This study tested the interaction between arterial stiffening and a genetic susceptibility risk factor for AD (APOE genotype) on neuropsychological functioning among older adults.

Participants and Methods: Vanderbilt Memory \& Aging Project participants with normal cognition (NC, $\mathrm{n}=162,72 \pm 7$ years, $29 \%$ APOE- $\varepsilon 4$ carrier) and mild cognitive impairment (MCI, $n=121,73 \pm 8$ years, $42 \%$ $A P O E-\varepsilon 4$ carrier) were studied. Participants completed neuropsychological assessment and cardiac magnetic resonance imaging to assess central arterial stiffening of the aortic arch using pulse wave velocity $(\mathrm{PWV}, \mathrm{m} / \mathrm{s})$. Linear regression models stratified by cognitive diagnosis (NC, MCI) related aortic PWV x APOE- 44 status to neuropsychological performances. Models adjusted for age, race/ethnicity, education, body mass index, and Framingham Stroke Risk Profile.

Results: In NC, PWV x APOE- $\varepsilon 4$ was unrelated to all neuropsychological performances (p-values $>0.08$ ). In MCI, PWV x $A P O E-\varepsilon 4$ related to performances on California Verbal Learning Test-II Recognition ( $\beta=-$ $0.09, p=0.02)$, Tower Test $(\beta=-0.44, p=0.04)$, Hooper Visual Organization Test $(\beta=-0.43, p=0.002)$, Biber Figure Learning Test (BFLT) Long Delay Free Recall $(\beta=-0.91, p=0.04)$ and BFLT Recognition $(\beta=-0.03$, $\mathrm{p}=0.008)$. Among MCI participants with higher aortic PWV, APOE- $\varepsilon 4$ carriers had worse performances than non-carriers. Excluding participants with cardiovascular disease (CVD) and atrial fibrillation yielded similar effects.

Conclusions: In prodromal AD, presence of the APOE- $\varepsilon 4$ allele modifies the association between central arterial stiffening and neuropsychological performances. These deleterious effects correspond to over 3 years of advancing age and cannot be explained by shared vascular risk factors or CVD. Results add to a growing body of evidence for geneenvironment interactions on neuropsychological functioning in adults at highest risk for $\mathrm{AD}$.

Correspondence: Francis E. Cambronero, Vanderbilt University, 1207 17th Avenue South, Suite 204, Nashville, TN 37212, United States. E-mail: francis.cambronero@vanderbilt.edu

C.L. CROOK, G. PAPANDONATOS, B. OTT \& G. TREMONT. Refinement of a Telephone Screening for Mild Cognitive Impairment.

Objective: Accessible screening measures are important for identifying amnestic Mild Cognitive Impairment (aMCI), which is associated with increased risk for Alzheimer's disease. The Minnesota Cognitive Acuity Screen (MCAS), a telephone-administered cognitive test, has good sensitivity $(86 \%)$ and adequate specificity $(78 \%)$ for distinguishing between healthy controls (HC) and aMCI. To improve accuracy, we added learning and recognition memory components to the original scale (MCAS-M). This study compared accuracy of the original and modified instruments for discriminating between HC and aMCI.

Participants and Methods: We examined $30 \mathrm{HC}$ and $30 \mathrm{aMCI}$. Groups did not differ in education ( $\mathrm{HC} \mathrm{M}=15.87$; aMCI M=14.80), but the aMCI group was older (HC M = 65.73; MCI M = 72.37). Participants completed measures two weeks apart; half received the MCAS followed by two administrations of the MCAS-M and half received the MCAS-M followed by two administrations of the MCAS. ROC analyses were conducted on both measures to determine accuracy for distinguishing $\mathrm{HC}$ and aMCI

Results: The MCAS had an AUC of $.98(95 \% \mathrm{CI}=.96-1.00)$ and the MCAS-M was .99 (95\% CI = .97-1.00). Sensitivity and specificity for the MCAS was $91 \%$ and $93 \%$, respectively. For the MCAS-M, sensitivity was $96 \%$ and specificity was $98 \%$, respectively.

Conclusions: Findings demonstrate that both tests performed very well in distinguishing between HC and aMCI, although the optimal sensitivity and specificity of the MCAS-M was higher than for the MCAS. This modified screening measure may be useful for early identification of individuals at heightened risk for dementia.

Correspondence: Cara L. Crook, Neuropsychology, Rhode Island Hospital, 593 Eddy Street, Neuropsychology Program, Physicians Office Building, Suite 430, Providence, RI 02903, United States. E-mail: cara. crook@gmail.com

J. BEAVER, M. BOEGE, K. DAVIS, R. BRALEY \& M. SCHMITTEREDGECOMBE. Characterizing Omission Errors in Everyday Task Completion and Cognitive Correlates in Individuals with Mild Cognitive Impairment and Dementia.

Objective: Functional ability declines with age and cognitive impairment. This study investigated errors of omission made by community-dwelling older adults completing everyday tasks in a naturalistic setting. Identifying types of task steps left incomplete during everyday task completion can aid in early detection of cognitive impairment and compensatory and assistive technology development.

Participants and Methods: Sixty-five cognitively healthy older adults (HOA), 52 individuals with mild cognitive impairment (MCI), and 13 individuals with dementia completed neuropsychological measures of memory, processing speed, and executive functioning, as well as eight different activities of daily living (e.g., watering plants) in a naturalistic environment. Task steps were divided into preparatory, action-oriented, and concluding steps, and proportion of omission errors was calculated for each step type across the eight activities, and compared across groups.

Results: For action-oriented steps, the number of omission errors increased with cognitive impairment (HOA $<$ MCI $<$ dementia). In contrast, for preparatory and concluding steps, the dementia group committed more omission errors than both the MCI and HOA groups, which did not differ. Memory consistently correlated with all three types of omission errors for the neurologically impaired group (MCI and dementia).

Conclusions: The results suggest that action-oriented steps in everyday task completion may be the first to be affected in the process of cognitive decline, whereas preparatory and concluding steps may be preserved longer, only beginning to show declines in later stages of impairment (dementia). Memory was found to be related to all types of omission errors in everyday activity completion in the neurologically impaired group.

Correspondence: Kaci Davis, Pullman, WA, United States. E-mail: kacibdavis@gmail.com 
D.A. DENNEY \& G.P. PRIGATANO. Differences in Awareness of Memory Function Among Persons with Amnestic MCI vs Subjective Memory Complaints but Normal Memory Function.

Objective: Patients with mild cognitive impairment of the amnestic type (MCI) and those with subjective memory complaints (SMC) but normal memory performance were asked to make subjective ratings of their cognitive and affective functioning. It was predicted that MCI patients would report less memory impairment than SMC patients. The MCI group was also expected to underreport level of memory impairment when compared to informant ratings. It was predicted the SMC patients would show the opposite pattern. It was also predicted that SMC patients would report greater mood disturbance than MCI patients.

Participants and Methods: 23 patients were studied and grouped based on diagnosis [10 with ICD-10 G31.84 (MCI); 13 with R41.3 (SMC)]. They rated their cognitive and affective dimensions on a 10 point scale and underwent neuropsychological examination as part of standard clinical care. Informants independently rated the patients' cognitive and affective functioning along the same dimensions. Cognitive performances and subjective ratings were compared between groups. Results: The MCI group was older $[M$ age $=79.60(9.52)]$ than the SMC group $[M$ age $=49.85(18.69)]$ but did not differ on other demographics. The MCI group reported less memory difficulties than the SMC group despite worse performance on memory measures compared to the SMC group. Groups did not differ on IQ, processing speed or set-shifting measures. Compared to informant ratings, the MCI group underestimated and SMC group overestimated memory difficulties. The SMC group reported higher levels of depression, anxiety, and fatigue than the MCI group.

Conclusions: The findings indicate that subjective ratings of cognitive and affective functioning obtained from patients and informants may have diagnostic value in separating patients with MCI vs SMC. The difference in ratings may reveal subtle, but important divergent disturbances in self-awareness of neurocognitive functioning between these two groups of patients.

Correspondence: David A. Denney, Ph.D., Clinical Neuropsychology, Barrow Neurological Institute, 222 West Thomas Road, Suite 315, Phoenix, AZ 85013, United States.E-mail: david.denney@dignityhealth. org

K.N. DEVLIN, L. SAAD, T. GIOVANNETTI, D.A. WOLK \& D. MECHANIC-HAMILTON. Diagnosing Mild Cognitive Impairment: Comparison of Conventional, Actuarial, and Statistical Methods.

Objective: Actuarial and statistical methods have been proposed as alternatives to conventional methods of diagnosing mild cognitive impairment (MCI), with the aim of enhancing diagnostic and prognostic validity. We compared the agreement of conventional, actuarial, and statistical MCI diagnostic methods and their relationship to demographic and prognostic indicators.

Participants and Methods: Nondemented older adults (n=366; M age $=70 ; 69 \%$ white) were diagnosed with MCI or normal cognition (NC) according to clinical consensus diagnosis based on Petersen/Winblad criteria, actuarial neuropsychological criteria (Jak/Bondi), and latent class analysis (LCA), using neuropsychological scores adjusted for age, sex, and education. Associations with demographics, subjective cognition, everyday function, and change in global cognition (median follow-up time $=39$ months) were examined.

Results: MCI rates by consensus, actuarial criteria, and LCA were $45 \%$, $64 \%$, and $41 \%$, respectively. LCA identified 3 MCI subtypes (memory only, $24 \%$; memory/language, $10 \%$; memory/executive, $7 \%$ ) and $2 \mathrm{NC}$ classes (low NC, 38\%; high NC, 21\%). Consensus and LCA largely agreed $(90 \% ; \kappa=0.79)$, whereas actuarial criteria agreed only moderately with consensus and LCA $(75 \% ; \kappa=0.52 ; \kappa=0.51)$. Cases classified as MCI by actuarial criteria but NC by consensus tended to be low NC by LCA, nonamnestic, nonwhite, and similar to NC participants in subjective cognition, everyday function, and global cognitive change. By contrast, participants classified as MCI by consensus or LCA consistently differed from NC participants in prognostic indicators.

Conclusions: Clinical consensus diagnoses were supported by LCA and demonstrated good prognostic validity. In diverse or demographically representative samples, actuarial criteria may be susceptible to false positive error, highlighting the importance of demographic corrections for race and/or education quality. Group differences in additional etiologic and prognostic indicators should be investigated further.

Correspondence: Kathryn N. Devlin, Sc.B., Department of Psychology, Temple University, Weiss Hall 1701 North 13th Street, Philadelphia, PA 19122, United States.E-mail: kathryn.devlin@temple.edu

A.L. DIAZ SANTOS, A. HEIDEL, D. LOEWENSTEIN, R. CURIEL, E. CROCCO, A. RAFFO, A. PEÑATE \& J.G. MELO. The LowensteinAcevedo Scales of Semantic Interference and Learning (LASSI-L), and The Short-Term Memory Binding Task (STMBT) as Predictors of Mild Cognitive Impairment (MCI).

Objective: It is essential to identify early stages of Alzheimer's Disease (AD) to facilitate treatment before multi-system degeneration occurs in the brain. The LASSI-L assesses free versus cued recall, semantic interference, and uniquely, recovery from proactive semantic interference (PSI). The STMBT assesses immediate recognition of shapes and shape-color bindings. We examined subtests of these novel measures, the LASSI-L and the STMBT in the detection of early MCI.

Participants and Methods: Sixty-four older adults (M=72.7, SD=9.4 years); 34 MCI and 30 cognitively normal (CN) controls were studied. Participants were presented with List A of the LASSI-L (15 semantically-related words). After free and cued recall trials, List A was presented again followed by another cued recall trial. Subsequently, List B (a content-equivalent wordlist) was administered and recall was assessed in the same manner. Finally, delayed free and cued recall for List A was assessed. The STMBT assessed accuracy in detecting changes between two consecutively presented arrays of shapes and shape-color bindings. Results: Stepwise logistic regression models of free and cued recall measures of maximum storage, vulnerability to PSI, recovery from PSI, and STMBT subtests indicated that a combination of Free Recall A $(p<.04)$, Free Recall B $(p<.02)$ and STMBT $(p<.03)$ memory for shapes was most predictive of separating MCI from CN groups. Sensitivity was $97.1 \%$, specificity was $96.7 \%$. The overall correct classification rate was $96.9 \%$.

Conclusions: Combining subtests of the LASSI-L and STMBT resulted in high levels of sensitivity and specificity in detection of early MCI, potentially leading to the clinical benefit of earlier treatment.

Correspondence: Ana L. Diaz Santos, Psy.D., Doctoral-Clinical Psychology, Carlos Albizu University, 9688 Fontainebleau Blvd, Apt. 504, Miami,FL33172, United States.E-mail: aldiaz.santos@gmail.com

S. EMRANI, M. LAMAR, C.C. PRICE, T.B. GINSBERG, K. GIFFORD, T.J. HOHMAN, A.L. JEFFERSON, M. KERWIN, R. SWENSON \& D.J. LIBON. Visual Versus Verbal Working Memory - I: Differing Between Subtle and Mild Cognitive Impairment.

Objective: To assess the capacity of clinically available visual versus verbal working memory (WM) tests to differentiate between statistically- determined patients with mild cognitive impairment (MCI) and pre-clinical or subtle clinical cognitive impairment (SCI).

Participants and Methods: Memory clinic outpatients were tested. Visual working memory was assessed with the Wechsler Memory Scale-IV Symbol Span; verbal working memory was assessed with the Wechsler Adult Intelligence Test- IV Digit Backward subtests. Jak, Bondi et al., (2009) criteria diagnosed single and multi-domain MCI using tests other than outcome measures assessing memory, language, and executive control. MCI was diagnosed when scores fell below $<1$ sd on at least two measures within at least one cognitive domain. SCI (Edmonds et al., 2016) was diagnosed in patients who scored below $<1$ sd on one of two measures in different cognitive domains. MCI $(\mathrm{n}=9)$ and SCI $(n=15)$ groups did not differ on age $(M=76.70 \pm 5.62)$; education 
$(\mathrm{M}=14.29 \pm 2.56)$, or MMSE $(26.62 \pm 2.07)$. WM test performance was expressed as age corrected z-scores.

Results: Analyzing the entire sample with a single group t-test (criterion, $\mathrm{z}=0.0)$ Symbol Span performance was statistically below expectation (M Symbol Span $=-0.55 \pm .069 ; \mathrm{p}<.001$ ). Digit Span Backward $(\mathrm{M}=0.30 \pm 0.98 ; \mathrm{ns})$ performance was not significant. When tests were assessed between-group differences were not found. However, when assessed within-group SCI patients scored lower on visual WM versus verbal WM tests (Symbol Span M= $-0.53 \pm 0.80$, Digit Backward $\mathrm{M}=$ $0.42 \pm .096, \mathrm{p}<.016)$. No within-group difference was observed for MCI patients (Symbol Span $\mathrm{M}=-0.50 \pm 0.49, \mathrm{M}=0.26 \pm 1.18, \mathrm{~ns}$ ).

Conclusions: Visual WM tests are not routinely used to assess for MCI and the determination of prodromal dementia. Visual WM tests could be more sensitive than verbal WM tests in identifying potentially emergent illness.

Correspondence: Sheina Emrani, Rowan University, 16 Halco drive, Paramus, NJ 07652, United States.E-mail: sheinaemrani@gmail.com

S. EMRANI, M. LAMAR, C.C. PRICE, T.B. GINSBERG, K. GIFFORD, T.J. HOHMAN, A.L. JEFFERSON, M. KERWIN, R. SWENSON \& D.J. LIBON. Visual Versus Verbal Working Memory - II: Serial Order Position Effects in Subtle and Mild Cognitive Impairment.

Objective: To assess how graphomotor visual working memory versus verbal working memory (WM) serial order test performance can differentiate between patients statistically- determined as presenting with mild cognitive impairment (MCI) and pre-clinical or subtle cognitive impairment (SCI).

Participants and Methods: Graphomotor visual WM was assessed with the Object Span Test. Patients were shown four geometric shapes and asked to draw stimuli in order of presentation. Verbal WM was assessed with the Backward Digit Span Test where patients repeated five digits backward. Tests test contained seven trials. MCI (Jak, Bondi et al., 2009) was diagnosed when scores fell below $<1$ sd on at least two measures within at least one cognitive domain. SCI (Edmonds et al., 2016 ) was diagnosed in patients who scored below $<1$ sd on one of two measures in different cognitive domains. Groups (MCI n=11; SCI n=16) did not differ for age $(M=76.70 \pm 5.62)$; education $(M=14.29 \pm 2.56)$, or MMSE (26.62 \pm 2.07$)$. Test performance was expressed as z-scores. Results: Between-group total Backward Digits serial order (M MCI= $-0.66 \pm 1.27$; SCI $=-0.12 \pm 1.01, \mathrm{~ns})$ did not differ; however, total Object Span serial order was lower for MCI $(\mathrm{M} \mathrm{MCI}=-2.07 \pm 0.82$; $\mathrm{M} \mathrm{SCI}=$ $-1.12 \pm 1.27, \mathrm{p}<.052)$. Within- group worse visual versus verbal WM performance was obtained for both groups (MCI, $p<.045$; SCI, $p<$ .003). Backward Digit Span for all five serial order positions did not differ between-group. Object Span serial order position yielded attenuated recency (serial position 4) with MCI scoring lower than SCI patients $(\mathrm{p}<.005)$.

Conclusions: Results found that total graphomotor visual WM test performance was more challenging for both groups; and (2) attenuated recency effects for visual versus verbal stimuli in MCI versus SCI suggesting greater sensitivity for graphomotor visual WM tests to identify emergent cognitive impairment.

Correspondence: Sheina Emrani, Rowan University, 16 Halco drive, Paramus, NJ 07652, United States.E-mail: sheinaemrani@gmail.com

J. EPPIG, D.A. NATION, I.B. MEIER, A.M. BRICKMAN, L.M. CAMPBELL, M. SANDERSON-CIMINO, K.J. BANGEN, L. DELANO-WOOD \& M.W. BONDI. APOE- 44 Moderates the Relationship between Lobar Microbleeds and a Diagnosis of Mild Cognitive Impairment.

Objective: Cerebral microbleeds (MBs) are associated with cognitive decline in older adults, and thought to reflect cerebral amyloid angiopathy (CAA) when distributed in lobar regions. This study investigated the prevalence of lobar MBs and their relationship to APOE- $\varepsilon 4$ positivity in healthy older adults and individuals with mild cognitive impairment (MCI).
Participants and Methods: Fifty-five older adults ( 44 healthy controls [HCs]; 11 MCIs) underwent APOE genotyping and susceptibility-weighted imaging for lobar MB detection. MCI was diagnosed using Jak/ Bondi comprehensive criteria across five cognitive domains. Lobar MBs were counted by visual inspection and classified as present or absent.

Results: HC and MCI groups did not differ in demographics or APOE$\varepsilon 4$ positivity (all $p$ 's $>.21$ ). The MCI group was more likely to have lobar MBs than HCs (MCI: 46\%; HC: 14\%; $p=.018$ ). Among those with lobar MBs, the APOE- $\varepsilon 4$ allele was overrepresented in the MCI group compared with HCs (MCI: $80 \%$; HCs: $17 \% ; p=.036$ ). A logistic regression predicting MCI diagnosis with lobar MBs, $\mathrm{APOE}$, and the lobar MBs x APOE interaction $\left(p=.017\right.$, Pseudo $\left.R^{2}=0.185\right)$ showed that APOE- $\varepsilon 4$ moderated the relationship between lobar MBs and MCI $(\mathrm{OR}=46.15 ; p<0.05)$; in the presence of APOE- $\varepsilon 4$, there was a 20 -fold increase in the likelihood of an individual with lobar MBs having an MCI diagnosis. Overall, the model correctly predicted $97.7 \%$ of HCs and $36.4 \%$ of MCI participants.

Conclusions: Lobar microbleeds are more prevalent in MCI than cognitively normal older adults. Importantly, the presence of lobar MBs in combination with APOE- $\varepsilon 4$ positivity is associated with significantly increased risk of MCI with great specificity. Our findings support recent work suggesting that APOE- $\varepsilon 4$ is associated with development of CAA, which increases risk of Alzheimer's disease. Future research should investigate the presence of lobar MBs across MCI subtypes.

Correspondence: Joel Eppig, B.A. in psychology, Clinical Psychology, SDSU/ UCSD, 1205 Colusa St, Apt \#3, San Diego, CA 92110, United States.E-mail: joel.eppig@temple.edu

L. FIELDS, K. WILMOTH, J. STRAIN, C. LOBUE, L.S. HYNAN, K. WOMACK, J. HART, M. CULLUM \& N. DIDEHBANI. Cognitive Functioning in MCI Patients with and without a History of SportsRelated Concussion.

Objective: Head injury has been associated with risk and earlier onset of MCI; however, it remains unclear whether sports-related concussions uniquely influence cognitive performance later in life in individuals who develop MCI. Thus, we compared cognitive performance in MCI patients with and without a history of sports-related concussion.

Participants and Methods: Participants were recruited from two separate studies of aging and included12 retired professional athletes with MCI and history of concussion recruited from a longitudinal athlete study compared to 12 non-athlete MCI patients without history of concussion recruited from the Alzheimer's Disease Center. Groups were similar in age (Athletes: $\mathrm{M}=69.75$; Non-athletes: $\mathrm{M}=66.82$ ), education (Athletes: $\mathrm{M}=15.58$; Non-athletes: $\mathrm{M}=15.92$ ), and race (11 Caucasian, 1 African American). Neuropsychological assessments differed by study, but all included Boston Naming Test (BNT), Phonemic and Semantic Fluency, and Trail Making Tests (A and B). Concussion history was gathered through interview and MCI diagnosis was made by multidisciplinary consensus. Independent sample T-tests (corrected for multiple comparisons) examined group differences in cognitive performance.

Results: Athletes scored relatively lower on all tests. T-tests revealed significant differences on BNT (Athletes: M=38.58; Non-athletes: $\mathrm{M}=50.33 ; \mathrm{p}=.024$ ), Trails A (Athletes: $\mathrm{M}=45.92$; Non-athletes: $\mathrm{M}=55.42 ; \mathrm{p}=.01$ ), and Trails $\mathrm{B}$ (Athletes: $\mathrm{M}=43.67$; Non-athletes: $\mathrm{M}=52.67 ; \mathrm{p}=.023)$, but not verbal fluency ( $\mathrm{p}>.05)$. All means were within normal limits for both groups except for athletes' BNT scores. Conclusions: Retired athletes with MCI scored relatively lower than non-athletes on all tests, and significantly lower on naming and attention. Our findings suggest a history of concussion in retired professional athletes with MCI may be associated with lower cognitive performance on some measures.

Correspondence: Lindy Fields, M.A., Psychology, Southern Methodist University, Psychology Department, P.O. Box 750442, Dallas, TX 75275-0442,United States. E-mail: lloneman@smu.edu 
S. GARCIA, A. LESNOVSKAYA, O.C. CALHOUN, A. BHAUMIK \& B.M. HAMPSTEAD. Preliminary Investigation of Gaze Pattern Differences in MCI and Healthy Older Adults.

Objective: Patients with mild cognitive impairment (MCI) have difficulty remembering the location of objects compared to healthy counterparts (HC). While eye fixations are known to differ in dementia patients, it is unclear whether 1) such differences exist in MCI compared to healthy controls (HC), and 2) if older adults with MCI have different gaze patterns outside of simple eye fixation count.

Participants and Methods: Seventeen older adults (MCI=7) completed an object location association task which required them to encode a series of objects within commonly encountered rooms. Eye tracking software simultaneously recorded eye movement and calculated eve fixations within immediate areas around the object (AOIs) and the rest of the environment. Duration of eye fixations was compared as a commonly used measure of eve movement. Gaze pattern differences were evaluated by: 1) comparing the number of times individuals shifted their fixations between $\mathrm{AOI}$ and RE (gaze shifts), and 2) by comparing average areas of fixation (pixel clusters).

Results: There were no significant differences between the two groups with regard to duration of eye fixation $(p=0.15, d=0.73)$. Similarly, MCI and HC participants did not differ in their overall gaze shifts $(p=0.71, d=0.20)$, nor did they differ in their pixel clusters $(p=0.37, d=0.43)$.

Conclusions: While statistically significant differences in gaze measures were not present, effect sizes suggest duration of eye fixations and cluster volume may hold promise. Future work with larger samples should investigate these and other measures of gaze patterns (e.g. area of the screen viewed rather than just fixated upon) since they may help explain the deficits that MCI patients demonstrate in object location memory. Correspondence: Sarah Garcia, PhD, Neuropsychology-Psychiatry, University of Michigan, 2100 Commonwealth, Ann Arbor, MI 48105, United States.E-mail:slga@med.umich.edu

M. GONZALES, P. INSEL, C. NELSON, D. TOSUN, N. MATTSSON, S. MUELLER, S.F.SACUIU, D. BICKFORD, M. WEINER, S. MACKIN \& T. ALZHEIMER'S DISEASE NEUROIMAGING INITIATIVE. Cortical Atrophy is Associated with Accelerated Cognitive Decline in Mild Cognitive Impairment with Subsyndromal Depression.

Objective: To investigate the association between cognitive decline and cortical atrophy in individuals with mild cognitive impairment (MCI) and chronic subsyndromal symptoms of depression (SSD) over a fouryear period.

Participants and Methods: Data from 101 individuals carrying an MCI diagnosis with cognitive and neuroimaging data were selected from the Alzheimer's Disease Neuroimaging Initiative repository. The Neuropsychiatric Inventory was used to identify individuals with stable endorsement (SSD group $\mathrm{N}=32$ ) or no endorsement (non-SSD group $\mathrm{N}=69$ ) of depressive symptoms across timepoints. Repeated measures of cognitive outcomes, cortical atrophy, and their associations were evaluated with mixed effects models adjusting for age, education, gender, and $A P O E$ genotype.

Results: The SSD group demonstrated accelerated decline on measures of global cognition (Alzheimer's Disease Assessment Scale $(\beta=1.580$, $\mathrm{p}=0.003)$ ), memory (Wechsler Memory Scale-Revised Logical Memory II Recall $(\beta=-0.691, p=0.003)$ ), information processing speed (Trail Making Test Parts $\mathrm{A}(\beta=4.681, p=0.002)$ and $\mathrm{B}(\beta=12.150, p=0.003))$, and semantic fluency (Category Fluency $(\beta=-0.855, p=0.002)$, as well as accelerated frontal lobe $(\beta=-0.020, p=0.008)$ and anterior cingulate $(\beta=-0.036, p<0.001)$ atrophy. No group differences were observed for rate of decline on measures of attention, learning, and confrontation naming or for rate of atrophy in any other regions. Accelerated frontal lobe and anterior cingulate atrophy was associated with cognitive decline on measures of global cognition, information processing speed, and semantic fluency (all $p<0.05$ ), but not memory

Conclusions: Individuals with chronic SSD may represent an MCI subgroup that is highly vulnerable to accelerated cognitive decline, an effect that may be governed by frontal lobe and anterior cingulate atrophy

Correspondence: Mitzi Gonzales, Psychology, University of Texas Austin, 2908 Pearl St Unit D, Austin, TX 78705, United States. E-mail: mitzi.m.gonzales@gmail.com

S.F. GUINEA, D. JIMÉNEZ, E. GARCÍA, A. JUNQUERA \& M.A. PARRA. Can Subtle ADL Impairments be Traced Along the Continuum of MCI?

Objective: Accumulating evidence and criteria of MCI due to Alzheimer's disease recognize that individuals with MCI commonly have mild problems performing complex functional tasks. It has been proposed that IADL problems could be a significant predictor of future decline and dementia onset. Characterizing functional disability in MCI and its relationship with cognitive deficits is critical.

Participants and Methods: 127 subjects participated in this study. Of these 43 were healthy older adults (Controls) and 84 were MCI patients [naMCI $(n=18)$, aMCI $(n=20)$, maMCI $(n=46)]$. They were assessed by a comprehensive neuropsychological battery including MMSE, GDS, CDR, NPI, TAVEC, FCSRT, ROF, BNT, FAS, TMT, LN, ZOO, Similarities, and ADL questionnaires such as Barthel Index, Lawton \& Brody Scale and Everyday Cognition Scale (ECOG).

Results: There were statistically significant differences between Controls and MCI patients in Lawton \& Brody and ECOG scales, but not in Barthel Index. After controlling for age, sex and education, there were statistically significant differences among MCI subgroups only in ECOG Scale. Post-hoc contrasts showed that maMCI but not aMCI patients were significantly different from both healthy controls and naMCI in ECOG domains such as memory, visuospatial, and total score. There were no significant differences between aMCI and maMCI subgroups. These ECOG domains significantly correlated with neuropsychological tests that tax abilities linked to these subjective experiences (e.g., TAVEC, ROF).

Conclusions: MCI is associated with subtle difficulties in complex everyday life tasks which are overlooked by traditional IADL scales (e.g., Lawton \& Brody). There is need of ADL tools than can detect such impairments in a way that is not accounted for by the disease severity. In that regard, memory and visuospatial deficits as assessed by ECOG appears to inform about these subtle impairments. ECOG is a more sensitive scale to identify those functional impairments in MCI which are mapped along the continuum of $\mathrm{AD}$.

Correspondence: Sara F. Guinea, UNIVERSIDAD COMPLUTENSE MADRID, Facultad de Psicologia, Madrid 28223, Spain. E-mail: sguinea@psi.ucm.es

S.F. GUINEA, E. GARCÍA, D. JIMÉNEZ, A. JUNQUERA \& M.A. PARRA. Short-Term Memory Binding Deficits Across Subtypes of MCI And Memory Load.

Objective: Short-term memory binding (STMB) declines in subjects with mild cognitive impairment (MCI). The extent to which these deficits are linked to factors such as memory load or MCI subtypes is little understood. It is necessary to reveal which form of MCI shows the greatest sensitivity to the STM binding test and with which setting these impairments can be detected.

Participants and Methods: 127 participants (43 healthy older adults and 84 with MCI) were grouped into a total of 4 subgroups: amnestic (aMCI, $\mathrm{N}=20$ ), amnestic multidomain (DCLma, $\mathrm{N}=46$ ), multidomain non-amnestic (DCLna, $N=18$ ) and controls $(\mathrm{N}=43$ ). All were assessed with a battery of neuropsychological tests and two versions of the STMB test. The latter asked participants to study arrays of two or three shapes or coloured shapes and detected changes in subsequent arrays. We measured the proportion of correct recognition across task condition and memory load for the different investigated groups.

Results: We found significant differences between controls and subjects with MCI in all the conditions of STMB test. aMCI showed worse performance than controls only in the binding task with 3 items. Whereas 
maMCI showed impairments regardless of the memory load and the task condition.

Conclusions: STM binding is sensitive to the very early stages of MCI (aMCI) when the task is sufficiently taxing. As the disease progress in stages prior to dementia, the task sensitive increases and becomes less dependent on memory load. STMB binding can help trace MCI in the prodromal stages of $\mathrm{AD}$.

Correspondence: Sara F. Guinea, UNIVERSIDAD COMPLUTENSE MADRID, Facultad de Psicologia, Madrid 28223, Spain. E-mail: sguinea@psi.ucm.es

B.L. DECK, A. HAGERTY, E. ROLL \& T. GIOVANNETTI. Do as I Do, Not as I Say: Relations between Narrative Script Production and Everyday Action Performance.

Objective: Research has shown that subtle errors during everyday tasks (i.e., micro-errors) are sensitive to mild cognitive difficulties. The goal of this study was to identify similar micro-errors during the generation of narratives describing everyday tasks (i.e., script generation) and determine whether action micro-errors and script micro-errors reflect a weakness in a domain-general cognitive process. If subtle script errors reflect a single construct, then script generation could serve as a proxy for performance.

Participants and Methods: 28 individuals with MCI (M age=73.1; M education=12.1) were asked to describe all steps required to pack a lunch with a sandwich, snack and a drink (script). Participants also enacted the lunch task (action). Performance/narrative recordings were scored for overt errors (e.g., step omission, sequence errors, etc.) and micro-errors (e.g., action - object moved but not used; script- pause or filler words -"uh" or "um"). Participants also completed the MMSE, $\mathrm{P}[\mathrm{r}]$ VLT, FAS, and Digits Backwards.

Results: Script and action errors were not significantly correlated (overt $\mathrm{r}=.18$, micro-error $\mathrm{r}=.04$ ). Script micro-errors correlated only with measures of verbal fluency (FAS- $r=-.43, \mathrm{p}=.03$; all other $\mathrm{r}<.14$, $\mathrm{p}>$.48), whereas action micro-errors correlated only with a measure of episodic memory $(\mathrm{P}[\mathrm{r}]$ VLT immediate recall $\mathrm{r}=-.45, \mathrm{p}<.02)$.

Conclusions: Subtle dysfluencies in language production and subtle disruptions of everyday action do not represent a single cognitive construct. The association between subtle dysfluencies in language and an independent test of verbal fluency suggests that script micro-errors likely reflect a weakness in a language-specific process that does not meaningfully overlap with the cognitive weaknesses associated with dysfluent everyday action performance. Script performance should not be substituted for performance-based measures of everyday function with older adults.

Correspondence: Ashley Hagerty, B.S., 125 North Tyson Avenue, Glenside, PA 19038, United States. E-mail: ashenhagerty@gmail.com

\section{J.Y. JANG, J.K. HO, A.E. BLANKEN \& D.A. NATION. Affective Neuropsychiatric Symptoms and Alzheimer's Disease Biomarkers in Non-demented Older Adults.}

Objective: The present study sought to identify the role of affective neuropsychiatric symptoms (aNPS: depression, anxiety, apathy, and irritability) in the early clinical manifestation of Alzheimer's disease (AD).

Participants and Methods: Participants included 963 individuals $[$ mean age $=73.0(7.0)]$ from the Alzheimer's Disease Neuroimaging Initiative who were cognitively normal or MCI at baseline. Hierarchical cluster analysis classified participants according to cerebrospinal fluid (CSF) biomarkers, amyloid beta $\left(\mathrm{A} \beta_{1-42}\right)$ and total tau, and aNPS (Neuropsychiatric Inventory). Empirically-derived aNPS-biomarker subgroups were compared on neuropsychological measures (memory, executive function, language) and rates of progression to either MCI or $\mathrm{AD}$.

Results: Four clusters were identified: Bio-aNPS- (N=346; biomarker negative, aNPS negative), Bio+aNPS- (N=434; biomarker positive, aNPS negative), Bio+Anxiety $+(\mathrm{N}=96$; biomarker positive with predominant anxiety), and Bio+Apathy+ $(\mathrm{N}=87$; biomarker positive with predominant apathy). There was no difference in age among the four clusters. Individuals with Bio+aNPS-, Bio+Anxiety+, and Bio+Apathy+ profiles had a greater proportion of apolipoprotein E4 (APOE4) carriers, worse cognition, and higher rate of progression to MCI or AD, relative to the Bio-aNPS- group. The Bio+aNPS-, Bio+Anx$i e t y+$, and Bio+Apathy+ groups did not differ in APOE 4 status, cognitive ability, or rate of progression $(34 \%, 33 \%$, and $38 \%$, respectively). Both Bio+Anxiety+ and Bio+Apathy+ groups had significantly higher levels of CSF A $\beta_{1-42}$, compared with Bio+aNPS-. The Bio+Apathy+ group also had lower levels of p-tau, compared with Bio+aNPS-.

Conclusions: The present study found no difference in clinical progression between individuals with and without aNPS, despite the fact that those with aNPS exhibited less AD pathology on CSF biomarker analysis. Together, these findings suggest that aNPS may lower the threshold of AD pathology required for the manifestation of cognitive decline. Correspondence: Jung Y. Jang, Psychology, University of Southern California, 3620 S McClintock Ave, SGM 501, Los Angeles, CA 90089, United States.E-mail: jungyjan@usc.edu

J.Z. KIRKLAND CALDWELL, J. BERG, J. CUMMINGS \& S.J. BANKS. Sex Differences in Verbal Memory and Hippocampal Volume: The Impact of Amyloid Imaging Measures.

Objective: To examine sex differences in impact of fibrillar amyloid as shown by florbetapir-PET amyloid positivity $(\mathrm{FB}+)$ on verbal memory performance and hippocampus volumes, accounting for apolipoprotein E epsilon 4 (APOE-4) carrier status.

Participants and Methods: We examined 742 normal control and mild cognitive impairment (MCI) participants from Alzheimer's Disease Neuroimaging Initiative (ADNI2 and ADNI-GO). All had baseline florbetapir-PET; 526 also had screening MRI hippocampal volumetry. We used ANOVA to examine main effects of sex, FB+/-, and their interaction on Rey Auditory Verbal Learning Test (RAVLT) scores and hippocampal volumes. Additional ANOVAs examined effects of APOE-4 carrier status. Age, cognitive status at screening, and intracranial volume served as control variables.

Results: For the full sample, men, FB+ individuals, and APOE-4 carriers showed poorer verbal learning and memory. However, for people with MCI, sex differences were observed only in non-AD MCI (i.e., FB- MCI). In addition, women with prodromal AD (i.e., FB+ MCI) performed more poorly than women with non-AD MCI, but men did not show this pattern. Within the full sample and MCI participants, women and $\mathrm{FB}+$ individuals had smaller hippocampal volumes. No effect of APOE-4 status was observed for hippocampal volume.

Conclusions: FB+ and sex each predicted learning, memory, and hippocampal volumes in normal aging and MCI. Women's memory advantage in learning and memory appears limited to non-AD MCI. Separately, memory detriments in prodromal $\mathrm{AD}$ versus non-AD MCI were found only in women. APOE-4 carriers performed more poorly on memory measures only for the full sample, and no APOE-4 effects were observed for the hippocampus.

Correspondence: Jessica Z. Kirkland Caldwell, Ph.D., Cleveland Clinic Lou Ruvo Center for Brain Health, 888 W. Bonneville Ave, Las Vegas, NV 89106, United States. E-mail: caldwej5@ccf.org

A. LESNOVSKAYA, S. GARCIA, O.C. CALHOUN \& B.M. HAMPSTEAD. Gaze Fixations are Associated With Object Location Memory in Older Controls and Patients With Mild Cognitive Impairment.

Objective: Patients with mild cognitive impairment (MCI) frequently report, and empirically demonstrate, difficulty remembering the location of objects (OL). Eye-tracking may provide important information about how OL are processed; yet little work has been performed in this area. The current study: (1) compared the number and distribution of gaze fixations during an 
OL encoding task in healthy controls (HC) and MCI; (2) determined whether gaze fixations were associated with memory performance. Participants and Methods: Twenty patients with MCI, (age 71.35 \pm 6.18 years) and $20 \mathrm{HC}$ (age 68.65 \pm 7.29 years) completed our OL task Participants were instructed to remember the locations of 15 objects, while a TOBII eye-tracker recorded gaze fixations. After a 15-minute delay, participants indicated each object's location using a touchscreen monitor during free recall (blank screen), cued recall (image of the empty room), and standard recognition (three possible locations) conditions. Fixations were quantified within the immediate area around the object $(\mathrm{AO})$ and the rest of the environment (RE).

Results: As expected, patients with MCI performed significantly worse on all phases of the memory test (all $p<0.001$ ). There were no significant differences in the total number of fixations within $\mathrm{AO}$ and RE $(p>0.22)$. Across all participants, Pearson correlations revealed a relationship between $\mathrm{AO}$ fixations and cued recall error $(r=0.43, \mathrm{p}=$ $0.006)$, as well as an inverse relationship between $\mathrm{AO}$ fixations and recognition accuracy $(r=-.35, p=0.027)$.

Conclusion: Findings suggest that increased attention to the object and the immediately surrounding area is detrimental to memory for the object's location. Results also reinforce our earlier findings that rehabilitative techniques should train individuals to integrate local (i.e., $\mathrm{AO}$ ) details with those from the larger context (i.e., RE). Correspondence: Alina Lesnovskaya, University of Michigan, $425 \mathrm{E}$ washington St, Apt 1215, Ann Arbor, MI 48104, United States. E-mail: askaya@umich.edu

\section{A. REALE-CALDWELL, R. SEVER, R.S. RUM \& M. SCHOENBERG. Comparison of Performance Validity Tests for the RBANS.}

Objective: Current performance validity measures of the Repeatable Battery for the Assessment of Neuropsychological Status (RBANS) are variable within the literature. The present study's objective is to compare performance validity algorithms developed for the RBANS in a sample of individuals with mild cognitive impairment (MCI). We compared the Effort Index (EI) (Silverberg et al., 2007) and the Effort Scale (ES) (Novitski et al., 2012) which provide embedded validity indicators of malingering effects.

Participants and Methods: The sample was 222 MCI patients. The EI uses digit span and list recognition subtest scores, the ES uses list recognition, list recall, story recall, figure recall, and digit span. We computed EI and ES scores, then evaluated possible false positives via two standalone performance validity tests (PVT): Green's Word Memory Test (GWMT) and the Victoria Symptom Validity Test (VSVT).

Results: Using the recommended EI cutoff of $>3$, we found $22(9.9 \%)$ subjects in the insufficient effort group. Using the ES algorithm alone, 142 participants showed suboptimal effort. A significant difference in proportion of participants identified as insufficient effort was obtained between the EI and ES PVT algorithms $\left(X^{2}=139.2334, p<.001\right)$. When ES was only used for those who showed insufficient effort on the EI, one participant was found to be invalid. A subsample of 68 participants were given the GWMT or the VSVT. When EI scores were compared to these PVTs, 6 false positives were discovered (14\%). In the ES insufficient group, when EI was not considered prior to utilizing ES, 41 false positives were found $(100 \%)$. When utilizing both ES and EI no false positives were discovered.

Conclusions: Caution should be used when applying ES alone due to its significant rate of false positives. Additionally, the ES should only be used when insufficient effort is first suggested by the EI. However, when these PVTs are utilized in a stepwise fashion they appear to be a good indicator of possible insufficient effort for MCI patients.

Correspondence: AmberRose Reale-Caldwell, MA, University of South Florida, 5506 Fulmar Drive, Tampa, FL 33625, United States. E-mail: amberrose47@yahoo.com
G.O. REYNOLDS \& G. TREMONT. Neuropsychiatric Symptoms and Awareness of Cognitive Deficits in Mild Cognitive Impairment. Objective: Reduced awareness of cognitive deficits is prevalent in dementia, but less commonly studied in mild cognitive impairment (MCI). Neuropsychiatric symptoms are common in MCI and may predict conversion to dementia. The relation between these symptoms in MCI is not well understood, as extant research has reported mixed findings. This study examined the relation between caregiver-rated awareness and neuropsychiatric symptoms in MCI, given that these symptoms may be comorbid and may predict future disease course.

Participants and Methods: Participants were 26 adults with MCI (11 men, 15 women; mean age $=73.96$; mean $\mathrm{MMSE}=26.73)$ and their caregivers. Caregivers rated their significant other on the Neuropsychiatric Inventory, and on a scale of awareness of cognitive problems (Likert scale 1-5, with higher ratings indicative of poorer awareness).

Results: Reduced awareness was reported among $42.3 \%$ of MCI participants. Independent samples t-tests revealed that MCI participants who endorsed difficulty with anxiety $(p=.003)$, impulsivity $(p=.049)$, sleep $(p=.027)$, and appetite $(p=.019)$, showed reduced awareness of cognitive problems, relative to participants who denied these neuropsychiatric symptoms. There were no between-group differences in awareness among participants with and without other neuropsychiatric symptoms (delusions, hallucinations, agitation, depression, mania, apathy, irritability, repetitive behaviors).

Conclusions: Poorer awareness of cognitive deficits in MCI is associated with anxiety and impulsivity, as well as with sleep and appetite concerns. These findings suggest that awareness of deficits and specific neuropsychiatric symptoms in MCI may share underlying neural correlates, e.g., frontal-subcortical dysfunction. Future research should continue to examine neuropsychiatric symptoms and awareness of cognitive deficits in MCI in order to elucidate potential mechanisms and inform treatment. Correspondence: Gretchen O. Reynolds, Alpert Medical School of Brown University, Department of Psychiatry, Rhode Island Hospital, Providence, RI 02912,United States.E-mail: gretchen_reynolds@ brown.edu

G.D. SANTORELLI, M. MATHER, J. SWEARER \& R. READY. Executive Function and Emotion Reactivity to Negative Mood Induction in Older Adults with Mild Cognitive Impairment/Mild Alzheimer's Disease.

Objective: Older adults (OAs) with mild cognitive impairment (MCI) and mild Alzheimer's disease (AD) experience difficulties with emotion regulation and subsequent neuropsychiatric symptoms. Difficulties with emotion regulation in MCI and mild $\mathrm{AD}$ may be due to deficits in executive function (EF). Theoretical models, including the Model of Cognitive Control of Emotion, postulate that EF is essential to the active regulation of emotions. Poorer EF is associated with heightened reactivity to negative mood induction in healthy adults. This study determined differences in emotional response to a negative mood induction in OAs with MCI or mild $\mathrm{AD}$ and cognitively healthy $\mathrm{OAs}$; associations between $\mathrm{EF}$ and emotion outcomes in the patient group were determined.

Participants and Methods: Participants (MCI/AD $n=11$, control $n=$ 12) watched a video montage depicting interpersonal loss. Self-reported emotions were assessed prior to and immediately after the video. EF was assessed via WAIS-IV Digit Span, Trail Making Test, Stroop ColorWord, and verbal fluency tasks.

Results: There was a trend for $\mathrm{OAs}$ with $\mathrm{MCI} / \mathrm{mild} \mathrm{AD}$ to report greater reactivity to the film in negative affect $(\mathrm{NA} ; M=8.82, S D=6.51 ; p=$ $.097 ; d=0.72)$ and sadness $(M=6.91, S D=3.99 ; p=.063 ; d=0.82)$ compared to controls (NA: $M=4.33, S D=5.87$; sadness: $M=3.83$. $S D=3.54)$. In the MCI/AD group, greater NA reactivity was strongly correlated with poorer performance on Trails B $(r=.51, p=.13)$ and moderately correlated with poorer performance on Stroop Color-Word Condition $(r=-.48, p=.14)$.

Conclusions: Findings from this pilot study suggest that OAs with $\mathrm{MCI} / \mathrm{mild} \mathrm{AD}$ may be more reactive to negative stimuli than healthy OAs. Cognitive inflexibility and behavioral disinhibition may be linked 
to emotion overreactivity in $\mathrm{OAs}$ with $\mathrm{MCI} /$ mild $\mathrm{AD}$, placing impaired OAs with EF deficits at risk for neuropsychiatric symptoms. A better understanding of the etiology of emotion dysregulation in persons with $\mathrm{MCI} / \mathrm{mild} \mathrm{AD}$ can lead to more effective interventions.

Correspondence: Gennarina D. Santorelli, Psychology, University of Massachusetts Amherst, 135 Hicks Way, Amherst, MA 01003, United States.E-mail: gsantorelli@psych.umass.edu

C.A. SOFKO, L.R. SAMUELS, D. LIU, K.E. OSBORN, F.E. CAMBRONERO, J.E. BOGNER, R. MARTIN-WILLETT, C. SEABOLT, M. BABICZ, M. THURSBY, L. WALLJASPER, J. THOMPSON, E. GORDON, K. GIFFORD, S.P. BELL, T.J. HOHMAN \& A.L. JEFFERSON. APOE genotype may modify the association between inflammatory biomarkers and neuropsychological functioning in older adults: The Vanderbilt Memory \& Aging Project.

Objective: To relate inflammatory biomarkers, including interleukin-6 (IL-6), tumor necrosis factor- $\alpha$ (TNF- $\alpha$ ), and high-sensitivity C-reactive protein (hs-CRP) to neuropsychological functioning among older adults.

Participants and Methods: Vanderbilt Memory \& Aging Project participants free of dementia $(n=335,73 \pm 7$ years, $59 \%$ male) completed a fasting blood draw and neuropsychological assessment. Least-squares regression related log-transformed plasma inflammatory biomarker concentrations to neuropsychological performances, adjusting for age, sex, education, race/ethnicity, cognitive diagnosis, and $A P O E-\varepsilon 4$ status (carrier, non-carrier). Models were repeated testing for inflammatory marker x $A P O E-\varepsilon 4$ interactions.

Results: Increased IL-6 related to lower Montreal Cognitive Assessment $(\beta=-0.44, p=0.02)$, California Verbal Learning Test-II (CVLT-II) Recognition $(\beta=-0.15, p=0.02)$, Biber Figure Learning Test (BFLT) Recognition $(\beta=-0.04, p=0.007)$, and FAS performances $(\beta=-2.23$, $\mathrm{p}=0.009)$. Higher TNF- $\alpha$ related to lower CVLT-II Total Learning $(\beta=-2.47, p=0.04)$, CVLT-II Interference Trial $(\beta=-0.68, p=0.0005)$, CVLT-II Recognition $(\beta=-0.22, p=0.03)$, and BFLT Interference Trial performances $(\beta=-1.60, p=0.02)$. Main effects for hs-CRP were null ( $p$-values $>0.08)$. APOE $\mathrm{x}$ IL-6 interactions emerged for Hooper Visual Organization Test $(\beta=-1.07, p=0.04)$ and Delis-Kaplan Executive Functioning System (DKEFS) Number Sequencing $(\beta=-9.73, p=0.002)$. $A P O E \times$ TNF $-\alpha$ interactions were observed for CVLT-II Recognition $(\beta=-0.42, p=0.047)$. Increased inflammatory biomarkers related to worse performance among $A P O E-\varepsilon 4$-carriers, with the exception of DKEFS Number Sequencing.

Conclusions: Increased plasma IL- 6 and TNF - $\alpha$ related to worse neuropsychological functioning (especially episodic memory performances) among older adults without dementia. hs-CRP may be less sensitive in this context. Future research should further assess interactions between Alzheimer's disease genetic risk and systemic inflammation on neuropsychological and brain health markers.

Correspondence: Channing A. Sofko, M.A., Psychology, University of South Alabama, 1201 17th Avenue South, 2nd Floor, Suite 204, Nashville, TN 37212, United States.E-mail: channing.sofko@ vanderbilt.edu

K.R. SUHRIE, B.C. ALLRED. DALLEY, T.J. ATKINSON, D.B. HAMMERS \& K. DUFF. Association Between a Brief Telephone Screening Measure and the Repeatable Battery for the Assessment of Neuropsychological Status (RBANS).

Objective: Cognitive screening of older adults over the telephone has the potential to increase accessibility for both patients and research participants, though research on the comparability between telephone screens and in-person cognitive assessment is limited. The current study compared the modified Telephone Interview of Cognitive Status (mTICS) to those obtained from in-person cognitive testing in subjects with Mild Cognitive Impairment (MCI).
Participants and Methods: Eighty older adults with amnestic MCI were screened with the mTICS over the telephone and were administered the Repeatable Battery for the Assessment of Neuropsychological Status (RBANS) in person approximately 20 days later.

Results: Scores on the mTICS (range: $20-42$ ) were significantly correlated with performance on the RBANS Total Index $(r=.71$, $p<.001)$, RBANS Immediate Memory Index $(r=.69, p<.001)$, RBANS Language Index $(r=.58, p<.001)$, RBANS Attention Index $(r=.44$, $p<.001)$, and RBANS Delayed Memory Index $(r=.57, p<.001)$, but not with the RBANS Visuospatial Constructional Index $(p=.38)$. The mTICS scores were significantly associated with pre-morbid intellect $(r=.33 p=.003)$. However, mTICS scores did not correlate with age $(p=.92)$ or education $(p=.07)$, and there were no differences between sexes $(p=.47)$.

Conclusions: In older adults with amnestic MCI, telephone cognitive screening using the mTICS provides valuable information about a range of cognitive abilities, and performance does not appear to be influenced by demographic variables. Using similar screening measures can save critical resources in clinical and research settings.

Correspondence: Kayla R. Suhrie, B.S., University of Utah, 650 Komas Drive, Ste 106A, Salt Lake City, UT 84108, United States. E-mail: kayla.suhrie@hsc.utah.edu

K.R. SUHRIE, T.J. ATKINSON, B.C. ALLRED. DALLEY, D.B. HAMMERS \& K. DUFF. Does Performance on the Modified Telephone Interview for Cognitive Status (mTICS) Tell Us Anything About Functional Abilities?

Objective: Although functional impairment is critical in discriminating Mild Cognitive Impairment (MCI) from dementia, it can be challenging to assess functional abilities. This study aimed to determine if a brief telephone screening measure was related to functional abilities on objective and self-report functional instruments administered in person.

Participants and Methods: Eighty older adults with amnestic MCI were administered the modified Telephone Interview for Cognitive Status (mTICS), a brief telephone screen of cognition; the Alzheimer's Disease Cooperative Study-Activities of Daily Living Scale adapted for MCI (ADCS-ADL MCI), a subjective functional measure; and the Independent Living Scales (ILS), an objective functional measure.

Results: Scores on the mTICS significantly correlated with scores from the following subscales of the ILS: Managing Money $(r=.47, p<.001)$, Managing Home and Transportation $(r=.36, p=.001)$, and Health and Safety $(r=.46, p<.001)$. Significant correlations were also noted between the mTICS and the 18-item ADCS-ADL MCI $(r=.37, p=.001)$ and the 24-item ADCS-ADL MCI $(r=.32, p=.004)$.

Conclusions: Even in this sample of older adults with amnestic MCI, who by definition are not functionally impaired, the mTICS was moderately correlated with daily functioning. This relationship may aid in the diagnosis of late life cognitive disorders. Similar telephone screening measures may be used as time- and resource-efficient tools to identify those patients in need of additional assessment.

Correspondence: Kayla R. Suhrie, B.S., University of Utah, 650 Komas Drive, Ste 106A, Salt Lake City, UT 84108, United States. E-mail: kayla.suhrie@hsc.utah.edu

C. SUMIDA, T.T. VO \& M. SCHMITTER-EDGECOMBE. Differences Between Healthy Older Adults and Individuals with Mild Cognitive Impairment on the Medication Management Abilities Assessment: Overdose and Underdose.

Objective: The aging population has an increased risk for polypharmacy and poor medication management. The Medication Management Abilities Assessment (MMAA) was developed to assess medication management abilities within the laboratory (Patterson, 2009). The present study sought to examine differences in MMAA performance between healthy older adults and patients with mild cognitive impairment (MCI). 
Participants and Methods: HOA (N=133) and individuals with MCI $(\mathrm{N}=32)$ were administered the MMAA. In addition to the MMAA total score, two new error scores were calculated that reflect the conditions of taking too much medication (e.g., extra pills; overdose) and too little medication (e.g., missing pills; underdose).

Results: A Mann-Whitney U test was computed between MCI and HOA for the MMAA total score, underdose and overdose. The MCI group performed significantly poorer on the MMAA original total, overdose and underdose scores compared to the HOA group $(p s<.05)$. Evaluation of the individual data revealed that $51.5 \%$ of the MCI participants took more than the necessary number of any of the four types of pills or took more attempts than required compared to $25.5 \%$ of the HOA. In addition, $66.7 \%$ of the MCI compared to $36.1 \%$ took less than the required amount of pills for one of the four pill types or took less attempts than required.

Conclusions: Using the MMAA test, we demonstrated that individuals with MCI were more likely to make overdose and underdose mistakes when asked to spontaneously plan out a daily medication routine compared to HOA.

Correspondence: Catherine Sumida, M.A., Psychology, San Diego State University, 1630 NE Valley Rd., L104, Pullman, WA 99163, United States. E-mail: catherinesumida@gmail.com

K.R. THOMAS, E.C. EDMONDS, L. DELANO-WOOD \& M.W. BONDI. Longitudinal Trajectories of Informant-Reported Daily Functioning in Empirically-Defined Subtypes of Mild Cognitive Impairment (MCI).

Objective: Within the Alzheimer's Disease Neuroimaging Initiative (ADNI) MCI cohort, we have previously used cluster analysis to identify MCI subtypes as well as participants initially diagnosed with MCI but found to have normal neuropsychological profiles, lower rates of progression, and normal biomarker profiles. The current study examined the rate of functional change over time by cognitive group status. Participants and Methods: ADNI participants (mean age $=73.44$ years) were classified using cluster analysis as dysexecutive MCI $(n=65)$, dysnomic MCI $(n=115)$, amnestic MCI $(n=217)$, or cluster-derived normal (CDN; n=256). Robust normal controls (NCs: n=284) who did not progress to MCI over a follow-up period of 1-7 years were also examined. The Functional Activities Questionnaire (FAQ), which evaluates activities of daily living (ADLs), was completed by the participants' informant at baseline, 6-, 12-, 18-, 24-, 36-, 48-, and 60-month follow-ups. Linear mixed effects modeling, adjusting for age and education, was used to examine FAQ trajectories by cognitive group.

Results: All groups showed greater functional decline relative to the robust NCs (all $p$-values <.01). The dysexecutive MCI subtype showed the fastest rate of decline relative to the other subgroups $(p<.001)$. The amnestic and dysnomic MCI subtypes also showed steeper rates of decline relative to the CDN and robust NC groups. While the CDN group showed more functional decline than the robust NCs, the CDN's predicted mean FAQ score remained below the suggested MCI cutoff at all visits.

Conclusions: The dysexecutive MCI group had a faster rate of decline in ADLs over a 5-year period when compared to other subgroups, which demonstrates the importance of executive dysfunction when predicting future functional decline in at-risk elders. While the CDN group had more functional difficulty than the robust NCs, the CDN group showed a slower rate of change in ADLs than the MCI groups, providing further support that their initial MCI diagnosis may have been a "false positive." Correspondence: Kelsey R. Thomas, PhD, VA San Diego Healthcare System, 3350 La Jolla Village Drive (116B), San Diego, CA 92161, United States. E-mail: kelseyrthomas@gmail.com

G. MANCIOPPI, L. TOMMASINI \& M.R. TIMPANO SPORTIELLO. MCI: A Study on Progression to Dementia in High-Risk Individuals. Objective: Mild Cognitive Impairment (MCI) has proven extremely effective in tackling dementia, both as a prevention and rehabilitation tool. The current definition of MCI is the result of decades of clinical researches, starting with Reisberg's studies (1988), followed by Petersen $(1999,2005)$. The most recent development has been provided by the Summers and Saunders hypotheses (2012) and by Edmonds (2014). The aim of this research is to combine the gradual shift over time in diagnostic category put forth by Summers and Saunders with the hypothesis of the triple partition of the MCI construct which stems from the neuropsychological actuarial analysis undertaken by Edmonds.

Participants and Methods: 150 out-patients (85 females), diagnosed with MCI according to the Petersen criteria. We used a battery of neuropsychological tests, including: long and short term memory tests (both visual and verbal tasks), attention tests, tests to assess executive functions, language and visual-perceptive tests, supported by neuroimaging techniques. These out-patients have been re-classified according to the Edmonds criteria and monitored over time, in order to follow the progress of the disease.

Results: Our research showed that in many cases (67\%), disesecutive impairment is more than amnesic one. This suggested that the amnesic and disesecutive sub-types of MCI are simply two different stages of the same pathology.

Conclusions: This theoretical approach proves to be extremely useful, as the early diagnosis of high-risk individuals allows an intervention to take place before the biological damage becomes irreversible. Early diagnosis enables prevention, thus proving to be a key tool to tackle dementia.

Correspondence: Marco R. Timpano Sportiello, Professor, of Psychology, University of Pisa-Italy, via P.Maroncelli, 73-Viareggio, Viareggio 55049, Italy.E-mail: viatene@alice.it

V. SANBORN, D. PUTCHA \& G. TREMONT. Recognition Memory Heterogeneity in Amnestic Mild Cognitive Impairment.

Objective: Determining whether an individual with amnestic Mild Cognitive Impairment (aMCI) has Alzheimer's disease (AD) is challenging. Recognition memory (RM) performance could be helpful in identifying individuals with underlying $\mathrm{AD}$. We examined RM discriminability and its associations with cognitive domains impaired in $\mathrm{AD}$ in individuals with aMCI to better identify those at highest risk of progression to AD.

Participants and Methods: Participants were 99 individuals diagnosed with aMCI (mean age $=74.48$ years) who underwent comprehensive neuropsychological evaluation. Hierarchical linear regression analyses were conducted to determine associations between discriminability on the HVLT-R and executive function (EF) and language tasks, controlling for age and education. We also examined group differences between impaired and unimpaired RM groups.

Results: While $100 \%$ of the aMCI group showed impaired delayed recall on a word list, we found $67 \%$ had RM discriminability impairment while $33 \%$ did not. For the entire group, language $(\beta=.357 p=.000)$ and EF $(\beta=.285 p=.006)$ domains significantly predicted RM discriminability. Individual tests of language and EF also predicted RM discriminability, including: naming $(\beta=.424, p=.000)$, initiation/perseveration $(\beta=.235$, $p=.023)$, category fluency $(\beta=.208, p=.044)$, and set switching $(\beta=.236$, $p=.024)$. Discriminability impairment groups did not differ on global cognitive measures, but significantly differed on naming performance $(t=2.07, p=.042)$.

Conclusions: Our results demonstrate individuals with aMCI are heterogeneous and show variability in RM discriminability. RM performance was associated with measures of executive functioning and language, elucidating mechanisms by which individuals with RM impairment may have underlying $\mathrm{AD}$ and be at heightened risk for progression. Future studies need to address this finding in a longitudinal sample. Correspondence: Geoffrey Tremont, PhD, Psychiatry \& Human Behavior, Brown Medical School, Rhode Island Hospital, 593 Eddy Street, Neuropsychology Program, Department of Psychiatry, Physician's Office Building, Suite 430, Providence, RI 02903, United States.E-mail: gtremont@lifespan.org 


\section{PM Coffee Break}

$$
\text { 2:15-2:45 p.m. }
$$

\section{Plenary C. Developmental Amnesia: Memory Formation in the Absence of Remembering}

\author{
Presenter: Faraneh Vargha-Khadem \\ 2:45-3:45 p.m.
}

F. VARGHA-KHADEM. Developmental Amnesia: Memory Formation in the Absence of Remembering.

Developmental Amnesia, a disorder resulting from early bilateral damage to the hippocampus, is characterized by four dissociations in memory processes, viz: severe impairment of episodic and autobiographical memory, spatial navigation, recall, and recollection, in the presence of spared semantic memory, perception, recognition and familiarity. This lecture will (a) review the history of cognitive memory research in adults and children, (b) examine the evidence for the neural circuits serving different components of memory processes, (c) relate the findings in humans to results of lesion studies in non-human primates, and (d) provide preliminary evidence on new methods of learning and memory retrieval in patients with developmental amnesia. The lecture aims to differentiate between neural systems that support the development of intelligence and knowledge acquisition versus memory and learning. As a result of attending this lecture, the audience will learn how to (1) diagnose the syndrome of developmental amnesia in children and adolescents, (2) use neuroimaging evidence to determine which components of cognitive processes are compromised, and (3) become familiar with translational research techniques for learning new information in the presence of early damage to the hippocampus.

Correspondence: Faraneh Vargha-Khadem, GOS Institute of Child Health, University College London, 30 Guilford Street, London WC1N 1EH, United Kingdom.E-mail: f.vargha-khadem@ucl.ac.uk

\section{Plenary D (Birch Memorial Lecture). Cognitive Neural Prosthetics to Overcome Brain and Spinal Cord Injury}

\author{
Presenter: Richard A. Andersen
}

4:00-5:00 p.m.

\section{R.A. ANDERSEN. Cognitive Neural Prosthetics to Overcome Brain and Spinal Cord Injury.}

Neural prosthetics are designed to assist patients paralyzed from spinal cord injury, peripheral neuropathies, and stroke. Neural activity is recorded and decoded to determine the subjects' intent, which can then be used to control assistive devices such as robots or computers. Initial proofs of concept can be traced back to studies in animals as early as the late 1950s and early 1960s.

At the turn of this century there have been a handful of clinical studies in humans in which implants of arrays of microelectrodes were made in the motor cortex of tetraplegic participants. On the other hand, posterior parietal cortex (PPC) provides high-order intent signals to motor cortex that are then used by motor cortex to control the muscles. We reasoned that the high-level intent signals of PPC could be easily interpreted by "smart" robotic systems, enhancing the versatility and intuitiveness of brain control.

In the course of PPC recordings with tetraplegic humans we have uncovered remarkable cognitive features that we have used for prosthetic control. Imagined goals and sequences can be decoded extremely rapidly, both sides of the body are represented which promises bilateral control, and complete hand shapes are encoded by single neurons allowing grasp control with very few cells. Individual finger movements are well represented and have even allowed a subject to perform brain controlled typing on a virtual keyboard and playing a simple melody on a virtual piano. Neurons are selective for very high order cognitive features such as both imagined and observed movements, and the representation of numerical quantities and simple mathematical operations. These wide-ranging findings point to future advanced neuroprosthetic applications in which PPC and other cognitive cortical areas are tapped for the unique cognitive variables they represent.

Following this lecture, the learner should be able to: 1) Learn how neural prosthetics can help people with paralysis; 2) Learn what distinguishes a motor prosthetic from a cognitive prosthetic; and, 3) Understand how touch sensation is important for manipulation of objects with the hand and how this sensory feedback might be recreated for people with paralysis and loss of somesthesis.

Correspondence: Richard A. Andersen, PhD, Biology and Biological Engineering, California Institute of Technology, 1200 E California Blvd, Pasadena, CA 91125, United States.E-mail: andersen@vis.caltech.edu

\section{THURSDAY EVENING, FEBRUARY 2, 2017}

Invited Presentation. From the Laboratory to the
Clinic and Back: The Role of Science and Policy
Development in Shaping Clinical Neuropsychology

Presenter: Antonio E. Puente

$$
\text { 5:00-6:00 p.m. }
$$

A.E. PUENTE. From the Laboratory to the Clinic and Back: The Role of Science and Policy Development in Shaping Clinical Neuropsychology.

As the Affordable Care Act goes into full effect, shifting paradigms in the provision of health care are occurring, many of which impact neuropsychologists. This presentation will focus on the specific challenges that impact our specialty including integrated care as well as dementia screening and assessment. The role of scientific evidence as the primary and initial step in determining how ideas evolve into policy will be presented. Subsequently, the role of policy influencing reimbursement and, in turn, how reimbursement affects practice (and, subsequently, science) will be outlined. Beyond the pragmatic analysis of lab to clinic, the general future of clinical neuropsychology as a profession and a science will be considered over the next 5-10 years based on the analyses presented.

Correspondence: Antonio E. Puente, PhD, Psychology, University of North Carolina, Wilmington, 601 South College Road, Wilmington, NC 28403, United States.E-mail: puente@uncw.edu 


\section{Symposium 4. Historical Perspectives in the Study of Neurotrauma: Progress and Pitfalls Over 40 Years of Research}

\author{
Chair: Frank G. Hillary
}

5:00-6:30 p.m.

\section{H.S. LEVIN, E.D. BIGLER \& F.G. HILLARY. Historical perspectives in the study of neurotrauma: progress and pitfalls over 40 years of research.}

The goal of this symposium is to uncover the progress and pitfalls during the last half-century of research in the area of traumatic brain injury (TBI). The TBI literature is one of the richest in neuropsychology so this ambitious symposium aims to provide an overview of the many dimensions to TBI-related research starting with early attempts to classify injury severity (Glasgow coma scale; Coma Data Bank) and posttraumatic amnesia in severe TBI in both animal models and humans. This early research dovetailed with work to understand the primary consequences of TBI including early coma emergence prediction and then a generation of research designed to understand the behavioral sequela of injury. We then follow the trajectory of TBI research into an era where magnetic resonance imaging was central to understanding brain and brain lesion changes. Most recently we examine recent advancements and integration of methods to include functional brain imaging and brain connectivity modeling, genetics, and big data sharing. In the process of uncovering the primary scientific perspectives within TBI research, we aim to discover those research tendencies that have been met with success and as well as with failure with the goal of creating a framework for the next generation of TBI studies. The primary speakers for this symposium. Drs. Harvev Levin and Erin Bigler, have been at the center of multiple TBI literatures over the past 30 years and bring their combined experience to bear on the issue of forging a new perspective for understanding the consequences of TBI. Dr. Hillary has been involved in one of the latest movements in TBI work (and neuropsychology more broadly) related to functional brain imaging and connectivity modeling to understand system-level neuroplasticity. Together this trio of speakers brings historical perspective as well as continued involvement in the latest developments in TBI research in an effort to bind the past with the present.

Correspondence: Frank G. Hillary, PhD, Psychology, Pennsylvania State University, 313 Moore Building, University Park, PA 16802, United States.E-mail: fhillary@psu.edu

\section{H. LEVIN. Milestones in Traumatic Brain Injury Research: A Neuropsychological Perspective.}

This talk will take a historical perspective to review early TBI research designed to diagnose and characterize injury severity including the development of landmark clinical observational indices such as the Glasgow Coma Scale and the use of markers such as posttraumatic amnesia and acute imaging findings to index injury severity and predict patient outcome as measured by the Glasgow Outcome Scale. These efforts resulted in the NIH Coma Data Bank, CT studies, and early investigations of MRI organized around understanding the relationship between coma duration, brain imaging findings, and outcome which now included neuropsychological measures. Translational efforts in the 1970s and 80s were conducted in animal models to determine the injury mechanisms observed in humans (e.g., diffuse axonal injury, intracranial hypertension, neurotransmitter alterations), and conduct preclinical trials of drugs and other interventions. An important outcome of this work revealed that the location and nature of brain lesion were heterogeneous, but not consistently related to patient outcome, with a few outstanding examples (e.g., depth of lesion, Marshall's CT classification). The field introduced clinical markers including genetics and premorbid characteristics (cognitive reserve) and later, Common Data Elements for imaging and outcome measures. The field takes a much longer view of outcome as life-long consequences. Today TBI research integrates imaging, genetics, neurochemistry, health, and individual factors to understand recovery and risk for later neurodegeneration. This approach is consonant with identifying individualized treatments. These efforts are organized around new data sharing mechanisms and Common Data Elements within research which holds the promise of understanding the myriad factors that contribute to TBI injury, recovery, and long-term outcome.

Correspondence: Harvey Levin, Ph.D., One Baylor Plaza MS637, Houston, TX 77030,United States.E-mail: hlevin@bcm.edu

\section{E.D. BIGLER. From Volumetrics to Brain Function, Lessons from TBI Neuroimaging.}

The goal of this talk is to take a historical perspective on the use of imaging to understand the consequences of brain injury on cognitive deficit and recovery of function. First, I will review the early structural imaging and brain volumetric studies from the 1970s and $80 \mathrm{~s}$ designed to document the consequences of TBI and predict patient outcome. Given the limitations of volumetrics/structural imaging alone, we then discuss developments of advanced functional and structural brain imaging in the late 1990s and 2000s including functional MRI (fMRI) and diffusion tensor imaging (DTI). There are now dozens of studies using these approaches to understand the structural and functional consequences of TBI, and it is a goal in this talk to review the primary findings, but also to outline the challenges to this work. One important development is the movement toward multi-modal imaging which provides novel metrics for trauma-induced brain changes in white matter, brain function and neurometabolism. Led by data sharing efforts (e.g.. FITBIR. TRACK II) to integrate the outcome of large-scale studies using multi-modal imaging, we now enter a new era in the use of brain imaging methods to understand the consequences of TBI.

Correspondence: Erin D. Bigler, Ph.D., Psychology and Neuroscience, Brigham Young University, 1001 SWKT, Provo, UT 84602, United States.E-mail: erin_bigler@byu.edu

H.S. LEVIN, E.D. BIGLER \& F.G. HILLARY. TBI in the age of the human connectome: a critique for a brave new small world.

In the new era of big data and brain science, connectivity modeling has become a focus within the clinical neurosciences with the goal of understanding how brain networks are organized and give rise to complex behavior (Biswal, 2010; PNAS). The goal of this talk is to introduce the concepts of network science and how these approaches have been used recently examine the consequences of traumatic brain injury. In this review we focus on one of the most commonly used mathematical applications to understand brain networks, graph theory, a branch of discrete mathematics. Graph theory has been leveraged over the past decade to examine the consequences of TBI on neural network functioning and in this review we focus on the implementation of graph theory with RSFC methods in fMRI datasets. We survey the connectivity literature to determine how graph theory is being used to form networks and the types of conclusions being drawn from those networks when clinical and healthy samples are juxtaposed. It is my goal to provide an overview of methods used in the TBI literature and recommendations for the role connectivity modeling methods may play in advancing diagnostics and prognostication in the various forms of brain injury Correspondence: Frank G. Hillary, PhD, Psychology, Pennsylvania State University, 313 Moore Building, University Park, PA 16802, United States.E-mail:fhillary@psu.edu 


\section{Paper Session 5. Vascular Disease and Injury}

\section{Moderator: Angela L. Jefferson}

$$
\text { 5:00-6:30 p.m. }
$$

S.B. PILLAY, D. BOOK \& J. BINDER. Where is Wernicke's area? A voxel-based lesion-symptom mapping study of spoken language comprehension in chronic aphasia.

Objective: Classical aphasia models localize "speech comprehension" to the posterior superior temporal gyrus (pSTG). Modern evidence suggests this region is involved in phonological processing for speech production, and that language comprehension engages frontal, temporal, and parietal networks involved in semantic, syntactic, and general executive processes. Spoken language consists primarily of multi-word utterances rather than single words. Comprehension of such stimuli places demands on the ability to combine word meanings using semantic and syntactic processes. Here we use VLSM to localize these processes that are unique to spoken comprehension, with the aim of providing a more meaningful definition of "Wernicke's area".

Participants and Methods: Participants were 51 right-handed patients with chronic LH stroke. They performed an Auditory Description Naming (ADN) task in which they heard a description and had to name the object described, a picture naming (PN) task, and an auditory sentence comprehension (ASC) task. Lesions were mapped using high-resolution MRI. VLSM analyses identified the lesion correlates of ADN and ASC impairment, first with no control measures, then adding PN impairment as a covariate to control for language and domaingeneral processes.

Results: ADN and ASC deficits were associated with lesions in a distributed frontal, temporal, and parietal language network. When PN impairment was included as a covariate, both ADN and ASC deficits were uniquely correlated with damage localized to the mid-to-posterior portion of the middle temporal gyrus (MTG).

Conclusions: The region most uniquely associated with spoken comprehension impairment is the mid-to-posterior MTG. This region appears to be critically necessary for the ability to integrate multi-word utterances during comprehension of spoken language. We propose that this function and its localization in the MTG provide a meaningful and clinically useful modern definition of Wernicke's area.

Correspondence: Sara B. Pillay, Medical College of Wisconsin, 8701 Watertown Plank Road, Center for Imaging Research, MEB 4670, Milwaukee, WI 53226, United States. E-mail: sara.berentsen@gmail. com

E.C. MIOTTO, B.M. HAMPSTEAD, P.R. BAZAN, A.B. XAVIER, M. CASTRO, M.M. MARTIN, E.G. FIGUEIREDO, A.B. CONFORTO, S.S. SIMON, C.M. BOTTINO, E. AMARO JUNIOR \& M.J. TEIXEIRA. Resting State Functional Connectivity and Neural Correlates of Face-Name Encoding in Patients with Left Frontoparietal Stroke. Objective: Face-name association impairment after stroke affects social interactions. To our knowledge, no study examined functional connectivity and face-name encoding after left frontoparietal stroke (LFPS), areas engaged in this ability. We investigated resting state functional connectivity (RSFC) and encoding of face-name in LFPS patients and healthy controls (HCs) using fMRI

Participants and Methods: 12 chronic LFPS patients and $18 \mathrm{HCs}$ were scanned $(3.0 \mathrm{~T})$ during encoding of face-name pairs and at resting-state. We created lesion masks to reduce the impact of affected regions during linear registration (MNI_152 2mm). BOLD signal at encoding of face-name pairs against a baseline fixation cross and recognition after scanning were evaluated. Left inferior frontal gyrus lesion patients (LIFG $n=7$ ) were compared to those without lesions in this area $(\mathrm{nLIFG} \mathrm{n}=5)$. In order to assess differences in RSFC we used ICA and dual regression approach

Results: LFPS and HC groups showed activated and deactivated areas within the default mode (DMN), frontoparietal (FPN) and visual networks (VN). The LIFG and nLIFG groups differed in activation mainly in the left inferior and precentral gyri during encoding and the LIFG group showed the poorest recognition performance. RSFC indicated differences in two networks between LFPS and HCs: left FPN showed higher correlation to the right supplementary motor area, parahippocampal and temporal pole in HCs; VN showed higher correlation to bilateral precuneus, posterior cingulate cortex and right insula in HCs Conclusions: Differences in activation between the LIFG and nLIFG groups were associated to reduced recognition performance in the LIFG group and suggest a crucial role of this region for face-name encoding. Higher correlation in HCs in the right hemisphere indicated reduced interhemispheric connectivity in LFPS patients, even in regions not directly affected by the stroke

Correspondence: Eliane C. Miotto, PhD, Department of Neurology, Hospital das Clinicas, University of Sao Paulo, Alameda Casa Branca 978 apt 41, Sao Paulo01408-000,Brazil.E-mail: ecmiotto@usp.br

K.J. BANGEN, A.L. CLARK, E.C. EDMONDS, N.D. EVANGELISTA, M.L. WERHANE, Z.Z. ZLATAR, D.A. NATION, M.W. BONDI \& L. DELANO-WOOD. Cerebral Blood Flow and Amyloid- $\beta$ Interact to Affect Memory Performance in Cognitively Normal Older Adults.

Objective: Cerebral blood flow (CBF) alterations and amyloid- $\beta$ (A $\beta)$ accumulation have been independently linked to cognitive deficits in older adults at risk for dementia. Less is known about how CBF and A $\beta$ may interact to affect cognition in cognitively normal older adults. Therefore, we examined CBF $\mathrm{x} A \beta$ interactions in regions typically affected in Alzheimer's disease (AD) within a sample of older adults from the Alzheimer's Disease Neuroimaging Initiative (ADNI) study.

Participants and Methods: 60 cognitively normal participants (mean age $=72$ years) underwent neuroimaging and memory testing. Arterial spin labeling MRI was used to quantify CBF and florbetapir PET amyloid imaging was used to measure $A \beta$ deposition. Amyloid positivity versus negativity was determined based on established cutoffs (Landau et al., 2013). The Rey Auditory Verbal Learning Test (RAVLT) was used to assess memory.

Results: Linear regression models adjusted for age, education, and pulse pressure, demonstrated significant CBF x A $\beta$ interactions on memory performance. Among A $\beta$-negative older adults, higher CBF in the hippocampus, precuneus, posterior cingulate, and pericalcarine cortices was significantly associated with better verbal memory performance $(p$-values $<0.05)$. In contrast, among A $\beta$-positive older adults, there were trends toward significant associations between higher CBF in these regions and poorer memory performance.

Conclusions: Our findings demonstrate that higher CBF supports memory performance in cognitively normal older adults free of significant $A \beta$ accumulation but not for those who exceed the threshold for amyloid positivity. Results suggest that CBF alterations and differential associations with cognition can be identified in healthy, asymptomatic $\mathrm{A} \beta$-positive older adults. Findings indicate that $\mathrm{CBF}$ and its associations with cognition may have utility as a reliable marker of brain function early in the $\mathrm{AD}$ process when interventions are likely to be beneficial. Correspondence: Katherine J. Bangen, VA San Diego Healthcare System/UC San Diego, Department of Psychiatry, UC San Diego, 9500 Gilman Drive, mail code 151B, La Jolla, CA 92093, United States. E-mail: kbangen@ucsd.edu 
E. MOORE, J.E. NEAL, D. LIU,J.G. TERRY, S. NAIR, K.R. PECHMAN, J. WATCHMAKER, E. GORDON, F.E. CAMBRONERO, K.E. OSBORN, J.E. BOGNER, R. MARTIN-WILLETT, C. SEABOLT, M. BABICZ, J. THOMPSON, M. THURSBY, L. WALLJASPER, K. GIFFORD, T.J. HOHMAN, S.P. BELL, T.J. WANG, J.J. CARR, M. DONAHUE \& A.L. JEFFERSON. Arterial stiffness is related to decreased cerebral blood flow and increased cerebrovascular reactivity in cognitively normal older adults: The Vanderbilt Memory \& Aging Project.

Objective: Arterial stiffening is associated with clinical Alzheimer's disease (AD), which may be due to alterations in cerebral hemodynamics. We related arterial stiffening to cerebral blood flow (CBF) and cerebrovascular reactivity (CVR) in older adults with normal cognition $(\mathrm{NC})$. We also tested the interaction between arterial stiffening and a genetic susceptibility factor for AD (APOE) on CBF and CVR.

Participants and Methods: Vanderbilt Memory \& Aging Project participants free of stroke with $\mathrm{NC}$ were studied ( $\mathrm{n}=153,72 \pm 7,29 \%$ $A P O E-\varepsilon 4$ carriers). Cardiac magnetic resonance assessed central arterial stiffening of the aortic arch using pulse wave velocity $(\mathrm{PWV}, \mathrm{m} / \mathrm{s})$. Pseudo-continuous arterial spin labeling MRI quantified CBF $(\mathrm{mL}$ blood $/ 100 \mathrm{~g}$ tissue/min) and CVR (\% change in response to a hypercapnic stimulus). Linear regressions related $\mathrm{PWV}$ to $\mathrm{CBF}$ and $\mathrm{CVR}$ regions of interest (ROIs), adjusting for age, race/ethnicity, education. body mass index, Framingham Stroke Risk Profile, $A P O E-\varepsilon 4$ status, and corresponding ROI tissue volume. Models were repeated testing a PWV x $A P O E-\varepsilon 4$ interaction.

Results: Increased PWV related to decreased CBF in the frontal lobe $(\beta=-0.43, p=0.04)$ and increased $C V R$ in the temporal $(\beta=0.11, p=0.03)$ and occipital lobes $(\beta=0.13, \mathrm{p}=0.02)$. PWV $\mathrm{x} A P O E-\varepsilon 4$ related to $\mathrm{CBF}$ $(\beta=-1.62, p=0.01)$ and CVR in the temporal lobe $(\beta=0.31, p=0.04)$. Among APOE- $\varepsilon 4$ carriers, higher PWV was related to lower $\mathrm{CBF}$ and increased CVR. Excluding participants with atrial fibrillation and cardiovascular disease yielded similar effects.

Conclusions: In cognitively normal older adults, increased central arterial stiffening corresponds to lower CBF but increased CVR (likely reflecting a compensatory mechanism). The regional specificity of results varies by $A P O E$ genotype such that e4 carriers with increased stiffening have lower CBF and increased CVR localized to the temporal lobe. These effects, which exist in the absence of cognitive symptoms or $\mathrm{AD}$, cannot be explained by shared vascular risk factors, cardiovascular disease, or atrophy.

Correspondence: Angela L. Jefferson, PhD, Department of Neurology, Vanderbilt University Medical Center, 1207 17th Avenue Sout, Suite 204, Vanderbilt Memory \& Alzheimer's Center, Nashville, TN 37212, United States. E-mail: angela.jefferson@Vanderbilt.edu

M.L. WERHANE, K.R. THOMAS, E.C. EDMONDS, K.J. BANGEN, A.L. CLARK, D.A. NATION, M.W. BONDI \& L. DELANO-WOOD. Elevated pulse pressure and apolipoprotein-E genotype interact to affect functional decline in cognitively normal older adults.

Objective: The apolipoprotein-E (APOE)- $\varepsilon 4$ allele and increased vascular risk have been independently linked to Alzheimer's disease (AD) risk. However, few studies have characterized how these risk factors may interact to affect functional status over time, and those that do exist have focused primarily on clinical samples. Thus, we investigated the longitudinal relationship between APOE- $\varepsilon 4$ genotype and elevated pulse pressure (PP) on functional status in a well-characterized sample of cognitively normal older adults from the Alzheimer's Disease Neuroimaging Initiative (ADNI).

Participants and Methods: Participants (mean age $=74$ years) included 205 cognitively normal older adults who underwent APOE genotyping and blood pressure assessment, which was used to calculate baseline PP (systolic blood pressure - diastolic blood pressure). The Functional Activities Questionnaire (FAQ), which evaluates activities of daily living (ADLs), was completed by participants' informant at baseline, 1-, 2-, 3 -, and 4-year follow-up visits.
Results: Adjusting for age, sex, and education, multiple regression revealed that $\mathrm{APOE}$ genotype and $\mathrm{PP}$ were not significantly related to FAQ scores at baseline; however, repeated measures ANCOVA revealed that APOE genotype significantly modified the relationship between baseline PP and FAQ scores over a 4-year period. Specifically, elevated baseline PP was associated with greater functional decline over time in APOE-e4 carriers, but not non-carriers $(p=.006)$.

Conclusions: Our results demonstrate that the presence of the APOE$\varepsilon 4$ allele exacerbates the deleterious effect of elevated PP on functional decline in older adults. Given that the sample consisted of healthy, asymptomatic older adults at baseline, these findings have important implications for the early identification of individuals at risk for functional decline, as well as the development of treatment targets for improved independence and quality of life in at-risk older adults. Correspondence: Madeleine L. Werhane, PhD, SDSU/UC San Diego Joint Doctoral Program in Clinical Psychology, 4930 Del Mar Ave, 106, San Diego, CA 92107, United States. E-mail: mlwerhane@gmail.com

B.C. SCHNEIDER, H.A. KRESGE, J.E. NEAL, D. LIU, K.E. OSBORN, F.E. CAMBRONERO, K.R. PECHMAN, E. GORDON, F.S. BADAMI, B.C. JASPER, M. BABICZ, J.E. BOGNER, R. MARTIN-WILLETT, C. SEABOLT, J. THOMPSON, M. THURSBY, L. WALLJASPER, K. GIFFORD, T.J. HOHMAN, L.T. DAVIS \& A.L. JEFFERSON. Enlarged perivascular spaces and white matter hyperintensities more strongly relate to neuropsychological functioning than other neuroimaging markers of small vessel disease: The Vanderbilt Memory \& Aging Project.

Objective: Small vessel disease (SVD) increases risk for clinical Alzheimer's disease and dementia. This study tested the association between different neuroimaging markers of SVD and cognition among older adults free of dementia and stroke.

Participants and Methods: Vanderbilt Memory \& Aging Project participants free of clinical dementia and stroke $(\mathrm{n}=322,73 \pm 7$ years) underwent 3T neuroimaging acquisition (T1, FLAIR, SWI) and neuropsychological assessment. White matter hyperintensities (WMHs) were quantified via an automated algorithm. Lacunar infarcts, perivascular spaces (PVS) in the basal ganglia, and microbleeds were manually coded by a board-certified neuroradiologist. Linear regression related each SVD marker to neuropsychological performances, adjusting for age, sex, education, race/ethnicity, diagnosis, and $A P O E-\varepsilon 4$ status.

Results: Increased $\log$-WMHs related to slower Inhibition $(\beta=1.96$, $p=0.008)$, Number Sequencing $(\beta=1.20, p=0.046)$, and log-Letter Number Sequencing $(\beta=0.02, p=0.04)$, and worse Digit Symbol $(\beta=-$ $0.84, p=0.03$ ), Hooper Visual Organization Test (HVOT, $\beta=-0.23$, $p=0.02)$, and Animal Naming performances $(\beta=-0.45, p=0.004)$. A higher density of PVS related to slower log-Letter Number Sequencing $(\beta=0.14, p=0.001)$ and worse Tower $(\beta=-1.41, p=0.01)$, Digit Symbol $(\beta=-7.21, p<0.0001)$, HVOT, $(\beta=-0.88, p=0.02)$, and Biber Figure Learning Test Recognition performances $(\beta=-0.06, p=0.01)$. Increased microbleeds related to slower log-Number Letter Sequencing performance $(\beta=0.01, p=0.004)$. All infarcts models were null ( $p$-values $>0.07)$. Conclusions: In our cohort free of dementia and stroke, increased WMH burden corresponded to poorer cognition, but lacunar infarcts and microbleeds were mostly unrelated to cognition. PVS, previously considered a clinically benign imaging feature in older adults, related to worse cognition across several domains. Enlarged PVS may reflect arteriolar narrowing or interstitial fluid drainage impairments. Further research is needed to understand the clinical relevance of increased PVS density.

Correspondence: Brittany C. Schneider, TN, United States. E-mail: brittany.c.schneider@vanderbilt.edu 


\section{Paper Session 6. Substance Abuse}

\section{Moderator: Rosemary Fama}

$$
\text { 5:00-6:30 p.m. }
$$

R. FAMA, C. HARDCASTLE, S.A. SASSOON, N. ZAHR, A. PFEFFERBAUM \& E.V. SUllivan. Neurological and Nutritional Biomarkers of Cognitive Impairment in Alcoholics.

Objective: Alcohol dependence is associated with selective cognitive and motor impairment, which is heterogeneous in extent and pattern of deficits. Causes of heterogeneity are elusive and inconsistently accounted for by demographic or alcohol consumption differences. Here, we sought neurological and nutritional factors as possible contributors to heterogeneity in alcoholism-related cognitive impairment.

Participants and Methods: We tested 96 alcoholics (ALC: 27-75 yr) and 42 controls (NC: 28-76 yr) on attention/working memory (ATT/ WM), production (PROD), immediate memory (IM), delayed memory (DM), visuospatial (VS), and upper motor functioning (MOT), from which education-corrected composite scores were calculated. We also obtained data to operationalize criteria established by Caine et al. to detect signs and symptoms consistent with past subclinical Wernicke's encephalopathy (WE). Caine criteria were based on dietary deficiency, cerebellar dysfunction, low general cognitive functioning, and oculomotor abnormalities.

Results: ALC ( $\mathrm{n}=96$ ) scored significantly lower than NC on ATT/WM, PROD, IM, and DM. Of the 86 ALC with Caine criteria, 21 met 0 criteria, 39 met 1 criterion, 24 met 2 criteria, 2 met 3 criteria, and 0 met all 4 criteria. Examination of Caine subgroups (age-matched 44-75 $\mathrm{yr}, \mathrm{n}=70)$ revealed that ALCs meeting 2 or more criteria $(\mathrm{n}=26)$ scored lower in IM, DM, and MOT than ALCs meeting $0(n=18)$ or 1 criteria $(\mathrm{n}=26)$. In addition, lifetime alcohol consumption was correlated with ATT/WM ( $\mathrm{r}=-.60)$, PROD ( $\mathrm{r}=-.58)$, IM ( $\mathrm{r}=-.53)$, DM $(\mathrm{r}=-.59)$, and MOT $(r=-60)$ in ALCs who met 2 or more Caine criteria (Bonferroni correction $\mathrm{p}<.008)$.

Conclusions: Neurological, particularly balance impairment, and historical nutritional status were valuable in distinguishing subgroups of alcoholics with cognitive impairment and thus may serve as biomarkers of cognitive impairment and the observed heterogeneity in cognitive functioning in alcoholism. (Support: AA010723, AA017168, AA005965)

Correspondence: Rosemary Fama, Ph.D., Department of Psychiatry and Behavioral Sciences, Stanford University School of Medicine, 401 Quarry Road, Stanford University School of Medicine, Stanford, CA 94305, United States. E-mail: rfama@stanford.edu

\section{K.M. FRAZER, J.J. MANLY, G. DOWNEY \& C. HART. Assessing cognitive functioning in long-term cocaine users.}

Objective: Cocaine is the second most widely used illicit substance in the United States and concern has been raised about potential cognitive deficits associated with its pathological use. Empirical evidence evaluating the cognitive consequences of regular cocaine use, however, has been mixed. This study employed a comprehensive cognitive battery to compare the performance of individuals diagnosed with cocaine use disorder against two control groups: 1) non-drug users and 2) drug users who report no cocaine use.

Participants and Methods: This one-session, outpatient study was conducted at the New York State Psychiatric Institute. Sixty research volunteers completed the study. The National Institutes of Health (NIH) Toolbox Cognition Battery was used to assess cognitive functioning across six domains: executive functioning, attention, episodic memory, working memory, processing speed and language. Each participant's score was also compared against a normative database adjusted for age, education and sex.

Results: There were no group differences across cognitive tasks with one exception: the drug-using control group outperformed cocaine use disorder and non-drug-using control groups on a measure of cognitive flexibility. The mean cognitive scores for all groups fell within the normal range for all tasks.

Conclusions: Cognitive functioning of individuals diagnosed with cocaine use disorder is similar to control group participants and overwhelmingly falls within the normal range when compared against normative data.

Correspondence: Kirsten M. Frazer, MA, Psychology, Columbia University, 575 Riverside Drivei, New York, NY 10031, United States. E-mail:kmf2143@columbia.edu

B.W. REYNOLDS, M.R. BASSO, L. LAU, A.K. MILLER, D. COMBS \& D. WHITESIDE. Ideational Fluency, Sensation Seeking, and Anxiety: A Recipe for Binge Drinking.

Objective: Binge-drinking is a significant problem among young adults (National Institute of Alcohol Abuse and Alcoholism [NIAAA], 2016), and corresponds with brain damage (Crews et al., 2000), and changes in mood, attention, memory, and executive functioning (Parada et al., 2012). However, existing studies have largely neglected to address relative contributions of executive function and personality to bingedrinking behavior. The current study addressed these shortcomings.

Participants and Methods: Forty-nine healthy undergraduates were administered D-KEFS Design Fluency to assess mental flexibility. Risky decision-making was assessed with the Iowa Gambling Task (IGT; Bechara et al., 1994). Impulsive personality was measured with the UPPS-P (Cyders et al., 2007). The Mood and Anxiety Symptom Questionnaire (MASQ; Watson \& Clark, 1991) assessed distress. Additionally, the Marlowe-Crowne Social Desirability Scale (MCSDS; Crowne \& Marlowe, 1960) and Reliable Digit Span from the WAIS-IV (RDS; Greiffenstein et al., 1994) assessed response bias. Binge-drinking was defined by consumption of 5 drinks if male, or 4 drinks if female over a 3 -hour period at least once in the past two months (NIAAA, 2016). Results: The data yielded 26 non-binge drinkers and 23 binge drinkers. Binge drinking occurrence significantly correlated with D-KEFS Design Fluency, the Sensation Seeking Scale from the UPPS-P, IGT performance, and MASQ Anxious Arousal. Forced entry logistic regression revealed that all but the Sensation-Seeking variables significantly predicted binge-drinking status, with $78 \%$ overall classification, $83 \%$ sensitivity, and $73 \%$ specificity.

Conclusions: These results suggest that binge consumption corresponds with diminished executive function in ostensibly healthy young adults. Importantly, our research uses well-established indicators of constructs implicated in alcohol misuse. Future research should seek to qualify predictors that effectively classify those whom engage in binge-drinking over time.

Correspondence: Bradley W. Reynolds, PhD, Psychology, University of Tulsa, 800 S Tucker Road, Lorton Hall 308, Tulsa, OK 74104, United States.E-mail:bradley-reynolds@utulsa.edu

V. OKUNEYE, A. SMITH, E. MORGAN, S. KEEDY, S.J. HUNTER \&. J. SCHNEIDER. Evidence of increased reward sensitivity in Young Black Men Who Have Sex With Men (YBMSM) who are heavy cannabis users.

Objective: Marijuana use appears to be an important factor for the engagement in risk behavior by YBMSM. Understanding its use may lead to improved strategies for preventing HIV transmission. We examined the association between cannabis use and neurocognitive systems supporting decision making and reward sensitivity in YBMSM.

Participants and Methods: 10 daily cannabis users and 10 non-users, all YBMSM between 18-30 yrs of age, performed a modified version of the Iowa Gambling Task during fMRI scanning. Task included 100 trials of selecting 1 card from 4 decks. 2 decks were "risky," associated with net loss of money over time but infrequent large rewards, and 2 decks were "safe," with net gain over time. Subjects were given immediate feedback on amount won or lost. Neural response was modeled during the deck selection and feedback evaluation. All image processing was conducted with AFNI. 
Results: Behaviorally, heavy marijuana users implicitly learned to choose riskier decks compared with non-users. Neuroimaging demonstrated that relative to non-users, heavy users had greater left dorsal caudate, ventral basal ganglia, and anterior cingulate response to "win" feedback relative to loss feedback. Non-users had less differential response. Choice of risky decks was associated with increased activation in parahippocampal and orbital frontal cortex in heavy users compared to non-users. Non-users showed greater dorsolateral prefrontal activation during risky relative to safe deck choice.

Conclusions: Findings suggest that in YBMSM, heavy cannabis users have stronger neural response to reward relative to punishment, and utilize different neural systems to support decision-making and/or learning about risky vs. safe choices. This is consistent with previous findings of heightened reward sensitivity and decreased response to loss in cannabis users more generally. Further confirmation of these findings is needed, given small sample size, and may have important implications regarding learning and sexual decision-making in YBMSM.

Correspondence: Scott J. Hunter, PhD, Psychiatry \& Behavioral Neuroscience, University of Chicago, UCM PBN, MC 3077, $5841 \mathrm{~S}$ Maryland Ave, Chicago, IL 60637, United States. E-mail: shunter@ uchicago.edu

R. MIGLIORINI, A.C. MAY, J. STEWART, M.P. PAULUS \& S. TAPERT. Exaggerated Aversive Interoceptive Processing in Adolescent Substance Users: An Early Risk Factor for Addiction? Objective: Aberrant neural processing of information from inside the body (i.e., interoception) may play a role in addiction neurobiology. Individuals with substance use disorders (SUD) exhibit altered patterns of activation in interoceptive brain regions, including insula, anterior cingulate cortex (ACC), and striatum. To investigate whether abnormal interoception precedes clinically significant substance use, this study examines functional magnetic resonance imaging (fMRI) response to unpleasant interoceptive sensations in adolescents with varied substance use histories.

Participants and Methods: Three groups (ages 15-17y) were compared on fMRI activation while experiencing periods of an aversive sensation (inspiratory breathing load): (1) substance experimenters without a SUD (EXP, n=16), (2) users who met criteria for SUD (SUD, n=17), and (3) non-using controls (CON, n=19). Mixed effects models were used to examine main effect of group and group by condition (anticipation vs. experience of breathing load) interactions across a priori regions of interest: insula, $\mathrm{ACC}$, and caudate $(\alpha=.05)$.

Results: Group main effects indicated that SUD exhibited greater left ACC activation than EXP and CON, whereas EXP showed lower left caudate activation than SUD and CON. Group by condition effects revealed that in response to the change from anticipation to breathing load: (1) SUD exhibited greater right posterior insula activation than CON; (2) SUD and EXP showed greater right mid-insula activation than CON; and (3) CON exhibited greater right ACC activation than SUD and EXP.

Conclusions: Both EXP and SUD demonstrated aberrant neural responses during aversive interoceptive stimulation compared to CON, which may reflect exaggerated negative reinforcement processing. The presence of neural alterations in sub-clinical alcohol and drug "experimenters" preliminarily suggests that abnormal interoception could be an early risk factor for maladaptive substance use. Supported by NIDA 5P20DA027843, NSF DGE-1247398

Correspondence: Robyn Migliorini, SDSU/UCSD Joint Doctoral Program in Clinical Psychology, 6363 Alvarado Court, Suite 103, San Diego, CA 92120, United States. E-mail: robyn.migliorini@gmail.com
J. SCOTT, D.H. WOLF, M.E. CALKINS, T.M. MOORE, J.D. JONES, K. RUPAREL, C.T. JACKSON, R.E. GUR \& R.C. GUR. Adolescent and Young Adult Cannabis Users Do Not Show Declines in Neuropsychological Performance: A Two-Year Longitudinal Study.

Objective: Cannabis use has been associated with alterations in cognitive functioning in adolescents and young adults, although few studies have used community-based samples to examine changes in neurocognitive functioning associated with initiating or continuing use, especially in less frequent cannabis users.

Participants and Methods: We examined associations between cannabis use and change in cognitive functioning over an average of 726 days $(\mathrm{SD}=216)$ using repeat assessment with the Penn Computerized Neurocognitive Battery (CNB). 279 youths from the Philadelphia Neurodevelopmental Cohort, ages 14-21 at baseline, were classified by cannabis use into Non-Users ( $\mathrm{n}=162)$, Occasional Users ( $\mathrm{n}=77$; use 1/ week to $1 /$ month), and Frequent Users ( $n=40 ; 3-4 /$ wk or daily). Mixed models examined main effects of group and time, as well as group $\mathrm{x}$ time interactions for age-normed cognitive domain accuracy scores in executive control, complex reasoning, episodic memory, and social cognition. Results: Main effects of cannabis group were found in memory and social cognition $(p<.01)$; follow-up analyses showed that Occasional Users were more accurate than Non-Users at both visits. Significant group by time interactions were found in memory and complex reasoning $(p<.01)$; follow-up analyses showed Frequent Users had a greater increase (i.e., steeper slope) in cognitive functioning across time. Results did not appreciably change in post hoc analyses examining only those who were adolescents at baseline, those who initiated or increased use, or Frequent Users who initiated use before age 16.

Conclusions: We found no evidence for cognitive decline over two years in frequent cannabis users, who surprisingly showed greater performance increases over time in some cognitive abilities. Occasional cannabis users showed stronger social cognition and memory functioning at both timepoints. Taken together, our results do not support a dose-response relationship between cannabis use and cognitive decline in adolescents and young adults over this time frame.

Correspondence: J. Cobb Scott, Ph.D., Psychiatry, Perelman School of Medicine at the University of Pennsylvania, 3400 Spruce Street, 10th Floor, Gates Building, Philadelphia, PA 19104, United States. E-mail: scott1@upenn.edu

\section{Paper Session 7. Sleep}

\section{Moderator: Melissa Lamar}

$$
\text { 5:00-6:30 p.m. }
$$

\footnotetext{
A. TSAPANOU \& N. SCARMEAS. Memory and Sleep Problems in the Elderly.

Objective: Sleep plays a crucial role to cognition. The aim of the present study is to examine specifically the association between memory performances and sleep problems in a Greek elderly population.

Participants and Methods: Cross-sectional design. Part of the Hellenic Longitudinal Investigation of Aging and Diet (HELIAD). 1338 elderly (over 65 y.o.) participants free of dementia and sleep medication, took part in the study. Sleep problems were estimated by using the Sleep Medical Outcomes scale. Additionally, a full neuropsychological assessment examining memory was conducted for each participant. Linear regression analysis was used to examine the association between memory and sleep problems, with the later as the independent variable. Age, sex, education, depression, and BMI were included as covariates. We conducted further analyses stratified by Apolipoprotein E- $\varepsilon 4$, as well as by sex.

Results: In the unadjusted model, there was a significant negative association between memory and sleep problems $(\beta=-0.11, p=0.001)$. Furthermore, memory was negatively associated with sleep duration
} 
$(\beta=-0.001, p=0.039)$, sleep adequacy $(\beta=-0.030, p \leq 0.001)$, as well as daytime sleepiness $(\beta=-0.017, p=0.017)$.

After controlling for the covariates, the importance between memory and sleep problems remained significant $(\beta=-0.007, p=0.011)$. Moreover, there was still an association between memory and sleep duration $(\beta=-0.001, p=0.004)$ as well as memory and sleep adequacy $(\beta=-0.014$, $p=0.013)$ but memory was no longer associated with daytime sleepiness. Conclusions: Worse memory was associated with sleep problems, independent of demographic and clinical factors, in a large sample of older Greek adults. Further research examining the underlying biological mechanisms is needed in order to shed more light into the present findings.

Acknowledgments: "IKY Fellowships of Excellence for Postgraduate Studies in Greece - Siemens Programme"

Correspondence: Angeliki Tsapanou, Taub Institute, Columbia University Medical Center, 630 West 168th Street, New York, NY 10032, United States.E-mail:at2859@cumc.columbia.edu

\section{J.T. FISCHER, H. HANNAY, P.R. SWANK \& L. EWING-COBBS. Sleep Disturbances and Internalizing Behavior Problems After Pediatric Traumatic Brain Injury.}

Objective: The current study aimed to investigate chronic sleep disturbance (SD) after traumatic brain injury (TBI) and extracranial/bodily injury (EI) in children and adolescents in comparison to typically developing children, and to better characterize longitudinal relations between SD and internalizing behavior problems.

Participants and Methods: Participants $(N=87)$ aged 8-15 included those with TBI, EI, and typically developing children (TD), and were assessed at pre-injury/baseline, 6-months, and 12-months. The parent-report Sleep Disturbance Scale for Children was used to assess SD. Internalizing behavior problems were measured via the Child Behavior Checklist.

Results: Controlling for age, the TBI and EI group experienced significantly higher levels of SD $(p=.042)$ and internalizing $(p=.024)$ than the TD group, but did not differ from each other. Injury severity was not strongly related to SD but was positively associated with internalizing behavior problems in the EI group. Cross-lagged panels examining longitudinal relations between internalizing and SD indicated that internalizing predicted later SD but not vice-versa, such that an increase in internalizing predicted an increase in SD. Mediation analyses demonstrated 6 month internalizing significantly mediated level of SD for both injury groups.

Conclusions: Traumatic injury predicted higher SD and internalizing. Injury severity was only positively significantly associated with SD and internalizing in the EI group. Although bidirectional relations between SD and internalizing disorders have been reported, the current study only identified significant pathways from internalizing to SD. Longitudinally, early internalizing problems predicted chronic SD. This study provides further evidence that internalizing problems may be a focus of intervention and highlights the importance of injury comparison groups in future studies investigating pediatric TBI. R01NS046308

Correspondence: Jesse T. Fischer, M.A., Psychology, University of Houston, 2111 Holly Hall St, Apt 3902, Houston, TX 77054, United States.E-mail: jessetfischer@gmail.com

\section{D.W. BEEBE, E. SLATTERY \& P.J. GUBANICH. Multi-night Sleep Restriction Increases Total Symptom Score on a Concussion Screener in Healthy Adolescents.}

Objective: Athlete-reported symptoms on concussion screeners correlate with short sleep the night before, even during pre-injury evaluations. This could be particularly important to understand in adolescents, who often get inadequate sleep on school nights. Here we study the effect of experimental sleep restriction (SR) on healthy adolescents completing a commercially-available concussion screening tool.

Participants and Methods: Twenty typically-developing 14-17 yearolds who had never experienced a head injury with loss of consciousness underwent a 3-week within-subject randomized cross-over protocol: a baseline week followed by 2 experimental weeks, in counterbalanced order, with 5 nights of 6.5 hours in bed (SR) vs. 9.5 hours in bed (Healthy Sleep; HS). Protocol adherence was verified via actigraphy. On the mornings following each week, adolescents completed ImPACT, a computerized assessment which is used to evaluate cognition and symptoms.

Results: Teens averaged 61/4 hours of sleep during SR and $81 / 2$ hours during HS, $p<.001$. Wilcoxon tests showed no changes on any ImPACT index score from cognitive tests, $\mathrm{p}>$.10, but adolescents reported significantly more symptoms during SR (raw=5.6) than HS (1.4), p=.006. Although notable effects were seen on items relating to sleep duration, fatigue, and drowsiness, there remained a significant effect $(p=.006)$ on the symptom composite after excluding five items manifestly related to sleep. Other items endorsed more during SR included headache, irritability, and feeling "slowed down."

Conclusions: Experimentally-induced short sleep, similar to what roughly $1 / 5-1 / 4$ of adolescents experience regularly on school nights, can increase report of symptoms often associated with concussion. Clinicians should consider whether short sleep might impact patient reports, timing of evaluation, or interventions that increase sleep.

Correspondence: Dean W. Beebe, Ph.D., Beh Med \& Clin Psychology, Cincinnati Children's Hosp Med Cntr, 3333 Burnet Avenue, MLC 3015, Cincinnati, OH 45229, United States.E-mail: dean.beebe@cchmc.org

A.M.K. MILEY-AKERSTEDT, J. SPAT, F. VIQAR, E. ROMAN, B.E. FRANK, M. BEDERSON, L. MARCUSE, M. FIELDS, J. YOO \& H.A. BENDER. Sleep Quality Negatively Impacts Cognition in Older Adults with Temporal Lobe Epilepsy.

Objective: Poor sleep quality in temporal lobe epilepsy (TLE) increases the likelihood of seizures and negatively impacts cognition. However, the effect of poor sleep on cognitive functioning in older adults with epilepsy remains unclear, despite the increased risk of co-occurring neurodegenerative disorders, such as Alzheimer's disease (AD). The current study aimed to examine the relationship between sleep quality and cognitive functioning among older adults with TLE.

Participants and Methods: Twelve older adults (mean age $=67.5 \pm 9.6$, mean education $=14.3 \pm 2.9 ; 76.9 \%$ female) with TLE were recruited from the Mount Sinai Epilepsy Center between 2012-2013. Participants met International Classification of Epilepsies (ILAE) criteria for TLE and those with co-occurring neurological and psychiatric illness, or history of alcohol/drug use/abuse were excluded. Participants underwent neuropsychological evaluation using tests culled from the NINDS Common Data Elements Battery. Self-reported measures of depression, anxiety, and health-related quality of life were completed. Sleep was assessed using the Pittsburgh Sleep Quality Index (PSQI; PSQI $\geq 5=$ poor sleep).

Results: Participants who were found to have poor sleep quality (PSQI $\geq 5, N=6$, mean PSQI=8.2 \pm 3.4 ) demonstrated a greater reduction in complex attention, verbal learning, verbal delayed recall, category fluency, nonverbal abstract reasoning, and mental flexibility on testing when compared to those with high sleep quality (PSQI $<5, \mathrm{~N}=5$, mean PSQI $=2.4 \pm 1.3$ ).

Conclusions: The results of the current study indicate that poor sleep quality is a prevalent problem in older adults with TLE and likely contributes to diminished neurocognition. In turn, data indicate that untreated sleep problems can exacerbate existing cognitive impairments in elderly individuals with TLE and should be evaluated, and potentially treated, during initial presentation.

Correspondence: Fawad Viqar, M.A., M.S., Psychology, Fordham University, 5 East 98th Street, New York, NY 10029, United States. E-mail:fawad.viqar@mssm.edu 
A. GOSSELIN, C.M. GECK, A. BENOIT, A. DOUGLASS \& J. DE KONINCK. Impact of Obstructive Sleep Apnea on Executive Functioning in Forensic Patients with Schizophrenia-Spectrum Disorders.

Objective: Obstructive sleep apnea (OSA) is a disorder with multiple negative neurobehavioral consequences. It is highly prevalent in individuals diagnosed with schizophrenia due to different risk factors (e.g., antipsychotics, obesity) and, particularly in forensic settings, patients with schizophrenia tend to display more impulsivity, which could be exacerbated by untreated OSA. Using Beebe and Gozal's (2002) model of executive dysfunction (ED) as a primary adverse outcome in untreated OSA, we hypothesized that forensic patients at higher risk of OSA will display more ED (e.g., higher impulsivity, emotionality and poorer cognitive performance) than those at lower risk of OSA.

Participants and Methods: Thirty male forensic patients diagnosed with schizophrenia-spectrum disorders from two forensic facilities in Ontario completed various subjective measures (i.e., Sleep Disorders Questionnaire 2-sleep apnea subscale (SDQ-2-SA), Difficulties in Emotion Regulation Scale (DERS), Barratt Impulsiveness Scale (BIS), Depression Anxiety and Stress Scale (DASS)) and a computerized Stop Signal Task (SST).

Results: Higher risk of OSA correlated with higher impulsivity (BIS, $\mathrm{r}=0.35, \mathrm{p}=0.056$ ), depression/anxiety (DASS, $\mathrm{r}=0.45, \mathrm{p}=0.01$ ), and marginally more emotion dysregulation (DERS, $r=0.32, p=0.08$ ). Higher risk of OSA also correlated with more errors on the SST number of hits: $r=-0.038, p=0.04$ and missed targets: $r=0.40 ; p=0.03)$.

Conclusions: Present findings support existing literature demonstrating associations between poor sleep and increased impulsivity and affect dysregulation among forensic patients (Kamphuis et al., 2014) and Beebe and Gozal's model of ED leading to daytime symptoms (e.g., poor behavioral control, increased emotional lability and impulsivity). Treating OSA among forensic patients could improve recovery and community reintegration outcomes although causality remains to be established.

Correspondence: Anik Gosselin, Ph.D., Forensic Treatment Unit, Brockville, The Royal Ottawa, 1600 County Road 2 West, Prescott, ON K0E1T0, Canada.E-mail: anik.gosselin@theroyal.ca

S.L. MARTINDALE, S. MORISSETTE, J. ROWLAND \& S.L. DOLAN. Sleep Quality Affects Cognitive Functioning in Returning Combat Veterans beyond Combat Exposure, PTSD, and Mild TBI History. Objective: Sleep disturbance is a frequent complaint among veterans and is common in the presentations of PTSD and TBI. Each condition affects cognitive functioning, however, it is unclear how sleep quality uniquely impacts cognitive functioning in combat veterans. The purpose of this study was to determine how sleep quality affects cognitive functioning in combat veterans beyond combat experiences, posttraumatic stress disorder (PTSD), and history of mild traumatic brain injury (mTBI) history.

Participants and Methods: Participants were 114 male veterans ages 22-55, deployed to the wars in Iraq and Afghanistan after 2001. Participants completed the DRRI-2 Combat Experiences Scale, the Mini International Neuropsychiatric Inventory, a TBI history interview, Pittsburgh Sleep Quality Index, and a neuropsychological assessment battery including the CVLT-II. WAIS-IV Digit Span Forward and Backward, D-KEFS Color Word Interference, Tower Test, Trails, and Verbal Fluency.

Results: Using partial least squares structural equation modeling, combat experiences and mTBI history were not directly associated with sleep quality. PTSD was directly associated with sleep quality, which contributed to deficits in neuropsychological functioning independently of and in addition to combat experiences, PTSD, and mTBI history. Combat experiences were negatively associated with motor speed and PTSD was positively associated with motor speed.

Conclusions: Sleep was associated with cognitive function independently of combat experiences, PTSD, and mTBI history. Sleep quality also contributed to cognitive deficits beyond effects of PTSD.
An evaluation of sleep quality may be a useful point of clinical intervention in combat veterans with cognitive complaints. Improving sleep quality could alleviate cognitive complaints, improving veterans' ability to engage in treatment.

Correspondence: Sarah L. Martindale, Ph.D., W.G. "Bill" Hefner VA Medical Center, 4801 Asherton Pl NW, Concord, NC 28027, United States.E-mail: Sarah.Martindale-Supak@va.gov

\section{Paper Session 8. Cross Cultural}

\author{
Moderator: To Be Announced \\ 5:00-6:30 p.m.
}

S. NA \& T.Z. KING. Disentangling Race-Related Differences on the Boston Naming Test: Contributions of Socioeconomic Status, Reading and Vocabulary on the Boston Naming Test in a Healthy College Sample.

Objective: The Boston Naming Test (BNT) is one of the most commonly used naming measures in neuropsychology. Research has shown, however, that BNT performance is affected by demographic factors (e.g., race, socioeconomic status (SES)). Although racial minority (MI) groups often perform more poorly on the BNT than racial majority (MA) groups, the mechanisms behind these differences are still being explored. BNT performance differences in MI and MA groups were hypothesized to be fully accounted for by factors such as SES and literacy.

Participants and Methods: 106 participants were collected from a southern urban university and divided into 2 groups: MA (50 Caucasians) vs. MI (33 African-American, 12 Asian, 7 Latino/a, 4 other). Groups were matched by age $(M=23.9(7.1))$, education $(M=14.3(1.6))$ and SES (Hollingshead, mother's education). The BNT, measures of verbal intelligence (WASI Verbal IQ), reading (WJ Letter Word ID), and vocabulary (WASI Vocabulary) were administered. Performance was age-normed and transformed into $\mathrm{z}$-scores.

Results: Despite being matched by SES and education, the MI group performed more poorly on the BNT $(\mathrm{z}=-.94(1.05))$ than the MA group $(\mathrm{z}=-.28(.92)), t(104)=3.44, p<.01$. Average BNT performance was lower than average VIQ (i.e., MI was $1.4 \mathrm{z}$-scores lower, MA was $1.1 \mathrm{z}$-scores lower). Both vocabulary (MI $\mathrm{z}=.53(.76), \mathrm{MA} \mathrm{z}=.88(.67), t(100)=2.42$, $p<.05)$ and reading $(\mathrm{MI} \mathrm{z}=.15(.56), \mathrm{MA} \mathrm{z}=.37(.50), t(99)=2.11, p<.05)$ were significantly different yet within normal limits for both groups. Two separate hierarchical regressions showed that race predicted additional variance in BNT after controlling for reading $\left(\beta=.19, \mathrm{R}^{2} \Delta=0.04\right.$, $p<.05)$ but not after controlling for vocabulary $(p>.05)$.

Conclusions: BNT performance was significantly different between groups after controlling for SES and reading ability. Differences between groups were not significant after controlling for vocabulary. Clinicians should interpret poor scores on the BNT with caution, especially in MI groups, and within the context of vocabulary skill.

Correspondence: Sabrina Na, Georgia State University, 235 Lincoln Court Avenue, Atlanta, GA 30329, United States.E-mail:s.diana.na@ gmail.com

S. SHAIR, V. KAVCIC, S. GARCIA, H. DODGE, S. NAVA, P. LICHTENBERG, H. PAUlSON, J. OVERALL, E. ROSE, S. CAMPBELL, C. TEBOE, A. BHAUMIK, B.M. HAMPSTEAD \& B. GIORDANI. Reliability of the CogState Brief and NIH Toolbox Cognition Batteries in African American Elders with Subjective Memory Complaints.

Objective: Limited research exists for test-retest reliability among computer-based assessments that are of interest in clinical trials; this is especially true for African Americans. The purpose of this study was to examine the relative and absolute reliabilities of the CogState Brief Battery and the NIH Toolbox Cognition Battery in a sample of African Americans. 
Participants and Methods: Participants were 46 community-dwelling African Americans (89\% female; age range $=61-85, M=71 \pm 5.3$; MMSE range $=24-30$ ) recruited through the Wayne State University Healthier Black Elders Center and the University of Michigan Alzheimer's Disease Center. Consensus diagnosis yielded 28 Normal Control (NC) and 18 Mild Cognitive Impairment (MCI) participants. Test batteries were administered twice across a four-month interval, with intraclass correlations (ICC) and magnitude of change measures (ANOVAs and effect sizes, $d$ ) calculated to derive relative and absolute reliability for the total sample and diagnostic groups.

Results: ICC showed an adequate to high degree of relative reliability between assessments for both computerized batteries (0.54-0.97), with the exception of Toolbox List Sorting Working Memory $(0.66, \mathrm{NC}=0.81$, MCI=0.36). In terms of absolute reliability, in the total sample, magnitude of change measures demonstrated significant retest score changes for Toolbox Total and Fluid composites, Flanker Inhibitory Control and Attention, List Sorting Working Memory, Picture Sequence Memory, and CogState Detection, with differences also evident on scores for NC and MCI groups separately.

Conclusions: Both of the computerized batteries showed reasonable relative reliability in a sample of African Americans with subjective memory complaints. Overall, these results support the use of computer-based assessments in the early detection of cognitive difficulties and for the monitoring of change over time, though care in interpretation should be taken when considering absolute change values for subtests reflecting working memory and attention.

Correspondence: Sarah Shair, MA, Neurology, University of Michigan, 2101 Commonwealth Blvd., Suite D, Ann Arbor, MI 48105, United States.E-mail:sshair@med.umich.edu

\section{J.L. PONSFORD, M. DOWNING \& H. PECHLIVANIDIS. The influence of cultural factors on outcome following traumatic brain injury.}

Objective: Most traumatic brain injury (TBI) outcome studies focus on white, English speaking patients who identify with the dominant health care system. Little is known of the experience of TBI individuals from Culturally and Linguistically Diverse (CALD) backgrounds. The present study aimed to compare outcomes following TBI in individuals from English-Speaking Backgrounds (ESB) with those from CALD backgrounds.

Participants and Methods: 104 ESB and 99 CALD participants with TBI were assessed an average 22.3 months post-injury on the Brief Acculturation Scale, Craig Handicap Assessment and Reporting Technique (CHART), Activities of Daily Living scale, Coping Scale for Adults, and Hospital Anxiety and Depression Scale.

Results: Results showed no significant group differences in most demographic and injury-related variables, although CALD participants showed lower pre-injury employment. There was no significant difference between groups in therapy costs. At post-injury follow-up, CALD participants were significantly less independent than the ESB group in light domestic duties, shopping and financial management, and reported lower cognitive independence, mobility and participation in occupational and social activities on the CHART after controlling for pre-injury employment. CALD participants reported heightened awareness of post-injury deficits relative to ESB participants, and held different beliefs regarding injury consequences and factors that would aid their recovery. The CALD group also reported greater anxiety symptoms and less problem-focused coping than the ESB group. Regression analyses showed that holding values other than Australian was a strong predictor of poorer outcome on the CHART, along with older age, lower education and longer time in hospital.

Conclusions: Poorer outcomes in CALD individuals with TBI are not simply reflective of socio-demographic factors. TBI clinicians need to consider their differing beliefs about injury and recovery in order to maximize outcomes in CALD individuals.
Correspondence: Jennie L. Ponsford, School of Psychological Sciences, Monash University, Apt 2001, 469 St kilda Road, Melbourne, VС 3004, Australia.E-mail: jennie.ponsford@monash.edu

A.E. WERRY, M. DANIEL \& B. BERGSTRÖM. Influence of Demographic Variables on Measures of Attention and Working Memory in Older Adults of Different Races.

Objective: Although research suggests demographic factors are differentially correlated with performance on attention and working memory (WM) tests, few studies examine this influence in older adults or consider specific racial groups. The objective of the current study was to determine the impact of demographic variables on attention and WM in neurologically healthy older adults of different races.

Participants and Methods: Subjects were 5311 Whites and 1098 African Americans from the National Alzheimer's Coordinating Center (gender: $36 \%$ male; mean/SD: age $=75.26 / 6.98$; education $=$ 15.70/2.91). Dependent variables for a series of multiple regression analyses included measures of Attention and WM - Digit Span Forward (DF), Digit Span Backward, Trail Making Test A \& B, and Digit Symbol (DS). Independent variables were age, education, and gender. Regression analyses were performed separately for White and Black groups.

Results: Age and education were statistically significant predictors on all attention and WM measures in both racial groups. Education was a stronger predictor and accounted for more variance on all attention and WM measures in the Black group than the White group. Age was a stronger predictor and accounted for more variance in the White group than the Black group on all attention and WM measures except DF. In both racial groups, gender was a statistically significant predictor only for DS.

Conclusions: Demographic variables predict attention and working memory performance differently for Black and White groups. Performance in the White group is more impacted by age on speeded measures and education for non-speeded measures while education has the largest influence on performance across all measures in the Black group. These findings highlight the importance of considering ethnicity and education when developing, norming, and interpreting neuropsychological tests. Correspondence: Amy E. Werry, Pacific University, 17610 NW Cornell Road \#9, Beaverton, OR 97006, United States.E-mail: amywerry@ pacificu.edu

G. FELIX, L.B. ZAHODNE, E. DALCHAND, K.M. FRAZER, M.N. MARTINEZ, H.L. SHOUEL \& J.J. MANLY. Illiteracy and Disparities in Cognitive Decline and Alzheimer's Disease among Spanish-Speaking Older Adults.

Objective: We sought to determine whether illiteracy was associated with more rapid cognitive decline, and conferred higher risk for Alzheimer's Disease (AD) compared to matched literates.

Participants and Methods: Participants were 674 Medicare eligible adults age 65 and older in the Washington Heights/Hamilton Heights Inwood Aging Project. Eligible participants were Hispanic, Spanish-speaking immigrants, attended $\leq 4$ years of school, and were not demented at baseline. Literacy was self-reported by asking: "Did you ever learn to read or write?" Neuropsychological tests measured language, visuospatial skill, speed, and memory at baseline and $18-24$ month follow-ups, and average follow-up was 4.96 years. At each visit, functional, cognitive, and medical data were reviewed and a diagnosis of $\mathrm{AD}$ was made using standard clinical criteria. Covariates included age at baseline, years of school, sex, nationality, medical conditions, and stroke. Growth curve models were used to determine the relationship of literacy to intercept and change in cognitive function. The relationship of literacy status to age of incident $\mathrm{AD}$ was tested using Cox proportional hazards models.

Results: Illiterates obtained lower scores on a composite neuropsychological test score than illiterates, but literacy had no effect on rate of decline. However, there was a significant, independent effect of literacy 
on incident AD risk, such that illiterates were $66 \%$ more likely to develop $\mathrm{AD}$ than illiterates $(\mathrm{OR}=1.66,95 \% \mathrm{CI}=1.15-2.39)$.

Conclusions: Illiteracy is independently associated with a higher risk of developing AD. This is due to lower initial level of cognitive function among illiterates that begins closer to the diagnostic threshold for dementia, but not due to more rapid rate of cognitive decline.

Correspondence: Gloria Felix, 630 West 168th St., P\&S Box 16, New York, NY 10032, United States. E-mail: gloriafelix2007@yahoo.com

L.D. MEDINA. Hippocampal Volume is Related to Cognitive Function in Non-Hispanics but not in Hispanics in a Case-Control Matched Sample.

Objective: Cross-cultural imaging research in the study of Alzheimer's disease is limited, but tends to support a relationship between smaller hippocampal volume and dementia in both Hispanics and nonHispanics. In Hispanics, this relationship appears to be partly mediated by the presence of white matter hyperintensities. However, extant research has been limited by baseline ethnic group differences. The current study aimed to address these limitations by examining this brain-behavior relationship in a case-control matched sample.

Participants and Methods: Data from 72 participants (36 Hispanic, 36 non-Hispanic) in the National Alzheimer's Coordinating Center (NACC) database were analyzed. Groups were matched on gender, handedness, and age. Matched groups did not differ in hippocampal or white matter hyperintensity volumes, or Clinical Dementia Rating level as well as in cognitive and subjective daily function scores. All participants were tested in English. Linear regression models were used to test the relationship between hippocampal volume and global cognitive ability (Mini Mental State Exam, or MMSE), episodic memory (Logical Memory, or LM), and informant-reported daily function abilities (Functional Activities Questionnaire, or FAQ).

Results: In non-Hispanics, hippocampal volume was related to MMSE $\left[\mathrm{F}(1,34)=10.772, p=0.002, \mathrm{R}^{2}=0.241\right]$ and $\mathrm{LM}[\mathrm{F}(1,34)=9.048$, $\left.p=0.005, \mathrm{R}^{2}=0.210\right]$. This relationship was not found in Hispanics: MMSE $\left[\mathrm{F}(1,34)=1.334, p=0.256, \mathrm{R}^{2}=0.038\right]$; $\mathrm{LM}[\mathrm{F}(1,34)=2.425$, $\left.p=0.129, \mathbf{R}^{2}=0.067\right]$. FAQ was not significantly related to hippocampal volume in non-Hispanics, $\mathrm{F}(1,34)=3.033, p=0.091, \mathrm{R}^{2}=0.082$, or Hispanics, $\mathrm{F}(1,34)=0.773, p=0.386, \mathrm{R}^{2}=0.023$.

Conclusions: Findings suggest that brain-behavior relationships may not be the same across ethnic groups. Interpretation is limited by a small sample size, but these results challenge the validity of current guidelines in the clinical diagnosis of cognitive impairment and Alzheimer's disease dementia in Hispanics. Further studies are needed to expand these findings.

Correspondence: Luis D. Medina, PhD, Neurosurgery, University of Colorado School of Medicine, 12631 E. 17th Ave., C307, Aurora, CO 80045, United States.E-mail:LUIS.MEDINA@ucdenver.edu

\section{Poster Session 5. Poster Symposia, Genetics, Cross Cultural}

$$
\text { 5:15-6:30 p.m. }
$$

\section{Behavioral Neurology/Cerebral Lateralization/Callosal Studies}

\section{J. SALVADOR-CRUZ, C.G. ARMENGOL \& D.S. TOVAR VITAL. Challenges in the Development of Lateralization: Implications for the Acquisition of Reading and Writing Skills.}

Objective:

To determine whether atypical development of lateralization, body schema, spatial organization and left/right discrimination are associated with reading and writing difficulties in elementary school children.
Participants and Methods: Forty-nine Mexican elementary school children (27 boys, 22 girls) aged 6-12 were selected from a pool of volunteers. Positive psychiatric history disqualified children from participating, based on responses to Salvador \&. Galindo's Neurological and Psychiatric History Questionnaire.

Lateralization was masured with Portellano's Neuropsychological School Maturity Questionnaire (NSMQ-CUMANES in Spanish). Matute et al's Neuropsychological Evaluation of Children (NEC; Spanish ENI) provided reading and writing skills data.

Results: Most participants (77.5 \%) exhibited non-homogenous lateralization (NHL). Sixty-seven percent were cross-dominant; $10.2 \%$ undefined). Full/homogeneous lateralization (F/HL) was $22.4 \%$ (right $=20.4 \%$, left- $2.0 \%$ left). No inter-group lateralization-associated differences were obtained for reading speed, but F/HL children's reading comprehension (low-medium to high-medium) surpassed that of cross-dominant children (very low to medium). Writing proficiency was higher for F/HL: most scores were "low" or "very low", but some "high" and "very high" scores were obtained). Scores for most cross-dominant children were "very low"; "medium" was their highest level attained. An age effect was noteworthy: cross-dominance was more frequent among 8-11 year-olds.

Conclusions: This study lends further documentation of the important link between brain development and lateralization and the broad spectrum of deficits that ensue from its disruption. Developing Interventions that aid in the acquisition of correct lateralization patterns and address anomalous patterns is a priority.

Correspondence: Carmen G. Armengol, Ph.D., Applied Psychology, Northeastern University, 280 Chestnut Ave, Jamaica Plain, MA 02130, United States. E-mail: c.armengol@neu.edu

\section{T. FRIEDRICH, A. SMITH \& L. ELIAS. The Impact of Age on} Navigation Asymmetry in Naturalistic Settings.

Objective: Pseudoneglect has been identified with tasks that involve navigation in extrapersonal space, as rightward veering and collisions have been found when walking or navigating motorized vehicles through apertures. The objective was to examine the effect of age on asymmetries in navigation within a naturalistic setting by examining the frequency of the location of impact on the participant's vehicle during near-crashes and crashes, as the magnitude of leftward bias demonstrated in pseudoneglect has been found to differ between younger and older adults.

Participants and Methods: The location of impact following nearcrashes and crashes, and participant's age and gender were retrieved from the SHRP2 NDS database. Over the course of the study 35 million vehicle miles were collected from 3546 participants, resulting in 2722 near-crashes and 1465 crashes.

Results: During near-crashes and crashes, irrespective of age, the location impact was most often in front of the participant vehicle. Unlike data reported in laboratory environments, in each age category, crashes and near-crashes on the right side of the participants' vehicle were not over-represented compared to the left of the vehicle.

Conclusions: This study is the first to examine age-related effects on asymmetry in navigation using crash analysis in a naturalistic setting. However, unlike rightward veering and collisions found in laboratory settings and differences in pseudoneglect between age groups, the location of impact on the participants' vehicle was not overrepresented on the right, irrespective of age category. These findings inform future research that attempts to apply laboratory research, regarding asymmetry in navigation, to naturalistic settings.

Correspondence: Trista Friedrich, PhD, Psychology, University of Saskatchewan, \#103-920 9th street east, Saskatoon, SK S7HON1, Canada.E-mail:ten505@mail.usask.ca 
A.K. HOLLAND, A. ROSA, C. BLANCO \& D.W. HARRISON. Support for the Capacity Model of Hostility Using a Dichotic Listening Paradigm: Reductions in Cerebral Laterality for Phoneme Detection Indicate Compromised Cognitive Control in High Hostile Men.

Objective: The capacity model (Holland et al., 2012) states that high hostility results from reduced right frontal regulatory control over posterior regions under dual task demands. For the current research, a dichotic listening paradigm was used to examine the hypothesis that high hostiles would perform poorly on this task relative to low hostiles. Diminishment in performance would be especially evident in the Focus Left condition, which is conceptualized as requiring cognitive control (Hugdahl et al., 2009), or the ability to inhibit the predominant tendency to report phonemes presented to the right ear (left hemisphere).

Participants and Methods: High hostile ( $n=17)$ and low hostile $(n=17)$ men completed the No Focus (NF), Focus Left (FL), and Focus Right conditions of the dichotic listening task before and after hearing a recording of angry infant vocalizations. Heart rate measures were taken across experimental conditions.

Results: A main effect for Condition was found for $\operatorname{HR}(F(1,32)=4.87$, $p=.03$ ), indicating that HR was higher after exposure to affective stress. A Hostile $\mathrm{x}$ Condition $\mathrm{x}$ Focus interaction was also found $(F(1,32)=4.1$, $p=.05)$, revealing that $\mathrm{HR}$ was higher post affective stress in the FL condition for high but not low hostile men. Finally, a Hostile x Condition $x$ Focus $x$ Ear interaction was found for phonemes reported $(F(6$, $96)=2.2, p=.04)$, indicating that low but not high hostile men showed a significant reduction in the phonemes reported at the right ear in the FL condition post stress.

Conclusions: The interaction found for phonemes reported indicates reduced cerebral laterality for high hostile men relative to low hostile men. This reduction in laterality is an indication of diminished right frontal regulatory capacity as a function of exposure to affective stress. High hostile men showed a reduced capacity to suppress the predominant tendency to report words presented to the right ear (left hemisphere), indicating compromised cognitive control.

Correspondence: Alissa K. Holland, Ph.D., Psychology, University of South Carolina Lancaster, 476 Hubbard Drive, Lancaster, SC 29721,

United States.E-mail: akhollan@mailbox.sc.edu

\section{Cross Cultural}

\section{J. AVILA. Socio-Cultural Impact on Stroop-Interference} Performance in Spanish-English Bilinguals.

Objective: Stroop Color-Word Interference (CWI) measures the ability to suppress an overlearned response in favor of an unusual response. While, consistent cross-language interference-suppression may advantage bilinguals on this task, cognitive, demographic and socio-cultural factors tend to mitigate superior performance. The purpose of this study was elucidate the role of socio-cultural factors in CWI performance in Spanish-English bilinguals.

Participants and Methods: Adult participants, aged 18 - 75 years, participated in this study: 41 Spanish-English Bilingual Hispanics (SEBH) and 77 monolingual English-speaking European Americans (MESEA). Stroop Test conditions assessed participants' processing speed (Color-Naming, CN, and Word-Reading, WR) and interference-suppression (CWI) performance. Additionally, reading ability (WRAT-3 Reading subtest), demographic (age), social (educational attainment, SES), and cultural (age acquired English, acculturation) characteristics were assessed.

Results: SEBH demonstrated significantly poorer performance in processing speed and interference-suppression compared to the MESEA. Hierarchical multiple regressions were conducted on each group separately, with CWI as the DV. After accounting for age (step 1) and processing speed (step 2 ), the addition of reading ability and socio factors (step 3 ) revealed significant $R^{2}$ changes in the MESEA model $\left(\Delta \mathrm{R}^{2}=10.3 \%\right)$, but not the SEBH model $\left(\Delta \mathrm{R}^{2}=2.9 \%\right)$. However, a significant change in $\mathrm{R}^{2}$ was revealed when cultural factors (step 4 ) were added to the SEBH model a $\left(\Delta \mathrm{R}^{2}=21.7 \%\right)$.

Conclusions: Findings suggest the bilingual advantage may depend on language acquisition and familiarity with US culture. Factors traditionally presumed to impact CWI performance may be confounded by cultural factors in non-MESEA groups. Failure to account for the socio-cultural experiences of SEBH individuals can result in inaccurate interpretation of cognitive ability.

Correspondence: Justina Avila, M.A., Psychology, University of New Mexico, 3003 Adams St. NE, Apt \# M74, Albuquerque, NM 87110, United States.E-mail: jfavila@unm.edu

D. BANUELOS, K. GONZALEZ, M. NAKHLA, K. KAUZOR \& J. RAZANI. The relationship between verbal and nonverbal neuropsychological tests and aspects of English fluency in ethnically diverse individuals.

Objective: The purpose of this study was to examine what aspect of English proficiency (with an objective language measure) best relates to the various neuropsychological tests in ethnically diverse individuals. Participants and Methods: Ninety-six Hispanic, Asian and MiddleEastern participants served in this study. Sixty percent were female, were on average 34.25 years and 14.36 years of education. Neuropsychological (NP) tests requiring verbal mediation (FAS, Animal naming, Trailmaking, Boston Naming Test) and no verbal mediation (Rey-Osterreith copy \& 3 minute delay, Wisconsin Card Sorting Test. Matrix Reasoning, Block Design) were administered. The Woodcock Language Proficiency Battery- Revised (WLPB-R) subscales of written and reading subscales were administered to assess English fluency.

Results: Bivariate correlation analyses were performed. The results revealed that while there were significant relationships between both the written and reading comprehension subscales of the WLPB-R and verbal and nonverbal NP tests, there were some subtle differences. Trails A did not correlate with writing subscale. Interestingly, the majority of the nonverbal NP tasks also significantly related to both the written and reading comprehension subscales, with the exception of the Rey-O. Conclusions: The findings of this study highlight the importance of the relationship between English fluency and verbally mediated NP tests. As importantly, English proficiency is also related to nonverbally mediated $\mathrm{NP}$ tests in ethnically diverse, immigrant populations. These findings are important to both research and clinical neuropsychologist for assessing NP skills of ethnically diverse immigrant groups.

Correspondence: Dayana Banuelos, Psychology, California State University Northridge, 18301 Chatsworth St., Northridge, CA 91326, United States.E-mail: dayana.banuelos.140@my.csun.edu

A.T. FLOWERS, S. MICHEL, G.M. CASTILLO, K. KAUZOR \& J. RAZANI. Socioeconomic Status and Neuropsychological Assessment Performance.

Objective: There is limited research examining the relationship between socioeconomic status (SES) and neuropsychological performance in ethnically diverse populations. Due to the difficulty to objectify SES across different ethnic and immigrant groups studies have explored subjective measures of SES in health research with considerable success. The purpose of this study was to determine if there were any differences in performance on neuropsychological measures amongst individuals based on their own, subjective assessment of their SES.

Participants and Methods: 134 healthy individuals were recruited from the Greater Los Angeles area from Caucasian, Hispanic, Middle Eastern, and Asian backgrounds. A subjective measure of SES was utilized which asked participants to rate their SES status while growing up from the following 3 categories (lower, middle, and upper class). Participants were administered tests of language (FAS and animal fluency; Boston Naming Test), and executive functioning (Wisconsin Card Sorting Test; Color Trails; Trailmaking, Stroop).

Results: A MANCOVA, with education as the covariate revealed significant differences across all three SES groups performance on all 
neuropsychological measures. Post hoc analysis revealed middle SES group performed better than lower SES group on the Stroop B (color naming test). However, the results revealed that the upper SES group performed poorer than the middle and lower SES on the Boston Naming Test.

Conclusions: These findings revealed somewhat counterintuitive results that highlight the difficulty in assessing SES accurately, particularly as it relates to understanding neuropsychological performance in ethnically diverse groups. These findings, implications and possible interpretations will be further explored.

Correspondence: Amina T. Flowers, Clinical Psychology, Psychology, California State University, Northridge, 5454 Brynhurst Avenue, Los Angeles, CA 90043, United States. E-mail: flowersamina@gmail.com

K. KAUZOR, A.T. FLOWERS, G.M. CASTILLO, M. NAKHLA, J. HERRERA, D. BANUELOS \& J. RAZANI. Hispanic Performance on Verbal and Non-verbal Neuropsychological Tests.

Objective: Previous research has demonstrated a relationship between acculturation and neuropsychological test performance in Hispanic individuals. However, which specific component of acculturation best predicts neuropsychological performance has not yet been examined. The present study aimed to examine the relationship between specific aspects of acculturation in a Hispanic group on standard verbal and non-verbal neuropsychological measures.

Participants and Methods: 50 Hispanic individuals from Mexican, Central American, and South American backgrounds participated. Participants were administered routine neuropsvchological tests. and verbal and nonverbal composite z-scores were created. Additionally, all participants completed a measure which assed 4 aspects of acculturation: preference, ethnic identity, exposure to culture, ethnic interaction. Results: Bivariate correlation revealed that the verbal scores were significantly correlated with ethnic identity $(r=.335, p=.040)$, ethnic interaction $(\mathrm{r}=.585, p<.001)$ and years educated outside the U.S. $(\mathrm{r}=$ $-.543, p<.001)$. Non-verbal scores were significantly correlated with only ethnic identity $(r=349, p=.032)$. Simultaneous regression, using education, years of education outside of the U.S. and the 4 acculturation factors revealed that these variables significantly predicted verbal scores. However, in the presence of all factors, ethnic identity was the only significant predictor $(\beta=.555, t(19)=2.470, p=.019)$ of the verbal scores. Conversely, none of the factors significantly predicted the non-verbal scores.

Conclusions: Overall, these results suggest that specific aspects of acculturation, such as how one identifies with their ethnicity or the amount of interactions one has with their culture can impact the neuropsychological performance for Hispanic individuals.

Correspondence: Kaitlyn Kauzor, psychology, California State University, Northridge, 28716 Kathleen Ave., Santa Clarita, CA 91390. United States.E-mail:kaitlyn.kauzor.943@my.csun.edu

S. LEONG, J. NGWA, J. TURNER, S. WOLDAY, O. OGUNLANA, S.P. JOHNSON, R. MURRAY, D. GAMBLE, L. GRAHAM, J. ALLARD, O. NTEKIM, T. FUNGWE, C. CASTOR, R. GILLUM \& T. OBISESAN. Exploring the Mini-Mental Status Exam in an African American Sample in a Primarily Urban Setting.

Objective: The Mini-Mental Status Exam (MMSE) is a 30 item questionnaire used to initially assess cognition in the clinical settings and to establish a neuropsychological threshold for subjects' inclusion into clinical trials on Alzheimer's disease (AD). Given the paucity of data on MMSE sensitivity when evaluating ethnic minorities or those with lower levels of education, a disproportionately higher number of cognitively-normal ethnic minorities may be inaccurately considered cognitively impaired. In particular, this may result in an overestimation of disease severity for age-related dementias in African Americans (AAs). Although education and age adjusted population-derived estimates for the MMSE are available, data is lacking on estimates derived from predominantly AA samples. Therefore, we aim to compare current raw and adjusted
MMSE scores in a sample of AAs in a primarily urban setting to the currently available estimates in the literature.

Participants and Methods: Of 3266 participants, 2933 (86.5\%) were AAs recruited from the Washington DC metropolitan area. Age and education adjusted MMSE scores are presented using published methods (Mungas et al. 1996). We additionally computed raw means by gender, categories of age and education, and used ANOVAs to examine expected education and age-based differences in raw and adjusted MMSE scores. SPSS was used to analyze the data and generate means \pm standard deviation.

Results: Overall the unadjusted mean $(27.11 \pm 3.46)$ for the entire sample, was similar to that of AAs $(27.11 \pm 3.47)$, probably because AAs constituted the majority of the sample. Whereas, AAs in our sample had an overall age and education-adjusted mean MMSE score of 26.11 \pm 3.19 , the means differed between men $(26.38 \pm 0.07)$ and women $(25.56 \pm 3.4)$

Conclusions: Our observation suggests that age and education-adjusted MMSE scores incorporate variance from social demographic factors that may account for discrepancies in current literature and provide a more accurate assessments of the cognitive phenotype in AAs.

Correspondence: Sharlene Leong, MA, Psychology, Howard University, 40 Upper Rock Circle, \#322, ROCKVILLE, MD 20850, United States. E-mail:shar.leong320@gmail.com

Z.A. MELIKYAN, A. MEJIA, S. RUBINO, H. KUWABARA, I. NAGORSKAYA, M. NOSOVITSKAYA, Y. WANG, A. PHELPS \& A.E. PUENTE. Rural Russian and American Populations' Performance on Color Trails Test and Trail Making Test.

Objective: Explore differences between rural healthy adults from Russia and the United States. Analyze group differences in performance on Trail Making Test (TMT) A and B and Color Trails Test (CTT) 1 and 2. Participants and Methods: Healthy adults who lived all their lives (except for own college years if any) in rural areas (population<50,000) and were native Russian/English speakers participated in the study. 52 Russian (21 male, 31 female) 18-85 years old and 28 American (12males, 16 females), 20-73 years old, with education $\leq$ Master's level were tested.

TMT A\&B and CTT $1 \& 2$ were administered. Completion time (sec.) and number of errors were analyzed using t-test, significant $(\mathrm{p}<0.05)$ results are reported.

Results: American group was significantly faster in completion of TMT A\&B. Russian group made more errors in TMT A\&B with TMT B being statistically significant. American group was significantly faster in completion of CTT $1 \& 2$. For CTT 2 number of non-completed tests and number of prompts was significantly higher in the Russian group, as was number of errors and near misses for both CTT 1\&2, which did not reach significance.

Conclusions: This is the first study to compare rural Russian and American populations on neuropsychological tests performance. Faster and more accurate performance in American group corresponds to results obtained previously for urban samples. Differences can be explained by less concern with timeliness and less experience with timed and standardized tests in Russian population, as well as less experience in Russian group with the alphabetical sequence.

Correspondence: Antonio E. Puente, PhD, Psychology, University of North Carolina, Wilmington, 601 South College Road, Wilmington, NC 28403,United States.E-mail: puente@uncw.edu

A. SEKHON, S.G. PATEL \& J. LEE. Cross-Cultural Differences and Acculturation Effects on WISC-IV Performance in Punjabi Children: A Pilot Study.

Objective: Cross-cultural neuropsychology research shows differences in cognitive assessment scores between ethnic minority and majority groups, with minority groups performing lower. However research focused on Punjabi youth remains inconclusive. This study will compare scores of Punjabi children on select subtests of the Wechsler Intelligence 
Scale for Children - Fourth Edition (WISC-IV) with its normative data. Participant acculturation styles will also be analyzed to determine whether acculturation relates to scores.

Participants and Methods: Participants were 25 Punjabi children (ages 6-16) recruited from Punjabi community centers near a major urban area of the United States. Participants were born and educated in the US (Age $=11.99$, SD: 2.9; Gender $=11 \mathrm{M} / 14 \mathrm{~F})$. Measures administered were the WISC-IV (Digit Span, Matrix Reasoning, Symbol Search, and Vocabulary), and the Acculturation, Habits, and Interests Multicultural Scale for Adolescents (AHIMSA). Statistical analyses included t-tests to compare means of the groups and a spearman rank correlation to determine the relationship between acculturation and WISC-IV performance.

Results: Significant differences were found between the scores of Punjabi children and the WISC-IV normative data on Vocabulary $(\mathrm{t}=$ $\left.2.46^{*}\right)$, Digit Span $\left(t=2.68^{*}\right)$ and Symbol Search $\left(t=2.42^{*}\right)$. A significant negative correlation was found between performance on Digit Span $(\mathrm{r}=-.48 * *)$, Vocabulary $(\mathrm{r}=-.68 * *)$ and acculturation style. Symbols note significance at $\left..01{ }^{* *}\right)$ and $.05\left(^{*}\right)$ level.

Conclusions: Results suggest when compared to normative data, Punjabi children had lower verbal fluency and visual scanning, but better working memory. Specific acculturation styles were also associated with subtest performance. While developing normative data for each ethnic group is unlikely, these results indicate ethnicity and acculturation should strongly be considered when using cognitive assessments and developing treatment recommendations.

Correspondence: Amardeep Sekhon, M.S., Clinical Psychology, Palo Alto University, 1791 Arastradero Rd, Palo Alto, CA 94304, United States.E-mail:asekhon@paloaltou.edu

\section{A.M. STRUTT, J. LAFOSSE \& M. MAHONEY. Assessment of language fluency and confrontation naming in monolingual Spanish speaking older adults.}

Objective: Although several translations and scoring modifications of fluency and confrontation naming measures have been proposed, a comparison between available versions in monolingual Spanish-speaking healthy controls has not been made. This study compared classification accuracy of the extant norms for phonemic and semantic fluency tasks. Confrontation naming tasks were reviewed, alternative linguistic responses were developed, and the sequence of stimuli presentation was compared between Spanish and English speakers.

Participants and Methods: One hundred sixty-eight primarily Spanish-speaking, cognitively intact U.S. resident volunteers (ages 50-80; 6-18 years of education) from 14 Spanish-speaking countries and with low levels of acculturation were administered these measures. Results: For phonemic fluency tests, participants scored highest on PMR versus FAS and ABS and age and education were not significant predictors. For semantic fluency tests, participants scored highest on the supermarket task; performance was related to age, but not education. Use of modified Boston Naming Test (BNT) norms resulted in 4-6\% fewer healthy Spanish-speaking controls being classified as impaired and $2-6 \%$ more being classified as within normal limits. Moreover, age and education predicted BNT score (Age $\beta=-.21, p=0.001$; Education $\beta=.372, p<0.001)$. New stimuli order of presentation is recommended following item analysis. Normative data are provided.

Conclusions: There are clear discrepancies between variations of phonemic and semantic fluency and confrontation naming tasks for monolingual Spanish-speakers residing in the U.S. Culturally and linguistically responsive use of these tasks along with appropriate normative data will aide in the differential diagnosis of neurodegenerative conditions.

Correspondence: Adriana M. Strutt, Ph.D., Neurology, Baylor College of Medicine, 7200 Cambridge, Houston, TX 77030, United States. E-mail: adrianam@bcm.edu
J.Z. STUART, T.J. FARRER \& S.E. COOK. Racial Differences in the Utility of the TOPF in a Hospital Based Outpatient Clinic.

Objective: Reading recognition measures are widely utilized as premorbid estimates in neuropsychological test batteries. Some measures, including the Test of Premorbid Functioning (TOPF), use demographically adjusted norms, in part, to account for racial differences which could be caused by educational factors. A recent study found that WTAR reading recognition accounted for the same variance in performance as race and education combined. The aim of the current study was to examine how well TOPF standard score predicts verbal abilities for Caucasians and African Americans in a diverse hospital based outpatient clinical sample.

Participants and Methods: A total of 457 outpatients, 70\% Caucasian (education mean $=14.9$ years, $\mathrm{SD}=2.94$, range $4-20$; age mean $=58.2$, $\mathrm{SD}=16.77$, range 16-89) and $30 \%$ African American (education mean $=13.88$ years, $\mathrm{SD}=2.83$, range $8-20$ years; age mean $=53.8, \mathrm{SD}=$ 15.4, range 21-87) were administered the TOPF and the WAIS-IV from late 2013 through 2015 as part of a flexible clinical neuropsychological evaluation. Paired correlations by race were utilized to examine the relationship of TOPF with the WAIS-IV Verbal Comprehension Index (VCI), controlling for education.

Results: The TOPF was significantly correlated with the VCI and all of its subtests for both the African American sample and the Caucasian sample. However, there was significant difference in education level by race $t(455)=3.1, p=.002$. After controlling for education, paired correlations of the TOPF and VCI remained significant for Caucasians $(r=.614, p<.001)$ and African Americans $(r=.581, p<.001)$. Similar findings are observed for individual subtests within the VCI.

Conclusions: Results suggest that the TOPF is significantly correlated to the WAIS-IV VCI and its components for both groups, even after controlling for education. Furthermore, our results demonstrate positive correlations for our African American sample which is different than the TOPF manual's suggestion of a negative correlation in their non-clinical sample.

Correspondence: Jill Z. Stuart, Ph.D., Neurology, Duke University Medical Center, 932 Morreene Rd., DUMC 3333, Durham, NC 27702, United States.E-mail: jill.stuart@duke.edu

S. THOMAS, M. MORRALL, E. BENNETT, J. LIMOND, G. POGGI, M. CHEVIGNARD \& R. GRUNDY. Challenges of Developing a Neurocognitive Test Protocol for a Multilingual International Trial : The SIOP Ependymoma II Program.

Objective: To devise a comprehensive neurocognitive assessment battery suitable for multilingual administration within an international trial for long term follow-up of childhood brain tumour survivors.

Participants and Methods: Ependymomas are one of the most frequently occurring malignant brain tumours in childhood. They present significant clinical management challenges and prognosis is often poor. SIOP Ependymoma II is an international program aiming to evaluate outcomes and improve diagnostic accuracy. It incorporates phase II and III treatment trials, centralised imaging and pathology review, and evaluation of quality of survival (QoS) including neurocognitive and psychological morbidity. Consensus discussions between key QoS representatives of participating countries were undertaken over a period of 10 years. These were informed by protocols established by the SIOP QoS working group (Limond et al 2015) and existing tumourspecific literature, with the aim of establishing a targeted minimum dataset augmented by optional broader data collection.

Results: A comprehensive battery of tests suitable for administration in multiple languages and cultural settings is presented. Obstacles encountered, including differing standard clinical practices across the sixteen participating countries, and difficulties identifying tests for administration in around fourteen European languages, are presented alongside solutions generated. This includes the 'Core Plus' model which provides a two tier approach to assessment, allowing core domains to be prioritised where test availability and resources are limited, and more comprehensive assessment where feasible. 
Conclusions: A European consensus has been reached for an internationally accepted test battery suitable for follow-up of childhood ependymoma survivors. This large European brain tumour trial opened in summer 2016. It will be the first to comprehensively measure long term cognitive outcomes and will do so across sixteen European countries. Correspondence: Sophie Thomas, DClinPsy, Paediatric Neuropsychology, Nottingham Children's Hospital, 8 Abbey Crescent, Sheffield S7 2QX, United Kingdom. E-mail: sophie.thomas@nhs.net

V.L. TORRES, M. LANG, I. VELEZ-URIBE, D. CHRISTOPHER, M. ROSSELLI \& F. ARRUDA. The Big Five Inventory (BFI) and Emotion Word Valence: Does Personality Influence the Appraisal of Emotion Words in Bilinguals?

Objective: Previous studies have found that higher scores on extraversion and openness in personality scales correlate with increased use of a second language. Frequency of use has been shown to be an important factor in the appraisal of valence in emotion words. In addition, differences in the way bilinguals appraise the emotional intensity of words in both languages have been found. We included three (openness, extraversion and conscientiousness) of the five dimensions assessed by the BFI, a scale that describes participants on five dimensions of personality, and analyzed them as predictors for valence ratings in Spanish and English. Interindividual differences in personality traits were expected to partially account for the variance in valence scores, with higher levels of openness, extraversion and conscientiousness correlating with lower differences between languages when appraising emotion words in both languages.

Participants and Methods: The sample consisted of 101 active Spanish-English bilinguals (69 females).

120 words in three categories (positive, negative and taboo) were presented visually and were appraised in a 1-9 valence scale in Spanish and English.

Results: The model was significant in predicting differential scores in the positive $\left(R^{2}=.097, p=.022\right)$ and taboo $\left(R^{2}=.124, \mathrm{p}=.006\right)$ word categories, with extraversion being the only significant factor $(p=.004)$. When examining taboo words, the domains of extraversion $(p=.002)$ and openness $(p=.006)$ were also significant factors. Consistent with the hypothesis, higher levels of extraversion were negatively correlated with decreased differences in scores between languages in the positive and taboo words category.

Conclusions: Bilinguals who were more extroverted rated positive and taboo words more similarly in both languages. This finding could be a result of extraverted individuals seeking out more social experiences in both languages.

Correspondence: Valeria L. Torres, BA, Psychology, Florida Atlantic University, 6230 SW 24th PL Apt 306, Davie, FL 33314, United States. E-mail:vtorres2015@fau.edu

I. VELEZ URIBE, V. ARANA, J. CONNIFF \& M. ROSSELLI. Testing a Predictive Model for the Appraisal of Valence of Emotion Words in Spanish-English Bilinguals.

Objective: Previously found differences in the perception of the emotional intensity of emotion words between languages in bilinguals, raise questions about which variables might influence these differences. In a regression model, seven variables were tested: Gender, age of acquisition of English, percent of life lived in the United States, English proficiency, Spanish proficiency, Latino cultural identity and US cultural identity scores. It was hypothesized that age of acquisition of L2 (English) would be one of the highest contributing variables to the models.

Participants and Methods: The sample included 101 Spanish-English bilinguals (69 females). After giving informed consent and responding to the linguistic, educational and cultural background questionnaires, participants performed a word rating task. This required them to rate 120 words in three categories (positive, negative and taboo), in both languages, (English and Spanish) and two sensory modalities (visual and auditory). Words were rated in an emotional valence scale ranging from 1 to 9 .

Results: The regression model for visual positive words was significant, $F(7,92)=3.50, p=.002$, accounting for $21 \%$ of the variance in scores. Only Spanish proficiency had significant weight over the model, $\beta=.28, t(100)=2.60, p=.01$. In the auditory positive category, the model was significant, $F(7,92)=4.38, p<.001$, accounting for $25 \%$ of the variance in scores. Two of the predictors were significant: English proficiency, $\beta=-.24, t(100)=-2.11, p=.037$, and, Spanish proficiency, $\beta=.36, t(100)=3.46, p=.001$.

Conclusions: The influence of level of proficiency is consistent with previous findings. Higher levels of proficiency in English seemed to have an attenuating effect in the differences between languages in the positive category, whereas a higher level of proficiency in Spanish seemed to have the opposite effect, as indicated by the positive direction of the correlation.

Correspondence: Idaly Velez Uribe, Psychology, Florida Atlantic University, 6812 sw 11 st, Pembroke Pines, FL 33023, United States. E-mail:ivelezur@fau.edu

K. VINCK \& S. MONCATA. Cognitive, Academic, and Behavioral Functioning of First and Second Generation Hispanic Children in the Child Welfare System: Findings using the National Survey of Child and Adolescent Well-Being (NSCAW II).

Objective: Studies of Hispanic children with histories of maltreatment typically report poor cognitive, behavioral, and academic outcomes (Vasilevski \& Tucker, 2016; Crozier and Barth, 2005). However, within-group differences of the Hispanic population have not been studied. The present study aims to identify the differences between first and second generation Hispanic children with maltreatment histories on cognitive, academic, and behavioral outcomes.

Participants and Methods: The data was drawn from the National Survey of Child and Adolescent Well-Being II (NSCAW-II), which included 450 Hispanic children were given the cognitive functioning assessment (44\% first generation; 56\% second generation), 407 Hispanic children academic functioning assessment ( $46 \%$ first generation; $54 \%$ second generation), 300 Hispanic children were given the behavioral functioning assessment (47\% first generation; $53 \%$ second generation). The K-BIT, Woodcock-Johnson, and CBCL were used to measure cognitive, academic, and behavioral functioning.

Results: Independent samples T-tests were conducted to compare means across standard scores of cognitive, behavioral, and academic measures. This study found no significant difference in cognitive and academic functioning between the first and second generation Hispanic children. Significant differences were observed on the standardized measurement of behavioral functioning with second generation Hispanic children scoring higher on sleep problems standard score $(t(298)=1.500, p=$ $0.015)$, attention problems standard score $(t(298)=1.515, p=0.010)$, and aggressive problems standard score $(\mathrm{t}(298)=1.321, p=0.022)$.

Conclusions: The results are consistent with the existing literature suggesting that acculturated second generation Hispanic children have been observed to have increased behavioral problems compared to less acculturated first generation Hispanic children (Gonzales, Knight, Morgan-Lopez, Saenz, \& Sirolli, 2002). These differences are attributed to a range of factors. Together, these findings support the need for services that are developed and delivered with attention to the unique histories of Hispanic children that can prevent the risk of missing critical needs and avert compromising the relevance of the services.

Correspondence: Krizia Vinck, MA, Clinical Psychology, William James College, 11 Evelyn St, Lynn, MA 01902, United States. E-mail: krizia_ vinck@williamjames.edu 


\section{Genetics/Genetic Disorders}

C.J. ALEXANDER, R. ROMERO, E. SHAPIRO, K. HATHAWAY \& J. MCLEOD. Using the Vineland Adaptive Behavior Scale to Assess Adpative Functioning Differences Between Subtypes of Mucopolysaccharidosis I.

Objective: Mucopolysaccharidosis (MPS) Type I is a rare recessive genetic disorder involving errors in metabolism. The decreased enzyme activity characteristic of this disease results in numerous medical complications involving almost every organ system, including the central nervous system. Due to the various rates of disease progression and variation of symptoms between subtypes, treatment options are limited and time sensitive. Age, diagnosis, Vineland Adaptive Behavior Scale-II (VABS-II) scores, and physical symptoms score (PSS) were used as data. This study assessed adaptive functioning of children, to provide data to inform the childrens' treatment teams.

Participants and Methods: This current study utilized an archival dataset collected by the Lysosomal Disease Network (LDN) and participating centers, including the University of Minnesota, Twin Cities Campus. The sample consisted of 47 participants with MPS I.

Results: A one-way multivariate analysis of variance (MANOVA) was conducted to compare the VABS-II scale means for both groups of MPS participants. The analyses of variance (ANOVA) of the VABS-II composite and the two subdomains were not significant. There was a negative correlation between the PSS and VABS-II composite and Daily Living Skills subdomain (DLS).

Conclusions: A significant difference of adaptive functioning between MPS subgroups was found. A negative relationship between PSS scores and overall adaptive functioning was found. As participants experience more physical symptoms, their ability to complete their activities of daily living is negatively impacted. An understanding of the impact of medical symptoms on adaptive functioning can help to target specific interventions on measured deficits, thus increasing the child's overall quality of life.

Correspondence: Carly J. Alexander, PsyD Clinical Psychology, Pediatrics, University of Minnesota, 597 Holly Ave, Apt A, Saint Paul, MN 55102, United States. E-mail: carly.jo.alexander@gmail.com

\section{CASNAR, B. YUND, K. LEE \& B. KLEIN-TASMAN. ASD Symptomatology and Related Variables in Children with Neurofibromatosis type 1.}

Objective: Social problems are a common concern of parents of children with neurofibromatosis type 1 (NF1). Recent research has demonstrated elevated rates of Autism Spectrum Disorder (ASD) symptomatology in children with NF1; however, few studies have examined variables that may contribute to those elevations. The primary aim of this study was to examine possible variables that may contribute to social responsiveness difficulties and restrictive and repetitive behaviors (RRB) in children with NF1.

Participants and Methods: Participants included 25 children (ages 9-13) with NF1, along with their parent. The Social Responsiveness Scale, Second Edition (SRS-2) was used to assess ASD symptomatology. Selected measures were used to assess intellectual functioning (Differential Ability Scales-II), attention (Cogstate, NEPSY-II), social cognition (Cogstate), and pragmatic language skills (Clinical Evaluation of Language Fundamentals- $\mathrm{V}$ : Metalinguistics).

Results: Thirty percent of parents observed mild to moderate social responsiveness difficulties and RRB on the SRS-2. Relations between SRS-2 Total Score and intellectual functioning, $r=-.53, p=.01$; social cognition, $r=-.46, p=.02$; and pragmatic language skills, $r=-.53$, $p=.01$, were found. Multiple linear regression analysis indicated that these three variables together explained $36 \%$ of the variance in social responsiveness difficulties reported by parents, Adjusted $R^{2}=.36, F(3$, $23)=5.31, p<.01$. A pragmatic language task (Making Inferences) significantly predicted parent report of social responsiveness and RRB, $\beta=-.44, p=.03$, and uniquely explained $38 \%$ of the variance.
Conclusions: Results indicate that children with NF1 are demonstrating elevated rates of ASD symptomatology based on parental report, and that intellectual functioning, social cognition, and pragmatic language skills are all contributing variables. Future research is needed to better explore these relations and develop interventions to address the social difficulties seen in children with NF1.

Correspondence: Christy Casnar, M.S., Clinical Psychology, University of Wisconsin - Milwaukee, P.O. Box 413, Garland Hall, Rm 325, Milwaukee, WI 53201, United States.E-mail: clcasnar@uwm.edu

S.M. COX, G. DOME, T. KENNEDY, A. GIOIA, K.K. HARDY \& K.S. WALSH. Neuropsychological and social functioning in children with Neurofibromatosis Type 1, ADHD, and Autism Spectrum Disorder.

Objective: Children with Neurofibromatosis Type 1 (NF1) exhibit executive function (EF) and social deficits that overlap with features of both Attention Deficit Hyperactivity Disorder (ADHD) and Autism Spectrum Disorder (ASD). Indeed, higher rates of both disorders are documented for children with NF1. Yet, to date there have been few studies comparing the neuropsychological profiles of these populations. Participants and Methods: Retrospective data from 114 children (38 per group) ages $6-18(\mathrm{M}=10.34, \mathrm{SD}=3.64 ; 50 \%$ male) was used with children matched on age and gender. Assessments measuring IQ (Wechsler) and EF (TEA-Ch, Tower of London-Dx) were included. Parent and teacher report of EF, attention, and social functioning were also collected (BRIEF, ADHD Rating Scale, CBCL/TRF).

Results: Nonverbal IQ was significantly lower in children with NF1, $(p=.012)$, along with lower Full Scale IQ $(p=.022)$. Parents reported children with NF1 to demonstrate better attention $(p<.001)$ and flexibility ( $p=.008$ ) than both other groups; however, performance-based measures in these areas indicate that the NF1 group demonstrates the same level of impairment or more compared to children with ASD and ADHD. No significant between-group differences in working memory or social functioning were reported by parents, although teachers reported children with NF1 to have more social problems $(p=.016)$ than those with ASD or ADHD.

Conclusions: Children with NF1 appear to exhibit profiles of impairment on performance-based measures of attention and EF that are similar to children with ADHD and ASD. In contrast, parents report children with NF1 to have fewer difficulties in these areas than their ADHD and ASD counterparts. Thus, some children with NF1 may not be identified by primary providers as needing services. Moreover, NF1specific interventions for cognitive and social difficulties are lacking. Results suggest that adapting existing education, monitoring, and interventions that are provided to families with ADHD or ASD may be appropriate for those with NF1.

Correspondence: Stephany M. Cox, PhD, Neuropsychology, Children's National Health System, 15245 Shady Grove Road, Suite 350, Rockville, MD 20850,United States.E-mail:scox@childrensnational.org

R.J. FEE, E.B. LEAFFER \& V.J. HINTON. Underlying contribution of attention and executive functioning to cognition in individuals with dystrophinopathy.

Objective: Dystrophinopathy is a genetic neuromuscular disorder that causes the lack of the protein dystrophin in muscle and brain, resulting in progressive weakness and cognitive deficits. Findings across studies indicate IQ is one SD below the population mean, with evidence of a verbal weakness, and poor digit span. Attention and executive functions that may contribute to overall IQ performance have not been fully examined.

Participants and Methods: 42 boys with dystrophinopathy, 5-17 years of age, diverse ethnicity and SES, were included. Measures: Peabody Picture Vocabulary Test-4 (PPVT-4, Single-word Comprehension), Comprehensive Test of Nonverbal Intelligence (CTONI, Visuospatial reasoning); NIH Toolbox: Flanker Test (Visual attention), Dimensional Change Card Sort Test (Set-Shifting), List Sorting Working Memory 
Test (Working Memory), and Pattern Comparison Speed (Processing Speed). Paired-samples t-test were conducted to compare performance. Results: Measures of crystallized verbal and nonverbal skills were found to be average (PPVT-4: $\mathrm{M}=96.26 \pm 17.63$; CTONI: $\mathrm{M}=97.38 \pm 11.36$ ). In contrast, mean performance on all attention/executive measures was low average and significantly lower than both single-word comprehension and visuospatial reasoning: NIH toolbox measures [visual attention $(\mathrm{M}=82.57 \pm 16.40)$, processing speed $(\mathrm{M}=84.58 \pm 14.95)$, working memory $(\mathrm{M}=86.40 \pm 13.89)$, and set shifting $(\mathrm{M}=86.17 \pm 14.11)]$. Conclusions: Isolated crystallized abilities (both verbal and nonverbal) are intact, but generalized attention/executive weaknesses were found and not limited to the verbal domain. These results suggest a possible weaknesses in overlapping frontal systems contributing to attention and executive functions, likely impacting overall cognitive efficiency. Correspondence: Robert J. Fee, Sergievsky Center, Columbia University/ Queens College, 630 West 168th St. PH19, New York, NY 10032, United States.E-mail:rf237@columbia.edu

\section{J. FRANK, M. MARCANGELO \& M. LACY. Neuropsychological Functioning of an Adult with Fanconi Anemia.}

Objective: Fanconi anemia (FA) is caused by a genetic defect in proteins responsible for DNA repair resulting in bone marrow failure and cancer. Historically average survival age was 19 , yet recent advances have extended survival. Common physical congenital deficits include short statue, with abnormalities of skin, eyes, kidneys, and ears (Mohanty, 2016). Only older case series (Prindull et. al., 1975; Nilsson, 1960) referencing intellectual disabilities been published to date.

Participants and Methods: A 22 year old African American male diagnosed with acute myeloid leukemia completed a brief cognitive battery: Wide Range Achievement Test-IV, Repeatable Battery for the Assessment of Neuropsychological Status, Wechsler Abbreviated Scale of Intelliegnce- $2^{\text {nd }}$; Neuropsychological Assessment Battery, with normative references calculated and presented.

Results: Full Scale IQ was estimated to be in the moderately to severely impaired range [WASI-II FSIQ=61], with commiserate verbal and visual intellectual deficits. On a single word reading test, his performance was at the $2^{\text {nd }}$ grade level [ $\left.\mathrm{T}=25\right]$, consistent with intellectual estimates. On a measure of immediate verbal memory, his performance was in the severely impaired range [RBANS Index $=49 ;(<.1 \%$ tile $)$ ]. Low average to moderately/severely impaired sustained attention $[\mathrm{Z}$ score $=-2.2]$ was also documented. On a visual spatial judgment task he performed in the severely impaired $[\mathrm{Z}$ score $=-4.58]$. His ability to vocalize solutions to life situations (e.g., what does it mean if your doctor says there is a $25 \%$ chance of having side effects from a treatment) was in the moderately impaired [NAB Judgment $\mathrm{T}=26$ ] range.

Conclusions: This case report is the first to present neuropsychological data on an adult with Fanconi anemia. DNA repair dysfunction and related issues leading to CNS dysfunction will be discussed, along with implications for consenting to treatment and limitations.

Correspondence: Joel Frank, MA, Clinical Psychology, The Chicago School of Professional Psychology, 1685 Overland Trail, Deerfield, IL 60015, United States. E-mail: joelmfrank@gmail.com

V.J. HINTON, R.J. FEE, K. ENGELSTAD \& D. DE VIVO. The developmental trajectory associated with Glut 1 Deficiency Syndrome.

Objective: To characterize the neuropsychological trajectory associated with Glut-1 Deficiency Syndrome (Glut1 DS). Glut1 DS results from an SLC2A1 disease-causing gene mutation that affects the transport of glucose across the blood-brain barrier. Affected individuals present with infantile-onset epilepsy refractory to medications, other paroxysmal events, deceleration of head growth, developmental delays, and disorders of movement. As a group, mean intellectual functioning clusters in the mild intellectually delayed range, but there is variability across patient population. Current standard of care focuses on the use of a ketogenic (high fat) diet to provide an alternative brain fuel source. To determine the developmental course of the disorder, cognitive performance and adaptive behavior were evaluated across time.

Participants and Methods: 32 children with Glut1 DS (5-12 years at baseline) who were on the ketogenic diet were given a battery of tests that included the Ravens Coloured Matrices (RCM), Peabody Picture Vocabulary Test (PPVT), Expressive Vocabulary Test (EVT), Beery Test of Visual Motor Integration (VMI) and Vineland Scales of Adaptive Behavior (VABS) at three sequential time points over 6 years.

Results: Overall, mean scores on the measures clustered in the mild intellectually delayed to borderline range. Nonverbal reasoning and receptive vocabulary were relative strengths and visual-motor integration and expressive vocabulary were relative weaknesses. Adaptive behaviors were low, yet variable, with socialization skills as strengths. Repeated measures ANOVA indicated there were no differences across standard scores over the three time points.

Conclusions: Individuals with Glut 1 DS have lower cognitive and adaptive skills than the general population, yet those who are maintained on the ketogenic diet make developmental gains at a comparable rate as their unaffected peers, such that standardized scores remain stable over time.

Correspondence: Veronica J. Hinton, PhD, Sergievsky Center \& Department of Neurology, Columbia University, P\& S Box 16, Columbia University, 630 West 168th Street, New York, NY 10025, United States. E-mail:vjh9@columbia.edu

E.B. LEAFFER, V.J. HINTON, K. ENGELSTAD, D. SHUNGU, S. DIMAURO \& D. DE VIVO. The relationship between cerebral lactic acidosis and memory performance in Mitochondrial Encephalomyopathy, Lactic Acidosis, and Stroke-like Episodes (MELAS).

Objective: To examine memory function in individuals with Mitochondrial Encephalomyopathy, Lactic Acidosis, and Stroke-like Episodes (MELAS). MELAS is a maternally inherited progressive multisystemic disorder of the central nervous system resulting from an Adenosine (A) to Guanine (G) transition at nucleotide $\mathrm{mt} 3243 \mathrm{~A}>\mathrm{G}$ in the tRNA. In addition to neurological and muscular impairment, cerebral lactic acidosis is a classic biomarker of the $\mathrm{mt} 3243 \mathrm{~A}>\mathrm{G}$ population; and increased lactate has been linked to neuropsychological deficits. We hypothesized that individuals with high lactate will demonstrate worse verbal and visual memory performance compared to individuals with normal lactate levels.

Participants and Methods: Verbal and visual memory skills were examined in 84 individuals carrying the $\mathrm{mt} 3243 \mathrm{~A}>\mathrm{G}$ mutation and 20 controls (9-76 years). Measures included: Selective Reminding Test (verbal) and the Benton Visuospatial Retention Test (visual). Test scores were converted to percentiles. Data above the $16^{\text {th }}$ percentile are "within normal limits," data falling between the $16^{\text {th }}$ and $2^{\text {nd }}$ percentile are "mildly impaired," while those falling below the $2^{\text {nd }}$ percentile are "severely impaired." Lactate levels were represented as "within normal limits" ( $\leq 5.6$ i.u.), "high" (5.7-7.6 i.u.) and "very high" ( $\geq 7.7$ i.u.).

Results: Performance on both memory measures is sensitive to increasing lactate level (Visual: Pearson $r=-0.40, p=0.00$; Verbal: Pearson $r=$ $-0.23, p=0.02$ ). However, only visual memory performance discriminates the groups across the lactate levels (Visual chi-square $=27.45$, $\mathrm{p}<0.0001$; Verbal chi-square $=4.34, \mathrm{~ns})$.

Conclusions: All individuals with high lactate levels perform poorly on the visual memory test, yet some individuals in this range are still able to perform within normal limits on the verbal memory test.

Correspondence: Emily B. Leaffer, MPH, Columbia University, 630 West 168th Street, PH 19, New York, NY 10019, United States. E-mail: ebl2121@cumc.columbia.edu 
A.L.M. LERNER, V.C. MERRITT \& P. ARNETT. Examination of COMT Genotype on Verbal and Visual Memory Following SportsRelated Concussion.

Objective: It is well understood that a number of variables impact post-concussion neuropsychological performance; however, the extent to which genetic factors influence cognition following concussion remains largely unknown. Limited work on the COMT gene has been done within the context of brain injury, and no study to date has examined the relationship between COMT polymorphisms and memory performance following sports concussion. The purpose of this study was to determine whether the polymorphisms associated with the COMT gene influence memory performance following sports-related concussion. It was hypothesized that athletes carrying a Met allele would outperform athletes homozygous for the Val allele on memory-related tasks.

Participants and Methods: Participants included 59 concussed college athletes (73.6\% male, $72.9 \%$ Caucasian) who underwent neuropsychological testing and provided a buccal sample for determination of their COMT genotype. The main outcome measures included the BVMT-R (Total and Delayed Recall) and HVLT-R (Total and Delayed Recall) to assess visual and verbal memory, respectively.

Results: The sample was divided into two groups based on the presence or absence of the Met allele. Met/Met and Met/Val carriers were grouped together (Met+; $\mathrm{N}=41$ ) and compared to $\mathrm{Val} / \mathrm{Val}$ carriers (Met-; $\mathrm{N}=18$ ). The groups did not differ on demographic and injury severity characteristics. Independent samples t-tests showed that the Met+ group demonstrated greater performance than the Met- group on the BVMT-R Total Recall index $(t(57)=-2.05, \mathrm{p}<.05, d=0.57)$, but no other significant differences we found on the other memory indices (all p>.05, $d=0.24-0.41)$.

Conclusions: These findings suggest that possession of a COMT Met allele may provide some protection against visual memory decline following concussion. Continuing to examine the relationship between the COMT gene and cognitive performance following brain injury is necessary and may have implications for the management of sportsrelated concussions.

Correspondence: Alexandria L.M. Lerner, B.S., 372 Moore Building, University Park, PA 16802, United States.E-mail: all5526@psu.edu

\section{A. LUCCHETTI \& T. BROWN. Neurocognitive Variability in Twin Adolescent Males with Williams Syndrome.}

Objective: Williams Syndrome is a neurodevelopmental genetic disorder with known cognitive impairments that occurs in 1 in every 10,000 live births. Medically, these children present with hypertension, facial dysmorphology, and endocrine, cardiac, musculoskeletal, and genitourinary abnormalities. Neuropsychological profiles of children with Williams Syndrome are most notable for mild to moderate Intellectual Disability and associated global delays across domains. This study highlights unexpected variability in twin adolescent males with Williams Syndrome.

Participants and Methods: Participants include two 13-year, 8-month-old twin males with Williams Syndrome. Both presented for comprehensive neuropsychological assessment at a major Midwestern medical institution as part of comprehensive multispecialty medical evaluations.

Results: Both males demonstrated variability across domains, with scores ranging from impaired to above average. Many scores clustered in the borderline to low average range. Neither met criteria for Intellectual Disability. Strengths were in rote auditory memory, some aspects of language, and sustained visual attention. Weaknesses were in efficient problem-solving, behavioral inhibition, reading, and fine motor coordination. There were concerns for anxiety and behavioral dysregulation in both males, although one male had a more pronounced psychiatric history.

Conclusions: This study provides novel information about twin males with neurocognitive profiles inconsistent with Williams Syndrome, notably with some cognitive skills in the average to above average ranges. Future directions should include identification of resiliency factors and ongoing serial assessments to track cognition over time. Correspondence: Amanda Lucchetti, MA, Psychiatry and Behavioral Sciences, Children's Hospital Colorado, 5100 Leetsdale Drive, Unit 401, Denver, CO 80246,United States.E-mail: amandalucchetti@gmail.com

WITHDRAWN: K.L. MCCABE, C. DURDLE, A. POPA, M. CABARAL, L. WONG, D. HARVEY \& T. SIMON. Quantifying the Resolution of Spatial and Temporal Representation in Children With 22q11.2 Deletion Syndrome.

Objective: Spatial and temporal information processing impairment likely underpins the non-verbal cognitive impairments observed in 22q11.2 deletion syndrome (22q11DS). The study quantified spatial and temporal processing abilities in children with 22q11DS, sex chromosome aneuploidy (SCA) and a typically developing (TD) control group.

Participants and Methods: Children $(22 q=70, S C A=49, T D=46)$ responded to spatial, temporal and pitch stimuli with varying difference ratios. Participant's task was to identify which of two sequentially presented stimuli were larger (spatial stimuli) or longer (temporal stimuli). Temporal duration detection tasks consisted of both visual and auditory stimuli. In addition, a pitch comparison task measured participant's ability to detect difference between non-spatial and non-temporal stimuli. Detection threshold was calculated as the minimum difference between stimuli required to achieve $75 \%$ accuracy.

Results: 22q11DS participants displayed higher spatial detection threshold (\%) than the TD and SCA groups, requiring greater difference between stimuli for accurate identification (\%: 22q11DS = 13.6; TD: $6.8 ; \mathrm{SCA}=7.9 ; \mathrm{F}(2,149)=8.42, \mathrm{p}<0.001)$. Temporal detection threshold was also higher for the 22q11DS group to visual (\%: 22q11DS $=14 ; \mathrm{SCA}=8 ; \mathrm{TD}=7 ; \mathrm{F}(2,86)=8.33, \mathrm{p}<0.001)$ and auditory stimuli $(\%: 22 q 11 D S=23 ;$ SCA $=12 ;$ TD: $8 ; \mathrm{F}(2,94)=8.99, \mathrm{p}<0.001)$ compared to both the SCA and TD groups. The SCA and TD groups displayed equivalent performance on all measures ( $p$ 's $>0.05)$. Pitch detection threshold did not differ between groups ( $p$ 's $>0.05$ ).

Conclusions: The observation of higher detection threshold to spatial and temporal stimuli in the 22q11DS group demonstrates further evidence for reduced resolution in both spatial and temporal discrimination, that does not extend to frequency estimation (pitch detection), and which is not explained by generalized cognitive impairment.

Correspondence: Kathryn L. McCabe, PhD, Psychiatry and Behavioral Sciences, University of California (Davis), 2825 50th St, Sacramento, CA 95816, United States. E-mail: kmccabe@ucdavis.edu

\section{S.E. MCKNIGHT, M. DRISCOLL, R. FULTON \& B. WILSON. A} Rare Case of Spinocerebellar Ataxia Type 16.

Objective: Spinocerebellar ataxia (SCA) is a genetic disorder with over 30 identified mutations. We present a rare case of SCA type 16, an autosomal recessive variant with fewer than twenty identified cases worldwide. In addition to cerebellar atrophy and ataxia, symptoms of SCA16 can include dysarthria, spasticity, nystagmus, and cognitive impairment, among others. SCA is thought to be progressive; however, less is known about the specific nature or course of type 16 .

Participants and Methods: A 25-year-old Caucasian female presented for neuropsychological evaluation for characterization of cognitive abilities in the context of SCA16 (diagnosed in 2014). The patient and family reported a stepwise decline in cognitive abilities, mood, speech, gait, and handwriting since 2012. Methods included a comprehensive neuropsychological and psychological evaluation, neurologic exam, and review of medical records and neuroimaging.

Results: Neuroimaging revealed diffuse cerebellar atrophy. Neurologic exam was significant for dysarthria, nystagmus, dysmetria, and gait disturbance. Primary deficits on neuropsychological testing were in the areas of attention, working memory, and psychomotor processing speed. Assessment of mood revealed depression and anxiety. 
Conclusions: This case study contributes to research and the clinical community at large by providing additional insights into this rare genetic disorder, as well as lending further support to the hypothesis that damage to the cerebellar circuitry can contribute to non-motor cognitive and emotional processing difficulties. By integrating clinical neuropsychology and neurology along with modern neuroimaging and genetic testing techniques, this case also highlights the need for a synergistic and holistic multidisciplinary approach to patient care in the era of integrated medicine.

Correspondence: Sarah E. McKnight, PsyD, Geisinger Medical Center, 227 Port Noble Drive, Bloomsburg, PA 17815, United

States.E-mail:sem.mcknight@gmail.com

\section{T.W. OLIVIER, A. AMARI \& K. SLIFER. Systematic Desensitization in a Young Adult with Pelizaeus-Merzbacher Disease.}

Objective: This poster will examine the use of systematic desensitization for use of a continuous positive airway pressure (CPAP) machine in a young adult with a history of Pelizaeus-Merzbacher disease (PMD), obstructive sleep apnea (OSA), and associated challenges.

Participants and Methods: An 18-year-old male with a history of PMD presented to a major hospital's outpatient sleep clinic and was subsequently referred to an outpatient pediatric psychology program for CPAP desensitization following a diagnosis of OSA. The patient had difficulty adhering to CPAP usage, as he was sensitive to items on or near his face. He presented with significant motor difficulties (e.g., needed a wheelchair, limited functioning of upper extremities), expressive and receptive language concerns (e.g.. mostly nonverbal), nystagmus, and cortical blindness. The patient was seen for an initial evaluation and 12 subsequent desensitization and parent training sessions.

Results: Desensitization through coping skills and other behavioral strategies (e.g., distraction, positive reinforcement for compliance) was utilized to elicit CPAP adherence. Parent training, which was seen as essential given the patient's developmental level, was also used. A 15 -step task analysis was constructed. The patient completed $50 \%$ of the steps halfway through treatment and $100 \%$ by termination. He was able to tolerate the entire machine (e.g., mask, air hose connected and turned on) for over 30 minutes in session (e.g., reported latency to sleep onset). Conclusions: This case study highlights the effectiveness of systematic desensitization with an individual with significant neurological and developmental challenges, using modifications and adaptations as needed throughout treatment. Although the patient reached his initial treatment goals, generalization to home was difficult and remained ongoing.

Correspondence: Traci W. Olivier, M.S., Neuropsychology, Kennedy Krieger Institute/Johns Hopkins School of Medicine, $101 \mathrm{~N}$. Wolfe Street,Apt.318, Baltimore, MD 21231,United States.E-mail: olivier@ kennedykrieger.org

L.V. TRAVERS, A. LIN, E. MCNAMARA, A.K. MORGAN \& M.K. COLVIN. The Neuropsychological Profile of Girls and Women with Turner Syndrome Across the Lifespan.

Objective: Turner syndrome (TS) is a genetic disorder characterized by complete or partial monosomy-X in phenotypic females. Prior research has established that cognitive profiles of girls/women with TS are characterized by intact intellectual functioning and verbal abilities, with relative weaknesses in visual-spatial, executive, and social domains. However, few researchers have explored the subtleties of this profile or its stability across the lifespan.

Participants and Methods: 26 patients (100\% female) ages 3-35 $\left(M_{\text {a ge }}=16.66\right)$ completed neuropsychological testing as part of routine care. $50 \%(n=13)$ had karyotypes of $45 X ; 50 \%$ were mosaic, ring $\mathrm{X}$, isochrome $\mathrm{Xq}$, or other. Analyses examining for age effects were conducted by dividing the sample into under 18-years-old $(n=12)$ and $18+(n=14)$.

Results: Across patients, overall IQ fell within the average range. One-sample-tests (with Bonferroni corrections) were conducted to compare our population's profile to the normative population. Significant weaknesses were found in perceptual reasoning and spatial skills, working memory, processing speed (including graphomotor speed and verbal fluency), and fine motor control/dexterity (all $p$ 's<.001). There were no differences in neuropsychological profile based on age.

Conclusions: The neuropsychological profile of TS was stable across the lifespan. Within the context of normal intellectual functioning, verbal abilities were generally stronger than visual abilities, including perceptual reasoning and spatial skills. Processing speed was slow on both visual and verbal tasks. Dexterity was bilaterally weak. Working memory was also weak, although this was only statistically significant for auditory material. Verbal knowledge/reasoning, cognitive flexibility, set-shifting, inhibition, and sustained attention were all intact. Future studies should compare profiles by karyotype, as preliminary findings suggest lower verbal abilities and overall IQ for ring $\mathrm{X}$ as compared to mosaic karyotypes.

Correspondence: Lea V. Travers, Ph.D., Psychiatry, Massachusetts General Hospital, 309 W. 4th Street, Boston, MA 02127, United States. E-mail: leatravers@gmail.com

C. VEGa, A. PROHL, C. WAN, S. ClanCY \& S. WARFIELD. Neuropsychological Comorbidities in Tuberous Sclerosis Complex and Autism Spectrum Disorder.

Objective: To characterize the neuropsychological profiles of patients with Tuberous Sclerosis Complex (TSC), autism spectrum disorder (ASD), and healthy controls. TSC is a genetic disorder that affects multiple organ systems, leading to neuropsychological comorbidities that may present as developmental delays, intellectual disability, ASD, ADHD, or specific learning disorders. The impact of TSC is exacerbated by epilepsy, which presents in up to $90 \%$ of patients. The current study is a preliminary report of patients diagnosed with TSC with and without ASD, idiopathic ASD, and healthy controls (HC) that were assessed as part of a prospective longitudinal study examining neuroimaging and behavioral biomarkers.

Participants and Methods: The sample included 17 TSC $($ mean age $=$ 7.9; $\mathrm{SD}=3.5), 16 \mathrm{ASD}$ (mean age $=11.3 ; \mathrm{SD}=4.5)$, and $32 \mathrm{HC}$ (mean age $=9.8 ; \mathrm{D}=3.9)$. The test battery included intelligence, language, executive functions, socialization, and adaptive skills. Results from the Autism Diagnostic Observation Scale, Second Edition (ADOS-2), were used as inclusion criteria for ASD. Results were analyzed using a MANCOVA that accounted for age differences

Results: There was a significance discrepancy between the HC and the two clinical populations. Post hoc analyses revealed better performance across all measures in the HC. The ASD and TSC groups performed similarly on measures of IQ and expressive language; however, the ASD group had more problems with executive functions, socialization, and adaptive skills.

Conclusions: Our study shows that TSC is associated with a significant risk of neuropsychological comorbidity. Cognitive performance was bimodally distributed, as reported is previous studies, and may be similar to ASD patients. However, TSC patients present with better overall environmental adjustments, when specifically accounting for executive function skills, socialization, and adaptive skills

Correspondence: Clemente Vega, Yale University School of Medicine, 300 Longwood Ave Fegan 9, Boston, MA 02115, United States. E-mail: clemente.vega@childrens.harvard.edu

\section{B. YUND, C. CASNAR, K. LEE \& B. KLEIN-TASMAN. Relations Between Parent Report of Attention and Sleep in Children with Neurofibromatosis type 1 .}

Objective: Despite variability in the neurofibromatosis type 1 (NF1) cognitive phenotype, attention difficulties are often described (Hyman et al, 2006). Considering attention skills are crucial to academic and daily living skills, and children with sleep problems frequently display attention difficulties (Blunden et al, 2005), exploration of potential relations is warranted. The present study investigates the prevalence of 
sleep problems and their association with attention problems in children with NF1.

Participants and Methods: Participants included 27 children (ages 9-13) with NF1 and their parents. Schedule for Affective Disorders and Schizophrenia for School Aged Children ADHD section was used to assess ADHD symptomatology. The Inattention and Hyperactivity/ Impulsivity content scales from The Conners-3 Parent Short Form were used to assess attention. The Sleep-Related Breathing Disorder (SRBD) scale from the Pediatric Sleep Questionnaire, which has been validated against polysomnography, was used to assess parent reported sleep problems.

Results: Thirty percent of children met current criteria for ADHD (ADHD-inattentive=5; ADHD-combined=3). Thirty-seven percent of parents endorsed sleep problems suggestive of high risk for a pediatric sleep disorder. Relations between SRBD scale and Conners-3 Inattention scale, $r h o=.475, p=.01$ and Hyperactivity scale, $r h o=.391, p=.04$ were found. Children meeting criteria for ADHD-inattentive were marginally more likely to display symptoms indicative of high risk for a sleep disorder, $\left.X^{2}(1, \mathrm{n}=27)=7.38, p=.06\right)$.

Conclusions: Results indicate that children with NF1 demonstrate attention and sleep difficulties based on parent report. Inattention and hyperactivity symptoms are associated with sleep problems. Children meeting criteria for ADHD-inattentive type tend to display symptomology associated with sleep disorders. Future research is needed to further characterize the relations between sleep and attention problems in children with NF1, to allow for development of targeted interventions. Correspondence: Brianna Yund, Psychology, University of WisconsinMilwaukee, 4008 N Morris Blvd, Apt 3, Milwaukee, WI 53211, United States.E-mail:bdyund@uwm.edu

\section{Acquired Brain Injury (TBI/ Cerebrovascular Injury \& Disease - Child)}

WITHDRAWN: R. ASARNOW, T. BABIKIAN, E. DENNIS, A. OLSEN, E.A. WILDE \& H.S. LEVIN. Indicators of Recovery/ Repair and Neurodegeneration After Moderate-Severe TBI Pediatric Traumatic Brain Injury: Potential Mechanisms.

Symposium Summary: Immediately following moderate-severe pediatric TBI there are well documented adverse changes in brain structure and function. We will present results from two recently completed longitudinal studies that use cutting edge brain imaging and behavioral neuroscience methods to detail changes in brain structure and function during the first 18 months post-TBI. These studies reveal that there is a complex mixture of evidence of both recovery/repair and neurodegeneration during this time frame. Dr. Asarnow will introduce the symposium by providing a historical overview of theories of the effects of TBI on the developing brain and also present potential mechanisms of recovery/repair (e.g. remyelination, compensatory processes) and neurodegeneration (eg. neuroinflammation). Subsequent presentations will describe evidence implicating specific mechanisms and predictors of long term outcomes. Dr. Babikian will present Magnetic Resonance Spectroscopy evidence of a chronic neuroinflammatory response in a subgroup of patients with slow interhemispheric transfer time. Dr. Dennis will present structural MRI data showing evidence of progressive white matter loss in this subgroup, while patients with normal interhemispheric transfer times show normal brain development post-TBI. Dr. Olsen will present fMRI data showing that this subgroup of patients shows widespread BOLD hyperactivation. Dr. Wilde will present MRI data showing that changes are particularly apparent in late-developing frontal brain regions, and may account for some of the frontallymediated cognitive and behavioral changes such as behavioral regulation and executive functioning often found in individuals with pediatric TBI. Dr. Levin will provide a historical overview of studies of the social brain following TBI and present fMRI data showing reorganization of the brain network for social cognition. Dr. Asarnow will discuss the data implicating different potential mechanisms of repair/recovery and neurodegeneration.

Correspondence: Robert Asarnow, Ph.D., Psychiatry, UCLA, 740 Westwood Blvd., Los Angeles, CA 90024, United States. E-mail: rasarnow@mednet.ucla.edu

WITHDRAWN: T. BABIKIAN, J. ALGER, E. DENNIS, C. GIZA \&. R. ASARNOW. Whole Brain MR Spectroscopic Correlates of Microstructural Abnormalities and Functional Outcomes in Pediatric Moderate/Severe TBI.

Diffuse axonal injury plays a role in functional morbidity following pediatric moderate/severe traumatic brain injury (msTBI). This study sought to delineate the course of brain disruption and repair during the first year following injury. Pediatric msTBI patients underwent brain imaging and cognitive testing at two time points (post-acutely at 2-4 months post injury, $\mathrm{n}=35$ and chronically at 14 months post injury, $n=27)$. Gender and age-matched healthy controls $(n=39)$ were evaluated twice, 12 months apart. Whole brain Proton Magnetic Resonance Spectroscopic Imaging (EPSI/MIDAS 1H-3D-MRSI) was used to measure neurometabolite levels in brain structures sensitive to injury (cortical lobes, corpus callosum [CC], and hippocampus). The association between metabolite biomarkers and functional measures (cognitive functioning and interhemispheric transfer time [IHTT] using an ERP paradigm, a measure of CG function) was explored. Post-acutely, in patients, there were elevations in choline (marker for inflammation and/or altered membrane metabolism) in all four brain lobes and the $\mathrm{CG}$, and decreases in $\mathrm{N}$-acetylaspartate (NAA; marker for neuronal and axonal integrity) in the CC compared to controls. Of note, lobar choline remained elevated in an msTBI subgroup that showed normal IHTT relative to controls. This subgroup also showed higher levels of CG NAA at the chronic time point. Post-acute NAA in the CC, but not lobar choline, explained a significant percentage of variance in both post-acute and chronic cognitive performance. Abnormalities in neurometabolites were more pronounced in brain regions with compromised white matter integrity based on DTI. Whole brain metabolic evaluations show a distinct pattern of neurochemical changes varying by subgroups of patients with different outcomes, which suggest a dynamic relationship between CC function, prolonged inflammatory responses to brain damage, reparative processes, remyelination, and subsequent neurobehavioral changes.

Correspondence: Talin Babikian, PhD, Child and Adolescent Psychiatry, Pediatrics, UCLA, 760 Westwood Plaza, Semel Institute, Room 47438B, Los Angeles, CA 90095, United States. E-mail: tbabikian@ mednet.ucla.edu

WITHDRAWN: E. DENNIS, F. RASHID, M. ELLIS, T. BABIKIAN, J. VILLALON-REINA, J. FASKOWITZ, R. VLASOVA, Y. JIN, A. OLSEN, R. MINK, C. BABBITT, J. JOHNSON, C. GIZA, P. THOMPSON \& R. ASARNOW.

Multi-Modal Imaging in Pediatric TBI: A Longitudinal Study.

There is considerable heterogeneity in post-TBI outcomes in children with moderate/severe traumatic brain injury (msTBI). Acute injury variables only account for some of this variance. In our study a measure of corpus callosum (CC) function, interhemispheric transfer time (IHTT), differentiated patients into two groups only a few months post-injury. We studied 21 children $(16 \mathrm{M} / 5 \mathrm{~F})$ with msTBI, assessed 2-5 months and 13-19 months post-injury, and 20 well-matched healthy control children. We assessed CC function through IHTT, measured using eventrelated potentials. We examined white matter (WM) microstructure using diffusion-weighted magnetic resonance imaging (dMRI) and regional brain volume using tensor-based morphometry (TBM). Half of the TBI patients had significantly slower IHTT at the first time-point (TBI-slow-IHTT, $\mathrm{N}=11$ ), and half were in the normal range (TBI-normal-IHTT, N=10). The TBI-normal-IHTT group did not differ significantly from healthy controls in WM integrity, either cross-sectionally or in the longitudinal trajectory of WM integrity between evaluations. In contrast, the WM integrity of the TBI-slow-IHTT group was 
significantly lower than healthy controls across a large portion of the WM. Longitudinal analyses showed the TBI-slow-IHTT group experienced a progressive decline in WM integrity throughout the brain from 2-5 to 13-19 months post-msTBI. TBM analyses showed volume loss in the TBI-slow-IHTT group in the WM, while the TBI-normal-IHTT group appeared to return to a normal developmental trajectory. We have discovered a biomarker that identifies a subset of patients with impaired $\mathrm{CC}$ integrity in the first months post-injury who experience widespread continuing and progressive degeneration, both in WM integrity and in regional brain volume, in the first year post-injury. Our hope is that identifying patients at risk for poorer outcomes will help clinicians know which patients might benefit from more aggressive treatment.

Correspondence: Emily Dennis, PhD, Imaging Genetics Center, University of Southern California, 4676 Admiralty Way, Suite 200, Marina del Rey, CA 90292, United States.E-mail: emily.dennis@ini. usc.edu

\section{WITHDRAWN: E.A. WILDE. Structural Brain Alteration in Children and Adolescents after Moderate to Severe TBI.}

Structural neuroplasticity following pediatric traumatic brain injury has been of significant interest in recent years, particularly as neuroimaging techniques have evolved to allow serial monitoring of post-injury changes and how these may impact subsequent development. Using neuroimaging and cognitive testing, we examined a group of children and adolescents aged $7-17$ years who had sustained moderate to severe TBI $(n=20)$ and a comparison group of children with orthopedic injury (OI) $(n=21)$. Children were evaluated at 3 and 18 months post-injury. Using volumetric analysis and diffusion tensor imaging, we describe complex and dynamic changes occurring in both white matter and gray matter over this time period, and how these changes diverge from expected development. Changes are particularly apparent in the latedeveloping frontal brain regions, and may account for some of the frontally-mediated cognitive and behavioral changes such as emotional control, behavioral regulation and executive functioning that often described by individuals with TBI sustained during childhood and adolescence. Better understanding the course of these changes in structural imaging may be important in TBI for monitoring development and facilitating advances in management and intervention.

Correspondence: Elisabeth A. Wilde, Ph.D., Physical Medicine and Rehabilitation, Neurology and Radiology, Baylor College of Medicine, 1709 Dryden Rd., Ste 1200, Houston, TX 77025, United States. E-mail: ewilde@bcm.edu

\section{WITHDRAWN: A. OLSEN, M. ELLIS \& R. ASARNOW. BOLD Hyper Activation after Pediatric Moderate-Severe Traumatic Brain Injury (msTBI) is Linked to Slow Inter-Hemispheric} Transfer Time as Measured with Scalp Event-Related Potential. Increased task-related BOLD activation is a common observation after msTBI. However, little is known about how such BOLD alterations are linked to electrophysiological measures of brain function. In this study we investigated how BOLD activation after msTBI was related to inter-hemispheric transfer time (IHTT), which is a scalp event-related potential measure of corpus callosum function. As our previous findings demonstrated that a sub-group of patients with slow IHHT had an increased risk of long-term neurodegeneration, our main focus was on investigating differences between a slow IHTT msTBI group and a normal IHTT msTBI group, compared to healthy controls. BOLD fMRI was acquired during performance of a spatial working memory task. For each trial, 1-5 pictures (items) were presented for $800 \mathrm{~ms}$ each within 4 locations on the screen. Participants were instructed to as quickly as possible after each trial reproduce the order of the presentation by using a response box with 4 buttons corresponding to the respective spatial locations. Task demands were manipulated in a parametric manner giving 4 trial types with different working memory load (WML); Baseline (1 item), Low WML (3 items), Intermediate WML ( 4 items) and High WML (5 items). A main contrast of interest representing the linear increase of BOLD activation as a function of WML was computed and then compared between groups. SPMs were corrected for multiple comparisons by applying a cluster threshold of $\mathrm{Z}>2.3$, and a cluster significance threshold of $\mathrm{P}<0.05$. The slow IHTT msTBI group exhibited widespread BOLD hyper activation as compared to controls. In contrast, the normal IHTT msTBI group did not differ from controls. This is the first study to link the typical BOLD hyper activation after TBI to an electrophysiological measure of brain function. and supports the relevance of IHTT as a biomarker after msTBI. Our future work will focus on linking these results to measures of functional and structural connectivity.

Correspondence: Alexander Olsen, PhD, Department of Psychology, Norwegian University of Science and Technology, Innherredsveien 56, Trondheim 7042, Norway. E-mail: alexander.olsen@ntnu.no

WITHDRAWN: H. LEVIN, M. NEWSOME, R. SCHEIBEL \& E.A. WILDE. Reorganization of Social Information Processing After Moderate to Severe TBI in Adolescents.

Objective: Study reorganization of the social brain following moderate to severe TBI in adolescents. The rationale was that disruption of social cognition and social functioning is common after TBI in adolescents, an age group whose peer relations and social interactions typically increase. Participants and Methods: Nine adolescents with moderate/severe TBI 2.5 years prior to study and 9 controls without TBI performed social perspective taking (SPT) and a social attribution task (SAT) during fMRI. On SPT task the target (self vs other) of the question about a trait and the perspective (third person or self) of the response were orthogonal; on each trial the adolescent responded to questions such as "Are you cheerful"? or "Does your father think you're cheerful?". In the SAT the adolescent viewed 2 animated, moving triangles and judged on each trial whether the triangles were "friends" with each other based on their movement and spatial position.

Results: In the PTT, brain activation did not differ between groups for analysis of target or perspective, but we found a target $\mathrm{x}$ perspective interaction. When adolescents with TBI were asked to evaluate statements about themselves from a third person's perspective, their activation exceeded controls in posterior brain regions (e.g., posterior cingulate) that are not activated by this task. On the SAT, the TBI group had less mesial frontal activation than controls and activation was negatively related to their white matter integrity on diffusion tensor imaging. Conclusions: We infer that reorganization of the brain network for social cognition occurs in adolescents at about 2.5 years following moderate to severe TBI and appears related to the integrity of white matter tracts implicated in the social brain network.

Correspondence: Harvey Levin, Ph.D., One Baylor Plaza MS637, Houston, TX 77030, United States. E-mail: hlevin@bcm.edu

\section{Cognitive Intervention/Rehabilitation}

WITHDRAWN: B.M. SANDROFF, J. BEST, P. TOMPOROWSKI \&. J. DELUCA. Exercise and Cognition: A Multidisciplinary Approach.

Symposium Summary: Over the past 40 years, it has been consistently hypothesized that exercise is beneficial for cognition. This includes approaches to examine the effects of exercise on cognition in the general population, across the lifespan, as well as in persons with neurological disorders (i.e., stroke, Parkinson's disease, schizophrenia, multiple sclerosis). The vast majority of literature studying the association among exercise and cognition in the general population has focused on older adults, given the high prevalence and impact of age-related cognitive decline. This large body of literature suggests that exercise is associated with improvements in several key cognitive domains and concomitant improvements in brain structure/function. This will be presented by Dr. John Best. There further is evidence that exercise can enhance cognition/brain structure and function in the developing brain. Indeed, there is a rapidly expanding body of literature that suggests that exercise 
actually improves academic achievement, cognition, and brain structure/function in children. Those data will be presented by Dr. Phillip Tomporowski. Based on the wealth of evidence supporting exercise for improving cognition in the general population, a more recent body of literature has emerged that suggests that exercise might benefit cognition and brain structure/function in persons with neurological disorders. This application of exercise and neuropsychological research is critical, given that cognitive impairment is highly prevalent, poorly-managed, and disabling in those populations. Relevant findings will be presented by Dr. Brian Sandroff. Collectively, this symposium will present a multidisciplinary, historical framework for understanding the effects of exercise on cognition and brain health across several key populations by linking past and present approaches with future directions for research in this area. A summary of the research and future directions will be led by Dr. John DeLuca.

Correspondence: Brian M. Sandroff, PhD, Kessler Foundation, 300 Executive Drive, Suite 70, West Orange, NJ 07052, United States. E-mail: bsandroff@kesslerfoundation.org

\section{P. TOMPOROWSKI. Exercise and Children's Cognition: Interpretation of Current Research.}

The belief that exercise is good for both body and mind can be traced back to ancient Greek philosophers and early physicians. Until relatively recently, empirical evidence to support a causal relation between exercise and mental processing was meager. The results of controlled experiments conducted with older adults that supported the exercisecognition relation published in the early 2000 s, led to a surge of interest in mental-health promoting effects of exercise training. Data obtained from exercise-training studies conducted with children over the past 15 years have yield mixed results, however. In this symposium, recent studies that have evaluated the effects of single, acute bouts of exercise and the effects of chronic exercise training on children's cognitive performance will be reviewed. A summary will be provided that focuses on neurological, cognitive, and social mechanisms that have been hypothesized to mediate the relation. Recommendations concerning clinical applications of exercise for children will be provided.

Correspondence: Phillip Tomporowski.E-mail: ptomporo@uga.edu

\section{WITHDRAWN: B.M. SANDROFF. Exercise and Cognition in Persons with Neurological Disorders.}

Cognitive impairment is highly prevalent, disabling, and poorly-managed in persons with neurological disorders such as stroke, Parkinson's disease (PD), schizophrenia, and multiple sclerosis (MS). This highlights the importance of examining exercise training as a rehabilitative approach for possibly managing cognitive dysfunction in those populations. Such an approach is in its infancy, and is largely based on the wealth of evidence documenting robust, beneficial effects of exercise on cognition and brain health in the general population across the lifespan. Using the literature in the general population as a backdrop, this symposium will discuss emerging data on the effects of exercise, physical activity, and physical fitness on cognition, brain structure, and brain function in persons with neurological disorders. Research in persons with stroke, PD, schizophrenia, and MS will be reviewed. A novel framework for optimizing future exercise interventions for improving cognition will further be presented. This involves the systematic development of exercise training interventions (i.e., randomized controlled trials) based on cross-sectional examinations of physical fitness and cognition, as well as the effects of single bouts of exercise (i.e., acute exercise) on cognition in those populations. Such a systematic approach has the promise to ultimately improve and optimize exercise training interventions on cognition for those who need it most.

Correspondence: Brian M. Sandroff, PhD, Kessler Foundation, 300 Executive Drive, Suite 70, West Orange, NJ 07052, United States. E-mail: bsandroff@kesslerfoundation.org
J. BEST. Exercise to Promote Cognition in Older Adults: Current and Future Research to Define Exercise Type and Target Populations.

Historically, it has been hypothesized that exercise can improve cognition in older adults. An early meta-analysis containing a heterogeneous set of experimental studies suggested that aerobic exercise training (e.g., progressive-intensity walking) produces significant benefits to cognition in older adults; however, a recent meta-analysis with more restrictive inclusion criteria suggests the effects are more modest among healthy older adults. Based on the current state of the science, it is unclear whether aerobic exercise training might be more beneficial in older adult populations with cognitive impairment and whether alternative types of exercise training, namely resistance training, might positively impact older adults' cognitive performance. Recent data will be discussed on the effects of 6-months of thrice-weekly aerobic exercise compared to a health education program on memory performance and task-based neural activation in regions of the temporal and occipital lobes in older adults with mild vascular cognitive impairment. To further clarify the state of the science, randomized controlled trials testing the effects of resistance training on executive functions in older women with and without baseline cognitive impairment will be discussed. Finally, future directions for research on the effects exercise on cognition in older adults will be presented. This involves direct examinations of the impact of aerobic training, resistance training, and combined aerobic and resistance training on cognitive performance in older women and men with mild cognitive impairment.

Correspondence: John Best.E-mail: john.best@ubc.ca

\section{Drug/Toxin-Related Disorders (Including Alcoholism)}

A.E. THORNTON, K. GICAS, T.A. O'CONNOR, T. WILLI, K. WACLAWIK, N.Y. WANG, W. PANENKA, D. LANG, A.A. JONES, T. BUCHANAN, F. VILA-RODRIGUEZ, A. BARR, G.N. SMITH \& W.G. HONER. Neuropsychological Aspects in Marginalized Persons with Multimorbidity.

Symposium Summary: Structural inequality is an inherent feature of society that is associated with large sub-populations of marginalized and vulnerable individuals who experience social, cultural and economic disadvantage. The challenges posed by marginalization have huge costs to societies, governments, health providers and individuals. At the individual level, marginalized persons suffer from numerous ailments including trauma, addiction, viral infection, brain injury, and mental illness. Consequently, marginalized individuals are at high risk for neurocognitive disorder. Yet, very few investigations have been conducted to address neuropsychological aspects of marginalization. This symposium will elucidate the neuropsychology of marginally housed persons with multimorbidies by describing their neurocognitive features and by linking these features to: a) risk factors for impairment (e.g., mental illness, brain injury, viral infection); b) structural brain findings (cortical thickness, gyrification, white matter integrity); and c) real world functioning. This work clarifies the important role that neuropsychology can play in addressing the consequences of marginalization for the individual.

Correspondence: Allen E. Thornton, Ph.D., Psychology, Simon Fraser University, 8888 University Drive, Burnaby, BC V5A 1S6, Canada. E-mail:aethornt@sfu.ca

T. WILLI, A.E. THORNTON, A. BARR, D. LANG, W. PANENKA, W. SU \& W.G. HONER. Structural Correlates of SubstanceInduced Psychosis: a Comparison Between Psychostimulant Dependent Individuals With and Without Psychosis.

Objective: After prolonged psychostimulant abuse, some individuals develop transient psychotic symptoms referred to as substance induced psychosis (SIP). The psychotic presentation closely resembles that of the 
schizophrenias, suggesting that similar underlying neural deficits may contribute to the emergence of psychosis across these disorders. This study aimed to characterize structural alterations in SIP, utilizing gray matter (GM) and white matter (WM) indices.

Participants and Methods: From a population of psychostimulantdependent polysubstance users without a DSM-IV diagnosis of idiopathic psychosis, groups were formed between individuals with cocaineassociated psychosis (GM n=29: WM n=24) and cocaine dependent nonpsychotic individuals ( $\mathrm{GM} n=74 ; \mathrm{WM} \mathrm{n}=43$ ). GM differences were investigated in a voxel-based analysis of subcortical structures, while tract based spatial statistics was used to investigate group differences in WM diffusion indices [i.e. fractional anisotropy (FA)].

Results: The cocaine-associated psychosis group had significantly smaller volumes of the thalamus and left hippocampus, controlling for age, total brain volume, and current methamphetamine / marijuana dependence. Additionally, the cocaine-associated psychosis group showed significantly lower FA values $(p<0.05)$ in voxels within WM tracts of fronto-temporal, fronto-thalamic, and interhemispheric pathways.

Conclusions: Collectively, these results suggest several neuroanatomic characteristics that differentiate psychostimulant users who develop psychosis from those who do not. These differences were found to be multimodal, present in both GM and WM, and similar to differences previously identified as abnormal in the schizophrenia literature. These data contribute to the characterization of structural alterations in the expression of psychostimulant associated psychosis, suggesting there may be shared neuroanatomical correlates in the expression of different forms of psychosis.

Correspondence: Taylor Willi.E-mail: taywilli12@gmail.com

\section{K. WACLAWIK, C. GIESBRECHT, W.G. HONER \& A.E. THORNTON. Predictors of One-Year Cognitive Decline in a Marginally Housed, Multimorbid Sample.}

There is a paucity of research on the nature and course of cognition in marginally housed populations, thus there is limited understanding of the relationship between this population's numerous, comorbid health risks and their cognitive functioning. Viral infections and substance use are prevalent in marginally housed populations and have wellestablished relationships with cognitive decline. However, the impact of comorbidity is often unaddressed in the literature. The present study aimed to characterize the nature of one-year cognitive decline in a marginally housed, multimorbid sample, and to assess associations between decline and common morbidities. Participants $(N=288)$ were recruited from single room occupancy hotels in the Downtown Eastside neighbourhood of Vancouver, Canada, and were followed for approximately one year. Neuropsychological functioning was assessed at baseline and at follow-up. Psychiatric diagnosis and infection status for HIV and hepatitis C were determined at baseline. Use of alcohol, cannabis, stimulants, and heroin was ascertained monthly via interview for the follow-up duration. In the overall sample, generally stable cognitive performance was observed in most cognitive domains, but HIV seropositive status was associated with declines in verbal memory $(p<.05)$ and response inhibition $(p<.05)$. Self-reported antiretroviral medication adherence did not mitigate this cognitive decline. These findings suggest that, among the numerous morbidities present in a marginally housed sample, HIV infection may be particularly detrimental to cognitive functioning. Future work should elucidate the degree to which these early indicators of cognitive deterioration are associated with progressive decline over longer time frames in multimorbid samples. Given the negative impact of cognitive impairment on functioning, these results can inform prioritization of treatment targets in populations presenting with multiple health risks.

Correspondence: Kristina Waclawik, MA, Psychology, Simon Fraser University, 1844 MacDonald St, Vancouver, ON V6K 3X9, Canada. E-mail:kwaclawi@sfu.ca
K. GICAS, A. Cheng, W. PANENKA, D. LANG, G.N. SMITH, W.G. HONER \& A.E. THORNTON. Structural Brain Markers are Differentially Associated with Neurocognitive Profiles in Socially Marginalized Persons with Multimorbid Illness.

Objective: Marginally housed persons are vulnerable to brain insult as a function of neurodevelopmental aberrations and environmental risk exposures, yet little is known about structural brain integrity and neurocognition in this context. This study aimed to delineate structurefunction associations to better understand the bases of neuropsychological impairments in a multimorbid population.

Participants and Methods: We conducted a cluster analysis to confirm three previously derived subgroups with distinct neurocognitive profiles in a large sample of socially marginalized persons $(N=299)$. Next, we examined the association between fronto-temporal cortical brain measures (gyrification, cortical thickness) and neurocognitive profiles using multinomial logistic regression. Chi-square tests and ANOVAs differentiated subgroups on proxy measures of neurodevelopment and acquired brain insult/risk exposure. In follow-up, we examined the association between white matter integrity and neurocognitive profiles using multinomial logistic regression and Tract-based Spatial Statistics. Results: We found that the lowest functioning neurocognitive cluster (Cluster 3, $\mathrm{n}=103$ ) was associated with greater frontal and temporal gyrification, widespread decreases in white matter integrity, and more proxies of aberrant neurodevelopment. The clusters characterized by selective decision-making impairment (Cluster 2, n = 109) and higher functioning (Cluster 1, $\mathrm{n}=87$ ) were associated with greater acquired brain insult/risk exposure. Finally, age moderated the association between cortical thickness and neurocognition, with positive associations in older adults only.

Conclusions: Our findings highlight the unique pathways to neurocognitive impairment in a heterogeneous population and help to clarify the vulnerabilities confronted by different subgroups. This is important for developing targeted interventions that better address the differential healthcare needs of marginalized persons.

Correspondence: Kristina Gicas, M.A., Psychology, Simon Fraser University, 8888 University Dr, Burnaby, BC V5A 1S6, Canada. E-mail: kgicas@sfu.ca

T.A. O'CONNOR, D. LANG, W. PANENKA, N.Y. WANG, R.R. PROCYSHYN \& A.E. THORNTON. The Impact of Traumatic Brain Injury and Aggregate Comorbidities on Cognition and Functioning in a Marginally Housed Sample.

Marginally housed individuals face numerous health risks that impair cognition and functioning, and may result in less capability to deal with further brain insult. Participants recruited from single-room occupancy hotels $(\mathrm{N}=436)$ underwent clinical and neurocognitive testing. In Study 1, bi-annual ratings of social and occupational functioning (SOF) were made $(\mathrm{n}=326)$ from which a composite functioning score was derived. A statistically weighted SOF-burden index was created, reflecting the aggregate extent to which demographics and non-TBI comorbidities (vascular, neurological, mental health; substance use; infection) were associated with SOF. Hierarchical linear regressions examined the effects of traumatic brain injury (TBI) on SOF in persons with differential SOF-burden levels. The SOF-burden index was a significant predictor of SOF, accounting for $9.4 \%$ of the total variance. Objective TBI (MRI defined) was a significant predictor of SOF $\left(R^{2}\right.$ Change $\left.=.019\right)$, however self-reported TBI (LOC $\geq 30$ mins; PTA $\geq 24 \mathrm{hrs)}$ was not. Neither SOF-burden by TBI interaction term was predictive of SOF. In Study 2 , similar analyses were run predicting cognition. Participants underwent tests of attention, inhibition, and verbal learning and memory $(\mathrm{n}=$ 293) from which a composite cognition score was derived. The neurocognitive-burden index was a strong predictor of cognition, accounting for $26 \%$ of the total variance. Neither self-reported nor objective TBI accounted for additional variance, regardless of burden level. In this complex sample, non-TBI comorbidities were strong associates of SOF and cognition. Only objective TBI was associated with SOF. Regardless of neurocognitive-burden level and classification method, TBI was not 
associated with cognition. Findings indicate that TBI history has very limited value in signifying cognitive dysfunction in multimorbid marginally housed individuals, however objective TBI may aid in predicting social and occupational functioning.

Correspondence: Tiffany A. O'Connor, Psychology, Simon Fraser University, 8888 University Drive, Burnaby, BC V5A1S6, Canada. E-mail:toa4@sfu.ca

N.Y. WANG, K. WACLAWIK, T.A. O'CONNOR, D. LANG, W. PANENKA \& A.E. THORNTON. The Role of Neurocognition, Psychiatric Symptoms, and Multimorbid Illness In Predicting Everyday and Social Functioning in Marginally Housed Persons. Multimorbid illness, consisting of substance use, psychiatric illness, viral infection, and traumatic brain injury (TBI), is prevalent in marginally housed persons, but it is unclear how these problems influence everyday functioning in this population. In Study 1, we conducted mediation analyses in the entire sample $(\mathrm{N}=216)$ to assess the effects of illness on ratings of everyday and social functioning at 6-month follow-up, and to determine whether the effects were mediated by neurocognition and psychiatric symptoms. Study 2 used multiple regression analyses to evaluate the extent to which neurocognition, psychiatric symptoms, and illnesses differentially predicted follow-up ratings of functioning in participants with $(n=37)$ and without $(n=180)$ schizophrenia. Study 1 found that neurocognition, as well as positive and negative symptoms, accounted for nearly $45 \%$ of the total effect of having schizophrenia on lower ratings of functioning. Neurocognition also mediated $11 \%$ of the effect of TBI on lower ratings of functioning. Additionally, greater frequency of heroin was significantly associated with lower ratings of functioning $\left(c^{\prime}=-.15\right)$, but this effect was not mediated by neurocognition. Study 2 revealed that neurocognition, psychiatric symptoms, and illnesses differentially predicted functioning in participants with and without schizophrenia. Greater heroin use $(p=.02)$ and the presence of TBI $(p=.02)$ predicted lower ratings of functioning in participants without schizophrenia, while neurocognition and major depression were marginally predictive. In persons with schizophrenia, the presence of more psychotic/disorganized symptoms significantly predicted poorer functioning ( $p=.02$ ). Our findings highlight the selective role of neurocognition in mediating the relationship between illness and functional outcome, and emphasize the differential impact of neurocognition, psychiatric symptoms, and illness in marginally housed persons with and without schizophrenia.

Correspondence: Nena Y. Wang, Psychology, Simon Fraser University, \#210-601 North Road, Coquitlam, BC V3J1P1, Canada. E-mail: nenaw@sfu.ca

\section{Historical}

N.C. MURRAY, M. STATUCKA, D.L. MCCABE \& W. LU. Concussion: Past, Present, and Future.

Symposium Summary: The majority of brain injuries that occur in the United States are from concussion, which has been defined as a biomechanical event that temporarily alters brain-related functions. The evaluation and management of concussion has perpetually changed based on the literature. An integrative approach that includes a review of historical literature, and addresses future trends in research, will serve to highlight the key aspects of neuropsychological practice as well illuminate persisting challenges to concussion treatment. With this in mind, this multi-presenter symposia aims to: 1) Review previous misconceptions regarding concussion which have led to critical barriers in treatment and explore current evidence-based guidelines that shape our understanding of concussion; 2) Provide a historical and up-to-date examination of the role of neuropsychology in the acute assessment of concussion; 3) Illustrate how this might be used to optimize patient management by discussing previous, current and future interventions resources and protocols; and 4) Specifically highlight the common recommendation of sleep and rest and discuss the shortcomings of previous research, while shedding light on the current relationship between concussion and rest. Speakers have been carefully selected on the basis that, they each have a track record in adopting a multi-disciplinary approach to traumatic brain injury that has enabled the application of discoveries in clinical care. We anticipate that participants will obtain additional knowledge of how past and present research can accelerate our knowledge base of concussion and how that knowledge base can be effectively applied to patient care.

Correspondence: Nicole C. Murray, Psy.D., Rusk Rehabilitation, NYU Langone Medical Center, 240 East 38th St., Fl. 17, New York, NY 10016, United States. E-mail: nicole.murray@nyumc.org

\section{N.C. MURRAY. Acute Concussion Assessments: The Role of Neuropsychology.}

There are approximately 750,000 non-sports related concussions reported each year as well as an extended range of sports that include risk for concussion (CDC, 2016). Emergency room assessment and sideline testing are environments that represent a significant departure from office-based, outpatient evaluation for the concussed individual. The importance of neuropsychologists in acute concussion evaluations (i.e. sideline assessments and emergency medicine) and the administration of appropriate diagnostic tools in the evaluation of concussion will be addressed during the assessment portion of the presentation. The goal of this presentation is to discuss the previous roles and increasing opportunities for neuropsychologists in the evaluation and management of acute concussion evaluations. The second goal is to share past, present and future diagnostic tools in the assessment of concussion. Reviewing past and present diagnostic techniques serve to highlight the gaps in our field and illustrate the importance of acute assessments post-concussion. Objectives: This part of the symposia includes 3 specific objectives. After the presentation, participants will be able to: 1 . Describe the importance of acute assessment by neuropsychologists on the sidelines and in the emergency room department. 2. List 4 significant differences between the past and present ways we assess concussion. Participants will also recognize the persisting challenges in our approaches to assessment. 3. Identify 3 useful assessment tools that are critical to completing immediate assessment post-concussion.

Correspondence: Nicole C. Murray, Psy.D., Rusk Rehabilitation, NYU Langone Medical Center, 240 East 38th St., Fl. 17, New York, NY 10016, United States.E-mail: nicole.murray@nyumc.org

D.L. MCCABE. The Role of Psychology in Concussion Management. The available evidence suggests a critical role for psychologists, especially neuropsychologists, in navigating the complex interplay between the psychological, physical, and psychosocial factors implicated in the emergence and maintenance of persistent post-concussive symptoms. Arguably, the modern role of psychology in the management of post-concussion symptoms was articulated by Mittenberg and Burton's (1994) survey of neuropsychologists' treatment practices for post-concussion syndrome. In their study, neuropsychologists identified several practices - including head injury education, reassurance (that symptoms are a part of normal recovery), psychotherapeutic support, gradual resumption of activity, and cognitive restructuring - as valuable components of concussion management. To date, the literature generally supports these approaches to treatment, but also suggests the need for interdisciplinary collaboration (e.g., to ensure adequate management of sleep, pain, and other symptoms, which may negatively impact quality of life as well as neuropsychological performance), and raises questions as to the optimal allocation, mode of delivery, and even definition of concussion "treatment." Objectives: This section of the symposium includes 3 specific objectives. After the presentation, participants will be able to: 1 . Describe the importance of time sensitivity when providing concussion interventions. 2 . Review, and reevaluate the role of psychology in concussion management. 3. Access resources related to concussion management. 
Correspondence: David L. McCabe, B.A., Psychology, CUNY Graduate Center and Queens College, 240 East 38th St., Psychology Dept. 17th Floor, Manhattan, NY 10016, United States.E-mail: davidlmc@gmail. com

\section{W. LU. Assessing and Treating Sleep Disturbance and Fatigue after a Concussion(s).}

A common symptom following traumatic brain injury (TBI), of all severities, is sleep disturbance and fatigue, affecting many of those who experience chronic TBI-related symptoms. A majority of these individuals experience either sleep apnea or insomnia and subsequently complain of feeling fatigued and/or somnolent during the day. However, little is known about the consequences of a concussion(s) on sleep function and daytime wakefulness. Furthermore, even less is known about the appropriate recommendations regarding rest post injury. The role of neuropsychologists and rehabilitation psychologists in treating concussion-related sleep difficulties and daytime fatigue is a newly emerging field and is in need of further discussion and research. Objectives: This section of the symposium includes 4 specific objectives. After the presentation, participants will be able to: 1. Explain the limitations of knowledge regarding this area of interest based on shortcomings of previous research. 2. Describe what we know currently about the relationship of post concussion syndrome on sleep and fatigue. 3. Understand how concussion-related sleep disorders and fatigue can be assessed and treated, presently and in the future, by neuropsychologists and rehabilitation psychologists. 4. Describe whether rest or increased mental activity is recommended after sustaining a concussion.

Correspondence: William Lu, PsyD, New York, NY, United States. E-mail:William.Lu@nyumc.org

\section{STATUCKA. Concussion: Myths and Misconceptions.}

There are numerous misconceptions about concussion, which unfortunately, continue to be perpetuated by the media, and well-meaning teachers, coaches, and healthcare professionals. Many of these misconceptions stem from the very definition of concussion as a transient disruption of brain function that quickly and fully recovers. Traditionally this definition has helped to differentiate concussion from more serious forms of traumatic brain injury, which in turn allowed healthcare professionals to make treatment recommendations and prognoses about recovery including making decisions about when it was safe for the individual to return to daily activities such as work, school, and participate in sports. However, recent research and guidelines are changing our understanding of what exactly a concussion is, its long-term implications and repercussions, as well as elucidating potential avenues for treatment and rehabilitation. Objectives: This section of the symposium includes 3 specific objectives. After the presentation, participants will be able to: 1. Identify the most common misconceptions about concussion.

2. Describe the historical findings, context, and theories that are at the root of these beliefs about concussion.

3. Appraise current, cutting-edge research which calls into question our beliefs about concussion. Some of these findings will be expanded upon in the following sections of the symposium.

Correspondence: Marta Statucka, Ph.D., 399 Bathurst Street, Fell Pavilion, 4F-409, Toronto, ON M5T 2S8, Canada. E-mail: marta. statucka@uhn.ca

\section{Memory Functions}

\section{K. OSIPOWICZ, N. KESHAVARZIAN, K. MCWILLIAMS, J. PETERSEN \& J. WILLIAMS. fMRI of Hippocampal Function: Old insights from New Studies.}

Symposium Summary: The hippocampus remains one of the most interesting, enigmatic, and frustrating brain structures. We all know that the hippocampus has some role in memory, but it is still unclear what that role is -- neuropsychological patients with hippocampal damage show prospective and retrospective amnestic syndromes (while maintaining the ability to encode and retrieve certain types of memories); in addition to a number of other underreported effects, such as anosmia and expressive dysphasia. Neuroimaging studies readily show activation of the posterior hippocampus during the encoding of visual stimuli, while consistently failing to activate the anterior hippocampus. This symposium will address the difficulty in fMRI investigations of the hippocampus by: (1) providing a clinical neuroimaging demonstration of the unconsidered functions of the hippocampus (2) reviewing the results of a series of failed hippocampal fMRI tasks, and an anatomical review and methodological correction to explain hippocampal activation failure, (3 and 4) presenting the results of two successful fMRI investigations of hippocampal activation, and reviewing our research in the context of hallmark historical findings in the study of the hippocampus. Correspondence: J Michael Williams, PhD, Philadelphia, PA, United States.E-mail:jw37@drexel.edu

\section{K. OSIPOWICZ \& J.I. TRACY. mTLE and Verbal Fluency.}

This presentation will review an fMRI study of verbal fluency changes in a sample of mesial temporal lobe epilepsy patients who undergo en bloc resection of the dominant temporal lobe including the mesial structures. Patients are compared against themselves and a sample of matched controls. Results show subtle changes before and after surgery, with a distinct neuroanatomical deviation from normal activation patterns. This study illustrates (1) the need to consider hippocampal function outside of the realm of memory and (2) the need for a clinically relevant, and robust fMRI task for the assessment of hippocampal function. Correspondence: Karol Osipowicz, Ph.D., Psychology, Drexel University, 3141 Chestnut St, 204 Stratton Hall, Philadelphia, PA 19104, United States.E-mail:kzo22@drexel.edu

K. OSIPOWICZ, N. KESHAVARZIAN, K. MCWILLIAMS, J. PETERSEN, M. BARROW, D. PANFILI \& J. WILLIAMS. Smelly Scenes: Role of the Hippocampus in Multisensory Integration of Memories.

This study examined the role of the hippocampus as a multisensory memory integration center. The role of the hippocampus in both memory storage and retrieval is well documented. Additionally, anatomical models of the hippocampus support a sensory input organization along the lateral axis, with the posterior hippocampus being enervated by the amygdala and olfactory cortex, the middle section receiving inputs from somatosensory and auditory cortices, and lastly the posterior hippocampus being connected to visual cortices. Here, we hypothesize that the hippocampus serves a role in the coding and decoding of multisensory information in episodic memory. We test this hypothesis using a novel fMRI paradigm where participants were asked imagine the smell of a olfactorily salient visual scene (e.g. garbage dump). Activation was observed in the bilateral hippocampi (extending from the anterior to the posterior sections), bilateral occipital lobe, and left inferior frontal gyrus. These findings support our hypothesis that the hippocampus plays an important role in multisensory memory coding. Additionally, we observed bilateral hippocampal activation in 19 of 21 subjects, suggesting that this task can be used as an effective clinical test of hippocampal function.

Correspondence: Karol Osipowicz, Ph.D., Psychology, Drexel University, 3141 Chestnut St, 204 Stratton Hall, Philadelphia, PA 19104, United States.E-mail:kzo22@drexel.edu

J. WILLIAMS, N. KESHAVARZIAN, K. MCWILLIAMS, J. PETERSEN \& K. OSIPOWICZ. fMRI Stimuli and Methods.

This presentation will review fMRI methods used to assess hippocampal activation in twenty-one neurologically healthy adults between the ages of 26 to 37. Tasks involving emotional and neutral pictures, words and environmental sounds were alternated during a single scanning session. The fMRI analyses revealed a robust bilateral occipital and posterior hippocampal activation associated with picture stimuli, and language 
areas associated with words and sounds (with additional primary auditory activation). Consistent with many studies, these results suggest that fMRI methods detect the dominant influence of the visual system on hippocampal activation. An additional analysis was conducted in an attempt to overcome issues with the tonicity of hippocampal function, problems with its location, and its size relative to fMRI resolution. This technique involved normalizing utilizing the standard algorithm, but each participant's T2 data was normalized to his own T1 space, instead of normalizing the data to AAL Atlas. This allowed resolution to increase from the standard $2 \times 2 \times 2 \mathrm{~mm}$ per voxel to $0.5 \times 0.5 \times 1$ $\mathrm{mm}$ per voxel. The AAL Atlas was then coregistered and normalized to each participant's T1 data, giving each participant their own atlas based ROIs. Data within the hippocampus, parahippocampal gyrus, and amygdala were smoothed; each region then underwent first level modeling, as data for each region was extracted from the functional data and modeled across time for each event. A sample-specific template was generated and second level comparisons were made according to the standard method. Widespread activation in both hippocampi was observed when emotional and neutral pictures were contrasted. This presentation will also include a discussion of the effects of processing on fMRI activation.

Correspondence: J Michael Williams, PhD, Philadelphia, PA, United States.E-mail:jw37@drexel.edu

K. MCWILLIAMS, J. PETERSEN, N. KESHAVARZIAN, J. WILLIAMS \& K. OSIPOWICZ. The Unique Role of Time in Autobiographical Memory Consolidation.

One of the enduring mysteries of hippocampal function is the representation of time as an aspect of memory. Memories form the basis for structuring subjective time constructions; however the mechanism by which this occurs is still unknown. The goal of this study was to examine the role of time in the consolidation and retrieval of autobiographical episodic memories. Twenty healthy adult subjects underwent functional magnetic resonance imaging to assess brain activity during visually cued autobiographical memory retrieval. In the scanner, participants viewed self-supplied photographs from four time periods: 1 year, 2 years, 6 years and 14 years prior. Participants were asked to recollect the memory associated with each photograph. Multi-voxel pattern analysis revealed three patterns of activation identifiable by the chosen construct of time, photograph year. Parietal, frontal, parahippocampal and thalamic activation increased with the remoteness of the memory. Similarly, medial temporal lobe activation, although present during all time points, was more pronounced in the left hemisphere for the recollection of older memories. In contrast, the putamen and caudate nucleus were more active during recent memory recall. As memories age the network that supports them changes to promote efficiency and adaptability. Our findings suggest that recent memories rely heavily on medial temporal and basal ganglia structures to give pliability to the memory trace, allowing it to be recalled, updated or incorporated with ease. As the memory ages however the need for pliability decreases. Older memories showed greater cortical, thalamic and asymmetric hippocampal activation during recall. These regions allow for the preservation of a rigid memory trace that is less susceptible to interference, but is more semanticized and laborious to recall.

Correspondence: Kellie McWilliams, BA, Psychology, Drexel University, 1708 Melon st, Philadlephia, PA 19130, United States. E-mail: kam392@gmail.com

\section{Student Social, Hosted by the INS Student Liaison Committee}

\section{FRIDAY MORNING, FEBRUARY 3, 2017}

\section{CE Workshop 9. Autism: Clinical and Translational Insights from Brain Mapping \\ Presenter: Rajesh Kana \\ 7:20-8:50 a.m.}

\section{R. KANA. Autism: Clinical and Translational Insights from Brain Mapping.}

Although a biological origin for autism spectrum disorders (ASD) has been proposed several decades ago, a firm and reliable neurobiological marker has rather been elusive. Of late, neuroimaging studies have provided converging findings on disruptions in brain connectivity as a common signature in the pathobiology of autism. Work from our group has tried to address two important questions, First about the diagnostic utility of neuroimaging-based markers of autism; and Second, about the efficacy of intervention programs in changing the brain circuitry underlying impaired functions in children with autism. Our findings reveal that brain abnormalities in autism spans multiple levels of organization, such as function, anatomy, connectivity, and chemical concentration. The use of multimodal neuroimaging may provide an avenue to assess these indices and generate a comprehensive explanation of this disorder. Some of our studies used machine learning techniques to understand abnormalities in which of these indices best predict autism. In testing the impact of an intense language intervention on children with autism who have below average reading comprehension, we found significant changes in brain connectivity of the reading network as a result of intervention. These findings provide significant insights and promising new directions in moving neuroscience research closer to clinic.
After this lecture, the audience will be able to: 1) Assess the state of brain research, particularly neuroimaging-based research, in autism spectrum disorders; 2) Analyze the preliminary steps in exploring the translational potential of neuroimaging research in autism; 3) Discuss brain plasticity and the potential of intense interventions in changing the brain circuitry in children with autism; and 4) Assess the distance between laboratory and clinic in neuroscience research and learn about the attempts to bridge this gap.

Correspondence: Rajesh Kana, Ph.D., AL, United States. E-mail: rkana@uab.edu

\section{CE Workshop 10. Not All Aging Processes Are Created Equal: Cognitive Aging Among Culturally Diverse Groups}

\author{
Presenter: April Thames
}

\section{7:20-8:50 a.m.}

\section{A. THAMES. Not All Aging Processes Are Created Equal: Cognitive Aging Among Culturally Diverse Groups.}

The US population aged 65 and older has increased, including the number of racial and ethnic minorities. It is increasingly important to understand specific risk factors that may be linked to ethnicity/race and psychosocial factors that contribute to pathologic brain/cognitive aging. This course will first review the research on ethnic and racial groups (focused on African Americans and Hispanic/Latinos) and risk 
for cerebrovascular disease, and cognitive impairment. We will cover the research findings that indicate biological risk factors in African Americans and Latino/Hispanics. Next, this course will review the psychosocial risk factors that may contribute to poor cognitive aging outcomes, including education, racial/ethnic discrimination, stressors related to immigration, poverty, loneliness/lack of social support. Finally, this course will cover the next steps for research and assessment, which include establishing mechanistic links between specific cardiovascular risks and cerebral sequelae through neuroimaging, hallmark indicators of biological aging, and focused cognitive and psychosocial assessments. The content is geared towards intermediate and advanced knowledge levels, and the following objectives will be achieved: (1) have a deeper understanding of how to study mechanisms that contribute to cognitive aging among racial and ethnically diverse groups, and (2) be familiar with key considerations in assessment of cognitive aging.

Correspondence: April Thames, Ph. D., CA, United States. E-mail: athames@mednet.ucla.edu

\section{Poster Session 6. Adult 2}

\section{8:00-9:15 a.m.}

\section{Cancer}

\section{A. AILION \& T.Z. KING. Childhood brain tumors: A systematic review of the structural neuroimaging literature.}

Objective: Due to medical advances, a large portion of children survive brain tumor diagnosis and treatment; therefore, it is important to identify the neuroanatomical and neurocognitive outcomes associated with survivorship. Recent neuroimaging research developments can help understand brain-behavior relationships in this population. This review will evaluate a range of structural neuroimaging studies, describe the neuroimaging techniques, and highlight methodological advantages and disadvantages in this population.

Participants and Methods: Searches were conducted in Pubmed, PsycInfo, and Medline databases in 05/2016 using the following terms: 'neuroimaging, 'structural magnetic resonance, 'MRI,' 'DTI,' 'tractography,' 'fiber tracking, 'TBSS,' 'white matter,' 'grey matter,' 'volume, 'volumetrics,' 'cortical thickness,' or 'atrophy,' and 'pediatric' or 'childhood,' and 'brain tumor,' and 'cognitive.' Only peer-reviewed studies on human populations in English were considered. The search identified a total of 131 articles, of which 31 met inclusion criteria. Articles were be grouped based on neuroimaging method. Six of these studies used semi-qualitative neuroimaging rating scales, 3 used lesion mapping, 6 used volumetric brain measures, and 16 used diffusion weighted imaging techniques.

Results: Across studies, damage is commonly reported near the cerebellum, brain stem, and subcortical regions, as well as the frontal lobe. Damage to these regions impacts a broad range of neurocognitive outcomes, as well as a number of specific neurocognitive domains. For instance, damage to more ventral and subcortical brain regions corresponded with lower motor speed, processing speed, attention, and memory.

Conclusions: Relevant limitations and future directions will be discussed with a focus on disentangling the complex and multifaceted factors associated with the consequences of brain tumor survivorship. Correspondence: Alyssa Ailion, B.S., Georgia State University, 934 Bridgegate Drive, Marietta, GA 30066, United States.E-mail:aailion1@ student.gsu.edu
S. AMEDORO, C.L. ARMSTRONG \& M. FISHER. Exploratory Analysis of Inductive Reasoning Abilitiesin a Pediatric Neurofibromatosis Type 1 Population.

Objective: Neurofibromatosis Type 1 (NF1) is associated with a high incidence of neurocognitive impairments. The current study examined patterns of inductive reasoning ability and potential implications for academic achievement.

Participants and Methods: Retrospective chart reviews were completed for 41 pediatric outpatients diagnosed with NF1 who were referred for neuropsychological evaluation by their neuro-oncologist. Three participants were excluded due to age/outlier score $(\mathrm{N}=38$, aged $11.21 \pm 3.28$, $53 \%$ female). Measures of inductive reasoning included: Biber Cognitive Estimation Test (BCET), Comprehension Test (COMP) and Similarities Test (SIM) from the WISC-IV, Affect Recognition Test (AFFR) and Theory of Mind Test (TOM) from the NEPSY-II, and Rey-Osterrieth Complex Figure Test (ROCF) Copy. Measures of academic achievement included: Word Reading and Math Computation from the WRAT-IV. Patterns of inductive reasoning were assessed via hierarchical cluster analysis, MANOVA, and discriminant analysis. Relationship to academic achievement was assessed using backwards stepwise regression.

Results: Cluster analysis indicated those with/without impairment in inductive reasoning $(\mathrm{N}=23$ and $\mathrm{N}=15)$. MANOVA $(\mathrm{F}(5,31)=11.39$, $\mathrm{p}<0.000)$ showed that twenty-three patients performed significantly lower on all measures of inductive reasoning, excluding AFFR. Discriminant analysis revealed that BCET and SIM discriminated with an overall accuracy of $97.4 \%$. Regression analyses predicted $77 \%\left(R^{2}\right)$ of the variance in math, and $32 \%\left(R^{2}\right)$ of the variance in reading.

Conclusions: Results revealed a significant problem with inductive reasoning in a pediatric NF1 population, as $61 \%$ of the sample demonstrated extensive impairment. Findings support thorough assessment of this domain because inductive reasoning deficits were associated with poorer math and reading achievement, and will inform methods of academic support.

Correspondence: Sarah Amedoro, Graduate Psychology, Immaculata University, 1611 Greenspring Dr, Lutherville, MD 21093, United States. E-mail:sarah.amedoro@gmail.com

A. CHILD, M. RIS, L.H. MUTHS \& L.S. KAHALLEY. Significant Reading Difficulties in a Pediatric Patient with Left Thalamic Tumor: A Case Report.

Objective: This case report examined the neurocognitive effects of left thalamic tumor and associated right homonymous hemianopsia. Thalamic tumors are linked to a range of effects, including impaired contralateral sensory or motor functioning and abnormal eye movements. Treatment often includes surgery, chemotherapy, and radiation therapy, which are associated with neurocognitive deficits.

Participants and Methods: An 8-year-old male with left thalamic pilomyxoid astrocytoma and right homonymous hemianopsia underwent a neuropsychological assessment. The patient had previously had a biopsy, two shunts placed, multiple shunt revisions, two courses of chemotherapy, and radiation therapy.

Results: The patient's neuropsychological testing revealed borderline intellectual functioning with relatively strong visuospatial skills as compared to verbal, working memory, or processing speed abilities. His profile was remarkable for a total inability to read or write words and some letters, resulting in extremely low performance on reading and writing tasks. Despite this, he solved basic math problems and had low average math skills relative to same-grade peers. Cognitive weaknesses included verbal memory and fine motor skills, while visual-motor skills were relatively preserved. Parent-report measures reflected a well-adjusted child with average adaptive functioning abilities.

Conclusions: Left thalamic tumors and associated treatments are associated with significant risk for neurocognitive impairment in children. This case report examined an 8-year-old with left thalamic tumor and homonymous hemianopsia who had undergone multiple surgical procedures, chemotherapy, and radiation therapy. His overall cognitive functioning was impaired, but further examination of his profile 
revealed significant verbal and reading deficits contrasted with relatively preserved visuospatial and math skills. This case report illustrates the global and specific neurocognitive risks of thalamic tumors and treatment.

Correspondence: Amanda Child, Psychology, University of Houston, TIMES Developmental Neuropsychology Lab, 4811 Calhoun Rd, Mailroom 365, Houston, TX 77204, United States. E-mail: amandachild11@gmail.com

B.E. CLARK, P. BEAN, E. CARROLL, T. COOK, M. HOFFMAN, M. MCCARVEL, J. MCFARLAND \& S. HALL. Cancer Patients' Perceptions Of Their Cognitive Functioning After Treatment Is Impacted By Comments From Others.

Objective: Cancer patients often cite negative cognitive symptoms during and after receiving chemotherapy for the treatment of cancer. This study examined the influence of negative expectations on selfreports of cognitive functioning in patients who had completed chemotherapy for the treatment of cancer. Understanding the multifaceted etiology of chemotherapy-related cognitive impairment (CRCI) is critical in order to facilitate the highest levels of patient quality of life.

Participants and Methods: Adult participants $(\mathrm{n}=53)$ who had completed chemotherapy treatment were recruited from an outpatient cancer center in the Northwest. Participants in the experimental group read a prompt stating that many cancer patients report difficulties with thinking during and after chemotherapy. Participants in the control group received a neutral prompt. Both groups completed a self-report measure of cognitive functioning (FACT-Cognitive Function-Version 3). Results: While the two groups did not differ significantly in the extent to which they reported cognitive symptoms, a significant correlation of $r=$ $.56(p<0.01)$ was found between the 'Perceived Cognitive Impairments' and 'Comments from Others' subscales for both groups.

Conclusions: This finding indicates a strong relationship between the content of what others say to cancer patients about their cognitive functioning and how cancer patients perceive their own cognitive functioning. Well-intentioned remarks from others may contribute to patients' negative beliefs about their cognitive functioning. These negative self-perceptions may adversely affect important aspects of cancer recovery. Understanding the mechanisms of CRCI may help health care professionals and close others interact with cancer patients in ways that support the best recovery possible.

Correspondence: Brook E. Clark, PhD, Psychology, University of Montana, 4924 Potter Park Loop Unit A, Missoula, MT 59808-5311, United States.E-mail: brook.clark@umontana.edu

H.M. CONKLIN, J.M. ASHFORD, B.T. HAREL, M. SWAIN, G. ROBINSON \& A. GAJJAR. Computerized Assessment of Cognitive Impairment among Children Undergoing Treatment for Medulloblastoma.

Objective: Advantages to computerized cognitive assessment include increased precision of response time variables and greater availability of alternate forms facilitating longitudinal assessment. Cogstate is a flexible computerized cognitive system developed for monitoring attention, memory and processing speed over time. Despite evidence that core areas of deficit in children treated for medulloblastoma are among those assessed by Cogstate, it has not previously been used with this population.

Participants and Methods: Children participating in an ongoing prospective trial of risk-adapted therapy for newly diagnosed medulloblastoma $(\mathrm{N}=37$; mean age $=12.6 \pm 5.1 ; 26$ males $)$ were administered Cogstate at baseline (after surgery, prior to adjuvant therapy) and three months later (six weeks after irradiation). Additional clinicallyestablished measures of neuropsychological functions were administered at baseline.

Results: One sample t-tests revealed performance within age expectations at baseline across Cogstate tasks (Detection, Identification, One Back and Groton Maze Learning). Females were slower than males across timed tasks (ps< .05). Pearson correlation coefficients revealed significant associations among Cogstate tasks and clinical measures of the same construct (e.g., Detection and Identification with Conners' CPT; One Back with Digit Span Backward; rs= .36-.61, ps<.05). Paired $\mathrm{t}$-tests revealed slower reaction times after radiation therapy (Processing Speed Composite, $p=.03$ ), with slower performance on Identification $(p=.02)$ and more errors on Groton Maze Learning $(p=.02)$ relative to normative data.

Conclusions: Cogstate is sensitive to cognitive changes of children undergoing treatment for medulloblastoma with predictors of performance consistent with the literature. Correlations with neuropsychological measures provide evidence for criterion-oriented validity. Findings offer initial support for Cogstate for monitoring of cognitive late effects. Correspondence: Heather M. Conklin, Ph.D., Psychology, St. Jude Children's Research Hospital, 262 Danny Thomas Place, Mail Stop 740, Memphis, TN 38105-2794, United States.E-mail: heather.conklin@ stjude.org

L.E. COX, J.E. SCHREIBER, K.A. CHRISTOFF, S.L. PALMER, M.A. SWAIN, D.J. MABBOTT, M.J. BONNER, C.L. ARMSTRONG, M. CHAPIESKI, S. KNIGHT, L. HUANG, H. ZHANG \& A. GAJJAR. Psychosocial Trajectories Among Survivors of Pediatric Brain Tumors: A Growth Mixture Modeling Approach.

Objective: Children treated with CNS-directed therapy for brain tumor are at significant risk for behavioral and social difficulties. The risk profile is not homogenous, suggesting that some survivors experience late effects that complicate survivorship. The aim of this study was to investigate the presence of latent classes based on psychosocial growth trajectory to inform identification of those at greatest risk.

Participants and Methods: Survivors $(n=166)$ treated on an international, multi-site protocol for newly diagnosed embryonal tumors were included. Participants were approximately 9 years at enrollment $(\mathrm{SD}=3.25)$, mostly male $(61.5 \%)$, Caucasian $(75.9 \%)$, and treated for average risk disease $(75.3 \%)$. The majority was diagnosed with medulloblastoma $(83.7 \%)$. Caregivers completed measures of their child's psychosocial functioning on the Child Behavior Checklist (CBCL) at baseline and up to five years post diagnosis. Growth mixture modeling (GMM) was employed to determine the number and trajectory of latent classes with respect to Internalizing, Externalizing, Total Behavior Problems, and Social Competence.

Results: GMM revealed distinct latent classes that were mostly stable over time. For Internalizing Problems, there were low (26.4\%) and medium $(38.8 \%)$ trajectories that remained within the normal range and a high class $(34.8 \%)$ trajectory in the at-risk range. For Externalizing and Total Problems, there were low and medium trajectories that fell within the normal range; a high trajectory had baseline scores in the normal range but moved into the at-risk range by year two. For Social Competence, there were moderate $(59.3 \%)$ and high $(40.7 \%)$ trajectories; both within the normal range.

Conclusions: Findings suggest that a subset of pediatric BT survivors experience psychosocial difficulties that persist over the follow-up period. Identifying those at risk for long-term difficulties is paramount, so that resources can be efficiently utilized.

Correspondence: Lauren E. Cox, PhD, Psychology, St. Jude Children's Research Hospital, Mail Stop 740, Rm R4017, 292 Danny Thomas Place, Memphis, TN 38105-3678, United States.E-mail:lauren.cox@ stjude.org

N.A. DASHER, A. SYLVIA \& K. VOTRUBA. Effect of Types of GvHD on Patient Mood and Functional Abilities following Bone Marrow Transplant.

Objective: Acute graft versus host disease (aGvHD) affects between $35 \%-50 \%$ of allogeneic hematopoietic stem cell transplant (HCT) recipients and about half of these individuals later develop chronic GvHD (cGvHD). GvHD has been shown to negatively impact patient mood and functional status. Our analyses investigated the relationship between 
site and severity of GvHD with patient and provider reports of patient functioning and mood.

Participants and Methods: Participants were evaluated repeatedly with the Patient Health Questionnaire - 9 (PHQ9) at three medical evaluations following bone marrow transplant. Patient functional status was evaluated both by self-report and provider report.

Results: Model comparison controlling for gender, donor type, GvHD severity, and patient reported functional decline predicted PHQ9 scores across all visits [Visit 1: $b=-.82, p=.01$; Visit 2: $b=-.39, p=.04$; Visit 3: $b=-.674, p=.01]$. Of those with $\mathrm{cGvHD}$, analysis of variance (ANOVA) showed significant differences between PHQ9 scores and symptom severity across all visits $[$ Visit $1: \mathrm{F}(3,129)=6.21, p=.001$; Visit 2: $\mathrm{F}(3.127)=5.59, p=.001 ;$ Visit $3: \mathrm{F}(3.73)=3.66, p=.02]$. Post hoc tests found that the mean PHQ9 score was significantly higher in those with severe vs. mild or no symptoms across all visits. ANOVA investigating the site of GvHD showed significant differences between PHQ9 scores and severity in those with cGvHD of the eyes (severe > mild or none) across all three visits [Visit 1: $\mathrm{F}(3.129)=4.51, p=.01$ : Visit $2: \mathrm{F}(3,127)=3.27, p=.02$; Visit $3: \mathrm{F}(3,73)=3.17, p=.03]$. No significant differnces in $\mathrm{PHQ} 9$ scores were found in those with cGvHD of the liver, skin, mouth, or lungs.

Conclusions: Results highlight the strong relationship between GvHD and resulting mood and functional abilities in this population and the importance of patient reports in accurately identifying potential barriers to treatment. Results also show the importance of the site of GvHD in identifying those most at risk for depression and functional decline. Correspondence: Nickolas A. Dasher, Psychiatry, University of Michigan, 1323 Natalie Lane, Unit 203, Ann Arbor, MI 48105. United States. E-mail:ndasher@gmail.com

K.R. NOLL, E. ESTEVIS, M.E. BRADSHAW, J.S. WEFEL, S. KESLER, J. REXER, C. HARMON \& S. RHINES. Driving Safety in Patients with Primary Brain Tumors.

Objective: Operating a motor vehicle involves multiple cognitive and sensorimotor faculties. Neurological conditions pose driving risk, but this has not been examined in primary brain tumor (PBT) patients. Participants and Methods: Sixtv-five patients with PBT [32 left: 33 right hemisphere; 62\% GBM; Age=53.2(15.9); Edu=14.9(2.5)] completed the Cognitive Behavioral Driver's Inventory (CBDI). A composite classifies patients as Passing, Failing, or Borderline and is predictive of behind-the-wheel performance. A subset also completed broader cognitive testing. The Borderline group was collapsed with the Failing group. Patient characteristics were compared across Passing/ Failing groups with t-tests or $\chi^{2}$ tests. CBDI subtests were compared across groups with ANCOVA controlling for significant demographic characteristics. Logistic regression identified characteristics predictive of CBDI outcome.

Results: CBDI scores were Passing for $70 \%$ of patients. Failing patients were older than Passing [62.6(12.9) vs. 49.1(15.4), p=.001]. Patients with GBM Failed more often than those with lower grade PBT $[43 \% \mathrm{vs.}$ $8 \%, p=.013]$. Covarying for age, Failing patients performed significantly below those who Passed across most subtests, particularly on measures of attention to detail (Picture Completion, $\eta^{2}=.41$ ), simple reaction speed (CBDI Task 1 time, $\eta^{2}=.38$ ), and mental flexibility (CBDI Task 4 times, $\eta^{2}=.37-42$. Age and histology accounted for $31 \%$ of the variance in CBDI outcome. No other characteristics were significant predictors. In those with broader testing, CBDI outcome was significantly associated with graphomotor speed, manual dexterity, and memory.

Conclusions: A sizeable proportion of patients with PBT appear at risk of driving difficulty, particularly those with GBM and older age. Visual attention and executive difficulties on the CBDI are most salient in at-risk patients, though failure was also associated with measures of dexterity and even memory. This highlights the importance of screening PBT patients for driving safety.
Correspondence: Eduardo Estevis, Ph.D, Neuropsychology, University of Texas MD Anderson Cancer Center, 1 Hermann Museum Circle \#3106, Houston, TX 77004, United States.E-mail:eestevis@mdanderson.org

A. GIOIA, T. KENNEDY, I. TAORMINA, G. DOME, K. OLSON, K.S. WALSH \& K.K. HARDY. Associations Between Depressive Symptoms and Neuropsychological Functioning in Pediatric Oncology Patients with Brain Tumors (BT) and Acute Lymphoblastic Leukemia (ALL).

Objective: Pediatric cancer survivors are at risk for developing symptoms of depression and anxiety. While these are known to impact neuropsychological performance in typically developing children, associations between such symptoms and neuropsychological functioning in survivors are unclear.

Participants and Methods: Retrospective data of 87 survivors of acute lymphoblastic leukemia (ALL; 49.4\%) and brain tumors (BT; 50.6\%) aged 3-19 (M=10.77, SD=4.23, 58.6\% Male) were reviewed. Anxious/ Depressed and Withdrawn/Depressed subscales from the CBCL and TRF were used to measure anxiety and depression. Measures of IQ, attention, and memory were also analyzed.

Results: Parents rated survivors of ALL $(M=58, S D=8.54)$ as having greater symptoms of depression than survivors of BT $(t=2.03, p<.05)$. Parent ratings of anxious and depressive symptoms were generally unrelated to neuropsychological test performance, though they correlated significantly with ratings of hyperactivity $(r=.32, p<.01$ and $r=.35$, $\mathrm{p}<.01$, respectively). Teacher reported ratings of withdrawn/depressed symptoms were significantly correlated with Wechsler intelligence subscales FSIQ $(\mathrm{r}=-.27, \mathrm{p}<.01)$, WMI $(\mathrm{r}=-.37, \mathrm{p}<.01)$, and PSI $(\mathrm{r}=-.50$, $\mathrm{p}<.01)$. Attention and memory measures were significantly correlated with teacher reported ratings of withdrawn/depressed symptoms, specifically the TEA-Ch Sky Search Attention score $(\mathrm{r}=-.36, \mathrm{p}<.05)$, and immediate recall, short, and long delay from the CVLT-C $(-.32<\mathrm{r}<-.39)$. Conclusions: Teacher reports of depressive symptoms were significantly associated with neuropsychological performance in pediatric cancer survivors, whereas parent ratings were generally unrelated to neurocognitive outcomes. Teacher ratings are obtained in few studies with this population; yet, this data suggests that teachers may perceive mental health symptoms that are more directly related to survivors' neuropsychological difficulties than ratings from parents. This highlights the need to obtain ratings from multiple informants in clinical practice and research.

Correspondence: Anthony Gioia, Psychology (BS), Neurology, Children's National Medical Center, 2804 Deer Trail Court, Ellicott City, MD 21042,United States.E-mail: agioia@childrensnational.org

A.M. HENNEGHAN \& S. KESLER. Executive Function and Emotional Distress Prior to Breast Cancer Treatment.

Objective: Patients with breast cancer (BC) often perceive cognitive dysfunction, which is strongly associated with psychological distress. However, these two constructs are very broad, and a better understanding of the association between cognitive dysfunction and psychological status is needed. Further, while effects of chemotherapy on cognitive dysfunction are well known, the effects of cancer pathogenesis remain unclear. Therefore, the objective of this study was to examine the relationships between different aspects of psychological and executive functioning in patients BC prior to any cancer treatment.

Participants and Methods: We enrolled 31 women with primary BC, ages 29-64, prior to any treatment, including surgery. Participants were administered standardized self-rating questionnaires: CAD, a measure of depression, anxiety and fatigue, and BRIEF, a measure of executive function. Two-sided Spearman correlations with Bonferroni correction were used to examine relationships between CAD and BRIEF subscales. Results: BRIEF subscale T scores ranged from 49.35(8.4) to 56.77(9.7) and CAD subscale T scores ranged from 49.58 (8.4) to 54.33 (9.2), all within the clinically normal range, Depressed mood was significantly positively correlated with executive emotional control and behavioral 
regulation difficulties $(p<.0001)$; and diminished interest as well as fatigue were correlated with executive behavioral regulation difficulties $(\mathrm{p}<0.001)$.

Conclusions: Higher levels of depressed mood, diminished interest, and cognitive fatigue may be related to reduced emotional control and behavioral regulation. However, psychological distress was not broadly associated with all aspects of executive function. Additionally, distress and cognitive dysfunction were not clinically elevated despite patients being very newly diagnosed with cancer.

Correspondence: Ashley M. Henneghan, MSN, School of Nursing, University of Texas at Austin, 3101 S. Lamar Blvd, Apt 1307, Austin, TX 78704, United States. E-mail: ashleyhenneghan@gmail.com

\section{J. IRISH, S.K. TADROUS-FURNANZ, S.K. PATEL \& A.A. TURK NOLTY. The Relationship Between Executive Functioning and Adaptive and Maladaptive Behavior in Childhood Cancer} Survivors.

Objective: Difficulties with executive skills often result from white matter frontal lobe deficits of childhood cancer treatment. Connections between executive functioning $(\mathrm{EF})$ and behavior problems have been established in child survivors of acute lymphoblastic leukemia (ALL), but not adaptive behaviors. Our aim was to examine the relationship between aspects of EF and adaptive and maladaptive behavior.

Participants and Methods: The sample included 44 children aged 6 to $18(M=11.9)$ who were survivors of ALL or other cancers with CNS involvement. Exclusion criteria included a brain tumor or developmental disability preceding cancer. Children underwent neuropsychological evaluation that included the California Verbal Learning Test (CVLT-C), and parent versions of the Behavior Rating Inventory of Executive Function (BRIEF) and Behavior Assessment System for Children (BASC-2).

Results: According to parents' BASC-2 responses, our sample had fewer externalizing behavior problems than the normative sample, $T=47.3, S D=7.8, p=.03$, but similar levels of internalizing problems, behavioral symptoms, and adaptive skills. BRIEF Behavioral Regulation Indexes correlated with Externalizing Problems, $r(42)=.57$, Internalizing Problems, $r(42)=.35$, Behavioral Symptoms, $r(42)=.65$, and Adaptive Skills, $r(42)=-.39$, all $p s<.05$. BRIEF Metacognition Indexes correlated with Behavioral Symptoms, $r(42)=.43$, and Adaptive Skills, $r(42)=-.45, p s<.01$. CVLT-C perseverations and false positives correlated with Internalizing Problems, $r(42)=.32$ and -.32 , respectively, $p s<.05$.

Conclusions: Childhood survivors with fewer behavioral regulation or metacognition difficulties seem to exhibit stronger adaptive skills, which strengthens our understanding of the connection between EF and behavioral functioning for childhood survivors of CNS-involving cancers. External scaffolding of EF may allow children who are not manifesting behavioral problems to increase adaptive skills, thus facilitating their navigation of social and academic settings.

Correspondence: Jennifer Irish, Fuller Graduate School of Psychology, 3762 E. California Blvd, Pasadena, CA 91107, United States. E-mail: jenniferirish@fuller.edu

L.M. JACOLA, D. ANGHELESCU, L. HALL, L. WHITE, K. RUSSELL, J.E. SCHREIBER, M. ROSSI, A. MOORE \& A. GAJJAR. The Relationship between Procedural Sedation during Treatment and Neurocognitive Outcomes in Survivors of Pediatric Medulloblastoma.

Objective: Survivors of pediatric medulloblastoma treated with radiation therapy (RT) are at risk for neurocognitive deficits. Variability in outcomes is not well understood, despite known risk factors (i.e., greater therapy intensity and younger age at diagnosis $[\mathrm{dx}]$ ). Treatment involves repeated exposure to procedural sedation with general anesthesia [GA]. GA exposure has been associated with later attention and learning problems in the general childhood population. We examined the association between GA exposure during risk-adapted protocol therapy for pediatric medulloblastoma and neurocognitive outcomes 3 years post-treatment (3YP).

Participants and Methods: GA frequency was abstracted from the medical record for 153 patients (mean age at $\mathrm{dx}[M]=9.4 \mathrm{yrs}, 33 \%$ high risk). Of these, $57 \%(n=87 ; M=10.1$ yrs, $25 \%$ high risk $)$ completed 3 YP assessment (WJ3 Cognitive: General Intellectual Ability, Broad Attention, Working Memory, and Processing Speed). We used linear regression to examine the association between GA exposure and neurocognitive outcomes. All $p$-values are two-sided.

Results: The overall group experienced 1-52 GA events during therapy $(M=22.3)$, and was increased in younger $(M$-young=31.8) and high risk patients $(M$-high=27.1, $p s \leq .01)$. At $3 \mathrm{YP}$, mean scores were in the Average range, with the exception of Processing Speed $(M=82.2$, $p<.001)$. Scores did not differ by risk group, but younger age at $\mathrm{dx}$ predicted lower GIA $(r=.23)$, Broad Attention $(r=.22)$, and Processing Speed $(r=.33, p s \leq .04)$. Increased GA exposure predicted worse scores on all measures ( $\mathrm{r}=.40-.50, p s \leq .001)$. After controlling for associated risk factors with semi-partial correlation, GA exposure predicted $8-19 \%$ of the variance in neurocognitive scores ( $p s \leq .007)$.

Conclusions: Increased GA exposure during therapy independently predicts decreased neurocognitive outcomes in survivors of pediatric medulloblastoma three years later, with modest effect sizes. When feasible, interventions promoting non-sedated procedures should be considered (i.e., during RT).

Correspondence: Lisa M. Jacola, Ph.D., Psychology, St. Jude Children's Research Hospital, 262 Danny Thomas Place, Memphis, TN 38105, United States.E-mail: Lisa.Jacola@STJUDE.ORG

T. KENNEDY, A. GIOIA, G. DOME, K.K. HARDY \& K.S. WALSH. The Relationship Between Processing Speed and Working Memory in Pediatric Brain Tumor and Acute Lymphoblastic Leukemia.

Objective: Impairments in processing speed (PS) and working memory (WM) in pediatric cancer has been well documented. Research suggests that PS may be a key cognitive resource in executive functioning. This concept has not yet been explored in pediatric cancer. We aim to examine the relationship between PS and WM in children with a history of brain tumor (BT) or leukemia (ALL), and explore differences in disease and age-related factors.

Participants and Methods: Retrospective data from 82 children with pediatric cancer ages 3 to $19(\mathrm{M}=10.4 \mathrm{SD}=4.11 ; 56.5 \%$ male; $56.5 \%$ brain tumor) was used. Intellectual (Wechsler) and executive function $(\mathrm{EF})$ data $(\mathrm{BRIEF})$ was collected. Processing speed and working memory was dichotomized at $1 \mathrm{SD}<$ mean to classify impairments. Age at testing was classified into 3 categories (3-7, 8-12, >13). Pearson correlations, chi square, and general linear models were run to examine study hypotheses.

Results: Pearson correlation revealed a significant relation between PSI and WMI $(r=.56, p=.000)$ and with working memory on the parent BRIEF $(r=-.48, p=.000)$. These relationships remain when examining the disease groups independently (BT $r=.63, p=.000 ; r=-.53, p=.000$; ALL $r=.49, p=.001 ; r=-.44, p=.004)$. A significant relation between diagnosis and PSI impairment was found ( $p=.04$, Phi $=.24 ; 59 \%$ impaired in BT; $34 \%$ in ALL). Controlling for diagnosis, statistically significant relationships were found between PS status (impaired v. not) and WM (WMI $F=25.41, p=.000, p E t a 2=.251$; Parent WM $F=7.21$, $p=.011, p$ Eta2 $=.082$ ).

Conclusions: The findings of this study support prior research that suggests that PS is important to WM functions. We found that survivors were more likely to have poorer WM when PS was impaired regardless of disease. White matter integrity is integral to both processes, and known to be affected in pediatric cancers secondary to medical intervention. Moving forward, examining the timing of onset for deficits in these areas will be important to understanding this relationship better. 
Correspondence: Tess Kennedy, B.A., Neuropsychology, Children's National Medical Center, Children's National Health System, 111 Michigan Ave. NW, Washington, DC 20010, United States. E-mail: tkennedy2@childrensnational.org

\section{J. LENIHAN, J. OPPENHEIM, G. LAM \& K. KAYSER. Utility of the BRIEF parent report as a screener for executive dysfunction in patients with pediatric brain tumors.}

Objective: Pediatric patients with medulloblastoma are at increased risk for cognitive late-effects due to post-treatment residua related to disease of the posterior fossa, which adversely affect overall neuropsychological functioning and executive functioning $(\mathrm{EF})$ in particular. This study examined whether the BRIEF-Parent report could be utilized to effectively predict EF on performance-based measures within this specific population.

Participants and Methods: Neuropsychological data were collected from patients aged 8-16 years following treatment for medulloblastoma. Hierarchical regression analyses were used to determine whether parent ratings on the BRIEF inhibit and shift subscales significantly predicted performance on Inhibition and Inhibition/Switching trials from the DKEFS Color-Word Interference Test (CW). Regression of demographic and treatment variables represented the baseline model. Rapid color naming was added as a measure of naming speed in model 2, followed by BRIEF inhibit and shift subscales in separate full models. Results: Rapid color naming accounted for a statistically significant increase in the variance explained in both the shift and inhibit outcome measures when controlling for demographic and treatment variables. However, the BRIEF subscales did not result in a statistically significant change in variance accounted for in the outcome measure above and beyond that explained by demographic variables and naming speed [Inhibit $\mathrm{R}^{2}$ change $=.02, \mathrm{~F}(1,2)=.42, p>.05$; Shift $\mathrm{R}^{2}$ change $=.01$, $\mathrm{F}(1,1)=.19, p>.05]$

Conclusions: While the BRIEF is a well-validated measure of everyday $\mathrm{EF}$, it alone may not be sufficient to predict EF performance in this population, possibly due to parental underreporting. The BRIEF inhibit/ shift scales and CW both measure EF; however, they may capture different aspects of this construct, as CW relies heavily on speed of processing. Future research exploring utility of other measures more sensitive to EF and those without the confound of speed is indicated. Correspondence: Jessica Lenihan, Department of Psychology, Loma Linda University, 1601 Barton Road Apt 1706, Redlands, CA 92373, United States.E-mail: jlenihan@llu.edu

E. OLSEN, J. ZEAL, J. KOOP, L. LOPEZ \& A. HEFFELFINGER. Academic Performance as Predicted by Working Memory, Processing Speed and Radiation Therapy in Pediatric Brain Tumor Survivors.

Objective: Disruptions in cognitive development are well characterized in pediatric brain tumor (PBT) populations. Children receiving radiation therapy (RT) may be particularly vulnerable to deficits in processing speed, attention, and working memory. The present study examined the relationship between these cognitive sequelae and academic functioning in PBT treated with $(n=16)$ and without $(n=36)$ radiation therapy. Participants and Methods: Participants included 52 PBT children ages 5 to 17 years (posterior fossa: $n=15$; supratentorial tumors: $n=36$ ) evaluated 8.71 years $(S D=5.23)$ post diagnosis. Participants completed measures of verbal intelligence (VIQ), working memory (WMI) and processing speed (PSI) (Wechsler Intelligence Test battery composites), sustained attention (Conners' Continuous Performance Test, Omission Errors), and academic achievement. A series of linear regressions examined the relationship between each cognitive predictor (PSI, WMI, CPT) and academic functioning. RT was entered as a dichotomous predictor variable. VIQ and years since diagnosis were included as covariates in all regression analyses.

Results: Children with a history of RT demonstrated lower academic performance $(M=86.96, S D=12.22)$ compared to non-RT children
$(M=97.5, S D=12.26)$. Regressions indicated WMI and RT accounted for a significant portion of the variance in academic performance $\left(\mathrm{R}^{2}\right.$ $=.59, F(1,44)=5.33, p<.05)$. WMI performance $(\beta=.49, p<.001)$ and history of RT were uniquely associated with academic performance $(\beta=-.22, p<.05)$. A model including PSI and RT failed to reach significance $\left(\mathrm{R}^{2}=.50, F(1,45)=3.41, p=.07\right)$. Models examining sustained attention were not associated with academic performance.

Conclusions: Reduced academic performance is an consequence of radiation therapy. WMI and PSI also appear to effect academic achievement which may reflect the impact of radiation on white matter integrity. Children receiving RT stand to benefit most from interventions and accommodations which target these deficits.

Correspondence: Emily Olsen, PhD, Neuropsychology, Medical College Of Wisconsin, MCW Clinic at Froedtert, 9200 West Wisconsin Ave, Milwaukee,WI 53226-3596, United States.E-mail: eolsen@mcw.edu

M. PARSONS, J. BIARS, M. AHLUWALIA, S. MOHAPATRA \& G. BARNETT. Cognitive Deficits in Older Adults with Glioblastoma (GBM).

Objective: GBM is the most common primary malignant brain tumor. Incidence increases with age, peaking in the $65+$ age group. Recent studies have shown that combined radiation and chemotherapy is the most effective treatment for older patients with GBM in terms of survival, but there was no assessment of cognitive outcome in these patients. Because older GBM patients have a poorer prognosis than younger patients, considering the risk of cognitive impairment and its impact on quality of life when making treatment decisions is of critical importance. We undertook a descriptive analysis of cognitive impairment in older GBM patients as a preliminary step towards understanding cognition in this population.

Participants and Methods: A single institution neuro-oncology/neuropsychology (NP) database was queried for this study. 30 older patients with GBM (age > 65) who had undergone NP assessment between 2010 and 2016 were identified. Individual test scores were classified as impaired at -2 SD or less. A composite score (CS) was calculated based on mean $\mathrm{Z}$ score across several tests in the battery and was similarly classified as impaired at -2 SD.

Results: $83 \%$ of older GBM patients showed cognitive impairment on at least one task. The most common impairments were in memory (visual delayed recall $67 \%$; verbal delayed recall $62 \%$ ) and executive function $(50 \%)$. The CS was impaired in $47 \%$ of patients. Based on standard clinical criteria, $77 \%$ of patients would be defined as having Mild Cognitive Impairment and $47 \%$ met criteria for dementia.

Conclusions: Cognitive problems in older adults with GBM are common and significant. Neuropsychological assessment facilitates identification and intervention for these issues, and is an important component of their overall care. Future studies will examine the relevance of individual difference variables (e.g., age, cognitive reserve, medical comorbidities) and tumor variables (location, size). A model for clinical neuropsychology service provision in this population will also be presented. Correspondence: Michael Parsons, Ph.D., Neurologic Institute, Cleveland Clinic, 9500 Euclid Ave, P-57, Cleveland, OH 44195, United States.E-mail: parsonm2@ccf.org

J.B. PETERS, D. ANGHELESCU, H.M. CONKLIN, L. HALL, B.L. MEANS, J.E. SCHREIBER, M. ROSSI, B. WRIGHT, A. GAJJAR \& L.M. JACOLA. The Impact of Sedation for Radiation Therapy on Performance Measures and Caregiver Ratings of Attention in Survivors of Pediatric Medulloblastoma.

Objective: Survivors of pediatric medulloblastoma are at risk for attention problems. Many patients require general anesthesia (GA) for procedures such as radiation therapy (RT) while on treatment. Preliminary evidence in the general childhood population suggests an association between GA exposure and attention problems. This study investigated whether GA for RT during therapy predicted attention outcomes 3 years after therapy (3YP). 
Participants and Methods: Sedation data were abstracted from medical records for 153 patients treated on protocol with risk-adapted RT (33\% high risk, mean age at diagnosis $[\mathrm{M}]=9.4 \mathrm{yrs})$. Of these, $63 \%$ completed $3 \mathrm{YP}$ assessments that included performance (CPT2) and rating (CBCL) attention measures. We compared the frequency of at-risk scores (i.e., scores 1 SD from the normative mean) between patients who completed RT with $\mathrm{GA}(\mathrm{GA} ; \mathrm{n}=41,37 \%$ high risk, $\mathrm{M}=6.9$ yrs) and without GA (noGA; n=56, 20\% high risk, $\mathrm{M}=12.2$ yrs) and used linear regression and semi-partial correlation to examine the relationship after controlling for associated factors (age at diagnosis). All $p$-values are two-sided.

Results: The GA group was sedated for RT an average of 24.7 times. Comparisons between the GA and noGA groups showed no differences in the frequency of high risk patients $(p=.06)$, but the mean age at $\mathrm{dx}$ was lower in the GA group $(p=.00)$. Compared to the noGA group, the GA group had a greater frequency of at-risk scores on the CPT2 (e.g., Omissions 4 v. 36\%; Variability: 15 v. 43\%) and CBCL Attention Problems (9 v. 39\%, $p s \leq .001)$. Increased GA exposure predicted worse attention outcomes $(|\mathrm{r}|=.34-.63, p \mathrm{~s} \leq .002)$. After adjusting for age at diagnosis, the frequency of GA exposure predicted $15 \%$ of variance in CPT2 Omissions $(p=.001)$.

Conclusions: At $3 \mathrm{YP}$, survivors who were sedated for RT were at greater risk for attention problems. Increased GA exposure independently predicted worse attention performance. These preliminary findings support systematic investigation of interventions to promote non-sedated RT.

Correspondence: Joanna B. Peters, Psy.D., Psychology, St. Jude Children's Research Hospital, 545 South Main Street, Apt. 702, Memphis, TN 38103, United States. E-mail: joanna.peters@stjude.org

\section{A.E. RICHARD, E.K. HODGES \& K.P. HEINRICH. Attentional Control and Math Performance in Pediatric Cancer Survivors. \\ Objective: Deficits in attention and academic performance, particularly math, are common in pediatric cancer survivors. Of cognitive deficits experienced by pediatric cancer survivors, attention deficits may be particularly responsive to intervention. However, it is unknown whether deficits in particular aspects of attention are associated with deficits in math skills. The current study investigated relationships between math calculation skills and performance on an objective test of attention and parent- and teacher-reported attention difficulties.}

Participants and Methods: 67 pediatric cancer survivors $\left(M_{\text {age }}=\right.$ 14.0 years) completed a computerized test of sustained attention and response control (Integrated Visual and Auditory Continuous Performance Test; IVA) and a written test of math calculation skills, as well as an IQ test, in the context of a full clinical neuropsychological evaluation. Parent and teacher ratings of inattention and impulsivity were obtained. Results: IVA visual response control accounted for $12.1 \%$ of the variance observed among math performance scores after controlling for full scale IQ, which accounted for $24.9 \%$ of the observed variance in math scores $(p<.005)$. IVA auditory response control was correlated with parent-rated inattention $(r=-.36, p<.005)$. However, parent-rated inattention was not a significant predictor of math calculation scores after controlling for FSIQ $(p=.38)$. Processing speed alone was also not a significant predictor of math calculation scores $(p=.31)$.

Conclusions: Consistency of responses to visual stimuli on a computerized test of attention is a unique predictor of variance in math performance among pediatric cancer survivors. In contrast, processing speed and parent-rated inattention were not significant predictors of math performance. Future research should investigate whether interventions for children with attentional difficulties can mitigate potential academic difficulties before they become apparent.

Correspondence: Annette E. Richard, Ph.D., Psychiatry, University of Michigan, University of Michigan, 2101 Commonwealth Blvd. Suite C, Ann Arbor, MI 48105, United States.E-mail: annette.e.richard@ gmail.com
J.E. ROSEBERRY, C.M. CONSIDINE \& D. SABSEVITZ. Lateralized Cognitive Functioning in the Frontal Lobes: A Brain Tumor Lesion Study.

Objective: Prior research has demonstrated that frontal lobes (FL) influence numerous cognitive abilities, including working memory, attention, planning, organizing, and inhibition. However, less is known about lateralization of cognitive abilities within the FL. Using a postoperative brain tumor sample as a lesion model, this study examines lateralized cognitive abilities of the FL.

Participants and Methods: Demographically matched patients with left $(\mathrm{n}=19)$ and right $(\mathrm{n}=21)$ WHO Grade III or IV FL tumors were given a comprehensive cognitive battery 3 to 4 weeks post-operatively, but before any other treatment(s).

Results: T-tests revealed group differences suggesting patients with FL tumors demonstrate poorer cognitive performance compared to normative samples on HVLT-R Total Recall $[t(39)=5.13, p=0.00)]$, BVMT-R Total Recall $[t(37)=6.70, p=0.00)]$, Category Fluency $[t(39)=2.26, p=0.015)]$, Letter Fluency $[t(39)=6.00, p=0.00)]$, SpatialSpan $([t(37)=1.93, p=0.0 .31)]$, Digit-Span $[t(39)=2.63, p=0.006)]$, TMT-A $[t(38)=2.63, p=0.006)]$, and TMT-B $[t(37)=3.91, p<0.001)]$.

ANOVA revealed group differences suggesting patients with left FL tumors performed worse than those with right FL tumors on HVLT-R Total Recall $([\mathrm{F}(1,38)=3.98, \mathrm{p}=0.05, \mathrm{~d}=0.62])$, BVMT Total Recall $[F(1,37)=5.36, p=0.026, d=0.80)]$, Category Fluency $([F(1,38)=8.27$, $\mathrm{p}=0.007, \mathrm{~d}=0.90])$, and Letter Fluency $[\mathrm{F}(1,38)=5.01, \mathrm{p}=0.031$, $\mathrm{d}=0.70)]$. The remaining cognitive performances did not differ between groups ( $p$ 's $=0.072$ to $0.361 ; \mathrm{d}$ 's $=0.29$ to 0.60$)$, notably including TMT-A, suggesting processing speed was not a contributor for other cognitive abilities.

Conclusions: Results support bilateral contributions of the FL to executive functions as measured by standard neuropsychological tasks. Also demonstrated is that some executive tasks are differentially impacted by lesion lateralization, suggesting left lesions have more negative impact on these tasks. Limitations of this study include lack of precise lesion location and variety in tumor size, which are considered for future research

Correspondence: Jarett E. Roseberry, PhD., Neurology, Medical College of Wisconsin, 9200 W. Wisconsin Avenue, Milwaukee, WI 53226, United States.E-mail: jarett.roseberry@my.rfums.org

C.M. SHARKEY, A. GIOIA, T. KENNEDY, I. TAORMINA, K.S. WALSH \& K.K. HARDY. Suicidal Ideation and Executive Functioning in Children with Pediatric Cancer.

Objective: Children diagnosed with cancer are at elevated risk for difficulties with neuropsychological functioning and psychosocial distress. Extant literature has indicated elevated rates of suicidal ideation (SI) in cancer survivors and a link between cognitive deficits and SI in the general population. The relationship between SI and cancer-related neuropsychological effects has not yet been studied.

Participants and Methods: Participants were 166 children with cancer (57.5\% Brain Tumor, 30.6\% ALL, 11.9\% other cancers) aged 6-19 years $(\mathrm{M}=11.21, \mathrm{SD}=3.75 ; 54.2 \%$ male). SI was measured by parent, teacher, or self endorsement of self-harm items on the CBCL, BASC-II, CDI, or BDI. Executive functioning (EF; BRIEF), ADHD symptoms (ADHD Rating Scale), and performance-based measures of EF were compared between those with and without SI.

Results: SI was endorsed for $17.4 \%$ of the sample ( $\mathrm{N}=29,55.2 \%$ Male, $\left.\mathrm{M}_{\mathrm{age}}=12.15\right): 31.0 \%(\mathrm{~N}=9)$ with self-endorsement only, 37.9\% ( $\left.\mathrm{N}=11\right)$ with parent endorsement only, $6.9 \%(\mathrm{~N}=2)$ with teacher endorsement only, $13.7 \%(\mathrm{~N}=4)$ with self and teacher or parent endorsement, and $10.3 \%(\mathrm{~N}=3)$ with parent and teacher endorsement. Those with SI had significantly greater impairments in parent-reported general executive composite scores $(\mathrm{M}=63.4, \mathrm{SD}=12.63)$ than those without $\mathrm{SI}(\mathrm{M}=54.30$, $\mathrm{SD}=11.16 ; t(155)=-3.66, p<.001)$. Those with SI had greater parent-reported behavior regulation problems $(\mathrm{M}=59.04, \mathrm{SD}=11.85)$ than those without $(\mathrm{M}=50.88, \mathrm{SD}=11.07 ; t(157)=-3.40, p<.01)$. Parents of children with SI endorsed significantly more inattention symptoms $(\mathbf{M}=6.1$, 
$\mathrm{SD}=2.49)$ than those without $\mathrm{SI}(\mathrm{M}=3.28, \mathrm{SD}=2.72 ; t(140)=-4.35$, $p<.001)$. In contrast, SI was unrelated to performance-based measures of EF

Conclusions: A significant portion of children with cancer experience SI, which may be associated with perceived deficits in neuropsychological functioning. Screening for SI and further assessment of the connection between EF and SI in pediatric cancer patients is necessary. Correspondence: Christina M. Sharkey, Ph.D., Psychology, Oklahoma State University, $4599 \mathrm{~N}$ Washington St. Apt 6L, Stillwater, OK 74075, United States.E-mail: christina.sharkey@okstate.edu

\section{A. HOLLAND, T. TARKENTON, B. OSCARSON \& P.L. STAVINOHA.} School Performance in Pediatric Medulloblastoma Survivors.

Objective: Pediatric medulloblastoma survivors are known to often experience academic difficulties; however, existing research generally shows survivors' performance on measures of academic skill development to be broadly average. This study was the first to examine school-reported grade point average (GPA), parent-reported GPA and School Competence, standardized school examination passing rates, and school services for pediatric medulloblastoma survivors. It was hypothesized that age at diagnosis, school services, and School Competence would predict school-reported GPA and test scores, and that schoolreported GPA and parent-reported GPA would be correlated.

Participants and Methods: Thirty-six pediatric medulloblastoma survivors ages 8-18 (M=14.07; $\mathrm{SD}=3.46)$ completed neuropsychological screening. A parent/guardian completed the Child Behavior Checklist (CBCL), yielding a School Competence score. Report cards, standardized testing scores, and school services documentation were coded to enable comparisons across different grade levels, scoring systems, and test versions. Stepwise multiple regression was conducted to examine the first hypothesis, and chi-square examined the second.

Results: School Competence was the only significant predictor for school-reported GPA $(b=-.34, p<.05)$ and state standardized testing scores in language arts $(\mathrm{b}=-.55, \mathrm{p}<.05)$ and math $(b=-.35, p<.05)$. Parent-reported GPA did not significantly correlate with schoolreported GPA $(X 2=90.48, p=.47)$. Frequencies are reported for GPA, standardized testing pass rates, and school services utilization.

Conclusions: Findings support the use of CBCL School Competence as a predictor of medulloblastoma survivors' objective school performance as reflected in school records. Parents may not accurately report survivors' GPA. Clinically, when school records are unavailable, CBCL School Competence may provide a meaningful estimate of the child's academic performance, potentially preferable to parent-reported grades. Limitations and further directions are discussed.

Correspondence: Tahnae Tarkenton, Masters in Clinical Rehabilitation Counseling, Neuropsychology, UT Southwestern Medical Center, 5940 Forest Park Road, Dallas, TX 75235, United States. E-mail: tahnae. tarkenton@utsouthwestern.edu

\section{A. VERHAAK, C. ARBONA, S. DAY \& J.S. WEFEL. Prediction of Subjective Memory Ability and Patient Self-Report Accuracy Before Resection of High Grade Glioma.}

Objective: Subjective reports of cognitive functioning are often unrelated to objective neuropsychological test results in medical populations. Predictors of subjective memory ability and correlates of congruence between self-reported and performance-based tests of memory function are largely unknown in the glioma population.

Participants and Methods: Patients $(\mathrm{n}=138)$ with high-grade glioma were assessed with the Hopkins Verbal Learning Test-Revised, Functional Assessment of Cancer Therapy-Brain, and Beck Depression Inventory-II. Multiple linear regression of subjective memory ability was conducted with sociodemographic, clinical, and neuropsychological variables as predictors. Patients were dichotomized into High or Low Subjective Ability based on memory self-report, and Impaired or Not Impaired based on memory testing, and categorized into one of four groups with these subjective/objective memory combinations.
Differences were determined between groups using one-way ANOVA and chi-square tests.

Results: Worse rating of subjective memory ability was predicted by a model $\left(p<.001, \mathrm{R}^{2}=.23\right)$ that included older patient age $(\beta=-.22 ; p<$ $.01)$ and greater depressive symptomatology $(\beta=-.30 ; p<.01)$. One-way ANOVA revealed that patients with congruent subjective/objective memory impairment were older [Welch's $\mathrm{F}(3,53.49)=10.46, \mathrm{p}<$ $.01]$ than patients who over- or underestimated their memory abilities relative to their memory test results, and had more depressive symptomatology $[\mathrm{F}(3,134)=2.99, \mathrm{p}<.05]$ than patients with no objective/ subjective memory impairment. Patients with congruent subjective/ objective memory impairment were more likely to have a posterior rather than anterior brain tumor $\left[\chi^{2}(3, \mathrm{~N}=138)=8.29, \mathrm{p}<.05\right]$.

Conclusions: Older patients with more depressive symptomatology reported worse subjective memory ability. Congruence of subjective/ objective memory impairment was better in older patients with more depressive symptomatology and posterior lesions.

Correspondence: Allison Verhaak, M.Ed. in Counseling, Psychological, Health, and Learning Sciences, University of Houston, 5539 Huisache St, Bellaire, TX 77401, United States.E-mail: allie.m.sawyer@gmail. com

K.L. VICKERS, D.R. ROALF, V. KAMATH, J.P. CHRISTODOULEAS, S.M. KEEFE \& P.J. MOBERG. The Neuropsychological Impact of Androgen Deprivation Therapy: A Meta-Analytic Review.

Objective: Prostate cancer is the most commonly diagnosed cancer among men in the US, and nearly half of men diagnosed receive Androgen Deprivation Therapy (ADT). Studies investigating the longterm cognitive impact of ADT are mixed; however a prior meta-analysis indicated only a small reduction in visuomotor functioning. Here, we report data from an updated meta-analysis of the effects of ADT on cognition in men with prostate cancer and include analysis of moderators thought to affect cognitive functioning following ADT.

Participants and Methods: Nineteen publications ( $k=120$ effects) were identified using PubMed, MEDLINE and Google Scholar. Included articles reported neuropsychological testing following at least 6 months of ADT and a control group (either non-ADT prostate cancer patient or healthy control). Moderators included neuropsychological domain. ADT treatment type, age, and education level.

Results: Analyses revealed poorer cognition in men on ADT, $d=-0.25$. $95 \% \mathrm{CI}=-0.32<\delta<-0.18$, that was significantly heterogeneous, $Q_{B}[119]=225.08, \mathrm{p}<.001$. Moderator analyses revealed a significant effect of neuropsychological domain, $Q_{B}[10]=20.63, p=.02$. Deficits in attention, $d=-0.45,95 \% \mathrm{CI}=-0.63<\delta<-0.275$, executive functioning, $d=-0.38,95 \% \mathrm{CI}=-0.53<\delta<-.23$, and global functioning, $d=-0.36$. $95 \% \mathrm{CI}=-0.674<\delta<-0.052$ were prominent. Meta-regression analyses identified age as a moderator of cognitive performance in the ADT group, Z [119] = -2.5, p = 0.011, but not among controls. Control group type, treatment type, and education were not significant moderators. Conclusions: Meta-analytic results suggest that frontal lobe functions are most affected by ADT, and indicate these may be important targets for remediation. Results of this analysis also identify a need for additional investigation of other potential moderators of cognition, including testosterone levels and mood.

Correspondence: Kayci L. Vickers, MS, Psychology, Drexel University, 421 S. 15th Street, Apt 3F, Philadelphia, PA 19146, United States. E-mail:kayci.vickers@gmail.com

K.S. WALSH, K.K. HARDY, P. CULLEN, L. EMBRY, R. ANNETT, K. MURASZKO, E. HWANG, K. MOORE, S. PERKINS, J. MICHALSKI \& R. NOLL. Neuropsychological Functioning in Children with Medulloblastoma: The Impact of Post-Operative Pediatric Cerebellar Mutism Syndrome Within the First Year Following Diagnosis.

Objective: Cerebellar mutism (CM) is a complication seen in $25-40 \%$ of children with medulloblastoma. Characteristic features include 
acute-onset mutism, ataxia, and emotional dysregulation. While there is evidence that children with CM demonstrate greater long-term cognitive impairments than children without CM, there is little information about when these differences become evident and what cognitive domains are affected.

Participants and Methods: We present a sample of 110 children with average risk medulloblastoma treated on a Children's Oncology Group protocol ACNS0331 who received neurocognitive testing within the first year following diagnosis. Children with $\mathrm{CM}(\mathrm{N}=55)$ were matched by gender (44\% male), race $(74 \%$ White), age at testing (Mean=8.9. $\mathrm{SD}=3.78$, range $4-17$ years), and diagnostic age (Mean=8.7, $\mathrm{SD}=3.82$, range $3-17$ years) to 55 children without $\mathrm{CM}$.

Results: Children with CM showed significantly poorer cognitive efficiency/processing speed (Wechsler Processing Speed Index $p=.016$ ). There was also a trend toward poorer rote verbal memory (CVLT Total $p=0.052$ ) for the CM group. Global intellect, attention, contextual verbal memory, and visual memory were not significantly different between groups. There were no group differences on parent reports of executive function.

Conclusions: This is the largest sample of uniformly-treated CM patients receiving robust treatment presented to date. Results suggest that children with CM are at greater risk for earlier and more intense cognitive effects than non-CM matched peers, particularly for skills requiring intact white matter pathways. This aligns with current theories about the role of disrupted cerebrocerebellar pathways in the presence of CM in some children with medulloblastoma.

Correspondence: Karin S. Walsh, Psy.D., Neuropsychology, Children's NationalHealth System, Children's NationalHealth System, Washington, DC20010,United States.E-mail: kwalsh@childrensnational.org.

\section{A.M. WHITAKER, L. BAVA \& D.R. FREYER. Bilingualism as a Potential Protective Factor Against Cognitive Late-Effects following Treatment for Childhood Acute Lymphoblastic Leukemia (ALL).}

Objective: Current treatment approaches for childhood ALL may contribute to executive dysfunction. While bilingualism has been associated with stronger inhibition and cognitive flexibility in typically developing children, it has historically been overlooked as a potential protective factor against treatment-related cognitive deficits. The aim of this study was to explore possible differences in inhibition and cognitive flexibility between monolingual (English) and bilingual (Spanish/ English) childhood ALL survivors.

Participants and Methods: Patients who were administered the D-KEFS Color-Word Interference Test through our cancer survivorship service $(n=94,49 \%$ male) were assigned to monolingual $(n=20)$ or bilingual $(n=74)$ groups depending on reported spoken home language(s). Participants were 8-18 years of age $(\mathrm{M}=11.74, \mathrm{SD}=2.86)$ and identified as Latino $(83 \%)$, Caucasian $(10 \%)$, or Other $(7 \%)$. Family income was estimated based on zip code.

Results: There were no significant differences between groups with regard to demographic, clinical, or CNS-directed treatment factors, except that estimated family income was significantly higher in the monolingual group ( $\mathrm{t}=3.92, p=.001)$. Inhibition and cognitive flexibility were average for both monolingual (M scaled scores=10.9 and 9.85, respectively) and bilingual ( $\mathrm{M}$ scaled scores $=9.58$ and 9.31 , respectively) survivors. No significant group differences were found after controlling for income and naming/reading fluency in either inhibition $(\mathrm{t}=.957, p>.05)$ or cognitive flexibility $(\mathrm{t}=.475, p>.05)$.

Conclusions: Although bilingualism has been associated with stronger inhibition and cognitive flexibility, bilingual survivors in this study performed comparably to monolingual survivors. Since groups were assigned based on reported language(s) as opposed to objective measurement and income was estimated based on zip code, additional longitudinal research is needed to further explore potential interactions between oncology treatment factors, bilingualism, economic disparity, and cognitive sequelae.
Correspondence: Ashley M. Whitaker, Ph.D., Children's Center for Cancer and Blood Diseases, Children's Hospital Los Angeles, $3033 \mathrm{~S}$ Kerckhoff Ave, San Pedro, CA 90731,United States.E-mail: awhitaker@ chla.usc.edu

S. WITHROW, M. GLODÉ, A. BREGA \& J. GRIGSBY. Verbal Learning and Memory Among Prostate Cancer Patients Undergoing Androgen Deprivation Therapy.

Objective: Associations between reductions in testosterone (T) and impairments in memory and other areas of cognition among prostate cancer patients undergoing Androgen Deprivation Therapy (ADT) have been found. However, different domains of cognition have been inconsistently implicated. This study sought to examine verbal learning and memory performance within this population.

Participants and Methods: This study recruited 73 prostate cancer patients aged 50 to 81 undergoing continuous or intermittent ADT. Verbal learning and memory was assessed after 24 to 36 months after treatment initiation using the Rey Auditory Verbal Learning Test (RAVLT) and T levels were converted into biologically available $\mathrm{T}$ levels (BioT).

Results: Significant group differences emerged on all five learning trials $(p<0.05)$, with lower performance seen among continuous ADT patients. The two ADT groups significantly differed with regard to the total number of correctly recalled words, $\mathrm{t}(58)=-2.49, \mathrm{p}=0.01$. However, no significant group differences were seen for delayed recall performance $(p=0.12)$ or free recall on List $B(p=0.22)$. Continuous and intermittent ADT patients also did not differ with regard to retention $(p=0.08)$, and intrusions $(p=0.48)$. Significant positive correlations between BioT levels and RAVLT scores trials 1 through 5 emerged. Conclusions: Prostate cancer patients receiving continuous ADT showed a flattened learning curve during the acquisition trials on the RAVLT. Despite differences in learning rates, no differences were seen in retention and delayed recall performance. Implications of the detected differences in learning curves will be discussed.

Correspondence: Susanne Withrow, MA, University of Colorado Denver, University of Colorado, Denver, Denver, CO 80206, United States. E-mail: SUSANNE.WITHROW@UCDENVER.EDU

J. ZEAL, E. OLSEN, J. KOOP \& A. HEFFELFINGER. Impact of Medical and Treatment Variables on Adaptive Functioning within Eighteen Months of Pediatric Brain Tumor Diagnosis.

Objective: Previous work examining associations between neurological severity and adaptive functioning in pediatric brain tumor (PBT) survivors have shown mixed results. Further research is needed to understand the timing of the effect of the accumulation of neurological insults on adaptive functioning. The current study examines the impact of medical and treatment variables across areas of adaptive functioning in a heterogeneous sample of PBT survivors within 18 months of diagnosis.

Participants and Methods: The parents of thirty children (aged $\mathrm{M}=6.6$ years, $\mathrm{SD}=5.0)$ completed a measure of their child's adaptive functioning (Vineland Adaptive Behavior Scales or Adaptive Behavioral Assessment System) within eighteen months of PBT diagnosis. Correlations between adaptive domains and individual medical and treatment variables and the calculated Neurological Predictor Scale (NPS) (Micklewright,J.L.,2008) scores were conducted. Analysis of covariance (ANCOVA) was conducted to examine the relationship between radiation and Practical/Daily Living Skills when controlling for time since diagnosis.

Results: After controlling for time since diagnosis, radiation remained significantly associated with Practical/Daily Living Skills domain $(r=$ $-.38, p=.044)$, but not Conceptual/Communication $(r=-.13, p=.28)$ or Social/Socialization $(r=.087, p=.35)$ within the first year after diagnosis. Tumor grade and age at diagnosis were not significantly associated with adaptive functioning. There was a significant effect of radiation on Practical/Daily Living Skills after controlling for time since diagnosis, $F(1,30)=6.48, p<.05$. 
Conclusions: Individual medical and treatment variables did not have an effect on overall adaptive functioning in PBT survivors one year post-diagnosis. Radiation appears to have an effect on the Practical/ Daily Living Skills domain within this time period. This may be a direct result of radiation-induced cell death and white matter damage involving motor systems and/or parents lessening physical demands of their children.

Correspondence: Jamie Zeal, BS, Medical College of Wisconsin, 5314 SudburyWay, Madison, WI 53714, United States.E-mail: jzeal@mcw. edu

\section{Medical/Neurological Disorders/Other} (Adult)

A.D. BONO, E. MURRAY, M. HALFACRE, K. ALTERESCU, K. SCORPIO, J.T. TWAITE, R.J. STAFFORD, R. ISLAM, J. SPIELMAN, L. RAMIG \& J.C. BOROD. Facial Expressivity and Depression in Parkinson's Disease (PD) with Lateralized Motor Impairment Onset.

Objective: The hemiparkinsonism (HP) literature varies, with some studies finding that PD individuals with right-sided motor symptom onset have higher levels of depression than those with left-sided onset and others finding no differences (Cummings, 1992). To date, there are no studies about HP and facial expression, though there is a large literature on right- vs. left-hemisphere tasks and HP (St. Clair et al., 1998). Participants and Methods: Participants included 54 individuals with PD: 26 with right-onset (RPD; 54\% male; $M$ age $=62.7$ ) and 28 with left-onset (LPD; $75 \%$ male; $M$ age=68.4). To examine facial expression, PDs were videotaped while producing emotional (happy [H], sad [S], \& angry [A]) monologues, using New York Emotion Battery procedures (Borod et al., 1992). Monologues were evaluated by 24 naïve raters for aspects of facial expression: emotional frequency (EF), emotional variability $(\mathrm{EV})$, emotional intensity (EI), and facial mobility (FM).

Results: Two-way ANOVAs for Group (2: RPD \& LPD) and Gender (2), covarying for age, were conducted on depression scores (BDI-II) and on the 4 facial expression variables for each monologue. There were significant effects $(p<.05)$ or trends $(p<.10)$ for Group for $\mathrm{S}$ for $\mathrm{EF}$, EV, EI, and FM and for A and H for FM, with RPDs higher than LPDs. There were also significant Gender effects across variables, with women higher than men. Significant or trend-level 2-way interactions for the sad monologue for all 4 variables showed that HP effects occurred for women, but not for men. For depression, there was a trend for Group. Overall, RPDs displayed higher levels of facial expressivity and depression than LPDs.

Conclusions: In sum, individuals with right-onset PD displayed higher levels of depression and higher levels of facial expressivity than did left-onset PDs. Findings are congruent with earlier studies suggesting higher levels of negative affect in RPD and with neuropsychological studies regarding right-hemisphere specialization for facial emotional expression (Borod, 2000).

Correspondence: Amanda D. Bono, Ph.D. Clinical Psychology, Psychology, CUNY Graduate Center, 152-72 Melbourne Ave. Apt. 1P, Flushing, NY 11367, United States.E-mail: adbono922@gmail.com

A.D. BONO, K. ALTERESCU, M. HALFACRE, E. MURRAY, J.T. TWAITE, K. SCORPIO, R.J. STAFFORD, J. SPIELMAN, L. RAMIG \& J.C. BOROD. Outcomes and Predictors of the Lee Silverman Voice Treatment (LSVT LOUD) on Facial Mobility and Emotional Expressivity in Parkinson's Disease (PD).

Objective: Impairments in facial expression are common in PD and can impair communication. Earlier analyses with the data from this project (Alterescu et al., 2013; Halfacre, 2012) found that PD patients receiving LSVT (Ramig et al., 2001) were rated as more expressive after treatment. Using control measures, we examined LSVT effects on emotional frequency $(\mathrm{EF})$, variability $(\mathrm{EV})$, intensity $(\mathrm{EI})$, and facial mobility
(FM) in PD. Further, we examined whether demographic (gender, age, \& education) and clinical factors (cognitive status, depression, illness duration, disease stage, \& side of initial motor symptoms) can predict LSVT improvement.

Participants and Methods: Participants were 39 PDs, randomly assigned to LSVT ( $\mathrm{n}=12: 75 \%$ male: M age $=66.7$ ), Articulation Treatment (Spielman et al., 2012; $\mathrm{n}=14 ; 64 \%$ male; $\mathrm{M}$ age $=68.4$ ), or a control group ( $\mathrm{n}=13 ; 69 \%$ male; $\mathrm{M}$ age $=65.3)$, and 15 healthy controls (HCs; $60 \%$ male; $\mathrm{M}$ age $=64.3)$. Demographic and clinical data were collected at baseline. Facial expression was elicited via an emotional expression task (Borod et al., 1992) involving 4 videotaped monologues (happiness, sadness, anger, \& neutral) at baseline and post treatment. Facial expressions were rated for EI, EF, EV, and FM by 18 naïve judges.

Results: Group (2) x Time (2) x Gender (2) x Monologue Type (4) ANCOVAs, controlling for depression, were conducted for the 4 face variables. There were significant Group $\mathrm{x}$ Time $\mathrm{x}$ Gender interactions for EI $(p=.03)$ and $\mathrm{EV}(\mathrm{p}=.02)$ and a trend for FM $(p=.08)$, with the LSVT group changing more over time for women than men. There were also 4-way interactions for EI $(p=.04), F M(p=.06)$, and EF $(p=.07)$, with the LSVT women changing more over time than men for sadness. Multiple regression analyses for the 8 demographic/clinical factors were not significant for any face variable.

Conclusions: LSVT had significant or trend-level effects on ratings of EI, EV, EF, and FM. No demographic/clinical variables predicted treatment change, suggesting that LSVT is relatively robust to individual differences.

Correspondence: Amanda D. Bono, Ph.D. Clinical Psychology, Psychology, CUNY Graduate Center, 152-72 Melbourne Ave. Apt. 1P, Flushing, NY 11367, United States. E-mail: adbono922@gmail.com

\section{A.M. BRYANT, J. SUHR, E. DAWSON \& L. BOXLEY. Visuospatial Memory Performance in Parkinson's Disease and Essential Tremor.}

Objective: Parkinson's disease (PD) is characterized by motor symptoms resulting from basil ganglia dysfunction. PD is associated with deficits including executive dysfunction and impaired visuospatial memory, which may be related to disruption in the basal ganglia-thalamo-frontal pathways. The degree to which deficits in visual memory may be accounted for by motor difficulties, such as psychomotor slowing or executive dysfunction, is unclear. Essential tremor (ET) is a common type of movement disorder. The pathophysiology of ET is less clear; however, pathways from the cerebellar-thalamic-frontal motor regions are involved. Specific alterations in the cerebellar-thalamic-prefrontal circuitry may influence cognitive performance in ET. To our knowledge, there have been no studies of visual memory in this group. The purpose of the present study was to examine visuospatial memory in PD and ET, controlling for general cognitive impairment, motor symptoms, psychomotor slowing, and verbal memory ability.

Participants and Methods: Participants were 30 PD and 32 ET patients who presented for neuropsychological evaluation to determine candidacy for deep brain stimulation surgery. We compared groups on Brief Visuospatial Memory Test (BVMT-R) learning, delayed recall. recognition, and copy, controlling for cognitive impairment (MiniMental Status Exam), motor function (tapping test), and psychomotor slowing (Trail Making Test-B) and verbal memory performance (California Verbal Learning Test).

Results: Groups did not differ with respect to age, sex, or education (all $p s>.22)$. Groups did not differ on BVMT-R learning $(p=.158)$ or copy $(p=.239)$. PD patients performed worse on delayed recall $(F(1)=4.913$, $p=.031)$, but not recognition $(p=.270)$.

Conclusions: Results suggest relative deficits in visuospatial memory in PD, which cannot be explained by general cognitive impairment, motor difficulties, psychomotor slowing, or verbal memory ability. Future studies should examine performance based on laterality of symptom presentation. 
Correspondence: Andrew M. Bryant, M.A., Psychology, Ohio University, 269 Porter Hall, Athens, OH 45701, United States.E-mail:ab859013@ ohio.edu

C.M. BURNS, D. TUPPER, C. DAVEY, D. KNOPMAN \& A. MURRAY. Cognitive Impairment in Advanced Chronic Kidney Disease.

Objective: Most studies investigating cognitive impairment (CI) in chronic kidney disease (CKD) have focused on mild CKD (estimated glomerular filtration rate [eGFR] $\left.45-59 \mathrm{in} \mathrm{ml} / \mathrm{min} / 1.73 \mathrm{~m}^{2}\right)$. We report the prevalence of moderate to severe cognitive impairment (CI) in advanced stages of CKD (eGFR <45; non dialysis) in the Brain In Kidney Disease (BRINK) cohort.

Participants and Methods: This cross sectional analysis included 565 BRINK participants (mean age 69.2 \pm 9.8 ; edu $14.3 \pm 2.8$ years; $50.1 \%$ male) at baseline. CI severity was classified based on the average cognitive domain $\mathrm{T}$ scores (memory, executive functioning and language) and a priori established cutoff $\mathrm{T}$ scores (e.g., moderate $\mathrm{CI}=\geq 1$ domain with an average score $30 \leq \mathrm{T}<35$; severe $\mathrm{CI}=\geq 1$ domain with an average T score $<30$ ). Chi-square and logistic regression models measured the relation between eGFR group and CI severity.

Results: The prevalence of moderate or severe CI was higher in those with severely reduced eGFR $<30$ compared with eGFR $\geq 30(40.4 \%$ vs $22.9 \%$ respectively; $\mathrm{p}<0.0001)$. In adjusted logistic regression models, the odds of moderate to severe CI were significantly lower for those with eGFR $\geq 30$ compared with eGFR $<30(\mathrm{OR}=0.55, \mathrm{p}=0.006)$, but not for $e G F R \geq 45$ vs. eGFR $<45$ ( $p=0.107)$.

Conclusions: The risk of prevalent moderate to severe CI was highest for non dialysis CKD patients with eGFR $<30$ in this large study cohort. Further studies are needed to further elucidate the pathophysiology and nature of CI in advanced stages of CKD.

Correspondence: Christine M. Burns, Ph.D., BERMAN CENTER, MINNEAPOLIS MEDICAL RESEARCH FOUNDATION, Berman Center, 701 Park Avenue; Suite PPC 4-465, Saint Paul, MN 55117, United States.E-mail: cmburns@email.arizona.edu

N. DEZHKAM, D. WHITESIDE \& K. HOTH. A Review and Neuropsychological Profile of Anti-NMDA-Receptor Encephalitis: A Case Study.

Objective: Anti-N-methyl-D-aspartate receptor encephalitis (antiNMDAR) is an autoimmune disorder which affects glutamate-gated ion channels resulting in increased extracellular glutamate and symptom profile including neuropsychiatric symptoms, dyskinesia, autonomic instability, seizures, and decreased consciousness (Lancaster et al. 2011, Tuzun et al. 2009, and Kleinig et al. 2008).

This paper describes the neuropsychological presentation and course of anti-NMDAR in a 33-year-old female. Her encephalitis was secondary to a paraneoplastic syndrome due to mature cystic teratoma in her left ovary, which is a commonly reported etiology. A paraneoplastic panel in cerebrospinal fluid was positive for anti-NMDA receptor antibody. She developed seizures, tachycardia, and hot flashes. Behavioral issues included agitation, akinetic mutism, inappropriate laughing, catatonia, and agitation. She also demonstrated confusion and notable cognitive decline. She was initially on first line immunotherapy with methylprednisolone bolus and IVIG. She was also treated with Keppra, phenytoin, and Vimpat for seizure prevention.

Participants and Methods: A 33-year-old Caucasian female was administered a neuropsychological evaluation shortly after diagnosis/ treatment. She was partially engaged and cooperative, and her insight was very limited and she demonstrated significant cognitive deficits.

Results: The patient was severely impaired in memory, attention, and executive functioning. Language functioning was variable. Visual spatial abilities were a relative strength and remained intact. Results indicated that she was incapable of safely living alone or engaging in most IADLs until she can be reassessed to determine the degree of cognitive improvement.
Conclusions: The pattern of results was consistent with the research literature on short term cognitive sequelae of anti-NMDA receptor encephalitis. Long term follow-up is indicated. This case adds to the small but growing body of literature on the neurocognitive deficits associated with this disorder.

Correspondence: Naseem Dezhkam, Psy.D., University of Iowa Hospitals and Clinics, 1000 Oakcrest St. Apt 210, Iowa City, IA 52246, United States.E-mail:naseem.dezhkam@gmail.com

K.E. DOROCIAK, L.E. PIPER, T. KELLEHER, R. MOLOKIE \& J.K. JANECEK. Executive Function Moderates the Relationship between Pain Severity and Physical Quality of Life in an Outpatient Sample of Adults with Sickle Cell Disease.

Objective: As patients with sickle cell disease (SCD) live longer, there is a need for greater attention to quality of life and neurocognitive functioning (Feliu, 2012). Pediatric research on SCD highlights that inattention and executive dysfunction are especially prevalent in this population (Berkelhammer et al., 2007). Additionally, pain is one of the primary manifestations of SCD that affects quality of life and cognitive functioning. Thus, the purpose of the present study is to examine if executive functioning impacts the relationship between pain severity and physical quality of life in adults with SCD.

Participants and Methods: Participants were 59 consecutive outpatients with SCD referred for neuropsychological evaluation at the University of Illinois at Chicago $\left(M_{\text {ago }=} 41.12, S D=12.57\right)$. Participants were administered measures of executive function (EF) (WAIS-IV Similarities and Matrix Reasoning; WCST Perseverative and Total Errors; TMT-Part B), pain severity (Brief Pain Inventory), and physical quality of life (QOL) (WHOQOL-Bref).

Results: Pain severity predicted poorer physical QOL $(p<.001)$. This relationship was significantly moderated by three measures of EF: WAIS-IV Matrix Reasoning ( $\beta=-.65, p=.012)$, WCST Perseverative Errors $(\beta=-.574, p=.026)$, and WCST Total Errors $(\beta=-.63, p=.031)$. Results of post-hoc analyses with conditional moderators (set at $+/-1$ SDs from the mean) indicated that in adults with better EF, pain severity predicted physical QOL, $p<.001$; that is, in the face of greater pain severity those with better EF reported significantly lower physical QOL. For adults with lower EF, pain severity did not predict physical QOL. Conclusions: Conclusions:

These findings suggest that EF moderates the relationship between pain severity and physical QOL, such that pain severity predicts QOL for SCD patients with high, but not low, EF. Psychoeducation and increased coping skills for those with better EF could potentially optimize QOL. Future research should explore factors that underlie this relationship. Correspondence: Katherine E. Dorociak, M.A., Clinical Psychology, Loyola University Chicago, 10 E Ontario St., Apt. 3909, Chicago, IL 60611, United States. E-mail: katiedorociak@gmail.com

K.E. DOROCIAK, L.E. PIPER, T. KELLEHER, R. MOLOKIE \& J.K. JANECEK. Neuropsychological Profile in an Outpatient Sample of Adults with Sickle Cell Disease.

Objective: Pediatric patients with sickle cell disease (SCD) demonstrate cognitive deficits across many domains, including attention, executive functioning, memory, language, and IQ (Berkelhammer et al., 2007). Few studies have examined the neurocognitive functioning of adults with SCD and results are limited to asymptomatic adults without history of stroke, abnormal neurologic exam, cognitive impairment, depression, or medication use that could affect neurocognitive functioning (e.g., Vichinsky et al., 2010). The purpose of the present study was to characterize cognitive functioning in a broader, outpatient sample of adults with SCD.

Participants and Methods: Participants were 59 consecutive patients with SCD referred for routine neuropsychological evaluation at the University of Illinois at Chicago $\left(M_{\text {age }=} 41.12, S D=12.57\right)$. Neuropsychological tests were administered to examine intellectual functioning (WAIS-IV); verbal and nonverbal memory (HVLT-R, BVMT-R); 
verbal fluency (FAS/Animals); naming (BNT-2); executive functioning (WCST); and timed visual scanning and sequencing (TMT). Average hemoglobin level was $9.45 \mathrm{~g} / \mathrm{dL}$ and $29.8 \%$ experienced $\geq 1$ stroke. Most participants were diagnosed with HbSS genotype $(n=43,72.9 \%)$. No participants were excluded based on physical or psychological functioning.

Results: Mean performances on all measures were lower than those of age and education matched peers using published normative data. Responses to questionnaires indicated severe pain and elevated symptoms of depression, anxiety, and sleep disturbance.

Conclusions: This study was the first to report the neuropsychological profile of an outpatient sample of adults with SCD. The profile evidenced a pattern of low average performances across most cognitive domains. Visual memory was a relative weakness whereas verbal fluency and naming were relative strengths. Future studies should examine the relationships between cognition, physical health, and psychological functioning in this population in order to better inform research and practice.

Correspondence: Katherine E. Dorociak, M.A., Clinical Psychology, Loyola University Chicago, 10 E Ontario St., Apt. 3909, Chicago, IL 60611, United States. E-mail: katiedorociak@gmail.com

R.P. FELLOWS \& M. SCHMITTER-EDGECOMBE. Independent and Differential Effects of Cardiometabolic Variables on Executive and Physical Functioning in Older Adults.

Objective: Older age is associated with an increased risk of developing cardiovascular and metabolic conditions that may contribute to cognitive and physical limitations. The primary aim of this study was to examine the extent to which hypertension, obesity, diabetes and treated hypercholesterolemia are associated with an executive functioning composite (DKEFS Color Word Interference Test-Inhibition, Design Fluency-Switching, Letter Fluency, Trail Making Test Part B), self-reported executive skills (Dysexecutive Questionnaire [DEX]) and everyday activities that require physical capability (Patient Reported Outcome Measurement Information System [PROMIS]-Physical Function).

Participants and Methods: Participants were 100 community-dwelling cognitively intact older adults (aged 50+). The presence of cardiometabolic conditions was ascertained from self-report and/or review of medications.

Results: After controlling for age, sex, education and depression, cardiometabolic conditions predicted performance on the executive functioning composite $\left[R^{2}=.084 ; F(4,91)=2.66 ; p=.038\right]$, DEX score $\left[R^{2}=.145 ; F(4,91)=5.04 ; p=.001\right]$, and PROMIS-Physical Function $\left[R^{2}=.158 ; F(4,91)=4.99 ; p=.001\right]$. Hypertension emerged as the only significant cardiometabolic predictor of the executive functioning composite $(\beta=-.276, p=.007)$. In contrast, only obesity $(\beta=-.329, p=$ $.001)$ was a significant cardiometabolic predictor of poorer self-reported executive dysfunction. Obesity $(\beta=-.249, p=.011)$ and hypertension $(\beta=-.213, p=.036)$ were independent predictors of poorer everyday activities that require physical functioning, whereas hypercholesterolemia treatment $(\beta=.204, p=.041)$ predicted better physical functioning.

Conclusions: Taken together, these findings suggest independent and differential effects of cardiometabolic conditions on executive abilities, self-reported dysexecutive functioning, and physical limitations in everyday tasks.

Correspondence: Robert P. Fellows, Washington State University, 515 SW Winter Cir, Pullman, WA 99163, United States. E-mail: robert. fellows@wsu.edu

N.E. GARCIA, H.L. COMBS, A. ROACH, D.T.R. BERRY \& S.C. SEGERSTROM. Wisconsin Card Sorting Test subscales in Parkinson's disease and Amyotrophic Lateral Sclerosis.

Objective: Executive dysfunction is prevalent in most neurodegenerative disorders, and is particularly common in Parkinson's disease (PD) and Amyotrophic Lateral Sclerosis (ALS). Despite similar cognitive concerns, the underlying pathology and course of these diseases are quite disparate. PD is characterized by prominent subcortical degeneration whereas ALS is associated with frontal lobe degeneration. The Wisconsin Card Sorting Test (WCST) is a well-validated measure of executive dysfunction that taps into set-shifting and problem-solving abilities.

Participants and Methods: The current study sought to investigate patterns of impairment on the WCST subscales (128 card version) between patients with PD $(n=24 ; M$ age $=65 ; M$ edu $=16 ; 67 \%$ male $)$ and $\operatorname{ALS}(n=37 ; M$ age $=60 ; M$ edu $=14 ; 54 \%$ male $)$.

Results: Results suggest the WCST is able to differentiate between patients with PD and ALS. Patients with PD $(M=2.25, S D=1.39)$ completed significantly fewer categories than patients with ALS $(M=4.11, S D=2.16 ; t(59)=4.09, p<.001)$. Patients with ALS $(M=1.35, S D=1.13)$ failed to maintain set significantly more often than patients with $\mathrm{PD}(M=.62, S D=.97 ; t(59)=2.67, \dot{p}=.01)$. The two groups did not differ on T-scores for conceptual level responses, total, perseverative, and non-perseverative errors, or raw scores for trials to complete the first category.

Conclusions: Limitations include the use of raw scores for secondary subscales; T-scores are not produced for these measures. The ALS group also had significantly lower education than the PD group. Results provide preliminary evidence for the primary WCST subscales (e.g., total errors, perseverative errors) to be less useful in differentiating between PD and ALS than certain secondary subscales (e.g., fail to maintain set). Although patients with ALS tended to complete more categories than patients with PD, they were also more likely to fail set. This pattern suggests greater distractibility within ALS and greater problem solving impairment in PD.

Correspondence: Natasha E. Garcia, B.A., Psychology, University of Miami, University of Kentucky, 111B Kastle Hall, Lexington, KY 405060044, United States. E-mail: natasha.garcia@uky.edu

\section{GHAZI SAIDI, R. WALSH \& S.J. BANKS. Biomarkers of AD, MCI and NCS: MMSE Cortical Thickness, Volumetric and CSF.}

Objective: Biomarkers are essential for diagnosis and novel therapeutics in Alzheimer's Disease (AD). Diagnosis of AD is confirmed by misfolded proteins on autopsy. Tau, A $\beta 1-42$, PTau181P, found both in brain and cerebrospinal fluid (CSF), can be AD biomarkers. AD is associated with changes to cortical thickness (CT) and cortical and subcortical volumes (V). Neuroimaging techniques which detect such changes are candidate biomarkers. We studied the value of CSF, CT, and V measures (in isolation \& combination) to predict cognitive ability (CA), as well as AD, MCI, and normal cognitive status (NCS).

Participants and Methods: Participants: 822 healthy controls, AD, and MCI patients (age M=77, SD=6.9); CA tested by Mini Mental State Exam (MMSE). Predictors: CSF Tau, Abeta142, PTau181P; CT of 32 brain regions (ROIs) and $\mathrm{V}$ of 18 ROIs prone to structural changes in AD or MCI. Using regression models, we tested if a CSF protein or any single ROI's CT or V could predict CA. Variables with predictability $>10 \%$ were utilized in combined models. Strongest variables were used to predict disease status.

Results: CSF proteins explain 7\%-10\% of MMSE score variations, $\mathrm{p}<.0001$. The best combined ROI predictors of MMSE include $\mathrm{V}$ of $\mathrm{L}$ hippocampus and R \& L entorhinal cortices $(24 \%, p<.0001)$; and the CT of R middle temporal, L entorhinal, L inferior parietal gyri $(11 \%$ to $21 \%, p<.0001)$. Some regions (e.g. $\mathrm{R}$ hippocampus) were excluded to avoid collinearity. Best predictors include Amyloid, Tau, V of L hippocampus, $\mathrm{V}$ of $\mathrm{L}$ entorhinal and CT of $\mathrm{R}$ middle temporal gyri for AD (79.5\%, p<0.001); Amyloid, CT of L inferioparietal gyrus and L entorhinal \& $\mathrm{V}$ of $\mathrm{R}$ entorhinal cortices for NCS by $80.4 \%, \mathrm{p}<0.0001$ and Amyloid for MCI by $55.7 \%$, p $<0.01$.

Conclusions: CSF samples in isolation have poor predictability for CA and AD diagnosis. However, A $\beta$ 1-42 predicts MCI. CT and V measures of hippocampus and entorhinal cortex are also strong predictors of AD and of ruling out disease. Overall, combined CSF, CT, and V measures offer promise for AD biomarker development. 
Correspondence: Ladan Ghazi Saidi, Ph.D, Cleveland Clinic Lou Ruvo Centre for Brain Health, 888 W Bonneville, Las Vegas, NV 89012, United States.E-mail: ghazisl@ccf.org

L. GHAZI SAIDI, S.J. BANKS \& R. WALSH. Striatal Binding Ratios and CSF Biomarkers of Cognition in Parkinson's disease.

Objective: Loss of dopaminergic neurons in the substantia nigra is a hallmark of Parkinson's disease (PD), and results in nigrostriatal dopamine deficiency (detected by Striatal Binding Ratio (SBR)). The relationship of dopamine depletion to motor and non-motor symptoms (e.g. Mild Cognitive Impairment (MCI)) and how oligomer accumulation impacts cognition in PD is vague. This study examines the relationship of MCI in PD to dopamine depletion, CSF-oligomer and SBR levels. Participants and Methods: Participants (P): 408 drug naïve PD patients and 159 healthy controls (HC) assessed by Montreal Cognitive Assessment (MoCA), CSF oligomers (t-Tau, p-Tau, A $\beta$-Amyloid, $\alpha$-synuclein) and L \& R putamen and caudate nuclei SBRs.

Pearson correlation and regression models were used to examine the correlation of CSF levels and SBR levels with MoCA scores, and predicted value of each biomarker individually and combined. All analyses were performed on P, PD, HC, P-MoCA $>26$, P-MoCA $<26$, $\mathrm{P}-\mathrm{MoCA}<24, \mathrm{P}-\mathrm{MoCA}>24$.

Results: In P-MoCA $\leq 24$ (24.5\%), $\alpha$-synuclein predicts MoCA by $22 \%$, $\mathrm{F}(1,26)=4.8, \mathrm{p}<0.001$. In $\mathrm{P}-\mathrm{MoCA}>24, \mathrm{HC}$, and in PD with greater dopamine deficiency in one hemisphere (2SDs less than the $\mathrm{M}_{\mathrm{HC}}$ ), especially on the right side, right putamen SBR predicts MoCA by up to $9 \%$. In P-MoCA $<24, \alpha$ synuclein predicts MoCA by $22 \%$. In PD, none of the CSF oligomers nor SBR levels predict MoCA. Right putamen SBR, alone, is a predictor of PD by $95.4 \%$.

Conclusions: $R$ putamen-SBR correlates with $\mathrm{MoCA}>24$. This is in line with evidence showing MCI in PD is worse in patients with greater left motor symptoms. $\alpha$-synuclein predicts MCI in PD patients. The spread of $\alpha$-synuclein from the brainstem to limbic and neocortical structure has been documented as the strongest neuropathological correlate with MCI in PD. Dysfunction of the putamen in PD is well known and there is further evidence for this in $\alpha$-synuclein transgenic rats. More work is warranted, but putamen-SBR and $\alpha$-synuclein levels may be considered as biomarkers of PD-MCI.

Correspondence: Ladan Ghazi Saidi, Ph.D, Cleveland Clinic Lou Ruvo Centre for Brain Health, 888 W Bonneville, Las Vegas, NV 89012, United States.E-mail:ghazisl@ccf.org.

\section{A. GURNANI \& J.E. HORWITZ. Adult Man Presenting with Acute Disseminated Encephalomyelitis (ADEM): A Case Study.}

Objective: Acute disseminated encephalomyelitis (ADEM) is a rare autoimmune disease caused by intense inflammation in the brain and spinal cord that damages the myelin. Since ADEM is rare and typically develops in children and young adults, a cognitive profile for adults has not been sufficiently established. Therefore, using a case presentation format, the present study seeks to provide information regarding the cognitive profile of ADEM based on findings from a 57-year-old white man ("EL") diagnosed with the condition.

Participants and Methods: Neuropsychological evaluations were conducted before and after discharge from acute rehabilitation. All major cognitive domains were assessed.

Results: Neuropsychological testing completed approximately two weeks after EL's ADEM diagnosis revealed impairments in memory, executive function, language, and visuospatial ability, with mild anxiety and depression noted. At six-month follow-up, he displayed continued impairments in memory, executive function, and visuospatial ability, but with improvements demonstrated in encoding and retrieval processes, processing and psychomotor speed, fluency, focused attention, cognitive flexibility, set maintenance, judgment, and visuospatial functioning, as compared with initial evaluation. Language performance at follow-up was within normal limits. Psychological functioning was characterized by continued mild anxiety.
Conclusions: Results from the current study suggest that ADEM in adults is characterized by impairments in memory, language, visuoconstructional ability, and executive functioning, with improvement in all domains over time. It is noteworthy, however, that EL had a very complex medical history characterized in part by multiple autoimmune conditions, with these conditions likely impacting his cognitive presentation. Overall, more research is needed to establish a cognitive profile in adults with ADEM.

Correspondence: Ashita Gurnani, University of Colorado at Colorado Springs, 1510 Dublin Blvd, Apt 160, Colorado Springs, CO 80918, United States.E-mail: agurnani@uccs.edu

M. HARCIAREK, J. MICHALOWSKI, B. BIEDUNKIEWICZ, J.B. WILLIAMSON, A. DEBSKA-SLIZIEN \& K.M. HEILMAN. Attentional-Intentional Brain Networks of Dialyzed Patients With End-Stage Renal Disease Are Not Entirely Normalized Following Kidney Transplant: Evidence From Event Related Potentials.

Objective: Dialyzed patients with end-stage renal disease (ESRD) often present with cognitive impairments. In particular, recent behavioral and event related potentials (ERPs) studies have showed that the core deficit in this population is a dysfunction of the anterior attentional-intentional system. Although there is evidence a successful kidney transplant improves the cognitive functions of dialyzed patients, the impact of a kidney transplant on the attentional-intentional networks has never been verified. Thus, this study was designed to investigate whether successful kidney transplantation improves deficits in these anterior attentional-intentional networks.

Participants and Methods: Sixteen dialyzed patients with ESRD, 16 patients with a successful kidney transplant and 15 matched healthy controls were assessed using reaction time tasks from the ROtmanBaycrest Battery to Investigate Attention and ERPs to provide insight into neural mechanisms underlying attentional-intentional dysfunctions. Specific ERP components were analyzed for the following attentional-intentional processes: response preparation (Contingent Negative Variation - CNV); perceptual preparation (P1); selective attention and monitoring (P300/Late Positive Potential).

Results: In patients with a kidney transplant, the amplitude of the CNV was similar to that of the controls; however, it was reduced in dialyzed patients under the long preparation period condition. Both patients' groups had increased P300 amplitudes in response to imperative stimuli in the Simple, Choice and Prepare 3 tasks. Also, Late Positive Potential amplitudes were increased in both of these patient groups during the Choice and Prepare 3 tasks.

Conclusions: Although a successful kidney transplant reduces the response preparation deficit seen in dialyzed patients, kidney transplantation does not alter the increased allocation of attentional resources toward stimulus analysis seen in individuals receiving dialysis.

Correspondence: Michal Harciarek, Psychology, University of Gdansk, Bazynskiego 8, Gdansk 80-309, Poland. E-mail: psymh@ug.edu.pl

M. HARCIAREK, J. MICHALOWSKI, B. BIEDUNKIEWICZ, J.B. WILLIAMSON, A. DEBSKA-SLIZIEN \& K.M. HEILMAN. Disorders of The Attentional-Intentional System in Dialyzed Patients With End-Stage Renal Disease: Should We Blame Kidney Disease, Dialysis or Both?

Objective: Recent studies have demonstrated that the core cognitive problems seen in patients receiving dialysis for end stage renal disease (ESRD) are disorders of the attentional-intentional system that produce deficits such as psychomotor slowing and an impaired ability to sustain attention. However, it remains unclear whether these patients' attentional-intentional disorders directly result from their chronic kidney disease and associated factors (e.g. neurotoxicity) or if the deficits are induced by chronic dialysis or by both. Thus, the goal of this study was to address this question.

Participants and Methods: Twenty-three non-demented adequately dialyzed patients with ESRD as well as 19 clinically and demographically 
matched patients with ESRD but no dialysis (examined before the initiation of dialysis or kidney transplant) were compared with 25 demographically matched controls on the performance on four reaction time (RT) subtests from the ROtman-Baycrest Battery to Investigate Attention. These included measures of Simple, Choice, and Prepare RTs as well as a Concentrate task.

Results: The analysis revealed that, whereas patients receiving dialysis presented with impairments of psychomotor speed and sustained attention, these problems were not observed in individuals with ESRD but no dialysis. In addition, patients on dialysis did have a significantly longer duration of their disease; however, neither duration of the disease nor the time on dialysis was associated with these patients' attentionalintentional problems.

Conclusions: This study indicates that disorders of the attentionalintentional system seen in well-dialyzed patients with ESRD may not directly result from the kidney disease itself. In contrast, it seems that they can be an adverse effect of the metabolic changes associated with chronic dialysis or the interaction between ESRD and dialysis. Correspondence: Michal Harciarek, Psychology, University of Gdansk, Bazynskiego 8, Gdansk 80-309,Poland.E-mail:psymh@ug.edu.pl

C. HERNAIZ ALONSO, P. SINHA, A. CHOI, N.A. SCHWAB, M. RICE, H. PARVATANENI \& C.C. PRICE. Memory Ability Predicts Anesthesia Response in Older Adults During Total Knee Arthroplasty.

Objective: Individual variability in susceptibility to anesthetics complicates initiation and maintenance of hypnotic depth during surgery. Moreover, cumulative deep hypnotic time and patient comorbidity have been found as significant predictors of mortality. Here we examined how pre-surgery cognitive and memory composites predict anesthesia induction response and hypnotic depth associated with anesthesia EEG profiles as measured by a Bispectral Index Monitor $\left(\mathrm{BIS}^{\mathrm{TM}}\right)$.

Participants and Methods: Older adults $(n=47$; age $=69.8 \pm 7.1$ ) electing Total Knee Arthroplasty (TKA) by the same surgeon and anesthesia protocol completed pre-surgery cognitive assessment and intraoperative monitoring using a Bispectral Index monitor $\left(\mathrm{BIS}^{\mathrm{TM}}\right.$ ) in order to measure hypnotic depth and frontal activation. Cognitive domains examined included processing speed, working memory, language, memory, reasoning, and motor function. EEG variables chosen for analysis were area over the curve (AOC) assessing rate of brain deactivation during the induction of anesthesia and cumulative time in which a patient fell below threshold of anesthesia maintenance as indicated by BIS score < 45. Pearson Product Moment Correlations assessed cognitive-EEG associations after controlling for age, comorbidity, and education.

Results: Only participant's baseline (presurgery) memory function positively associated with rate of anesthesia induction $(\mathrm{r}=.32 ; \mathrm{p}=.04)$. Baseline memory and language $\left(r^{\prime} s=0.30, p<0.05\right)$ composites positively associated with longer time under deep hypnotic state.

Conclusions: Our findings suggest there are presurgical cognitive markers of anesthesia responsiveness in older adults electing total knee replacement surgery with general anesthesia. The presentation will discuss individual BIS ${ }^{\mathrm{TM}}$ profiles relative to cognitive profiles.

Correspondence: Carlos Hernaiz Alonso, Health Science, Clinical and Health Psychology, University of Florida, 2101 NE 32ND Avenue, Fort Lauderdale,FL 33305,United States.E-mail: carlosha26@gmail.com

\section{HIZEL, M.G. LOPEZ, C.C. PRICE \& F.T. BILLINGS. Working Memory and Recall Domains of the Mini-Mental Status Examination Predict Postoperative Delirium.}

Objective: Delirium occurs in at least 10 to $24 \%$ of the general patient population, with reports up to $50 \%$ of hospitalized older adults over age 65. Pre-surgical cognitive vulnerabilities in memory and executive domains were investigated as predictors of this negative postoperative outcome.
Participants and Methods: A set of 594 adults electing cardiac surgery at Vanderbilt University Medical Center were administered the Minimental State Examination (MMSE) preoperatively as part of a NIH funded clinical trial. Research personnel assessed postoperative delirium twice daily using the Confusion Assessment Method for the ICU. The MMSE was subdivided into domains of orientation (range 0-10), attention/working memory $(0-5)$, delay recall $(0-3)$, language $(0-8)$, and visuoconstruction (0-1). Logistic regression and receiver operating characteristics evaluated predictive values and sensitivity/specificity of variable cut-points.

Results: Nearly a quarter of the sample experienced postoperative delirium $(137 / 594,23.1 \%)$. A model of age, sex, history of stroke, and MMSE domain scores predicted $20.7 \%$ of the variance and classified $78.9 \%$ of the sample ( $95 \%$ no delirium, $24 \%$ delirium). From the MMSE, only the domains of attention/working memory and delay recall were significant predictors (attention/working memory: $\mathrm{AUC}=0.573$, 95\% CI: $0.515-0.632, p=0.010$ with optimal score of 3 ; delay recall: $\mathrm{AUC}=0.59,95 \%$ CI: $0.533-0.647, \mathrm{p}=0.001$ with optimal score of 2 ). Each error of attention/working memory was associated with a .74 increase in the odds of delirium. Each error of delay recall was associated with a .71 increase in the odds of delirium.

Conclusions: These data underscore previous work indicating attention/working memory and episodic memory domains are sensitive predictors of negative perioperative outcomes. These screener domains, as well as age, female sex, and history of stroke, increased the odds of detecting delirium.

Correspondence: Loren Hizel, BA, Clinical and Health Psychology, University of Florida, $1810 \mathrm{NW}$ 23rd Blvd Unit 160, Gainesville, FL 32605,United States.E-mail: Ihizel@phhp.ufl.edu

D.K. HORTON, H. WADSWORTH, K. DHIMA, M. CULLUM, J. WHITE \& R. RUCHINSKAS. Utility of Cognitive and Balance Measures in Predicting Ventriculoperitoneal Shunting Recommendation in Normal Pressure Hydrocephalus.

Objective: Ventriculoperitoneal shunting (VPS) is used to treat normal pressure hydrocephalus (NPH). Assessment of cognition and balance pre- and post-external lumbar drain (ELD) is used to determine the potential benefit of VPS. The present study aimed to determine which measures are best at distinguishing between those who do and do not receive a VPS recommendation post-ELD.

Participants and Methods: 88 participants were administered the Berg Balance Scale (BBS) and a brief neuropsychological battery pre- and post-ELD that included the MMSE, Trail Making Test, Animal Fluency, Hopkins Verbal Learning Test - Revised, and Digit Span. Subjects were divided into groups based on whether or not VPS was recommended post-ELD. Paired samples t-tests were used to assess changes pre- and post-ELD. Mean change scores for each measure were used as predictors in logistic regressions to determine which combination of variables best predicted VPS recommendation.

Results: Paired samples t-tests revealed significant improvements $(p<.05)$ on BBS, Trails B, and Animal Fluency in the "VPS recommended" group. Regression analyses revealed that VPS recommendation could be accurately predicted for $80 \%$ of the sample using BBS alone. Accuracy increased to $85 \%$ when using BBS and Trails B as predictors. The addition of Animal Fluency or other predictors did not increase accuracy beyond $85 \%$.

Conclusions: Results identified BBS. Trails B. and Animal Fluencv as the best measures at distinguishing between patients who are or are not recommended to undergo VPS post-ELD. While improved balance post-ELD appears to be the strongest predictor of VPS recommendation, improved visual attention and cognitive flexibility increases predictability. Although Animal Fluency did not increase prediction accuracy, verbal fluency improved in those who were recommended for VPS. These findings may have implications for procedures used to select appropriate candidates for VPS among individuals with suspected NPH. 
Correspondence: Daniel K. Horton, PhD, Psychiatry, Children's Medical Center Dallas, 4533 Cedar Springs Rd., \#425, Dallas, TX 75219, United States.E-mail: daniel.horton@childrens.com

L. KEATING, D. FOSSATTI, B. COSTABILE, M. YAKOBSON, A. PAGUYO \& K.A. WALKER. The Effect of Pre-ICU Depression on Cognition and Emotional Functioning After Critical Illness.

Objective: Post-intensive care unit (ICU) depression, post-traumatic stress disorder (PTSD), and cognitive impairment are common following critical illness. Whereas post-ICU depression has been associated with poorer cognitive functioning and elevated PTSD symptoms following ICU discharge, the contribution of pre-ICU depressive symptoms remains unclear. The current study sought to determine whether a diagnosis of major depressive disorder (MDD) at the time of critical illness predicted memory functioning, depression, and PTSD symptoms at ICU discharge and 4-month follow-up.

Participants and Methods: Fifty-seven ( $\mathrm{n}=57$ ) individuals with no pre-existing neurologic or cognitive impairment admitted to the ICU and diagnosed with septic shock and/or respiratory failure were enrolled. Memory was assessed at ICU discharge and 4-month follow-up using the Repeatable Battery for the Assessment of Neuropsychological Status (RBANS). Medical records and the MINI International Neuropsychiatric Interview were used to confirm pre-ICU MDD. Post-ICU depression and PTSD were assessed using the Center for Epidemiologic Studies Depression Scale (CES-D) and the PTSD Checklist (PCL), respectively. Results: Ten of the 57 patients $(18 \%)$ were diagnosed with MDD at the time of ICU admission. A diagnosis of pre-ICU MDD predicted poorer memory at ICU discharge $(\mathrm{rpb}=-.311, \mathrm{p}=.040)$, but was not predictive of memory functioning at 4 months following ICU discharge $(\mathrm{rpb}=.095, \mathrm{p}>.05)$. A diagnosis of MDD at the time of ICU admission predicted elevated depressive symptoms at four months post-discharge $(\mathrm{rpb}=.383, \mathrm{p}=.049)$, but was unrelated to PTSD symptoms ( $\mathrm{rpb}=.189$, p>.05). Level of depressive symptoms at 4-month follow-up did not predict memory functioning at 4-month follow-up $(\mathrm{r}=-.059, \mathrm{p}>.05)$. Conclusions: Findings suggest patients with MDD at the time of critical illness onset are more likely to have memory deficits in the early stage of recovery and persistent post-ICU depressive symptoms.

Correspondence: Luke Keating, St. John's University, 1048 Johnston Ave, Wantagh, NY 11793, United States.E-mail: luke.keating14@ stjohns.edu

N. KURNIADI, A. ELY, C.E. WIERENGA \& W. KAYE. Neurocognitive Profiles of Individuals Remitted from Eating Disorders.

Objective: Anorexia nervosa (AN) and bulimia nervosa (BN) are life-threatening illnesses marked by extreme eating behaviors and rigid beliefs about body and weight. While evidence suggests neuropsychological dysfunction among individuals with eating disorders (EDs), the majority of studies examine indiviuals in the acute stages of illness, rendering it difficult to determine whether such deficits are secondary to malnutrition or pre-existing traits. The current study addresses this issue by investigating neurocognitve functioing among women remitted from anorexia nervosa (RAN) and bulimia nervosa (RBN).

Participants and Methods: Ninety-one women (28 RAN, 37 RBN, and 27 healthy controls) participated in the study. The Verbal Fluency subtest of the Delis Kaplan Executive Funciton System (DKEFS), the California Verbal Learning Test-II (CVLT-II), and the Wisconsin Card Sort Test (WCST) were used to assess performance in verbal learning and fluency, inhibition, and problem solving. Analyses of variance were used to examine between-group differences in task performance and the relationship to illness duration.

Results: On the DKFES, RBN demonstrated significantly more repetition errors $(p=.001)$ and total errors $(p=.016)$ on Verbal Fluency, and greater repetitions $(p=.012)$ and total intrusions $(p=.043)$ on the CVLT relative to controls. Longer BN illness was significantly and negatively associated with performance in strategic planning (WCST) and cognitive inhibition (DKEFS).
Conclusions: Individuals remitted from EDs demonstrated significant impairments in executive and verbal learning and memory relative to controls, with the most pronounced deficits seen among the RBN group. The combination of poor inhibitory control and reduced ability to learn from prior experiences may contribute to the maintenance of binge/purge behaviors when ill. Findings suggest that neurocognitive impairments persist after remission from ED's. Such deficits may reflect pre-existing traits that contribute to the development of $\mathrm{AN}$ and $\mathrm{BN}$. Correspondence: Natalie Kurniadi, M.A., Clinical Psychology, California School of Professional Psychology, PO Box 7471, San Diego, CA 92167, United States.E-mail:natalie.kurniadi@alliant.edu

J.J. MAHONEY, S. BAJO, A. DE MARCO, D. BROSHEK \& R.C. HILSABECK. Referring Providers' Preferences and Satisfaction with Neuropsychological Services.

This study aims to expand upon previous research by characterizing the attitudes/preferences of referring providers who utilize neuropsychological services. A 31-question survey was disseminated across several professional listservs and data was collected from individuals across different medical and non-medical professions who refer for neuropsychological services $(\mathrm{N}=81)$. Most referring providers prefer shorter reports (2-4 pages), in bullet-point/table format for ease of readability, and receipt of the completed report within two weeks. Only $50 \%$ of respondents reported reading the entire neuropsychological report, with the background, developmental/medical, and educational histories being the least frequently read sections. Nearly all respondents indicated that they are satisfied with neuropsychological services overall and agree that the referral question is typically satisfactorily answered, the findings are communicated clearly, and they agree with the diagnostic impressions. Referring providers appreciate most of the neuropsychological recommendations, with the exception of those regarding laboratory work, medications, and other medical procedures. Few differences in preferences and satisfaction were noted across provider specialties, patient populations, or practice settings. The most useful aspects of neuropsychological services included the thoroughness and integration of the evaluation/report, along with the impressions, diagnoses, and recommendations. Recommendations included shorter reports, increased availability of neuropsychological services, and more concise impressions and recommendations. These findings are consistent with and expand upon prior literature regarding referring provider preferences and satisfaction. Illuminating the most and least useful aspects of neuropsychological services provides valuable information to practitioners, particularly in the context of rapidly-changing institutional and healthcare demands.

Correspondence: James J. Mahoney, PHD, Psychiatry and Behavioral Sciences, University of Virginia, 1417 Madison St, Waynesboro, VA 22980, United States. E-mail: jamesjmahoney@gmail.com

A. MANKOWSKA, M. HARCIAREK, J.B. WILLIAMSON, B. BIEDUNKIEWICZ \& K.M. HEILMAN. Leftward Bias of Visual Attention in Patients With End-Stage Renal Disease Receiving Dialysis: a Neglected Phenomenon.

Objective: Individuals with end-stage renal disease (ESRD) receiving dialysis typically present with cognitive difficulties, including problems sustaining attention. Prior studies have revealed that the right hemisphere appears to be dominant for mediating both sustained attention and the allocation of spatial attention. Whereas these patients' defective ability to sustain attention may be a consequence of right hemisphere dysfunction, the allocation of spatial attention has never been investigated in these patients. Thus, the aim of this study was to test the hypothesis that dialyzed patients might also have a spatial attentional bias.

Participants and Methods: Thirteen non-demented patients with ESRD receiving adequate dialysis but without any neurological diseases (age range: 20-60) and 15 demographically matched healthy controls were the participants of this study. Participants performed a standard 
line bisection task using 24 horizontal lines $(24 \mathrm{~cm}$ long and $2 \mathrm{~mm}$ thick) that were sequentially placed on a white board placed at eye level. Results: The analysis revealed that both groups deviated their bisections leftwards, patients receiving dialysis had a significantly greater leftward bias than healthy controls.

Conclusions: To our knowledge, this is the first study indicating that patients with end-stage renal disease may present with impaired ability to correctly allocate their spatial attention (spatial neglect). Although the reason for this leftward bias needs to be elucidated, it is possible that ESRD and/or dialysis may have induced right frontal-subcortical dysfunction that disinhibited the right parietal lobe, inducing a left sided attentional bias; however, further studies will have to test this hypothesis.

Correspondence: Aleksandra Mankowska, Master, Institute of Psychology, University of Gdansk, Jana Bazynskiego 8, Gdansk 80309, Poland.E-mail: aleksandra.wojtowic@gmail.com

T.S. PATERSON, N. O'ROURKE, W.J. L. THORNTON \& R.J. SHAPIRO. Modelling Medication Adherence in Renal Transplant Recipients: Cognitive and Psychosocial Impacts.

Objective: Estimates indicate 20-70\% of renal transplant recipients (RTR) are non-adherent to medications (Chisholm-Burns, et al., 2012). Past findings indicate medication adherence decreases in relation to worse everyday problem solving (EPS; Gelb et al., 2010), increased depressive symptoms, and reduced self-efficacy (SE) in RTR (Schoenthaler et al., 2009; Tucker et al., 2001). However, these associations have previously been examined independently. To provide better understanding of adherence in RTR, we used structural equation modelling (SEM) to provide a comprehensive examination of relationships between EPS and traditionally measured cognition, depression, SE, and adherence in RTR.

Participants and Methods: RTR $(\mathrm{N}=211)$ underwent transplant at least 6 -months prior to participation. Adherence was measured via the Transplant Effects Questionnaire. Cognitive measures assessing intelligence, working memory, executive functions, and memory were utilized, as were EPS measures. Depressive symptoms were assessed using the subscale scores of the Centre for Epidemiological Studies Depression Scale, and SE was measured with multiple questionnaires. SEM was used to assess the fit of our model to collected data.

Results: Results indicate SE and EPS have direct positive associations with adherence, while other cognitive abilities were positively related to SE, and inversely related to depression; depression was also negatively related to SE. Our final model provided a good fit to the data $(\mathrm{CFI}=0.97$; SRMR=0.072; RMSEA=0.031).

Conclusions: We examined relationships between cognitive and psychosocial factors and adherence in RTR. Findings indicate the importance of EPS and SE in predicting medication adherence, and suggest that influences of depression and other cognitive variables are more complex than previously reported, with SE mediating associations between these variables and adherence. These findings are of import to the future development of treatments to improve adherence among RTR.

Correspondence: Theone S. Paterson, M.A., Psychology, Simon Fraser University, 92 Wolfrey Ave., Toronto, ON M4K1K8, Canada. E-mail: theonep@sfu.ca

R.J. PIERS, A. NISHTALA, J. HIMALI, A. BEISER, J.S. SACZYNSKI, D.D. MCMANUS, E.J. BENJAMIN \& R. AU. Atrial Fibrillation and Cognitive Decline: the Framingham Heart Study.

Objective: There is a paucity of longitudinal research investigating the relations between atrial fibrillation $(\mathrm{AF})$ and cognitive performance. Our study investigated the association between $\mathrm{AF}$ and longitudinal change in cognitive performance in a community-based sample.

Participants and Methods: Participants of the Original and Offspring cohorts were dementia- and stroke-free at the time of baseline neuropsychological (NP) assessment (between 1999-2001), and underwent at least one additional NP assessment with a one year or greater inter-test interval. 244 participants from the Original cohort (mean age $=83 \pm 3$ years; $63 \%$ female) and 2454 participants from the Offspring cohort (mean age $=61 \pm 9$ years; $54 \%$ female) met criteria for analyses. Using linear regression, we examined the association between prevalent $\mathrm{AF}$ and cognitive performance adjusted for age and sex, and for vascular risk factors and APOE 4 .

Results: At time of baseline cognitive assessment, 24 (11.5\%) Original cohort and 57 (3.1\%) Offspring cohort participants had prevalent $\mathrm{AF}$. AF was associated with lower neuropsychological test performance across multiple domains in the Offspring cohort, but after adjusted for covariates, the only association that remained significant was for a test of abstract reasoning (Similarities; Beta \pm standard error $[\mathrm{SE}]$ )= $-1.06 \pm 0.39, p<0.006)$. Longitudinally, prevalent $\mathrm{AF}$ was associated with annual change in neuropsychological test performance for executive function (Trail Making B) for both Original and Offspring cohorts, respectively (Beta $\pm \mathrm{SE}=-1.24 \pm 0.33, \mathrm{p}<0.001 ; \mathrm{Beta} \pm \mathrm{SE}=-0.09 \pm 0.03$, $\mathrm{p}<0.001)$.

Conclusions: $\mathrm{AF}$ was related to poorer cognitive function, particularly for measures associated with frontal lobe function. These results suggest that managing risk for atrial fibrillation could potentially also have positive effects on brain aging.

Correspondence: Ryan J. Piers, Neurology, Framingham Heart Study, 19 Harvard Avenue, Brookline, MA 02446, United States. E-mail: rpiers@bu.edu

E.E. QUASNEY, J. HOELZLE, T. HAMMEKE \& A. STARSKY. The Impact of Balance Disturbance on an Auditory Sustained Attention Task.

Objective: Cognitive and balance deficits are two of the most prevalent consequence of mTBI. There is some indication that a challenge to one or both of these functions can result in cognitive detriments due to constraints on attentional capacity. However, the evidence remains both conflicting and sparse. This study examined the impact of increasing balance challenge on attention.

Participants and Methods: Forty-three healthy adults completed three balance tasks of varying difficulty levels while also engaging in an auditory sustained attention task. Attention task trials were completed while participants stood on force plates to measure postural sway during the three respective stances. Balance errors, derived from the Balance Error Scoring System (BESS), were also recorded as a measure of postural stability.

Results: While no differences in cognitive performance were evident based on level of balance challenge, sustained attention was predicted by both postural sway as measured by the force platform and by errors on a modified version of the BESS. Specifically, with greater the sway area, participants evidenced fewer correctly identified targets and more variability in reaction time. Additionally, worse BESS performance was associated with fewer correct responses to targets and a greater number of omissions.

Conclusions: These findings reveal a significant relationship between balance perturbation and sustained attention, suggesting that impairments in balance may contribute to attentional impairments, even among otherwise healthy young adults. This highlights the importance of considering balance impairment as a contributing factor in cognitive symptoms among individuals with mTBI and, more broadly, among patients with various other neurologic and complex medical conditions. Correspondence: Erin E. Quasney, Department of Behavioral Medicine and Psychiatry, WVU School of Medicine, $610 \mathrm{~N} 112 \mathrm{th}$ Street, Wauwatosa, WI 53226, United States. E-mail: equasney@gmail.com

C. SEMERJIAN, S.A. PARIKH, T. NADER, D. FRIM \& M. LACY. Repeatable Battery for the Assessment of Neuropsychological Status (RBANS): Cognitive Profile of Adult Patients with Chiari Malformation Type 1.

Objective: Chiari malformation type 1 (CM1) is characterized by cerebellar tonsillar herniation below the level of Foramen Magnum 
(deSouza, Zador, \& Frim, 2011). Increasing research has been directed at investigating the role of the cerebellum in cognition (Kriziol and colleagues, 2014). In a large recent survey on neurocognitive symptomatology, CM1 adults endorsed difficulties with memory (43.88\%) and language (43.75\%) (Fischbein et al., 2015). At present, only self-report surveys, case studies, and small cohorts have been presented examining the potential cognitive sequelae of CM1. Thus, the present study aims to identify the cognitive profile in adults with CM1.

Participants and Methods: Following consent, twenty pre-surgical adults presenting for routine neurosurgical appointment completed the Repeatable Battery for the Assessment of Neuropsychological Status (RBANS). Data for participants were analyzed as standard scores.

Results: The cohort had a mean age of 28.29 (SD=8.93) with $85 \%$ females. Compared to manual based norms, adults displayed average current global functioning (RBANS Total $M=98.2, S D=13.9$ ), immediate and delayed memory (RBANS Imm. Memory $M=91.40, S D=12.3$, Del. Memory $M=94.15, S D=15.2$ ), language (RBANS Lang $M=92.2$, $S D=12.9$ ), visuoconstruction (RBANS Vis Cons $M=103.65, S D=16.1$ ) and attention (RBANS Attn $M=93.2, S D=20.7$ ).

Conclusions: The current preliminary data indicated that adults with CM1 display average global cognitive functioning. Based on discrepancy between reported and objective performance on cognitive tasks, transient causes should be investigated (e.g., sleep, pain, mood state). Limitations include small sample size and use of a screening tool.

Correspondence: Claire Semerjian, M.S., Psychology, Roosevelt University, 206 E Chestnut Street, Apt 902, Chicago, IL 60611, United States.E-mail: csemer319@gmail.com

\section{STEED, M. HAINES, M. BONNELL, A.M. MYERS-FABIAN \& M.C. HUDSON. Cognitive Improvements Persist Post Left Ventricular Assist Device Placement.}

Objective: Either as bridge to transplant or destination therapy, left ventricular assist devices (LVADs) serve many patients with advanced heart failure (AHF). Despite reports of improvement in multiple body systems following LVAD placement, including increased cerebral perfusion, research examining longitudinal outcomes of cognition is sparse. In prior research $(\mathrm{n}=12)$, we found improvements in verbal memory 6 months and 12 months post-placement and in visual memory between 12 and 24 months. The purpose of the current follow-up study was to examine trends in neurocognition in an increased sample $(n=18)$ and after additional follow-up testing.

Participants and Methods: We again compared cognition pre- and post-LVAD placement across time points (3-6 months; 6-12 months; and 2 years) for 18 patients with advanced heart failure who had LVAD placement, using the Repeatable Battery for the Assessment of Neuropsychological Status and the Trail Making Test. We used linear mixed models to assess change over time, which account for intraindividual variance, handle missing data, and model trends over time.

Results: Delayed list learning memory showed a significant quadratic trend following LVAD placement, with initial improvement stabilizing over time. Speed of processing showed a significant cubic trend of initial improvement, stabilization, and improvement between years 1 and 2 post-placement. Visual memory showed a cubic trend of improvement, decline, and improvement—all time points were above clinical means. Delayed story memory showed a significant linear trend. List recognition and semantic fluency showed nearly significant quadratic trends. All other cognitive functions remained stable up to two years post placement.

Conclusions: Findings demonstrate cognition generally improves, and these improvements are stable, up to two years post-LVAD placement. Correspondence: Drake Steed, Psy.D., Rehab Psychology Services, The University of Toledo Medical Center, 3000 Arlington Ave., Toledo, OH 43614, United States. E-mail: drake.steed@gmail.com
J. STELMOKAS, A. RAHMAN, K.A. KITCHEN ANDREN, J.M. RECKOW, D.M. UKUEBERUWA, E. JONES, Z. CRESPI \& L.A. BIELIAUSKAS. The Influence of Cognitive Status and Depression on Duration of Hospital Stay in Post-Acute Rehabilitation.

Objective: Cognitive status effectively predicts length of stay (LOS) in hospitalized older patients, though which specific cognitive domains are the most important, relative to other medical and psychiatric variables, has not been clarified.

Participants and Methods: Medical records of 188 older $(M=66.03$ years, $S D=11.06)$, primarily male $(94.7 \%)$ inpatient Veterans admitted to a post-acute rehabilitation unit $(\operatorname{LOS} M=36.34$ days, $S D=24.00)$ were reviewed.

Results: Univariate analyses indicated that LOS significantly correlated with the number of inpatient medications, depressive symptoms (DSM-IV-TR Checklist), and memory performance (Addenbrooke Cognitive Examination - Revised, Memory subscale), but did not correlate with other demographic (age, education, living arrangement), cognitive (ACE-R Total/subscales or executive functioning) and admission-related characteristics (primary reason for admission, prior living status, quality of goals). Hierarchical regression analyses revealed that inpatient medications alone accounted for $8.3 \%$ of the variance in LOS $(F(1,181)=16.30, p<.001)$, and the addition of memory functioning and depressive symptoms accounted for an incremental $4.0 \%$ of the variance in $\operatorname{LOS}(F(2,179)=8.31, p=.019)$. Partial correlations indicated that medications $\left(r_{\mathrm{sp}}=.258, p<.001\right)$ and memory $(r=-.141$, $p<.05)$ significantly predicted LOS, while depressive symptoms did not. Conclusions: Impaired memory performance may predict incremental variance in rehabilitation progress and outcome beyond that captured by other markers of medical status.

Correspondence: Julija Stelmokas, Psy.D., Ann Arbor VA, 2215 fuller road, Ann Arbor, MI 48104, United States. E-mail: jstelmok@med. umich.edu

R. VENEZIA, D. FOSSATTI, B. COSTABILE, N. SILVERSTEIN, S. SCHAFFER \& K.A. WALKER. Opioid Dosage in Critically Ill ICU Patients is Associated with Attention Deficits, but not Memory Deficits, at Follow-up.

Objective: Sedatives (e.g., benzodiazepines) and analgesics (e.g., opiates) are commonly used to modulate anxiety/agitation and pain in critically ill patients on the intensive care unit (ICU). However, the effect of these therapeutics on post-ICU cognitive functioning is unclear. The goal of the current study was to examine how post-ICU memory and attention are affected by ICU analgesic and sedative administration.

Participants and Methods: Fifty-seven $(n=57)$ individuals without pre-existing neurological or cognitive impairment, who were admitted to the ICU for greater than 72 hours and diagnosed with septic shock and/or respiratory failure, were enrolled. Memory and attention were assessed at 4-month follow-up using subscales from the Repeatable Battery for the Assessment of Neuropsychological Status (RBANS). Benzodiazepine and opioid administration, and depth of sedation (Richmond Agitation Sedation Scale; RASS) were continuously monitored during the ICU stay.

Results: Cumulative opioid dosage $(\mathrm{r}=481, \mathrm{p}=.001)$ and cumulative benzodiazepine dosage $(\mathrm{r}=.341, \mathrm{p}=.025)$ were independently associated with depth and length of sedation. In a model that covaried for disease severity and level of medical comorbidity, cumulative opioid dosage predicted attention deficits at 4-month follow-up $(B=-.467$, $\mathrm{p}=.027$ ), but was unrelated to memory functioning. In the same model, cumulative benzodiazepine dosage demonstrated a trend association with attention at 4-month follow-up $(B=-.364, p=.076)$, but not with memory. Cumulative depth and length of sedation did not predict attention or memory deficits at follow-up.

Conclusions: The findings provide preliminary evidence suggesting that high levels of ICU opioid and benzodiazepine administration may have prolonged detrimental effects on attention abilities after ICU 
discharge. These cognitive deficits do not appear to be the result of prolonged sedation.

Correspondence: Rachel Venezia, Ph.D. candidate, Clinical Psychology, St. John's University, 76-66 Austin St. Apt. 2K, New York City, NY 11375, United States. E-mail: rachel.Gaia.venezia@gmail.com

K.A. WALKER, K. HAPPER, R. VENEZIA, A. HIRALALL, A. KAUR \&. S. SCHAFFER. Neuroimaging findings and cognitive functioning in patients with sepsis-associated encephalopathy.

Objective: Sepsis-associated encephalopathy (SAE) is the most common form of encephalopathy on the medical and surgical intensive care unit (ICU). Currently, little is known about the underlying pathogenesis of SAE, and it is unclear whether the cognitive effects of SAE persist. The present study used neuroimaging to examine the brain changes associated with SAE and determine the relationship between SAE and post-ICU cognitive functioning.

Participants and Methods: Charts from 60 patients with no preexisting neurologic conditions, who developed SAE on the ICU and underwent neuroimaging, were retrospectively examined. MRI and CT scans were rated by two neuroradiologist using a standardized rating protocol. An additional sample of 30 patients with no pre-existing neurologic or cognitive impairment, who developed septic shock, was prospectively enrolled. In this group, cognition was assessed on the day of ICU discharge and 4-month follow-up using the Repeatable Battery for the Assessment of Neuropsychological Status.

Results: Among patients who developed SAE, periventricular white matter lesions $(73 \%)$, subcortical white matter lesions (54\%), cerebrovascular calcification $(63 \%)$, and lacunar infarcts $(33 \%)$ were most common. Acute infarcts $(15 \%)$, acute hemorrhage $(3 \%)$, and cerebral edema $(13 \%)$ were less prevalent. Acute neuroimaging findings (i.e., cerebral infarcts and edema) were associated with greater risk of mortality (OR, 5.25. $p=.02$ ) and disability (OR, 5.27. $p=.03$ ). In a hierarchical regression, which covaried for disease severity and medical comorbidity, SAE was associated with cognitive impairment at ICU discharge $(\beta=.74, p=.002)$ and at follow-up $(\beta=.54, p=.024)$.

Conclusions: Although white matter lesions and cerebrovascular calcification are most common in patients with SAE, acute neuroimaging abnormalities are associated with worse outcomes. Furthermore, development of SAE increases the likelihood of cognitive deficits months after ICU discharge.

Correspondence: Keenan A. Walker, M.A., Psychology, St. John's University, 101-01 67th Dr., APT 4J, Forest Hills, NY 11375. United States.E-mail:kwalke26@gmail.com

E. WARNER, S. AMINI, L. HIZEL, S. LEVY, N.A. SCHWAB, S.J. CROWLEY, J.J. TANNER, C. HERNAIZ ALONSO, A. CHOI, H. PARVATANENI \& C.C. PRICE. Speed of Clock Drawing is Reduced After Orthopaedic Surgery in Older Adults.

Objective: Older adults are at an increased risk for negative postoperative cognitive changes. The Digital Clock Drawing Test (dCDT) mav be a valuable tool for assessing pre and post-operative cognitive differences. The primary aim of this prospective study was to examine pre to post-surgery 3 -week and 3 -month change in performance on the dCDT in older adults (age > 65) electing total knee arthroplasty (TKA) relative to non-surgery peers with osteoarthritis.

Participants and Methods: Participants included 38 non-demented TKA individuals (mean age $=69.6$ ) and 31 non-surgery peers (mean age $=68.9)$ with osteoarthritis. There were no group differences at baseline in age, education, and premorbid intellectual estimates. Clock drawing time to completion was assessed at baseline (pre-surgery), 3-weeks, and 3-months post orthopedic surgery. T-tests examined 3-week and 3 -month change in total time to completion relative to baseline for surgery vs. non-surgery peers.

Results: Groups were not statistically different in baseline clock drawing time $(p=0.54)$. At 3 -weeks post surgery, surgery participants' time to completion increased (became slower) relative to the non-surgery peers $(p=0.009)$. Specifically, relative to baseline, surgery participants were slower on average by 3 seconds, while non-surgery peers were faster on average by 7 seconds. By 3 -months, the non-surgery peers were still 7 seconds faster on average while the surgery participants were largely unchanged from baseline (trend 3 -month group differences, $p=0.11$ ). Conclusions: Following surgery, our sample of older adults did not show an expected decrease in clock drawing time to completion (i.e.. practice effect) relative to non-surgery peers with orthopedic pain. These findings suggest sensitivity of millisecond speed (dCDT) measures in revealing cognitive changes among patients electing TKA relative to non-surgery peers with osteoarthritis. Support: NINR-014181(CP) Correspondence: Eric Warner, B.S., Department of Clinical and Health Psychology, University of Florida, 631 NW 34th Drive, Gainesville, FL 32607,United States.E-mail: ericwarner@ufl.edu

M.K. YEE, P. JANULEWICZ-LLOYD, K. SULLIVAN \& M. KRENGEL. Diagnostic Criteria for Gulf War Veterans Illness: CDC Versus Kansas Criteria.

Objective: Veterans from the 1990-1991 Gulf War (GW) consistently report chronic health symptoms and functional impairments. Symptoms engage multiple organ systems, including the central nervous system in particular. Multiple research and clinical criteria are documented, but the two most common are Fukuda's CDC Chronic Multisymptom Illness (CMI) and the Kansas Criteria. It is unclear if either of these criteria are sensitive and specific enough to be considered the gold standard. This analysis investigates the incidence of illness defined by the two criteria, and compares rates of health svmptoms.

Participants and Methods: $382 \mathrm{GW}$ Veterans responded to a survey as part of the Fort Devens Longitudinal Cohort Study. Veterans were separated into one of three groups: no diagnosis (ND), CMI criteria only (CD), or both CMI and Kansas criteria (CK). The chi-square test of independence assessed differences in health svmptom rates among the three groups. If initial tests revealed significant differences, additional tests adjusted for multiple comparisons were run.

Results: 323 (84.6\%) Veterans met CMI and 241 (63.1\%) met Kansas Criteria. $59(15.4 \%)$ were in the ND group, $82(21.5 \%)$ in the CD group, and $241(63.1 \%)$ in the CK group. Groups were similar in age, education and gender $(p>0.05)$. Significant differences $(p<0.0001)$ were found across all three groups for 32 of 34 health symptoms. The CK group reported significantly higher rates for 30 symptoms compared to the ND group and the CD group $(\mathrm{p}<0.0002)$. However, the $\mathrm{CD}$ group only reported significant differences for 7 symptoms compared to the ND group $(p<0.0002)$.

Conclusions: Veterans continue to report high rates of symptoms meeting criteria for deployment related illness. More Veterans meet CMI criteria, but Veterans meeting Kansas criteria reported significantly higher rates of health svmptoms. This suggests that the CMI criteria mav be too broad and the Kansas criteria may provide increased sensitivity. These results could impact the diagnosis and research of GW Veterans Illness.

Correspondence: Megan K. Yee, M.A., Environmental Health, Boston University, 715 Albany Street, T4W, Boston, MA 02118, United States. E-mail:meganyee@bu.edu

\section{Multiple Sclerosis/ALS/Demyelinating Disorders}

\section{CADDEN \& P. ARNETT. Acute and Chronic Pain and Cognitive Functioning in Multiple Sclerosis.}

Objective: Research suggests individuals with chronic pain demonstrate diminished attention, learning and memory, processing speed, and executive functioning. However no obvious pattern of common effects is established. Furthermore, less is known about the differential impact of acute pain versus chronic pain. The goal of the current study was to 
examine the relationship between measures of acute and chronic pain with cognitive functioning in individuals with Multiple Sclerosis (MS). Participants and Methods: Fifty-three individuals with MS participating in a neuropsychological research study of MS-related factors were examined. Pain was measured using the Brief Pain Inventory (BPI). Participants completed a comprehensive neuropsychological test battery. Demographic and illness-related variables (EDSS) were collected. Correlations between pain measures and sample-normed composites of processing speed, memory, and executive functioning were examined.

Results: Acute pain did not correlate with any of the cognitive composites. Chronic pain significantly correlated with the memory composite $(\mathrm{r}=-.28, \mathrm{p}=.04)$. This relationship dropped to marginal significance once EDSS was controlled for statistically $(r=-.24, p=.09)$. Examination of individual memory tests revealed that chronic pain was significantly and specifically related to measures of verbal learning and memory (e.g., CVLT-II) as opposed to spatial memory (e.g., BVMT-R) or working memory (PASAT).

Conclusions: Chronic pain, as opposed to acute pain, associates negatively with verbal learning and memory. Although causal relationships are unclear, it may be that chronic pain specifically interferes with auditory verbal learning and memory. Neuropathological and treatment implications of these findings will be explored.

Correspondence: Margaret Cadden, Psychology, The Pennsylvania State University, 372 Moore Building, University Park, State College, PA 16802, United States. E-mail:mhc147@psu.edu

D. CALVO, J. PETERSEN, B. POLLOCK, H. GERHART, A. RIDGEL \& M. SPITZNAGEL. Can one week of moderate intensity aquatic exercise improve cognition and fitness in MS?

Objective: Cognitive impairment exists in approximately $50 \%$ of individuals with MS. Research suggests exercise can improve cognition. Aquatic exercise may facilitate exercise engagement by reducing commonly cited barriers (e.g., pain, fatigue) in MS. We hypothesized that 1 week of aquatic exercise would improve fitness and cognition in MS, and that higher intensity during exercise would correlate with greater cognitive/fitness outcomes.

Participants and Methods: 38 individuals participated in a weeklong aquatic exercise intervention $(n=19)$ or control condition $(n=19)$. Cognition (D-KEFS Sorting, TMT, COWAT) and fitness (2MST) were assessed 24 hours pre- and post-intervention. Heart rate (HR) data was collected throughout exercise sessions. Reliable change indices (RCI) were calculated to identify statistically reliable change.

Results: 2MST improved for exercisers $(F(1,15)=20.65, p<0.0$ $\left.1, \eta^{2}=0.57\right)$, but not controls $(F(1,15)=1.69, p=0.21)$. Although COWAT $\left(F(1,33)=8.29, p<0.01, \eta^{2}=0.20\right)$ and TMT $(F(1,33)=10.89$ $\left.. p<0.01, \eta^{2}=0.24\right)$ performances improved for both groups, exercisers demonstrated greater RCI on the 2MST (37\% of participants), Sorting Test $(16 \%)$, and COWAT $(11 \%)$. Lower rates of RCI were noted in controls ( $5 \%$ for 2MST, Sorting Test, and COWAT). Reliable change did not occur on TMT for either group. Average HR during exercise correlated with post-intervention 2MST $(r=0.49, p=0.03)$, Sorting Test $(r=0.47, p=0.04)$, and TMT $(r=0.47, p=0.04)$ performances.

Conclusions: Fitness improved in exercisers, but not controls. Aspects of cognition improved for both groups, suggesting possible practice effects; however, reliable change occurred at higher rates in exercisers. Higher HR correlated with better fitness and cognitive outcomes. Results suggest a single week of moderate intensity aquatic exercise can improve both fitness and cognition in MS. Future studies using similar interventions with varying intensities and duration should explore optimal dose for maximal outcomes.

Correspondence: Dayana Calvo, Kent State Psychology, 3877 Lake Run Blvd, Stow, OH 33777, United States.E-mail: dcalvo1@kent.edu
A.R. CARLEW \& D.B. SALISBURY. Occupational Attainment as a Proxy of Cognitive Reserve in Patients with Multiple Sclerosis.

Objective: Recently, support for the cognitive reserve hypothesis has emerged in the multiple sclerosis (MS) literature (Sumowski, Chiaravalloti, \& DeLuca, 2009). While abundant evidence for the value of word reading tests as a proxy of cognitive reserve exists (e.g., Benedict et al., 2010), some researchers have begun investigating measures of "lifetime enrichment" including hobbies, educational attainment (EA), and occupational attainment (OA; eg., Pinter et al., 2014). Only one study to our knowledge has investigated the predictive validity of occupational attainment on cognitive functioning in MS (Ghaffar, Fiati, \& Feinstein, 2012). In the present study, we investigate the contribution of occupational attainment to cognitive functioning in patients with MS. Participants and Methods: Sixty-two individuals diagnosed with MS were administered a neuropsychological battery including the wordreading tests and the Symbol Digit Modalities Test (SDMT). Patients' highest attained occupation was coded using the Census Industrial and Occupational Category Codes. OA was then effect coded into high (executive and specialty), medium (support positions or skilled labor), and low (manual or unskilled labor).

Results: A hierarchical regression was conducted to determine if $\mathrm{OA}$ predicted more preserved (i.e., higher) performance on the SDMT over and above that of word reading and EA. While EA and word reading did not account for significant variance in the first model, the full model for all three variable was significant, $R^{2}=.167, F(4,57)=2.864, p=.031$; adjusted $R^{2}=.109$. The addition of $\mathrm{OA}$ to the model led to a statistically significant increase in $R^{2}$ of $.118, F(2,57)=4.047, p=.023$.

Conclusions: $\mathrm{OA}$ was predictive of more preserved cognitive functioning on a test traditionally used to measure cognitive decline in patients with MS. While this is consistent with previous findings, the predictive value of $\mathrm{OA}$ beyond word reading scores and EA is a novel finding.

Correspondence: Anne R. Carlew, Psychology, Univerisity of North Texas, 940 West Round Grove Rd, 1425, Lewisville, TX 75067, United States.E-mail: anne.carlew@gmail.com

S.L. COSTA, J. DELUCA, K. COSTANZA \& N. CHIARAVALLOTI. Understanding difference between the California Verbal Learning Test (CVLT) and Selective Reminding Test (SRT).

Objective: The California Learning Verbal Test II (CVLT) and the Selective Reminding Test (SRT) are among the most commonly used tests to assess learning and memory in Multiple Sclerosis (MS). The current study compares performance on CVLT and SRT.

Participants and Methods: 124 participants with MS completed a evaluation assessing executive functions (EF), working memory (WM), processing speed (PS) and pre-morbid IQ. The SRT and CVLT were administered on 2 different days in a fixed order. Total correct response (TR), and consistent long term retrieval (CLTR) slopes for trials 1-2 and 1-4 were calculated for both measures. Semantic and serial clustering on the CVLT were calculated.

Results: All participants were impaired on SRT (i.e. needed more than 8 trials to recall 10 words twice), however only 41 participants (33\%) were impaired on CVLT (i.e. total correct responses in 5 trials). The sample was thus divided into 2 groups based on CVLT performance: impaired $(Z=<-1.0$ at TR trials $1-5 ; n=41)$ and not impaired $(Z>-1.0$; $\mathrm{n}=83$ ).

The two groups did not differ on SRT TR slopes, or SRT CLTR slopes. The impaired group showed poorer performances on EF $(p<.01)$ and PS $(p=.01)$ as compared with the not impaired group. The not impaired group showed a higher score on semantic clustering than the impaired group $(p<.01)$. EF was correlated with CVLT TR slopes [trials 1-2 $(r=.2, p=.03)$; and $1-4(r=.34, p<.01)$ ], and CVLT CLTR slopes [trials $1-2(r=.29, p<.01)$, and $1-4(r=.37, p<.01)]$. EF was correlated with SRT CLTR slope trial 1-4 $(r=.3, p<.01)$. PS was correlated with CLTR slope trial 1-4 on CVLT $(r=.28, p=.01)$ and SRT $(r=.3, p<.01)$.

Conclusions: Although the CVLT and SRT are both thought to be sensitive to learning impairments in MS, this study shows that observed impairment levels on the two tests are significantly different. Data 
indicates that the observed differences in performance on the 2 tests are likely to be associated with the increased role of executive functioning in supporting new learning and memory on the CVLT but not on the SRT. Correspondence: Silvana L. Costa, Kessler Foundation, 300 Executive Drive, West Orange, NJ 07052-3390, United States. E-mail: scosta@ kesslerfoundation.org

H. DUNCANSON, E. MOES, M. JERRAM \& S. ORSILlo. Pilot study of an internet based self-guided mindfulness program for individuals with Multiple Sclerosis.

Objective: Psychological stress and emotional preoccupation have been found to be associated with new brain lesions and exacerbations of symptoms in multiple sclerosis. Research has further suggested that problems with attention may be directly related to poor emotion regulation skills in individuals with MS. Mindfulness is one intervention that has been suggested to enhance both attention to the present moment as well as emotion regulation skills. The current study evaluated the effectiveness of an 8-week online meditation training program delivered to individuals diagnosed with Multiple Sclerosis.

Participants and Methods: The eight week intervention was delivered online. Participants were emailed MP3 files along with written instructions for the meditative practice each week. The meditations were presented in a progressive manner from mindfulness of breath to a loving/kindness meditation. Twenty-six individuals with MS were assessed at baseline and follow-up on measures of mindfulness, emotion regulation, quality of life, inhibitory control, and contrast sensitivity (a proxy measure of dopamine).

Results: Findings revealed that individuals with MS reported significantly increased levels of mindful awareness, emotion regulation skills, and quality of life post-intervention. Inhibitory control, as measured via the Stop Signal Task, was moderately improved at follow-up. Proxy measurements of dopamine level did not significantly increase at follow-up, although this was potentially confounded by methodological issues. Proxy measurements of dopamine and inhibitory control also failed to mediate the relationship between mindfulness and improved emotion regulation.

Conclusions: In sum, the current study provides preliminary evidence that a short, self-guided meditation training program was feasible and acceptable when delivered to a group of individuals diagnosed with MS. Further, the findings suggest that brief meditation training may improve cognition, emotion regulation, and quality of life in individuals diagnosed with MS.

Correspondence: Haley Duncanson, MA, Psychology, Suffolk University, 253 center st, Randolph, MA 02368, United States. E-mail: haleyduncanson28@gmail.com

H.M. GENOVA, Y. GOVEROVER, J. LENGENFELDER, C. BOBER, J. DELUCA \& N. CHIARAVALLOTI. Cognitive Reserve Protects Against Social Cognition Impairments in Multiple Sclerosis.

Objective: Emerging research suggests that individuals with Multiple Sclerosis (MS) experience deficits in social cognition, including impairments in facial affect recognition and Theory of Mind. However, there is little understanding of why these deficits occur, how prevalent these deficits are, and the factors that predict social cognitive abilities. The current study examines the degree to which cognitive reserve is predictive of social cognitive abilities in MS.

Participants and Methods: 43 individuals with MS participated. Cognitive reserve was assessed using The Vocabulary subtest of the Wechsler Abbreviated Scale of Intelligence-Second Edition, as well as years of education. Social cognition was assessed using the Reading the Mind in the Eyes Test. A hierarchical linear regression analysis was used to examine the influence of CR on social cognition after controlling for demographic (age) and disease related variables (i.e. ambulation index). Results: Cognitive reserve measures significantly predicted social cognition, accounting for $29.8 \%$ of the variance in Reading the Mind in the Eyes score, after controlling for the contributions of demographic/ disease-related variables.

Conclusions: The current study found that cognitive reserve is predictive of social cognitive ability in that individuals who had higher vocabulary attainment had better social cognitive ability. These findings extend a growing body of literature demonstrating a strong relationship between cognitive reserve and classic cognitive domains by showing that cognitive reserve is also predictive of social cognitive impairments. Correspondence: Helen M. Genova, Ph.D., Kessler Foundation, 300 Executive Drive, West Orange, NJ 07052, United States. E-mail: hgenova@kesslerfoundation.org

Y. GOVEROVER, S. HAAS \& J. DELUCA. Exploring Money Management in Persons with MS: A Pilot Study.

Objective: Managing one's own finances is an instrumental activity of daily living (IADL) crucial to independent functioning and requires adequate executive-attentional abilities as well as retrospective and prospective memory. This study examined whether persons with Multiple sclerosis (MS) have problems in managing finances versus healthy controls (HC), and the variables that may contribute to these problems.

Participants and Methods: Twenty-three healthy and 30 persons with MS were included in the study. Participants completed a battery of neuropsychological tests and the money management survey. The survey asked participants to provide basic responses to thirteen questions about their money management problems.

Results: significant difference between the two groups was noted on the total money management score. Overall, individuals with MS reported more problems managing money than healthy controls. Participants with MS reported more problems paying their bills on time compared to HC. Tests of prospective memory, executive functions, and self-report of functional status were significantly correlated with money management. Conclusions: To our knowledge, this is the first study to examine money management in MS. Money management is an important IADL that present problems for individuals with MS. Additional studies are needed to explore this area and understand the nature of the problem.

Correspondence: Yael Goverover, New York Univeristy, 82 Washington Sq East, 6th floor, NYC, NY 10003, United States.E-mail:yg243@ nyu.edu

J. LENGENFELDER, H.M. GENOVA, D. VASQUEZ, S. LAVRADOR \&. N. CHIARAVALLOTI. Remediation of Facial Affect Deficits in Multiple Sclerosis: A Pilot Study.

Objective: To examine preliminary efficacy for a training program to improve facial affect deficits in individuals with multiple sclerosis (MS). Participants and Methods: Participants consisted of 10 individuals with clinically definite MS, with a mean age of 52.8 (range 35-65), mean education of 15.7 (range 13-19); 70\% were female. Facial affect was assessed with a Facial Emotion Identification Test (FEIT), adapted from Ekman where individuals are presented with standardized photographs of people expressing one of six basic emotions. Individuals completed a 12 session intervention utilizing the Training of Affect Recognition (TAR) program developed by Wölwer and Frommann and previously utilized in schizophrenia. Individuals also utilized mimicry and generated of stories from their own life to demonstrate each of the six emotions.

Results: Results indicate that $100 \%$ of trained individuals demonstrated improved performance on the FEIT, as indicated by an improvement of one standard deviation or more. There was a significant difference from pre and post treatment on measures of the FEIT (Kerr and Neale, 1993; $t(9)=-7.12, p=.000)$. More importantly, all participants were considered to be clinically impaired at baseline, but performed within normal limits following the intervention. Significant differences were also seen on a 55 item version of the FEIT, $t(9)=-3.43, p=.011$, with individuals receiving higher number of correct responses following the intervention. 
Conclusions: These findings demonstrate the potential to remediate facial affect deficits in persons with MS. Additional work is necessary with larger samples to more reliably examine the efficacy of this treatment for facial affect deficits. Additional work also is also needed to examine the impact of this intervention on emotional processing more generally, including facial affect recognition, theory of mind and empathy, in addition to social integration and quality of life. Correspondence: Jean Lengenfelder, Kessler Foundation, 300 Executive Dr., Suite 70, West Orange, NJ 07052, United States. E-mail: jlengenfelder@kesslerfoundation.org

\section{K. MORDECAI, M. DUX, T. LEE-WILK, C. TYNER, M.C. ADLER, M. BEDMINSTER, L. SKALINA \& W.J.CULPEPPER. Computerized Cognitive Training for Veterans with Multiple Sclerosis.}

Objective: Despite the high prevalence of cognitive dysfunction in multiple sclerosis (MS), relatively few studies have investigated the efficacy of interventions designed to address cognitive deficits. Telemedicine initiatives are needed for this patient group given frequent motor impairment and subsequent transportation difficulties. The goal of this pilot study was to investigate a home-based, computerized cognitive training program in Veterans with relapsing-remitting MS.

Participants and Methods: Sixteen participants (63\% male, 63\% African American, age=47.8 (8.9), education=15.3 (2.5) years) trained with Lumosity in their homes for 10 weeks ( 4 sessions/week). Cognitive outcomes included the Minimal Assessment of Cognitive Function in MS (MACFIMS, Benedict et al., 2002) battery prior to training and 3 months post-training.

Results: Results revealed significant improvements after Lumosity training in composite scores for executive functioning $(\mathrm{p}=.013)$ and verbal memory $(p=.002)$, while a trend toward improving attention/ working memory was noted $(p=.065)$. Composite scores for visual memory and processing speed did not change significantly.

Conclusions: These findings provide initial evidence that home-based cognitive training programs may be an effective intervention for some cognitive deficits experienced by patients with MS. Future studies with more rigorous methodology (e.g., randomized clinical trials, longer post-training testing intervals) are needed to best assess the utility of Lumosity and other computerized training programs to improve or stabilize cognitive functioning in patients with MS.

Correspondence: Kristen Mordecai, Ph.D., Neuropsychology, VA Maryland Medical Center, VA Maryland Medical Center, 10 N. Greene St., Annex Neuropsychology, Baltimore, MD 21201, United States. E-mail: kristen.mordecai@va.gov

\section{NICCOLAI, A. GERSTENECKER, K. TRIEBEL, R. MARTIN \& D. MARSON. Medical Decision-Making Capacity and its Cognitive Predictors in Multiple Sclerosis.}

Objective: To investigate medical decision-making capacity and its neurocognitive predictors in progressive multiple sclerosis (MS).

Participants and Methods: Participants were 22 people with progressive MS and 18 healthy controls who completed neuropsychological testing and a performance-based measure of medical decision-making capacity (Capacity to Consent to Treatment Instrument; CCTI). All diagnoses were made by a board-certified neurologist with experience in MS. Group comparisons on the CCTI standards of Appreciation, Reasoning, and Understanding, and the neuropsychological measures were performed using independent $t$-tests. The association between the CCTI standards of Appreciation, Reasoning, and Understanding and neuropsychological measures was evaluated using Pearson's product moment correlations. Neuropsychological variables found to be significantly associated with the CCTI standards of Appreciation, Reasoning, and Understanding were then used to construct three regression models. For the models, a stepwise linear regression was conducted to predict performance on the CCTI standards of Appreciation, Reasoning, and Understanding.
Results: Performance on three medical decision-making consent standards (i.e., Appreciation, Reasoning, Understanding) was significantly poorer for people with progressive MS than for healthy controls. Verbal fluency was found to be the primary cognitive predictor for both Reasoning and Understanding in the progressive MS group, with short-term verbal memory emerging as the primary cognitive predictor of Appreciation.

Conclusions: Medical decision-making capacity is a complex cognitively mediated functional ability that is compromised in many people with progressive MS.

Correspondence: Lindsay Niccolai, M.A., Medical/Clinical Psychology, University of Alabama at Birmingham, 335 Mobile Avenue, Trussville, AL 35173, United States.E-mail:niccolai@uab.edu

J. NUNAN-SAAH, S. PAUlRAJ, L. POSECION, B. NOURBAKHSH, E. WAUBANT, L. JULIAN, J. GRAVES, S. IM-WANG \& R. GOMEZ. The Impact of Emotional and Psychosocial Factors on Executive Functioning in Pediatric Multiple Sclerosis.

Objective: Multiple sclerosis (MS) is associated with executive dysfunction, anxiety, depression, fatigue, and lowered quality of life in adults. Although these issues can occur in pediatric MS, the relationships among them have vet to be been investigated. This study examines the associations among some of the most salient cognitive (executive functioning), psychological (anxiety, depression), and psychosocial (fatigue, quality of life, externalizing symptoms) factors that affect children with MS.

Participants and Methods: Sixty-five MS patients ages 6 to 18 were evaluated through the UCSF Regional Pediatric Multiple Sclerosis Center. Participants completed a neuropsychological assessment battery that included the D-KEFS Verbal Fluency Test and Trail Making Test. Parents and children also completed rating forms assessing executive functioning (BRIEF), emotional functioning (BASC-2), quality of life (PedsQL), and fatigue (PedsQL Multidimensional Fatigue Scale).

Results: After controlling for significant demographic variables, higher levels of anxiety and depressive symptoms were partially predictive of executive dysfunction, both on testing and self-report. In addition, higher reported executive dysfunction was predictive of psychosocial difficulties, including lower quality of life, higher levels of fatigue, and more severe externalizing symptoms.

Conclusions: These results further our understanding of the psychological and psychosocial factors associated with cognitive outcomes in pediatric MS. An appreciation of the relationships among these variables can inform medical and psychological treatment, as well as interventions in the home and community, in order to better meet the needs of children with MS.

Correspondence: Julia Nunan-Saah, MS, PhD Program in Clinical Psychology, Palo Alto University, Palo Alto University, 1791 Arastradero Road, Palo Alto, CA 94304, United States.E-mail: jnunan-saah@ paloaltou.edu

M. PITTERI, H.M. GENOVA, J. LENGENFELDER, J. DELUCA, V. ROSSI \& M. CALABRESE. Facial Affect Recognition Deficits In Early Multiple Sclerosis Patients Without Cognitive Dysfunction. Objective: Multiple sclerosis (MS) is a chronic inflammatory disease of the central nervous system associated with accumulating multifocal tissue damage, early onset, and variable and unpredictable course. Cognitive and emotional impairments are frequently observed in MS patients, and interfere with interpersonal relationships and adversely affect family and social life. Previous studies indicate that emotion recognition is a complex cognitive process that requires the integrity of several neural circuits that subserve several cognitive domains. We examined whether facial affect recognition deficits exist in early relapsing remitting (RR) MS patients without cognitive dysfunction.

Participants and Methods: We studied a group of 31 early RRMS patients without cognitive dysfunction (Age: mean $36.26 \pm 7.6$ years $(\mathrm{y})$; Education: mean $13.4 \pm 3.4 \mathrm{y}$; Gender: $\mathrm{F}=24)$ and compared them to 39 matched healthy controls. MS patients were administered the Brief 
Repeatable Battery of Neuropsychological tests (BRB-N) and a task of facial affect recognition on which they were required to identify 6 basic emotions.

Results: Results showed that all MS patients obtained scores above the cut-off $\left(5^{\text {th }}\right.$ percentile $)$ in all the BRB-N subtests. Nonetheless, the MS patients recognized significantly fewer facial emotional expressions than controls $(p<0.001)$. Among the emotions, MS patients get worse in identifying fear $(p<0.001)$ and anger $(p=0.012)$. No correlation was found with age, education, and disease duration.

Conclusions: Our preliminary results suggest that facial affect recognition might be impaired in patients with MS in the early stages of the disease and even without cognitive dysfunction. The relationship between emotion recognition difficulties and poor social and psychological quality of life indicate that emotional processing skills should be considered when evaluating function in MS early on in the disease course, before cognitive difficulties emerge.

Correspondence: Marco Pitteri, Ph.D., Department of Neurosciences, Biomedicine and Movement Sciences, University of Verona, Piazzale L.A. Scuro 10, Verona 37134, Italy. E-mail: marco.pitteri@univr.it

\section{A. RAPHAIL \& M. SCHULTHEIS. Cognitive Correlates of Driving Using a Virtual Reality Driving Simulator in Individuals With Multiple Sclerosis.}

Objective: Individuals with multiple sclerosis (MS) have been found to have impaired driving ability, but it is unclear whether they have difficulty driving because of either physical or cognitive deficits. The aim of the present study was to examine the relationship between cognitive ability and driving on a highway course using a virtual reality driving simulator (VRDS)

Participants and Methods: Participants included 11 individuals with MS and 26 healthy individuals who were active drivers. Participants were excluded if they had an Expanded Disability Status Score (EDSS) of 5 or more to limit the impact of physical disability. Participants were asked to drive at a constant speed on an empty highway on the VRDS. Variability in speed and lane position was compared to a battery of neuropsychological tests to determine whether driving is related to cognitive ability.

Results: No significant differences were found between the MS and control groups for either variability in speed or lane position. In the MS group, greater variability in speed was related to poorer processing speed on the Symbol Digit Modalities Test (SDMT; $r=-.70, p=.02$ ) and poorer executive functioning on Trail Making Test B (TMT-B; $r=$ $.73, p=.01)$. Increased variability in lane position was associated with slower processing speed $(r=-.69, p=.02)$ and poorer executive functioning $(r=.73, p=.01)$. There were no significant relationships in the control group between variability in speed or lane position and either SDMT or TMT-B.

Conclusions: While there were no significant differences in ability to maintain constant speed and lane position between the MS and control groups, driving was significantly associated with processing speed and executive functioning in the MS group. These relationships were not found in the control group, indicating that driving may place a greater cognitive load on individuals with MS and that their cognitive resources may potentially be depleted more quickly.

Correspondence: Ann-Marie Raphail, BA, Psychology, Drexel University, 136 N 21st St., 2R, Philadelphia, PA 19103, United States. E-mail: ar3223@drexel.edu

F. SACCÀ, T. COSTABILE, A. CAROTENUTO, R. LANZiLlo, M. MOCCIA, C. PANE, C. RUSSO, A. BARBARULO, S. CASERTANO, F. ROSSI, E. SIGNORIELLO, G. LUS \& V. BRESCIA MORRA. The EDSS integration with the Brief International Cognitive Assessment for Multiple Sclerosis and Orientation Tests.

Objective: While neurological examination is sufficient to assess most functional scores (FS) of the Expanded Disability Status Scale (EDSS), no objective tool is available to determine the cerebral FS (CFS).
Landgod et al. developed the Brief International Cognitive Assessment for MS (BICAMS) that requires only 15 minutes. Aim of our study was to integrate the BICAMS in the CFS calculation, and to evaluate its impact on a large cohort of MS patients.

Participants and Methods: We used the Brief International Cognitive Assessment for MS (BICAMS) and orientation tests (OTs) to measure the Cognitive Functional System (CFS) score. To appreciate the implementation of neuropsychological tests we calculated both the EDSS before (Native-EDSS) and after the use of the BICAMS/OTs (NPS-EDSS) and compared them with a paired t-test.

We performed a multivariate logistic regression analysis to test the effect of age, age at onset, disease duration, gender, education, and nativeEDSS on the probability of having one impaired BICAMS test.

Results: We tested 604 MS patients with BICAMS, OTs, and EDSS. 384 patients $(63.6 \%)$ had at least one altered test at the BICAMS. Older age, higher education, higher native-EDSS, male gender, longer disease duration, and secondary progressive MS disease course, were associated with at least one impaired BICAMS test. Native-EDSS was different from NPS-EDSS $(-0.112 ; \mathrm{p}<0.001)$ in 99 patients $(16 \%)$. When considering patients with a Native-EDSS $\leq 4.0$, the proportion of miscalculated EDSS was 25\%.

Conclusions: Despite BICAMS is a brief assessment tool, it shows similar predictive factors of cognitive impairment as more complex batteries. Its use leads to a more accurate CFS assessment in two-thirds of MS patients, and a more accurate EDSS calculation in 25\% of patients with a score $\leq 4.0$. This may help clinicians to better recognize cognitive impairment in everyday clinical practice, especially in the case of isolated cognitive worsening.

Correspondence: Francesco Saccà, MD, Neuroscience, Reproductive and Odontostomatological Sciences, University Federico II, Via Pansini, 5, Edificio 17, piano terra, Napoli 80131,Italy. E-mail: francesco.sacca@ unina.it

\section{J. SANDRY, M.D. ZUPPICHINI, J. ROTHBERG \& J. DELUCA. Evaluating a Consolidation and Interference Hypothesis in Multiple Sclerosis.}

Objective: Difficulty learning and remembering is common in multiple sclerosis (MS). Past evidence suggests that MS participants have difficulty acquiring new information, however, the mechanism that underlies acquisition deficits (encoding vs consolidation) remains understudied. The main aims were to (1) investigate what extent memory impairment is a result of increased susceptibility to post-learning interference, (2) evaluate whether minimizing interference will improve memory acquisition and (3) to investigate what acquisition mechanism is impaired. Participants and Methods: 24 MS participants (12 memory-impaired and 12 memory-unimpaired) and 15 Healthy Controls (HC) were presented with 4 separate list learning blocks. Interference onset was manipulated and counterbalanced across the retention interval of each block such that interference occurred Early, in the Middle, Late or did not occur (no interference).

Results: Proportion retention scores were analyzed using a 3 Group X 4 Interference Onset mixed ANOVA. There was a main effect of Group $(p=.017)$ with MS memory-impaired participants performing worse (poorer consolidation) than the memory-unimpaired and HC groups. There was no main effect of Interference Onset and no interaction (p's>.68). A follow-up embedded process model analysis on immediate memory scores revealed that memory-impairment in MS may also be related to working memory inefficiency during encoding.

Conclusions: MS memory-impaired participants lost proportionally more information during the consolidation/retention interval and initially encoded less information. Dissimilar from other research, minimizing interference did not improve retention for the memoryimpaired participants or for the memory-unimpaired groups. These findings suggest that minimizing post-learning interference is not a useful memory remediation strategy for MS participants and that multiple impaired acquisition processes contribute to the observed memory deficits in this population. 
Correspondence: Joshua Sandry, PhD, Psychology Department, Montclair State University, 303 N Franklin Ave, Floor 2, Nutley, NJ 07110, United States.E-mail: joshsandry@gmail.com

L. STROBER, A. BECKER \& J. RANDOLPH. Cognitive health in multiple sclerosis (MS): Impact on fatigue, sleep, well-being, and overall quality of life.

Objective: It is well appreciated that symptoms such as fatigue, sleep disturbance, and pain hinder daily functioning in individuals with multiple sclerosis (MS). Engagement in positive health-related behaviors (e.g., exercise) and other activities that foster cognitive health may mitigate the effects of MS-related symptoms. The present investigation aimed to examine the role of activities associated with cognitive health on disease symptoms, well-being, and quality of life (QOL).

Participants and Methods: 248 individuals with MS completed a cognitive health questionnaire (CHQ) consisting of previously derived Nutrition/Exercise and Social/Intellectual Activity factors and measures assessing disease symptoms, psychological functioning, and QOL.

Results: Engagement in positive nutritional habits and exercise was inversely related to reports of fatigue, sleep problems, pain, poor medical adherence, depression, and anxiety ( $r$ 's ranging from -.16 to - -23 , p's $\leq .01$ to .001), and positively related to disease management selfefficacy $(r=.19, p=.003)$. Participation in social and intellectual activities did not have such effects on disease symptoms but was positively correlated with QOL $(r=.36, p \leq .001)$ and general self-efficacy $(r=.24$, $\mathrm{p} \leq .001)$. Social and intellectual engagement was negatively correlated with depression and anxiety ( $\mathrm{p}$ 's $=-.19$ to $-.21, \mathrm{p}<.005)$.

Conclusions: Results suggest that engagement in healthy lifestyle behaviors known to promote cognition may result in fewer symptoms of fatigue, sleep disturbance, pain, depression, and anxiety among individuals with MS. Engaging in social and intellectual activities may also result in improved overall well-being and QOL. Interventions aimed at improving individuals' awareness of activities that promote cognitive health appear warranted.

Correspondence: Lauren Strober, Ph.D., Neuropsychology \& Neuroscience, Kessler Foundation, 300 Executive Drive, Suite 70, West Orange, NJ 07052, United States. E-mail: lstrober@kesslerfoundation. org

\section{Stroke/Vascular Cognitive Impairment}

G. ARMSTRONG, M.E. CAMPBELL, L. OHLHAUSER, D. LEITNER, M. LIBBEN, J. UPSHAW, A.F. PARKER \& H. MILLER. Quick and Easy: Confirming the Utility of the PHQ-9 in a Stroke Population. Objective: The Patient Health Questionnaire (PHQ-9), a nine-item self-report questionnaire that assesses the severity of depression, has been found to be sufficiently sensitive in detecting depression in stroke patients. The Personality Assessment Inventory (PAI) is a 344-item self-report measure, which is a well-established measure of depression. The present pilot study aims to investigate the convergent validity of the PHQ-9 with the depression subscale of the PAI in a stroke population. Participants and Methods: The participants in this study consisted of stroke patients receiving treatment on the Rehabilitation Unit at the Kelowna General Hospital $(N=18)$. Participants completed the PHQ-9 and PAI on their own time during their stay as an inpatient.

Results: The PHQ-9 was significantly correlated with the depression subscale of the PAI $(r=.78, p<.001)$. This study also aims to investigate the contribution of functional impairments resulting from stroke in the overestimation of depression severity in self-report ratings on the PHQ-9. A high correlation was also noted between the anxiety subscale of the PAI and the PHQ-9 $(r=.73)$ and thus this study may also address relationships between this subscale of the PAI and PHQ-9.

Conclusions: The significant correlation of the PHQ-9 and the depression subscale of the PAI indicate that they measure a similar construct. Results suggest the PHQ-9 produce a similar outcome as the more intense PAI assessment. Ease of administration, time efficiency and decreased strain on the patient could lend to the value of the PHQ-9. Correspondence: Graham Armstrong, Undergraduate, Pyschology, University of British Columbia - Okanagan, 3930 Patten Drive, Armstrong, BCVOE 1B2, Canada.E-mail: g_armstrong@live.com

\section{S. CHAPMAN, G. COCCHINI, L.E. COLVIN, M.S.V. ELKIND \& S. COSENTINO. Reality monitoring in unawareness of memory deficits.}

Objective: Patients who suffer memory deficits following stroke differ in their awareness of their difficulties. The severity of the mnemonic deficit has been hypothesized to contribute to unawareness; however, there is an inconsistent association between these constructs, raising the question of whether compromise to specific mnemonic mechanisms leads to the deterioration of awareness. In this pilot study, we examine the extent to which reality monitoring, a mnemonic mechanism by which individuals discriminate between information arising from internal versus external sources, is associated with awareness of memory deficits. Participants and Methods: 16 individuals suffering from memory impairment following stroke underwent assessment of general cognitive functioning, episodic memory, and awareness of memory difficulties. Reality monitoring was assessed with an experimental paradigm in which participants had to discriminate between information that was either seen or imagined. Dependent variables included stimuli recognition, source identification, and response bias. Responses were analyzed within the Two-High-Threshold Theory.

Results: 11 participants were aware of their memory difficulties and 5 were unaware. Mann-Whitney tests showed that the unaware group performed more poorly on recognition of stimuli $(U=3, p=.003, r=$ $.69)$ and identifying the source of stimuli $(U=7, p=.019, r=.58)$. Source identification differences persisted when adjusting for memory differences $(F(1,13)=5.83, p=.031)$.

Conclusions: Findings suggest that difficulty monitoring the source of a memory may contribute to unawareness of memory deficits following stroke, and raise potential avenues for rehabilitation of memory awareness.

Correspondence: Silvia Chapman, PhD, Psychology, University of London, 7 REGENCY PLACE, Weehawken, NJ 07086, United States. E-mail:ps201sc@gold.ac.uk

S.I. DEV, T.T. NGUYEN, A.N. SUTHERLAND-OWENS \& L.T. EYLER. Intra-individual variability in processing speed is related to systolic blood pressure in bipolar disorder.

Objective: Bipolar Disorder (BD) is associated with worse processing speed (PS) and greater vascular risk. Increased intra-individual variability (IIV) in cognitive performance is related to future cognitive decline, and BD has been shown to be associated with increased IIV in cognition compared to psychiatrically-healthy comparators (HC). However, less is known about the potential relationship between vascular health and IIV in this population. The objective of this preliminary analysis was to examine group differences in IIV of PS and the relationship between blood pressure (BP), a marker of vascular risk, and IIV.

Participants and Methods: A small sample of $14 \mathrm{BD}$ and $34 \mathrm{HC}$ participants were assessed for $\mathrm{BP}$ at a baseline visit and were then administered the Brief Assessment of Cognition in Schizophrenia (BACS) symbol-coding subtest on three occasions over 2 weeks. IIV of the BACS symbol-coding score was calculated for each participant after accounting for differences in group means and linear trends. Independent samples t-test investigated group differences in IIV and Pearson's correlations determined the relationship between BP and IIV within each group.

Results: BD participants exhibited slower PS ( $t=-7.11 ; p<0.001)$, but no group differences were found in IIV of PS or BP. Within the BD group, higher systolic BP was related to greater PS IIV ( $r=0.82$; $p=0.001$ ), but this association was not observed in the HC group. 
Conclusions: The results of this preliminary investigation suggest that $\mathrm{BD}$ patients with higher BP, even within the clinically normal range, also have more week-to-week variability in their PS. Thus, cognitive variability may be particularly sensitive to sub-clinical individual differences in BP in this population, and these together may possibly portend future cognitive decline. Larger studies including long-term follow-up are required to better elucidate the contributions of vascular health to cognitive IIV, and cognitive decline with age, in BD.

Correspondence: Sheena I. Dev, M.S., Clinical Psychology, SDSU/UCSD Joint Doctoral Program, 3350 La Jolla Village Drive, Building 13, MC 151B, San Diego, CA 92161, United States.E-mail: sdev25@gmail.com

M.G. DILORENZO, T. WILLIAMS, K. MCDONALD \& R. WESTMACOTT. Preschool Executive Functioning Abilities Predict Later Academic Achievement in Children with Arterial Ischemic Stroke.

Objective: Executive functions (EF) are vulnerable to the effects of early stroke due to their protracted development. The current study investigated: (1) the link between preschool EF and school-age EF in children with a history of arterial ischemic stroke (AIS), and (2) the predictive role of preschool EF in later academic achievement.

Participants and Methods: Twenty-six children with unilateral AIS were retrospectively identified for this study based on their completion of neuropsychological assessments at preschool ( 4 years to 5 years 11 months) and at school-age (6 years 1 month to 14 years). Reports of executive function were obtained from parents at both time points using the Behaviour Rating Index of Executive Function. Academic achievement was measured at school-age using the Weschler Individual Achievement Test-III or the Woodcock-Johnson Tests of Achievement-III.

Results: Preschool EF skills were consistently unrelated to counterpart EF skills at school-age. Preschool EF skills (inhibit, shift and working memory) accounted for a significant proportion of variance in school-age spelling and reading skills, after accounting for preschool FSIQ and lesion size. The preschool EF factors did not collectively explain a significant proportion of variance in school-age math skills when controlling for preschool FSIQ and lesion size. However, inhibitory control and working memory skills at preschool emerged as significant unique predictors of school-age math outcomes.

Conclusions: Preschool EF skills emerged as reliable predictors of later academic performance in children with a history of AIS. The current research helps clinicians predict long-term cognitive and academic outcomes in pediatric stroke patients.

Correspondence: Miranda G. DiLorenzo, Psychology, Sick Kids Hospital, 27 Columbus Avenue, Woodbridge, ON L4L 6V9, Canada. E-mail:mgdilo@yorku.ca

J. CRUMEDY, M. OYETEJU, S. JEEVAN, J. LAI, A. CHAN, P. DESAI, M. FRANDO, T. KALDIS, L. NGUYEN, L. TASTARD, G. BRITZ \& M. DULAY. Predictors of Cognitive Impairment After Cerebrovascular Accident (CVA).

Objective: Stroke is a major cause of difficulties with memory, language, visual-spatial abilities and executive functioning. In individuals without a history of stroke, research has shown that other factors can contribute to cognitive problems including fatigue, pain, sleep difficulties, depression, and reduced attention focus. The purpose of this study was to examine the relative contribution of various factors in explaining cognitive test performance in individuals who sustained a stroke.

Participants and Methods: One-hundred and twelve patients $(53 \%$ women, average age of 61 vears) underwent neuropsychological assessment approximately 6 months after stroke. Tests included age-corrected measures of verbal memory, visual memory, naming, working memory, mental flexibility, and phonemic fluency. Sleep difficulties, history of depression, fatigue, gender, location of stroke, and side of stroke were used as predictors of cognitive test performance.

Results: Stepwise regression analyses indicated significant predictors of cognitive test performance. For example, location of stroke $(24 \%)$ and sleep difficulties (14\%) predicted $38 \%$ of the variance in phonemic fluency test performance $(p<0.0001)$. Further review of the data indicated that patients with frontal and cerebellar strokes were the patients with prominent phonemic fluency difficulties compared to other stroke locations. Location of stroke, sleep difficulties, and history of depression predicted $43 \%$ of the variance in story memory test performance.

Conclusions: Results replicate the finding that location of stroke predicts cognitive performance, and extends previous research by demonstrating that sleep difficulties and history of depression uniquely contribute to cognitive difficulties after CVA. Results highlight the importance of interpreting cognitive test performance in light of poor sleep and mood state.

Correspondence: Mario Dulay, Houston methodist Hospital, 3414 Legends Garden Drive, Spring, TX 77386, United States. E-mail: mdulay@houstonmethodist.org

A. FEDOR, A. KOZLOWSKI, M. LUDWIG \& F. HYLAND. Is stepcount in exoskeleton-assisted locomotor training associated with cognitive functioning after stroke?

Objective: Approximately 800,000 cerebrovascular accidents (i.e. stroke) occur each year in the United States. Cognitive dysfunction is a common impairment following stroke. Exercise has been associated with improved cognitive functioning in other neurological conditions. Clinicians are increasingly using assistive technology (i.e. robotic walking devices) to help individuals resume function after an injury. The current study sought to examine the association between the number of steps taken with a robotic-assistive walking device and recovery of cognitive functioning following stroke.

Participants and Methods: A sample of 56 participants was collected as part of a larger project using secondary analysis of existing data. Participants had an average age of $68.5( \pm 12.8)$, and were $39 \%$ female. Cognitive functioning was determined using scores on the FIM instrument at admission and discharge. Total step count while using the powered exoskeleton was recorded by clinicians. Linear regression analyses were conducted in block format with discharge cognitive functioning serving as the dependent variable.

Results: Results indicated the initial model (i.e. age, sex, length of stay, admission Cognitive FIM score) for predicting discharge cognitive functioning was significant $(F(5,50)=6.87, p<0.001)$. However, addition of the total step count did not provide significant predictive ability over the initial model $\left(\Delta \mathrm{R}^{2}=0.013, p=0.31\right)$.

Conclusions: Despite previous literature demonstrating positive associations between exercise and cognitive functioning, this relationship may not be applicable to the stroke population. Several limitations such as small sample size, basic measurement of step count, and an inherent relationship between length of stay and step count (i.e. those with longer length of stays have more opportunity to accumulate steps) may also be attenuating the current results. Perhaps the most important limitation is the ability of the Cognitive FIM to accurately measure cognitive functioning at admission and discharge.

Correspondence: Andrew Fedor, Kent Hall, Kent, OH 44242, United States.E-mail:AFedor1@kent.edu

T. FERLAND, N. SCHWARZ, L. NORDSTROM, L. PAGEN, W.P. MILBERG, R. MCGLINCHEY \& E. LERITZ. Association of Metabolic Syndrome with Cognitive Function in Adults.

Objective: Metabolic Syndrome (MetS), characterized by three or more co-occurring risk factors for cerebrovascular disease, represents a significant health concern, affecting nearly $45 \%$ of US adults over the age of 50. The impact of individual risk factors, such as high blood pressure (BP), high cholesterol and obesity on cognitive function has been fairly well established. However, less is known about their cumulative effect on cognition. The purpose of this study was to examine how patterns of risk factors impact neuropsychological function.

Participants and Methods: Sixty adults (mean age 57.50) underwent a comprehensive battery of neuropsychological tests, as well as 
measurement of BP, cholesterol, weight, and fasting glucose. Eighteen participants were diagnosed with MetS according to NCEP-III guidelines. A factor analysis of raw neuropsychological scores revealed three factors comprising memory, executive, and attention domains. Groups were then compared on resulting factor scores, and physiological measures were related to cognitive domains.

Results: The MetS group exhibited significantly worse performance on the executive function factor score. Within the whole sample, higher blood pressure, cholesterol, and glucose, were significantly associated with worse performance on measures of executive function; this appeared to be driven primarily by the MetS group.

Conclusions: These results demonstrate that MetS is associated with significant alterations to cognitive function and that the clustering of blood pressure, cholesterol, and glucose may have a specific impact on executive function.

Correspondence: Tori Ferland, VA Boston Healthcare System, $150 \mathrm{~S}$ Huntington Street, Boston, NH 02130, United States. E-mail: tori. ferland@gmail.com

M.W.M. FONG, J. SIEGEL, L. RAMSEY, G. SHULMAN \& M. CORBETTA. Neuroanatomic and Neuropsychological Correlates of Post Stroke Functional Status.

Objective: Stroke outcome measures assess different dimensions of functional status. This study aimed to explore the underlying dimensions of a range of outcome measures in patients with first clinical stroke and examine neuroanatomic and neuropsychological predictors of outcome. Participants and Methods: 132 first time stroke subjects completed a neuropsychological evaluation comprising of 5 domains (i.e., language, verbal memory, visual memory, attention, and motor) and neuroimaging within 2 weeks post stroke. 103 returned for a follow-up assessment at 3 months when they also completed a battery of outcome measures. A principal component analysis was used to examine the dimensions underlying functional outcome. Separate linear regression models were used to predict functional status at 3 months from neuropsychological scores and lesion characteristics.

Results: The principal component analysis revealed a two-factor structure among the functional measures, accounting for $79.7 \%$ of the variance. Factor 1 loaded on physical and cognitive tasks. Factor 2 loaded on emotional/well-being questions. Motor impairment was the only neuropsychological domain that significantly predicted Factor 1 . After controlling for demographics and lesion size, it explains $40.9 \%$ of the variance. None of the neuropsychological domains were significantly associated with Factor 2. Damage to parietal deep white matter and subcortical regions (thalamus, basal ganglia) were significantly associated with poorer physical/cognitive function at 3 months post-stroke, explaining $10.9 \%$ of the variance. Frontal and temporal cortical damage was associated with better outcome.

Conclusions: Two dimensions were found across a wide range of stroke outcome measures. Motor function and lesion characteristics at 2 weeks post-stroke predict physical/cognitive function at 3 months post stroke. Correspondence: Mandy W.M. Fong, Neurology, Washington University in St. Louis, 4444 Forest Park Avenue, Campus Box 8518, St. Louis, MO 63108, United States. E-mail:wfong@wustl.edu

I. K THIRUSELVAM, J. ROBBINS, L. MILLER \& M. HAUT. Anterograde Amnesia for Explicit and Implicit Information in a Case of Bilateral Hippocampal Stroke.

Objective: Case report of a neuropsychological evaluation of a female with infarcts involving the bilateral hippocampi and occipital lobes, the right parietal lobe, and thalamus, in addition to a remote left middle cerebral artery stroke affecting the left frontal lobe.

Participants and Methods: A 69-year-old, right-handed, Caucasian female with 18 years of education and bilateral hippocampal infarcts was administered standard clinical measures of verbal and visual memory, language, executive functions, intelligence, and experimental measures of implicit memory.
Results: This patient demonstrated severe anterograde amnesia for explicit information, both on standard verbal and visual memory measures, and observation (e.g., no recall of examiner after leaving the room briefly). She also demonstrated impaired implicit memory on a verbal priming task and a procedural motor learning task. Specifically, she showed learning over repeated trials, but could not retain the motor learning over a delay. She demonstrated retrograde amnesia for events in the past two years, impaired working memory, significant word-finding difficulties, and deficits in cognitive flexibility, inhibition, and abstraction. General intelligence, basic attention, visual problem-solving, and word-reading remained intact. The patient had limited insight into the degree of her memory impairment, and showed confabulation.

Conclusions: While it was expected that this patient would have amnesia for explicit material given her bilateral hippocampal stroke, her impaired implicit memory was an unexpected finding. In retrospect, her amnesia for implicit material was likely the result of prior lesions involving the association cortex and thalamus. These findings suggest lesions in the association cortex and/or the thalamus, in addition to the hippocampus, may produce amnesia for both explicit and implicit information.

Correspondence: Indrani K Thiruselvam, MA, Psychology, Marquette University, 751 W. Bode Circle, Unit 209, Hoffman Estates, IL 60169, United States.E-mail: tindrani@gmail.com

D. LEITNER, H. MILLER \& M. LIBBEN. Neuropsychological Evaluation Following Subsequent Bilateral Thalamic Infarct - A Case Study.

Objective: The occurrence of bilateral thalamic infarct (BTI) is rare, and prognosis is typically poor, often resulting in death or severe impairments. Here, we present a case of a middle-aged woman with subsequent BTIs. We compare and contrast the results of two neuropsychological evaluations over a one year period, and compare these findings to other reports of similar etiology.

Participants and Methods: The patient was a 57 year-old, righthanded, married, Caucasian female who suffered a right thalamic hemorrhage subsequent to lacunar infarcts in the left thalamus. The neuropsychological evaluation included review of her medical records, interview (and follow-up) with the patient and her husband, comprehensive neuropsychological testing, as well as assessment of functional status using the Mayo-Portland Adaptability Inventory - Fourth Edition (MPAI-4).

Results: The significant impairments noted across multiple cognitive domains were nearly identical to the initial evaluation one year prior. Emotional and social dysfunction appeared to be secondary to cognitive impairments. Scores on the MPAI- 4 suggested deficits ranging from moderate to severe for functional independence in the home and community. Discrepancies between self- and significant other-ratings were noted on the Participation subscale of the MPAI-4.

Conclusions: Cognitive deficits due to subsequent BTIs are complex and appear stable over time. Higher levels of function (e.g., IADLs) appear to be profoundly affected. While strong social supports help circumvent deficits, impaired self-awareness and emotional sequelae may increase risk of caregiver burden. Sequelae of subsequent BTI show some similarity to those found in simultaneous BTI (e.g., severe memory impairment) that occur in the P1 segment of the posterior cerebral artery. This case expands previous findings by showcasing the longitudinal effects of subsequent BTIs on cognitive, emotional, and social functions.

Correspondence: Damian Leitner, BSc. (Hons) Psychology, Kelowna General Hospital, 2268 Pandosy Street, Kelowna, BC V1Y 1T2, Canada.E-mail:Canadian_02@hotmail.com 
M.J. MOORE, N. SHALEV, J. LEVENSTEIN, C. GILLEBERT \& N. DEMEYERE. Dissociations Between Visual Neglect and Neglect Dyslexia.

Objective: Visuo-spatial neglect is a common neuropsychological syndrome characterized by lateralized attentional deficits. Neuropsychological evidence suggest that neglect can selectively impair perception in multiple reference frames, but any presumptive relationship between visuospatial neglect and Neglect Dyslexia (ND) is still unclear. The purpose of this study is to determine whether dissociations exist between visuo-spatial neglect and ND.

Participants and Methods: We investigated ND using a sentence reading task and spatial and object neglect using the Hearts Cancellation and Apple Cancellation tasks (OCS and BCoS respectively). Both tests were administered to 535 subacute stroke patients.

Results: ND occurred in $5.6 \%$ of patients and was characterized by consistently lateralized errors when reading words. We identified 30 patients with clear cases of ND. Of these patients, 22 exhibited right lateralized ND and 8 left lateralized ND. 50\% of ND patients exhibited neglect towards the contralesional side of the perceived space ('Egocentric' neglect) and $37.5 \%$ neglected the contralesional part of a perceived object ('Allocentric' neglect). 45.8\% ND patients exhibited no visuo-spatial neglect. ND was found to be dissociated from both allocentric $(\mathrm{ND}=15)$ and egocentric neglect $(\mathrm{ND}=12) .9 \mathrm{ND}$ patients were found to perform normally when asked to read pronounceable pseudowords, suggesting that ability to read words with lexical content may be selectively damaged in some cases of ND.

Conclusions: Cumulatively, these findings suggest that ND is caused by a reading-specific attentional/perceptual cognitive deficit which is dissociated from both allocentric and egocentric neglect.

Correspondence: Margaret J. Moore, Neuroscience, B.S., Psychology, B.S. (in progress), Experimental Psychology, University of Oxford, 14815 Van Pelt Drive, Goshen, IN 46526, United States. E-mail: mooremar@imail.iu.edu

\section{Invited Symposium 3. Advances in Pediatric Mild TBI: Toward a Neurobiopsychosocial Model}

\section{Chair: Keith O. Yeates}

\section{Discussant: H. Gerry Taylor}

\section{9:00-10:30 a.m.}

K.O. YEATES, A. PTITO, V.A. ANDERSON, M. KIRKWOOD \& H. TAYLOR. Advances in Pediatric Mild TBI: Toward a Neurobiopsychosocial Model.

Symposium Summary: Pediatric mild traumatic brain injury (mTBI), including concussion, is a significant public health problem, with the number of children seeking medical care rising dramatically. This symposium will summarize research on pediatric mTBI, including concussion, with the aim of highlighting the need for a multi-level, multi-dimensional approach to understanding and managing children with these injuries. The symposium will begin with a brief introduction by Keith Yeates, to provide a historical context, and then be followed by four speakers presenting on various aspects of assessment and management of mTBI and a discussant. Alain Ptito will discuss neuroimaging as a diagnostic and prognostic tool. Keith Yeates will discuss neuropsychological testing as a predictor and outcome of pediatric mild TBI. Vicki Anderson will address psychosocial predictors of and influences on outcomes. Michael Kirkwood will describe neuropsychological assessment as an intervention model in pediatric concussion. Gerry Taylor will act as discussant, integrating the themes that emerge from the individual presentations and highlighting future directions for research on mTBI. The symposium will conclude with an audience question-and-answer period. The goal of the symposium is to provide insights into recent advances in this important and developing area of research and to stimulate further scientific progress by promoting a neurobiopsychosocial model of pediatric mTBI.

Correspondence: Keith O. Yeates, PhD, University of Calgary, 2500 UniversityDrNW, Calgary, AB T2N1N4, Canada.E-mail: KYeates@ ucalgary.ca

\section{A. PTITO. Neuroimaging as a Diagnostic and Prognostic Tool in Pediatric Concussion.}

Functional magnetic resonance imaging (fMRI) studies offer new information about mild traumatic brain injury or concussion that cannot be obtained solely from conventional morphological imaging approaches. The goals of the talk will be to present the clinical picture of concussion and mild head injury in adults and children (aged 10-17 years), with an emphasis on sports. fMRI data obtained from athletes and children with persistent post concussive symptoms will be described and compared. Serial functional neuroimaging studies suggestive of recovery will also be introduced. We used fMRI to investigate the neural mechanisms underlying working memory (frontal lobe function) and topographical orientation (hippocampal function) in a group of symptomatic concussed athletes and children. We have shown that the fMRI tasks that were developed in adults could be validly used in children and that the working memory task that was previously proven to be useful in adults showed a similar attenuation of fMRI signals in concussed children. However, unlike the concussed adults, the children had a diminished ability to recruit other brain regions and performed significantly worse than controls on the working memory task, a finding not seen in adults. We also used a navigational memory task sensitive to functioning of the medial temporal region, including the hippocampus and parahippocampal gyrus. An attenuation of fMRI signal was seen in the concussed subjects, with additional peaks in the hippocampus, suggesting that these subjects remained in a "learning phase" of the task. Taken together, these studies demonstrate that, not only can these tasks be applied in children; they also show potential as an assessment tool in the context of pediatric concussion.

Correspondence: Alain Ptito, PhD, Department of Psychology, McGill University Health Center, 3801 Université, Montréal, QC H3A 2B4, Canada.E-mail: alain.ptito@mcgill.ca

K.O. YEATES, E.D. BIGLER, A. BACEVICE, B. BANGERT, D. COHEN, L. MHHALOV, N. ZUMBERGE \& H. TAYLOR. Neuropsychological testing as an outcome and predictor in pediatric mild traumatic brain injury.

The role of neuropsychological testing in the management of pediatric mild traumatic brain injury (mTBI) remains controversial. Neuropsychological functioning can be viewed as an outcome in its own right, but also as a predictor of other outcomes, such as postconcussive symptoms (PCS). We recently examined neuropsychological test scores as both outcomes and predictors using data drawn from an ongoing prospective cohort study involving children 8-16 years of age with $\mathrm{mTBI}$ $(n=107)$ or orthopedic injuries $(\mathrm{OI}, \mathrm{n}=66)$, recruited from emergency departments at two large children's hospitals in the Midwestern United States. They completed the NIH Toolbox cognitive battery at 10 days and 3 months post-injury. They and their parents also rated PCS at both occasions. Children with mTBI displayed significant deficits in fluid cognitive skills at 10 days compared to the OI group, and more limited deficits at 3 months post injury. The groups did not differ on crystallized cognitive skills. The groups also differed in PCS at 10 days, but not at 3 months. Post-acute fluid cognitive skills were a significant predictor of post-acute PCS, even when controlling for pre-injury symptoms. They were not as consistently a significant predictor of PCS at 3 months. These preliminary findings suggest that neuropsychological testing can differentiate children with mTBI from those with OI and also predict concurrent PCS during the post-acute period, but is less likely to show group differences or predict other outcomes at longer intervals postinjury. Neuropsychological testing may play a role in assessing recovery from pediatric mTBI during the post-acute period. 
Correspondence: Keith O. Yeates, PhD, University of Calgary, 2500 University Dr NW, Calgary, AB T2N1N4, Canada. E-mail: KYeates@ ucalgary.ca

V.A. ANDERSON, M. TAKAGI, S. BRESSAN, G. DAVIS, C. CLARKE, N. ANDERSON, S. HEARPS, K. DUNNE \& F. BABI. Psychosocial Predictors of and Influences on Outcomes of Pediatric Concussion. Objective: Over recent years, in the context of wide media focus, the field of child concussion has become highly controversial, resulting in public concern for the safety of children participating in contact sports. Evidence documenting the consequences of these injuries in children and factors contributing to poor outcomes is beginning to emerge. This presentation aims to consider the role of psychological factors for delayed recovery, and potential for intervention.

Participants and Methods: We conducted a prospective, longitudinal study following children and adolescents $(n=138)$ with concussive injuries, recruited from acute presentation to Emergency medical services (T0) and followed-up at 1-4 days (T1), 2 weeks (T2), 1 month (T3) and 3 months (T4) post-injury. Our primary outcome was persistent post concussive symptoms (Post Concussive Symptom Inventory) at 2 weeks post-concussion, when symptom resolution is generally expected, and this was used to divide the sample into symptomatic $(\mathrm{n}=75)$ and asymptomatic $(n=59)$. These two groups were compared on the following variables: injury-related, cognition, child mental health, quality of life, and parent mental health.

Results: The two groups did not differ with respect to physical or cognitive symptoms, but the symptomatic group reported significantly more pre- and post-concussion internalizing problem behaviors ( $\mathrm{p}=.001)$. At 1 month follow-up the symptomatic group also demonstrated poorer quality of life $(p<.05)$ and poorer parent mental health $(p<.05)$.

Conclusions: These findings highlight the importance of considering child and family mental health pre- and post-concussion when formulating causes of delayed recovery. This has implications for including psychological treatment for persisting post-concussive symptoms in children and adolescents.

Correspondence: Vicki A. Anderson, PhD, Child Neuropsychology, Level 4 East, Murdoch Childrens Research Institute, Flemington Road, Parkville,VIC 3052, Australia.E-mail: vicki.anderson@rch.org:au

\section{KIRKWOOD. Neuropsychological Assessment as an} Intervention Model in Pediatric Concussion.

Although most youth recover well after mild TBI, a sizable minority display postconcussive symptoms that persist for months or longer. Yet a paucity of research has focused on how to treat persistent problems. Neuropsychological assessment has become widely recognized as important in the clinical management of TBI. Such assessment has the potential to improve outcomes after mild TBI in two primary ways. First, clinical neuropsychologists are well positioned to provide sound education after mild TBI, correcting common misconceptions held by patients, caregivers, and professionals. Second, given expertise in, and instrumentation to thoroughly evaluate, brain injury, psychosocial functioning, and typical and atypical development, neuropsychologists are also well positioned to differentiate among the many neurological, socioemotional, and developmental factors that have been found to influence symptom reporting following pediatric mild TBI. Disentangling the multiple factors that drive reported symptoms is key to finding the most effective intervention approaches. We recently completed a project using a prospective interrupted time series design that demonstrated the potential value of a one-time neuropsychological consultation in reducing postconcussive symptoms that had persisted for months in 8-17 year olds after mild TBI. The consultation was also associated with high rates of parental satisfaction. As the study was quasi-experimental in nature, future work will require methodologically more rigorous designs to rule out other explanations (e.g., spontaneous change) for the positive outcomes.
Correspondence: Michael Kirkwood, Children's Hospital Colorado, 13123 E. 16th Ave, Aurora, CO 80045, United States. E-mail: michael. kirkwood@childrenscolorado.org

\section{H. TAYLOR. Discussion of Issues and Future Directions.}

Despite the high incidence of mild traumatic brain injury (mTBI) in children, we have much to learn about the natural course of post-injury consequences, factors related to variability in outcome, and mechanisms of effect. Three of the presentations focus on ways to identify children at heightened risk for persisting post-concussion symptoms (PCS). The findings suggest that persisting symptoms are related to pre- and post-injury internalizing problems, parent mental health, and post-injury quality of life (Anderson), as well as the acute effects of concussion as assessed in the ED and post-acute measures of cognitive ability (Yeates). The findings also demonstrate that persisting PCS are associated with altered patterns of neural activation during tests of memory and executive function (Ptito), suggesting a potential neural basis for these problems. These findings highlight the multiple neural and psychosocial factors that predispose youth to persisting PCS. Another presentation (Kirkwood) suggests that children and families may benefit from early neuropsychological assessments that examine these multiple risk factors and manage outcomes for individual youth in accordance with this more complex array of considerations. The discussion will highlight themes raised by the presentations, review methodological challenges of research in this area, and outline needs for further studies of the factors responsible for variability in outcomes and ways to improve assessment and management of pediatric mTBI.

Correspondence: H. Gerry Taylor, Department of Pediatrics, Case Western Reserve University and Rainbow Babies \& Children's Hospital, University Hospitals Cleveland Medical Center, W.O. Walker Building Suite 3150, 10524 Euclid Avenue, Cleveland, OH 44106, United States. E-mail:hot2@case.edu

\section{Symposium 5. Interdisciplinary Approaches to Understanding Post-Operative Cognitive Complications in Older Adults}

\author{
Chair: Catherine C. Price \\ Discussant: Steve DeKosky \\ 9:00-10:30 a.m.
}

C.C. PRICE, J. BROWNDYKE, T. GIOVANNETTI, T.F. FLOYD \& S. DEKOSKY. Interdisciplinary Approaches for Understanding Post Operative Cognitive Complications in Older Adults.

Since mid- $20^{\text {th }}$ century there has been increasing concern for older adults' risk of cognitive change, delirium, dementia and mortality after major surgery. Yet there are still no accepted mechanisms for why some older adults experience post-operative cognitive complications. The topic is also of increasing concern given rising numbers of older adults electing major surgeries. This symposium will highlight recent NIH funded interdisciplinary clinical and animal based research addressing the complex and controversial topic of post-operative cognitive changes and considerations for dementia thresholds. Tania Giovannetti, Neuropsychology/ Temple University, will summarize her team's findings that pre-surgical cognitive function in very ill older adults having aortic valve replacement may be a more significant risk factor for outcome than the surgery itself. Dr. Jeffrey Browndyke, Neuropsychology/ Duke University, will present his team's NIH funded neuroimaging investigations in cardiac surgery patients showing pre-post functional connectivity changes in cognitively "well" older adults and the relationship of these imaging changes to short- and long-term global cognitive outcomes. Dr. Catherine Price, Neuropsychology/ University of Florida, will incorporate data from $\mathrm{NIH}$ investigations and argue that it is time to embrace 
pre-operative cognitive and neuroimaging assessments for the purpose of risk assessment, mechanism exploration, and intervention design. Dr. Thomas Floyd, Anesthesiology/Stony Brook University, will summarize one probable perioperative mechanisms of insult via his team's animal based research studying anesthesia, hypoxia, and associated hippocampal and cognitive insults. Dr. Steve Dekosky, Neurology/ University of Florida, well known for his seminal work on Alzheimer's disease, will integrate key findings of the four speakers and comment upon the importance of interdisciplinary research for perioperative wellness and associated dementia prevention.

Correspondence: Catherine C. Price, Ph.D., Clinical and Health Psychology, University of Florida, PO Box 100165, Gainesville, FL 32610,United States.E-mail: cep23@PHHP.UFL.EDU

T. GIOVANNETTI, C.C. PRICE, M. FANNING, S. MESSE, S.J. RATCLIFFE, A. LYON, S. KASNER, G. SEIDEL, J.E. BAVARIA, W. SZETO, W. HARGROVE, M. ACKER \& T.F. FLOYD. Baseline Cognitive Function is Significantly Associated with Postoperative Death and Stroke in Aged Adults Following Aortic Valve Replacement.

Aortic valve replacement (AVR) for aortic stenosis (AS) improves cardiac function and increases longevity but rare negative outcomes can be devastating. This study evaluated baseline demographic and cognitive predictors of perioperative stroke, study drop out and death over 1 year in elderly AVR patients. Participants undergoing AVR (n $=185 ; \mathrm{M}_{\text {age }}=76 \pm 6$ ) for calcific AS and 198 non-surgical, comparison participants with vascular disease $\left(\mathrm{M}_{\mathrm{age}}=74 \pm 6\right)$ were evaluated at baseline, 4- 6 weeks, and 1 year post-surgery on the MMSE and measures of episodic memory, language, visuoconstruction, working memory/ inhibition, and attention. Surgery vs. non-surgery group differences in drop out and mortality were evaluated. Surgery participants were subgrouped by perioperative stroke status (i.e., clinical stroke, silent infarct, no stroke). Among the surgical group, baseline demographic and cognitive differences were examined between participants who survived versus those who died and between those who suffered a peri-operative stroke versus those who did not. Compared to the non-surgery group, surgery participants were significantly more likely to drop or die (both $p<.01)$. Surgery participants who died had significantly fewer years of education, more medical comorbidities, reported more symptoms of depression, and lower cognitive scores on the MMSE and all cognitive composites than surgical participants who completed the study (all $\mathrm{p}<$ $.05)$. Only medical comorbidities $(\mathrm{p}<.02)$ and tests of working memory $(p<.02)$ and language $(p<.02)$ were significant predictors of mortality in a multinomial logistic regression model. Surgical participants with perioperative clinical stroke had lower baseline MMSE and cognitive scores, though only the difference on the language tests was statistically significant $(p<.05)$. These results indicate that poor pre-surgical cognition should be considered a risk marker for mortality, clinical stroke, and need for intensive post-surgical follow-up care.

Correspondence: Tania Giovannetti, PhD, Psychology, Temple University, Weiss Hall, Psych Dept, 1701 N 13th St, Philadelphia, PA 19121, United States. E-mail:tgio@temple.edu

J. BROWNDYKE. Postoperative Resting-state and Task-based Functional Connectivity Changes and Cognition Following Cardiac Surgery.

Postoperative cognitive dysfunction (POCD) occurs frequently in elderly patients undergoing cardiac and non-cardiac surgery. POCD is typically characterized by reductions in mental flexibility, information processing and memory. Estimates vary, but on average, POCD is present in $15-25 \%$ of elderly patients at 4-6 weeks after surgery and importantly, can persist for years and increase the risk for later dementia. It is being recognized that certain pre-operative factors may predispose individuals to POCD, but what is still less understood are the functional neurological underpinnings of transient and/or chronic POCD. This presentation will present results from a NIH-funded multidisciplinary study of resting-state and task-based functional connectivity changes in elderly patients undergoing aortic valve replacement and/or cardiac bypass $(\mathrm{n}=27)$ and non-surgical control participants with a history of cardiac disease $(n=28)$. Postoperative resting-state functional connectivity alterations at 6 -weeks relative to presurgical baseline were found to be positively associated with postoperative global cognitive outcomes in patients, but not controls. These postoperative resting-state functional connectivity changes were observed in areas associated with the default mode network. Additional alterations in task-based functional connectivity were detected at 6-weeks in these cardiac surgery patients, which were also associated with postoperative global cognitive outcomes. The surgery-related task-based functional connectivity alterations occurred in posterior midline and subcortical regions and suggest possible maladaptive compensatory action in brain networks governing cognitive control and memory.

Correspondence: Jeffery Browndyke.E-mail: j.browndyke@duke.edu

\section{C.C. PRICE. The Challenge and Promise of Pre-Surgical Cognitive Profiles.}

Despite numerous attempts to identify anesthetic or surgical mechanisms for cognitive decline after surgery, there are no definitive conclusions to date. Pre-operative patient characteristics such as age and education are, however, repeatedly reported as important predictors for postoperative cognitive impairment and complications. Here, the speaker will argue that it is time for interdisciplinary teams to explore mechanisms of perioperative cognitive decline based on the hypothesis that baseline cognitive vulnerabilities interact with specific anesthetic and surgical approaches. The speaker will present data from two data sources. First, data from the University of Florida pre-surgical center show that 1) a substantial number $(\sim 33 \%$; based on sample of $>700$ older adults visiting the pre-surgical center) of older adult patients having major surgery are cognitively compromised relative to non-surgery matched peers and that 2) certain surgical groups are more cognitively impaired than others. Second, data from an interdisciplinary prospective NIH investigation studying neuroimaging predictors of cognitive decline after total knee replacement surgery (surgery $n=65$; non-surgery $n=65$ ) show that pre-operative cognitive weaknesses in combination with measurements of baseline neuroanatomical integrity contribute to severity of acute post-operative functional connectivity change. The data will stimulate the audience to consider the viability of cognitive assessments within pre-surgical centers and the challenging new frontier of cognitive neuroscience research within the perioperative setting.

Correspondence: Catherine C. Price, Ph.D., Clinical and Health Psychology, University of Florida, PO Box 100165, Gainesville, FL 32610, United States.E-mail: cep23@PHHP.UFL.EDU

\section{T.F. FLOYD. The Hypoxia Inducible Factor and Aging-Related} Postoperative Cognitive Dysfunction.

One probable mechanism to post-operative cognitive dysfunction is hypoxia. Hypoxia's role in postoperative organ dysfunction in every other organ system (heart, liver, kidney) is well accepted, and the brain, most certainly, has greater oxygen demands and lesser tolerance for inadequate oxygenation than any of these other organ systems. At one end of the spectrum, severe hypoxia yields hypoxic encephalopathy. At the other end of the spectrum, voluminous evidence documents that mild-moderate hypoxia yields cognitive impairment, even in healthy individuals. Indeed, mild-moderate hypoxia is surprisingly common and persistent postoperatively and is more frequent with aging. The Hypoxia Inducible Factor (HIF) transcription system is the "master" regulator of the cellular response to hypoxia. HIF contributes to the transcriptional regulation of approximately 2000 genes involved in regulating a host of activities focused upon maintaining oxygen delivery, metabolic homeostasis, and survival, to include glucose transport, glycolysis, and oxygen transport, angiogenesis. Coincidentally, the HIF controlled, hypoxia driven transcriptional responses supporting cell function and survival appear to be markedly impaired by aging, placing aging cells and the 
maintenance of efficient cell function at risk during hypoxic stress. Using animal based cognitive and postmortem data, the speaker will discuss 1) the association between disease-related hypoxia and cognitive function, 2) the HIF system and its management of cellular hypoxia, and 3 ) the evolving role of HIF in supporting memory under hypoxic stress and the implications for POCD in the aged.

Correspondence: Thomas F. Floyd, NY, United States. E-mail: Thomas. Floyd@stonybrookmedicine.edu

\section{Symposium 6. Neuropsychology and Technologies: Taking the lead on new opportunities for understanding brain-behavior relationships}

\author{
Chair: Maria T. Schultheis
}

9:00-10:30 a.m.

M.T. SCHULTHEIS, A. RAPHAIL, J. ZAMZOW, K.L. VICKERS, J. TESSIER \& K.J. MANNING. Neuropsychology and Technologies: Taking the lead on new opportunities for understanding brainbehavior relationships.

Technologies have contributed to the growing changes in the field of neuropsychology- with some like neuroimaging significantly changing the role of the neuropsychologist. In our innovative-driven society, new technologies are being developed every day. For neuropsychology, this offers a unique opportunity for expanding our understanding of brain-behavior relationships. Most notably, technologies offer the opportunity to integrate our current assessment measures with other factors influencing cognition, resulting in a comprehensive evaluation of brain-behavior relationships. Given the "real world" demands for complex and dynamic cognitive processing, it is arguable that technologies may offer a novel venue for improving overall ecological validity in neuropsychology. The current symposium brings together research that highlights the use of innovative technologies for examining cognitive performance in neurological populations. This includes 1) Eye Tracking technology for examining the cognitive and physical demands of visual spatial skills in adults with MS, 2) Actigraphy for defining the relationship between sleep and cognition among adults with relapse-remitting MS, 3) Virtual reality driving simulation for examining cognitive load and dual task driving among individuals with brain injury, and 4) Using body sensors for measuring physiological responses and their role in anxiety and cognition in veterans with TBI/PTSD. Each study will define both the strengths and the challenges of working with novel technologies, the unique metrics generated and how they vary from traditional measures of cognition. The session will conclude with a panel discussion about the future implications of integrating technologies into neuropsychological research and training. Specifically focusing on opportunities for interdisciplinary collaboration and training and considerations for matching technologies with the needs of neurological populations. Correspondence: Maria T. Schultheis, PhD, Psychology, Drexel University, 3141 Chestnut Street, Stratton Building Suite 123, Philadelphia,PA 19104,United States.E-mail: schultheis@drexel.edu

\section{K.L. VICKERS, K.J. MANNING, N.Z. RATZON \& M.T. SCHULTHEIS. Testing the Limits: Using VR to Quantify the Impact of ABI on Driving.}

Objective. Virtual Reality allows neuropsychologists to examine complex, real-world behaviors with high ecological validity. The current study employed a virtual reality driving simulator (VRDS) to examine the effects of imposed secondary tasks on driving performance.

Participants and Methods. 17 participants with acquired brain injury $\left(\mathrm{ABI} ; M_{\text {age }}=39.45,35 \%\right.$ Female $)$ and 27 healthy controls $\left(\mathrm{HC} ; M_{\text {age }}=\right.$ $37.45,45.2 \%$ Female) were recruited for the current study. All participants were licensed and drove regularly. Participants completed two standardized VRDS drives: (1) a baseline drive with no distractions, and (2) the same route with three secondary tasks (Conversation, Radio Tuning, and Coin Sorting). Order of tasks was counterbalanced across participants.

Results. Independent samples t-tests revealed no significant betweengroup differences during the baseline drive. A series of 3 (Task) x 2 (Group) ANOVAs revealed that while the ABI group tended to go slower than the HC group in the presence of a distractor, $F(1,111)=6.24$, $p=.01,=.05$ (small), this did not depend on task, $F(2,111)=.40$, $p=.67$. Importantly, the ABI group also showed greater variability in speed, $F(1,110)=10.97, p<.01,=.09$ (medium), and lane position, $F(1,109)=8.99, p<.01,=.08$ (medium), across all secondary tasks. This effect was driven primarily by performance on measures requiring both a cognitive and motor load (Radio Tuning and Coin Sorting).

Conclusions. These results indicate that long-term driving difficulties following $\mathrm{ABI}$ are subtle and are most likely due to reduced cognitive resources. Clinically, VRDS provides a safe way to test the limits of cognitive capacity during difficult driving situations, and provides information about situations most likely to cause difficulty following neurological compromise.

Correspondence: Kayci L. Vickers, MS, Psychology, Drexel University, 421 S. 15th Street, Apt 3F, Philadelphia, PA 19146, United States. E-mail:kayci.vickers@gmail.com

J. TESSIER, E. WHIPPLE, S. WINTER, K. ROBINSON \& M.T. SCHULTHEIS. Subjective and Objective Measurement of Distress During VR Driving in Veterans with PTSD.

Objective: Examine subjective and objective distress in multiple driving conditions in Veterans with posttraumatic stress disorder (PTSD).

Participants and Methods: Eighteen post-9/11 combat Veterans with PTSD were enrolled. Virtual Reality Driving Simulator (VRDS) was used to measure driving performance in baseline and challenge conditions. Subjective distress was measured with the Subjective Units of Distress Scale (SUDS) nine times during the session. Objective distress was measured with heart rate variability (HRV), which was collected continuously using the Empatica E4 wristwatch.

Results: The average of the three challenge condition SUDS ratings was utilized in analyses. Veterans with PTSD reported significantly higher SUDS during the challenge condition $(M=48.19, S D=23.01)$ than to the baseline condition $(M=33.50, S D=19.02), t(17)=-2.67, p=$ .02 . They demonstrated significantly higher HRV during the challenge condition $(M=.18, S D=.05)$ than to the baseline condition $(M=.15$, $S D=.03), t=2.44, p=.02$.

Conclusions: As expected, Veterans with PTSD experienced more distress during the challenge condition, which includes stimuli designed to increase anxiety (i.e., oncoming traffic). Contrary to expectation, they demonstrated greater HRV in the challenge condition. PTSD is associated with reduced HRV, which reflects an inflexible and unresponsive autonomic nervous system. Veterans in this sample demonstrated an adaptive physiological response to distress (increased HRV). Thus, continuous measurement of objective distress enables a nuanced examination of response to anxiety and a combined impact on performance. This promising technology offers several novel clinical applications. For example, exposure therapies supplemented with VRDS coupled with noninvasive continuous measurement of distress examine individual performance in "dangerous" environments in a controlled but vivid setting and allow for integrating biofeedback to adaptively respond. Correspondence: Jillian Tessier, Psychology, Drexel University, $133 \mathrm{~S}$ 22nd St., Patio Apartment, Philadelphia, PA 19103, United States. E-mail: jt672@drexel.edu

E. VAKIL, M. OHAYON \& O. AVIV. Direct and indirect measures of context in older versus young adult: The additive contribution of eye tracking.

Objective: The facilitation of memory for target stimuli due to similarity of context in the learning and testing phases is known as the "Context Effect" (CE). Previous studies reported that while memory 
for contextual information in older adults was impaired when measured directly CE was preserved. In an attempt to obtain a better understanding of the underling cognitive processes while performing this task, eye movements were monitored for participants in both groups.

Participants \& Methods: Twenty five older adults (mean age 83.71) and 24 matched controls (mean age 22.50) participated in this study. We employed a local-context stimulus array, presenting participants with photographs of trial-unique male faces shown wearing distinctive. trial-unique hats. Throughout the test, eye movements were recorded by the SensoMotoric Instruments (SMI) RED-M remote eve-tracker that allowed free head movements, with a sampling rate of $120 \mathrm{~Hz}$ and high accuracy of $0.8^{\circ}$

Results: Consistent with previous results memory in older adults was impaired when measured directly but preserved when measured indirectly (i.e. CE). For both groups, eye tracking showed longer dwell-time on targets during the learning phase for the stimuli correctly answered. Conclusions: The finding that eye movement patterns were similar for the two groups during learning but different during the test phase indicates that the source of the differences in memory performance between the groups is at the retrieval rather than the encoding stage of learning. This study demonstrates the potential benefits of eye tracking to enhance understanding of underling cognitive processes.

Correspondence: Eli Vakil, PhD, Psychology, Bar Ilan University, Bar Ilan University Ramat Gan, Ramat Gan 52900, Israel. E-mail: vakile@ mail.biu.ac.il

\section{J. YUAN \& R. HOLTZER. HbO , Variability During Single- and Dual-Task Gait in Older Adults.}

Objective: Evidence that mobility and cognition are interrelated is robust, especially in older adults. To date, functional neuroimaging studies of gait have exclusively focused on average patterns of neural activity. Emerging neuroscience research suggests that variability of neural activity may also have intrinsic meaning. As such, examining variability of neural activity during locomotion would offer novel insights into the inherently dynamic nature of the brain. The objective of this study was to compare variability of neural activity during simple and attention-demanding gait in older adults.

Participants \& Methods: Functional near-infrared-spectroscopy (fNIRS) was used to assess oxygenated hemoglobin $\left(\mathrm{HbO}_{2}\right)$ levels in the prefrontal cortex (PFC) during locomotion in 347 non-demented older adults (mean age $=76$ years; $\%$ female $=55$ ). $\mathrm{HbO}_{2}$ variability was operationalized as the standard deviation of $\mathrm{HbO}_{2}$ levels across six continuous straight walks under single-task (normal walk) and dualtask conditions (walking-while-talking). Reliability and validity for this walking paradigm have been previously established.

Results: A linear mixed effect model revealed significantly higher $\mathrm{HbO}_{2}$ variability in dual-task compared to single-task gait (estimate $=-.841$, p >.000; 95\% CI [-.125, -.043]). The analysis controlled for gender, age, education, and disease comorbidity.

Conclusions: This study provided the first evidence that $\mathrm{HbO}_{2}$ variability is higher in the PFC during dual-task compared to single-task gait. Our results are in line with accumulating evidence from studies of behavioral variability that demonstrate increased variance in cognitive or gait performance during tasks requiring greater executive control processes. These initial findings encourage the future use of variability measures to examine functional neural correlates of gait in older adults. Correspondence: Jennifer Yuan, Yeshiva University, 720 Greenwich Street \#8F, New York, NY 10014, United States. E-mail: jenniferdyuan@ gmail.com

\section{Paper Session 9. Epilepsy \\ Moderator: Joseph I. Tracy \\ 9:00-10:30 a.m.}

\section{J.I. TRACY. Functional Connectivity in Epilepsy.}

This talk will focus on resting state functional connectivity ( $\mathrm{rsFC}$ ) in epilepsy. Three recent findings will be highlighted. First, depending on the age of seizure onset brain network modularity and connectivity can be disrupted in distinct ways both at the global and local level, with mesial temporal sclerosis a mediating factor in these effects. Such perturbations may have different implications for regional plasticity and adaptive reorganization. Second, data will be presented to challenge the view that the resting state fMRI signal in epilepsy is stable over time, generating insights into the large scale temporal dynamics of network organization. Lastly, we will examine data addressing the relationship between task-fMRI and intrinsic resting state networks, focusing on the domain of language. Our findings suggest that intrinsic networks capture a broad swath of bilateral activity, reflecting a set of pre-potent language regions. Task-related fMRI can then be understood as a winnowing down and selection of the larger, subsuming language-ready RSN. Such data provides a mechanistic explanation of how language network spatial representations shift between an unfocused condition (resting-state) and a focused/task constrained covert speech task. Correspondence: Joseph I. Tracy, Ph.D./ABPP, Neurology, Thomas Jefferson University/Sidney Kimmel Medical College, 901 Walnut Street, Health Sciences Bldg Ste 447, Philadelphia, PA 19107, United States. E-mail: joseph.tracy@jefferson.edu

D.E. MARRA, L. GLASS, D. SABSEVITZ, E.E. QUASNEY, J. BINDER, W. MUELLER, M. RAGHAVAN \& S. SWANSON. Cognitive Reserve Predicts Post-Operative Cognitive Outcomes in an Epilepsy Population.

Objective: Cognitive reserve (CR), or the brain's resiliency to injury or disease, has been understudied in an epilepsy sample. Thirty to sixty percent of individuals who undergo anterior temporal lobectomy (ATL) to treat intractable epilepsy experience cognitive decline. This study examined how educational attainment (EA) predicts post-operative (PO) cognitive decline.

Participants and Methods: Two hundred forty-three patients. $\left(M_{\text {education }}=13.00,52 \%\right.$ right-TLE, $48 \%$ left-TLE $)$ underwent comprehensive neuropsychological examinations pre-and-post ATL. Hierarchal regressions were conducted to examine the ability of EA to predict cognitive changes after controlling for pre-operative scores. The odds of experiencing clinically significant decline for those with Low EA (LEA; $\geq 12$ years education) vs High EA (HEA; $13+$ years education) were determined via logistic regression. These findings were further explored for patients with left-ATL versus right-ATL.

Results: After controlling for pre-operative scores, higher EA predicted better PO test scores for measures of verbal and visual memory, language. and executive functioning $(p$ 's $<.05)$. For all patients, LEA had higher odds (2.00) of experiencing decline in verbal memory. LEA/left-ATL resections had higher odds of decline compared to HEA/left-ATL for tests of verbal memory (odds range: 2.51-3.28) and confrontation naming (odds $=5.37$ ). Whereas, LEA/right-ATL patients had higher odds of decline for visual memory only (odds $=2.49$ ).

Conclusions: $\mathrm{CR}$ is a significant predictor of PO cognitive outcomes and should be further investigated in an epilepsy population. Furthermore, post-secondary education appears to be protective of post-operative decline in the verbal domain (naming and verbal memory) for left-ATL and visual memory for right-ATL patients.

Correspondence: David E. Marra, MS, Psychology, Marquette University, 1616 N. 50th St, Milwaukee, WI 53208, United States. E-mail: david. marra@marquette.edu 
K. OSIPOWICZ \& J.I. TRACY. Normative Anticorrelation Inhibits Seizure Generalization in Mesial Temporal Lobe Epilepsy.

Objective: Previously, we demonstrated anticorrelated connectivity from the epileptogenic mTL to the contralateral hemisphere in unilateral mTLE patients, and saw a marked absence of this anticorrelated connectivity in those with bilateral interictal activity (Tracy et al., 2014). We postulated that this anticorrelated connectivity serves a neuroprotective function, inhibiting the spread of seizure activity to the epileptic mTL. Here, we sought to extend our previous findings and to test the hypothesis that this contralateral anticorrelated connectivity is neuroprotective.

Participants and Methods: We used a large homogenous sample (all candidates for mesial temporal lobe resection) of mTLE patients $(\mathrm{n}=111)$ classified according to the presence of seizure generalization and matched controls $(n=114)$. Voxelwise comparisons of resting state connectivity of the mesial temporal lobe was compared between patient groups and controls; additionally, we analyzed the relationship of connectivity with pathology and cognitive variables.

Results: When focal patients were compared to controls, we found few differences in mTL connectivity, however, when patients with evidence of generalization were compared against either the control or focal groups, we observed a conspicuous absence of contra-ictal frontal and parietal anticorrelation. Additionally, the association between epileptogenic zone connectivity and epilepsy duration showed an inverse correlation between the negative connectivity to these frontal and parietal regions.

Conclusions: These results replicate and extend our previous findings of anticorrelated connectivity and clarify our theory that this is a normative inhibitory function, which in the context of mTLE may be neuroprotective serving to inhibit the spread and generalization of seizure activity. Correspondence: Karol Osipowicz, Ph.D., Psychology, Drexel University, 3141 Chestnut St, 204 Stratton Hall, Philadelphia, PA 19104, United States.E-mail:kzo22@drexel.edu

W. SCHRAEGLE, N. NUSSBAUM \& J.B. TITUS. Hippocampal Sclerosis is a Risk Factor for Depression Features in Youth with Temporal Lobe Epilepsy.

Objective: Temporal lobe epilepsy (TLE) increases the risk for depression, but the role of hippocampal sclerosis (HS) is unknown. Because impairments in executive functioning (EF) are known to be associated with depression, we explored the relationship between HS, EF, and depression in pediatric TLE.

Participants and Methods:

The study included 62 patients with TLE (HS, n=17) age 8-16 years. EF was assessed with the D-KEFS (Verbal Fluency), WISC-IV (Digit Span), WCST (Perseverative Errors), and the BRIEF (MI \& BRI). Depression features were assessed with the BASC-2:PRS.

Results:

Correlational analyses revealed associations between depression and WCST, BRI, and HS $(p \leq 0.01)$. HS correlated with WCST $(p<0.05)$. ANOVA revealed higher depression ratings and more perseverative errors on the WCST in youth with HS $(p<0.05)$. No differences were found for the BRI variable $(p>0.10)$. This difference in WCST was found to mediate the significant relationship between HS and depression. Logistic regression indicated HS was associated with a fourfold increase in depression $(\mathrm{p}<0.05)$.

Conclusions: The current work identifies HS as a risk factor for depression in pediatric TLE and suggests a relationship between HS and poor executive functioning. HS may be associated with greater limbic system disruption, which may lead to impaired executive control and poor coping and emotional adjustment. As HS is not modifiable, these findings suggest EF intervention may be a potential modality for improving HRQOL in youth with TLE.

Correspondence: William Schraegle, The University of Texas, Austin, 1307 Kinney Ave \#114, Austin, TX 78704, United States. E-mail: waschraegle@gmail.com
R.C. BREWSTER, T.Z. KING, B. CROSSON, J.A. TURNER \& D.L. DRANE. White Matter Correlates of Verbal Memory in Left Temporal Lobe Epilepsy: A Study of Structural Connectivity.

Objective: Although verbal memory deficits are prominent cognitive sequelae in left temporal lobe epilepsy (LTLE), relationships between verbal memory and left temporal lobe white matter integrity (WMI) are unclear. Presumed measures of WMI, fractional anisotropy (FA) and mean diffusivity (MD) were determined along the left fornix (FRX), uncinate fasciculus (UF), and parahippocampal cingulum (PHC) in participants with LTLE, right TLE (RTLE), and controls. We hypothesized that the LTLE group would demonstrate the lowest FA and highest MD of the three groups, and predicted relationships between these measures and verbal memory performance in the TLE groups.

Participants and Methods: Mean FA and MD were extracted from diffusion-weighted images of 23 participants with LTLE (mean age $=39.35, S D=16.92 ; 69 \%$ female), 21 with RTLE (mean age $=40.62$. $S D=11.64 ; 62 \%$ female) and 20 controls (mean age $=37.50, S D=13.88$; $75 \%$ female) along three left hemisphere target tracts and the left corticospinal tract (CST; a control region). All TLE participants were left-language dominant presurgical candidates. TLE participants completed word list recall, story recall, confrontation naming, vocabulary, and motor dexterity (control task) measures.

Results: PHC FA was lower in the LTLE group $(M=0.34, S D=0.07)$ than in the RTLE group $(M=0.35, S D=0.08)$, and controls $(M=0.38$, $S D=0.04), F(2,47.94)=4.71, p<.05, \omega^{2}=.040$. LTLE PHC FA was negatively correlated with vocabulary $(\mathrm{r}=-.53, \mathrm{p}<.05)$ and naming $(\mathrm{r}=-.51, \mathrm{p}<.05)$. RTLE PHC FA was positively correlated with list learning $(r=.64, p<.05)$, and PHC MD was negatively correlated with list learning $(\mathrm{r}=-.65, \mathrm{p}<.05)$. There was no relationship between $\mathrm{FA}$ or MD and the control task for either group.

Conclusions: Current results highlight left temporal PHC WMI as affected in LTLE and differentially related to verbal memory functioning based on TLE group. These findings support previous literature describing white matter disruption in LTLE and related verbal memory dysfunction.

Correspondence: Ryan C. Brewster, Georgia State University, 11070 Strathmore Drive, Los Angeles, CA 90024, United States. E-mail: rbrewst@gmail.com

J. SPAT, E. ROMAN, E. RAMIREZ-COOMBS, S. MANDELBAUM, V. GUZMAN, L. LANGAN, L. MARCUSE \& H.A. BENDER. Healthcare Disparities and Cognitive Performance Among Minority Patients with Epilepsy.

Objective: The literature examining healthcare disparities in epilepsy suggests that historically under-represented racial and ethnic minority groups are at higher risk of receiving sub-optimal care when compared to their non-minority counterparts. Such disparities are closely related to low SES and higher rates of poverty, which contributes to poorly understood symptom management and lack of access to specialized care. As a direct result of these factors, a prolonged duration of untreated seizures and/or poorly controlled seizures can result in significant cognitive deficits. The present case series examines this alarming public health concern in under-represented racial and ethnic minority patients with a history of life-long, un-/under-treated epilepsy.

Participants and Methods: Consecutive case series ( $N=6$ ) referred for pre-surgical neuropsychological evaluation from 2010-2016 at Mt. Sinai Epilepsy Center. All patients were non-U.S. born, ranged in age from 25 to 67 years, self-identified as having low SES and had a life-long history of seizures beginning in childhood.

Results: Though all the patients had been experiencing seizures since a very young age, all were diagnosed 5-10 years after seizure onset, attributing this delay in adequate medical care to ongoing psychosocial stressors, poor access to healthcare, and low health literacy. Cognitively, patients who had a longer delay in diagnosis exhibited the most global and profound cognitive impairments, despite being evaluated in their native language via culturally-sensitive batteries. 
Conclusions: In this case series, racial and ethnic minorities with epilepsy had several year lag from seizure onset to treatment, thereby contributing to increased risk of cognitive impairments. These results highlight the clear need to conduct high-quality, high-impact research focused on historically- under-studied and under-represented racial and ethnic populations in an attempt to reduce the gap in service delivery and improve quality of life.

Correspondence: Jessica Spat, Ph.D., 1468 Madison Avenue, NY 10029.

United States.E-mail: jessica.spat@mssm.edu

\section{Paper Session 10. Medical / Infectious Disease}

\section{Moderator: Marc Norman}

9:00-10:30 a.m.

D.P. SHEPPARD, S.M. TIERNEY, V.M. KORDOVSKI, M.P. FAYTELL, G. AVCI \& S.P. WOODS. A Comparison of the Sensitivity, Reliability, and Stability of Three Diagnostic Criteria for HIV-Associated Neurocognitive Disorders.

Objective: $\mathrm{HIV}$-associated neurocognitive disorders (HAND) are present in an estimated $50 \%$ of $\mathrm{HIV}$-infected individuals, yet the multiple diagnostic criteria currently available can yield varying prevalence rates. The present study examined the frequency, sensitivity, and reliability of three diagnostic criteria (DSM-5, Frascati, Gisslen) for HAND.

Participants and Methods: Participants included 361 adults with HIV disease and 199 seronegative comparison subjects with broadly comparable demographics who completed a comprehensive research neuropsychological battery to determine neurocognitive status as defined by the DSM-5, Frascati, and Gisslen neurocognitive criteria for HAND. Participants were assessed for everyday functioning impairment using self-report and clinician-ratings. A subset $(n=146)$ of the HIV+ group was assessed $14.3(.2)$ months later using an identical battery.

Results: Across a series of logistic regressions, HIV serostatus was associated only with Frascati-defined neurocognitive impairment ( $p=.027, \mathrm{OR}=1.7[1.1,2.7])$, but not DSM-5 or Gisslen-defined neurocognitive impairment ( $p \mathrm{~s}>.05)$. Results showed DSM-5 and Frascati criteria agreed on $71 \%$ of observations, Frascati and Gisslen showed agreement on $80 \%$, and DSM-5 and Gisslen criteria showed agreement on 46\%. Only Frascati-defined neurocognitive impairment was a significant predictor of everyday functioning impairment ( $p=.002$. $\mathrm{OR}=2.3[1.4,3.8]$ ). DSM-5 criteria evidenced significantly fewer incident (ps<.001) and significantly more remitting (ps <.001) neurocognitive disorders compared to using Frascati and Gisslen criteria.

Conclusions: Reliability across the three diagnostic criteria was poor, while Frascati criteria evidenced the best sensitivity and specificity to $\mathrm{HIV}$ and accuracy for predicting everyday functioning outcomes. Future studies should aim to examine gold standard biological markers (e.g., ApoEع4) and clinical health outcomes that might be associated with specific diagnostic criteria.

Correspondence: David P. Sheppard, Psychology, University of Houston, 1800 El Paseo St. Apt. 414, Houston, TX 77054, United States. E-mail: dsheppard.uh@gmail.com

T. KUHN, Z. MAHMOOD, T. WILLIAMSON, S. BOOKHEIMER, C. HINKIN \& A. THAMES. Accelerated Brain Aging and Cognitive Decline in HIV.

Objective: Many studies have found that older HIV-positive adults are more vulnerable to cognitive problems than healthy elderly controls with recent evidence supporting the contention that HIV accelerates brain aging. Therefore, this study sought to determine whether (1) HIV infection results in accelerated brain aging; (2) Accelerated brain aging is associated with cognitive decline.

Participants and Methods: Accelerated brain aging was defined as the difference between expected age-based DTI metrics of brain integrity [fractional anisotropy (FA), diffusivity (MD)], determined using a regression equation using age, gender and education to predict FA and MD in HIV - controls $(\mathrm{n}=36)$, and the observed FA/MD in our entire HIV+ sample $(n=72)$. Paired-samples t-tests were conducted to evaluate whether observed and predicted FA/MD values differed in the HIV+ group. Correlation analyses were conducted to determine the association between FA/MD discrepancy scores and age as well as cognitive performance.

Results: MD in the HIV+ group was significantly higher than expected for their age $(t(1,71)=4.230, p<0.001)$. Age was correlated with both FA $(r=0.302, p=0.010)$ and $\mathrm{MD}(\mathrm{r}=-0.673), \mathrm{p}<0.001)$ discrepancy scores, suggesting that as they age, $\mathrm{HIV}+$ participants displayed greater age-related brain changes. Further, MD discrepancy scores were significantly correlated with global cognitive function $(r=-0.321$, $p=0.007)$ which was driven by an association with learning $(r=-0.250$, $p=0.037)$. FA discrepancy scores were also significantly correlated with global cognitive performance $(r=0.352, p=0.002)$ and learning $(\mathrm{r}=0.277, \mathrm{p}=0.020)$.

Conclusions: HIV infection can result in accelerated brain aging and this HIV-associated accelerated brain aging was associated with worse cognitive performance in multiple domains.

Correspondence: Taylor Kuhn, M.S., University of Florida, 317 SW 54th Dr, Gainesville,FL 32607, United States.E-mail: tkuhn@phhp.ufl.edu

E. LOJEK, A. PLUTA, B. SZYMANSKA, N. GAWRON, M. SOBANSKA, A.R. AMBROZIAK, T. WOLAK, P. BIENKOWSKI, H. SIENKIEWICZ-JAROSZ, A. SCINSKA-BIENKOWSKA, S. RAO \&. R. BORNSTEIN. Neurocognitive and Brain Functions in Highly Functioning and Successfully Treated Young HIV Seropositive Men.

Objective: Despite successful treatment, neurocognitive and brain dysfunction can be still present in $\mathrm{HIV}(+)$ individuals for unclear reasons. This study aimed to examine a wide variety of neuropsychological, neurophysiological and social functions in highly functioning, successfully treated young $\mathrm{HIV}(+)$ men.

Participants and Methods: Thirty $\mathrm{HIV}(+)$ men with an undetectable viral load and no comorbidities and $20 \mathrm{HIV}(-)$ healthy controls aged 25-35 participated in the study. The groups were comparable on age, sex, education, work status, social and physical activity, social support, dieting and mood. Drug users, patients with chronic or progressive diseases were excluded from the study. The assessment included a comprehensive battery of neuropsychological tests and MRI/fMRI examinations (N-back task).

Results: Despite preserved abilities on general intelligence, reasoning, language, psychomotor skills and mood, $\mathrm{HIV}(+)$ individuals showed significant impairment compared with the controls in the domains of attention, working memory and executive function (VMS Forward and Backward, Digit Span Backward, perseverations in the WCST and the RFFT). HIV (+) group did not differ from the controls on the subjective estimation of their cognitive skills. No marked grey matter differences were found. The number of correct of answers in the N-back task was comparable in both groups, but there were differences in the patterns of brain activation during the performance. Despite similarities between the groups, $\mathrm{HIV}(+)$ individuals demonstrated significantly higher activation compared with the controls in inferior frontal gyrus bilaterally and middle frontal gyrus in right hemisphere. They also showed markedly decreased activation in superior parietal lobule in right hemisphere in comparison with HIV (-) subjects.

Conclusions: This study showed that even in successfully treated, highly functioning, young $\mathrm{HIV}(+)$ men, there are subtle neurocognitive deficits most possibly compensated by the activation of neural and cognitive reserves.

Correspondence: Emilia Lojek, Ph.D., Psychology, University of Warsaw, Stawki 5/7, Warsaw00-183, Poland.E-mail: emilia@psych.uw.edu.pl 
M. HARCIAREK, A. MANKOWSKA, J. MICHALOWSKI, J.B. WILLIAMSON, B. BIEDUNKIEWICZ, A. DEBSKA-SLIZIEN \&. K.M. HEILMAN. Allocation of Spatially Directed Focal Attention in Patients With End-Stage Renal Disease Receiving Dialysis: Attentive But Too Engaged.

Objective: Patients receiving dialysis often reveal cognitive impairments and the improper allocation of directed attention may play a role in their cognitive dysfunction. There are several possible attention functions that may be impaired including distractibility, sustained attention and spatial allocation of attention. The allocation of spatially directed focal attention has not been fully studied in this population. Thus, this research was designed to investigate if patients receiving dialysis may have problems spatially allocating and/or disengaging focal attention. Participants and Methods: Thirteen non-demented adequately dialyzed patients with end-stage renal disease and 15 demographically matched healthy controls performed a Posner-type visual attention task, where to the left and right of the central point two boxes were displayed in which an imperative stimulus could appear. In each trial, this stimulus was preceded by a right or left preparatory cue (box highlighting) that was valid in $80 \%$ of the trials and invalid in $20 \%$.

Results: In comparison to healthy controls, the patients receiving dialysis responded more slowly to trials with invalid cues. In contrast, there was no group difference for trials with valid cues. There were also no group differences in error rates, regardless of the cues' validity.

Conclusions: The results of this study suggest that patients with end-stage renal disease need more time to disengage from invalid cues, but they do not seem to have difficulties allocating spatially directed focal attention in response to valid cues. The mechanism that could account for these patients' defective ability to disengage (attentional grasp) is not known. However, disengagement disorders are often associated with frontal-subcortical brain dysfunction, and impairments of these networks are frequently reported in this population. Correspondence: Michal Harciarek, Psychology, University of Gdansk, Bazynskiego 8, Gdansk 80-309, Poland. E-mail: psymh@ug.edu.pl

\section{J. EVANS, E. BUCHARD-MACDONALD \& L. PAUL. Balancing the Demands of Two Tasks: An Investigation of Cognitive-Motor Dual- Tasking in Relapsing Remitting Multiple Sclerosis.}

Objective: People with relapsing remitting MS (PwRRMS) suffer disproportionate decrements in gait performance when walking is combined with a cognitive task. There has been much less investigation of the impact of cognitive demands on balance. We investigated whether: (1) PwRRMS show disproportionate decrements in postural stability under dual-task conditions compared to healthy controls; (2) dual-task decrements are associated with everyday dual-tasking difficulties; (3) mood/ fatigue are associated with dual task decrements.

Participants and Methods: 34 PwRRMS and 34 matched controls completed cognitive (backward digit span) and balance (movement of centre of pressure on a Biosway, on stable and unstable surfaces) tasks under single and dual-task conditions. Everyday dual-tasking was measured using the Dual-Task Questionnaire. Mood was measured by the Hospital Anxiety and Depression Scale. Fatigue was measured via the Modified Fatigue Index Scale.

Results: The groups were well-matched on age, gender, years of education, pre-morbid IQ and digit span. Compared to controls, under dualtask conditions PwRRMS showed a significantly greater decrement in digit span on stable and unstable surfaces (both $p<0.001$ ), and in postural stability on an unstable surface $(p=.007)$, but not a stable surface. PwRRMS reported higher levels of everyday dual-tasking difficulties $(p<.001)$. Decrement scores were not correlated with everyday difficulties or fatigue. Stable surface balance decrement scores were significantly associated with levels of anxiety (rho=.527, p=.001) and depression (rho=.451, p=.007).

Conclusions: RRMS causes difficulties with dual tasking, impacting balance, particularly under challenging conditions, which may increase risk of gait difficulties and falls. The striking relationship between anxiety/depression and dual-task decrement suggests that worry may be contributing to dual-task difficulties, and raises the possibility that therapeutic interventions aimed at managing worry may improve cognitive-motor dual-tasking.

Correspondence: Jonathan Evans, University of Glasgow, mental health \& wellbeing, Glasgow G12 OXH, United Kingdom. E-mail: Jonathan. Evans@glasgow.ac.uk

R.M. BOWLER, S.W. ADAMS \& U. DYDAK. Neuropsychological Test Performance in Relation to MRI Manganese Deposition in the Brain.

Objective: There is a long history of welding exposure leading to elevated levels of manganese (Mn), causing a Parkinson's-like syndrome in those overexposed. This study examines the results of neuropsychological testing of 30 active welders from a semi-trailer manufacturing plant in Indiana with Mn exposure, and 21 demographically similar but unexposed controls, individually administered brief clinical neuropsychological test battery.

Participants and Methods: Tests chosen for assessments included the domains reported to be impaired from Mn exposure: Cognitive Flexibility and Executive Function, Working Memory/Attention \& Concentration and Learning, Visuospatial Memory, Visuomotor Tracking Speed, Verbal Skills, Motor Function and Effort.

Results: Welders were exposed to $\mathrm{Mn}$ in air (ACGIH TLV $=0.02 \mathrm{mg}$ / $\mathrm{m}^{3}$ ) of $0.12 \mathrm{mg} / \mathrm{m}^{3} \pm 0.03 \mathrm{mg} / \mathrm{m}^{3}$, for an average duration of 13.3 years. Manganese accumulation in the brain was measured using MRI T1 relaxation times in 8 brain regions (ROIs): bilateral globus pallidi, frontal lobes, caudate nuclei, and substantia nigra. The welders scored significantly worse than controls on Rey-Osterrieth Copy, Digit Span total and longest forward, Fruit Naming, and the Parallel Lines test evaluating graphomotor tremor, and shorter MRI T1 relaxation times than controls in the bilateral globus pallidi, right substantia nigra, left caudate nucleus, and the bilateral frontal lobes. Tests of verbal abilities (Animal \& Fruit Naming), verbal learning (WHO-UCLA AVLT), working memory and verbal recall (Digit Span), fine tactile manipulation (Grooved Pegboard), and graphomotor tremor (Parallel Lines) were associated with increased Mn deposition in all but the right frontal lobe and right substantia nigra.

Conclusions: The relationship between MRI Mn deposition and neuropsychological test performance substantiates early clinical dysfunction associated with Mn exposure.

Correspondence: Rosemarie M. Bowler, SFSU, 8371 Kent Dr, El Cerrito, CA 94530, United States. E-mail: rbowl@sfsu.edu

\section{Poster Session 7. Neuropsychiatry}

9:30-10:45 a.m.

\section{Drug/Toxin-Related Disorders (Including Alcoholism)}

L. COLEMAN, K. WOOTEN \& D.G. NEMETH. Prenatal Opioid Abuse (POA) Causes Children's Learning/Attentional Problems. Objective: According to the 2014 Prescription Drug Abuse During Pregnancy website, children exposed to prenatal opioid abuse are "at risk of abnormal brain development throughout early childhood," which includes learning difficulties and poor academic skills. These children are also likely to exhibit behavioral and attentional problems (Minnes, 2011). Children exposed to prenatal opioid abuse (POA) tend to be more impulsive, have difficulty regulating their emotions, and have lower processing speed skills than non-exposed children (Mathias, 1998). The purpose of this presentation is to examine the effects maternal opioid abuse during pregnancy has on the learning and attentional abilities of the children. 
Participants and Methods: POA children ages 5-7, two female and male, evaluated at The Neuropsychology Center of Louisiana, LLC, were compared with the current literature on behavioral, attentional, and learning styles of POA children.

Results: Based on testing results, our POA children have all been diagnosed with ADHD, combined type (Mean TOVA Attention Comparison Score of -6.59); exhibit Learning and Impulsivity problems; and present with low Processing Speed (Mean Processing Speed Index score of $6.67 \%$ ). They also had difficulties in academic skills, such as reading, math, and written language.

Conclusions: Our POA children's data was consistent with the literature, which suggested that prenatal opioid exposure does affect children's learning, attentional, and behavioral development. Their developmental difficulties are being treated by behavior therapy and medication. The results of these evaluations will be highlighted in this poster presentation.

Correspondence: LaJae Coleman, B.S., 4611 Bluebonnet Blvd., Suite B, Baton Rouge, LA 70809, United States.E-mail: lajae.coleman@gmail. com

E. GILBART, N.E. WRIGHT \& K.M. LISDAHL. Marijuana Use, Aerobic Fitness, Mood, and Disinhibition in Emerging Adults.

Objective: $44.4 \%$ of $12^{\text {th }}$ graders report lifetime marijuana (MJ) use (Johnston et al., 2015). Repeated CB1 binding due to THC results in downregulation of the endocannabinoid system in cortex and limbic regions (Hirvonen et al., 2012), while aerobic exercise may upregulate CB1 activity (Ferreira-Vieira et al., 2014). Here we examine the influence of young adult MJ use on anxiety, depression, reward response, and disinhibition and whether aerobic exercise moderated these effects. Participants and Methods: 85 subjects (36 MJ, 49 controls) aged 16-25 were balanced for gender (39F); 67\% were Caucasian. Exclusion criteria included: MRI contraindications, left handed, Axis-I disorders. major medical/neurologic disorders, prenatal issues, prenatal drug exposure, or $>20$ times other drug use in lifetime. Subjects completed the Frontal Systems Behavior Scale, BIS/BAS, State-Trait Anxiety Inventory (State), and BDI-II. Participants also completed a $\mathrm{VO}_{2}$ maximum test to measure of aerobic fitness. Multiple regressions were run to predict anxiety, depressive symptoms, reward respond, and disinhibition from past year MJ use, $\mathrm{VO}_{2}$ max, and $\mathrm{MJ}^{*} \mathrm{~V} \mathrm{O}_{2}$ max interactions, while controlling for past year alcohol use, gender, and cotinine.

Results: MJ use significantly predicted increased depressive symptoms $(p=.02)$, decreased reward response $(p=.05)$, and increased disinhibition $(\mathrm{p}=.003) . \mathrm{V}_{2}$ max marginally predicted disinhibition $(\mathrm{p}=.06)$ and significantly moderated MJ effects $\left(\mathrm{MJ}^{*} \mathrm{VO}_{2}\right.$ max, $\left.\mathrm{p}=.001\right)$. Fun-seeking was marginally predicted by alcohol use $(\mathrm{p}=.07)$.

Conclusions: Similar to previous findings (Wright et al., under review; Medina \& Shear, 2007), increased MJ use significantly predicted greater depressive symptoms, greater disinhibition and reduced reward response. Notably, improved aerobic fitness moderated the impact of MJ on disinhibition, in that more fit MJ users demonstrated superior performance compared to low-fit MJ users. Therefore, aerobic fitness may present a viable and low-cost intervention for MJ users to improve executive functioning.

Correspondence: Erika Gilbart, BA, Psychology, University of Wisconsin Milwaukee,WI,United States.E-mail: egilbart@uwm.edu

K. JENNETTE, E. GILBART \& K. LISDAHL. The Relationship Between Marijuana Use, Inhibitory Control, and Learning Strategy in Adolescents and Young Adults.

Objective: Verbal learning and memory deficits are common in young marijuana (MJ) users (Lisdahl et al., 2014); however few studies have addressed the impact of learning strategy on list-learning tasks. Further, no studies to date examined whether inhibitory control moderates learning strategy in young MJ users. This study compared the relative use of "higher-order" semantic clustering versus serial clustering on a trial-by-trial basis during encoding on the CVLT-II in adolescent and young adult MJ users and controls. The contribution of inhibitory control on learning strategy in this sample was also assessed.

Participants and Methods: 138 demographically matched adolescents (51 MJ users, 87 controls) were recruited from the community and administered the CVLT-II and D-KEFS Color Word Interference task as part of a larger neuropsychological battery. An organization strategy ratio (OSR) score was calculated by dividing semantic by serial clustering score for each trial. Repeated Measures ANOVA was run for OSR scores on trials 1-5 by group (MJ vs. control), controlling for gender, alcohol, nicotine use, and D-KEFS inhibition score.

Results: No differences in organizational strategy across trials were observed between MJ users and controls. However, D-KEFS Inhibition performance was a marginal moderator of organization strategy use $(\mathrm{p}=0.07)$.

Conclusions: No differences were observed between MJ users and controls in the relative use of semantic versus serial clustering during encoding, indicating that the differential use of organization strategy does not account for previously reported verbal learning deficits in MJ users. However, inhibitory control performance was a marginal moderator of organization strategy, indicating an association between better cognitive control and the use of higher order organization strategy during encoding.

Correspondence: Kyle Jennette, MA, Psychology, University of Wisconsin-Milwaukee, 2717 E. Hartford Ave, Milwaukee, WI 53211, United States.E-mail: kjtommasi@gmail.com

\section{KIM. Deficits of decision-making in college students who participate in binge drinking.}

Objective: Individuals with binge drinking (BD) show deficits in reallife decision-making, but the nature of these deficits is not fully understood. This study investigated deficits of decision-making in college students with BD using Iowa Gambling Task (IGT) and Prospect Valence Learning (PVL) Model.

Participants and Methods: Participants: Based on scores of Alcohol Use Disorder Identification Test and Alcohol Use Questionnaire, BD $(n=30)$ and control $(n=31)$ groups were determined.

Measurement and PVL analysis: IGT was administered to evaluate the decision-making. Participants were required to select one card from four decks in each trial. Each selection resulted in monetary gains, which is sometimes coupled with simultaneous losses. Decks A and B are disadvantageous decks with long-term losses and decks C and D are advantageous decks with long-term losses. The PVL model estimates four parameters; utility shape (subjective evaluation for selection outcome based on gains \& losses), loss aversion (sensitivity to losses), recency (selection based on feedback of previous trials), and consistency (consistent selection of cards which individual prefers) parameters. PVL parameters were estimated with Markov Chain Monte Carlo (MCMC) sampling scheme in OpenBugs and BRUGS, its interface to R.

Results: The BD group received significantly lower total net scores $(F(1,59)=5.45, p<.05)$, block net scores in the third $(t(59)=-2.39$, $p<.05)$ and fourth $(t(59)=-1.97, p<.05)$ blocks on the IGT than the control group. BD group also selected cards from deck B more frequently than control group $(t(59)=2.45, p<.05)$. In terms of PVL analysis, BD group scored significantly lower on the loss-aversion parameter than control group $(U=313.00, p<.05)$.

Conclusions: College students with BD showed difficulties in decision-making, possibly due to failures in contingency learning and sacrificing immediate rewards in favor of long-term benefits.

Correspondence: Myung sun Kim, Ph.D, Psychology, Sungshin Women's University, Bomun-ro sungbuk-gu, Seoul 02844, Korea (the Republic of).E-mail:kimms@sungshin.ac.kr

C. KOHEN \& S. TAPERT. The Neurocognitive Effects of Changing Hazardous Drinking Behaviors in Adolescents and Young Adults. Objective: Adolescence is a time of considerable neurodevelopment and increased substance use. Alcohol remains among the most commonly 
used intoxicants during adolescence with $21 \%$ of high school students having consumed 5 or more drinks on one occasion in the last 30 days. Neuropsychological and neuroimaging studies have shown that binge drinking during adolescence may provoke deleterious neurocognitive effects in the domains of learning, memory, visuospatial skills, working memory, and executive functions. Longitudinal studies have found that these deficits in cognitive functioning may remit with abstinence in both adults and adolescents, yet few studies have examined if adverse neurocognitive sequelae abate after individuals modestly decrease their hazardous drinking patterns. The objective of this study was to determine if a reduction in frequency of binge drinking episodes corresponds to improved neurocognitive functioning on tasks of memory, attention, visuospatial skills, and executive functioning.

Participants and Methods: Analyses were conducted on participants in a large longitudinal study with neuropsychological tests completed at two or more time points (T1 \& T2) after the age $18(N=72)$, representing a range of drinking levels and change with time.

Results: Multiple regression analyses showed no significant predicted relationships between a change in binge drinking episodes and change in neuropsychological test variables. However, a post-hoc examination of heavier drinkers $(n=36)$ showed trends of recovered performance on memory, attention, and executive functioning tasks in those who reduced drinking.

Conclusions: Neuropsychological deficits of heavy drinkers may dissipate with a moderate decrease in hazardous drinking episodes.

Correspondence: Casey Kohen, MA, Psychology, San Diego State University, 6867 Golfcrest Dr Apt 8, San Diego, CA 92119, United States.E-mail: caseykohen1@gmail.com

K.E. MAPLE, A. THOMAS, M. KANGISER, E. GILBART \& K.M. LISDAHL. Anterior Cingulate Volume Reductions in Adolescent and Emerging Adult Cannabis Users: Association with Affective Processing Deficits.

Objective: Cannabis has previously been associated with aberrant affective and neural functioning (e.g., Gruber et al., 2009; Spechler et al., 2015). The current study sought to examine the relationship between cannabis use and brain structure. Secondly, it assessed whether structural differences associated with cannabis use were related to affective processing.

Participants and Methods: Individuals were excluded for comorbid psychiatric and neurological disorders, psychotropic medication use, and excessive other drug use. Participants (32 cannabis users and 43 non-users, ages $16-25$ years) completed an affective processing battery (PennCNP; Erwin et al., 1992; Gur et al., 2002) and underwent magnetic resonance imaging. Structural images were processed using FreeSurfer (Fischl et al., 2002; Desikan et al., 2006). Principal components analysis (PCA) was used to reduce affective processing variables. A series of multiple regressions investigated whether past year cannabis use and cannabis $\mathrm{x}$ gender predicted volumes in bilateral prefrontal, temporal, limbic, and cerebellar regions. Covariates included gender, alcohol and nicotine use, and intracranial volume. False Discovery Rate (FDR) corrections were conducted (Benjamini \& Hochberg, 1995).

Results: Greater cannabis use predicted smaller left rostral anterior cingulate (rACC) volumes [beta=-.29, $p<.01$; FDR corrected: $p=0.01$ ] A cannabis $\mathrm{x}$ gender interaction was observed in the left rACC; greater cannabis use predicted smaller left $\mathrm{rACC}$ volumes only within females, although this did not survive FDR corrections [beta=-.19, $p=.04$; FDR corrected: $p=.25]$. Within the cannabis-using group, Pearson correlations revealed that smaller left rACC volumes were associated with lower Emotion Discrimination Correct scores $[\mathrm{r}=.37, p=.04]$.

Conclusions: These findings may be one mechanism underlying the high prevalence of comorbid affective disorders amongst cannabis users (e.g., Lev-Ran et al., 2013). Additional interpretations and implications will be discussed.
Correspondence: Kristin E. Maple, M.S., Psychology, University of Wisconsin-Milwaukee, 1512 N. Warren Ave., APT. 207, Milwaukee, WI 53202, United States.E-mail: kemaple@uwm.edu

M. MCDONNELL, J.S. GOLDBERG, A.J. JACOBSON, G.J. LEE \& R. WHYTE. Changes in Cognitive Functioning in Patients Receiving Intensive Outpatient Treatment for Alcohol Abuse.

Objective: The objective of this study is to evaluate if those in alcohol addiction treatment population experience improvements in cognitive functioning with treatment.

Participants and Methods: Outpatient alcohol addiction patients ( $n=18 ; 56 \%$ Female; 44\% Male) were recruited from the Chemical Dependency Department at the Loma Linda University Behavioral Medicine Center. Participants were evaluated using the Repeatable Battery for the Assessment of Neuropsychological Status (RBANS) within two days of admittance, and again three weeks later. A Repeated Measures Analysis of Covariance (ANCOVA), controlling for age, was conducted to evaluate changes in cognitive functioning between baseline and 3-week follow-up for those receiving treatment for alcohol abuse at an intensive outpatient chemical dependency program.

Results: A Repeated Measures ANCOVA, controlling for age, was conducted to evaluate changes in cognitive functioning of patients with a recent history of alcohol abuse before and after completion of a chemical dependency program. There was an increase in their Total RBANS scores, $\mathrm{F}(1,16)=4.657, p=.046$, and in the delayed memory index score, $\mathrm{F}(1,16)=5.448, p=.033$. On individual subtests, there were significant increases in scores for list learning $\mathrm{F}(1,16)=5.830, p=.028$, list recall $\mathrm{F}(1,16)=5.276, p=.035$, and story recall $\mathrm{F}(1,16)=12.480$, $p=.003$. Additionally, there was an interaction effect of age and story recall $\mathrm{F}(1,16)=10.221, p=.006$.

Conclusions: In conclusion, participants engaged in an intensive outpatient treatment program experienced improvements in overall cognitive function, and more specifically in learning and memory. This suggests that individuals can experience improvements in their cognitive functioning, even after just three weeks of treatment.

Correspondence: Michelle McDonnell, Loma Linda University, 6605 Palm Avenue, Riverside, CA 92506,United States.E-mail:mmcdonnell@ llu.edu

E.E. MORGAN, K.L. DOYLE, R.K. HEATON, J. VILLALOBOS \& I. GRANT. Higher Levels of Emotion Dysregulation in Methamphetamine Users Compared to Non-Users Relates to Neurobehavioral Deficits and Craving.

Objective: The role of emotion dysregulation in the development and maintenance of addiction is well-studied, but little evidence exists regarding the degree of emotion dysregulation experienced by methamphetamine (MA) users despite an established link between MA and negative emotions. The present study hypothesized that MA users would evidence greater difficulty regulating emotions relative to controls. Correlates of emotion dysregulation were explored in the MA group, including MA use characteristics (i.e., craving, withdrawal; quantity, frequency, recency of use; age of first use) and "frontal systems" behaviors (i.e., apathy, impulsivity, disinhibition).

Participants and Methods: All participants $(N=80)$ completed a comprehensive neurobehavioral and psychiatric assessment. The MA group $(n=25)$ had a lifetime MA dependence history with MA abuse or dependence within 18 months of the exam. Participants completed the Difficulties with Emotion Recognition Scale (DERS), plus a timeline follow-back interview for MA use history, MA Craving Questionnaire-Brief, Barratt Impulsivity Scale, UPPS Impulsive Behavior Scale, and the Frontal Systems Behavior Scale.

Results: Controlling for demographics as well as psychiatric and medical risk factors, a main effect of MA on the DERS was observed: the MA group reported greater emotion dysregulation than the Non-MA group $(p=.006$; Cohen's $d=1.2)$. In the MA group, DERS was significantly correlated with measures of MA craving, disinhibition, and impulsivity 
$(p s<.05$; medium to large effect sizes); apathy and all other MA use characteristics were nonsignificant ( $p \mathrm{~s}>.05)$.

Conclusions: Elevated emotion dysregulation observed among MA users may play an important role in the real world consequences of MA use, including the addiction cycle. Our pattern of correlates suggests that poor emotion regulation may perpetuate MA addiction via increased craving and a greater tendency to be disinhibited or act impulsively. As such, emotion regulation skills may be a key target for MA addiction treatment.

Correspondence: Erin E. Morgan, PhD, University of California, San Diego, 220 Dickinson Street, HNRP/TMARC Suite B 8231, San Diego, CA 92104, United States. E-mail: eemorgan@ucsd.edu

K. MULHAUSER, K.L. ZANE, N.A. EMMERT, R. VAN PATTEN, N. ELROD \& J. WEINSTOCK. Changes in Cognition Over the Course of Residential Treatment for Alcohol Use Disorder.

Objective: Chronic heavy use of alcohol is associated with broad neuropsychological deficits. Extended abstinence (i.e., $\geq 6$ weeks) has been shown to improve cognitive abilities. However, less is known about the effects of an acute period of abstinence on cognition. The present study evaluated changes in neuropsychological status over the course of 14-day residential treatment for alcohol use disorder (AUD). We hypothesized that (a) multiple neuropsychological deficits would be evident at baseline and that (b) participants would demonstrate limited improvement in cognitive abilities over the evaluated period.

Participants and Methods: Participants $(N=27)$ were adult patients receiving hospital-based residential treatment for AUD. Participants were evaluated upon admission to the program (following detoxification) and then reevaluated prior to discharge, approximately 10 days later. Immediate and delayed memory, visuospatial abilities, semantic fluency, and attentional abilities were assessed using select subtests from the RBANS.

Results: At baseline, $81 \%$ of participants demonstrated borderline or impaired functioning $\left(<9^{\text {th }}\right.$ percentile) on one or more tests of neuropsychological functioning, with an average impairment on two of eight tests. Participants demonstrated significant improvement over the evaluation period on Figure Copy $(p=.018)$, Sematic Fluency $(p=.020)$, Coding ( $p=.012)$, Story Recall $(p=.014)$, and Figure Recall $(p<.001)$. No significant changes were observed for List Learning, Story Memory, or Digit Span.

Conclusions: These findings suggest that acute abstinence early in recovery can have a positive impact on cognition in individuals with AUD. The relatively responsive changes in visuospatial abilities, fluency, processing speed, and delayed memory may be used to guide treatment programing. These findings suggest important clinical implications for residential treatment effectiveness during the initial stages of recovery from AUD.

Correspondence: Kyler Mulhauser, MA, Psychology, Saint Louis University, 1 North Grand Blvd, Department of Psychology, Saint Louis, MO 63109, United States.E-mail:mulhauserk@slu.edu

I.M. PACHECO-COLON, S. COXE, J. DUPERROUZEL, J.M. ROSS \& R. GONZALEZ. The Association Between Cannabis Use and Motivation Among Adolescents.

Objective: Decreased motivation is often noted as a consequence of cannabis use (CU). Prior research reports mixed findings, relies on adult samples, and may not account for confounds. This study examines relationships among CU and several motivation indices among adolescent cannabis users.

Participants and Methods: Participants were 79 adolescent cannabis users ages 14-18, classified as recent regular cannabis users (RC; CU $\geq 10$ times in the past month, $\mathrm{n}=36$ ) or as light users (LC; had used but never regularly, $n=43$ ). Frequency and amount of substance use were assessed across participants' lifetime and during the past month. Motivation was measured through the Apathy Evaluation Scale (AES) and the 4 global quotients of the Motivation and Engagement Scale
(MES), which assess thoughts and behaviors affecting motivation in the high school setting. Between-group differences were examined with a series of hierarchical multiple regressions (one per motivation index) using the motivation measures as DVs. Covariates found to correlate with any of the motivation measures were entered on step 1 (i.e., IQ, sex, age, ADHD symptoms, depression, anxiety, alcohol and nicotine use) and user group (i.e. RC vs. LC) was entered on Step 2. To examine associations between amount of $\mathrm{CU}$ and motivation, we conducted a second set of regressions, with covariates on step 1 and either lifetime or past month amount of CU on step 2.

Results: After controlling for confounds, no significant differences were observed between RC and LC on any of the motivation measures, $p \mathrm{~s}>$ .05. Also, no associations between lifetime amount of $\mathrm{CU}$ and motivation were observed, $p s>.05$. Unexpectedly, greater past month amount of CU was associated with lower AES score and scores reflecting more motivation on 3 MES indices, $p \mathrm{~s}<.05$.

Conclusions: Our findings do not support a link between reduced motivation and CU among adolescents. It is possible that our participants have not been using cannabis long enough for differences in motivation to manifest. Supported by R01DA031176.

Correspondence: Ileana M. Pacheco-Colon, Psychology, Florida International University, 5091 NW 7 th St, Unit 1011, Miami, FL 33126, United States.E-mail: ipach008@fiu.edu

A. PRITCHETT \& Y. NOMURA. Effects of Prenatal Cannabis and Tobacco Exposure on Birth Outcome and Temperament.

Objective: Cannabis and tobacco are often used among women during pregnancy, yet their effects on offspring neurobehavior are still largely unknown.

Participants and Methods: The Stress in Pregnancy Study (SIPS) screened pregnant women at two urban hospital sites. Birth outcome data were recorded by the labor and delivery nurse at birth and deposited to the electronic medical record. Among the participants ( $\mathrm{N}=232)$, 38 had self-reported tobacco and/or cannabis use during pregnancy. In the follow-up, mothers were asked to prospectively report on infant temperament (Infant Behavior Questionnaire Revised; Gartstein \& Rothbart, 2003) at six months of age. A multivariable General Linear Model was used to assess differences in infant temperament between exposure groups with a priori determined potential confounders, including child's race, birth weight, in utero alcohol exposure, maternal education, marital status and maternal trait anxiety.

Results: There were no deviations in birth outcomes between groups. Tobacco exposed infants were reported to be significantly more difficult to soothe after becoming distressed $(p=.03)$. When controlling for potential confounders, tobacco exposure alone was associated with increased fearfulness $(p=.04)$ and sadness $(p=.05)$ in infants. Infants of women who reported both cannabis and tobacco use were rated significantly higher on fearfulness $(\mathrm{p}=.01)$.

Conclusions: This study confirms previous findings that prenatal tobacco exposure is associated with negative affect in infancy. Additionally, polysubstance exposure may have a synergistic effect on emotional dysregulation in offspring. Infants who display higher fearfulness/ sadness and an inability to self-regulate are at an increased risk for developing future mood disorders. This study thus reports a phenotypic expression of possible underlying limbic dysfunction in infancy due to maternal substance use during pregnancy, and highlights an early time point for possible interventions.

Correspondence: Alexandra Pritchett, B.S., Psychology, Queens College, 57 Hausman St, Brooklyn, NY 11222, United States. E-mail: lexi. pritchett@gmail.com

E. SUllivan, B. ADINOFF, M. CULluM, R. WALKER \& C. NEUMANN. Assessing Impulsivity in Cocaine Users Utilizing Hot and Cool Measures of Executive Functioning.

Objective: Prolonged cocaine use may be linked with executive functioning (EF), specifically in the areas of impulsivity and inhibition. In 
addition to examining the absolute level of $\mathrm{EF}$ across healthy individuals and substance abusers, the associations between "hot" and "cool" EF processes may shed light on underlying connectivity and overall integrity of higher-order neurobiological systems. The present study examined measures of hot and cool EF in cocaine users and controls to better understand how these two processes relate to substance abuse.

Participants and Methods: Sixty-five cocaine users (mean age $=44.1$ years; $\mathrm{SD}=6.92$ ) and 27 controls (mean age $=38.9$ years; $\mathrm{SD}=9.17$ ) completed a cognitive battery, which included measures of cool EF: Stroop Task, Trail Making Task (TMT), Wisconsin Card Sort (WCST), and Continuous Performance Task (CPT), and measures of hot EF: Iowa Gambling Task (IGT) and Balloon Analogue Risk Task (BART). Results: Cocaine and control groups differed on IGT performance. Within-group analyses revealed an inverse correlation between hot IGT performance and cool CPT commission errors for the control group $(\mathrm{r}=-.393 ; \mathrm{p}<.05)$, but not the cocaine group $(\mathrm{r}=-.003 ; \mathrm{p}=.981)$. Performance on two hot EF tasks was correlated within both cocaine ( $\mathrm{r}=.254$; $\mathrm{p}<.05)$ and control $(\mathrm{r}=.451 ; \mathrm{p}<.05)$ groups. Measures of cool EF (Stroop Task, TMT, and WCST) were more closely correlated for cocaine users than for controls.

Conclusions: Hot EF differed between groups, and patterns of hot and cool EF associations presented differently within each group. Some overlap was found between performance on hot and cool measures of response inhibition for the control group, but there was no overlap on these measures of cocaine users. Cool measures of EF were more closely related for cocaine users than for controls. This study suggests there are potential differences between substance users and controls on EF measures, and emphasizes the importance of identifying measures of hot and cool EF to best assess neurocognition in substance users.

Correspondence: Erin Sullivan, Clinical Psychology, University of North Texas, 2025 Lakepointe Drive, Apt 28D, Lewisville, TX 75057, United States.E-mail: ErinSullivan2@my.unt.edu

\section{Emotional Processes}

\section{A. ALKOZEI, R. SMITH, L. DEMERS, S. DIVATIA, M. WEBER, S. BERRYHILL \& W.D.S. KILLGORE. Emotional Intelligence Can Be trained via an Online Training Program and is Associated with Better Performance on the IGT.}

Objective: The aim of this study was to test the effectiveness of an online emotional intelligence (EI) training program for improving EI scores, as measured with self-report and performance based EI measures. In addition, we investigated whether changes in EI corresponded to changes in emotionally guided decision-making ability, as measured in performance on the Iowa Gambling Task (IGT).

Participants and Methods: Fifty-nine non-clinical men and women were randomized to receive either a 3 -week online training program targeted to improve EI $(n=29)$ or a placebo control training program $(\mathrm{n}=30)$. Participants' EI scores were measured using the Mayer-Salovey-Caruso Emotional Intelligence Test (MSCEIT) and the Bar-On Emotional Quotient Inventory (EQ-i). Participants also completed the Wechsler Abbreviated Scale of Intelligence (WASI) as a measure of IQ, and the Beck Depression Inventory (BDI-II), which were used as covariates in the analysis. Finally, participants completed the Iowa Gambling Task both at pre- and post-training.

Results: Compared to placebo, participants in the EI training group showed increased performance in total MSCEIT scores $(\mathrm{F}(1,55)=4.36$, $\mathrm{p}=.04)$, as well as on the subscales of Perceiving Emotions $(\mathrm{F}(1,55)$ $=4.65, \mathrm{p}=.04)$ and Facilitating Thought $(\mathrm{F}(1,55)=21.54, \mathrm{p}<.001)$. In addition, the EI training group showed better emotion-guided decision-making at post-training, as indexed by a steeper learning curve on the Iowa Gambling Task (IGT) $(\mathrm{F}(1,50)=3.89, \mathrm{p}=.05)$. Finally, greater increases in MSCEIT scores from pre- to post-training positively correlated with changes in performance on the $\operatorname{IGT}(\mathrm{r}=.43, \mathrm{p}=.04)$. Conclusions: The present study is the first to provide preliminary evidence that EI can be trained with the help of an online training program. In addition, such changes in EI as a result of such a program can lead to improved performance on an emotionally guided decision-making task over and above the effects of IQ.

Correspondence: Anna Alkozei, Ph.D., Department of Psychiatry, University of Arizona Medical Center, University of Arizona Medical Center, Department of Psychiatry, 1501 N Campbell Ave, Tucson, AZ 85724, United States.E-mail: aalkozei@psychiatry.arizona.edu

S. ANDERSON, A.E. WERRY \& M. DANIEL. Depression Influence on Executive Functioning and Processing Speed after accounting for Cerebrovascular Risk in Non-Demented Older Adults.

Objective: Past research suggests depression affects executive functioning (EF) and processing speed (PS). Few studies have examined this influence in older adults while accounting for cerebrovascular risk factors, the effects of different classes of antidepressants, and ruling out the effects of prodromal dementia. The objective was to determine the impact of depression on EF and PS in older adults excluding those who developed dementia within 5 years after data collection and accounting for cerebrovascular risk factors and antidepressant use.

Participants and Methods: Subjects were 822 individuals from the National Alzheimer's Coordinating Center (mean/SD: age = 74.48/6.48; education $=15.67 / 3.36)$. A series of multiple regression analyses were completed. Dependent variables included measures of PS: Digit Symbol, Trail Making Test A; and EF: Trail Making Test B, Digit Span Backwards (DSB), Animal Fluency, and Vegetable Fluency. Predictor variables were scores on the Geriatric Depression Scale-Short Form (GDS$\mathrm{SF}$ ) and clinical diagnosis of depression. Covariate variables included imaging evidence of cerebrovascular lesions (e.g., one or more strategic infarcts and/or pervasive white matter ischemia), current diagnoses of diabetes or hypertension, and current use of one or more of 4 classes of antidepressant medications (i.e., selective serotonin reuptake inhibitors, serotonin norepinephrine reuptake inhibitors, tricyclic antidepressants, and bupropion).

Results: After accounting for cerebrovascular risk factors and antidepressant use, the GDS-SF was a statistically significant predictor of scores on all EF and PS measures except DSB. Clinical diagnosis of depression was a statistically significant predictor of only DSB. However, no combination of depression variables accounted for more than three percent of the variance in scores on neuropsychological measures.

Conclusions: Although statistically significant, symptoms of depression accounted for little variance in scores on measures of EF and PS.

Correspondence: Sarah Anderson, Neuropsychology, Portland VA, 18350 NW Cornell Rd, Apt 350D, Beaverton, OR 97006, United States. E-mail: sanderson@pacificu.edu

M. BEZDEK, Z. TAIWO, G. MIRABITO \& S.N. LIGHT. Does Emotion Recognition Ability Predict Neural Responses of Empathic Happiness?

Objective: We investigated the association between performance on tasks of appraising emotions and neural responses of empathic happiness (i.e. vicarious happiness) and empathic concern (i.e. vicarious sadness) in response to viewing an emotionally evocative television program.

Participants and Methods: While functional MRI volumes were collected, 20 participants viewed a reality television program (Extreme Makeover: Home Edition) that was found to elicit empathic happiness and concern in a previous study. The program was divided into 18 sections, and participants rated their evoked happiness and concern after each segment. Participants also completed the "Happy Faces" task, which called for them to recognize neutral and various intensities of positively-valenced human facial expressions while undergoing fMRI. Results: The correct recognition of happy facial expressions elicited greater activation in the posterior cingulate cortex, relative to neutral faces. Video segments that evoked greater empathic happiness elicited increased activation in sensory and frontal regions, including in dorsomedial prefrontal cortex (DMPFC). The percent signal change in DMPFC for empathic happiness - empathic concern eliciting film 
segments was correlated with accuracy on the "Happy Faces" task $\left(R^{2}=31 \%, p=.01\right)$.

Conclusions: Judging positive emotions and experiencing vicarious happiness in response to a reality program elicit brain activation in regions recruited for social cognition. The strength of response may be associated with greater ability to infer emotional states in others.

Correspondence: Matt Bezdek, Ph.D., Psychology, Georgia Institute of Technology, Center for Advanced Brain Imaging, 831 Marietta St., Atlanta, GA 30318, United States. E-mail:mbezdek@gmail.com

S. JEEVAN, J. CRUMEDY, M. OYETEJU, T. CHATMAN, R. CHACKO, Y. ZHANG, G. BRITZ \& M. DULAY. Emotion Affects Decision Making and Reasoning After Cerebrovascular Accident (CVA).

Objective: Stroke causes significant impairments in memory, language, attention, and executive functioning. In addition, depression may occur due to functional limitations in daily activities or secondary to damage to areas of the brain involved in emotion regulation. In patients without a stroke, previous studies find that depression is associated with cognitive impairments. This study related clinical diagnosis of major depression to cognitive test performance after CVA.

Participants and Methods: Eighty-four patients (33\% female; mean age of 59.7 years) were tested an average of 4 months after stroke. Groups included unilateral cerebellar $(\mathrm{N}=25)$, frontal lobe $(\mathrm{N}=23)$, pons ( $\mathrm{N}=17)$, and thalamic $(\mathrm{N}=19)$ stroke. DSM-IV-TR diagnosis of major depressive disorder (MDD) was evaluated. Tests included age-corrected measures of abstract reasoning, phonemic fluency, problem solving, mental flexibility, and organization.

Results: The frequency of MDD ranged from $28-40 \%$ as a function of location of stroke (no between-group percentage differences). Frontal lobe patients with CVA and MDD had the highest percentage of impairments in reasoning $(38 \%)$, phonemic fluency $(62 \%)$, problem solving $(44 \%)$ and mental flexibility $(67 \%)$ compared to all other groups including frontal lobe CVA without MDD (chi-square p values $<0.05$ for each analysis). Cerebellar CVA patients with MDD had the second highest percentage of impairments compared to other groups. Individuals with pons or thalamic strokes showed no effect of depression on cognitive test performance.

Conclusions: Results were consistent with previous studies in other patient populations that indicated that patients with depression perform poorer on cognitive tasks compared to patient without depression. Disruption in brain regions involving both emotion regulation and cognition may account for these associations. Our results suggest that the presence of depression should be taken into account when evaluating executive functioning after CVA.

Correspondence: Mario Dulay, Houston methodist Hospital, 3414 Legends Garden Drive, Spring, TX 77386, United States. E-mail: mdulay@houstonmethodist.org.

P. FIgUEROA, A. VAILLANCOURT, K. BURNETT, L.K. PAUL \& W.S. BROWN. Self-Perception of Compassion in Individuals with Agenesis of the Corpus Callosum.

Objective: Previous research demonstrated that adults with agenesis of the corpus callosum (ACC) have deficits in social cognition, including theory of mind and recognizing negative emotions in the facial expressions of others (Syminton et al., 2010; Bridgman, et al., 2014). However, there is currently no research regarding how, given these social processing deficits, individuals with ACC perceive their relationships with others. The objective of the current study was to determine if individuals with ACC have a normal level of self-understanding with respect to their compassion towards others.

Participants and Methods: The Compassion for Others Scale (Pommier, 2011) was administered to 17 individuals with ACC (age: 19-55; FSIQ: 88-116) and to 25 age-and IQ-matched controls (age: 19-64; FSIQ: 98-115). This scale is a self-report measure of compassion involving six constructs of compassion: Kindness, Indifference, Common Humanity, Separation, Mindfulness, and Disengagement.
Results: Multivariate analysis of variance across the six constructs revealed greater levels of self-reported Kindness $\left(p=.045, \eta_{\mathrm{p}}{ }^{2}=.10\right)$ and Common Humanity $\left(p=.035, \eta_{\mathrm{p}}{ }^{2}=.12\right)$ for individuals with ACC when compared to neurotypical controls. However, they did not differ on the other scales.

Conclusions: The self-reports of a greater sense of kindness and solidarity provided by individuals with ACC should be considered in light of previous MMPI-2 results showing that persons with ACC have deficits in social cognition and diminished self-awareness (Brown \& Paul, 2000). Thus, the current data suggest that persons with ACC tend to report of themselves what is most socially acceptable, representing aspiration rather than behavioral self-understanding.

Correspondence: Paul Figueroa, M.A., Fuller Grad Sch of Psych, 180 N. Oakland Ave., Pasadena, CA 91101, United States. E-mail: paulfigueroa@fuller.edu

E.S. HALLOWELL, J.T. MCMAINS, S.W. LIEBEL, B. DUDA, U.S. CLARK, X. XU, R.S. NIAURA \& L.H. SWEET. The Effect of Severity of Cigarette Smoking and Early Life Adversity on Current Affect.

Objective: Exposure to early life stress (ELS) has been associated with numerous negative outcomes, including increased smoking. As exposure to negative experiences has been linked to increased negative affect, one possible explanation for this association is the use of smoking for affect regulation. Specifically, individuals exposed to higher levels of ELS may report more smoking throughout their lifetime, the use of which may lead to subsequently improved mood compared to non-smoking counterparts. The present study examined the possibility that smoking may be a mechanism to attenuate unpleasant emotional states associated with early life stress, thus emotional states may, in part, be moderated by smoking. In this study, the predictive role of early life stress and chronic smoking was examined in order to elucidate their impact on multiple measures of adult affect.

Participants and Methods: 92 individuals from the Northeastern United States $(\mathrm{M}=37.96, \mathrm{SD}=12.61)$ were sampled based smoking status (non-smokers, former smokers, current smokers). Affect and ELS were assessed using the Positive and Negative Affect Scales (PANAS) and the Early Life Stress Questionnaire (ELSQ). Pack years were calculated based on self-report of smoking intensity (e.g., number of estimated packs per day) and years smoking. Linear regression was conducted to determine the impact of pack years and ELS on current affect (i.e., positive versus negative, respectively).

Results: Pack years significantly predicted current positive affect $(B=$ $.377, \mathrm{p}<.000)$ but ELS $\operatorname{did} \operatorname{not}\left(B=-.050, \mathrm{p}=.621 ; \operatorname{model} 1 \mathrm{r}^{2}=.135\right)$. Neither pack years nor ELS predicted current negative affect $\left(\mathrm{r}^{2}=.006\right)$. Conclusions: The results suggest that smoking may serve as a means of positive affect regulation across the lifespan within a sample of individuals exposed to ELS. A better understanding of smoking as a coping mechanism for stress may inform future interventions to reduce smoking.

Correspondence: Emily S. Hallowell, M.A., Psychology, University of Georgia, 110 Pinyon Pine Circle, Apt 12, Athens, GA 30606, United States.E-mail: esmhallowell@gmail.com

L.A. KAIS, F. BRYANT, F. DOMOKOS \& R.L. SILTON. Inhibition and Shifting Processes Influence the Relation Between Savoring Beliefs and Positive Affect.

Objective: Savoring beliefs (SB) reflect an individual's perception about their capacity to enjoy past, present and future events. Positive affect (PA) refers to the extent to which an individual subjectively experiences positive mood (e.g. joy and alertness). The present study is a preliminary investigation designed to advance understanding of how individual differences in executive function (EF) influence the relation between SB and PA.

Participants and Methods: Emerging adults $(N=738)$ enrolled in an urban university completed a portion of the BRIEF-SR (Guy, Isquith, 
\& Gioia, 2004), assessing related but distinct EF domains of inhibition, shifting, and updating (Miyake et al., 2001). The Savoring Beliefs Inventory (SBI; Bryant, 2003) was administered to assess SB regarding the ability to enjoy past, present and future positive events. PA was assessed with the MASQ-AD-14 (Clark \& Watson, 1991). Nine moderation analyses were conducted using PROCESS (Hayes, 2013). The following conceptual model was used for each analysis: $\mathrm{X}=\mathrm{SB}$ [past/ present/future], M=EFs [inhibition/shifting/updating], Y=PA.

Results: The relations between SB-past/SB-present and PA were moderated by inhibition and shifting. Increased PA was evident at low levels of SB-past and SB-present when increased disinhibition was reported. Decreased PA was observed at high levels of SB-past and SB-present when increased shifting difficulties were reported. The relation between SB-future and PA was moderated by shifting, with decreased PA observed at high levels of SB-future when increased shifting difficulties were reported. Updating was not a significant moderator.

Conclusions: Results suggest that select components of EF influence the relationship between SB and PA. Additional research using experimental methods to further elucidate the relationship between SB, PA, and the role of EFs is warranted. Future positive psychology interventions may benefit from implementing strategies that target engagement of cognitive shifting during savoring to further boost PA.

Correspondence: Lorri A. Kais, MA, Clinical Psychology, Loyola University Chicago, 2337 N Spaulding Ave, Apt 1, Chicago, IL 60647, United States.E-mail: lorrikais@gmail.com

\section{A. KARSTENS, O. AJILORE, S. SHANKMAN, S. YANG, A. ZHANG, A. LEOW, A. KUMAR \& M. LAMAR. Brain-Behavior Profiles Distinguishing Psychological Resiliencefrom Depression After Trauma in an Urban Dwelling Sample of Adults: The Possible Role of Rumination.}

Objective: Depression often develops after trauma exposure and can be particularly severe and/or chronic when accompanied by rumination. Not all trauma-exposed individuals develop depression, some exhibit psychological resilience. Differences in brain-behavior relationships associated with rumination including poor inhibitory control and overactivation of self-evaluative brain networks involving the posterior cingulate may explain differences in depression risk versus resilience following trauma.

Participants and Methods: Using the SCID and the Hamiliton Depression Rating Scale, 134 trauma-exposed individuals were divided into Depressed ( $\mathrm{n}=70$, age 53 years, $34 \%$ white, $53 \%$ female) and Resilient ( $\mathrm{n}=64$; age $\sim 59$ years, $42 \%$ white, $43 \%$ female) groups. The Stroop Interference Score measured inhibitory control. Posterior cingulate was evaluated using tract-based structural connectomics in all participants. The Depressed group was younger than the Resilient group, $\mathrm{F}(1,138)=7.59, \mathrm{p}=.01$, but did not differ on other demographics.

Results: Statistical analyses adjusted for age, sex and estimated IQ. An ANCOVA for inhibitory control revealed the Depressed group had lower Stroop Interference Scores than the Resilient group, $\mathrm{F}(4,129)=5.01, \mathrm{p}=.04$. Post-hoc analysis within the Depressed group determined results were driven by multiple trauma exposures. Connectome analyses revealed the 'centrality' or importance of the left posterior cingulate to regional network communication was greater in the Depressed versus the Resilient group $(p=0.003)$; and similarly driven by multiple trauma exposures.

Conclusions: This study revealed that trauma-exposed individuals with depression, particularly those with multiple exposures, showed differences in cognition and brain structures previously associated with rumination when compared to trauma-exposed individuals resilient to depression. Future longitudinal studies are needed to determine whether these differences predict the onset of depression versus resilience following trauma.

Correspondence: Aimee Karstens, Bachelors of Science, University of Illinois, Chicago, 2134 N Maplewood Ave, Apt. 2, Chicago, IL 60647, United States. E-mail: karsten2@uic.edu
H. LiU, C. SARAPAS, L. Lieberman, E.S. STEVENS \& S. SHANKMAN. Relationships Between Emotion Regulation and Executive Functions.

Objective: Executive dysfunctions and emotion dysregulation have both been demonstrated in individuals with panic disorder (PD). However, few studies have examined whether and how these two constructs may be related in this population. One possibility is that emotion regulation deficits stem from specific problems in executive functioning only under stress. We therefore examined the association between executive function and emotion regulation in both threatening and safe contexts. Participants and Methods: Participants were 38 individuals with current panic disorder and 37 healthy controls $(70.7 \%$ female, mean age $=28.4, S D=9.2)$. Participants completed an emotion regulation task in which they were asked to increase, maintain, or decrease emotional response to each trial while eye blink startle reflex to acoustic probes was recorded. Participants also completed two computerized tasks yielding three measures of executive functioning: speeded set-shifting and inhibitory control from the Parametric Go/No-Go/Stop task, and attentional control from the Attention Network Test. To investigate whether executive functions are differentially disrupted under stress among anxious individuals, tasks were administered under both Safe (no shock) and Threat (mild electric shock to wrist at any time) conditions.

Results: Condition (safe, threat) X Group (control, PD) ANCOVAs adjusted for age did not reveal main effects of Condition or Condition $\mathrm{X}$ Group interactions for any of the three executive functioning variables $(F \mathrm{~s}<1)$. Thus, threat of shock did not affect task performance. Startle suppression ability during the emotion regulation task was positively associated with set-shifting $(r=.27, p<.05)$, inhibitory control $(r=$ $.40, p<.001)$, and attentional control $(r=.25, p<.05)$.

Conclusions: Results suggest that executive functioning was not affected by specifically threatening contexts. Instead, emotion dysregulation may be related to more general deficits in executive functioning. Correspondence: Huiting Liu, University of Illinois at Chicago, 1007 W Harrison St, M/C 285, Chicago, IL 60607, United States. E-mail: hliu74@uic.edu

\section{Z. MAHMOOD, T. KUHN, R.A. NUNEZ \& A. THAMES. Neural Correlates of Coping and Perseverance.}

Objective: Coping style and grit/perseverance are understood as independent dynamic psychological constructs that confer resiliency in the face of stress/adversity. Less is known, however, about whether these constructs are associated with one another, and the underlying neural substrates. This study investigated relationships between coping styles and grit, as well as their underlying neural correlates.

Participants and Methods: Participants consisted of 53 adults recruited from the Greater Los Angeles community. Coping styles were assessed using the BRIEF Cope questionnaire. Perseverance was examined as the total score on the Grit scale. Participants underwent MRI. Results: Grit score was positively correlated with active, planning, and positive reinterpretation and growth (PRG) coping styles ( $p$ 's $\leq .05)$, but inversely correlated with denial coping and behavioral and mental disengagement coping styles $(p ’ s \leq .01)$. Significant associations were found between total grit score and supramarginal gyrus, frontal pole, putamen and pars orbitalis (PORB), $(p\urcorner s \leq .05)$. Linear regression demonstrated that PORB $(\mathrm{B}=-.21)$, putamen $(\mathrm{B}=-.18)$, and frontal pole $(\mathrm{B}=-.18)$ each uniquely contributed to perseverance. Supramarginal $(B=-.38)$ and lingual $(\mathrm{B}=.37)$ volumes were related to active coping. Caudate $(\mathrm{B}=-.19)$, inferior temporal gyrus $(\mathrm{B}=-.18)$ and supramarginal $(\mathrm{B}=$ -.14) volumes were associated with PRG coping style. Fusiform uniquely contributed to planning $(\mathrm{B}=-.26)$ and mental disengagement coping ( $\mathrm{B}$ $=-.17)$. Inferior temporal gyrus $(\mathrm{B}=-.18)$, caudal anterior cingulate $(\mathrm{B}=-.15)$ and cuneus $(\mathrm{B}=-.10)$ volumes also contributed to mental disengagement coping.

Conclusions: Findings support past literature findings on coping and perseverance. We found both independent and overlapping gray matter regions associated with these behaviors. Understanding co-occurring 
psychological factors and their underlying mechanisms can inform therapeutic intervention.

Correspondence: Zanjbeel Mahmood, Psychiatry \& Biobehavioral Sciences, University of California, Los Angeles, 740 Westwood Plaza, 28-255, Los Angeles, CA 90095, United States. E-mail: zmahmood@ ucla.edu

M. MATHER, I. TANG, K.D. EDWARDS, G.D. SANTORELLI \& R. READY. Associations Between Alexithymia and Emotion Dysregulation.

Objective: Alexithymia has been linked to emotional dysregulation. However, the specific types of emotion dysregulation that are associated with alexithymia are not established. This study determined whether alexithymia is associated with specific difficulties with emotion regulation, controlling for depressive symptoms. We determined if links between alexithymia and emotion dysregulation were moderated by age. Older adults have more skills in emotion regulation than younger persons and alexithymia may have less impact on emotion regulation for older persons.

Participants and Methods: Younger adults $(n=49$; age 18-23; $M=$ $19.79, S D=1.25)$ and older adults $(n=42$; age 60-83; $M=66.19, S D$ $=4.83$ ) completed the 20-Item Toronto Alexithymia Scale (TAS-20; Bagby et al., 1994), the Beck Depression Inventory II (BDI-II; Beck et al., 1996), and the Difficulties in Emotion Regulation Scale (DERS; Gratz \& Roemer, 2004).

Results: In moderated multiple regressions, alexithymia significantly predicted total DERS score $(\beta=.34, p<.001)$, controlling for depression. With regard to DERS subscales, alexithymia significantly predicted lack of awareness $(\beta=.74, p<.001)$, lack of clarity $(\beta=.56, p<.001)$, and nonacceptance $(\beta=.37, p=.001)$ of emotions. Age moderated the relationship between alexithymia and lack of awareness of emotions $(p=.02)$, such that the association was stronger for younger adults. Conclusions: The contribution of alexithymia to emotion dysregulation may be limited to aspects of regulation that involve a poor understanding or acceptance of one's emotional experiences. The link with nonacceptance of emotions provides a novel perspective from which to understand the impact of alexithvmia on emotion dysregualtion. Alexithymia does not seem to be related to more instrumental problems with emotion regulation, such as difficulty carrying out goal-directed behavior. Findings indicate that alexithymia may be more detrimental to the emotion functioning of younger compared to older persons. Correspondence: Molly Mather, University of Massachusetts Amherst, Department of Psychological and Brain Sciences, University of Massachusetts, Tobin Hall, 135 Hicks Way, Amherst, MA 01003, United States.E-mail:mmather@umass.edu

R. NG, F. ROGOSCH \& D. CICCHETTI. Associations Between Memory Functioning and Internalizing Symptomatology in Children Exposed to Chronic Maternal Depression.

Objective: Young children experience marked stress when exposed to maternal depression. It has been postulated that the chronic exposure to stress adversely impacts the functional and anatomical development of neural substrates involved with stress regulation, i.e., hippocampus and prefrontal cortex, leaving the child vulnerable to developing psychiatric disorders. The goal of this study was to examine the relationships between chronic exposure to maternal depression and severity of mother's recent depressive episode with later memory and psychological functioning.

Participants and Methods: A total of 50 children with depressed caregivers (DC) and 50 comparison youth with non-depressed caregivers (NC) participated in this investigation. DC mothers were coded into chronic, recurrent, or non-recurrent depression subgroups based on longitudinal clinical data. At 9 years, child participants completed the CVLT-C and WRAML as indices of verbal and nonverbal memory, and the Children's Depression Inventory. Caregivers also completed the Child Behavior Checklist to assess their child's internalizing and externalizing behaviors. Multiple hierarchical regressions were used to examine associations between chronic maternal depression, recent depression severity, and children's later memory and emotional functioning.

Results: Chronic but not recent maternal depression was associated with later verbal memory performance (Chronic Depression: $\mathrm{B}=-0.23$, $\mathrm{p}=0.03$; Recent Depression Severity: $\mathrm{B}=-0.11, \mathrm{p}=0.26)$ and internalizing and externalizing symptoms in children $(\mathrm{Bs}>0.31, \mathrm{ps}<0.005)$. Memory indices were not associated with child's concurrent emotional adjustment.

Conclusions: Chronic rather than acute/recent exposure to maternal depression impacts hippocampal functioning, as indicated by poorer memory performance. However, memory functioning is not a strong predictor of concurrent psychological functioning. Our results broadly support postulations that prolonged exposure to parent psychopathology contributes to the allostatic load in offspring.

Correspondence: Rowena Ng, M.A., Institute of Child Development, University of Minnesota, 836 South Bundy Drive \#203, Los Angeles, CA 90049, United States.E-mail: rowenang@umn.edu

\section{WITHDRAWN: I. REIFE, N. RUSSO-PONSARAN \& C. MCKOWN. Self-Efficacy as a Moderator for the Relationship Between Theory of Mind and Social Problem Solving.}

Objective: Crick and Dodge's (1994) theory of Social Information Processing suggests that one's own effectiveness, or self-efficacy, at carrying out a desired response factors into the decision of how to respond to social encounters. Previous research suggests that perspective-taking skills are needed for effective problem solving (McKown, Allen, Russo-Ponsaran \& Johnson, 2013). This study hypothesized that that self-efficacy moderates this association such that more self-efficacy would show a stronger relation between perspective taking and theory of mind than less self-efficacy.

Participants and Methods: The current study used archival data from a larger social emotional learning study. Students (9-14 years) were recruited from a suburban Chicago school. Assessments included the NEPSY, Second Edition Theory of Mind task (Korkman, Kirk \& Kemp, 2007), a lab-developed self-efficacy self-report, and a social problem-solving assessment (McKown, Gumbiner, Russo, \& Lipton, 2009). The NEPSY measures social perspective-taking skills. The self-efficacy measure requires children to rate their abilities to execute social tasks (e.g., I am sure I can make friends). The social problem-solving assessment is a vignette-based interview in which children answer questions about challenging social situations (Bauminger, Edelsztein, and Morash, 2005).

Results: After controlling for age and IQ, self-efficacy significantly moderated the relation between perspective-taking and social problem-solving skills $\left(\mathrm{R}^{2}=.16, \mathrm{~F}(5,63)=2.41, \mathrm{p}=.05\right)$, accounting for $5.9 \%$ of the variance in social problem solving $(\mathrm{F}(1,63)=4.41, \mathrm{P}=.04)$. Conclusions: Findings show that self-efficacy moderated the relation between perspective-taking and social problem-solving; lower self-efficacy more strongly predicted this relationship than higher self-efficacy. Results suggest that when children have less confidence in their abilities to execute social interactions adequately, their ability to take another's perspective is more influential on their problem-solving abilities.

Correspondence: Ilana Reife, M.S., Illinois Institute or Technology, 1419 N Wicker Park Ave, Unit Ground, Chicago, IL 60622, United States. E-mail: igr211@gmail.com

J. RENSBERGER, E. AN, N.M. HENNIG, A.A. TURK NOLTY \& J. BACKWATER. Cortisol, DHEA, and Heart Rate Variability in Army National Guard Special Forces.

Objective: Autonomic nervous system flexibility is important for an adaptive stress response. Power spectrum analysis of heart rate variability (HRV) allows measurement of the ratio of high frequency (HF) to low frequency (LF) components of HRV, thus expressing the output of autonomic flexibility through the interaction of sympathetic and 
parasympathetic activity on the sinoatrial node of the heart. Increased parasympathetic activity is thought to be related to increased relience. A high degree of stress also activates the hypothalamic pituitary adrenal (HPA) axis, causing the adrenal cortex to secrete cortisol and dehydroepiandrosterone (DHEA) concurrently. The antiglucocorticoid effects of DHEA counter the deleterious effects of prolonged cortisol release and promote resilience to stress. Our aim was to examine the relationship between the ratio of DHEA to cortisol and HRV as both are thought to contribute to physiological and psychological resilience.

Participants and Methods: Twenty-four members of the Army National Guard Special Forces underwent virtual reality scenarios designed to induce varying stress responses. Blood DHEA, saliva cortisol, and HRV data were collected.

Results: With age controlled, we found trends toward correlations between HRV LF/HF and the ratio of DHEA to cortisol. These associations were negative following exposure to brief stressors, and positive while participants were engaged in combat-related activities and following intense. emotionally laden stressors.

Conclusions: HRV appears to measure resilience, specifically by reflecting the capacity for emotional regulation and flexible adjustment to changing situational demands. Parasympathetic dominance was related to a weaker ratio of DHEA to cortisol, whereas sympathetic dominance was related to a stronger ratio of DHEA to cortisol. These findings suggest that stress reactivity in biological systems such as the autonomic nervous system is connected with the capacity to adaptively manage environmental stressors.

Correspondence: Jared Rensberger, Fuller School of Psychology, 1436 E Orange Grove Blvd., Apt. 4, Pasadena, CA 91104, United States. E-mail: jaredrensberger@fuller.edu

\section{A. SCAVONE, M. KADZIOLKA \& C. MILLER. The Influence of Alexithymia and Mindfulness on Perceived Social Support. \\ Objective: Greater activity in the amygdala, anterior cingulate cortex (ACC), and prefrontal cortex is associated with social threat perception (Dedovic et al., 2009). Increased activity in these areas has also been noted in individuals with alexithymia, who may struggle with social connectedness (Kano \& Fukudo, 2013). In contrast, mindfulness is asso- ciated with the inhibition of evaluative processing in cortical regions/ amygdala and increased connectivity with the ACC (Farb, Anderson, \&. Segal, 2012). Thus, mindfulness training may act as a buffer against social stress for alexithymic individuals. The current study examined whether mindfulness is associated with alexithymia and social support in a non-clinical sample.}

Participants and Methods: Participants were 232 undergraduate students. They completed self-report measures: The Toronto Alexithymia Scale, The Social Provisions Scale (social support), and The Mindful Attention Awareness Scale. Linear regression analyses identified associations between alexithymia and social support, and mindfulness and social support.

Results: Alexithymia predicted social support $\left(\mathbf{R}^{2}=0.09-0.18\right)$, independent of depression scores. Mindfulness predicted alexithymia $\left(\mathbf{R}^{2}=0.32\right)$ independent of depression scores, and social support $\left(R^{2}=0.04-0.08\right)$. Conclusions: The results suggest that individuals with elevated levels of alexithymia are less likely to experience meaningful social connections and are less likely to focus on the present moment. In contrast, mindfulness was associated with a greater social connectedness. The underlying issue may be the cortico-limbic network primed to strongly react to social threat. These findings support the use of mindfulness training to enhance processing of social information at the cortical level in alexithymic individuals, which may reduce interpersonal stress. Correspondence: Antonette Scavone, Psychology, University of Windsor, 805-166 University Ave W, Windsor, ON N9A5N9, Canada. E-mail: scavone@uwindsor.ca
Z. TAIWO, M. BEZDEK \& S.N. LIGHT. The Role of Executive Function in Empathic Processing of Positive versus Negative Emotions.

Objective: Theoretical models have implicated executive function in empathy (to vicariously share the emotion of another), however, the specific contribution of executive function to empathic processing remains unclear. This study uses fMRI methodology to explore the role executive function may play in empathy for positive ("empathic happiness") versus negative ("empathic concern") emotions.

Participants and Methods: Sixteen healthy adults $\left(\mathrm{M}_{\text {age }}=20.6, S D\right.$ $=7.06,62.5 \%$ female) completed an empathy induction fMRI paradigm consisting of empathic concern, empathic happiness and neutral emotion eliciting conditions in which participants viewed video clips from a television show. In addition, participants completed neuropsychological testing. fMRI data processing was completed with AFNI.

Results: Better verbal fluency (phonemic) performance was related to stronger activation in the right insula $(p=.007)$, anterior cingulate cortex $(p=.006)$, amygdala $(p=.093)$ and nucleus accumbens shell $(p=$ $.03)$, during empathic concern eliciting video clips relative to empathic happiness eliciting video clips. However, stronger activation of the nucleus accumbens core during empathic happiness eliciting video clips was associated with better forward digit span $(p=.046)$. Furthermore, stronger activation of the frontopolar cortex during empathic happiness video clips relative to empathic concern video clips $(p=.007)$ was associated with better cognitive flexibility measured via the DKEFS semantic fluency switching subscale.

Conclusions: These findings suggest that specific facets of executive function map onto empathic concern versus empathic happiness; with complex generative motivational/ideational processes requiring cognitive flexibility and the ability to hold information in an online state possibly playing a stronger role in empathy for positive emotions, whereas simpler, evolutionarily older generative emotional/ideational processes may be more essential for empathic concern.

Correspondence: Zinat Taiwo, Psychology, Georgia State University, 1015 WASHINGTON ST SW, APT 7, ATLANTA, GA 30315, United States.E-mail: zinattaiwo@gmail.com

S. TOCCHINI, M.R. TIMPANO SPORTIELLO \& F. BENUZZI. Emotional recognition: which are differences in the same perceptual modality?

Objective: To inquire visual and auditory recognition of basic emotions (anger, sadness, happiness, disgust and fear) in patiens with lesions in cortical areas involved in emotional processing.

Participants and Methods: The patients (n.20) are composed by: Traumatic Brain Injury (12; 4 females) with temporal, frontal and fronto-temporal lesions; mesial or anterior temporal (n. 2; 1 male), amygdala and insular tumours ( 1 female), MTLE ( 2 females) and Herpetic Encephalitis (3 males). The control group is costituted by 15 subjects (9 females). To assess recognition of basic emotions we used two batteries for facial and prosody emotional recognition: Facial Emotion Recognition Battery (FERB) and the Emotional Prosody Recognition Battery (EPRB). Moreover we used a redoub and revised form of International Affective Picture System (IAPS-R) and of International Affective Digitized Sounds (IADS-R) inquiring respectively basic emotions through imagery and complex scenes and linguistic and not linguistic sounds.

Results: Four patients with mesial-temporal lesions are impaired in visual recognition of fear, disgust and sadness assessed by IAPS-R. A patient with insular- fronto- temporal tumour recognized less accurately auditory fear assessed through IADS-R. In two batteries of emotional recognition by faces and prosody (FERB and BRUE) the patients showed no impairment.

Conclusions: Our results allow to conjecture a dissociation in emotional recognition if the stimuli are primary, innate and with a direct path processing (FERB and EPRB) or if they are more complexe requiring deep processing (IAPS-R and IADS-R). These data are interesting to lay out a rehabilitation program in patients impaired in emotional recognition. 
Correspondence: Stefania Tocchini, PhD, Public Health Authority ASL Nordovest Toscana, University of Pisa and of Modena and Reggio Emilia, Cittadella della salute-via ospedale, 1, Lucca 55100, Italy. E-mail:stefania.tocchini@hotmail.it

J.T. TWAITE, H.A. BOKHARI, A.D. BONO, R.J. STAFFORD, E. ARONOVA, S.D. GOLDE, J.L. STORBECK \& J.C. BOROD. Examining Relationships between Basic Emotion Perception and Musical Training in the Prosodic, Facial, and Lexical Channels of Communication and in Music.

Objective: Intensive musical training is positively associated with a range of perceptual (e.g., auditory processing) and cognitive (e.g., visuospatial) abilities. However, less is known about relationships between musical training and emotion processing abilities, such as emotion perception. Based on preliminary literature, we hypothesized that individuals with intensive musical training would be more accurate than non-musicians in perceiving basic emotions within the prosodic, lexical, and facial channels of communication and in music.

Participants and Methods: Participants were 58 musically-trained individuals (55\% female; $M$ age $=22.0$ yrs, $M$ duration of training $=13.3$ yrs) and 61 demographically comparable healthy controls ( $48 \%$ female, $M$ age=21.1). All participants were screened for psychiatric, medical, and neurological history. They were administered measures of prosodic, lexical, facial (Borod, Welkowitz, \& Obler, 1992), and musical (Eerola \&. Vuoskoski, 2011) emotion perception, as well as additional measures to control for non-emotional (e.g., perceptual, cognitive, \& personality) factors that could influence emotion perception.

Results: Musicians scored significantly higher than non-musicians on measures of prosodic $\left(F_{(1,115)}=6.91, p=0.010\right)$ and music $\left(F_{(1,115)}=\right.$ 6.72, $p=0.011)$ emotion identification. These findings remained after controlling for relevant group differences (e.g., personality \& alexithymia). No significant group differences were obtained for the lexical or facial channels of emotion communication or for non-emotional prosodic and lexical control measures.

Conclusions: We found that musicians were more accurate than non-musicians in identifying prosodic and musical emotions, but not lexical or facial emotions. This study has implications for how musicians process emotion as compared to non-musicians. Evaluation of causal mechanisms is needed to ascertain if musical training might represent an effective intervention for emotional deficits.

Correspondence: Jamie T. Twaite, Psychology Department, CUNY Graduate Center, 35-30 Kissena Blvd, Psychology Department, Flushing, NY 11367, United States. E-mail: twaitej@gmail.com

\section{B. VAN MEURS, M. HARTNELL \& S. LISSEK. Sex Differences in} Avoidance Behavior and Associated Neural Correlates.

Objective: Females are known to suffer from anxiety disorders at higher rates than males, accounting for $55-60 \%$ of individuals with GAD and having nearly twice the risk of developing PTSD. One pathway to increased rates of anxiety disorders could be differences in avoidance behavior. Females are quicker to learn avoidance responses and have slower extinction of the avoidance response. This project analyzed a subset of data from a larger project in order to assess sex differences in maladaptive avoidance behavior and associated brain mechanisms. Participants and Methods: 22 Participants (13 Female; 9 Male) with a range of trait anxiety (Spielberger STAI) participated in an approach avoidance conflict task following Pavlovian discrimination fear conditioning. During the test phase, participants were shown stimuli with varying degrees of threat information based on the initial discrimination conditioning. Half of the trials assessed fear to these stimuli when there was no chance to avoid, and the other half assessed behavioral avoidance to the stimuli. Brain activation was measured using fMRI for each type of trial.

Results: Females demonstrated higher overall avoidance rates and higher maladaptive avoidance rates. Additionally, there were sex differences in several brain regions including posterior insula, anterior cingulate, and bilateral precentral gyrus when the avoidance response was not available. There were also sex differences in the right superior temporal gyrus and the cerebellum during the avoidance decision.

Conclusions: These results are consistent with previous work demonstrating sex differences in the processing of fear conditioning cues. Females show greater activation in somatomotor and somatosensory networks, suggesting greater anticipation of pain. This increased anticipation of pain then contributes to sex differences in valuation and decision making for the approach-avoidance conflict, leading to higher rates of avoidance in females.

Correspondence: Brian van Meurs, University of Minnesota, 942 Monroe Ave, River Forest, IL 60305, United States. E-mail: vanm0049@umn.edu

B.G. ZUCCATO \& C. ABEARE. The Role of Emotion Regulation in the Relation Between Social Integration and Stress: A Pilot Study. Objective: Stress can have deleterious effects on mental and physical health. However, a variety of factors related to social integration have been shown to mitigate the effects of stress on health outcomes. The purpose of the current study was to investigate the role of emotion regulation in the relation between several social integration variables (social isolation, loneliness, perceived and enacted social support) and stress, and to demonstrate an effect in healthy adults for future studies in traumatic brain injury (TBI) populations.

Participants and Methods: Participants were 96 undergraduate students, who completed nine questionnaires inquiring about demographic variables, perceived and objective stress, social network size, loneliness, perceived and enacted social support, emotional symptoms, and general difficulties with emotion regulation. The social variables were amalgamated into one composite social integration variable, and a mediation analysis was conducted to determine whether emotion regulation mediates the relation between social integration and stress. Results: Results revealed correlations between all variables in directions consistent with the literature, and that emotion regulation ability partially mediates the relation between social integration and stress.

Conclusions: Effective emotion regulation appears to mitigate the adverse effect of lack of social integration on stress in healthy adults. Given this finding, future research will investigate this question in TBI patients with the goal to improve management strategies for those who suffer from chronic stress and/or social isolation/loneliness, particularly after serious illness or injury, such as TBI.

Correspondence: Brandon G. Zuccato, B.A., Psychology, University of Windsor, 2258 County Rd. 46, Woodslee, ON NOR1V0, Canada. E-mail: zuccatob@uwindsor.ca

\section{Forensic Neuropsychology}

S. BENDER, B. ARREDONDO, J.J. MAHONEY \& B.A. MARCOPULOS. A Forensic Case Study Involving Unequivocal Severe Brain Injury and Unequivocal Response Bias.

Objective: We present a unique forensic case to illustrate the value of neuropsychological assessment in disentangling the effects of unequivocal severe brain injury and malingering in determining competency to stand trial.

Participants and Methods: When first evaluated, Mr. J was a 58-year-old man charged with first degree murder. Mr. J sustained a self-inflicted gunshot wound to the head. The bullet entered his head in the left submandibular region and exited at the top of the left frontal bone. GCS score was 8 . Head CT showed extensive damage to the left and right frontal lobes with midline shift. Mr. J was evaluated by multiple examiners regarding his mental status at the time of the offense, competency to stand trial, and mitigating factors at sentencing. Multiple cognitive remediation and competency restoration attempts were made across institutions.

Results: Initial competency to stand trial evaluation revealed profound aphasia and neurocognitive dysfunction, with no indications of response 
distortion or poor effort. However, subsequent CST exams showed nonsensical speech and symptom exaggeration. Evidence of malingering in the setting of brain damage is now known as "secondary malingering" and raises questions about motivations, ability to inhibit behavior, and accountability. He was later found competent but cognitive remediation and competency restoration efforts were ineffective.

Conclusions: This case study is unique, as it involves both unequivocally severe brain injury and unequivocal response bias consistent with "secondary malingering." It also raises technical and conceptual issues regarding malingering assessment, and exposes both flaws to and advances in the revision of the Slick criteria for malingering. Gaps in the current knowledge base will be reviewed, as well as discussion of ethics/professional issues specific to competency restoration/cognitive remediation, managing consultation/boundaries with other colleagues, and directions for future malingering research.

Correspondence: Scott Bender, Charlottesville, VA, United States. E-mail:SB4QK@hscmail.mcc.virginia.edu N. BUESO-IZQUIERDO, J.C. DAUGHERTY, N. HIDALGO-
RUZZANTE, M. RUBIO-GIMBERT, M. GARCÍA-LEÓN \&. M. PEREZ
GARCIA. Impulsivity and external versus internal attributions in
male batterers of intimate partner violence.
Objective: To study the relationship between reaction time in response to a task change on a test of impulsivity in executive functioning and the external or internal attribution in batterers.

Participants and Methods: Data were obtained from a sample of 75 male batterers from different prisons in Andalucia (Spain). An extensive neuropsychological protocol was administered. For this study, we present data for the Go-no go task with an interview about sociodemographic and psychological variables. Participants had a mean age of 39 years $(S D=8.391)$, and all were matched in education. Participants were divided into two groups according to answers on the following Yes or No questions related to the type of attribution (Culpability and Fairness):"Do you think your ex-partner (from the police report) is most responsible for the current situation in which you are living?" and: "Do you think the police report solicited by your ex-partner is unjust?". Student t-tests were conducted to compare these groups

Results: In terms of culpability, 44 batterers believed their ex- partners were responsible for their current situation (external), while 31 did not attribute responsibility to their partner (internal). With regard to fairness, 63 men reported believing that their sentence was unjust, while 12 believed it was fair. Results demonstrated that batterers who made external attributions performed with statistically lower reaction times on the motor inhibition component of the items related to culpability $(t=2.025, p<.047)$. Those who thought the crime was unjust performed with significantly slower reaction times $(t=2.157, p<.034)$ in response to a task change on the test for impulsivity. Nevertheless, results demonstrated that were no differences between groups on total errors or correct answers.

Conclusions: Our results suggest that executive functioning must be studied in batterers in depth, with a consideration to their behavioral reactions and responses on objective tasks. Research may allow us to better predict their behavior.

Correspondence: Natalia Bueso-Izquierdo, Personality, Evaluation and Psychological Treatment, University of Granada (Spain) [Universidad de Granada], Campus Universitario de Cartuja C.P. 18071 Granada (Granada), Granada 18071, Spain.E-mail: nbueso@ugr.es

C.V. DOMBROWSKI, K.J. YOUNG, C.A. CHEN \& K. TREADWELL. Validity Performance in an Anxious Undergraduate Sample.

Objective: Despite the prevalence of anxiety and its effect on cognitive performance, the relationship between anxiety and performance validity is rarely studied. Moreover, experimental manipulation studies frequently use undergraduate samples, a population with inconsistent patterns on performance validity tests (PVTs). This study aims to investigate validity performance in undergraduates with and without sub-clinical anxiety.

Participants and Methods: Undergraduates were prescreened into an elevated sub-clinical anxiety group and a control group based on General Anxiety Disorder-7 Scale. A total of 87 participants (mean age $=19.2$ years, $\mathrm{SD}=1.0 ; 51 \%$ female; $53 \%$ Caucasian, $40 \%$ Asian, 9\% Latino, 2\% black, and 5\% other; mean WTAR $=106$, $\mathrm{SD}=12.2$ ), split between both groups, completed a general neuropsychological battery with embedded and stand-alone PVTs (i.e., TOMM, RDS, DCT, and Rey-15); they were given encouraging instructions (i.e., "do your best") prior to testing. Standard pass/fail cutoffs were used for PVTs.

Results: Overall, $13 \%$ of the undergraduate sample failed either the RDS or the DCT; no failures were observed TOMM Trial 2 or Rey-15 Item Recall. The high anxiety group was more likely to fail the RDS, whereas the low anxiety group tended to fail the DCT (Fisher's exact test $p=.024)$. Group status was not predictive of higher failure rates overall. Conclusions: The relatively high frequency of PVT failures in this study suggest that PVTs should be incorporated into cognitive assessment of undergraduates. RDS failures in anxious individuals should be interpreted cautiously, particularly in the absence of other failures. This study adds to the mounting evidence that undergraduate study participants and anxious individuals rarely fail the TOMM.

Correspondence: Caitlin V. Dombrowski, University of Connecticut, 46 Brooksdale Rd, Brighton, MA 02135, United States. E-mail: caitlin. dombrowski@gmail.com

S. SAGAR, J. HURTUBISE, C. CHARRON, A. ZOUGARI, K. SEKE, R.M. ROTH \& L.A. ERDODI. Gender and Lateral Dominance Influences Likelihood of Failure on Performance Validity Tests.

Objective: Cutoffs on performance validity tests (PVT) may have to be adjusted based on demographic factors to minimize bias. Although research in this area is limited, Kim et al. (2010) found a gender effect on the Recognition Memory Test (RMT), and suggested different cutoffs for males $(\leq 42)$ and females $(\leq 43)$. However, the effect of lateral dominance on RMT or the Word Choice Test (WCT) is unknown. The current study aimed to replicate previously reported gender differences on the WCT, and examine the effect of lateral dominance on these measures. Participants and Methods: Archival data were collected from a mixed clinical sample of 220 patients (44.5\% male, $89.7 \%$ right-handed) medically referred for neuropsychological assessment. Mean age was 45.5 years $(\mathrm{SD}=15.7)$. Mean level of education was 13.9 years $(\mathrm{SD}=$ 2.6). Independent $t$-tests and likelihood ratios (LR) were computed for both RMT and WCT as a function of gender and lateral dominance.

Results: Females had significantly lower Perceptual Reasoning and Working Memory Index. They also were more likely to fail the RMT than males (LR: $1.40-1.65)$ at more conservative cutoffs $(\leq 37-\leq 41)$. However, failure rates were comparable (LR: 1.04-1.22) at more liberal cutoffs $(\leq 42-\leq 43)$. A similar trend was observed on WCT, although gender differences were slightly more pronounced (LR: 1.39-2.31). Left-handed patients had significantly lower FSIQ, Verbal Comprehension Index and Perceptual Reasoning Index. They also were about twice more likely to fail the RMT than right-handed patients (LR: 1.682.15). However, no significant difference in failure rates was observed on WCT (LR: 1.03-1.39).

Conclusions: To our knowledge, this is the first empirical investigation reporting a clinically significant relationship between gender and lateral dominance and PVT failure rate. If this finding is replicated using different samples and instruments, cutoffs may need to be adjusted for women and left-handed examinees to protect them against an inflated false positive rate.

Correspondence: Laszlo A. Erdodi, EMU, 174 King St, London, ON N6A1C6, Canada.E-mail:lerdodi@gmail.com 
K. SEKE, S. SAGAR, C. CHARRON, A. ZOUGARI, J. BAKER, R. ROTH \& L.A. ERDODI. The Stroop Test as a Measure of Performance Validity in Adults Clinically Referred for Neuropsychological Assessment.

Objective: This study was designed to develop performance validity indicators embedded within the Delis-Kaplan Executive Function Systems (D-KEFS) version of the Stroop task.

Participants and Methods: Archival data from a mixed clinical sample of 132 patients $\left(50 \%\right.$ male; $\left.M_{\text {Age }}=43.4 ; M_{\text {Education }}=14.1\right)$ medically referred for neuropsychological assessment were collected. Criterion measures included the Recognition Memory Test and two composites based on several independent validity indicators.

Results: An age-corrected scale score of $\leq 6$ on any of the four trials reliably differentiated psychometrically defined credible and non-credible response sets with high specificity (.87-.94) and variable sensitivity (.34-.71). An inverted Stroop effect was less sensitive (.14-.29), but comparably specific (.85-.90) to invalid performance. Aggregating the newly developed D-KEFS Stroop validity indicators further improved classification accuracy (.61-.81 sensitivity). Failing five of the new indicators was associated with a zero false positive rate. Although failing the validity cutoffs was unrelated to self-reported depression or anxiety, it was associated with higher self-reported somatic symptoms.

Conclusions: In addition to processing speed and executive function. the present findings suggest that the D-KEFS version of the Stroop task can also function as a measure of performance validity. Furthermore, a multivariate approach to performance validity assessment is generally superior to univariate models. Non-credible presentation on neuropsychological testing may be related to psychogenic factors. This finding warrants further investigation, as it may provide insight into the neuropsychiatric mechanisms behind invalid performance during cognitive testing.

Correspondence: Laszlo A. Erdodi, EMU, 174 King St, London, ON N6A1C6,Canada.E-mail:lerdodi@gmail.com

T.J. FARRER, V.STABILE, M. BROOKS \& E.D. BIGLER. Fail Rate of Performance Validity Tests in Academic Accommodation Seeking College Students: The Role of Diagnosis on Effort Measurement. Objective: Performance validity tests (PVT) are a standard component of neuropsychological assessment and are often used when testing college students who are seeking academic accommodations for suspected neurocognitive disorders. Previous studies in general patient populations find that PVT performance is attenuated by certain conditions, such as dementia or brain injury. A broad failure rate of PVTs is reported among college students seeking accommodations but no study has determined if diagnosis can impact PVT outcomes in this population.

Participants and Methods: Study sample consisted of 540 students, all of whom presented at a university clinic for assessment to determine eligibility for accommodations. As part of their evaluation 473 students were administered PVTs, which included the Word Memory Test (WMT), the Test of Memory Malingering (TOMM), and Reliable Digit Span (RDS). Following assessment, students were either found to not meet criteria for a diagnosis or were diagnosed with a condition based on DSM-IV criteria and clinician group consensus.

Results: Failure rates ranged from 2\% for TOMM Trial 2 to $21 \%$ for RDS. Those with a diagnosis ( $79 \%$ of sample) performed significantly worse on TOMM Trial 1 and RDS compared to those without a diagnosis $(p<.05)$. Groups did not differ on WMT or TOMM Trial 2. Of the total sample, $21 \%$ failed at least one PVT with RDS being the most commonly failed test. Of those that passed all other PVTs, 9\% continue to fail the RDS suggesting RDS is consistently more difficult compared to other PVTs. In fact, of those that passed all other PVTs but failed RDS, 96\% met criteria for a diagnosis and $4 \%$ did not.

Conclusions: There is a relatively low fail rate on stand-alone PVTs among academic accommodation seeking college students and fail rates depend on the test used and the diagnostic status of the examinee. Embedded PVTs, such as RDS, have higher failure rates but this appears to be influenced by diagnosis suggesting that certain PVTs require more cognitive demand than others.

Correspondence: Thomas J. Farrer, Duke University Medical Center, 153 Carden Place Drive \#C, Mebane, NC 27302, United States. E-mail: thomasfarrer@yahoo.com

K.N. FIELDS, T. STEFURAK \& B.D. HILL. Predicting Juvenile Recidivism with the Wechsler Index Scores and Personality Assessment Inventory Scores: The Role of Intraindividual Variability.

Objective: Research has demonstrated an inverse relationship between IQ and criminal behavior (Templer, 2009; Walsh, Swogger \& Kosson. 2004), as well as links between personality traits (particularly psychopathy) and offending (Hare, Clar, Grann, \& Thornton, 2000). Recent research has also shown that intraindividual variability in cognitive test performance may serve as a marker for overall neurological health (Burton, Strauss, Hultsch, Moll, \& Hunter, 2004), which Moffitt (1993) cites as a key factor in the life-course persistent offending trajectory. The present study examined the links between intelligence, personality, and intraindividual variability indicators in both domains with offending.

Participants and Methods: The study sample consisted of 301 juvenile offenders in a mid-size southeastern city in the U.S. Each youth had scores on either the WISC-IV, WISC-V or WAIS-IV, and a PAI profile. Regression analyses were conducted predicting several different types of offending using the Wechsler and PAI scores as well as intraindividual variability indicators for each participant on each test. Specifically, the standard deviation for each youth on their respective test profile was used. Age, sex, Reliable Digit Span and the PAI validity scales were entered as covariates for all analyses.

Results: The dominant predictors across different analyses of offending were the Verbal Comprehension Index and the Antisocial, and Aggression scales of the PAI. Of note is that the Working Memory Index and the Wechsler intraindividual variability score predicted drug/alcohol offending significantly. This is the only domain in which the latter factors exhibited significant relationships with offending.

Conclusions: The difficulties in predicting recidivism from factors inside the youth are highlighted here. The effect sizes were small across the analyses. The linking of intraindividual variability intelligence factor to drug/alcohol offending is intriguing and deserves further study. Correspondence: Kelly N. Fields, M.A. Psychology, Psychology, University of South Alabama, 2889 Sollie Road Apartment 1320, Mobile, AL 36695, United States.E-mail:kelly.n.fields@gmail.com

J. FOX, M. BROOK, J. STRATTON \& R. HANLON. Neuropsychological Profile and Descriptive Classifications of Mass Murderers.

Objective: As mass murders become more prominent throughout the United States, the ability to predict and prevent these crimes becomes imperative. Research indicates that mass murderers (MM) experience increased incidence of psychosocial stressors, psychiatric issues, and head trauma. However, few scientific studies of MM exist, and no prior study has examined the associations between cognitive abilities and mass murder. We examined demographic, neurologic, psychiatric, substance use, criminal, and cognitive characteristics of MM and compared MM to single murderers (SM) on these characteristics. Additionally, we proposed a sub-classification of MM based on the degree of relationship with their victims and explored group differences in the aforementioned characteristics.

Participants and Methods: MM $(n=23)$ and SM $(n=103)$ completed a clinical interview, cognitive testing, and review of records. For the classification analysis, we divided MM into three groups: family MM $(n$ $=6)$, acquaintance MM $(n=8)$, and classic MM $(n=9)$. We evaluated group differences in descriptive and cognitive chracteristics using t-tests or Wilcoxon Rank-Sum tests (continuous variables) and chi-square tests or Fisher's exact test (categorical variables). We used demographically adjusted standardized test scores to generate a cognitive profile for MM. 
We estimated magnitude of group difference among the classification groups in decriptive and cognitive characteristics with correlation coefficient and Cohen's $w$.

Results: Results suggested that MM exhibit low average abilities across cognitive domains. MM exhibited higher levels of premeditation and better cognitive abilities than SM. Subgroups of MM were distinguishable based on demographic and psychiatric variables but exhibited similar cognitive profiles.

Conclusions: Our findings suggest that MMs may possess the cognitive ability to engage in preventative or rehabilitative efforts, and that subtyping based on the degree of victim relationship may be important in this group of offenders.

Correspondence: Jaclyn Fox, Psychiatry and Behavioral Sciences, Northwestern University Feinberg School of Medicine, 3765 N. Lakewood Ave, Apt 2, Chicago, IL 60613, United States. E-mail: jaclyn.fox@northwestern.edu

C. LADUKE, J. GALLO, D. DEMATTEO, K. HEILBRUN \& T. SWIRSKY-SACCHETTI. The Neuropsychological Assessment of Justice-Involved Men: A Case for Group-Specific Norms.

Objective: Neuropsychological expertise will continue to play an increasing role in criminal legal decision making. Valid neuropsychological evidence in criminal forensic contexts requires normative data that is representative of justice-involved individuals. Unfortunately, existing normative data appears unlikely to represent justice-involved individuals due to relevant demographic (e.g., age and multicultural identity) and clinical factors (e.g.., learning deficits, ADHD, substance use, TBI, trauma). As a result, the interpretation of neuropsychological performance within justice-involved individuals using existing normative data may increase the risk of inaccurate description, invalid clinical conceptualization, and misdiagnosis of impairment. The goal of the current study is to improve the validity of neuropsychological assessment with justice-involved men.

Participants and Methods: Ninety-five men in a private correctional facility in a large mid-Atlantic state completed a battery of demographic items, neuropsychological measures (i.e., BADS, CWIT, COWAT, PMT, Ruff $2 \& 7$, SDMT, TMT), and clinical measures (i.e., intellectual functioning, educational quality, depression, ADHD, substance use, TBI, trauma).

Results: Descriptive analyses demonstrated the demographic and clinical diversity of justice-involved men. Statistical analyses, effect size calculations, and clinical analyses demonstrated that justice-involved men perform significantly below existing normative data across neuropsychological measures of intellectual functioning, attention, verbal fluency, and executive functioning. As a result, preliminary normative data are presented to inform the use of the selected measures in the neuropsychological assessment of justice-involved men.

Conclusions: Justice-involved men appear to represent a unique neuropsychological population of interest that requires group-specific normative data to help ensure neuropsychological opinions about these individuals are relevant, valid, and admissible within criminal legal decision making.

Correspondence: Casey LaDuke, PhD, School of Medicine, University of Virgina, 932 Michie Tavern Lane, Apt A, Charlottesville, VA 22902, United States.E-mail: claduke3@gmail.com

P. MARTIN, B.P. HUNTER, A.M. RACH, R.J. HEINRICHS \& R.W. SCHROEDER. Excessive Decline from Premorbid Functioning (EDPF): Assessing Performance Validity with the WAIS-IV and TOPF.

Objective: This study examined the effectiveness of two indicators of Excessive Decline from Premorbid Functioning (EDPF), calculated by comparing scores from the WAIS-IV with TOPF demographic premorbid estimates, as embedded performance validity tests (PVTs). Participants and Methods: Patients referred for neuropsychological evaluations were classified according to whether they failed 0 PVTs $(n=147)$ or $\geq 2$ PVTs $(n=41)$. Patients failing only 1 PVT or those diagnosed with dementia, intellectual disability, or left-sided cerebral vascular accident were excluded. Two new validity indicators were then created. EDPF-FSIQ was derived from the discrepancy between obtained and demographically predicted WAIS-IV FSIQ, and EDPF-V was created by adding the obtained versus predicted discrepancies for WAIS-IV WMI and VCI. Independent sample t-tests were used to examine group differences, and ROC analyses were conducted to determine classification accuracy of EDPF-FSIQ and EDPF-V.

Results: Mean (SD) EDPF-FSIQ and EDPF-V in the $\geq 2$ PVT fail group were 18.4 (11.4) and 31.8 (17.7), respectively, whereas mean EDPF-FSIQ and EDPF-V in the 0 PVT fail group were 3.5 (10.3) and 5.1 (18.3), respectively. These differences were significantly different at $p<.001$. EDPF-FSIQ resulted in an AUC of .837, classifying patients with $56 \%$ sensitivity at $\geq 90 \%$ specificity. EDPF-V resulted in an AUC of .850 , classifying patients with $61 \%$ sensitivity at $\geq 90 \%$ specificity. Conclusions: Both EDPF-FSIQ and EDPF-V demonstrated excellent discrimination between patients providing valid versus invalid test performance. These results suggest that EDPF might potentially outperform traditional embedded validity measures. Additional advantages of EDPF include incorporation of demographic estimates of premorbid ability and performance on multiple tests spanning different cognitive domains.

Correspondence: Phillip Martin, PhD, Psychiatry and Behavioral Sciences, University of Kansas School of Medicine - Wichita, 7714 E. Morrist, Wichita, KS 67207, United States.E-mail:pmartin820@gmail. com

T.J. SWIFT, R.C. NICKS, R.R. SWIFT, R. JUAN \& L.E. AGUERREVERE. Detecting Simulated Memory Malingering with Eye-Tracking technology.

Objective: Eye-tracking is a growing field used to detect eye movements and analyze human processing of visual information for interactive and diagnostic applications. Different domains in scientific research such as neuroscience, experimental psychology, computer science, and human factors have benefitted from eye-tracking methods and techniques to unobtrusively investigate the quantitative evidence underlying cognitive processes. The purpose of the current study was to determine if eye tracking technology could differentiate simulated memory malingering from legitimate performance.

Participants and Methods: The present study used a "simulator" design to determine if eye tracking variables (i.e. fixation and saccade numbers) can differentiate persons known to be intentionally feigning from legitimate response on the Test of Memory Malingering (TOMM). In the current study, 40 non-clinical volunteers' eye movement was measured while they were performing the TOMM under one of two conditions: under standard instructions (controls); under instructions to simulate memory deficit in pursuit of personal injury litigation (malingerers).

Results: Results indicated that malingerers demonstrated greater time looking at the stimulus overall (Mdiff $=0.50)$, greater time fixating on the correct target stimulus (Mdiff $=0.44)$, and greater time between first fixation and responding to the stimulus $(M d i f f=0.72)$ than the control group $[F(2,37)=3.2$ to $3.8 ; p<.05)$. Areas under the Curve $(\mathrm{AUC})$ between malingerers and control ranged from .75 to .90 , indicating that eye tracking indicators have a good discrimination between the groups. Conclusions: Therefore, eye tracking variables are capable of accurately differentiating malingering from non-malingering memory performance. Although much research is needed in the area, eye tracking technology could potentially contribute to the identification of exaggerated cognitive symptoms among patients in a variety of health care settings.

Correspondence: Timothy J. Swift, 2100 N Raguet,HSTC Bldg 105D, Nacogdoches, TX 75965, United States. E-mail: swifttj@jacks.sfasu.edu 
D.E. TRAHAN. False Positive Rates for Reliable Digit Span in Individuals with Alzheimer's Disease or Other Dementias.

Objective: Reliable Digit Span (RDS) (Greiffenstein, Baker, \& Gola, 1984) has been used to detect suboptimal effort and potential malingering in litigants claiming neurocognitive dusfunction. Studies have supported the effficacy of RDS in detecting potential malingering in litigants versus younger patients with mild TBI. Others, however, have reported false positive (FP) rates as high as $69 \%$ when using RDS in nonlitigating clinical samples, especially for individuals over age 60 (Trahan \& Ross, 2005). This study extends research on RDS by examining FP rates in individuals with Alzheimer's Disease (AD) or other dementias (OD).

Participants and Methods: The AD group included 51 individuals (23 male, 28 female) with probable Alzherier's Disease (Age: $M=76.73$, $S=7.12$ ). The OD group included 32 individuals (15 male, 17 female) with other dementias (i.e., Vascular, NPH, Trauma) (Age: $M=74.34$, $S=12.53)$. None were in litigation. All were administered the WAIS-IV along with a battery of other neuropsychological tests.

Results: Data analysis revealed RDS mean scores of $7.32(S=3.09)$ for the AD group and $7.13(S=1.43)$ for the OD group. Mean RDS scores for the two groups were not significantly different $(t=.54, p=.59)$. FP rates were examined using two different cutoff scores: RDS $<8$ and RDS $<7$. Using the higher cutoff, FP rates were $59 \%$ for the AD group and $59 \%$ for the OD group. Using the lower cutoff, FP rates were $37 \%$ for the AD group and 34\% for the OD group.

Conclusions: Although studies have demonstrated efficacy of RDS in detecting malingered neurocognitive dysfunction in relative young samples, other studies have revealedd unacceptably high FP rates in more impaired patients, especially those over age 60 (Loring et al., 2016; Trahan \& Ross, 2005). Results from this study also revealed high FP rates for dementia patients, especially when the higher cutoff of RDS $<8$ was applied. Caution is strongly advised when using RDS in dementia patients over age 60 , especially in the absence of other validity measures.

Correspondence: Donald E. Trahan, Center for Behavioral Studies, 3560 Delaware, Suite 105, Beaumont, TX 77706, United States. E-mail: dtrahan@swbell.net

D.E. TRAHAN. Specificity of the CVMT Symptom Validity Scale in Adults with Alzheimer's Disease or Other Dementias.

Objective: The Continuous Visual Memory Test (CVMT) (Trahan \& Larrabee, 1988) measures visual memory using complex, ambiguous designs and a recognition format. Larrabee (2008) developed a Symptom Validity Scale (SVS) for the CVMT to assist in detecting potential malingering in litigants claiming neurocognitive dysfunction. Studies have found moderate sensitivity in individuals with definite malingered neurocognitive dysfunction, but high specificity in people with moderate to severe TBI (Larrabee, 2008) and in normal adults of all ages (Trahan, 2015). This study extends research on the SVS by examining specificity in individuals with Alzheimer's Disease (AD) or other dementias (OD).

Participants and Methods: The AD group included 51 individuals (23 male, 28 female) with probable AD (Age: $M=76.73, S=7.12$ ). The OD group included 32 individuals (15 male, 17 female) with other dementias (i.e., Vascular, NPH. Trauma) (Age: $M=74.34, S=12.53$ ). All were administered the CVMT along with a battery of other neuropsychological tests. Testing was conducted in an outpatient office setting for all individuals.

Results: Data analysis revealed SVS Mean scores of $11.48(S=3.98)$ for the AD group and $12.53(S=4.27)$ for the OD group. Group means were not significantly different $(t=.75, p=.46)$. Using the recommended cutoff score of SVS < 14, (Larrabee, 2008), specificity rates were .35 for the AD group and .53 for the OD group.

Conclusions: Although the CVMT SVS has demonstrated high specificity for normal adults of all ages and clinical samples of younger adults with TBI, specificity rates were definitely lower for older patients with
Alzheimer's Disease or other dementias. Results suggest caution when using the SVS with individuals over age 60 with dementia.

Correspondence: Donald E. Trahan, Center for Behavioral Studies, 3560 Delaware, Suite 105, Beaumont, TX 77706, United States. E-mail: dtrahan@swbell.net

D. WHITESIDE, A. HAHN-KETTER, O. GAASEDELEN \& M.R. BASSO. Logistically Derived Embedded Performance Validity Measures Using Tests of Executive Functioning in a Mild Traumatic Brain Injury Sample.

Objective: The development of increasingly sophisticated embedded performance validity tests (PVTs) is critical for the practice of forensic neuropsychology. One recent study (Whiteside et al., 2015) utilized several cognitive measures to develop a logistically-derived cross domain embedded PVTs with acceptable classification accuracy. The current study utilized the same methodology to develop an embedded PVT utilizing three executive functioning measures, the Trailmaking Test Part B (TMT-B), Wisconsin Card Sorting Test Perseverative Responses (WCST-PR), and the Stroop Color-Word Test to study the classification accuracy of this single domain embedded PVT.

Participants and Methods: Participants included 155 consecutive neuropsychological patients with a diagnosis of mild traumatic brain injury (MTBI) who completed all the study measures. This included 95 MTBI patients with no known external incentives (mean age $=44.0$, $\mathrm{SD}=12.56$, mean education $=13.45, \mathrm{SD}=2.23,45 \%$ female, $97 \%$ Caucasian) who passed all PVTs and 60 MTBI patients who failed at least 2 PVTs (mean age $=44.1, \mathrm{SD}=15.47$, mean education $=13.05, \mathrm{SD}=2.58$, $58 \%$ male, 93\% Caucasian), including at least one free-standing measure. No significant differences between groups were found on any demographic variable except gender.

Results: A logistically derived combined variable was calculated and had excellent classification accuracy $(\mathrm{AUC}=.82$, with sensitivity at $57 \%$ with specificity set at $90 \%$ ), which was higher than any individual measure. Among the individual measures, the Stroop Color-Word test had the highest overall classification accuracy (AUC=.80, 43\% sensitivity), while the WCST-PR had the lowest (AUC=.67, 35\% sensitivity). Conclusions: The study supports the use of this logistically derived variable as an embedded PVT based upon executive functioning tests. The results also support the use of the Stroop Color-Word measure as an embedded PVT.

Correspondence: Douglas Whiteside, Ph.D., Department of Psychiatry, University of Iowa, 200 Hawkins Drive, Iowa City, IA 52242, United States.E-mail:douglas-whiteside@uiowa.edu

\section{Psychopathology/Neuropsychiatry (Including Schizophrenia)}

D.M. AASE, J.M. BABIONE, A. OSBORN, L. EVERETT, I. HASSAN, J.A. DIGANGI, E. PROESCHER, J. GREENSTEIN, R. WALTERS \& K.L. PHAN. PTSD Severity Predicts Working Memory Performance in OEF/OIF/OND Veterans.

Objective: Posttraumatic stress disorder (PTSD) is associated with poorer performance in several neuropsychological domains among U.S. military veterans. In particular, moderate effect sizes for PTSD have been observed in the domain of working memory (Scott et al., 2015). although many prior studies have not attended to subthreshold PTSD symptoms. The present study evaluated working memory performance among veterans of Operations Enduring Freedom, Iraqi Freedom and New Dawn (OEF/OIF/OND) as a function of overall PTSD severity.

Participants and Methods: Subjects were 94 (16 female, 78 male) OEF/OIF/OND veterans who were assessed using a brief neuropsychological battery, structured clinical interviews (CAPS and M.I.N.I.), and self-report instruments.

Results: When controlling for IQ, combat exposure, current alcohol use disorder, and current major depressive disorder or anxiety disorder. 
CAPS Total score was a significant predictor of Digit Span forward (Beta=-.28, $p=.024)$. Using the same covariates, CAPS Total score was also a significant predictor of Digit Span backward (Beta=-.27, $p=.041)$. While the model predicting BVMT-R T1 was significant $[\mathrm{F}(5,88)=3.16, p=.011]$, CAPS Total score was only a marginally significant predictor of BVMT-R T1 (Beta=-.25, $p=.067$ ). The model predicting CVLT-2 T1 was not significant.

Conclusions: Severity of PTSD symptoms was a significant predictor of multiple indicators of working memory performance, even when accounting for relevant covariates. These findings add to the extant literature by exploring PTSD severity as a continuous construct in a heterogeneous sample of OEF/OIF/OND veterans. Limitations of the study, clinical implications, and directions for future research are discussed. Correspondence: Darrin M. Aase, Jesse Brown VA Medical Center, 820 South Damen Avenue, Chicago, IL 60612, United States. E-mail: daase@psych.uic.edu

K. ANGERS, S. ASSARI, D. MARSHALL, P. BABU, R.E. EASTER, B. PESTER, K.H. HINRICHS, M. MCINNIS, S.A. LANGENECKER \& K. RYAN. Cognitive performance over five yearsamong individuals with bipolar disorder and unaffected controls using latent growth modeling.

Objective: Cognitive dysfunction is a key feature of bipolar disorder (BD). However, not much is known about the temporal stability of this cognitive dysfunction as some studies have demonstrated a neurodegenerative model in $\mathrm{BD}$, while others have shown no change in cognitive functioning over time. Building upon our prior work, which examined the natural course of executive functioning, the current study aimed to investigate the natural course of memory, emotion processing, and fine motor dexterity over a five year period in BD.

Participants and Methods: Using a five-year longitudinal cohort, 90 individuals with $\mathrm{BD}$ and 17 healthy controls $(\mathrm{HC})$ were administered a battery of neuropsychological tests at study baseline, 1 year, and 5 years after study entry that captured four areas of cognitive performance: visual memory, auditory memory, emotion processing, and fine motor dexterity.

Results: Latent Growth Curve Modeling showed no group differences in the slopes of any of the cognitive factors between the BD and HC groups. Age at baseline was negatively associated with visual memory, emotion processing, and fine motor dexterity. Education level was positively associated with auditory and visual memory and fine motor. Female gender was negatively associated with emotion processing.

Conclusions: Extending our prior work on executive functioning, individuals with $B D$ show similar linear change in other areas of cognitive functioning including memory, emotion processing, and fine motor, as compared to unaffected, healthy controls. Age, education, and gender may have some differential effects on cognitive changes.

Correspondence: Kaley Angers, B.S., Psychiatry-Neuropsychology, University of Michigan, 4050 Grand Oaks Trail, Burton, MI 48519, United States.E-mail: kangers@umich.edu

J.M. BABIONE, A. OSBORN, L. EVERETT, I. HASSAN, C. SCHROTH, J.A. DIGANGI, D.M. AASE \& K.L. PHAN. PTSD Symptom Severity Predicts Verbal Encoding and Retrieval in Combat-Exposed OEF/ OIF/OND Veterans.

Objective: Individuals with PTSD often demonstrate deficits in verbal memory (VM) functioning (Scott et al., 2015). While many studies have examined VM performance between individuals with PTSD and controls, there is a need for increased attention to the impact of symptom severity. In this study, we examined the relationship between PTSD symptom severity and VM performance in a group of U.S. military veterans. We hypothesized there would be a negative relationship between PTSD symptom severity and VM performance.

Participants and Methods: The sample included 81 U.S. military veterans who were deployed as part of Operations Enduring Freedom, Iraqi Freedom, and/or New Dawn (OEF/OIF/OND). Participants were assessed for combat exposure and PTSD symptoms with the CAPS-IV and were administered a brief test battery, which included the CVLT-II, BDI-II, and WTAR.

Results: Four covariates (i.e., age, education, intelligence, depression) were employed in four linear regressions to assess the impact of CAPS-IV Total score and specific PTSD symptom clusters on VM performance. Verbal encoding was predicted by Total PTSD severity $[F(5,57)=2.47$, $p=.02]$ and PTSD re-experiencing symptom severity $[F(5,57)=3.37$, $p=.01]$, while long delay free recall was predicted by PTSD avoidance symptom severity $[F(5,57)=2.45, p=.04]$.

Conclusions: These results indicate that the severity of specific PTSD symptoms may differentially impact individual domains of VM performance. It would be beneficial for future studies to address the role of these individual PTSD symptom clusters. Further research in needed to verify these findings and determine potential clinical implications when working with this population.

Correspondence: Joseph M. Babione, Jesse Brown VA Medical Center, Mental Health Service Line, 5136 S. Blackstone Ave. Apt. 207, Chicago, IL 60615, United States. E-mail: josephbabione@gmail.com

P. BABU, R.E. EASTER, K. ANGERS, J. PATEL, K. RYAN, S. ASSARI, M. KAMALI, E. SAUNDERS, M. MCINNIS, S.A. LANGENECKER \& D. MARSHALL. Impact of gender and history of childhood trauma on cognitive functioning in patients with bipolar disorder.

Objective: Few studies have investigated the potential impact of trauma and gender on cognitive functioning in Bipolar Disorder (BD). This study aimed to investigate the effect of gender and history of childhood trauma on cognitive functioning in patients with BD.

Participants and Methods: Participants completed diagnostic interviews, the Childhood Trauma Questionnaire (CTQ), symptom severity questionnaires, and neuropsychological testing resulting in eight cognitive domains (e.g. executive functioning, attention, memory, fine motor function, and emotion processing). Participants were equivalent on age, education, and IQ. Participants were classified as positive or negative for a history of trauma using CTQ cut points previously established by Heim et al. (2009). 231 individuals diagnosed with BD (46 males with trauma, 28 males no trauma, 110 females with trauma, and 47 females no trauma) were included.

Results: While controlling for depression, there was a significant group effect for fine motor function $(p=.004)$ and auditory memory $(p<.001)$ with both male groups performing significantly worse than females. There was a significant group effect for verbal fluency and processing speed $(p=.02)$ and conceptual reasoning/set-shifting $(p=.006)$, as males with trauma demonstrated significantly poorer performance compared to the other three groups. The male with trauma group also demonstrated significantly poorer performance compared to the female no trauma group on processing speed and interference resolution $(p=.02)$. Conclusions: Our findings show that male bipolar patients may be more susceptible to the cognitive consequences of childhood trauma compared to females.

Correspondence: Pallavi Babu, BA, Psychiatry - Neuropsychology, University of Michigan, 2101 Commonwealth Blvd, Ann Arbor, MI 48105, United States. E-mail: pababu@med.umich.edu

M.R. BASSO, E. ESTEVIS, D. COMBS, D. WHITESIDE \& B.W. REYNOLDS. Inpatient Depressives' Responses to Reward and Punishment Correlate with Distinct Facets of Executive Function.

Objective: Depressed patients often show an attenuated response to reward on tasks such as the Iowa Gambling Task and probabilistic reward tasks (Must et al., 2006; Pizzagalli et al., 2009), presumably reflecting abnormal mesial and ventral frontal lobe function. Few studies have evaluated the relationship between measures of executive function and response to reinforcement. Fewer still have simultaneously evaluated depressives' responses to reinforcement and punishment. We hypothesized that depressive severity would correlate with diminished 
response to both reward and punishment. We further expected that better executive function would correlate with robust response to reward and punishment.

Participants and Methods: 21 inpatients diagnosed with major depressive disorder and 19 healthy individuals participated. Mood was assessed with the Mood and Anxiety Symptom Questionnaire. Reinforcement responsivity was assessed using a probabilistic reinforcement task developed by Pizzagalli et al. (2009). The Wisconsin Card Sorting Test, Iowa Gambling Test, D-KEFS fluency and Stroop tests, and the CVLT-2 were administered.

Results: Using a $p<.01$, depression and anxiety correlated with diminished response to reward and an enhanced response to punishment on the probabilistic reinforcement task. Stroop and semantic fluency correlated with better responsivity to reward ( $r$ 's $>$.4), whereas Iowa Gambling Test correlated with better responsivity to punishment $\left(r^{\prime} s=.5\right)$.

Conclusions: These data replicate previous work involving outpatients, revealing attenuated response to reward in inpatients. Extending previous work, they show that depression enhances response to punishment. Additionally, these data show distinct relationships between reinforcement responses and aspects of executive function. Mental flexibility correlated uniquely with response to reward, whereas risky decision-making correlated with response to punishment. These data imply that different neural substrates underlie depressed patients' responses to reward and punishment.

Correspondence: Michael R. Basso, Ph.D., Psychology, University of Tulsa, 800 South Tucker Drive, Tulsa, OK 74104, United States. E-mail:michael-basso@utulsa.edu

K.L. BESSETTE, L.M. JENKINS, J.P. STANGE, S.R. DELDONNO, K. SKERRETT, L.R. KLING, P.M. MAKI, S. WEISENBACH \& S.A. LANGENECKER. Comorbid Depression and Anxiety Has Greater Top-Down and Bottom-Up Neural Emotional Processing than Depression Alone in the Remitted State.

Objective: Major Depressive Disorder (MDD) is linked with increased top-down neural activity, whereas anxiety disorders have been associated with increased bottom-up neural activation during emotion processing tasks. Studies attempting to examine the effects of comorbidity of these internalizing disorders risk confounding the effects of the active state of depression with illness-based neural emotional processing, which may have led to ambiguous results. The current study examines the effect of comorbidity (Comorbid) while the depressive illness is in remission (rMDD)

Participants and Methods: Seventy-eight young adults $(36=$ Healthy, $27=$ rMDD, $15=$ Comorbid , ages $18-23$, completed a facial emotion perception task during a functional MRI. A 3 Group (Healthy, rMDD, Comorbid) by 5 Emotions (Fear, Angry, Sad, Happy, Neutral) ANCOVA, controlling for sex, site, and movement translations, was conducted to identify significant group differences in important emotion regulation regions of interest (ROIs) for bilateral amygdalae, middle frontal gyri (MFG) and inferior frontal gyri (IFG) during accurate detection of angry, happy and neutral faces $(p<.05, \mathrm{k}=15)$.

Results: The comorbid group showed increased activation for angry, happy and neutral facial expressions in the right MFG, and bilateral IFG in comparison to rMDD. Comorbid also demonstrated increased activation in left and right amygdala during happy and neutral facial expressions, respectively, in comparison to rMDD. Comorbid demonstrated increased activation for angry and happy facial perception compared to Healthy in frontal gyri. However, Healthy showed increased activation to neutral faces compared to both comorbid and rMDD groups in right MFG and left IFG

Conclusions: Comorbidity demonstrated increased neural activity in both bottom-up and top-down happy and neutral processing. Increased clarity through subtyping within depression may better illustrate causes, mechanisms and treatments.
Correspondence: Katie L. Bessette, Department of Psychiatry, University of Illinois at Chicago, $1601 \mathrm{~W}$ Taylor Street, Room 307, Chicago, IL 60612, United States. E-mail: kbessette@psych.uic.edu

S.A. BOBHOLZ, J.L. CRAWFORD, M. VARVARIS, A. SAWA \& D.J. SCHRETLEN. Pallidal hypertrophy as a putative neural compensatory response to schizophrenia: Findings in firstepisode schizophrenia.

Objective: Globus pallidus hypertrophy is a reliable finding of brain magnetic resonance imaging (MRI) of persons with chronic schizophrenia. If this is a risk factor for schizophrenia, then it should be present from disease onset, but if it is a compensatory neural response to the disease, then it might appear only in attenuated form or not at all early in the disease. We aimed to determine whether young adults with first-episode schizophrenia (FES) show globus pallidus hypertrophy.

Participants and Methods: Structural MRI scans of 36 outpatients with FES and 68 healthy controls (HC) matched for age, sex, and race were processed with FreeSurfer 5.3.0 to estimate grey matter volume and cortical thickness measures of multiple regions of interest across the brain. Multivariate analysis of covariance was used to compare mean differences between the FES and HC groups with age, sex, and intracranial volume included as covariates and Sidak correction for multiple comparisons.

Results: As expected, the FES group showed significantly $(\mathrm{p}<.05)$ reduced grey matter volume and cortical thickness or area in several regions across the cortical surface, most extensively in frontal lobe regions bilaterally. The FES group also showed larger globus pallidus volumes bilaterally, although the differences were not statistically significant (LH FES mean (SD) in mm3 = 1925 (278) mm3, Control = 1827 (394); RH FES = $1881(276)$, Control = $1839(365)$ ).

Conclusions: Consistent with prior research, we found several areas of reduced gray matter volume and cortical thickness/area in young adults with first-episode schizophrenia. Conversely, while the FES group also showed enlarged globus pallidi bilaterally, the differences were not statistically significant. These findings are more consistent with the possibility that pallidal hypertrophy is a compensatory neural response to schizophrenia than with the idea that it is a risk factor for the disease. Longitudinal investigation of pallidal changes over the course of the disease could help clarify this.

Correspondence: Samuel A. Bobholz, 600 North Wolf Street, Baltimore, MD 21287,United States. E-mail: sabobholz@wisc.edu

\section{A. BOUCHARD \& W. SPAULDING. Anticholinergic Drug Burden Predicts Levels of Community Functioning in Outpatients With Serious Mental Illness.}

Objective: Although drugs with anticholinergic properties are understood to have deleterious effects on cognition, relatively little is known of their long-term consequences, particularly with respect to higher-order processes such as social behavior. The present study seeks to determine whether anticholinergic burden predicts treatment response among outpatients with serious mental illness (SMI), as measured by trajectories of community functioning.

Participants and Methods: The study utilized archival data from 88 outpatients with SMI who received psychiatric rehabilitation services between 2003 and 2012. The Multnomah Community Ability Scale was administered on multiple occasions over the normal course of treatment, with an average delay of 276 days between administrations. Medication data and measures of illness severity were gathered via chart review, and anticholinergic burden scores were calculated for each participant using the Anticholinergic Cognitive Burden Scale. Analyses were conducted with growth curve modeling techniques.

Results: Community functioning improved, on average, over time, $t(87)$ $=2.00, p=.049$. Anticholinergic burden was associated with community functioning at baseline, $t(86)=-2.18, p=.032$, but not with rates of change in community functioning over time, $t(86)=-0.39, p=.695$. 
When measures of illness severity were added to the model as covariates, the reported pattern of results held.

Conclusions: The overall linear trajectory for community functioning was higher or lower depending on levels of anticholinergic burden. To the extent that participants were prescribed more drugs with anticholinergic properties, they had lower levels of community functioning, even when controlling for illness severity. The findings extend the relatively sparse literature on anticholinergic burden and real-world functioning to SMI populations and psychiatric rehabilitation outcomes.

Correspondence: Axel Bouchard, Psychology, University of NebraskaLincoln, 238 Burnett Hall, Lincoln, NE 68588, United States. E-mail: axelb2@gmail.com

\section{C.Z. BURTON, K. RYAN, M. MCINNIS \& I.F. TSO. Cognition in Bipolar Disorder With and Without Psychosis.}

Objective: The presence of psychotic symptoms (hallucinations or delusions) in bipolar disorder is presumed to reflect a greater severity of illness, though the empirical literature in this area is limited and mixed. This study aimed to examine neuropsychological, clinical, and demographic differences between individuals diagnosed with affective-only bipolar disorder (BP-A) and bipolar disorder with psychosis (BP-P) in a large sample of participants followed naturalistically.

Participants and Methods: Participants were enrolled in the University of Michigan Longitudinal Study of Bipolar Disorder between 2006 and 2010; these analyses included baseline neuropsychological, clinical, and demographic variables comparing $168 \mathrm{BP}-\mathrm{A}$ participants and 213 BP-P participants matched for duration of illness.

Results: The groups did not differ in any of the eight neuropsychological domains, including auditory memory, visual memory, verbal fluency and processing speed, processing speed with interference resolution, conceptual reasoning and set-shifting, inhibitory control, fine motor skills, and emotion processing. As expected, BP-A participants had longer chronicity of affective disorder and more rapid cycling. There were no other significant demographic or clinical differences. Bayesian statistics also supported the general absence of group differences.

Conclusions: These cross-sectional results do not support the notion that bipolar disorder with history of psychosis represents a more severe illness than bipolar disorder without psychosis. In particular, in this large sample the presence of psychosis was not associated with worse neuropsychological functioning. Although identification of psychotic symptoms in bipolar disorder is important in determining pharmacological and psychotherapeutic targets for treatment, it may not factor in to prognosis as much as clinical intuition would suggest. Longitudinal and prospective follow-up will be helpful to further explore these relationships.

Correspondence: Cynthia Z. Burton, PhD, Psychiatry, University of Michigan, 4250 Plymouth Road, Rachel Upjohn Building, Ann Arbor, MI 48109, United States. E-mail: czburton@med.umich.edu

\section{CAIRNCROSS, Y. KOTELNIKOVA \& A. LAZOSKY. Insight Into Neurocognitive Functioning in Psychiatric Illness: A Comparison of Self-Report, Case Manager Report, and Objective Neuropsychological Test Data.}

Objective: Poor insight for symptoms is common in individuals with psychiatric illness. With an increased interest in implementing treatments to improve cognitive functioning, determining whether lack of insight extends to neurocognition is imperative. This study explores the convergence of ratings obtained from self-report, case manager report, and neuropsychological tests of neurocognition.

Participants and Methods: Participants with chronic mental illness (schizophrenia $[n=11]$, MDD $[n=2]$, schizoaffective disorder $[n=3]$, delusional disorder with atypical depression $[n=1]$, and psychotic disorder NOS $[n=2]$ ) were recruited from an outpatient mental health program. They completed a 4-hour neuropsychological battery and self-report measures assessing mood, sensation/perception, motor, and cognitive abilities. Case managers' assessments of the patients neurocognition were based on their observations and knowledge of the patients' daily functioning.

Results: The pattern of bivariate associations indicated that agreement between patients and case managers was poor; they agreed only on the level of tension experienced by the patients and their hearing. Patients were accurate at reporting their own problem solving ability, reading comprehension, word reading, and aspects of mood when compared to neuropsychological tests. Case managers were accurate at reporting patients' coordination, spelling, pronunciation, and verbal memory when compared to neuropsychological tests. Mean differences across groups were further analyzed using a one-way ANOVA. It is notable that patients tended to over-estimate and case managers under-estimate patients' executive functioning.

Conclusions: Results emphasize the importance of using neuropsychological assessment to more accurately determine patients' neurocognitive abilities given the limited insight of patients and discrepancy between case manager ratings and patients' actual performance on neuropsychological tests prior to designing or implementing cognitive interventions in this population.

Correspondence: Molly Cairncross, MA, Psychology, University of Windsor, Psychology Department, University of Windsor, 401 Sunset Avenue, Windsor, ONN9B3P4, Canada. E-mail: cairncrm@uwindsor. ca

B.M. CERNY, L.R. KLING, L.B. GABRIEL, N. CRANE, S.A. LANGENECKER \& A. PASSAROTTI. Convergence between scores on the BIS-11 and measures of executive function in individuals with remitted major depression.

Objective: This study examined correlations between neuropsychological measures of executive function and a self-reported measure of impulsivity in healthy controls and individuals with remitted major depression (RMD). Impulsivity, including as measured by the Barrett Impulsivity Scale (BIS-11) has been linked to poor executive function (Zhou et al., 2014 and Choi et al., 2013). Several scales on the BIS-11 have also been linked to major depression (Swann et al., 2008). This study aimed to establish a relationship between poor neuropsychological performance on measures of executive function and greater self-report of impulsivity on the BIS-11 among individuals with RMD.

Participants and Methods: 47 healthy controls and 63 individuals with RMD were administered Trail Making Test B (or D), the Stroop Color Word Test, the Parametric Go/No Go task and the Barratt Impulsivity Scale (BIS-11).

Results: Neuropsychological measures of executive function (EF) performance measures were significantly intercorrelated. BIS-11 scales were significantly intercorrelated internally and across EF measures as well. This was observed in healthy controls, with stronger performance on neuropsychological measures related to lower impulsivity scores with the BIS-11 total scale and several primary and secondary scales. This relationship was not observed in the RMD group.

Conclusions: This study did not observe a relationship between poor performance on measures of executive function and increased self-report of impulsivity on the BIS-11 in individuals with RMD. The BIS-11 has been shown to be dependent on the clinical state of respondents (Corruble et al., 2003), and self-reported EF may be less accurate. The BIS-11 therefore may not reliably detect impulsivity in RMD, demonstrating the importance of obtaining performance measures of EF to objectively establish difficulty. More research is needed to establish this relationship in individuals currently experiencing a major depressive episode.

Correspondence: Brian M. Cerny, Psychiatry, University of Illinois at Chicago, 1963 W Winona, Chicago, IL 60640, United States. E-mail: bcerny@psych.uic.edu 
S.V. CLARK, J.A. BERNARD, A. AHMADI, T.Z. KING, R.D. LATZMAN, J.A. TURNER \& V.A. MITTAL. Clinical and Cognitive Insight in Youth at Ultra High-Risk of Psychosis: Relationships with Cognition, Symptoms, and Default Mode Connectivity.

Objective: Clinical insight is awareness of having a mental disorder; cognitive insight is ability to self-reflect (self-reflectiveness; SR) and certainty in cognitions (self-certainty; SC). Insight impairment is considered a form of anosognosia and is related to prognosis. In schizophrenia it is associated with brain function and improving insight is a potential early intervention point. We investigated whether insight is impaired in youth at ultra high-risk (UHR) for psychosis, and if it is related to cognition, symptoms, and brain networks. Our goal was to identify if these processes are affected before psychosis onset and associated with neurological biomarkers that may predispose individuals to psychosis. Participants and Methods: Fifty-five UHR adolescents and 55 controls were assessed with the Structured Interview of Prodromal Symptoms, MATRICS, Scale to Assess Unawareness of Mental Disorder, and Beck Cognitive Insight Scale. Participants also underwent functional MRI scans. We tested group differences in SR and SC, and correlations among insight dimensions and clinical and cognitive measures. We also explored relationships between insight dimensions and functional connectivity of major brain networks.

Results: SR was higher in UHRs, but the groups did not differ in SC. Among UHR, poorer clinical insight was related to greater symptom severity. Functional connectivity revealed default mode (DMN) connectivity correlated negatively with SR and clinical insight in UHR, but SR did not correlate with DMN connectivity in controls.

Conclusions: Greater SR in UHR contradicts previous research in UHR and psychosis, but may be explained by either hyper-reflectiveness in UHR or an underreporting control group. As expected, clinical insight correlated with symptomatology, so increasing clinical insight may decrease symptom severity. DMN hyperconnectivity may be related to low self-reflectiveness and impaired clinical insight in UHR, suggesting a biomarker for self-disturbance before psychosis onset.

Correspondence: Sarah V. Clark, PhD, Psychology, Georgia State University, 800 Peachtree St. NE, \#8513, Atlanta, GA 30308, United States.E-mail: sclark55@student.gsu.edu

T. COMBS, J.E. ROSEBERRY \& S. HILL. Working Memory Impairments in Schizophrenia: Single Item Maintenance is Compromised Following Intact Updating.

Objective: Serial order processing deficits are evident in schizophrenia; however, it is unclear whether this deficit is a result of degradation of memory stores, a loss of the corresponding serial position information, retrieval deficits, or a vulnerability to internal processes such as updating. This study examined the vulnerability of working memory stores to updating.

Participants and Methods: Schizophrenia patients (n=33) and demographically similar healthy controls $(n=42)$ were assessed using a novel updating/maintenance task. Participants were presented with a single item to maintain and were periodically instructed to update the target item and indicate whether new items matched the target item.

Results: Mixed effects regression for overall updating accuracy revealed a significant group difference based on diagnosis $[F(1,219)=4.56 . p=.03$; $\mathrm{B}=.06]$, indicating that individuals with schizophrenia performed worse than healthy controls. Despite a similar performance pattern, the significant group by level of processing interaction was observed $[\mathrm{F}(3,219)=3.07, \mathrm{p}=.03]$. This interaction was characterized by similar accuracy after the initial response followed by a steeper decline in accuracy of subsequent responses in patients only.

Conclusions: This finding supports the notion that working memory stores are vulnerable to disruption by other internal processes, suggesting a use it and lose it phenomenon. Specifically, this pattern suggests that, in this sample of schizophrenia patients, accessing the information is sufficient to disrupt working memory maintenance.
Correspondence: Tarra Combs, M.S., Rosalind Franklin University, 7305 314th Ave, Salem, WI 53168, United States. E-mail: tarra. carrathers@my.rfums.org

J.L. CRAWFORD, C.M. FIGUEROA, C.J. ZOLLIECOFFER, S.A. BOBHOLZ \& D.J. SCHRETLEN. Effects of Demographic Characteristics on Cognitive Test Performance in Schizophrenia versus Bipolar Disorder.

Objective: Elsewhere we report that adding a predictor for the presence vs. absence of neuropsychiatric illness alters, but does not eliminate. the impact of one's demographic background and premorbid ability on cognitive performance in a diagnostically heterogeneous sample. Here we ask whether the same predictors consistently affect cognitive performance in persons with schizophrenia (SZ) or bipolar disorder (BD).

Participants and Methods: Our sample consisted of 210 outpatients with SZ and 152 with BD. The SZ group ranged in age from 15 to 63 $(\mathrm{M}=34)$ years; the BD group from 17 to $64(\mathrm{M}=38)$ years. The BD group completed more years of schooling ( $\mathrm{M}=14$; range 8 to 21$)$ than the SZ group $(M=13$; range 7 to 19$)$. Most persons with SZ were black $(57 \%)$ men $(69 \%)$. Most persons with BD patients were white $(62 \%)$ women $(61 \%)$. Premorbid ability estimates were based on the Hopkins Adult Reading Test (HART). We used multiple linear regression modeling (LRM) to estimate the amount of variance in 14 cognitive measures explained by age, sex, race, years of education, and HART scores in the $\mathrm{SZ}$ and BD groups separately.

Results: Across 14 cognitive measures, demographic characteristics accounted for a mean of $20 \%$ of the variance among patients with SZ (range: $2.6 \%$ to $35.2 \%$ ) and $19 \%$ among those with BD (range: $5.4 \%$ to $41.8 \%$ ). However, LRMs explained more variance in the SZ than BD group on tests of executive functioning $\left(\mathrm{R}_{\text {Diff }}^{2}=.17\right)$ and phonemic word fluency $\left(\mathrm{R}_{\text {Diff }}^{2}=.07\right)$. They explained more variance in the $\mathrm{BD}$ than SZ group on tests of visual memory $\left(R_{\text {Diff }}^{2}=.10\right)$, attention $\left(R_{\text {Diff }}^{2}\right.$ $=.09)$, and semantic word fluency $\left(\mathrm{R}_{\text {Diff }}^{2}=.08\right)$. On average, HART scores contributed the most (Beta $\mathrm{M}=0.30$ ) and race the least (Beta $\mathrm{M}=0.08)$ to LRMs

Conclusions: Personal and premorbid characteristics make meaningful contributions to cognitive test performance in both SZ and BD. However, the two diseases may differentially modify the effects of these characteristics, reflecting the contributions of both host and disease to cognitive performance.

Correspondence: Jeffrey L. Crawford, Bachelor of Arts in Psychology, Medical Psychiatry, Johns Hopkins University, 3307 Pinole Valley Road, Baltimore, MD 21231, United States. E-mail: jcrawf29@jhmi.edu

S.R. DELDONNO, B.J. MICKEY, A. WELDON, P. PRUITT, L.M. JENKINS, K. SKERRETT, L.R. KLING, J. ZUBIETA \& S.A. LANGENECKER. The Influence of Childhood Trauma and Affective Personality Traits on Neural Correlates of Reward Anticipation.

Objective: Childhood trauma is associated with an increased vulnerability for developing depression later in life. Additionally, certain positive and negative affective personality traits are related to deficits in reward learning that can be observed during a depressive state. The current study examined the moderating influence of childhood trauma history and affective personality traits on neural activation during the anticipation of reward.

Participants and Methods: Twenty-three participants with Major Depressive Disorder (MDD; 16 female, mean age $=26.5$, sd $=0.47$ ) and 23 healthy controls (HC; 19 female, mean age $=28.5$, sd $=8.44$ ) completed the Childhood Trauma Questionnaire (CTQ) and Behavioral Inhibition Scale/Behavioral Activation Scales (BIS/BAS; affective personality measure). Participants underwent fMRI at 3T while performing a Monetary Incentive Delay Task. The contrast of interest was win-minus-neutral (W-N) during anticipation of a $\$ .20$ or $\$ 5$ reward. Whole brain analysis was done at $\mathrm{p}=.005, \mathrm{k}=55$. 
Results: Higher CTQ scores related to greater activation in the left insula for $\mathrm{W}-\mathrm{N}$, across groups. Trait behavioral inhibition (negative affect) was negatively associated with $\mathrm{W}-\mathrm{N}$ activation in the amygdala, hippocampus, and inferior frontal gyrus, across groups. Trait reward-responsiveness (positive affect) was positively related to $\mathrm{W}-\mathrm{N}$ activation in the fusiform gyrus and dorsolateral prefrontal cortex, across groups. HCs showed greater $\mathrm{W}-\mathrm{N}$ activation than MDDs in the dorsal anterior cingulate, precentral gyrus, and precuneus.

Conclusions: History of childhood trauma, trait negative affect, trait positive affect, and diagnosis modulated neural response to reward anticipation. It is possible that childhood trauma has an influence on the development of affective personality traits and responsiveness to reward, which could increase risk of developing MDD.

Correspondence: Sophie R. DelDonno, M.A., University of Illinois at Chicago, 1601 W Taylor St, Chicago, IL 60612, United States. E-mail: sdeldonno@psych.uic.edu

R.E. EASTER, K.H. HINRICHS, P. BABU, K. ANGERS, E. ROSENZWEIG, D. MARSHALL, B. PESTER, M. MCINNIS, S.A. LANGENECKER \& K. RYAN. Clinical predictors of decline in cognitive functioning in bipolar disorder.

Objective: Our prior work has shown that individuals with bipolar disorder (BD) do not demonstrate accelerated cognitive decline over five years compared to a control group. In this study, we investigated which clinical features of BD are related to changes in cognitive functioning over five years.

Participants and Methods: Participants included 167 individuals diagnosed with BD who completed diagnostic interviews and neuropsychological testing at baseline and at a five-year follow-up. Difference scores were created between follow-up and baseline neuropsychological tests to determine patients' overall change. Participants were categorized as "decliners" versus "not decliners" based on clinically meaningful differences from a control sample. Clinical illness features were taken from the baseline diagnostic interview.

Results: In correlation, number of years with illness was negatively related to change in performance on Purdue Pegboard, California Verbal Learning Test, and Trail Making Test - Part B (TMT-B). Several clinical features, including age of onset, chronicity of illness, rapid cycling, history of mixed episodes, and number of depressive episodes were positively associated with decline on the Wisconsin Card Sorting Test and Stroop. Independent sample t-tests showed those in the TMT-B decliner group had more mood episodes per year with the illness and a younger age of mania onset. Further, decline in Purdue Pegboard performance was associated with years of illness, chronicity of psychosis, and general impact of the BD illness.

Conclusions: Changes in executive functioning, fine motor functioning, and verbal memory were associated with years with bipolar illness. Additional clinical illness features were related to changes in executive functioning, suggesting this area is most vulnerable to the bipolar disorder. Decliner groups for executive and fine motor functioning also showed worse course of illness. Future studies may pursue aspects of illness that contribute to these declines at the neural systems level.

Correspondence: Rebecca E. Easter, University of Michigan, 2101 Commonwealth Blvd, Ann Arbor, MI 48105, United States. E-mail: reaster@med.umich.edu

L.T. EYLER, S.I. DEV, T.T. NGUYEN, J. TANTIONGLOC \& T. COLEMAN. Accelerated Brain Aging in Bipolar Disorder.

Objective: Bipolar disorder (BD) has been characterized as neuroprogressive. One study found that "brain age" based on structural measures was significantly older than chronological age in BD; we aimed to extend this work by examining brain age based on multiple imaging modalities as well as clinical correlates.

Participants and Methods: Magnetic resonance images of brain structure and function were collected from 46 euthymic individuals with BD and 80 healthy comparisons (HC; age range $=30-80$ yrs). Multi-modal measures were entered into a Linear Support Vector Regression to create a predictive model of chronological age in the HC group, and, using the 100 best regressors based on recursive feature estimation, calculate a "brain age". Cross-validation with 1000 iterations was conducted to yield multiple estimates that were averaged. Chronological age was subtracted from brain age to create an age discrepancy score. Within a matched subsample ( $\mathrm{HC}$ n=53; BD n=46), we compared group discrepancy scores and calculated correlations with clinical variables.

Results: Age of the HC group was estimated within +/- 8.8 years; the most predictive measures were regional brain size, cerebral blood flow and white matter hyperintensities, and, less commonly, white matter tract integrity, regional functional response, or functional connectivity. BD brain age was older than chronological age by about 7 years, significantly different from HC $(\mathrm{p}=0.02)$. Among BD, those with more lifetime manic episodes had older brain age compared to their actual age, after accounting for actual age $(p=0.02)$. There was no relationship with gender, symptom severity, duration of illness, lifetime depressive episodes, illegal drug use, or lithium use.

Conclusions: Our finding of older brain age in relation to chronological age in BD, particularly among those with more manic episodes, is consistent with neuroprogression. Ongoing analyses will examine performance of other predictive models and determine which brain features contribute most to group differences.

Correspondence: Lisa T. Eyler, Ph.D., Psychiatry, University of California, San Diego, Mail Code 0931, 9500 Gilman Drive, San Diego, CA 92093-0931, United States.E-mail: Iteyler@ucsd.edu

\section{FORTE, D. NEWELL \& P. NESTOR. Mechanisms of Cognitive} Disturbance in Schizophrenia.

Objective: We examined the role of attentional control and processing speed in the neuropsychological disturbance in schizophrenia (SZ), as compared to healthy controls (HC). We hypothesized that attentional control and processing speed will each uniquely account for a significant portion of the variance in neuropsychological functioning across measures of intelligence and memory.

Participants and Methods: Eighty-five SZ (Mean age $=41.81$, $\mathrm{SD}=9.41)$ and $76 \mathrm{HC}($ Mean age $=41.55, \mathrm{SD}=7.99)$ completed a comprehensive neuropsychological evaluation. Trails B of the Trail Making Test (TMT) and perseverative errors (PE) of the Wisconsin Card Sorting (WCS) served as measures of attentional control, Trails A of the Trail Making Test (TMT) and the Processing Speed Index (PSI) of the Wechsler Adult Intelligence Scale-Third Edition (WAIS-III) served as measures of processing speed and WAIS-III, full-scaled IQ $($ Mean $=99.20, \mathrm{SD}=16.69)$ and Wechsler Memory Scale-Third Edition WMS-III (Mean= 95.34, SD=18.03) general memory index served as outcome measures.

Results: Multiple regression analyses indicated both attentional control and processing speed each accounted for a significant portion of variance in neuropsychological outcome measures of intelligence and memory. Attentional control measures of Trails B response time and WCS PE accounted for $25.85 \%$ of the variance in full-scale IQ for HC, compared to $19.27 \%$ for SZ. Processing speed measures of Trails A and WAIS-III PSI regressed over general memory indicated that only WAIS-III PSI accounted for a significant portion of variance in general memory $6.3 \%$ for HC, and $10.24 \%$ for SZ.

Conclusions: Attentional control measures exert a stronger influence on cognition in HC compared to SZ.

Correspondence: Mayte Forte, B.A., Neurology, University of Miami Miller School of Medicine, 1120 NW 14th Street, Miami, FL 33136,

United States.E-mail: Mayte.Forte001@umb.edu 
K.M. GRIMES, L. VOJTILA \& K.K. ZAKZANIS. The Development of Subsyndromal Positive Symptomatology: Neurocognitive Performance and Cognitive Biases in Young Adults with Schizotypal Traits.

Objective: It is well established that patients with schizophrenia demonstrate neurocognitive deficits. Furthermore, these patients exhibit evidence of cognitive biases, which have been linked to positive symptomatology. Recent research suggests that neurocognitive deficits may increase the risk of developing cognitive biases and as such, we posit that there would exist evidence of this prior to the development of a first-episode psychosis. Accordingly, we examined individuals with elevations in schizotypal traits, which some research suggests precedes a first-episode psychosis. We hypothesized that decreased neurocognitive performance would predict elevations in schizotypal traits, which in turn would lead to greater attention to threat, belief inflexibility, and jumping to conclusions biases and hence, would result in subsyndromal positive symptomatology.

Participants and Methods: Undergraduate students ( $\mathrm{N}=115)$ completed the Schizotypal Personality Questionnaire and DAVOS Assessment of Cognitive Biases. They also completed a computerized neuropsychological assessment battery (BRAINscreen).

Results: Schizotypal traits significantly predicted an attention to threat bias (medium effect), belief inflexibility bias (medium effect), and subsyndromal positive symptomatology (large effect). None of the biases were predictive of subsyndromal positive symptomatology. Neurocognitive performance and the jumping to conclusions bias were nonsignificant.

Conclusions: The mechanism explaining subsyndromal positive symptoms in patients with schizotypal traits may be different than the mechanism in patients with schizophrenia given that patients with schizophrenia demonstrate neurocognitive impairment and that cognitive biases have been shown to be related to positive symptomatology. These findings bring into question whether the presence of schizotypal traits can be considered equivalent to the schizophrenia prodrome and if different mechanisms are present.

Correspondence: Kyrsten M. Grimes, M.A., Psychological Clinical Science, University of Toronto Scarborough, 35 Wynford Heights Cres Apt 1407, Toronto, ON M3C1K9, Canada.E-mail:kyrsten.grimes@ mail.utoronto.ca

\section{L.D. HAISLEY, B.C. KAVANAUGH, J. DUPONT-FRECHETTE, C.E. GAUDET, P. TELLOCK \& K.A. HOLLER. Neuropsychological Assessment to Predict Risk for Physical Restraint and Seclusion on Children's Psychiatric Inpatient Unit.}

Objective: The American Academy of Child and Adolescent Psychiatry recommended utilizing assessment in order to predict which children are at risk for restraints and seclusions within psychiatric settings. Previous predictive assessment focused on factors such as age, history of aggression, and child diagnoses. The current study examined the potential utility of neurocognitive assessment to predict which children are at greater risk for restraints and seclusions within a psychiatric inpatient setting.

Participants and Methods: A medical chart review was conducted for 271 children who received consecutive neuropsychological assessments within a psychiatric inpatient unit from 2010-2015. Memory, fine motor, language, executive, and IQ domains were assessed with standard neuropsychological tests (z-scores). Number of restraints (holds) and seclusions were systematically documented, within the medical record, and thus tallied for each child's stay. Negative binomial regression (NBR) analyses, accounting for exposure (length of stay) and controlling for age, examined the relationship between neurocognitive scores and holds/seclusions

Results: The NBR model predicting the number of holds was significant (Likelihood Ratio $\left.X^{2}(11)=29.71, p=.002\right)$; Pseudo $R^{2}=0.107$. Trails B (incident rate ratio $=0.56)$, Stroop Color-Word $(0.95)$, CPT Comissions (1.09) and Omissions (0.96) were significant contributors. NBR model predicting seclusions was significant (Likelihood Ratio $X^{2}(11)=19.75$, $\mathrm{p}=.048)$; Pseudo $\mathrm{R}^{2}=0.072$, with Trails B (0.57) and CPT Omissions (0.96) reaching significance.

Conclusions: Controlling for age and length of stay, executive skills were related to rates of holds and seclusions within a children's psychiatric inpatient setting. Neurocognitive assessment may be helpful, in conjunction with psychiatric screening, to predict which children are at higher risk for experiencing a restraint/seclusion. Thus neurocognitive assessment in these settings may inform targeted treatment planning. Correspondence: Lauren D. Haisley, B.A., University of Connecticut, 12 Stone Pond RD, Tolland, CT 06084, United States. E-mail: lauren. haisley@uconn.edu

R.S. HUBER, D.G. KONDO, X. SHI, A.P. PRESCOT, P.F. RENSHAW \& D. YURGELUN-TODD. Relationship of Executive Functioning Deficits to N-acetyl Aspartate (NAA) and Gamma-aminobutyric Acid (GABA) in Youth with Bipolar Disorder.

Objective: Previous investigations have reported cognitive deficits in adults with bipolar disorder; however, there are few studies involving bipolar youth. Limited data on the relationship between in vivo brain chemistry and bipolar illness suggests that unique metabolite patterns may be characteristic of bipolar illness. This study utilized proton magnetic resonance spectroscopy ( ${ }^{1} \mathrm{H}$ MRS) to evaluate the relationship between neurocognitive performance and brain metabolites in youth with bipolar disorder.

Participants and Methods: Thirty participants, twenty depressed bipolar participants and 10 healthy comparison participants, ages 13 to 21, completed mood measures (CDRS, MADRS, YMRS), and a measure of executive function (WCST). Additionally, proton MRS data were acquired from a single voxel in the anterior cingulate cortex (ACC). Two-dimensional (2D) J-resolved 1H MRS sequence was used to measure ACC metabolites including $\mathrm{N}$-acetyl aspartate (NAA), a measure of neuronal integrity, and gamma-aminobutyric acid (GABA), an inhibitory neurotransmitter.

Results: There were significant relationships between WCST performance and NAA $(p<.001)$ and GABA $(p<.01)$ in the ACC in bipolar youth, such that as performance on the WCST increased, both NAA and GABA levels increased. This relationship was not observed for mood measures in bipolar youth or for neurocognitive or mood measures for healthy comparisons participants.

Conclusions: These findings build on previous observations of biochemical alterations associated with bipolar disorder and indicate that executive deficits in bipolar youth are correlated with NAA and GABA. These results suggest that cognitive deficits occur early in the course of illness and may reflect risk factors associated with altered brain metabolites. Correspondence: Rebekah S. Huber, PhD, Department of Psychiatry, University of Utah, 383 Colorow Drive, Salt Lake City, UT 84108, United States.E-mail:Rebekah.Huber@utah.edu

I.J. HUNT, M. LARSON, B. KIRWAN, E.D. BIGLER \& S.D. GALE. Cognitive control disruption and quality of life in individuals with obsessive-compulsive disorder.

Objective: Obsessive-compulsive disorder (OCD) is associated with diminished quality of life and cognitive control dysfunction. Conflict adaptation, a process underlying cognitive control, is the ability to continuously detect conflict in previous trials and adjust performance on current trials. Conflict adaptation involves interplay between the anterior cingulate cortex (ACC) and dorsolateral prefrontal cortex (dIPFC) for detecting conflict and signaling for increases in control, respectively. We hypothesized that individuals with OCD would show reduced conflict adaptation effects in response times, ACC activation, and dIPFC activation. We also expected diminished conflict adaptation to be associated with poorer quality of life.

Participants and Methods: 19 individuals with OCD and 20 healthy controls completed a Stroop task while response times and fMRI data were recorded. 2-Group (OCD, control) x 2-Previous Trial Congruency (congruent, incongruent), x 2-Current Trial Congruency (congruent. 
incongruent) ANOVAs were conducted for both response times and fMRI data. Indices of conflict adaptation were correlated with quality of life scores.

Results: There was a significant response time conflict adaptation effect collapsed across groups; however, there were no between-groups interactions or main effects. For the fMRI, the ANOVA revealed a significant cluster in the ACC with control participants showing greater ACC conflict adaptation effects relative to individuals with OCD. No differences were seen in the dIPFC. Conflict adaptation was not significantly related to quality of life.

Conclusions: Response time conflict adaptation was seen when data were collapsed across groups, but no difference was seen between groups. Diminished ACC conflict adaptation showed neural differences between groups as expected. Individuals with OCD may use different neural processes to achieve similar behavioral results to those of healthy controls.

Correspondence: Isaac J. Hunt, Ph.D., Psychology, Brigham Young University, 1709 Kitty Hawk Dr. Apt \#3, Columbia, MO 65202, United States.E-mail: isaacjameshunt@gmail.com

\section{S. HWANG \& M. KIM. Associations between depression severity and neurocognitive functions in adult patients with major depressive disorder.}

Objective: Severity of depressive symptoms is believed to predict neuropsychological functions in patients with major depressive disorder. This study investigated associations between severity of depressive symptoms and neurocognitive functions in adult patients with major depressive disorder using comprehensive neuropsychological tests.

Participants and Methods: Ten patients with mild depressive symptoms (MD) and 22 patients with severe depressive symptoms (SD) participated. Severity of depressive symptoms was determined by both self-reported (Beck Depression Inventory: BDI) and clinician-assessed (Hamilton Depression Rating Scale-17 items: HDRS-17) questionnaires. Additionally, state and trait anxiety were measured by Korean version of State-Trait Anxiety Inventory (STAI-KYZ). For the assessment of neurocognitive functions, 10 subtests of Korean-Wechsler adult intelligence scale (K-WAIS), evaluating verbal, nonverbal ability, social judgment, psychomotor speed, and attention, were administered. Executive functions were measured by Stroop test, word fluency, and design fluency tests.

Results: The MD and SD groups did not differ in any of measures evaluating neuropsychological functions. However, MD and SD group differed in the scores of the STAI-KYZ, with SD group obtaining significantly higher scores on the state $(t(30)=-3.96, p<.001)$ and trait $(t(30)=-4.13, p<.001)$ of the STAI-KYZ than MD group. Significant interaction of trait anxiety and depression severity was observed on Picture Arrangement subtest of K-WAIS.

Conclusions: Results of this study suggest that other factors, such as previous history of hospitalization, rather than severity of depressive symptoms, may predict neuropsychological deficits in patients with depression. In addition, trait anxiety possibly moderates the effect of current symptom severity on social judgment in these patients. Correspondence: Seon hee Hwang, M.A., Psychology, Sungshin Women's University, 118-3 Anam-ro, Dongdaemun-Gu, Seoul 02474, Korea (the Republic of).E-mail: antonyfor@naver.com

T. KARPOUZIAN, H. MELTZER \& J. REILLY. Eye Movement Biomarkers of High vs. Low dose Lurasidone on Prefrontal Abilities in Treatment-Resistant Schizophrenia.

Objective: Eye movement (EM) paradigms are used to examine the brain systems involved in cognitive and sensorimotor processes that are compromised in clinical populations. Recently they are used as biomarkers of pharmacological treatment effects on these systems. In recent onset schizophrenia, antipsychotic treatment has been shown to improve response latencies and antisaccade error rate and decrease memory guided saccade accuracy. Antipsychotic treatment effects on these measures in chronic, treatment-resistant schizophrenia (TRS) are less clear. This study evaluated the effects of high vs. low dose Lurasidone, an atypical antipsychotic, on EM performance in TRS.

Participants and Methods: Twenty-seven TRS patients completed EM testing after: 1) 6 weeks of $80 \mathrm{mg}$ of Lurasidone, 2) 12 weeks randomized to low dose $(80 \mathrm{mg})$ or high dose $(240 \mathrm{mg})$ Lurasidone, and 3$) 24$ weeks of randomized treatment. EM testing included: 1) a prosaccade task in which they were to shift gaze to visual targets, 2) an antisaccade task in which they were to inhibit a response towards a target and shift gaze in the opposite direction, and 3) a memory guided saccade task in which they were to shift gaze to the remembered location of a prior target.

Results: Linear mixed-effects models evaluating change over time revealed in the high dose group an increase in correct antisaccade latency $(p<.001)$, a relative improvement in antisaccade error rate, and a decrease in memory guided saccade accuracy $(p=.01)$. Only an initial $(p=.04)$ but not sustained reduction in antisaccade error rate was observed in the low dose group.

Conclusions: Among these TRS patients, high dose Lurasidone treatment resulted in decreased speed of voluntary attentional shift but improved antisaccade error rate, which may reflect a speed-accuracy trade-off. Working memory accuracy decreased after high dose treatment, similar to effects in recent onset patients. These findings suggest dissociable dose-dependent effects of antipsychotic treatment on prefrontal abilities assessed by EM testing.

Correspondence: Tatiana Karpouzian, M.S., Psychiatry and Behavioral Sciences, Northwestern University, $710 \mathrm{~N}$ Lake Shore Dr, Chicago, IL 60611, United States. E-mail: t-karpouzian@northwestern.edu

J.G. KEILP, M. GORLYN, A. BURKE, M.A. OQUENDO \& J.J. MANN. Independent Contribution of Neurocognitive Dysfunction to the Risk for Suicidal Behavior in the Context of Other Clinical Predictors.

Objective: Neurocognitive deficits in cognitive control and memory have consistently been associated with suicidal behavior in depression, but little is known about their relative importance in the context of other risk clinical risk factors. Do they contribute to risk independently, or are they a proxy for other risk factors?

Participants and Methods: Participants were 138 unmedicated, currently depressed patients with a past history of suicidal behavior and 131 depressed patients with no past suicidal behavior. Patients were well-characterized clinically and neuropsychologically (see Keilp et al., 2001, 2013). A variety of demographic and clinical severity measures, as well as neurocognitive measures previously found to distinguish past attempters - cognitive control (Stroop interference) and memory (Buschke Selective Reminding) - were used in a logistic regression, to determine the most efficient set of classifiers of past attempt status.

Results: Stepwise logistic regression revealed significant contributions of Borderline Personality Disorder $(\mathrm{OR}=4.3)$, Bipolar vs. Unipolar Depression $(\mathrm{OR}=3.0)$, and current suicidal ideation $(\mathrm{OR}=1.1)$ as significant, well-known classifiers of past suicide attempters. However, poor performance on Stroop interference measure $(\mathrm{OR}=1.3)$ and memory (Selective Reminding, OR=1.3) also contributed to the final equation, which correctly classified $71.6 \%$ of depressed patients as past attempters or not. Variables including age, sex, education level, depression severity, impulsiveness, aggression, hopelessness, substance abuse, or PTSD did not enter the equation. Neurocognitive measures were independent of suicidal ideation, and, in a supplemental analysis, distinguished past attempters from those with ideation alone.

Conclusions: More severe deficits in cognitive control and memory during a depressive episode contribute independently to the risk for suicidal attempt. They do not contribute directly to suicidal ideation, but appear to contribute to the likelihood that ideation will be enacted. Correspondence: John G. Keilp, Ph.D., Psychiatry/MIND, Columbia University/NYSPI, Box 42, 1051 Riverside Drive, New York, NY 10032, United States.E-mail:jgk13@cumc.columbia.edu 
L.R. KLING, K.L. BESSETTE, K. SKERRETT, M. PHILLIPS \& S.A. LANGENECKER. Cluster Analysis-Defined Symptom Subtypes in remitted Major Depressive Disorder.

Objective: Strategies to define more homogeneous subsets of individuals with major depressive disorder have experienced limited success. The Mood Spectrum Self-Report Scale (MOODS-SR) provides a measure of lifetime subscales of depression, mania and related symptoms. We hypothesized that the MOODS-SR might be a useful tool to define clinical subtypes (in currently remitted, RMDD) and that we could corroborate the validity of these subtypes with clinical, personality and neuropsychological measures.

Participants and Methods: A 3 solution k-cluster analysis was run with healthy control and remitted MDD participants (HC N=35, RMDD $\mathrm{N}=54$ ). MOODS-SR factor scales ( 9 depression and 7 mania scales) were used to conduct cluster analysis. One-way ANOVAs were performed to determine cluster differences in clinical, personality, and neuropsychological measures.

Results: There are 3 clusters based upon lifetime symptoms: Minimal (Min), Moderate (Mod) and Severe (Sev). Min had a balance of HC $(\mathbf{N}=35)$ and RMDD $(\mathbf{N}=8)$. The $\operatorname{Mod}(\mathrm{N}=33)$ and $\operatorname{Sev}(\mathrm{N}=13)$ were clusters of all rMDD participants. The Sev cluster had elevated MOODS bipolar scales relative to Min/Mod. Min was different from Mod, Sev on Global Assessment of Functioning (better), number of episodes (fewer), and Rumination Responsiveness Scale (lower). Mod had higher Childhood Trauma Questionnaire relative to Min, Min, and Mod had better performance in Purdue Pegboard and inhibitory control relative to Sev. Sev was better in detecting angry and fearful faces.

Conclusions: Clusters of participants defined by lifetime symptoms, and not current symptoms, may lead to more homogeneous subsets; herein a small subset with higher lifetime bipolar symptoms, more impulsivity, poorer bimanual coordination, and better detection of fearful and angry faces. Greater homogeneity in subtyping could, in turn, then lead to early detection and or more specific treatments. The MOODS-SR measures various facets of mood disorders and even in young adults can be used to define subgroups by lifetime symptoms. Correspondence: Leah R. Kling, B.S. in Psychology, Psychiatry, University of Illinois at Chicago, $1601 \mathrm{~W}$ Taylor Street, Chicago, IL 60612, United States. E-mail: leahrkling@gmail.com

J.K. LAI, N. COHEN, C. TOBIN, B. MEFFERT, D. BLONIGEN, M. BONN-MILLER \& A.J. HEINZ. Subjective and Objective Measures of Impulsivity: Relations with Clinical Symptom Severity and Psychosocial Functioning Among Military Veterans with Alcohol Use Disorder and Posttraumatic Stress Disorder.

Objective: Impulsivity, a multi-faceted construct characterized by unplanned actions and disregard for long-term consequences, is associated with a host of negative outcomes. This study examined how subjective (self-report) and objective (neuropsychological) measures of impulsivity, which tend to correlate weakly, were associated with clinical symptom severity and psychosocial functioning among veterans with Alcohol Use Disorder (AUD) and Posttraumatic Stress Disorder (PTSD). Participants and Methods: Fifty two veterans with AUD and PTSD completed self-reported (Difficulties in Emotion Regulation Scale, Impulsivity subscale; UPPS-P Impulsive Behavior Scale) and neuropsychological (Conners Continuous Performance Test, CPT-3; Iowa Gambling Task, IGT; Balloon Analogue Risk Task, BART; Delis-Kaplan Executive Function System Color-Word Interference Test, DKEFS $\mathrm{CW}$ ) measures of impulsivity along with clinical and psychosocial functioning indices (Clinician Administered PTSD Scale; Alcohol Use Disorder Identification Test; Inventory of Psychosocial Functioning). Zero-order correlations were conducted to examine relations among clinical symptoms, psychosocial functioning, and subjective and objective measures of impulsivity.

Results: Higher levels of self-reported impulsivity were associated with poorer psychosocial functioning and elevated PTSD symptoms. Elevated intrusive PTSD symptoms were associated with earning more money on IGT; heightened cognitive/mood alterations were associated with more CPT-3 commission errors. Minimal relations emerged between subjective and objective measures of impulsivity. Lack of perseverance (UPPS-P) was correlated with poorer inhibition-switching (DKEFS $\mathrm{CW}$ ). Sensation seeking (UPPS-P) was correlated with more balloons popped (BART).

Conclusions: Different facets of impulsivity conferred risk for different aspects of impairment, suggesting that multimodal assessment of impulsivity may aide in treatment planning for this high-risk population.

Correspondence: Jennifer K. Lai, M.S., Palo Alto University, 1791 Arastradero Road, Palo Alto, CA 94304,United States. E-mail: jlai@ paloaltou.edu

H. LUU, M.L. MILLER, O. GAASEDELEN, A.E. HAHN-KETTER, J.D. BAYLESS, D. MOSER \& D. WHITESIDE. Long-Term Cognitive Functioning in Post-Electroconvulsive Therapy (ECT) Patients.

Objective: Electroconvulsive therapy (ECT) is an effective treatment for affective disorder, especially for those resistant to other therapies, but ongoing cognitive complaints remain a prevalent concern among patients and the public. Studies revealed cognitive deficits associated with ECT typically resolve within a few days posttreatment and individuals even demonstrate improvement after ECT. Limited studies have investigated post-acute effects of ECT across multiple cognitive domains and none have studied individuals several years post-ECT. Thus, this study examined changes in cognitive functioning up to 14 years post-ECT.

Participants and Methods: Individuals who completed baseline neuropsychological assessment pre-ECT within the last 14 years were recruited for follow-up neuropsychological assessment. Participants $(N$ $=33$ ) were administered the RBANS both pre and post-ECT. Separate regression models were constructed predicting post-ECT RBANS domain scores (e.g., Immediate Memory, Delayed Memory, Visual Spatial, Language, and Attention), while controlling for baseline scores. Additional predictor variables included: baseline domain scores, interval since last ECT, age, education, and primary psychiatric diagnosis (Major Depressive Disorder or Bipolar Disorder).

Results: After controlling for baseline cognitive functioning and demographic variables, results indicated significant (i.e., $\mathrm{p}<0.05)$ gains in post-ECT score in Immediate Memory (delta $R$ squared=0.037) and Language (delta $\mathrm{R}$ squared=0.057), and reduction in Visuospatial/ Constructional score (delta $\mathrm{R}$ squared $=0.043$ ).

Conclusions: To date, this is the first long-term follow-up study to investigate cognitive performance following ECT. These findings suggest that memory and other cognitive domains tend to remain stable even several years later post treatment and are consistent with the current literature. Future studies should examine subjective memory complaints and its correlation with objective data.

Correspondence: Hien Luu, Psychiatry, University of Iowa Hospitals and Clinics, 17 N. Dearborn, Chicago, IL 60602, United States. E-mail: hluu@my.adler.edu

M. MILLER, V. ZEMON, D. GOFF, L. BALCER, E. DIMINICH, L. HASANAJ, R. NOLAN \& P. BUTLER. Optical Coherence Tomography of the Retina in Schizophrenia: Relationships with Perceptual Function.

Objective: To examine thickness of the retinal nerve fiber layer (RNFL, includes ganglion cell axons), ganglion cell/inner plexiform layer (GCL+IPL), and macular volume in people with schizophrenia (SCZ). We also sought to evaluate the relation of these structural measures to visual function.

Participants and Methods: This study included six participants with SCZ and six healthy controls (CON). RNFL thickness, macular volume, and GCL+IPL thickness were assessed with spectral-domain optical coherence tomography (SD-OCT). The King-Devick test (K-D), a test of rapid number naming, assessed efferent visual function, capturing saccadic eye movements, attention, and speed of processing. 
Results: There was no significant difference between groups in RNFL thickness and macular volume. However, the GCL+IPL was significantly thinner in SCZ compared to CON $(p=.02)$. K-D time scores were significantly longer (worse) in SCZ compared to CON $(p=.03)$. For people with SCZ, longer K-D scores were significantly correlated with decreased GCL+IPL thickness $(p=.006)$.

Conclusions: Our findings suggest that neuronal degeneration may be present in the retinas of people with SCZ, and this may contribute to visual impairments found in the disorder. While speculative, a volumetric loss in ganglion cell bodies and dendrites (seen in GCL+IPL findings) might be detected more readily than axonal loss in SCZ, and therefore, may be a more sensitive measure of early degeneration in the disease.

Correspondence: Margaret Miller, M.A., Ferkauf Graduate School of Psychology, Yeshiva University, 170 E. 108 St., Apt. 3, New York, NY 10029, United States. E-mail: MargaretArlene@gmail.com

M.L. MILLER, H. LUU, O. GAASEDELEN, J.D. BAYLESS, D. MOSER \& D. WHITESIDE. Depression, Anxiety, and Quality of Life in Individuals who have undergone Electroconvulsive Therapy.

Objective: Electroconvulsive therapy (ECT) is associated with positive outcomes for treatment-resistant mood disorders (Dierckx et al., 2012). However, there is limited research on long-term cognitive and psychological changes beyond one year post-ECT. Thus, this study evaluated whether longstanding improvement in anxiety, depression and quality of life occurred following ECT.

Participants and Methods: Participants who completed a brief pre-ECT neuropsychological assessment within the last 14 years were recruited for a long-term follow-up evaluation. At follow-up, participants $(N=34)$ were administered cognitive, emotional measures (Beck Depression Inventory [BDI-II], Beck Anxiety Inventory [BAI]) functioning measures, and the WHO Quality of Life (QoL) Measure.

Results: Controlling for baseline BDI score, age, education, time post-ECT, and diagnoses (Major Depressive Disorder and Bipolar Disorder), significant reductions in depression were observed at follow up (Beta $=-17.34 ; \mathrm{R}$ squared $=0.41)$. A MANOVA indicated significant differences across QoL domains, including significantlymhigher levels of environment resources $(\mathrm{M}=70, \mathrm{SD}=15.7)$ but lower levels of psychological well-being $(M=42, S D=22.4)$. Significant negative correlations $(\mathrm{r}<-0.40)$ were observed between QoL domains and BDI/ BAI scores. Conclusions: This is the first study to examine long-term psychological well-being in individuals who have undergone ECT. These findings suggest significant decline in depressive symptoms following ECT that appear to remain stable across time, consistent with the current literature showing that ECT improves depressive symptoms (Bayless et al., 2010). However, participants were still endorsing lower levels of psychological well-being, especially individuals with elevated anxiety and/or depressive symptoms. Future research should continue to examine how to best help individuals with treatment-resistant depression as well as examine which variables may predict depressive symptoms after ECT. Correspondence: Michelle L. Miller, PhD Candidate, Psychology, University of Iowa, 11 Seashore Hall E., Iowa City, IA 52245, United States.E-mail:michelle-miller-2@uiowa.edu

T.T. NGUYEN, S.I. DEV, A.N. SUTHERLAND-OWENS, C.A. DEPP \& L.T. EYLER. Relationship Between Short-term Intra-individual Variability in Affective Symptoms and Cognitive Performance in Bipolar Disorder.

Objective: Mood/affect variability and neurocognitive dysfunction are hallmark features of bipolar disorder (BD). Although it is generally understood that cognitive impairment persists across various mood states, the association between day-to-day affect regulation and cognition is unclear. This study evaluated intra-individual variability (IIV) in affect, collected via ecological momentary assessment, and how it relates to cognition in BD and healthy individuals.
Participants and Methods: Outpatients with BD $(n=17)$ and healthy comparison subjects (HC; $n=46$ ) were administered a neuropsychological battery. They were then provided with mobile devices for 2 weeks and completed 3x-daily surveys about their affect. IIV in momentary affect ratings across time was calculated for each subject. We examined the association between positive and negative affect IIV and cognition (processing speed, executive function, working memory) using multiple regressions with an interaction term to test for differential correlations between groups.

Results: BD patients had significantly greater positive and negative affect IIV compared to HC and worse cognitive performance in all domains. For negative affect, a significant GroupxIIV interaction was observed for processing speed and a trend for executive function $(p=.07)$; HCs with greater negative affect IIV had slower processing speed $(p=.001)$ and poorer executive function $(p=.02)$, but no relationships were found in BD. Conversely, a marginally significant interaction was observed for positive affect $(p=.08)$, such that BDs with greater positive affect IIV had worse working memory $(p=.04)$, but not HCs.

Conclusions: BD lacked the normal relationship between negative affect variability and poorer cognition seen in HC; instead, greater positive affect variability was related to worse working memory in BD. Negative affect variability appears to be independent of cognitive deficits in BD, suggesting uncoupled neural systems for information processing compared to negative affect regulation among patients.

Correspondence: Tanya T. Nguyen, Ph.D., Psychiatry, University of California, San Diego, 3350 La Jolla Village Drive, San Diego, CA 92161, United States. E-mail: ttn050@ucsd.edu

\section{A. OSBORN, J.M. BABIONE, L. EVERETT, I. HASSAN, C. SCHROTH, J.A. DIGANGI, D.M. AASE \& K.L. PHAN. An Exploratory Analysis of PTSD Symptomatology, Gender, Working Memory, and Attention in OIF/OEF/OND Veterans.}

Objective: Recent literature has established that posttraumatic stress disorder (PTSD) symptomatology is associated with several domains of cognitive dysfunction (Scott et al., 2015). Many of the primary symptoms of PTSD are conceptualized within an attentional framework, and thus are hypothesized to have a strong relation to attention and working memory. Although literature to date has presented evidence for such an association (Vasterling et al., 2002), additional research may help differentiate how attention and working memory relate to specific clusters of PTSD symptoms. Additionally, many of the studies' samples are comprised of male veterans, and the results may not be generalizable to other populations. Therefore, the present study seeks to explore the relationship between attention, working memory, and PTSD symptomatology in both male and female OIF/OEF/OND veterans.

Participants and Methods: Subjects were 95 participants (16 women, 79 men) volunteering for a research study. Participants were given brief structured interviews and neuropsychological battery. Measures included CPT-2, Digit Span, and the Stroop Color Word Test.

Results: Differential patterns of correlations between PTSD symptom clusters and attention/working memory were apparent based on gender. For men, re-experiencing symptoms were negatively correlated with working memory $(r=-.37, p<.01)$. For women, overall symptom severity was significantly related to attention $(r=56, p<.05)$, as were symptoms for re-experiencing $(r=-.60, p<.05)$, avoidance $(r=-.60, p<.05)$, and hyperarousal $(r=.60, p<.05)$.

Conclusions: Findings suggest that there may be different patterns of association between PTSD symptom clusters attention and working memory based on gender. Such differences may have important implications for course and treatment of those with PTSD. Future research should explore possible causal relationships.

Correspondence: Amanda Osborn, Master of Science, Psychology, Illinois Institute of Technology, 3631 S Winchester Ave Apt 2, Chicago, IL 60609, United States. E-mail: aosborn@hawk.üt.edu 
M. PARK. Cut-off scores of the Schizotypal Personality Questionnaire for screening of high-risk psychosis.

Objective: Various cut-off scores of the Schizotypal Personality Questionnaire (SPQ) are used to screen individuals who are at high-risk for schizophrenia. We investigated the cut-off scores of the SPQ, which reliably discriminate those at high-risk for schizophrenia.

Participants and Methods: Participants: Based on the scores of the SPQ, three groups were determined: those who obtained the highest $5 \%$ of scores of the SPQ, highest $10 \%$ of scores, and average scores were included in the $5 \%(n=24), 10 \%(n=50)$ and control $(n=48)$ groups, respectively.

Measurement: The performances of the three groups on the Wisconsin Card Sorting Test (WCST) were compared with those of patients with schizophrenia $(n=17)$.

Results: The schizophrenia and 5\% groups showed significantly lower performances on the WCST than $10 \%$ and control groups, however, there were no significant differences between schizophrenia and 5\% groups and between $10 \%$ and control groups on the performances of the WCST. Specifically, the $5 \%$ group showed significantly more errors than $10 \%(t(28.77)=2.63, p<.05)$ and control $(t(30.01)=2.66, p<.05)$ groups. Additionally, $5 \%$ group obtained fewer completed categories than $10 \%(t(29.77)=-2.37, p<.05)$ and control $(t(26.71)=-2.60, p<.05)$ groups. However, the schizophrenia and the $5 \%$ groups did not differ in terms of completed categories $(t(26.84)=1.99, p>.05)$, but total numbers of error $(t(39)=-2.52, p<.05)$. The $10 \%$ and control groups did not differ in terms of total numbers of error $(t(96)=-.12, p>.05)$ and completed categories $(t(96)=.33, p>.05)$.

Conclusions: The results of this study suggest that highest $5 \%$ scores of the SPQ can be used as reliable cut-off scores for screening individuals who are at high-risk for schizophrenia.

Correspondence: Min-Seok Park, Sungshin Woman University, 100, Jingwan 4-ro, Seoul 03304, Korea (the Republic of).E-mail: poemuze@ gmail.com

R.D. SHURA, J. ROWLAND, S.L. MARTINDALE, T.W. BREARLY \& H.M. MISKEY. Evaluating the Motor Slowing Hypothesis of Depression.

Objective: The purpose of this study was to evaluate the hypothesis that processing speed deficits are the primary cognitive deficits in individuals with depression.

Participants and Methods: Research volunteers were enrolled at a Veterans Affairs medical center. The final sample $(n=161)$ included participants who served in the US military since September 11, 2001, passed cognitive and symptom validity measures, and denied a history of moderate or severe traumatic brain injuries. Depression was measured using a structured interview, subscales from the Personality Assessment Inventory, and the Beck Depression Inventory-II. Outcomes were objective cognitive performance on 10 processing/motor speed variables. Results: There was no consistent pattern of slowed processing speed in participants with current depressive diagnoses compared to those without. After removing participants with other psychiatric diagnoses, only non-dominant fine motor dexterity was significantly correlated with depressive symptom burden $(r=.29, p=.002)$. Based on depressive symptom burden, non-dominant fine motor dexterity was also significantly slower in participants with high burden compared to those with low burden $(p=.001, d=0.76)$.

Conclusions: These results do not support a generalized cognitive slowing hypothesis in individuals with depression. Cognitive deficits reported in prior depression research with adults might be attributable to invalid performance, additive effects of comorbid conditions, or deficits in more complex abilities such as executive functions. However, the relation of fine motor dexterity to depressive symptoms was a consistent finding, which might be further considered in future studies.

Correspondence: Robert D. Shura, PsyD, Hefner VAMC, MA-MIRECC, 11M-2/MH\&BS, 1601 Brenner Ave., Salisbury, NC 28144, United States.E-mail:shura@marshall.edu
G.R. SILVA, M. BUJAK, R. BATISTA, D. ALMEIDA, R.P. FONSECA \& C.H. KRISTENSEN. Verbal Executive Dysfunction in Posttraumatic Stress Disorder: The Role of Clinical Symptoms in Process-Specific Executive Deficits.

Objective: Comparisons between verbal and visuospatial executive dysfunction in Posttraumatic Stress Disorder (PTSD) are not sufficiently explored. This study aims (1) to explore differences in inhibitory control (IC) and cognitive flexibility (CF) between two groups of trauma victims (PTSD and trauma) and (2) to investigate the relationship between clinical symptoms and executive functions. Participants and Methods: Assessments were conducted with 49 trauma victims (18 to 50 years old). Clinical measures were the PCL-5, SPTSS, PTCI, BDI and BAI. Executive functions IC and CF were measured (1) verbally, through the Hayling Sentence Completion Test (HSCT) and (2) visuospatially, through the Trail-Making Test (TMT). Z scores were obtained from a national sample stratified by age and education. A verbal/visuospatial index was generated by subtracting $Z$ scores on the TMT from the HSCT. Measures were compared between the PTSD $(\mathrm{n}=32)$ and trauma $(\mathrm{n}=17)$ groups through chi-square or Mann-Whitney $\mathrm{U}$ tests. Relationships between clinical symptoms, IC and CF were investigated through the

Spearman's rho.

Results: Participants with PTSD had higher Z scores on HSCT part B response times $(\mathrm{U}=367.5, \mathrm{Z}=2.01, \mathrm{p}=.04, \mathrm{r}=0.29)$ than controls. Deficits in verbal IC $(\mathrm{Z}$ scores $<1.5)$ were present in $50 \%(\mathrm{n}=16)$ of the PTSD group and in $23.5 \%(n=4)$ of the trauma group $(\chi 2=3.22$, $\mathrm{p}=.07, \mathrm{r}=0.25$ ), while $9.4 \%$ of the PTSD group and $11.8 \%$ of the trauma group had deficits in visuospatial IC. The verbal/visuospatial index of IC was lower in participants with PTSD $(\mathrm{U}=176, \mathrm{Z}=-2.02$, $\mathrm{p}=.44, \mathrm{r}=0.29)$, indicating a specific trend to perform worse in verbal IC tasks. PTCI scores were related to worse verbal $\mathrm{CF}(\mathrm{rs}=.28$, $\mathrm{p}=.04)$.

Conclusions: PTSD symptoms and diagnosis were specifically related to verbal deficits in our sample. Future longitudinal studies should utilize this verbal/visuospatial index to verify if (1) premorbid verbal deficits influence PTSD manifestation or (2) PTSD symptoms hinder verbal executive functioning.

Correspondence: Gustavo R. Silva, Graduate Program in Psychology, Pontifical Catholic University of Rio Grande do Sul, Av. Ipiranga 6681, prédio 11, sala 915, Porto Alegre 91619900, Brazil. E-mail: silva.gustavoramos@gmail.com

A. SWIFT \& V. WALSH. How Time Flies: The Perception, Perspective and Experience of Time in Bipolar Affective Disorder. Objective: This study examined time perception, time experience and time perspective in bipolar disorder, comparing these constructs across different mood states (depression, mania and euthymia).

Participants and Methods: In a cross sectional, quasi-experimental design, 58 bipolar participants completed a clinical interview to assess mood and were assigned to one of three mood state groups: euthymia. depression or mania. Furthermore, 20 health service personnel without a diagnosis of bipolar disorder were recruited as a control group. Therefore, 78 participants in total completed a temporal generalisation computer task (Wearden, 1992), a visual analogue scale of time experience (Blewitt, 1992) and the Zimbardo Time Perspective Inventory (ZTPI, Zimbardo and Boyd, 1999).

Results: Results indicated that the manic group were significantly less accurate on the temporal generalisation task indicating a deficit in time perception. However, no significant differences were found between the control, euthymic and depressed groups. Furthermore, in an unfilled duration, depressed participants rated the subjective passing of time as significantly slower and the manic group rated time as passing significantly faster than the other groups. However, when focussed upon a task (filled duration) this effect was reduced to the extent that no significant differences between the groups were found. Finally, significant differences were found between the group profiles on the ZTPI, indicating that different mood states are characterised by differences in temporal perspectives.

Conclusions: The differences in time perception and experience of time across mood states are considered with direct clinical implications 
for treatment. The results are also examined in relation to the Interactive Cognitive Subsystems model of bipolar disorder (Teasdale and Barnard, 1993) and other leading clinical theories. Additionally, the neurobiological basis of time perception is considered along with ideas for future research.

Correspondence: Anna Swift, D Clin Psy, Clinical Psychology, Norfolk and Norwich University Hospital, Norfolk and Norwich University Hospital NHS Trust, Colney Lane, Norwich NR4 7UY, United Kingdom. E-mail: anna.swift@nnuh.nhs.uk

I. TORRES, S.A. MACKALA, C. HIDIROGLU, S.S. AHN \& L.N. YATHAM. Metamemory Monitoring in Bipolar Disorder.

Objective: Patients with bipolar disorder (BD) show diminished awareness on ratings of their cognitive functioning in daily life, but less is known about patient's awareness of their performance while completing specific cognitive tasks, especially in domains in which patients show deficits, such as memory. We hypothesized that patients would show diminished metamemory monitoring accuracy on an item-to-item basis during performance of verbal and nonverbal recognition memory tasks. Participants and Methods: Eighty clinically stable patients diagnosed with DSM-IV BD and 55 demographically similar healthy volunteers were administered verbal and nonverbal multiple choice recognition memory tasks. Subjects provided retrospective confidence judgments that quantified the degree to which they believed that each response was correct. Primary measures included 1) relative accuracy: the relationship between confidence ratings and performance across test items, 2) absolute accuracy: the degree of discrepancy between confidence ratings and performance, and 3) bias: the degree of over- or under- confidence. Results: On verbal recognition, patients showed comparable performance on all metamemory indices relative to controls. On the nonverbal task, patients exhibited similar relative accuracy and bias, but lower absolute accuracy, $\mathrm{t}(123)=2.0, \mathrm{p}<.05$. In addition, the correlation between mean confidence ratings and nonverbal recognition performance was lower in patients than controls.

Conclusions: Metamemory deficits in BD may be domain/task specific and involve specific components. In this study patients demonstrated intact awareness of verbal recognition memory performance. Although patient's relative accuracy of nonverbal recognition memory performance was also intact (i.e. across items ratings corresponded to performance), absolute accuracy was diminished, but with no evidence of systematic over- or under-estimation. Further study and treatment of metamemory deficits may improve patient's daily functioning.

Correspondence: Ivan Torres, University of British Columbia, Room 2C7 - 2255 Wesbrook Mall, Dept of Psychiatry, UBC, Vancouver, BC V6T 2A1, Canada.E-mail: ivan.torres@ubc.ca

C. WILSON, C. DEMRO, E. THOMPSON, P. RAKHSHAN, Z. MILLMAN, J. GOLD, S. AUGUST, J. WALTZ, K. BUSSELL, G. REEVES \& J. SCHIFFMAN. Association of Neurocognition and Psychosis-Risk Symptoms.

Objective: Previous research has observed neurocognitive deficits among individuals at risk for psychosis. Few studies have examined the association of specific psychosis-risk symptoms and neurocognitive tasks. The aim of the present study was to explore the association of neurocognitive performance with psychosis-risk symptoms.

Participants and Methods: Nineteen help-seeking youth (12-25 years) completed the Structured Interview for Psychosis Risk Syndromes (SIPS), a psychosis-risk interview. Neurocognitive performance was assessed using the MATRICS Consensus Cognitive Battery for schizophrenia (MCCB). Correlations between psychosis risk symptom domain sum scores and MCCB tasks were examined. We hypothesized that individuals with higher symptom severity would correlate with lower neurocognitive performance.

Results: Partial correlations controlling for age and gender demonstrated that only negative symptoms were significantly correlated with specific neurocognitive tasks (WASI Vo: $r(14)=-.50, p=.04$; WMS
Spatial Span: $r(13)=-.66, p=.007)$. Neurocognitive task performance was not significantly correlated with positive, disorganized or general symptoms. Of specific negative symptoms, increased difficulty with ideational richness was significantly associated with lower performance on multiple neurocognitive measures.

Conclusions: Correlation of negative symptoms with specific neurocognitive tasks supports prior research suggesting that negative symptoms may be associated with neurocognitive deficits, even at the clinical high-risk phase of psychosis. Fine-grained analysis of specific negative symptoms indicates that reduced ideational richness in comparison to other symptom domains may correlate most strongly with lower neurocognitive task performance. Generalizability of findings for the current study is limited by small sample size. Future studies should examine whether these findings hold for a larger sample and further explore how specific negative symptoms relate to neurocognitive deficits.

Correspondence: Camille Wilson, M.A., Department of Human Services Psychology, University or Maryland, Baltimore County, 1000 Hilltop Circle, Baltimore, MD 21250, United States.E-mail: cwilson4@umbc. edu

C.J. ZOLLIECOFFER, C.M. FIGUEROA, J.L. CRAWFORD \& D.J. SCHRETLEN. The Impact of Disease on the Contribution of Demographic Characteristics to Cognitive Test Performance.

Objective: Demographic characteristics and premorbid ability contribute to normal inter-individual differences in cognitive test performance, but the impact of illness on their contribution remains unclear. Here we examine how accounting for disease alters the contribution of demographic characteristics and premorbid ability to cognitive performance in a mixed clinical sample (CS) and healthy adults (HA).

Participants and Methods: The 897 participants included 526 adult outpatients with schizophrenia, bipolar disorder, or another disease (CS) and 371 neurologically normal adults (HA). Participants ranged from 15 to 91 years of age $(\mathrm{M}=41)$ and completed 3 to 21 years of schooling $(\mathrm{M}=14)$. Most participants were white $(58 \%)$ or black $(38 \%)$, and $50 \%$ were male. Premorbid estimates were based on the Hopkins Adult Reading Test. We first used multiple linear regression modeling (LRM) to estimate the variance in 14 cognitive measures explained by five predictors: age, sex, race, years of education, and estimated premorbid ability. We then added a term for the presence vs. absence of disease to the LRM and examined the impact of this on the 14 models. Results: Adding a term for disease improved the LRM (doubling the R-square for some) and weakened the contribution of most predictors (i.e., reduced their beta weights) for every cognitive measure. However, demographic variables and estimated premorbid ability still accounted for significant unique variance in performance for every cognitive measure after adding a term for the presence of disease to the models. Conclusions: Having a disease attenuates but does not eliminate the impact of a person's demographic background and estimated premorbid ability on neurocognitive test performance. Knowing whether the person being tested has a disease increased the explained variability in the score distribution of every test administered.

Correspondence: Chandler J. Zolliecoffer, Bachelor of Science, Psychiatry, Johns Hopkins School of Medicine, 12 W Mt Vernon Place, Baltimore, MD 21201, United States. E-mail: czollie1@jhmi.edu 
AM Coffee Break

10:30-10:45 a.m.

\section{Plenary E. Behavioral Clusters and Brain Network Mechanisms of Impairment and Recovery}

\section{Presenter: Maurizio Corbetta}

$$
\text { 11:00 a.m.-12:00 p.m. }
$$

M. CORBETTA. Behavioral Clusters and Brain Network Mechanisms of Impairment and Recovery.

A long-held view is that stroke causes many distinct neurological syndromes due to damage of specialized cortical and subcortical centers. However, in recent studies on a large cohort of first time stroke subjects studied longitudinally at 2 weeks, 3 and 12 months, we showed that a few clusters of behavioral deficits spanning multiple functions explained neurological impairment. These clusters are stable across recovery indicating that they represent a stable solution to describe impairment. It has been also proposed that focal lesions cause remote physiological abnormalities, but the behavioral relevance of these changes vis-a-vis structural damage is unknown. In separate studies we measured resting functional connectivity fMRI (FC), lesion topography, and behavior in multiple domains (attention, visual memory, verbal memory, language, motor, and visual), and used machine-learning models to predict neurological impairment in individual subjects. We found that visual memory and verbal memory were better predicted by $\mathrm{FC}$, whereas visual and motor impairments were better predicted by lesion topography. Attention and language deficits were well predicted by both. These results link key organizational features of brain networks to brain-behavior relationships in stroke.

After attending the lecture, participants will be able to: 1) Describe the three factors that explain human cognitive performance post-stroke; 2) Differentiate between anatomical and functional analysis of brain connectivity and topography; 3) Explain the main mechanism of dysfunction of brain networks post-stroke; 4) Explain the main mechanisms of recovery at the brain network level post-stroke.

Correspondence: Maurizio Corbetta, M.D., Department of Neurology and Neurological Surgery, Washington University School of Medicine, 660 South Euclid Avenue, Box 8111, St. Louis, MO 63110, United States.E-mail:mcorbetta@wustl.edu

\section{Lunch (On Own)}

$$
\text { 12:00-1:00 p.m. }
$$

\section{FRIDAY AFTERNOON, FEBRUARY 3, 2017}

\section{Benton / Mid-Career Awardee Presentation: Subtle Brain-Behavior Biomarkers of Modifiable Cardiovascular Disease Risk Factors: Implications for Minority Health Disparities, Aging and Dementia}

\section{Award Recipient: Melissa Lamar}

$$
\text { 1:00-2:00 p.m. }
$$

\section{LAMAR. Subtle Brain-Behavior Biomarkers of Modifiable Cardiovascular Disease Risk Factors: Implications for Minority Health Disparities, Aging and Dementia.}

Mid-life cardiovascular disease risk factors (CVD-RFs) such as hypertension (HTN) and diabetes (DM) and associated cerebrovascular disease contribute to late-life risk and development of dementia including Alzheimer's disease (AD). Dr. Alzheimer himself was one of the first to speculate on the role of cerebrovascular disease on brain aging. The US population has changed since initial work in this area was conducted. For example, 55 million Hispanics live in the US, representing $17 \%$ of the population; these numbers will more than double by 2060 to 130 million or $31 \%$ of the US population. While Hispanics have a lower prevalence of the APOE 4 allele, a known genetic risk for AD, they have some of the highest prevalence rates of DM and uncontrolled HTN in the US. Given HTN-related treatment and control in Hispanics lags behind US trends by 10-15\%, higher CVD-RF prevalence combined with treatment-related health disparities may predispose Hispanics not only to an earlier and increased risk for AD but a more protracted course of dementia. Thus, work to identify preclinical markers of accelerated brain aging earlier - in early to mid-life before the clinical onset of dementia and its extensive neuropathology - is critical. Dr. Arthur Benton dedicated his career to the promotion of novel and objective neuropsychological techniques to promote understanding of neurological impairment. In that spirit, I will review work to (1) expand neuropsychological outcomes to reveal subtleties of behavior related to CVD-RFs and associated cerebrovascular disease in non-Hispanic whites, (2) apply these techniques in Hispanics including results related to treatment-related control of CVD-RFs and cognition, and (3) advance neuropsychological assessment and neuroimaging toward identifying brain vulnerability as opposed to overt brain damage associated with CVD-RFs in Hispanics. Only with subtle brain-behavior biomarkers will we be able to detect vulnerability to pathological aging in Latinos at increased risk for earlier adverse outcomes.

Correspondence: Melissa Lamar, PhD, Rush Alzheimer's Disease Center, Rush University Medical Center, 600 S Paulina St, Chicago, IL 60612, United States.E-mail:Melissa_Lamar@rush.edu

\section{Invited Symposium 4. A Summit on Cognitive Rehabilitation: Mapping the Past, Defining the Present and Imagining the Future}

\author{
Chair: Anthony Y. Stringer
}

$$
\text { 1:00-2:30 p.m. }
$$

A.Y. STRINGER, B.A. WILSON \& K.D. CICERONE. A Summit on Cognitive Rehabilitation: Mapping the Past, Defining the Present and Imagining the Future.

Symposium Summary: While the treatment of persons with traumatic brain injury can be traced back to the ancient Egyptians, the modern era of cognitive rehabilitation began with World War I and the pioneering work of Kurt Goldstein. Subsequent international conflicts continued to spur the development of rehabilitation programs and strategies into contemporary times. Early work in the field was equal parts art and science, yet such work yielded enduring principles that continue to guide the treatment of persons with neurocognitive impairment. Contemporary clinicians have a growing armamentarium of empirically-supported treatments to address cognitive impairment in an expanding range of patient populations. The future will see an increasing use of pharmacological, genetic, electronic, digital, and neuroprosthetic tools, in addition to the cognitive strategies and techniques that have long been our staple. In this symposium, Dr. Barbara A. Wilson will trace the historical 
development of cognitive rehabilitation and provide an overview of the enduring principles from the past; Dr. Keith D. Cicerone will review the empirical efficacy of current cognitive rehabilitation strategies and techniques; and Dr. Anthony Y. Stringer will discuss the future of cognitive rehabilitation in an era of advancing pharmacological, genetic, electronic, digital, and neuroprosthetic tools.

Correspondence: Anthony Y. Stringer, PhD, ABPP/ABCN, Department of Rehabilitation Medicine, Emory University, 1441 Clifton Road NE, Atlanta, GA 30322,United States.E-mail: astring@emory.edu

\section{B.A. WILSON. The History of Cognitive Rehabilitation.}

The earliest known description of the treatment of brain injury is from an Egyptian document of 2500-3000 years ago. The papyrus was discovered by Edwin Smith in Luxor in 1862. It describes the treatment of 48 cases of injury of which 27 were brain trauma cases. Modern rehabilitation as we know it today, however, began during World War One with Kurt Goldstein, a major influence in modern brain injury rehabilitation. The first book on brain injury rehabilitation was written by Poppelreuter in 1917. In World War Two, Luria, in what was then the Soviet Union, made great contributions. In the United Kingdom, Zangwill and Newcombe were major players while in the USA Aita set up a unit based on interdisciplinary care. The Yom Kippur War in 1973 resulted in Ben-Yishay developing what were to become modern holistic rehabilitation programs. Around the same time, Diller and colleagues published studies on the treatment of unilateral neglect and Prigatano set up a holistic program influenced by the work of Goldstein and Ben-Yishay. Current neuropsychological rehabilitation (NR) is concerned with the amelioration of cognitive, emotional, psychosocial and behavioural deficits caused by an insult to the brain. Rehabilitation is NOT synonymous with recovery (i.e getting back to what one was like before the injury or illness), it is NOT synonymous with treatment (treatment is something we do to people or give to people), it IS a two way interactive process whereby people who are disabled by injury or disease work together with professional staff, relatives and members of the wider community to achieve their optimum physical, psychological, social and vocational well-being.

Correspondence: Barbara A. Wilson, OBE, Ph.D., D.Sc., CPsychol, BRPsSC, AcSS, Oliver Zangwill Centre, Princess of Wales Hospital, Lynn Road, Ely, Cambridgeshire CB6 1DN, United Kingdom. E-mail: barbara.wilson00@gmail.com

K.D. CICERONE. The Present Status of Cognitive Rehabilitation. A considerable body of evidence has accumulated to demonstrate the effectiveness of cognitive rehabilitation since Dr. Harvey Levin noted this intervention to be "unproved but promising" (Levin, 1990). Evidence based cognitive rehabilitation attempts to incorporate the best available scientific evidence with clinical judgment and patient preferences and values. Even when well controlled randomized trials exist, their application may be limited by variations in patient characteristics, and single-subject research can help to develop innovative interventions. This lecture will review the evidence supporting specific interventions for acquired cognitive impairments; the integrated treatment of cognitive, emotional and social limitations; and the relevance of cognitive interventions to real-world functioning. There is sufficient evidence from systematic reviews of cognitive rehabilitation to support specific algorithms and guidelines for clinical decision making. Technical advances have begun to inform principles of neuroplasticity-based treatment and may identify the active ingredients and mechanisms of action for cognitive rehabilitation. However, the clinical application of evidence-based practice remains limited by inadequate descriptions of specific interventions and limited opportunities for formal training. Both basic and translational research will be needed to foster the evolution of cognitive rehabilitation.

Correspondence: Keith D. Cicerone, PhD, ABPP/ABCN, JFK-Johnson Rehabilitation Institute, 2048 Oak Tree Road, Edison, NJ 08820, United States.E-mail: kcicerone@jfkhealth.org.
A.Y. STRINGER. The Future of Cognitive Rehabilitation.

While cognitive rehabilitation as a clinical art can be traced back a century (or perhaps even millennia) and as an empirically-validated clinical treatment for about a decade, its future lies far beyond our grasp. Yet we can imagine what that future might be. While long held historical principles of rehabilitation and empirically-supported interventions will be a part of that future, we are also likely to see pharmacological, genetic, electronic, neuromodular, and neuroprosthetic tools take their place alongside the traditional cognitive exercises and strategies. It will be tempting to think that such tools will be all that is necessary to rehabilitate persons with damaged or dysfunctional brains, but this will be a mistake. There will be no substitute for the creative and empirically-guided clinician working in partnership with the motivated patient. This lecture will explore the principles -- past and present -- that will shape the future of cognitive rehabilitation.

Correspondence: Anthony Y. Stringer, PhD, ABPP/ABCN, Department of Rehabilitation Medicine, Emory University, 1441 Clifton Road NE, Atlanta, GA 30322, United States. E-mail: astring@emory.edu

\section{Panel Discussion, Presented by the INS Student Liaison Committee: International Cross-Cultural Considerations in Research}

\author{
Presenters: Anita Sim, Jonathan Evans, Tedd Judd, \\ Robert K. Heaton
}

1:00-2:30 p.m.

\section{Symposium 7. Locus Coeruleus-Norepinephrine System, Cognitive Effort, and Early Risk for Alzheimer's Disease}

\author{
Chair: William S. Kremen \\ Discussant: Mark W. Bondi
}

1:00-2:30 p.m.

W.S. KREMEN. Locus Coeruleus-Norepinephrine System, Cognitive Effort, and Early Risk for Alzheimer's Disease.

Symposium Summary: Early identification of risk for Alzheimer's disease (AD) is of great public health importance. This symposium will address the integration of phenomena linked to the locus coeruleus (LC) and early risk for AD. Tau pathology is a hallmark feature of AD, and the LC has also been identified as the earliest site of abnormal tau. The $\mathrm{LC}$ is the brain's major norepinephrine (NE) projection site. The LC modulates brain and parasympathetic activity via NE. Loss of $\mathrm{NE}$ has proinflammatory effects. The LC drives pupil dilation responses during cognitive testing. Pupil dilation is a validated psychophysiological indicator of cognitive effort; if two people have the same test score, the one requiring more effort is hypothesized to be at higher risk for decline The LC-NE system dysfunction may, thus, be a risk indicator for very early stages in the progression to AD. Dr. Kremen will provide an overview theory and data regarding the LC system and its links to cognition and $\mathrm{AD}$, and data showing that pupil dilation differentiates cognitively normal and mild cognitive impairment (MCI) groups $(\mathrm{n}=918)$. Mr. Sanderson-Cimino will present new findings showing that MRI-assessed LC neuromelanin contrast (an index of LC integrity) is associated with tau and with pupil response. Dr. Elman will show differences in task-related pupil dilation associated with processing speed, inflammation, and resting state fMRI that are consistent with tonic/phasic rate of activity and signal to noise in the LC $(\mathrm{n}=358)$. Dr. Mather will demonstrate that MRI-assessed LC neuromelanin contrast is associated with higher cognitive reserve and parasympathetic control over heart rate. Finally, Dr. Mark Bondi will serve as discussant. He will provide an integration of 
the findings suggesting that task-related pupil response and LC contrast are indices of LC-NE system dysfunction, which-given the very early appearance of tau deposits in the LC-hold promise as biomarkers of the earliest stages of risk for AD.

Correspondence: William S. Kremen, Psychiatry, UCSD, 9500 Gilman Dr. (MC 0738),La Jolla, CA 92093, United States.E-mail:wkremen@ ucsd.edu

W.S. KREMEN, M.S. PANIZZON, E. JEREMY, A.J. JAK, R.L. HAUGER, M.W. BONDI, M. LYONS \& C.E. FRANZ. TaskEvoked Pupil Response: A Novel Biomarker of Locus Coeruleus Dysfunction Indicating Early Risk for MCI and Alzheimer's Disease.

Objective: The locus coeruleus (LC) drives pupil dilation responses and has been identified as the earliest site of tau deposition, a hallmark feature of Alzheimer's disease (AD). We hypothesized that taskedevoked pupil dilation (TEPD) would serve as a biomarker of LC function and of early risk for mild cognitive impairment (MCI) or AD. TEPD indexes cognitive effort; effort (dilation) increases as cognitive load increases, but declines when load is well beyond capacity. Given the same test score, a person requiring more effort should be at higher risk for decline. We predicted that people with single-domain (SD) MCI would show greater TEPD to achieve the same score as cognitively normal (CN) individuals; those with multiple-domain (MD) MCI would have reduced TEPD and poorer performance.

Participants and Methods: 918 men ages 56-65 in the Vietnam Era Twin Study of Aging (VETSA) underwent pupillometry during digit span at low, moderate, and high loads (3, 6, \& 9 digits). Neuropsychologically-defined MCI was based on scores that were also adjusted for cognitive ability assessed at age 20 .

Results: As proof of principle, we grouped subjects by maximum (max) span from 4 to 9 . At low load, as max span increased, TEPD decreased. All increased TEPD at moderate loads. TEPD decreased at high loads, except for the 8-9 max span groups who were not well beyond capacity. The SD-MCI group did not differ in digit span performance, but had greater TEPD than the CN group. The MD-MCI group had poorer performance and failed to modulate TEPD to changing load. TEPD was normally distributed in the CN group.

Conclusions: Results suggest some remaining compensatory capacity in SD-MCI, but failure to adequately compensate in MD-MCI. CN individuals with higher TEPD prior to cognitive decline are predicted to be at higher risk for MCI. Our results in middle-aged adults suggest that TEPD—an index of LC system function—is a useful biomarker for very early identification of risk for MCI and AD.

Correspondence: William S. Kremen, Psychiatry, UCSD, 9500 Gilman Dr. (MC 0738), La Jolla, CA 92093, United States.E-mail:wkremen@ ucsd.edu

M. SANDERSON-CIMINO, J. EPPIG, S.F. SORG, L.M. CAMPBELL, E. GRANHOLM, W.S. KREMEN \& M.W. BONDI. The Relationship Between Locus Coeruleus Integrity and Biomarkers of Alzheimer's Disease: A Magnetic Resonance Imaging Pilot Study.

Objectives: The locus coeruleus (LC) drives pupil response dilation and has been associated with cognitive decline in older adults. Research suggests the locus coeruleus (LC) may be one of the first structures to accumulate hyperphosphorylated tau (pTau), a neuropathological biomarker of Alzheimer's Disease (AD). Fast Spin Echo (FSE) MRI provides a proxy measure of the LC's integrity and degradation through the imaging of neuromelanin, a polymer present in the structure. We investigated the relationship between FSE-derived LC integrity, pupil response (PR) and cerebrospinal fluid (CSF) biomarkers of AD, pTau and amyloid beta (A $\beta 42)$.

Participants and Methods: 5 non-demented older adults underwent T1-weighted FSE MRI, lumbar puncture, and pupillometry. The LC and a section of the pontine tegmentum (PT) were manually traced on the second and third slices below the inferior colliculus (Clewett et al.,
2016). A contrast ratio (CR) was calculated with the following formula: (LC - PT) / PT. Higher CR values indicate greater LC intensity (an indicator of LC integrity) compared to the PT.

Results: There was a moderate to large negative correlation between LC-CR and CSF $p$ Tau a $(r=-.684, p=.203)$ and a large positive correlation between LC-CR and CSF A $\beta 42(r=.802, p=.055)$. LC values were greater in cognitively normal subjects than those with MCI ( $p=.072)$. Considerable associations were also observed between PR and LC-CR as well as PR and pTau. These correlations were not statistically significant in this small pilot sample, but the effect sizes indicate strong associations.

Conclusions: Our pilot data support the use of FSE derived LC-CR as an index of neuronal integrity of the LC, as well as a potential neuroimaging marker of preclinical AD. The LC-CR suggests that neuromelanin intensity is correlated with both PR and CSF biomarkers of AD. Larger studies examining associations of LC-CR with PR ptau and A $\beta 42$ in late middle-aged and older adults are currently underway.

Correspondence: Mark Sanderson-Cimino, B.S. Psychology, VMRF Psychology, 4347 Hamilton street, San Diego, CA 92104, United States. E-mail:mesandci@gmail.com

E. JEREMY, M.S. PANIZZON, D.J. HAGLER, C. FENNEMANOTESTINE, C.E. FRANZ, L.T. EYLER, E. GRANHOLM, A.J. JAK, M.J. LYONS, A. DALE \& W.S. KREMEN. Convergent Evidence of Pupillary Response as an Early Indicator of Locus Coeruleus Dysfunction and Risk for Mild Cognitive Impairment. Objective: There is great need for early screening tools for Alzheimer's disease $(\mathrm{AD})$ as pathology may be present decades before symptom onset. Task-evoked pupil dilation (TEPD) is a well-validated measure of cognitive effort that may detect declines in cognitive efficiency prior to impaired performance. TEPD increases with cognitive load but decreases after capacity is exceeded. TEPD is linked to function of the locus coeruleus ( $\mathrm{LC}$ ) which has been proposed as an initial site of abnormal tau. LC damage may also contribute to key AD-related pathology: inflammatory response and amyloid deposition. We examined whether TEPD is related to measures of resting-state functional MRI (rs-fMRI), inflammation, and cognitive performance, all of which can be linked to LC function.

Participants and Methods: 980 men ages 56-65 in the Vietnam Era Twin Study of Aging were defined as cognitively normal or as having mild cognitive impairment (MCI). Pupil dilation was measured during digit span tasks. We also assessed processing speed and measured C-reactive protein (CRP), a marker of inflammation. During rs-MRI $(\mathrm{n}=358)$, blood-oxygenation-level-dependent (BOLD) signal variance was measured in regions selected a priori that are modulated by the LC. Results: Participants with both MCI and lower working memory ability had greater drop-off in TEPD between moderate and high task loads, consistent with system capacity being overwhelmed. Higher BOLD variance, higher CRP, and slower processing speed were associated with more dilation at low loads and less task appropriate increase in dilation at moderate loads.

Conclusions: TEPD reflects altered brain activity, inflammatory state, and cognitive effort. This provides converging evidence for involvement of the LC, an early site of AD pathology that may provide the neural substrate linking these measures together. Given the relatively young age of the sample, TEPD may be a useful psychophysiological biomarker for early identification of $\mathrm{AD}$ risk.

Correspondence: Elman Jeremy, Ph.D., Psychiatry, University of California, San Diego, 9500 Gilman Dr. (MC0738), University of California San Diego, La Jolla, CA 92093, United States. E-mail: jaelman@ucsd.edu

M. MATHER. Locus Coeruleus Neuromelanin MRI Contrast Correlates With Cognitive and Cardiovascular Factors.

The locus coeruleus (LC) is a small nucleus in the brainstem that is the source of much of the brain's norepinephrine (NE). The LC-NE system 
plays a key role in helping us to focus on what matters most and ignore distractions, especially under arousing or demanding situations (Mather, Clewett, Sakaki, \& Harley, 2015). Recent findings indicate that locus coeruleus (LC) decline likely plays a larger role in cognitive decline in aging and Alzheimer's disease than previously appreciated (Mather \& Harley, 2016). One reason its role has been mostly ignored to date is that it is a challenging region to assess in living humans. However, the fact that LC neurons accumulate neuromelanin permits a specialized magnetic resonance imaging (MRI) sequence that allows the $\mathrm{LC}$ to be imaged (Keren et al., 2015). We have found that, in older adults, higher LC-MRI signal is associated with higher cognitive reserve scores. In addition, in both younger and older adults, lower measures of LC-MRI are associated with lower indicators of parasympathetic influences over heart rate variability, consistent with the LC role in suppressing parasympathetic control over heart rate. These and other on-going projects highlight the utility of the LC-MRI measure as an indicator of individual differences in LC-NE system function.

Correspondence: Mara Mather, Leonard Davis School of Gerontology, University of Southern California, 3715 McClintock Ave, Los Angeles, CA 90089,United States.E-mail:mara.mather@usc.edu

\section{Symposium 8. Clinical Applications of Functional Neuroimaging for Presurgical Functional Mapping: The Past, Present, and Future Roles for Neuropsychologists}

\section{Chair: Christen M. Holder}

Discussant: Andrew Papanicolaou

$$
\text { 1:00-2:30 p.m. }
$$

\section{C.M. HOLDER, N. SHAY, R. REZAIE, S. NARAYANA, A. BABAJANI- FEREMI, B. POTTER \& A. PAPANICOLAOU. Clinical Applications of Functional Neuroimaging for Presurgical Functional Mapping: The Past, Present, and Future Roles for Neuropsychologists.}

Summary: Functional neuroimaging is concerned with mapping the cortical regions of somatosensory, motor, and language functions, and for assessing hemispheric dominance for both language and memory prior to surgery, with outcomes measured as functional skills on neuropsychological measures. In the past, questions of localization and dominance were addressed exclusively through invasive methods, specifically the WADA procedure and direct Cortical Stimulation Mapping (CSM). Although these methods have been called the "gold standard," they both carry risks of increased morbidity and high cost, and are associated with several shortcomings. Dr. Nicole Shay will review these procedures, including the role of the neuropsychologist, and discuss limitations of the procedures, particularly in young and impaired patients. Dr. Roozbeh Rezaie, Dr. Shalini Narayana, and Dr. Abbas Babajani-Feremi will review the noninvasive procedures of magnetoencephalography (MEG), transcranial magnetic stimulation (TMS), and functional magnetic resonance imaging (fMRI) and their clinical applications. They will present techniques for combining data from these non-invasive methods to provide detailed information for surgical planning, and the arguments in favor of the use of the non invasive alternatives, as made by Papanicolaou et al. in Epilepsia (2014) will be presented. Dr. Christen Holder and Dr. Brian Potter will review a series of cases where a combination of MEG, TMS, and fMRI provided information that informed surgical interventions when invasive techniques were not an option or where a combination of non-invasive techniques offered concordant information to invasive techniques, and associated neuropsychological outcomes. Dr. Andrew Papanicolaou will serve as discussant, giving an introduction to these topics, providing commentary and answering questions.
Correspondence: Christen M. Holder, PhD, Department of Pediatrics, University of Tennessee Health Science Center, 51 N Dunlap, Suite 320, Memphis, TN 38105, United States.E-mail: cholder7@uthsc.edu

N. SHAY \& A. PAPANICOLAOU. The Traditional Use of Invasive Procedures for Determining Localization and Lateralization.

Two invasive brain mapping techniques have long been considered the "gold standard" of presurgical planning. The first of these is the intracarotid sodium amytal method, also known as the Wada test, whose primary purpose is to determine hemispheric dominance of language and memory. The second is direct Cortical Stimulation Mapping (CMS) performed intraoperatively or in some cases extraoperatively through electrode arrays (grids or strips) placed below the dura matter for the primary purpose of determining the seizure onset zone for subsequent resection, and identifying the somatosensory, motor and language regions within the dominant hemisphere. Neuropsychologists traditionally play an important role in both procedures, although those working outside a surgical center seldom have an opportunity to observe or participate in them. Both an overview of the procedures and video presentations of the neuropsychologist role will be provided. When put to the empirical test, studies suggest that often, postsurgical neuropsychological outcomes do not support the idea that these techniques should be considered a "gold standard." Additionally, despite the long-standing reign of these invasive techniques, many shortcomings have been identified. Morbidity levels, infection, and patient discomfort are among the most conspicuous of these. In addition, issues such as questionable efficacy of identifying memory areas, narrow time frames, restricted use of repetition for establishing reliability, and inability to control for situational variables are problematic. These concerns are particularly salient in centers that see a high number of pediatric patients, as well as highly impaired patients. Examples illustrating these shortcomings in WADA and CMS procedures will be provided.

Correspondence: Nicole Shay. E-mail: Nicole.Shay@lebonheur.org

R. REZAIE, S. NARAYANA, A. BABAJANI-FEREMI \& A. PAPANICOLAOU. Non-Invasive Procedures as the Future of Functional Mapping.

Functional MRI, the most well-known of the non-invasive techniques, measures brain activity by detecting changes associated with local blood flow, and has recently become a routine procedure to map the motor cortex, expressive language, and receptive language in a large number of clinical centers. It has been found to be particularly useful in determining lateralization and intrahemispheric localization for both language and memory mechanisms. TMS can be used in mapping the motor cortex or for localizing the language-specific cortex, and has been used successfully in mapping both adult and pediatric populations. MEG is a method used primarily for somatosensory motor, receptive, and expressive language mapping. While fMRI, MEG, and TMS have been used in laboratories to explore the functional architecture of the brain for quite some time, the past ten to fifteen years have seen an increase in the uses of these techniques in major epilepsy and tumor surgery centers. Specifically, they are used to supplement the invasive methods used in pre-surgical planning, and in some cases replace them in an effort to reduce morbidity. We will offer an introduction to these techniques and provide photo and video demonstrations of the protocols. In addition to an overview of these procedures, we will discuss how they are employed for the purpose of (a) supplementing (or replacing) the Wada procedures for determining hemispheric dominance, (b) for identifying the central sulcus and specifying the motor cortex, and (c) for identifying the expressive and receptive language cortices, thus supplementing (or replacing) direct Cortical Stimulation Mapping. The evidence of efficacy of these procedures used alone or in combination as compared to the efficacy of the invasive techniques will be reviewed.

Correspondence: Roozbeh Rezaie,TN, United States.E-mail: rrezaie@ uthsc.edu 
C.M. HOLDER, B. POTTER, R. REZAIE, S. NARAYANA \& A. BABAJANI-FEREMI. Outcomes of Non-Invasive Presurgical Planning.

MEG, fMRI, and TMS have been used successfully as adjunct means of assessing localization and lateralization of brain functions, such as language. The question that remains is whether the non-invasive methods could replace CSM and the Wada. It appears that concordance of non-invasive techniques for estimating language may provide a better estimate of hemispheric specialization as it will be shown by the example of a patient series in our center: Nine patients ranging in age from 14 to 37 will be presented. Three cases are presented where CSM was not a viable option. Six other patients represent cases where both invasive and non-invasive techniques were used during the presurgical stage to demonstrate compatibility of the results. Of the three cases where invasive methods were not a viable option, a combination of fMRI, MEG, and TMS were used and resulted in adequate information for surgical planning. In the other six cases reviewed, patients underwent Wada and CSM, as well as fMRI. MEG, and TMS. In these cases, lateralization and localization data were concordant between the two sets of techniques. Five of the patients had no declines in their post-surgical neuropsychological data, and one patient showed an expected mild decline in verbal memory. Cases like those described lead to the conclusion that a combination of noninvasive methods can assess laterality of language and memory with the comparable accuracy to that of invasive methods and reduce postsurgical morbidity. Moreover, given that MEG can provide concordant localization estimates for receptive language to those of CSM and that fMRI and TMS can specify the expressive language cortex, the need for awake craniotomy can be drastically reduced.

Correspondence: Christen M. Holder, PhD, Department of Pediatrics, University of Tennessee Health Science Center, $51 \mathrm{~N}$ Dunlap, Suite 320, Memphis, TN 38105,United States.E-mail: cholder7@uthsc.edu

\section{Paper Session 11. Mental Illness}

\section{Moderator: Derin J. Cobia}

\section{1:00-2:30 p.m.}

\section{A.I. FORD. History of Depression: From Possession to Organic Brain Disorder.}

Objective: Historical overview of the conceptualization of depression, from ancient supernatural belief to today's neuroscience perspective. Participants and Methods: Reviews of historical writings on the diagnostic conceptualization and etiological evidence of depression.

Results: Ancient cultures across the world had initial conceptualizations of mental illness, including depression, as resulting from possession or other supernatural influence. Western understanding of an organic basis-posited as melancholy by Hippocrates and elaborated by Galen-emerged fairly early but was followed by alternating periods of regression and progression in scientific thinking. The Middle Ages brought a return to demonology as the etiology for mental ailments, which persisted until the end of the Renaissance when Robert Burton's The Anatomy of Melancholy (1621) marked a return to the biological perspective.

The Age of Enlightenment brought the concept of heritability of melancholy and this was soon followed by a rise in psychological conceptualizations of depression. From Freudian psychoanalytic theory, to behaviorism, and then cognitive theory, the 1900 s saw a proliferation of psychological explanations for depression. These progressed now alongside organic investigations, particularly following the serendipitous discovery of early pharmacologic treatment for depression.

Conclusions: The conceptualization of depression has an interesting history of organic vs. non-organic etiology that is culminating now into a complex integration of biological and psychological processes.
Correspondence: Alicia I. Ford, PhD, Psychiatry and Behavioral Sciences, Oklahoma State University Center for Health Sciences, 1111 W.17th St.,Tulsa, OK 74107, United States.E-mail: alicia.ford@ okstate.edu

E. WEBER, J. STUDENY, B.C. KAVANAUGH, N.E. COOK, C. GAUDET, K. MCCURDY \& K.A. HOLLER. The Relationship between Depression and Executive Functioning in Child Inpatient Outcomes and Neuropsychological Deficits.

Objective: Several studies have identified the importance of executive functions (EF) for academic functioning in childhood. However, little is known about the implications of executive dysfunction in the context of comorbid mood symptoms and depression. This study examined the association between clinically significant self-reported depressive symptoms and EF deficits on cognition and psychiatric outcomes in a child inpatient setting.

Participants and Methods: A medical chart review was conducted for 98 children aged 6-12 years with self-reported depressive symptoms $(\mathrm{n}=36)$, EF deficits $(\mathrm{n}=24)$, or both $(\mathrm{n}=38)$ who received a neuropsychological evaluation during their course of treatment at a children's psychiatric inpatient hospital from 2010-2015. Clinically significant depressive symptoms were defined by a T-Score $\geq 1.5$ standard deviations (SD) above the normative mean on at least 1 (or more) CDI-2 subdomain. Executive dysfunction was defined by a T-Score $\geq 1.5 \mathrm{SD}$ below the normative mean on at least neuropsychological test of EF (i.e., the Stroop C-W, TMT-B, or COWAT-FAS).

Results: Slightly more than one-third (38\%) of the sample had co-occurring EF deficits and clinically significant depressive symptoms. The combination of depression with EF deficits was associated with longer psychiatric hospitalization $(\mathrm{d}=0.63, \mathrm{~d}=0.69)$, greater prevalence of comorbid $\mathrm{AD} / \mathrm{HD}(\mathrm{OR}=4.52$; $\mathrm{OR}=2.11)$, as well as poorer test performance on measures of executive functioning $(\mathrm{d}=-2.17 ; \mathrm{d}=-0.69)$ and immediate memory $(\mathrm{d}=-0.97 ; \mathrm{d}=-.83)$, compared to either clinically significant depressive symptoms only and EF deficits only, respectively. Conclusions: The results of this study suggests that, among psychiatric inpatients, not all children with clinically significant self-reported depressive symptoms present with EF difficulties. However, those who do are likely to experience more significant psychiatric and cognitive difficulties beyond what can be accounted for by depression alone.

Correspondence: Elyssa Weber, MA, Clinical Psychology, University of Massachusetts, Boston, 100 Morrissey blvd, Boston, MA 02143, United States.E-mail: elyssa.weber001@umb.edu

J.G. KEILP, S. BEERS, N. MELHEM, A. BURKE, M.A. OQUENDO, D. BRENT \& J.J. MANN. Familial Transmission of Neurocognitive Deficits Associated with Suicidal Behavior Risk.

Objective: Deficits in cognitive control, memory, and decision making have been associated with risk for suicidal behavior in depression (Richard-Devantoy et al., 2014, 2015). These deficits are evident both within and and outside the depressed state (Jollant et al., 2005; Keilp et al., 2014), and appear to be trait markers of risk. If traits, do they run in families and contribute to the familial aggregation of suicidal behavior risk?

Participants and Methods: Offspring of parents with a history of major depression and no past suicidal behavior $(n=215$, age $19.4+/-7.6$ ) and offspring of parents with a history of both depression and suicide attempt (n=192, age $19.0+/-6.4$ ) were compared on a battery of neuropsychological tests including measures of cognitive control (computerized Stroop task), memory (Buschke Selective Reminding, SRT), and decision making (Iowa Gambling Task, IGT). Participant families were recruited from two sites, in New York and Pittsburgh, as part of the Familial Pathways to Early Suicidal Behavior project.

Results: Offspring of past suicide attempter parents performed more poorly than offspring of non-attempter parents on Stroop interference $(p=.049)$ and IGT $(p=.047)$; difference on SRT did not reach significance $(p=.11)$. After removing offspring who had themselves made a 
suicide attempt ( $\mathrm{n}=19$ in each sample), difference in Stroop interference remained significant $(p=.03)$; IGT difference was marginal $(p=.08)$. Differences were not related to other clinical factors such as intelligence, history of depression, substance abuse, or PTSD - all of which were equivalent between the groups.

Conclusions: Data provide evidence for the familial transmission of neurocognitive risk factors for suicidal behavior, and their existence prior to the onset of this behavior. Deficits in cognitive control and decision making may represent vulnerability factors, that interact with other clinical conditions to raise the likelihood of suicide attempts. Correspondence: John G. Keilp, Ph.D., Psychiatry/MIND, Columbia University/NYSPI, Box 42, 1051 Riverside Drive, New York, NY 10032, United States.E-mail:jgk13@cumc.columbia.edu

F. STEFFEN-ALLEN, R.P. SO, L.S. KEGELES \& C.A. CHEN. Increased Delta as a Compensatory Mechanism During Working Memory in High-Performing Patients with Schizophrenia.

Objective: Working memory (WM) impairment is a core cognitive deficit in schizophrenia (SZ) and has been linked to dysfunctional oscillatory activity, especially in high-frequency bands $(>30 \mathrm{~Hz})$. WM deficits in SZ have also been linked to GABAergic interneuron dysfunction in the dorsolateral prefrontal cortex (DLPFC) in magnetic resonance spectroscopy (MRS) studies. This study aims to understand how high-performing patients, who perform equivalently as controls, execute WM tasks.

Participants and Methods: High-performing, SZ patients (n=7) and controls $(n=9)$ had scalp EEG recorded during a modified Sternberg WM task with two difficulty levels. Task-induced oscillatory amplitudes were computed across the frequency range for three electrodes $(\mathrm{Fz}, \mathrm{Cz}$, and $\mathrm{Oz}$ ). Averaged amplitudes were then computed in one-second steps across the task for each participant. MRS measurements of GABA in left DLPFC and anterior cingulate cortex (ACC) were also obtained. Repeated-measures ANOVAs were conducted for each frequency band. Results: As predicted, diagnosis did not predict WM performance. Patients and controls showed similar oscillatory activity in gamma, beta, alpha, and theta. In delta, patients showed greater occipital delta during encoding $(t=-2.68, d f=14, p=0.018, d=-1.43)$. Patients also showed lower levels of GABA in the left DLPFC $(t=2.980, d f=14, p=0.010$, $d=1.55)$, but not the ACC $(t=1.133, d f=13, p=0.227, d=0.59)$. Conclusions: Our results suggest increased occipital recruitment as a compensatory mechanism for working memory performance in highperforming individuals with SZ that may compensate for GABAergic interneuron dysfunction in the frontal cortex.

Correspondence: Faith Steffen-Allen, B.A., Psychology, University of Connecticut, 85 Squaw Hollow Road, Ashford, CT 06278, United States.E-mail:faith.steffen@uconn.edu

\section{Poster Session 8. Aging \& Dementia 2}

$$
\text { 1:30-2:45 p.m. }
$$

\section{Dementia (Alzheimer's Disease)}

\footnotetext{
S.L. AGHJAYAN, E.C. MORMINO, R. BUCKLEY, D.M. RENTZ, R. SPERLING, K. JOHNSON \& R. AMARIGLIO. Consistent Report of Subjective Cognitive Decline Longitudinally is Associated With Amyloid Burden.

Objective: Though subjective cognitive decline (SCD) is increasingly considered an early marker of Alzheimer's disease (AD), it has also been associated with other etiologies, such as mood, reducing its specificity for $\mathrm{AD}$. Given the progressive nature of $\mathrm{AD}$, we hypothesized that clinically normal older individuals $(\mathrm{CN})$ who consistently report SCD over the course of 4 years would be more likely to exhibit abnormal amyloid-beta $(\mathrm{A} \beta)$ burden than those who inconsistently report SCD.
}

Participants and Methods: Over the course of 4 years, we examined $201 \mathrm{CN}$ (age $=73.7 \pm 6.1,57 \%$ females, baseline $\mathrm{CDR}=0$ ) enrolled in the Harvard Aging Brain Study who underwent PiB-PET imaging in year 4. Subjects were asked annually if they had "recently experienced a change in their ability to remember things," coded as yes or no. Consistent SCD was measured as a report of "yes' for each of the four time points collected. Individuals were grouped according to whether they endorsed consistent or inconsistent SCD. A $\beta$ burden was measured continuously across an aggregate of cortical regions. Using a linear regression model, we assessed whether consistent SCD predicted amyloid deposition, controlling for age, sex, education, and depression.

Results: In our sample, $12.9 \%$ were considered to endorse consistent SCD. Consistent SCD significantly predicted amyloid levels at year 4 $(\mathrm{b}=.38, \mathrm{p}<.001)$ compared to inconsistent SCD.

Conclusions: These findings suggest that those who consistently report SCD are more likely on the early AD trajectory, compared to those who are less stable in their report. Consistent SCD may serve as a tool to detect those likely to manifest greater $\mathrm{A} \beta$ burden, of particular utility in secondary prevention trials.

Correspondence: Sarah L. Aghjayan, Bachelor of Arts, Neurology, Brigham \& Women's Hospital, 111 Park Drive, Apartment 11, Boston, MA 02215, United States. E-mail: saghjayan@partners.org

S. ALLiSON, C. ROE, G. BABULAL, S. STOUT, A. FAGAN, D. HOLTZMAN, J. MORRIS \& D. HEAD. Alzheimer Disease Biomarkers and Driving Space in Clinically Normal Older Adults: Role of Spatial Navigation Abilities.

Objective: Individuals frequently modify their driving habits as they age, including limiting the area in which they travel. Numerous studies have identified several factors, including spatial navigation skills, related to restricted driving space in older adults. However, no study to date has examined how Alzheimer disease (AD) pathology in clinically normal (CN) older adults (i.e., preclinical AD) may affect driving space, or the role of spatial navigation skills. This study examined whether AD biomarkers (i.e., concentrations of amyloid-beta ${ }_{42}\left(A \beta_{42}\right)$ as a marker of amyloid plaques, and tau as a marker of neuronal injury in the cerebrospinal fluid (CSF)) were associated with driving space and spatial navigation abilities, and whether spatial navigation abilities mediated the relationship between $\mathrm{AD}$ biomarkers and driving space.

Participants and Methods: CN older adults $(n=112)$ aged 65 and older completed the Santa Barbara Sense-of-Direction Scale and the Driving Habits Questionnaire as part of a longitudinal study on preclinical AD and driving performance. All participants had a lumbar puncture for the purpose of CSF.

Results: Neither CSF levels of $A \beta_{42}$ nor tau were significantly associated with driving space. CSF levels of $A \beta_{42}$, but not tau, were associated with self-reported navigation abilities. In addition, lower self-reported navigation ability was associated with reduced driving space. Lastly, spatial navigation abilities mediated the relationship between CSF A $\beta_{42}$ and driving space.

Conclusions: These findings suggest that cerebral amyloid deposition is associated with a lower perceived ability to navigate the environment, which may lead older adults to limit their driving area.

Correspondence: Samantha Allison, MA, Psychology Department, Washington University, 5445 Odell Street, Saint Louis, MO 63139, United States.E-mail: sallison@go.wustl.edu

W.A. ALVERSON, P.J. MASSMAN \& R.S. DOODY. Longitudinal Cognitive Asymmetry and Decline in Alzheimer's Disease Patients. Objective: A significant minority of patients with Alzheimer's disease (AD) exhibit asymmetric cognitive profiles. The present study aims to investigate stability of asymmetry as well as cognitive decline across subgroups of AD patients, classified by their asymmetry profile, in a large sample of patients.

Participants and Methods: 323 patients with probable AD were classified "Low Verbal," "Low Visuospatial," or "Symmetric" based 
on an Asymmetry Index (AI) derived from cognitive test performance at their initial evaluation. Patients underwent annual evaluations $\left(2^{\text {nd }}\right.$ evaluation $\mathrm{N}=234,3^{\text {rd }}$ evaluation $\mathrm{N}=111$ ). Proportions of profile classification, consistency of asymmetry indices, Mini Mental Status Exam (MMSE) scores, and a test performance composite were compared across available time points.

Results: $27.6 \%$ of patients had asymmetric profiles (11.8\% Low Verbal; 15.8\% Low Visuospatial) at initial evaluation, and this proportion decreased across time points $\left(18.9 \%\right.$ at $3^{\text {rd }}$ evaluation). Classification consistency across evaluations was fair to moderate (Cohen's Kappa $=.307-.442 ;$ all $p<.01)$. No patients changed "direction" of asymmetry between any evaluations. Correlations of AI across evaluations ranged from .61 to .73 , and were all significant (all $p<.01$ ). Declines in composite neuropsychological test performance did not differ between groups. Low Verbal patients had a significantly greater decline in MMSE score between the first two evaluations. MMSE scores otherwise did not differ between groups. Low Visuospatial patients displayed worse visuospatial memory performance at initial evaluation compared to other patient groups. No other group differences in memory performance were found.

Conclusions: Cognitive asymmetry was relatively stable across time, with a trend toward patients becoming more symmetric over time, consistent with past literature. With the exception of the Low Verbal patients exhibiting greater MMSE decline the first year, the subgroups showed grossly similar trajectories of cognitive decline.

Correspondence: William A. Alverson, B.S., Psychology, University of Houston, 2496 Bering Dr, Houston, TX 77057, United States. E-mail: waalverson@gmail.com

M. AZAR, S. CHAPMAN, E. BERTRAND, S. LAWLESS, Y. GU, O. TATARINA, Y. STERN \& S. COSENTINO. Quality of Life in an Ethnically Heterogeneous Community-Based Cohort of Patients with $\mathrm{AD}$.

Objective: Quality of life encompasses psychological, emotional and functional domains. Clinic-based research in Caucasian patients with Alzheimer's disease has suggested that multiple leisure-based and affective indicators of QOL decline with decreasing cognitive functioning. We examined the extent to which the association between QOL indicators and dementia severity extends to an ethnically diverse, community-based sample of individuals with AD.

Participants and Methods: Cross-sectional analyses examined informant report of patient $\mathrm{QOL}(\mathrm{n}=128, \mathrm{CDR} \geq 1)$ including the frequency, opportunity, and enjoyment of 15 non-ADL tasks indicative of QOL (Pleasant Events Schedule-AD), and frequency of positive and negative affect (Lawton's Apparent Emotions Scale). Partial correlations examined the association between these QOL indicators and dementia severity (MMSE), adjusting for education and informant type (i.e., family or trained caregiver) and compared across previous and current cohorts. Results: \% of participants with high QOL was similar between both cohorts $(23.1 \%$ vs $16.2 \%)$. However, MMSE was not correlated with summary indicators of QOL (opportunity $(r=0.00, p=0.98)$, frequency $(r=0.03, p=0.80)$ and enjoyment of daily activities $(r=-0.01, p=0.94))$ in this community. Indicators of positive affect (pleasure $(r=0.00 . p=0.98)$ and interest $(r=-0.06, p=0.55)$ were not associated with MMSE score, nor were indicators of negative affect (anger $(r=0.09, p=0.33)$, and depression $(r=-0.11, p=0.29)$ ). Associations between QOL indicators and MMSE $(p<0.01)$ emerged when expanding the cohort $(\mathrm{CDR} \geq 0.5)$. Conclusions: Associations between QOL and dementia severity were not observed in this community-based sample unless individuals at the very mildest stages $(\mathrm{CDR}=0.5)$ were included. The differential associations between QOL and cognition by cohort raise questions about how cultural and other factors influence QOL in $\mathrm{AD}$, and how diagnostic setting may affect relationship between disease markers.

Correspondence: Martina Azar, B.S., Taub Institute, Columbia University Medical Center, 119 Tioga Street, New York, NY 10032, United States.E-mail:ma523@cornell.edu
A.Y. BAENA, D. NORTON, D. COSIO, A. SCHULTZ, J. GATCHEL, F. LOPERA, K. JOHNSON, R. SPERLING \& Y.T. QUIROZ. Neuroticism is Associated With Tau Accumulation in Preclinical Autosomal Dominant Alzheimer's Disease.

Objective: Neuroticism, a personality trait characterized by the tendency to experience negative emotions, is associated with increased risk for dementia and cognitive impairment. However, it is unclear whether this is because neuroticism worsens cognitive performance independently of dementia, or because dementia-related processes contribute to neuroticism. We investigated whether neuroticism is associated with genetic predisposition to Alzheimer's disease (AD), and to biomarkers of AD pathology. We hypothesized that cognitively normal individuals with autosomal-dominant $\mathrm{AD}$ would have elevated levels of neuroticism, and that these would be related to increased tau accumulation.

Participants and Methods: Eighteen cognitively-normal individuals of the Colombian kindred with early-onset Autosomal-dominant AD participated (aged 25 to 44). Eight were carriers of the Presenilin-1 (PSEN-1) E280A mutation and ten were age matched non-carriers. All participants were blind to genetic status, and all carried a $50 \%$ risk of developing early onset $\mathrm{AD}$. All participants underwent tau PET imaging and completed the NEO Five-Factor Inventory.

Results: Mutation carriers had higher levels of neuroticism compared to non-carriers $(p=0.05)$, and neuroticism in carriers only was associated with increased levels of tau in entorhinal $(r=0.77, p=0.04)$ and inferior temporal cortices $(\mathrm{r}=0.87, \mathrm{p}<0.001)$. Other personality dimensions did not relate to $\mathrm{AD}$ pathology or differ between groups.

Conclusions: Neuroticism is elevated in cognitively normal individuals with autosomal-dominant $\mathrm{AD}$, and is related to tau accumulation. Further research with a larger sample size is needed to understand the underlying mechanisms explaining these associations.

Correspondence: Ana Y. Baena, Psicología, Grupo de Neurociencias de Antioquia, CALLE 62 \# 52-59, MEDELLIN 05, Colombia. E-mail: anitabaena713@gmail.com

\section{J.E. BAGGER, J. DERIGHT \& J. BRANDT. The Effect of Generation Gap on Informant Ratings using the IQCODE.}

Objective: In order to better understand the relationship between dementia patients and their informants, we aimed to better understand age and/or generational factors that may play into informant responses. Participants and Methods: Participants who completed the informant version of the online Dementia Risk Assessment $2.0 \quad(N=617)$ for patients over the age of 50 were included in this analysis. Each informant completed the IQCODE about a loved one to assess for perceived cognitive change over the past decade. The present study explored the effects of patient age group $(\leq 64,65-77$, and $\geq 78)$ and generation gap (e.g., sibling (0), parent (1), grandchild (2)).

Results: As the average age of the patient increased, the average IQCODE increased, regardless of generation gap. IQCODE was significantly lower for the youngest age group when there were two generations between the patient and the informant and was significantly higher for the same generation in the oldest age group.

Conclusions: Across all age groups, the generations closest to the patient (e.g,. sibling or parent) were relatively consistent in their IQCODE ratings. However, the IQCODE ratings from relatives from two generations younger were more variable, as they rated younger patients as less impaired and older patients as more impaired as compared to other generations. The present study reinforces existing literature on patient-informant relationships and suggests that it may be important to consider the generation of an informant rating.

Correspondence: Justina E. Bagger, Psychiatry, Johns Hopkins University School of Medicine, 721 St. Johns Rd., Baltimore, MD 21210, United States.E-mail: justinabagger@icloud.com 
D. CAHN-WEINER, M. GONZALES, S. FARIAS \& D. HARVEY. Cognitive Components of Everyday Functioning in Alzheimer's disease and Lewy Body Dementia.

Objective: Distinct cognitive profiles of patients with Alzheimer's disease (AD) and Lewy Body Dementia (LBD) have been described, but less is known about differences in their ability to carry out instrumental activities of daily living. The objective of this study was to characterize the functional limitations in AD and LBD by examining informant-rated everyday functioning using the Everyday Cognition scale (ECog).

Participants and Methods: Subjects underwent clinical assessment at the University of California, Davis Alzheimer's Disease Center and were diagnosed with Probable AD ( $\mathrm{N}=454)$ or Probable LBD ( $=44)$. Functional limitations were measured using the ECog (Farias et al., 2008), which provides global and domain-specific scores of everyday tasks (i.e. memory, language, visual perception, executive function). Diagnostic group differences in ECog scores were assessed with tobit models adjusting for age, gender, and dementia severity (MMSE score). Results: After accounting for age, gender, and dementia severity, the groups demonstrated similar levels of global functional impairment on the ECog. The LBD patients were rated as being more impaired than the $\mathrm{AD}$ patients in everyday language skills $(\beta=0.153, \mathrm{SE}=0.057, \mathrm{p}=0.007)$. There were no other significant group differences.

Conclusions: Patients with AD and LBD did not differ in overall functional impairment when accounting for dementia severity. The finding of greater everyday language impairment in the LBD group was unexpected, but may be explained by the importance of verbal initiation and generation skills in carrying out everyday language tasks. Verbal fluency skills have been reported in some studies to be more impaired in LBD than in $\mathrm{AD}$ patients. These findings suggest that a somewhat different pattern of everyday functional difficulties can be seen in subtypes of dementia and have implications for the development of intervention strategies to help patients with dementia sustain independence.

Correspondence: Deborah Cahn-Weiner, UC Davis, 100 N. Wiget Lane, Suite 150, Walnut Creek, CA 94598, United States. E-mail: dcahnweiner@ucdavis.edu

\section{B. CERBONE \& P.J. MASSMAN. The Benefits of Phonemic Cueing in Alzheimer's Disease Patients' Naming Performance.}

Objective: Deficits in confrontation naming vary among Alzheimer's disease (AD) patients, including the extent to which phonemic cueing is helpful in generating the target word. We examined the extent of phonemic cuing benefit on the Boston Naming Test (BNT) in probable AD patients and how it is associated with sex, premorbid intellectual functioning, ApoE genotype, dementia severity, and neuropsychological functioning.

Participants and Methods: Participants were 1107 individuals with mild to moderate Alzheimer's disease (mean MMSE $=20.0$ ). A Phonemic Cue Index (PCI), adjusting for the number of cues given, was calculated to determine the extent of benefit from BNT phonemic cueing, with higher values indicating greater benefit. Patients produced an average of 31.6 percent $(S D=21.9)$ correct responses on the items for which they were given phonemic cues.

Results: ANCOVA (with MMSE as the covariate) showed that females' PCI was significantly higher than males' $(p<.001)$, even though they did not differ in BNT total raw score with and without phonemic cues. An independent samples t-test revealed that the Mild AD group's PCI was significantly higher than the Moderate AD group's $(p<.001)$. ANCOVA (with age, MMSE, and AMNART as covariates) demonstrated that patients with zero ApoE $\varepsilon 4$ alleles benefited less from phonemic cueing than patients who had one $(p=.003)$ or two $(p=.002) \varepsilon 4$ alleles. Even with MMSE partialed out, PCI was moderately correlated with semantic functioning measures, such as WAIS Vocabulary, Similarities (both $r$ 's $=.30, p$ 's <.001), and Information $(r=.25, p<.001)$, but not with non-semantic measures such as Block Design and Digits Backward. PCI was also associated with premorbid verbal intellectual functioning. as measured by the AMNART $(r=.29, p<.001)$.
Conclusions: These findings suggest that examining phonemic cueing benefit, in addition to the traditional spontaneous naming BNT score, provides useful information.

Correspondence: Brittany Cerbone, B.A., Psychology, University of Houston, 4100 Southwest Freeway, Apt 459, Houston, TX 77027, United States. E-mail: brittany.cerbone@gmail.com

K.N. CHILDS \& B. CANNON. Verbal Fluency Discrepancies: Are They Pathognomonic Indicators of Alzheimer's Type Dementia? Objective: Identification of the early warning signs of neurodegenerative conditions, such as Alzheimer's disease, is critical to maintaining quality of life for an ever-growing population of older adults. The present research sought to explore the sensitivity and specificity of verbal fluency measures in identifying and differentiating several neuropsychological conditions that are common to aging populations.

Participants and Methods: Neuropsychological data was collected from a clinical sample of older adults. Information was collected for 41 patients who had been diagnosed with mild cognitive impairment (MCI) upon an initial evaluation and were subsequently re-evaluated to monitor interval changes. Patterns in semantic and phonemic verbal fluency performance were examined within and between diagnostic groupings to identify potential pathognomonic trends.

Results: While phonemic fluency performance was found to be largely consistent within diagnostic groups across administrations, semantic fluency was noted to significantly decrease upon re-evaluation for groups that demonstrated evidence of cognitive decline. Semantic fluency performance was also found to be significantly different between the group of patients who maintained a diagnosis of MCI and groups that demonstrated evidence of cognitive decline. There was no statistically significant trend in verbal fluency patterns that differentiated likely dementia due to Alzheimer's disease from other forms of neurodegenerative conditions.

Conclusions: Findings from this research suggest that semantic fluency performance can be thought to have implications for differentiating individuals who demonstrate progression to a degenerative condition, from those who do not demonstrate progression. However, contrary to the expectations of the proposed research, semantic fluency performance was not found to have diagnostic utility in differentiating degenerative etiologies.

Correspondence: Kathleen N. Childs, Marywood University, 2220 Sunburst Drive, Hazle Township, PA 18202, United States. E-mail: kathleenchildspsyd@gmail.com

L.R. CLARK, S. BERMAN, L. RIVERA, S. HOSCHEIDT, B. DARST, C. ENGELMAN, H. ROWLEY, C. CARLSSON, S. ASTHANA, P. TURSKI, O. WIEBEN \& S.C. JOHNSON. Relationship Between MRI Measures of Cerebral Arterial Flow and Perfusion in Asymptomatic Adults At-Risk for Alzheimer's Disease and Older Adults with Cognitive Impairment.

Objective: The current study aimed to (1) determine regional associations between MRI measures of cerebral artery blood flow (via four-dimensional (4D) flow) and capillary perfusion (via arterial spin labeling; ASL) in adults at increased risk for Alzheimer's disease (AD) and older adults with mild cognitive impairment and dementia and (2) test potential moderators of the relationship between arterial flow and capillary perfusion.

Participants and Methods: 187 participants (mean age 63.04y; 155 healthy, 32 cognitively impaired) completed MRI scans including ASL and phase contrast vastly undersampled isotropic projection imaging (PC VIPR) 4D flow sequences. Voxelwise multiple regression models investigated relationships between mean arterial blood flow (middle cerebral arteries (MCA); internal carotid arteries (ICA)) and cortical perfusion. Subsequent voxelwise regression models tested hypothesized moderators of the above relationship via interaction terms of mean arterial flow $\mathrm{x}$ age, mean arterial flow $\mathrm{x}$ cardiovascular risk, mean arterial 
flow x genetic risk for AD, and mean arterial flow x cognitive status on cortical perfusion.

Results: Broad relationships were observed between mean flow in the MCA and ICA and cortical perfusion, most strongly in parietal and frontal regions ( $p<.05$, FWE-corrected). Trends ( $p<.001$, uncorrected) suggested stronger relationships between arterial flow and perfusion in the presence of advanced age and greater vascular risk. Although mean flow was attenuated in individuals with dementia, the relationship between arterial flow and perfusion was similar across cognitively healthy and impaired groups.

Conclusions: Widespread positive relationships were observed between mean cerebral arterial blood flow and cortical perfusion across cognitively healthy and impaired adults. These relationships may become altered in the presence of advanced age and cardiovascular risk factors. Future studies will examine the contribution of altered vessel flow on trajectories of cognitive decline in preclinical $\mathrm{AD}$.

Correspondence: Lindsay R. Clark, PhD, Geriatrics, University of Wisconsin-Madison, 600 Highland Ave, J5/1 Mezzanine, Madison, WI 53792, United States.E-mail: Irclark@medicine.wisc.edu

\section{CLEM, K. DHIMA, C. LOBUE, H. WADSWORTH \& M. CULLUM. Factor Structure of the 15-Item Geriatric Depression Scale and Predicting Progression to Alzheimer's Disease.}

Objective: Depression has been reported as a risk factor for Alzheimer's disease (AD), although few studies have examined the prognostic ability of depression rating scales to predict the development of $\mathrm{AD}$. This study examined the factor structure of the Geriatric Depression Scale 15-item version (GDS-15) among subjects with Normal Cognition (NC) and Mild Cognitive Impairment (MCI), as well as the utility of those factors in predicting progression to a diagnosis of $\mathrm{AD}$.

Participants and Methods: National Alzheimer's Coordinating Center data were obtained for subjects diagnosed as $\mathrm{NC}(\mathrm{N}=10,587)$ or MCI $(\mathrm{N}=4,299)$ at baseline. Exploratory (EFA) and confirmatory factor analyses (CFA) were performed on the GDS-15 for each group using a varimax rotation. Factor scores were calculated from the extracted factor structure for NC and MCI groups. ANOVA was used to determine if mean factor scores differed between NC and MCI groups. Logistic regression analyses were performed to examine factor scores as predictors for progressing from NC/MCI to $\mathrm{AD}$ (over 11 years), while adjusting for demographic covariates.

Results: EFA identified a two-factor structure (hopelessness, dissatisfaction) for both NC and MCI subjects. CFA demonstrated good to excellent model fit (RMSEA=.04-.05; Adjusted GFI=.96-.97). MCI subjects had higher levels of hopelessness and dissatisfaction $(p$ 's<.001) compared to NC subjects, with a small effect $(d=.2-.3)$. However, cumulative factor scores were not predictive of progression to $\mathrm{AD}$ for $\mathrm{NC}$ or MCI groups ( $p$ 's $>.05)$.

Conclusions: The GDS-15 appears to measure similar constructs in NC and MCI populations. While MCI subjects reported higher levels of hopelessness and dissatisfaction, these factors did not predict progression to AD. Though depression has been found to be a risk factor for progression to $\mathrm{AD}$, these findings suggest that the constructs assessed by the GDS- 15 cannot be used to reliably distinguish those who will progress from NC or MCI.

Correspondence: Matthew Clem, MEd, Psychiatry, UT Southwestern Medical Center, 11348 Lippitt Ave, Dallas, TX 75218, United States. E-mail:matthew.clem@utsw.edu

L.E. COLVIN, S. COSENTINO, A. MACKAY-BRANDT \& S. CHAPMAN. Mood and Personality Characteristics Influence Metamemory Accuracy in Healthy Older Adults.

Objective: Disordered metacognition has significant functional implications for cognitively impaired older adults. Emerging work is revealing the neuroanatomic changes that compromise metacognition; however, little is known about whether premorbid factors also influence metacognition. Research suggests that psychological variables influence the perception of cognition, but whether they affect the accuracy of those perceptions (i.e., metacognition) has not been explored. The current study examined whether discrete personality and mood classes are associated with metamemory in healthy older adults.

Participants and Methods: Utilizing Latent Class Analysis (LCA), we tested for discrete personality (NEOFFI) and mood (STAI, BDI-II, and GDS) classes among 154 cognitively healthy older adults, ages 50-85, enrolled in the NKI-Rockland study. Metamemory was calculated using a calibration score comparing subjective memory ratings (revised Cognitive Function Questionnaire) to objective memory (Rey Auditory Verbal Learning Test) to determine the degree to which a person was overconfident, underconfident, or accurate in his or her self-assessment. ANCOVA was used to examine whether metamemory differed across the emergent classes, covarying for age, race, and ethnicity.

Results: Two discrete classes emerged in the LCA: Class A was characterized by predominantly high neuroticism, low extraversion, and high anxiety; Class B was characterized by predominantly low neuroticism, high extraversion, and low anxiety. Metamemory differed as a function of Class Membership $(\mathrm{F}(1,152)=6.533, p=.012)$ with individuals in Class A demonstrating under-confidence and Class B demonstrating over-confidence in their memory.

Conclusions: The association between psychological traits and metamemory suggests that premorbid characteristics of an individual may be important to consider in the conceptualization, assessment, and treatment of metacognitive disturbance in individuals with MCI or dementia. Correspondence: Leigh E. Colvin, PhD, Clinical Psychology, Columbia University, 1185 park avenue, New york, NY 10128, United States. E-mail:lec2151@tc.columbia.edu

C.M. CONTRASTANO, J. GUAY, J. KEAVENEY \& M. MCGRATH. The Relationship Among the Judgment of Line Orientation Test, Spatial Abilities, and Executive Functioning.

Objective: To examine if executive dysfunction predicts impaired performance on the Repeatable Battery for the Assessment of Neuropsychological Status (RBANS; Randolph, 1998) Judgment of Line Orientation (JOLO) subtest, independent of spatial and memory abilities, in geriatric adults diagnosed with either cognitive disorder or dementia. Participants and Methods: The researcher obtained data from the charts of 64 clients who had completed neuropsychological assessments. Half of the participants were diagnosed with Cognitive Disorder NOS and half were diagnosed with probable dementia of the Alzheimer's type according to the criteria of the Diagnostic Statistical Manual-Fourth Edition-Text Revised (DSM-IV-TR; American Psychiatric Association, 2000). The participants included 57 females and 7 males, ages 68-91. This study utilized a stepwise multiple linear regression. The criterion variable was performance on the RBANS JOLO. The predictor variables included the level of executive dysfunction, as measured by the Trail Making Test B (Reitan, 1958), Wechsler Memory Scale, Fourth Edition Clock Drawing (Wechsler, 2009), and Controlled Oral Word Association Test (Suhr et al., 1998); spatial abilities, as measured by the RBANS Figure Copy and Necker Cube (Nasreddine et al., 2005); and memory, as measured by the RBANS Delayed Memory Index Score.

Results: Results showed that while impaired performance on the Necker cube drawing was the strongest predictor of impaired performance on the RBANS JOLO, the number of errors on Trail Making Test B, a measure of executive functioning, accounted for an additional $5 \%$ of variance in performance.

Conclusions: Clinicians working with this population should consider that poor performance on the RBANS JOLO might indicate difficulties with executive functioning and therefore further assess executive functioning using additional measures. Determining the presence of executive dysfunction is particularly important for differential diagnosis and further recommendations for this population.

Correspondence: Christina M. Contrastano, Clinical Psychology, Immaculata University, 41 Reilly Ct, Apt 2, Metuchen, NJ 08840, United States.E-mail: clindsay1@mail.immaculata.edu 
E. DALCHAND, L.B. ZAHODNE, G. FELIX, K.M. FRAZER, M.N. MARTINEZ, H.L. SHOUEL, A.M. BRICKMAN \& J.J. MANLY. Early Parental Death and Sibship Size: Investigating the Causal Relationship to Alzheimer's Disease in a Multiethnic Cohort.

Objective: Early childhood adversity is associated with cognitive decline in old age, but few studies have included ethnically diverse cohorts. We hypothesized that early paternal death and larger sibships would lead to higher AD risk, but not maternal death. We expected childhood adversity would have stronger effects among ethnic minorities and women due to relatively sparse social and financial buffers.

Participants and Methods: Participants were adults age 65 and older in the Washington Heights/Hamilton Heights Inwood Aging Project. Early maternal and paternal death was operationalized as retrospective report of parent's death before the participant was age 18. Sibship size was determined by adding the number of full siblings reported. Tests measured language, visuospatial skill, speed, and memory at baseline and $18-24$ month follow-ups. At each visit, a diagnosis of AD was made using standard clinical criteria. The relationship of early parental death and sibship size to age of incident $\mathrm{AD}$ was tested using race-and sex-stratified Cox proportional hazards models, adjusting for recruitment cohort.

Results: Early maternal death was associated with incident AD among females $(\mathrm{HR}=1.60,95 \% \mathrm{CI}=1.25-2.29)$ but not males. There was no relationship between early paternal death and $\mathrm{AD}$. In the whole cohort, compared to participants with no siblings, participants with 4 or more siblings were $72 \%$ more likely to develop incident AD $(95 \%$ $\mathrm{CI}=1.21-2.42)$; however, stratified models showed that the effect was only significant among women $(p<.006)$ and not men, and specific to non-Hispanic Blacks $(p<.016)$ but not Whites or Hispanics.

Conclusions: Early maternal death was found to increase AD risk while early paternal death did not. A large sibship confered higher AD risk later in life. Women and African Americans may be more susceptible to deleterious early life conditions because of limits on social resources that buffer lower family socioeconomic status.

Correspondence: Elizabeth Dalchand, 630 West 168th St., P\&S Box 16, New York, NY 10032, United States.E-mail: edalchand@gmail.com

B.L. DEFEIS, M.E. MEINERDING, P. SUNDERARAMAN, Y. GU, C. ZHU, M. AZAR, S. LAWLESS, O. TATARINA, E. HUEY, Y. STERN \& S. COSENTINO. Neurocognitive correlates of psychotic symptoms in a community-based cohort of Mild Cognitive Impairment and Alzheimer's Disease.

Objective: Psychotic symptoms are known to be present in both Mild Cognitive Impairment (MCI) and Alzheimer's Disease (AD) and result in increased functional impairment. The extent to which such individual psychotic symptoms reflect specific cognitive changes is not clear. For example, delusions may reflect the construction of beliefs in the face of memory loss. We examined domain-specific cognitive performance of participants with and without psychotic symptoms in a community-based cohort of MCI and AD to evaluate possible neurocognitive correlates of psychotic symptoms.

Participants and Methods: 256 elders categorized as having MCI or $\mathrm{AD}$, with Clinical Dementia Rating (CDR) scores higher than 0.5, were enrolled in the heterogeneous, community-based Predictors 3 cohort. Cognitive factor scores were derived measuring memory, language, speed, and visual-spatial ability. Psychotic symptoms (hallucinations, delusions, and illusions) were assessed with the Columbia University Scale for Psychopathology in AD. Presence or absence of psychotic symptoms and demographic variables were used in linear regressions to predict each cognitive factor.

Results: Psychotic symptoms were present in 51\% of participants, delusions being the most prevalent $(48 \%)$, followed by hallucinations $(22.8 \%)$ and illusions $(2.8 \%)$, and were more common among those with higher CDR scores $(p<.001)$, lower global cognition $(p<.001)$, Hispanic ethnicity $(p=.002)$ and lower education $(p<.001)$. Controlling for age, gender, education, and ethnicity, delusions $(\beta=-.178 ; \mathrm{p}=.017)$ and illusions $(\beta=-.141 ; p=.039)$ predicted poorer memory performance, and illusions $(\beta=-.112 ; p=.002)$, predicted poorer language performance. Conclusions: Selective associations were found between specific psychotic symptoms and cognitive scores. The associations of psychosis with ethnicity and education suggest that cultural factors may influence psychosis in AD. The pattern of associations may inform the nature and etiology of psychotic symptoms in AD.

Correspondence: Brittany L. DeFeis, MSc Candidate, The Taub Institute for Research on Alzheimer's Disease and the Aging Brain, Columbia University Medical Center, 3333 Broadway, C8G, New York, NY 10031, United States.E-mail: bld2130@cumc.columbia.edu

N.A. EMMERT, K. MULHAUSER, L. SCHWARZ \& J. GFELLER. Using the RBANS, Premorbid Intelligence, and Educational Attainment to Classify Dementia Patients as Normal or Impaired on the ILS Health and Safety Scale.

Objective: Previous research has demonstrated that variables such as demographics, cognition, and depression are related to performance on the Independent Living Scales (ILS). Additionally, these variables have discriminated between individuals with normal and impaired scores on the ILS Full Scale score. However, there is minimal research investigating these relationships using individual ILS scales, such as the ILS Health and Safety (ILS HS) scale. The present study examined the ability of the RBANS Total Scale, demographic variables, estimated premorbid intelligence, and depression to classify patients above and below cutoffs for normal $(\mathrm{T}>30)$ or impaired ( $\mathrm{T} \leq 30)$ ILS HS performance.

Participants and Methods: The present study utilized archival data from a clinical sample $(N=73)$ of patients with various types of dementia (e.g.., Alzheimer's disease), who were administered the WTAR, RBANS, GDS, and ILS HS scale as part of a larger neuropsychological evaluation at an outpatient service.

Results: Results of a discriminant function analysis demonstrated that years of education, WTAR FSIQ, and RBANS Total Scale correctly classified $75.3 \%$ of cases. Classification errors occurred more frequently in the normal ILS HS group, demonstrating a $28 \%$ false positive rate. Conclusions: In summary, global cognitive ability, estimated premorbid intelligence, and level of education made independent contributions towards discriminating dementia patients with normal from impaired functional abilities in the domain of health and safety. These results differ from previous research, which found that depression severity was predictive of ILS group membership in a sample of patients with normal and impaired cognitive functions.

Correspondence: Natalie A. Emmert, M.S., Psychology, Saint Louis University, 3359 Summerset Court, North Tonawanda, NY 14120, United States. E-mail: emmertna@slu.edu

S. FARIAS, M. SCHMITTER-EDGECOMBE, A. WEAKLEY, D. HARVEY, K. DENNY, C. BARBA, T. GIOVANNETTI \& S. WILLIS. Compensation Strategy Use Among Older Adults: Association with Diagnostic Status, Neuropsychological Function, and Everyday Function.

Objective: The effective use of compensation strategies, behaviors aimed at mitigating or adapting to actual or perceived loss, may be one factor that contributes to greater resilience among older adults in the face of declining cognition. Few studies have examined the relationship between compensation and functional outcomes. The goal of the present study was to better understand how compensation strategy use among older adults with varying degrees of cognitive impairment impacts everyday functioning.

Participants and Methods: Participants included 125 older adults (including those with normal cognition, Mild Cognitive Impairment (MCI) or dementia) who were enrolled in a longitudinal observational study. The Everyday Compensation Questionnaire (EComp), a new instrument that assesses frequency of compensation strategy use when completing various activities of daily living (e.g., managing 
appointments, shopping, managing finances, etc.), was completed by participants' informants. Objective measures of neuropsychological functioning were also collected and the primary outcomes consisted of ratings of performance in everyday abilities including instrumental activities of daily living (IADLs).

Results: Cognitively normal elders and those with MCI had equivalent levels of compensation use, which was greater than those with dementia. Higher levels of neuropsvchological function were associated with more frequent compensation use. Most importantly, greater frequency of compensation strategy use was associated with higher levels of independence in everyday function, and this relationship remained significant even after accounting for cognition.

Conclusions: This is one of the first studies to document that spontaneous use of compensation strategies is associated with a higher level of functioning in daily life among older adults. Findings provide strong rational for further development of interventions that directly target the enhancement of such strategies.

Correspondence: Sarah Farias, Ph.D., Neurology, University of California, Davis, 15890 Highway 124, Plymouth, CA 95669, United States.E-mail:farias@ucdavis.edu

J. TALON-CROTEAU, A. GIGUÈRE-RANCOURT, D. LAURIN \& M. SIMARD. Nutritional Supplementation in Prevention and Treatment of Cognitive Impairments Associated with Alzheimer's disease.

Objective: The objective of this work was to assess the efficacy of nutritional supplements on cognition in healthy elderly, patients with Mild Cognitive Impairment (MCI) and with Alzheimer's disease (AD).

Participants and Methods: The following databases were searched to identify studies written in English and French, and published from April $1^{\text {st }} 1999$ to June $1^{\text {st }}$ 2014: Cochrane Library, Current Contents, EBSCO, EMBASE, PubMed and PsycNet. Only randomized controlled trials (RCT) investigating the effects of omega-3, vitamins B6, B9, B12, $\mathrm{C}$ and $\mathrm{E}$ on older adults with and without cognitive impairment were included.

Results: Nineteen RCT assessed the effects of omega-3, vitamins B6, B9, B12, C and E on cognition of healthy elderly, MCI and AD patients. MCI patients registered the strongest effect sizes $(d \geq 0.8)$ on measures of episodic memory, depressive symptoms when taking omega-3. AD patients recorded the strongest effect sizes $(d \geq 0.8)$ on measures of global cognitive function and activities of daily living while taking omega- 3 and combination of omega- 3 supplements. Omega- 3 were thus the most effective supplement to improve cognition in MCI and to slow cognitive decline in AD. All supplements were well tolerated and safe. Conclusions: Conclusions about the specific effects of each nutriment were limited by the heterogeneity of tests, although there were some positive results. In future trials, clinical results shall be supported by changes in biological markers. The development of guidelines is mandatory to standardize research on these interventions for prevention and treatment of cognitive impairment associated with $\mathrm{AD}$.

Correspondence: Ariane Giguère-Rancourt, PhD student, École de Psychologie, Université Laval, 2325, rue des Bibliothèques, Québec, QC G1V 0A6, Canada. E-mail: ariane.giguere-rancourt.1@ulaval.ca

K. HACKETT, C. SHIH, A. SEIFAN, J. SHUM, E.E. CAESAR \& R.S. ISAACSON. NIH Toolbox Cognition Battery and PROMIS Measures for Detecting Preclinical Alzheimer's Disease.

Objective: Describe the performance of patients with suspected preclinical Alzheimer's disease (AD) on NIH Toolbox Cognition Battery (NIHTB-CB) and Patient-Reported Outcomes Measurement Information System (PROMIS) measures.

Participants and Methods: 121 Weill Cornell Memory Disorders/ Alzheimer's Prevention Clinic patients (52 males, 69 females, mean age $59.50 \pm 14.59$ ) completed baseline neuropsychological testing and self-report PROMIS measures (sleep disturbance, anxiety, depression). All received consensus diagnoses (case reviewed by neuropsychologists and neurologists specializing in dementia). Subjects were classified as Detectable Cognitive Impairment due to AD (DCI-AD) if amnestic cognitive deficits were present, below the Mild Cognitive Impairment (MCI) threshold, and the subject had at least one non-cognitive symptom ( $\mathrm{mood} / \mathrm{motor} / \mathrm{sleep} / \mathrm{smell})$ and family history of AD, without other explainable etiologies. NIHTB-CB and PROMIS scores, adjusted for age, gender, ethnicity and education, were compared across groups using ANOVAs and post-hoc analyses.

Results: Subjects were classified as Cognitively Normal (CN) ( $n=23)$, DCI-AD $(n=10)$, DCI-non-AD $(n=35)$, MCI-AD $(n=14)$, AD $(n=7)$. or Other $(n=32)$. Rey Auditory Verbal Learning Test (Immediate \& Delayed Recall), Dimensional Change Card Sort, Flanker Inhibitory Control \& Attention, and Pattern Comparison Process Speed were significantly more impaired in DCI-AD than in both DCI-non-AD $(p<.05)$ and $\mathrm{CN}(p<.05)$. There were no measures where MCI-AD was significantly more impaired than DCI-AD. Neither PROMIS measures nor Odor Identification showed differential impairment in DCI-AD when compared to CN. DCI-non-AD or MCI-AD.

Conclusions: Select NIHTB-CB tests measuring learning, memory and executive function, but not PROMIS measures or Odor Identification. can help clinicians identify patients at higher risk of preclinical AD. Further research will require larger sample sizes to reveal the utility of other measures, as well as validation of the DCI-AD diagnostic approach using AD biomarkers.

Correspondence: Katherine Hackett, BA, Neurology, Weill Cornell Medicine, 346 East 18th Street Apt. 2C, New York, NY 10003, United States.E-mail: hackettk22@gmail.com

D.B. HAMMERS, T.J. ATKINSON, B.C. ALLRED. DALLEY, K.R. SUHRIE, K. HORN, K. RASMUSSEN, B. BEARDMORE, L. BURRELL, K. DUFF \& J. HOFFMAN. Amyloid Positivity using [18F]Flutemetamol-PET and Cognitive Deficits in Non-Demented Community-Dwelling Older Adults.

Objective: $[18 \mathrm{~F}]$ Flutemetamol is a radioactive diagnostic agent indicated for Positron Emission Tomography (PET) imaging of the brain to estimate beta-amyloid neuritic plaque density in adult patients with cognitive impairment. While it is considered a promising clinical agent that can optimize amyloid binding along with possessing an extended half-life, there has been little research examining its relationship with cognition. The purpose of the current study was to compare cognitive performances among individuals having either increased amyloid deposition (Flute+) or minimal amyloid deposition (Flute-).

Participants and Methods: Twenty-seven non-demented community-dwelling adults over the age of 65 underwent [18F]Flutemetamol amyloid- positron emission tomography imaging, along with cognitive testing using the RBANS and select behavioral measures. Multi-variate analysis of variance was used to identify differences between the $[18 \mathrm{~F}]$ Flutemetamol groups for the cognitive and behavioral measures.

Results: Flute+ participants performed significantly worse than Flute- participants on RBANS Indexes of Immediate Memory $(p<.001$, $\left.\eta^{2}=0.43\right)$, Language $\left(p<.05, \eta^{2}=0.25\right)$, Delayed Memory $(p<.001$ $\left.\eta^{2}=0.63\right)$, and Total Scale score $\left(p<.005, \eta^{2}=0.39\right)$, but no differences in the endorsed level of depression $\left(p>.05, \eta^{2}=0.00\right)$ or subjective report of cognitive difficulties $\left(p<.05, \eta^{2}=0.03\right)$ were observed. Conclusions: Although these results are preliminary, [18F]Flutemetamol accurately tracks with cognition in a non-demented elderly sample, which may allow for better prediction of cognitive decline in late life.

Correspondence: Dustin B. Hammers, Ph.D., ABPP, University of Utah, 650 Komas Dr. \#106-A, Salt Lake City, UT 84108, United States. E-mail:dustin.hammers@hsc.utah.edu

A.L. HARMELL, G.M. PEAVY \& B. PALMER. Neuropsychiatric and Cardiovascular Predictors of Alzheimer's disease.

Objective: Presently, over 5 million Americans live with Alzheimer's disease (AD); this number is projected to grow to over 7 million by 
2025. In the absence of effective treatments, and difficulty reversing the neuropathological changes once they emerge, finding modifiable risk factors early in the course of the disorder is one of the most promising strategies to delay or prevent the progression of dementia. The purpose of this study was to explore whether neuropsychiatric and cardiovascular symptoms influence phenotype and rate of progression to AD.

Participants and Methods: This study included 82 volunteers with Mild Cognitive Impairment from the UC San Diego Alzheimer's Disease Research Center (ADRC). Participants were seen annually and underwent a one-hour neurological examination, ApoE genotyping, and assessments of medication use, cardiovascular symptoms (diabetes, hypertension, high cholesterol, smoking), and neuropsychiatric functioning. Cox proportional hazards regression models were used to estimate hazard ratios (HRs) and 95\% confidence intervals; ApoE-e4 interaction terms were tested.

Results: Fifty-seven individuals converted to AD during the course of the study (average follow-up of 4.6 years). After adjusting for age, sex, and education, individuals endorsing agitation at baseline were more than two times as likely to convert to $\mathrm{AD}(\mathrm{HR}=2.118,95 \%$ CI $[1.062$, 4.226], $\mathrm{p}=0.033$ ) and individuals with at least mild depression were 2.5 times as likely to convert to $\mathrm{AD}(\mathrm{HR}=2.481,95 \% \mathrm{CI}[1.163,5.294]$, $\mathrm{p}=0.019)$. There were no significant interactions between neuropsychiatric symptoms and ApoE-e4; cardiovascular risk factors did not predict change in cognitive status.

Conclusions: Results suggest that agitation and depression at baseline in subjects with MCI are associated with increased risk of future AD. As such, clinicians should monitor individuals presenting with both cognitive complaints and new neuropsychiatric symptoms closely, as these individuals may be at higher risk of developing AD.

Correspondence: Alexandrea L. Harmell, M.S., Psychiatry, SDSU/UCSD Joint Doctoral Program in Clinical Psychology, 8950 Villa La Jolla Drive B101, La Jolla, CA 92037, United States.E-mail: aharmell@ucsd.edu

\section{A.J. JACOBSON, J.S. GOLDBERG, E. JIMENEZ, P.H. LU, G.J. LEE \& M.F. MENDEZ. Deep Gray Matter Correlates of Symptoms on the Frontal System Behavior Scale in Behavioral Variant Dementia and Early-Onset Alzheimer's Disease.}

Objective: Patients with behavioral variant frontotemporal dementia (bvFTD) often present with profound behavioral and frontal-executive symptoms compared to similar aged patients with early-onset Alzheimer's disease (EOAD). The purpose of the present study was to examine the relationship between deep gray matter (DGM) volumes and caregiver ratings on the Frontal System Behavior Scale (FrSBe) in bvFTD and EOAD patients.

Participants and Methods: Fifteen bvFTD $\left(M_{\mathrm{age}}=59.5, S D=11.4\right)$ and $20 \mathrm{EOAD}\left(M_{\text {age }}=59.1, S D=5.3\right)$ patients underwent MRI and caregivers completed the FrSBe, yielding three subscale scores (Apathy, Disinhibition, Dysexecutive) and a Total score. Automated segmentation of MRI scans estimated volumes of DGM structures. Volumes were then corrected for total brain size to account for variability of intracranial volume. Diagnostic groups were compared on FrSBe ratings and subcortical volumes. Correlations adjusted for age were used to assess the relationship between volumes and FrSBe scores within each group. Results: BvFTD patients had higher ratings on the FrSBe Total score $(p<.001)$ and on each individual subscale score $(p s<.001)$ compared to EOAD patients. Additionally, bvFTD patients showed significantly greater bilateral atrophy in the thalamus $(p s<.001)$, nucleaus accumbens $(p s<.01)$, and the right putamen $(p<.05)$. Analysis of the bvFTD group revealed a significant negative correlation between Apathy score and right thalamus volume $(r=-.69, p=.006)$. The remaining correlations between FrSBe scores and volumes were non-significant. In EOAD, correlations between FrSBe scores and DGM structures were non-significant.

Conclusions: Compared to EOAD, bvFTD patients show greater changes to DGM structures - specifically, the thalamus, nucleus accumbens, and putamen. Symptoms of apathy were associated with atrophy of the right thalamus in bvFTD patients, suggesting that parts of the frontal-subcortical circuits may be damaged in bvFTD rather than EOAD.

Correspondence: Aron J. Jacobson, PhD, Psychology, Loma Linda University, 11130 Anderson St., Loma Linda, CA 92350, United States. E-mail: aronjacobson@gmail.com

C.D. KAY, M. SEIDENBERG, S. DURGERIAN, K.A. NIELSON, J. SMITH, J.L. WOODARD, L. MOURANY, G. LOSINSKI \&. S. RAO. Are Measures of Intraindividual Variability Sensitive to the Preclinical Stage of Alzheimer's Disease in Elders at Genetic Risk? Objective: Increased intraindividual variability (IIV) within tasks of cognitive control has been sensitive in discriminating healthy elders from individuals in the early stages of Alzheimer's disease (AD), including those with mild cognitive impairment (MCI). However, there has been limited research examining the relationship between IIV and genetic risk factors for $\mathrm{AD}$. We examined the influence of the apolipoprotein E-epsilon 4 (APOE- $\varepsilon 4)$ allele and familial AD risk in healthy elders on motor timing and processing speed IIV.

Participants and Methods: Seventy-one healthy elders $\left(M_{\text {age }}=67.3\right)$ comprised two AD risk groups: high-risk ( $n=42$; AD family history in a first-degree relative and APOE- $\varepsilon 4$ positive) and low-risk ( $n=29$; no AD family history and APOE- $\varepsilon 4$ negative). Participants completed a finger-tapping time reproduction task (TRT) presented in a synchronization-continuation paradigm and a processing speed task (PST). IIV measures included coefficient of variation values of inter-response intervals.

Results: No significant differences emerged in motor timing or processing speed IIV between high- and low-risk participants ( $p$ 's>0.05). Mean performance on the TRT and PST also did not vary as a function of $\mathrm{AD}$ risk $(p$ 's $>0.05)$.

Conclusions: Consistent with the limited existing research, results suggest that measures of IIV are not sensitive to the preclinical stage of $\mathrm{AD}$ in healthy elders at high versus low genetic risk for this disease. Longitudinal data is necessary to more clearly determine the predictive value of IIV for progression to MCI and clinical AD.

Correspondence: Christina D. Kay, PhD, Psychology Assessment Center, Massachusetts General Hospital, 1 Bowdoin Square, 7th Floor, Boston, MA 02114,United States. E-mail: cdkay@mgh.harvard.edu

\section{E. KEMP, H. MICHALAK, J. MCDONALD, E. PUGH, M. BECKER \& A.P. MECCA. Sleep Disturbance and Risk of Cognitive Decline in the ADNI Cohort.}

Objective: To investigate the role of disrupted sleep on change in cognition, including memory and executive function $(\mathrm{EF})$, over time, as well as risk for diagnostic conversion in Alzheimer's Disease (AD).

Participants and Methods: 1735 older adult and geriatric participants with cognitively normal (CN), Early Mild Cognitive Impairment (EMCI), Late Mild Cognitive Impairment (LMCI), and Alzheimer's Disease (AD) diagnoses were followed for up to 24 months. Sleep disturbance was assessed with the Neuropsychiatric Inventory (NPI), and overall cognition, memory sub-scores, and executive function sub-scores were measured with the Alzheimer's Disease Assessment Scale-Cognitive Sub-scale (ADAS-Cog 11). Age, sex, APOE genotype, education, and sedative use were included as covariates.

Results: A linear repeated measures mixed effects model was used to evaluate the effect of sleep disturbance on change in cognition over time. Presence of sleep disturbance bore no significant effect on measures of memory, EF, or overall cognition in the CN, EMCI, or AD diagnostic groups. The LMCI group showed no significant difference in change in memory or EF subscores, but did for overall change in cognition (Parameter Estimate $=-0.047, p=0.018$ ), with those positive for sleep disturbance declining at a slower rate than those without. A Cox Hazard Model showed no significant effect of sleep disturbance on risk of diagnostic conversion from either CN to either EMCI or LMCI, or EMCI or LMCI to AD. 
Conclusions: The findings of this study largely contradict previous research which asserts that sleep disturbance is a risk factor for cognitive decline. This is notable as our measures of cognitive decline were more robust and sensitive than that of previous cross-sectional research. Similarly, our study is one of the first to use follow-up NPI data, rather than just baseline, thus capturing sleep disturbance more sensitively than previous prospective studies. It may be that the focus on sleep disturbance as a risk factor for decline has been misplaced.

Correspondence: Emily Kemp, B.S., Psychiatry, Vale University, 83 Olive Street, New Haven, CT 06511, United States. E-mail: emily.kemp@ yale.edu

T. KIELY, C. MOORE, A. LEE, L. SHAUGHNESSY, A. ATRI \& C. MARREIRO. The Contribution of Neuropsychological Performance, Behavioral and Functional Measures in High- \& Low-likelihood Alzheimer's Disease Profiles.

Objective: Randomized clinical trials (RCTs) rely on diagnostic criteria including patterns of cognitive impairment as well as behavioral and functional features to select participants. Without amyloid/ tau biomarkers, $20 \%$ of clinically diagnosed patients lack AD pathology at autopsy. Inclusion of these individuals in RCTs hinders success. The objective of this study is to examine which measures (neuropsychological, behavioral, functional) increase accuracy of high-likelihood $\left({ }_{\mathrm{H}} \mathrm{AD}\right)$ or low-likelihood $\left({ }_{\mathrm{L}} \mathrm{AD}\right) \mathrm{AD}$ pathological diagnosis.

Participants and Methods: Participants came from the National Alzheimer's Coordinating Center (NACC) database. Inclusion criteria included: stable etiologic diagnosis of AD across first two visits; CDR global score $<=1$, and autopsy. Propensity analysis was used to form two matched groups (on education, sex, race, ethnicity) of $\mathrm{H} \mathrm{AD}(\mathrm{n}=71)$ and ${ }_{\mathrm{L}} \mathrm{AD}(\mathrm{n}=71)$, defined by Braak and CERAD neuritic plaque staging. Baseline instruments from the NACG Uniform Data Set included the neuropsychological battery, Neuropsychiatric Inventory (NPI), and the Functional Activities Questionnaire. A logistic regression examined the degree to which selected measures predicted autopsy group.

Results: The final logistic model included Digit-Symbol Coding, Logical Memory II (LM-II), and NPI, and was an adequate fit to the data $\left(\mathrm{x}^{2}(3)=23.72, \mathrm{p}<0.001 ;\right.$ McFadden's $\left.\mathrm{R}^{2}=0.12\right)$. NPI $(\mathrm{z}=2.35, \mathrm{p}<0.001)$ and LM-II $(\mathrm{z}=3.59, \mathrm{p}<0.01)$ each made a significant contribution to prediction. A one-unit increase in NPI score decreased the odds of ${ }_{\mathrm{H}} \mathrm{AD}$ classification by 1.12 , whereas a one-unit decrease in LM-II increased the odds of ${ }_{\mathrm{H}} \mathrm{AD}$ classification by 1.23.

Conclusions: These preliminary analyses suggest, in the absence of biomarker data, a combination of LM-II and NPI scores increase accuracy of diagnostic classification of $\mathrm{AD}$. Future work should examine the use of these variables to create propensity scores that may be helpful in making inclusion decisions for RCTs

Correspondence: Thomas Kiely, Ph.D., Neuroscience, Ray Dolby Brain Health Center, 45 Castro Street; Suite: 220, San Francisco, CA 94114 , United States. E-mail: kielyt1@sutterhealth.org

B.S. LAST, N. SCHUPF, J.J. MANLY \& A.M. BRICKMAN. Sibling history of dementia is associated with cortical thickness in older adults.

Objective: Family history of dementia is known to increase risk of Alzheimer's disease (AD). In the current study, we examined whether a reported sibling history of dementia is associated with cortical thickness, a magnetic resonance imaging (MRI) marker of neurodegeneration, among community-dwelling older adults in upper Manhattan.

Participants and Methods: Participants in the Washington Heights Inwood Columbia Aging Project with at least one biological sibling and an available 3T T1-weighted MRI scan were included in the analysis $(\mathrm{n}=533)$. Sibling history of dementia was ascertained by self-report and considered as present or absent. Regional cortical thickness was derived from high resolution structural MRI scans with FreeSurfer and a summary cortical thickness variable was created by weighted averages of regional data. Participants were evaluated with a comprehensive neuropsychological battery and a summary measure was derived to represent overall cognitive functioning. With linear multiple regression, we examined the association of sibling history with cortical thickness after controlling for age, race/ethnicity, sex, education, and global cognition.

Results: Fifty participants reported a positive sibling history of dementia. Sibling history was significantly associated with lower overall cortical thickness. Among the covariates, increased age, male sex, and lower overall cognition were associated with lower overall cortical thickness. Conclusions: Positive sibling history of dementia is associated with lower overall cortical thickness independent of participant's own cognitive status. Decreased cortical thickness may be an endophenotype of $\mathrm{AD}$ and/or an early marker of neurodegeneration.

Correspondence: Briana S. Last, B.A., Taub Institute, Columbia University Medical Center, 80 Bennett Avenue, Apt 5E, New York, NY 10033, United States.E-mail: briana.last@gmail.com

Y. LEE, E. UMUCU, L. HANCOCK, A. GILMORE-BYKOVSKYI, D. NORTON, H. BLAZEL, S.C. JOHNSON, C. CARLSSON, D. WASHINGTON, S. ASTHANA \& C. GLEASON. Neuropsychological Correlates of Apathy in Cognitively Healthy Middle-Aged Individuals at Risk for Alzheimer's Disease.

Objective: Apathy is a well-established prodromal behavioral symptom for Alzheimer's disease (AD). However, little is known about apathy in healthy individuals who are at increased risk of acquiring AD. This study examines the presence and nature of apathy in these individuals, along with the associations between apathy and neuropsychological measures. Participants and Methods: 316 cognitively normal participants $\left(M_{\text {age }}=57.23, S D=5.18,70.3 \%\right.$ female $)$ from a sub-set of the $\mathrm{W}$ isconsin Alzheimer's Disease Research Center's Clinical Core: The Investigating Memory in People at Risk, Causes and Treatments (IMPACT) cohort. Participants completed the Apathy Evaluation Scale Self-Report (AESS), Mini Mental State Examination (MMSE), Center for Epidemiologic Studies Depression Scale (CES-D), and several neuropsychological tests, including Trails A \& B, Rey Auditory Verbal Learning Test (RAVLT), and Boston Naming Test (BNT).

Results: Participants reported several symptoms consistent with apathy $(M=24.85, S D=5.94$, range 18-62, higher scores indicate more apathy). AES-S scores were strongly related to depression (CES-D; $r=.46 p<.000$ ) and MMSE total score $(r=-.12 p<.03)$. Trails B and RAVLT Delayed Recall predicted $47 \%$ of the variance in AES-S $(F=7.73, p=.001)$. Adding Trails A, BNT, RAVLT Initial Learning, and RAVLT List B Recall predicted $57 \%$ of the variance in AES $(F=3.09, p=.006)$.

Conclusions: Our findings suggest apathy is present at low levels in cognitively normal individuals who have an increased risk of AD. Neuropsychological tests explained a wealth of variation in apathy scores, suggesting that AES-S scores could potentially reveal underlying neuropsychological changes. These findings are consistent with recent research, which suggests that behavioral changes may be one of the first signs of progression toward Mild Cognitive Impairment or AD. Correspondence: Yun-Pai (Beatrice) Lee, University of WisconsinMadison, 2924 Harvey Street, APT 7H, Madison, WI 53715, United States.E-mail:lee@neurology.wisc.edu

E. UMUCU, Y. LEE, L. HANCOCK, A. GILMORE-BYKOVSKYI, D. NORTON, H. BLAZEL, S.C. JOHNSON, C. CARLSSON, D. WASHINGTON, S. ASTHANA \& C. GLEASON. The Relationship Between Apathy and MMSE in Cognitively Healthy Middle-Aged Individuals at Risk for Alzheimer's Disease.

Objective: Apathy is a well-established prodromal behavioral symptom for Alzheimer's disease (AD). However, little is known about apathy in healthy, middle aged individuals who are at increased risk of acquiring AD. The current study examines the relationship between apathy and global cognition in this population.

Participants and Methods: 316 middle-aged cognitively normal participants $\left(M_{\text {age }}=57.23, S D=5.18,70.3 \%\right.$ female $)$ from a sub-set 
of the Wisconsin Alzheimer's Disease Research Center's Clinical Core: The Investigating Memory in People at Risk, Causes and Treatments (IMPACT) project were recruited in this study. Participants completed the Apathy Evaluation Scale, Self-Report (AES-S), Mini Mental State Examination (MMSE), and the Centers for Epidemiologic Studies-Depression Scale (CES-D).

Results: Simultaneous regression results indicated a strong relationship between MMSE and AES-S $(B=-.65, p<.05)$. The unstandardized regression coefficient between MMSE and CES-D was significant $(B=-1.70, p<.001)$, as was the unstandardized regression coefficient between CES-D and AES-S $(B=.39, p<.001)$ when controlling for MMSE. MMSE was no longer significant predictor of AES-S $(B=.02$, $p=.95)$. The relationship between MMSE and AES-S was fully mediated by CES-D.

Conclusions: Although the relationship between apathy and depression is controversial, this study indicates depression as a strong predictor of apathy in healthy individuals at increased risk of developing AD, suggesting that mood mediates the relationship between apathy and MMSE.

Correspondence: Yun-Pai (Beatrice) Lee, University of WisconsinMadison, 2924 Harvey Street, APT 7H, Madison, WI 53715, United States.E-mail:lee@neurology.wisc.edu

S. LIU, F. CHANG, C. KREISEL, K. GOMEZ, E. WOO, J. RINGMAN, L. APOSTOLOVA, S. WOLF \& C. NUNEZ. Cognition, Neuropsychiatric Symptoms and Everyday Functioning in Latino Older Adults.

Objective: The purpose of this study was to examine neuropsychiatric symptoms (NPS) and activities of daily living (ADLs) in elderly Latinos with and without dementia. We also evaluated relationships between NPS and ADLs with executive function.

Participants and Methods: 560 elderly, community-dwelling participants, comprised of non-Latino whites $(n=423)$ and Latinos $(n=137)$, were participants in this study. The Neuropsychiatric Inventory-Questionnaire (NPI-Q) and Functional Activities Questionnaire (FAQ) were administered to all participants, with a subset $(\mathrm{n}=55)$ also administered Trail-Making-Test B (TMT-B). ANOVA and partial correlations were used to evaluate relationships between these measures in Latino and non-Latino whites with normal cognition (NC), mild cognitive impairment (MCI), and dementia.

Results: Latinos with NC and dementia had significantly higher NPS compared to non-Latino whites, with increased frequency of agitation, anxiety, depression, and nighttime disturbances for all diagnosis groups. Latinos with NC and MCI had poorer ADLs than non-Latinos, with increased dependence for all diagnosis groups in completing taxes, tracking current events, traveling out of the neighborhood alone and remembering appointments. There was no significant difference in TMT-B completion time between ethnic groups and no correlation between NPI total score and TMT-B completion time. There was a strong, positive correlation between FAQ total scores and TMT-B completion time for Latinos, and a moderate correlation for non-Latino whites.

Conclusions: Our findings support current literature of Latinos reporting more neuropsychiatric symptoms, despite diagnostic status. We found a difficultly in performing ADLs in Latinos overall and a stronger relationship between difficulty performing ADLs and poor executive skills in Latinos when compared to non-Latino whites. These findings suggest that cultural differences may significantly contribute in the assessment of dementia.

Correspondence: Serena Liu, CA, United States.E-mail: liuseren@usc. edu

D.A. LOWE, L.A. BRENNER, D.M. SZELES, A. WALKER \& M.T. WAGNER. Are Changes in Metacognition the First Sign of Prodromal Alzheimer's?

Objective: To test whether metacognition is an early sign of prodromal Alzheimer's disease (AD), we assessed family presence during the initial dementia work-up as a marker of metacognition to differentiate prodromal AD from normal aging.

Participants and Methods: In a retrospective chart review, 53 patients (Age: $M=63.58, S D=7.31$ years) presented with memory complaints to a university outpatient neurology clinic. Comprehensive neuropsychological testing was conducted along with CSF biomarkers. Twenty-five patients were clinically diagnosed with prodromal $\mathrm{AD}$ and 28 were classified as worried well/normal aging. Integrity of metacognition was operationalized as family presence to help explain concern and symptoms to the doctor during the initial diagnostic visit. In a mean follow-up of 46.48 months, the clinical diagnosis of prodromal AD versus normal aging had a sensitivity of $95.8 \%$ and a specificity of $93.1 \%$.

Results: The major finding from this study was that $84 \%$ of those diagnosed with prodromal $\mathrm{AD}$ were accompanied to the clinic by a collateral informant, while only $21 \%$ of worried well were accompanied, $\chi^{2}=79.58, p<.05$.

Conclusions: Early diagnosis of prodromal AD based on psychometric data is fraught with methodological issues, particularly when baseline data is not available. The findings from this study showed that collateral presence may be a valuable marker in the detection of prodromal AD. Often, family members are keenly aware of patients' earliest symptoms, reporting that the patient's "personality is slowly disappearing." It is our assumption that family presence at an initial dementia work-up is a reflection of subtle change in metacognition relating to the patient's ability to self-reflect. This data suggests that further investigation into subtle changes in metacognition may be a useful marker in distinguishing prodromal AD from normal age-associated change in cognition. Correspondence: Deborah A. Lowe, M.S., Department of Psychology, Texas A\&M University, MUSC Department of Psychiatry \& Behavioral Sciences, 67 President St., MSC 861, Charleston, SC 29425, United States.E-mail:dalowe@tamu.edu

\section{D.A. LOWE \& S. BALSIS. Sex Differences in Cognitive Dysfunction} Due to Alzheimer's Disease.

Objective: Alzheimer's disease (AD) seems to affect women differently than men. Almost two-thirds of those afflicted by AD are women. Several studies have found that women with AD demonstrate worse cognitive deficits and faster global cognitive decline than men with $\mathrm{AD}$. However, we still do not fully understand how AD influences a range of cognitive functions in women relative to men, particularly over time. Participants and Methods: Two hundred forty-nine participants (102 women) from the Alzheimer's Disease Neuroimaging Initiative were analyzed. Participants ranged from 55 to 90 -years-old $(M=74.54$, $S D=7.28)$ and were highly educated $(M=15.59, S D=2.85)$ and predominantly White $(n=237,95 \%)$. All participants were diagnosed with $\mathrm{AD}$ within two years of their baseline evaluation, with the majority converting from mild cognitive impairment at baseline $(n=153,61 \%)$. Participants completed a neuropsychological battery. Item response theory-derived latent scores for memory, language, visuospatial, executive/processing speed, and global cognitive domains were analyzed at three time points (baseline, month 12, and month 24 evaluations). Results: At baseline women and men had equivalent memory performance; however, women's memory performance was worse than men's at month $12, t(247)=3.45$, and month $24, t(240.68)=2.12, p \mathrm{~s}<.05$. Indeed, a mixed-model ANOVA confirmed the Time by Sex interaction, $F(1,245)=7.50, p<.01$, showing that women's memory performance declined more quickly than men's, particularly in the first 12-month period.

Conclusions: Among individuals on the AD spectrum, women seem to demonstrate more severe memory impairment and different rates of memory decline than men. There were no significant cross-sectional or longitudinal sex differences in other cognitive domains, though trends were noted. Additional research should continue to analyze sex differences in a range of cognitive functions, particularly among more demographically representative samples. 
Correspondence: Deborah A. Lowe, M.S., Department of Psychology, Texas A\&M University, MUSC Department of Psychiatry \& Behavioral Sciences, 67 President St., MSC 861, Charleston, SC 29425, United States.E-mail: dalowe@tamu.edu

M. MAPSTONE, M. FIANDACA, A. CHEEMA, K. MAPSTONE, J. GONZALEZ, R. PADILLA, R. MALHAS, R. MIRAMONTES \& H. FEDEROFF. Non Standardized Plasma Collection and Handling Procedures Represent Significant Challenges for Blood Based Metabolomic Biomarkers of Alzheimer's Disease.

Objective: Alzheimer's disease (AD) pathology is characterized by alterations of cell membrane lipids. The utility of blood-based lipid biomarkers of $\mathrm{AD}$ will rely on the quality of the source blood sample, as many lipid species are susceptible to oxidation. Here, we examined the effect of two sources of oxidation: 1) the age of a stored plasma sample, and 2) collection and processing methods on lipid peroxidation in human plasma.

Participants and Methods: We performed an assay of lipid peroxidation (Thiobarbituric Acid Reactive Substances - TBARS) on 39 stored plasma samples from three groups of thirteen age-, education-, and sex-matched older adults (mean age $=81.2$ ). Twenty preclinical $\mathrm{AD}$ and 19 normal controls were split equally between the three groups. Group A samples were "fresh" (average age $=3.9$ years) and were collected under "optimal" conditions including morning phlebotomy, overnight medication and food withholding, and cooling the blood immediately after collection. Group B samples were also "fresh", but were not collected under optimal conditions. Group C samples were "old" (average age $=10.9$ years) and also were not collected under optimal conditions.

Results: Kruskal-Wallis ANOVA revealed that TBARS florescence values differed across the three groups $(H=6.79, p=0.034)$. Fresh samples collected under optimal conditions had significantly lower peroxidation levels when compared to fresh samples not collected under optimal conditions $(p=0.011)$. However, peroxidation levels did not differ between fresh and old samples collected under the same (not optimal) conditions. There was no effect of disease (preclinical AD vs control) on peroxidation levels.

Conclusions: Plasma sample quality, as determined by TBAR assay is not affected by prolonged sample storage, at least up to 13 years. However, optimal collection methods do diminish evidence of lipid peroxidation. Blood collection and processing variables may impact biomarker sensitivity and specificity and should be considered when deriving blood lipid biomarker panels.

Correspondence: Mark Mapstone, PhD, Neurology, University of California, Irvine, 318 Sprague Hall, 835 Health Sciences Road, Irvine, CA 92697, United States. E-mail: mark.mapstone@uci.edu

M.N. MARTINEZ, L.B. ZAHODNE, E. DALCHAND, G. FELIX, K.M. FRAZER, D.L. SANCHEZ, S. SEEHRA, H.L. SHOUEL \& J.J. MANLY. Marital Status and Dementia Risk among Ethnically Diverse Older Adults.

Objective: We hypothesized that marriage would be protective against incident MCI and dementia. Prior research shows that the benefits of being married are greater for women, and that cultural differences in stigma of unmarried status, financial resources, and traditionality of roles influence health benefits of marriage. We therefore expected that sex and race/ethnicity would moderate relationships between marital status and incident cognitive impairment.

Participants and Methods: Participants were 1,633 non-Hispanic Blacks, 1,370 non-Hispanic Whites, and 2,362 Hispanics in the Washington/Hamilton Heights/Inwood Columbia Aging Project, age 65 years and older ( $67 \%$ female) and not demented at baseline. Marital status was self-reported at baseline as married, never married, widowed, or divorced/separated. Diagnoses of MCI and AD were made at baseline and $18-24$ month follow-ups from functional, cognitive, and medical data using standard research criteria. Race-and sex-stratified logistic regression models tested the relationship between marital status and incident dementia or MCI, adjusting for age at baseline, length of follow-up, years of education, and medical burden, and income and depressed mood were tested as potential mediators.

Results: Widowhood $(\mathrm{OR}=1.39,95 \% \mathrm{CI}=1.12-1.73)$ and divorced/ separated status were associated with higher incidence of MCI and AD $(\mathrm{OR}=1.27,95 \% \mathrm{CI}=1.01-1.59)$ compared to married participants. "Never married" participants were not at higher risk. The protective effect of marriage was strongest among Hispanic women, and weakest among Blacks. AD/MCI risk among divorced/separated and widowed participants was mediated by depressed mood and income, and there was an additional direct effect of widowhood.

Conclusions: Social, psychological, and financial interventions could help to maintain cognitive function among older people who have experienced divorce or widowhood. Interventions should take into account cultural factors that influence impact of marital status on cognition.

Correspondence: Michelle N. Martinez, Columbia University Medical Center, 630 West 168th St., P\&S Box 16, New York, NY 10032, United States.E-mail:Michelle.N.Martinez.17@dartmouth.edu

A. MASEY, M.A. GLENN, S.T. MOELTER, R.S. WILSON \& K. GOLDBERG. Moderating Effects of Cognitive Reserve on Primacy Recall and Alzheimer's Disease Pathology.

Objective: Delayed primacy recall has been found to predict medial temporal lobe (MTL) tangles and hippocampal volume in cognitively normal older adults (Bruno et al, 2015; Glenn, 2016). This study examined whether cognitive reserve modifies this relationship.

Participants and Methods: The present study analyses were performed on archival data obtained from the Rush Alzheimer's Disease Center. Subjects included 54 normal older adults who participated in the Religious Orders Study or Memory and Aging Project. Cognitive reserve was measured by a single, word reading test, an abbreviated, 10-item version of the National Adult Reading Test (NART, e.g. Langbaum et al., 2014). Multiple linear regression analyses were performed to examine the interaction between baseline NART scores $(M=8, S D=2)$ and medial temporal lobe tangle pathology in predicting CERAD Word List delayed primacy proportion scores proximate to death, controlling for age, sex, and education.

Results: Both NART scores $(b=.045, p=.042)$ and MTL tangles scores $(b=-.010, p=.021)$ were related to delayed primacy proportion scores. When an interaction term between NART scores and medial temporal lobe tangles was added to the model, the association was not significant $(b=-.001, p=.498)$.

Conclusions: The association between MTL tangles and delayed primacy proportion scores does not appear to be moderated by a measure of cognitive reserve. This bolsters support for delayed primacy proportion score as being a sensitive cognitive marker of early neurodegeneration in cognitively normal older adults. This measure may be useful in the detection of early cognitive changes associated with preclinical AD. Correspondence: Alison Masey, M.A., Bruce Hall, Suite 201, Widener University, 1 University Place, Chester, PA 19013, United States. E-mail:masey.alison@gmail.com

M.E. MEINERDING, B.L. DEFEIS, P. SUNDERARAMAN, M. AZAR, S. LAWLESS, O. TATARINA, Y. GU, Y. STERN \& S. COSENTINO. Correlates of Dependency in a Community Cohort of Individuals with Alzheimer's Disease.

Objective: Dependence level offers unique information about a patient's functional status not directly provided by other markers of disease severity. Previous research using clinic-based cohorts of Alzheimer's disease $(\mathrm{AD})$ patients has documented the validity of the Dependence Scale through its association with markers of disease severity, cost and progression. This study examined whether such associations are replicated in a more ethnically diverse, community-based, population.

Participants and Methods: 149 elders with AD enrolled in the Predictors 3 cohort (82\% Hispanic, 12\% black, $5 \%$ white, $1 \%$ other) were assessed with the Dependence Scale, modified Mini-Mental State 
Examination (mMMS), the Blessed Dementia Rating Scale (BDRS), and Clinical Dementia Rating (CDR) Scale, and were assigned an Equivalent Institutional Care (EIC) rating. Cross-sectional associations were examined using bivariate correlations. Fisher-z tests examined differences in the strength of associations across a previous clinic-based cohort and the current community-based cohort.

Results: The Dependence Scale was associated with CDR $(r=0.32$; $p<.001)$, mMMS $(r=-0.25 ; p<.01)$, BDRS-IADL $(r=0.40 ; p<$ $.001)$, BDRS-BADL $(r=0.66 ; p<.001)$, and EIC $(r=0.52 ; p<.001)$, indicating an increased need for care and the relationship between poorer cognition and cognitive function with increased dependency. The strength of all associations were comparable across cohorts except the correlation with BDRS-BADL was stronger in the community cohort $(r=0.66$ vs $r=0.26$, respectively, $z=4.72 ; p<.001)$.

Conclusions: Findings confirm most previous clinic-based observations, extending the validity of the Dependence Scale to an ethnically diverse community-based cohort of individuals with AD. The strength of the association between dependence and BDRS-BADL ratings varied by cohort, however, raising questions about cultural and other factors that may differentially influence dependence in different $\mathrm{AD}$ populations. Correspondence: Maria E. Meinerding, MS, The Taub Institute for Research on Alzheimer's Disease and the Aging Brain, Columbia University, 630 West 168th Street, PH19-123, New York, NY 10032, United States.E-mail:mm4817@cumc.columbia.edu

\section{A. RITTER, N. HAWLEY \& J.B. MILLER. Sensitivity of the Montreal Cognitive Assessment Memory Scores to Hippocampal Volume in a Neurodegenerative Disease Sample.}

Objective: The Montreal Cognitive Assessment (MoCA) is frequently used to screen for both Alzheimer's and non-Alzheimer's dementia. Despite widespread use, there has been very little research into the neuroanatomical correlates associated with performance on the MoCA. This study examined the relationship between memory scores on the MoCA and hippocampal volume (HV), a well-established Alzheimer's disease (AD) biomarker. An additional aim of the study was to determine if the newly devised MoCA Memory Index Score (MIS), designed to better capture encoding deficits seen in $\mathrm{AD}$, was more closely correlated with hippocampal volume than the delayed recall score.

Participants and Methods: Records from 268 individuals seen in an outpatient neurodegenerative disease clinic who underwent brain MRI with automated volumetric analysis clinic were reviewed. The MoCA was administered as part of the intake with neurology and the Total Score, delayed recall (DR) and MIS were calculated. Pearson correlations were calculated between memory scores and HV, and regression models were fit predicting left and right HV from memory indices. Demographic variables were added to account for effects of age, education, sex, and estimated intelligence.

Results: Correlations between memory and $\mathrm{HV}$ were positive and significant, ranging from weak to moderate $(\mathrm{r}=0.37-0.42, p<.001)$; Fisher's R-to-Z transformations comparing MIS to DR were not significant, though correlations for the MIS were slightly larger. Incorporating memory indices significantly improved model fit predicting both left and right $\mathrm{HV}$.

Conclusions: Low scores on the MoCA memory section were associated with smaller hippocampal volumes, and thus when encountered in clinic, should raise concern for hippocampal atrophy. These findings provide additional evidence supporting sensitivity of the MoCA to neuroanatomical functioning in neurodegenerative disease.

Correspondence: Justin B. Miller, Ph.D., Cleveland Clinic Foundation, 888 West Bonneville Ave, Las Vegas, NV 89106, United States. E-mail: justin.b.miller@gmail.com

A. MINOR, A.L. JEFFERSON \& K. GIFFORD. Family history and
subjective cognitive decline in non-demented older adults.
Objective: Subjective cognitive decline (SCD) may represent an early
marker of Alzheimer's disease (AD). While family history of dementia increases the risk of $\mathrm{AD}$, it may also heighten one's worry about cognitive changes. Little research has examined whether this worry could manifest as SCD. The current study examined if a family history of memory problems was associated with SCD in older adults.

Participants and Methods: Vanderbilt Memory \& Alzheimer's Center registry members with no self-reported family history of memory problems (FH-; n=753, 72 \pm 9 years) and self-reported family history of memory problems $(\mathrm{FH}+\mathrm{n}=634,68 \pm 8$ years $)$ completed a brief interview, telephone-based cognitive screener (Telephone Interview for Cognitive Status; TICS), Center for Epidemiologic Studies Depression Scale (CESD), and 196-item SCD survey. FH- was defined as 0 family members with memory problems (e.g., mild cognitive impairment, dementia, AD) and $\mathrm{FH}+$ as $\geq 1$ family member(s) with memory problems. General linear models related family history to total SCD score, adjusting for age, sex, race, education, TICS score, and CESD. Models were repeated stratified by cognitive function (intact functioning=TICS $>32$, impaired functioning $=$ TICS $\leq 32$ ).

Results: FH+ individuals had greater total SCD score than FH- $(\mathrm{F}=6.4$, $\mathrm{p}=0.01)$. Stratified analyses revealed that in individuals with intact cognitive functioning, $\mathrm{FH}+$ was related to greater total SCD score $(\mathrm{F}=5.6, \mathrm{p}=0.02)$. However, family history showed no associated with total SCD score in individuals with impaired cognition $(\mathrm{F}=1.7, \mathrm{p}=0.19)$. Conclusions: Family history of memory impairment may make individuals more sensitive to changes in their memory, thus increasing a perception of SCD specifically in individuals with better cognition. Further research is needed to more clearly understand the interplay between a family history of memory problems and SCD as predictors of cognitive decline and $\mathrm{AD}$.

Correspondence: Alexandra Minor, Vanderbilt University Medical Center, Nashville, TN, United States.E-mail: alexandra.p.minor@ vanderbilt.edu

C. MOORE, T. KIELY, C. MARREIRO, A. LEE, L. SHAUGHNESSY \& A. ATRI. Diagnostic Accuracy of Pathologically Confirmed Alzheimer's Disease in the NACC UDS: Computational Classification Using Psychometric Measures.

Objective: Revised diagnostic criteria for Alzheimer's disease (AD) rely on clinical features, such as patterns of cognitive, behavioral and functional features, that can be further supported by biomarker data to improve diagnostic certainty of AD-pathology. In the absence of amyloid/tau biomarkers, 20-33\% of patients diagnosed with AD do not have evidence of AD pathology. The inclusion of subjects without $\mathrm{AD}$ pathology in AD clinical trials can significantly contribute to failure. This study explores the potential utility of quantitative methods integrating cognitive, functional, and mood factors to improve AD classification accuracy.

Participants and Methods: Of 34,924 participants from the National Alzheimer's Coordinating Center UDS database, 800 met inclusion criteria ( $\mathrm{AD} \mathrm{dx}, \mathrm{CDR} \leq 1$, autopsy data). Using propensity analysis, 2 matched (on sex, race, education, ethnicity, and age) groups were created for AD-pathological HIGH vs LOW likelihood;AD-pathology likelihood was based on Braak and CERAD neuritic plaque staging. Discriminant function analysis (DFA) was conducted using neuropsychological performance, functional abilities, and mood/behavior to assess classification in AD HICH vs LOW groups.

Results: $12.2 \%$ of 800 subjects were misdiagnosed as having AD. In the matched-group analysis $(n=142)$, DFA using all variables correctly classified $70 \%$. Post-hoc analyses using only memory and mood variables did not improve classification accuracy (66\% accuracy).

Conclusions: In this large clinical research sample, $12.2 \%$ of subjects with the AD clinical phenotype (but without amyloid/tau biomarker) did not have high likelihood of AD pathology on autopsy. Analyses using quantitative methods that integrated cognitive/behavioral/ADL measures showed modest ability to further correctly classify subjects into those with high vs low likelihood of having AD-brain pathology. These results support the need for amyloid/tau biomarkers and/or longitudinal 
assessments to improve diagnostic classification in individuals with AD clinical phenotype.

Correspondence: Caitlin Moore, Boston University, 506 Wisconson St, San Francisco, CA 94107, United States.E-mail: caitlin.s.moore@gmail. com

N.W. NELSON, D.M. VOCK \& F. YU. Relative predictive values of the MMSE and ADAS-Cog for dementia stage and daily function in Alzheimer's disease.

Objective: The current study examined the predictive values of the Mini Mental State Examination (MMSE) and Alzheimer's Disease Assessment Scale - Cognitive Subscale (ADAS-Cog) for dementia stage and daily function in older adults with probable Alzheimer's disease (AD).

Participants and Methods: Fifty-four primarily college-educated $(M$ $=15.6, S D=3.0$ years $)$ septuagenarians $(M=78.0, S D=6.3$ years $)$ participated in the current study. Participants were evenly distributed by gender $(n=25$ women; $46.3 \%)$. All participants presented with clinician-confirmed diagnoses of probable AD. Participants were recruited through a multi-year study that examines benefits of aerobic exercise in probable AD. Current assessments were completed prior to intervention. Participants completed the MMSE and ADAS-Cog to assess cognitive functioning. The Clinical Dementia Rating Scale (CDR) and Disability Assessment for Dementia (DAD) Scale assessed impairment severity and everyday function.

Results: While the MMSE and ADAS-Cog were individually associated with CDR and DAD $(p<0.01)$, both offered only moderate prediction of the $\mathrm{CDR}(\mathrm{CDR}<1$ versus $\geq 1$; $\mathrm{C}$-index $=0.73$ and 0.72 , respectively $)$ and DAD $\left(R^{2}=0.19\right.$ and 0.17 , respectively). Use of both instruments resulted in marginally improved overall predictive ability $(\mathrm{C}$-index $=$ 0.75 for $\mathrm{CDR}$, and $\mathrm{R}^{2}=0.22$ for $\left.\mathrm{DAD}\right)$.

Conclusions: The MMSE and ADAS-Cog demonstrated modest predictive value for dementia stage and daily functioning in the current sample of participants with probable AD. Despite the more time-intensive nature of the ADAS-Cog, this instrument was not incrementally predictive of impairment severity or daily functioning. Clinical and research implications are discussed.

Correspondence: Nathaniel W. Nelson, Ph.D., GSPP, University of St. Thomas, University of St. Thomas, Minneapolis, MN 55403, United States.E-mail:nels0600@stthomas.edu

S. OLESON, D. EAGAN, S. KAUR, W.J. HERTZING, M. ALKATAN, J. DAVIS, H. TANAKA \& A. HALEY. Dietary polyunsaturated fat and cerebral glutamate: interaction with Apolipoprotein $\mathrm{E}$ genotype.

Objective: Epidemiological data support a link between dietary polyunsaturated fatty acid (PUFA) intake and reduced risk of Alzheimer's disease (AD). However, some studies suggest dietary PUFA do not exert the same benefits for individuals carrying the $\varepsilon 4$ allele of the apolipoprotein $\mathrm{E}(\mathrm{ApoE})$ gene, a genetic risk factor for $\mathrm{AD}$. One potential mechanism through which dietary PUFA may exert cognitive benefits is through regulation of glutamatergic synapses, and previous work has demonstrated greater PUFA intake to be associated with lower cerebral glutamate $(\mathrm{Glu})$. The purpose of the present study was to investigate whether ApoE genotype moderates the relationship between dietary PUFA and cerebral glutamate in cognitively healthy middle-aged adults. Participants and Methods: Sixty-six middle-aged adults were grouped according to ApoE genotype ( $\varepsilon 4$ carrier or $\varepsilon 4$ non-carrier) and asked to record dietary intake for three consecutive days. All participants subsequently underwent a proton magnetic resonance spectroscopy $\left({ }^{1} \mathrm{H}\right.$ MRS) scan to assess Glu in the posterior cingulate cortex.

Results: An analysis of covariance (ANCOVA) revealed a significant interaction between ApoE genotype and PUFA intake when controlling for age, gender, education level, total caloric intake, body mass index, and carotid atherosclerosis, suggesting that greater PUFA intake was associated with lower cerebral Glu for $\varepsilon 4$ non-carriers, but not for $\varepsilon 4$ carriers, $F(1,57)=5.308, p=.025$.
Conclusions: Greater PUFA intake was associated with lower cerebral Glu concentrations in healthy middle-aged adults who were ApoE $\varepsilon 4$ non-carriers, but not for ApoE $\varepsilon 4$ carriers. These findings support the idea that the action of PUFA on the brain differs according to ApoE polymorphism and demonstrates cerebral Glu to be a sensitive marker to genetic risk factors. Early treatment consisting of dietary fat modification that is tailored to patient genetic risk factors may be an important area of intervention for the prevention of cognitive decline.

Correspondence: Stephanie Oleson, San Diego State University, 108 E Dean Keeton St, Austin, TX 78712, United States. E-mail: stephanieoleson@utexas.edu

\section{A.A. OLIVEIRA. Neuropsychological performance differences} between two groups of probable-AD patients from different areas of Brazil.

Objective: The current study examined the performance of two different groups of patients diagnosed with probable Alzheimer's disease (AD) on a neuropsychological test battery

Participants and Methods: : Twenty-two AD patients from Brasília-DF (AD1) and thirty-four AD patients from Palmas-TO, northern Brazil (AD2), were selected and a short neuropsychological battery administered. To verify the reliability of these previous diagnoses of $\mathrm{AD}$, both groups of patients were compared with a group of healthy controls.

Results: AD patients showed cognitive deficit but scores were lower for the AD2 group compared with the AD1 group considering the cut-off point. Notably, patients from the AD1 group were older $(\mathrm{p}=0.004)$ and had less formal education $(p<0.001)$ than those from the AD2 group. Comparing different cognitive domains between AD groups, post hoc analysis showed that the AD1 group was characterized by deficits in episodic memory retrieval $(p<0.001)$, semantic memory $(p<0.001)$ and verbal fluency $(p<0.001)$. In contrast, the AD2 group showed lower scores in attention $(p=0.007)$, executive functioning $(p<0.001)$ and working memory $(p<0.001)$.

Conclusions: This pattern suggests that the Palms group of patients had a neuropsychological profile that was inconsistent with AD. Although the results of this study have important clinical implications, the effects of age, education, and gender on cognitive performance should be explored further.

Correspondence: Analucy A. Oliveira, GRADUATE, PSYCHOLOGY, UNIVERSIDADE SALGADO OLIVEIRA, RUA 1131 OD 242 CASA 2, GOIANIA 74180100, Brazil. E-mail: oliveiraanalucy1@gmail.com

K.E. OSBORN, T.J. HOHMAN, K. GIFFORD \& A.L. JEFFERSON. Adverse Vascular Risk Relates to CSF Biomarker Evidence of Axonal Injury Among Amyloid Positive Older Adults.

Objective: Vascular risk factors and damage to white matter tracts composed of large-caliber myelinated axons are associated with Alzheimer's disease (AD) pathology. Potential links between vascular health and axonal injury in AD are poorly understood. This study tests interactions between amyloid deposition and vascular risk on cerebrospinal fluid (CSF) concentrations of neurofilament light (NFL), a biomarker of large-caliber axonal injury.

Participants and Methods: Data were drawn from Alzheimer's Disease Neuroimaging Initiative participants with normal cognition (NC, $n=115$. $76 \pm 5$ ), mild cognitive impairment (MCI, $n=195,75 \pm 7$ ), and AD ( $\mathrm{n}=99$, $75 \pm 8$ years). Vascular risk was calculated using a modified Framingham Stroke Risk Profile (FSRP), including age, systolic blood pressure, anti-hypertensive medication usage, diabetes, smoking, atrial fibrillation, and cardiovascular disease. Amyloid status was defined with a CSF $A \beta_{42}$ cut point (amyloid $+\leq 192 \mathrm{pg} / \mathrm{mL}$, amyloid- >192 pg/mL). Linear regression related FSRP $\mathrm{x}$ amyloid status (amyloid+, amyloid-) to CSF NFL (pg/mL), adjusting for age, education, sex, and diagnosis (NC, MCI, AD). Models were repeated stratified by amyloid status.

Results: FSRP $x$ amyloid status was unrelated to NFL $(\beta=-8.6, p=0.75)$. In stratified models, higher FSRP (more vascular risk) related to higher NFL (more axonal injury) among amyloid+ participants $(\beta=42.2$. 
$\mathrm{p}=0.007)$. Excluding participants with clinical AD ( $\mathrm{n}=99)$ yielded similar results $(\beta=34.2, p=0.04)$. FSRP was unrelated to NFL among amyloid- participants $(\beta=43.3, p=0.29)$.

Conclusions: Study results suggest increased vascular risk relates to greater biomarker evidence of axonal damage among individuals who are also biomarker positive for significant amyloid deposition. These findings were maintained when excluding participants with clinical AD. Though cross-sectional, results collectively suggest that prior to conversion from NC or MCI to AD, the concomitant presence of amyloid may increase vulnerability to axonal injury with increasing vascular risk. Correspondence: Katie E. Osborn, Psy.D., Neurology, Vanderbilt Memory \& Alzheimer's Center, 1207 17th Avenue South, 2nd Floor, Suite 204, Nashville,TN 37212, United States.E-mail:katie.osborn@ vanderbilt.edu

M.P. PASE, J. HIMALI, P. JACQUES, C. DECARLI, C. SATIZABAL, H.J. APARICIO, R.S. VASAN, A. BEISER \&. S. SESHADRI. Sugary Beverage Intake and Preclinical Alzheimer's Disease in the Community.

Objective: The long-term effects of sugary drinks on the brain are poorly understood. We examined whether habitual sugary beverage intake was associated with markers of preclinical Alzheimer's disease (AD) and/or vascular brain injury (VBI).

Participants and Methods: We performed cross-sectional analysis of participants from the community-based Framingham Heart Study. We studied 4276 participants with neuropsychological outcomes and 3846 participants with brain MRI, all free from overt stroke and dementia (mean age 54 [SD, 11] years; $46 \%$ men for larger sample). Intake of total sugary beverages, fruit juice and sugar-sweetened soft drink were quantified using a food frequency questionnaire. Markers of preclinical AD included total brain volume, hippocampal volume and tests of episodic memory. Markers of VBI included white matter hyperintensities, silent brain infarcts and tests of executive function, abstract reasoning and visual memory.

Results: In multivariable models adjusted for age, sex and caloric intake, higher intake of total sugary beverages was associated with lower total brain volume $(<1 /$ day, reference; $1-2 /$ day, $\beta \pm \mathrm{SE}=-0.55 \pm 0.14$ mean percent difference, $\mathrm{p}=0.0002 ;>2 /$ day, $\beta \pm \mathrm{SE}=-0.68 \pm 0.18, \mathrm{p}<0.0001)$, and poorer episodic memory, both for immediate $(<1 /$ day, reference; $1-2 /$ day, $\beta \pm S E=-0.41 \pm 0.12$ mean score change, $p=0.001$; and $>2 /$ day, $\beta \pm \mathrm{SE}=-0.67 \pm 0.16, \mathrm{p}<0.0001)$ and delayed recall $(<1 /$ day, reference; $1-2 /$ day, $\beta \pm \mathrm{SE}=-0.37 \pm 0.12$ mean score difference, $p=0.003$; and $>2$ day, $\beta \pm \mathrm{SE}=-0.69 \pm 0.16, \mathrm{p}<0.0001)$. Relative to $<1$ sugary beverage/ day, the difference in total brain volume and episodic memory associated with consuming $>2$ sugary beverages/day were equivalent to 2 and 11 years of brain aging, respectively. Daily fruit juice intake was associated with poorer outcomes across all AD markers (all $p<0.05$ ). Sugary beverage intake was not associated with VBI.

Conclusions: Higher intake of sugary beverages was associated cross-sectionally with markers of preclinical AD.

Correspondence: Matthew P. Pase, PhD, Neurology, Framingham Heart Study, 72 East Concord St, Boston, MA 02118, United States. E-mail: matthewpase@gmail.com

R.J. PIERS, T. ANG, Y. LIU, S. DEVINE, S.H. AUERBACH \& R. AU. How Much Risk is Genetic Risk for Dementia? Framingham Offspring Study.

Objective: Apolipoprotein E genotype and family history of dementia are well known risk factors for development of dementia. However, few studies have looked systematically at the independent and combined effects of both.

Participants and Methods: Four thousand one hundred and eighty-three offspring participants underwent ApoE genotype (mean age $=75.26 \pm 9.29 ; 52.04 \%$ female). Both Offspring and their parents were followed for incident dementia. To have a positive family history of dementia, participants needed to have at least one parent with diagnosed dementia. Using survival analysis (Cox Proportional Hazards Regression Model, Poisson Regression Model), we examined the association between ApoE4+ and family history of dementia, independently and in combination, and the development of dementia, adjusting for age, gender, and education.

Results: Eight hundred and eighty-nine participants were ApoE4+ and 991 had a positive family history of dementia, while 254 had both risk factors. $244(5.83 \%)$ participants developed incident dementia over a median follow-up of 42 years. 414 participants had ApoE genotype available for both parents. Having both parents ApoE4+ resulted in about $75 \%$ ApoE 4+ in the Offspring. After controlling for familial history of dementia, ApoE4+ was associated with rate of dementia (Beta \pm standard error $[\mathrm{SE}]=1.52 \pm 0.20, \mathrm{p}<0.0001)$. After controlling for ApoE4+, family history of dementia was not associated with rate of dementia $(p=0.2149)$. After controlling for genetic predisposition to ApoE4+, ApoE4+ was associated with rate of dementia $($ Beta $\pm \mathrm{SE}=$ $1.67 \pm 0.81, \mathrm{p}=0.0326)$.

Conclusions: Our results suggest that the ApoE4+ gene is a stronger predictor of subsequent development of dementia than a family history of dementia in the absence of ApoE4+ parental transmission.

Correspondence: Ryan J. Piers, Neurology, Framingham Heart Study, 19 Harvard Avenue, Brookline, MA 02446, United States. E-mail: rpiers@bu.edu

S. PILLEMER, J. DAVIS \& G. TREMONT. Gender Differences in Factors of Burden and Depression Among Dementia Caregivers. Objective: Previous literature has examined burden and depression predominately as unitary constructs in relation to dementia caregiving. Little is known concerning gender differences in the specific factors of burden and depression in dementia caregivers. The current study examined whether empirically validated dimensions of caregiver burden, as measured by the Zarit Burden Interview (ZBI), and depression, as measured by the Center for Epidemiologic Studies Depression Scale (CESD) differed by gender for dementia caregivers.

Participants and Methods: The sample consisted of 211 community-residing dementia caregivers (mean age $=62.8 ; 77 \%$ female) who reported some degree of distress and were enrolled in a longitudinal intervention study. Dementia severity was mild-moderate. Baseline levels of burden were assessed using the ZBI, and baseline levels of depression were assessed using the CESD.

Results: Confirmatory factor analysis $\left(\chi^{2}(2) p<.001\right)$ revealed 3 facets of burden: impact of caregiving on caregivers' lives, guilt, and frustration/embarrassment, and 4 facets of depression: depressed affect, somatic and retarded activity, positive affect, and interpersonal feelings. Overall burden $(p<.001)$ and impact of caregiving on caregivers' life $(p<.001)$ were significantly higher in females. Additionally, overall levels of depression $(p=.018)$ and both the depressed affect $(p=.005)$ and positive affect $(\mathrm{p}=.012)$ factors were significantly higher in females. There were no significant gender differences with care recipient's dementia severity at baseline.

Conclusions: Findings suggest that distressed male and female dementia caregivers experience burden and depression differently. Results from this study could potentially be used to identify gender-specific interventions related to different subtypes of burden and depression to optimize quality of life for dementia caregivers.

Correspondence: Sarah Pillemer, Ferkauf Graduate School of Psychology, 15 Whiting Street, Apt 3, Providence, RI 02906, United States.E-mail: sarah.pillemer@gmail.com

A.A.M. RAHMAN-FILIPIAK, J.L. WOODARD, A. NORMAN, E.Z. GROSS \& T.S. HALSTEAD. The Anticipatory Dementia Inventory (ADI): An application of the Health Belief Model to fear of dementia in middle- and older-aged adults.

Objective: Fear of Alzheimer's disease (FAD) or Anticipatory Dementia is a healthy adult's misinterpretation of everyday memory failures as indicators of developing dementia. Existing FAD assessments measure 
only the presence, but not the severity or etiology of FAD symptoms, using atheoretical approaches. The current study aimed to contextualize FAD within the Health Belief Model through development of a new scale that measures perceived vulnerability and perceived self-efficacy. Psychometric properties of the resulting Anticipatory Dementia Inventory (ADI) are examined in a sample of healthy middle- and older-aged adults.

Participants and Methods: 50 ADI items were selected based on prior conceptualizations of FAD. 94 cognitively intact, community-dwelling adults (aged 52-96) with and without a family history of dementia completed FAD measures, including the ADI. Initial psychometric properties of the ADI were assessed. A principal components analysis was performed to characterize the underlying component structure of the ADI.

Results: The ADI demonstrated excellent internal consistency $(\mathrm{a}=.896)$ and construct validity $\left(r_{(86)}=.697, p<.001\right)$ as compared to other measures of FAD. PCA revealed a six-component structure of anticipatory dementia including general negative affect, cognitions and physical symptoms related to FAD, beliefs in dementia treatment efficacy, self-efficacy in reducing dementia risk, perception of personal vulnerability to dementia, and resilience to FAD.

Conclusions: Initial scale development and evaluation suggests that the ADI has strong psychometric properties for the assessment of FAD. The ADI has six factors that mirror dimensions of the Health Belief Model. These results suggest that interventions for FAD should focus on increasing public knowledge of prevalence and risk for AD, and strengthening self-efficacy and motivation for health and lifestyle changes to reduce $\mathrm{AD}$ risk.

Correspondence: Annalise A.M. Rahman-Filipiak, M.A., Clinical Psychology, Wayne State University, 26596 Vassar Ave., Redford, MI 48240, United States.E-mail:ec9198@wayne.edu

J. ROBBINS, K. STAKE, I. K THIRUSELVAM, K.R. BRYANT, L. MILLER, M. HAUT \& J.R. WIENER. The Clinical Utility of the Neurobehavioral Examination in Alzheimer's Disease.

Objective: Examine differences on a novel measure of neurobehavioral functioning, the Neurobehavioral Examination (NBE), between healthy controls, patients with mild cognitive impairment (MCI), and Alzheimer's disease (AD).

Participants and Methods: Twenty-four healthy community volunteers (Education $M=16.96, S D=2.97$; Age $M=69.38 ; S D=6.21$ ) were administered the NBE, a novel measure of cognitive functioning with a strong executive component. Seventy-seven clinical patients (MCI N = 56, Education $M=14.18, S D=3.19$, Age $M=66.62, S D=8.22$; AD $\mathrm{N}=21$, Education $M=12.19, S D=2.84$, Age $M=76.24, S D=6.91$ ) were administered the NBE. We examined total score as well as total number of frontal signs.

Results: Between-groups (diagnosis) ANCOVA was used to examine differences in total score on the NBE with covariates of age and education. There was a significant effect of diagnostic group on total score, $F(2,96)=22.22, p<.000, n_{\mathrm{p}}{ }^{2}=.316$. Posthoc pairwise comparisons showed significant mean differences among all groups. Secondary ANCOVA with the same covariates was used to examine group differences in frontal signs on the NBE, revealing a significant main effect, $F(2,96)=10.9, p<.000, n_{\mathrm{p}}{ }^{2}=.185$. Posthoc pairwise comparisons showed significant mean differences among all groups.

Conclusions: The NBE showed stepwise, significant differences between healthy controls, MCI, and AD groups based on total score and total frontal signs, supporting its clinical utility as a novel, brief measure of cognition and executive functioning.

Correspondence: Jessica Robbins, West Virginia University, 30 th Street NE, \#101, Atlanta, GA 30308, United States.E-mail: jessirobbins@ gmail.com
I.G. RODRIGUEZ, A.L. DIAZ SANTOS, T. ALVES, M. RODRIGUEZ, I. TOURGEMAN, D. LOEWENSTEIN, R. DUARA \& M. GRIEG. The Impact of Cardiovascular Risk Factors (CVRF's) on Neurocognitive Performance in Mild Cognitive Impairment (MCI) and Alzheimer's Disease (AD).

Objective: This study examined neurocognitive performance of MCI and AD patients with multiple CVRF's.

Participants and Methods: Participants included MCI ( $n=64)$ and AD $(\mathrm{n}=82)$ patients with history of CVRF or the presence of hypertension. MCI groups included: nonclinical hypertension ( $n=50$; age $M=76.76$, $\mathrm{SD}=6.02$; education $\mathrm{M}=13.82, \mathrm{SD}=3.71)$, clinical hypertension $(\mathrm{n}=14$; age $\mathrm{M}=78.57, \mathrm{SD}=6.26$; education $\mathrm{M}=14.21, \mathrm{SD}=4.31)$, nonclinical $(2$ or less) CVRF ( $\mathrm{n}=33$; age $\mathrm{M}=77.79, \mathrm{SD}=6.60$; education $\mathrm{M}=13.24$, $\mathrm{SD}=4.10)$, and clinical ( 3 or more) $\mathrm{CVRF}(\mathrm{n}=31$; age $\mathrm{M}=76.48, \mathrm{SD}=$ 5.47; education $\mathrm{M}=14.61, \mathrm{SD}=3.42)$. $\mathrm{AD}$ groups included: nonclinical hypertension ( $\mathrm{n}=66$; age $\mathrm{M}=77.80, \mathrm{SD}=7.76$; education $\mathrm{M}=12.39$, $\mathrm{SD}=$ .30 ), clinical hypertension ( $\mathrm{n}=16$; age $\mathrm{M}=78.25, \mathrm{SD}=7.55$; education $\mathrm{M}=11.25, \mathrm{SD}=4.04)$, Nonclinical CVRF $(\mathrm{n}=42$; age $\mathrm{M}=77.57, \mathrm{SD}=$ 8.30; education $\mathrm{M}=11.90, \mathrm{SD}=4.33$ ), and clinical $\mathrm{CVRF}(\mathrm{n}=40$; age $\mathrm{M}=78.24, \mathrm{SD}=7.06$; education $\mathrm{M}=12.45, \mathrm{SD}=4.20$ ). Logical Memory, Visual Reproduction (VR), Hopkins Verbal Learning Test-delayed (HVLT), Trail Making Test-B, Verbal and Category Fluency (Cat-Fl), and Boston Naming Test were examined.

Results: A 2x3 (CVRF by hypertension) ANCOVA for cognitive performance controlling for age and education were conducted for each diagnostic category. In AD, there was a significant difference between hypertension groups in the HVLT $[\mathrm{F}(1,52)=5.36, \mathrm{p}=.025]$. In the MCI group, there was an interaction effect between hypertension and CVRF for Cat-Fl $[\mathrm{F}(1,62)=6.55, \mathrm{p}=.013)$, differences between the CVRF groups $[\mathrm{F}(1,63)=6.08, \mathrm{p}=.017]$ and an interaction effect $[\mathrm{F}(1,63)=4.87, \mathrm{p}=.031]$ for HVLT, and an interaction effect for VR-I $[\mathrm{F}(1,62)=7.32, \mathrm{p}=.009]$.

Conclusions: Results indicate that CVRF's contribute to poorer cognitive performance in $\mathrm{MCI}$ and $\mathrm{AD}$, particularly in delayed verbal memory. Multiple CVRF's and hypertension may impact semantic fluency and visual information processing in MCI. These results should be further explored longitudinally. This study was supported by NIA, NIH, Bethesda, MD, Grant\#1P50 AG025711-05.

Correspondence: Ivan G. Rodriguez, PsyD, Clinical Psychology Program, PsyD., Carlos Albizu University, Miami Campus, 9375 Fontainebleau Blvd, Apt L-405, Miami, FL 33172, United States. E-mail: irodriguez712@sunmail.albizu.edu

\section{E. ROLL \& T. GIOVANNETTI. Semantic Knowledge and Everyday} Function in People with Dementia.

Objective: Novel tests of everyday action semantics were developed and piloted in healthy participants and then administered to individuals with Alzhiemer's disease (AD) and Parkinson's disease dementia (PDD). Relations between action semantics and everyday function as measured by performance-based tests and collateral questionnaires of everyday function were explored.

Participants and Methods: Healthy adults $(\mathrm{n}=53)$ were recruited from the community and 41 participants with dementia were recruited from university-affiliated specialty clinics $(A D n=20$; PDD $n=21)$. Three action semantic tests were developed: 1) probe test - forced, two-choice response option assessed knowledge of everday tasks; 2) picture sequencing task - required accurate ordering of cards depicting task steps; 3) script task - assessed task knowledge through an openended verbal response. The Naturalistic Action Test (NAT), a performance-based test of everyday action, was administered; performance was scored for omission (i.e., failure to complete steps) and commission (i.e., inaccurate performance of task step) errors. Neuropsychological measures and collateral reports of functioning also were obtained.

Results: The AD and PDD groups performed worse than healthy participants on all semantic tasks. AD and PDD groups significantly differed only on the Script Test, such that AD participants produced fewer task steps than PDD participants. Within the dementia groups, performance 
on each of the action semantic tests significantly correlated with omission errors on the performance-based test of everyday function. In regression models, only the Sequencing Task significantly predicted omissions, commissions, and collateral report of everyday functioning in the home.

Conclusions: Semantic task knowledge is crucial for the completion of steps in everyday tasks. Everyday serial ordering abilities is a skill that is crucial for multiple aspects of everyday function and may be easily assessed with picture arrangement.

Correspondence: Emily Roll, Psychology, Drexel University, Temple Univeristy/Psychology Dept, 1701 N 13th St (Weiss Hall), Philadelphia, PA 19122, United States.E-mail: eer44@drexel.edu

H.L. SHOUEL, E. DALCHAND, G. FELIX, K.M. FRAZER, M.N. MARTINEZ, J.J. MANLY \& L.B. ZAHODNE. Independent Associations Between Objective Versus Subjective Social Support and Cognition in a Racially Diverse Cohort.

Objective: Objective social support (social network size, OSS) may protect against cognitive decline through mental stimulation, while subjective social support (perceived quality, SSS) may protect against cognitive decline by buffering stress. This study addresses these nonmutually exclusive hypotheses by comparing associations between OSS versus SSS and cognition in older adults. We predicted that SSS would relate most strongly to cognition due to evidence for negative cognitive effects of stress. We further predicted stronger associations among African Americans and Hispanics due to the presumed adaptive function of these groups' prominent social systems.

Participants and Methods: 408 non-demented participants (31\% White; 39\% African American; 29\% Hispanic) in the Washington Heights-Inwood Columbia Aging Project completed emotion and cognition modules of the NIH Toolbox and additional tests. Multiple regressions estimated cross-sectional associations between social support and a cognitive composite score and seven individual scores. Models controlled for age, sex, education, race/ethnicity, chronic illnesses, and depression. Stratified analyses followed to determine if there are differences in the relationship across race/ethnicity.

Results: OSS and SSS were correlated ( $r=0.284)$. Both African Americans and Hispanics reported more OSS than Whites, but only Hispanics reported more SSS. OSS and SSS were independently related to better cognition in Whites but worse cognition in Hispanics, with SSS showing stronger associations. Social support was not related to cognition in African Americans.

Conclusions: Results are consistent with previous studies showing positive associations between social support and cognition in Whites but suggest that these relationships may not be generalizable to African Americans and Hispanics. Longitudinal research is needed to determine if negative associations between social support and cognition among Hispanics reflect negative effects of social support (e.g., social strain) or reverse causation.

Correspondence: Heather L. Shouel, University of Wisconsin-Madison, 630 West 168th Street, P\&S Box 16, New York, NY 10032, United States.E-mail:shouel@wisc.edu

E.E. SUNDERMANN, A. BIEgON, L. RUBIN, R. LIPTON, S. LANDAU \& P.M. MAKI. Does the Female Advantage in Verbal Memory Mask Alzheimer's Disease Pathology?

Objective: There is a growing recognition of sex differences in Alzheimer's disease (AD). Females show an advantage over males on tests of verbal memory, which are used to diagnose $\mathrm{AD}$ and its precursor, amnestic mild cognitive impairment (aMCI). Women retain this advantage in aMCI despite reduced hippocampal volume and temporal lobe glucose metabolism. Here we examined whether this female advantage endures despite evidence of $\mathrm{AD}$-specific pathology, temporal lobe and cortical amyloid- $\beta$ (A $\beta)$ deposition measured with [18F]AV 45 (florbetapir) positron emission tomography.
Participants and Methods: Participants with normal cognition $(\mathrm{N}=304)$, aMCI $(\mathrm{N}=515)$, and AD dementia $(\mathrm{N}=175)$ were drawn from the Alzheimer's Disease Neuroimaging Initiative (ADNI). Across and within diagnostic groups, we conducted linear regressions to examine the interaction of sex with temporal and cortical $A \beta$ burden on immediate and delayed recall on the Rey Auditory Verbal Learning Test (RAVLT) adjusting for age, education, and APOE4.

Results: Across groups, the sex-temporal A $\beta$ interactions were significant for both RAVLT outcomes and the sex-cortical A $\beta$ interaction was significant for delayed recall only. Women outperformed men among those with low to moderate $A \beta$ burden, but not among those with high $A \beta$ burden. In diagnosis-stratified analyses, a significant sex-A $\beta$ (temporal and cortical) interaction was observed for delayed recall in the aMCI group, but not in the normal or AD dementia groups.

Conclusions: Thus, women maintain a verbal memory advantage over men in aMCI despite similar levels of AD pathology. Although this advantage may benefit women by delaying verbal memory impairment until more advanced pathology, it may delay diagnosis of aMCI and treatment intervention.

Correspondence: Erin E. Sundermann, PhD, Neurology, Albert Einstein College of Medicine, 1207 Herschel Woods Ln., Cincinnati, OH 45208, United States.E-mail: erin.sundermann@einstein.yu.edu

U.M. VENKATESAN, E.K. FESTA, B.R. OTT \& W.C. HEINDEL. What Drives Driving: Differences in the Relationship of Visual Search and Sensory Binding to Driving Performance between Healthy Aging and Alzheimer's Disease.

Objective: Patients with Alzheimer's disease (AD) demonstrate deficits in cross-cortical visual feature binding that are distinct from age-related changes in selective attention. This may have consequences for driving performance given the demands placed on multisensory integration. The current study examined the relationship of visuospatial search and sensory binding to driving in elderly controls (EC) and patients with early AD.

Participants and Methods: Patients $(\mathrm{n}=71)$ and EC $(\mathrm{n}=40)$ completed a visual search task requiring either luminance-motion ( $\mathrm{L}-\mathrm{M})$ or color-motion (C-M) integration, analogs of intra- and cross-cortical binding, respectively. Standardized road test and naturalistic (video recorded) driving data were collected along with clinical screening measures commonly used in driving evaluations (e.g., Trail Making A\&B, Clock Drawing).

Results: Visual search and clinical test scores were differentially related to driving behavior in each group. Both $\mathrm{L}-\mathrm{M}$ and $\mathrm{C}-\mathrm{M}$ visual search and Trails B were associated with standardized road test performance in EC, while C-M integration. Trails A, and Clock Drawing were associated with naturalistic driving performance in $\mathrm{AD}$. Stepwise regressions indicated that Trails B and L-M measures best predicted standardized road test performance in EC, while C-M integration and Clock Drawing best predicted naturalistic driving performance in $\mathrm{AD}$. Backward logistic regression analyses also indicated that $\mathrm{C}-\mathrm{M}$ integration and Trails A best predicted group membership.

Conclusions: Findings suggest that while selective attention is most relevant to driving behavior in EC, multisensory integration may be most sensitive to driving in AD. This latter relationship may only emerge in naturalistic settings, which better reflect patients' driving behavior. Measures of visual integration may offer distinct insights into driving behavior. The study findings thus have important implications for the assessment of driving competency in both healthy elders and those with early AD.

Correspondence: Umesh M. Venkatesan, MS, Psychiatry and Human Behavior, Alpert Medical School of Brown University, 190 Thayer St., Providence, RI 02912, United States. E-mail: umi414@gmail.com 
C. VILA-CASTELAR, J.J. LY, L. KAPLAN, K. VAN DYK, E. GAMMADA, J. BERGER, L.O. MACINA, J. STEWART \& N.S. FOLDI. Long-term cognitive effects of Donepezil treatment in patients with Alzheimer's disease: The role of attention.

Objective: Cholinesterase inhibitors (ChEIs), widely used in the treatment of Alzheimer's disease (AD), increase the level of available acetylcholine, a neurotransmitter linked to attentional functions. Our group demonstrated that a sensitive measure of attention. the Attentional Blink (Blink), detected treatment response and predicted 6-month global and neuropsychiatric function after only 6 weeks of treatment, while global (i.e., Alzheimer's Disease Assessment Scale-Cognitive; ADAS-Cog) and domain-specific measures did not. However, to date, the Blink has not been used to assess long-term effects of ChEIs. We hypothesized that responders to treatment would also maintain blink accuracy under highload demands after 6 months of Donepezil.

Participants and Methods: 23 participants newly diagnosed with $\mathrm{AD}$, treated with de novo Donepezil (5mg), were assessed at baseline (T1) and after $\approx 6$ months of treatment (T2). Treatment 'responders' $(n=9)$ vs. 'non-responders' $(n=14)$ were categorized on the basis of ADAS-Cog change score. The Blink targeted top-down accuracy under high load intervals (133 \& 266ms). Global cognition (ADAS-Cog; Mini Mental Status Exam, MMSE), memory (Hopkins Verbal Learning Test, HVLT), and language (Category Fluency Test) were also assessed. Wilcoxon signed-rank tests compared baseline to 6-month performance. Results: Responders maintained baseline performance on all measures. Non-responders decreased Blink accuracy of the 266ms interval $(69 \%$ (T1) to $54 \%$ (T2), $z=-2.22, p=.03, d=.94$ ) and global cognition (MMSE, 24.9 (T1) to 22.5 (T2), $z=-2.37, p=.02, d=.96$ ), but did not deteriorate at the $133 \mathrm{~ms}$ interval or on memory or language.

Conclusions: Long-term Donepezil treatment prevented decay of MMSE global function and top-down attention in responders to 6-month treatment. These results further support the utility of sensitive measures of attention to detect, evaluate and predict short and long-term treatment effect of ChEIs.

Correspondence: Clara Vila-Castelar, Ph.D. in Clinical Psychology, Psychology, Queens College and The Graduate Center, CUNY, 6530 Kissena Boulevard, Psychology Department, Queens, NY 11367, United States.E-mail: clara.vilacastelar@qc.cuny.edu

G. WAGNER, G. HOLGUIN, T. SLONIM, J. ROSEN \& L. HAASEALASANTRO. Pilot Study Examining the Neuropsychological Profiles of Dementia with Lewy Body and Alzheimer's Disease.

Objective: Dementia with Lewy Body (DLB) is the second most common form of dementia after Alzheimer's disease (AD). DLB is characterized by cognitive decline (e.g., memory, visuospatial skills), as well as clinical features including fluctuations in awareness, visual hallucinations, and extrapyramidal symptoms. There can be considerable overlap in the neuropsychological profiles of DLB and AD; therefore DLB can be difficult to differentiate from AD. Neuropsychological testing can aid in differential diagnoses between DLB and AD. For example, recent literature demonstrates that patients with DLB perform significantly better on verbal memory tests than patients with AD. However, DLB patients perform significantly worse on tests of visuospatial skills and attention compared to AD patients.

Participants and Methods: The pilot study examined differences in neuropsychological performance in community-based neurology center patients diagnosed with probable DLB $(n=8)$ and probable AD $(n=8)$. Patients were matched based on age, education, and gender. Each patient underwent a neurological exam, clinical interview and completed a battery of standardized neuropsychological tests measuring attention, language, executive functions, processing speed, memory, visuospatial skills, and motor abilities.

Results: Our preliminary findings suggest that DLB patients performed significantly better on tests of verbal memory and worse on tests of figural memory compared to AD patients. Additionally, DLB patients performed significantly worse on tests of visuospatial skills, attention, and processing speed compared to $\mathrm{AD}$ patients.
Conclusions: These pilot findings, duplicated in a community-based neurology center sample, support prior research suggesting differences in performance of memory, visuospatial skills, and attention/processing speed between DLB and AD. These pilot findings provide additional support for the generalizability of neuropsychological findings from a research setting into a community-based sample.

Correspondence: Gabrielle Wagner, The Neurology Center, 1533 Lorraine Drive, Encinitas, CA 92024, United States. E-mail: gabriellemwagner@gmail.com

C.W. WATSON, J.J. MANLY, A.M. BRICKMAN \& L.B. ZAHODNE. Racial Discrimination is Associated with Cortical Thinning in Alzheimer's Disease Signature Regions in African American Older Adults.

Objective: Elderly African Americans and Latinos often exhibit worse cognitive and brain health outcomes than Whites and have a higher incidence of Alzheimer's disease (AD). Chronic stress is also associated with poorer brain health, but the potential impact of stressful experiences related to race/ethnicity such as persistent discrimination is not well understood. This cross-sectional study examined how racial discrimination and socioeconomic status relate to $\mathrm{AD}$-related structural brain outcomes in a multi-ethnic cohort of older adults.

Participants and Methods: 216 dementia-free older adults (22\% Latino, 42\% African-American, 36\% White) in the Washington Heights-Inwood Columbia Aging Project underwent structural magnetic resonance imaging and responded to questionnaires on major life event discrimination and socioeconomic status. We quantified cortical thickness in nine Alzheimer's disease signature regions. We evaluated associations between racial discrimination, financial assets, and a cortical thickness composite across racial/ethnic groups, adjusting for age, gender, education, and reading level.

Results: African-Americans reported more racial discrimination than Latinos and Whites. Racial discrimination was negatively associated with cortical thickness in African Americans, but not Whites or Latinos. A significant interaction between racial discrimination and financial assets revealed that reporting lower financial assets was more associated with cortical thinning among African Americans who reported experiencing racial discrimination.

Conclusions: Our findings suggest that chronic negative social experiences are associated with worse outcomes in brain areas tied to AD in elderly African-Americans. Brain outcomes appear to be worst for African Americans who report both racial discrimination and low socioeconomic status.

Correspondence: Caitlin W. Watson, Psychology and Urban Studies, Department of Neurology and Taub Institute for Research on Alzheimer's Disease and The Aging Brain, Columbia University Medical Center, 450 West 149th Street, Apt \# 32, New York, NY 10031, United States. E-mail: caw2153@columbia.edu

G. WEISSBERGER, J. STRONG, M.W. BONDI \& N. STRICKER. Diagnostic accuracy of memory measures in Alzheimer's dementia and Mild Cognitive Impairment: A systematic review.

Objective: With an increasing focus on biomarkers in the field of dementia research, it is important to highlight the role of neuropsychological testing in detecting mild cognitive impairment (MCI) and Alzheimer's dementia (AD). This systematic review summarizes sensitivity and specificity of memory measures in identifying patients with MCI or AD relative to healthy elderly controls (HC).

Participants and Methods: In line with PRISMA (Preferred Reporting Items for Systematic reviews and Meta-Analyses) standards, we ran a PubMed search of studies published before 1/24/2015. Key words included in the search were: [Neuropsychological Tests] or [Neuropsychology] and [Alzheimer] or [MCI] and [sensitivity] or [specificity] or [ROC]. Additional studies were identified based on prior knowledge and record review. Studies that did not report diagnostic accuracy data for episodic memory measures, did not implement widely accepted 
diagnostic criteria for MCI or AD, or had flawed methodology were excluded. In total, 66 studies were identified.

Results: AD vs. HC ( $n=39$ studies) and MCI vs. HC ( $n=31$ studies) comparisons revealed high sensitivity and specificity $(\geq 80 \%$ for AD comparisons, based on guidelines put forth by the 1998 consensus report of the Working Group on Molecular and Biochemical Markers of AD; $\geq 70 \%$ for MCI comparisons) for measures of immediate and delayed memory, especially those involving word-list recall. Reviewing measures that differentiated $\mathrm{AD}$ from other dementias ( $n=9$ studies) yielded mixed results, with generally high sensitivity in the context of low or variable specificity across studies and measures.

Conclusions: Diagnostic accuracy values for differentiating AD and MCI from HCs are promising. Results of the review highlight memory measures that hold promise for detection of preclinical AD. Emphasizing diagnostic test accuracy statistics over null hypothesis testing in future studies will promote the ongoing use of neuropsychological testing as the broader Alzheimer's field relies increasingly upon biomarkers. Correspondence: Gali Weissberger, Ph.D., Geriatric Research Education and Clinical Centers, West Los Angeles Veteran's Administration, 15133 Dickens St, Apt. D, Sherman Oaks, CA 91403, United States. E-mail: gweissbe@gmail.com

R. TAIPA, C. PINTO, J. FERNANDES, S. NEVES, P. PINTO, L. SOUSA, A. CORREIA, E. SANTOS, N. SOUSA \& S. CAVACO. Higher levels of CSF phosphorylated tau correlate with younger age and poorer memory in Alzheimer's disease.

Objective: Increasing age is the most important risk factor for developing Alzheimer's disease (AD) and memory impairment is the most important cognitive marker of the disease. Cerebrospinal fluid (CSF) levels of b-amyloid (Ab 1-42), total Tau (hTau) and phosphorylated Tau (pTau) have been accepted as valid biomarkers in the diagnosis of AD. Our aim was to explore the associations between age, neuropsychological performance, and CSF biomarkers in AD.

Participants and Methods: Twenty-four consecutive patients with probable AD ( 15 women; mean age at onset=62.5, sd=8.5; mean age at study=64.8, $\mathrm{sd}=8.7$; mean Global Deterioration Scale=3.8, $\mathrm{sd}=0.6$ ) according to the NIA recent criteria (McKhann et al. 2011) from our dementia outpatient clinic were included in the study. Data regarding age at onset, age at study, performance on the Dementia Rating Scale-2 (DRS-2), and CSF biomarkers (pTau, total Tau and Ab 1-42) were collected contemporaneously. DRS-2 scores were adjusted to the patients' age and education according to national norms. Pearson's correlations and multiple linear regressions were used for data analyses. Results: pTau correlated negatively with age at onset $(\mathrm{r}=-0.483)$ and with age at study $(r=-0-500)$. Both total Tau $(r=-0.442)$ and $p$ Tau $(r=-0.508)$ correlated negatively with the memory subscale of the DRS-2, but not with the total score. pTau remained statistically associated $(\mathrm{p}<0.05)$ with age at onset (and age at study) and memory subscale score when these variables were considered as covariates.

Conclusions: The study results provide support to previous reports that younger AD patients have higher levels of CSF Tau and that CSF pTau levels may reflect the neuropathological basis of memory impairment in $\mathrm{AD}$.

Correspondence: Sara Cavaco, Neurology, Centro Hospitalar do Porto Portugal, Largo Prof. Abel Salazar, Porto 4099-001, Portugal. E-mail: smcavaco@icbas.up.pt

\section{S. ROGERS \& C.E. HARRISON. Clarifying the Types of Memory Deficits in Alzheimer's Disease.}

Objective: Some studies have shown that memory deficits related to Alzheimer's disease (AD) are more prominent for verbal relative to nonverbal information. However, this study examines if it might be more accurate to make a distinction between learning and retrieval/recall, and between types of verbal and nonverbal memory.

Participants and Methods: 50 older adults (27 women, $M$ age $=78.20, M$ education $=14.18)$ with $\mathrm{AD}$ completed the
BVMT-R, ROCF, HVLT-R, and subtests of the WMS-IV as part of outpatient neuropsychological evaluations.

Results: Paired samples $t$-tests showed that those with AD performed significantly worse on BVMT-R Trials 1-3 than ROCF 3', ROCF 30', Logical Memory I \& II, and HVLT-R Trials 1-3. BVMT-R Delayed Recall scores were significantly worse than ROCF 3' \& 30', and Logical Memory I \& II. Performance on HVLT-R Delayed Recall was significantly worse than Logical Memory I \& II, HVLT-R Trials 1-3, and ROCF 3 ' \& 30'. Scores on Logical Memory II were significantly lower than Logical Memory I and ROCF 3', and ROCF 30' performance was worse than Logical Memory I and ROCF 3'. All $p$ s $<.05$.

Conclusions: The memory measures most sensitive to AD are delayed recall versions of verbal supraspan lists and both the learning and delayed recall paradigms of nonverbal tasks with time constraints. Within verbal memory, performance on delayed recall is differentially worse than learning. There is no difference in patients' ability to learn stories versus a list of words, but their list recall is significantly worse than their story recall. Within visual memory, delayed recall is significantly worse than learning only when time constraints are absent, and there is worse visual learning and delayed recall when restricted by time constraints. To understand the memory deficits in AD, it appears most accurate to make distinctions between learning and delayed recall and between types of verbal and nonverbal abilities.

Correspondence: Steve Rogers, Ph.D., Psychology, Westmont College, 955 La Paz Road, Santa Barbara, CA 93108, United States. E-mail: sarogers@westmont.edu

\section{Movement and Movement Disorders}

K.D. MAHDAVI \& S. ROGERS. Examining the Effects of Anxiety on Cognition Among Those with Parkinson's Disease.

Objective: Studies have shown that anxiety negatively impacts cognitive performance. There is also considerable documentation of patients with Parkinson's disease (PD) who suffer from anxiety. This study explores the relationship between anxiety and cognitive performance among those with parkinsonian pathology.

Participants and Methods: 115 patients (38 women, $M$ age $=74.58$, $M$ education = 15.26) participated in comprehensive neuropsychological assessment as part of outpatient neurology evaluations. Measures completed included HVLT-R, BVMT-R, Trailmaking, BNT, RCFT, and subtests from WAIS-IV, WMS-IV, and DKEFS.

Results: There were significant negative correlations between overall anxiety and WAIS-IV Arithmetic, Coding, and Block Design, Trails A, and HVLT-R Delayed Recall, $p \mathrm{~s}<.05$. When anxiety was divided into levels, $t$-test analyses showed that those with any level (mild-to-severe) of state anxiety performed significantly worse on WAIS-IV Arithmetic, Coding, and Block Design, Trails A, DKEFS Color Naming, WMS-IV Logical Memory I, and DKEFS Inhibition, $p s<.02$, than those without any state anxiety. Those who had moderate-to-severe levels of state anxiety performed significantly worse than those with mild levels of state anxiety on HVLT-R Delayed Recall and WAIS-IV Picture Completion, $p$ s $<.05$.

Conclusions: The cognitive profiles of those with parkinsonian pathology appear to directly vary with levels of anxiety. Greater levels of anxiety correlate with worse working memory, visual processing speed, verbal delayed recall, and visuospatial construction. Even those with mild anxiety exhibit worse executive functions, visual processing speed, verbal memory for contextual information, and visuospatial construction than those who do not endorse any anxiety. And the more severe the degree of state anxiety, the greater the reduction in nonverbal delayed recall and visual perception. In part, anxiety appears to exacerbate some of the deficits already associated with PD.

Correspondence: Steve Rogers, Ph.D., Santa Barbara, CA 93108, United States.E-mail: strogers@westmont.edu 
A. GONCALVES, A. MENDES, N. VILA-CHÃ, I. MOREIRA, J. FERNANDES \& S. CAVACO. Criterion validity of UPDRS-I in the detection of cognitive impairmentin Parkinson's disease.

Objective: To explore the validity of the first item of Unified Parkinson's Disease Rating Scale-section I (UPDRS-I) to identify cognitive impairment (CI) in Parkinson's disease (PD) patients.

Participants and Methods: 269 consecutive patients with PD (52\% men: age $=68 \pm 11$; education $=6 \pm 4$; disease duration $=9 \pm 6$; levodopa equivalent dose $=874 \mathrm{mg} \pm 548$; UPDRS-III-Off $=33 \pm 11$; Hoehn \&. Yahr $=2.7 \pm 0.8$ ) performed the Dementia Rating Scale-2 (DRS-2). The $5^{\text {th }}$ percentile of age and education adjusted scores (based on normative data) was used to identify impaired performance on DRS-2 and was used as criterion for CI. Based on a clinical interview, and blinded to the neuropsychological assessment, a neurologist answered the first item of UPDRS-I.

Results: 181 patients $(67.3 \%)$ scored above the cut-off of impairment on DRS-2 and 88 (32.7\%) were identified with CI. According to the neurologist assessment. 155 patients $(57.6 \%)$ did not have cognitive difficulties (score 0 ), 88 (32.7\%) had just mild memory loss (score of 1 ); and $26(9.7 \%)$ had moderate or severe cognitive difficulties (scores 2-4). In this assessment, scores $>0$ had a specificity of $67.4 \%$ and a sensitivity of $62.5 \%$ to detect CI, whereas scores of $2-4$ had a specificity of $99.4 \%$ and a sensibility of $28.4 \%$. The positive predicted value of the $\geq 2$ cut-off was $96.2 \%$ and the negative predicted value was $74.1 \%$.

Conclusions: This study points to the limited validity of a clinical judgment without formal assessment tools in the identification of CI in PD. These findings reinforce the need for formal neuropsychological assessment in movement disorders settings.

Correspondence: Sara Cavaco, Neurology, Centro Hospitalar do Porto Portugal, Largo Prof. Abel Salazar, Porto 4099-001, Portugal. E-mail: smcavaco@icbas.up.pt

L.R. ALAMEDDINE, K.A. WYMAN-CHICK, S.A. SPERLING \& C.A. MANNING. Depression Does Not Impact Verbal Fluency in Individuals with Early-Stage Tremor-Dominant Parkinson's Disease.

Objective: Studies examining the effects of depression on verbal fluency in patients with tremor-dominant Parkinson's disease (TDPD) have yielded mixed results. Verbal fluency is mediated by the frontal lobes, to varying degrees depending on the type of fluency. Depression has also been linked to frontal lobe dysfunction and associated with reduced fluency. We hypothesized that depressed individuals with TDPD would have significantly lower verbal fluency compared to non-depressed individuals with TDPD.

Participants and Methods: Data were obtained from the Parkinson's Progression Markers Initiative (PPMI). We analyzed 148 tremor-dominant individuals diagnosed with early-stage PD (age $=67.6 \pm 5.3$, education=15.6 \pm 3.2$)$. Depression was defined as a score of $>5$ on the Geriatric Depression Scale-Short Form (depressed n=53, not depressed n=95). Two one-way ANOVAs were conducted to assess for differences in letter (F) and category (Animals) verbal fluency between the two groups. Results: Groups did not differ in age, education, or scores on the Montreal Cognitive Assessment (MoCA). Levene's test of equality was not significant for either condition, therefore equal variances were assumed. Group differences for letter fluency, $\mathrm{F}(1,147)=0.14, p=.71$, and category fluency, $\mathrm{F}(1,147)=0.13, p=.72$, were not significant.

Conclusions: There was no significant difference between verbal fluency in depressed vs. non-depressed individuals with early-stage TDPD. Although depression is often thought to negatively impact performance on cognitive measures with a processing speed component, depression does not play a significant role in verbal fluency in early-stage TDPD. The clinical implications of these findings indicate that verbal fluency in this population can be interpreted independent of depression.
Correspondence: Lama R. Alameddine, Psy.D., Neurology, University of Virginia, 1215 Lee St., Charlottesville, VA 22903, United States. E-mail: Ir.alameddine@gmail.com

E.R. APPLEMAN, J. HIMALI, A. CRONIN-GOLOMB, S. FRANK \& R. AU. Using Multigenerational Longitudinal Research to Examine for Cognitive Differences Pre-Diagnosis in Parkinson's Disease.

Objective: Marked heterogeneity in neuropsychological (NP) deficits characterizes Parkinson's disease (PD). Little research has been conducted on pre-diagnostic cognitive changes despite reports of deficits in newly diagnosed patients. Current conceptualization of neurodegeneration in PD predicts that the earliest deficits in attention and arousal may arise from brainstem pathology that precedes progression to midbrain substantia nigra: hence, precede PD diagnosis. Identifying cognitive changes pre-diagnosis would add to known PD biomarker risk factors and may lead to treatments that significantly slow progression, improve prognosis, and allow opportunities for prevention.

Participants and Methods: This project capitalized on the Framingham Heart Study (FHS) 6+ decades of data, with its rigorous prospective follow-up of a community-based sample. Study sample consisted of 33 participants with PD who had a NP assessment between 1-3 years pre-PD diagnosis and 3 control participants for each PD case, matched for age, sex, and calendar year in which NP data were obtained. Performance on the Mini-Mental State Examination (MMSE) and NP tests of verbal and visual memory, attention and executive functioning, abstract reasoning, and verbal fluency was compared for control participants and PD cases tested pre-diagnosis.

Results: No significant differences were found between control participants and PD cases tested pre-diagnosis for either the MMSE or any of the NP assessment variables.

Conclusions: Traditional NP tests did not detect cognitive deficits prior to PD diagnosis. Future research employing more sensitive measures with the ability to capture changes in attention and arousal may be required to reveal the early presence and trajectory of cognitive changes in PD.

Correspondence: Erica R. Appleman, M.A., Psychology, Boston University, 134 Horace Street, Boston, MA 02128, United States. E-mail:ericaa@bu.edu

S.J. CROWLEY, J.J. TANNER, N.A. SCHWAB, L. HIZEL \& C.C. PRICE. Contribution of Cortical White Matter to Motor Sequence Learning in Parkinson's Disease.

Objective: Parkinson's disease (PD) might be associated with motor sequence learning (MSL) deficits, but the neurophysiological mechanism causing this impairment is not well defined. Hélie et al (2015) proposed that the basal ganglia (BG) initiates procedural motor learning and transfers learned sequences to cortex over time, implying dysfunction of the BG in PD may impair coordination of MSL in cortex. Thus, individuals with PD mav be more reliant on connectivity in the cortex for MSL. This study assessed the correlation between MSL and cortical white matter integrity, with the hypothesis that connectivity would positively correlate with task performance in PD participants but not in controls.

Participants and Methods: 80 participants (40 early to mid-stage. non-demented PD, 40 control) performed the Digit Symbol Copy (DS-Copy) task, with improvement over 15 second intervals used to measure MSL. White matter fractional anisotropy (FA) of the left supplementary motor area (SMA) was used as a measure of cortical white matter integrity. An ANCOVA compared PD patients and controls on DS-Copy performance over time. DS-Copy performance was also compared between right side and left side svmptom onset groups. A logistic regression compared the association between FA and DS-Copy improvement (Time 6-Time 2) in each group.

Results: PD participants trended towards completing fewer symbols overall $(p=.087)$ but with no group by time interaction. SMA FA did not differ between groups and white matter integrity did not predict 
DS-Copy performance for either group. PD with left side onset did not differ from controls in DS-Copy performance, while right side onset $\operatorname{did}(p=.048)$.

Conclusions: This outcome supports several studies that have found intact MSL in PD and provides new evidence of differentiation of DS-Copy performance by side of onset. Future studies should expand connectivity measures beyond motor cortex and use a more variable sample of PD individuals both on medication and off medication. Correspondence: Samuel J. Crowley, Clinical and Health Psychology, University of Florida, $2220 \mathrm{SW} 34$ th Street Apt 207, Gainesville, FL 32608, United States.E-mail:samjcrowley@ufl.edu

A.N. DEVITO \& J. MOHLMAN. Neuropsychological Outcomes of a Combined CBT and Executive Skills Training Intervention for Anxious Parkinson's Patients.

Objective: A novel 12-week combined cognitive behavioral therapy (CBT) and cognitive training intervention for neurocognitive functioning and mood was tested in 14 patients with Parkinson's Disease (PD).

Participants and Methods: Patients with primary diagnosis of an anxiety disorder and mild-to-moderate PD were recruited through an urban hospital. Participants completed measures of anxiety (BAI, STAI, HAM-A) and depression (BDI, HAM-D) and a neuropsychological battery that measured memory, processing speed, verbal and nonverbal executive functions, and cognitive flexibility. Participants then completed an 8-week waiting phase followed by a second baseline alternate form assessment. Immediately thereafter, participants began CBT and Attention Process Training (APT) provided by supervised graduate-level trainees. APT is an EF skills program that progresses in a hierarchical manner, with each session's tasks increasing in complexity. After treatment, participants were assessed to determine if the intervention was successful in reducing anxiety and improving neurocognitive functioning. Clinicians completed the HAM-A at 3-month follow-up to determine if any reduction in anxiety symptoms was maintained over time. Paired t-tests were employed to compare pre- and post-intervention measures of anxiety and neuropsychological functioning.

Results: Results indicated no significant changes in anxiety or depression between baseline 1 and baseline 2 . From baseline 2 to post-treatment, there were significant improvements on the STAI, BDI, and HAM-D, and at 3-month follow-up, the HAM-A. Results indicated that there were no significant changes between baseline assessments or at the post-treatment time point on any neuropsychological domain.

Conclusions: The results of this pilot study suggest that this intervention may be successful at reducing anxiety symptoms, but may need to be modified to improve neurocognitive functioning. Larger, randomized control trials are needed to determine the efficacy and limitations of this intervention.

Correspondence: Alyssa N. DeVito, M.A., Child and Adolescent Psychiatry, Columbia University, 635 W. 165th Street, 6th Floor, New York, NY 10032, United States. E-mail: and2129@tc.columbia.edu

K. DHIMA, M. CLEM, H. WADSWORTH, R.B. DEWEY, P.E. O'SUILLEABHAIN \& L. LACRITZ. Hyperlipidemia in Parkinson's Disease: Protective Against Cognitive Decline?

Objective: Various oxidative stress conditions (OSC) have been linked to increased risk of cognitive impairment in the general population. However, this association remains unclear in Parkinson's disease (PD). Previous analysis of the current dataset identified hyperlipidemia (HLD) as protective against cognitive decline in a longitudinal PD cohort. This study examined the additive effect of OSC on cognitive change over time, while attempting to parse out the role of HLD.

Participants and Methods: Fifty-one individuals with PD $\left[\mathrm{M}_{\mathrm{age}}=63.98(9.17)\right]$ underwent cognitive assessment at baseline (T1) and 5 years (T2) as part of a larger study. Forward regression analyses were performed to measure the predictive value of total OSC and covariates on cognitive outcomes. OSC included hypertension, diabetes, heart disease, stroke, rheumatic disease, cancer, and elevated plasma homocysteine. Covariates included HLD, PD age of onset, and PD duration. Cognitive change scores were calculated (T2-T1) for selected measures: Rey-Osterrieth Complex Figure (Rey-O; immediate and delayed recall), Block Design, Digit Span, and Hopkins Verbal Learning Test-Revised (delayed recall). Bonferroni correction for multiple comparisons was applied $(\alpha=.01)$.

Results: Analyses revealed no impact of total OSC on cognitive outcomes (all $p>.01$ ). However, beta weights identified HLD and younger age of onset as significant protective factors for Rey-O immediate and delayed recall performance (both $p=.005$ ).

Conclusions: An additive effect of oxidative stress on cognitive change was not detected in this PD cohort. However, hyperlipidemia diagnosis appeared to be a protective factor against cognitive decline, particularly in visual memory. These findings are supported by previous studies demonstrating protective effects of hyperlipidemia as well as statins on rate of disease progression and cognitive outcomes in PD. Further efforts are warranted to characterize the specific role of hyperlipidemia and associated treatments on cognition in PD.

Correspondence: Kaltra Dhima, Clinical Psychology, Psychiatry, University of Texas Southwestern Medical Center, University of Texas Southwestern Medical Center, 5323 Harry Hines Blvd., Dallas, TX 75390-9044, United States. E-mail: Kaltra.Dhima@UTSouthwestern. edu

S. ELLIS, N. EDELSTYN \& T. SHEPHERD. A Study Designed to Examine the Feasibility of a Randomized Single-Blind CrossOver Trial That Will Assess the Effects of the Second Generation Dopamine Agonists, Pramipexole Prolonged Release and Ropinirole Modified Release, on Cued Recall Memory in Idiopathic Mild or Moderate Parkinson's Disease Without Cognitive Impairment.

Objective: The aim of this 24 month study was to assess the feasibility of a cross-over design to explore the effects of ropinirole modified release (RPR) and pramipexole prolonged release (PPX) on cued-recall memory in Parkinson's disease (PD). As the design required patients to switch from their prescribed dopamine agonist (PPX to RPR, or RPR to PPX), our first objective was to examine safety of switching between the investigative medicinal products (IMPs); our second primary objective was to obtain estimates of cued-recall to inform a power calculation for a definitive trial.

Participants and Methods: Patients were randomised in a 1:1 ratio to 2 treatment arms and administered each IMP for 8 weeks. The arms differed only in the sequence the IMPs were administered. Memory was assessed ON- and, following a washout period resulting in $93.75 \%$ IMP elimination, OFF-medication.

Results: 220 patients were screened for eligibility. 81 patients were invited to take part, of whom 53 declined to participate or did not respond. 145 were excluded because they did not meet the inclusion criteria or failed at screening. 22 patient-participants consented, of which 16 completed the study (defined as completed the memory assessment sessions on their assigned IMP).

There were no serious adverse events. Rates of non-serious adverse events and adverse reactions were equivalent between the IMPs.

A comparison of cued-recall difference scores between the ON vs OFF conditions for patients entering the study on PPX vs RPR $(0.034,95 \%$ confidence intervals: -0.07-0.13; and -0.02, CIs: -0.09-0.06) indicated that with a statistical power of 0.95 , an effect size of 0.41 and a significance level of 0.5 , a total of 128 patients would be needed to conduct a fully powered RCT.

Conclusions: This study presented challenges to recruitment both in design and execution and while it was a major aim of the study to assess this, evaluation of these challenges also provided the opportunity to explore how these could be overcome for future studies in this area. 
Correspondence: Simon Ellis, MD, Neurology, University Hospitals of North Midlands NHS Trust, Neurology Research Unit, First Floor, Block B, Royal Stoke Hospital, Stoke on Trent ST4 6QG, United Kingdom. E-mail:simon.ellis@uhns.nhs.uk

\section{R.J. FEE, B. YAFFE \& P.J. MATTIS. Poor recognition memory in Parkinson's disease may indicate the comorbidity of Alzheimer's disease.}

Objective: Substantial evidence supports that Parkinson's disease (PD) is associated with a cognitive deficit in memory retrieval. This deficit is likely related to PD specific variables such as focal cell death, imbalance in dopamine-acetylcholine or disruption in neural networks. Our data suggest that recognition memory on the BVMT is relatively preserved in $74 \%$ of the sample, which leaves $26 \%$ as outliers. The origin of these differences is not clear, however it is possible that there is a second pathology. Specifically studies have found that up to $50 \%$ of patients with PD have Alzheimer's disease (AD) pathology at the time of autopsy. We hypothesized a significant relationship between recognition and general cognitive decline and that overall cognitive functioning would be significantly different for those with a deficit in recognition suggesting that poor discriminability is related to the comorbidity of AD.

Participants and Methods: 74 participants (54 males and 20 females) diagnosed with PD ranging in age from 42 to $81(M=62.05 \pm 9.04)$ with 8 to 20 years $(M=15.19 \pm 2.76)$ of education were included in the study. Measures: Dementia Rating Scale-2 (DRS) total scores and the Brief Visuospatial Memory Test-Revised (BVMT) Recognition Discrimination Index were analyzed using Spearman correlation. BVMT discriminability scores were categorized into preserved $(\geq 5)$ and impaired $(<5)$ groups and performance was compared on DRS using a one-way ANOVA

Results: A significant correlation between BVMT and DRS scores $\left(r_{s}(72)=.38, \mathrm{p}<.01\right)$ was found. There was also a significant difference between BVMT groups $(F(1,72)=17.65, \mathrm{p}<.01)$ such that the BVMT poor discriminability group performed significantly lower on overall cognitive functioning.

Conclusions: Results revealed a recognition memory deficit in PD is associated with general cognitive decline. These findings suggest a recognition deficit in $\mathrm{PD}$ may indicate the comorbidity of $\mathrm{AD}$ and potential differences in the neuropathological correlates of PD patients with dementia.

Correspondence: Robert J. Fee, Sergievsky Center, Columbia Universityl Queens College, 630 West 168th St. PH19, New York, NY 10032, United States.E-mail:rf237@columbia.edu

\section{C.E. HARRISON \& S. ROGERS. Specifying the Nonverbal Memory Impairments that Characterize Parkinson's Disease.}

Objective: Studies have shown that memory deficits related to Parkinson's disease (PD) are more prominent in nonverbal relative to verbal information. Less clear are the particular types of nonverbal memory problems. Are the deficits in learning, recall, recognition, or time-based memory skills? Dopaminergic and cholinergic neuronal systems influence acquisition and retrieval, but not storage, in PD, so there might be worse learning relative to recall. This study attempts to clarify the specific type of nonverbal memory impairments associated with PD. Participants and Methods: 115 older adults (38 women, $M$ age $=$ 74.58, $M$ education $=15.2$ ) who had been diagnosed with PD completed the BVMT-R and ROCF as part of outpatient neuropsychology evaluations. All patients were taking carbidopa-levodopa.

Results: Paired samples $t$-tests showed that those with a parkinsonian pathology performed significantly worse on BVMT Trials $1-3$ Total than ROCF 3', $t(66)=-5.48, p<.001$, and ROCF 30', $t(53)=-3.52, p$ $<.001$, but not BVMT Delayed Recall. Their scores on BVMT Delayed Recall were significantly worse than ROCF $3^{\prime}, t(66)=-5.62, p<.001$, and ROCF $30^{\circ}, t(53)=-3.58, p<.001$. Moreover, they demonstrated worse ROCF 30' than ROCF 3', $t(57)=-3.52, p<.001$.
Conclusions: Results indicate those with PD have significantly worse nonverbal learning and memory when limited by time than when time constraints are absent. However, when time constraints are lifted, delayed recall seems to be worse than initial learning. This suggests that the nonverbal memory deficits related to PD are not necessarily related to learning, but may differentially impact time-based memory performance, perhaps due to problems with processing speed, but when time constraints are absent, delayed recall seems to be worse than initial learning. These findings clarify the specific types of nonverbal memory problems associated with PD, which can aid differential diagnoses compared to other pathologies and provide targets for cognitive intervention.

Correspondence: Cory E. Harrison, PhD, Neuropsychology, Pacific Neuroscience Medical Group, 515 W Foothill Blvd, Unit A, Monrovia, CA 91016, United States. E-mail: cburns12@apu.edu

T. HENDERSHOTT, D. ZHU, S. LLANES \& K. POSTON. Predictive Validity of the Mini Mental State Examination, Montreal Cognitive Assessment, and Mattis Dementia Rating Scale-2 in Parkinson's Disease.

Objective: Individuals with Parkinson's disease (PD) have an increased risk of developing cognitive impairment (CI). Global assessments such as the Mini Mental State Examination (MMSE), the Montreal Cognitive Assessment (MoCA), and the Mattis Dementia Rating Scale-2 (DRS) are widely used to assess overall cognitive function in PD. It is imperative to understand which of these tests accurately detect CI in PD. Although there are several studies comparing the MMSE and MoCA, no study compares the sensitivity and specificity of the MMSE, MoCA, and DRS in the same cohort of subjects. In this study we assess the predictive ability of these tests to identify CI in PD.

Participants and Methods: The MMSE, MoCA, and DRS were administered to 85 PD participants ages 42 to 85 , along with a neuropsychological battery that assessed five cognitive domains: attention/working memory, executive function, episodic memory, language, and visuospatial. Using published criteria, we defined CI as $\geq 1.5$ standard deviations below the mean on at least two tests. Participants were categorized as PD without CI (PD-noCI, n=45) or PD with CI (PD-CI, n=40).

Results: In a logistic regression predicting global CI, MoCA $(p=0.003)$ and DRS $(p=0.01)$, but not MMSE $(p=0.54)$, were unique predictors. MoCA and DRS comparatively predict CI (area under the ROC curve, 0.90 and 0.88 respectively). MoCA recall $(\mathrm{p}=0.002)$ and visuospatial/ executive scores $(p=0.0002)$ predicted episodic memory and visuospatial scores, whereas the DRS memory $(p=0.07)$ and construction scores $(p=0.36)$ did not.

Conclusions: These results demonstrate that the MoCA and DRS are sensitive in the detection of general cognitive impairment in PD. In addition the MoCA is sensitive in detecting selective episodic memory and visuospatial impairment. Detection of general and select cognitive impairment highlights the possible neural correlates of the disease and can inform clinical treatment.

Correspondence: Taylor Hendershott, B.A., Neurology and Neurological Sciences, Stanford University School of Medicine, 1901 Emory Street, San Jose, CA 95160, United States. E-mail: trhend15@stanford.edu

\section{D.C. HERGERT, J. SANCHEZ-RAMOS, R. WEST, K. ELLIOTT \&} C.R. CIMINO. Awareness in Huntington's Disease.

Objective: The purpose of the study is to examine if anosognosia of cognition in Huntington's disease (HD) is domain specific or non-specific. Both Metacognitive Knowledge (the overall knowledge and beliefs about the self or impairments) and Online Awareness (activated within tasks and situations) of memory and executive functions were explored. Participants and Methods: Fifty-six HD patients and fifty informants were recruited from the HD Center of Excellence at USF. Patients and informants completed the FrSBe and Everyday Memory-R rating scales to measure metacognitive knowledge of executive function and memory. For online awareness, patients were asked to rate their performance 
before and after the NAB Judgment, Driving, and Daily Living Memory tasks. They were also administered tasks of executive functioning (i.e. Trail Making Test, Stroop, EXAMINER Unstructured Task) and Memory (HVLT-R).

Results: Metacognitive Knowledge: The relationship between patients' self-report of memory was more strongly related to memory performance than informant report. Informants' report of executive functioning was associated with executive functioning performance, while patient report was unassociated with any executive performance. Online Awareness: Patients' predictions of executive performance were unrelated to actual performance, while memory predictions were related. Memory evaluation was more strongly associated with performance than executive function evaluation. Patients tended to provide higher evaluation ratings for executive tasks versus memory tasks.

Conclusions: HD patients appear to have better appreciation of their memory functioning versus executive dysfunction. This suggests awareness is domain dependent and is perhaps governed by local monitoring systems of memory and executive function.

Correspondence: Danielle C. Hergert, M.A., Clinical Psychology, University of South Florida, 6200 Eubank Blvd. NE, Apt 122, Albuquerque, NM 87111, United States.E-mail:dblinkoff@mail.usf.edu

J.A. LAFO, P. MANGAL, B.M. SCOTT, E. TRIFILIO, M.S. OKUN, K. FOOTE \& D. BOWERS. Construct Validity of the University of Florida DBS Cognitive Rating Scale: What cues neuropsychology to raise a red flag for DBS candidacy?

Objective: Deep brain stimulation (DBS) is a common treatment option for medication-refractory motor symptoms of Parkinson's disease (PD) and essential tremor (ET). At the University of Florida, selection of appropriate DBS candidates involves independent input and consensus by an interdisciplinary team, including neuropsychology. To ease team communication, we developed a DBS "Cognitive Rating Scale" (DBSCRS), which reflects clinical judgement based on the neuropsychological exam. The current study evaluated the construct validity of this scale. We hypothesized that poor performance in cognitive domains least susceptible to typical PD/ET progression (i.e., episodic memory, language) would have the greatest influence on DBS-CRS ratings.

Participants and Methods: Participants included a DBS-naïve sample of 106 PD patients and 46 ET patients who underwent pre-DBS neuropsychological exams (attention, episodic memory, language, executive function, visuospatial). An expert neuropsychology team made DBS-CRS ratings on a $1-5$ scale, with $1 / 2$ point intervals. Composite scores were computed for each domain. Separate hierarchical regressions for each group examined the influence of domain-specific scores on the DBS-CRS, controlling for demographic and disease variables.

Results: Models including each domain as a predictor were significant $(p<.001)$ and accounted for $71.6 \%$ of variance in the DBS-CRS in the PD group and $71.5 \%$ of variance in the ET group. In both analyses, the strongest predictor of DBS-CRS ratings was delayed memory recall, followed by executive function.

Conclusions: Our findings support the construct validity of the DBS-CRS. Key psychometric factors contributing to worse DBS-CRS ratings were worse delayed memory and executive function scores. Indeed, the co-occurrence of delayed recall and executive dysfunction should raise concern for superimposed dementia or other degenerative processes. Future studies will examine the predictive validity of the DBS-CRS in terms of post-operative complications and cognitive decline.

Correspondence: Jacob A. Lafo, B. A., University of Florida, 550 NE 6th Ave., Gainesville,FL 32601, United States.E-mail: jlafo@phhp.ufl.edu
F.V. LOPEZ, M. SPLIT, V. FILOTEO, I. LITVAN, R. MOORE, E. PIROGOVSKY-TURK, S. LESSIG, D. SONG \& D. SCHIEHSER. Does the Geriatric Depression Scale Measure Depression in Parkinson's Disease?

Objective: The Geriatric Depression Scale (GDS) is recommended for screening purposes in Parkinson's disease (PD) primarily because of its minimal focus on motor and somatic symptoms. However, empirical evidence is limited regarding its validity in PD. The aim of the current study was to evaluate the convergent and divergent validity and factor structure of the GDS in PD.

Participants and Methods: Non-demented individuals with PD $(n=160)$ completed the GDS and items were subjected to a Principle Component Analysis (PCA). The GDS total score and factors were correlated with the Unified Parkinson's disease Rating Scale depression item (UPDRSd), Hamilton Rating Scale for Depression (HAM-D), Apathy Scale (AS), State-Trait Anxiety Inventory-Trait scale (STAI), Modified Fatigue Impact Scale (MFIS), and a subjective measure of cognition (SMC). Multiple regression analyses were performed to determine the best predictors of GDS.

Results: The GDS total score was strongly correlated with the "divergent" measures of AS $(r=.58)$, STAI $(r=.73)$, and MFIS $(r=.59)$, while only moderately correlated with the UPDRSd $(r=.38)$ and HAM-D $(r=.41)(p \mathrm{~s}<.05)$. Regression analyses revealed the best predictors of GDS total were all measures, except the UPDRSd and HAM-D. The PCA resulted in six factors denoting Dysphoria, Despair, Anxiety, Apathy, Fatigue, and Cognition. The GDS subscale of Dysphoria was predicted by UPDRSd, AS, and STAI; Despair and Anxiety by STAI; Apathy by AS and STAI; Fatigue by MFIS and AS; and Cognition by SMC, AS, and MFIS $(p s<.05)$.

Conclusions: The GDS was best predicted by measures of apathy, anxiety, and fatigue, but not depression. The GDS subscales had some association to respective convergent measures, yet none of the affective depression GDS subscales were uniquely associated with depression measures. Thus, the findings question the validity and utility of the GDS in assessing depression as an independent construct in PD. Further investigation into the psychometric properties of the GDS is warranted to discern its merit in PD.

Correspondence: Francesca V. Lopez, Veterans Affairs San Diego Healthcare System, 128 Wild Horse Loop, RSM, CA 92688, United States.E-mail: francesca.v.lopez@gmail.com

K.F. MCINERNEY, C.I. BERMUDEZ, L. SEGALA, N. SUNSUSLOW, J. ROOKS, C. LUCA, H. MOORE, C. SINGER, J.R. JAGID, S. ANDERSON \& B.E. LEVIN. Does Exposure to Toxins Influence Cognition in Parkinson's Disease.

Objective: There is a well documented association between lifetime exposure to toxins and Parkinson's disease (PD). Less is known whether toxins also influence PD related cognitive and emotional changes. The current study explored whether self-reported exposure to toxins were linked to diminished cognitive functioning in idiopathic PD.

Participants and Methods: Three hundred ten PD patients ( $M$ age $=64.91, M$ educ $=13.31 ; M$ disease duration = 10.61, $)$ completed a self-report questionnaire assessing history and length of exposure to toxins (air pollutants, pesticides, metals, chemicals, minerals, radiation, and consumption of well water). Participants also underwent a comprehensive neuropsychological evaluation assessing language, visuospatial, verbal and visual memory, processing speed, attention, executive functioning and depression/anxiety.

Results: Fifty nine percent of the PD sample reported a history of contact with toxins, ranging from short term to chronic exposure. The most frequent exposures included pesticides $(n=54)$ and air pollutants $(n=35)$. Biserial correlations were calculated to assess whether exposure to toxins was associated with cognitive performance in patients. No significant relationships were observed between reported toxin exposure and any of the cognitive variables. Depression was linked to exposure but the association disappeared after controlling for education. Type of toxin exposure or number of toxins were also unrelated to 
outcome. Length of exposure was inconsistently reported and could not be analyzed.

Conclusions: The finding that over half the PD sample endorsed significant toxin exposure during their lifetime, adds to the growing body of research showing toxin exposure is a risk factor in the development of PD. However, these findings argue against the notion of exposure to toxins having a compounding or deleterious effect on PD related cognition and mood.

Correspondence: Katalina F. McInerney, PhD, Neurology, University of Miami, 1150 NW 14th Street, Suite 604, Miami, FL 33136, United States.E-mail:kfm42@med.miami.edu

\section{K. MOFFETT \& S. PENNA. Corticobasal Syndrome: A Unique Neuropsychological Profile Involving Unilateral Left Ideomotor Apraxia.}

Objective: Corticobasal syndrome (CBS) is a rarely occurring neurodegenerative disorder of the cortex and basal ganglia, characterized by the insidious onset and gradual progression of asymmetric motor impairments (especially rigidity and apraxia), cortical sensory deficits, and alien limb behavior. Behavioral, personality, and cognitive changes (especially language dysfunction) may accompany or precede these motor changes, and often present in a highly heterogenous fashion across patients. Ideomotor apraxia (IMA) is arguably the most common feature of CBS, and prevailing theories of IMA in CBS posit that it stems from left-hemisphere disruption of frontoparietal networks (Huey et al., 2009). The fact that IMA so commonly overlaps with language dysfunction in CBS is further evidence that it is often secondary to left-hemisphere degeneration in this population.

Participants and Methods: In the current case report, we present a 75-year-old, right-handed woman with highly asymmetric motor impairments including unilateral left-limb IMA, as well as fully intact language functioning.

Results: Indeed, her overall cognitive and motor profile were reflective of right-hemisphere pathology, with little evidence of left-hemisphere involvement. The source of her left-limb IMA was therefore suspected to involve a disconnection between left- and right-hemisphere frontal-motor cortices, a phenomenon first described by Liepmann and Maas in 1907 and then Geschwind and Kaplan in 1962.

Conclusions: This case provides evidence of unilateral left-sided IMA in a patient with right-lateralized CBS, demonstrating an uncommon mechanism by which ideomotor apraxia may manifest in such patients. It also contributes to the CBS literature by reporting on the comprehensive neuropsychological profile of an individual with unilateral right-hemisphere dysfunction.

Correspondence: Kristin Moffett, Ph.D., Rehabilitation Medicine, Emory University, 219 Briarvista Way NE, Apt 219, Atlanta, GA 30329, United States.E-mail:kristn.l.moffett@emory.edu

C.F. PLUIM, V. FILOTEO, R. MOORE, E. PIROGOVSKY-TURK, I. LITVAN, S. LESSIG, D. SONG \& D. SCHIEHSER. Changes in Self- and Caregiver-Reported Frontal Behaviors in Parkinson's Disease: A Longitudinal Study.

Objective: Parkinson's disease (PD) patients and their caregivers often report patient-related behavioral changes associated with executive dysfunction, disinhibition, and apathy. While self- and caregiver reports are often used interchangeably, research suggests these reports may not be concordant. Moreover, examination of self vs. informant report of frontal behavior has yet to be examined over time in PD. Such information has the potential to aid in clinical assessment and monitoring. Participants and Methods: Fifty-two non-demented PD patients and their caregivers completed the Frontal Systems Behavior Scale (FrSBe) self- and family-versions, respectively, at baseline and a two year follow-up evaluation. Four 2 (group) x 2 (time) mixed model repeated measures ANOVAs were conducted to evaluate changes in self vs. caregiver report of patient 1) executive dysfunction, 2) disinhibition, 3) apathy, and 4) overall frontal behavior over time. Behavior changes were correlated with baseline symptoms, including patient and caregiver depression, caregiver burden, and patient cognition, motor function, and disease severity.

Results: Both PD patients and their caregivers reported significant increases in patient executive dysfunction, apathy, and overall frontal behavior over time, with no differences in ratings between groups. There were no significant group, time, or interaction effects for disinhibition. Worse baseline patient cognition and caregiver burden predicted greater increases in disinhibition, while worse baseline motor function predicted greater increases in apathy and disinhibition.

Conclusions: Both caregivers and patients report consistent and significant worsening of frontal behavior specific to executive dysfunction and apathy in PD. Patient cognition, motor function and caregiver burden may be critical to specific behavioral changes; future research is needed to elucidate other predictive factors of frontal behavioral change in PD. Correspondence: Celina F. Pluim, VA San Diego Healthcare System, 2640 Figueroa Blvd, San Diego, CA 92109, United States. E-mail: celpluim@gmail.com

B. ROHL, K. COLLINS, S. MORGAN, S. COSENTINO, E. HUEY \& E. LOUIS. Sleepiness Across the Cognitive Spectrum in Essential Tremor.

Objective: Increasing evidence suggests that essential tremor (ET) is a complex disorder with nonmotor features including cognitive deficits and sleep problems, but the interrelation of such symptoms is unknown. Excessive daytime sleepiness without nighttime sleep dysfunction, shown to be associated with cognitive impairment in Parkinson's disease, has been proposed to reflect a specific distribution of neuropathology rather than a secondary symptom of hyposomnia. This study examined whether a similar phenotype was present in ET by examining indicator of daytime and nighttime sleep quality across the spectrum of cognitive functioning in ET.

Participants and Methods: Cross-sectional data on self-reported nighttime sleep dysfunction and excessive daytime sleepiness were prospectively collected using the Pittsburgh Sleep Quality Index (PSQI) and the Epworth Sleepiness Scale (ESS), respectively, in 96 ET cases. Cases underwent comprehensive neuropsychological assessment, and were classified as ET with normal cognition (ET-NC), mild cognitive impairment (ET-MCI), or dementia (ET-D).

Results: PSQI scores did not differ by ET cognitive groups $(p=0.22)$. However, ESS scores were highest in the ET-MCI group $(10.1 \pm 5.0$ [10.0]), followed by the ET-D $(6.8 \pm 2.9[6.0])$ and ET-NC groups $(6.1 \pm 4.0[5.0]),($ Kruskal-Wallis test $=8.320, p=0.016)$. In a linear regression adjusted for age, tremor severity, cognition enhancing medication, antidepressant use, body mass index and depression, cognitive diagnosis was no longer associated with ESS score $(\beta=0.02, p=0.13)$. Conclusions: Excessive daytime sleepiness, but not nighttime sleep dysfunction, is greatest in ET-MCI. Pathologic subtypes of ET associated with excessive daytime sleepiness may track with mild, rather than severe, cognitive dysfunction.

Correspondence: Brittany Rohl, Bachelor's of Science in Psychology, Neurology, Columbia University Medical Center, 710 West 168 th Street, New York, NY 10032, United States.E-mail:br2454@cumc.columbia. edu

J.C. ROTHLIND, M. YORK, K. CARLSON, P. LUO, W. MARKS, F. WEAVER, M. STERN, K. FOLLETT, J. DUDA, E. LAI \& D. REDA. Predictors of Multi-Domain Cognitive Decline following Deep Brain Stimulation Surgery for Treatment of Parkinson's Disease. Objective: Objectives Deep Brain Stimulation (DBS) to treat Parkinson's Disease (PD) is associated with higher rates of a) statistically reliable decline on single tests and b) multi-domain cognitive decline (MCD; Rothlind et al 2014). We examine the association between three variables that may serve as risk factors for MCD following DBS, including older age, greater baseline neuropsychological impairments, 
and incidence of serious adverse events (SAE) documented during the six-month study interval.

Participants and Methods: Participants: 316 patients with PD, screened to exclude individuals with clear signs of dementia, enrolled as part of VA-NINDS Cooperative Study 468. N=281 completed 6 month follow-up and became the focus of these analyses. One-hundred-sixty-four were randomized to receive DBS at either subthalamic nucleus ( $\mathrm{n}=80)$, or globus pallidum $(\mathrm{n}=84)$, and 117 were randomized to best medical therapy (BMT) during this phase of the study. Method: All participants received a comprehensive baseline neuropsychological evaluation which was repeated six months after treatment with DBS or BMT. SAE were documented as part of the study protocol.

Results: Results: MCD showed a modest but statistically significant association with age $>70$ years old and baseline Mattis Dementia Rating Scale Raw Total $<130 / 144$. SAE (including, but not limited to infections and small intracranial hemorrhages) documented in the study interval was a robust predictor of MCD following DBS, but not in the best medical therapy arm of the study.

Conclusions: Conclusions: Findings offer further support for consideration of baseline age and cognitive impairment in evaluating potential risks and benefits of DBS, as well as the importance of assessing any variables that increase risk of $\mathrm{SAE}$.

Correspondence: Johannes C. Rothlind, Ph.D., MHS, San Franisco VAMC, San Francisco VAMC 116B, 4150 Clement, San Francisco, CA 94121, United States. E-mail: johannes.rothlind@va.gov

\section{P. BHATIKAR, R. SALAZAR, O. BARTHELEMY \& A. CRONIN- GOLOMB. Parkinson's Disease Affects Category Switching.}

Objective: Parkinson's disease (PD) is associated with impairments in executive function arising from fronto-striatal dysfunction, including reductions in verbal fluency. Because set shifting deficits are common in PD, the task demands of category switching may be particularly difficult to meet. We hypothesized that, relative to healthy age-matched control adults (HC), category switching for individuals with PD would be characterized by fewer correct words and more repetitions and intrusions. We predicted a group-by-condition interaction such that the impact of category switching, relative to phonemic and semantic fluency, would be greater for PD than HC.

Participants and Methods: Fifty two individuals with PD and $43 \mathrm{HC}$ received three conditions of the D-KEFS verbal fluency test: phonemic fluency (FAS 3-letter average), semantic fluency (Animals), and category switching (Fruits-Furniture). Independent samples t-tests assessed PD-HC differences, and mixed design analysis of variance assessed the group-by-condition interaction.

Results: The PD group generated fewer words than HC on phonemic fluency $(p=.005)$ and category switching $(p=.041)$. The PD group generated more intrusions than $\mathrm{HC}$ on category switching $(p=.007)$, and there was a trend for phonemic fluency $(p=.051)$. PD did not differ from HC on repetitions or on any aspect of semantic fluency. The group-bycondition interaction (semantic fluency, category switching) was significant $(p=.041)$; the impact of category switching was greater for PD than HC.

Conclusions: The higher executive task demands of category switching over semantic fluency had a significant impact on PD performance, resulting in fewer words and more intrusions on switching. PD performance was specifically marked by more intrusions (and not repetitions), which may reflect limitations in developing successful search and retrieval strategies, an expected consequence of disruption to prefrontal-striatal networks that are important to maintaining set and inhibiting inaccurate responses.

Correspondence: Robert Salazar, M.A., Boston University, 1 Fairland St, Roxbury, MA 02119, United States. E-mail: rdsalaz@bu.edu
E. WEIZENBAUM, R. SALAZAR, T. ELLIS \& A. CRONIN-GOLOMB. Predictors of Self-Perceived Stigma in Parkinson's Disease.

Objective: Though Parkinson's disease (PD) has traditionally been characterized as a motor disorder, the disease burden arising from subcortical-cortical dysfunction extends far beyond physical limitations to aspects of cognition, mood, and other non-motor symptoms. The extensive symptomatology often leads to disease-related stigma, which in turn may further affect activities of daily living (ADLs) and quality of life. Quantitative evidence of the factors that contribute to self-perceived stigma in PD is limited. We assessed predictors of stigma in PD, including demographic and disease characteristics.

Participants and Methods: Hierarchical linear modeling was used to assess predictors of self-perceived stigma in 364 non-demented individuals with idiopathic PD (164 women, 200 men). Self-perceived stigma was measured by the four-item stigma subscale of the Parkinson's Disease Questionnaire (PDQ-39). We considered age, gender, disease duration, severity, motor function, ADLs, and depression. Predictor variables were chosen based on significant correlations with the stigma subscale. Because men and women may respond differently to psychosocial challenges, further analyses were conducted separately for these subgroups.

Results: For the total sample, the full model accounted for $13 \%$ of the variance in stigma perception $(p<.001)$. Younger age and higher depression scores were the only significant predictors, with minimal contributions of ADLs and motor severity. For both women and men, depression significantly predicted stigma; for men, age was also a significant predictor.

Conclusions: Age and depression were the primary predictors of self-perceived stigma in PD. These results highlight the impact of non-motor symptoms on the subjective experience of PD, with implications for potential treatment targets. Psychological interventions directed toward depression, especially in younger individuals with PD, may prove effective in alleviating self-perceived stigma.

Correspondence: Robert Salazar, M.A., Boston University, 1 Fairland St, Roxbury, MA 02119,United States.E-mail:rdsalaz@bu.edu

C. XU, R. SALAZAR, T. ELLIS \& A. CRONIN-GOLOMB. Social Support Mediates the Relation between Depression and Motor Limitations of Parkinson's Disease.

Objective: Parkinson's disease (PD), an age-related neurodegenerative disease, is most recognized for its motor symptoms, but disabling non-motor symptoms such as depression are also prevalent. In fact, researchers have found that lower quality of life in PD is driven by such non-motor symptoms. The question is raised as to what factors may reduce depression in PD to improve quality of life. We predicted that depression would be directly related to social support, as indexed by the social support subscale of the widely-used Parkinson's Disease Questionnaire-39 (PDQ-39). We also predicted that social support would mediate the relation between motor activities of daily living (ADLs) and depression.

Participants and Methods: Participants included 348 non-demented individuals with PD (150 F, 198 M). The Unified Parkinson's Disease Rating Scale (UPDRS) assessed motor ADLs, and the Geriatric Depression Scale (GDS) assessed depression.

Results: As predicted, social support correlated with depression $r(348)=.35, p<.001$ and with ADLs $r(348)=.27, p<.001$, and depression correlated with ADLs $r(348)=.50, p<.001$. The regression model indicated that the total effect of depression on ADLs was significant $[\mathrm{B}=.36, \mathrm{p}<.001,95 \%$ confidence intervals $(\mathrm{CI})(.28, .43)]$. When social support was entered into the model, the indirect effect was $\mathrm{B}=.06,95 \%$ CI $(.03, .10)$, indicating that social support partially mediated the relation between depression and ADLs.

Conclusions: Future interventions can focus on strengthening the social support an individual with $\mathrm{PD}$ receives, thereby reducing the effect of the relation between depression and ADLs. Social support interventions are a controllable aspect of an individual's life that may improve overall quality of life. 
Correspondence: Robert Salazar, M.A., Boston University, 1 Fairland St, Roxbury, MA 02119,United States. E-mail: rdsalaz@bu.edu

B.M. SCOTT, J.A. LAFO, P. MANGAL, M. BRADLEY, M.S. OKUN \& D. BOWERS. Emotion-semantic priming and electrocortical reactivity in Parkinson's disease.

Objective: Previous studies using various physiological approaches (SCR, startle, ERP) have observed blunted reactivity to aversive stimuli in nondemented individuals with Parkinson's disease (PD). A key issue pertains to identifying methods for improving PD emotional blunting. This study tested the hypothesis that an 'exo-evoked' emotion-semantic priming technique might prompt normal reactivity to emotional pictures, as measured by the late positive potential (LPP), an electrocortical index of arousal/attention.

Participants and Methods: Participants included 24 nondemented PD and age/education matched controls, who viewed standard sets of neutral and aversive pictures (IAPS) while EEG was recorded from EGI-64 electrode net. Immediately prior to each picture, Ss heard a brief sentence whose content was either neutral (e.g., this is a picture from a gun safety video) or negative (e.g., this is a picture of gun used in a violent murder). These 'priming' sentences were delivered via headphones and designed to bias emotional responses (LPP) to the upcoming picture. Priming effects reflected difference in LPP amplitude to pictures preceded by negative vs neutral primes. Data were analyzed using mixed models ANOVA.

Results: As predicted, the LPP amplitudes were significantly greater for pictures that were preceded by a negative vs. neutral cue $(p<0.01)$. This LPP priming effect was similar for both the PD and control groups (i.e., no group differences or interactions). Participants with lower scores on executive function tasks were more susceptible to priming effects for aversive pictures. Priming effects were unrelated to mood or motor severity in PD.

Conclusions: The similar priming effects observed in both patients and controls suggests that PD patients benefit from external cues that bolster emotional reactivity. These findings have important implications in terms of apathy, executive function, and development of targeted treatment therapies for mood/motivational disorders in PD.

Correspondence: Bonnie M. Scott, M.S., Clinical and Health Psychology, University of Florida, $538 \mathrm{NW}$ 39th Road, Unit 305, Gainesville, FL 32607, United States. E-mail: bonnie.m.scott@gmail.com

L. SEGALÀ, K.F. MCINERNEY, J. ROOKS, S. ANDERSON, N. SUN-SUSLOW, C. LUCA, H. MOORE, C. SINGER, C.I. BERMUDEZ \& B.E. LEVIN. The Role of Hypertension on Cognition in Parkinson's Disease.

Objective: Cardiovascular risk factors, in particular hypertension, are linked to diminished cognition in older age. Research has shown a relationship between hypertension and Alzheimer's disease. Yet little is known about the impact of hypertension on mental status in Parkinson's disease (PD), a condition associated with a wide range of non-motor symptoms resulting from frontal subcortical dysfunction. The purpose of this study was to examine the associations between hypertension and cognition in a sample of patients with PD.

Participants and Methods: Eighty five patients with PD (66\% males) without severe cognitive impairment (MMSE $M=27$ ) participated in this study. Participants were administered a battery assessing attention and working memory (WAIS-III Digit Span), semantic fluency (animals), verbal learning and memory (CVLT-II), and depression (BDI-II). Additional neuropsychological data were obtained from a subgroup of patients included in the larger sample, including phonemic fluency (FAS) and visuospatial ability (HVOT and JLO).

Results: A series of multiple regression analyses revealed that the presence of hypertension significantly contributed to decreased performance in semantic and phonemic fluency $(p<.001)$, verbal learning and memory $(p<.008)$, and visuospatial skills $(p<.01)$ even after controlling for disease severity (Hoehn-Yahr scale), age and education.
Hypertension had no effect on measures of attention, working memory and symptoms of depression.

Conclusions: These findings suggest that among non-demented PD patients, hypertension plays an important role in cognitive but not in affective changes. More research is needed to understand the relationship between cardiovascular risk and cognition in PD, and to identify early vascular biomarkers that may be targeted for interventions.

Correspondence: Laura Segalà, Neurology, University of Miami, 1120 NW 14th St., Miami, FL 33136, United States. E-mail: segala.laura@ gmail.com

J. SPAT, H. MURPHY, M. BEDERSON, S. MANDELBAUM, J. SUZUKI, E. SMITH-HYLE, L. LANGAN, J. CIARDULLO, A. DRANIKOV \& H.A. BENDER. Neuropsychological Assessment Evaluating Candidacy for Deep Brain Stimulation in Children with Pharmaco-Resistant Movement Disorders: A Case Series.

Objective: Children with congenital movement disorders are receiving treatment with Deep Brain Stimulation (DBS) with increased regularity. Thus, it is incumbent upon neuropsychologists to develop comprehensive, age-appropriate batteries aimed at determining surgical candidacy and providing data with prognostic value for post-surgical neurocognitive, psychological and academic functioning. The present study reviews the commonalities in assessment methods needed to develop test batteries tailored to the needs of this often difficult-to-assess population. Participants and Methods: Three cases referred from a movement disorders center from 2013-2016 were reviewed. They include a 16 year old bilingual patient with genetic torsion dystonia; a 17 year old, bilingual patient with an unspecified, rapidly progressing dystonia; and 12 year old patient with atypical myoclonus dystonia. All were administered a global measure of intellectual functioning (or proxy measure, if non-native English speaking), word reading, confrontation naming, semantic fluency, attention, processing speed, verbal-/non-verbal learning and memory. Parent-report questionnaires were given.

Results: For each patient, assessments were severely limited by dyarthria, dysfluent speech output, psychomotor slowing, diminished fine motor control, fatigue and variable attention due to uncontrolled motor movements. Though non-standardized assessment techniques were employed (i.e., assistive technology for communication), gross interpretations did not yield any obvious contraindications for successful DBS placement, such as profound intellectual disability or severe psychiatric distress.

Conclusions: Considerable value may be added by comprehensive assessment of children with pharmaco-resistant movement disorders prior to DBS placement. Beyond assessing candidacy, baseline assessments can help gauge expectations for post-surgical cognitive changes, if any, as well as to help better inform school re-entry and/or provide accommodations for entry into the workforce.

Correspondence: Jessica Spat, Ph.D., 1468 Madison Avenue, NY 10029, United States.E-mail: jessica.spat@mssm.edu

M. SPLIT, F.V. LOPEZ, V. FILOTEO, R. MOORE, E. PIROGOVSKYTURK, I. LITVAN, S. LESSIG, D. SONG \& D. SCHIEHSER. Rapid Eye Movement Sleep Behavior Disorder and Daytime Sleepiness are related to Poor Attention and Executive Function in Parkinson's disease.

Objective: Non-motor symptoms, such as fatigue, daytime sleepiness, and Rapid Eye Movement Behavior Disorder (RBD) are common in Parkinson's disease (PD); however, it is unclear how these symptoms are associated with cognitive dysfunction often experienced by individuals with PD. Thus, the aim of this study was to investigate the relationship between fatigue, daytime sleepiness, RBD, sleep quality, and cognitive performance in PD.

Participants and Methods: Sixty-six non-demented individuals with PD completed a comprehensive neuropsychological battery. Composite scores were generated for five domains representing Attention, Executive Function, Memory, Visuospatial, and Motor/Disease Severity and 
were correlated with self-report measures of fatigue (Modified Fatigue Inventory Scale; Fatigue Severity Scale), daytime sleepiness (Epworth Sleepiness Scale), RBD (RBD Sleep Questionnaire), sleep quality (Pittsburgh Sleep Quality Index), anxiety (State-Trait Anxiety Inventory), and depression (Geriatric Depression Scale). Multiple regression analysis was conducted to determine the best predictor(s) of cognition controlling for significant demographic and disease characteristics as well as psychiatric symptoms.

Results: Poorer attention $(r=-.34 ; p=.005)$, executive function $(r=-.26$; $p=.04)$, and memory $(r=-.37 ; p=.003)$ were associated with greater RBD symptoms. Fatigue, sleepiness, sleep quality, depression, and anxiety were not related to any cognitive domain, with the exception of sleepiness, which was the only significant predictor of executive function controlling for gender and RBD $(p=.005)$.

Conclusions: This study demonstrates that in PD, poorer memory and attention performances are associated with greater RBD symptoms and executive function is associated with sleepiness; these relationships cannot be accounted for by depression, anxiety, disease severity, or motor function. Future research is needed to examine the unique role of RBD and sleepiness in cognition and whether they are predictive of future cognitive decline in PD.

Correspondence: Molly Split, Veterans Affairs San Diego Healthcare System, 4236 4th Avenue, San Diego, CA 92103, United States. E-mail: molly.split11@gmail.com

J. STIVER, P. PRESSMAN, K. VOSSEL, S. DUTT, M. WYNN, R. SALONER \& J. KRAMER. Cognitive and Motor Correlates of Depressive Symptoms in Parkinson's Disease.

Objective: Research shows that depression is a prevalent non-motor feature in Parkinson's disease (PD), but few studies have examined the relative contribution of different predictors. The purpose of this study was to evaluate the relative associations of parkinsonian symptom severity and cognitive deficits with depression in non-demented, idiopathic PD patients. We hypothesized that motor symptom severity and cognitive diagnosis would both independently predict depressive symptoms in PD.

Participants and Methods: Forty-eight PD participants (52\% male; mean age $=67.4)$ underwent a comprehensive neuropsychological evaluation and received a cognitive diagnosis of normal (PD-Normal; $\mathrm{n}=27$ ) or mild cognitive impairment in PD (PD-MCI; $n=21$ ) according to Litvan et al. (2012) criteria. Participants also completed a neurological examination of motor symptom severity (Unified Parkinson's Disease Rating Scale-III) and an assessment of depressive symptoms (Beck Depression Inventory-II).

Results: Multiple linear regression, controlling for age, gender, and education, showed that while both risk factors were independently associated with depressive symptoms, cognitive diagnosis more strongly predicted depressive symptoms $(b=0.47, p<0.01)$ than motor symptom severity $(b=0.31, p<0.05)$.

Conclusions: Results show that cognitive diagnosis is more strongly associated with depressive symptoms than motor symptom severity in PD. These findings are consistent with recent research suggesting that depression in PD is not only associated with motor impairment, but may track more closely with cognitive impairment, highlighting the importance of non-motor symptoms in functional status of PD. It remains less clear if there is any causal relationship between cognition and depression in PD, and how they might interact with motor disease severity. Correspondence: Jordan Stiver, University of California, San Francisco, 106 Cardenas Ave., San Francisco, CA 94132, United States. E-mail: jordan.stiver@ucsf.edu
N. SUN-SUSLOW, K. MCINERNEY, C.I. BERMUDEZ, H.L. SCHNEIDER, S. ANDERSON, L. SEGALÀ, C. LUCA, J.R. JAGID, C. SINGER \& B.E. LEVIN. The Role of Social Support on Cognitive Functioning after Deep Brain Stimulation in Parkinson's disease. Objective: Lack of social support is a risk factor for disease development and progression. How these social factors affect Parkinson's disease (PD) patients undergoing Deep Brain Stimulation (DBS) is unknown. This study examined whether social support is associated with cognitive and emotional functioning in PD before and after DBS surgery.

Participants and Methods: Thirty-seven PD patients $\left(\right.$ Mean $_{\text {age }}=63.23$, $\mathrm{SD}_{\text {age }}=7.67 ;$ Mean $_{\text {edu }}=13.32 ; \mathrm{SD}_{\text {edu }}=4.37 ; 34 \%$ Female) underwent a neuropsychological evaluation, including CVLT-II, HVOT, FAS/PTM, BDI-II, and BAI. The average household was 2.59 people and $70 \%$ were married. Multiple regression and ANCOVA were applied to examine whether marital status (MS) and number of individuals in the household (NIH) were associated with cognition and emotion before and after surgery.

Results: After accounting for age, education, and language of evaluation, neither MS nor NIH was associated with cognition or emotion before DBS. However, there was a significant main effect of marital status on several change scores post DBS, including HOVT $(p=0.008)$, CVLT-II total recall, short and long delay ( $p$ value range=.03-.001), FAS/PTM $(p=0.005)$. There was no main effect of MS on BDI-II and BAI. Sex, disease duration, and number of medications did not significantly contribute to outcome.

Conclusions: These findings suggest that, although social support may not contribute to cognition or emotion in PD individuals pre-DBS, it is an important contributor to outcome post DBS. Specifically, MS is linked to better verbal fluency, verbal learning and memory, and visual integration. This highlights the importance of social support as a buffer against cognitive decline after DBS surgery.

Correspondence: Ni Sun-Suslow, M.S., Psychology, University of Miami, 5665 Ponce de Leon Blvd., Coral Gables, FL 33124-0751, United States.E-mail: n.sun1@umiami.edu

S.M. SZYMKOWICZ, V.M. DOTSON, J. JONES, M.S. OKUN \& D. BOWERS. Symptom Dimensions of Depression and Apathy and their Relationship with Cognition in Parkinson's Disease.

Objective: Parkinson's disease (PD) is neurodegenerative disorder characterized by both motor and non-motor symptoms. Both depression and apathy, alone and in combination, have been shown to negatively affect cognition in patients with PD. However, the influence of specific symptom dimensions of depression and apathy on cognition is not well understood. The current study investigated the relationship between symptom dimensions of depression and apathy, based on factors identified by factor analysis in Kirsch-Darrow et al. (2011), and memory and executive function in PD.

Participants and Methods: A sample of 138 non-demented individuals with PD (mean age $=64.51 \pm 7.43$ years; mean $\mathrm{MMSE}=28.37$ \pm 1.58 ) underwent neuropsychological testing and completed the Beck Depression Inventory, $2^{\text {nd }}$ edition and Apathy Scale. Separate hierarchical regression models examined the relationship between symptom dimensions of depression and apathy (apathy, depressive symptoms, loss of interest/pleasure, and somatic symptoms) and three cognitive domain composites: immediate verbal memory, delayed verbal memory, and executive function.

Results: After adjusting for general cognitive status, disease variables, and the influence of the other symptom dimensions, depressive symptoms predicted delayed verbal memory performance $(p=0.028)$, such that higher depressive symptoms were associated with worse delayed verbal memory performance. No symptom dimension was associated the immediate verbal memory or executive function composites.

Conclusions: Findings from the current study suggest that depressive symptoms, rather than other symptom dimensions of depression or apathy, influence specific domains of cognitive functioning in patients with PD. Further research is needed to better understand possible 
mechanisms through which specific symptom dimensions of depression and apathy are associated with cognition in PD.

Correspondence: Sarah M. Szymkowicz, MS, Clinical \& Health Psychology, University of Florida, Dept of Clinical \&. Health Psychology, PO Box 100165, Gainesville, FL 32610-0165, United States. E-mail: smszymkowicz@phhp.ufl.edu

B.P. TAYLOR, E.J. VAN ETTEN, H.M. HOLDEN, L. GRAVES, F.V. LOPEZ, L. NGUYEN, E. PIROGOVSKY-TURK, J. COREY-BLOOM, V. FILOTEO \& P. GILBERT. CVLT-II Performance in Huntington's Disease and Parkinson's Disease.

Objective: Using the original version of the California Verbal Learning Test (CVLT), Massman et al. (1990) found that individuals with Huntington's disease (HD) show significant deficits compared to those with Parkinson's disease (PD) on verbal memory indices including total immediate recall, short delay free/cued recall, long delay cued recall, and perseverations. However, the groups did not differ on immediate recall span, long delay free recall, semantic/serial clustering, recognition memory and intrusions. No studies have compared verbal memory abilities in individuals with HD or PD using the second version of the CVLT (CVLT-II).

Participants and Methods: The CVLT-II was administered to individuals with PD $(\mathrm{n}=72)$ or $\mathrm{HD}(\mathrm{n}=77)$. Standardized scores on trial 1 immediate recall, total immediate recall, short and long delay free recall, short and long delay cued recall, total recognition discriminability, semantic/serial clustering, intrusions, repetitions, and learning slope were compared between groups using ANCOVA, controlling for education and Dementia Rating Scale (DRS) scores.

Results: After controlling for multiple comparisons, the HD group performed significantly worse than the PD group on total immediate recall, short and long delay free recall, long delay cued recall ( $\mathrm{ps}<.01)$, and total recognition discriminability $(\mathrm{p}<.05)$. However learning slope and trial 1 immediate recall did not differ. The HD group committed more repetition errors $(p<.01)$ but not intrusion errors. The HD group demonstrated poorer serial clustering $(p<.01)$ but semantic clustering did not differ.

Conclusions: Our findings using the CVLT-II to assess verbal memory in a relatively large sample of individuals with HD or PD, while controlling for DRS scores, are largely consistent with a previous study using the original CVLT in smaller samples. Notable differences include our finding that individuals with HD perform worse than those with PD on long delay free recall and recognition discriminability.

Correspondence: Brad P. Taylor, M.A. Student, Psychology, San Diego State University, 4776 Filipo Street, San Diego, CA 92115, United States.E-mail:bptaylor@mail.sdsu.edu

\section{B. TRAN. Neuropsychological Predictors of Functional Decline in} Non-demented Parkinson's Disease.

Objective: Impairment in processing speed, verbal memory, and executive abilities are evident early in Parkinson's disease (PD) progression (Weintraub et al., 2015) and significantly predict performance of instrumental activities of daily living (IADLs) (Cahn et. al., 1998; Burton, Strauss, Hultsch, \& Hunter, 2006; Gross, Rebok, Unverzagt, Willis, $\&$ Brandt, 2012). We sought to examine whether change in performance of neuropsychological measures can predict decline in performance of IADLs in a non-demented PD cohort across two years.

Participants and Methods: We retrospectively examined clinical, functional, and neuropsychological data of 100 PD patients from the Parkinson's Disease and Movement Disorders Center at University of Pennsylvania. The following neuropsychological measures were categorized into the following domains: memory: Hopkins Verbal Learning Test-Revised (HVLT-R); processing speed: Symbol Digit Modalities Test (SDMT) and Trail Making Test-A (TMT-A); and executive functioning/working memory: Letter-Number Sequencing, Controlled Oral Word Association (COWA), animal fluency, and Trail Making Test-B (TMT-B). IADLs were assessed using the Penn Parkinson's Daily
Activities Questionnaire-15 (PDAQ-15), an informant-based measure of daily function.

Results: There was a significant correlation between change in performance of IADLs and change in the following measures: HVLT-R delayed recall $(\mathrm{r}=0.346, \mathrm{p}<0.01)$ and HVLT-R recognition discriminability $(\mathrm{r}=0.276, \mathrm{p}<0.01)$. A hierarchical regression analysis revealed that change in HVLT-R delayed recall, COWA, and TMT-A were significant predictors of change in performance of IADLs $\left(R^{2}=0.385, F(22,77)\right.$ $=2.192, \mathrm{p}<.01)$.

Conclusions: These findings support the use of neuropsychological measures to predict subtle declines in performance of IADLs in non-demented PD. Furthermore, health providers can identify individuals at risk of functional decline and provide recommendations, including cognitive remediation, to promote neurocognitive abilities and improve quality of life

Correspondence: Baochan Tran, Psychology, University of Pennsylvania, 1733 Christian St., Unit 1, Philadelphia, PA 19146, United States. E-mail:btran86@gmail.com

E.J. VAN ETTEN, L. GRAVES, B.P. TAYLOR, H.M. HOLDEN, F.V. LOPEZ, L. NGYUEN, E. PIROGOVSKY-TURK, J. COREY-BLOOM, V. FILOTEO \& P. GILBERT. Recall and Recognition Discriminability in Parkinson's Disease and Huntington's Disease.

Objective: Studies using the original California Verbal Learning Test (CVLT) to examine recall and recognition abilities in individuals with Parkinson's Disease (PD) and Huntington's Disease (HD) produced mixed findings. Some suggested that individuals with HD demonstrate worse recall and recognition, whereas others revealed comparable performance. The California Verbal Learning Test-Second Edition (CVLT-II) provides several novel indices of recall and recognition discriminability in an effort to provide a more thorough assessment of memory abilities. In the present study, we examined differences between individuals with PD or HD on CVLT-II indices of recall and recognition discriminability. Participants and Methods: The CVLT-II was administered to individuals with PD $(\mathrm{n}=72)$ or HD $(\mathrm{n}=77)$. Standardized scores on subtypes of recall discriminability (immediate, short delay free and cued, long delay free and cued) and recognition discriminability (total, source, semantic, and novel) were compared between the groups using analysis of covariance tests, controlling for education and Dementia Rating Scale scores. Results: Following Bonferroni corrections for multiple comparisons, the HD group performed significantly worse than the PD group on short and long delay free recall and long delay cued recall discriminability indices $(p s<.01)$. Additionally, the HD group performed significantly worse on source recognition discriminability $(p<.0125)$. Group differences were associated with large Cohen's $d$ effect sizes.

Conclusions: When accounting for intrusions, we found that individuals with $\mathrm{HD}$ exhibit significantly worse recall than those with PD following a short or long delay. However, recognition discriminability did not differ between the groups, with the exception of source recognition discriminability. These new CVLT-II measures may provide a more thorough assessment of recall and recognition abilities and mav improve efforts to characterize and compare profiles of memory dysfunction in different neurodegenerative populations.

Correspondence: Emily J. Van Etten, San Diego State University, 8732 Pacheco Avenue, Westminster, CA 92683, United States. E-mail: emilyjoyvanetten@gmail.com

A.M. VANDEBUNTE \& S. ROGERS. The Effect of Depression on the Cognition of those with Parkinson's Disease.

Objective: Previous research has shown a negative association between depression and cognition. Many of those with Parkinson's disease (PD) also suffer from depression, but less clear is how the presence of state depression affects the cognitive abilities of patients with PD.

Participants and Methods: A total of 115 adults ( 75 women, $M$ age $=$ 74.58 years, $M$ education $=15.26$ years with parkinsonian pathology 
completed subtests of the WAIS-IV, WMS-IV, DKEFS, Trailmaking, BNT, COWAT, ROCF, HVLT-R and BVMT-R. They indicated any history of depression on an objective questionnaire, and state depression was measured through the Geriatric Depression Scale.

Results: Mild levels of state depression were endorsed by $54 \%$, moderate depression by $31 \%$, and severe state depression by $8 \%$. Significant negative associations were found between state depression and performance on Logical Memory I, $r(23)=-.70, p<.001$, Logical Memory II, $r(23)$ $=-.59, p<.01$, ROCF 3' delay, $r(23)=-.42, p<.05$, and ROCF 30 'delay, $r(23)=-.49, p<.02$. Among those being treated for a depressive condition, there were significant negative associations between state depression and performance on Block Design, $r(32)=-.47, p<.01$, and Picture Completion, $r(35)=-.48, p<.01$.

Conclusions: State depression seems to impact cognitive functioning among those with PD, regardless if there is a history of depression. Patients with greater levels of state depression exhibit worse contextual verbal learning and memory, as well as worse nonverbal learning and memory for complex visual material. Patients with an underlying depressive condition and higher levels of state depression exhibit worse visual perception and construction relative to those with lower state depression. Minimizing the state depression of those with PD may therefore reduce problems with learning and memory, and if a patient is already receiving treatment for depression, attenuating state levels of depression may improve visuospatial functions.

Correspondence: Anna M. VandeBunte, Psychology, Westmont College, 312 Mellifont Drive, Santa Barbara, CA 93103, United States. E-mail: avandebunte@westmont.edu

\section{J. WERTHEIMER, J.S. MILLER, K. MAXWELL \& A. GOTTUSO. Cognition and Parkinson's Disease: The Patient's Perspective.}

Objective: Cognitive dysfunction is expected and often debilitating for people with Parkinson's disease (PWP). A better appreciation of and awareness about the patient's perspective of cognitive difficulties and the impact these challenges have on daily life is indicated. Objective: To understand the patient's perspective about cognitive changes and how their cognitive difficulties impact daily life.

Participants and Methods: 1,548 PWP participated in a cross sectional research design study. Participants completed the Everyday Cognition Scale (ECog), The Parkinson Alliance Cognitive Questionnaire (PACQ), and Lawton Instrumental Activities of Daily Living Scale.

Results: ECog: Language, Memory, Attention, and Organization had the highest frequency of perceived change within age and disease duration-matched groups. The Attention domain had the highest reports of "consistent worsening" over time. PACQ: $65 \%$ of PWP reported mild to mild-to-moderate cognitive difficulties, and $16 \%$ reported moderate to severe difficulties. The most frequently reported difficulties were memory and expressive language skills, followed by slowed processing speed and difficulties with executive functioning. Difficulties in all cognitive domains were reported in greater frequency with age and disease progression. Slowed processing speed and increased difficulties with executive functions appear to be the most notable observed dynamic change as PD progresses. Across age and disease duration groups, the majority of participants' reported experiencing an adverse impact of cognition on daily functions. 22\% (Younger and Early Disease Duration PD Group) to $43 \%$ (Older and Late Advanced PD Group) reported a moderate to extreme impact of cognitive difficulties on daily functions. Conclusions: Cognitive deficits are prevalent for PWP. Despite research identifying reduced appreciation of cognitive deficits as a challenge in self-report (Copeland, et al., 2016), understanding the patient's perspective about his or her personal experience has important implications. Correspondence: Jeffrey Wertheimer, Ph.D., Physical Medicine and Rehabilitation, Cedars-Sinai Medical Center, 8700 Beverly Blvd., Los Angeles, CA 90048, United States. E-mail:Jeffreycwertheimer@yahoo. com
A. STEVENS, M.E. WIGGins, J.J. TANNER, A. AMADOR, N.A. SCHWAB, T. GIOVANNETTI \& C.C. PRICE. Category Fluency Association Index is Sensitive to Temporal Lobe Atrophy in Parkinson's Disease.

Objective: Previous literature has demonstrated that some individuals with Parkinson's disease (PD) have brain atrophy in temporal lobe regions with this reflecting cognitive impairment in PD (Tanner et al., 2015; Goldman et al., 2012, Weintraub et al., 2011). This investigation studied temporal thickness and its relative contribution to verbal fluency semantic integrity in PD relative to non-PD peers.

Participants and Methods: All participants were recruited through studies at the University of Florida. Participants included non-demented individuals with PD ( $\mathrm{n}=81,68 \pm 4.9785$ years, $27 \%$ female $)$ and control participants ( $\mathrm{NC} ; \mathrm{n}=50,67 \pm 6.2671$ years, $26 \%$ female). All participants completed the category fluency (animal; 60 second output) with consecutive responses scored using the association index (AI; Carew et al., 1997). T1-weighted MRI data were used to assess hypotheses that left temporal lobe and left frontal lobe thickness would associate with AI versus raw output, respectively.

Results: PD and control participants were matched in age and education. PD showed thinner temporal cortex relative to peers $(p<0.01)$. PD left temporal thickness positively associated with AI when controlling for age (partial $r=0.24, p<0.01$; no association for non-PD peers; $p=0.75$ ). There were no significant associations between frontal or temporal thickness for raw animal output for either group.

Conclusions: These findings suggest that reduced temporal thickness in PD associates with a metric of semantic integrity as measured by the association index from animal fluency. The findings will be discussed as a potential indicator of cognitive progression in PD.

Correspondence: Margaret E. Wiggins, Clinical and Health Psychology, University of Florida, $4455 \mathrm{SW}$ 34th Street, Apartment QQ226, Gainesville, FL 32608, United States.E-mail: ellewiggins@ufl.edu

\section{K.A. WYMAN-CHICK, C.A. MANNING \& S.A. SPERLING. Neuropsychological Test Performance in Parkinsonism Without Dopaminergic Deficiency on [123I]-FP-CIT SPECT Imaging.}

Objective: Scans Without Evidence of Dopaminergic Deficiency (SWEDD) is a term that refers to normal dopamine uptake on [123I]-FP-CIT SPECT imaging in individuals who are clinically diagnosed with Parkinson's disease (PD). It has been argued that SWEDD represents a benign form of parkinsonism from a motor perspective. Little is known about the cognitive functioning of individuals in this group. The purpose of this study was to compare neuropsychological test performance between individuals with SWEDD, individuals with PD who demonstrated dopamine deficiency, and Healthy Controls (HC). Participants and Methods: Data were obtained from the Parkinson's Progression Marker Initiative. The sample included 59 participants with SWEDD, 411 with PD, and 114 HC. Tests included Judgment of Line Orientation, Letter-Number Sequencing, Symbol Digit Modalities, Hopkins Verbal Learning Test-Revised, and Letter and Category Fluency. MANOVA was used to compare standardized scores between the groups.

Results: Box's Test of Equality of Covariance Matrices was significant $(p<.001)$, therefore Pillai's Trace was used. There was a statistically significant difference in performances between the three groups, $F(14$, $1154)=5.04 ; p<.001 ;$ partial $\eta^{2}=.058$. To control for Type I error across univariate tests, alpha was set at .007 . There were significant univariate main effects for Category Fluency, $F(2,582)=6.91: p=.001$; partial $\eta^{2}=.023$, and Symbol Digit Modalities $F(2,582)=24.09$ : $p<.001$; partial $\eta^{2}=.076$. Pairwise comparisons revealed significant differences in Category Fluency between SWEDD (M=0.22, $S D=1.08)$ and HC $(\mathrm{M}=0.86$, $S D=1.15)$ and in SDMT performance between SWEDD $(M=45.09$, $S D=11.54)$ and HC $(\mathrm{M}=51.75, S D=9.79)$. No significant differences between SWEDD and PD were found.

Conclusions: Individuals with SWEDD demonstrate significantly worse non-motor cognitive symptoms than HC. The neuropsychological test performances were similar between the SWEDD group and PD 
group, which may reflect a common pathology that is not mediated by dopamine.

Correspondence: Kathryn A. Wyman-Chick, PsyD, Department of Neurology, University of Virginia, PO BOX 800394, Charlottesville, VA 22908-0394, United States. E-mail:kwyman@pacificu.edu

B. YAFFE, R.J. FEE \& P.J. MATTIS. The Relationship of Motor Dysfunction and Memory Recognition Deficit in Parkinson's Disease.

Objective: Parkinson's disease (PD) is associated with motor and memory retrieval deficits that are related to disease specific factors. Preliminary data demonstrated impairment beyond retrieval based on a lack of improvement using recognition in PD and its association with general cognitive decline in this sample. We previously hypothesized that recognition deficits in PD may indicate the comorbidity of Alzheimer's disease (AD), considering that AD pathology was found in a number of PD patients at the time of autopsy. Accordingly, given that motor impairment is the most prominent feature of PD, we examined whether it is related to recognition deficits, which would suggest that the recognition deficit is related to PD specific pathology. We hypothesized a significant relationship between motor dysfunction and general cognitive decline in patients with PD, without a relationship between recognition and motor dysfunction.

Participants and Methods: 65 participants (51M and 14F) with PD ranging in age from 42 to $81(M=61.94 \pm 9.40)$ with 12 to 20 years $(M=15.03 \pm 2.58)$ of education were included. Unified Parkinson's Disease Rating Scale (UPDRS) motor scores, Dementia Rating Scale-2 (DRS) total scores and the Brief Visuospatial Memory TestRevised (BVMT) Recognition Discrimination Index were analyzed using Spearman correlation. BVMT discriminability scores were categorized into preserved $(\geq 5)$ and impaired $(<5)$ groups, and performance was compared on UPDRS using Kruskal Wallis.

Results: There was a significant negative correlation between DRS and UPDRS scores $\left(r_{s}(63)=-.40, p<.01\right)$. A significant relationship between UPDRS and BVMT was not found.

Conclusions: Greater motor dysfunction is associated with poorer cognitive functioning in PD. However, the relationship between cognitive decline and recognition deficits is not due to level of motor impairment in PD. Therefore, motor dysfunction is not related to recognition deficits, indicating that the deficit is not PD disease specific.

Correspondence: Beril Yaffe, M.A., Psychology, The Graduate Center, CUNY, $247 N$ 7th St Apt 506, Brooklyn, NY 11211, United States. E-mail: beryaf@gmail.com

K.L. ZANE, L. SCHWARZ, P. RUPPERT, K. MULHAUSER \& J. CHIBNALL. Cognitive Slowing and Motor Slowing in Parkinson's Disease.

Objective: Parkinson's disease (PD) is traditionally categorized as a movement disorder; however, it is clear it can also lead to significant cognitive dysfunction (Cosgrove, Alty, \& Jamieson, 2015). Previous research has estimated that approximately $19 \%$ of $\mathrm{PD}$ patients have some form of cognitive impairment (Lieberman, 1998). Still, it remains unclear whether there is a decline in cognitive processing speed. The present study examined the associations of cognitive and motor slowing in PD patients. In particular, we exmained much variance in performance on measures of reaction time is attributable to motor slowing as opposed to cognitive slowing.

Participants and Methods: Archival data from an academic medical center was used to examine mental processing speed and motor abilities in adults with PD ( $\mathrm{N}=102)$. Sample demographics were as follows: mean age $=65(40.2 \%$ female and $59.8 \%$ male $)$; mean education $=13$; and estimated premorbid intelligence mean $=101.68$. Pearson correlations and multiple linear regressions were performed for analyses.

Results: Significant relationships were found between measures of cognitive and motor processing. Stepwise linear regressions showed that performance on motor tasks (i.e., Grip Strength, Grooved Pegboard) predicted performance on non-motor measures of processing speed (i.e., Stroop Color Word Test). In addition, grip strength was also predictive of performance on a verbal fluency task and a simple substitution task (i.e., Symbol Digit Modalities Test).

Conclusions: The current findings suggest that motor abilities accounts for a significant amount of variance on non-motor measures of mental processing speed. Thus, in spite of the dissociation of motor and cognitive domains, cognitive slowing may actually be meaningfully related to motor decline. Further research of cognitive and motor slowing in PD patients is recommended.

Correspondence: Katherine L. Zane, Ph.D., Psychology, Saint Louis University, 3124 Olive Street, Saint Louis, MO 63103, United States. E-mail: zanekl@slu.edu

\section{PM Coffee Break} 2:30-3:00 p.m.

\section{Plenary F. Contributions to Understanding the Dynamic Course of Alcoholism: An INS Legacy}

\section{Presenter: Edith V. Sullivan}

$$
\text { 3:00-4:00 p.m. }
$$

\section{E.V. SULLIVAN. Contributions to Understanding the Dynamic Course of Alcoholism: An INS Legacy.}

Alcohol Use Disorder (AUD) has been a major cause of family, social, and personal strife for centuries, with current prevalence estimates of $14 \%$ for 12 -month and $29 \%$ lifetime AUD. Neuropsychological testing of selective cognitive, sensory, and motor functions complemented with in vivo brain imaging has enabled tracking the consequences of AUD, which follows a dynamic course of development, maintenance, and recovery or relapse. Controlled studies of alcoholism have revealed evidence for disruption of selective functions involving executive, visuospatial, mnemonic, emotional, and balance abilities and brain systems supporting these functions, notably, frontocerebellar, frontostriatal, and frontolimbic circuitry. On a hopeful front, longitudinal study provides convincing evidence for improvement in brain structure and function following sustained sobriety. These discoveries have a strong legacy in INS, starting from its early days when assumptions regarding which brain regions were disrupted relied solely on patterns of functional sparing and impairment deduced from testing. Today's work using refinements in assessment and multi-modal neuroimaging builds on that legacy, moving the field toward examination of compensatory processes to overcome impaired functions.

Following this lecture, the learner should be able to: 1) Recognize that alcohol dependence disrupts selective brain structures and functions; 2) Appreciate that alcoholism-related functional brain changes are a form of neuroadaptation that may underlie dysfunction, making alcoholism a self-perpetuating disorder; 3 ) Learn that sustained sobriety can result in improvement in brain structure and function, indicative of damage reversal or compensatory mechanisms that can be identified with formal neuropsychological testing and longitudinal, quantitative structural and functional brain imaging.

Correspondence: Edith V. Sullivan, Ph.D., Psychiatry \& Behavioral Sciences, Stanford University School of Medicine, 401 Quarry Road, Stanford,CA 94305-5723, United States.E-mail: edie@stanford.edu 


\section{Invited Symposium 5. The Next Generation: A Look at Cohort Studies of People at Risk for Alzheimer's Disease}

\author{
Chair: Sterling C. Johnson
}

4:00-5:30 p.m.

S.C. JOHNSON, T. BARRY-TANNER, J. HASSENSTAB, A. SOLDAN, J.J. MANLY \& A.L. JEFFERSON. The Next Generation: A Look at Cohort Studies of People at Risk for Alzheimer's Disease.

Symposium Summary: The pathological processes underlying Alzheimer's disease (AD) begin years or perhaps decades prior to onset of overt dementia. New findings are emerging from several longitudinal AD-risk enriched cohorts that indicate brain and cognitive changes exist during the preclinical and prodromal phases of AD. The symposium will begin from the 'at-risk' participant's perspective using clips from an upcoming independent documentary film offering an intimate look at AD research entitled "Will I be Next?". The film follows three middleaged women who volunteer as research participants in the longitudinal Wisconsin Registry for Alzheimer's Prevention (WRAP) study. Each of these women has a family history of $\mathrm{AD}$ and is at high risk for developing $\mathrm{AD}$ in the future ("the next generation").

The symposium will transition to a discussion of several select riskenriched longitudinal cohort studies, including a summary of each study's design, seminal findings, and future directions. Cohorts include the WRAP study (University of Wisconsin, PI: Sterling Johnson, PhD), the Adult Children Study (Washington University, PI: John Morris MD; presented by Jason Hassenstab, PhD), the BIOCARD Study (Johns Hopkins University, PI: Marilyn Albert; presented by Anja Soldan, PhD), the Offspring Study (Columbia University, PI: Jennifer Manly. $\mathrm{PhD}$ ), and the Vanderbilt Memory and Aging Project (Vanderbilt University Medical Center, PI: Angela Jefferson, PhD). The presentation of seminal findings from these cohorts will focus on neuropsychological and biomarker changes in people at risk for $\mathrm{AD}$, characteristics that may pose resilience against cognitive decline, and implications for participant selection in future prevention trials.

Correspondence: Sterling C. Johnson, PhD, Wisconsin ADRC, University of Wisconsin-Madison, 600 Highland Avenue, Madison, WI 53792, United States.E-mail: scj@medicine.wisc.edu

\section{T. BARRY-TANNER. Will I Be Next?}

Will I Be Next? is a documentary about the quest for a cure to Alzheimer's disease as told by human research subjects in the Wisconsin Registry for Alzheimer's Prevention (WRAP) longitudinal observational cohort study. For the past 15 years, 1550 middle-aged subjects have offered their brains and bodies for repeat investigation to the WRAP study. At the heart of the study are the test group participants - middleaged adults with a deceased or living parent with Alzheimer's disease. Parental history makes the test group 2 to 3 times more likely to get the disease than those without a history of it in their families. The three main characters in the film are test group participants who are followed as they grapple with the disease and its impact on their families, and what they are doing to lower their own risk. A 23 minute clip of the documentary will be shown by one of the producers of the film. For more on the film, see http://willibnext.com/.

Correspondence: Therese Barry-Tanner, c/o INS, 2319 S Foothill Dr Ste 260, Salt Lake City, UT 84109, United States.E-mail: tbarry-tanner@ hotmail.com

\section{S.C. JOHNSON. The Wisconsin Registry for Alzheimer's Prevention (WRAP).}

The Wisconsin Registry for Alzheimer's Prevention (WRAP) is a longitudinal observational cohort study enriched with persons who have a biological parent with Alzheimer's Disease (AD). Parental history was used as a risk factor since persons with parental history are at twice the risk for sporadic AD compared to the general population. Since late 2001, WRAP enrolled 1548 people at a mean baseline age of 54 . The second visit was four years after baseline and subsequent visits are every two years. The average length of follow-up is 10 years and $83 \%$ of participants remain active in the cohort. Serial cognitive measurement, self-report lifestyle and medical data, together with data collection from several ancillary linked studies involving amyloid and tau imaging, CSF and other direct measures have yielded many potentially important findings which will be reviewed. Methods and results for capturing early reliable indicators of cognitive decline (prior to classic mild cognitive impairment) will be presented including use of internally derived robust norms and graphical modeling techniques. The cohort is now 65 years of age on average and approximately $13-18 \%$ of the cohort are experiencing meaningful decline (depending on how its defined) beyond normal aging. While decline is associated with biomarkers of $\mathrm{AD}$, co-occurring pathologies such as vascular disease also account for a proportion of the variance in cognitive decline and evidence for the interplay between pathologies will be discussed. The preclinical phase of $\mathrm{AD}$ in late middle age is a poorly understood time frame that WRAP is addressing. Of paramount importance is establishing the endophenotypes of $\mathrm{AD}$ and neurovascular disease to which cognitive outcomes can be linked.

Correspondence: Sterling C. Johnson, PhD, Wisconsin ADRC, University of Wisconsin-Madison, 600 Highland Avenue, Madison, WI 53792, United States.E-mail:scj@medicine.wisc.edu

\section{J. HASSENSTAB, A. FAGAN \& J. MORRIS. Correlating Rates of Change Between Cognition and Biomarkers in Middle Aged Adults at Risk for Alzheimer's Disease: The Adult Children Study.}

Among older adults at risk for Alzheimer's disease (AD), crosssectional and longitudinal associations across multiple modalities of $\mathrm{AD}$ biomarkers and cognition have been well characterized, particularly among those who have developed clinically significant symptoms. What is less understood is how and to what extent longitudinal changes in AD biomarkers and cognition are correlated in asymptomatic middle-aged individuals. This was the impetus for the Adult Children Study (ACS), which began in 2005 with the intent to develop and validate biological markers, imaging features, and cognitive profiles of preclinical Alzheimer's disease (AD). Participants are enrolled in two groups: 1) Adult children in middle age who have at least 1 parent who developed AD and 2) Adult children in middle age with parents that never developed AD. To date, the ACS has enrolled nearly 275 individuals aged 43-77 with a mean follow-up interval of approximately 7 years. All participants undergo an annual or triennial clinical assessment, including a comprehensive neuropsychological battery, cerebrospinal fluid (CSF) collection, amyloid PET imaging with Pittsburgh compound B (PETPIB), and structural MRI. In this presentation, we will present methods and some initial results from examinations of the correlations between longitudinal rates of change in cognition, MRI volumetrics, CSF concentrations of Alzheimer-related proteins, and molecular imaging of cerebral fibrillar amyloid with PET. We used multivariate mixed models for repeated measures to assess the correlations between rates of change across the biomarker modalities and cognition. Our initial results indicate that a decrease in CSF $\beta$ - amyloid $_{1-42}\left(A \beta_{1-42}\right)$ is associated with a faster increase in PET-PIB mean cortical standardized uptake value ration (MCSUVR), but not with other biomarkers. The rate of change in CSF tau and CSF phosphorylated tau ${ }_{181}$ is correlated with the rate of change in PET-PIB, hippocampal volume, and 3 cognitive composites (episodic memory, attentional control, and global cognition). The rate of change in hippocampal volume is correlated with rates of change across the 3 cognitive composites. Similar analyses were completed using baseline data, however, only 3 significant correlations were observed thus far: CSF $A \beta_{1-42}$ and PET-PIB, CSF Tau and PET-PIB, and CSF $A \beta_{1-42}$ and global cognition. We will discuss interpretation of these findings and their relevance for disease progression models and their implications for secondary prevention trials that rely on AD biomarkers and cognition to track disease modification. 
Correspondence: Jason Hassenstab, PhD, Neurology, Washington University in St. Louis, 4488 Forest Park Ave, Suite 130, St. Louis, MO 63130,United States. E-mail: hassenstabj@abraxas.wustl.edu

A. SOLDAN, C. PETTIGREW, Q. CAI, M. WANG, A. MOGHEKAR, A. FAgAN, C. FOWLER, C. CARLSSON, C. MASTERS, S.C. JOHNSON, S. ASTHANA, J. MORRIS, A. GROSS \& M. ALBERT. Hypothetical Preclinical Alzheimer Disease Groups and Longitudinal Cognitive Change.

The goal of this study was to use two different datasets to determine which group of cognitively normal individuals shows the greatest cognitive decline over time based on their cerebrospinal fluid (CSF) biomarker profile. In the first analysis, 222 cognitively normal participants from the BIOCARD study (mean baseline age 57 years, mean follow-up 11 years) were classified into one of the following 4 hypothetical preclinical AD groups using baseline CSF levels of A $\beta 42$ and tau or $A \beta 42$ and phosphorylated tau (p-tau): stage 0 (high $A \beta 42$ and low tau), stage 1 (low A $\beta 42$ and low tau), stage 2 (low A $\beta 42$ and high tau), and suspected non-AD pathology (SNAP) (high A $\beta 42$ and high tau). Cognitive performance was measured using a composite score composed of tests previously shown to predict progression from normal cognition to Mild Cognitive Impairment. The results showed that individuals in stage 2 had lower baseline cognitive scores and a greater decline in the cognitive composite score relative to the other 3 groups (all $p<.001$ for the rate of decline). Individuals in stage 0 , stage 1 , and SNAP did not differ from one another in cognitive performance at baseline or over time and showed practice-related improvement in performance. The rate of change in the cognitive composite score did not differ by $\mathrm{APOE} \varepsilon 4$ genotype. Comparable results were obtained in an analysis that combined BIOCARD data with four additional independent cohorts that also enrolled and followed cognitively normal adults over time: Adult Children Study (ACS), Wisconsin Registry for Alzheimer's Prevention (WRAP) and Wisconsin IMPACT cohorts, and Australian Imaging, Biomarker, and Lifestyle Study (AIBL) (total N=881 with baseline CSF, mean baseline age $=59$ years, mean follow-up 7 years). Taken together, these results suggest that abnormal levels of both CSF Ab42 and tau in midlife are necessary for observing a marked decline in cognition among cognitively normal individuals.

Correspondence: Anja Soldan, Cognitive Neuroscience Division, Johns Hopkins School of Medicine, 1620 McElderry Street, Baltimore, MD 21205, United States. E-mail: asoldan1@jhmi.edu

\section{J.J. MANLY \& A.M. BRICKMAN. Offspring Study of Mechanisms for Racial Disparities in Alzheimer's Disease.}

Alzheimer's Disease (AD) is a clinically heterogeneous disorder, resulting from multiple etiologic pathways. The rationale for the Offspring Study of Mechanisms for Racial Disparities in $\mathrm{AD}$ is that causes of disparities in $\mathrm{AD}$, and possible racial/ethnic differences in mechanistic pathways to $\mathrm{AD}$, can be identified through evaluation of diverse middle-aged cohorts with and without an objectively-determined family history of AD. Mechanisms of disparities are difficult to study if samples are limited to age 65 and older because several factors attenuate the relationships of these risk factors with $\mathrm{AD}$ when measured in old age. Prior findings are that racial disparities in cognitive function narrow with age due to selective survival. In addition, there is increasing evidence for the importance of cardiovascular and cerebrovascular risk factors for AD, but these relationships appear to be strongest when cardiovascular function is measured in middle age. The mid-life preclinical phase is particularly relevant for studying racial disparities in $\mathrm{AD}$ because cardiovascular risk factors are more prevalent and have an earlier age of onset among minorities, in whom the potential influence of cardiovascular factors on the development of AD may take place at an earlier age. The Offspring Study leverages a well-characterized, diverse, and longitudinally followed parent cohort to test whether the primary mode of transmission of parental AD risk to offspring cognition differs across race/ethnicity. We will test the hypothesis that social and vascular factors play a bigger role in the mechanistic pathway to AD among minorities, while genetic pathways that affect amyloid deposition in the brain play a larger role among Whites. As a result, we expect that social and vascular factors are more likely to influence the relationship between parental risk and offspring cognitive function among minorities, and will account for the racial/ethnic disparities in $\mathrm{AD}$ and cognitive aging.

Correspondence: Jennifer J. Manly, PhD, Neurology, Columbia University, 630 West 168th St., P\&S Box 16, New York, NY 10032, United States. E-mail: jjm71@cumc.columbia.edu

\section{A.L. JEFFERSON. The Vanderbilt Memory \& Aging Project: Study Design, Findings, and Future Directions.}

The Vanderbilt Memory \& Aging Project was established as an observational longitudinal study investigating vascular health and brain aging. Specifically, the study aims to provide insights into complex associations among systemic vascular health, microvascular and microstructural changes in the brain, and cognitive decline and dementia among older adults. From September 2012 to November 2014, 335 participants 60-92 years of age and free of clinical dementia and stroke were enrolled. At baseline, these participants represented the cognitive spectrum ranging from normal cognition to prodromal dementia (i.e., mild cognitive impairment; MCI). Participants are seen every 18-months for 3 years, and at each time point participants undergo physical and frailty examination, fasting blood draw, clinical and medical history interview, neuropsychological assessment, echocardiogram, 1.5T cardiac MRI, and multimodal 3T brain MRI emphasizing cerebrovascular structure and function. Optional procedures include 24-hour ambulatory blood pressure monitoring, actigraphy, and lumbar puncture for cerebrospinal fluid (CSF) collection. Diverse sources of baseline and longitudinal data from this cohort are providing our investigative team and collaborators with rich opportunities to investigate pathways linking vascular and cerebrovascular health, clinical and pathological AD, and neurodegeneration contributing to novel strategies to delay or prevent cognitive decline. This presentation will review cohort characteristics and highlight important outcomes to date that enhance knowledge about the association between systemic vascular health and brain health in aging. Correspondence: Angela L. Jefferson, PhD, Department of Neurology, Vanderbilt University Medical Center, 120717 th Avenue Sout, Suite 204, Vanderbilt Memory \& Alzheimer's Center, Nashville, TN 37212, United States. E-mail: angela.jefferson@Vanderbilt.edu

\section{Symposium 9. Depression in Clinical Conditions: Impact on Behavior, Neural Mechanisms and Quality of Life}

\section{Chair: Ekaterina Dobryakova}

$$
\text { 4:00-5:30 p.m. }
$$

E. DOBRYAKOVA, R. KAMAT, K. BIJANKI, Y. GOVEROVER \& L. STROBER. Depression in Clinical Conditions: Assessment, Impact on Behavior, Neural Mechanisms and Quality of Life.

Symposium Summary: Depression, a psychiatric disorder, has been shown to negatively impact an individual's quality of life as well as cognition, thus leading to alterations in neural mechanisms. The occurrence of depression in clinical populations is a lot higher than in the general public. Compared to rates of depression in the general population $(6.7 \%)$, up to $77 \%$ of individuals with traumatic brain injury (TBI) are being diagnosed with clinical depression, and up to $50 \%$ of individuals with multiple sclerosis (MS) are reported to have depression. Given this, it is surprising that the impact of depression within clinical conditions is largely neglected, with very few investigations examining the impact of depression on behavioral and neural measures in disease. The current symposium constitutes a collection of multidisciplinary work and spans several clinical populations. The overall goal of the 
symposium is to explore the impact of depression on behavior, neural mechanisms and quality of life, as well as depression assessment measures. The first talk will examine neural mechanisms associated with depression in TBI. The second talk will characterize functional correlates and the course of depression in individuals with Human Immunodeficiency Virus. The subsequent talk will examine mechanisms of positive emotional experience in a single epilepsy patient undergoing a deep brain stimulation. The next talk of the symposium will examine the relationship between age, quality of life and depression in individuals with MS. Finally, the symposium will end with a talk that explores clinical characterizes and etiology of depression in five clinical populations - Alzheimer's, Parkinson's, stroke, epilepsy, and MS. Thus, the current symposium has multidisciplinary representation. It is anticipated that the current symposium will appeal to an interdisciplinary audience and will draw attention to the study of depressive symptomology in clinical conditions.

Correspondence: Ekaterina Dobryakova, Ph.D, Kessler Foundation, 300 Executive dr, West Orange, NJ NJ, United States.E-mail: edobryakova@ kesslerfoundation.org

\section{E. DOBRYAKOVA. Depressive Symptomology Modulates Cortico- Strtiatal Activation During Feedback Presentation in Individuals with Traumatic Brain Injury.}

Objective: Compared to rates of depression reported in the general population $(6.7 \%)$, the occurrence of depression after traumatic brain injury (TBI) is substantially higher, with studies reporting between $50 \%$ to $77 \%$ of individuals with TBI being diagnosed with clinical depression. Depression has been shown to negatively impact cognition, as well as overall quality of life of individuals with TBI. However, the influence of depression on neural mechanisms in individuals with TBI remains unexplored. Here we explored neural mechanisms of individuals with TBI who reported high and low depression (as assessed with the Chicago Multiscale Depression Inventory). Specifically, we examined how the level of depression modulates brain activity during a task that presents participants with positive and negative feedback, since the neural mechanism associated with feedback processing has been shown to be impaired in individuals with depression without TBI. Based on prior findings in individuals with depression, we expected hypoactivation in the ventromedial prefrontal cortex (VMPFC) and the striatum in individuals with TBI scoring high on the CMDI.

Methods \& Results: Participants underwent MRI of the head while performing a gambling task where they could either win $\$ 1$ for correctly guessing a value on the card (positive feedback) or lose $\$ 0.50$ for incorrectly guess a value on the card (negative feedback). CMDI scores ranged from 38 to 96 and when divided on two groups, the difference between high and low CMDI scores group was significant. Contrary to our expectations, individuals with TBI who scored higher on the CMDI exhibited increased activation in the VMPFC and striatum. Previous investigations showed similar findings in association with anxiety.

Conclusion: Current findings describe how the neural mechanisms associated with feedback presentation are modulated by depressive symptomology in individuals with TBI and highlight the importance of examining depressive symptomology.

Correspondence: Ekaterina Dobryakova, Ph.D, Kessler Foundation, 300 Executive dr, West Orange, NJ NJ, United States. E-mail: edobryakova@ kesslerfoundation.org

\section{R. KAMAT. Depression and its Correlates in the Context of HIV Infection.}

Objective: Depression is the most prevalent HIV-associated psychiatric disorder with a lifetime prevalence of $40-60 \%$ in the HIV-infected population. It commonly co-occurs with apathy, which is a distinct and clinically significant sequela of HIV infection. Across many patient populations, these two syndromes increase risk of disability, but their comorbidity has received limited attention in HIV+ cohorts. We aimed to characterize the course and functional correlates of HIV-associated depression in the context of apathy.

Methods and Results: In a cohort of HIV+ persons ( $\mathrm{n}=225$ ), we examined the neuromedical correlates and longitudinal progression of major depressive disorder in the context of pre-existing apathy. We found that lower nadir and current CD4 counts were associated with the development of a new or recurrent episode of major depression. Additionally, in HIV + persons without pre-existing apathy at baseline, new onset of major depression was associated with a significant increase in apathy at the second visit. In a separate study, we investigated the role of comorbid depression and apathy in health-related quality of life (HRQoL) in HIV+ individuals $(\mathrm{n}=80)$. Independent of apathy, depression was associated with mental (but not physical) health related HRQoL. With regard to the subcomponents of HRQoL, depression was associated with physical role limitations, energy/fatigue, social functioning, and emotional well-being.

Conclusions: Our findings provide a greater understanding of the etiology as well as psychiatric and functional consequences of depression in the context of HIV-associated neurobehavioral disturbance. These data may inform HIV-treatment specific interventions to mitigate the risk of developing depression and have implications for identifying patients at particular risk for poor health outcomes. Thus, there is a clear need to consider mood disorders in the assessment of functional declines and diagnosis of HIV-associated neurocognitive disorders.

Correspondence: Rujvi Kamat, Ph.D., UCSD, 220 Dickinson St, San Diego, CA 92103, United States. E-mail: rkamat@ucsd.edu

K.R. BIJANKI, N.P. PEDERSEN, D.L. DRANE, C. INMAN \& J.T. WILLIE. Stimulation of the Dorsal Cingulum Produces Euphoria and Positive Emotional Bias in an Epilepsy Patient Undergoing Invasive Presurgical Evaluation.

Objective: We sought to elucidate the mechanisms of positive emotional experience by leveraging a serendipitous finding of evoked laughter and euphoria in an epilepsy patient undergoing a deep brain stimulation research paradigm.

Participants and Methods: The patient was a 25-year-old woman with grid, strip and stereotactic depth electrode implantation for pre-surgical evaluation for medically-intractable epilepsy. Electrical stimulation was delivered to the dorsal anterior cingulate via a current-controlled cerebral stimulator focally at three discrete locations.

Results: Stimulation at one of three sites (dorsal cingulum) produced involuntary laughter, happiness, and relaxation that persisted over the course of twenty minutes of stimulation. Stimulation-induced euphoria was accompanied by a statistically significant positive shift in emotional bias during the evaluation of happy, neutral, and sad facial expressions $(\mathrm{t}=-2.334, \mathrm{df}=59, \mathrm{p}=.023)$, as well as reliable transient increases in skin conductance and heart rate. Anatomical and diffusion tensor neuroimaging reconstruction of the stimulated locations revealed patterns of connectivity differentiating between the three stimulated regions. The patient's clinical team (surgeon, neurologist, psychologist, and anesthesiologist) determined that the dorsal cingulum stimulation provided substantive relief to the patient's anxiety during surgery, and it was applied in the operating room during a subsequent awake resection. The stimulation successfully promoted a self-reported feeling of relaxation and happiness as the patient underwent a novel language mapping procedure during resective neurosurgery.

Conclusion: The current findings significantly expand the literature on the role of the anterior cingulate on positive emotional function. The novel application of this stimulation to assist during the patient's subsequent awake resection suggests it may be a novel target for anxiety management, including for awake neurosurgery.

Correspondence: Kelly R. Bijanki, Ph.D., $4127 b$ Woodruff Memorial Research Building, 101 Woodruff Circle, Emory University, Atlanta, GA 30322, United States.E-mail: kbijank@emory.edu 


\section{Y. GOVEROVER. Is Being Younger Better? Age, Depression And QOL in MS.}

Objective: Multiple sclerosis (MS) is a chronic autoimmune disease of the central nervous system. MS has a heterogeneous presentation, affecting motor, cognitive, visual, and affective domains. The frequency of major depression in MS has been calculated as up to $50 \%$ and has been identified as a significant predictor of quality of life (QOL). The present study examined the relationship of age with affective symptomology and QOL in individuals with MS.

Methods: Participants included 30 individuals with MS. Outcome measures included the Chicago Multiscale Depression Inventory (CMDI), and the Multiple Sclerosis Quality of Life Instrument (MSQOL-54).

Results: Increased age was associated with decreased affective symptomology and increased QOL in adults with MS. The effect of age on both physical and mental QOL was mediated by symptoms of depression. Conclusions: The relationship of age with subjective well-being reflects the aging paradox observed in the general population, such that younger adults with MS report increased affective svmptomology and decreased QOL. Although age is not amenable to clinical intervention, it affects QOL via affective symptoms, suggesting that addressing symptoms of depression can increase physical and mental QOL even in younger adults. Younger adults with MS may benefit from education on coping strategies to prevent and/or decrease symptoms of depression as a means of increasing QOL

Correspondence: Yael Goverover, New York Univeristy, 82 Washington Sq East, 6th floor, NYC, NY 10003, United States.E-mail:yg243@ nyu.edu

\section{STROBER. Doctor, Am I Depressed? How to Appreciate the Intricacies of Depression in Neurological Populations and a Guide to Aide in Its Assessment.}

Objective: Depression is extremely common in neurological illnesses. with staggering rates as high as $87 \%$. Whether reactionary in nature or biological, controversy exists as to how best to understand and assess what is "typical" or indicative of depression in neurological populations. Depression may at times be minimized, particularly in elderly individuals in which less is known as to what is "normal" aging. On the flip side, depression can be overdiagnosed given the overlap of neurovegetative symptoms (e.g., fatigue, sleep difficulties, appetite change), which are common to neurological illness but also cardinal features of depression. Moreover, use of common self-report measures are hindered by the fact that they were designed for their young. healthy counterparts and do not take into consideration the above mentioned issues. To date, there have been many efforts to remedy this situation when assessing depression in medical populations (e.g., raising cutoffs on measures, removing neurovegetative items, development of disease specific measures). Despite this, practitioners may frequently feel that they are lacking in their ability to accurately assess depression in their patients.

Methods \& Results: The present talk will provide a better understanding of the risk factors, unique clinical characteristics, and etiology of depression in five clinical populations - Alzheimer's, Parkinson's, stroke, epilepsy, and multiple sclerosis. Additionally, we will explore the use of self-report measures in these populations and provide guidelines regarding the specific measures and cutoffs that are most appropriate. Conclusions: It is hoped that a greater appreciation for the intricacies in assessing depression in these populations will be obtained. Correspondence: Lauren Strober, Ph.D., Neuropsychology \& Neuroscience, Kessler Foundation, 300 Executive Drive, Suite 70, West Orange, NJ 07052, United States. E-mail: lstrober@kesslerfoundation. org

\section{Symposium 10. Comorbidities Associated with Neurocognitive Performance in Sports Concussion and MS}

\author{
Chair: Peter Arnett \\ 4:00-5:30 p.m.
}

\section{P. ARNETT. Comorbidities Associated with Neurocognitive Performance in Sports Concussion and MS.}

Symposium Summary: Comorbidities are common in neurological conditions, and often include sleep disturbances, psychiatric disorders, headache, and fatigue. Given the salience of direct influences on cognitive difficulties among neurological populations, comorbidities that impact cognitive functioning, like those mentioned above, have historically been overlooked, controlled for, or used as exclusionary criteria. In recent years, however, there has been an increased appreciation for the study of these variables as something often intrinsic to neurological conditions. With these considerations in mind, this symposium will examine comorbidities commonly associated with sports-related concussion and multiple sclerosis (MS), and their association with cognitive dysfunction. Peter Arnett will open the symposium by providing an overview and brief history of these issues. Then sports-related concussion will be discussed, where Jessica Meyer will show that depression is related to cognitive problems post-concussion both cross-sectionally and in relation to decline from baseline. Erin Guty will demonstrate that there is a strong relationship between cognitive problems and a most persistent and disabling symptom post-concussion, headache. Concerning MS, Jessica Zamzow will show that sleep disorder symptoms, but not sleep duration and efficiency, are associated with poorer cognitive function. Margaret Cadden will review MS data showing that chronic, but not acute, pain is associated with cognitive dysfunction. Lastly, Cristina Roman will illustrate common structural neuroimaging links underlying both depression and cognitive problems in MS. Subsequent to these talks, Maria Schultheis will serve as a discussant regarding the clinical and theoretical implications of these findings.

Correspondence: Peter Arnett, Ph.D., Psychology, Penn State University, 352 Moore Building, University Park, PA 16802, United States. E-mail: paa6@psu.edu

E. GUTY \& P. ARNETT. The Relationship between Headache and Cognitive Impairment following Sports-Related Concussion.

Objective: Headache is one of the most commonly reported and longestlasting symptoms following concussion. Problems with cognitive functioning are also common following concussion. The goal of this study is to explore how headache symptoms may relate to cognitive impairment following sports-related concussion.

Participants and Methods: 136 participants were included from a university-based sports concussion program. Headache was assessed using the headache symptom item from the PCSS. Individuals with a score of 1 or greater were placed into the "headache" group while individuals with scores of zero were in the "no headache" group. Group means were then compared on various cognitive indices. Additionally, impairment groups were compared, with impaired groups defined as having 2 or more test indices below a standard score of 80 .

Results: Compared with the no headache group, individuals in the headache group had poorer performance on 3 indices of memory (HVLT-R Delayed Recall, t $(120)=2.00, p=.048$; HVLT-R Total Recall, $\mathrm{t}(120)=2.40, \mathrm{p}=.018$; ImPACT Visual Memory Composite, $\mathrm{t}(113)=4.27, \mathrm{p}<.001)$, and 2 indices of attention/processing speed (ImPACT Visual Motor Speed Composite, $\mathrm{t}(115)=3.58, \mathrm{p}=.001$ and the SDMT, $\mathrm{t}(119)=2.90, \mathrm{p}=.005)$. When examining impairment across the test battery, $37 \%$ of patients from non-impaired group had headache symptoms compared with $64 \%$ of the impaired group $(p<.005)$. When examining only memory impairment, $39 \%$ of patients from the non-impaired group had headache symptoms compared with $72 \%$ from the impaired group $(\mathrm{p}<.005)$. Finally, when exploring attention/processing 
speed impairment, $39 \%$ of patients from non-impaired group had headache symptoms compared with $81 \%$ from the impaired group $(\mathrm{p}<.005)$. Conclusions: Our results demonstrate a significant relationship between headache and difficulties in cognition following concussion. Future work should explore potential functional outcomes associated with this relationship.

Correspondence: Erin Guty, Pennsylvania State University, 850 Toftrees Ave., Apt. 515, State College, PA 16803, United States. E-mail: erin. guty@gmail.com

N.A. GRIMA, S.M.W. RAJARATNAM, D. MANSFIELD, T. SLETTEN, G. SPITZ \& J.L. PONSFORD. Melatonin Supplementation Improves Sleep Disturbance Following Traumatic Brain Injury: Preliminary Results from a Randomized Controlled Trial.

Objective: Traumatic brain injury (TBI) is a leading cause of disability worldwide. Although $50 \%$ of patients experience sleep disturbance, effective and safe pharmacological treatments are lacking. Our previous research has demonstrated that melatonin production is attenuated and delayed in TBI patients. We performed a clinical trial to determine the effectiveness and safety of melatonin for sleep disturbance in patients with TBI and insomnia.

Participants and Methods: Randomised, double-blind, placebo-controlled, 2-sequence, 2-treatment (melatonin and placebo) crossover study was conducted in 26 outpatients with mild to severe TBI (mean Post Traumatic Amnesia 37.92 days), on average 66 months post injury. Primary endpoints included the Pittsburgh Sleep Quality Index (PSQI) global scores indicative of subjective sleep quality, and sleep onset latency derived from wrist actigraphy.

Results: Melatonin reduced global PSQI scores (melatonin, 7.77 vs placebo, 8.86; difference $-1.09 ; 95 \%$ CI -1.95 to $-0.22 ; p=.02)$, and a strong trend suggested sleep onset latency was reduced with melatonin supplementation (melatonin, 25.71 minutes vs placebo, 34.43 minutes; difference -8.72 minutes; $95 \%$ CI -17.51 to $0.08 ; p=.05$ ). Adverse events were comparable across placebo and melatonin treatments.

Conclusions: The present study suggests melatonin supplementation is effective in improving both subjective and objective sleep quality in patients with TBI and clinically diagnosed chronic insomnia.

Correspondence: Natalie A. Grima, Doctor of Psychology (Clinical Neuropsychology), Cognitive Neurology, Beth Israel Deaconess Medical Centre, 112 Jersey Apt 15, Boston, MA 02215, United States. E-mail: nataliegrima@gmail.com

B.M. ASKEN, J.R. CLUGSTON, D.N. DUBOSE, J.K. BOONE, M.A. MCCREA \& R.M. BAUER. Physiological Effects of Delayed Removal from Activity Following Sport-Related Concussion as Evidenced in Serum Biomarkers.

Objective: Recent evidence suggests delaying concussion symptom reporting and continuing athletic activity immediately post-injury may be associated with protracted clinical symptom resolution. The present analyses investigated the effect of delayed removal from athletic activity after concussion on concentration of blood biomarkers associated with brain injury.

Participants and Methods: We examined serum UCH-L1 ( $\mathrm{N}=29)$, $\mathrm{S} 100 \mathrm{~B}(\mathrm{~N}=32), \mathrm{A} \beta 42(\mathrm{~N}=29)$, and tau $(\mathrm{N}=29)$ drawn at baseline and acutely post-concussion (median $=4.0 \mathrm{hrs}$ from injury) in male and female collegiate athletes (mean \pm SD age at injury $20.3 \pm 1.0$ years). Multivariate analysis of variance (MANOVA) was used to evaluate average post-concussion concentration changes from baseline $(\Delta[$ biomarker]) in athletes who were removed from play immediately after injury and athletes who delayed symptom reporting and thus remained in play for varying intervals after injury. Follow-up hierarchical regression analyses examining the unique predictive effect of immediate-vs. delayed reporting on $\Delta$ [biomarker], controlling for sex, time between injury and blood draw, and sport type.

Results: We observed a significant omnibus effect of reporting group on $\Delta[$ biomarker $](\mathrm{F}[4,22]=3.748, \mathrm{p}=.018)$. Univariate analyses indicated delayed reporting was associated with greater $\Delta \operatorname{tau}(\mathrm{F}[1,26]=6.48$, $\mathrm{p}=.017)$ and greater $\Delta \mathrm{S} 100 \mathrm{~B}(\mathrm{~F}[1,26]=13.68, \mathrm{p}=.001)$. Delayed reporting did not significantly affect $\Delta \mathrm{UCH}-\mathrm{L} 1(p=.110)$ or $\Delta \mathrm{A} \beta 42$ $(\mathrm{p}=.817)$. Regression analyses indicated delayed removal from athletic activity predicted a $0.89 \mathrm{pg} / \mathrm{mL}$ greater $\Delta \operatorname{tau}(\beta=.430, \mathrm{p}=.017)$ and a $27.9 \mathrm{pg} / \mathrm{mL}$ greater $\Delta \mathrm{S} 100 \mathrm{~B}(\beta=.405, \mathrm{p}=.021)$ than athletes immediately removed from activity.

Conclusions: Delayed reporting of concussion symptoms and continued participation in athletic activity may result in compounded physiological effects, as evidenced by greater elevations from baseline in serum tau and $\mathrm{S} 100 \mathrm{~B}$. These findings supplement previous data suggesting associations between delayed removal from activity and prolonged clinical recovery.

Correspondence: Breton M. Asken, MS, ATC, Clinical and Health Psychology, University of Florida, Dept. of Clinical and Health Psychology, P.O. Box 100165, Gainesville, FL 32610, United States. E-mail: baskens@phhp.ufl.edu

J. ZAMZOW, E. CULNAN, A. RAPHAIL, A. GENCARELLI, J.D. KOSS \& M.T. SCHULTHEIS. Sleep and cognitive function in relapsing-remitting multiple sclerosis.

Objective: This study aimed to examine the relationship between sleep and cognitive function in individuals with relapsing-remitting multiple sclerosis (MS).

Participants and Methods: Participants $(\mathrm{n}=51)$ completed sleep diaries and wore an actigraph for two weeks to record sleep duration and efficiency. Participants were administered the Insomnia Severity Index (ISI), STOP-BANG questionnaire for sleep apnea, and Global Sleep Assessment Questionnaire for restless leg syndrome (RLS) and periodic limb movement disorder (PLMD). Participants also completed a battery of cognitive tests measuring psychomotor vigilance, visual attention (UFOV), set-switching (D-KEFS), inhibition (Stroop Color and Word test), working memory (Paced Serial Auditory Attention Task, N-Back), processing speed (Symbol Digit Modalities Tets: oral), verbal fluency (FAS, Animals), and memory (Selective Reminding Test).

Results: One-tailed Pearson's correlations were used to assess the relationship between sleep and cognitive function. Partial correlations were used to control for age, mood, and sleep medication use as needed. The ISI was associated with attention $(r=.27, p=.03)$, vigilance $(r=-.32, p$ $=.02)$, and working memory $(r=-.33, p=.01)$. The STOP-BANG was associated with processing speed $(r=-.31, p=.03)$, working memory $(r=-.57, p<.01)$, vigilance $(r=-.44, p<.01)$, phonemic fluency $(r=$ $-.41, p<.01)$, and inhibition $(r=-.42, p<.01)$. RLS and PLMD were associated with working memory $(r=-.29, p<.01)$ and attention $(r=$ $.52, p<.01)$. All correlations reflected greater symptomology associated with poorer performance. No significant associations emerged between sleep duration or efficiency and cognitive function.

Conclusions: Sleep disorder symptoms were associated with poorer cognitive function, but sleep duration and efficiency were not. Sleep disorders are highly prevalent in MS, but are treatable, and may pose as a modifiable risk factor for cognitive impairment in MS.

Correspondence: Jessica Zamzow, Ph.D., UCLA, 4557 1/4 Lexington Ave., Los Angeles, CA 90029, United States.E-mail:JZamzow@mednet. UCLA.edu

C.A. F. ROMAN \& P. ARNETT. Structural Neural Correlates of Cognitive Functioning and Depression in Multiple Sclerosis: Examining Similarities Across Primary and Secondary Factors.

Introduction: Cognitive impairment is a frequent and often devastating consequence of MS that is driven by various sequelae primary to the disease (i.e., neuropathology). Depression, a secondary factor in MS, can also influence cognitive functioning. While both cognitive impairment and depression have been shown to have associations with neuropathology, it is still unclear whether they share common neural underpinnings. The purpose of the current study is to investigate the 
possible neural correlates that underlie both depression and cognitive impairment in MS

Method: Forty-nine individuals with MS were administered a neurocognitive battery, evaluated for depression, and scanned during a DTI protocol. Standard scores for all cognitive tests were calculated using a sample-based control group. All DTI data processing and analyses were carried out using FMRIB Software Library's (FSL) DTI/TBSS pipeline. Using cognitive performance and depression as predictors, voxel-wise regression analyses were run on $\mathrm{FA}, \mathrm{RD}, \mathrm{AD}$, and $\mathrm{MD}$ maps separately using FSL's Randomise tool. Lesion and ROI analyses, specifically in deep gray matter, were also conducted.

Results: After correcting for multiple comparisons, performance on attentional tasks was found to be significantly associated with FA in various tracts. Trending toward significance were tracts associated with both cognitive functioning and depression (i.e., the corpus callosum and superior longitudinal fasciculus), particularly when measured by RD. Additionally, the association between white matter damage and depression appeared to be lateralized to the left hemisphere of the brain, while cognitive functioning was more right lateralized or diffuse.

Discussion: This study provides evidence of white matter damage common to depression and cognitive functioning in MS. However, it also points to possible lateralization of contributing brain areas, suggesting some differing neural correlates for secondary and primary factors. Correspondence: Cristina A. F. Roman, Psychology, The Pennsylvania State University, 372 Bruce V. Moore Building, The Pennsylvania State University, University Park, PA 16802, United States.E-mail: car342@ psu.edu

\section{Paper Session 12. Memory}

\section{Moderator: Roy P.C. Kessels}

$$
\text { 4:00-5:30 p.m. }
$$

K.E. OSBORN, L.R. SAMUELS, D. LIU, F.E. CAMBRONERO, J.E. BOGNER, R. MARTIN-WILLETT, C. SEABOLT, M. BABICZ, M. THURSBY, L. WALLJASPER, J. THOMPSON, E. GORDON, K. GIFFORD, S.P. BELL, L. ACOSTA, T.J. HOHMAN, H. ZETTERBERG \& A.L. JEFFERSON. Cognitive Diagnosis Modifies the Effect of Cerebrospinal Fluid Biomarkers of Alzheimer's Disease, Neurodegeneration, and Axonal Injury on Episodic Memory Performance: The Vanderbilt Memory \& Aging Project.

Objective: To test whether cognitive diagnosis interacts with cerebrospinal fluid (CSF) biomarkers of amyloid deposition $\left(\mathrm{Ab}_{42}\right)$, tau aggregation ( $\mathrm{p}$-tau), neurodegeneration (total tau), and axonal injury (neurofilament light, NFL) on episodic memory among older adults. Participants and Methods: Vanderbilt Memory \& Aging Project participants with normal cognition ( $\mathrm{NC}, \mathrm{n}=83,72 \pm 7$ years) and mild cognitive impairment (MCI, $n=58,73 \pm 6$ years) completed a fasting lumbar puncture and neuropsychological protocol. Least-squares regression related diagnosis (NC, MCI) x CSF biomarker $(\mathrm{pg} / \mathrm{mL})$ interactions (including $\mathrm{A} \beta_{42}, \mathrm{p}$-tau, total tau, and NFL) to memory performances. Models adjusted for age, sex, education, race/ethnicity, cognitive diagnosis, and $A P O E$-e4 status. Follow-up models stratified results by diagnosis.

Results: Diagnosis x A $\beta_{42}$ interactions emerged for all memory variables, including California Verbal Learning-II (CVLT-II) Total Learning $(\beta=0.017, p=0.02)$, Long Delay Free Recall (LDFR, $\beta=0.007, p=0.009)$, and Recognition $(\beta=0.002, p=0.008)$, and Biber Figure Learning Test (BFLT) Total Learning $(\beta=0.051, p=0.04)$, LDFR $(\beta=0.020, p=0.001)$, and Recognition $(\beta=0.0003, p=0.009)$. Results were identical for diagnosis $\mathrm{x}$ p-tau $(\mathrm{p}$-values $<0.04)$ and diagnosis $\mathrm{x}$ total tau interactions ( $p$-values<0.04). In all models, enhanced biomarker related to worse memory performance only in MCI $(p$-values<0.036). Diagnosis $x$ NFL interactions only emerged for CVLT-II Total Learning $(\beta=0.007$, $p=0.02)$ and LDFR $(\beta=0.002, p=0.03)$, but the association was present only in NC (p-values<0.03).

Conclusions: While episodic memory impairments relate to lower $\mathrm{A} \beta_{42}$ and higher $\mathrm{p}$-tau and total tau in MCI, CSF NFL corresponds to early changes in verbal memory in cognitively normal elders. Collectively, findings suggest large-caliber axonal injury may affect memory processes in an asymptomatic phase $(\mathrm{NC})$ that precedes clinical symptom onset (MCI) driven by amyloid deposition, tau aggregation, and neurodegeneration.

Correspondence: Katie E. Osborn, Psy.D., Neurology, Vanderbilt Memory \& Alzheimer's Center, 1207 17th Avenue South, 2nd Floor, Suite 204, Nashville, TN 37212, United States. E-mail: katie.osborn@ vanderbilt.edu

M.D. GRILLI, A.A. WANK \& M. VERFAELLIE. The Life Stories of Adults with Amnesia: Insights into the Contribution of the MTL to the Higher-Order Organization of Autobiographical Knowledge.

Objective: Facts about one's personal life are not stored in isolation but rather are organized into chapter memories, which represent major themes that capture the arch of one's life story. Neuropsychological studies have revealed that autobiographical fact knowledge is impaired in adults with medial temporal lobe (MTL) damage. However, whether such impairment compromises the organization and retrieval of chapter memories has not been studied.

Participants and Methods: Therefore, we had 10 adults with MTL amnesia and 20 control participants narrate their life stories, and we extracted chapter memories from these narratives using a novel scoring protocol. We examined both the quantity and quality of chapter memories, evaluating separately retrograde and anterograde chapter memories. Additionally, to investigate the idea that formation of life chapter memories occurs on a protracted time scale, we separated the amnesic participants into an early-life and a later-life onset subgroup.

Results: Intriguingly, results revealed that early-onset, but not lateronset, amnesic participants generated fewer retrograde chapter memories than controls. As expected, they also generated fewer anterograde chapter memories than controls. Higher-order temporal and thematic relations of chapters were impaired in both amnesic subgroups. Moreover, although both amnesic subgroups generated retrograde chapters that were as rich in content as those of controls, anterograde chapters were less rich in content in amnesic participants.

Conclusions: Findings reveal that the organization of autobiographical knowledge into chapter memories occurs on a protracted time scale and depends on the MTL not only for integration and organization of new content, but also for retrieval of higher order relations among chapter memories.

Correspondence: Matthew D. Grilli, PhD, Psychology, University of Arizona, 1503 E. University Blvd., Tucson, AZ 85721-0068, United States.E-mail:mdgrilli@email.arizona.edu

K.B. CASALETTO, G. MARX, S. DUTT, J. NEUHAUS, R. SALONER, L. KRITIKOS, B. MILLER \& J. KRAMER. Is "Learning” Episodic Memory? Distinct Cognitive and Neuroanatomic Correlates of Immediate Recall During Learning Trials Among Healthy Aging and Neurodegenerative Cohorts.

Objective: Although commonly interpreted as a marker of episodic memory, very little is known regarding the neurobehavior of immediate recall during learning trials. Medial temporal lobes importantly contribute to delayed recall, yet immediate recall may necessitate networks beyond and ultimately may be distinct from episodic memory. We aimed to operationalize cognitive and neuroanatomic correlates of total immediate recall in healthy and neurodegenerative groups.

Participants and Methods: Demographically-matched healthy older adults ( $\mathrm{n}=91$ ), individuals with Alzheimer's disease ( $\mathrm{n}=566)$, logopenic variant primary progressive aphasia $(\mathrm{PPA})(\mathrm{n}=34)$, behavioral variant frontotemporal dementia $(n=97)$, semantic variant PPA $(n=71)$, or nonfluent/agrammatic variant PPA ( $n=39)$ completed a neurocognitive 
battery, including the CVLT-Short Form Trials 1-4 Total Immediate Recall; a subset also completed a structural brain MRI. Regressions covaried for age and sex, and MMSE in cognitive and total intracranial volume in neuroanatomic models.

Results: Healthy adults demonstrated a hetergeneous pattern of cognitive associations with total immediate recall (executive, speed, episodic memory), such that no singular cognitive or neuroanatomic correlate uniquely predicted performance. Within the clinical cohorts, syndrome-specific cognitive and neural systems contributed to total immediate recall; e.g., semantic processing was the strongest cognitive correlate in svPPA (partial $r=0.41$ ), while frontal volumes was the only meaningful neural correlate in bvFTD (partial $r=0.21$ ). Medial temporal lobe was not independently associated with total immediate recall in any study group ( $\mathrm{ps}>0.05)$.

Conclusions: Multiple distinct neurobehavioral systems are important for immediate recall during learning trials unique to the brain organization of the individual. Conventional memory networks may not be sufficient or even importantly contribute to total immediate recall in many syndromes. Use of "total learning scores" as a metric of episodic memory may be erroneous.

Correspondence: Kaitlin B. Casaletto, PhD, Psychiatry, University of California, San Francisco, 675 Nelson Rising Lane, San Francisco, CA 94115, United States. E-mail: kaitlin.casaletto@gmail.com

R. SALONER, K.B. CASALETTO, S. DUTT, M. WYNN, J. STIVER, E. FOX \& J. KRAMER. Worth the Wait: Performance on a OneWeek Delayed Recall Task is Associated With Medial Temporal Lobe Structures and Subjective Memory Complaints in Normal Adults.

Objective: Traditional episodic memory tests employ a delayed recall length ranging from 10-30 minutes. However, these tests may be insensitive to subtle neuroanatomical changes found in early disease or age-related decline. We aimed to determine the sensitivity of a 1-week delayed recall paradigm to both medial temporal lobe (MTL) structure and subjective memory symptoms among cognitively normal older adults.

Participants and Methods: 159 functionally intact, older adults (mean age $=76.0$ ) completed a story recall task (Fishermen Story; FS) in which all subjects learned to a criterion of $90 \%$. Recall was tested after 30-minutes and 1-week. Subjects also received the CVLT-II and a subset of participants $(\mathrm{n}=137)$ completed a structural brain MRI and the Everyday Cognition Self-Report questionnaire. Primary neuroanatomical ROIs were the hippocampus and entorhinal cortex (ERC).

Results: FS 30-min and CVLT-II 20-min recalls showed little-to-no relationship with MTL after controlling for age and total intracranial volume. FS correlated .17 with ERC $(p=.048)$ but .14 (ns) with hippocampus. CVLT-II delayed recall did not correlate with either structure. In contrast, 1-week delayed recall of FS demonstrated significant, meaningful relationships with ERC $(r=.28 ; p=0.001)$ and hippocampus $(r=.20, p=0.02)$. Furthermore, poorer FS performance at 1 -week correlated with increased subjective memory symptoms ( $r=-.23$; $\mathrm{p}=0.009)$. CVLT-II and 30-minute FS recall were not associated with memory symptoms (ps>0.05).

Conclusions: Memory paradigms that utilize one-week delays are more sensitive than standard paradigms to MTL volumes and subjective memory symptoms in normal older adults. Findings suggest that longer delay periods may improve detection of memory consolidation changes associated with age-related neurobehavioral decline. Correspondence: Rowan Saloner, Neurology, University of California, San Francisco, 120 El Capitan Drive, San Rafael, CA 94903, United States.E-mail: rowan.saloner@ucsf.edu
E. BERTRAND, M. AZAR, A.M. BRICKMAN, E. HUEY, C.G. HABECK, J. LANDEIRA-FERNANDEZ, D.C. MOGRABI \& S. COSENTINO. Cortical thickness and metamemory in cognitively diverse older adults.

Objective: Impaired memory awareness (i.e., metamemory) is a common characteristic of Alzheimer's disease (AD) with important clinical implications (e.g., increased engagement in high-risk situations, higher caregiver burden). The mechanisms contributing to impaired awareness are still debated, partly because its cognitive and neuroanatomic substrates have not been fully articulated. This study examined objective metamemory testing in relation to cortical thickness.

Participants and Methods: A modified Feeling of Knowing (FOK) task was used to measure metamemory in 14 individuals with mild AD and 20 cognitively healthy older adults. The accuracy of metamemory judgments (i.e., calibration) was examined at the local (i.e., item) and global level wherein individuals made predictions about overall performance. Structural MR images were acquired and cortical thickness was calculated using Freesurfer software for 14 ROIs. The association between metamemory and regional cortical thickness was analyzed using partial correlations adjusted for gender and diagnosis.

Results: Item calibration was associated with right posterior cingulate thickness $(\mathrm{r}=-.42, \mathrm{p}=.02)$ while global calibration was associated with right medial prefrontal thickness $(r=-.39, p=.029)$. Steiger's z tests revealed that each type of calibration had comparable associations with PCC (Steiger's $z=-0.35, p=0.726$ ) and prefrontal thickness (Steiger's $z=-.65, p=.51)$.

Conclusions: To our knowledge, this was the first study to examine metacognition in relation to cortical thickness. Both global and local metamemory appear to rely on the integrity of right sided midline regions (PCC and $\mathrm{mPFC}$ ) known to be important for processing self-referential information. Findings are conceptualized with regard to the Default Mode Network, and also considered in relation to the role of the insula whose structural integrity has been linked to awareness in volumetric studies.

Correspondence: Elodie Bertrand, Psychology, Pontifícia Universidade Católica do Rio de Janeiro, Rua Marquês de São Vicente 225, Edifício Cardeal Leme, $2^{\circ}$ Andar - Sala 201, Rio de Janeiro 22451-900, Brazil. E-mail: elodie.bertrand1@gmail.com

S. VOGEL, G. SHAN, C. BIRD, A. BONNER-JACKSON, J.B. MILLER \&. S.J. BANKS. The Relationship Among Memory Performance and Hippocampal Subregion Volumes in a Memory Clinic Population. Objective: Medial temporal lobe structures, including the hippocampus, are important for the formation and recollection of memories. Regions of the hippocampus have been found to be associated with specific aspects of memory. Specifically, the CA4 has been implicated in pattern separation, encoding, and recognition of visual information, while the presubiculum has been implicated in memory retrieval. The current study examined the relationship between these hippocampal subregions bilaterally and standard measures of verbal and nonverbal memory in order to evaluate whether these regions predict such memory performance.

Participants and Methods: Participants were drawn from a clinical sample of older adults (mean age $=69.9$ ) seen in a memory disorders clinic $(n=651)$. All participants underwent a brain MRI as well as a neuropsychological evaluation that included measures of verbal, the Hopkins Verbal Learning Test-Revised, and nonverbal, the Brief Visuospatial Memory Test-Revised, memory.

Results: Multivariate regressions were conducted in order to assess whether subregions of the hippocampus predict verbal and/or nonverbal memory, after accounting for the influence of age, gender, and total intracranial volume. Results indicated that age, total intracranial volume, left and right presubiculum and CA4 volumes were significant predictors of verbal and nonverbal memory encoding and retrieval (all $\mathrm{p}$ $<.005)$. Gender was not a significant pedictor of memory performance. Conclusions: Results suggest that the volumes of bilateral presubiculum and CA4 are predictive of both verbal and nonverbal memory 
encoding and retrieval in a memory clinic population. Future research examining other hippocampal subregions and specific diagnostic categories will inform understanding of the contributions of these and other subfields to memory function.

Correspondence: Sally Vogel, PhD, Psychiatry \& Psychology, Cleveland Clinic, 9500 Euclid Ave, Cleveland, OH 44195, United States. E-mail: sallyjvogel@live.com

\section{Paper Session 13. Updating Neuropsychological Practice}

\section{Moderator: Adam M. Brickman}

$$
\text { 4:00-5:30 p.m. }
$$

T.W.BREARLY, R.D.SHURA, S.L.MARTINDALE, R.A.LAZOWSKI, D. LUXTON, B. SHENAL \& J. ROWLAND. Neuropsychological Test Administration by Videoconference: A Systematic Review and Meta-Analysis.

Objective: The purpose of this meta-analysis was to assess the effect of videoconference $(\mathrm{VC})$ technology on neurocognitive testing by comparing results to those acquired through onsite administration. Specifically, we investigated the significance and magnitude of the mean difference between scores acquired in each condition.

Participants and Methods: Included studies assessed adult participants using a counterbalanced cross-over design to allow comparison of results. Based on PRISMA guidelines, studies were systematically identified and risk of bias was assessed. A random effects model was used to calculate an overall effect of $\mathrm{VC}$ administration, followed by subgroup analyses by test type. Mean effects were calculated using Hedges' $g$. Results: Eleven studies met inclusion criteria resulting in a total of 468 participants and 74 test scores. The mean effect for $\mathrm{VC}$ administration was small and non-significant $(g=-0.027, p=.341)$; however, significant heterogeneity between scores precluded meaningful interpretation. Subgroup analyses indicated that there was no significant difference for phonemic fluency, semantic fluency, digit span, and list learning tests: however, there was a small effect for the Boston Naming Test for VC administration $(g=-0.115, p<.001)$. It was not possible to calculate an interpretable effect for the Clock Drawing Test or the Mini-Mental State Examination due to substantial heterogeneity of scores.

Conclusions: This meta-analysis was the first to assess the effects of $\mathrm{VC}$ administration on neuropsychological test results. Verbally mediated tests were not significantly affected by $\mathrm{VC}$ administration when compared to onsite administration, suggesting that they may be validly administered using synchronous video technology. Substantial variability across studies for some visually-mediated tests obscured results, as mean scores for the $\mathrm{VC}$ condition suggested that factors such as display quality or distance of visual stimuli from the camera may contribute to heterogeneity in the literature.

Correspondence: Timothy W. Brearly, PsyD, Mental Health and Behavioral Science, Salisbury VAMC, 807 Lincolnton Road, Salisbury, NC 28144, United States. E-mail: tbrearly@gmail.com

R. ELBIN, D. LIPINSKI, A. SUFRINKO \& A.P. KONTOS. Comparison of Patient Satisfaction following Face-to-Face and Telehealth Clinical Visits for Sport-Related Concussion.

Objective: Multi-disciplinary management for sport-related concussion (SRC) is recommended standard of care, especially for patients at risk for prolonged recovery. However, many patients lack access to specialty referral, such as neuropsychology, for SRC. Improving access to specialized care is critical to mitigate adverse concussion related outcomes. Telehealth may offer an effective approach for extending specialized care for SRC to remote areas, as it has shown promise in similar clinical areas (e.g., mental health). This study compared patient and parent ratings of the therapeutic alliance and patient satisfaction between telehealth and face-to-face visits for youth with SRC.

Participants and Methods: Twenty adolescent patients $(M=15.20$, $S D=1.55 \mathrm{yrs}$ ) with SRC and their accompanying parent/guardian completed a series of surveys assessing therapeutic alliance and patient satisfaction following their first neuropsychological consultation. Patients were randomly assigned to either a telehealth (i.e., interactive videoconferencing) visit or face-to-face visit condition. Mean differences for survey scores between conditions were analyzed with t-tests and ANOVA (Bonferroni-corrected $p \leq .05$ ).

Results: Therapeutic alliance scores did not differ between telehealth and face-to-face conditions $(p=.21)$, and patient satisfaction scores were also similar between conditions $(p=.19)$.

Conclusions: Neuropsychological consultation for SRC using telehealth is comparable in patient and parent reports of rapport/satisfaction to face-to-face consultation.

Correspondence: RJ Elbin, PhD, University of Arkansas, Human Performance Lab, University of Arkansas, HPER 321, Fayetteville, AR 72701, United States. E-mail: rjelbin@uark.edu

H.E. FEENSTRA, J. MURRE, I.E. VERMEULEN \& S.B. SCHAGEN. Reliability and Validity of an Online Tool for Self-Administered Cognitive Assessment: the Amsterdam Cognition Scan.

Objective: Traditional, supervised neuropsychological assessment can be time-consuming and labor-intensive. In research there is a need for efficient neuropsychological tools that allow for large-scale data collection. Thus far, few online tools are available with adequate psychometric properties and accompanying reference data. Our aim was to develop a new self-administered online test battery for the oncology setting.

Participants and Methods: The Amsterdam Cognition Scan was developed based on seven traditional neuropsychological tests. Convergent validity was assessed using Spearman correlations. Using a counterbalanced design, 201 (112 female) cancer patients (mean age 53.5 years) completed an unsupervised online assessment and a supervised traditional assessment. Test-retest reliability of the online battery was assessed using intraclass correlation coefficients (ICCs). 96 cancer patients (57 female, mean age 51.8) participated.

Results: Comparing online and traditional test scores, we observed moderate to strong convergent validity ( $r=.36$ to.70; battery sum score $r=.78)$. Correlations were influenced - as expected - by the similarity between the traditional test and its online counterpart. ICCs of the online tests ranged from .29 to .76 , with an ICG of .78 for the battery sum score. These correlations are generally comparable with the test-retest correlations of the traditional tests as reported in the literature.

Conclusions: The Amsterdam Cognition Scan is one of the first online self-administered test batteries with thorough studies on psychometric properties. Our results indicate adequate validity and reliability for the vast majority of the online tests. Reference data is available. As the Amsterdam Cognition Scan measures a wide range of cognitive functions, its application can go beyond the oncology setting.

Correspondence: Heleen E. Feenstra, Msc, Plesmanlaan 121, Amsterdam 1066 CX, Netherlands. E-mail: h.feenstra@nki.nl

T. GIOVANNETTI, T. YAMAGUCHI, E. ROLL, H. FUJINO, T. MAETA, S.S. RYCROFT, J. HULSWIT, B. GREENSTEIN, K. LEUNG, L.H. THOMPSON, R. DIVERS \& C.C. TAN. The Virtual Kitchen: Preliminary Data from A Novel Virtual Reality Test of Mild Difficulties in Everyday Function.

Objective: Methods for standardized and efficient early detection and characterization of mild functional difficulties in older adults are lacking. We present preliminary data from a novel standardized virtual reality (VR) task, the Virtual Kitchen (VK), designed to assess subtle functional difficulties.

Participants and Methods: 14 healthy older adults and 21 healthy young adults completed the VK, a non-immersive VR task involving manipulation of virtual objects using a touch screen for two everyday 
tasks (breakfast, lunch). In young adults, the VK was also administered under a Dual Task condition (VK + Oral Trail Making Test) to simulate mild cognitive difficulties. Both groups also completed the tasks (breakfast, lunch) using real objects (Real Kitchen; RK). The VK program generated the number of steps accomplished and completion time. Trained coders tallied omissions, commissions, micro-errors (i.e., mild inefficiencies), and inefficient mis-reaching to objects/screen from video of the VK and RK. Tests of episodic memory and executive function were administered. Older adults also rated their everyday functioning. Results: Older adults took significantly longer and made more micro-errors and inefficient mis-reaches (all $\mathrm{p}<.01$ ) than young adults. Young adults showed the same pattern of longer completion time and more subtle errors in the Dual VK versus the Standard VK (all $p<.05)$. VK variables were significantly related to cognitive tests (all $r>35$ ) and RK variables (all $\mathrm{r}>.36$ ); VK variables were significantly associated with self-rated function in older adults (all $\mathrm{r}>.47$ ).

Conclusions: The VK is sensitive to mild difficulties and is associated with real task performance, episodic memory and executive function abilities, and functional self-ratings. Thus, VR, and specifically the VK, is a promising tool for efficient and valid performance-based, functional assessment, even when functional difficulties are mild. Automated scoring may be improved with methods to capture micro-errors and mis-reaching toward the screen.

Correspondence: Tania Giovannetti, PhD, Psychology, Temple University, Weiss Hall, Psych Dept, 1701 N 13th St, Philadelphia, PA 19121, United States.E-mail: tgio@temple.edu

J. STELMOKAS, L.J. YASSAY, B. GIORDANI, H. DODGE, I. DINOV, A. BHAUMIK, K. SATHIAN \& B.M. HAMPSTEAD. Quantitative and Normative Volumetry Using Neuroquant: Association With Memory Performance in Healthy Older Adults and Mild Cognitive Impairment.

Objective: MRI-derived volumetry is well established in research, but has yet to translate into clinical practice. NeuroQuant (NQ) is a fullyautomated program that may bridge this critical gap via widespread access and normative data. The original version (NQ1) was recently updated (NQ2) to include additional brain regions and normative data (age and sex based z-scores for each region). The current study evaluated the relationship between (1) NQ versions and (2) Volumes of the hippocampus (HP), inferior lateral ventricle (ILV), isthmus/posterior-cingulate (IPC) using both percent of intracranial volume (ICV) and z-scores and memory performance in healthy older controls (HOC) and those with mild cognitive impairment (MCI)).

Participants and Methods: 122 participants (MCI n=61) underwent MRI and neuropsychological testing. Each structural scan was processed using both NQ versions. The primary cognitive variables were the Immediate and Delayed Memory Indices (IMI/DMI) of the Repeatable Battery for the Assessment of Neuropsychological Status (RBANS).

Results: (1) Volumes were significantly correlated between NQ versions in all participants $(r=0.400-0.998)$ as well as HOC $(r=0.521-0.998)$ and MCI ( $r=0.295-0.998)$ individually. Subcortical regions and the cerebellum typically demonstrated lower or even non-significant correlations. Mean medial temporal volumes differed from 0.01 to 0.03 across all participants. (2) Regarding memory and ICV volumes: the HP correlated with DMI in all participants $(r=0.36)$ and in the MCI group alone $(r=0.33)$; the ILV correlated with DMI in all participants $(r=-0.23)$. HP z-scores were significantly related to both IMI $(r=0.24)$ and DMI $(r=0.44)$ in all participants and with the DMI in the MCI group $(r=0.40)$.

Conclusions: NQ1 and NQ2 provide similar volumetric data, though region specific differences exist. Medial temporal relationships with memory indices are generally consistent with our previous findings but differences exist between ICV and normative (z-scores) values. Correspondence: Julija Stelmokas, Psy.D., Ann Arbor VA, 2215 fuller road, Ann Arbor, MI 48104, United States.E-mail: jstelmok@med. umich.edu
C.G. WONG, L. RAPPORT, B. BILLINGS, M. DE IORIO, B. VERMILION, B. STACH \& M. KASSA. Age-Related Hearing Loss and Verbal Memory Assessment.

Objective: Prior research has found that adults with hearing loss perform worse on cognitive testing than adults without hearing loss, and some studies have suggested that hearing loss is associated with dementia. Heavy emphasis on tests involving auditory stimuli for memory assessment may result in overdiagnosis of cognitive impairment in individuals with hearing loss. The present study compared visual and auditory versions of a verbal memory test among adults with and without hearing loss.

Participants and Methods: Thirty-seven adults with moderate-to-severe, sensorineural hearing loss (HL) and 46 adults with normal hearing (NH) participated. Age ranged from $55-82$ years. They completed a neuropsychological battery that included auditory and visual versions of the Hopkins Verbal Learning Testing-Revised (HVLT-R).

Results: Mixed-model ANOVA indicated significant group (HL vs. NH) by condition (visual vs. auditory HVLT-R) interactions $(p<.001)$ with large effect sizes $\left(\mathrm{h}_{\mathrm{p}}{ }^{2}=.25\right.$ to .36$)$. Post hoc contrasts showed the groups did not differ significantly on the visual version and showed small effect sizes $(d=0.2$ to 0.3 ), but the HL group performed significantly worse than the NH group on the auditory version $(p<.001)$ with large effect sizes $(d=1.1$ to 1.6$)$. Auditory and visual versions of immediate recall were highly correlated for the NH group $(r=.74)$ but not for the HL group $(r=.19)$. Groups did not significantly differ on other neuropsychological tests and showed small effect sizes $(d=0.0$ to 0.4$)$. Moreover, for the HL group, the HVLT-visual version was strongly correlated with other neuropsychological tests whereas the standard auditory version was not.

Conclusions: Findings indicate that auditory verbal memory tests substantially overestimate memory impairment among adults with hearing loss. Visual assessment of verbal memory shows evidence of superior validity and is a viable alternative method to assess memory function especially in older populations.

Correspondence: Christina G. Wong, Wayne State University, 5057 Woodward, Detroit, MI 48202, United States.E-mail: cgwong@wayne. edu

\section{Poster Session 9. ABI \& Intervention 4:15-5:30 p.m.}

\section{Acquired Brain Injury (TBI/ Cerebrovascular Injury \& Disease - Adult)}

L. BENNETT, J. BURKE \& D. LECHUGA. The Role of Athletic Exposure on Neurocognitive Performance in Disability-Seeking, Retired National Football League Players.

Objective: The NFL concussion rate is greater than most sports. Further, players incur 900 - 1500 subconcussive impacts each season. Playing position (PP) is a principle risk factor, as some positions have a higher risk of sustaining both concussive and subconcussive impacts. Athletic exposure (AE), calculated using years of NFL-play and risk associated with primary PP (based on magnitude and frequency of impacts), approximates a player's exposure to potentially damaging collisions. Given the cognitive declines associated with repetitive sub/ concussive impacts, many retired NFL players are pursuing worker's compensation claims, which include neurocognitive assessment. To date, population-specific norms for applicable neuropsychological (NP) measures have not been established. Appropriateness of currently utilized, US population-based norms when assessing retired players and the role of $\mathrm{AE}$ on long-term cognitive functioning across domains are explored. Participants' performances across measures were predicted to be significantly discrepant from general population norms. Additionally, 
significant differences between various levels of $\mathrm{AE}$ were expected on all NP measures.

Participants and Methods: 97 retired NFL players were assessed. ANOVAs and MANOVAs were used to examine differences between $\mathrm{AE}$ levels on all measures. Various levels of AE were compared to existing norms on all NP outcome variables using Cohen's $d$ and Hedge's $g$.

Results: Overall, findings did not evidence significant discrepancy from test-publisher norms or predicted significant differences between $\mathrm{AE}$ levels; however, trends not reaching statistical significance emerged on language, memory, and motor functioning measures.

Conclusions: Given discrepancies on select measures, population norms may be of limited use when interpreting NP data of retired NFL players. Neuropsychologists serving this population should consider the role of biological, psychosocial, and alternative etiological factors when determining diagnostic impressions and treatment recommendations.

Correspondence: Lauren Bennett, CSPP at Alliant Int. University, 201 E. Angeleno Ave., Unit 103, Burbank, CA 91502, United States. E-mail: lbennett@alliant.edu

T.F. BERGQUIST, A.T. WEHDE, K. KRISHNAN, M.R. POWELL \& A.W. BROWN. Relationship of Clinical Characteristics to Functioning in a Sample of Person with Mild Traumatic Brain Injury (TBI) Seeking Treatment.

Objective: Most people with mild TBI return to their previous level of functioning within a few weeks; however, a minority of individuals continues to experience problems for an extended period of time. In this study, we measured mood, life satisfaction, functional status, and level of symptom complaint in a treatment seeking sample of patients with diagnosed with mild TBI with extended recovery periods.

Participants and Methods: Participants. Patients were referred to an outpatient brain treatment program with a diagnosis of mild TBI. All 20 participants were presenting for treatment to an interdisciplinary outpatient treatment clinic. Mean age of patient was 44.15 years, Range: $25-67$ years; females $=14$, males $=6$; Mean time since injury $=$ 9.50 months, Range: 1-71 months.

Method. Measures were administered to assess individual differences in mood, life satisfaction, participation, and level of symptom complaint. Measures: All patients were classified for injury severity using the Mayo Classification System (MCS). Participants completed the Mayo Portland Participation Index (M2PI), the Satisfaction with Life Scale (SWLS), the Patient Health Questionnaire (PHQ-9), and the Neurobehavioral Symptom Inventory (NSI-22).

Results: Using Spearman's Rho correlations the following measures correlated significantly:

Time since injury and the PHQ-9 $\left(r_{s}=.551, \mathrm{p}<0.05\right)$

M2PI and the PHQ-9 $\left(r_{s}=.524, \mathrm{p}<.05\right)$

M2PI and SWLS $\left(r_{s}=-.595, \mathrm{p}<.01\right)$ : as functional disability went down, life satisfaction went up

Conclusions: Within a treatment-seeking sample diagnosed with mild TBI, increased depressive symptoms are associated with longer time to onset of treatment. In addition, higher disability ratings are associated with higher levels of depression and lower life satisfaction. Mood and life satisfaction are associated with level of functioning after Mild TBI and targeting these areas in a timely manner as part of treatment may help to both better understand persons presenting for treatment and improve treatment outcome.

Correspondence: Thomas F. Bergquist, Ph.D., Physical Medicine and Rehabilitation, Mayo Clinic, 200 First Street SW, Rochester, MN 55905, United States.E-mail:Bergquist.Thomas@mayo.edu

F. BINEY. A Case of Significant Retrograde Amnesia and Loss of Autobiographical Memories Following Traumatic Brain Injury.

Objective: Anterograde amnesia following traumatic brain injury (TBI) is well documented (Vakil, 2005). The occurrence of dense retrograde amnesia (RA) and severe loss of autobiographical memories (AM) following TBI is less common. Both are linked to anterior temporal lobe injuries in conjunction with frontal lobe involvement. The current case study describes the cognitive recovery course of a TBI survivor with significant retrograde and anterograde memory deficits. The long term recovery potential of post-TBI memory deficits are discussed.

Participants and Methods: MP was a 67-year-old, right handed man when he was struck by a vehicle while riding a bicycle in 2013 . He sustained a severe TBI with left temporal and left putaminal parenchymal hematomas and left anterior temporal lobe encephalomalacia. EEG noted abnormal waking, drowsy, and sleeping states due to mild transient diffuse slowing over the left frontal temporal leads. MP underwent neuropsychological evaluations at 10 months, 16 months, 24 months, and 36 months post-injury. Standardized scores (SS) were obtained from appropriate age and education corrected normative data Results: At 10 months post-injury, MP demonstrated impaired performance ( $\geq 2$ SD below mean) in verbal learning, recall, and recognition, visual memory, verbal fluency, confrontation naming, bilateral motor speed and executive functions. Using the criterion of $\geq 1$ SD improvement $(\mathrm{z}>1)$ in standardized scores, MP showed improvements in perceptual reasoning/intellectual skills and delayed verbal memory in the first 24 months post-injury. Additional gains were seen from 24 to 36 months in verbal learning and recall, visual memory, and verbal learning for stories

Conclusions: MP's impairments are consistent with other reports in the literature of significant RA and AA memory impairment following fronto-temporal lobe injury. Most of his improvements in memory functioning occurred beyond the typical 24-month time frame during which the majority of cognitive recovery is expected.

Correspondence: Fedora Biney, neuropsychology, The Institute for Research and Rehabilitation (TIRR), 20511, Houston, TX 77433, United States.E-mail:febiney@aol.com

M.E. CAMPBELL, D. LEITNER, L. OHLHAUSER, M. LIBBEN \& H. MILLER. A Comparison of the Buschke Selective Reminding Test and the California Verbal Learning Test- Second Edition in a Stroke Population.

Objective: There are many assessments of learning and memory, however not all of them yield analogous results and thus are not necessarily interchangeable. The current study examined the relationship between the California Verbal Learning Test- Second Edition (CVLT-II) and the Buschke Selective Reminding Test (SRT) in a stroke population. Participants and Methods: This study used archival data from stroke patients $(N=104)$ on the rehabilitation unit at Kelowna General Hospital. Patients were administered both the SRT and the CVLTII, in a counterbalanced order, as part of a larger neuropsychological assessment. Analyses were conducted on $Z$-scores from the List Total, Learning, and Long Delay Free Recall (LDFR) subscales of the two measures.

Results: Performance on the SRT and CVLT-II was significantly correlated (all $p$ values < .001). However, impairment was indicated as significantly worse by the SRT compared to the CVLT-II (all $p$ values $<.001)$. On both the SRT and the CVLT-II, patients with left hemisphere stroke tended to perform worse than those with right hemisphere stroke, however this trend was not significant after Bonferroni adjustment. In assessing the ability of the CVLT-II and the SRT to distinguish stroke hemisphere, the SRT model was found to be a good fit based on the Hosmer and Lemeshow criteria $(p=.31)$. Specifically, the LDFR portion of the SRT demonstrated the ability to significantly differentiate between stroke hemisphere $(p=.003)$.

Conclusions: The results suggest greater sensitivity to impairment of the SRT, thus indicating that the SRT and CVLT-II should not be used interchangeably. Such results warrant further research on the relation between tests of verbal learning and memory to yield optimal clinical practice.

Correspondence: McKenzie E. Campbell, BSc. (Hons), Psychology, University of British Columbia - Okanagan, 4890 Lakeshore Rd, Kelowna, BCV1W4H7, Canada.E-mail: mckenzie-campbell@live.com 
A.P. CHAPLIN, R.T. LANGE, T.A. BRICKELL, L.M. FRENCH \& S.M. LIPPA. Comparing Neuropsychological Outcome in Active Duty Soldiers Following Complicated, Uncomplicated, and Equivocal Mild Traumatic Brain Injury.

Objective: Mild traumatic brain injury (mTBI) occurs on a broad spectrum, ranging from a brief alteration of consciousness to clearly detectable intracranial abnormality. The purpose of this study was to examine neuropsychological outcome across three severity sub-groups following $\mathrm{mTBI}$.

Participants and Methods: Participants were 64 U.S. military service members recruited from Walter Reed National Military Medical Center who had sustained an mTBI. Participants completed a 3-4 hour neuropsychological evaluation (on average 5.6 months post-injury) that included several common neurocognitive and neurobehavioral measures. Participants were classified into three severity sub-groups: (a) Complicated mTBI $(\mathrm{n}=17)$, (b) Uncomplicated mTBI $(\mathrm{n}=31)$, and (c) Equivocal mTBI $(n=16)$.

Results: Pairwise comparisons revealed paradoxical differences between Complicated and Uncomplicated mTBI groups on tasks of attention, processing speed, memory, and executive functioning (Complicated $>$ Uncomplicated; $\mathrm{d}=.29-94)$. In addition, the Complicated mTBI group had higher levels of psychological distress (e.g., anger, anxiety) compared to the Uncomplicated mTBI group $(\mathrm{d}=.32-.56)$. When comparing Uncomplicated and Equivocal mTBI groups, neurocognitive outcomes were in the expected direction on tasks of attention, executive functioning, and auditory delayed memory (Equivocal > Uncomplicated; $\mathrm{d}=.29-.47$ ). The Equivocal mTBI group also had higher scores on anxiety, headaches, and pain $(\mathrm{d}=.33-.37)$. There were few differences on neurocognitive variables between the Complicated and Equivocal mTBI groups. However, the Complicated mTBI group reported increased levels of psychological distress and fewer cognitive complaints ( $\mathrm{d}=.29-.46)$. Conclusions: These results suggest that there were some differences in neuropsychological outcome following complicated, uncomplicated, and equivocal mTBI in this military sample. However, the effect sizes of these differences were only small-medium.

Correspondence: Andre P. Chaplin, Psy.D., Neuropsychology, Walter Reed National Military Medical Center, 807 Franklin St., Alexandria, VA 22314, United States.E-mail: andrechaplin@gmail.com

K.S. CHIOU, H.M. GENOVA, J. LENGENFELDER \& N. CHIARAVALLOTI. Investigation of Response Time as a Process Variable in Metacognitive Functioning After Traumatic Brain Injury.

Objective: There has been an emergence of clinical interest in metacognition, a form of self-awareness specifically involving one's online, in-the-moment ability to monitor cognitive performance. Previous studies in traumatic brain injury (TBI) have focused on identifying weaknesses in metacognitive accuracy. However, little remains known about how the process of making a metacognitive decision is altered after injury. The goal of this study was to contrast the temporal aspects of the metacognitive decision-making process between adults with TBI with healthy peers.

Participants and Methods: Adults with moderate to severe TBI and matched healthy volunteers (total $n=30$ ) were recruited. Reaction times (RTs) were collected as participants made metacognitive retrospective confidence judgments (RCJs) during a computerized memory task. Independent samples t-test was used to determine between group differences in RCJ RT on the task. Correlation analyses were used to investigate relationships between RCJ RT and performance in the domains of processing speed and attention.

Results: Adults with TBI were just as accurate as healthy participants in assessing their recognition performance; however, they were significantly slower in judging their performance $(p=0.03)$. For adults with TBI, slower RCJ RTs were significantly associated only with better performance on an attention task $(p<0.05)$.

Conclusions: The results indicate that adults with TBI take significantly longer to achieve the same metacognitive accuracy as healthy volunteers.
Interestingly, the extended time required to make metacognitive judgements was related to attention rather than processing speed deficits. The findings suggest that temporal process variables should also examined when assessing metacognitive functioning after TBI.

Correspondence: Kathy S. Chiou, PhD, Psychology, University of Nebraska, 218 Burnett Hall, Lincoln, NE 68588, United States. E-mail: kchiou2@unl.edu

A.L. CLARK, K.J. BANGEN, S.F. SORG, M.L. WERHANE, D. SCHIEHSER, M.W. BONDI \& L. DELANO-WOOD. Repetitive Mild Traumatic Brain Injury Moderates the Association Between Age and Cerebral Blood Flow of Medial Temporal Lobe Structures. Objective: Traumatic brain injury (TBI) — even when mild in severityis a risk factor for poor long-term outcomes and neurodegenerative changes, and vascular pathology may play an important role. Although cerebral blood flow (CBF) alterations have been observed in both TBI and aging, little is known about how history of repetitive TBI may influence $\mathrm{CBF}$ in regions that are also vulnerable to aging. We therefore sought to clarify the influence of repetitive TBI and age on CBF, as well as associations between CBF and cognition.

Participants and Methods: 37 Veterans with history of TBI (mean age $=35$ ) underwent neuroimaging, completed psychiatric symptom inventories, and were administered a comprehensive neuropsychological battery. Participants were divided into two groups: (1) those with history of 1 or 2 TBIs $(n=19)$ and (2) those who have sustained $3+$ TBIs $(n=18)$. Resting CBF was measured using multiphase pseudocontinuous arterial spin labeling and averaged across FreeSurfer-derived parcellations of the medial temporal lobe (MTL).

Results: Regression analyses, adjusting for symptoms of posttraumatic-stress disorder (PTSD), revealed a significant Group x Age interaction for resting $\mathrm{CBF}$ in the right fusiform $(p=.03)$ and parahippocampal gyri $(p=.008)$, and a trend was observed in bilateral hippocampi $(p$ 's=.08). Examination of simple main effects revealed that increased age was significantly associated with decreased CBF in those with 3+ TBIs, but not in those with fewer injuries. Across the sample, regression analyses adjusting for PTSD revealed that reduced CBF was associated with poorer verbal memory performance $(p ' s<.05)$.

Conclusions: Lifetime history of repetitive TBIs exacerbates age-related CBF reductions of the MTL. Given this region is known to be susceptible to neurodegenerative diseases, our findings suggest that CBF alterations following multiple TBIs may contribute to the deleterious pathological processes that give rise to poor cognitive outcomes.

Correspondence: Alexandra L. Clark, M.S., UCSD/SDSU Joint Doctoral Program in Clinical Psychology, 3350 La Jolla Village Dr., San Diego, CA 92161, United States. E-mail: alexandra.leigh.cl@gmail.com

M. ETTENHOFER, K. LUNSFORD, D. BARRY \& J. KEGEL. Neurocognitive Eye Tracking in Moderate-to-Severe Traumatic Brain Injury: Evidence for Enhanced Sensitivity to Impairment.

Objective: Moderate-to-severe traumatic brain injury (TBI) often produces long-term cognitive impairment. However, patterns of recovery may vary considerably, and the sensitivity of individual neuropsychological tests may vary. This study was conducted to investigate effects of moderate-to-severe TBI on BEAM, a continuous performance test that includes multiple cognitive metrics from saccadic eye movements, and a battery of neuropsychological tests. Previous research has demonstrated increased rates of BEAM saccadic impairment in chronic mild TBI, but patterns of impairment in moderate-to-severe TBI remain unknown. Participants and Methods: Participants included 17 adults with remote history of moderate-to-severe TBI and 52 uninjured controls. Neuropsychological tests included WAIS-IV Digit Span and Symbol Search, CVLT-II, Trail Making Test (A\&B) and Grooved Pegboard. Saccadic metrics from BEAM included response time (RT) to five different cues; full and partial commission errors, and fixation loss errors. Analyses were conducted using ANCOVA (controlling for age) and Mann-Whitney U tests. 
Results: Average effect size of TBI for the eight BEAM saccadic metrics was $d=.96$. Significant impairments were shown in RT for directional $(d=1.26)$, misdirectional $(\mathrm{d}=.77)$, and gap $(d=.84)$ cues, commission errors $(d=.70)$, partial commission errors $(d=1.44)$, and fixation loss errors $(d=2.04)$. Among the nine conventional neuropsychological metrics, average effect size of TBI was $d=.42$. Individuals tests that were significantly impaired in the TBI group included CVLT-II (Trials 1-5 total, $d=.70$ ) and Grooved Pegboard (nondominant hand, $d=.63$ ). Conclusions: These results suggest that, in addition to conventional tests of memory and motor function, saccadic measures of attention, executive function and spatial processing may be especially sensitive to impairment after TBI. Overall, this study provides additional evidence that neurocognitive eye tracking may offer enhanced sensitivity to chronic impairment after brain injury.

Correspondence: Mark Ettenhofer, NMCSD, DVBIC, 4837 E. Alder Dr., San Diego, CA 92116-2325, United States.E-mail: ettenhofer@gmail. com

N.D. EVANGELISTA, A.L. CLARK, K.J. BANGEN, S.F. SORG, M.L. WERHANE, D. SCHIEHSER \& L. DELANO-WOOD. Brain Derived Neurotropic Factor (BDNF) Val66Met Moderates the Association Between PTSD and Cortical Thickness in Veterans with History of Traumatic Brain Injury.

Objective: Post-traumatic stress disorder (PTSD) has been linked to cortical thinning of frontal and temporal regions in Veterans with history of traumatic brain injury (TBI). However, it remains unclear how brain-derived neurotrophic factor (BDNF) - a protein that plays a role in neuronal growth, maturation and maintenance-may potentially influence the effects of PTSD on the brain. We therefore sought to clarify the associations between BDNF genotype, PTSD, and cortical thickness in Veterans with history of mild TBI (mTBI).

Participants and Methods: 59 Veterans with history of mTBI underwent BDNF genotyping and were divided into (1) Met+ carrier ( $n=29)$ and (2) Met- carrier ( $n=30)$ subgroups. Participants also completed psychiatric symptom questionnaires (PCL-M) and structural MR imaging. Cortical thickness values were derived from parcellated regions of interest (ROIs) using FreeSurfer.

Results: Analysis of covariance controlling for age, revealed a significant PTSD x Genotype interaction for cortical thickness in the cuneus, precuneus, and the rostral anterior cingulate $(p$ 's $<.05)$. Examination of simple main effects revealed that Met+ carriers with greater PTSD symptomology demonstrated significantly thinner cortices relative to Met- carriers with comparable levels of symptoms ( $p$ 's $<.05)$.

Conclusions: Results show that BDNF genotype differentially affects the relationship between PTSD and cortical thickness in Veterans with history of mTBI. Specifically, mTBI Met+ carriers-who may have lower levels of this critical neurotrophin available in the central nervous system- appear to be especially vulnerable to the negative effects of PTSD on cortical thickness of several brain regions. These findings suggest that BDNF genotype plays an important role in modulating brain structure in the context of comorbid TBI and PTSD. Future studies should further evaluate the epigenetic effects of BDNF on recovery and treatment outcomes in Veterans with comorbid TBI and PTSD.

Correspondence: Nicole D. Evangelista, B.S., VA San Diego Health Care System, Veterans Medical Research Foundation, 3350 La Jolla Village Dr., San Diego, CA 92161, United States.E-mail:nevangel@ucsd.edu

S. FATOORECHI, A. WALKER, H. RAD, E. ROMERO, T. YEH, P. VESPA, D. MCARTHUR, D. HOVDA, D. PLURAD, E. WOO, K.B. BOONE, J. FUSTER \& M.J. WRIGHT. Perceived Workload on Performance Validity Tests in Persons with and without Traumatic Brain Injury.

Objective: Performance validity tests (PVTs) are used to determine the validity of neuropsychological performances in clinical and forensic assessments. It is assumed that healthy and clinical populations perceive PVTs similarly in terms of task difficulty. Our goal was to determine if persons with and without TBI perceive PVTs similarly and if they perceive them as equally taxing as neuropsychological measures of ability.

Participants and Methods: TBI $(n=19)$ and comparison $(n=21)$ groups consented to voluntary participation in this IRB approved study. The groups were well matched on demographic variables. The TBI group suffered moderate-to-severe injuries primarily due to accelerationdeceleration events (79\%). All participants completed a brief neuropsychological battery and passed 2/2 PVTs. After each test, including the Rey-15 Memory Test (RMT) and Dot Counting Test (DCT; the PVTs in the current study), they provided a ratings of workload. Workload rating for tests of ability were averaged to create a global cognitive workload rating.

Results: The TBI group demonstrated impaired memory ( $p=.023)$, attention/executive ability $(p=.015)$, and global cognitive ability $(p=.037)$ in contrast to the comparison group. Mixed ANOVAs with global cognition ratings and PVT ratings as a within subjects factor revealed that both groups perceived greater workload on tests of ability in contrast to PVTs $(p s<=.002)$ and TBI participants provided higher task load ratings across test types in contrast to comparison participants ( $p$ s=.021-.033). No interactions between group and task load ratings were observed.

Conclusions: Our data suggest that persons with and without TBI perceive tests of ability as more taxing than PVTs and that persons with TBI generally perceive both types of tests as more demanding than healthy participants do. These data indicate that examinees may be able to discern the difference in difficulty between PVTs and tests of ability, suggesting that embedded indicators should also be used when assessing performance validity.

Correspondence: Sarah Fatoorechi, Clinical Psychology, Alliant International University, 1124 W. Carson St., B-4 South (490), Torrance, CA 90502, United States.E-mail:sfatoorechi@alliant.edu

A.A. FEDIO, A. PATTERSON, M. THYEN, A. ARCURI \& P. FEDIO. Bringing the Real World Into the Classroom: Individuals With Brain Injury Educate Clinical Psychology Graduate Students.

Objective: The study examined how clinical psychology graduate students with higher, or lower, self-rated clinical skills benefited from listening to personal narratives reported by individuals with acquired brain injury $(\mathrm{ABI})$.

Participants and Methods: Thirty-four clinical psychology graduate students completed questionnaires before and after listening to presentations by a small group of individuals who were members of an ABI speakers' bureau. Speakers described the nature of their brain injuries and their recovery process. Using 5-point Likert scales, students rated their own clinical skills across several areas and answered questions about common problems and effects on functioning following ABI. Median split analyses were performed, and student groups scoring high versus low on measures were compared using independent samples t-tests.

Results: Students demonstrated a general understanding of ABI symptomatology and increased their factual knowledge about ABI after listening to the speakers. Students with lower (than higher) baseline self-rated clinical skills regarding helping clients to take an active role in their own health gained significantly more knowledge about ABI sequelae regarding changes in sense of self, motivation, fatigue, and family relationships. After hearing the speakers, these students also rated themselves as feeling more proficient if they were to interview a brain-injured patient; they also believed an ABI patient would feel more comfortable being interviewed by them.

Conclusions: Individuals with ABI can effectively educate cliniciansin-training by sharing experiences regarding their recovery process. Benefits to clinical psychology graduate students of listening to ABI speakers included increased factual knowledge about ABI sequelae. The experience also improved students' confidence in working with individuals with brain injury. Results support advocacy work for individuals 
with $\mathrm{ABI}$ which may ultimately improve mental health services for the brain injury population.

Correspondence: Alison A. Fedio, PhD, PsyD, American School of Proessional Psychology, Argosy University, 1550 Wilson Boulevard, Suite600,Arlington,VA 22209, United States.E-mail: afedio@argosy. edu

A.A. FEDio, J.N. STILlMAN, J.L. PRILUCK, C.L. ROPER, S. CUMMINGS \& P. FEDIO. Functional Correlates of Patients' Current and Predicted Future Satisfaction With Their Aggression Following Traumatic Brain Injury.

Objective: Aggression is not an uncommon sequel of moderate/severe traumatic brain injury (TBI). The purpose of this study was to examine how patients' satisfaction with their post-TBI aggression related to their own, as well as their family members', assessment of their recovery.

Participants and Methods: Fourteen individuals with moderate/severe TBI $(8 \mathrm{M} / 6 \mathrm{~F}$, mean age: $36 ; 4$ years post injury), and a family member for each, rated the patients' physical, cognitive, and emotional level of functioning (Neurobehavioral Functioning Inventory [NFI]). Patients also completed the Tennessee Self-Concept Scale (TSCS2), and estimated their current and future satisfaction with their recovery.

Results: Patients' self-rating of aggression was within the average range. Their current satisfaction with their aggression related to their social $(r=.87)$ and moral ( $r=.79)$ self-concept, as well as their self-satisfaction with recovery of attention, depression, motor functioning, and reduced somatic symptoms ( $r=.71-.77)$. Patients' current satisfaction with their aggression also related to their family members' evaluation of the patients' motor and somatic functioning $(r=.77-.82)$. Patients' predicted (2-3 years into the future) satisfaction with their aggression related to their future satisfaction with recovery of attention, communication, and depression $(r=.73-.94)$

Conclusions: Patients' current satisfaction with their aggression related directly to their sense of self and aspects of their physical, cognitive, and emotional functioning, but related only to family members' ratings of patients' physical functioning. In contrast, the patients' predicted future satisfaction with aggression related to their future satisfaction regarding cognition and emotions, but not their physical functioning. These findings underscore the importance of physical functioning at the time of evaluation for both patients and their families, in contrast to the patients' emphasis on more existential areas of cognition and emotions as they predict their future recovery.

Correspondence: Alison A. Fedio, PhD, PsyD, American School of Proessional Psychology, Argosy University, 1550 Wilson Boulevard, Suite600,Arlington,VA 22209, United States.E-mail: afedio@argosy. edu

B.H. WING, B.J. TUCKER, M.D. ALLEN \& A.K. FONG. Functional NeuroCognitive Imaging: Rehabilitation of Neurovascular Uncoupling in Concussion Treatment.

Objective: Typical treatment of mild traumatic brain injury (mTBI) recommends limited physical exertion, cognitive activity, and exposure to symptom-aggravating stimuli until baseline normative function returns. But with its limited effectiveness for post-concussion syndrome (PCS), there is a clear need for novel approaches to standardized therapeutic assessment, particularly ones that incorporate established clinical and pathophysiological understanding of mTBI with modern neurocognitive imaging. We demonstrate the application of functional neurocognitive imaging (fNCI), which combines the conventional power of neuropsychological testing with the objectivity of functional magnetic resonance imaging (fMRI). We demonstrate fNCI's reliability in the assessment and localization of neurovascular uncoupling (NVU) to guide treatment of mTBI, with particular effectiveness in relief of persistent PCS symptoms.

Participants and Methods: Degrees of NVU in task-associated regions of activation were statistically quantified to establish pre-treatment benchmarks for 270 concussed patients using fNCI. Using identified neurocognitive biomarkers, a targeted, sustained, cyclical, and multimethod therapeutic approach was structured for each patient and conducted over a consecutive, multiday period.

Results: The same fNCI-directed measures were applied to assess therapeutic outcome. Reported findings showed significant, accelerated improvement of neurovascular recoupling (NVR) using fNCI-directed therapy in persistent models of mTBI. Patient averages revealed $73.7 \%$ decrease in NVU as measured by fNCI, and $65.8 \%$ improvement on conventional post-concussion symptom scale (PCSS) scores.

Conclusions: We provide practice-based evidence demonstrating the effectiveness of fNCI in clinical assessment and therapeutic guidance for chronic models of concussion. These outcomes are consistent with theoretical pathophysiological involvement of NVU in PCS.

Correspondence: Alina K. Fong, UT, United States. E-mail: alinafong@ yahoo.com

L.S. GAYNOR, M. SULLAN, J.M. GULLETT, M. AMIN, A. BOHSALI, R.M. BAUER, T. MARECI \& D. FITZGERALD. Alteration and/or loss of consciousness is associated with increased symptom report of PTSD, depression, sleep-related anxiety, and larger amygdala volume in OIF/OEF/OND Veterans.

Objective: Mild Traumatic Brain Injury (mTBI) and PTSD are highly prevalent in Veterans from recent theaters, and are associated with substantial cognitive and neuropsychiatric morbidity. This high degree of comorbidity suggests common biobehavioral substrates. Previous research has shown associations between PTSD and mTBI with volumetric changes in limbic regions associated with fear responding. This study evaluated associations between prior TBI indicators (alteration or loss of consciousness history; AOC/LOC) and symptoms of PTSD, depression, sleep-related anxiety, and amygdala volume $\geq 6$ months post-injury.

Participants and Methods: 8 Veterans with history of mTBI and 9 Veterans without history participated in the study. Symptoms were assessed with several self-report questionnaires (PTSD: PTSD Checklist-Military Version (PCL-M); Depression: Beck Depression Inventory. $2^{\text {nd }}$ Edition (BDI-II); Sleep-related Anxiety: Fear of Sleep Inventory (FOSI)). Spearman's rho and partial correlations controlling for whole brain volume were used to determine associations between variables. Mann-Whitney U Test was used to determine significance of PCL-m cluster scores between Veteran groups. Age and education did not differ significantly between groups.

Results: Frequency of LOCs and AOCs was positively associated with the FOSI (LOC: $\rho=.792, p<.001$; AOC: $\rho=.729, p=.002)$, PCL-M (LOC: $\rho=.630, p=.009 ;$ AOC: $r=.611, p=.012)$ BDI-II (LOC: $r=.599, p=.014$; AOC: $\mathrm{r}=.532, \mathrm{p}=.034)$, and amygdala volume (LOC: $\mathrm{r}=.571, \mathrm{p}=.033$; AOC: $r=.169, p=.563)$. Two PCL-m clusters significantly differed between Veteran groups (Cluster B, Re-experiencing: $p=.002$; Cluster C, Avoidance/Numbing: $\mathrm{p}=.011$ )

Conclusions: History of AOC/LOC was significantly associated with PTSD, depression, sleep-related anxiety, and increased amygdala volume. These findings suggest previous AOC/LOC should be considered when assessing and treating Veterans. The relationship between amygdala volume following TBI and mood and sleep -related symptoms requires further investigation.

Correspondence: Leslie S. Gaynor, Neuropsychology, Clinical and Health Psychology, University of Florida, 25 SW 5th Terrace, Apt 4331, Gainesville, FL 32601, United States.E-mail: lesliesg2@ufl.edu

H.M. GENOVA, K.S. CHIOU, Z. JIANG, M. HOPTMAN, J. DELUCA \& N. CHIARAVALLOTI. Longitudinal White Matter Changes Associated with Executive Functioning Outcome in Chronic Moderate to Severe TBI.

Objective: Cognitive outcome in Traumatic Brain Injury (TBI) is variable and a full understanding of this variability has eluded scientists for decades. Further, clinicians and researchers have yet to identify variables that consistently predict cognitive outcome in chronic TBI. The 
current study will examine longitudinal white matter integrity changes (assessed by Diffusion Tensor Imaging; DTI) in chronic TBI and their relationship with cognitive outcome.

Participants and Methods: The current study consisted of 23 participants: 15 persons with moderate to severe chronic TBI and 8 healthy controls. All participants were assessed at two time points, separated by 3 years. Assessments included neuropsychological testing (during which DKEFS Trail-Making, Color Word Inhibition and Verbal Fluency were administered) and a DTI scan. Tract Based Spatial Statistics (TBSS) of the DTI data was performed with FSL.

Results: In the TBI group, a significant positive correlation was observed between change in performance in DKEFS Trail Making and changes in Fractional Anisotropy (FA) over time in the left forceps minor and cingulum, as well as the right superior longitudinal fasciculus, forceps major, and inferior longitudinal fasciculus. That is, improvement in performance in Trail Making over time was found to be associated with increases in FA in those tracts. No significant associations were found between areas of FA change over time and change in performance on the DKEFS Color Word Inhibition or Verbal Fluency subtests.

Conclusions: The finding that changes in white matter integrity over time is associated with longitudinal improvements in cognitive functioning in chronic TBI offers promising potential for clinical application. Understanding the mechanisms underlying cognitive outcome allows for development of specifically targeted interventions that would ultimately increase the efficacy of rehabilitation efforts.

Correspondence: Helen M. Genova, Ph.D., Kessler Foundation, 300 Executive Drive, West Orange, NJ 07052, United States. E-mail: hgenova@kesslerfoundation.org

S.M. GREIF, A.R. SNYDER, L.J. ROTBLATT, L.S. GAYNOR, A.M. SVINGOS, G. RUIZ \& R.M. BAUER. Acute Symptom Recall and Sub-Acute Sleep and Mood Dysfunction in Mild Traumatic Brain Injury.

Objective: The purpose of this study was to investigate the relationship between symptoms recalled from the first 24 hours after mild traumatic brain injury (mTBI) and self-reported sleep and mood symptoms 1-2 weeks post-injury. Self-reported symptoms scales are frequently used for diagnosis, but they may also be valuable for helping to identify patients who are at-risk for increased sleep and emotional dysfunction within later stages of injury.

Participants and Methods: Participants included 20 individuals between the ages of 18-32 who were recently diagnosed with an mTBI. Participants were administered the Post Concussion Scale and asked to recall the symptoms they experienced within the first 24 hours after their injury. Sleep difficulties were assessed using the Medical Outcomes Study Sleep Scale (MOS-Sleep), mood symptoms were measured using the Beck Depression Inventory (BDI) and the State-Trait Anxiety Inventory (STAI). Multiple Linear regression was used to examine the relationship between 24 hour symptom recall, sleep, and mood.

Results: 24 hour symptom recall significantly predicted BDI, Trait STAI, and multiple sleep indices on the MOS-Sleep at $p<0.05$. Symptom severity ratings were directly related to sleep and mood dysfunction. Effect sizes were strongest for the MOS-Sleep, followed by STAI trait scores, then BDI.

Conclusions: In this preliminary sample, symptoms that participants recalled experiencing within 24 hours after mTBI significantly predicted levels of anxiety, depression, and sleep dysfunction 1-2 weeks postinjury. These results suggest that diagnostic tools like the PCS may also be used as screeners for more specific areas of mTBI dysfunction in later stages of recovery. Future research should examine 24 hour symptoms taken immediately after the injury and longer-term outcomes. Correspondence: Sarah M. Greif, BS, Clinical and Health Psychology, University of Florida, 507 NW 39th Rd, APT 202, Gainesville, FL 32607 , United States.E-mail:sgreif1021@phhp.ufl.edu
E.C. GROSSNER, K.S. CHIOU, R.A. BERNIER, E. BRENNER \& F.G. HILLARY. Examining Metacognitive Accuracy in Moderate and Severe TBI.

Objective: Metacognition (MC) can be impacted by traumatic brain injury (TBI). This study examined MC during an abstract reasoning task after TBI and correlated those measurements with brain volume alterations. We also examined the influence of task anchoring (item difficulty order) on task accuracy and MC judgment.

Participants and Methods: 36 TBI and 38 matched healthy controls underwent the same procedure, including BOLD FMRI scan and neuropsychological testing. The battery included modified Matrix Reasoning tasks, which required confidence judgments. Participants received this subtest twice, once with the trials in order of difficulty (ordered list) and once randomized for difficulty (random list). Gamma coefficients were calculated as a measure of MC accuracy. Whole brain volumes were also calculated using T1-weighted structural images in VBM8.

Results: TBI and HC groups were comparable on Matrix Reasoning performance. T-tests revealed no significant difference in MC accuracy between healthy control and TBI participants. Regardless of group, MC accuracy was higher for the ordered than the random list, $p=0.068, d$ $=0.30$. Healthy controls were more accurate for the ordered list than the random list, $p=0.036, d=0.49$. TBI participants did not differ in accuracy between the two lists. Additionally, a Pearson correlation revealed no significant relationship between MC accuracy and total gray matter volume.

Conclusions: There was a difference in MC accuracy for ordered and random lists, with healthy controls performing better on the ordered list than the random list. TBI participants did not show any difference in MC ability between the differently anchored lists, demonstrating that they did not benefit from the same anchors as the HC participants. There was no significant relationship between total gray matter volume and MC accuracy and we anticipate that more specific analyses (e.g., frontal cortical thickness) could provide greater sensitivity to these relationships.

Correspondence: Emily C. Grossner, Psychology, The Pennsylvania State University, 650 Toftrees Ave., Apt. 212, State College, PA 16803, United States. E-mail: epg5130@psu.edu

J. HAMMOND, J. REIS, A.A. HOMAECHEVARRIA \& D. HENDERSON. Trends in Helmet Use and Concussion Among Skiers and Snowboarders: Using an Innovative Data Collection Technique for Injury Prevention.

Objective: This study examined trends in helmet use and concussions among skiers and snowboarders from 2011 to 2016. Given the increasing awareness of sports-related brain injuries, it was hypothesized that helmet use would increase and concussion incidence would decrease over time.

Participants and Methods: Using an innovative data collection method, data (demographic factors, helmet use, injury characteristics, trail features) were entered by ski patrol at three ski resorts immediately after an injury. From 2011 to 2016, 2,656 incidents were recorded (age 3 - 85 years; $58.2 \%$ male, $41.8 \%$ female).

Results: A significant increase in helmet use was observed from 2011 $(49.4 \%)$ to $2016(63.3 \%), \chi^{2}(3, n=2645)=31.28, p<.001$. Ability, age, equipment type [TYPE] and ownership [OWN], history of professional instructions, medical insurance [INSURANCE], previous trail exposure, season pass status [PASS], and trail rating [TRAIL] were significantly associated with helmet use. Logistic regression analyses indicated that OWN $(O R=2.74)$, INSURANCE $(O R=2.29)$, and PASS $(O R=2.23)$ significantly contributed to the model. In contrast, there was no significant difference in the number of concussions from 2011 (10.3\%) to 2016 $(9.4 \%), \chi^{2}(3, n=2656)=0.46, p=.93$. Age, TYPE, OWN, and TRAIL were significantly associated with sustaining a concussion. Logistic regression analyses indicated that $O W N(O R=1.53)$, age $(O R=0.99)$, and TYPE $(O R=0.72)$ significantly contributed to the model.

Conclusions: Rates of helmet use changed in a positive direction for the resorts in the study. Because the severity of concussion was not 
recorded, it is unknown whether this increase was associated with less serious head injury. Demographic, behavioral, and trail factors may be useful to predict helmet use and concussions. This research extends our current understanding of ski helmet use behaviors and brain injury prevention, minimizes limitations noted in the literature (e.g., recall bias), and enhances the role of neuropsychology in injury prevention research and practice.

Correspondence: Julia Hammond, MA, University of Montana, Department of Psychology Skaggs Building Room 143, Missoula, MT 59812, United States. E-mail: julia.hammond@umontana.edu

L. HARIK, G. WHITENECK, M. SHERER, E. NGAN \& L. NOVELO. Comparison of the Neurobehavioral Symptom Inventory and the Rivermead Postconcussion Questionnaire.

Objective: Traumatic brain injury (TBI) results in a constellation of symptoms that include emotional, behavioral, physical, and cognitive complaints. The Neurobehavioral Symptom Inventory (NSI) and the Rivermead Post-Concussive Symptoms Questionnaire (RPQ) are two measures used to assess subjective ratings of these complaints. The goal of the current study was to determine the similarity of constructs measured by the two scales.

Participants and Methods: Inclusion criteria were (a) medical documentation of TBI, (b) age 18 to 64 years, (c) capacity to give informed consent, (d) ability to complete all study measures in English, (e) absence of interfering medical or psychiatric condition. Participants were selected from a convenience

sample of persons with TBI who were living in the community and were able to complete the study assessment. Mean time since injury was six years. Exploratory factor analysis was conducted including all 36 items from both measures.

Results: Total $n=497$. The correlation between total scores for the NSI and RPQ was 0.89. Exploratory factor analysis revealed that items of similar content between the two measures loaded similarly within factors and appeared to define similar constructs. Factors reflected the categorical nature of complaints into emotional, physical, sensory, and cognitive complaints.

Conclusions: Evidence from the exploratory factory analysis indicated that the NSI and RPQ assess highly similar constructs. Indeed, items that assessed the same type of complaint (e.g., emotional distress) loaded on the same factors. These data support development of a "crosswalk" between these measures so that existing literature that includes findings from either measure can be easily compared.

Correspondence: Lindsey Harik, Ph.D., Psychology, TIRR, 421 Harvard St, Unit G, Houston, TX 77007, United States.E-mail: linharik@gmail. com

S.N. HOFFMAN, S.M. JURICK, L.D. CROCKER, N.J. STEELE, A.V. KELLER, A.A. RAUCH, N.E. DEFORD, M. SANDERSONCIMINO \& A.J. JAK. Patterns of Performance and Symptom Validity Tests in Iraq and Afghanistan Veterans with Mild Traumatic Brain Injury History.

Objective: Treatment-seeking Operation Enduring and Iraqi Freedom (OEF/OIF) Veterans with mild TBI (mTBI) history have higher than expected over-reporting rates on symptom validity tests (SVT) and suboptimal performance on neuropsychological tests of performance validity (PVT). The present study examines OEF/OIF Veterans who fail SVTs but pass PVTs (SV-Fail), fail PVTs but pass SVTs (PV-Fail), fail both PVTs and SVTs (B-Fail), or pass both (B-Pass).

Participants and Methods: A neuropsychological exam, including the Minnesota Multiphasic Personality Inventory Second Edition Restructured Form (MMPI-2-RF) and the Test of Memory Malingering (TOMM), was given to $48 \mathrm{OEF} / \mathrm{OIF}$ Veterans with mTBI history. The SV-Fail group was divided into subgroups based on elevations on psychopathological (F-scales), somatic/cognitive (RBS/FBS scales), or both types of scales. SV-fail and PV-fail groups were compared via one-way ANOVA on neuropsychological memory tests, MMPI-2-RF scales, and self-reported psychological measures.

Results: 21 of the 48 Veterans performed below expectation on one or more MMPI-2-RF validity scales (1 on F-scales only, 3 on RBS/FBS scales only, and 17 on both types). Of those 21, 15 performed at or above cutoff on TOMM. This SV-Fail group $(n=15)$ had significantly higher F-scale scores and self-reported anxiety compared to PV-Fail $(n=10)$ and B-Pass ( $n=17)$ groups and higher self-reported PTSD symptoms compared to B-Pass. The PV-Fail group had significantly lower cognitive performance and lower F-scale scores compared to SV-Fail, lower cognitive performance compared to B-Pass, and lower self-report depression and anxiety symptoms compared to B-Fail $(n=6)$. The B-Fail group had significantly higher depression and anxiety symptoms, lower PVT scores, and higher F-scale scores compared to the B-Pass group. Conclusions: $65 \%$ of the sample had poor SVT and/or PVT scores. However, distinctions between PVT (21\%) and SVT (31\%) failures hold important implications for interpreting neuropsychological and symptom self-report measures

Correspondence: Samantha N. Hoffman, Psychiatry, VA San Diego Healthcare System, Veterans Medical Research Foundation, 3350 La Jolla Village Drive, Bldg. 13, 3rd Floor MC151B, San Diego, CA 92161, United States. E-mail: samantha.hoffman94@gmail.com

S.M. JURICK, L.D. CROCKER, M. SANDERSON-CIMINO, A.V. KELLER, S.N. HOFFMAN, A.A. RAUCH, L.S. TRENOVA, B. BOYD, R. AUPPERLE, E.W. TWAMLEY, C. RODGERS \& A.J. JAK. Contributions to Executive Dysfunction in Operation Enduring Freedom/Operation Iraqi Freedom (OEF/OIF) Veterans with Post-Traumatic Stress Disorder and Traumatic Brain Injury History.

Objective: Post-traumatic stress disorder (PTSD) and mild traumatic brain injury (mTBI) history are highly comorbid in OEF/OIF veterans and associated with executive functioning (EF) deficits. The present study examined contributions of injury details, processing speed/attention (PS/A), and PTSD symptoms to EF.

Participants and Methods: Veterans with PTSD and mTBI history $(\mathrm{N}=65)$ underwent neuropsychological assessment within a treatment study. An EF-deficit group $(n=34)$ was comprised of Veterans with impaired scores (>1 SD below a normative mean) on one or more tests of perseveration (Wisconsin Card Sorting Test-64 perseverative responses), working memory (WAIS-IV digit span sequencing), switching (D-KEFS number-letter switching), or inhibition (color word inhibition or inhibition/switching). The EF-intact group $(n=31)$ did not fall below $1 \mathrm{SD}$ on any measure. One-way ANOVA and linear regressions were used to assess relationships between EF and PS/A, injury details, and PTSD symptoms.

Results: The EF deficit group had greater PTSD symptoms and poorer PS compared to the EF-intact group. Worse PS/A was related to poorer performance on nearly all tests of EF. Greater PTSD symptoms were related to worse switching, inhibition, and perseveration controlling for injury variables and PS/A. History of greater than three mTBIs was associated with worse performance on switching. Finally, the relationship between PS and inhibition/switching was stronger for those with a history of three or more mTBIs and loss of consciousness (LOC).

Conclusions: PS/A and most tests of EF were related, and PTSD contributed to switching, inhibition, and perseveration controlling for PS/A and injury details. History of greater than three mTBIs was associated with poorer switching performance, and along with LOC history, influenced the relationship between PS/A and inhibition/switching. Given the comorbidity and overlapping presentation of PTSD and $\mathrm{mTBI}$, present findings may provide insight into underlying processes of EF and inform treatment planning.

Correspondence: Sarah M. Jurick, B.A., Psychiatry, University of California San Diego, 9500 Gilman Drive, Mail Code 0603, La Jolla, CA 92037-0603, United States. E-mail:smjurick@gmail.com 
E. KAPLAN, L. SPIELMAN \& W. GORDON. Self-Discrepancy and Perfectionism: Two Factors Associated with Depression in Individuals with Traumatic Brain Injury.

Objective: The phenomenology of post-traumatic brain injury (TBI) depression is poorly understood. The purpose of this study was to examine relationships among self-discrepancy, perfectionism and depression in individuals with TBI.

Participants and Methods: Surveys were completed by 60 adults with mild $(56.7 \%)$, moderate $(15.0 \%)$ or severe $(28.3 \%)$ TBI between 6 months and 51 years post injury $(\mathrm{M}=11.05, \mathrm{SD}=9.6)$. Self-discrepancy, defined as the difference between one's pre-TBI self-perception and current self-perception, was measured using an adapted version of the Integrated Self-Discrepancy Index. Perfectionism and depression were measured using the Multidimensional Perfectionism Scale and Beck Depression Inventory-II

Results: Age was significantly associated with perfectionism and anxiety was significantly associated with post-TBI depression. Self-discrepancy and perfectionism were not associated with one another. Multiple regression analyses adjusting for age and anxiety indicated that self-discrepancy $(\beta=.25, p<.05)$ and perfectionism $(\beta=.37, p<.01)$ were significantly associated with depression. A comprehensive model that included age, anxiety, self-discrepancy and perfectionism explained $44.1 \%$ of the variance in depressive symptoms $(\mathrm{F} \Delta=9.35, \mathrm{p}<.01)$. an increase of $16.4 \%$ from the percentage of variance explained by age and anxiety alone.

Conclusions: Self-discrepancy and perfectionism are uniquely associated with post-TBI depression and the presence of both factors results in the strongest explanatory model. Relationships among self-discrepancy. perfectionism and depression should be further explored across injury severity. Potential impacts of these factors on cognitive interventions should also be considered

Correspondence: Erica Kaplan, PhD, Rehabilitation Medicine, Mount Sinai, 201 Avenue A Apt 1, New York, NY 10009, United States. E-mail: erica.r.kaplan@gmail.com

A.R. KAUP, R. TOOMEY, K.J. BANGEN, L. DELANO-WOOD, K. YAFFE, M.S. PANIZZON, M.J. LYONS, C.E. FRANZ \& W.S. KREMEN. Lifetime Traumatic Brain Injury with Current Psychiatric Symptomatology Impacts Cognition among Late Middle-Aged Men: Findings from the Vietnam Era Twin Study of Aging (VETSA).

Objective: Traumatic brain injury (TBI), posttraumatic stress disorder (PTSD), and depression are each known to increase risk for cognitive impairment in aging. We investigated whether these conditions interact to influence cognition among late middle-aged men from the Vietnam Era Twin Study of Aging (VETSA).

Participants and Methods: 1018 VETSA participants [mean age $=61.6$ (range 56-66)] were interviewed to ascertain lifetime TBI, and completed the PTSD Checklist, Center for Epidemiologic Studies Depression Scale, and a comprehensive cognitive test battery. In mixed effects models, we tested the effect of TBI on cognition. including interactions between TBI and current psychiatric svmptomatology (PTSD and/or depression) on cognition. All models were adjusted for demographics, education, premorbid cognitive ability (Armed Forces Qualification Test at mean age of 20), site, APOE- 4 , cardiovascular comorbidities, and alcohol/ drug abuse.

Results: $31 \%(n=314)$ of the sample reported history of at least one TBI. $24 \%(n=73)$ of this TBI group endorsed either current PTSD and/ or depression, compared to $18 \%(n=118)$ of those without TBI. Results showed significant TBI $x$ PTSD/depression interactions on executive function (Abstract Reasoning: $p=.03$; Trail Making Switching: $p=.045$; Stroop Inhibition: $p<.001$ ), such that men with both TBI and current PTSD/depression demonstrated executive deficits, while men with TBI alone or PTSD/depression alone did not. There was no effect of TBI (nor interactions) on any other cognitive domain (all $p>.05$ ).

Conclusions: Among community-dwelling middle-aged men, the combination of lifetime TBI and current psychiatric symptomatology
(PTSD/depression)—but neither alone-appears to increase risk for executive dysfunction. Future study is needed to understand how these factors interact to impact brain aging and whether emotional well-being may buffer the effects of TBI.

Correspondence: Allison R. Kaup, PhD, Research / Psychiatry, San Francisco VA Healthcare System / University of California San Francisco, 4150 Clement St, 116B, San Francisco, CA 94121, United States.E-mail: allison.kaup@ucsf.edu

E. KEATLEY, C. ABEARE, B.G. ZUCCATO, T.L. MCAULEY \& J.E. CASEY. Use of the Depression Anxiety and Stress Scale (DASS) in Varsity Athletes for Baseline Concussion Assessment: Psychometric Properties, Comparison with Non-Athletes, and Frequency of Item Endorsement.

Objective: The clinical utility of the Depression, Anxiety, and Stress Scale (DASS) was evaluated for use in collegiate athletes during pre-season baseline concussion testing. Standard baseline concussion evaluations utilize post-concussion symptom questionnaires that typically have only a few items related to psychiatric functioning. Psvchiatric functioning is related to athletic performance as well as recovery from concussion, making it an important area of assessment in athletes as part of baseline concussion evaluations.

Participants and Methods: The DASS was administered to 366 collegiate athletes during baseline concussion testing as well as 119 undergraduate non-athlete comparison group. Comparative analyses and internal consistency reliabilities were calculated for each DASS subtest, for both the full version of the DASS (DASS-42) as well as the abbreviated version (DASS-21). Patterns of item endorsement were examined. Results: Results demonstrated good internal consistency reliability of the DASS-42 for both groups. However, the DASS-21 had adequate reliability for only the non-athlete group. Consistent with previous research, athletes had lower DASS scores in general. Across both groups, the stress subscale had the highest scores.

Conclusions: The DASS- 42 was shown to have adequate internal consistency reliability for collegiate athletes during baseline concussion testing. Athlete performance on the DASS following concussion can be compared to the reported frequency of item endorsement in the current study.

Correspondence: Eva Keatley, BS, Clinical Neuropsychology, University of Windsor, 401 Sunset Ave, Windsor, ON N9B 3P4, Canada. E-mail: keatley@uwindsor.ca

R.E. KEELAN, L. RAPPORT, S. KROHNER, R. KANSER, N. BILLINGS, J. BASHEM, B. VERMILION, R. HANKS, M. LUMLEY \& S.A. LANGENECKER. The Role of Experienced Affect on Facial Emotion Perception Accuracy in Moderate to Severe Traumatic Brain Injury.

Objective: People with TBI experience high rates of emotion perception difficulties and mood changes after injury; however, studies exploring links between experienced affect and emotion perception after TBI are sparse.

Participants and Methods: Participants were 50 adults with moderate-to-severe TBI and 39 healthy comparisons (HC). Participants completed a neuropsychological assessment, including measures of experienced affect (depression and affect intensity) and a face emotion perception task (FEPT). The FEPT requires participants to identify happy, sad, angry, fearful, and neutral faces.

Results: For the TBI group, there were significant inverse relations between affect intensity and FEPT accuracy. However, curve estimation revealed a significant quadratic model, indicating inverse relation between affect intensity and FEPT among low FEPT performers and positive relation between affect intensity and FEPT among high FEPT performers. No significant correlations were observed for the HC group. Significant linear and quadratic trends also were observed between depressive symptoms and FEPT for the total sample. 
Conclusions: Among people with TBI with impaired emotion perception, high levels of affect intensity may disrupt accurate recognition of others' emotions, whereas acknowledging one's own depressive experience may heighten sensitivity in recognizing others' emotions. Consistent with the Yerkes-Dodson Law, emotional arousal and depressive symptoms can become disruptive to accurate identification as they surpass an asymptote. Additionally, given simultaneous demands of monitoring internal and external emotion. individuals with TBI mav have difficulty dual tasking in the presence of high affect intensity and depression. Future research should examine benefits of psychoeducation with patients and families, as well as teaching emotion regulation strategies for decreasing adverse effects of intense emotional experience and improving emotion perception.

Correspondence: Rachel E. Keelan, M.A., Clinical Psychology, Wayne State University, 5057 Woodward Ave, Detroit, MI 48202, United States.E-mail:kayrach@wayne.edu

A.V. KELLER, L.D. CROCKER, S.M. JURICK, J. BOMYEA, N.E. DEFORD, C.C. HAYS, E.W. TWAMLEY \& A.J. JAK. The Role of Cognitive Functioning in Suicidal Ideation in Veterans with a History of Mild Traumatic Brain Injury and Comorbid Psychiatric Conditions.

Objective: Suicidal ideation is highly prevalent in Iraq and Afghanistan-era (OEF/OIF) Veterans with mild traumatic brain injury (mTBI) history and comorbid psychiatric conditions. Neuropsychological abilities may be fundamental for how individuals think about suicide and generate and/or utilize alternative behaviors and coping strategies, though to date few studies have examined the role of cognitive functioning in suicidal ideation. Therefore, we examined associations between cognitive performance and suicidality in Veterans with a history of mTBI.

Participants and Methods: OEF/OIF Veterans with mTBI history seeking outpatient services ( $\mathrm{N}=258$ ) completed a clinical neuropsychological assessment, and measures of PTSD, depression, and neurobehavioral symptoms. Participants were divided into two groups based on responses to an item on a depression measure: suicidal ideation endorsers $(\mathrm{SE}=43)$ and non-endorsers $(\mathrm{NE}=215)$. T-tests and logistic regressions were used to examine relationships between cognitive performance and group membership.

Results: Groups did not differ on demographic variables, premorbid intellectual functioning, or TBI injury characteristics. The SE group endorsed more severe PTSD, depression, and neurobehavioral symptoms and performed significantly worse on tests of delayed verbal and visuospatial memory compared to the $\mathrm{NE}$ group. There were no group differences in other cognitive domains. Logistic regressions revealed that a composite of the memory measures uniquely predicted suicidal ideation above PTSD, depression, and neurobehavioral symptoms.

Conclusions: Cognitive dysfunction specific to memory confers additional risk of suicidal ideation beyond emotional distress and neurobehavioral symptoms in OEF/OIF Veterans. These findings suggest that implementing treatment strategies that target verbal and visuospatial memory difficulties (e.g., cognitive rehabilitation) may reduce suicide risk.

Correspondence: Amber V. Keller, Bachelor of Arts in Psychology, Research, VA San Diego Healthcare System, 3350 La Jolla Village Drive, Building 13 MC151B, San Diego, CA 92161, United States. E-mail: ambervkeller@gmail.com

J. FRANCO, M. MILLAN, B.R. SHANE, A. CASTELlanOS \& W. KILLGORE. Blue Wavelength Light Therapy Increases Thalamic Grey Matter Volume Following Mild Traumatic Brain Injury.

Objective: Approximately $50 \%$ of patients with traumatic brain injury (TBI) complain of sleep disturbances. Current research shows that bright light therapy (BLT), which has been used in the past to treat depression, is also effective in reducing fatigue in patients with TBI.
However, the underlying mechanisms are still unknown. We hypothesized that six weeks of blue BLT would lead to improved sleep and induce structural changes in the brains of individuals with mild TBI. Participants and Methods: In a double blind design, 28 subjects (18-45 years of age) with mild TBI were randomly assigned to either the blue BLT treatment group or the amber BLT placebo group. Actigraphy watches were used for at-home monitoring of sleep and wake patterns, and the Epworth Sleepiness Scale (ESS) was administered to measure daytime sleepiness. MRI scans of each subject's brain were collected and voxel based morphometry (VBM) was utilized to measure changes in grey matter (GM) volume following treatment.

Results: Six weeks of blue BLT significantly shifted subjects' circadian rhythms towards earlier bedtimes $(p<0.05)$, and reduced davtime sleepiness by $30 \%$ when compared to pre-treatment values $(\mathrm{p}<0.01)$. Blue BLT also caused a significant increase in thalamic GM after controlling for age, gender, and total intracranial volume. Specifically, the thalamic pulvinar nucleus had a significantly larger GM volume after treatment when compared to that of TBI subjects who had undergone the placebo therapy ( $p<0.01$ for difference in amber to blue post; $\mathrm{p}<0.0001$ for difference in blue pre to post).

Conclusions: Blue BLT improved timing of sleep by causing subjects to go to bed an hour earlier than their pre-treatment bedtime. It also reduced daytime sleepiness and significantly increased thalamic GM volume. These findings represent a first step to understanding how BLT works on a neurological level. In the future, light therapy may prove to be a novel and effective way to treat sleep disturbance and improve neurocognitive recovery following a mild TBI.

Correspondence: William D. "Scott" Killgore, PhD, 1501 N Campbell Ave, Tucson, AZ 85724, United States.E-mail:killgore@email.arizona. edu

R.T. KIM, S.F. SORG, K.A. HOLIDAY, L. DELANO-WOOD, M. MELOY, A.L. CLARK, M. TRAN, E. LOCANO, A.J. JAK, L.T. EYLER \& D. SCHIEHSER. Brain Function and Task Performance Predict Self-Reported Disinhibition and Executive Function in Veterans with Mild-Moderate Traumatic Brain Injury. Objective: Veterans often report neurobehavioral and cognitive changes following mild-moderate traumatic brain injury (mmTBI); however it is unclear whether self-reported complaints including disinhibition and executive dysfunction (EDF) are related to objective cognitive performance and brain function, especially in the context of PTSD. We therefore examined whether objective cognitive performance, brain activation, and/or PTSD symptoms best predicted self-reported disinhibition and EDF in mmTBI Veterans.

Participants and Methods: 56 Veterans with mmTBI and 35 Veteran controls (VC) completed the Frontal Systems Behavior Scale (FrSBe), a measure of subjective disinhibition and EDF, and PTSD Checklist-Military. Participants also completed the Go/NoGo task, a cognitive inhibition task, while undergoing fMRI.

Results: Groups did not differ on Go/NoGo performance (d') or BOLD signal change, but the mmTBI group reported higher FrSBe scores and PTSD $(p$ 's<.05) compared to VCs. In the mmTBI group, subjective disinhibition and EDF were related to worse d' and PTSD $\left(p^{\prime} s<.02\right)$. Greater levels of subjective disinhibition were associated with increased signal change in the bilateral rostral anterior cingulate (AC; $p$ 's<.01), while EDF was associated with increased signal change in the bilateral caudal AC, left rostral AC, and precentral gyrus ( $p$ 's<.03). In regression analyses, increased signal change in the right rostral AC, d', and PTSD all predicted subjective disinhibition; EDF was predicted by PTSD and increased signal change in the left precentral gyrus $(p ' s<.04)$.

Conclusions: This study represents one of the first to empirically link subjective disinhibition and executive dysfunction with objective cognition and brain function in mmTBI. Subjective disinhibition and EDF appear to be dissociated by differing neural mechanisms and possible subsequent decrements in task performance. Findings underscore the importance of detailed measurement and highlight the potential utility of neuroimaging to elucidate neurobehavioral changes in mmTBI. 
Correspondence: Russell T. Kim, B.S. - Psychology, Research, VA San Diego Healthcare System, Veterans Medical Research Foundation, 3350 La Jolla Village Dr., San Diego, CA 92161, United States. E-mail: russelltkim@gmail.com

\section{J.W. KIRTON \& J. SADEK. Differential diagnosis of repetitive mild traumatic brain injury and posttraumatic stress disorder: Three illustrative cases.}

Objective: Mild traumatic brain injury (mTBI) and posttraumatic stress disorder (PTSD) are a common differential diagnoses. With warriors returning from a decade of war the physical, mental, and neurological scars are with the focus of long-term treatment. Over the past decade the amount of research on TBI has exploded, with controversy surrounding whether repeated mTBI can cause neurological damage. Through our 3-case series we explore differential diagnosis of mTBI and PTSD. We explore current theories of mTBI and of PTSD including psychological and physiological models of PTSD. We review the neuroanatomy of mTBI and PTSD and discuss how it explains similar cognitive profiles in etiologically different cases.

Participants and Methods: 3 OEF/OIF combat veterans referred for neuropsychological testing in the context of mild TBI and PTSD. Clinical interview, review of medical records, and identical neuropsychological test batteries administered to each patient.

Results: Two patients' clinical history and neuropsychological profiles suggested PTSD as the etiology of cognitive impairment. One patient's clinical history and neuropsychological profiles suggested repetitive mild TBI as the likely etiology.

Conclusions: PTSD and mTBI can have overlapping neuropsychological profiles but can have distinct etiologies. More research is needed to determine whether repetitive mTBI can cause cognitive impairment independently from PTSD. Repetitive mTBI as a source of long lasting cognitive changes remains a controversy.

Correspondence: Joshua W. Kirton, M.S., Clinical and Health Psychology, University of Florida, P.O. Box 100165, Gainesville, FL 32610, United States. E-mail: jwkirton13@yahoo.com

E. KORNBLITH, T. NOVAKOVIC-AGOPIAN, G. ABRAMS, J. BURCIAGA \& K. MARTON. Neurocognitive Profiles Among Veterans with History of Traumatic Brain Injury.

Objective: Traumatic brain injury (TBI) is common among Veterans of all eras. A wide range of neurocognitive and functional outcomes has been documented in TBI patients, especially for mild and moderate TBI. The goal of the current study is to describe neuropsychological status and associated functional outcomes in a cohort of Veterans with history of chronic TBI.

Participants and Methods: Thirty-two Veterans with a history of chronic TBI (mild-severe; average time since injury $=11$ years) completed a battery of measures which included neuropsychological evaluation, functional assessment of executive function [Goal Processing Scale (GPS), Novakovic-Agopian et al., 2014], and self-report measures of emotional and functional status. Neuropsychological composite domain scores of Overall Attention/Executive Function (OA/EF), Auditory Working Memory (AWM), Sustained Attention, Mental Flexibility, Inhibition, Composite Memory (CM), Total Recall (TR), and Delaved Recall (DR) were used to place participants into homogeneous clusters. Results: Two distinct clusters were identified: "Functionally Impaired" (FI) and "Non-impaired." The FI cluster was characterized by mean Z scores lower than -.75 on measures of OA/EF, AWM. CM. TR, and DR. The Non-impaired cluster exhibited stronger performance on the GPS functional assessment of executive function. The clusters differed on re-experiencing PTSD symptoms and post-TBI community integration, with the FI cluster endorsing more problems in these domains. Clusters did not differ on demographic variables or injury severity.

Conclusions: These results suggest that in a sample of Veterans with chronic TBI, neurocognitive outcomes may be independent from injury severity. Neuropsychological measures, especially of complex attention. executive function, and memory, may aid in identifying TBI patients with functional impairment and assist with treatment planning. Support from VA Rehabilitation Research and Development Correspondence: Erica Kornblith, PhD, San Francisco VAMC, 4150 Clement St, San Francisco, CA 94121, United States. E-mail: eschimbor@gmail.com

J. LARA-RUIZ, D. DELGADO, E. ROMERO, S. AMAYA, E. WAITE, D. HARDY, P. VESPA, D. MCARTHUR, D. HOVDA, C. WANG, R. SWERDLOFF, D.F. KELLY, K.M. GUSKIEWICZ \& M.J. WRIGHT. Anxious and Depressive Symptoms in Retired Professional Football Players: A Contrast with Persons With and Without Moderate-to-Severe Traumatic Brain Injury.

Objective: Traumatic brain injury (TBI) has been associated with anxiety and depression (Ponsford et al., 2008; Bombardier et al., 2010). Sports-concussion is also associated with mood disturbances (McCrory et al., 2013). We sought to compare reported depressive and anxious symptoms in retired professional football players (FB), persons with moderate-to-severe TBI, and healthy participants.

Participants and Methods: Data from two IRB approved studies were combined for the current analysis. Data from male FB $(n=36)$, TBI $(n=16)$, and comparison $(n=20)$ participants were used. The FB participants all suffered at least 3 sports-related concussions during their professional career, but were free from other neurological injuries. The TBI participants suffered moderate-to-severe injuries primarily due to acceleration-deceleration events. All participants completed the Hospital Anxiety and Depression Scale (HADS).

Results: The FB group was somewhat older and more educated than the other groups $(p<<.001)$. ANCOVAs, covarying for age and education, failed to reveal any group differences in depression ratings, but an effect of group was detected for anxiety ratings. Pairwise comparisons showed that the FB group had greater anxiety ratings than the TBI and comparison groups. The TBI and comparison group did not significantly differ in their anxiety ratings. FB participant's game play characteristics (position, seasons played, and concussion frequency) did not correlate with their anxiety ratings.

Conclusions: Our data suggest that retired professional football players experience significant symptoms of anxiety that may be greater that those experienced by individuals with moderate-to-severe TBI, although our TBI sample was relatively small and may not be very representative of the TBI population. Nevertheless, the FB group reported clinically significant symptoms of anxiety. It is unclear if their experience of sports-related concussion or other factors are driving this relationship. Correspondence: Jose Lara-Ruiz, CA, United States.E-mail: jlara.ruiz@ gmail.com

C. LEVITCH, M.E. ZIMMERMAN, N. WAKSCHLAG, N. KIM, R. LIPTON, W. STEWART, M. SLIWINSKI \& M. LIPTON. Recent and Long-Term Soccer Heading Exposure is Differentially Associated with Cognitive Impairment in Adult Amateur Players. Objective: The goal of this study was to examine the relationship between neuropsychological function and recent or long-term heading (repetitive sub-concussive head impacts during soccer play) in amateur adult soccer players.

Participants and Methods: Soccer players (N=257) completed previously validated questionnaires to estimate long-term heading (LTH: prior $12 \mathrm{mos}$; both games and practices) and recent heading (RH; prior 2 wks). CogState was used to assess neuropsychological function. For LTH, we divided participants into quartiles: Low Heading (LH), Moderate Heading (MH), High Heading (HH), and Very High Heading (VHH). RH was defined as four groups: No Heading (NH), and the remaining groups were tertiles (MH, HH, VHH). Linear regression was used to examine the relationship between LTH or RH group and neuropsychological function, controlling for age, race, ethnicity, sex, premorbid reading ability, years of heading exposure, and concussion history. 
Results: Participants had an average age of $26 \mathrm{yrs}(\mathrm{sd}=8)$ and headed the ball a median of 677 times/yr and 3 times/2wks. For LTH, participants with $\mathrm{VHH}$ in games had poorer performance on tests of verbal learning $(p<.001)$ and memory $(p=.019)$ compared to the LH group. For RH, participants with VHH had poorer performance on tests of processing speed $(p=.020)$ and attention $(p=.020)$ compared to the other RH groups. Previous concussions were reported by 88 (34.2\%) participants. Concussion history was not related to cognitive function and did not modify the association of heading and cognition.

Conclusions: Adult amateur soccer players with high levels of longterm heading had poorer performance on a test of memory, while players with high levels of recent heading performed poorly on tests of processing speed and attention. These findings were independent of the effects of concussion. They suggest short-term cognitive consequences of repetitive sub-concussive impacts may not persist over time, whereas verbal learning and memory are adversely affected by cumulative longterm exposure.

Correspondence: Cara Levitch, BAS, Psychology, Fordham University, 151 East 31st Street, Apt 17G, New York, NY 10016, United States. E-mail: clevitch@fordham.edu

L.M. FRENCH, T.A. BRICKELL, S.M. LIPPA, R. GARTNER, A.E. DRISCOLL, M.M. WRIGHT, B.N. PIZZANO, L. JOHNSON, D.E. NORA, H.S. MAHATAN, J.K. SULLIVAN, D.M. THOMPSON, A.C. SNELLING, B.R. YANOS, T.J. CASTILLO, C.E. SIMON \& R.T. LANGE. A Case Series Analysis of the Natural History of Neurocognitive Performance following Moderate, Severe, and Penetrating Traumatic Brain Injury in U.S. Military Service Members.

Objective: The purpose of this study was to examine neurocognitive performance trajectories following moderate, severe, and penetrating TBI.

Participants and Methods: Participants included 16 U.S. military service members (age: $\mathrm{M}=30.6$ years, $\mathrm{SD}=8.5$ ) with moderate-severe $(n=13)$ or penetrating $(n=3)$ TBI who passed performance validity tests, prospectively enrolled in a longitudinal study at Walter Reed National Military Medical Center. Participants were assessed at baseline (M=16.8 months post-injury $[\mathrm{SD}=27.6])$ and follow-up $(\mathrm{M}=35.8$ months post-injury $[\mathrm{SD}=34.8])$. From a larger test battery, 24 measures were selected to create six cognitive summary scores.

Results: At both baseline and follow-up, processing speed and visual spatial skills were the most common domains with multiple low scores. In contrast, attention, learning, memory, and executive functioning were least likely to be impaired at baseline or follow-up. Over time, attention $(n=7)$ and processing speed $(n=7)$ were most likely to decline, memory was most likely to improve $(n=8)$, and learning $(n=7)$ and executive functioning ( $\mathrm{n}=9$ ) were most likely to remain stable. When considering individual cognitive profiles, 5 participants declined over time, 8 improved, and 3 demonstrated variable performance. Both participants in the chronic stage of recovery at baseline declined over time. Twelve of the 16 cases $(75.0 \%)$ met DSM-IV criteria for mild neurocognitive disorder (NCD) at baseline and 10/16 participants (62.5\%) met criteria for mild NCD at follow-up. Over time, 8 participants met criteria for mild NCD at both time points, 4 improved, 2 developed mild NCD, and 2 never met criteria for mild NCD.

Conclusions: Service members with moderate-severe and penetrating TBI demonstrated a number of different neurocognitive performance trajectories. In general, processing speed and visuospatial skills were the most common areas of low performance. Over time, attention and processing speed was most likely to decline and memory was most likely to improve.

Correspondence: Sara M. Lippa, PhD, Boston VA Hospital, Walter Reed National Military Medical Center, Bethesda, MD 20889, United States. E-mail:saralippa@gmail.com
R.T. LANGE, L.M. FRENCH, S.M. LIPPA \& T.A. BRICKELL. A Cross-sectional Perspective of Neurocognitive Outcome from Military-related Mild-moderate TBI in the Sub-acute Recovery Period and 1-year post-injury.

Objective: To examine neurocognitive outcome following mild-moderate traumatic brain injury (TBI) in the sub-acute recovery phase, and at 1-year post-injury, using a cross-sectional study design.

Participants and Methods: Participants were 83 U.S. military service members who had sustained a mild-moderate TBI, and 15 service members who had sustained orthopedic injuries without brain injury (i.e., Trauma Controls [TC]). Participants completed a 5-6 hour neuropsychological test battery within 6-months of injury $(\mathrm{M}=3.6, \mathrm{SD}=2.8)$ and/or at 1-year post-injury $(\mathrm{M}=11.9, \mathrm{SD}=1.1)$.

Results: In the sub-acute recovery phase, compared to the TC group, lower scores (i.e., meaningful effect sizes $[\mathrm{d}>.30]$ ) were found in the TBI group on measures of verbal fluency, executive functioning, perceptual reasoning, and immediate/delayed memory (particularly auditory memory $[\mathrm{d}=.31-.81])$. At 1-year post-injury, lower scores were found in the TBI group on measures of verbal fluency, executive functioning, and immediate/delayed memory (including visual/auditory memory $[\mathrm{d}=.32-.47])$. When considering the number of low scores (i.e., $<16^{\text {th }}$ percentile) across all measures simultaneously, there were few group differences in the sub-acute period. However, at 1-year post-injury, the TBI group had more low scores than the TC group (e.g., 5-or-more low scores: $\mathrm{TBI}=32.7 \%$; $\mathrm{TC}=7.5 \%)$.

Conclusions: Compared to a TC group, the mild-moderate TBI group had worse neurocognitive performance in the sub-acute recovery phase and 1-year post-injury. Given our understanding of the natural history of recovery from mild TBI, it is unclear why these differences exist. Nonetheless, regardless of etiology, these results suggest that long-term evaluation and treatment of service members following military-related mild-moderate TBI is necessary.

Correspondence: Sara M. Lippa, PhD, Boston VA Hospital, Walter Reed National Military Medical Center`, Bethesda, MD 20889, United States. E-mail: saralippa@gmail.com

S.M. LIPPA, R.T. LANGE, T.A. BRICKELL, R. GARTNER, A.E. DRISCOLL, M.M. WRIGHT, B.N. PIZZANO, L. JOHNSON, D.E. NORA, H.S. MAHATAN, J.K. SULLIVAN, D.M. THOMPSON, A.C. SNELLING, B.R. YANOS, T.J. CASTILLO, C.E. SIMON \& L.M. FRENCH. Neuropsychological Outcome Following Moderate, Severe, and Penetrating Traumatic Brain Injury in U.S. Military Service Members.

Objective: Examine neuropsychological outcome following Moderate, Severe, and Penetrating military-related Traumatic Brain Injury (TBI). Participants and Methods: Participants were 39 U.S. military service members who had sustained a Moderate-Severe $(n=29)$ or Penetrating TBI $(n=10)$, and 37 Trauma Controls (TC) who were prospectively enrolled in a nation-wide 15-year longitudinal study. Participants completed a 5-6 hour neuropsychological test battery on average 16.3 months ( $\mathrm{SD}=25.4$ ) post-injury, that included the MMPI-2-RF and 29 common neurocognitive measures. Participants were excluded for invalid performances. Time since injury ranged from 1-119 months and was included as a covariate.

Results: Pairwise comparisons across the three groups (Mod-Severe, Penetrating, TC) revealed that: (a) the Moderate-Severe TBI group performed better than the TC group on 5/29 neurocognitive measures and 4/9 MMPI-2-RF clinical scales ( $d=.31-.71$; small-moderate effect sizes); (b) the TC group performed better than the Moderate-Severe TBI group on 4/29 neurocognitive measures and 1/9 MMPI-2-RF scales ( $\mathrm{d}=.31-.37)$; and $(\mathrm{c})$ the Penetrating TBI group performed worse than the TC group on measures in all cognitive domains $(\mathrm{d}=.33-1.75$; small-very large effect sizes), but particularly on measures of attention/ processing speed ( $\mathrm{d}=.46-1.39)$, visuospatial reasoning $(\mathrm{d}=.44-.86)$, and psychological functioning $(\mathrm{d}=.33-.85)$.

Conclusions: Compared to a TC group, Penetrating TBI generally performed worse across most individual cognitive tests, psychological 
measures, and in terms of number of low scores. In contrast, Moderate-Severe TBI demonstrated similar or better neurocognitive and psychological outcomes than TCs on most individual measures: however, there was a subset of Moderate-Severe TBI that performed consistently poorly across cognitive tests. These results emphasize the need for continued evaluation and treatment of service members following Moderate-Severe and Penetrating TBI.

Correspondence: Sara M. Lippa, PhD, Boston VA Hospital, Walter Reed National Military Medical Center', Bethesda, MD 20889, United States. E-mail:saralippa@gmail.com

S.M. LIPPA, T.A. BRICKELL, L.M. FRENCH \& R.T. LANGE. Predictors of Neurobehavioral Outcome 2-Years Following MildModerate Traumatic Brain Injury.

Objective: Recovery from mild-moderate traumatic brain injury (TBI) can be affected by numerous factors. The purpose of this study was to examine predictors of outcome at 2 -years following mild-moderate TBI. Participants and Methods: Participants were 168 U.S. military service members (Age: $\mathrm{M}=34.8$ [SD=9.3]; 86.6\% male) with mild-moderate TBI $(n=74)$ or no TBI $(n=94)$. Participants completed the TBI Quality of Life (TBI-QOL), Neurobehavioral Symptom Inventory (NSI), Posttraumatic Stress Disorder (PTSD) Checklist, and Combat Experiences Scale at 2-years post-injury. Using regression analyses, PTSD, depression, resilience, combat exposure, TBI severity, age, and gender were used to predict 13 outcome variables (e.g., anger, headaches, fatigue, postconcussion symptoms).

Results: Posttraumatic Stress (beta=.602), depression (beta=.192), TBI severity (beta $=.175$ ), and resilience (beta $=-.100)$, all significantly contributed to NSI total score (Adjusted $\mathrm{R}^{2}=.734$ ). The models explained over $45 \%$ of the variance in all but two of the TBI-QOL subscales (Adjusted $\mathrm{R}^{2}=.463-.699$ ). PTSD and depression were the most consistent predictors of TBI-QOL subscale scores, followed by TBI severity and resilience. PTSD (betas $=.206-.518$ ) contributed to all 12 TBI-QQOL subscales; depression (betas=.205-.576) contributed to all but one of the subscales. TBI severity contributed to 5 TBI-QOL subscales (betas $=.133-.275$ ) and resilience contributed to 4 TBI-QOL subscales (betas=.138-.573).

Conclusions: At 2-years post-injury, PTSD and depression were most consistently and strongly related to neurobehavioral symptoms and health-related quality of life. TBI severity and resilience were modestly related to neurobehavioral symptoms and some quality of life scales. Self-reported PTSD and depression 2-years post-injury should be considered strong risk factors for poor long-term neurobehavioral outcome that warrant early intervention.

Correspondence: Sara M. Lippa, PhD, Boston VA Hospital, Walter Reed National Military Medical Center', Bethesda, MD 20889, United States. E-mail: saralippa@gmail.com

T.A. BRICKELL, S.M. LIPPA, R.T. LANGE \& L.M. FRENCH. The Natural History of Psychological Symptoms following Moderate, Severe, and Penetrating Traumatic Brain Injury in Military Service Members: A Case Series Analysis.

Objective: Examine psychological symptom trajectories following moderate, severe, and penetrating Traumatic Brain Injury (TBI) in military service members.

Participants and Methods: Participants included 15 U.S. military service members with moderate-severe $(n=12)$ or penetrating $(n=3)$ TBI prospectively enrolled in a longitudinal study at Walter Reed National Military Medical Center. Average age was 28.7 years $(\mathrm{SD}=7.5)$ at baseline. Average time since injury was 12.2 months $(\mathrm{SD}=19.9)$ at baseline and 31.7 months $(\mathrm{SD}=17.1)$ at follow-up. Participants completed the MMPI-2-RF at baseline and follow-up.

Results: At both baseline and follow-up, somatic symptoms were the most commonly reported, with 5 participants having multiple elevated scales at baseline and 11 participants having multiple elevated scales at follow-up. Of all the individual scales, Neurological Complaints $(\mathrm{n}=7)$ and Anger Proneness $(\mathrm{n}=5)$ were the most commonly elevated at baseline. At follow-up, Malaise $(n=9)$, Head Pain $(n=8)$, Neurological Complaints ( $n=8)$, Cognitive Complaints $(n=7)$, RC1 $(n=7)$, and Anger Proneness $(n=7)$ were most commonly elevated. Over time, three participants did not have a meaningful change in their profiles, one had an improvement in behavioral dysfunction, and $12 \mathrm{had}$ an increase or variability in 1-2 groups of scales. Of the latter 12 participants, 7 reported increased somatic symptoms, followed by increased emotional dysfunction $(n=6)$, increased $(n=2)$ or variable $(n=2)$ interpersonal symptoms, increased thought disorder $(\mathrm{n}=1)$, and increased behavioral dysfunction $(n=1)$.

Conclusions: Service members with moderate-severe and penetrating TBI demonstrated a number of different psychological symptom trajectories; however, there was a clear trend of increased symptoms at follow-up relative to baseline, especially with regard to somatic complaints and emotional dysfunction. This demonstrates a clear need for psychological symptom assessment and management not only in the acute recovery phase, but also many years following the injury.

Correspondence: Sara M. Lippa, PhD, Boston VA Hospital, Walter Reed National Military Medical Center`, Bethesda, MD 20889, United States. E-mail: saralippa@gmail.com

R.T. LANGE, J. GILL, S.M. LIPPA, T.A. BRICKELL \& L.M. FRENCH. The relationship between Self-reported Postconcussion Symptoms with Tau and Amyloid-beta 42 Levels following Military-related Mild-Moderate Traumatic Brain Injury.

Objective: To examine the relation between levels of tau and amyloidbeta 42 with postconcussion symptom reporting in participants with and without mild-moderate traumatic brain injury (TBI).

Participants and Methods: Participants were 47 U.S. military service members who had sustained a mild-moderate TBI and 21 service members who had sustained orthopedic injuries without TBI (i.e., Trauma Controls [TC]). Participants completed a baseline assessment that included the Neurobehavioral Symptom Inventory (NSI) and a blood draw on average 5.0 months post-injury ( $\mathrm{SD}=3.7)$. Thirty-five participants also completed a 1-year follow-up evaluation. Measures of Tau and Amyloid-beta 42 were obtained using an ultra-sensitive immune-assay. The TBI group was classified into two categories based on ICD-10 criteria for Postconcussion Disorder (PCD): (a) PCD-Negative $(\mathrm{n}=34)$, and (b) PCD-Positive $(\mathrm{n}=13)$.

Results: At baseline (a) the PCD-Negative group had higher amyloidbeta 42 levels compared to the TC $(\mathrm{d}=.32)$ and PCD-Positive $(\mathrm{d}=.44)$ groups, and (b) the PCD-Positive group had higher tau levels compared to the PCD-Negative (d=.27) and TC (d=.24) groups. At follow-up, (a) the PCD-Negative group had higher amyloid-beta 42 levels compared to the TC $(\mathrm{d}=.52)$ and PCD-Positive ( $\mathrm{d}=.60)$ groups, and (b) the PCD-Positive group had higher tau levels compared to the PCD-Negative group $(\mathrm{d}=.27)$. However, PCD symptom trajectories from baseline to follow-up were not consistently associated with changes in tau or amyloid-beta 42 levels.

Conclusions: These results suggest that there may be some association between tau/amyloid levels and postconcussion symptoms following mild-moderate TBI for some people. However, a larger sample size is needed to establish the strength and clinical meaningfulness of this association.

Correspondence: Sara M. Lippa, PhD, Boston VA Hospital, Walter Reed National Military Medical Center`, Bethesda, MD 20889, United States. E-mail: saralippa@gmail.com

W. LOPEZ, J. LARA-RUIZ, F. MARTINEZ, D. DELGADO, H. RAD, D. BUDDING, D. HARDY, P. VESPA, D. MCARTHUR, D. HOVDA, D. PLURAD, E. WOO, J. FUSTER \& M.J. WRIGHT. Role of Encoding and Retrieval in Activity Memory Following Traumatic Brain Injury.

Objective: Persons with traumatic brain injury (TBI) exhibit memory deficits for the content (recall), but not the context [temporal order 
memory (TOM)] of completed activities in an incidental learning paradigm (Schmitter-Edgecombe \&. Wright, 2003; Wright et al., 2014). Our goal was to evaluate the role of encoding, consolidation, and retrieval in content and contextual memory for performed activities following TBI. Participants and Methods: TBI $(n=25)$ and comparison ( $n=24)$ groups consented to voluntary participation in this IRB approved study. The groups were well matched on education, premorbid intellect, sex, race, and ethnicity, but differed in age. The TBI group suffered moderate-to-severe injuries that were largely due to acceleration-deceleration events $(84 \%)$. All participants completed a brief neuropsychological battery. After each test, they provided ratings of workload. The test performances served as to-be-remembered activities and the ratings served to aid incidental memory for the activities. After the last rating, participants completed free recall, recognition, and TOM reconstruction trials for the activities. Indices of encoding, consolidation, and retrieval were obtained via the Item Specific Deficit Approach (Wright et al. 2009).

Results: ANCOVAs, covarying for age, showed the TBI group evidenced poorer content memory $(p=.008)$, but equivalent TOM for the activities in contrast to the comparison group. Hierarchical regressions showed that encoding predicted content memory $\left(R^{2}=.22 p=.018\right)$, but both encoding $\left(R^{2}=.16, p=.046\right)$ and retrieval $\left(R^{2}=.19, p=.023\right)$ predicted TOM for activities.

Conclusions: Our data indicate that TBI-related memory deficits for the content of performed activities are related to encoding deficits, while their intact TOM performances were predicted by encoding and retrieval. It appears that TBI-related encoding deficits impact memory for activity content, but the order of activities are, to some degree, automatically encoded, as retrieval difficulties play a greater role in TOM for them.

Correspondence: Walter Lopez, CA, United States. E-mail:wdlopez31@ gmail.com

\section{A. LOYNING, S. KEY-DELYRIA \& J. YOTS. Comparing Artificial Grammar Learning and Natural Language Learning in Adults with Agrammatic Aphasia.}

Objective: This study examines the relationship between artificial grammar learning (AGL) and natural language learning (NLL) in individuals with agrammatic aphasia (AA) due to damage to the left inferior frontal gyrus (LIFG). While there are studies observing AGL in people with $\mathrm{AA}$, comparing AGL to NLL in individuals with $\mathrm{AA}$ is novel. Using AGL allows for the isolation of non-linguistic sequencing. Previous studies have found impaired AGL performance in individuals with AA, supporting the idea that the role of the LIFG is not limited to language processing. However, it is unclear how AGL relates to NLL in adults with AA. We hypothesize control groups will perform better on AGL and NLL than adults with AA. Furthermore, we expect the AGL and NLL results to correlate in each group, supporting the notion that the LIFG is domain-general.

Participants and Methods: Participants are a group of younger and older adult controls and a group of people with AA. Participants complete cognitive and language processing tasks, including computerized N-back, AGL, and NLL tasks. AGL involves exposing participants to a block of strings that follow an artificial grammar and pre- and post-testing to determine whether they learned the grammar implicitly. The NLL task mirrors the AGL task but uses natural language rather than an artificial grammar and has been carefully designed for use with individuals with AA.

Results: Preliminary findings indicated no evidence of learning on the NLL or AGL tasks. Changes between pre- and post-test performance were insignificant. Further, surprisingly inconsistent timing within the training block suggested the need for methodological improvements.

Conclusions: Measures will be implemented to minimize fatigue, which is particularly important in individuals with AA who often have motor impairments. Methodological considerations for individuals with AA will be discussed.
Correspondence: Ashley Loyning, Speech And Hearing Sciences, Portland State University, 1908 NE 46th Ave., Portland, OR 97213, United States. E-mail: aloyning@pdx.edu

M. MALLECK, R. RICHARDSON, C.M. CONSIDINE \& C. ABEARE. Baseline Postconcussive Symptom Patterns in Athletes and NonAthletes.

Objective: Effective decision-making about return-to-activity following concussion is dependent on accurate baseline estimates. Clinically, there is an emphasis on preventing early return for safety reasons. However, mistaken delays in return-to-activity are also associated with poorer functional outcome. Base rates for individual postconcussive symptoms (PCS) in the general population assist in differentiating persisting abnormal symptoms versus a "good old days bias" presentation. However, less is known about the base rates for the number of co-occuring PCS.

Participants and Methods: Sixty-eight athletes (men=34) were given the Post-Concussion Symptom Scale (PCSS) questionnaire as part of their varsity team baseline assessments. They were matched by age and gender with non-athlete undergraduate controls who also completed the PCSS. ANOVA and descriptive analyses were conducted to investigate group differences and symptom base rates.

Results: There was a main effect of athlete status on the number of symptoms endorsed $[\mathrm{F}(1,132)=33.677, p<.001]$ such that athletes endorsed fewer symptoms than non-athletes. No differences were found for sex or the interaction (all $p$ 's $>.05$ ).

Despite non-athletes reporting a higher frequency of different symptoms, the overall pattern of symptoms reported by both groups was similar. The symptoms endorsed most and least frequently were generally the same between the two groups.

Base rates for the number of comorbid-endorsed symptoms in the overall sample, as well as for athletes and non-athletes independently, were also computed.

Conclusions: Despite similarities in overall symptom reporting patterns, athletes reported fewer symptoms at baseline. This suggests different return-to-baseline standards be used for students after concussion. The normal base rates of different numbers of co-occurring postconcussive symptoms allows better detection of return-to-baseline in concussion patients, as well as provides a psychoeducational tool to address the "good old days" bias in persisting PCS cases.

Correspondence: Malayna Malleck, Psychology, University of Windsor, 3770 Vaughan Street, Windsor, ON N9C1Z5, Canada.E-mail:malleck@ uwindsor.ca

\section{J. MASSA. The Utility of Guided Relaxation Meditation in Stroke Rehabilitation.}

Objective: The present study examined whether the use of guided muscle relaxation meditation improves the efficacy of motor relearning in rehabilitation of stroke patients.

Participants and Methods: Twenty participants with a history of a single unilateral stroke affecting motor performance. Ten participants received guided muscle relaxation meditation instruction and EEG training on alpha wave awareness. Ten participants engaged in conversation to maintain rapport. During baseline and reassessment, the following tests were administered to measure motor abilities: FugleMeyer Test, Jebson Hand Function Test, and Motor Activity Log. EEG was collected during baseline, intervention, and reassessment using MindBand.

Results: Main effect was found for non-dominant hands in a 2x3 repeated measures ANOVA. No main effect was found for dominant hands; but, participants reported perceived motor gains in the use of their dominant hands after guided meditation. In addition, participants reported feeling less tension and stress about their physical limitations. Brain wave changes also showed significant differences that support less stress and more alertness. 
Conclusions: The finding of improvements in non-dominant hand performance upon reassessment suggests that meditation increases functioning in motor areas that are not impacted by stroke. More important is the perceived improvement in the dominant hands suggesting that meditation helped them to re-evaluate their physical limitations. Meditation offers potential for cost effective clinical application with lasting benefits for stroke survivors.

Correspondence: Jacqueline Massa, Ph.D., Psychology, Middle East Technical University - Northern Cyprus Campus, Kalkanli-Guzelyurt, Psychology Program R-132, Mersin 10 Turkey 99738, Turkey. E-mail: jmassa@metu.edu.tr

K.L. MCCLINTOCK, T. ANG, R.J. PIERS, D.J. EBLE, E.M. JOHNSON, R. DAVIS, D. PENNEY, M. MCCLEAN, J. GRAFMAN, C. DECARLI \&. R. AU. Cognitive Effects of Traumatic Brain Injury Assessed with the Digital Clock Drawing Test at the Framingham Heart Study. Objective: The digital Clock Drawing Test (dCDT) permits precise, reliable, and objective assessment of higher-order decision-making processes that may be used to track early emerging neurocognitive decline well before current assessment methods. We sought evidence of persistent cognitive changes associated with a history of traumatic brain injury (TBI) in a young, non-demented community-based population. Participants and Methods: 713 Framingham Heart Study Generation 3 participants were administered the dCDT (Command and Copy conditions). A retrospective medical record review categorized participants as 'Probable TBI' $(n=121)$ or 'No TBI' $(n=592)$. We compared novel dCDT latency measures (including total time to completion, pre- $1^{\text {st }}$ hand latency, pre- $2^{\text {nd }}$ hand latency, post-clock face latency, average inter-digit latency, total ratio of 'think' time to 'ink' time) as well as MRI measures (including ratio of hippocampal volume to total cranial volume and total white matter hyper intensity volume) between TBI groups. Results: In the Command condition, 'Probable TBI' participants demonstrated longer total times to completion $(+3.06$ seconds, $p=0.009)$ and pre- $2^{\text {nd }}$ hand latencies $(+0.25$ seconds, $p=0.039)$ than 'No TBI'. 'Probable TBI' participants also demonstrated a higher load of total white matter hyper intensity $\left(+0.1361 \mathrm{~cm}^{3}, \mathrm{p}=0.0238\right)$ and smaller adjusted hippocampal volumes $(-0.0002, \mathrm{p}=0.0122)$ as compared to "No TBI'. Additionally, within the 'Probable TBI' group, participants whose total time to completion and pre- $2^{\text {nd }}$ hand latencies were in the slower $50 \%$ demonstrated smaller hippocampal volumes $(-0.0002, p=0.013$ and $-0.0002, p=0.0451$, respectively) as compared to participants with latencies in the faster $50 \%$. There were no significant findings in the Copy condition.

Conclusions: The dCDT can detect subtle cognitive differences with a previous TBI, corroborated by neuroanatomical biomarkers. This suggests potential chronic effects associated with TBI that increase the risk for later life accelerated cognitive decline and dementia.

Correspondence: Kaeden L. McClintock, Framingham Heart Study - Department of Neurology, Boston University School of Medicine, 64 Winslow Road, Duxbury, MA 02332, United States. E-mail: kaedenmcclintock@gmail.com

\section{Z. MERZ, R. VAN PATTEN, J. LACE \& T. GREIF. Current Public} Knowledge Pertaining to Traumatic Brain Injury (TBI): Influence of Demographic Factors, Social Trends, and Sport Concussion Experience on the Understanding of TBI Sequelae.

Objective: The current study aimed to assess current broad TBI-related knowledge in the general public, as well as understanding regarding specific TBI-related conditions including post-concussive syndrome (PCS) and chronic traumatic encephalopathy (CTE).

Participants and Methods: Data were collected from 307 individuals via online researcher-developed survey instrumentation utilizing the Amazon Mechanical Turk marketplace, a recently-developed website that allows for a streamlined process of survey-based participant recruitment and data collection. Participants completed background demographics questions and a 31-item true/false questionnaire pertaining to TBI-related knowledge.

Results: The overall accuracy rate of our U.S. sample was $61 \%$. No accuracy differences were present for gender or geographic region $(p$ 's $>.05)$. Participants who self-reported a prior concussion diagnosis, who reported receiving formal concussion training, and who endorsed participation in collegiate, semi-professional, or professional athletic competition, all exhibited lower accuracy rates than the respective comparison groups $(p$ 's <.001). Finally, individual item analysis revealed the presence of significant misconceptions pertaining to PCS and CTE.

Conclusions: Misconceptions regarding TBI remain highly prevalent within the general public and may be explained, to some extent, by inefficiencies in current TBI-education practices. Moreover, misconceptions regarding PCS and CTE are also prevalent and likely reflect inconsistencies in the scientific literature, coupled with misleading media reports. To combat these trends, greater emphasis must be placed on construct definition within the field and streamlined, efficient communication with the general public.

Correspondence: Zachary Merz, Saint Louis University, 9906 heritage drive, apt c, Saint Louis, MO 63123, United States. E-mail: merzz@ slu.edu

\section{J. MEYER \& P. ARNETT. Post-Concussion Depression and Cognitive Functioning in Collegiate Athletes.}

Objective: Depressive symptoms are commonly reported following concussion and depression is often associated with neuropsychological deficits across several cognitive domains. As such, post-concussion depression may increase the likelihood of post-concussion cognitive deficits. The present study aimed to evaluate the association of depression with post-concussion neuropsychological test performance.

Participants and Methods: 132 athletes tested at baseline and post-concussion were included from a university-based sports concussion program. The BDI-Fast Screen and a comprehensive battery of neuropsychological measures were administered at both testing sessions. Depressed and nondepressed groups were formed using BDI-FS scores of 4 or higher for the depressed group and scores of 0 or 1 for the non-depressed group.

Results: Depressed athletes tested post-concussion performed worse than non-depressed athletes on tests of verbal memory (HVLT-R: $\mathrm{t}(130)=02.27, \mathrm{p}=0.025)$ and visual memory (ImPACT Visual Memory Composite: $\mathrm{t}(127)=-2.49, \mathrm{p}=0.014)$. Depressed athletes were also more likely to demonstrate clinically significant decline on tests of verbal memory (ImPACT Verbal Memory composite: Fisher's Exact Test, $p=0.058$ ) and processing speed (SDMT: Fisher's Exact Test, $p=0.024$ ). More specifically, on the ImPACT Verbal Memory composite, $25 \%$ of depressed athletes showed a reliable decline compared to $8 \%$ of non-depressed athletes. $27 \%$ of depressed athletes showed a reliable decline on the SDMT, compared to $7 \%$ of non-depressed athletes.

Conclusions: Our findings suggest depressive symptoms are associated with cognitive functioning post-concussion. Clinicians should evaluate for post-concussion depression and consider its possible impact when interpreting neuropsychological test performance. Treatment for post-concussion depressive symptoms could improve cognitive test performance and facilitate the return to play of athletes.

Correspondence: Jessica Meyer, Penn State, 373 Moore Building, University Park, PA 16801, United States.E-mail: jem508@psu.edu

R.D. MOORE, C. ALARIE, V. SICARD \& D. ELLEMBERG. Slow-torecover athletes exhibit worse emotional status, reduced cognitive performance, and decreased neurophysiological function relative to asymptomatic athletes with and without a history of concussion. Objective: $20 \%$ of concussed athletes will experience persisting symptoms (McCrory, 2013), but little is known about the specific psycho-affective, cognitive and neurophysiological alterations of slow-to-recover athletes (SRA) relative to asymptomatic concussed athletes (ACA) and 
athletes without a history of concussion (NC). We sought to compare the psycho-affective states, cognition, and neurophysiological function of SRA, ACA and NC athletes.

Participants and Methods: Twenty SRA were matched for age, gender, time since injury (mean $=49.3, \mathrm{SD}=40.0$ days) and number of injuries $(\mathrm{m}=2.2, \mathrm{SD}=1.3$ concussion) with $20 \mathrm{ACA}$, and for age and gender with 20 NC. All participants completed the Beck Depression Inventory-II (BDI-II), the Profile of Mood State (POMS), an N-Back task, and an odd-ball task while event-related brain potentials (ERPs) were recorded. Results: Analyses of BDI-II scores revealed that SRA exhibited greater depressive symptoms than ACA and NG $(p=<0.05)$. ACA did not differ from NC. Analyses of the POMS revealed that SRA differed from ACA and NHC in terms of Depression-Dejection, Anger-Hostility, Vigor-Activity, Confusion-Bewilderment and total mood disturbance $(p=<0.05)$. ACA did not differ from NC on any POMS subscale or total mood disturbance. Analyses of the N-back task revealed that SRA took longer to respond, and responded less accurately relative to NHC $(p=<0.05)$, but not ACA. ACA did not differ from NC athletes. Lastly, analyses ERPs revealed that NC exhibited reduced P3-ERP amplitude relative to $\mathrm{ACA}$ and $\mathrm{NC}$ ( $\mathrm{ps}=<0.05)$.

Conclusions: Our results suggest that athletes with persistent symptoms exhibit pervasive deficits indicative of worse emotional status, reduced cognitive performance, and decreased neurophysiological function relative to asymptomatic athletes with and without a history of concussion. More comprehensive evaluations are warranted to adequately identify and track deficits in slow-to-recover athletes.

Correspondence: Robert D. Moore, University of South Carolina, 2100 Boulevard Edouard-Montpetit, H3T1J4, QC H3T1J4, Canada. E-mail: moorerd3@mailbox.sc.edu R.D. MOORE, V. SICARD \& D. ELLEMBERG. The Moderating
Influence of Learning Disabilities on the Neuropsychological
and Neurophysiological Health of Athletes with a History of
Concussion.
Objective: A significant portion of athletes will experience persistent neurophysiological and neuropsychological alterations following a concussion. Research suggests that a priori conditions may be key factors moderating injury outcomes. Accordingly, we sought to evaluate the relation between learning disorders (LD) and concussion outcomes.

Participants and Methods: Seventy-seven asymptomatic university athletes participated in this study ( 24 concussed w/out LD, 24 concussed w/LD, 26 controls). Athletes with a history of concussion were $\sim 1$ year from injury (range: 6 months- 2 years). Tests included an oddball task during which event-related brain potentials (ERPs) were recorded, a modified CogState battery, the Beck Depression Inventory-2 (BDI-II), and the Profile of Mood States (POMS).

Results: Concussed athletes w/LD exhibited prolonged P3 latency. indicative of delayed information processing, relative to concussed athletes w/out LD and controls. Irrespective of LD, concussed athletes exhibited decreased ERN and Pe amplitudes relative to controls, indicative of deficits in action monitoring and error awareness. CogState: concussed athletes w/LD exhibited decreased accuracy on the 1-Card Learning Task and the N-back Task relative to controls, indicative of deficits in learning and memory. No differences were observed between concussed athletes w/out LD and any other group. BDI-II: Concussed athletes w/LD exhibited greater depressive symptoms than controls, but concussed athletes w/out LD did not differ from any other group. POMS: Concussed athletes w/LD exhibited greater tension-anxiety, angerhostility, and overall mood disturbance than controls. Further, concussed athletes w/out LD exhibited increased anger-hostility than controls.

Conclusions: Having LD may be a significant factor moderating the neurophysiological, cognitive, and psycho-affective outcomes of concussion, and may explain a significant portion of the persistent deficits observed by researchers and clinicians.
Correspondence: Robert D. Moore, University of South Carolina, 2100 Boulevard Edouard-Montpetit, H3T1J4, QC H3T1J4, Canada. E-mail: moorerd3@mailbox.sc.edu

C.C. MORENO, B.M. ASKEN, J.R. CLUGSTON, D.N. DUBOSE, J.K. BOONE \& R.M. BAUER. Effects of Sport Type and Concussion History on Baseline Serum Biomarker Concentrations in Collegiate Athletes.

Objective: Research suggests blood-based biomarkers may provide more objective markers for traumatic brain injury and, potentially, indicators of exposure to repetitive subclinical brain trauma. We evaluated concentration variability in baseline blood-based biomarkers considered indicative of brain trauma, and specifically examined associations between sex, concussion history, and sport type.

Participants and Methods: We analyzed baseline serum concentrations of ubiquitin carboxy-hydrolase L1 (UCH-L1), S100 calcium-binding protein B (S100B), amyloid beta peptide 42 (A 442$)$, and tau collected via venipuncture from male $(\mathrm{n}=28)$ and female $(\mathrm{n}=27)$ University of Florida varsity athletes (mean \pm SD age at baseline $20.0 \pm 1.3$ years). Mean serum biomarker concentration $(\mathrm{pg} / \mathrm{mL})$ differences based on sex, concussion history $(0,1$, or $2+$ ), and sport type (moderate-high collision vs. no-low collision were analyzed with multivariate analyses of variance (MANOVAs).

Results: We found a significant omnibus effect of collision-sport type $\left(\mathrm{F}[4,50]=6.730, \mathrm{p}<.001\right.$, partial $\left.\mathrm{eta}^{2}=.350\right)$. Univariate analyses indicated moderate-high collision sport athletes had higher mean baseline concentration of serum $\mathrm{A} \beta 42(12.37 \mathrm{pg} / \mathrm{mL}$ vs. $9.47 \mathrm{pg} / \mathrm{mL})$ and lower mean baseline concentration of serum total tau $(1.57 \mathrm{pg} / \mathrm{mL}$ vs. $2.19 \mathrm{pg} / \mathrm{mL}$ ) than no-low collision sport athletes. Mean baseline concentration of UCH-L1 and S100B did not differ between collision sport types. We observed no statistically significant omnibus effects of sex $(p=.198)$ or concussion history $(p=.303)$.

Conclusions: Differential exposure to repetitive subclinical brain trauma may be associated with variable baseline concentrations of serum $A \beta 42$ and serum total tau. Serum concentrations of $A \beta 42$ and total tau could provide insight into the physiological effects of exposure to repetitive brain trauma even in the absence of clinically observable concussion.

Correspondence: Charles C. Moreno, BA, Clinical and Health Psychology, University of Florida, 4455 SW 34th St, Apt QQ226, Gainesville, FL 32608, United States. E-mail: charles.moreno@ufl.edu

C. MOSTI \& M. SPIERS. Does the NCAA Concussion Management Plan Matter? How Concussion Education Source Influences Symptom Knowledge in College Athletes.

Objective: Pre-season concussion training is required for most college athletes. However, education programs remain unregulated and it is unclear whether the source of concussion education affects athlete concussion knowledge. We aim to report if the type and number of educational resources influence athlete symptom knowledge.

Participants and Methods: A total of 164 college athletes $(65.2 \%$ female, $M=20.52$ years, $S D=3.1$ ) completed a 66 -item questionnaire regarding concussion incidence, symptom knowledge and education.

Results: Only $65.6 \%$ of athletes reported receiving any concussion education and only $60.1 \%$ of athletes reported signing the mandated NCAA pre-season concussion form. The top three sources of concussion education in order of self-reported importance were 1) Athletic Trainers 2) Coaches and 3) Physicians. Concussion symptom knowledge varied by educational source $(\mathrm{F} 5,115)=2.38, p=.04$. Athletes who completed an in-person concussion training program scored significantly higher on symptom knowledge ( $75 \%$ correct, $M=24.00, S D=1.11)$ versus athletes who only received education from athletic trainers $(\mathrm{M}=21.38, S D=0.42$, $p=.03)$, physicians $(\mathrm{M}=19.54, S D=0.75, p=.001)$ or the NCAA concussion form $(\mathrm{M}=21.52, S D=0.40, p=.04)$. Both total number of formal education resources such as coaches or trainers $(\mathrm{r}=-.041, p=.65)$ and 
informal sources such as parents or teammates $(\mathrm{r}=-.01, p=.93)$ were unrelated to symptom knowledge.

Conclusions: Despite increased regulation of concussion training. athlete symptom knowledge varies considerably based on source of concussion education. Although neither required nor identified as a top source of concussion information among athletes, formalized in-person concussion training yields the highest symptom knowledge among college athletes and should be prioritized as an educational intervention for concussion awareness

Correspondence: Caterina Mosti, M.S., Psychology, Drexel University, 857 W. Diversey Parkway, Chicago, IL 60614, United States. E-mail: caterina.mosti@gmail.com

S.A. PARIKH, C. SEMERJIAN, T. NADER, D. FRIM \& M. LACY. Pre-surgical Executive Functioning in Adult Chiari Malformation Type 1.

Objective: Chiari Malformation type 1 (CM1) is a developmental malformation leading to tonsillar ectopia and descent inferior to the foramen magnum (Rozenfeld et al 2015). Our prior research (DeDois et al., 2016) documented cognitive deficits in children with CM1. Allen and colleagues (2014) documented inhibition deficits in a small CM 1 adult cohort $(\mathrm{N}=24)$ following decompression surgery compared to controls. The current investigation is one of the few to examine executive functioning in a presurgical cohort of adults with CM1.

Participants and Methods: Following consent, twenty adult patients with CM1 documented by a board certified neurosurgeon completed a battery of neuropsychological assessment tools including the Trail Making Test, Stroop Color Word Test, and Delis Kaplan Executive function Scales - Sorting subtest. Normative references were utilized to calculate means and standard deviations.

Results: The mean age was 28.29 years with 13.57 years of education, with the majority of the sample female $(85 \%)$. Mean performances across mental flexibility (Trails B M = 45.24), problem solving (DKEFS Card Sort $\mathrm{M}=10$; DKEFS Sort Free $\mathrm{M}=9.6$ ), and response inhibition abilities (Stroop Interference $\mathrm{M}=54.20$ ) tasks were in the average range.

Conclusions: The current study is one of the first to assess cognition in a cohort of adults with CM1 prior to surgical intervention. The findings of average executive functioning will be discussed in the context of current limited literature addressing the possible neurocognitive impact of decompression surgery in CM1 and recommendations for further research. Limitations include small sample size and biased surgical sample.

Correspondence: Shravan A. Parikh, Psychology, Adler University, 215 W Washington St, Apt 1501, Chicago, IL 60606, United States. E-mail: sparikh@my.adler.edu

\section{E. POLEJAEVA, L.G. AlTOMARE, J. DEMERY, M. LARSON \& W.M. PERLSTEIN. Task-Irrelevant Interference Mimics Effects of Traumatic Brain Injury on Cognitive Control.}

Objective: Moderate-to-severe traumatic brain injury (m/sTBI) survivors exhibit impaired maintenance and updating of task-relevant context information, a critical function of cognitive control. Using a version of the AX-CPT sensitive to context processing, we previously showed that chronic m/sTBI survivors exhibit a pattern of behavioral performance consistent with a deficit in actively maintaining context information; namely, impairment in the active representation, maintenance and utilization of context representations in guiding task-appropriate behavior (i.e., context-induced $[\mathrm{CI}]$ and context-failure $[\mathrm{CF}]$ errors; Larson et al., 2006). Here we aimed to determine if, by adding delay-period interference to degrade context representations, we could induce healthy controls to perform similarly to m/sTBIs by facilitating decay of context representations.

Participants and Methods: Chronic m/sTBI patients and healthy controls ( $n=26 /$ group) performed the AX-CPT; some trial blocks included delay-period task-irrelevant distractors. Trial-wise reaction time (RT) and error rates (ERs) were acquired and statistically analyzed using mixed-model analyses of variance (ANOVAs); planned contrasts examined conditions reflecting $\mathrm{CI}$ and $\mathrm{CF}$ errors.

Results: Delay-period interference precipitated a pattern of task performance in controls similar to that seen in $\mathrm{m} / \mathrm{sTBI}$ patients: Both groups showed greater ERs under interference conditions ( $p \mathrm{~s}<.0001)$; controls' ERs did not differ from $\mathrm{m} / \mathrm{sTBIs}$ under interference conditions $(p>.10)$. Additionally, interference caused greater CI $(p<.015)$ than CF $(p>.5)$ errors.

Conclusions: Task-irrelevant delay-period interference induced a pattern of behavioral performance in controls that caused them to largely mirror the pattern seen in m/sTBI survivors, suggesting that rapid decay of context representations in patients may underlie aspects of their impaired cognitive control.

Correspondence: Elena Polejaeva, Clinical \& Health Psychology, University of Florida, PO Box 100165, Gainesville, FL 32610-0165, United States.E-mail: polejaeva@ufl.edu

H. RAD, W. LOPEZ, S. FATOORECHI, A. WALKER, J. LARA-RUIZ, D. HARDY, P. VESPA, D. MCARTHUR, D. HOVDA, D. PLURAD, E. WOO, J. FUSTER \& M.J. WRIGHT. The Role of Cognitive Speed and Control in Encoding Deficits Following Traumatic Brain Injury.

Objective: Previous work has suggested that memory impairment following traumatic brain injury (TBI) is largely due to encoding deficits (Wright et al., 2010; Wright \& Schmitter-Edgecombe, 2011; Tayim et al., in press). Cognitive speed and control have been suggested to be responsible for these encoding deficits. Our objective was to determine the role of cognitive speed and control in encoding deficits following TBI. Participants and Methods: TBI $(n=26)$ and comparison $(n=30)$ groups consented to voluntary participation in this IRB approved study. The groups were well matched on education, premorbid intellect, sex, race, ethnicity, and age. The TBI group suffered moderate-to-severe injuries that were largely due to acceleration-deceleration events (81\%). All participants completed a brief neuropsychological battery, including the California Verbal Learning Test-Second Edition and measures used to construct cognitive speed and control scores. Also, indices of encoding, consolidation, and retrieval were obtained via the Item Specific Deficit Approach (Wright et al. 2009).

Results: The TBI group demonstrated impaired memory $(p s=.004-$ $.023)$, attention ( $p s=.015-.039)$, and executive ability $(p=.007)$ in contrast to the comparison group. Additionally, the TBI group exhibited deficits in encoding and consolidation $(p s=.005-.013)$ relative to the comparison group. Consistent with previous work, encoding deficits $\left(R^{2}=.57\right)$, followed by consolidation deficits $\left(R^{2}=.08\right)$, predicted delayed recall in the TBI group $(p<.001)$. Stepwise regression showed that cognitive speed alone predicted TBI-related encoding deficits $\left(R^{2}=.32\right.$, $p=.003)$.

Conclusions: Our data indicate that encoding deficits following TBI are predicted by reduced cognitive speed. Also, these data suggest that improving the processing speed of to-be-remembered material might greatly enhance memory performances following TBI.

Correspondence: Heleya Rad, CA, United States. E-mail: heleyak@ gmail.com

S. RASKIN, E. AIKEN, O. DEJOIE \& H. VOSSLER. A Systematic Approach to Prospective Memory Treatment.

Objective: Prospective memory (PM) has recently been identified as one of the areas of cognitive functioning that has a significant effect on the daily lives of people with traumatic brain injury (TBI). Attempts at treatment have been limited but promising

Participants and Methods: Twenty individuals with TBI participated in a randomized controlled trial using modules created for the treatment of PM deficits. These include training moducles for visualizaiton, increasing cognitive load, increasing the cue-intention relatedness, increasing the focal nature of the cue, implementation intentions, and 
acting out the intention. In each module, the time between the cue and the intention is slowly increased. Ongoing tasks include puzzles and games. The active control was a brain injury education program. All participants attended 6 one hour sessions over a period of 6 weeks. All participants were given a series of neurospsycholgocial measures (Memory for Intentions Test, Hopkins Verbal Learning Test, Brief Test of Attention, Stroop Color Word Interference Test) and a series of generalization measures (Comprehensive Assessment of Prospective Memory (CAPM), Prospective Memory Diary Study, WHO Quality of Life-Brief). Results: Participants who received the treatment condition demonstrated a significant change in performance on the MIST pre-treatment versus post-treatment $(\mathrm{p}<.01)$. Althought the paticipants in the active control condition also showed improvement it was not significant. There were no significant differences measured pre-treatment to posttreatment for either group on the other neuropsychological measures. The treatment group demonstrated significant improvement on the PM Diary $(p<.001)$ and the CAPM $(p<.01)$. The active control group demonstrated significant improvement on the CAPM $(p<.01)$ but not the diary.

Conclusions: This package of PM Training modules appears to be effective in ameliorating some of the difficulties with prospective memory in people with brain injury. In addition, this appears to generalize to daily life.

Correspondence: Sarah Raskin, Ph.D., Psychology, Trinity College, 300 Summit Street, Hartford, CT 06119, United States. E-mail: sarah. raskin@trincoll.edu

S. RASKIN, M. GINGER, O. DEJOIE \& H. VOSSLER. The Effect of the Cue-Intention Relationship on Prospective Memory Performance in Individuals with Traumatic Brain Injury.

Objective: Prospective memory has been identified as an area with significant impact on daily life after traumatic brain injury, including functions such as medication adherence and compliance with appointments. One standard measure of prospective memory, the Memory for Intentions Test (MIST), intentionally uses cues that are naturally related to the action to be performed (e.g., "when I hand you an envelope, please self-address it.") However, more experimental tasks tend ot use items that are not related (e.g., "when you see a word written in purple, please press the C key.") This study measured the effect of cue-intention relatedness to see if this had an effect on prospective remembering in healthy adults and those with traumatic brain injury.

Participants and Methods: Twenty individuals with traumatic brain injury (TBI) and twenty age-matched healthy adults (HA) (mean age for $\mathrm{TBI}=40.2$ years, male/female $=15 / 10$; mean age for $H A=41.55$, male/ female $=12 / 13$ ) participated in the study. All participants were given a set of tasks taken from the MIST and a set of similar tasks that are have low cue-intention relatedness (e.g., "when I hand you an envelope, please draw a circle.") Total testing time was approximately one hour. Results: Two-way analysis of variance (ANOVA) indicated a significant effect for group (TBI, HA) for both conditions, with the individuals with TBI participating more poorly $(\mathrm{p}<.001)$. There was also a significant effect for condition (related, unrelated) whereby both groups performed more poorly in the unrelated condition $(\mathrm{p}<.01)$. Finally, there was an interaction that indicated that the TBI group was more greatly effected by the relatedness than the HA group $(p<.01)$.

Conclusions: The data confirmed the hypothesis that the relationship between the cue and the intention to be performed has an effect on prospective remembering. This effect was greater for those with TBI, suggesting that this could be a useful method in daily life and in rehabilitation efforts.

Correspondence: Sarah Raskin, Ph.D., Psychology, Trinity College, 300 Summit Street, Hartford, CT 06119, United States. E-mail: sarah. raskin@trincoll.edu
M. REYNOLDS, B.E. CLARK, E. CARROLL, J. MCFARLAND, M. MCCARVEL \& S. HALL. Personal Experience with mTBI on Symptom and Recovery Expectations.

Objective: The terms mild traumatic brain injury (mTBI) or concussion may evoke different expectations. Expectations are important because previous researchers have demonstrated that expected symptoms at the time of injury were the best predictors of actual symptoms post-injury. A personal history with TBI may affect expectations surrounding the injury. The current study investigated the effect of terminology, as well as personal history of TBI, on various outcome expectations.

Participants and Methods: Participants $(n=197)$ were grouped according to their history of TBI. They then read a vignette depicting a person sustaining an mTBI in a motor vehicle accident. They were told that the person had either sustained a concussion, mTBI, or there was no diagnosis. Participants reported expectations regarding outcomes, as well as their familiarity with the terminology. Expectations were compared between those with and without a reported history of TBI.

Results: There was a main effect of terminology on expected symptoms, $F(5,191)=4.49, p=.012$. The diagnosis of "concussion" was associated with greater expected vestibular, somatic, and cognitive symptoms. There was an interaction for expected positive changes in life perspective, $F(5,191)=3.62, p=.03$. The term "mTBI," was associated with greater positive changes in life perspective for people with no history of TBI, but worse changes in life perspective for those with a history of TBI. Participants were more familiar with the term "concussion" relative to "mTBI," but greater familiarity had no effect on expectations.

Conclusions: Findings from this study reveal that a concussion is viewed as a more serious condition than mTBI. Expectations for positive changes in life perspective were effected by personal experience with mTBI. It appears that people with no history of TBI may have an overly optimistic view of the consequences following a concussion whereas those with a history of TBI have a negative view.

Correspondence: Meredith Reynolds, University of Montana, 301 Kiwanis Street, Apartment 9, Missoula, MT 59802, United States. E-mail: meredith.reynolds@umontana.edu

A.A. ROY, S. FOSTER \& A.H. WICKLUND. Characterization of concussion: Injury, symptoms, and reporting behaviors in professional rodeo athletes.

Objective: Rodeo athletes have been underrepresented in concussion research literature, despite the high incidence of injury in the sport. Rodeo culture and the compensatory structure encourages competing despite injury (sitting out forfeits entry fees and the chance of winnings) and there is no central organization for communication between medical providers, making it nearly impossible to track an athlete's injury. The goal of this study is to develop a characterization of rodeo athletes and their experience with concussions, as a step towards effective concussion management strategies for this population.

Participants and Methods: 50 professional rodeo athletes (46 males, 4 females; mean age $=27$ ) completed an anonymous questionnaire pertaining to concussion history, education, treatment, and symptom-reporting.

Results: In regards to concussion history, $90 \%$ reported previous diagnosis of concussion (58\% reported 1-5 concussions; $17 \%$ reported 6-10; $15 \%$ reported 10 or more). The most commonly reported postconcussive symptoms were headache $(74 \%)$, dizziness $(66 \%)$, and memory problems $(50 \%)$. Despite the high prevalence rate, $32 \%$ indicated they had never received any form of concussion education. Of those with a previous concussion diagnosis, $14 \%$ reported never receiving any postinjury treatment.

Participants were also asked to report on their use of protective equipment: $64 \%$ reported never wearing a helmet, $56 \%$ reported never wearing a neck roll. Further, 27\% reported never to both forms of protection. This is in contrast to the $2 \%$ who reported always using both forms. Regarding symptom-reporting, $32 \%$ indicated having previously hid symptoms to avoid missing events and $38 \%$ indicated they currently would hide symptoms to avoid missing events. 
Conclusions: Preliminary data from this ongoing study indicate that concussions are a frequent injury within the rodeo, demonstrate a lack of consistent education and treatment for concussion in rodeo athletes, and highlight a culture of resistance to symptom-reporting and protective equipment.

Correspondence: Ashley A. Roy, Ph.D., Clinical Psychology, Illinois Institute of Technology, 114 Frankfort Sq, Columbus, OH 43206, United States.E-mail: agretencord@gmail.com

S.L. SAlinas \& S. PENNA. Patients with Post-Concussion Syndrome: Impact of Duration of Cognitive Complaints on Neuropsychological Testing.

Objective: The current study examined the relationship between duration of cognitive complaints in relation to performance on objective measures of traditional neuropsychological testing in adolescents and adults with post-concussion syndrome (PCS).

Participants and Methods: All patients were referred to a neuropsychology testing clinic due to persistent cognitive complaints after sustaining a concussion. Each patient underwent a comprehensive neuropsychological battery assessing specific cognitive domains (e.g. memory, attention, executive functioning). Fifteen of the twenty-nine patients had cognitive complaints more than six months after their concussion, while the remaining fourteen had persistent cognitive complaints six months or less post injury. Using t-tests with Bonferroni correction, the current study evaluated whether or not duration of cognitive complaints had an impact on objective measures of neuropsychological testing.

Results: Number of Failure to Maintain Sets on the Wisconsin Card Sorting Test (WCST), a measure of ideation and problem solving (i.e. executive functioning), were negatively impacted by patients who had persistent cognitive complaints longer than six months. $t(12)=-2.64$, $p=.022$.

Conclusions: Patients with persistent cognitive complaints longer than six months exhibited more impulsive behavior on the WCST than patients who had cognitive complaints six months or less. These findings indicate that PCS patients with persistent cognitive complaints longer than six months exhibit behavior that is indicative of disruption in the frontal-subcortical area of the brain. In addition, this pattern of performance on the WCST further suggests susceptibility to distraction (Figueroa \& Youmans, 2013). Dysexecutive behavior of this nature can negatively influence independent goal-directed behavior, as well as overall functioning (Rabinowitz \& Levin, 2014).

Correspondence: Stephanie L. Salinas, MS, Department of Clinical Psychology, Georgia School of Professional Psychology at Argosy University, 980 Hammond Dr., Suite 100, Atlanta, GA 30328, United States.E-mail: steph.salinas34@gmail.com

\section{W. SAUVE, R.D. MOORE \& D. ELLEMBERG. Sex Differences in Psycho-affective Outcome following Concussion in University Athletes.}

Objective: Women's participation in contact sports has increased during the last decade and relative to men, they are at greater risk for concussions and they report more intense symptoms following a concussion (Covassin, 2007). Concussions or mild traumatic brain injuries are known to cause psycho-affective alterations (Jorge, 2004). This longitudinal study assessed the influence of sex on psycho-affective health of athletes following a concussive injury at two time points.

Participants and Methods: Thirty university athletes ( 14 females, age $=20.79 \pm 1.37 ; 16$ males, age $=20.93 \pm 1.12)$ completed the Beck's Depression Inventory-II (BDI-II) and the Profile of Mood States (POMS) at 7 and 30 days post-concussion.

Results: The analyses revealed that females had significantly higher total scores on the BDI-II relative to males at 7 days post-concussion $(p=0.05)$. The analyses also revealed that all athletes reported significantly higher scores on the somatic $(p=0.02)$ and affective $(p=0.01)$ sub-factors and the total score $(p=0.02)$ of the BDI-II at day 7 compared to day 30. On the POMS subscales, all athletes reported greater anger $(p=0.05)$, vigor $(p=0.03)$, fatigue $(p=0.01)$, confusion $(p=0.01)$ and total mood disturbances $(p=0.03)$ at day 7 compared to day 30 . However, the analyses failed to reveal significant sex differences at either time point for any of the POMS subscales and the total mood disturbance scale.

Conclusions: The current results suggest that sex differences in the alteration of psycho-affective health following a concussion are selective to depressive symptoms in the acute phase of the injury. Therefore, time since injury might be the most important factor influencing psychoaffective symptoms reported by concussed athletes.

Correspondence: William Sauve, Universite de Montreal, 2100 boul. Edouard-Montpetit, Montreal, QC H3T 1J4, Canada. E-mail: sauve. w91@gmail.com

\section{S.L. SCHILLING \& J. HENNING. Suicidality in Military Veterans with Traumatic Brain Injuries.}

Objective: Suicide is a significant public health issue, and one of the world's leading causes of death (CDC, 2015). The presence of a mild traumatic brain injury (mTBI), in combination with other factors, can pose additional challenges with respect to risk of suicidality. Both suicide and TBIs impact the military/veteran population at a very high rate (Okie, 2005). The aim of this study was to investigate the relationship between suicidal ideation (SI), depression, and neuropsychological test results in a population of military veteran individuals with mTBI.

Participants and Methods: This study explored the relationship between cognitive impairments and reported SI or depression in military veterans $(n=89)$ with mTBI. Pearson's $r$ and Spearman's rho correlations, t-tests, and regressions were used to explore relationships, compare means, and predict associations between neuropsychological test results in specified domains (i.e. memory, attention, processing speed, and mental flexibility), and depression (i.e. BDI-II score) and/or reported suicidal ideation (MMPI-2; Suicide Potential Scale [SPS] score; level of SI endorsed in the case report) in military personnel with mTBIs. Results: Results showed a trend toward significance in a U-shaped curve with respect to BDI-II and Trails A scores. A significant negative relationship was observed between BDI-II total score and Delayed Memory. and was also seen between the SPS and Delayed Memory scores. Delayed Memory therefore showed the most consistent relationship with suicidality and depression.

Conclusions: Further research is required to clarify this observed relationship between delayed memory, SI, and depression, especially in military/veteran populations.

Correspondence: Samantha L. Schilling, MA, Military Psychology, Adler University, 961 Boston Way, Unit 11, Coralville, IA 52241, United States. E-mail: schilling.sam@gmail.com

B. SEAMAN, A.B. DODD, E.D. CLAUS, A.R. MAYER \& R. YEO. The Effects of Comorbid Mild Traumatic Brain Injury and Alcohol Use Disorder.

Objective: Sustaining a mild traumatic brain injury (mTBI) may influence alcohol consumption. The current study investigated the impact of self-reported mTBIs on alcohol use in a sample of individuals with alcohol use disorder (AUD) or a history of seeking treatment.

Participants and Methods: 173 individuals recruited for a neuroimaging/genetic study of alcohol abuse completed the Alcohol Use Disorders Identification Test (AUDIT) to assess consequences of consumption, and the Time Line Follow-back to assess average drinks per drinking day (ADPDD). The Rivermead Concussion Scale was completed for each injury reported. TBI history was characterized by multiplying injury severity by injury recency, summed across all reported mTBI's, to provide an overall measure of "mTBI impact".

Results: $60.7 \%$ of individuals reported a history of at least one mTBI and some had up to four prior injuries. Among those with any mTBI, the partial correlation between overall mTBI impact and AUDIT total score (controlling for sex) was $\mathrm{r}=-.313, \mathrm{p}=.002$. mTBI impact and 
ADPDD correlated at $\mathrm{r}=-.284, \mathrm{p}=.004$. Two general linear models (using all participants grouped into four levels of mTBI impact: "none" and four levels of severity) revealed significant effects for overall mTBI impact on the AUDIT $(\mathrm{p}=.003)$ and ADPDD $(\mathrm{p}<.001)$. Additionally, individuals with no mTBI drank less than those with the mildest of mTBI histories $(\mathrm{p}=.01)$.

Conclusions: As overall mTBI impact severity increased, alcohol use and adverse consequences decreased. Those with milder injuries, perhaps receiving less medical attention and advice, may be most at risk for alcohol abuse.

Correspondence: Brandi Seaman, Psychology, University of New Mexico, 1801 Gibson Blvd SE, Apt. 1098, Albuquerque, NM 87106, United States.E-mail: bseaman@unm.edu

V. SICARD, J. LORTIE, R.D. MOORE \& D. ELLEMBERG. Persistent Executive Dysfunction Following a Sport-Related Concussion Assessed with a Task-Switching Paradigm.

Objective: Cumulative research suggests persistent deficits in aspects of higher cognition such as cognitive flexibility following a sportrelated concussion (Ellemberg et al., 2007; Howell et al., 2013; Mayr et al., 2014). The current study aims to prospectively examine executive functions in athletes with a history of concussion.

Participants and Methods: 46 university student-athletes (22 history of concussion and 24 controls) completed a color-shape switch-task. All participants were asymptomatic at time of testing. Athletes with a history of concussion sustained only one concussion and were $6+$ months from injury. In the switch task, participants first performed two task conditions (homogeneous conditions), one in which they respond to color of the stimuli, and the other in which they respond to the shape. Then, participants perform a heterogeneous condition, which requires them to respond to either the color or the shape rule set depending on the cue provided. Three different costs are computed from the raw scores: global switch cost, which is thought to be a measure of global cognitive control abilities; local switch cost, which is believed to be a measure of cognitive flexibility; and working memory cost.

Results: No group difference was observed on the homogeneous condition (ps > .47). Athletes with a history of concussion exhibited a significantly greater reaction time ( $979.02 \pm 144.38 \mathrm{~ms})$ on the heterogeneous condition of the switch task relative to control athletes (849.56 \pm 133.74 $\mathrm{ms} ; \mathrm{p}=.01)$. No group difference was observed for response accuracy $(p>.65)$. Moreover, no difference was observed on any of the switch costs (ps > .07).

Conclusions: These results suggest that a single concussion may be sufficient to produce subtle, but persistent delays in information processing while performing the switch task. These findings suggest that the switch task, which requires multiple executive functions, may be useful for detecting subtle, but persistent cognitive deficits, even after the resolution of clinical symptoms.

Correspondence: Veronik Sicard, Kinesiology, Université de Montréal, 2100 Boulevard Edouard-Montpetit, Laboratoire des Neurosciences du développement, de l'exercice et de la vision, Montréal, QC H3T1J4, Canada.E-mail:veronik.sicard@gmail.com

A.R. SNYDER, S. GREIF, G. RUIZ, J.R. CLUGSTON, D. FITZGERALD \& R.M. BAUER. Symptom Tolerability of Brief Aerobic Exercise After Mild Traumatic Brain Injury.

Objective: The purpose of this study was to investigate the effect of a brief, 7-day aerobic exercise intervention administered 14 to 25 days post injury on the self-reported symptom experience of participants diagnosed with mild traumatic brain injury (mTBI). This study aimed to provide empirical evidence to help assess the translational relevance of similar preclinical research in animal models of mTBI and to generate pilot data from which to design larger-scale efficacy studies.

Participants and Methods: A longitudinal, randomized-controlled clinical trial was used to study 30 participants. Those with mTBI were randomized to either aerobic $(n=11)$ or non-aerobic $(n=10)$ exercise interventions each lasting for 7 days. Symptom reports using the 22-item Post-Concussion Scale (PCS) were taken before and after both the intervention period and each exercise day. A non-injured healthy group $(n=9)$ provided reference values for symptom change. Multilevel modeling was used to model symptoms over the 7-week intervention period and analysis of covariance was used for pre-post analyses.

Results: Exercise group had no effect on self-reported symptom change across all comparisons. Participants with and without mTBI were also not significantly different in their symptom response after a single session of exercise.

Conclusions: In a small sample, daily aerobic exercise does not increase symptom experience in participants with mTBI during the post-acute time period after injury. These data support current return-to-exercise guidelines and indicate that limiting exercise in this time frame may not be necessary to prevent injury exacerbation. Our findings are also consistent with preclinical models that demonstrate safety and tolerability of aerobic exercise within a similar time frame post-mTBI. Future analyses will examine longer-term outcomes of the intervention.

Correspondence: Aliyah R. Snyder, B.S., Clinical and Health Psychology, University of Florida, 1225 Center Drive, Room 3151, Gainesville, FL 32611, United States. E-mail: aliyahsnyder@phhp.ufl.edu

S.F. SORG, A.L. CLARK, M.L. WERHANE, R.T. KIM, D. SCHIEHSER, M.W. BONDI \& L. DELANO-WOOD. Elevated Intra-individual Variability on Tests of Executive Functions in Veterans with Mild Traumatic Brain Injury.

Objective: Although cognitive disruption in mild TBI (mTBI) may be non-uniform, few studies have explored within-subject variations across cognitive tests. Given that efforts investigating mTBI outcomes have largely focused on comparison of mean levels of performance which may obscure group differences, we examined whether intra-individual variability (IIV) across tests of executive functions (EF) is increased in mTBI relative to controls in a sample of military Veterans. We also explored relationships among EF-IIV, PTSD symptoms and white matter integrity.

Participants and Methods: 78 Veterans $(\mathrm{mTBI}=44$, Military Controls [MCs]=34) completed PTSD ratings, diffusion tensor imaging (DTI), and cognitive testing with optimal effort testing. EF-IIV was calculated as the standard deviation across 5 tests of EF (DKEFS Trails Letter/ Number Switching, Verbal Fluency Switching, Letter Fluency and Design Fluency Switching; and WCST Perseverative Responses). Fractional anisotropy (FA) was extracted from selected white matter ROIs. Results: Compared to MCs, the mTBI group had higher EF-IIV $(p<.05)$, worse mean EF scores $(p<.05)$, and greater PTSD symptoms $(p<.01)$. The EF-IIV difference remained after adjusting for the EF mean and PTSD symptoms. Across groups, PTSD symptoms negatively correlated with mean EF $(\mathrm{r}=-.34, p<.01)$, but not with EF-IIV $(p=.8)$. There were no group differences in FA $(p ' s>.05)$. EF-IIV negatively correlated with FA in multiple frontal white matter regions $\left(\mathrm{r}^{\prime} \mathrm{s}=-.28-39, p^{\prime} s<.05\right)$, while mean EF did not ( $p$ 's>.05).

Conclusions: Results show that Veterans with mTBI have greater variability across EF tests compared to MCs above and beyond what may be attributed to mean group differences on those measures or psychiatric symptoms. Findings suggest that, in contrast to conventional analyses that average scores across tests, discrepancy analyses may more sensitively capture cognitive impairment and demonstrate tighter associations with imaging indices in Veterans with head injury histories.

Correspondence: Scott F. Sorg, VA San Diego/UC San Diego, 3350 La Jolla Village Dr., San Diego, CA 92161, United States. E-mail:ssorg@ ucsd.edu 
M. SULLAN, J.M. GULLETT, M. AMIN, A. BOHSALI, R.M. BAUER, T. MARECI \& D. FITZGERALD. The relationship between loss of consciousness, sleep-related disorders, and volumetric brain changes in veterans with mild traumatic brain injury.

Objective: Research suggests that sleep is a key element in establishing and maintaining homeostasis within the central nervous system. Sleep-related disorders may be associated with reductions in whole brain volume (WBV), specifically in disorders such as obstructive sleep apnea (OSA). In traumatic brain injury (TBI) patients, sleep disorders are often reported, but the neural bases for these abnormalities have not been established. The present study investigates structural changes in mild-TBI patients with sleep disturbances to identify potential neural mechanisms related to sleep abnormalities this population.

Participants and Methods: 8 veterans with mTBI and 9 veterans with no history of brain injury were analyzed with an overnight polysomnography study and whole brain MRI. Brain volume was determined using FSL SIENA-X, which derives normalized volume metrics for each participant. A partial correlation was performed, controlling for PTSD and depression. Age and education were not significantly different between groups.

Results: Sleep efficiency was positively associated with WBV ( $\mathrm{r}=.777$. $\mathrm{p}=.002$ ). Increased OSAs, calculated using the apnea/hypopnea index (AHI), were associated with reduced WBV ( $\mathrm{r}=-.607, \mathrm{p}=.028)$, which remained significant after controlling for body mass index $(r=-.70$, $\mathrm{p}=.011)$. AHI was positively related to the number of loss of consciousness (LOC) events in our sample $(\mathrm{r}=.764, \mathrm{p}=.004)$. Reduced percentage of peripheral gray matter (pGM) volume and reduced time spent in stage 3 sleep approached significance $(r=.53, p=.062)$, as did the relationship between increased LOC events and reduced WBV $(\mathrm{r}=-.502, \mathrm{p}=.081)$. Conclusions: These results suggest that WBV and pGM are associated with the ability to fall and stay asleep, and that reductions in WBV and pGM are associated with an increased number of LOCs in a population of head-injured veterans. Further research into the relationship between LOCs and these metrics is needed.

Correspondence: Molly Sullan, BS, Clinical and Health Psychology, University of Florida, 230 SW 2nd Ave, Apt 403, Gainesville, FL 32601 , United States. E-mail: mollysullan@gmail.com

V. TERWILLIGER, N. KRAMER, D. COLEGROVE, K. ABBOTT, J. ALEXANDER, H. BREITER, A. HERROLD \& J. REILLY. The Role of Oral Contraception on Outcomes Following Concussion in Female Collegiate Athletes: A Pilot Study.

Objective: Prior research (Mihalik et al., 2009) indicates women taking oral contraception pills (OCP) may have better outcomes following sports-related concussion (SRC) than non-OCP users but this finding has not been replicated nor has the role of prior concussions been evaluated. Our objective was to investigate whether oral contraception use in collegiate female athletes affects concussion outcomes after accounting for menstrual cycle (MC) phase and prior concussions.

Participants and Methods: Nineteen OCP $(n=13)$ and non-OCP $(n=6)$ female varsity collegiate athletes who sustained SRC were enrolled. Outcome measures included: length of recovery, days between injury and clearance for full contact participation after completing return to play protocol; SCAT2 peak total number of symptoms; and SCAT2 peak symptom severity score. MC phase was determined by date of last menstrual period onset and classified as follicular (day 1-14) or luteal (day 15-28). All women enrolled reported regular periods (approximately $12 /$ year).

Results: OCP and non-OCP groups did not significantly differ by age, race, or injury characteristics. A MANOVA revealed a main effect of group (OCP vs. non-OCP) $(\mathrm{p}<.05)$ after accounting for MC phase and history of prior concussions. Post-hoc analyses revealed OCP users had a lower peak total number of symptoms $(\mathrm{p}<.05, d=3.62)$, lower peak symptom severity $(p<.05 ; d=3.32)$, and a trend for longer recovery time $(\mathrm{p}=.15 ; d=2.18)$ than non-OCP users.

Conclusions: These pilot data build on prior findings regarding the potential protective effects of OCP-use in females who sustain SRC.
Larger studies are needed to further investigate the role of estrogen-regulating medication in decreased symptom burden and duration in women post-SRC.

Correspondence: Virginia Terwilliger, Psychiatry \& Behavioral Sciences, Northwestern University Feinberg School of Medicine, 100 W Chestnut St., Apt 2505, Chicago, IL 60610, United States. E-mail: virginia.k.terwilliger@gmail.com

WITHDRAWN: M. TROYANSKAYA, N.J. PASTOREK, A. WALDER, G.S. VOGT, N.J. PETERSEN \& R.S. SCHEIBEL. Community Functioning, Emotional Status, and Social Cognition Following Combat Deployment.

Objective: There have been numerous reports of problems with community functioning, social interactions, and emotional disturbances among individuals who were deployed to Iraq and Afghanistan. This study investigates the relations between perceived limitations in community integration, current emotional status, and performance on a social cognition measure.

Participants and Methods: Participants included 76 recently deployed veterans; 58 individuals who reported symptoms consistent with deployment-related traumatic brain injury (TBI) and 18 who had no history of blast exposure or TBI. The Penn Emotional Recognition Test (ER-40) was used to assess social cognition. Current emotional status was evaluated using the Brief Symptom Inventory (BSI). The Community Integration Questionnaire (CIQ) was used to assess current level of community functioning and participation. Demographics and deployment- related information were also collected.

Results: The groups did not differ significantly in their demographic characteristics. Participants in both groups performed within average range on the measure of social cognition. Participants in the TBI group had a significant negative correlation between level of anxiety (BSI Anxiety) and ability to accurately classify faces depicting "Happy" and "Neutral" expressions on the ER-40 $(p=0.02)$. Better community functioning in the TBI group positively correlated with ability to accurately classify faces depicting "Happy" $(p=0.05)$ and "Fear" ( $p=0.03)$ expressions. There were no significant findings in a comparison group. Conclusions: Although participants did not exhibit any deficits in social cognition, current emotional status appears to impact the ability to recognize emotions among those with a history of deployment- related TBI.

Correspondence: Maya Troyanskaya, MD, PMR, Baylor College of Medicine, 6550 Fannin \# NB 410, Houston, TX 77025, United States. E-mail:mayat@bcm.edu

WITHDRAWN: M. TROYANSKAYA, N.J. PASTOREK, N.J. PETERSEN, A. WALDER, G.S. VOGT \& R.S. SCHEIBEL. Post-Deployment Community Integration and Participation.

Objective: Problems with social and family functioning, productivity, and initiation are common among combat-deployed veterans. This study investigates how limitations in participation and community integration change over time and to what extent they are influenced by demographic and deployment-related characteristics.

Participants and Methods: Participants included 85 recently deployed veterans; 67 individuals who reported symptoms consistent with deployment-related traumatic brain injury (TBI) and 18 who had no history of blast exposure or TBI. The Participation Index of Mayo-Portland Adapt-ability Inventory-4 (M2PI) and the Community Integration Question-naire (CIQ) were used to assess current level of community functioning and participation. Demographics and deployment- related information were also collected. Participants completed two to five inperson assess-ments over several years.

Results: The groups did not differ significantly in their demographic characteristics. Participants with history of TBI reported more significant ongoing problems with community integration $(p=0.03)$ and participation $(p=0.001)$. Both groups showed over time improvement of reported problems. Total CIQ score in the TBI group 
changed from 16.1 to 17.5 and in the Comparison group from 18.7 to 20.7. The M2PI scores changed from 8.7 to 7.5 and from 1.8 to 1.3 respectively. Current employment status had a significant effect on community integration $(p=0.001)$ and participation $(p=0.0001)$. Conclusions: There is evidence of improvement over time in reported limitations in community functioning. History of deployment-related TBI increases the severity of those limitations, but does not affect improvement over time relative to those without a history of combat-related TBI. Employment plays significant role in post-deployment adjustment. Correspondence: Maya Troyanskaya, MD, PMR, Baylor College of Medicine, 6550 Fannin \# NB 410, Houston, TX 77025, United States. E-mail:mayat@bcm.edu

D.M. UKUEBERUWA, K.A. KITCHEN ANDREN \& L.A. BIELIAUSKAS. Persistent Symptom Clusters and Objective Cognitive Functioning among Veterans with TBI History.

Objective: Individuals with history of mild traumatic brain injury (mTBI) may continue to report a constellation of somatic, cognitive, and affective symptoms for years after injury. Previous research has not clearly demonstrated a relationship between these symptoms and objective cognitive testing. This project aims to identify the significance of persistent subjective symptoms and performance.

Participants and Methods: The sample included 111 veterans $\left(M_{\mathrm{age}}=30.22, S D=9.04 ; 94 \%\right.$ male $)$ with history of mTBI. Veterans who sought evaluation for Compensation and Pension or demonstrated poor effort on testing (i.e., Digit Span age-corrected scale score $<7$ ) were excluded. Veterans completed self-report measures, including the Neurobehavioral Symptom Inventory (NSI) and Positive and Negative Affect Scale (PANAS) as well as cognitive measures, including the Montreal Cognitive Assessment (MoCA), Trail Making Test (TMT) A\&B, Digit Span, and Peabody Picture Vocabulary Test.

Results: Poor concentration and forgetfulness were the most frequent and severe endorsed symptoms. NSI total score was significantly correlated with negative affect $(r=.36, p<.01)$ and MoCA total score. The NSI somatic factor was correlated with the MoCA, and the NSI cognitive factor was correlated with TMT-A\&B. All NSI factors correlated with negative affect at $r>30, p<.01$. Veterans who demonstrated impairment on TMT-A $(\mathrm{T}<35)$ reported more somatic $(F(1,106)=.18, p<.05)$ and cognitive symptoms than unimpaired veterans (cognitive: $F(1,106)=.72$, $p<.05)$, but they did not differ in affective symptoms on the NSI or PANAS.

Conclusions: Self-reported elevations in somatic and cognitive symptoms differentiated veterans who were impaired in an aspect of cognition (i.e., processing speed). Veterans with objective cognitive impairment did not differ in reported affective symptoms. Somatic and cognitive symptoms may be more important than the total number of symptoms (including affective) in the assessment of persistent mTBI sequelae related to impaired cognitive functioning.

Correspondence: Dede M. Ukueberuwa, Ph.D., Penn State University, 372 Moore Building, University Park, PA 16802, United States. E-mail: dedemu@gmail.com

G. VOELBEL, M. LAZAR, S. WU, C. OGEDEGBE, G. WYLIE \& H. AZMI. Prospective Longitudinal Investigation of White Matter Integrity in Mild Traumatic Brain Injury.

Objective: The objective was to prospectively investigate the change in integrity of cerebral white matter up to one-year post- mTBI.

Participants and Methods: Seventeen adults with a mTBI were recruited $(76 \%$ female, mean age $=38.10, S D=12.48$.

The healthy control group included fifteen healthy adults $(33.33 \%$ female, mean age $=30.53, S D=11.31)$.

Diffusion tensor imaging (DTI) data for the mTBI group was acquired 7 days, 4 months, and 12 months post-injury. The control group DTI data was collected at baseline and at 4-month follow-up.

Results: At baseline, the mTBI group had significantly lower FA of the left Cingulate Gyrus $(p=.024)$ and right Inferior Longitudinal
Fasciculus, $(p=.032)$ compared to control group. At 4-months post-injury, the mTBI group had a significantly lower AD of the Splenium $(p=.025)$ and right Inferior Longitudinal Fasciculus $(p=.039)$ compared to the control group. Analyses of longitudinal changes demonstrate the mTBI group had significantly lower MD of the right Cingulate Gyrus at 4 -months post-injury compared to baseline $(p=.01)$. Examining the slope of the means over the three time points demonstrated a significant decrease of FA $(p=.047)$ and significant increase of RD $(p=.03)$ of the left Anterior Thalamic Radiation.

The slope of the mean AD for the Splenium $(p=.005)$ and the right Inferior Frontal-Occipital Fasciculus $(p=.014)$ significantly decreased over the three time points.

Conclusions: The results demonstrate significantly reduced cerebral white matter tract integrity at baseline and 4-months post-injury of the mTBI group. The left Cingulate Gyrus, Splenium, right Inferior Longitudinal Fasciculus, and left Anterior Thalamic Radiation are the most affected white matter tracts in a mTBI. The results of the longitudinal analyses demonstrated significant reduced neural integrity may continue up to one-year post-injury in adults with their first mTBI.

Correspondence: Gerald Voelbel, New York University, 82 Washington Square East, Room 618, NY, NY 10003, United States. E-mail: gv23@ nyu.edu

A. WALKER, S. FATOORECHI, S. AMAYA, T. YEH, F. MARTINEZ, D. HARDY, P. VESPA, D. MCARTHUR, D. HOVDA, D. PLURAD, E. WOO, J. FUSTER \& M.J. WRIGHT. The Dunning-Kruger Effect and Traumatic Brain Injury.

Objective: People routinely overestimate their ability for tasks that they are less competent at. This is the Dunning-Kruger effect (Kruger \& Dunning, 1999). Persons with traumatic brain injury (TBI) often exhibit reduced awareness for their cognitive deficits (Prigatano, 2005). Our objective was to determine if the Dunning-Kruger effect could be a possible explanation for reduced awareness following TBI.

Participants and Methods: TBI $(n=22)$ and comparison $(n=26)$ groups consented to voluntary participation in this IRB approved study. The groups were well matched on education, premorbid intellect, sex, race, ethnicity, and age. The TBI group suffered moderate-to-severe injuries that were largely due to acceleration-deceleration events (82\%). All participants completed a brief neuropsychological battery. After each test, participants provided self-assessments of performance. Neuropsychological performances and performance ratings were averaged into memory, attention/executive, psychomotor, and language domain scores. These scores were centered, added to a constant to get rid of any negative values, and subtracted from each other by domain to reflect differences between ability and self-ratings.

Results: The TBI group demonstrated deficits in memory $(p=.011)$ and attention/executive ability $(p=.034)$ in contrast to the comparison group. Overall, performance ratings were not different between the groups. Moreover, ANOVAs revealed that individuals in the TBI and comparison group with deficit performances $\left(T_{\mathrm{s}}<40\right)$ significantly overrated their memory $(p<.001)$, attention/executive function $(p=.038)$, and psychomotor ability $(p=.019)$; no group by deficit interactions were detected.

Conclusions: Our data suggest that the Dunning-Kruger effect may help to explain reduced awareness of cognitive deficits following TBI. Our data also suggest that persons with and without TBI exhibit the Dunning-Kruger effect to a similar degree. Finally, our results indicate that the Dunning-Kruger effect can be acquired via brain injury.

Correspondence: Andrew Walker, CA, United States. E-mail: andrew. walker@pepperdine.edu 
E. WEBER, J. LENGENFELDER \& N. CHIARAVALLOTI. Frontal Systems Behavioral Dysfunction Predicts Employment Status in Moderate-to-Severe TBI.

Objective: To explore the independent contribution of frontal systems behavioral dysfunction on employment outcomes in individuals with traumatic brain injury (TBI), in the context of relevant predictors of work status.

Participants and Methods: Participants included 42 individuals with moderate-to-severe TBI, who were at least 1 year post-injury. Participants were classified into 2 groups by current employment status, defined as report of full- or part-time employment (Employed), or lack of paid employment (i.e., disability, unemployed, retired; Unemployed). Groups did not significantly differ on most demographic or disease variables, although the Unemployed sample reported greater depression, pain, and fatigue $(p s<0.05)$. Participants completed a neuropsychological evaluation emphasizing frontal systems difficulties as well as the Frontal Systems Behavior Scale (FrsBe) as a self- and other-report measure of neurobehavioral symptomatology.

Results: T-tests using all cognitive indicators of frontal dysfunction revealed no statistically significant differences between the Employed and Unemployed samples ( $p s>0.05)$. T-tests using behavioral indicators of frontal dysfunction revealed significant differences between Employed and Unemployed participants on both self- $[\mathrm{t}(40)=-2.06$, $p=0.046$, Hedge's $g=0.71]$ and family-rated $[\mathrm{t}(40)=-3.60, p=0.001$, Hedge's $g=1.12]$ FrSBe total T-scores. Two planned logistic regressions for each FrSBe total score were conducted, with other predictors of employment (i.e., depression, pain, fatigue) also in the model. Only the family-rated FrSBe T-score remained a significant predictor of employment (FrSBe family: $p=0.038$; R-squared change $=0.177$; FrSBe self: $p=0.322)$.

Conclusions: Results suggest that behavioral indicators of frontal systems dysfunction, particularly via proxy ratings, are predictive of employment status in individuals with TBI. Future work should aim to test the efficacy of strategies to reduce dysfunctional frontal behaviors as a means to gain and maintain employment in this population. Correspondence: Erica Weber, Kessler Foundation, 300 Executive Drive, Suite 70, West Orange, NJ 07052, United States. E-mail: eweber@ kesslerfoundation.org

E. WILLIAMSON, J. STEWART, D. HEYANKA \& Z. PROCTORWEBER. Premorbid IQ Predicts Persistent Postconcussive SelfReported Symptoms Among Returning Veterans.

Objective: Extant literature has demonstrated that symptoms of postconcussive syndrome (PCS) persist well beyond the expected 3-month post-injury recovery period in a minority of individuals with mild traumatic brain injury (mTBI). Suboptimal effort and pre- and post-injury psychosocial stressors - rather than actual mTBI or current cognitive functioning - have been identified as predictors of chronic PCS (Ponsford et al., 2012). Whether premorbid IQ has any influence on chronic PCS has been understudied. Therefore, the current study investigated whether premorbid IQ was related to chronic PCS.

Participants and Methods: Thirty-one veterans (mean age: 34.19 years) who underwent mTBI evaluations 6 or more months post-injury in a VA outpatient neuropsychology clinic were included. A multiple linear regression was conducted to examine the effects on the outcome variable, PCS (Neurobehavioral Symptom Inventory), of the following predictors: cognitive functioning (Repeatable Battery for the Assessment of Neuropsychological Status Total Index), effort (Reliable Digit Span), depression (Beck Depression Inventory-Second Edition), posttraumatic stress disorder (PTSD Checklist), quality of sleep (Pittsburg Sleep Quality Index), pain (Brief Pain Inventory), and Premorbid IQ (Wechsler Test of Adult Reading).

Results: The overall multiple linear regression model was statistically significant $R^{2}=.77, F(7,25)=11.98, p<.001$. Depression $(p<.05)$ and premorbid IQ $(p<.01)$ were the most salient predictors of chronic PCS. The WTAR explained an additional $12 \%$ of the variance in PCS after controlling for psychosocial stressors and current cognitive ability.
Conclusions: The findings support premorbid functioning as an important predictor of chronic PCS. Given the influence of premorbid intelligence on prolonged PCS, adapting postconcussive interventions to meet the specific needs of individuals with varying levels of intellect may be important in minimizing ongoing symptomatology.

Correspondence: Emily Williamson, MA, Clinical Psychology, William James College, 12100 Park Blvd, 2103, Seminole, FL 33772, United States.E-mail:emily_williamson@williamjames.edu

\section{Cognitive Intervention/Rehabilitation}

S. MACOUN, S. ALI, J.C. SHEEHAN, J. MACSWEEN \& K. KERNS. Investigating the Impact of a Computer-Based Cognitive Intervention on Children with Attention/Executive Function (EF) Problems.

Objective: Attention and executive function (EF) problems in children can result in long-term difficulties affecting quality of life. Early direct cognitive interventions for attention/EF have proven beneficial, helping children to meet their potential. This waitlist control study investigated the impact of a novel game-based cognitive intervention (The Caribbean Quest; CQ) on behavioural outcome (emotion regulation, self-monitoring, attention, and working memory) in children with attention/EF problems. The CQ, delivered with the support of an interventionist, consists of five hierarchically structured and self-adjusting tasks designed to target and strengthen aspects of attention/EF along with metacognitive strategies to support treatment generalization. Participants and Methods: Participants (ages 5-12) were randomly assigned to a waitlist control $(\mathrm{N}=30)$ or active intervention (CQ; $\mathrm{N}=26$ ) group. The $\mathrm{CQ}$ was delivered via trained interventionists over 6 weeks. with a minimum of 10 intervention hours required. Select scales from the parent and teacher versions of the Comprehensive Executive Function Inventory (CEFI) were administered pre- and post-intervention to investigate far transfer effects in the domains of emotion regulation, self-monitoring, attention, and working memory.

Results: Pre- and post-test analyses showed significant gains in parent and teacher ratings of emotion regulation, while improvements on self-monitoring were trending towards significance. Reported changes in attention and working memory were not significant.

Conclusions: The CQ intervention shows far transfer effects to the domains of emotional regulation and self-monitoring. While significant gains were not seen in attention and working memory, further analyses investigating the impact of age and severity of premorbid attention/EF problems will be discussed in addition to longitudinal outcomes.

Correspondence: Sheliza Ali, Clinical Psychology, Psychology, University of Victoria, Department of Psychology, P. O. Box 1700 STN CSC, University of Victoria, Victoria, BC V8W 2Y2, Canada. E-mail: sheliza@uvic.ca

S. MACOUN, J.C. SHEEHAN, S. ALI, J. MACSWEEN \& K. KERNS. Caribbean Quest: Investigating the Impact of a Computerized Cognitive Training Intervention for Children on Cognitive and Academic Outcomes.

Objective: Direct cognitive interventions for attention/executive function (EF) in children have shown benefits for improving cognitive and academic function. We utilized a waitlist control design to investigate the efficacy of a computerized cognitive training intervention (the Caribbean Quest; CQ) for attention/EF (e.g., working memory; WM) in children with these concerns. The CQ includes five 'games' that are self-adjusting and hierarchically more demanding, which aim to strengthen attention/ EF through massed practice. The CQ is delivered with the support of an interventionist, utilizing a process-specific approach that combines direct cognitive 'exercises' with individual instruction in metacognitive strategies.

Participants and Methods: 54 children (ages 5-12 years) identified with attention/EF difficulties were randomly assigned to either 
waitlist-first or CQ intervention-first groups, using a crossover design. Children received 10-12 hours of the CQ intervention over 6 weeks, supported by trained Educational Assistant interventionists within schools. Pre-post cognitive and academic outcome measures included tasks of auditory working memory, visual-spatial working memory, inhibitory control, attention, and reading fluency.

Results: Results of the first two testing times using this crossover design were mixed. A significant group by time interaction was found for visual-spatial working memory, with null effects seen on some other tasks. Results will be further expanded to discuss the longitudinal effects of the intervention, in addition to the impact of factors such as age and premorbid severity of attention/EF problems on intervention outcome. Conclusions: These findings provide preliminary support for the efficacy of the CQ cognitive training intervention for improving visuospatial working memory.

Correspondence: John C. Sheehan, M.S., Clinical Psychology, University of Victoria, 3286 Bellevue Rd., Victoria, BC v8x 1c1, Canada. E-mail: jsheehan@uvic.ca

J. BAldo, J. Muir, S. PAUlRaj, B. CURRAN \& M. COle. Feasibility of a Mindfulness-based Stress Reduction Program for Stroke Patients.

Objective: This pilot study sought to evaluate the feasibility and acceptability of a Mindfulness-Based Stress Reduction (MBSR) program in Veterans with mild-moderate post-stroke anxiety and depression. The pilot study was designed to evaluate the feasibility of all aspects of study implementation, including patient recruitment and retention, group randomization, blinding procedures, and treatment fidelity.

Participants and Methods: We recruited 20 individuals with a history of a single left or right hemisphere stroke, with no or only mild language impairment. The study employed a randomized clinical trial design with a clinical intervention (MBSR) and active control condition (Brain Health class). Participants underwent pre- and post-intervention assessments that included the Repeatable Battery for the Assessment of Neuropsychological Status (RBANS A and B), the Geriatric Depression Scale (GDS, short form), the State-Trait Anxiety Inventory (STAI), and a medical symptom inventory. During post-testing, participants were also given an anonymous survey to rate their satisfaction with the course and provide feedback.

Results: Overall, participants rated the MBSR course favorably, giving it an average rating of 3.8 out of 5 . The attrition rate was approximately $20 \%$, due to scheduling conflicts and medical emergencies. Class attendance was also high, with just a single patient in the MBSR condition missing two classes. Randomization and blinding procedures were sufficient to ensure relatively balanced groups and objective assessors, respectively.

Conclusions: Preliminary findings from this pilot study of MBSR in individuals with a history of stroke suggest that the program is feasible and acceptable in this patient population. Ongoing work is needed to establish the benefit of MBSR above and beyond a standard brain/stroke education course with respect to the improvement of psychiatric and cognitive changes associated with stroke.

Correspondence: Juliana Baldo, Ph.D., Research Service, VA Northern California Health Care System, 150 Muir Road (126R), Martinez, CA 94553, United States.E-mail: jvbaldo@gmail.com

T.T. VO, K.D. BROWN, B. COLE, J. DAHMEN, E. CHEN, D. COOK \& M. SCHMITTER-EDGECOMBE. Development of a UserFriendly Digital Memory Notebook: An Iterative User-Centered Development Process.

Objective: Paper-pencil memory notebooks are often used to help individuals experiencing memory difficulties maintain independence. We report on a Digital Memory Notebook (DMN) being developed to increase usability and frequency of note taking and reduce memory load of users.
Participants and Methods: Using an iterative, user-centered development process, eight healthy older adults were tested in Iteration1, two individuals with Traumatic Brain Injury, one individual with Mild Cognitive Impairment (MCI), and one caregiver in Iteration2. Participants completed several tasks using the DMN (add new event to a to-do list, schedule an event). After performing each task, the After Scenario Questionnaire was used to gather satisfaction ratings (1/strongly satisfied through 7/strongly dissatisfied) for ease of use and time to complete task. The Post-Study System Usability Questionnaire (PSSUQ) and open-ended feedback prompts about the DMN were completed at the end.

Results: In iteration1, all but one individual (aged 95) were generally very satisfied (ratings 1-2) with ease of use and task completion times. In iteration2, the caregiver (aged 90) and individual with MCI reported some dissatisfaction with the ease of use and time required to complete the tasks (ratings 4-6). These individuals also rated overall usability lower on the PSSUQ. Despite this, $87 \%$ of the participants in Iteration 1 and $100 \%$ in Iteration 2 said they would use the app if available.

Conclusions: Users found the app valuable, although continued work is needed. Participants who were younger and more comfortable with technology were more satisfied with the app. User feedback is being integrated into Iteration 3 of the DMN.

Correspondence: Katelyn D. Brown, M.A., Psychology, Washington State University, 200 NW True St Apt 1, Pullman, WA 99163, United States.E-mail: katelyn.brown@wsu.edu

R. CIABATTONI, K.L. VICKERS \& M.T. SCHULTHEIS. Impact of Strategy Type on Adherence to a Behavioral Regimen.

Objective: Adherence to recommendations is a predictor of long-term rehabilitation outcomes. Because psychological regimens are often behavioral and unstructured in nature, compensatory strategies are recommended to encourage adherence. The current study examined the relationship between strategy use and regimen adherence during a week-long stress monitoring task.

Participants and Methods: 33 healthy college students $\left(M_{\text {age }}=20.03\right.$, $63.6 \%$ Male) completed a brief online stress monitoring task. This was accessible via an online portal and was to be completed three times daily for seven days. Each monitoring event included a brief stress and mood questionnaire. Participants were not prescribed a schedule or strategy, but were asked to report any strategies used throughout the week during a follow-up survey. Strategies were coded based on received responses, and included Alarms $(n=5)$, Written Reminders $(n=5)$, Use of Anchors (i.e., after meals; $n=9)$, Mixed Strategy $(n=2)$, and No strategy $(n=$ 6). Six individuals did not complete the follow-up survey.

Results: A one-way ANOVA revealed an effect trending near significance for strategy type on adherence, $F(4,26)=2.44, p=.07$. Post-hoc analyses revealed that adherence for those who used Phone Alarms was significantly greater than individuals who did not use a strategy, $t(7.57)$ $=2.63, p=.03$. Similarly, individuals who used written reminders also had significantly greater adherence than those who did not endorse a strategy, $t(7.33)=2.41, p=.045$. Descriptive analyses indicate that individuals who used Alarms had the greatest consistency in adherence times.

Conclusions: These results indicate that the use of specific strategies (i.e., phone alarms and written reminders) may result in the greatest adherence to treatment recommendations. Future research is needed to understand these relationships in cognitively compromised populations. Correspondence: Riley Ciabattoni, PA, United States. E-mail: riley. marissa@knights.ucf.edu

J. HUSSEY, D.G. NEMETH \& T. OLIVIER. Meaningful recovery via long-term integrated care: A case study on West Nile Encephalitis. Objective: In survivors of West Nile Encephalitis, physical, occupational, and speech therapies are often necessary. Due to age and history, senior citizens do not often survive. Therefore, the long-term outcome of such patients has not been well studied. As a result of "behavioral 
alterations due to brain damage that compromise the quality of patients' lives...reintegration may take years or never occur at all" (Lezak, 1988). Cognitive rehabilitation is beneficial to those who have "prolonged functional decline" (Berner, Feldman, Spigel, Chowers, \& Finckeltov, 2005). Patients who receive cognitive rehabilitation are likely to improve across many areas of functioning.

Participants and Methods: This case study highlights the effects of cognitive rehabilitation on a 77 year old Caucasian male in an outpatient neuropsychological practice. After completion of physical, occupational, and speech therapies, 95 cognitive rehabilitation sessions were conducted once weekly for 60 minutes. A variety of techniques were utilized, including verbal and visual memory exercises, executive functioning tasks, processing speed trainings, and working memory exercises. Supportive and cognitive-behavioral therapies were utilized as needed. Psychopharmacological management by a medical psychologist was incorporated into the patient's treatment program.

Results: With this patient, it was important that functional improvements be made. Results from a 5 month re-evaluation show improvements in the areas of memory, processing speed, and executive functioning. Results after one year show continued improvements in the areas of memory and processing speed.

Conclusions: Cognitive rehabilitation was significant in increasing this patient's quality of life. Through such multidisciplinary care, this patient saw objective improvements in memory and processing speed as well as observed improvements in well-being. This patient's improvements provide evidence in support of cognitive rehabilitation in long term recovery and continuity of care.

Correspondence: Julia Hussey, B.S., Clinical Psychology, University of Nevada, Las Vegas, 4332 Flagship Ct., Las Vegas, NV 89121, United States.E-mail:jhuss325@gmail.com

F. LANGLOIS, H. IMBEAULT, J. DUVAL, N. DUVAL, M. DROUIN, L. LEFEBVRE, B. GILBERT, C. BOCTI, G. LACOMBE, T. FULOP \& S. BELLEVILLE. Impacts of a Multi-Domain Cognitive Training in Geriatric Patients Diagnosed with Mild Neurocognitive Disorder. Objective: Mild Neurocognitive Disorder (miNCD) describes a highly prevalent cognitive deficit state in aging that usually affects more than one cognitive domain, including working memory. Many studies have tested cognitive training in that population, but mostly focussing on episodic memory. Thus, working memory difficulties of these patients are often left unaddressed. Here, we developed a program designed to teach working memory compensatory strategies as well as retrieval techniques. The goal of this study was to pilot the program, in combination with an episodic memory training (MEMO), and examine its potential for patients with miNCD.

Participants and Methods: Fifteen patients diagnosed with miNCD were assigned to an experimental $(n=10)$ or a wait-list control group $(n=5)$. In the experimental condition, patients received 10 sessions of cognitive training in small groups ( 5 per group). In combination with the MEMO program, patients were trained in different working memory strategies such as active repetition, dual-coding (verbal and visual), and efficient management of interference. Outcome measures were administered before and after the intervention.

Results: Retention rate was $100 \%$. Pre-post-training comparisons show improvement in objective measures of working memory (Training $=15 \%$; Control $=3 \%$; Cohen's $d=0.57$ ), episodic memory (Training=59\%; Control $=-3 \%$; Cohen's $d=0.57$ ), and satisfaction and control over memory capacities (Training $=47 \%$; Control $=1 \%$; Cohen's $d=0.52$ ), although none of those results reach the statistical significance $(p>.05)$. Conclusions: The intervention was well tolerated, the satisfaction among participants were very high, and we found moderate effect sizes, suggesting that this multi-domain cognitive program might be a useful tool to enhance cognitive functioning in geriatric patients with miNCD. However, definitive conclusions require additional research involving more patients and further follow-ups to analyze maintenance of these effects over time.
Correspondence: Helene Imbeault, Ph.D., QC, Canada. E-mail: helene. imbeault@usherbrooke.ca

K. KIM. The efficacy of the Cogmed Working Memory Training ${ }^{\circledR}$ depending on the level of sluggish cognitive tempo (SCT).

Objective: Sluggish cognitive tempo (SCT) is an emerging construct predominantly found in the ADHD-I presentation and portrays behavioral symptoms such as drowsiness, lethargy, and hypoactivity (Carlson \& Mann, 2002). The present study investigates the role of SCT in predicting improvement in attention, working memory, and executive functions through the Cogmed Working Memory Training ${ }^{\circledR}$.

Participants and Methods: Twenty-two participants between the age of 5 and $14(\mathrm{M}=9.75, \mathrm{SD}=2.15)$ were enrolled in the study, and they participated in the 25 sessions of Cogmed Working Memory Training ${ }^{\circledR}$ The pre and post-neuropsychological assessments were comprised of measures of SCT, attention, working memory, and executive functioning. Results: The group of children with fewer SCT symptoms showed a significant increase and a trend toward significant improvement on some of attention measures, Working Memory Index, and some of lab measures and self-report executive functioning compared to those of children with more SCT symptoms.

Conclusions: The findings underscore the significance of SCT on the efficacy of the Cogmed Working Memory Training ${ }^{\circledR}$. High level of SCT hindered the benefits from the Cogmed Working Memory Training ${ }^{\circledR}$. Correspondence: Kyoungjun Kim, M.A., School of Psychology, Fuller Theological Seminary, 26 Alice st, Apt A, Arcadia, CA 91006, United States.E-mail:wisp3457@hotmail.com

\section{J. LEON-CARRION, U. LEÓN-DOMÍNGUEZ \& M. DOMINGUEZ MORALES. Neuropsychological markers for safe driving.}

Objective: Our aim was to use the point system driver's license to find differences in cognitive functions that could be markers for safe driving. Participants and Methods: This study uses the point system to assess cognitive and executive functions in drivers with full points, partial points or no points on their driver's license. We employed cognitive tests to assess attention processes, processing speed, planning, cognitive flexibility, and inhibitory control. Analyses for covariance (ANCOVAS) were used for group comparisons. The Bonferroni method was used for correcting for multiple comparisons.

Results: In general, drivers with the full points scored better than the other two groups. Significant differences were found in tasks assessing attention and planning.

Conclusions: Differences in reaction time on attention tasks could serve as neuropsychological markers for safe driving. Further study is required to ascertain the impact of impaired executive functioning on driving ability.

Correspondence: Jose Leon-Carrion, PH.D.., EXPERIMENTAL PSYCHOLOGY, UNIVERSITY OF SEVILLE, c/Camilo Jose Cela, $s / n$, Dept. de Psicología Experimental, Seville 41018, Spain. E-mail: leoncarrion@us.es

\section{J. LEON-CARRION \& M. DOMINGUEZ MORALES. Neuropsychological rehabilitation of dysfunctional sphincter control.}

Objective: To identify the cognitive functions associated with the autonomic nervous system to help determine the course of sphincter control rehabilitation in patients with traumatic brain injury (TBI).

Participants and Methods: A retrospective study was carried out on patients from the Centre for Brain Injury Rehabilitation (CRECER), Seville, Spain. Observers were blinded to patient groupings. A total of 58 patients ( 44 male, 14 female, median age 20) were selected based on the following criteria: severe TBI patient, Glasgow Coma Scale (GCS) score $\leq 8$, clinically confirmed neuropsychological disorders at admission and a minimum of four months of multidisciplinary rehabilitation. Exclusion criteria included previous TBI or stroke, a history of neurological or psychiatric disorders, and substance abuse. The median interval from 
brain injury to rehabilitation was 8 months. The median treatment time period was 10.5 months. Wilcoxon's test was used for the comparative analysis of functionality index (FIM+FAM) scores at admission and discharge to ascertain the efficacy of rehabilitation.

Results: Results show that patients with TBI and reduced sphincter control at admission tend to have longer hospital stays and require more ongoing special care at home after hospital discharge. A correlation was found between poorer sphincter control and reduced functionality in all the FIM+FAM subscales $(p<0.01)$. This correlation was also found for self-care, mobility, type of transfer, locomotion, communication, psychosocial adjustment and cognitive functions.

Conclusions: The functional level of patients with sphincter dysfunction limits independent living. Dysfunctional sphincter control is also associated with neuropsychological disorders, especially in attention and executive functioning.

Correspondence: Jose Leon-Carrion, PH.D.., EXPERIMENTAL PSYCHOLOGY, UNIVERSITY OF SEVILLE, c/Camilo Jose Cela, $s / n$, Dept. de Psicología Experimental, Seville 41018, Spain. E-mail: leoncarrion@us.es

\section{S. MACOUN, K. KERNS, J.C. SHEEHAN \& J. MACSWEEN. Cognitive rehabilitation in schools: training the paraprofessional trainer.}

Objective: The success of cognitive interventions in children is limited by accessibility to qualified professionals, affordability, and logistical factors (intervention frequency, etc.), supporting school-based delivery models. We investigated the feasibility of training Educational Assistants (EAs) to deliver an attention/EF cognitive intervention (Caribbean Quest/CQ) in schools and the impact of such training on their practice. Participants and Methods: Participants included 45 EAs across 13 schools, trained to deliver the CQ to 83 children (ages 5-12 years) identified as having attention/EF problems. The CQ is a computerized process-specific intervention consisting of cognitive exercises for remediating attention/EF and metacognitive strategy instruction, supported by an interventionist. Prior to delivering the intervention, EAs completed web-based training. Interviews/surveys (80\% response rate) were conducted to determine the feasibility of delivering the $\mathrm{CQ}$ and EA experiences during/following the CQ.

Results: Concerning feasibility, the majority of EAs delivered the minimum of 10-12 intervention hours required in a 1:1 or 1:2 format during the school day and felt the intervention was not disruptive $(57 \%)$ or only mildly disruptive in school. Most EA's felt their knowledge of metacognition and supports for attention/EF was good/excellent following the study (97\%) and that their knowledge/skills for supporting children with attention/EF problems significantly improved (78-97\%). Many EAs reported developing unanticipated new skills following the intervention $(83 \%)$

Conclusions: Results indicate that delivering such cognitive interventions in schools is feasible, with benefits extending to the paraprofessionals trained as interventionists. Data related to the impact on paraprofessionals' practice, logistical considerations, and strategies for further enhancing school-based delivery models and training paraprofessionals will be presented.

Correspondence: Sarah Macoun, Ph.D., Department of Psychology, University of Victoria, Department of Psychology, University of Victoria, PO Box 1700 STN CSC, Victoria, BC V8W 2Y2, Canada. E-mail: sjmacoun@uvic.ca

K. MARTON, E. KORNBLITH, T. NOVAKOVIC-AGOPIAN, G. ABRAMS \& J. BURCIAGA. Relationship Between Baseline Functional Status and Executive Function Training Outcome in Veterans with Chronic TBI.

Objective: Deficits in executive control functions are some of the most disabling consequences of brain injury. Goal-Oriented Attentional Self-Regulation (GOALS) training was designed to target these deficits with attention regulation and problem solving training applied to participant-defined goals. The purpose of this study is to explore the relationship between baseline functional status and intervention outcomes in Veterans with chronic traumatic brain injury (TBI).

Participants and Methods: Thirty-two Veterans with a history of chronic mild to severe TBI (average of 11 years post injury) underwent neuropsychological and functional evaluation before and after completing GOALS or a control psychoeducational training to which they were randomized. Based on their performance on baseline neuropsychological evaluation, participants were clustered into "Functionally Impaired" (FI) and "Non-impaired" (NI) groups in a prior analysis. The FI group demonstrated greater cognitive difficulty and PTSD symptoms, as well as reduced community integration. Participants' assessment performance at baseline and post-intervention was compared for the FI and NI groups using a MANOVA.

Results: In the FI group, neuropsychological domain scores in auditory working memory and composite executive function skills significantly improved post-GOALS when compared to the control training $(n=19$, $\mathrm{p}=.004$ and .002 , respectively). In the NI group, there was no significant change regardless of intervention type.

Conclusions: The FI group benefited significantly from GOALS but not a control intervention, while the NI group did not demonstrate significant change after either training. The challenges and importance of further research exploring the relationship between baseline functional characteristics and intervention outcomes in Veterans with chronic TBI will be discussed. Future research may help to predict which rehabilitation interventions are indicated given patient characteristics.

Correspondence: Kacey Marton, M.S., Palo Alto University, 1020 Jena Terrace, Sunnyvale, CA 94089, United States. E-mail: kaceymarton@ gmail.com

J. MCCARTHY \& A. BAIRD. The Use of Prospective Memory Training for Improving Academic Self-Efficacy in an Undergraduate Population: A Pilot Study.

Objective: Undergraduates often struggle to finish degree requirements due to factors such as poor academic self-efficacy and goal management. The cognitive rehabilitation literature has found that prospective memory (PM) skill training has improved goal attainment in older adult and brain-injured populations. Due to the ongoing brain development supporting PM in emerging adults, cognitive rehabilitation approaches also may help improve function and academic self-efficacy.

Participants and Methods: Thirty-nine undergraduate students (25 female) were randomly assigned to one of two conditions: PM skills training $(n=21)$ or relaxation training $(n=18)$. PM was assessed immediately before and 2 to 14 days after intervention using self-report and performance PM measures and mood and academic self-efficacy questionnaires. PM training emphasized implementation intention, cue monitoring, and use of external aids. Larger post-intervention gains were expected in the PM condition.

Results: PM performance and academic self-efficacy measures showed significant main effects for time $(p<.01)$. Effect sizes $\left(\omega^{2}{ }_{\text {partial }}\right)$ were .12 for an updated version of the Royal Prince Albert (RPA) PM test, .21 for Self-Efficacy for Learning form, .25 for a time-based PM measure embedded in a working memory test, and .37 for an event-based PM task incorporated into a semantic memory test. Only the RPA showed a significant interaction between time and condition; however, this interaction reflected a decline after relaxation and stability after PM training. The interaction for the time-based PM measure approached significance $(p=.07)$ and was in the expected direction. Self-reported PM did not show significant change.

Conclusions: A single-session PM intervention shows promise for improving PM performance and academic self-efficacy. Self-reported PM was not affected by this intervention in the current form. Further exploration of this intervention in a long-term context is warranted. Correspondence: Jeff McCarthy, Psychology, University of Windsor, 2-861 PELISSIER ST, WINDSOR, ON N9A4L6, Canada. E-mail: mccar112@uwindsor.ca 
M. PRIMOSCH, C. MAXSON, B. STEWART \& C.P. MCFARLAND. Enhancing Memory and Imagination Improves Problem-Solving Among Individuals with Depression.

Objective: Depression is associated with overgeneral memories and reduced ability to imagine future scenarios, both of which contribute to problem-solving deficits. Recent work has shown that problem-solving among healthy young and older adults can be enhanced by increasing access to recalled and imagined events. This study tested the hypothesis that individuals with depression would show improved problem-solving following a detailed interview.

Participants and Methods: Thirty-eight participants (19 depressed; 19 non-depressed) were enrolled. The study was completed in 2 sessions, 1 week apart. In both sessions, participants 1 ) watched a video of common kitchen activities, 2) answered questions about the videos in a general impressions interview or a detailed interview, 3) completed a problem-solving task, and memory and imagination tasks.

Results: Problem-Solving: A mixed ANOVA revealed an interview type by step type interaction, $F(1,36)=9.81, p<.005$. Post-hoc analyses revealed that depressed participants generated significantly more relevant steps following a detailed interview, $t(18)=4.28, p<.001$.

Memory and Imagination: An interview type by detail type interaction emerged for both memory, $F(1,36)=22.61, p<.001$, and imagination tasks, $F(1.36)=48.37, p<.001$. Post-hoc analyses revelaed that depressed participants generated significantly more internal details following the detailed interview for both the memory task, $t(18)=4.98$, $p<.001$, and the imagination task, $t(18)=4.32, p<.001$.

Conclusions: Problem-solving deficits represent a key target for intervention in the treatment of depression. This study reveals that a brief interview can lead to improved problem-solving and more detailed recall and imagination among individuals with depression. This brief intervention may advance the treatment of depression, both during and between episodes, by increasing access to alternative solutions to current problems.

Correspondence: Craig P. McFarland, Psychology, University of Montana, Skaggs Bldg Room 202, Missoula, MT 59812, United States. E-mail: craig.mcfarland@umontana.edu

C. MULLEN \& A.Y. STRINGER. Strategy Training Improves Route Recall: Preliminary Report from a Randomized Controlled Trial of Cognitive Rehabilitation.

Objective: We report preliminary findings of a randomized comparison of memory vs. executive strategy training on the ability of cognitively impaired patients to learn and recall a simulated route.

Participants and Methods: Participants were a memory-impaired mixed neurological adult sample with diagnoses including traumatic brain injury, vascular neurocognitive disorder, and malignant neoplasm of the brain, who were participating in a larger cognitive rehabilitation program. Participants were randomly assigned to either memory or executive strategy training. Memory strategy training (the active treatment) consisted of a four step method (write-organize-picture-repeat) patients learned to apply to remembering new routes. Patients receiving executive strategy training (the control condition) practiced strategies for improving attention and the ability to solve problems. Route recall was evaluated before and after strategy training using a simulated 9-turn route presented on computer. Two alternate forms of the route were used to avoid practice effects and the order of form administration was randomized.

Results: No statistically significant differences were seen between scores obtained on the two simulated route forms. Treatment and control groups also did not differ significant at pretest on route recall. Compared to the control condition, at posttest memory strategy training resulted in better immediate route recall when turns were presented in random order $[t(3)=6.20, p=.008]$ and better delayed recall when turns were presented in serial $[t(3)=3.87, p=.031]$ or random $[t(3)=7.309$, $p=.005]$ order.

Conclusions: As expected, memory strategy training was superior to the control condition in improving route recall. Findings were not due to test practice effects or differences in initial task performance, and support the benefits of strategy training in memory impaired neurological patients.

Correspondence: Christine Mullen, Mercer University, 6004 Druid Hills Reserve Drive NE, Atlanta, GA 30329, United States. E-mail: christinemariemullen@gmail.com

D. MURDAUGH, T.Z. KING \& K. O'TOOLE. Efficacy of an Individualized, Manualized Cognitive Remediation Program to Improve Adaptive Functioning in Pre-Adolescents with Neurological Impairments.

Objective: To prepare for transition of medical care and adult independence, developing programs to remediate neurocognitive deficits affecting adaptive skills is becoming increasingly important. The purpose of this study was to assess the efficacy of an individualized and manualized cognitive remediation program (CRP) to increase independence and to identify parental factors that may influence impact of treatment.

Participants and Methods: Thirteen patients, ages 9-15, with neurological diagnoses participated in CRP (8 sessions) consisting of three main components: individual goal-based therapy, group parent training session with weekly parent check-ins, and home skill practice. Parents were trained to use seven graduated levels of assistance and their performance was tracked. Patients were evaluated before and after the program using the Adaptive Behavior Assessment System 2nd Edition (ABAS-II), Comprehensive Executive Function Inventory Parent Response Form (CEFI - PRF), and Parenting Styles Questionnaire (PSQ). Parent engagement and involvement ratings and patient level of independence ratings were administered each week of the program. Results: Patients demonstrated significant improvement in overall adaptive skills (ABAS-II GAC; $p=0.027$ ), with specific improvement in self-care skills $(p=0.004)$. Parent engagement and parent involvement were significantly correlated $(\mathrm{p}=0.013)$, and were positively correlated with specific aspects of authoritative parenting style, self-confidence $(p<0.001)$. Case series analyses were used to assess individual improvement in adaptive domains and revealed improvement in self-care skills, daily routines/transitions, household chores, and emotional/behavioral and social skills.

Conclusions: These findings indicate the importance of individualized and manualized techniques to effectively improve adaptive skills and increase independence for successful transition of medical care and adult living. Results particularly emphasize the importance of parental involvement and engagement in the program.

Correspondence: Donna Murdaugh, PhD, Neuropsychology, Children's Healthcare of Atlanta, 2897 Jenny Way, Lawrenceville, GA 30044, United States. E-mail: donna.murdaugh@choa.org

T. NOVAKOVIC-AGOPIAN, G. ABRAMS, E. KORNBLITH \& J. BURCIAGA. Goal-Oriented Attentional Self-Regulation Training in Veterans with PTSD and $\mathrm{mTBI}$.

Objective: Difficulties in executive control functions are common sequelae of both TBI and PTSD. Goal-Oriented Attentional SelfRegulation (GOALS) training was designed to target these deficits with attention regulation training applied to participant-defined goals. In previous studies individuals with chronic TBI significantly improved post GOALS, but not control training, on measures of attention/executive function, functional task performance, and self-report measures of emotional regulation (Novakovic-Agopian et al 2011, Novakovic-Agopian et al 2015), and on goal-directed control over neural processing on fMRI (Chen, et al 2011). The objective of this ongoing study is to assess effectiveness of GOALS training in Veterans with comorbid PTSD and mTBI

Participants and Methods: Twenty two Veterans with diagnosis of PTSD, history of chronic mTBI and mild-moderate executive dysfunction were randomized to start with either 5 week of GOALS or matched control psychoeducation (EDU) training. Assessments at baseline and 
post training included neuropsychological, functional task performance, and self-report measures

Results: Post GOALS training, but not post EDU, participants significantly improved on measures of: attention/executive function (overall performance, sustained attention, and inhibition); complex functional task performance (overall performance, attentional maintenance, switching, execution, and memory); and emotional regulation (significant symptom decrease on BDI-II, PCL-M and POMS)

Conclusions: GOALS training may be promising in Veterans with concurrent PTSD and chronic mTBI. Training self-regulatory cognitive and emotional control strategies applied to actual situations in participant lives and personally relevant goals may provide meaningful improvements in cognitive, emotional, and occupational functioning. The challenges and importance of: a) assessing change in functioning at different levels, and b) using participant-defined goals applied to relevant training, will be discussed

Correspondence: Tatjana Novakovic-Agopian, Ph.D, Neurology, UCSF, SFVA, 661 Myra way, San Francisco, CA 94127, United States. E-mail: tna@cns-site.com

\section{M.E. O'CONNELL. Remote Neuropsychology for Rural Dementia Care: Feasibility of, and Adaptations Required for, Videoconferenced Support Group and Cognitive Rehabiliation.}

Objective: To detail the adaptations required for Telehealth videoconferencing for delivery of a support group and the feasibility of videoconferenced cognitive rehabilitation.

Participants and Methods: A small sample $(N=10)$ of rural spouses of persons with atypical or young-onset dementia, typically variants of frontotemporal dementia, were recruited from the Rural and Remote Memory Clinic for the videoconferenced support group, which was held monthly over seven years. For the cognitive rehabilitation trial, six urban participants with self-identified subjective memory impairment, amnestic mild cognitive impairment, or dementia due to Alzheimer disease were randomly assigned to eight weeks of individualized cognitive rehabilitation in-person $(n=3)$ or via videoconferencing $(n=3)$. Results: Multiple-baseline single case design demonstrated effectiveness of the cognitive rehabilitation irrespective of in-person versus videoconferenced delivery. For example, items trained with spaced retrieval demonstrated improvement, but untrained items remained stable, only improving after training. Adaptations for videoconferenced delivery were similar for the spousal support group and for cognitive rehabilitation: the clinician had to be more explicit with instructions and frequently check comprehension. Videoconferencing for a support group or for cognitive rehabilitation required that clients be more self-sufficient than would occur in-person. Cognitive rehabilitation by videoconferencing involved greater reliance on handouts and other on-site props.

Conclusions: Our small sample trials suggest videoconferencing, with some adaptations to practice, can be used for remote interventions to provide rural dementia care. Nevertheless, videoconferencing required more self-reliance, which would interact with degree of cognitive impairment, and a care partner would likely be needed for delivery of service to rural persons with more advance dementia.

Correspondence: Megan E. O'Connell, PhD, Psychology, University of Saskatchewan, 9 Campus Drive, Saskatoon, SK S7N 5A5, Canada. E-mail:megan.oconnell@usask.ca

\section{S. PATEL, K.L. VICKERS \& M.T. SCHULTHEIS. Simulator Discomfort Across Neurological Populations: Who is Most Affected?}

Objective: Virtual reality (VR) simulation is a promising tool for rehabilitation research; however, discomfort has been reported in the use of this technology among healthy participants. Less is known about the impact of VR on clinical populations. This study compared types of discomfort experienced by individuals with TBI, MS, and healthy controls (HC) while driving in a virtual reality driving simulator (VRDS).
Participants and Methods: Licensed drivers were recruited for 2 driving studies, including 21 with TBI $\left(M_{a g e}=40,66.7 \%\right.$ Male $), 44$ with MS $\left(M_{\text {age }}=47.23 ; 18 \%\right.$ Male $)$, and $69 \mathrm{HC}\left(M_{\text {age }}=40.95 ; 36.2 \%\right.$ Male $)$. In both studies, participants drove a standardized VRDS route and completed 29-item simulator sickness questionnaires (SSQ) pre- and post-drive. This questionnaire was then divided into subscales to assess changes in disorientation, nausea, oculomotor, and fatigue symptoms. Results: A 3 (group) x 4 (scale) x 2 (time point) mixed effects ANOVA revealed a significant interaction between group and scale, $F(6,1088)=$ $19.84, p<.01$, wherein the TBI group experienced increased oculomotor scores between pre- and post-drive, whereas the MS group experienced increases in disorientation, nausea, and fatigue. Also, group and time point comparisons revealed a significant interaction, $F(2,1088)=7.21$, $p<.01$, indicating that the degree of change in discomfort was dependent on group.

Conclusions: Individuals with TBI had the greatest baseline discomfort, but experienced the highest increases in oculomotor symptoms. Individuals with MS had low baseline discomfort, but experienced large increases in nausea, fatigue, and disorientation and were most susceptible to simulation sickness. The HC group were most consistent across scales at both pre and post measures. Combined, the findings indicate discomfort is variable and different populations experience different types of symptoms on VRDS, which may warrant individual clinical considerations.

Correspondence: Shyam Patel, PA, United States.E-mail:sdp76@ drexel.edu

\section{R. PERNA \& J. MCGEE. Memory Dysfunction Due to Hemolytic} Anemia and Hemochromatosis: A Case Study.

Objective: Hemolytic anemia and Hemochromatosis are disorders that may affect brain functioning and cause cognitive dysfunction. Both disorders may affect brain oxygenation and metabolism and cause memory impairment. This case study shows the clinical course of cognitive symptoms secondary to these disorders. The person acutely developed these disorders and acutely exhibited delirium, and then a diverse range of severe cognitive impairments for over one year. This case highlights symptoms that occur secondary to these disorders and the potential for recovery even after protracted brain dysunction.

Participants and Methods: Ms. T was 36 years old, married and healthy, until she woke up one morning with petechae and bruises all over her body, went to the ER and was diagnosed with Ideopathic thrombocytopenia (ITP). She was started on IVIG Imunoglobulin treatment and blood transfusions. After several rounds of IVIG, Ms. T developed hemolytic anemia, and had a hemoglobin level found to be 2 (female norm range 12.1-15.1). Head CT was negative, but brief delirium developed. Her IVIG was discontinued, but blood transfusions continued. She then developed hemochromatosis (iron accumulation) and persistent cognitive problems, specifically short-term memory impairment. More than a year after her diagnosis of hemolytic anemia, Ms. T continued to exhibit impaired memory.

Results: Ms. T had an estimated premorbid IQ in the average range or higher, but initially had below average memory testing until she had 3 months of cognitive rehabilitation and 3 months longer to fully recover from her anemia and hemochromatosis. Short-term verbal memory improved from low average to high average (RBANS, WMS-IV).

Conclusions: Hemolytic anemia and hemochromatosis may cause cognitive dysfunction even with normal brain imaging. Though there was cognitive impairment at more than one year post hospitalization there was subsequently significant cognitive recovery. This case presentation may have neuropsychologically diagnostic and prognostic implications.

Correspondence: Robert Perna, Neuropsychology/Psychology, TIRR, 9427 Greenwillow, Houston, TX 77070, United States. E-mail: dr.perna@juno.com 
WITHDRAWN: E. POUTIAINEN, P. PIETILÄ, J. NUKARI, J. SARAJUURI \& S. KOSKINEN. Technology Assisted Rehabilitation for Persons With Dementia and Their Family Members - A Controlled Intervention Study.

Objective: The study aims to find out whether an interactive multidisciplinary rehabilitation model at an early phase of dementia has a positive effect on Alzheimer disease (AD) patients' cognition, emotional wellbeing and daily living and whether it has an effect on emotional wellbeing and coping of their family members.

Participants and Methods: The intervention was designed for persons at an early phase of AD under 75 years of age, and their family members. The multi-methodological approach consisted of group intervention, telerehabilitation and cognitive training (FORAMENCognitive Tablet) at home via mobile technology. In this controlled study $28 \mathrm{AD}$ patients and 22 family members were randomized to the intervention and 25 AD patients and 20 family members to the control group. The outcome measures were cognitive performance (immediate and delayed memory, speed and attention, language, reasoning), mood (DEPS, CSDD), quality of life (QoL) (QoL-AD) and daily activities (ADL) (ADCD-ADL). Additionally family members' mood (BDI-II), QoL (WHOQoL-BREF) and coping (COPE) were evaluated. Outcomes were collected at baseline, after a 13 week rehabilitation period, and 6 months after the baseline measurement.

Results: After the 13 week rehabilitation period a significant increase was found in the AD patients' mood according to the patients (DEPS) $(p<.038)$ as well as according to the reports of their family members (CSDD) $(\mathrm{p}<.007)$. This effect remained positive during the three and half month follow-up period. No significant changes were found in rehabilitees' cognition, QoL or ADL. The AD controls did not show any changes. Further, in family members attending to the rehabilitation group a positive value in caregiving increased during the 6 month follow-up (COPE) $(\mathrm{p}<.023)$.

Conclusions: The multidisciplinary rehabilitation may be useful in alleviating AD patient's mood symptoms and in supporting coping of their family members.

Correspondence: Erja Poutiainen, Rehabilitation Foundation, Po.Box 39, Helsinki SF-00411 Helsinki, Finland.E-mail: erja.poutiainen@ kuntoutussaatio. $i$

S. PYNE, S. MACOUN, K. KERNS \& J. MACSWEEN. Effectiveness of a Cognitive Intervention on Metacognitive Awareness in Children with Attention, Self-Regulation, and Executive Function Difficulties.

Objective: This study evaluated whether participating in a 6-week cognitive intervention would increase children's metacognitive skills. Metacognition is the ability to use knowledge about one's cognitive processes to change behavior, including self-monitoring, self-evaluation, and cognitive control. These abilities are often poor in children with neurodevelopmental disorders, such as Attention Deficit Hyperactivity Disorder, Fetal Alcohol Spectrum Disorder, and Autism Spectrum Disorder.

Participants and Methods: Participants, recruited through schools, included children ages 5-11 years identified as having poor attention, self-regulation, and/or executive functioning. Intervention and control groups consisted of 27 (24 M:3 F) and 33 (24 M:9 F) children, respectively. Children completed a computerized attention/working memory intervention (Caribbean Quest/CQ), supported by educational assistants (EA's) who provided metacognitive strategies. Metacognition was assessed pre and post intervention using three child measures of metacognition (self-report, behavioural ratings, and open-ended questions) and parent/EA ratings. Follow up data was collected to assess maintenance of gains.

Results: Results showed increased metacognitive awareness on parent rating scales and child assessments. After participating in the CQ, children were better able to name metacognitive strategies on an openended questionnaire. Parents of younger children (5-7) reported higher metacognitive abilities. Older children (8-11) performed better on a behavioural monitoring task

Conclusions: These results suggest that participation in cognitive interventions may help children to improve their metacognitive abilities, translating into gains in their ability to monitoring and reflecting on their own cognition.

Correspondence: Sarah Pyne, University of Victoria, 431 Walter Ave, Victoria, BCV9A 2E6, Canada.E-mail: skpyne@uvic.ca

N. NEDLEY, F.E. RAMIREZ \& D.E. BYRKIT. Violation Conscience is Related to Worse Mental Health Among Geriatrics.

Objective: Violation of conscience, by going against one own value system has emotional repercussions. The study assesses the effect of violation of conscience on depression and anxiety among seniors.

Participants and Methods: Participants that finished an educational program and were 55 or older at baseline were included. Those who chose to participate met once a week for 2 hours for 8 weeks. The Nedley Depression Recovery Program Assessment Test (registration TX 7-398-022) was used. It assessed depression level based on standard criteria, demographics and anxiety with patient history including the question "Do you often do things that go against your conscience?". The depression was classified into 4 categories as none $(0-6)$, mild $(7-10)$. moderate (11-19) or severe (20 or more). The anxiety was classified into 4 categories as none (0-4), mild (5-8), moderate (9-12) or severe (13 or more). The program taught lifestyle changes, like diet, rest, diet. exercise, etc. One session taught the importance of not acting against their own conscience, in order to decrease stress level.

Results: From 5757 total participants from 4 continents, 2825 were geriatrics, the group had a mean age of 64.7 (SD 7.2), 70.2\% ( $\mathrm{n}=1998)$ were females and $88.9(\mathrm{n}=2512)$ were Caucasian.

At baseline 370 reported that they were violating their conscience, that group had a mean depression 15.6 (SD 6.5) and anxiety 8.9 (SD 4.2). At baseline those not violating their consciences $(n=2455)$ had a mean depression of 10.8 (SD 7.3) and anxiety 6.2 (SD 4.4).

At the end of the 8-weeks those that at baseline were violating their conscience and at the end stopped, had an end mean depression of 7.4 (SD 6.3). and anxiety of 4.7 (SD 4.1) while those that continue to violate their conscience by the end of the 8-weeks had a group mean depression of 9.8 (SD 6.4) and anxiety of 5.6 (SD 3.8).

Conclusions: Violating the conscience was related to higher levels of anxiety and depression. It seems that stopping conscience violation is important for mental health.

Correspondence: Francisco E. Ramirez, MD, Research, Nedley Clinic, 20601 West Paoli Lane, Weimar, CA 95736, United States. E-mail: eramirez@weimar.edu

\section{A. RAPHAIL. Eye-Tracking in Neuropsychology: Using the Eyes as the Window to the Mind.}

As the field of neuropsychology grows, neuropsychologists have continued to develop and refine numerous cognitive tests that allow us to learn about the brain and detect cognitive dysfunction in various patient populations. Simultaneously, related fields such as cognitive psychology and neuroscience have utilized technology to provide alternative methods for studying brain-behavior relationships. One commonly used instrument is the eye-tracker, a tool that records eye-movements during tasks to provide researchers with additional measures of cognition that are not possible with more traditional pencil and paper tests. Eye-trackers not only produce common variables such as completion time, but also provide implicit variables such as fixation duration and saccade latency that can reveal more specific information such as where the participant is looking, what kind of information is distracting, and what approach they used. Measuring these sensitive variables is particularly helpful during more complex tasks because it allows the examiner to better understand why a particular diagnostic group may perform poorly. Historically, eye-tracking has been minimally used in neuropsychological research, despite some unique advantages. For example, 
one method being employed in research examining visuo-spatial ability among individuals with multiple sclerosis is to compare eye-tracking variables with conventional neuropsychological variables to interpret performance on a visual search task. This combined approach offers novel measures of performance allowing comparison of cognitive and physical contributions during visuo-spatial tasks. This talk will discuss how integrating eye-tracking can enhance our research and assessment of patients by producing more specific information that is not possible with traditional paper tests.

Correspondence: Ann-Marie Raphail, BA, Psychology, Drexel University, 136 N 21st St., 2R, Philadelphia, PA 19103, United States. E-mail: ar3223@drexel.edu

C. Rossi, S. OTTINo, C. PARRINi, F. CECCHI, F. CaVAllo \& M.R. TIMPANO SPORTIELLO. The use of $b$ ioengineering techniques in cognitive rehabilitation: a pilot study.

Objective: Our research is a pilot study of a wider research project concerning the application of a new bioengineering technique in cognitive rehabilitation. The present study consists in two parts. The first one aims at evaluating the equivalence between classical re-habilitation instruments (i.e. exercises administered through paper and pencil/ through a personal computer) and novel ones (i.e. a parallel form of such exercises administered through a bioengineering device equipped with sensors and with sensorized cloth) (BDESC). The second part aims at evaluating the usibility of these devises in a pre-clinical group, MCI. Participants and Methods: 30 healthy subjects (HS) (13 M) and 10 MCI subjects $(6 \mathrm{M})$.

Two kinds of cognitive exercises were administered: a verbal paired associated learning task and a sustained attention task. All subjects performed both traditional cognitive tasks (TCT) (i.e. immediate and delayed recall of Verbal Paired Associated of WMS-IV and sustained Attention task, TAP 2.3) and the parallel forms of these cognitive exercises administered through the bioengineering device (BDESC). Half of the subjects performed before TCT and then BDESC, half of the subjects viceversa; each subject was randomly assigned to one of those designs. Results: The internal consistency for each exercise has been evaluated by Cronbach's alpha. Paired t-tests were run to exclude differences in cognitive performances between the parallel forms of exercises.

Conclusions: The alpha values obtained were above the cut-off of 0.70 . None significative differences in performances were found comparing parallel forms of exercises. Such findings showed that both instruments are substantially equivalent in re-habilitation of episodic memory and sustained attention. Futhermore, our findings demonstrated that innovative exercises like BDESC can train the same cognitive functions of TCT and that bioengineering breaks new ground to cognitive rehabilitation. Correspondence: Chiara Rossi, Dr, Via dei Reani, 27, Firenze 50125, Italy.E-mail: chiara.rss@gmail.com

K.L. VICKERS, R. CIABATTONI \& M.T. SCHULTHEIS. Defining the Relationship Between Executive Functioning and Consistency in Regimen Adherence.

Objective: Adherence to recommendations is an important predictor of rehabilitation outcomes. Research has shown that executive functioning (EF) relates to adherence, but little is known about this relationship. The current study hypothesized that reduced EF would be related to poorer consistency in adherence to a behavioral health regimen (i.e., variability in time of day regimen events are completed), and that both would relate to overall adherence (total events completed).

Participants and Methods: 33 healthy college students $\left(M_{\text {age }}=\right.$ $20.03 ; 63.6 \%$ Male) were recruited. Participants were administered EF measures, DKEFS Color-Word Interference Test (CWI), DKEFS Tower Test (TT), and the Trail Making Test (TMT), and completed an online stress monitoring regimen $3 \mathrm{x}$ daily for seven days, from which adherence and consistency were derived.

Results: Bivariate correlations revealed a significant association between regimen consistency and self-corrected errors on CWI Condition 4 (Inhibition/Switching), $r=.40, p=.04$ and a marginal association between consistency and TT Rule Violations, $r=.37, p=.06$. No relationship was found between consistency and errors on TMT B, $r=-.33, p=.10$, or between consistency and overall adherence, $r=.08, p=.70$.

Conclusions: Results indicated greater errors on EF tasks were related to worse consistency, though this did not relate directly to overall adherence. Importantly, these data represent the first systematic study of consistency in adherence to a behavioral regimen, and provide continued evidence that EF is related to some aspects of adherence. Contrary to past findings, consistency appeared to represent a separate construct from overall adherence, which may indicate that it is a moderator (rather than a necessary component) of treatment success. Future research aims to understand how the relationship between EF and consistency affects regimen adherence among individuals experiencing executive dysfunction.

Correspondence: Kayci L. Vickers, MS, Psychology, Drexel University, 421 S. 15th Street, Apt 3F, Philadelphia, PA 19146, United States. E-mail:kayci.vickers@gmail.com

\section{SATURDAY MORNING, FEBRUARY 4, 2017}

\section{CE Workshop 11. How Does Evidence-Based Practice Address the 'Replication Crisis' in Clinical Neuropsychology?}

Presenters: David Loring, Stephen C. Bowden

$$
\text { 7:20-8:50 a.m. }
$$

\section{LORING \& S.C. BOWDEN. How Does Evidence-Based Practice} Address the 'Replication Crisis' in Clinical Neuropsychology?

The 'replication crisis' in scientific research, including psychology, has cast doubt on the trustworthiness of research including clinical research. This course will initially review concerns voiced by proponents of the 'replication crisis' and then demonstrate how these concerns have been addressed, including by proponents of evidence-based clinical practice. Firstly, we will show that in evidence-based practice, not all published studies are of equal quality and the ranking of evidence-quality directly addresses the non-replication question. Secondly, we will illustrate how formalized critical-appraisal skills provide clinicians with the tools to implement a form of post-publication peer review to evaluate the methods-quality and clinical relevance of any published study. Clinicians who routinely undertake critical appraisal and seek to be guided by the highest quality of available evidence will minimize the impact of non-replication on their clinical practice and their research output. As a result of participation in this course, attendees will attain the following learning objectives: (1) identify levels-of-evidence; (2) describe the critical-appraisal approach for evaluating quality of published research; (3) find levels-of-evidence guidelines and learning-resources for critical appraisal. Prior knowledge - graduate level familiarity with research methods in psychology.

Correspondence: David Loring, GA, United States. E-mail: dloring@ emory.edu 


\section{CE Workshop 12. Constraint-Induced Therapies for Neurological Disorders: Contemporary Findings, Application to Disorders of Movement, Aphasia, and Visual Perception, and Increased CNS Neuroplasticity}

\section{Presenter: Victor W. Mark}

7:20-8:50 a.m.

V.W. MARK. Constraint-Induced Therapies for Neurological Disorders: Contemporary Findings, Application to Disorders of Movement, Aphasia, and Visual Perception, and Increased CNS Neuroplasticity.

The Constraint-Induced therapies (CI therapies) are a family of treatments for diverse neurological disorders that are disabling because of their associated Learned Nonuse or Misuse: the conditioned inhibition or maladaptation of purposive activity when compensatory but inefficient means are used instead for everyday activities. The resulting disability may be counter-conditioned by a CI therapy program. The CI Therapy Research Laboratory at the University of Alabama at Birmingham has conducted clinical trials of the CI therapies for over the past 25 years. In this course we will review: (1) the basic neuroscience foundation for CI therapies, (2) the theoretical model for Learned Nonuse, (3) the fundamental methods of CI therapies, (4) the immediate as well as long-term functional benefits of CI therapy for persons with stroke, cerebral palsy, TBI, multiple sclerosis, and spinal cord injury, including results from numerous Randomized Controlled Trials, (5) the validated measurement of learned nonuse in the real world as the preferred outcome measure of CI therapy, (6) the improvements in brain structure on MRI following CI therapies, and (7) the adaptation of CI therapy methods for disorders of leg use, aphasia, and visual perception. We will also review video demonstrations of the treatment methods for children and adults as well. This workshop will be appropriate for clinicians or researchers with intermediate/advanced knowledge of neuroscience, psychology, or rehabilitation.

Correspondence: Victor W. Mark, M.D., Physical Medicine and Rehabilitation, University of Alabama at Birmingham, 1720 2nd Avenue South, SRC 190, Birmingham, AL 35294-7330, United States. E-mail: vwmark@uab.edu

\section{INS Business Meeting (Business \& Beignets) \\ 9:00-9:30 a.m.}

\section{Poster Session 10. Peds 2}

9:00-10:15 a.m.

\section{ADHD/Attentional Functions}

J.R. ANDERSON, J. GUNSTAD \& M. SPITZNAGEL. Differential effects of coffee on sustained attention for good and poor sleepers: Is coffee truly the solution?

Objective: Poor sleep negatively impacts sustained attention/vigilance. Many individuals depend on caffeine (e.g., coffee) to counteract the cognitive sequelae of poor sleep. Although previous work demonstrates that caffeine improves vigilance following laboratory-induced sleep deprivation, few studies have naturalistically examined this relationship. We predicted that coffee would restore vigilance in individuals reporting a recent history of poor sleep to that of those with good sleep quality over a 120 -minute period.

Participants and Methods: Sixty-nine participants completed two randomized, counterbalanced trials consisting of $80 z$ water or coffee (100mg caffeine), followed by cognitive testing. The Pittsburgh Sleep Quality Index assessed recent sleep history, with the established cutoff separating individuals with good (GS) and poor sleep quality (PS). The Automated Neuropsychological Assessment Metrics-4 Standard Continuous Performance Test assessed vigilance at 30, 90, and 120 minutes. Vigilance outcomes were assessed via 2 (Beverage)*3(Time)*2(Sleep) ANOVA.

Results: A Beverage*Time*Sleep interaction $(F=3.48, p=.04)$ was found for commission errors; PS committed more errors than GS at 90 minutes following coffee $\left(F=6.57, p=.01, \eta_{\mathrm{p}}{ }^{2}=.140\right)$ but not water $(p=.13)$. Analyses also revealed a Time* Sleep interaction $(F=5.35$, $p=.007)$ for omission errors; while PS displayed more omission errors than GS regardless of beverage condition, a smaller difference in errors was observed following coffee $\left(F=4.64, p=.04, \eta_{\mathrm{p}}{ }^{2}=.067\right)$ compared to water $\left(F=12.33, p=.001, \eta_{\mathrm{p}}{ }^{2}=.159\right)$ at 90 minutes.

Conclusions: In combination, these findings suggest that consuming coffee to counteract poor sleep only partially restores vigilance and may reduce accuracy. Such practice may have a negative impact on daytime outcomes (e.g., work and school performance) in individuals with poor quality of sleep.

Correspondence: Jason R. Anderson, Clinical Psychology, Kent State University, 1282 Buckingham Gate Blvd, Cuyahoga Falls, OH 44221, United States.E-mail: jasreidan@gmail.com

E. HEBER, J. HALPERIN, B. KRONE, A. BEDARD, I. IVANOV \&. J. NEWCORN. Cognitive and Emotional Control in Youth With ADHD, and the Impact of Stimulant and Non-Stimulant Treatment.

Objective: To understand the effect of cognitive control on emotional control in ADHD youth following medication treatment.

Participants and Methods: ADHD youth $(\mathrm{N}=72)$ were randomized to receive atomoxetine(ATX) or methylphenidate(MPH). Diagnostic status was determined by KSADS-PL \& severity ratings on ADHD-RS 1.5SD greater than age+gender means. Conners' Continuous Performance Test II(2000) provided objective measures of cognitive control:omissions, commissions, mean response time(RT), RT variability\&detectability. Emotional control was measured by parent report on the Social Skills Rating Scale self-control subscale(Gresham\&Elliot,1990).

Results: Pre-treatment cognitive control accounted for $36 \%$ of the variance in improvement in emotional control following $\mathrm{MPH}(\mathrm{p}=.003)$, with detectability making the strongest contribution( $(\mathrm{p}=.004)$. Changes in cognitive control following MPH accounted for $23 \%$ of the variance in treatment response $(p=.04)$, with improvements in detectability accounting for a significant portion of improvement in emotional control $(p=.036)$. Pre-treatment cognitive control accounted for $28 \%$ of the variance in treatment response following $\operatorname{ATX}(p=.02)$. Omission errors and RT Variability made the strongest contributions $(p=.009 \&$ $\mathrm{p}=.003$, respectively). Improvements in cognitive control accounted for $33 \%$ of the improvements in emotional control following ATX $(\mathrm{p}=.02)$. Conclusions: Pre-treatment cognitive control is significantly associated with change emotional control following treatment with stimulant \& non-stimulant medications. Changes in cognitive control account for significant variance in changes in emotional control following treatment. Improvement in emotional control following MPH treatment was partially accounted for by improvements in measures of attentional control (i.e., detectability). Response to ATX was associated with improvements in measures of attention \& state regulation. Findings provide evidence for the complex relation between neural substrates of cognitive \& emotional control, \& indicate directions for future research. Correspondence: Anne-Claude Bedard, NY, United States. E-mail: ac.bedard@utoronto.ca 
S.E. SCOTT, S. DEASLEY, T.A. DUDA \& J.E. CASEY. Preliminary Validation of the BASC-2 in a Canadian Pediatric Sample with and without ADHD.

Objective: Adapting psychological tests for valid use with ethnocultural groups other than those with whom they were developed may require collection of new normative data (Grégoire et al., 2008). The 2nd edition of the Behavior Assessment System for Children (BASC-2) is an American norm-referenced test that is used widely in Canada, although no empirical studies have investigated this practice. The present study aimed to preliminarily validate the use of American normative data with Canadian youth who were typically developing or who had ADHD. Participants and Methods: Archival data from the Parent Rating Scale of the BASC-2 were used. The sample included 17 participants with $\mathrm{ADHD}$ and 30 typically developing participants between the ages of 9 and 16. A Canadian normative data set was formed using data from typically developing participants and a subset of participants with ADHD. One sample t tests were used to compare the mean T score of participants in the Canadian normative data set to a value of 50 , and to compare the mean T scores of the ADHD group on the 4 composites to the mean profile of the ADHD group in the standardization sample. Results: Mean T scores of participants in the Canadian normative data set did not significantly differ from 50 on the Externalizing Problems, Internalizing Problems (IP), and Behavioral Symptoms Index composites (all $p>.08$ ); the mean T score on the Adaptive Skills composite was significantly higher than 50 ( $p=.05)$, but normatively average. T scores of participants with ADHD generated from the Canadian normative data did not significantly differ from the mean $\mathrm{T}$ scores of the ADHD clinical group on 3 composites (all $p>$.33), but scores were significantly higher on the IP composite $(p<.05)$.

Conclusions: Participants in the Canadian normative data set produced similar scores using either set of normative data, and ADHD participants largely conformed to the expected profile of scores, providing preliminary evidence of the validity of using American normative data with the studied groups.

Correspondence: Joseph E. Casey, ON, Canada.E-mail: jecasey@ uwindsor.ca

\section{CASSILL \& D. TUCKER. Differences of Attentional Impairment} in Obstructive Sleep Apnea and ADHD.

Objective: By definition, Attention Deficit Hyperactive Disorder (ADHD) involves problems with attention. Although not inherent to the diagnosis, it has been shown that Obstructive Sleep Apnea (OSA) leads to problems with attention as well. What has not been clearly elucidated is how these problems with attention differ between diagnoses. The purpose of this study is to determine if OSA and ADHD lead to identifiably different patterns in performance on a task of attention. Participants and Methods: Out of 67 participants recruited from a clinical population, 30 were diagnosed with OSA (30 male) while 37 were diagnosed with ADHD (17 male). There were no significant differences for education (ADHD M=14.99, $\mathrm{SD}=2.83$; OSA $\mathrm{M}=14.58$, $\mathrm{SD}=2.66$ ) and WASI-2 FSIQ (ADHD $\mathrm{M}=103.43$, SD=15.38; OSA $\mathrm{M}=104.33, \mathrm{SD}=14.10$ ). The groups differed for age (ADHD $\mathrm{M}=38.78$, $\mathrm{SD}=13.69$; OSA M=53.87, SD=12.71). 32/37 ADHD Ss and 24/30 OSA Ss were right handed. Conners' Continuous Performance Test II (CPT-2) was used to assess attention.

Results: Logistical regression controlling for age was used to determine differences in performance on the CPT-2 between the ADHD and OSA group $($ chi square $=27.794, p=0.001, d f=9)$. Age was entered into the first block of the regression resulting in an $\mathrm{R}^{2}=0.329$. The CPT- 2 variables were entered in the second block resulting in an $R^{2}=0.454$. The resulting classification was correct for $81.1 \%$ for ADHD and $76.7 \%$ for OSA. Of the CPT-2 variables, hit reaction time block change showed a trend towards significance $\left(c^{2}=3.59, p=0.058\right)$ and standard error block change was significant $\left(c^{2}=5.157, \mathrm{p}=0.023\right)$.

Conclusions: Looking further at these relationships, the ADHD group had a slowing of reaction time over the course of the task, whereas the OSA group did not. Both groups showed and increase in response time variability over time. However, this was more pronounced in the OSA group potentially reflecting greater difficulty with the arousal components of attention in this group. Implications for diagnosis and treatment will be discussed.

Correspondence: Carolyn Cassill, B.S., Austin Neuropsychology, 711 W 38th Street, F2, Austin, TX 78705, United States. E-mail: carolyn. cassill@utexas.edu

E. CRAUN, S. KAPLAN \& M.M. WONG. Differential working memory abilities for youth with attention deficits.

Objective: Impairments in working memory (WM) have been suggested to play an important role in attention deficits (Barkley, 1997). WM provides temporary storage and manipulation of information needed to make goal directed decisions and is comprised of different domains (i.e., spatial, verbal, and nonverbal) (Baddeley, 1992). Research has demonstrated those with attention deficits exhibit shortfalls in some domains of WM over others (Martinussen et al., 2005). Therefore, the present study assesses whether youth with attention deficits symptoms demonstrate differential associations between verbal working memory (VWM) and nonverbal working memory (NVWM).

Participants and Methods: 110 community-dwelling youth and their parents completed diagnostic interviews and a neuro-battery, as part of a larger longitudinal study on sleep and health. For this study, the VWM and NVWM subtests of the Stanford Binet -5 and attention problems and ADHD subscales in the Child Behavioral Checklist were used.

Results: Multiple linear regressions were conducted to assess attention deficits and VWM and NVWM functioning. Attention problems and ADHD subscales were used to predict to VWM and NVWM. Significant associations were found among attention problems $\left(\mathrm{R}^{2}=0.08\right.$, $\mathrm{F}(1,109)=9.02, p<.005)$ and $\mathrm{ADHD}$ subscales $\left(\mathrm{R}^{2}=0.04, \mathrm{~F}(1,109)=4.12\right.$, $p<.05)$ and VWM. Attention problems and ADHD symptoms did not predict NVWM. Specifically, attention problems predicted VWM $(b=-0.18(0.06), p<.005)$, but not NVWM $(b=-0.01(0.08), p=.883)$. Similarly, ADHD subscale predicted VWM $(b=-0.15(-.191), p<.05)$, but not NVWM $(b=-0.04(.09), p=.663)$.

Conclusions: These results suggest that those with attention deficits have differential abilities in the realm of WM. As these neurocognitive deficits have been associated with functional impairments, such as lower academic performance and achievement, more research is needed to conceptualize cognitive abilities with youth with attention deficits. This study was supported by the National Institute of Health under award number R01AA020364.

Correspondence: Elizabeth Craun, Idaho State University, 275 La Valle Strada, Pocatello, ID 83201, United States.E-mail: craueliz@isu.edu

A.N. DEVITO, P. SINGH, M. TOMB \& B. TURNER. Do Anxiety Symptoms Moderate Working Memory Performance in Underserved Children?

Objective: To evaluate the effect of anxiety symptoms on the relationship between inattention and working memory in a clinical sample of underserved children.

Participants and Methods: This sample was a subset of a larger clinical sample referred for a neuropsychological evaluation due to primary concern of learning disability or ADHD ( $\mathrm{n}=107,68 \%$ male). Inclusion criteria consisted of FSIQ > 85, age between 8-16 years old $(\mathrm{M}=10.68$, $\mathrm{SD}=2.26$ ), and no history of severe psychiatric or neurological complications. Children were required to have the BASC-II, CPT, and NEPSY Auditory Attention (NAA) and Response Set Omissions (NRS) as part of their original testing battery to be included in the study. Approximately $95 \%$ of the sample endorsed diverse racial or ethnic backgrounds, with $70 \%$ endorsing Hispanic ethnicity. Children who were not English-dominant were excluded from analyses.

Results: Bivariate correlations among measures of inattention and the working memory (WM) index on the WISC-IV were examined. Child-reported BASC-II inattention scores, the CPT-II Omissions T-score, and NAA and NRS scaled scores were examined on WM through hierarchical 
linear regression analyses (HLM). Child-reported BASC-II anxiety scores were used as moderators. Non-verbal IQ, sex, and age were included as covariates.

HLM analyses revealed main effects that approached significance for child reports of inattention and anxiety on WM, and an interaction effect that approached significance $(B=70.67, p=0.07)$. A main effect for the NAA approached significance, and an interaction effect for NAA and child reported anxiety vielded significant results $(B=115.10, p=0.05)$. No significant main effects or interactions were found for the CPT or NRS.

Conclusions: This study offers a preliminary examination of neurocognitive data in a clinical sample of underserved children. Interaction effects of self-reported anxiety and attention depended on how attention was measured in this sample.

Correspondence: Alyssa N. DeVito, M.A., Child and Adolescent Psychiatry, Columbia University, 635 W. 165th Street, 6th Floor, New York, NY 10032, United States. E-mail: and2129@tc.columbia.edu

WITHDRAWN: D. DEWEY, A. VOLKOVINSKAIA \& C. MCMORRIS. Quality of Life in Adolescents with Developmental Coordination Disorder and Attention Deficit/Hyperactivity Disorder.

Objective: An Individual's perception of their own well-being, referred to as quality of life (QOL), can be influenced by physical, psychological, and social factors. Children with neurodevelopmental disorders such as Developmental Coordination Disorder (DCD) and Attention Deficit/ Hyperactivity Disorder (ADHD) have been found to have lower QOL than typically developing children. Few studies, however, have investigated QOL in adolescents with these neurodevelopmental disorders.

Participants and Methods: Forty-four adolescents participated. Based on performance on standardized measures, nine had DCD only, 10 evidenced ADHD only, nine met criteria for co-occurring DCD and ADHD, and 16 were typically developing. Participants completed the KIDSCREEN-52, a self-report measure that assesses ten dimensions of QOL: physical well-being, psychological well-being, moods and emotions, self perception, autonomy, parent relation and home life, financial resources, peers and social support, school environment, and bullying.

Results: No significant group differences were found for total raw score on the KIDSCREEN-52. Investigation of dimensions scores revealed a significant group difference on physical well-being, F $(3,40)=3.67$, $\mathrm{p}=.020$. Post hoc comparisons showed that adolescents in the DCD only $(p=.092)$ and co-occurring DCD and ADHD $(p=.067)$ tended to score lower than those in the typically developing group.

Conclusions: Adolescents with DCD and/or ADHD did not display lower overall QOL compared to typically developing peers. However, youth with DCD only and co-occurring DCD and ADHD perceived that their QOL in terms of physical activity, energy and fitness as lower than that of typically developing youth. This finding is consistent with research that has found that youth with DCD are less physically active and fit than those without DCD. As there is limited research on QOL in adolescents with DCD, studies with larger sample sizes are needed to confirm the findings of the present study.

Correspondence: Deborah Dewey, PhD, Paediatrics, University of Calgary, Owerko Centre, University of Calgary, 2500 University Dr. NW, Calgary, AB T2N 1N4, Canada.E-mail:dmdewey@ucalgary.ca

T.A. DUDA, J.E. CASEY, A.M. O'BRIEN, N. FROST \& A.M. PHILLIPS. Attenuated Graphomotor Procedural Learning in Children and Adolescents with ADHD.

Objective: Research indicates children, adolescents, and young adults with ADHD frequently present with motor difficulties, including handwriting problems and reduced automatization and procedural learning of motor skills (Adi-Japha et al., 2011: Brossard-Racine et al., 2015: Duda et al., 2014; Koziol et al., 2013). There are no studies, however, investigating the development of graphomotor procedural learning in children with ADHD. The present study sought to determine if children and adolescents with ADHD would demonstrate reduced procedural learning of a graphomotor program relative to typically developing peers as indicated by an objective measure of graphomotor automatization and fluency.

Participants and Methods: Thirty-two children and adolescents with $(n=16)$ and without ADHD $(n=16)$ who did not differ significantly in age, IQ, and SES participated in the study. Each group of participants practiced a novel grapheme on a digitizing tablet 30 times. Participants with ADHD discontinued stimulant medication $(n=2)$ or were medication naïve $(n=14)$.

Results: Control participants demonstrated a statistically significant improvement in graphomotor fluency from the beginning to the end of practice, $T=2, z=-2.534, p=.009, r=-0.448$, whereas participants with ADHD did not, $T=4, z=-1.810, p=.074, r=-0.32$.

Conclusions: Consistent with findings in adults with ADHD, results provide evidence that graphomotor procedural learning in children and adolescents with ADHD may be attenuated relative to typically developing peers. Attenuation of learning versus no learning is emphasized noting near-significant improvement in the ADHD group and a medium effect size. Findings have implications for future research that may inform remediation of handwriting difficulties, academic accommodations, and using digitizing technology as a neuropsychological assessment instrument.

Correspondence: Thomas A. Duda, PhD, Psychology, University of Windsor, 401 Sunset Avenue, Windsor, ON N9B 3P4, Canada. E-mail: dudat@uwindsor.ca

A. FEDER, S. COURREGE, K. BORESS \& R. SKEEL. Examination of Revisions to a Measure Designed to Detect ADHD Simulators.

Objective: The present study evaluated a revision of new self-report measure, the ADHD Symptom Infrequency Scale (ASIS), developed to identify individuals exaggerating symptoms in order to meet the criteria for Attention-Deficit/Hyperactivity Disorder (ADHD). The current work follows item analysis of a previous pilot study that provided initial evidence of the utility of the ASIS

Participants and Methods: A four group comparisons model (control, ADHD-diagnosed, an undiagnosed but possible, and analogue simulators) examined differences in endorsement rates across the measure. A total of 227 participants recruited online through Mechanical Turk completed questionnaires. Validity checks were included to screen for adherence to instructions and sufficient effort. Analyses were completed with 152 participants $(65 \%$ female, $81 \%$ Caucasian, mean age $=35$. $\mathrm{SD}=11.5)$. Participants completed both the Barkley Adult ADHD Rating Scale and the novel measure. Both the ADHD and control groups were instructed to answer honestly, and the simulator group was instructed to complete the questionnaires as if trying to convince a doctor they had ADHD

Results: Items meant to detect symptom exaggeration were endorsed at a significantly higher rate for simulators compared to the other three groups, $F(3,148)=87.71, p<.001$. The optimal cut point resulted in a positive predictive value of $90 \%$ and a negative predictive value of $80 \%$ for distinguishing simulators from ADHD-diagnosed. Item level analysis revealed a subset of items that were particularly effective in discriminating between groups.

Conclusions: This study suggests that the ADHD Symptom Infrequency Scale has potential as a reliable and valid measure of ADHD that is sensitive to malingering and exaggeration of symptoms.

Correspondence: Abigail Feder, Psychology, Central Michigan University, 126 Amor Lane, Saint Johns, MI 48879, United States. E-mail:helbl1aj@cmich.edu

N. FEIRSEN \& A. CHACKO. Executive Functioning and Hyperactivity in Youth with ADHD.

Objective: The literature suggests that basic cognitive processing deficits are related to the manifestation of hyperactivity in ADHD, but the exact nature of this relationship remains nebulous. As such, the goal 
of this study was to evaluate patterns of motor activity in children with ADHD as they completed behavioral inhibition (BI) and working memory (WM) tasks; specifically, this study aimed to identify a shared mechanism between cognitive and motoric impairment in children with ADHD.

Participants and Methods: 130 youth (ages 7-10) diagnosed with ADHD were included. The Automated Working Memory Assessment (AWMA) was used to objectively measure WM ability. The A-X Continuous Performance Test (AX-CPT) was used to objectively measure BI. Motor activity was recorded throughout the evaluations using two acceleration-sensitive devices (Actigraphs).

Results: There were no significant differences between groups (children with an identified WM deficit/no WM deficit) with regard to level of hyperactivity $(F(1,127)=0.30, p=0.58)$ when the entire sample was included. However, when medicated children were excluded, those with WM deficits displayed a significantly higher level of motor activity $(F(1,86)=5.59, p=0.02)$. There were no significant differences between groups (BI deficit/no BI deficit) with regard to level of hyperactivity $(F(1,125)=0.95, p=0.33)$, and this did not change after medicated children were excluded $(F(1,84)=0.70, p=0.40)$.

Conclusions: This study documented that unmedicated children with WM deficits display significantly higher levels of activity than children without a deficit in this area. However, this study failed to show any significant relationship between BI and hyperactivity in this population. This suggests that there may be shared underlying mechanisms between WM and hyperactivity, or that the two domains are functionally related. This could have clear behavioral treatment implications, which will be discussed in further detail.

Correspondence: Nicole Feirsen, Psychology, Graduate Center, CUNY, $205 \mathrm{~W} 88 T H$ ST, APT 7D, NEW YORK, NY 100242349, United States. E-mail:nicolefeirsen@gmail.com

L. FERENC, H. SCHNEIDER, M. RYAN \& E.M. MAHONE. ParentReported Adaptive Functioning in Preschoolers With or Without ADHD.

Objective: Children with ADHD demonstrate more psychosocial and adaptive difficulties than peers without ADHD. Adaptive skill deficits are especially prominent in school age children with ADHD, and occur beyond those predicted by IQ alone. Assessing adaptive skill development in young children with ADHD is imperative, so that treatment and intervention can be introduced at earlier ages. Nevertheless, studies assessing the relationships between adaptive functioning, IQ and executive control in preschoolers with ADHD are more limited.

Participants and Methods: Participants included 111 children ages $4-5$ years (60 with ADHD, 51 typically developing children-TD). The ADHD group was screened for language disorders and autism, and met modified DSM-IV criteria. Group differences were examined for the discrepancy scores between adaptive skills (Vineland Domain scores) and FSIQ. Associations with ADHD symptoms (Conners' Scales) and executive functions (BRIEF-P) were examined within the ADHD group for adaptive scales showing group differences.

Results: The ADHD group showed adaptive skill deficits (relative to IQ) compared to the TD group for Vineland Communication and Social domains, and the Adaptive Skill Composite (all p<.01). Within the ADHD group, all three discrepancy scores were significantly associated with ADHD symptom severity and overall executive dysfunction (BRIEF-P GEC), both $p<.01$.

Conclusions: Adaptive skill deficits (beyond those predicted by IQ) are present in preschool-age children with ADHD, and are associated with severity of ADHD symptoms and level of executive dysfunction. Social and communication skills appear most prominently affected, and (along with executive functions) may be a critical target for early intervention. Correspondence: Lisa Ferenc, MA, BS, AA, Neuropsychology, Kennedy Krieger Institute, 1750 E. Fairmount Ave, 3rd floor, Baltimore, MD 21231, United States.E-mail: ferenc@kennedykrieger.org
S. GAU. Comparison of neuropsychological functioning between adults with early- and late-onset DSM-5 Attention-Deficit/ Hyperactivity Disorder.

Objective: Diagnostic and Statistical Manual of Mental Disorders, $5^{\text {th }}$ Edition (DSM-5) elevated the age-of-onset criterion in ADHD. We compared neuropsychological functioning between adults with DSM-5 Attention-Deficit/Hyperactivity Disorder (ADHD) whose onset before and after 7 years old and non-ADHD controls.

Participants and Methods: Neuropsychological functions, measured by Cambridge Neuropsychological Test Automated Battery, were compared among (1) ADHD, onset $<7$ years $(\mathrm{n}=118)$; (2) ADHD, onset between 7 and $<12$ years $(\mathbf{n}=32)$; $(3)$ non-ADHD controls $(\mathbf{n}=117)$. Results: After adjusting for age, sex and psychiatric comorbidity, both ADHD groups showed poorer performance in several neuropsychological functions. Only the early-onset group performed worse in alertness (Reaction Time) and planning (Stocking of Cambridge) than non-ADHD controls. There was no difference in the neuropsychological functioning between the two ADHD groups.

Conclusions: DSM- 5 criteria for adult ADHD is not too lax in terms of neuropsychological functioning.

Correspondence: Susan Shur-Fen Gau, MD., Ph.D., Psychiatry, NATIONAL TAIWAN UNIVERSITY HOSPITAL \& COLLEGE OF MEDICINE, No 7, Chun-Shan South Road, Taipei 10002, Taiwan. E-mail: gaushufe@ntu.edu.tw

B. GRAVES, L.R. KIVISTO, T.A. DUDA \& J.E. CASEY. Predicting Academic Achievement Using Intelligence, Executive Functioning, and Socioeconomic Status in Children With and Without ADHD. Objective: Research has found that socioeconomic status (SES), intelligence (IQ) and executive functioning (EF) all contribute to academic achievement (AA). Considering the cognitive deficits in ADHD are primarily deficits of EF, this domain may have a greater impact on AA in children with ADHD, compared to their peers. No published study to date has examined the relation of these combined factors on AA in children with and without ADHD. The aim of the current study was to investigate the impact of IQ, SES, and EF on AA in children. It was hypothesized that the study variables would differentially predict AA for the ADHD and non-ADHD groups.

Participants and Methods: Children with $(\mathrm{n}=17)$ and without ADHD $(n=30)$ aged $9-15$ (38\% female) were recruited as part of a larger study. Children completed the Vocabulary, Digit Span, Coding, and Block Design subtests of the WISC-IV and the Word Reading, Spelling, and Math Computation subtest of the WRAT-4. EF was estimated by parent reports on the BASC-2 EF Index. Family SES was estimated using the Hollingshead Index.

Results: T-tests revealed the ADHD and non-ADHD group did not significantly differ on IQ, SES, age, gender, and AA (average of WRAT-4 subtests combined). The only significant difference revealed the ADHD group had greater EF impairment. Linear regression analyses were then conducted for the ADHD and non-ADHD groups using EF, IQ, and SES to predict AA. In the ADHD group, EF was a significant predictor of AA, but SES and IQ were not. For the non-ADHD group, both SES and IQ were significant predictors of AA, while EF was not.

Conclusions: The current study suggests that difficulties with AA may be influenced by different factors for children with and without ADHD. Findings suggest that EF, rather than IQ or SES, predicts AA in children with $\mathrm{ADHD}$, supporting the use of differential learning strategies and assessment tools.

Correspondence: Bryanna Graves, Clinical Neuropsychology, Psychology, University of Windsor, 466 Campbell Avenue, Windsor, ON N9B2H4, Canada. E-mail: graves.bryanna@gmail.com 
M. JORGENSON, H. TAM, M. VASSERMAN \& N. GUILLERY. Impact of ADHD on Receptive Language in Children With and Without Language Impairment.

Objective: ADHD and Language Impairment (LI) commonly co-occur. Children with ADHD alone may also have difficulty listening and following directions due to inattention. Research has found that children with ADHD without LI struggle with higher-level listening comprehension. Our aim was to compare receptive language skills in children with ADHD, LI, and comorbid ADHD/LI to examine the impact of inattention on these skills.

Participants and Methods: A clinically-referred sample of children ( $\mathrm{N}=189)$ completed a comprehensive neuropsychological battery that included measures of listening comprehension and following directions. Results: Listening comprehension and following directions composites were significantly correlated with FSIQ and digit span (DS), but not with parent reported working memory impairment. Few children with ADHD without LI demonstrated deficits in receptive language, while nearly a third of children with LI did, regardless of ADHD status. Group differences in performance on tests of listening comprehension were observed $(F(2)=8.339, p=0.001)$, where children with ADHD only performed better than those with LI. These group differences remained significant when FSIQ and DS were included as covariates. Approximately $13 \%$ of children with ADHD alone demonstrated significant difficulties following verbal directions as compared to $33 \%$ of children with LI and $27 \%$ of children with $\mathrm{ADHD} / \mathrm{LI}$, but between-group differences were no longer significant when FSIQ or DS scores were included as covariates. Conclusions: In a clinical sample of children with ADHD and/or LI, children with ADHD alone did not demonstrate significant deficits on receptive language tasks. Additionally, the presence of ADHD in addition to LI did not appear to further exacerbate difficulties with listening comprehension and following directions. These findings suggest that poor performance on measures of receptive language in children with ADHD should not be attributed to ADHD symptomatology alone, and may indicate more significant language problems.

Correspondence: Meghan Jorgenson, Psy.D., Child and Adolescent Psychiatry, NYU Langone Medical Center, 1 Park Avenue, 7th Floor, New York, NY 10016, United States.E-mail:meghan.jorgenson@nyumc. org

A. KANDASAMY, S. VOELKER, J. HAKIM-LARSON \& N. MCNEVIN. The Influence of Impulsivity on Standardized Digital Test Performance.

Objective: Technological advances are changing the landscape of the practice of psychology. Advances in assessment procedures, such as the digitization of popular cognitive tests, provides opportunities for adapting eye tracking tools to obtain additional information during testing. The purpose of this study was to determine if the administration of a digital standardized test can provide further insight into the multifaceted construct of impulsivity, a behaviour difficult to measure using traditional paper-and-pencil testing.

Participants and Methods: Participants were 64 undergraduate students (50 women, 14 men) at an ethnically diverse, medium-sized, university in southwestern Ontario. Eye-tracking data (fixation duration, pupil dilation, latency to initiate, and saccadic speed) as well as reaction time were recorded during administration of the Peabody Picture Vocabulary Test, Fourth Edition Digital Stimulus Book (PPVTIV) and were used to predict impulsivity, as measured by the Barratt Impulsiveness Scale, Eleventh Edition. Demographic factors such as ADHD diagnosis, gender, age, and SES were collected.

Results: Hierarchical regressions indicated slower reaction time and smaller pupil dilation predicted greater impulsivity; but not fixation duration, latency to initiate, or saccadic speed. Greater impulsivity predicted lower PPVT-IV Standard Scores, but not percent correct or number of self-corrections. Impulsivity was related to both gender and an ADHD diagnosis. PPVT-IV scores were associated with age and household income.
Conclusions: Findings lend insight into potential concerns about whether standardized test performance is representative of examinees' abilities, given the relationship between impulsivity and performance. The use of an impulsivity or reaction time measure in combination with the assessor's behavioural observations has the potential to increase objectivity and enhance test validity. The present study can help guide future research in evolving assessment processes and practical uses of eye tracking.

Correspondence: Abirami Kandasamy, Clinical Psychology, Psychology, University of Windsor, 2255 University Avenue West, Apartment 306, Windsor, ON N9B1E6, Canada.E-mail: kandasaa@uwindsor.ca

K. KINGERY, K. LEIKEN, C. SIDOL \& J. EPSTEIN. Brain Activity During Periods of Longer Reaction Times: Event-Related Potential Comparisons of Children With and Without ADHD.

Objective: The objective of the present study was to investigate the neurophysiological basis of long reaction times (RTs) that are a well-established phenomenon among patients diagnosed with Attention Deficit Hyperactivity Disorder (ADHD).

Participants and Methods: Children (ages 7-12) diagnosed with ADHD $(n=25)$ and typically developing controls $(n=26)$ completed an auditory Go/No-Go computerized task while electroencephalography (EEG) was collected. Terciles were created for each individual's reaction times on correct "Go" trials (i.e., fast RTs/medium RTs/slow RTs). EEG activity in parietal electrodes (P3/Pz/P4) between stimulus onset to 900 msec was averaged for all epochs within each individual's terciles and the magnitudes and latencies of the peak amplitudes for each tercile were identified. Main effects of group on tau, a measure of extended RTs, was investigated. Linear mixed models were conducted with group, tercile, and their interaction as independent variables separately for amplitude, latency, and area under the curve (AUC) for the parietal stimulus-locked waveform.

Results: There was no main effect of group for tau. A main effect of tercile was found for the peak amplitude but not the peak latency with long RT terciles having the lowest peak amplitude. There was also a significant main effect of tercile when comparing AUC across terciles indicating that longer RTs are associated with less positive activation overall within the specified time window. There were no group differences in peak amplitude or peak latency nor were there any significant group*tercile interactions.

Conclusions: Study results indicated that long RTs appear to be related to lower ERP peaks during stimulus processing. This seems to be true across children with ADHD as well as typically developing controls. This study adds to a growing body of literature documenting distinct cognitive processes during instances of long RTs.

Correspondence: Kathleen Kingery, M.A., 6611 Greenfield Drive, Cincinnati,OH 45224,United States.E-mail:kingery.kate@gmail.com

L.R. KIVISTO, N. FROST, T.A. DUDA \& J.E. CASEY. Sleep Problems in Children With and Without ADHD Using the BEARS Sleep Disorder Screening Tool.

Objective: Children with Attention-Deficit/Hyperactivity Disorder (ADHD) have been found to have more problems with falling asleep. sleep anxiety, awakenings during the night, and daytime sleepiness, in comparison to children without ADHD. The BEARS Sleep Screening Tool (BEARS) has been found to be a useful clinical screening tool to elicit specific information regarding children's sleep. There is currently very little research using the BEARS, and no literature assessing sleep problems in ADHD using the BEARS. The purpose of this study was to determine whether children with ADHD have more parent reported sleep problems than children without ADHD using the BEARS. It was predicted that children with ADHD would have more reported sleep problems in all five areas assessed by the BEARS than children without ADHD

Participants and Methods: Archival data collected from a community sample of children with $(n=17)$ and without ADHD $(n=31)$ ages 
9-15 were analyzed (38\% female). Children and their parents provided demographic information. Parents independently completed the BEARS and Conners-3.

Results: Logistic regression revealed that children with ADHD were 4.37 times more likely to have one or more sleep problems than children without $\mathrm{ADHD}$ on the BEARS, $\beta=-1.473$, SE $=0.651, p=.024$, Nagelkerke $R^{2}=.150, \chi^{2}(1)=5.537, p=.019$. Individual items of the BEARS were examined using Fisher's exact test and found that only one item (awakenings during the night) was endorsed significantly more for children with ADHD $(p<.001)$.

Conclusions: The study supports the BEARS as a useful tool for screening sleep problems in children with ADHD. Sleep problems are often not adequately assessed during intake interviews for children. ADHD symptoms in adolescents have also been found to be negatively correlated to duration of sleep. For children with ADHD it is critical that sleep problems are assessed, as their presence can impact symptomology, and advise treatment.

Correspondence: Lynette R. Kivisto, Honors BA Psychology, Psychology, University of Windsor, 401 Sunset Avenue, Windsor, ON N9B3P4, Canada.E-mail:kivistol@uwindsor.ca

C. LEVITCH, S.A. GODOVICH, E. MEYERS \& A.K. ROY. Emotion Attribution Impairments, Biases, and Development in Children with Attention Deficit/Hyperactivity Disorder and Severe Emotion Dysregulation.

Objective: The aim of this study was to examine emotion attribution development and accuracy as well as the presence of a hostile attribution bias in young children (5-9 yrs) with symptoms of emotion dysregulation and social impairment.

Participants and Methods: Three groups of children were determined based on a clinician-administered parent interview (KSADS-PL): 80 children with severe emotional dysregulation, as evidenced by frequent, impairing outbursts (SED), 61 children with ADHD without outbursts (ADHD), and 51 healthy controls (HC). Children completed the Assessment of Children's Emotion Skills (ACES). The ACES measures emotion attribution (EA) accuracy and biases and was divided into (1) social behaviors and situations and (2) labeling facial expressions. One-way ANOVAs were used to examine group differences in EA accuracy and biases and correlations were used to examine the relationship between EA accuracy and age.

Results: There were no significant differences among the groups on measures of EA accuracy in response to faces or behaviors/situations $\left(F\left({ }_{2,188}\right)=2.83, p=.061 ; F(2,189)=2.40, p=.093\right)$. However, children with SED demonstrated slightly worse EA accuracy for behaviors/situations than HCs, and children with ADHD demonstrated slightly worse EA accuracy for faces than children with SED. There were no group differences in anger biases $\left(F\left({ }_{2.189}\right)=2.84, p=.061\right)$. EA accuracy for behaviors/situations improved with age in all groups (HC: $r=.54, p=.000$; ADHD: $r=.56, p=.000$; SED: $r=.62, p=.000$ ); only children with SED improved in EA accuracy for faces with age $(r=.32, p=.005)$.

Conclusions: The results of this study indicate the continued development of emotion attribution from contextual scenarios throughout childhood. In addition, it provides partial support for impairments on context-based emotion attribution tasks among children with severe emotion dysregulation compared to healthy controls, which further informs our understanding of specific emotion attribution deficits in psychiatric populations of children.

Correspondence: Cara Levitch, BAS, Psychology, Fordham University, 151 East 31st Street, Apt 17G, New York, NY 10016, United States. E-mail: clevitch@fordham.edu
J. LONGORIA, D. MARICLE \& D. MILLER. A revised model of attentional constructs within the Test of Everyday Attention for Children: Results from exploratory factor analysis with a mixed clinical sample.

Objective: This study examined the latent variables that underlie the Test of Everyday Attention for Children (TEA-Ch) in a mixed clinical sample. Three attentional constructs-selective attention, sustained attention, and attentional control- are reported to exist within the TEA-Ch; however, no studies have examined construct validity using exploratory factor analytic (EFA) techniques.

Participants and Methods: Participants with the following primary diagnoses were included: ADHD $(N=59)$, learning or language disorder $(N=45)$, seizures $(N=25)$, head injury $(N=42)$, neurovascular trauma $(N=25)$, congenital $(N=17)$, and other $(N=33)$. The nine TEA-Ch subtests (Sky Search, Map Mission, Score!, Score DT, Sky Search DT, Code Transmission, Walk, Don't Walk, Creature Counting, and Opposite Worlds) were dependent variables. A secondary EFA was conducted after several variables cross-loaded in the initial EFA.

Results: A two-factor model emerged as the best fit for the data and explained $77 \%$ of the variance. The root mean square error of approximation fit index (.00) indicated good fit. Map Mission (.93) and Opposite Worlds (.52) loaded significantly on factor one. Score! (.82), Score DT (.92), and Code Transmission (.74) loaded significantly on factor two.

Conclusions: A three-factor attentional model did not emerge in a mixed clinical sample of disorders associated with attentional dysfunction. Factor one appears to represent selective visual attention, whereas factor two appears to represent sustained auditory attention. Only five of the nine subtests were significant measures of the two attentional constructs. These results suggest a more conservative model defined by attentional demands and sensory processing modality should be used to interpret the factor structure of the TEA-Ch. It will be beneficial to examine the construct validity of the newly released TEA-Ch2 with EFA techniques and a sample of clinical disorders associated with inattention. Correspondence: Jennifer Longoria, School Psychology, Psychology and Philosophy, Texas Woman's University, CFO 702, P.O. Box 425470, Denton, TX 76204-5470, United States.E-mail: jlongoria1@twu.edu

\section{LOTT \& D.W. BEEBE. The impact of sleep restriction on sustained attention among healthy adolescents.}

Objective: The influence of sleep on adolescents' sustained attention has been understudied with mixed results from correlational and experimental studies (de Bruin et al., 2016). The present study evaluated the impact of sleep restriction on adolescent sustained attention using a modified psychomotor vigilance task (PVT), a computerized measure of sustained attention and response speed.

Participants and Methods: One-hundred sixty-nine healthy adolescents ages $14-17$ years participated in a 3-week sleep manipulation protocol, involving a baseline week followed by cross-over, counterbalanced sleep conditions, including a sleep restriction (SR) condition (6.5 hours in bed per night for five nights) and an enhanced sleep (ES) condition (10 hours in bed per night for five nights). Adherence to sleep protocol was monitored using self-report measures and actigraphy (Micro Motionlogger Sleep Watch®).

Results: Linear mixed modeling was used to evaluate the effect of sleep on adolescents' PVT performance. Results showed an overall effect of sleep condition on PVT reaction times (RT; $F=8.63, p=.004$ ) and attentional lapses $(\mathrm{F}=7.526, \mathrm{p}=.007)$. However, effects were moderated by order. Those who completed the ES condition first showed faster average RT $(366.93 \mathrm{~ms}, \mathrm{SD}=53.75)$ and fewer lapses $(8.77, \mathrm{SD}=8.87)$ during the ER condition compared to adolescents who completed the SR condition first (average $\mathrm{RT}=399.13, \mathrm{SD}=66.18$; average lapses $=$ 13.16, SD = 11.86). Those who completed SR condition first showed minimal difference between the SR and the ES condition.

Conclusions: Sleep duration had an overall impact on sustained attention and response speed, but these effects were moderated by the order of conditions. Adolescents first assigned to the SR condition showed 
minimal improvement in PVT performance when the ES condition followed. This may be due to a carry-over effect, suggesting that adolescents may take longer than a week to recover from a restricted sleep condition.

Correspondence: Mark Lott, Ph.D., Division of Behavioral Medicine \& Clinical Psychology, Cincinnati Children's Hospital Medical Center, 3333 Burnet Avenue, MLC 3015, Cincinnati, OH 45229, United States. E-mail:mark.lott@cchmc.org

WITHDRAWN: A.J. LUNDERVOLD, J. MEZA, M. HYSING \& S. HINSHAW. Early inattentive behavior predicts high school academic achievement across two culturally and diagnostically diverse samples.

Objective: To investigate childhood inattentive behavior as a predictor of future academic success in two diagnostically and culturally diverse samples, taking the impact of the child's intellectual function and sex into account.

Participants and Methods: Samples: (a) a US all-female sample predominated by youth with ADHD (Berkeley Girls ADHD Longitudinal Study [(BGALS]), and (b) a sample recruited from a Norwegian population based sample (the Bergen Child Study [BCS]). Inattentive behavior and intellectual function were assessed via the same measures in the two samples; academic achievement scores in high school were country-specific.

Results: Childhood inattentive behavior predicted academic achievement in both samples, with the strongest effect in the BGALS sample including a large subgroup of children with ADHD. Intellectual function was another strong predictor, but left the effect of inattention statistically significant when covaried in regression analyses. A sex-specific analysis of the BCS sample revealed that the direct effect of inattention was not mediated by intellectual function in girls, while intellectual function fully mediated the effect in boys.

Conclusions: The effect of early signs of inattention on future academic success was found across the two samples, supporting arguments for not restricting remediation procedures to a specific diagnostic group. Future multicenter studies with pre-planned common inclusion criteria should be performed to increase our understanding of sex differences across culturally and diagnostically diverse samples.

Correspondence: Astri J. Lundervold, PhD, Department of Biological and Medical Psychology, University of Bergen, Jonas Lies vei 91, Bergen 5009, Norway.E-mail: astri.lundervold@uib.no

WITHDRAWN: A.J. LUNDERVOLD, T. BøE \& A.

LUNDERVOLD. Prediction of academic achievement in adolescents from teacher reports of inattention in childhood a classification study.

Objective: Inattentive behavior is associated with academic problems. The present study investigates primary school teacher reports on nine items reflecting different aspects of inattention, with an aim to reveal patterns of behavior predicting high-school academic achievement. To that end, we used different types of pattern analysis and machine learning methods.

Participants and Methods: The children $(\mathrm{n}=2397)$ participated in the Bergen Child Study (BCS). Teacher ratings on the inattention items from SNAP-IV was obtained when the children were 7 - 9 years old, and their academic achievements were available from an official school register when attending high-school (16 - 19 years old). Scores of inattention and academic achievement were at a categorical level, and were analyzed by including a multinominal logistic regression, classification and regression trees, random forest, support vector machine and multilayer neural networks. We used cross validation techniques, calculation of confusion matrices, and assessment of feature importance. For all methods we used the caret package in $\mathrm{R}$.

Results: Boys obtained higher inattention scores and lower academic scores than girls. Inattention related to sustained attention and distractibility turned out to have the highest predictive value of academic achievement level across the selected statistical analyses. The most successful classification rate was found between the inattention items and the highest level of achievement scores.

Conclusions: A pattern of early problems related to sustained attention and distractibility predicted academic achievement at high-school. This pattern was fairly consistent across different statistical classification models. A wider range of variables should be included in future studies to strengthen the predictive power of the statistical models.

Correspondence: Astri J. Lundervold, PhD, Department of Biological and Medical Psychology, University of Bergen, Jonas Lies vei 91, Bergen 5009, Norway.E-mail: astri.lundervold@uib.no

M. MARTINELLI, S. MOSTOFSKY \& K.S. ROSCH. Binge eating is associated with delay discounting and $B M I$ in children with ADHD. Objective: Attention-deficit/hyperactivity disorder (ADHD) is linked to increased rates of both obesity and binge eating in children, possibly due to overlap in behavioral characteristics (e.g., atypical response to reward) and neurobehavioral circuitry. For instance, greater delay discounting (i.e., preference for smaller-sooner rather than larger-later reward) has been observed in individuals with ADHD as well as those with obesity and/or binge eating, yet no study has investigated delay discounting as a transdiagnostic process for these disorders. Therefore, this study incorporated measures of binge eating (BE) and BMI to examine for differential associations with delay discounting (DD) among children with and without ADHD.

Participants and Methods: Children age 8-12 with ADHD ( $\mathrm{n}=53$ ) and typically developing (TD) controls $(\mathrm{n}=25)$ completed a monetary DD task. Partial correlations (controlling for intellectual reasoning ability) between DD, BE symptoms on the Children's Binge Eating Disorder Scale (C-BEDS), and BMI were examined within diagnostic group. Linear regressions were conducted to determine whether diagnostic group moderated the relationship between (1) DD and BMI and (2) BE symptoms and BMI.

Results: Greater DD was associated with greater BE symptoms for children with ADHD $(r=-.336, p=.015)$, but not for TD children $(r=-.233, p=.273)$. For children in the ADHD group, greater BE symptoms was associated with higher BMI z-score $(r=.366, p=.008)$, but this relationship was not present for TD children $(r=-.119, p=$ .578). Diagnosis marginally moderated the relationship between DD and BMI $(\beta=-.210, p=.077)$ and between BE symptoms and BMI $(\beta$ $=.202, p=.070)$.

Conclusions: These findings suggest that children with ADHD with greater BE symptoms display a stronger preference for immediate reward and higher BMI z-scores. Further research on delay discounting is necessary in order to inform our understanding of the behavioral overlap between ADHD, binge eating, and obesity.

Correspondence: Mary Martinelli, Kennedy Krieger Institute, $716 \mathrm{~N}$. Broadway St., Suite 331, Baltimore, MD 21205, United States. E-mail: marymartinelli12@gmail.com

M. MARTINELLI, S. MOSTOFSKY \& K.S. ROSCH. Investigating the Impact of Cognitive Load and Motivation on Response Control in Relation to Delay Discounting in Children with ADHD.

Objective: Attention-deficit/hyperactivity disorder (ADHD) is characterized by deficits in impulse control across a range of behaviors, from simple actions to those involving complex decision-making (i.e., preference for smaller-sooner versus larger later rewards). This study investigated whether changes in motor response control with increased cognitive load and motivational contingencies are associated with decision-making in the form of delay discounting (DD) among children with and without $\mathrm{ADHD}$.

Participants and Methods: Children with ADHD ( $\mathrm{n}=26)$ and typically developing (TD) controls $(n=40)$ completed a standard go/no-go (GNG) task, a GNG task with motivational contingencies, a GNG task with increased cognitive load, and two measures of delay discounting that differ in the extent to which the delays and rewards are experienced in real-time. 
Results: Children with ADHD exhibited greater DD than TD controls when making decisions about immediately consumable rewards delivered in real-time, but not when the choices involved money. A greater decline in response control with increased cognitive load was uniquely associated with greater DD on the real-time task for children with ADHD, but not TD children. The effect of motivational contingencies on response control was not associated with DD for either group.

Conclusions: The findings from this study help to inform our understanding of the factors that influence deficient self-control in ADHD, suggesting that impairments in cognitive control may contribute to greater delay discounting in ADHD.

Correspondence: Mary Martinelli, Kennedy Krieger Institute, $716 \mathrm{~N}$. Broadway St., Suite 331, Baltimore, MD 21205, United States. E-mail: marymartinelli12@gmail.com

\section{E. MCGLADE, M. LEGARRETA, E. BUELER \& D. YURGELUN- TODD. Speed and Attention in Veterans with and without a History of Suicide Attempts.}

Objective: Suicide rates of Veterans in 2000 were 20\% higher than would be anticipated after accounting for age and gender effects and 60\% higher than expected in 2010 (Hoffmire et al., 2013). A number of neurocognitive domains have been related to suicidal behaviors, including attention (Keilp et al., 2008). However, very limited data have been reported in veterans. The current study examined attention performance in veterans with and without a history of suicide attempts. Participants and Methods: Forty-nine veterans between the ages of 18 and 55 participated in the study. Veterans completed the Columbia Suicide Severity Rating Scales (CSSRS) to assess history of suicide attempts. Twenty-seven participants reported no history of suicide attempt (HC) and 22 reported a history of suicide attempts (SA). Participants completed additional clinical and neurocognitive measures including the Ruff $2 \& 7$ Selective Attention Test (Ruff 2\&7), Hamilton Depression Scale (HAM-D), and Hamilton Anxiety Inventory (HAM-A). Results: Participants in the SA group performed more slowly on the Ruff 2\&.7 Automatic Detection Speed ( $p=0.01$ ), Ruff $2 \& 7$ Controlled Search Speed $(p=0.004)$, and Ruff $2 \& 7$ Total Speed compared to HC $(p=0.01)$. Interestingly, clinical state symptoms of depression and anxiety (HAM-A/HAM-D scores) were not related to Ruff $2 \& 7$ speed scores.

Conclusions: Veterans with a history of suicide attempts showed decreases in automatic detection speed and controlled search speed compared to those without suicide attempts. Decreases in sustained attention were not related to symptoms of depression or anxiety, suggesting that current mood does not fully account for these differences in attention. Further research should examine the role of attention in decision making as it relates to suicide behavior and to further identify neurobiological differences that underlie cognitive performance and suicidality.

Correspondence: Erin McGlade, PhD, Diagnostic Neuroimaging Lab, University of Utah, 383 Colorow Dr, Salt Lake City, UT 84108, United States.E-mail:erin.mcglade@hsc.utah.edu

\section{A. MLODNICKA, J. HALPERIN, Y. NOMURA \& Y. HURD. Impact of Neurodevelopmental Genes on the Trajectory of ADHD severity: A Pilot Study.}

Objective: Attention-deficit/Hyperactivity Disorder (ADHD) is a chronic neurodevelopmental disorder characterized by symptoms of inattention and hyperactivity, and is associated with delays in neural development.

Participants and Methods: To assess the association of genes involved in neurodevelopment with symptoms of ADHD, saliva was collected and genotyped from 128 participants from a longitudinal study of preschoolers who were followed annually for 7 years. We examined four single nucleotide polymorphisms (SNPs) in genes associated with neurodevelopment: neuregulin-1 (NRG-1; SNP rs3924999), neurotropin-3
(NT-3; SNP rs6489630), brain-derived neurotrophic factor (BDNF; rs6265), and regulator of $\mathrm{G}$ protein signaling 4 (RGS4; rs951439).

Results: Hierarchical linear modeling revealed that neuregulin-1 and neurotropin-3 were associated with symptoms of inattention and hyperactive at age 3-4; however, these genes did not impact the trajectory of the inattentive and hyperactive symptoms over development. Early environmental factors such as maternal diabetes, maternal substance use (tobacco, alcohol, illicit drug) were also analyzed along with each of the genetic factors. Maternal gestational diabetes and NRG-1 risk allele, maternal gestational diabetes and BDNF, alcohol and NRG-1, and illicit drug use and NT-3 were all associated with greater inattentive and hyperactive symptoms at age $3-4$.

Conclusions: Although the sample size is small for genetics study, these findings can inform future investigations in understanding the neurodevelopment and heritability of ADHD.

Correspondence: Agnieszka Mlodnicka, Psychology, CUNY Graduate Center - Graduate Center, 65-30 Kissena Blvd, Department of Psychology, New York, NY 11367, United States.E-mail: amlodnicka@ gmail.com

J.M. NELSON, S. ODMAN \& N. NEGRI. Comparison of Adolescents and Adults With Purely Inattentive Type ADHD and Combined Type ADHD on Speeded Cognitive and Academic Measures: Implications for the Sluggish Cognitive Tempo Construct.

Objective: The purpose of this study was to investigate the presence of purely inattentive ADHD (i.e., no hyperactive-impulsive symptoms) in individuals with ADHD and compare these individuals to those with combined type ADHD on speeded cognitive and academic measures. The validity of purely inattentive ADHD has been questioned and its prevalence has been estimated to be low if it is a valid diagnostic category (Murphy \& Gordon, 2004). Purely inattentive ADHD is potentially more associated with underactivity than it is with hyperactivity and therefore these individuals may be particularly prone to sluggish cognitive tempo. Participants and Methods: Participants were individuals diagnosed with ADHD via a neuropsychological evaluation at a university-based clinic. Participants and parents completed measures of childhood and current ADHD symptoms. Dependent variables included the Integrated Auditory and Visual Speed Quotients, Wechsler Adult Intelligence Scale Processing Speed Index, Woodcock-Johnson Tests of Cognitive Abilities Retrieval Fluency, and Woodcock-Johnson Tests of Achievement Academic Fluency Cluster.

Results: Approximately 20\% met the criteria for purely inattentive ADHD, although prevalence varied based on informant type and time of symptom rating (i.e., childhood or current). Participants with purely inattentive ADHD demonstrated significantly slower retrieval fluency than did those with combined type ADHD. A marginally significant difference was found for processing speed. Groups did not differ on reaction time and academic fluency measures.

Conclusions: A substantial proportion of participants with ADHD were individuals who did not experience any hyperactive-impulsive symptoms. These participants demonstrated slower retrieval fluency and processing speed than did those with combined type ADHD, providing some support for the hypothesis that purely inattentive ADHD is associated with sluggish cognitive tempo.

Correspondence: Jason M. Nelson, Ph.D., Regents' Center for Learning Disorders, University of Georgia, 337 Milledge Hall, Athens, GA 30602, United States.E-mail:jmnelson@uga.edu

A.M. PHILliPS, A.M. O'BRIEN, T.A. DUDA \& J.E. CASEY. Symptoms of Hyperactivity/Impulsivity and Increased Error Rates on Graphomotor Tasks Using a Digitizing Tablet.

Objective: Children with ADHD often experience problems with writing, which can involve problems with spelling and written expression. In addition, these children tend to commit more non-linguistic errors related to motor programming (i.e., non-phonetic letter insertions/omissions/substitutions; Adi-Japha et al., 2007) and kinematic 
aspects of writing (i.e., illegible material and/or inappropriate speed; Brossard et al., 2008). The purpose of the present study is to investigate whether parent ratings of ADHD symptomology using the Conners 3 rating form correlates with the number of errors achieved on graphomotor tasks using a digitizing tablet.

Participants and Methods: Participants $(n=40)$ included a community sample of children and adolescents aged $9-15$ ( $M_{\text {age }}=11.8$ years $)$, of whom 16 met diagnostic criteria for ADHD. Participants completed three graphomotor tasks of varying complexity using a WACOM Cintiq 21UX digitizing tablet and MoveAlyzeR software. Completion of the Conners 3 parent rating form was used to assess ADHD symptomology, including hyperactivity/impulsivity (HI), inattention (IN), and executive functions (EF).

Results: A medium strength and significant positive correlation was found between number of errors made on the handwriting tasks and Conners $3 \mathrm{HI}$ scores, $r(38)=0.33, p=0.04$. No such relationship was found for IN, EF, or ADHD Index scores.

Conclusions: Children experiencing elevated symptoms of hyperactivity/impulsivity as reported by parents make more errors on digital graphomotor tasks. This study suggests some ecological validity for a performance-based measure (i.e., errors on a digital graphomotor task) significantly correlating with a clinical, parent report measure (i.e., Conners $3 \mathrm{HI}$ score) in a mixed sample of children with and without ADHD.

Correspondence: Amanda M. Phillips, University of Windsor, 401 Sunset Ave., Windsor, ON N9B 3P4, Canada.E-mail: phill11b@uwindsor.ca

K.S. ROSCH, K. HIRABAYASHI, D. CROCETTI \& S. MOSTOFSKY. Differential correlations between subcortical volumes and delay discounting in children with and without ADHD.

Objective: Attention-Deficit/Hyperactivity Disorder (ADHD) is characterized by increased delay discounting (i.e., preference for smaller, immediate over larger, delayed rewards) and reduced subcortical volume. This study examined the relationship between delay discounting and the volume of the globus pallidus, putamen, caudate, and thalamus in a large sample of children with ADHD and typically developing (TD) children.

Participants and Methods: Participants included 74 children with ADHD and 56 TD children. High resolution T1-weighted 3D MPRAGE images covering the whole brain were acquired and subcortical structures were automatically extracted. Participants also completed two delay discounting (DD) tasks involving choices between immediate and delayed rewards of either playing a preferred game in real-time (realtime DD) or a classic discounting task involving choices about money (classic DD). Partial correlations and linear regression were employed to determine whether the relationship between subcortical volumes and DD was moderated by diagnostic group after accounting for Age, intellectual ability, and total cerebral volume.

Results: In the full sample, greater delay discounting (i.e., less area under the curve) on the classic DD task was significantly associated with greater volume of the left and right putamen (left: $r=-.213, p=.016$; right: $r=-.265, p=.003)$ and the right globus pallidus $(r=-.198, p=.025)$. There were no significant correlations with the real-time DD task $(r s<.14, p s>12)$. Diagnostic group moderated the relationship between classic DD and volume of the left and right putamen ( $p s=.001$ and .006), left and right thalamus ( $p s=.001$ and .005 ), and left and right globus pallidus ( $p s=.040$ and .032 ), such that greater volume predicted greater delay discounting among TD children only.

Conclusions: These findings suggest that the relationship between subcortical volumes and delay discounting differs for children with ADHD and typically developing controls.

Correspondence: Keri S. Rosch, Ph.D., Neurology, Kennedy Krieger Institute, 716 North Broadway, Baltimore, MD 21205, United States. E-mail:ksrosch@gmail.com
K.S. ROSCH, K.L. SWEENEY, E.M. MAHONE \& S. MOSTOFSKY. Neuropsychological Correlates of Delay Discounting in Girls and Boys with ADHD.

Objective: Attention-Deficit/Hyperactivity Disorder (ADHD) is characterized by increased delay discounting (i.e., preference for smaller, immediate over larger, delayed rewards), and executive dysfunction, including deficits in response inhibition, working memory, and attention regulation. This study examined the relationship between delay discounting and executive dysfunction in a large sample of children with ADHD, oversampled for girls, compared to same-sex typically developing (TD) children.

Participants and Methods: Participants included 8-12 year-old children with ADHD (n=99; 29 girls) and their TD peers ( $\mathrm{n}=57,19$ girls). Participants completed two delay discounting (DD) tasks involving choices between immediate and delayed rewards of either playing a preferred game in real-time (real-time DD) or a classic discounting task involving choices about money (classic DD), as well as a computerized visuospatial working memory task (spatial span) and a go/no-go (GNG) task. Parent ratings of executive function in daily activities (BRIEF) were also examined.

Results: Sex moderated the relationship between commission error rate and real-time DD among children with ADHD ( $\beta=2.3, p=.023)$, such that greater delay discounting on the real-time DD task correlated with more commission errors among girls with ADHD ( $r=-.411, p=.033)$, but not among boys with ADHD $(r=.058, p=.634)$. However, sex did not moderate the relationship between parent-reported behavior regulation and real-time DD $(\beta=1.38, p=.171)$, although this relationship was robust among girls with ADHD ( $r=-.501, p=.009)$, and was not significant among the larger sample of boys with ADHD $(r=-.163, p=.201)$. Conclusions: These findings suggest that among girls with ADHD, those who display a stronger preference for immediate reward also display greater response disinhibition. Furthermore, parent rated behavior dysregulation in daily activities is related to delay discounting in children with ADHD, regardless of sex.

Correspondence: Keri S. Rosch, Ph.D., Neurology, Kennedy Krieger Institute, 716 North Broadway, Baltimore, MD 21205, United States. E-mail:ksrosch@gmail.com

\section{A.N. SIMONE \& J. HALPERIN. Persistence of ADHD Symptoms, not Remission, is Associated with Working Memory Difficulties at 8-years-old.}

Objective: To examine in a longitudinal sample of children whether working memory (WM) performance acts as a function of persistence or remission of symptoms of attention-deficit/hyperactivity disorder (ADHD). It was hypothesized that those children who persisted with ADHD from preschool through school-age, and not those who showed a diminution in symptoms, would perform significantly worse on WM at 8-years-old as compared to controls.

Participants and Methods: The sample consisted of 144 8-year-old children who were initially recruited at preschool age deemed as either "at-risk" for ADHD or "typically-developing" based on the presence or absence of inattentive and/or hyperactive/impulsive symptomatology. Seventy-one children demonstrated persistence of ADHD symptoms in which they were "at-risk" at preschool age and met formal DSM-5 criteria for a diagnosis of ADHD at 8-years-old (ADHD-Persisters); 21 children were "at-risk" for ADHD as preschoolers but showed a remission of ADHD symptoms at 8-years-old (ADHD-Remitters); and 52 children were "typically-developing" preschoolers and did not meet criteria for an ADHD diagnosis at 8-years-old (Controls). WM was obtained from the WMI of the Wechsler Intelligence Scale for Children Integrated - Fourth Edition (WISC-IV Integrated). Data were analyzed using ANOVA with Group (ADHD-Persisters vs. ADHD-Remitters vs. Controls) serving as the between-subjects factor and WMI score serving as the outcome measure.

Results: The groups significantly differed on WM ability at 8-years-old $(\mathrm{p}<0.001)$. Post hoc comparisons revealed that ADHD-Persisters performed significantly worse on WM as compared to Controls 
$(p<0.001)$, whereas ADHD-Remitters did not significantly differ from ADHD-Persisters $(p=0.12)$ or Controls $(p=.56)$ on WM performance. Conclusions: Findings indicate that improvement of WM is potentially related to $\mathrm{ADHD}$ symptom remission.

Correspondence: Ashley N. Simone, Psychology, The Graduate CenterCUNY, 335 Somerston Road, Yorktown Heights, NY 10598, United States.E-mail: ashleyn.simone@gmail.com

K.L. SWEENEY, M. RYAN, H. SCHNEIDER, L. FERENC, E.M. MAHONE \& M. DENCKLA. Developmental Trajectory of Motor Deficits in Preschool Children with and without ADHD.

Objective: Motor deficits persisting into late childhood ( $>7$ years) are associated with increased executive and cognitive dysfunction, likely due to shared neural circuitry. Identifying young children at risk for anomalous neurological development by examining motor deficits is critical. The study assessed developmental trajectory of motor deficits in children with ADHD during early childhood, compared to typically developing (TD) children.

Participants and Methods: Participants included 61 children (31 ADHD, $30 \mathrm{TD})$ ages $4-7$ years $(62 \%$ male) who participated in three visits (T1, T2, T3), each one year apart $(\mathrm{T} 1=48-71, \mathrm{~T} 2=60-83$, T3=72-95 months). Variables examined include Total Overflow, Finger Tapping Time, Finger Sequencing Time from the Revised Physical and Neurological Examination for Subtle Signs (PANESS). Main effects for group, visit and the group-by-visit interaction were examined through linear mixed effects (LME) models, with follow-up inquiry of visit and group differences at T1 vs. T2 and T2 vs. T3.

Results: There were significant main effects for group (favoring TD) for Overflow and Finger Tapping Time (both $\mathrm{p}<.05$ ), and for time (performance improving over time) for all 3 variables (all $p<.05$ ), but not significant group-by-time interactions for any variables. For Overflow $(p=.003)$, Finger Tapping $(p=.017)$, and Finger Sequencing $(p=.014)$, performance across groups improved significantly between T1 and T2. Performance continued to improve significantly between T2 and T3 for Finger Sequencing $(p<.001)$, but not for Overflow or Finger Tapping. Conclusions: By age 4 years, children with ADHD manifest significant deficits in motor control and speed. For ADHD and TD children, motor control and speed improve rapidly between ages 4 and 7 years, with the most rapid improvements occurring before age 6 . Motor overflow and skeletomotor speed (finger tapping) are sensitive markers of motor development in children as young as 4 years, but finger sequencing may be less sensitive to anomalous development due to delayed competency. Correspondence: Kristie L. Sweeney, Master of Science, Neuropsychology, Kennedy Krieger Institute, 124 Pleasant Hill Road, Owings Mills, MD 21117, United States. E-mail: Sweeney@kennedykrieger.org.

S.K. TADROUS-FURNANZ. Does processing speed contribute to the far transfer effects of Cogmed Working Memory Training on Reading Comprehension?

Objective: Reading skills are more strongly associated with processing speed than with working memory. Our aim was to examine whether children with attention deficits who showed more improvement in Reading following computerized working memory training would have faster processing speed.

Participants and Methods: We offered 20 students aged 7 to 17 who had been identified with difficulties in learning and attention, five halfhour sessions per week of Cogmed working memory training over a period of 5 weeks. There were 17 who met the inclusion criterion for this study of WIAT-III Pseudoword Decoding scores greater than 85. They underwent 6-hour neuropsychological evaluations before and after Cogmed $^{\text {TM }}$ Training that included the WISC-IV and WIAT-II. Cogmed Index Improvement, is a measure of effort and achievement from the computer-based adaptive system.

Results: There was a mild trend toward a significant improvement in Reading Comprehension from pre- to posttraining, $t(13)=1.63$, $p=.13$. Even with the Cogmed Index Improvement controlled, this gain in reading approached a correlation with processing speed, $r(12)=.50, p=.07$

Conclusions: Faster processing speed uniquely contributes to improvement in reading comprehension following working memory training with Cogmed. Students with adequate processing speed may therefore be able to benefit from the far transfer effects of working memory training on their reading comprehension

Correspondence: Sandy K. Tadrous-Furnanz, MA, Psychology, Fuller Theological Seminary, 670 N. Hill Avenue, Apt \# 6, Pasadena, CA 91106, United States. E-mail: sandytadrous@fuller.edu

E. VOGT, K.A. RITCHIE, D.E. MARRA, R. SCHNEIDER, J. HOELZLE \& P. MARSHALL. Convergence of ADHD Symptom Report and Neuropsychological Tests.

Objective: Invalid performance and symptom report confounds differential diagnosis. This study investigated the convergence of self-reported ADHD symptoms with performance on neuropsychological tests before and after participants with suspected invalid performance/symptom report are removed from analyses.

Participants and Methods: Three hundred thirty adults [ $M$ age 26.81 (8.02)] were referred for an evaluation to determine whether a diagnosis of ADHD is warranted. Patients completed symptom and performance validity, neuropsychological, and self-report measures. Ninety-nine adults were identified as giving invalid performance based on symptom and performance validity testing.

Results: When all patients were considered, self-report of inattention was significantly associated with ten measures of memory, attention, working memory, and processing speed $(r$ range $=-0.13$ to -0.24 , $p<.05)$. Self-report of hyperactivity/impulsivity was significantly associated with fifteen measures of memory, attention, working memory, and executive functioning $(r$ range $=-0.12$ to $-0.26, p<.05)$. In contrast, when individuals with suspected invalid performance were removed from analyses, the majority of the correlations between self-report of ADHD symptoms and neurocognitive functioning decreased and were non-significant (Fischer $\mathrm{r}$ to $\mathrm{z}$ transformation range -1.6 to -3.2 ; $p<.05)$. Color-word interference remained associated with self-reported inattention $(r=.16, p<.05)$ while symbol search and digit symbol coding were associated with self-reported hyperactivity $\left(r^{\prime} s=.19\right.$, $p<.05)$.

Conclusions: Many clinicians consider the convergence of data when deciding whether to diagnosis ADHD. Self-report of ADHD symptoms may appear to converge with performance on various cognitive measures, however this may be an artifact of failing to identify invalid performance or symptom report. This research adds to a growing literature documenting the importance of evaluating symptom and performance validity during clinical research.

Correspondence: Elisabeth Vogt, MS, Psychology, Marquette University, 2928 N 84th Street, Milwaukee, WI 53222, United States. E-mail: elisabeth.vogt@marquette.edu

\section{K. WOOTEN, L. COLEMAN \& D.G. NEMETH. Addressing the Sustained Attentional Problems of ADHD Ultra-rapid Metabolizers.}

Objective: The Genomind Study is a test used to determine an individual's genetic affinity for certain types of medications. According to Genomind, an ultra-rapid metabolizer is an individual who "breaks down medication very quickly to process it out of the body" (2016). These individuals are essentially at risk for low blood levels of the drug. In other words, they are less likely to respond to certain medications because the drugs are processed too quickly in their body (2016). This is quite disconcerting for an individual who has twice failed medication management. The purpose of this poster presentation is to explore this phenomenon; especially in relationship to Attention Deficit Hyperactivity Disorder (ADHD) ultra-rapid metabolizers.

Participants and Methods: The neuropsychological and metabolic profiles of 10 individuals diagnosed with ADHD will be presented. These 
physician- referred individuals were all evaluated and treated at The Neuropsychology Center of Louisiana (NCLA) in Baton Rouge, Louisiana. They were referred because of the lack of efficacy of previous medication management.

Results: These individuals were evaluated and placed on efficacious medication regimes with excellent academic and behavioral outcomes. These interventions will be highlighted.

Conclusions: These case studies point to the value of utilizing attentional and genetic testing to develop appropriate interventions. This approach is far less frustrating for neurodevelopmentally impaired individuals than using the more typical "trial-and-error" approach to psychopharmacology.

Correspondence: Kortney Wooten, B.S., 4611 Bluebonnet Blvd., Suite B, Baton Rouge, LA 70809, United States.E-mail:kortneywooten@ ymail.com

A. KIRSCH, K. DEANE, T. COMBS, S. AYLWARD \& E. HEIDEMAN. Discriminative Power of Rey-Osterrieth Complex Figure Test for Attention-Deficit/Hyperactivity Disorder Compared to Other Attention and Executive Functioning Measures.

Objective: The Rey-Osterrieth Complex Figure Test (ROCF), which requires reproduction of a detailed drawing, immediately and after a delay, relies on attention and executive functioning (Shin, Park, Park, Seol, \& Kwon, 2006). Previous studies have indicated that children with Attention-Deficit/Hyperactivity Disorder (ADHD) perform worse than controls on the ROCF (Seidman et al., 1995); however, the discriminative utility of the ROCF, as compared to other measures of attention and executive function, is unknown.

Participants and Methods: This study compared the discriminative function of the ROCF for ADHD, to other common measures of attention and executive function (CPT, DKEFS, BASC-Attention and Hyperactivity Scales) in a clinically referred sample of 262 children/adolescents (Range $=3-22$ years, $38 \%$ females, $49 \%$ ADHD).

Results: Results from logistic regression revealed that neither the ROCF immediate $(B=-.01, S . E .=.01, p=.301)$ nor the ROCF delayed $(B=-.02$, S.E. $=.01, p=.100)$ were predictive of whether children received ADHD diagnoses or not. However, the recognition subtest of the ROFC was predictive $(B=-.06, S . E .=.03, p=.038)$. CPT hit rate $(B=.02, S . E .=.01$, $p=.024)$, move accuracy on the tower subtest of the DKEFS $(B=-.12$, S.E. $=.05, p=.019)$, BASC attention problems $(B=.07, S . E .=.02, p<.001)$, and BASC hyperactivity $(B=.04, S . E .=.01, p=.002)$ were also significant predictors of diagnosis.

Conclusions: This study indicated that many measures of attention and executive functioning, as well as the immediate and delayed subtests of the ROCF, are not significantly or uniquely predictive of ultimate diagnosis. However, the ROCF recognition test may be useful in conjunction with other measures to diagnose attention problems in pediatric samples. Correspondence: Alexandra Kirsch, Psychology, Loyola University Chicago, 6301 N. Sheridan Rd.,, Apt 24P, Chicago, IL 60660, United States.E-mail:akirsch@luc.edu

\section{Assessment/Psychometrics/Methods (Child)}

\footnotetext{
A. KIRSCH, K. DEANE, T. COMBS, S. AYLWARD \& E. HEIDEMAN. Correlates of Rey-Osterrieth Performance Across Memory, Attention, and Visual-Spatial Functioning in a Referred Pediatric Sample.

Objective: The Rey-Osterrieth Complex Figure Test(ROCF), requires individuals to reproduce a complicated drawing, immediately and after a delay, and relies on numerous neurocognitive functions, including visuospatial abilities, memory, attention, planning, and working memory (Shin, Park, Park, Seol, \& Kwon, 2006). This study was designed to examine which of these functions are most related to performance on ROCF in a pediatric sample to better understand its clinical utility.
}

Participants and Methods: This study compared the strength of clinical correlates of ROCF performance, including visuospatial abilities(Beery VMI), memory(CMS/CVLT), attention(CPT), executive functioning(DKEFS-Color-Word, Verbal Fluency, Tower), and working memory (WISC-IV/V) in a clinically referred sample of 262 children/ adolescents (Range $=3-22$ years, $38 \%$ females).

Results: The ROCF immediate and delayed scores were correlated, in the order of strength, with the workingmemory subscale of the WISC $(r \mathrm{~s}=.26-.26$, $p \mathrm{~s}=.001-.002)$, the DKEFS verbal fluency subtest $(r \mathrm{~s}=.24-.25$, $p s=.001-.002)$, Beery VMI $(r s=.20-.26, p s=.001-.006)$, the CMS story-immediate $(r \mathrm{~s}=.20-21, p s=.011-.022)$, the DKEFS towerrule violations $(r s=.17-.20, p s=.011-.019)$, the CMS story-delayed $(r \mathrm{~s}=.16-.18, p s=.038-.048)$, and CPT commissions $(r \mathrm{~s}=.16-.17$, $p s=.023-.027)$. The ROCF immediate score, but not delayed, was correlated with DKEFS tower achievement $(r=.18, p=.016)$ and the CVLT-Total $(r=.17, p=.017)$. Neither subscale was correlated with other subscales of the CPT, DKEFS, or CMS.

Conclusions: This study highlights that performance on the ROCF most directly aligns with measures reliant on working memory, verbal fluency, impulsivity, planning visual-motor integration, and story memory. Thus, careful considerations should be made when interpreting this measure in populations with deficits in these areas.

Correspondence: Alexandra Kirsch, Psychology, Loyola University Chicago, 6301 N. Sheridan Rd.,, Apt 24P, Chicago, IL 60660, United States.E-mail: akirsch@luc.edu

C.A. AUSTIN, M. GERSTLE, K.T. BAUM \& D.W. BEEBE. Parent Expectations and Change in Parent Efficacy in Pediatric Neuropsychological Evaluations.

Objective: To better understand parent expectations for pediatric neuropsychological evaluation and the change in parent knowledge and efficacy over the course of the evaluation.

Participants and Methods: 131 parents of children receiving outpatient neuropsychological assessment participated in the study. Parents received questionnaires at 4 time points: before parent interview, day of child testing/interview, just before feedback, and a mailed follow-up several months after receiving the final report. Friedman's and Wilcoxon non-parametric tests were used to analyze changes across time points (alpha set at .01 due to multiple analyses).

Results: Parents reported significantly increased knowledge of how to help their child at Time 3, prior to hearing results of the evaluation. Parent efficacy also significantly increased on the follow-up questionnaire post-oral and written feedback (Time 4). Parents reported increased understanding of their child's medical condition, knowledge of why their child struggles, how to help and ability to help their child. Parents' top goals for the evaluation were to improve their child's life and services at school, and to better understand their child's problems. At follow-up, respondents rated the evaluation helped most with learning about treatment options, ways to deal with their child's problems, and gaining a better understanding of their child.

Conclusions: Parents perceive significant benefits from neuropsychological evaluation. The pattern is also consistent with clinical expectations, i.e., the majority of increase should occur following feedback. Interestingly, parents also showed higher ratings for knowing how to help their child just prior to that feedback. Parents indicated the most consistency in expecting and receiving improved understanding of their child as a high-ranking goal of the evaluation.

Correspondence: Cynthia A. Austin, Ph.D, Behavioral Medicine and Clinical Psychology, Cincinnati Children's Hospital and Medical Center, 3333 Burnet Avenue, Cincinnati, OH 45229, United States. E-mail:cynthia.austin@cchmc.org 
A. CANNON, C. STEPHAN, A. PRITCHARD \& T.A. ZABEL. Improvement or Inflation? Analysis of the Adaptive Behavior Assessment System (ABAS-II Versus ABAS-3) in a Clinical Sample. Objective: The ABAS is commonly used to assess adaptive skills and determine the functional impact of neuropsychological deficits. ABAS-II and ABAS-3 scores are strongly correlated in the normative sample (Harrison \& Oakland, 2015), but mean composite scores are higher on the ABAS-3. This study investigated the comparability of ABAS-II and ABAS-3 within a clinical population, and aimed to help anticipate potential instrumentation-related increases when different ABAS versions are used in serial assessment.

Participants and Methods: 4,233 patients evaluated in an outpatient pediatric neuropsychology clinic in an urban medical setting were administered the ABAS-II or ABAS-3 between 2012 and 2016. Groups of patients with parent-ratings on the ABAS-II ( $n=3110$; mean age $=10.41,66 \%$ boys, mean Verbal Composite Index $=92.29)$ or the ABAS-3 ( $n=1123$; mean age $=10.67,66 \%$ boys, mean Verbal Composite Index $=93.44)$ were compared. There were no between group differences in patient gender, age, or mean verbal intelligence. Additionally, paired t-tests were run on a subset of patients $(n=7)$ for whom parents completed ABAS-II and ABAS-3 forms during the same visit.

Results: Significant differences were found between the ABAS-II and ABAS-3 groups for the General Adaptive Composite ((GAC) $\mathrm{p}<.01$; mean=75.85 and 82.77) and the Practical Composite $(\mathrm{p}<.01 ; m e a n=73.29$ and 79.37). The Conceptual Composite difference approached significance $(p=.066)$. The paired t-test showed significant differences when the measures were administered at the same visit (GAC and Conceptual Composite ps<.05).

Conclusions: Findings suggest that the ABAS-3 may produce higher scores than the ABAS-II. Differences of nearly one-half of a standard deviation were observed. Transition between versions may result in instrumentation-related increases in scores and suggest greater improvement than has actually occurred.

Correspondence: Alicia Cannon, Ph.D., Neuropsychology, Kennedy Krieger Institute, 1750 East Fairmount Ave, Department of Neuropsychology, Baltimore, MD 21231, United States. E-mail: cannona@kennedykrieger.org

S. DEASLEY, A.M. O'BRIEN, L.R. KIVISTO \& J.E. CASEY. Concurrent Validity of the BDEFS-CA in a Canadian Sample of Children with and without ADHD.

Objective: The Barkley Deficits in Executive Functioning Scale - Children and Adolescent (BDEFS-CA) is a parent-completed behavior rating scale used to assess executive functioning. It includes five subscales of various aspects of executive functioning: Self-Management to Time, Self-Organization/Problem Solving, Self-Restraint, Self-Motivation, and Self-Regulation of Emotions. Since its release in 2012, no independent research has examined the validity of the BDEFS-CA. The purpose of the present study was to investigate the concurrent validity of the BDEFS-CA by determining its correlation with the Conners 3 , a wellestablished measure of ADHD symptoms. Given the relatedness of ADHD symptoms and executive dysfunction (Barkley, 2015), it was expected that the BDEFS-CA subscales would be highly positively correlated with the ADHD index of the Conners 3.

Participants and Methods: Participants $(n=100)$ included parents of Canadian children aged 8- to 14-years-old $\left(M_{a g e}=11.34,29 \%\right.$ female) recruited from the community as part of a larger study examining fine motor control and ADHD. Half of the children met criteria for ADHD. Parents completed the BDEFS-CA long form and the Conners 3.

Results: As expected the Conners 3 ADHD index was significantly, positively correlated with each of the five BDEFS-CA subscales: SelfManagement to Time $(r=.78, p<.001)$, Self-Organization/Problem Solving $(r=.71, p<.001)$, Self-Restraint $(r=.72, p<.001)$, Self-Motivation $(r=.78, p<.001)$, and Self-Regulation of Emotions $(r=.61, p<.001)$. The Conners 3 ADHD Index was also significantly, positively correlated with the BDEFS-CA summary score $(r=.80, p<.001)$.
Conclusions: The summary score and all subscales of the BDEFS-CA were significantly positively correlated with overall ADHD symptoms as measured by the well-established Conners 3 . Results provide evidence of concurrent validity of the BDEFS-CA. In addition, the findings support Barkley's (2015) notion that ADHD is a disorder of executive functioning.

Correspondence: Shanna Deasley, University of Windsor, 401 Sunset Avenue,Windsor, ONN9B3P4, Canada.E-mail: deasley@uwindsor.ca

C.E. GAUDET, B.C. KAVANAUGH \& K.A. HOLLER. Appraising Neuropsychological Impairment in Children with Severe Psychiatric Disorders: Applying Predicted Base Rates.

Objective: This study was designed to assess the performance of a pediatric population with severe psychiatric disorders (SPD) on neuropsychological testing through the examination of discrepancies between the actual and predicted rates of abnormal performances using a flexible battery of tests.

Participants and Methods: The study consisted of 99 children with SPD from a children's psychiatric inpatient unit (Age $=10.2 ; \mathrm{SD}=1.8$ ) and 161 children drawn from an outpatient sample referred for neuropsychological testing (Age $=11.4 ; \mathrm{SD}=2.6$ ). Both samples completed a neuropsychological battery consisting of 18 scores that were interpreted. The methodology to generate predicted rates of abnormal scores was derived from Crawford, Garthwaite, and Gault (2007) and incorporates correlations between test scores from the outpatient sample and Monte Carlo simulations.

Results: Children with SPD produced more abnormal scores at higher rates (i.e., 2 or more, etc.) than the predicted base rates and the outpatient group across varying criteria for abnormality $(<1 \mathrm{SD},<1.5 \mathrm{SD}$, $\&<2$ SDs) with the exception of 1 or more abnormal scores at $<1$ SD. For example, when the criterion for abnormality was defined as scores falling below $1.5 \mathrm{SDs}$, the predicted percentage of a 'normal' population generating 5 or more abnormal scores was $6.3 \%$, whereas it was $19.2 \%$ for children with SPD.

Conclusions: Characterizing neuropsychological functioning in children with SPD remains an elusive pursuit. These findings suggest that children with SPD exhibit both higher rates and a greater degree of impairment on neuropsychological assessment, as indicated by higher percentages of children with SPD obtaining abnormal results at higher rates than comparison groups. Full Scale Intelligence Quotient (FSIQ) scores and the presence of psychopathology may contribute to the observed rates of abnormal scores in this population.

Correspondence: Charles E. Gaudet, M.S., Psychology, University of Rhode Island, University of Rhode Island, Chafee Hall, 142 Flagg Rd., Kingston, RI 02881, United States.E-mail: chad_gaudet@my.uri.edu

C.E. GAUDET, B.C. KAVANAUGH \& K.A. HOLLER. Rates of Neuropsychological Test Completion Within a Pediatric Inpatient Sample.

Objective: The neuropsychological assessment of children with severe psychiatric disorders (SPD) during a psychiatric hospitalization presents unique challenges attributable to both the setting and examinee. This study examined test completion rates among an inpatient sample consisting of children with SPD.

Participants and Methods: Two-hundred thirty-six children with SPD $($ Age $=9.6 ; \mathrm{SD}=2.0)$ were administered a neuropsychological battery during psychiatric hospitalization consisting of the following tests: Wechsler Abbreviated Scale of Intelligence (WASI-I/II), Grooved Pegboard, Verbal Fluency, Wide Range Assessment of Memory and Learning-Second Edition (WRAML-II-Story Memory [Immediate \& Delayed], Recognition, and Sentence Memory), Beery Visual-Motor Integration (VMI), Rey Complex Figure Test (RCFT-Copy), Trail Making Test Parts A \& B, Stroop Color and Word Test, Continuous Performance Test-Second Edition (CPT-II), and Wisconsin Card Sorting Test (WCST). Completion was defined as producing a valid score on the test. 
Results: Only $33 \%$ of children completed the entire battery, $55 \%$ completed 9 or more tests, and $69 \%$ completed 8 or more tests. The WASI had the highest rate of completion (95\%) followed by the Beery VMI (92\%) and Grooved Pegboard (89\%). The CPT-II had the lowest rate of completion (62\%) followed by the WRAML-II (64\%) and RCFTCopy $(69 \%)$. On average, children were able to complete $7.9(\mathrm{SD}=2.3)$ tests within the battery.

Conclusions: Failure to complete the battery may be attributable to a broad range of causes such as discharge, behavioral dysregulation, or fatigue. These findings illustrate the challenge of obtaining estimates of neuropsychological functioning in children with SPD using existing tests in an inpatient setting.

Correspondence: Charles E. Gaudet, M.S., Psychology, University of Rhode Island, University of Rhode Island, Chafee Hall, 142 Flagg Rd., Kingston, RI 02881,United States. E-mail: chad_gaudet@my.uri.edu

S. HEARPS, M.H. BEAUCHAMP, S. HEARPS, J. DOOLEY, D. DAVID, J.R. CRAWFORD, L. TURKSTRA, S. MCDONALD \& V.A. ANDERSON. Pediatric Evaluation of Emotions Relationships and Socialization (PEERS): Interim Analysis of Clinical Data.

Objective: Specific social skills deficits are a key symptom of a number of clinical populations such as autism (ASD), attention deficits/hyperactivity disorder (ADHD) and acquired brain injury (ABI), however there are currently no theory-driven, ecologically valid, developmentally appropriate measures of social skills for children and adolescents that delineate these specific skills. The Pediatric Evaluation of Emotions Relationships and Socialization (PEERS) is a new measure of social competence administered via iPad that was developed to fill this gap. This study aimed to evaluate preliminary data to assess PEERS for clinical value.

Participants and Methods: 216 typically developing (TD) participants aged 5-14 years recruited from Australian primary and secondary schools completed a subset of 6 PEERS subtests and an abbreviated measure of IQ between June-August 2016. 14 participants with clinically diagnosed ABI $(n=2)$, ASD $(n=2)$, ADHD $(n=6)$ and $\operatorname{LD}(n=2)$ in the same age range were also assessed. $\mathrm{Z}$-scores were derived relative to TD data for each age.

Results: Clinical groups displayed relative deficits in specific social skills compared to the TD group. Areas of weakness included emotion recognition/perception and social information processing for the ABI group; selective attention, social information processing speed, emotion recognition/perception and social interpretation for the ASD group; inhibition for the ADHD group; processing speed and selective attention for the LD group.

Conclusions: Preliminary results suggest that PEERS may be a useful aid in the assessment of clinical diagnoses where specific social skills deficits are a key feature. Further analyses with larger sample sizes are warranted.

Correspondence: Simone Hearps, Child Neuropsychology, Murdoch Childrens Research Institute, Level 4 West, MCRI, The Royal Children's Hospital, Flemington Road, Parkville, VIC 3056, Australia. E-mail: simone.hearps@mcri.edu.au

H. HENNRICK, K. RINNA, M. GOLDSTEIN, K. BONEFF \& C. FREEDMAN-DOAN. Informant Reports of Children's Cognitive Abilities Affected by Relationship Factors.

Objective: Relationship factors impact informant reports of cognitive ability. This research examined relationship factors' influence on teachers' and parents' perceptions of children's attention, executive functioning, and working memory, beyond performance based indices. Participants and Methods: The sample consisted of 37 child-parent-teacher triads. Children were between the ages of 8-14 years $(\mathrm{M}=10.99, \mathrm{SD}=1.49) .37 .8 \%$ of the children were male. Performance based measures of children's cognitive abilities were assessed using the Conners CPT, WMI of the WISC-IV, and the Color-Word Interference Test of the D-KEFS. Teacher and parent informant evaluations included the Conners $3{ }^{\text {rd }}$ Edition Reports, Behavior Rating Inventory of Executive Function, Student Teacher Responsiveness Scale, and Parent Relationship Questionnaire.

Results: Regression analyses show that children's performance based measures were significant predictors of teachers' evaluations of children's attention $(\beta=.45, t(34)=2.96, p<.01)$, executive functioning $(\beta=-.33, t(34)=-2.04, p<.05)$, and working memory $(\beta=-.33$, $t(34)=-2.01, p<.05)$, and accounted for $20.5 \%\left(\mathrm{R}^{2}=.21\right), 10.9 \%$ $\left(R^{2}=.11\right)$, and $10.6 \%\left(R^{2}=.11\right)$ of the variance in reported abilities, respectively. Hierarchical regression analyses show that teacher-student conflict accounted for significantly more variance in teachers' evaluations of children's executive functioning $\left(R^{2}\right.$ change $\left.=.22, p<.01\right)$. Conflict in the parent-child relationship was the only significant predictor of parents' evaluations of children's attention $(\beta=.45, t(35)=2.96$, $p<.01)$, executive functioning $(\beta=.62, t(35)=4.68, p<.01)$, and working memory $(\beta=.42, t(35)=2.71, p<.01)$, and accounted for $20 \%\left(\mathrm{R}^{2}=.20\right), 38.5 \%\left(\mathrm{R}^{2}=.39\right)$, and $17.3 \%\left(\mathrm{R}^{2}=.17\right)$ of the variance in reported abilities, respectively.

Conclusions: Teachers more accurately report on children's cognitive abilities, while parent reports of cognitive functioning capture conflict in the relationship.

Correspondence: Heather Hennrick, Eastern Michigan University, 410 Ferris Street, Ypsilanti, MI 48197,United States.E-mail: hhennric@ emich.edu

S. JOELSON, M. KENNA, J. REMER, E. CROTEAU-CHONKA, T. HARRISON GOLDMAN, V. D'SA \& S. DEONI. Effects of Infant Social-Emotional Problems on Cognitive Performance.

Objective: Early childhood developmental evaluation may be crucial for detecting indications of behavioral issues, thereby aiding infants in need of interventions. While research on infant behavior and cognitive development is growing, little is known about the relationship between early social-emotional behavior and cognitive performance starting at 12 months. The purpose of this study was to examine the cognitive development in children stratified by parent-reported infant social-emotional problems from 12 to 35 months of age.

Participants and Methods: 119 children (38 female), 1-3yrs were included in this study. The Brief Infant Toddler Social Emotional Assessment (BITSEA) parent-form was collected, with the possible problem read-out used to categorize children into 1 of 2 groups: "Possible Problem" ( $\mathrm{n}=22,7$ female) or "Control" ( $\mathrm{n}=97,37$ female). The early learning composite (ELC) and Verbal and Non-Verbal Developmental Quotients (VDQ and NVDQ) from the Mullen Scales of Early Learning were used to assess general cognitive ability and verbal and non-verbal functioning, respectively. For each group, a bootstrap approach was used to examine the cross-sectional trend of cognitive score with age (e.g., ELC (Age) $=\alpha$ Age $+\beta$ ) and the $\alpha$ and $\beta$ terms compared between groups. Groups were matched for maternal socio-economic status $(p=0.219)$, age $(p=0.379)$, and gender $(p=0.579)$.

Results: Between groups, ELC, VDQ, and NVDQ displayed significant differences in slope $(\alpha)(p<0.001)$ and intercept $(\beta)(p<0.001)$. "Possible Problem" slopes $(\alpha)$ and intercepts $(\beta)$ were uniformly significantly lower than the control.

Conclusions: The results reveal differing trends in linear trajectories for infant cognitive development stratified by emotional behavior. However, further research is required to confirm these findings. Future studies should emphasize longitudinal analysis and the structural underpinnings of possible behavioral problems

Correspondence: Sarah Joelson, B.A., Engineering, Brown University's Advanced Baby Imaging Lab, 359 Llyod Avenue, Providence, RI 02906 , United States.E-mail:sarah_joelson@brown.edu 
E.F. JONES, C. STEPHAN, A. BENJAMIN, A. PRITCHARD, E.M. MAHONE \& T.A. ZABEL. Patient Experience of Neuropsychological Assessment: Preliminary Findings from a Pediatric (Ages 5-18) Patient Sample.

Objective: Parent report of satisfaction is an important outcome measure in the field of pediatric neuropsychology (Bodin et al., 2007; Kirkwood et al., 2016); however, pediatric patient experience of assessment has been under-investigated. The present study sought to examine the feasibility of acquiring child experience ratings immediately following neuropsychological and psychological testing.

Participants and Methods: The Child Experience Questionnaire (CEQ) is composed of 15 theoretically-derived items (eleven 5-point Likert-scale items, four ranking items) written at a Kindergarten reading level. Items query children's experience of their clinician and assessment procedures, and include two sample questions to assess response reliability (e.g., "Is a dog an animal?"). The CEQ was administered via computer (Qualtrics) in order to support "read aloud" functionality and permit independent completion by patients in the absence of parents/ clinicians. Thirty-six clinically-referred pediatric patients ( $\mathrm{M}$ age $=10.94$ years, $\mathrm{SD}=3.13$; range $=5-18 ; 23$ male) completed the $\mathrm{CEQ}$ following neuropsychological assessment.

Results: Overall, 91\% of patients reported positively upon their clinicians. Few (11\%) reported anxiety or boredom (22\%) during testing, but many indicated that the evaluation made them tired $(47 \%)$. Onehundred percent of children reported that they tried their best throughout the evaluation. With the "read aloud" functionality, the majority (98\%) of patients accurately completed the sample items, including a subset of patients referred with reading-related difficulties.

Conclusions: This study is a first step towards a reliable method of acquiring child-reported experience ratings of neuropsychological/ psychological assessment. These preliminary findings suggest that the CEQ may be a useful tool for refining the practice of clinical assessment and evaluating its utility.

Correspondence: TA.Zabel,PhD, MD, United States.E-mail: zabela@ kennedykrieger.org.

\section{LALIBERTE DURISH, K.O. YEATES \& B. BROOKS. Convergent and Divergent Validity for a Measure of Psychological Resilience in Children with Mild Traumatic Brain Injury.}

Objective: The Connor-Davidson Resilience Scale (CD-RISC) has not been validated in a pediatric population; therefore, this study examined the construct validity of the CD-RISC within children with mild traumatic brain injury (mTBI) or orthopaedic injury $(\mathrm{OI})$.

Participants and Methods: Participants $(N=65)$, ages 8-18 years, who sustained a mTBI $(n=46)$ or an OI $(n=19)$, were recruited from existing research databases at a children's hospital in Calgary, Alberta. Total sample and within-group correlations between the CD-RISC (25-item and 10-item versions) and measures of perfectionism, health-related quality of life, anxiety and depression, and general behaviour problems, were examined to explore convergent validity, as well as reaction time to explore divergent validity.

Results: In the overall sample, both the 25-item and 10-item versions of the CD-RISC were significantly negatively correlated with parent- and self-reported general behaviour problems $(r=-.31, p=.031$ and $r=-.39$, $p=.005$ for the 25 -item version; $r=-.40, p=.005$ and $r=-.43$, $p=.002$ for the 10 -item version) and positively correlated with self-reported quality of life $(r=.32, p=.010$ for the 25 -item version; $r=.40$, $p=.001$ for the 10 -item version). Contrary to validation studies in adult populations, the 25-item CD-RISC was positively associated with parent ratings of child depression $(r=.33, p=.021)$. Within-group correlations did not significantly differ between mTBI and OI groups. The CD-RISC was not significantly correlated with reaction time or perfectionism. Conclusions: The current results provide evidence of the construct validity of the CD-RISC when used in a pediatric population, based on significant correlations with self-reported quality of life as well as general behaviour problems. The 10-item CD-RISC may offer a more "pure" measure of psychological resilience than the 25-item CD-RISC given the unexpected correlation between to the 25-item measure and parent-reported child depression.

Correspondence: Christianne Laliberte Durish, M.Sc., Psychology, University of Calgary, 405-188 15 Ave SW, Calgary, AB T2R1S4, Canada.E-mail: christianne.lalibert@ucalgary.ca

E.B. LEAFFER, S. POWELL \& W.S. MACALLISTER. Is the WISC-V Yielding Lower Scores? Comparisons of the WISC-IV and WISC-V in Pediatric Epilepsy.

Objective: Since the release of the Wechsler Intelligence Scale for Children $-5^{\text {th }}$ edition (WISC-V), clinicians have speculated that the WISC-V has yielded lower scores than its predecessor (WISC-IV). This study compared WISC-IV and WISC-V performances in a pediatric epilepsy sample, accounting for known risk factors for cognitive deficits in epilepsy including age at seizure onset and number of anti-epileptic medications. We hypothesized that no significant differences would be identified between WISC-IV and WISC-V on composite indices.

Participants and Methods: Matched by age (Range: $6-15$ years, Mean: 9.8, SD: 2.7), age at seizure onset (Mean: 5.1, SD: 3.1), and number of anti-epileptic medications (Mean: 1.1, SD: 0.6-0.7), scores from 24 children with WISC-V were compared with 24 matched-children with WISC-IV. Descriptive statistics, t-tests $(\alpha=0.05)$, and Cohen's d effect sizes were calculated.

Results: WISC-V and WISC-IV composite scores did not differ significantly. In terms of effect sizes, no meaningful effects were observed for the Full Scale IQ ( $t=0.41$, ns; $d=0.1)$, Verbal Comprehension Index $(\mathrm{t}=-0.19, \mathrm{~ns}, \mathrm{~d}=0.1)$, Working Memory Index $(\mathrm{t}=0.39, \mathrm{~ns}, \mathrm{~d}=0.1)$, and Processing Speed Index ( $t=-0.48, n s, d=0.1)$. Small-to-medium effects were observed when the Perceptual Reasoning Index was separately compared with the Visuospatial Index $(t=1.11, \mathrm{~ns}, \mathrm{~d}=0.3)$ and the Fluid Reasoning Index ( $\mathrm{t}=1.08, \mathrm{~ns}, \mathrm{~d}=0.3)$, the two new WISC-V indices.

Conclusions: No significant differences were observed on any composite index of the WISC-IV compared with WISC-V. Based on a pediatric epilepsy sample, the WISC-V does not appear to be yielding lower scores than the WISC-IV.

Correspondence: Emily B. Leaffer, MPH, Columbia University, 630 West 168th Street, PH 19, New York, NY 10019, United States. E-mail: ebl2121@cumc.columbia.edu

K. ANSELL, T. BROWN, A. SCHRUM \& A. LUCCHETTI. A Pilot Study of Critical Flicker Frequency in a Clinical Pediatric Sample. Objective: With origins in psychophysics and visual perception, Critical Flicker Frequency (CFF) utilizes just noticeable differences to assess an individual's perceptual threshold of an increasing and decreasing visual flicker. CFF has been conceptualized as a measure of cognitive processing capacity in adults that correlates with attention and memory. Applicability in a pediatric population has not been well established. Using a clinical pediatric sample, we are interested in 1) examining $\mathrm{CFF}$ performance and 2) understanding the relationship between $\mathrm{CFF}$ and cognition.

Participants and Methods: Patients $(\mathrm{N}=17,68 \%$ male, $7-19$ years old) completed the CFF as part of the standard pediatric neuropsychological battery. Data collection is ongoing.

Results: CFF was unrelated to intellectual abilities and age in this initial pilot sample. Increasing $(\mathrm{M}=38.30 \mathrm{~Hz}, \mathrm{SD}=2.14)$ and decreasing $(\mathrm{M}=40.40 \mathrm{~Hz}, \mathrm{SD}=5.06) \mathrm{CFF}$ were highly variable. Left and right eyes were positively correlated when identifying increasing $(r(16)=.61$, $\mathrm{p}<.05)$ and decreasing $(r(16)=.67, \mathrm{p}<.01)$ flicker frequency.

Conclusions: The utility of CFF in pediatric assessment remains unclear; however, it does not appear to represent a strong developmental gradient with the available data. There seems to be a wider range of CFF in this pediatric sample than that which has been documented in adult samples. Increasing sample size is expected to improve predictive power to assess the potential clinical utility of CFF as a means to measure cognitive processing in children and adolescents. 
Correspondence: Amanda Lucchetti, MA, Psychiatry and Behavioral Sciences, Children's Hospital Colorado, 5100 Leetsdale Drive, Unit 401, Denver, CO 80246,United States.E-mail: amandalucchetti@gmail.com

E.N. MERCER, J. BECK, V. D'SA, S. DEONI \& T. HARRISON GOLDMAN. Mullen Early Learning Composite: A Predictor of Later Intelligence Quotient Scores.

Objective: The Mullen, an assessment of early cognitive development, continues to be in its orignial version, while other childhood assessments have seen numerous revisions. It is important to investigate whether early cognitive assessments, especially ones that have not been updated, are predictive of a child's later cognitive abilities, specifically when the later score is collected using assessments that have gone through multiple revisions. This study investigated whether children's Early Learning Composite (ELC) scores on the Mullen are correlated with future Full Scale Intelligence Quotient (FSIQ) scores, specifically on the Wechsler Preschool and Primary Scale of Intelligence-IV (WPPSI) and the Wechsler Intelligence Scale for Children-V (WISC).

Participants and Methods: The WPPSI was administered to 31 children between 48 and 71 months $(M=60.129 \pm 5.631)$ who had previously completed a Mullen assessment between the ages of 18 and 36 months $(M=23.935 \pm 5.790)$. The WISC was administered to 20 children between 74 and 105 months $(M=86.200 \pm 8.806)$ who had previously completed a Mullen assessment between the ages of 22 and 47 months $(M=35.150 \pm 8.312)$. The WPPSI and WISC FSIQ scores were each compared separately to the Mullen ELC score through paired-sample t-tests.

Results: While the ELC scores were significantly lower than the WPPSI FSIQ scores $(p=.023)$, they still significantly correlated with each other, $r=0.646, n=31, p<.001$. Also, the ELC scores were significantly higher than $(\mathrm{p}=.009)$, but still significantly correlated with the WISC FSIQ scores, $r=.778, n=20, p<.001$.

Conclusions: The results suggest that ELC scores of tests administered between 18 and 36 months are correlated to later WPPSI FSIQ scores. Similarly, between 22 and 47 months, ELC scores are correlated to later WISC scores. These findings indicate that the Mullen is predictive of children's cognitive development later in childhood. Future research should examine the predictive validity of ELC scores outside of the age ranges in these analyses.

Correspondence: Emily N. Mercer, B.A. in Psychology from Providence College, Engineering (Advanced Baby Imaging Lab), Brown University, 194 First Street, Pawtucket, RI 02861, United States. E-mail: emily mercer@brown.edu

\section{A.M. O'BRIEN, L.R. KIVISTO, S. DEASLEY \& J.E. CASEY. Confirmatory Factor Analysis of the BDEFS-CA in a Canadian Sample of Children with and without ADHD.}

Objective: The Barkley Deficits in Executive Functioning Scale-Children and Adolescents (BDEFS-CA) is a 70-item downward extension of the adult version, and consists of the same five scales: SelfManagement to Time, Self-Organization/Problem Solving, SelfRestraint, Self-Motivation, and Self-Regulation of Emotions. The BDEFS-CA scales were developed via a principal-components factor analysis with parents $(\mathrm{N}=1922)$ using the 14 highest loading executive function (EF) items from the adult BDEFS. Since its release in 2012, there has been no published independent research regarding the validity of the BDEFS-CA. As such, the purpose of the present study was to replicate the five factor structure of the BDEFS-CA in an independent sample using confirmatory factor analysis (CFA) to assess the construct validity of the measure.

Participants and Methods: Participants $(n=100)$ included parents of children aged 8 - to 14 -years old $\left(M_{\text {age }}=11.34,29 \%\right.$ female) recruited from the community. Half of the sample met diagnostic criteria for ADHD. Parents completed the BDEFS-CA long form as part of a study examining aspects of fine motor control in children with and without ADHD.
Results: Despite a large significant chi-squared value $\left[c^{2}(2335)=5137.91, p<.001\right]$, the $c^{2} / \mathrm{df}$ fell within acceptable parameters (i.e., $<3$ ). Root mean squared residual and the comparative fit index were also below suggested cut-offs $[\mathrm{RMR}=0.07$, CFI $=0.72]$, suggesting good model fit. High standardized factor weights were found for all observed variables $(0.66<\mathrm{b}<0.95)$. Latent variables of the BDEFS were strongly correlated $(0.67<r<0.96)$.

Conclusions: Although the model did not fit on all indices, criteria for three of six commonly reported indices were met. This provides evidence supporting the construct validity for the five scale structure of the BDEFS-CA in the current sample.

Correspondence: Amanda M. O'Brien, Psychology, University of Windsor, 401 Sunset Ave, Windsor, ON N9B 3P4, Canada. E-mail: obrie115@uwindsor.ca

\section{O'DESKY \& Z. GRATZ. WISC Disparity Can Rule In NLD Not Rule Out.}

Objective: The concept of a nonverbal learning disability (NLD) was first proposed in 1964. These individual's have strong verbal skills with weak visuospatial skills. Children are often determined to have a probable diagnosis of NLD by the school psychologist using a 10 point disparity between a child's performance on the verbal and visual subtests of the WISC (Dixon, nd, Hernadek and Rourke, 1994).

Participants and Methods: The current study attempted to determine if a disparity on the WISC is an appropriate predictor of NLD. Visuoconstructive skills were assessed using the Rey Complex Figure Test (RCFT). The initial sample included 224 children with a primary diagnosis of NLD or a secondary diagnosis of NLD with a primary diagnosis of ADHD. There were 133 males and 91 females, mean age 10 yrs. 3 mos. This group had a mean FSIQ of 101.67, sd 13.527, a VCI of 104.71, sd 13.088 and PRI 101.79, sd 14.809. Individuals were determined to have a normal performance if their copy score was above the 10th percentile; otherwise, their performance was considered to be impaired.

Results: Incidence of NLD varied by discrepancy of VIQ:PIQ $(\mathrm{c} 2(5)=18.81, p=.001)$. With a VIQ:PIQ discrepancy $<0, \mathrm{n}=34$ had a normal RCFT copy while $\mathrm{n}=39$ had an impaired performance on the RCFT. With a VIQ:PIQ discrepancy of $1-5$ points, $84.4 \%$ were impaired on the RCFT while $75 \%$ of individuals with either a $6-10$ point difference or 11-15 point difference were impaired on the RCFT. Similarly, $78.6 \%$ of individuals with a $16-20$ point difference were impaired on the RCFT but predictive significance was only reached when there was a >20 point VIQ:PIQ discrepancy. Then $94.4 \%$ were impaired on the RCFT suggesting that this is the discrepancy needed before a probable diagnosis of NLD is given.

Conclusions: Based on the current study, it is recommended that a 20 point disparity be used to give a primary diagnosis of NLD; this is particularly important in a school setting wherein the intelligence test is typically used to diagnose NLD.

Correspondence: Ilyse O'Desky, Psy.D., Psychology, Kean University, 24 Allenby Lane, Scotch Plains, NJ 07076,United States.E-mail: iodesky@ kean.edu

A. RACH, J.V. WETHE, M. HOWARD, A.J. STARLING, J. BOGLE \&. D.W. DODICK. Age-Based Child-SCAT3 and SCAT3 Normative Values Using a Youth Football Population.

Objective: Reliability studies for the Child-Standardized Concussion Assessment Tool (Child-SCAT3) are lacking. Further, the reliability studies for the adult version, the SCAT3 have focused on high school and collegiate athletes. The purpose of this study was to investigate practice effects and test-retest reliability of the Child-SCAT3/SCAT3 in youth athletes.

Participants and Methods: Pre- and post-season data were collected from 55 pediatric and 40 adolescent football players that were retested over an interval of three months. Four athletes with a diagnosis of ADHD were excluded. One player self-reported one previous concussion. 
Practice effects, pearson's r, and intraclass correlation coefficients (ICG) were investigated for the four components of the Standardized Assessment of Concussion (SAC) and three stances of the Modified Balance Error Scoring System (m-BESS).

Results: There was no evidence of practice effects on the SAC-Child, but pediatric athletes showed practice effects on the m-BESS tandem leg stance $\mathrm{t}(32)=2.96, p<.05$. There was also practice effects on the SAC-Adult orientation scale $\mathrm{t}(39)=3.14, p<.05$. The Child-SCAT3 immediate memory component achieved good reliability, whereas, the SCAT3 the orientation, immediate memory, and concentration components all achieved good reliability. The three stances of the m-BESS were not reliable.

Conclusions: Given that only the immediate memory component for the Child-SCAT3 met criteria for repeated use, caution is warranted when the tool is used in clinical decision making and our findings suggest that the Child-SCAT3 may require additional modifications to be appropriate used with athletes 12 years of age and younger.

Correspondence: Amanda Rach, Ph.D., Psychiatry, University of Virginia, 137 Yellowstone Drive, 205-A, Charlottesville, VA 22903, United States. E-mail: amandamrach@gmail.com

R. RAO, G. DURMUSOGLU, J.B. ALLEN \& D.W. BEEBE. The Relationship of Executive Function and Attention with Sleep Duration in Children with Obstructive Sleep Apnea.

Objective: Pediatric obstructive sleep apnea (OSA) affects $2-3 \%$ of children and may lead to deficits in attention and executive functioning (EF). However, even among children with OSA, outcomes may depend in part on sleep duration, both proximal (recent; e.g., as assessed via a prospective sleep diary) and habitual (long-term; e.g., as measured via retrospective questionnaires). Measures of attention and EF, can be similarly classified as proximal (e.g., office-based neuropsychological testing) and habitual (e.g., report of behaviors over several weeks to months). The aim of this study was to examine the relationship of proximal and habitual sleep duration with attention and EF in children with OSA.

Participants and Methods: We re-analyzed pre-treatment data from a large clinical trial studying 453 children 4.0 to 9.99 years of age. All had mild to moderate OSA per polysomnogram. All underwent neuropsychological assessment that included the NEPSY Attention/Executive Functions Domain. Around the same time, parents completed a prospective daily sleep diary, reported on habitual sleep duration, and completed the BRIEF and Conners scales. In addition, a subset had Teacher-report BRIEF and Conners scales.

Results: Fewer habitual attention and EF problems (Conners Parent and BRIEF Teacher) were significantly correlated with longer habitual sleep duration. However, no significant association was found among proximal sleep duration (Sleep Diary) and habitual attention and EF, nor among proximal attention and EF (NEPSY) and habitual or proximal sleep duration.

Conclusions: Habitual measures of sleep duration, obtained via retrospective questionnaires, may help identify patterns associated with habitual attention and EF problems. Results also suggest that when looking for sleep-related daytime impairments, clinicians should look more at habitual, day-to-day functioning rather than on tests of attention and EF administered in a one-on-one, controlled setting.

Correspondence: Roshni Rao, Doctor of Psychology, School of Professional Psychology, Wright State University, Roshni, Rao, Monroe, OH 45050,United States.E-mail: rao.27@wright.edu

\section{RYAN, H. SCHNEIDER, A. PRITCHARD \& E.M. MAHONE. Predictive Utility of Performance-Based and Rating Scale Measures of Inhibition in Preschool Children.}

Objective: Previous research investigating performance-based measures and ratings of executive functions (EF) in adolescents suggests that parent and teacher ratings are significant predictors of ADHD diagnostic status, while performance measures account for little additional variance (Toplak et. al, 2009). The development of inhibitory control is particularly critical for younger children, and problems with inhibition are especially prominent in young children with ADHD. This study examined the utility of performance-based and rating scale measures of inhibitory control as predictors of ADHD among preschoolers.

Participants and Methods: Children ages 4-5 years (66 ADHD, 56 control) were recruited as part of a larger study. ADHD diagnosis was determined using modified DSM-IV criteria based on structured interview and ADHD-specific rating scales. Three performance-based measures of inhibition ((NEPSY-2 Statue, Conflicting Motor Response Test, Auditory Continuous Performance Task for Preschoolers--Commissions), and caregiver ratings (parent, teacher) on the BRIEF-P Inhibit scale were used to predict ADHD status using logistic regression. Results: Logistic regression indicated that all three performance measures and both parent and teacher ratings were significantly associated with ADHD status in the expected directions at $p \leq .01$. Among the performance-based measures, Statue was most predictive of ADHD status $(p<.001, O R=.821)$; however, Statue did not account for variance in ADHD status once parent and teacher ratings were entered into the model $(\beta p=.729, \mathrm{OR}=.955)$.

Conclusions: Consistent with previous findings in adolescents, these results suggest that, among preschoolers, caregiver ratings of inhibition are better predictors of ADHD status than performance-based measures of inhibition.

Correspondence: Matthew Ryan, MS, Neuropsychology, Kennedy Krieger Institute, 1750 East Fairmount Ave., Baltimore, MD 21231, United States. E-mail: RyanM@kennedykrieger.org

\section{A.D. SCHELLER \& J.B. NEWMAN. Examination of Automatized Sequences Task as an Index of Performance Validity in General Pediatric Neuropsychological Evaluations.}

Objective: Examine the clinical utility of the Automatized Sequences Task (AST) as a performance validity test (PVT) in outpatient evaluations in an academic medical setting. AST performance was examined in relation to demographics, diagnoses, and compared to performance on basic cognitive indices (IQ, processing speed) and other PVTs (Test of Memory Malingering [TOMM], Reliable Digit Span [RDS]).

Participants and Methods: Retrospective analysis of 374 children ages 8-17 (57.8\% male) who completed evaluations that included the AST. TOMM and RDS were available for 79 and 120 children, respectively. RDS $\leq 6$ and TOMM $\leq 45$ were used as indicators of noncredible effort. Full Scale IQ and PSI indices on an age-appropriate Wechsler test were also examined.

Results: The sample was divided into two groups based on AST performance: Non-elevated (within expected performance on all trials, $\mathrm{n}=213$ ) and Elevated (beyond cut-offs on $\geq 1$ trials, $n=161$ ). Groups did not differ by gender, race, number of diagnoses, ADHD, or anxiety diagnosis. However, the Elevated group was more likely to be younger $\left(\chi^{2}=28, p<.001\right)$, medically referred $\left(\chi^{2}=5, p=.03\right)$, or have a learning disability $(\mathrm{LD})\left(\chi^{2}=6, p=.03\right)$. AST performance was associated with performance on $\operatorname{RDS}\left(\chi^{2}=13, p<.001\right)$ but not the TOMM. Elevated AST performance was also more likely in children with IQ $<80\left(\chi^{2}=14\right.$, $p<.001)$ or PSI $<80\left(\chi^{2}=12, p=.001\right)$. Similar findings were obtained when Elevated AST was classified as performance beyond cut-offs on $\geq 2$ trials; however, AST Total Time was associated only with age.

Conclusions: This is the first examination of the AST in a general pediatric neuropsychological sample. Particular caution should be exercised when interpreting AST performance in children that are younger ( $\leq$ 10 years), who are referred relative to a medical diagnosis, or who are found to have LD, below average IQ and/or processing speed. Additional research is needed to understand the role of the AST in evaluating older children, as it may be sensitive to effort in ways not measured by the TOMM.

Correspondence: Alexandra D. Scheller, M.S., Psychology, Loyola University Maryland, 552 Edwards Dr, Odenton, MD 21113, United States.E-mail: alexandra.scheller@gmail.com 
H. SCHNEIDER, M. RYAN \& E.M. MAHONE. Contrasting Parent vs. Teacher Ratings on the BRIEF-P in Preschool Children with and without ADHD.

Objective: Identifying ADHD symptoms in multiple settings is important in diagnosis; however, it is unclear if parents and teachers view common symptoms, such as executive weaknesses, similarly in early childhood. This study contrasted parent vs. teacher ratings of executive function in preschoolers with ADHD and typically developing (TD) preschool children. The BRIEF-P allows for a direct comparison of symptom ratings, since parents and teachers complete exactly the same measure.

Participants and Methods: Parent vs. teacher differences on 3 BRIEF-P Index scores (Inhibitory Self Control, Flexibility, \& Emerging Metacognition) ratings and correlations between parent/teacher ratings of each Index were examined separately in ADHD and TD groups. Participants included children ages 4-5 years (47 ADHD, 37 TD). Children with ADHD were diagnosed using modified DSM-IV criteria and screened for language and autism spectrum disorders

Results: Within the TD group, parent/teacher correlations for all 3 Index scores were small and non-significant (range $=.10-.26$ ); however, within the ADHD group, parent/teacher correlations were consistently larger and all statistically significant (all $p<.05$; range $=.29-.31$ ). Rater differences on Index scores within the TD group showed significantly higher ratings by teachers for 2 of 3 Index scores; conversely, within the ADHD group, 2 of 3 Index scores showed significant differences in which parents rated the child as more impaired than teacher.

Conclusions: In preschool children, discrepancies between parent and teacher ratings of executive function differ as a function of diagnosis. Among children with ADHD, parents tend to report more symptoms than teachers of the same child, even though parent/teacher correlations on the same scales remain moderately high. Lower associations in TD children may be due to restricted range of reported symptoms.

Correspondence: Heather Schneider, Neuropsychology, Kennedy Krieger Institute, 1750 E. Fairmount Ave, Baltimore, MD 21231, United States. E-mail:SchneiderH@kennedykrieger.org

E.M. SHIPLEY, R.A. LANDSMAN \& J. LINTON REESMAN. The Test of Memory Malingering (TOMM): Use with Deaf or Hard of Hearing Children Referred for Neuropsychological Assessment.

Objective: Symptom validity measures serve an integral component of neuropsychological evaluations (NAN). The Test of Memory Malingering (TOMM; Tombaugh, 1996) is a commonly accepted and widely used measure of symptom validity (Slick et al., 2004) with support for use across a variety of developmental, psychiatric, neurological disorders and with children as young as five years (Schneider et al., 2014). Following preliminary investigation of the utility of the TOMM with a D/HOH sample, the present study sought to examine the utility of the TOMM in a diverse, mixed clinical sample.

Participants and Methods: Retrospective review of a de-identified clinical data set of $\mathrm{D} / \mathrm{HOH}$ children ages 5 to 18 years $(\mathrm{N}=55, \mathrm{M}=12.3$, $\mathrm{SD}=3.8,65.5 \%$ male), who were referred for clinical evaluation in a large neuropsychology department with a specialty clinic for $\mathrm{D} / \mathrm{HOH}$ children. All children were administered at least Trial 1 the TOMM, with instructions delivered in ASL or spoken English, dependent on child preference, by examiners fluent in the language of administration. Results: $34(61.8 \%)$ obtained scores of 46 or higher during Trial 1 and $92.7 \%$ of the total sample earned a passing score by Trial 2. 7\% (N=4) children did not earn passing scores. Demographic, behavioral ratings, and cognitive functioning differences were examined.

Conclusions: The TOMM demonstrates promise as a clinically useful measure with $\mathrm{D} / \mathrm{HOH}$ children when considered among clinically relevant factors that may be of increasing importance in $\mathrm{D} / \mathrm{HOH}$ samples. This is the only symptom validity measure studied within this population to date.

Correspondence: Elizabeth M. Shipley, M.A., Neuropsychology, Johns Hopkins University School of Medicine, 707 N. Broadway, Baltimore, MD 21205, United States. E-mail: ShipleyE@KennedyKrieger.org
C. STEPHAN, E.M. MAHONE \& L. JACOBSON. Cross-setting rater agreement on ADHD symptomatology: What's a clinician to do?

Objective: Clinical diagnostic criteria for ADHD require cross-setting assessment of symptoms from multiple informants (per DSM-5). Extent of agreement between parent and teacher reports of ADHD symptomology was examined in a referred sample overall and by age and sex of child.

Participants and Methods: Youth referred for outpatient neuropsychological assessment $(\mathrm{N}=2871 ; \mathrm{M}$ age $=9.83$ years, $\mathrm{SD}=3.15 ; 64 \%$ male $)$ were included if complete ADHD Rating Scale-IV responses were available from both parent and teacher. Participants were classified by rater into diagnostic presentations, each requiring $\geq 6$ symptoms per DSM-5 criteria: ADHD-Inattentive, ADHD-Hyperactive, ADHD-Combined, not ADHD ( $\leq 3$ symptoms of either), and sub-threshold ADHD symptomatology ( $\geq 3 \& \leq 5$ symptoms). Overall categorical agreement between raters was examined: 1) for the overall sample, 2 ) by age (elementary [ $\leq 11$ years] vs. secondary [ $>11$ years]), and 3 ) by child's sex. Agreement was examined using Krippendorff's alpha and percent agreement was compared across groups with z transformations.

Results: Overall, parent and teacher agreement was $36 \%$ across the five categorical classifications $(\alpha=.149)$. Agreement was better for secondary $(\alpha=.15)$ vs. elementary $(\alpha=.14)$ students ( $34 \%$ vs. $40 \%$; $z=3.16, p=.002)$ and girls $(39 \% ; \alpha=.13)$ vs. boys $(35 \%, \alpha=.15)(\mathrm{z}=-2.13, \mathrm{p}=.03)$.

Conclusions: Overall, inter-rater agreement between parents and teachers for classification of participants was low. The somewhat better agreement for older youth and girls may be due to the reduction in hyperactivity seen in girls and across development, and subsequent lower incidence of ADHD-Hyperactive and Combined presentations in girls and older youth, resulting in a greater likelihood of raters consistently classifying participants into the three remaining categories. Given requirement for cross-setting symptomatology, findings have important implications for understanding cross-informant reports regarding ADHD symptomatology.

Correspondence: Catherine Stephan, BS, Neuropsychology, Kennedy Krieger Institute, 1750 East Fairmount Avenue, Department of Neuropsychology, Baltimore, MD 21231, United States. E-mail: stephan@kennedykrieger.org

C. STEPHAN, C. SACKETT, A. TOLLIVER, L. JACOBSON \& A. PRITCHARD. Does adaptive competence rely on better language-based or visually-based reasoning skills?

Objective: $40 \%$ of the WISC-V normative sample showed a significant ( $\geq 12$ points) split between language- and visually-based reasoning skills (Wechsler, 2014) which may have implications for day-to-day functioning. The present study examined whether the magnitude and direction of this discrepancy in cognitive abilities is related to youths' adaptive skills.

Participants and Methods: 1289 youth $(M$ age $=10.40, S D=2.74 ; 65 \%$ male) evaluated in an outpatient neuropsychology clinic, representing a mixed clinical sample. Via retrospective chart review, Wechsler Intelligence Scale for Children ( $5^{\text {th }}$ ed.) and Adaptive Behavior Assessment System $\left(2^{\text {nd }} \& 3^{\text {rd }}\right.$ ed. $)$ scores collected as part of routine clinical care were extracted from the clinical database.

Results: Language-based reasoning skills were more strongly associated $(z=2.67, p=.008)$ with adaptive skills (VCI-GAC $r=.23, p<.001)$ than visually-based reasoning skills (FRI-GAC $r=.13, p<.001)$. Controlling for full-scale IQ, the magnitude of youths' VCI-FRI split was significantly related to adaptive functioning $(\beta=.094, t(1286)=3.57, p<.001$, $\left.\Delta R^{2}=.009\right)$. Among youth with larger cognitive skill discrepancies, those with VCI<FRI demonstrate worse adaptive function than those with VCI>FRI. Youth with smaller cognitive skill discrepancies tend to have adaptive functioning abilities that fall between those of youth with considerably stronger skills in one area or the other.

Conclusions: Informant rating of adaptive functions is efficient but appears to rely more heavily on language-mediated skills than on visually-based abilities. Future research using performance-based measures of adaptive functions will help to elucidate whether this emphasis on 
language-based reasoning is a feature of the nature of adaptive skills or the measurement of adaptive functions and intelligence.

Correspondence: Catherine Stephan, BS, Neuropsychology, Kennedy Krieger Institute, 1750 East Fairmount Avenue, Department of Neuropsychology, Baltimore, MD 21231, United States. E-mail: stephan@kennedykrieger.org.

C. STEPHAN, E.F. JONES, L. JACOBSON, A. PRITCHARD, E.M. MAHONE \& T.A. ZABEL. Parent satisfaction ratings of pediatric neuropsychological assessment services.

Objective: Parent satisfaction ratings are an important means of demonstrating the utility of pediatric neuropsychological assessment. Studies have identified an impact of parent-related demographic variables (i.e., SES; maternal education) on satisfaction ratings. To expand upon past research, this study examined the impact of clinical operations on parent reported satisfaction.

Participants and Methods: Parents/Caregivers of youth undergoing neuropsychological assessment $(\mathrm{N}=310 ; \mathrm{M}$ age $=10.40$ years, $\mathrm{SD}=3.67$; $58 \%$ male) completed a satisfaction survey after receiving verbal feedback from the clinician regarding assessment results but prior to receipt of written report. The theoretically derived satisfaction scale (13 items; 5 point Likert scale) was modeled upon variables reported by Bodin et al. (2007). Internal structure of the items was examined via exploratory factor analysis (EFA, principal axis factoring, Promax rotation). Satisfaction was examined: 1) for the overall sample, 2) by appointment length (short [ $\geq 6 \mathrm{hrs}$.] vs. long $[<6 \mathrm{hrs}$. ]), and 3 ) by appointment waittime (short $[>100$ days $]$ vs. long $[\leq 100$ days $]$ ).

Results: Overall satisfaction was very high $(\mathrm{M}=4.80, \mathrm{SD}=0.50)$. EFA established 2 factors: Clinician Acceptance of Family ( 5 items; M=4.89; $\alpha=.99$ ) and General Satisfaction (8 items; $M=4.76 ; \alpha=.92$ ). Differences in ratings between factors were significant $(\mathrm{t}(309)=-5.42, \mathrm{p}<.001$, Cohen's d=.31). Satisfaction was higher $(\mathrm{t}(291)=1.65, \mathrm{p}=.01$, Cohen's $\mathrm{d}=.26)$ for shorter appointments $(\mathrm{M}=4.87)$ than longer ones $(\mathrm{M}=4.76)$, and for longer wait times $(M=4.83)$ than shorter waits $(M=4.76)(\mathrm{p}<.01$, Cohen's d=.14).

Conclusions: Overall, parents/caregivers were very satisfied with neuropsychological assessment services. Small but significant differences in satisfaction were found for operation-related variables, which may be related to patient age and/or severity. Additional and/or alternative approaches may be necessary to identify clinician and patient characteristics yielding greater variance in satisfaction ratings. Correspondence: Catherine Stephan, BS, Neuropsychology, Kennedy Krieger Institute, 1750 East Fairmount Avenue, Department of Neuropsychology, Baltimore, MD 21231, United States. E-mail: stephan@kennedykrieger.org.

E. WEBER, K. MCCURDY, B.C. KAVANAUGH, J. STUDENY \& K.A. HOLLER. Assessing effort with the TOMM and Automatized Sequences in a child inpatient setting.

Objective: Several studies have identified the importance of including effort measures during neuropsychological evaluations of children. However, the use of effort measures for child inpatient psychiatric samples has yet to be explored.

Participants and Methods: This study examined the results of administering the Test of Memory Malingering (TOMM) to all youths aged 5-12 $(\mathrm{n}=70)$ and Automatized Sequences (AS) to youth aged 8-12 $(\mathrm{n}=56)$ on a child inpatient unit. Per recommended cut rates suboptimal effort was defined as: TOMM Trial $2<45$, alphabet sequence $\geq$ 8 seconds, counting $\geq 6$ seconds, days of the week $\geq 4$ seconds, and months of the year $\geq 10$ seconds or a total time from all 4 sequence subtests $\geq 27$ seconds.

Results: The pass rate for the TOMM was $71.1 \%(\mathrm{~m}=47.8, \mathrm{sd}=4.0)$ and $61.2 \%$ for the overall AS cut off score $(\mathrm{m}=23.4, \mathrm{sd}=8.2)$. The concordance rate between the TOMM and AS total scores was 70.6\%: $60.1 \%$ passed both; $9.8 \%$ failed both; $19.6 \%$ passed the TOMM but failed the AS; $9.8 \%$ passed the AS but failed the TOMM. Both the TOMM and AS were unrelated to race, gender, family involvement with the department of child and family services, socioeconomic status, parent employment, psychiatric diagnosis, histories of prior in patient psychiatric hospitalization or a request for neuropsychological consult. However, counting inefficiency was associated with age $\chi^{2}(1, N=69)=4.7 p<.05$. Maltreatment history was associated with inefficacy reciting months of the year (fisher's exact test, $\mathrm{p}<.05$ ) and overall time to completion (fisher's exact test, $\mathrm{p}<.01)$.

Conclusions: Results suggest that caution should be used when administering AS to younger children or children with a maltreatment history. On the TOMM and AS, a large minority of children fell below standard cut-offs for suboptimal effort during psychiatric hospitalization. Further research is needed to determine whether this reflects genuine suboptimal effort, the substantial cognitive deficits of these children, or a combination of the two.

Correspondence: Elyssa Weber, MA, Clinical Psychology, University of Massachusetts, Boston, 100 Morrissey blvd, Boston, MA 02143, United States.E-mail: elyssa.weber001@umb.edu

I. WRIGHT \& A. MARSH. A Robust Statistical Approach to Classification of Developmental Change in Cognitive Test Scores. Objective: Classification of change in cognitive function over time is an important facet of paediatric neuropsychological practise. Changes in test scores may correspond to the emergence of impairment with the onset of neurological conditions or a positive response to treatment or intervention. This process is complicated by a naturally advancing baseline of absolute functioning (raw scores). However, statistically rigorous methods are lacking for classification of change in children's absolute functioning. Conventional determination of absolute change typically uses raw scores precluding statistical control for normal test-retest variation. This paper outlines a statistical approach for the classification of change profiles.

Participants and Methods: WISC-IV datasets using the Monte Carlo technique were created to simulate profiles of developmental change over a notional 24-month period. Regression techniques were applied in order to classify the nature of change and establish confidence parameters alongside sensitivity and specificity of judgements at various time intervals. Classifying change allows clinicians to confidently determine whether a child is following a normal trajectory, slow development, plateau or regression.

Results: The proposed statistical method allows for a statistically robust determination of developmental trajectory. These methods enable the clinician to detect normal versus abnormal development and distinguish between various patterns of abnormal development. Classification accuracy and error rates are compared to conventional methods.

Conclusions: The statistical method described in this paper allows for a robust classification of change, controlling for normal variance in performance and determining confidence and abnormality for any change in development. Compared to conventional techniques the suggested approach significantly reduces error rates and specifies uncertainty while improving classification accuracy.

Correspondence: Ingram Wright, MA(Cantab) PhD DClinPsychol, School of Experimental Psychology, University of Bristol, Bristol Children's Hospital, Upper Maudlin Street, Bristol BS2 8BJ, United Kingdom.E-mail: ingram.wright@bristol.ac.uk

\section{J.A. HOLDNACK. Does Cognitive Variability Differentiate Children} with Clinical Disorders from Controls.

Objective: Cognitive variability is thought to differentiate children with clinical conditions from controls. This study compares cognitive variability on the WISC-V in typically developing children versus children with developmental disorders.

Participants and Methods: Variability (i.e., highest versus lowest, significant pairwise differences, and standard deviation) among the 10 core subtests of the WISC-V was evaluated in 2022 undiagnosed children from the WISC-V standardization sample. Variability in clinical 
samples was evaluated in ADHD ( $\mathrm{n}=48$ ), Autism (AU: $\mathrm{n}=30$ ), Math Disorder (MD: n=28), Reading Disorder (RD: n=30), and Reading and Writing Disorder (RWD: $\mathrm{n}=22$ ).

Results: In the normative sample, the average lowest versus highest score was $7.1(+2.2)$ and the standard deviation was $2.2(+0.6)$. Over $97 \%$ of cases in the normative sample had at least 1 and $80 \%$ had 7 or more pairwise discrepancies of 4 or more $(12.2+6.2)$. AU was associated with a higher rate of significant pairwise discrepancies ( $p$ $<.05,14.8+6.2)$ compared to children in the normative sample. No other clinical group showed a significant difference from the normative sample on any variability measure. Variability was significantly associated with highest obtained subtest scaled score $(\mathrm{p}<.0001, \mathrm{r}=.45$ to .55 ) and modestly with ability (fsiq: $\mathrm{p}<.01, \mathrm{r}=.06$ to .07 ). Adjusting scores by highest obtained scored resulted in all clinical groups except ADHD and RD showing significant variability.

Conclusions: Typically developing children show high rates of cognitive variability. Generally, variability measures do not differentiate clinical samples from controls unless highest obtained score is considered. Correspondence: James A. Holdnack, Ph.D., PMG, The Psychological Corporation, 5 Rose Hill Drive, Bear, DE 19701, United States. E-mail: holdwork@yahoo.com

\section{Learning Disabilities/Academic Skills}

\section{J.A. HOLDNACK. Application of WISC-V Multivariate Base Rate Data to Children with Learning Disorders.}

Objective: The WISC-V contains cognitive measures to differentiate typically developing children from individuals with reading disorder. This study applies a multivariate base rate model for identifying risk for reading difficulties.

Participants and Methods: Multivariate base rates were derived from 2022 healthy individuals from the WISC-V standardization sample. Samples of children identified with reading disorders included: Reading Disorder (RD: n=30) and Reading and Writing Disorder (RWD: n=22). Two multivariate models were considered. A 4 subtest model consisting of Vocabulary, Digit Span, Naming Speed Literacy, and Delayed Symbol Translation and an 8 subtest model which added Similarities, Naming Speed Quantity, Picture Span, and Immediate Symbol Translation.

Results: The 4 subtest model yielded base rates of $14.7 \%$ and $6.5 \%$ ( 3 scores at 8 and 7 or less) and 8.6\% (2 scores 6 or less). The base rates for clinical samples are: $\mathrm{RD}(41.4 \%, 31.0 \%$, and $31.0 \%)$ and RWD $(57.1 \%, 34.7 \%$, and $47.6 \%)$. Likelihood ratio positive ranged from 2.8 (RD) to 5.3 (RWD) and .50 (RWD) to .70 (RD) for LR-. The 8 subtest model yielded base rates of $12.0 \%, 9.7 \%$, and $7.2 \%$ (cut-offs of 7,6 , and 5 at scaled scores of 9,8 , and 7 ). The base rates for clinical samples are: RD $(51.7 \%, 41.4 \%$, and $34.5 \%)$ and RWD $(52.4 \%$, $52.4 \%$, and $38.1 \%$ ). LR's ranged from $4.1(\mathrm{RD})$ to 5.4 (RWD) and .53 (RWD) to $.70(\mathrm{RD})$.

Conclusions: Children with Reading Disorders show higher rates of scores in the low average range on measures known to be associated with learning difficulties compared to controls.

Correspondence: James A. Holdnack, Ph.D., PMG, The Psychological Corporation, 5 Rose Hill Drive, Bear, DE 19701, United States. E-mail: holdwork@yahoo.com

P. ALBERT, M. ROMSKI \& R. SEVCIK. Receptive Language Moderates the Effects of AAC Intervention on Communication Skills in Children with Developmental Delays.

Objective: The purpose of this study was to examine language outcomes in children with developmental disabilities following augmentative and alternative communication (AAC) intervention and to consider the effects of comprehension ability on intervention efficacy. Specifically, we investigated whether receptive language ability moderates the relationship between language intervention type and children's language outcomes post-intervention.
Participants and Methods: The current study included 113 children with severe speech and language impairments defined as: (1) Expressive Language age equivalent score on the Mullen Scales of Early Learning of less than 12 months, and (2) parent report of less than 10 spoken words. All children participated in a longitudinal study investigating parent-implemented AAC interventions. All parent-child pairs were randomly assigned to an Augmented Communication intervention using a speech-generating device (SGD), or a Spoken Communication intervention. Researchers selected a set of target vocabulary words that would appear on the SGD's during intervention sessions. Interventions were implemented for 12 weeks, with two sessions per week. Sessions were recorded and coded for spoken and augmented target word use.

Results: Functional vocabulary use, the primary language outcome of interest, was defined by number of target words (spoken and augmented) used during session 24. Intervention type was a significant predictor of functional vocabulary; children who received Augmented Communication intervention had greater functional vocabulary than children who received Spoken Communication intervention. Receptive language moderated the relationship between intervention type and functional vocabulary.

Conclusions: A main goal of AAC is to provide an additional source of communication output for individuals with developmental disabilities (Romski \& Sevcik, 2005). It appears that based on the results of this study, receptive language may play an important role in how AAC promotes communication.

Correspondence: Phebe Albert, Clinical Psychology, Psychology, Georgia State University, 215 North Ave NE, Apartment 3410, Atlanta, GA30308, United States. E-mail: phebealbert@gmail.com

J.L. AMARAL, E.C. BOISELLE, J.M. GIRARD, P.W. FORBES \& D.P. WABER. Service Delivery Outcomes after Assessment in Children with Learning Disorders.

Objective: There are few studies of outcome after pediatric neuropsychological evaluation and they focus primarily on satisfaction. The principal aim of this study was to survey parents of children evaluated in a neuropsychologically based interdisciplinary learning disability clinic to ascertain a material outcome, changes in special education services. A secondary aim was to assess parent satisfaction and parent and school understanding of the child.

Participants and Methods: Parents of children evaluated over a threeyear period who lived in a single state $(\mathrm{N}=439)$ were invited to respond to an online survey. Of these, 137 responded and had children in public school. Children's mean age at evaluation was 9.95 years $(S D=2.24)$ and $60.0 \%$ were male. The survey assessed demographic background, parent satisfaction, parent and school understanding of the child, tone of school response, changes in special education services, and parent perception of any provided services.

Results: The majority of children $(70 \%)$ received greater intensity of services and/or different teaching approaches after evaluation. Parents nearly uniformly (98\%) saw these changes as helpful. Most parents $(87 \%)$ were satisfied with the evaluation, and a majority said they $(87 \%)$ and the school staff $(68 \%)$ understood the child better after the evaluation.

Conclusions: Children with learning problems can receive important material benefit in the form of special education services after pediatric neuropsychological evaluation. Although causation cannot be established, the study suggests clear benefit of neuropsychological evaluation for learning disorders and that change in special education services can be an important objective metric of outcome.

Correspondence: Joseph L. Amaral, Ph.D., Psychiatry, Boston Children's Hospital/Harvard Medical School, 1063 Beacon Street, Apt BR, Brookline, MA 02446,United States.E-mail: joseph.amaral@ childrens.harvard.edu 
J. SALVADOR-CRUZ, C.G. ARMENGOL, A. SEGURA VILLA \& L.A. LEDESMA TORRES. Neurological soft signs (NSS) as potential risk indicators of disrupted neurodevelopment in a Mexican sample of elementary school children.

Objective: Determining whether NSSs signal underlying developmental difficulties in healthy elementary school children.

Participants and Methods: Forty-nine Mexican elementary school children (27 boys, 22 girls) aged 6-12 participated. Exclusionary criteria were being held back a grade and psychiatric history (per Salvador \& Galindo's Neurological and Psychiatric History Questionnaire.

Portellano's Neuropsychological School Maturity Questionnaire (NSMQCUMANES in Spanish) was administered, yielding a neuropsychological development index (NDI). Neurological soft signs (NSS) were assessed with Matute et al.'s Children's Neuropsychological Evaluation (CNE; Spanish ENI)

Results: Obtained NDI's (neuropsychological maturity-chronological age quotients)showed this distribution : Very low $=18.4 \%$; Medium low $=12.2 \%$; Low $=40.8 \%$; Medium high $=4.1 \%$. Subtest scores paralleled this distribution, with a stronger overall performance on Auditory Comprehension relative to the rest (Audio-gnostic Writing, Visuoperceptual and Rhythm), with $30-50 \%$ children obtaining very low and medium low scores.

CNI/ENI yielded no clear quantitative evidence of NSSs. Qualitative analyses of three subtests (Visual Tracking, Right/Left Discrimination, Opposing Digits) revealed decreasing NSSs with age.

Conclusions: A high incidence of NSSs among healthy children, gradually tapering with age, has been documented in other studies. (Dazzan et al, 2006). Differential patterns of cognitive vulnerability suggest differential brain networks involvement (Zhao et al., 2013). Recent advances in the study of NSSs have underscored their importance for pediatric neurology. NSSs are best viewed as vulnerabilities to be targeted for early intervention aimed at fostering resiliencies (Levit-Binnun \& Golland, 2012).

Correspondence: Carmen G. Armengol, Ph.D., Applied Psychology, Northeastern University, 280 Chestnut Ave, Jamaica Plain, MA 02130, United States.E-mail: c.armengol@neu.edu

C. ARRINGTON, P. MOLFESE, B. BUIS, J.C. FRIJTERS, W. MENCL \& R. MORRIS. Pre-Intervention White Matter Microstructural Integrity Predicts Improvements in Reading Scores in Developmental Dyslexia.

Objective: Research suggests an association between developmental dyslexia and poor white matter integrity in tracts such as the superior longitudinal fasciculus (SLF), inferior longitudinal fasciculus (ILF), and uncinate fasciculus (UF). Little is known about their relation to the development of reading skills in developmental dyslexia.

Participants and Methods: The current study examined relations between white matter integrity and improvements in reading in 52 students involved in a reading intervention. TRACULA was used to extrapolate integrity values from DTI data for the ILF, temporal and parietal portions of the SLF, and the UF, bilaterally. Values were correlated with growth in basic reading, reading fluency, and passage comprehension. Regression analyses, accounting for age and preintervention reading, were performed for significant correlations.

Results: Improvements in basic reading and passage comprehension were significantly correlated with left ILF, bilateral SLF, and right UF. Growth in reading fluency was significantly correlated with right UF. Medial diffusivity in right UF predicted growth in basic reading, $t(48)=2.39, p=0.02$. Axial diffusivity and fractional anisotropy in right UF predicted reading fluency growth, $t(50)=-2.41, p<0.01$ and $t(50)=-2.53, p<0.01$ respectively. Improvements in passage comprehension was related to medial diffusivity in left ILF $(t(49)=2.41$, $p<0.01)$ and fractional anisotropy in left UF $(t(49)=2.27, p=0.02)$. Conclusions: Results indicate that pre-intervention white matter integrity predicts growth in reading outcomes above and bevond preintervention reading performance. White matter integrity may be useful in predicting response to intervention in children with developmental dyslexia.

Correspondence: C. Nikki Arrington, PhD, Psychology, Georgia State University, 831 Marietta St NW, Atlanta, GA 30318, United States. E-mail: carrington3@gsu.edu

A. CHILD, W.H. LACEY \& P.T. CIRINO. Shared and Unique Predictors of Math, Reading, and Attention Skills in Ninth Grade Children.

Objective: Math is a critical skill that predicts academic and vocational success. However, many high school students struggle with math, and it is not yet clear which cognitive factors contribute to math ability alone or the overlap between math, reading, and attention in this age group. The current study expects to find contributions of working memory and processing speed to all three outcomes and their overlap, while vocabulary will relate to reading, math, and their overlap. Numerosity is expected to solely relate to math outcomes.

Participants and Methods: $969^{\text {th }}$ grade students completed vocabulary (WASI Vocabulary), numerosity (Panamath), and working memory and processing speed (Automated Symmetry Span) tasks. Math (WRAT-3 Arithmetic), reading (WRAT-3 Reading), and attention (SWAN Teacher) abilities were also assessed. Analyses include multiple regression and partial correlation analyses.

Results: Zero-order correlations ranged from $|0.33|$ to $|0.44|$. The overall model for math, $F(4,86)=5.61, p<0.01, R^{2}=0.21$, was significant, with vocabulary, $p=0.04$, and spatial working memory, $p=0.02$, as significant predictors. Models for reading and inattention were not significant, and numerosity and processing speed did not relate to any outcomes. Vocabulary also contributed to the overlap between all pairs of outcomes, with proportion of variance shared between academic and attention variables decreasing $4-5 \%$ after controlling for this variable. Conclusions: The current study found that language skills contribute to overlap between math, reading, and attention outcomes in high school students in addition to a specific role of spatial working memory to math calculation ability (Swanson \& Jerman, 2006). Unlike in vounger children, numerosity did not relate to math calculations, and processing speed exhibited very weak relations to all outcomes. These findings have implications for understanding unique and shared cognitive skills that contribute to math, reading, and attention skills in high school students. Correspondence: Amanda Child, Psychology, University of Houston, TIMES Developmental Neuropsychology Lab, 4811 Calhoun Rd, Mailroom 365. Houston, TX 77204, United States. E-mail: amandachild11@gmail.com

P.T. CIRINO, T.D. TOLAR \& L.S. FUCHS. Longitudinal Algebra Prediction for Early versus Later Takers.

Objective: Algebra serves as a linchpin for later math achievement (e.g. NMAP, 2008). However, it is unclear whether to take algebra early (e.g., G8) or later (G9). Therefore, we assessed students in G6 on an array of cognitive, numerosity, and arithmetic measures, and followed these students through Algebra I. We expected significant correlations for each domain at both time points, with the strongest for arithmetic. We expected the skill-gap to shrink over time, given that later takers are older and have an additional year of math instruction.

Participants and Methods: There were 53 students; 27 took Algebra 1 early (G8), and 26 later (G9). 91\% had lunch assistance, and most were Hispanic (79\%), similar to area demographics. At both time points, we had 4 cognitive measures (of working memory, vocabulary, visualauditory learning, and rotation), 3 of number, 3 of arithmetic, and 2 of algebra. Analyses were correlations and ANOVA; we balanced small sample size with emphasis on effect sizes, given the unique data.

Results: Two correlational patterns emerged: (a) arithmetic was more related to algebra, median $r=.52$, than either cognitive, median $r=.27$, or number, median $r=.12$, measures; and (b) predictors closer in time were slightly stronger, working memory excepted. There were few differences between groups for number (all $d<0.45$, all $p>.05$ ); 
mixed differences for cognitive (vocabulary and working memory in G6, $d=0.64$ to 0.82 ); but large differences on arithmetic and algebra (e.g., $d=0.82$ to 1.81 , all $p<.001)$. All differences favored early takers, and the pattern of group differences was stable over time.

Conclusions: Results support a hierarchy of math, where earlier developed skills exert influence even much later. Taking algebra later does not close the gap with earlier takers, despite an extra year of math instruction. Results imply either that there is no disadvantage to all students taking algebra early, or that later takers students might profit from a different constellation of instruction prior to G9.

Correspondence: Paul T. Cirino, PhD, Psychology, University of Houston, UH TMC Annex, 2151 W Holcombe Blvd, 224a, Houston, TX77204-5053,United States. E-mail: pcirino@uh.edu

K. DAVIS, A.E. MARGOLIS, Z. HUO \& R. MARSH. Disrupted Amygdalar Subregion Functional Connectivity As A Biomarker For Anxiety Symptoms Associated With Reading Disorder.

Objective: To identify neurobiological factors underlying the anxiety symptoms associated with reading disorder (RD). We compared patterns of resting state functional connectivity ( $\mathrm{rsFC}$ ) from amygdala subregions across children with RD and typically developing (TD) children, and assessed associations of amygdala connectivity with self-reported anxiety.

Participants and Methods: We collected rs-fMRI data from 22 children with RD (and no diagnosis of anxiety disorder) and 21 TD children ages 7 to 13 years. Seeds were placed in the basolateral (BLA) and centromedial (CMA) subregions of the amygdala, and wholebrain connectivity maps were compared across groups. Associations of amygdalar connectivity with anxiety symptoms (RCMAS) and neuropsychological test performance (GORT, WJ-III-Ach, TOWRE, GatesMacginitie) were explored.

Results: Relative to TD children, those with RD showed increased rsFC from CMA to left frontal pole, right lateral occipital cortex, and right thalamus, and from LBA to left frontal pole $(p<0.01$, corrected). Across all subjects, connectivity[RM1] was positively associated with reading problems and self-reported levels of anxiety $(p<0.05$, corrected). Further, amygdala hyper-connectivity mediated the relationship between reading problems and anxiety.

Conclusions: Our findings are consistent with prior findings of hyper-connectivity between amygdala nuclei and regions in both the sensory/perceptual and saliency/emotion regulation systems in pediatric anxiety disorders. Further, aberrant amygdala-frontal rsFC may represent a shared neurobiological substrate for the two disorders or a marker for their comorbidity. While hyper-connectivity from amygdala to visual sensory areas may relate to hyper-vigilance during reading, and hyper-connectivity to frontal areas may suggest dysfunctional emotion regulation in children with both $\mathrm{RD}$ and anxiety.

Correspondence: Katie Davis, Psy.D., Department of Psychiatry, Columbia University, 141 E 89th St Apt 9H, New York, NY 10128, United States.E-mail: kadavis@nyspi.columbia.edu

E.H. GERST, P.T. CIRINO, J. MICIAK, H. YOSHIDA, M. GIBBS \& S.P. WOODS. Processing Speed in Late-Elementary School Children: Examination of the Structure and Its Relation to Reading.

Objective: The first aim of this study was to examine the structure of processing speed (PS) in children by comparing five theoretically driven models of PS: two conceptual models (a Unitary model, a Complexity model) and three methodological models (a Stimulus-Material model, an Output modality model, and a Timing modality model). The second aim was to evaluate how well these models predict key reading skills (decoding, fluency, and comprehension) for elementary-age children. Participants and Methods: Participants consisted of 844 children enrolled in urban public elementary schools. Average participant age was $9.92(\mathrm{SD}=0.89)$ and students were enrolled in $3^{\text {rd }}-5^{\text {th }}$ grades. Sixteen variables from 12 tasks differing in their demand characteristics captured PS. Confirmatory factor analyses and regression equations evaluated hypotheses.

Results: Two similarly-structured models fit best: a two-factor Timing model (Latency and Efficiency) and a two-factor Complexity model (Simple and Complex). Only the Efficiency/Complex factors were predictive of all key reading skills when considered alone and with relevant language and demographic variables.

Conclusions: The structure of PS in children was found to form a two-factor structure with separation apparent between simpler and more complex levels of timed processing. PS appears to be contributory to the prediction of key reading skills in the context of important predictors for late-elementary school children.

Correspondence: Elyssa H. Gerst, University of Houston, 8383 el Mundo St, Apt 411, Houston, TX 77054, United States. E-mail: elyssa.gerst@ gmail.com

L.M. HARDY, M. TOMB, A.N. DEVITO, G. LIVAI \& P. SINGH. Foundational Literacy Skills Among Diverse Children in a Clinic Sample.

Objective: Given the paucity of research among underserved children, the current study examined neurocognitive underpinnings of literacy in a clinic sample of low-income children referred for concerns of academic underachievement.

Participants and Methods: This sample included children with and without a reading $\mathrm{LD}$, ages seven to twelve $\left(n=313, M_{\text {age }}=9.1\right.$, $S D=1.59,70 \%$ Male, $76 \%$ Hispanic/Latino/a, $I Q \geq 80)$, who completed neuropsychological evaluations at a community clinic. Participants were further delineated into three groups based on language spoken in the home (English, mostly English/some Spanish, and mostly Spanish/ some English). Phonological memory, phonological processing, and rapid naming measures were administered as part of a comprehensive battery (CTOPP I,II: Phonological Memory, KTEA-II,3: Phonological Processing, KTEA-3: Letter Naming Facility, KTEA-3: Object Naming Facility).

Results: Participants with a Reading LD performed significantly poorer across measures compared to non-LD participants $\left(F_{1.240}=10.66\right.$, $p<.001 ; F_{1,130}=6.43, p<.05 ; F_{1,96}=5.40, p<.05 ; F_{1,97}=5.28$, $p<.05$, respectively). However, mean scores from the non-LD group also fell below average $(M=91.35 ; M=86.56 ; M=91.84 ; M=89.56$; respectively). Children exposed solely to English in the home demonstrated stronger performances on measures of phonological memory and phonological processing $\left(F_{2,221}=4.157, p<.05 ; F_{2,122}=3.57\right.$, $p<.05)$. Home language groups did not differ on Letter and Object naming performances $\left(F_{2,88}=.389, n s ; F_{2,89}=.216, n s\right.$, respectively). Conclusions: Understanding the neurocognitive correlates, including foundational literacy skills, in this diverse sample of underserved children may support culturally-competent interpretation of neuropsychological measures. Clinical implications will be discussed.

Correspondence: Lindsay M. Hardy, Psy.D., Psychiatry/Promise Program, Columbia University Medical Center/New York Presbyterian, 635 W. 165th St., \#636A, New York, NY 10032, United States. E-mail: hardyli@nyspi.columbia.edu

L. HOKKANEN, J. LAUNES, M. VIRTA, M. LAASONEN, A. TUULIOHENRIKSSON \& K. MICHELSSON. Midlife Cognition in a Birth Risk Cohort - 40 Year Follow-Up.

Objective: Perinatal complications such as preterm birth or hyperbilirubinemia are acknowledged risks of development causing e.g. cerebral palsy, intellectual disability and, in the mild end, learning disabilities. Studies of very low birth weight have revealed low cognitive performance in young adulthood. Long term outcome in other perinatal risks has been less studied.

Participants and Methods: Perinatal Adverse Events and Special Trends in Cognitive Trajectory (PLASTICITY) -project prospectively follows a cohort of subjects from birth. The cohort is a consecutive series of newborns from one maternity hospital in Helsinki Finland in 
1971-74 fulfilling one or several inclusion criteria: birth weight $<2000$ grams, APGAR score <7, hyperbilirubinemia, respiratory difficulties, neurological symptoms, maternal diabetes, hypoglycemia, or infection. Severe defects were excluded from follow-up. A group of controls was also followed.

Results: At the mean age of 42 (SD 1.3) years, 470 subjects (389 risk cohort, 81 controls) were assessed with a full neuropsychological battery and MRI. Participation rate was $55 \%$. Those who participated had performed better in the Wechsler Intelligence Scale for Children at the age of 9 as compared to nonparticipants.

The risk cohort performed less well in the Wechsler Adult Intelligence Scale- IV than the controls (mean FSIQ 105.5, SD 18.4 vs 113.4, SD 15.7 respectively, $t=3.5, p<.000)$. In early analysis of selected cognitive domains, the risk cohort perfomed worse in executive attention (Trail Making B mean 62.9 SD 22.1 vs 56.3, SD 14.3, $\mathrm{t}=-2.6, \mathrm{p}=.011$ ) and reading of non-words (mean 49.3, SD 16.5 vs 44.4, SD 11.7, $\mathrm{t}=-3.2, \mathrm{p}=.002$ ), while in many tests no difference appeared compared to controls.

Conclusions: On group level, perinatal risks impose subtle long term cognitive consequences visible still at midlife. Further analyses will reveal the impact of separate risk categories. Attrition is unavoidable in long follow ups, those who participated seem to have higher cognitive resources.

Correspondence: Laura Hokkanen, PhD, Department of Psychology, University of Helsinki, P.O.Box 9, Helsinki 00014, Finland. E-mail: laura.hokkanen@helsinki.fi

E.A. HUSTON-WARREN, P.T. CIRINO, C.A. ALFANO \&. P. TAYLOR. Executive Functions and Self-Regulated Learning as Predictors of Math Achievement: A Path Analytic Framework.

Objective: Despite considerable evidence for the hierarchical nature of math learning and the influence of executive functions in early math development, few studies have investigated the role of executive functions and related skills (i.e. self-regulated learning skills) in later elementary years. The goal of the present study was to comprehensively evaluate the role of executive functions and self-regulated learning skills as predictors of mathematical outcomes, proposing ways in which these predictive relationships may differ across elementary grades 3 through 5.

Participants and Methods: Directly examining the hierarchy of math learning, this study utilized a path analytic framework to assess the likely mediating role of early math skill mastery (e.g. fact fluency) and the hypothesized moderating effect of grade. Both direct and indirect effects were assessed in a large and diverse sample of students $(N=846)$ in third grade $(N=186)$, fourth grade $(N=484)$, and fifth grade $(N=176)$.

Results: While the moderating effect of grade was not significant, the final model showed good fit $\left(\chi^{2}=313.48, d f=256\right.$; CFI $=0.97$; RMSEA $=0.028,90 \% \mathrm{CI}=0.015$ to $0.038 ;$ SRMR $=0.074)$ and demonstrated predictive power for several considered variables. Additionally, a strong mediating role of math fact fluency was observed.

Conclusions: The results underscore the robust influence of executive functions and metacognition on math outcomes across grade level, thus supporting efforts to integrate findings across bodies of literature. Correspondence: Emily A. Huston-Warren, B.A., Psychology, University of Houston, 8383 El Mundo St., Apt. 411, Houston, TX 77054, United States.E-mail:emily.hustonw@gmail.com

J. GUERIN \& Q. MANO. Attentional control associated with sensitivity to the statistical regularity of subword orthography.

Objective: The objective of the present study was to examine whether attentional control is associated with sensitivity to the statistical regularity of subword orthography.

Participants and Methods: A total of 44 neurologically healthy participants were administered the Stroop Color-Word Test and two experimental measures that assess sensitivity to subword regularity: visual wordlikeness judgment and visual lexical decision. In the wordlikeness judgment task, participants viewed non-pronounceable letter strings in isolation and judged whether each looked like it could be a word. Within this task, three conditions of stimuli were created that varied along degrees of subword orthographic regularity (i.e., low reguarlity, intermediate regularity, high regularity). In the lexical decision task, participants viewed words and orthographically legal, pronounceable nonwords. Within the lexical decision task, orthographic neighborhood size and subword regularity were factorially manipulated.

Results: Results showed significant correlations among Stroop Color-Word Interference and sensitivity to subword regularity on both experimental tasks. Specifically, Stroop Color-Word Interference was associated with sensitivity to subword regularity on the visual wordlikeness judgment task, above and beyond Stroop Word Reading, Stroop Color Naming, and wordlikeness task difficulty. Moreover, there was a significant association between Stroop Color-Word Interference and the main effect of subword regularity on word target accuracy within the lexical decision task.

Conclusions: Sensitivity to the statistical regularity of subword orthography appears to be uniquely, positively, and significantly related to attentional control. Though preliminary, present results suggest that sensitivity to subword orthographic regularity is not a purely passive process but instead may be influenced by "top-down" attentional control. Results have the potential to shape new thinking about orthographic processing in developmental dyslexia.

Correspondence: Quintino Mano, Ph.D., Psychology, University of Cincinnati, 47 West Corry Blvd., Edwards 1 Center, Suite 4130, Cincinnati, OH 45243, United States.E-mail: quintino.mano@uc.edu

A.E. MARGOLIS, K. DAVIS, Z. HUO \& R. MARSH. Intrinsic Functional Connectivity Within The Left Hemisphere Reading Network And Associations With Phonological Ability In Children With And Without Reading Disorder.

Objective: To compare functional connectivity from the left hemisphere reading network in children with reading disorder (RD) and typically developing (TD) children, and to assess whether patterns of connectivity are associated with phonological ability.

Participants and Methods: Resting state fMRI data was collected from 22 children with RD and 21 TD children between 7 and 13 years old. Seeds were placed in eleven ROIs defining the reading network. Wholebrain connectivity maps were compared across groups and associations with phonological abilty (using WJ-III Word Attack, Spelling and TOWRE) were explored.

Results: Relative to TD children, those with RD showed reduced functional connectivity between left inferior frontal gyrus (IFG) and right middle temporal gyrus (MTG), $\mathrm{p}<0.05$, corrected. Children with RD showed negative, whereas TD children showed positive connectivity between left superior temporal gyrus (STG) and right caudate, $\mathrm{p}<0.05$, corrected. Across all subjects, left IFG-right MTG and left STG-right caudate connectivities were positively associated with phonological ability, $\mathrm{p}$ 's $<0.048$ and 0.001 , respectively.

Conclusions: Our finding of reduced connectivity from left hemisphere reading network regions to right hemisphere temporal and striatal regions in $\mathrm{RD}$ children is consistent with prior data pointing to the recruitment of right hemisphere regions during the early stages of learning to read in TD children. Further, increased connectivity was associated with better phonological processing. Thus, reduced bilateral fronto-temporal and temporal-striatal connectivity may underlie the difficulty that children with RD have in using phonological skills when reading.

Correspondence: Amy E. Margolis, PhD, Child and Adolescent Psychiatry, Columbia University Medical Center, 1051 Riverside Drive, New York, NY 10032, United States. E-mail:margola@nyspi.columbia. edu 
K. REESE, B. SHIEH, R. PLOTKIN \& J. LINTON REESMAN. As Easy as ABC? Deaf or Hard-of-Hearing Students' Alphabetic Knowledge and Recitation.

Objective: Alphabet knowledge and recitation $(A K / R)$ in children has been documented as an important predictor of literacy skills. Hearing children typically recite the alphabet correctly by age 6 years. For children who are deaf or hard-of-hearing (D/HOH), no study to date has systematically examined the age at which AK/R is mastered, or at what age these difficulties are associated with risks to literacy development. The present study examined the performance of clinically-referred D/ $\mathrm{HOH}$ students on an AK/R task within a specialty neuropsychology clinic

Participants and Methods: The present study was conducted via retrospective chart review of D/HOH students ages 6 to 21 years, $(\mathrm{N}=19$, mean age $=10.69$ years, $\mathrm{SD}=3.99,63 \%$ male $)$ referred for clinical evaluation in a neuropsychology specialty clinic for $\mathrm{D} / \mathrm{HOH}$ children. Performance on an alphabet recitation elicitation task was examined, including the mean number of letters spoken/signed, letters written, and letter sequential accuracy. During the task, 58\% utilized hearing aids (HA) or cochlear implants (CI), 79\% utilized spoken English, and 21\% used American Sign Language (ASL).

Results: Mean number of letters spoken or signed was $21(\mathrm{SD}=5)$. Seven participants $(36.84 \%)$ produced all 26 letters in the correct sequence. Mean number of letters written was $21(\mathrm{SD}=6)$, with most participants demonstrating sequencing errors $(72 \%)$. This sample demonstrated well-below expected performance compared with hearing children, who reportedly acquire alphabet recitation by age 6 .

Conclusions: Neural processing of language whether spoken or signed has demonstrated equivalence. Early fingerspelling skills predict emerging literacy and letter writing skills in children who are $\mathrm{D} / \mathrm{HOH}$. The present study identifies a possible area of skill weakness in AK/R in spoken or signed modalities that may serve as an early and easily identifiable marker for later literacy difficulties within this population, though further examination of this phenomena is needed in non-clinical samples.

Correspondence: Kaycee Reese, Kennedy Krieger Institute, 11 E Chase St. \#303, Baltimore, MD 34471, United States. E-mail: reesek@ kennedykrieger.org

E. RIGGALL, R. WINTER, L. BRANUM-MARTIN, R. SEVCIK, R. MORRIS \& S. FROST. Modeling Implicit Sequence Learning in Developmental Dyslexia with and without Specific Language Impairment.

Objective: This study examined implicit sequence learning (ISL), measured by the widely-used Serial Reaction Time Task (SRTT), in children who met criteria for Developmental Dyslexia (DD) with or without Specific Language Impairment (SLI). ISL, a type of procedural learning, has been shown to be deficient in both DD and SLI (Lum et al., 2010; Menghini et al., 2006). Though such procedural learning impairments as measured by the SRTT remain debated, with both positive and negative findings, ISL has been theoretically and objectively linked to levels of grammatical understanding across SLI and typically developing children (Lum, 2012). These relations between ISL and grammar abilities remain unexamined among children with DD. Children also learn implicitly during reading instruction that words are composed of phonemic patterns, potentially in a similar manner to learning about the patterns underlying grammar. Whether ISL is similarly related to phonological awareness also remains unexamined in these groups.

Participants and Methods: Using multi-level growth models of initial reaction time and slope of learning across the repeated sequence of the SRTT, we modeled ISL in a sample of elementary school children who met criteria for DD $(\mathrm{N}=53)$ or DD+SLI $(\mathrm{N}=20)$. We further examined the predictive contributions of grammatical understanding, vocabulary abilities, phonological awareness, and diagnostic groups (DD with and without SLI) on ISL on the SRTT.

Results: Results showed generally linear trends in improved reaction time, with minimal differences in rates of learning. The predictors of grammatical understanding, vocabulary ability, phonological awareness, or diagnostic group (DD or DD+SLI), did not relate strongly to rates of implicit learning.

Conclusions: Results are discussed in terms of the SRTT as an indicator of ISL in this sample, especially in relation to the contribution of language dimensions studied, in relation to diagnostic categorical results, and in relation to approaches to analyzing such SRTT learning task data.

Correspondence: Emily Riggall, B.A., Psychology, Georgia State University, 1110 Briarcliff Rd NE, Unit 1, Atlanta, GA 30306, United States.E-mail: emily.riggall@gmail.com

\section{A. ROBERTS, L. JACKSON \& R. TANGEN. Cognitive Correlates of} Academic Learning in Fetal Alcohol Spectrum Disorders.

Objective: Children with Fetal Alcohol Spectrum Disorders (FASD) are at risk for impairment in academic learning. Research has demonstrated below average reading and math skills, although comprehensive research across academic domains and across the FASD spectrum is limited. This study examined the impact of suspected prenatal alcohol exposure on academic learning across the FASD spectrum. In addition, the study examined cognitive correlates related to reading and math performance.

Participants and Methods: Participants included 59 children (Mean Age $=126$ months) who were evaluated through a multidisciplinary FASD Clinic. All participants completed a comprehensive neuropsychological assessment and were evaluated using the 4-digit-code (Astley \& Clarren, 2000). Twelve participants met criteria for full/partial Fetal Alcohol Syndrome (FAS), 26 for static encephalopathy (SE), and 21 for neurobehavioral disorder (ND).

Results: Results indicated there were significant $(p<.05)$ differences between the SE and ND group for all academic tasks and significant differences $(p<.05)$ between the FAS and ND groups for select academic tasks. No significant differences were found between the FAS and SE groups. Further analysis revealed children with FASD perform better on reading than math tasks $F(1,55)=8.205, \mathrm{p}=.006$, better on applied math than computational tasks $F(1,54)=11.195, \mathrm{p}=.001$, and better on word reading than reading comprehension $F(1,57)=25.444, \mathrm{p}=.000$. Finally, the cognitive predictors for reading and math differed, such that FSIQ was the best predictor of math performance, while working memory was the best predictor of reading performance.

Conclusions: Overall, findings suggest that within a group of children with FASD, there is relationship between level of severity of diagnosis and related academic outcomes. In addition, children with FASD have strengths and weaknesses on academic testing that could be used to develop specific intervention programs.

Correspondence: Alycia Roberts, PhD, Developmental/Behavioral Pediatrics \& Psychology, Rainbow Babies \&. Children's Hospital, W.O. Walker Center, 10524 Euclid Ave., Ste 3150, Cleveland, OH 44106, United States.E-mail: alycia.roberts@UHhospitals.org

J. SALVADOR-CRUZ, C.G. ARMENGOL, E.E. SANCHEZ VIELMA \& L.A. LEDESMA TORRES. Executive Function and Reading Comprehension: Performance of Mexican 9-year-olds on a NonLinguistic measure of inhibition.

Objective: To explore an understudied aspect of EF: inhibition, on reading comprehension. To examine performance of Spanish-speaking children when using a non-linguistic alternative to the color-word interference test.

Participants and Methods: Thirty 9 year-old school children ( 15 boys, 15 girls) with no neurological or psychiatric conditions were individually administered the Reading Comprehension (RC) subtest of PROLEC-RM (Mexican adaptation of a Spanish comprehensive battery of reading ability) and the Five Digit Test (FDT) - a non-linguistic alternative to the Stroop Color-Word Test (SCWT). Independent variables were correct responses for $\mathrm{RC}$ and response speed for the FDT interference 
condition. Given the non-normal distribution of the independent variables, data were analyzed via Spearman's Rho.

Results: Spearman's Rho=.068 was non-significant. No gender differences were observed. Mean correct RC score $=11.431$. Mean FDT interference RT- 75.13.

Conclusions: Findings showed no support for inhibitory control as an independent contributor to reading comprehension. Inhibition is an identified component of the triad of executive functions impacting reading, but no consensus regarding its impact on reading comprehension has been reached (Christopher et al., 2013). More sophisticated assessment instruments that capture this complex cognitive process and the impact of socio-cultural variables must be incorporated in its clinical and experimental investigation in order to arrive at a more complete model of its neuropsychological underpinnings.

Correspondence: Judith Salvador-Cruz, Ph,D., Mexico City, Mexico. E-mail: salvadcj@gmail.com

J. SALVADOR-CRUZ, C.G. ARMENGOL, A. SEGURA VILLA \& L.A. LEDESMA TORRES. Neurological Soft Signs (NSS) as Potential Risk Indicators of Disrupted Neurodevelopment in a Mexican Sample of Elementary School Children.

Objective: Determining whether NSSs signal underlying developmental difficulties in healthy elementary school children.

Participants and Methods: Forty-nine Mexican elementary school children (27 boys, 22 girls) aged 6-12 participated. Exclusionary criteria were being held back a grade and psychiatric history (per Salvador \& Galindo's Neurological and Psychiatric History Questionnaire.

Portellano's Neuropsychological School Maturity Questionnaire (NSMQCUMANES in Spanish) was administered, yielding a neuropsychological development index (NDI). Neurological soft signs (NSS) were assessed with Matute et al.'s Children's Neuropsychological Evaluation (CNE; Spanish ENI)

Results: Obtained NDI's (neuropsychological maturity-chronological age quotients)showed this distribution : Very low $=18.4 \%$; Medium low $=12.2 \%$; Low $=40.8 \%$; Medium high $=4.1 \%$. Subtest scores paralleled this distribution, with a stronger overall performance on Auditory Comprehension relative to the rest (Audio-gnostic Writing, Visuoperceptual and Rhythm), with $30-50 \%$ children obtaining very low and medium low scores.

CNI/ENI yielded no clear quantitative evidence of NSSs. Qualitative analyses of three subtests (Visual Tracking, Right/Left Discrimination, Opposing Digits) revealed decreasing NSSs with age.

Conclusions: A high incidence of NSSs among healthy children, gradually tapering with age, has been documented in other studies. (Dazzan et al, 2006). Differential patterns of cognitive vulnerability suggest differential brain networks involvement (Zhao et al., 2013). Recent advances in the study of NSSs have underscored their importance for pediatric neurology. NSSs are best viewed as vulnerabilities to be targeted for early intervention aimed at fostering resiliencies (LevitBinnun \& Golland, 2012).

Correspondence: Judith Salvador-Cruz, Ph,D., Mexico City, Mexico. E-mail:salvadcj@gmail.com

S. SEESE, J. SAFER-LICHTENSTEIN, A.D. VERBALIS, C. LUONG-TRAN, K.K. HARDY, L. FEDERIN, L. ANTHONY \&. L. KENWORTHY. What Do Children with ADHD and ASD Look Like in the Mainstream Classroom? Examining Executive Functions in School.

Objective: Executive function (EF) skills are critical for students to be successful in the classroom. EF deficits are prominent among children who have an Autism Spectrum Disorder (ASD) or Attention Deficit Hyperactivity Disorder (ADHD). This study aims to evaluate how teachers and observers report EF in the classroom among these two diagnostic groups.

Participants and Methods: A sample of mainstream school children either with an ASD or ADHD (N=149) participated in a school-based EF intervention study. Student behavior was observed by treatment-blinded research staff and by teachers who reported using a modified version of the SKAMP (Swanson, 1992). Classroom observations and SKAMPS yield scores on several EF skills, such as transitioning, attending to work, and following rules. Group differences were explored using independent sample t-tests.

Results: Total SKAMP and classroom observation scores were significantly correlated for both the ASD group $(r=-.486, p<.01)$ and for the ADHD group $(r=-.292, p<.01)$. However, children with ADHD exhibited more positive behaviors during observations than children with ASD, $(\mathrm{t}(290)=-2.79, \mathrm{p}=.006)$. Teachers reported students with ADHD as having more difficulty staying seated than children with ASD $(\mathrm{t}(179)=-3.50, \mathrm{p}=.001)$. Within the ADHD group, students observed not "following rules in the classroom" were more likely to have a negative overall teacher rating $(r=-.343, p<.01)$.

Conclusions: Overall, ratings were similar across diagnostic groups but several items stood out as more likely to be concerning in one group versus another. Given the difficulties identified by either observers or teachers, EF skills at school are an important target for treatment. Correspondence: Sydney Seese, Bachelors, Neuropsychology, Children's National Medical Center, 198 Halpine Rd Apt 1466, Rockville, MD 20852, United States. E-mail: sseese2@childrensnational.org.

H. TANAKA, R. HANCOCK, P. BEHROOZI, P. DUONG \& F. HOEFT. Slow Reading: A New Neurobiological Phenotype.

Objective: Dyslexia is often identified by untimed reading measures. Past studies found that poor readers struggle with reading fluency, even after intervention programs. Hence, it is an open question whether slow, accurate readers also have neurobiological signatures of dyslexia, and whether they would benefit from accommodations. This study aims to characterize brain differences between slow, accurate readers and those with typical reading fluency.

Participants and Methods: 51 native English-speaking $3^{\text {rd }}$ and $5^{\text {th }}$ graders, divided into two groups based on standard score (ss) discrepancy between timed (Phonemic Decoding Efficiency [PDE] of the Test of Word Reading Efficiency) and untimed (Word Identification [WID] of the Woodcock Reading Mastery Test-Revised) reading measures: SlowR (WID >80; WID-PDE >7.5ss; $\mathrm{N}=20$ ) and Control (WID-matched; WID $>80$; WID-PDE $<7.5 \mathrm{ss} ; \mathrm{N}=31$ ). Brain activation was induced by a visual real-word rhyme judgment fMRI task, and was compared between SlowR and Control groups, with PDE as a covariate. Results were thresholded at a corrected $(p<.05)$ voxelwise height of $p<.01$.

Results: Slow but accurate readers had significantly reduced activation in the bilateral precentral gyri and significantly increased activation in the left supramarginal gyrus compared to Control participants.

Conclusions: Slow but accurate readers show hyperactivation in the supramarginal gyrus, suggesting continued high reliance on phonological processing for real words, and reduced activation in the bilateral precentral gyrus, possibly reflecting differential subvocalization during rhyme judgments. Hence, slow but accurate readers have an atypical brain signature, distinct from both traditional dyslexics (low reading accuracy, low fluency) and typical readers (normal accuracy, normal fluency). While reading fluency is notoriously difficult to improve, slow, accurate readers may benefit from accommodations including increased time for reading assignments and tests; given the brain differences found here, further study is recommended.

Correspondence: Hiroko Tanaka, PhD, Psychiatry, University of California San Francisco, 401 Parnassus Ave, San Francisco, CA 94143, United States.E-mail: hiroko.tanaka@ucsf.edu

S.G. MASSONI, M. LENCIONI, M. TIMPANO SPORTIELLO \& S. TOCCHINI. Developmental Dyslexia and Working Memory. Objective: Background: Previous studies have suggested that dyslexic individuals exhibited difficulties in tasks involving working memory (WM), in particular in phonological loop (PL). Recent data have pointed to the central executive $(\mathrm{CE})$ system as another source of WM deficit in 
dyslexic readers. The visuo-spatial sketchpad (VS) does not seem to be implicated in reading difficulties.

Few studies investigated VS separating the visual components from spatial ones. We choose WMS IV to assess these components specifically. Objective: This study investigated associations between WM (PL, VS and CE - Baddeley and Hitch model) and developmental dyslexia.

Participants and Methods: Participants In our study we tested 30 subjects 16 to 19 years-old,(17 males), with reading and writing difficulties, but normal math abilities. All subjects had the diagnosis of developmental dyslexia and/or dysorthography.

Methods: We assessed the capacity of PL using the digit span subtest of WAIS-IV, the VS through the Symbol Span (SA) of WMS IV and the CE by the administration of Spatial Addition (SSP) of WMS IV and digit span forward of WAIS-IV. We used WMS IV to assess visual and spatial components separated.

Results: Subjects with developmental dyslexia totalized Digit Span and Digit Span Forward scores significantly lower $(p<0.05)$ than SSP and SA scores. Moreover differences between Working Memory Index (WMI) and Visual Working Memory Index (VWMI) are significant.

Conclusions: Our findings, in agreement with previous studies, suggest that working memory functioning is impaired in dyslexic readers. In detail dyslexic and dysorthographic subjects performed poorly in phonological loop and executive central task measurements. It will be interesting, for rehabilitation goals, to understand through further studies if poor performances in executive central task could depend on poor phonological capacity.

Correspondence: Marco Rossano Timpano Sportiello, Prof, Via P.Maroncelli 73, Viareggio 55049, Italy.E-mail: marco.timapano@ uslnordovest.toscana.it

\section{E.M. WESONGA, L. YALEM, S.K. GILLIGAN \& D. WHITE. Comorbidity of Learning Disabilities: Prevalence Rates of Co-occuring Reading Disability, Writing Disability, Math Disability, ADHD, and Other Psychiatric Disorders in a School Sample.}

Objective: Given the closely intertwined development of reading, writing, and arithmetic skills, children with learning disabilities (LDs) may present with difficulties across multiple academic domains. However, reading disability (RD), writing disability (WD), and math disability (MD) are often studied in isolation.

Participants and Methods: In order to investigate comorbidity in the LD population, we examined archival data from a sample of 274 children aged $6-14$ years $(38 \%$ girls; mean age $=9.8$ years $)$ who attended a private day school in the St. Louis, Missouri community for students with LDs. All students carried a diagnosis of at least one specific LD, and LD diagnoses were based on an ability-achievement discrepancy of at least 1.5 standard deviations. On the whole, students demonstrated average intelligence (WISC-IV; mean FSIQ $=104)$. Diagnostic information regarding the presence of $\mathrm{ADHD}$, mood disorders, anxiety disorders, and adjustment disorders was also examined.

Results: Within our sample, the majority of students were diagnosed with multiple LDs. Remarkably, about $25 \%$ of all students were diagnosed with all three specific LDs (RD + WD + MD), whereas about $33 \%$ were diagnosed with only one LD. RD and WD co-occurred in $65-85 \%$ of those with either disorder, RD and MD co-occurred in 37-89\% of those with either disorder, and MD and WD co-occurred in $41-74 \%$ of those with either disorder. ADHD was present in $57 \%$ of the sample, and other psychiatric disorders were present in $14 \%$ of the sample. Rates of co-occurring ADHD and other psychiatric disorders remained relatively consistent across groups with isolated LD, two comorbid LDs, and three comorbid LDs.

Conclusions: Overall, our results support previous findings indicating high prevalence rates of co-occurring LDs. In light of these findings, special attention must be given to ensure that children with isolated or comorbid LDs are properly identified and placed in educational programs which appropriately address their unique concerns.
Correspondence: Erika M. Wesonga, Psychology, Washington University in St. Louis, St. Louis, St. Louis, MO 63108, United States. E-mail: ewesonga@wustl.edu

R. WINTER, E. RIGGALL, P. ALBERT, C. ARRINGTON, J.A. TURNER, R. SEVCIK \& R. MORRIS. Functional MRI Overlap Between ADHD and Developmental Dyslexia on Alternative Force Choice Tasks: A Meta-Analytic Study.

Objective: The present study utilized meta-analytic methods to examine the activation overlap between subjects diagnosed with Attention Deficit Hyperactivity Disorder (ADHD) versus Developmental Dyslexia (DD). Despite current estimates of comorbidity reaching about $40 \%$ (Boada et al., 2012), the overlap between overused and underused brain networks in these disorders during functional in-scanner tasks remains unclear. This study conducted a systematic review and Activation Likelihood Estimation (ALE) analysis of studies that administered in-scanner two-alternative forced-choice tasks to ADHD or DD subjects.

Participants and Methods: Our search for DD or ADHD fMRI papers yielded 48 studies (22 DD and 26 ADHD) matching the following criteria: 1.Functional neuroimaging studies published between 20102016; 2.Reported Talaraich or MNI coordinates; 3.Recorded a direct comparison between DD/ADHD and control subjects; 4 . Used a forcedchoice button press or inhibition response task. ALE meta-analysis was performed via BrainMap's GingerALE software $(\mathrm{P}<0.05)$.

Results: Results indicated hypoactivation in the supplemental motor cortex, prefrontal cortex, associative visual cortex and the supramarginal gyrus on forced choice/inhibition response tasks across both ADHD and DD populations. Unfortunately, only five DD studies controlled for co-morbid ADHD, while none of the ADHD studies controlled for reading deficits, making it difficult to determine whether results were suggestive of deficits in a single, independent network, or shared overlapping networks relevant to each condition.

Conclusions: Our findings suggest significant overlap in cortical use across DD/ADHD populations during forced-choice tasks. The current fMRI literature does not allow for more robust cross population examination due to the lack of comparable cognitive paradigms used across the clinical populations. In order to better understand the nature of the co-morbidity between these highly prevalent disorders, future research should address this methodological lacuna.

Correspondence: Rebecca Winter, MA, Psychology, Georgia State University, 1337 Holly Lane, Atlanta, GA 30329, United States. E-mail: rwinter3@student.gsu.edu

\section{Epilepsy/Seizures}

K.D. SRNKA, J. JONES, D. ALMANE, B. HERMANN \& M. SEIDENBERG. Intraindividual Variability in Sustained Attention Mediates the Relationship between Age of Epilepsy Onset and IQ and Academic Achievement.

Objective: Earlier age of epilepsy onset is associated with increased neuropsychological impairment in childhood epilepsy (CE). We have previously reported that attentional ability, particularly intra-individual variability (IIV) in sustained attention, is impaired in CE. Given the importance of sustained attention ability for successful performance on measures of higher order cognitive functioning, we predicted that IIV would mediate the relationship between age of epilepsy onset and performance on measures of intellectual functioning and academic achievement.

Participants and Methods: 168 participants with recent onset CE, mean age 12 years, mean onset age 11.75 years, $51.2 \%$ generalized and $48.8 \%$ localized epilepsies, were administered the Connor's Continuous Performance Test- $2^{\text {nd }}$ Edition, the Wide Range Achievement Test- $3^{\text {rd }}$ Edition (WRAT), and Wechsler Abbreviated Scales of Intelligence. We conducted mediation analysis based on the Baron and Kenny (1986) conditions. Age of onset was the independent variable, IIV was the 
mediator variable, and the performance (PIQ) and verbal (VIQ) indices of IQ and the three indices of academic achievement (spelling, reading, arithmetic) were the dependent variables in five separate models. The Sobel test was used to determine the significance of the IIV in reducing the association between the predictor variable (age of onset) and the outcome measures.

Results: IIV was found to be a significant partial mediator for the relationship between age of onset and PIQ $(\mathrm{z}=2.87, \mathrm{p}<.05)$, WRATarithmetic $(\mathrm{z}=2.20, \mathrm{p}<.05)$, and WRAT-reading $(\mathrm{z}=2.03, \mathrm{p}<.05)$. Sobel's $\mathrm{Z}$ was not significant for VIQ $(\mathrm{z}=1.26, \mathrm{p}=.21)$ and WRATspelling $(\mathrm{z}=1.79, \mathrm{p}=.07)$.

Conclusions: These results suggest that early onset effects on cognition are mediated at least to some extent by increased IIV on a measure of sustained attention. Mediation analyses may provide an approach to examining the psychological mechanisms associated with cognitive impairment in childhood epilepsy.

Correspondence: Kyle D. Srnka, Psychology, Rosalind Franklin University of Medicine and Science, 3503 Green Bay Rd, \#307, North Chicago,IL 60064, United States.E-mail:kyle.srnka@my.rfums.org

\section{Invited Symposium 6. Translational Neuropsychology: Contemplating the Past and Looking Toward the Future}

\section{Chair: Adam M. Brickman}

10:00-11:30 a.m.

A.M. BRICKMAN, D.M. BARCH, R.M. BILDER, R. AU \& R.M. BAUER. Translational Neuropsychology: Contemplating the Past and Looking Toward the Future.

Symposium Summary: With the celebration of the $50^{\text {th }}$ year anniversary of the International Neuropsychological Society comes the opportunity to reflect on the history of our field and to contemplate the directions we are going. Neuropsychology, at its core, is a multi- and inter-disciplinary field that draws inspiration from cognitive psychology, behavioral neurology, and basic neuroscience to derive brain-behavior relationships and apply them to clinical settings. Early studies used lesion approaches in animals and humans to map behavioral correlates of regional brain damage and, by inference, normal brain functioning. The majority of contemporary studies in neuropsychology apply psychometric assessment to determine cognitive correlates of clinical conditions and developmental stages. Clinical and applied aspects of neuropsychology deploy similar psychometric assessments to aid in diagnosis and treatment recommendations. As we look toward our future, we propose embracing a translational neuropsychology approach, in which we apply methodological and theoretical innovation to elucidate the mechanistic bases of brain-behavioral relationships that support direct translation into clinical practice. This symposium will highlight translational approaches used in five neuropsychology laboratories. Dr. Brickman will discuss work on the integration of neuroimaging and experimental methods to dissociate hippocampal subfields, the cognitive processes they mediate, and associated treatment-focused clinical trials. Dr. Barch will focus on historical concepts of brain connectivity, current conceptions and methods, and future innovations and translations, including stimulation or feedback approaches to enhance connectivity and human/animal parallels. Dr. Bilder will discuss the principle analytic and conceptual models that have driven our understanding of brain and behavior, from past comparisons of "organic" disorders through classic psychometric analyses, to the development of causal models that span multiple biological scales. Dr. Au will discuss how technology integration provides immediate opportunities to create novel cognitive biomarkers and enable a future role for neuropsychology in the realm of big data. Dr. Bauer will discuss a simultaneous animal-human platform developed to examine cortical-hippocampal interactions using MRI, electrophysiology, viral vectors, and human/animal performance.
Correspondence: Adam M. Brickman, PhD, Taub Institute for Research on Alzheimer's Disease and the Aging Brain, Department of Neurology, Columbia University, P\&S Box 16, 630 West 168th Street, New York, NY 10032, United States. E-mail: amb2139@columbia.edu

\section{A.M. BRICKMAN, R.P. SLOAN \& S.A. SMALL. Dietary Flavanols, Hippocampal Subfields, and Cognitive Aging: A Translational Neuropsychology Story.}

Neuropsychologists are uniquely positioned to engage in work that translates basic neuroscientific observations into human studies and, ultimately, clinical practice. Over the past several years, we have been gaining insight into the complexity of the hippocampal circuit, how this complexity relates to Alzheimer's disease (AD) and normal aging, and whether there are treatment approaches that can target specific hippocampal-mediated behaviors. Computational and theoretical models point to specialized functional roles for the distinct molecular subfields that comprise the hippocampal formation and differential vulnerability to aging and disease across these subfields. Converging research suggests that the lateral entorhinal cortex (EC) is affected first in $\mathrm{AD}$ and relatively spared in normal aging, but that the dentate gyrus (DG) is affected in normal aging and relatively spared in AD. We used a translational neuropsychological approach to 1) develop and validate neuropsychological instrumentation that reflects specifically function of the EC and DG based on theoretical models, 2) test the extent to which EC dysfunction is uniquely involved in $\mathrm{AD}$ and the extent to which DG dysfunction is uniquely involved in normal aging using novel functional neuroimaging tools, and 3) design and implement clinical trials to test the extent to which dietary flavanol consumption can treat normal age-related, DG-mediated memory decline. We developed a novel object recognition task ('ModBent') and an episodic learning and memory test ('ModRey') designed to reflect DG and EC function, respectively; we validated these instruments by showing a double anatomical dissociation with cerebral blood volume (CBV) measures in the hippocampus formation obtained with high-resolution functional magnetic resonance imaging (fMRI). Next, we compared hippocampal CBV values between older adults who progressed to develop clinical AD and showed a selective AD-related CBV reduction in the lateral EC. Finally, among healthy adults we showed that normal aging is associated with DG function. We conducted a small clinical trial and showed that consumption of a dietary flavanol, known to stimulate neurogenesis in the DG, selectively increased DG CBV and performance on the ModBent. We implemented a large-scale $(n=4,000)$ clinical trial to test the effects of dietary flavanols on cognition in older adults. The DG and EC are neuropsychologically dissociable and play distinct roles in normal aging and AD. Normal aging appears to target DG and the cognitive processes it mediates selectively, whereas $\mathrm{AD}$ appears to target the EC selectively. By moving theoretical observations into approaches to instrument development, neuroimaging, and intervention, we highlight a successful example of translational neuropsychology.

Correspondence: Adam M. Brickman, PhD, Taub Institute for Research on Alzheimer's Disease and the Aging Brain, Department of Neurology, Columbia University, P\&S Box 16, 630 West 168th Street, New York, NY 10032, United States. E-mail: amb2139@columbia.edu

\section{D.M. BARCH. Connectomics and the Brain: Past, Present and Future.}

Much work on understanding the etiology of neurological and psychiatric disorders has been aided by advances in neuroimaging technologies that were novel for the time they were introduced, including the use of magnetic resonance imaging to assess both structural and functional imaging of the human brain. The focus of this talk will be on how we currently measure human brain functional and structural connectivity, the ways in which understanding brain connectivity has informed our knowledge of the mechanisms that lead to the development and maintenance of various behavioral impairments associated with psychiatric and neurological disorders, and where the field is heading in terms of 
novel measures and approaches. First, I will provide a brief history of the development of our current methods to measure and understand human functional and structural brain connectivity, with a particular focus on the methods developed as part of the Human Connectome Project. This overview will include some discussion of the relative pros and cons of different methods. Second, I will review the current knowledge about core "intrinsic" brain connectivity networks in humans developed using data from brain connectivity. Third. I will selectively describe some of the key work using brain connectivity to understand the neurobiology of neurological and psychological disorders, with a specific focus on psychosis, depression and neglect, as these are domains with large existing literatures on brain connectivity impairments. Lastly, I will focus on emerging innovations in the measurement of human brain connectivity. This will include work focused on enhancing connectivity through behavioral (e.g., cognitive training, biofeedback, mindfulness), pharmacological, and stimulation methods (tDCS, tACS). It will also include work on human-animal translation models for understanding mechanisms of brain connectivity, and work in non-human primates and rats.

Correspondence: Deanna M. Barch, Department of Psychological \& Brain Sciences, Washington University, Box 1125, One Brookings Drive, St. Louis, MO 63130,United States.E-mail: dbarch@wustl.edu

\section{R.M. BILDER. Neuropsychological Models: Past, Present and Future.}

The last half century has witnessed many changes in neuropsychology, including formalization of training models, the advent of modern neuroimaging methods, and the impact of information sciences and technologies on both data acquisition and analysis. Our discipline has further undergone major conceptual upheavals. The historical roots of neuropsychology drew heavily from $19^{\text {th }}$ century lesion models and assumed that partially modularized functions could be ablated. By the time the INS was founded there was a productive tension between modular and distributive theories, which persists in modern network models of the brain based on structural and functional connectivity findings. Nevertheless, an explicit mind-body dualism remained as studies distinguished "organic" from "functional" syndromes. Methodological refinements helped bring measurement methods from a coarse "sign" approach to a more nuanced and dimensional psychometric approach, and this in turn promoted a more robust statistical and actuarial basis for neuropsychological assessment. Simultaneously, evidence of biological anomalies linked to psychological dysfunction made less tenable the splitting of "mind" from "brain." Meanwhile, our assessment approaches are beginning to leverage modern psychometric theory to enable more efficient identification and assessment of latent constructs with desired precision; this already can be used to establish best practices from empirical data. Next steps will include further construction of causal models that go beyond psychometric definitions of latent constructs, leverage the temporal sequencing of component processes, and enable the construction of more definitive experiments to distinguish alternate theories about brain-behavior relations. An extension of these strategies to include other levels of analysis (from the genome to the syndrome) promises to reframe our most basic assumptions about mind and brain, with profound impacts on both human health and philosophy.

Correspondence: Robert M. Bilder, PhD, ABPP-CN, UCLA Semel Institute for Neuroscience \& Human Behavior, 740 Westwood Plaza, Room C8-849,Los Angeles, CA 90049,United States.E-mail: rbilder@ mednet.ucla.edu

\section{R. AU \& T. ANG. Next Generation Neuropsychology: Digital Biomarkers and Big Data.}

Technology is changing what we can do and how we can do it. E-health and the internet-of-things (IoT) have translated into a plethora of wearable sensors and smart home devices and offer solutions for monitoring and detecting changes at the earliest stages, enabling feedback and intervention strategies before modifiable behavioral patterns associated with these changes become intractable. The Aging Well Institute (AWI) is a global initiative initiated through Boston University's School of Public Health designed to catalyze groundbreaking discoveries for chronic disease prevention and identify determinants of sustaining lifelong physical, cognitive and mental health. The AWI platform establishes a new disruptive model that synergizes research, technology, education/ training and application by bringing together multiple sectors to develop solutions that would not otherwise be possible. The AWI e-Cognitive Health Initiative uses the foundation of the Boston University-based Framingham Cognitive Aging Study, which boasts arguably the richest database of its kind, with up to 45 years of prospective health and lifestyle measures, along with available genetic and other biomarkers to relate to brain health. Longitudinal assessment includes an extensive battery of neuropsychological testing as well as neuroimaging via MRI. More recently, the assessment of cognitive performance has also included digital capture of unique traits, starting with voice (2005) and written performance (2011). Starting in 2015, Framingham also began collecting e-health data using mobile devices and is expanding its real-time tracking of key health metrics. We present several case studies of how blending research and technology create innovative solutions for brain health. This new approach to neuropsychological research will enable forward thinking studies, create a research-to-marketplace pipeline and generate collaborations and resources necessary to thrive and fuel innovation and discovery.

Correspondence: Rhoda Au, PhD, Anatomy \& Neurobiology, Neurology and Epidemiology, Boston University Schools of Medicine and Public Health, 72 E Concord Street, B6, Boston, MA 02118, United States. E-mail: rhodaau@bu.edu

\section{R.M. BAUER. An Interactive Translational Platform for Investigating Age-Related Memory Decline.}

As the healthcare pendulum has swung from disease management to prevention, renewed attention has been given to developing sensitive tools to identify illness risk at a point at which primary or secondary prevention will be effective. In my presentation, I will describe a combined human-animal platform designed to (a) more precisely understand cortical and hippocampal contributions to normal and pathological aging, and (b) to develop neurocognitive probes that are sensitive to the complex transition between normal aging and very early preclinical disease. The platform attempts to achieve two-way translation using a "comparative neuropsychology" approach, wherein empirically validated rat models of spatial and object memory (perceptual "oddity" tasks) are adapted for use in humans, and wherein human paradigms are adapted to the rat (e.g., touch-screen discrimination). Methodological approaches include MRI, electrophysiology, viral vectors, and human/ animal performance. The presentation will focus on recent investigations of the role of the perirhinal cortex in complex object recognition, and will show how animal discrimination learning paradigms can be fruitfully adapted for use with humans. Our recent findings suggest that the specific computations performed by perirhinal cortex are highly susceptible to age-related decline, and, when evaluated in the context of other risk factors, can contribute highly sensitive information to the detection very early preclinical decline in the medial temporal memory system. Correspondence: Russell M. Bauer, PhD, ABPP, Clinical \& Health Psychology, University of Florida, PO Box 100165 HSC, Gainesville, FL 32610, United States.E-mail: rbauer@phhp.ufl.edu 


\section{Symposium 11. Development and Adaptation of Ässessment Instruments: Best Practices, Legal Issues, Training, and Lessons Learned}

\author{
Chair: Lisa Drozdick
}

10:00-11:30 a.m.

\section{DROZDICK, A. NAKONECHNY, S. KEMP \& D. SHAFER. Development and Adaptation of Assessment Instruments: Best Practices, Legal Issues, Training, and Lessons Learned.}

Summary: Historically, psychological assessments have been developed locally for use with individuals from the same country or geographic region. Practitioners utilizing an instrument in a different region than the one where the normative data was collected had to use clinical judgment to estimate the influence of a client's background, language differences, and other variables on test results and provide subjective interpretations of the results. Over time, greater sophistication and knowledge of the cultural influences on test performance has led to more extensive consideration of culture during the development of instruments. These efforts have led to greater portability of assessments across multiple regions; however, standard approaches have not been developed or implemented. One major change in the approach to instrument development has been the movement from pure translations of instruments to adaptation of instruments across languages and cultures. This symposium will focus on the changes in test development over time to adjust to uses across cultures and languages, with special attention to the translation and adaptation process for assessments. Dr. Lisa Whipple Drozdick will introduce the process of test development in the context of global use and provide case examples of cultural and language adaptation. Ms. Anna Nakonechny will describe the best practices for translating instruments, including recent work on the CNS trials which included translation work on manuals, videos, and other documentation. Dr. Sally Kemp will present her experiences in developing and implementing a neuropsychology training program in Columbia. The use of both English and Spanish instruments and the unique aspects of training practitioners in this emerging field in Columbia will be included. Finally, Mr. David Shafer will provide information on the legal quagmires and solutions around adapting copyrighted instruments.

Correspondence: Lisa Drozdick, Pearson, 19500 Bulverde Road, Suite 201, San Antonio, TX 78259, United States.E-mail: lisa.drozdick@ pearson.com

\section{DROZDICK. Developing Tests for Global Use.}

Psychological measurements assess specific constructs within the context of a test instrument. Item content, test construction, and decisions around how to measure the construct can be influenced by cultural experiences. While linguistic differences are often readily apparent, more subtle cultural differences can influence the utility of a measure. For example, the prototypical image of a tree differs across geographic regions and the selection of the type of tree could influence performance. The development of assessments has addressed regional differences in three main ways: developing a single global instrument with minimal cultural references or linguistic requirements, translating a single instrument into multiple languages but utilizing the same content otherwise, and adapting an instrument into multiple regional editions that modify items to fit to the varying regional needs. Each of these approaches addresses different needs and requires different development approaches. In addition, the utility of the assessment may vary depending upon the approach utilized. This presentation will describe the process for developing an assessment within the context of global use and regional variations. Case examples from the adaptation of the Differential Ability Scales-Second Edition, Early Years Spanish Edition and the translations of the Repeatable Battery for the Assessment of Neuropsychological Status will be presented.
Correspondence: Lisa Drozdick, Pearson, 19500 Bulverde Road, Suite 201, San Antonio, TX 78259, United States. E-mail: lisa.drozdick@ pearson.com

\section{A. NAKONECHNY. Translation of Assessments: Best Practices and Lessons Learned.}

Modifying an instrument for use across regions with different dialects or languages may involve modifying the language of administration. The decision to perform a translation versus a linguistic validation may greatly influence the utility and validity of the final product. Translation work typically involves the translation of an instrument form the original language to the second language and a back translation from the translated work back to the original language. Discrepancies are addressed before the final translation. Linguistic validation involves an additional step of validating the underlying meaning across languages to ensure the translation is conceptually equivalent to the original work. Several organizations have endorsed these steps in their standards and certification processes for translation work. Translations of cognitive and language assessments can be particularly difficult due to localization of language concepts and idioms. This presentation will focus on the translation process, including international guidelines and certifications, and present lessons learned from translating psychological assessments across languages. A case study with global CNS trials using videos, manuals, and other documentation will be presented.

Correspondence: Anna Nakonechny. E-mail: anakonechny@ transperfect.com

\section{S. KEMP. Developing Neuropsychology Training Programs in Colombia.}

The qualifications for becoming a neuropsychologist differ across countries. Some countries require a degree in clinical psychology while others require additional courses in neuropsychology beyond a four year psychology program, and others require a professional undergraduate degree. In many developing countries, the requirements are lower. Countries need to balance the need to provide services and the availability of trained practitioners to fill that need. Colombia was the first country in South America to establish an undergraduate training program for professional psychologists. An undergraduate degree in psychology in Colombia is a professional degree, comparable to an MS degree in the United States. Very few psychologists obtain a master's degree or a $\mathrm{PhD}$ as it is not required for practice. This talk describes the development of master's degree program in neuropsychology in Barranquilla. Colombia. It involved training master's level students in psychology on instruments developed for both English and Spanish speakers. Guidelines for selecting instruments and applying results appropriately in interpretation were key aspects of the training.

Correspondence: SallyKemp, MO, United States.E-mail:docsallykemp@ gmal.com

\section{SHAFER. Legal Requirements and Recommendations Around} Test Adaptation and Translation.

With the growing demand for test adaptations and translations, it is important to know the legal issues around the appropriate use of intellectual property. Legal definitions and rights vary across geographies and what is appropriate in the United States may not be appropriate in Germany. Understanding the legal ramifications of cross-country adaptations and translations is key to producing valid and ethical assessments. Identifying what is a public domain instrument versus protected intellectual property is the first step in the process as the legal issues vary around this definition. It is important to note that publication of an instrument in a journal does not mean it is a public domain instrument. Most major publication companies and many individual instrument authors maintain ownership of their products and provide legal agreements around use of those instruments. This presentation will provide an overview of legal definitions of public domain, differences in 
legal rights around intellectual property, and legal agreements around licensing or distribution of assessments.

Correspondence: David Shafer.E-mail: david.shafer@pearson.com

\section{Symposium 12. Neonatal Hypoxic-ischemic Encephalopathy in the Post-therapeutic Hypothermia Era: How does a Multi-disciplinary Approach from Bench to Bedside Help Us Understand a Shift in Brain Injury Patterns and Neurobehavioral Outcomes?}

\section{Chair: Gwendolyn J. Gerner}

\section{Discussant: Martha Denckla}

$$
\text { 10:00-11:30 a.m. }
$$

G.J. GERner, F. NORThington, E. GRAHAM, A. PORETTI, J. BURTON \& C. LIMPEROPOULOS. Neonatal Hypoxic-ischemic Encephalopathy in the Post-therapeutic Hypothermia Era: How does a Multi-disciplinary Approach from Bench to Bedside Help Us Understand a Shift in Brain Injury Patterns and Neurobehavioral Outcomes?

Symposium Summary: Asphyxia in the perinatal and neonatal periods resulting in hypoxic-ischemic encephalopathy $(\mathrm{HIE})$ was a leading cause of death and severe disability among children born full-term. The classic HIE brain injury pattern includes involvement of deep gray matter nuclei and peri-rolandic cortex, with brainstem tegmentum involvement in the most severe cases. Therapeutic hypothermia became a standard of clinical care in 2010 for perinatal HIE within six hours of birth, slowing the metabolic cascade and mitigating secondary brain injury. The benefits of therapeutic hypothermia are well documented from bench to bedside; however, animal and human neuroradiological studies in the post-therapeutic hypothermia era have elucidated patterns of white matter (WM) abnormalities and neurobehavioral correlates. Elevations in specific neonatal serum biomarkers of WM injury have also been observed. In this symposium Dr. Northington will present the basic science of spatiotemporal patterns of WM injury in HIE following therapeutic hypothermia. Dr. Graham will discuss the use of perinatal blood biomarkers to detect specific patterns of brain injury in this population. Dr. Poretti will discuss neuroradiological findings in the pre- and post-therapeutic hypothermia eras, contrasting quantitative versus qualitative methods of neuroradiological evaluation. Dr. Gerner will present on the relationship between patterns of neonatal WM abnormalities and specific neurobehavioral deficits in early childhood, while Dr. Burton will present how language development progresses following HIE and treatment with therapeutic hypothermia. Dr. Limperopoulos will conclude and discuss the historical context of these findings. Correspondence: GwendolynJ. Gerner, PsyD, Neurology \& Developmental Medicine, Kennedy Krieger Institute, 716 N. Broadway, Baltimore, MD 21205, United States. E-mail: gernerg@kennedykrieger.org

M. HOSSAIN, J. BURNSED, R. CHAVEZ-VALDEZ, J. ZHANG \& F. NORTHINGTON. Imaging the Spatiotemporal Progression of White Matter Injury after Neonatal Hypoxia Ischemia.

Background: Hypoxic-ischemic encephalopathy (HIE) is a leading cause of death in children under age 5. Hypothermia therapy (HT) provides incomplete neuroprotection and this protection may vary by brain region. Using a newly established model of HT in the neonatal mouse model of HIE, the extent of WM injury following HI and HT was determined with MRI and diffusion tensor imaging (DTI).

Methods: HI was induced in postnatal day10 mice using the Vannucci Model (unilateral carotid ligation \& 45 minutes of hypoxia $\left(\mathrm{FiO}_{2}=\right.$ $0.08)$ ). Following HI, mice recovered at $31^{\circ} \mathrm{C}(\mathrm{HT})$ or $36^{\circ} \mathrm{C}$ (Normothermia, NT) for 4 hours. T2-weighted and DTI MRIs were obtained from injured and control mice at P30. Volumes of major gray matter structures and volumes and mean fractional anisotropy (FA) values of related white matter tracts were obtained via manual delineation. Results: NT and HT mice had reduced ipsliateral hippocampal volumes $\left(5.85 \mathrm{~mm}^{3}\right.$ and $7.13 \mathrm{~mm}^{3}$ vs. $\left.12.07 \mathrm{~mm}^{3}, \mathrm{p}<0.001\right)$ and lower ipsliateral fimbria FA values $(0.44$ and 0.47 vs. $0.59, p<0.001)$. NT and HT mice had smaller ipsliateral cerebral cortex $\left(52.09 \mathrm{~mm}^{3}\right.$ and 54.63 $\mathrm{mm}^{3}$ vs. $\left.64.38 \mathrm{~mm}^{3}, \mathrm{p}<0.01\right)$ and lower corpus callosum $\mathrm{FA}$ values $(0.53$ and 0.55 vs. $0.59, \mathrm{p}<0.01)$. The fimbria volume/FA values and the hippocampal volumes were significantly correlated in the NT $\left(\mathbf{R}^{2}=0.54\right.$ and $0.74 ; \mathrm{p}<0.001$ for both $)$ and HT $\left(\mathbf{R}^{2}=0.45\right.$ and 0.78 ; $\mathrm{p}<0.001$ for both) mice, however, no correlation was found between the corpus callosum volume/FA values and cortical volumes. Stratifying for gender revealed that significance in hippocampal volumes and fimbria FA values are attributable to the male mice with no differences in female mice treated with NT and HT.

Discussion: The findings from this study show that important differences in WM volume and structure are detectable following neonatal HI, especially in male mice. The results from this study can be used to improve the quantitative evaluation of $\mathrm{HI}$ injury and therapeutic efficacy.

Correspondence: Frances Northington. E-mail: frances@jhmi.edu

\section{E. GRAHAM, I. BURD, A. EVERETT \& F. NORTHINGTON. Blood} Biomarkers for Evaluation of Perinatal Encephalopathy.

Recent research in identification of brain injury after trauma shows many possible blood biomarkers that may help identify the fetus and neonate with encephalopathy. Traumatic brain injury shares many common features with perinatal hypoxic-ischemic encephalopathy. Trauma has a hypoxic component, and one of the $1^{\text {st }}$ physiologic consequences of moderate-severe traumatic brain injury is apnea. Trauma and hypoxia-ischemia initiate an excitotoxic cascade and free radical injury followed by the inflammatory cascade, producing injury in neurons, glial cells and white matter. Increased excitatory amino acids, lipid peroxidation products and alteration in microRNAs and inflammatory markers are common to both traumatic brain injury and perinatal encephalopathy. The blood-brain barrier is disrupted in both leading to egress of substances normally only found in the central nervous system. Brain exosomes may represent ideal biomarker containers, as RNA and protein transported within the vesicles are protected from enzymatic degradation. Evaluation of fetal or neonatal brain derived exosomes that cross the blood-brain barrier and circulate peripherally has been referred to as the "liquid brain biopsy". A multiplex of serum biomarkers could improve upon the current imprecise methods of identifying fetal and neonatal brain injury (e.g., hypoxic-ischemic encephalopathy) within the context of events and/or markers associated with asphyxia, such as fetal heart rate abnormalities, meconium, cord gases at delivery, and Apgar scores. Quantitative biomarker measurements of perinatal brain injury and recovery could lead to operative delivery only in the presence of significant fetal risk, triage to appropriate therapy after birth and measure the effectiveness of treatment.

Correspondence: Ernest Graham. E-mail: egraham5@jhmie.edu

A. PORETTI. White Matter Injury in Neonatal Hypoxicischemic Injury After Therapeutic Hypothermia: Qualitative and Quantitative Analysis of Conventional and Advanced Neuroimaging Techniques.

Magnetic resonance imaging (MRI) is the neuroimaging technique of choice in the diagnostic work-up of neonatal hypoxic-ischemic injury (HII). In neonatal HII, four patterns of brain injury have been delineated including deep nuclear structures (putamen and thalamus), cerebral cortex, brainstem tegmentum, and cerebral white matter. Correlations have been shown between these imaging patterns and severity, temporal characteristics of the hypoxic-ischemic insult, and neurodevelopmental outcome. These data have been acquired in the pre-hypothermia era. In neonates with HII, therapeutic hypothermia has been shown to decrease 
brain tissue injury with a reduction in lesions of the basal ganglia and thalami. In the post-hypothermia era, T2-hyperintense signal of the periventricular white matter is one the most common neuroimaging findings in neonatal $\mathrm{HII}$ and is seen in up to $67 \%$ of affected neonates. Quantitative evaluation of diffusion tensor imaging (DTI) may detect mild abnormalities that remain undetected by qualitative evaluation alone. Quantitative DTI analysis in 32 neonates with HII and hypothermia therapy from our group revealed significantly lower fractional anisotropy (FA) and higher mean diffusivity (MD) and radial diffusivity (RD) values in the posterior centrum semiovale compared to neonatal controls. Lower apparent diffusion coefficient (ADC) values in the posterior centrum semiovale correlate with blood pressure deviation below the range with optimal autoregulation during hypothermia. In summary, white matter injury seems to be highly prevalent in neonatal HII after hypothermia therapy. Quantitative analysis of the white matter microstructure using DTI data may shed light onto the pathomechanism of white matter injury in neonatal HII after hypothermia and quantitative DTI scalars may be used as predictors of motor, cognitive, and behavioral outcome.

Correspondence: Andrea Poretti. E-mail: aporett1@jhmi.edu

G.J. GERNER, E. NEWMAN, J. BURTON, B. ROMAN, E. CRISTOFALO, M. ALLEN, M. LEPPERT, M. JOHNSTON, F. NORTHINGTON, T. HUISMAN \& A. PORETTI. Patterns of White Matter Injury and Neurobehavioral Outcomes Following Neonatal Asphyxia and Treatment with Therapeutic Hypothermia.

Objective: Perinatal hypoxic-ischemic encephalopathy (HIE) was historically associated with damage to deep gray matter (GM) nuclei, with relative sparing of central GM and cortical white matter (WM). Deep GM injury has diminished since inception of therapeutic hypothermia; however, WM involvement has become more recognized. The goal of this study was to explore patterns of WM injury and later neurobehavioral outcomes following neonatal HIE and therapeutic hypothermia.

Participants and Methods: DTI was obtained on 32 neonates with HIE after therapeutic hypothermia and 10 control patients. The Mullen Scales of Early Learning was administered to a subgroup of the HIE birth cohort ( $\mathrm{N}=14)$ between ages 20 and 32 months. Group differences in fractional anisotropy (FA), mean diffusivity (MD), axial diffusivity (AD), and radial diffusivity (RD) across multiple regions of interest (ROIs) were examined via t-tests. The relationship between DTI scalars in specific ROIs were correlated with Mullen scores.

Results: Neonates with HIE had significantly lower FA $(\mathrm{t}=-2.53$, $p=.016)$ and higher RD

$(\mathrm{t}=3.16, p=.003)$ in the splenium of the corpus callosum $(\mathrm{CC})$; and significantly lower FA

$(\mathrm{t}=-3.01, p=.012)$, higher MD $(\mathrm{t}=2.54, p=.015)$, and higher RD $(\mathrm{t}=2.92, p=.006)$ in the posterior centrum semiovale (PCS) compared to controls. Significant correlations were observed between lower FA (Spearman rho=.620, $\mathrm{p}=.018$ ), higher MD (Spearman rho=-.617, $\mathrm{p}=.019)$, and higher RD (Spearman rho= -.626, $\mathrm{p}=.017$ ) in the PCS and this correlated with worse performance on the Mullen Visual Reception subscale. Significant correlations with fine motor skills were also observed.

Conclusion: Therapeutic hypothermia may mitigate GM injury in HIE, but patterns of WM injury in posterior cortical regions with neurobehavioral correlates are present. Further investigation of WM injury and associated patterns of long-term neurobehavioral outcomes following perinatal HIE and therapeutic hypothermia treatment is warranted. Correspondence: GwendolynJ. Gerner, PsyD, Neurology \& Developmental Medicine, Kennedy Krieger Institute, 716 N. Broadway, Baltimore, MD 21205, United States. E-mail: gernerg@kennedykrieger.org.
J. BURTON, G.J. GERNER, N. REEDY, A. PORETTI, M. LEPPERT, M. ALLEN, C. PARKINSON, F. NORTHINGTON \& E. CRISTOFALO. Language Development Following Neonatal Hypoxic-Ischemic Encephalopathy Treated with Therapeutic Hypothermia.

Objective: Moderate to severe hypoxic-ischemic encephalopathy in the term infant prior to the advent of therapeutic hypothermia resulted in life-long disability. A number of studies have demonstrated a reduction in cerebral palsy and intellectual disability following treatment with therapeutic hypothermia; however less is known about changes in less severe outcomes such as language and learning disabilities.

Participants and Methods: A prospective convenience sample of language performance of 24 two year old children born at term with neonatal hypoxic-ischemic encephalopathy who were treated with therapeutic hypothermia was analyzed. Language performance on the Mullen and the MacArthur Bates CDI were examined. Correlations between $\mathrm{pH}$ and language outcomes were investigated. Preliminary correlations of FA and MD for white matter tracts implicated in language function with performance on the language measures were also performed for a subset of 10 children.

Results: Prevalence of language disability in the general population ranges between $2.63-8.04 \%$. In this study, $29 \%$ (9/24) of the participants had an expressive language T-score below 40 on the Mullen. $19 \%(4 / 21)$ had less than 50 words on the MacArthur Bates. When controlling for SES $(p=.014)$ and gender $(p=.001)$, there were no group differences based on severity of encephalopathy at birth on the expressive $(p=.868)$ or receptive language portions $(p=465)$ of the Mullen. Similarly, initial cord $\mathrm{pH}$ did not correlate significantly with the language measures ( $r=-0.282$ for Mullen expressive, $r=-0.148$ for receptive Mullen).

Conclusion: Therapeutic hypothermia has become standard of care for neonatal HIE in term infants with numerous studies demonstrating a reduction in severe neurodevelopmental outcomes at 18-24 months. In this study, we demonstrated increased risk for language impairment at age two that could not be predicted by severity of encephalopathy as a neonate.

Correspondence: Joanna Burton. E-mail: BurtonJ@kennedykrieger.org

\section{Paper Session 14. Cognitively Based Interventions in Aging}

\author{
Moderator: Sylvie Belleville \\ 10:00-11:30 a.m.
}

C. MEWBORN, C. LINDBERGH \& L. MILLER. Cognitive interventions for older adults: A systematic review and metaanalysis of randomized controlled trials.

Objective: Older adults experience age-related declines in cognition that are associated with decreased functional independence and psychological distress. Cognitive interventions have the potential to improve cognition, delay age-related cognitive declines, and improve quality of life for older adults. The current meta-analysis was conducted to update and expand previous work on the efficacy of cognitive interventions and to examine the impact of demographic and methodological variables on the efficacy of cognitive interventions to improve cognition in older adults. Participants and Methods: EBSCOhost, Embase online databases, and reference lists were searched to identify relevant randomizedcontrolled trials of cognitive interventions for older adults (60+ years). Participants were healthy or mildly cognitively impaired (MCI). Eligible interventions trained a single cognitive domain (e.g., memory) or were multi-domain training. Outcomes were assessed post-intervention using standard neuropsychological tests. Pre-specified data points were extracted, including study quality indicators.

Results: 279 effects from 97 studies were pooled based on a randomeffects model and expressed as Hedges' $g$. Results indicated that cognitive interventions improved cognition relative to active and passive 
control groups $(g=0.298, p<.001,95 \%$ CI $=0.248-0.347)$. Age, education, and cognitive status (healthy vs. MCI) were not significant moderators. Working memory interventions proved most effective $(g=0.479)$. Memory, processing speed, and multi-domain interventions also significantly improved cognition. Effects were larger for directly trained outcomes, but were also significant for non-trained outcomes (i.e., transfer effects). Following moderator analyses, small, but significant heterogeneity in observed effects remained.

Conclusions: Overall, cognitive interventions produce a small, but significant improvement in older adults' cognition which transfers to non-trained tasks. The project was pre-registered with PROSPERO (\#42016038386).

Correspondence: Catherine Mewborn, University of Georgia, 660 Barnett Shoals Road, Unit 421, Athens, GA 30605, United States. E-mail: cmewborn@uga.edu

S.S. SIMON, B.M. HAMPSTEAD, M.P. DA SILVA, L.F. mascarenhas, R. Ávila, F.H. PORTO, S. BRUCKI, C.B. MARTINS, F.L. DURAN, L.D. TASCONE, M.M. MARTIN, E. AMARO JUNIOR, G. BUSATTO FILHO \& C.M. BOTTINO. Cognitive and Neuroimaging Changes After Mnemonic Strategy Training in Amnestic Mild Cognitive Impairment: a Randomized, Single-Blind Study.

Objective: To evaluate the efficacy of mnemonic strategy training (MST) versus an active education control program (EP) in patients with amnestic mild cognitive impairment (aMCI).

Participants and Methods: Thirty participants with aMCI were randomized to MST for face-name associations or to EP. All patients completed 4 individual 1 -hour sessions, twice a week, over a 2 -week period. At baseline, participants completed clinical and neuropsychological assessments as well as CSF and MRI exams. During fMRI, the subjects underwent a face-name encoding task and then completed the Face-Name Recognition Task (FNRT) outside the scan following a 30-minute delay. One-week after the programs, fMRI and the FNRT were repeated. The FNRT was also repeated at 1 and 3 -month follow-ups.

Results: Groups were comparable at baseline. MST significantly improved memory for the trained face-name associations relative to $\mathrm{EP}(p<0.001, d>0.8)$, which remained significant after 1 and 3 -months $(p<0.001, d>0.8)$. fMRI results in the MST group revealed increased activation in parietal (bilateral precuneus and angular gyrus) and frontal regions (portion of right precentral gyrus and left middle frontal gyrus), compared to EP. Regarding transfer effects, the MST group showed a trend toward better performance on novel stimuli $(p=0.07$; $d=0.35$ after 1 -week; $d=0.62$ after 1 -month, $d=0.75$ after 3 -months), and were slower to recall names relative to EP after 1 -week $(p=0.02$, $d=0.79$ ); a finding that suggests the use of MST. These behavioral effects were again accompanied by increased frontal and parietal activation compared to EP.

Conclusions: MST effectively improved memory for specific content in patients with aMCI, with benefits persisting at 3 months. Patients also appeared to generalize training to novel stimuli.

Correspondence: Sharon S. Simon, Phd Candidate, Psychiatry, University of São Paulo, Rua Ovídio Pires de Campos, 785, São Paulo 05403010,Brazil.E-mail: sharon.sanzsimon@gmail.com

E. BRENNER, F.G. HILLARY, A. ROY, R.A. BERNIER, E.C. GROSSNER, K. SATHIAN \& B.M. HAMPSTEAD. Changes in Resting-State Neural Networks After Memory Training in Amnestic Mild Cognitive Impairment.

Objective: We used graph theory and functional MRI to examine local and global changes in resting state functional connectivity following two memory-training interventions in patients with amnestic mild cognitive impairment (aMCI).

Participants and Methods: A total of 58 aMCI patients were enrolled in a randomized controlled trial comparing mnemonic strategy training
(MST) and spaced-retrieval training (SRT) for object-location associations. Here, we present resting-state fMRI data from 37 participants $(\mathrm{MST}=19)$ that were collected before and after 3 training sessions. Regions of interest were examined using Power's 264 functionally defined regions (Power et al., 2011). Graph theory was used to examine specific connectivity profiles with a focus on communication between large-scale networks associated with dysfunction in aMCI, including the default mode network (DMN) and salience network (SN).

Results: MST resulted in greater behavioral improvement than SRT. There were no effects on measures of overall connectivity including average path length, local average transitivity, global transitivity, and weighted transitivity for either intervention. However, significantly decreased connectivity was observed in the right fronto-parietal SN of the SRT group $(d=0.93)$. In contrast, MST increased connectivity in the left temporal DMN $(d=0.51)$ and decreased connectivity in the left ventral attention network $(d=0.79)$. Directly comparing the groups post intervention, the MST group exhibited significantly more connectivity in the right frontal DMN $(d=0.77)$ and the right fronto-parietal SN $(d=0.93)$.

Conclusions: Findings suggest treatment specific changes in resting-state functional connectivity where MST resulted in significantly more connectivity in the DMN and fronto-parietal SN than SRT. Results illustrate the potential that cognitive interventions can alter connectivity within critical network hubs known to be dysfunctional in aMCI.

Correspondence: Einat Brenner, Psychology, Pennsylvania State University, 419 Bruce V. Moore Building, University Park, PA 16802, United States.E-mail: ebb5161@psu.edu

S. BELLEVILLE, C. HUDON, N. BIER, C. BRODEUR, S. GAUTHIER, B. GILBERT, S. GRENIER, M. OUELLET \& C. VISCOGLIOSI. Cognitive training in persons with MCI has durable effect on memory and generalizes to daily life: Results from the MEMO+ randomized controlled trial.

Objective: Cognitive training was found beneficial for healthy older adults. However, there is no consensus regarding its effect in persons with mild cognitive impairment (MCI) due to the paucity of well designed randomized controlled trials. The goal of this study was to assess the effect and durability of memory training on the cognition of persons with MCI, assess whether the effect generalizes to daily life and compare its effect with that provided by a psychosocial intervention.

Participants and Methods: One hundred and forty five older adults meeting criteria for MCI were randomized to memory training, psychosocial intervention or to a no contact control condition. Interventions were provided in small groups over eight $2-\mathrm{hr}$ sessions. Outcome measures included immediate and delayed composite memory scores, measures of psychological health (depression, anxiety and well-being) and generalization questionaires (self-reported measures of complex activities of daily living, memory complaint and strategy use in everyday life). Maintenance was assessed by repeating measurements 3 months and 6 months following training.

Results: Participants randomized to cognitive training showed improvement on composite memory scores and on self-reported measures of complex activities of daily living and strategy use in everyday life. Improvement was maintained 3 months and 6 months post-training for composite memory scores and strategy use in everyday life. Participants in the psychosocial and no contact conditions showed no improvement. None of the intervention had a significant effect on measures of psychological health.

Conclusions: This randomized controlled trial confirms that cognitive training improves memory in persons with MCI, that the effect is durable and that it can generalize to daily life. Psychosocial interventions showed no effect on any measures. This suggests that the effect of cognitive training cannot be explained by a non-specific social contact or mood effect. 
Correspondence: Sylvie Belleville, Ph.D., CRIUGM, 4565 Queen Mary, Montreal, QCH3W-1W5, Canada.E-mail: sylvie.belleville@umontreal. ca

R.P.C. KESSELS, S. VOIGT-RADLOFF, M.M.E. DE WERD, R. LEONHART, D. BOELEN, M.G.M. OLDE RIKKERT \& M. HÜLL. Structured Relearning of Everyday Tasks in Dementia: The Randomized Controlled REDALI-DEM Trial on Errorless Learning. Objective: Errorless learning (EL) is a structured learning method that has been used to teach patients with cognitive deficits new information or skills. Several studies have applied EL in dementia patients, showing promising results. However, studies so far suffer from the lack of control groups, did not train relevant tasks or used only small samples. This is the first multicenter large-scale RCT in dementia comparing EL to an errorful structured learning control group (trial and error learning; TEL).

Participants and Methods: 161 patients with Alzheimer's or mixedtype dementia (living at home, MMSE $\mathrm{M}=19.8, \mathrm{SD}=3.3$; age $\mathrm{M}=76.6$; $\mathrm{SD}=6.7$ ) were randomized into two groups (81 EL, $80 \mathrm{TEL}$ ). The intervention consisted of 13 one-hour task training sessions over 10 weeks using EL or TEL in the patient's own home, with trained therapists teaching patients 2 everyday tasks. As primary outcome task performance at week 16 was videotaped and rated by masked assessors. Task performance at week 26 was the secondary outcome, as well as satisfaction with treatment, need for assistance, challenging behavior, adverse events, resource utilization and treatment costs.

Results: Intention-to-treat analysis showed significant improved task performance at week 16 in both arms (effect size [95\%CI]: 0.61 [0.37$0.85]$ task A; 0.47 [0.23-0.71] task B), which was maintained at week 26. No significant interaction with treatment group was found on the primary or secondary outcome measures.

Conclusions: The first sufficiently powered, individually tailored RCT on the effectiveness of EL shows that structured relearning improved the performance of daily living tasks in dementia patients, an improvement that was maintained for 6 months. However, reduction of errors (EL) had no additional effect over traditional structured learning using TEL. Future research should examine if the severity of dementia and the selected everyday life task is of any effect on EL.

Correspondence: Roy P.C. Kessels, PhD, Radboud University Medical Center, Medical Psychology (Geriatrics 925), PO Box 9101, Nijmegen 6500 HB, Netherlands. E-mail: roy.kessels@radboudumc.nl

A. POLSINELLI, H. RITCHIE, S.A. MOSELEY, E.L. GLISKY \& S. FELD. Mindfulness Training For Improving Cognitive And Emotional Functioning In Healthy, Non-Meditating Older Adults. Objective: Mindfulness in young and middle-aged adults is associated with cognitive and emotional benefits, but is less commonly studied in older adults, despite the overlap between these benefits and domains of age-related changes. The aim of this study was to investigate the cognitive and emotional benefits of a brief online mindfulness training for older adults. A concurrent aim of the study was adherence to high methodological rigor, which is reflected in the use of objective measures of cognitive and emotional functioning, and a well-matched, active control group.

Participants and Methods: Forty-seven healthy, non-meditating older adults $\left(\mathrm{M}_{\text {age }}=75.8, \mathrm{SD}_{\text {age }}=5.6\right)$ were randomized to receive either 6-weeks of mindfulness (MT) or mind-wandering training (MWT). In a within subjects design, participants completed the same battery of tests prior to and after training, which included assessments of mindfulness (Five Facet Mindfulness Questionnaire), attention (Attentional Blink), updating (Keep Track), inhibition (Simon), and emotional regulation (Emotional Interference Task).

Results: For both the MT and MWT groups, accuracy on the Attentional Blink task improved $\left(F(1,45)=4.06, p=.05, \mathrm{~h}^{2}=.09 ; F(1,45)=11.46\right.$, $\left.p=.002, \mathrm{~h}^{2}=.21\right)$ and two facets of the FFMQ, Observe $(F(1,45)=6.95$, $\left.p=.01, \mathrm{~h}^{2}=.14\right)$ and Describe $\left(F(1,45)=4.31, p=.04, \mathrm{~h}^{2}=.09\right)$, increased, following training. MT did not improve updating, inhibition, or emotion regulation.

Conclusions: The present study found slight benefits of a brief, online mindfulness training for older adults on facets of self-reported mindfulness and attentional functioning but these findings were not specific to mindfulness training. Future studies will examine the potential impact of this improved attention on daily functioning in older adults. Correspondence: Angelina Polsinelli, MA, Psychology, University of Arizona, 1503 E University Blvd, Psychology, Room 314, Tucson, AZ 85721, United States. E-mail: apolsine@email.arizona.edu

\section{Paper Session 15. TBI Across the Lifespan}

\author{
Moderator: Suzanne Penna
}

10:00-11:30 a.m.

WITHDRAWN: N.P. RYAN, C. CATROPPA, M.H.

BEAUCHAMP, R. BEARE, T. SILK, K.O. YEATES \& V.A. ANDERSON. Uncovering the Neural Correlates of Cognitive, Affective, and Conative Theory of Mind in Paediatric Acquired Brain Disorder: Evidence from Traumatic Brain Injury.

Objective: Deficits in theory of mind ( $\mathrm{ToM})$ are common after neurological insult acquired in the first and second decade of life, however the contribution of large-scale neural networks to ToM deficits in children with brain injury is unclear. This study investigated the sub-acute effect of pediatric TBI on grey matter volume of three large-scale, domain-general brain networks (the Default Mode Network, DMN; the Central Executive Network, CEN; and the Salience Network, SN), as well as two domain-specific neural systems implicated in social-affective processes (the Cerebro-Cerebellar Mentalizing Network, CCMN, and the Mirror Neuron/Empathy Network, MNEN). We also evaluated prospective structure-function relationships between these large-scale neural networks and cognitive, affective and conative ToM.

Participants and Methods: 3D T1 weighted magnetic resonance imaging sequences were acquired sub-acutely in 137 participants (child TBI: $n=103$; typically developing (TD) children: $n=34$ ). Both groups were assessed on measures of Theory of Mind (ToM) at 24-months post-injury.

Results: Participants with severe TBI showed volumetric reductions of the CCMN, SN, MNEN, CEN and DMN, as well as reduced grey matter volume in several hub regions of these networks, including the cerebellum. Poorer cognitive ToM was associated with reduced volume overall CGMN package and several of its hub regions, including the cerebellum. In contrast, affective and conative ToM was associated with volumetric reductions in the SN and MNEN and their hub regions, respectively.

Conclusions: These findings suggest that several large-scale neural networks that may be important for cognitive, affective and conative theory of mind in the developing brain. These findings are discussed in relation to neurocognitive models of ToM. We conclude that detection of sub-acute structural abnormalities in large-scale neural networks and their hub regions may aid in the early identification of children at risk for chronic social impairments.

Correspondence: Nicholas P. Ryan, Child Neuropsychology, Clinical Sciences, Murdoch Childrens Research Institute, 50 Flemington Rd, ParkvilleVIC,VIC3052, Australia.E-mail:nicholas.ryan@mcri.edu.au

D.T. PULSIPHER, E.M. KRAPF, A.N. RABKIN, J.M. KLAVER \& L.D. STANFORD. Postconcussive Symptoms in Children and Adolescents Are as Common in Other Neurologic/ Neurodevelopmental Disorders as They Are in Concussion.

Objective: Acute and chronic symptoms typically seen following concussion (i.e., postconcussive symptoms [PCS]) occur frequently in individuals who have not experienced concussion including healthy samples 
and individuals with psychiatric diagnoses. Based on these findings, we hypothesized that PCS would occur frequently in non-concussed neurologic populations. The purpose of this study was to compare total number of PCS and PCS severity (i.e., frequency, intensity, and duration) between children with concussion and non-concussed children with another neurologic/neurodevelopmental disorder.

Participants and Methods: The sample included 420 clinically-referred youth ages $8-18$ vears $(\mathrm{M}=14.15$ years, $\mathrm{SD}=2.85$ years $)$ who underwent a neuropsychological evaluation in a medical setting. Included in the study were 272 children with concussion and 148 non-concussed children with another neurologic/neurodevelopmental disorder (e.g., epilepsy, prematurity). All participants completed the Postconcussion Checklist, which assesses total number of PCS, and the Postconcussion Syndrome Checklist, which assesses severity markers. Results: The concussion group was significantly older than the neurologic/neurodevelopmental group $(d=0.35)$. After controlling for age, the two groups did not differ on total number of PCS $(d=0.05)$, PCS frequency $(d=0.34)$, or PCS intensity $(d=0.45)$. The two groups significantly differed in terms of PCS duration $(d=0.62)$, with the concussion group reporting longer duration of symptoms.

Conclusions: PCS are common in non-concussed youth with another neurologic/neurodevelopmental disorder. This information, in combination with previous research, should continue to raise questions as to the diagnostic validity of PCS. Practitioners should be cautious in their interpretation of PCS in concussed individuals with the understanding that such symptoms are non-specific and occur frequently in healthy, psychiatric, and neurologic/neurodevelopmental samples.

Correspondence: Dalin T. Pulsipher, Ph.D., NeuroDevelopmental Science Center, Akron Children's Hospital, 215 W. Bowery St., Suite 4500, Akron,OH 44308, United States.E-mail:dpulsipher@chmca.org

\section{J. BELLEROSE, A. BERNIER, C. BEAUDOIN, J. GRAVEL \& M.H. BEAUCHAMP. Persistent socio-cognitive clouding following preschool mild TBI.}

Objective: A peak in the incidence of traumatic brain injury (TBI) occurs during the preschool period ( $0-5$ years). These early years also constitute a crucial period of social development, thus it is possible that TBI may affect social skills. Deficits in social cognition, namely theory of mind (ToM), have been reported after severe preschool TBI (Walz et al., 2009). It is not known whether preschool mild TBI (mTBI) disrupts ToM. The goal of the current study was to investigate the impact of preschool mTBI on ToM and examine associations with social functioning.

Participants and Methods: 72 children with accidental mTBI, 58 children with an orthopaedic injury (OI), and 83 typically developing children (TDC) aged between 2 and 5 years completed two ToM tasks (Desires Reasoning and False Belief Understanding. FBU) at 6 (T1) and 18 (T2) months post-injury. Parents completed the Adaptive Behaviour Assessment System (ABAS; Global and Social composites).

Results: The mTBI group performed significantly worse on desires reasoning compared to both comparison groups at T1 and T2 $(F(2,191)=4.93, p=.01$, post hoc: mTBI vs. OI, $p=.03 ; \mathrm{mTBI}$ vs. TDC, $p=.02)$. The Group effect for FBU approached significance $(F(2,188)=2.76, p=.066)$. Significant positive correlations were found in the mTBI group between ToM and social functioning (FBU and ABAS Social at T1, $p=.02$; Desires and ABAS Social at T1, $p=.04 ; \mathrm{FBU}$ T1 and ABAS Global T2, $p=.004 ; \mathrm{FBU}$ T1 and ABAS Social T2, $p=.01$ ). Conclusions: Preschool children who sustain mTBI display ToM difficulties, which are associated with less optimal social functioning. The results suggest a long-term, brain-injury-specific socio-cognitive effect of preschool mTBI.

Correspondence: Jenny Bellerose, BA, Psychology, University of Montreal, C.P. 6128, succursale Centre-ville, Montreal, QC H3C 3J7, Canada.E-mail: jenny.bellerose@umontreal.ca
S. MCDONALD, J. RUSHBY, K. DALTON, R. LANDIN-ROMERO, N. PARKS \& S. ALLEN. Social Cognition After TBI: Its All About Connections.

Objective: Severe traumatic brain injury (TBI), resulting in multifocal and diffuse injury often leads to deficits in social cognition, i.e. reading and interpreting social cues. Focal lesion research implicates frontal and temporal structures as critical to social cognition. However, white matter integrity is also likely to be necessary as social cognition typically requires processing dynamic visual, auditory and semantic information across the brain. In this study, we examined what white matter tracts are associated with poor social cognition in severe TBI, using track-based spatial statistics.

Participants and Methods: 17 participants (14 male: mean age 46 yrs, SD: 13) with mod-severe TBI (PTA: 50 days, SD: 35) and 17 age and gender matched controls underwent MRI scans using a 3T Tx scanner. Functional anisotropy (indexing white matter integrity) was registered to a common skeleton. Voxel-wise statistics $(p<0.025$ corrected for multiple comparisons) were then conducted, aligned across participants and covarying for cognitive function. Participants were also assessed on The Awareness of Social Inference Test: part 1 (emotion recognition) and part 2 (understanding social inference).

Results: Participants were impaired on both parts of TASIT relative to controls and had extensive white matter pathology. Emotion recognition was correlated with loss of white matter integrity in bilateral thalami, the external capsule and planum temporale on the right and the corpus callosum and fornix. Understanding social inference additionally involved bilateral fronto-occipital tracts, intraparietal sulci, uncinate fasiculi, corticospinal tracts as well as the superior longitudinal tract, putamen and cingulum on the left.

Conclusions: White matter integrity is likely to underpin complex social processing. This study highlights the potentially complex contribution played by white matter damage following TBI to everyday social judgements.

Correspondence: Skye McDonald, PhD, Psychology, University of NSW, School of Psychology, Unversity of NSW, Sydney, NSW 2052, Australia. E-mail:s.mcdonald@unsw.edu.au

N. PRESSON, S.R. BEERS, G. VAN EMAN, W. BIRD, K. EDELMAN, W. SCHNEIDER \& D.O. OKONKWO. Quantitative High Definition Fiber Tracking Metrics Differentiate Healthy Control and Chronic TBI Groups.

Objective: The current study used High Definition Fiber Tracking (HDFT), an advanced white matter tractography pipeline, to test two hypotheses: 1) quantitative HDFT metrics will differentiate chronic traumatic brain injury (TBI) and control groups in 13 brain white matter pathways; 2) within the chronic TBI sample, HDFT metrics will predict objective neuropsychological test performance.

Participants and Methods: 79 healthy controls and 61 chronic TBI subjects completed a high-b-value multishell diffusion MR scan and neuropsychological test battery. HDFT was used to generate tractography for 13 major white matter tracts and to quantify spread, symmetry, and anisotropy (GFA) of each tract.

Results: After controlling for age, hierarchical regression with random intercept for tract showed lower homologue correlation, lower tract spread, and lower GFA for chronic TBI relative to controls (all $\mathrm{p}<0.0001$ ); spread and GFA models showed an independent effect of age. Within the chronic TBI sample, replicating previous results from a smaller sample, Trail Making Part A\&B, WAIS-IV Processing Speed Index, ANAM Simple Reaction Time, and CVLT Short- and Long-Delay Free Recall significantly correlated with geometric HDFT metrics and GFA for multiple tracts; significant correlation coefficients ranged from .22 to .57 .

Conclusions: The current results replicate previous, small-sample results (Presson et al., 2014) showing 1) HDFT metrics of white matter pathway spread, symmetry, and GFA are reduced in the chronic TBI group relative to healthy controls, and 2 ) greater spread, symmetry, and 
GFA in specific tracts is associated with better neuropsychological test performance within the chronic TBI group.

Correspondence: Nora Presson, PhD, Learning Research and Development Center, University of Pittsburgh, 3939 O'Hara Street, University of Pittsburgh, Pittsburgh, PA 15260, United States. E-mail: presson@pitt.edu

A. TREBLE-BARNA, S.L. WADE, L. MARTIN, V. PILIPENKO, K.O. YEATES, H. TAYLOR \& B. KUROWSKI. Influence of Dopamine-Related Genes on Neurobehavioral Recovery following Traumatic Brain Injury During Early Childhood.

Objective: Unexplained heterogeneity in recovery is one of the most significant barriers to clinical management of traumatic brain injury. We examined associations of dopamine genes with short- and long-term neurobehavioral recovery, as well as neurobehavioral recovery trajectories over time, in children who sustained early childhood TBI relative to children who sustained orthopedic injuries (OI).

Participants and Methods: Participants were recruited from a prospective, longitudinal study evaluating outcomes of children who sustained a TBI $(n=68)$ or OI $(n=72)$ between the ages of 3 and 7 years. Participants completed assessments at the immediate post acute period ( 0 to 3 months after injury), six, twelve, and 18 months post injury, and an average of 3.5 and 7 years post injury. Thirty-two single nucleotide polymorphisms (SNPs) in dopamine genes (DRD2, SLC6A3, SLC18A2, COMT, and ANKK1) were examined in association with short- and long-term executive function (Behavior Rating Inventory of Executive Function) and behavioral adjustment (Child Behavior Checklist), as well as their trajectories over time.

Results: After controlling for premorbid child functioning, genetic variation within the ANKK1 (rs1800497 and rs2734849) and SLC6A3 (rs464049, rs460000, and rs1042098) genes was differentially associated with short- and long-term neurobehavioral recovery following TBI relative to OI. In addition, genetic variation within the SLC6A3 (rs464049 and rs460000) gene was differentially associated with neurobehavioral recovery trajectories over time following TBI.

Conclusions: The findings provide preliminary evidence that genetic variation in genes involved in dopamine receptor D2 expression and density (ANKK1) and dopamine transport (SLC6A3) plays a role in neurobehavioral recovery following pediatric TBI. Elucidation of biologic processes underlying neurobehavioral recovery has the potential to advance the field by facilitating individualized prognosis, development of novel targets for intervention, and reduced public health burden.

Correspondence: Amery Treble-Barna, PhD, Physical Medicine and Rehabilitation, University of Pittsburgh School of Medicine, 6816 Meade St \#2, Pittsburgh, PA 15208, United States.E-mail: amerytreblebarna@ gmail.com

\section{Paper Session 16. Subjective Cognitive Complaints Moderator: Sietske Sikkes \\ 10:00-11:30 a.m.}

\footnotetext{
S. SIKKES, P. CRANE, R. JONES \& L. RABIN. Subjective Cognitive Decline and Preclinical Alzheimer's Disease: Harmonization of Measurement Instruments.

Objective: Subjective Cognitive Decline (SCD) in the absence of objective cognitive deficits is a potential harbinger of cognitive decline and progression to Alzheimer's disease (AD). The key issue in the study of SCD is the vast diversity of its measurement instruments, which hampers comparising findings. Using advanced psychometric procedures we pooled international data from the SCD Initiative Working Group to identify the relationship of SCD to amyloid deposition and clinical progression.
}

Participants and Methods: A total of 11 international studies were included, representing 6 countries, 5 languages and a total sample size of over 4000 cognitively intact individuals. Amyloid deposition was measured using either PET scans (Florbetapir or Pittsburgh Compound B) or A $\beta 1-42$ in the cerebrospinal fluid, and clinical progression as a conversion to mild cognitive impairment (MCI) at follow-up. We used item response theory (IRT) to harmonize assessments across study, facilitating the direct comparison of participant data across studies.

Results: Clinical follow-up was available for 4185 subjects, of which $903(21.6 \%)$ converted to MCI over a median follow-up duration of 4 years. A total of $185(/ 701,26 \%)$ participants were amyloid positive. The harmonization procedure involved conceptual and statistical matching of items across studies. Multidimensional (bifactor) measurement models were estimated within each study and consistency with a common SCD trait is accomplished with equality constraints. Given a fitted measurement model individual SCD scores can be estimated and used to rank individuals on a theoretically relevant metric. The validity of the derived measures are established with consistency with the across and within association of SCD and evidence of clinical progression.

Conclusions: We demonstrated that SCD items meet assumptions for harmonization. Going forward, this common metric will allow investigating the relationship between $\mathrm{SCD}$ and preclinical $\mathrm{AD}$, and linking international studies that enroll SCD participants.

Acknowledgement: This abstract was submitted on behalf of the Subjective Cognitive Decline Initiative (SCD-I) Working Group.

Correspondence: Sietske Sikkes, PhD, Neurology, Alzheimer Center, IU University Medical Center, PO Box 7057, Amsterdam 1007 MB, Netherlands.E-mail:s.sikkes@vumc.nl

E. HESSEN, M. ECKERSTRØM, A. NORDLUND, I. ALMDAHL, M. GÖTHLIN, J.D. STÅLHAMMAR, C. ECKERSTRØM, M. BJERKE, I. REINVANG, T. FLADBY \& A. WALLIN. Subjective cognitive impairment is a predominantly benign condition in memory clinic patients followed for 6 years. The Gothenburg-Oslo MCI study.

Objective: In the quest for prevention or treatment there is a need to find early markers for pre-clinical dementia. The present study observed memory clinic patients with subjective cognitive impairment (SCI) and normal cognitive function at baseline and followed them for six years. The aim was to address the potential relation between (1) baseline CSF biomarkers and (2) decline in memory performance the first two years of follow-up, with cognitive decline or conversion to dementia after six years.

Participants and Methods: Eighty-one patients (mean age sixty-one years) with SCI were recruited from two university memory clinics and followed for six years.

Results: Eighty-six percent of the cohort remained cognitively stable or improved, nine percent developed MCI and only five percent converted to dementia. Regression analysis revealed that both low levels of A $\beta 42$ at baseline and memory decline during the first two years of follow up were significant and strong predictors of dementia at six-years follow up. When combined these variables were associated with fifty percent risk of conversion to dementia.

Conclusions: While not all the converters received an $\mathrm{AD}$ diagnosis, the findings support the notion that low Aß42 in CSF is a sensitive marker for development of dementia. Furthermore, the findings suggest that the SCI group mainly consisted of cognitively healthy persons. To increase the likelihood of the presence of preclinical dementia and make the concept of SCI more meaningful, future studies of SCI cohorts should include a stringent set of SCI features, as recommended by the Subjective Cognitive Decline Initiative (SCD-I).

Correspondence: Erik Hessen, PhD, University of Oslo, Krags vei 13, Oslo0783, Norway. E-mail: erik.hessen@nevropsykologi.no 
R. BUCKLEY, B. HANSEEUW, P. VANNINI, D.M. RENTZ, R. SPERLING, K. JOHNSON \& R. AMARIGLIO. Region-specific tau and $\beta$-amyloid effects on subjective cognitive concerns in the Harvard Aging Brain Study.

Objective: Subjective cognitive concerns (SCC) are associated with $\beta$-amyloid $(\mathrm{A} \beta)$ in the earliest phases of the Alzheimer's disease (AD) trajectory, but until now, SCC has not been investigated in relation to region-specific tau deposition. We examined relationships between entorhinal cortical (EC) and inferior temporal (IT) tau, neocortical A $\beta$ and SCC in older adults.

Participants and Methods: 126 older participants $(77 \mathrm{yrs}, S D=6.2$. CDR $0=118 /$ CDR $0.5=8$ ) participating in the Harvard Aging Brain Study underwent AV1451-PET and PiB-PET imaging. An SCC composite was created using $\mathrm{z}$-transformed subscales from three SCC measures administered in the Harvard Aging Brain Study (Amariglio et al., 2015); the Memory Functioning Questionnaire (MFQ), the Everday Cognition (ECog) battery and a seven-item concern measure.

Results: Three separate models were run, looking at the effects of EC and IT tau, and $\mathrm{A} \beta$ on SCC, after controlling for age, sex, education, and Geriatric Depression Scale score. Greater EC tau $(\beta=0.33, p<.001)$, IT tau $(\beta=0.18, p=.05)$ and $\mathrm{A} \beta(\beta=0.17, p=.05)$ were associated with greater SCC. In two final models investigating the interactive effects of tau and $\mathrm{A} \beta$ on SCC, an EC tau and $\mathrm{Ab}$ interaction was not significant $(\beta=0.04, p=.93)$, while a trend relationship was found between IT tau and $\mathrm{A} \beta(\beta=0.6, p=.14)$.

Conclusions: This is the first study to demonstrate region-specific patterns of association between tau and SCC. EC and IT tau and A $\beta$ were all singularly associated with greater SCC, with EC tau exhibiting the strongest relationship. When tau and $\mathrm{A} \beta$ were considered together, synergistic relationships were not convincingly apparent. We argue that while SCC is clearly related to neuroimaging markers of tau and A $\beta$, determining the temporal ordering and interactive effects is challenging, and can only be ascertained using longitudinal data.

Correspondence: Rachel Buckley, PhD, Neurology, Harvard Medical School, Athinoula A. Martinos Center for Biomedical Imaging, 13th Street, Building 149, Boston, MA 02129, United States. E-mail: rfbuckley@mgh.harvard.edu

K. GIFFORD, L.R. SAMUELS, D. LIU, T.J. HOHMAN, K.E. OSBORN, F.E. CAMBRONERO, M. BABICZ, J.E. BOGNER, R. MARTINWILLETT, C. SEABOLT, J. THOMPSON, M. THURSBY, L. WALLJASPER, L. ACOSTA, S.P. BELL, H. ZETTERBERG \& A.L. JEFFERSON. Relation of cerebrospinal fluid markers of Alzheimer's disease pathology and subjective cognitive decline; the Vanderbilt Memory and Aging Project.

Objective: Subjective cognitive decline (SCD) is related to early Alzheimer's disease (AD) pathology, including amyloid deposition on PET in cognitively normal older adults. Among older adults free of dementia, this study investigated the association between SCD and cerebrospinal fluid (CSF) amyloid deposition $\left(\mathrm{Ab}_{42}\right)$. We also investigated the association in other CSF markers of Alzheimer's disease (AD), including tau aggregation (ptau), neurodegeneration (total tau), and axonal injury (neurofilament light, NFL).

Participants and Methods: Vanderbilt Memory \& Aging Project participants with normal cognition ( $\mathrm{NC} ; \mathrm{n}=83,72 \pm 7$ years) and mild cognitive impairment (MCI; $n=58,73 \pm 6$ years) completed a multi-item SCD protocol and fasting lumbar puncture. Stratified by diagnosis, linear regression models related CSF concentrations $(\mathrm{pg} / \mathrm{mL})$ of $\mathrm{Ab}_{42}$, ptau, total tau, and NFL to total SCD score, adjusting for age, sex, education, race/ethnicity, depressed mood, and $A P O E-\mathrm{e} 4$ status.

Results: Decreasing $\mathrm{Ab}_{42}$ concentrations related to increasing total SCD in NC $(b=0.997, p=0.003)$ but not MCI participants $(b=1.00, p=0.86)$. However, no other CSF marker was related to total SCD score in NC ( $p$-values>0.10) or MCI ( $p$-values>0.07).

Conclusions: Consistent with previous neuroimaging studies, a possible preferential association between SCD and one of the first pathological processes in the proposed cascade of AD was noted. Surprisingly, SCD was not related to tau, a marker typically associated with objective cognitive impairment. Results provide continued support for the use of SCD as a marker of early AD pathology. Further research is needed to examine how SCD may be related to other markers of vascular disease. Correspondence: Katherine Gifford, PsyD, Vanderbilt University, 2525 West End Ave, 12th Floor, Suite 1200, Nashville, TN 37203, United States.E-mail: katie.gifford@vanderbilt.edu

S. COSENTINO. The role of domain-independent health perceptions in Subjective Cognitive Decline.

Objective: Subjective cognitive decline (SCD), the perception of cognitive deficits by older adults without clinically detectable cognitive impairment, has been considered a potentially early marker of dementia for over 20 years. New imaging technologies have reinforced the idea that SCD could reflect early AD pathology, but the relationship between SCD and dementia is imprecise. It is conceivable that SCD may in part reflect domain-independent health beliefs not specific to cognitive functioning (e.g., perceived level of control, health pessimism). In order to more comprehensively characterize the variables that contribute to SCD, this study examined whether subjective perceptions of cognition were linked with perceptions of physical health.

Participants and Methods: 417 individuals were drawn from a community dwelling sample of older adults (North Manhattan Aging Project also known as the Washington Heights and Inwood Columbia Aging Project). Cognition and physical function (i.e., hearing, gait) were both assessed subjectively with self-ratings and objectively upon exam. Bivariate correlations and linear regression examined whether SCD and subjective physical function (SPF) were associated independent of objective functioning in these domains, and apart from mood, a known contributor to SCD

Results: SCD was associated with depression $(r=.37, p<.001)$ and SPF $(r=.16, p=.001)$, but not objective cognition $(r=.04, p=.40)$ or physical function $(r=.02, p=.75)$. In a linear regression adjusted for depression, SPF was independently associated with SCD $(\beta=.26$, $p=.02)$. Stratified analyses showed that SCD and SPF were associated in those with low depression $(\beta=.43, p<.01)$ but not high depression $(\beta=.23, p=.18)$.

Conclusions: Results provide novel information that SCD in part appears to reflect domain-independent perceptions of health, primarily in the absence of depression. The meaning of SCD and its prognostic value for dementia may be fine-tuned by considering an individual's health perceptions more broadly.

Correspondence: Stephanie Cosentino, PhD, Neurology, Columbia University Medical Center, 630 West 168th, New York, NY 10804, United States.E-mail:sc2460@cumc.columbia.edu

A. APPLE, M. SCHROEDER, A. RYALS, L.I. WAGNER, D. CELLA, F. PENEDO, J.L. VOSS \& L. WANG. Elevated hippocampal functional connectivity related to memory in breast cancer survivors with self-reported cognitive concerns.

Objective: Between 35-75\% of patients experience cancer related cognitive impairment (CRCI) for months or years following treatment. The specific brain regions affected in CRCI remain largely unknown. Rodent models point to the hippocampus as a likely candidate however no study has investigated the functional connectivity of hippocampal networks in CRCI. We compared hippocampal-cortical connectivity in cancer survivors and controls and tested the relationship between functional connectivity differences and measures of cognition.

Participants and Methods: FMRI data were acquired during a memory task from 16 breast cancer survivors and 16 controls. The NIH Toolbox was used to assess cognitive performance and Neuro-QOL was used to measure self-reported cognitive concerns. The "background connectivity" method was used whereby task-related activity was removed from the fMRI data to provide state-related activity for the functional connectivity analysis. Whole-brain group-level comparisons identified regions with different connectivity with hippocampus in survivors 
versus controls. Average connectivity was extracted from regions of significant difference between the groups and correlated with cognitive performance.

Results: Survivors reported greater cognitive concern than controls $(\mathrm{p}=0.004)$, but objective cognitive performance did not differ. Cancer survivors showed increased connectivity of hippocampus with left lingual gyrus, left cuneus, and left precuneus compared with controls ( $p=0.001)$. In survivors, connectivity of these regions with hippocampus was positively correlated with episodic memory performance measured with NIH Toolbox $(r=0.52, p=0.045)$. No relationship between connectivity and cognition was found in the control subjects.

Conclusions: The observed increase in hippocampal-cortical connectivity in survivors compared to controls may be a compensatory response to cancer and its treatments. This compensation could explain, in part, the subjective feelings of cognitive impairment that were reported by survivors.

Correspondence: Alexandra Apple, Department of Psychiatry and Behavioral Sciences, Northwestern University Feinberg School of Medicine, 710 N. Lake Shore Drive, Abbott Hall, suite \#1306, Chicago, IL 94301, United States. E-mail: alexandraapple2013@u.northwestern. edu

\section{Poster Session 11. Cognition}

\section{0:30-11:45 a.m.}

\section{Cross Cultural}

\section{A.E. WERRY, M. DANIEL \& B. BERGSTRÖM. Influence of Demographic Variables on Measures of Memory and Language in Older Adults of Different Races.}

Objective: Although research suggests demographic factors are differentially correlated with performance on memory and language tests, few studies examine this influence in older adults or consider specific racial groups. The objective was to determine the impact of demographic variables on memory and language in neurologically healthy older adults of different races.

Participants and Methods: Subjects were 1098 African Americans and 5311 Whites from the National Alzheimer's Coordinating Center (gender: 36\% male; mean/SD: age $=75.26 / 6.98$; education $=15.70 / 2.91)$. Dependent variables for a series of multiple regression analyses included measures of: memory - Mini Mental State Exam (MMSE), Logical Memory Immediate Recall (LMI), and Logical Memory Delayed Recall (LMD); and language - Boston Naming Test (BNT), Animal Naming, and Vegetable Naming. Independent variables were age, education, and gender. Regression analyses were performed separately for Black and White groups.

Results: Age, gender, and education were statistically significant predictors on all memory and language measures in both racial groups. Education was a stronger predictor and accounted for more variance on all memory and language measures in the Black group than the White group. Age was also a stronger predictor and accounted for more variance in the Black group than the White group on measures of memory and language except semantic fluency.

Conclusions: With the exception of gender, demographic variables predict memory and language performance differently for Black and White groups. For language measures, age was a stronger predictor of performance in the White group and education was a stronger predictor in the Black group. Education was a stronger predictor than age on memory test performance in both groups; however, the strength of the relationship was greater in the Black group than the White group. These findings highlight the importance of considering ethnicity and education when developing, norming, and interpreting neuropsychological tests.
Correspondence: Amy E. Werry, Pacific University, 17610 NW Cornell Road \#9, Beaverton, OR 97006, United States. E-mail: amywerry@ pacificu.edu

\section{Executive Functions/Frontal Lobes}

N. ABRAHAM, M. BARBOZA, A. KRIVENKO, S. NAPOLITANO, H. O'HARA, G. OZINGA, S.E. TOLFO \& A. POREH. Examination of Cognitive Functioning Among Holocaust Survivors.

Objective: The present archival study investigated the effect of severe trauma on neurocognitive functioning in older adults.

Participants and Methods: A random sample of 196 elderly Israelis was administered a large battery of neuropsychological tests in addition to the Mini Mental Status Exam (MMSE). Within this sample, we identified 38 of the subjects as Holocaust survivors, most of whom reported being incarcerated in concentration camps, suffering from physical and emotional abuse, and at the time of the study, were suffering from nightmares. Their age, level of education, and mental status exam were similar to the larger sample.

Results: However, a more detailed analysis of the data shows that the Holocaust survivors were exhibiting a higher degree of verbal executive function deficits, characterized by a decline in phonemic but not semantic fluency and decreased performance on the interference portion of the Stroop Test, but not on the first and second portions of this test. No difference was found with regard to other measures, such as the Wechsler Memory Test Logical Prose and the California Auditory Verbal Learning Test.

Conclusions: The results of the study may suggest that traumatic experiences during the early years may have a lasting effect on executive functions. Limitations of the present study are discussed, including the effects of bilingualism on the above measures.

Correspondence: Natalie Abraham, Cleveland State University, 6100 Laurent Drive, apt 305, Parma, OH 44129, United States. E-mail: Natalie.Abraham@uhhospitals.org.

B.A. BAILEY, D. GARCIA, A.M. SVINGOS, D.M. BAGNER \& S.C. HEATON. Parent-Reported and Performance-Based Changes in Inhibitory Control Following Parent-Child Interaction Therapy (PCIT) in a Pediatric TBI Sample.

Objective: Traumatic Brain Injury (TBI) is a leading cause of disability in children under age five, resulting in persistent behavioral and cognitive deficits. Environmental factors, such as parenting behaviors, have been identified as relevant predictors of post-injury functioning. Thus, parent-training programs (e.g., Parent-Child Interaction Therapy; PCIT) may be beneficial for this population. Though previous research has shown that modified PCIT can reduce parent-reported inhibition problems, less is known about whether treatment effects are evident on performance-based measures. Thus, the current study examined parent-reported and performance-based changes in inhibitory control following PCIT in a pediatric TBI sample

Participants and Methods: Study participants $(n=10)$ were $2-5$ years old, had sustained a recent head injury $(90 \%$ mild severity), and exhibited elevated behavior problems. Participants received PCIT twice a week for 5 weeks. Measures of inhibitory control included the Inhibit subscale of the Behavioral Rating Inventory of Executive Functioning and the Head-Toes-Knees-Shoulders task.

Results: Paired sample $t$-tests revealed significant improvements in parent-reported inhibitory control between baseline and postintervention $(\mathrm{t}[1,9]=47, \mathrm{p}=.001, d=1.54)$, but these changes were not significant on the performance-based task $(t[1,8]=-1.437, p=.189$, $d=0.33)$.

Conclusions: Findings suggest that PCIT produces large significant effects on parent-reported inhibitory control after head injury, while performance-based measures indicate moderate, but non-significant, effects. Differences between parent-report and performance-based 
measures may occur for a variety of reasons. Parent report may be influenced by increased ability to manage the behavior problems associated with impairments. Conversely, changes in child behavior may not generalize to laboratory settings due to differing behavioral contingencies. Further research should examine factors influencing parent perceptions of child executive functioning.

Correspondence: Brittany A. Bailey, Bachelor of Arts, Clinical Health Psychology, University of Florida, 1225 Center Drive, Rm 3158, Gainesville,FL 32508, United States.E-mail: bbailey92@phhp.ufl.edu

M. BARBOZA, A. KRIVENKO, A.M. POREH, S. NAPOLITANO, N. ABRAHAM, S.E. TOLFO, G. OZINGA \& H. O'HARA. Preliminary data for comparison of written verbal and non-verbal fluency.

Objective: Previous research has shown that verbal and non-verbal fluency assessments are thought to utilize similar components of executive functioning. However, given that most of the nonverbal fluency include a graphomotor component one wonders how such measures correlate with writing fluency.

Participants and Methods: 37 were recruited and administered the Guildford Animal fluency, Regard's Five Point Test, and Barkley Deficits in Executive Functioning Scale,

Results: Spearman correlations were calculated to compare the Guildfornd Animal fluency and 5PT tests. No significant differences were found between the written verbal fluency measure and the 5PT for this preliminary data suggesting that the written adaptation for the verbal fluency measure is not as effective as the traditional spoken test when correlated with the results from nonverbal fluency tests. The results from the written verbal fluency measure were also correlated with the Barkley Deficits in Executive Functioning Scale and no significant differences were found.

Conclusions: In future studies we plan to modify the written fluency and attempt to improve it such that it would better correlate with the traditional oral verbal fluency test, allowing for its utilization in group settings.

Correspondence: Marina Barboza, Psychology, Cleveland State University, 7220 Green Valley Dr, Mentor, OH 44060, United States. E-mail: barboza.10@osu.edu

E.M. BOUTZOUKAS, A. SZE, L. SEPETA \& M. BERL. Executive Functioning Profiles in Children with Intellectual Disability.

Objective: Assessment of executive functioning (EF) within children who have intellectual disability (ID) is a challenge, but is an important endeavor as EF may moderate outcomes. This study examines EF profiles of children with IQ below 70 .

Participants and Methods: Using a clinical database of children referred for outpatient neuropsychological evaluation, children were included in the study if they had one or both verbal and nonverbal IQ $<70$ on any measure of intellectual functioning and a parent had completed a common parent questionnaire of EF, the Behavior Rating Inventory of Executive Functioning (BRIEF). Descriptive, chi square, and repeated measures analysis of variance were conducted to determine differences across subscales of EF and by IQ Group: FSIQ<70 (FS-ID); $\mathrm{VIQ}<70$ (V-ID); nonverbal IQ<70 (NV-ID).

Results: 200 children met inclusion criteria $(60 \%$ male; age range 5-19 years old (Mean=10.95 (3.24)). 67 participants were FS-ID (Mean PIQ=57(10), Mean VIQ= 58(10)), 51 were V-ID (Mean PIQ=81 (9), Mean VIQ=63(8)), and 82 were P-ID (Mean PIQ=64(6), Mean $\mathrm{VIQ}=84(10))$. MANOVA revealed a main effect of BRIEF subscale $(p<.05)$ and an IQ Group by BRIEF subscale interaction $(p<.05)$. Working Memory (Mean $\mathrm{T}=69$ ) was significantly higher than all other subscales, followed by Planning/Organization (Mean T=64), Shift (Mean T=63), Monitoring (Mean $\mathrm{T}=63$ ), and Initiation (Mean $\mathrm{T}=62$ ). Organization of Materials (OM) (Mean $\mathrm{T}=53$ ) was significantly lower than all other subscales. IQ groups differed by the percentage of children who had clinically elevated $(\mathrm{T}>65)$ scores for $\mathrm{OM}(\mathrm{p}=.01)$; Planning and Organization $(\mathrm{p}<.05)$; and Initiation $(\mathrm{p}<.05)$.
Conclusions: It is inaccurate to assume that children with ID have a flat profile of elevated scores given the variability within this study. Difficulties with working memory and inflexibility is similar to EF profiles seen in children with autism. Few concerns with emotional control and OM may relate to different parent expectations from non-ID children. Correspondence: Madison Berl, PhD, Children's National Medical Center, 111 Michigan Avenue NW, Washington, DC 20010, United States.E-mail:mberl@childrensnational.org.

J.S. CARMASIN, R. ROTH, L. RABIN, J. ENGLERT, L. FLASHMAN \&. A. SAYKIN. Stability of Self-Rated Executive Dysfunction in MCI and Older Adults With Subjective Cognitive Dysfunction.

Objective: Amnestic mild cognitive impairment (MCI) is characterized by subjective memory concerns and objective memory deficit, but patients with MCI also report concerns with other aspects of cognitive functioning including executive functions. While there is considerable research on the temporal stability of memory concerns in older adult populations, there is no such research with respect to executive functions in those with MCI. We evaluated the stability of subjective executive functioning in patients with MCI, older adults with subjective cognitive decline (SCD), and cognitively normal older adults (CN).

Participants and Methods: Participants were 21 older adult participants with amnestic MCI, $24 \mathrm{SCD}$, and $22 \mathrm{CN}$ matched for age [MCI $=73.9$ years $(\mathrm{SD}=5.5) ; \mathrm{SCD}=73.4(\mathrm{SD}=6.6) ; \mathrm{GN}=72.2(\mathrm{SD}=5.8)]$ and gender. Diagnostic categorization remained stable for all participants over the two assessment time-points (mean interval of 409 days; no group difference). Participants and their informants completed the Behavior Rating Inventory of Executive Function-Adult (BRIEF-A), assessing executive functions in everyday life over the past month. Mixed-model repeated measures analyses of variance comparing groups were conducted on the 9 BRIEF-A scales.

Results: MCI and SCD groups endorsed worse executive functions on most scales relative to the $\mathrm{CN}$ group, most prominently in working memory, initiation, and planning/organization. No Group by Time interactions were observed for any of the scales for the self or informant report forms, and the pattern of scores across the scales remained largely unchanged.

Conclusions: Problems with aspects of executive functioning are endorsed by both patients with MCI and older adults with SCD, as well as their informants, and these ratings are stable over the period of a year. This suggests that report of significant worsening of executive functions over a year in an older adult should receive further clinical attention. Correspondence: Jeremy S. Carmasin, PhD, Psychiatry, DartmouthHitchcock Medical Center, Dartmouth-Hitchcock Medical Center, One Medical Center Drive - Dept. of Psychiatry, Lebanon, NH 03756, United States.E-mail: jcarmasin@gmail.com

\section{J. CARVALHO \& B. SPRINGATE. Anxiety and Sleep Dysfunction} Predict Executive but not Memory Dysfunction in Healthy Adults. Objective: There is indication anxiety in older adults is associated with decreased memory and executive functioning (Yochim et al., 2013), and poor sleep quality has been associated with anxiety and executive functioning (EF) in healthy young adults (Benitez \& Gunstad, 2012). This study sought to explore the impact of anxiety and sleep disruption on memory and EF in community-dwelling adults

Participants and Methods: Data were obtained from 4615 participants who completed Wave II of the Midlife in US Study (MIDUS) surveys, a nationally representative sample of English-speaking adults within the US ( $M$ age $=54.52, S D=11.7$, range $=34-84,56.8 \%$ female). Cognitive variables were composite scores of EF (standardized mean scores for digits backward, category fluency, number series, backward counting, and mean switch and nonswitch trials in a stop and go switch task) and memory (immediate and delayed word list recall). Anxiety was measured via the Mood and Anxiety Symptom Questionnaire (MASQ) Anxious Arousal scale. Sleep was measured by participant report of the number of nights they slept soundly. 
Results: Multiple regression analyses revealed Anxious Arousal $(\beta=-.113, p=.001)$ and frequency of sleeping soundly at night $(\beta=.090$, $p=.006)$ predicted EF $\left(\mathbf{R}^{2}=.027\right)$. However, in a similar model, neither anxious arousal $(\beta=-.06, p=.06)$ nor frequency of sleeping soundly $(\beta=-.002, p=.96)$ predicted episodic memory $\left(R^{2}=.004\right)$.

Conclusions: This study explored whether sleep and anxiety predict cognitive performance. While EF was predicted by anxious arousal (negatively) and sleeping soundly at night (positively), neither of these factors predicted memory. Results suggest frontally-mediated tasks are more susceptible to effects of anxiety and sleep deprivation relative to temporally-mediated tasks.

Correspondence: Janessa Carvalho, Ph.D., Psychology, Bridgewater State University, 275 High Hill Road, Dartmouth, MA 02747, United States.E-mail:janessacarvalho@gmail.com

\section{H.L. COMBS, N.E. GARCIA, D.T.R. BERRY \& S.C. SEGERSTROM. Deep Brain Stimulation for Parkinson's disease: An Investigation of Post-surgical Self-regulation and Executive Functioning.}

Objective: Parkinson's disease (PD) is a common neurodegenerative disorder that attacks the basal ganglia and contributes to a range of motor, cognitive, and behavioral impairments. This dysfunction may contribute to self-regulatory impairment across several domains. Deep Brain Stimulation (DBS) is a procedure that allows for direct and reversible manipulation of brain activity in patients with PD. Preliminary studies suggest mild executive dysfunction follows DBS but as the literature is in its early stages, there is a need to examine further the range of executive deficits and self-regulatory impairment observed in PD following DBS.

Participants and Methods: In the present study, twenty-seven PD patients post-DBS completed a brief neuropsychological test battery and provided measures of heart rate variability (HRV). Patients also completed measures regarding their ability to self-regulate emotions and thought patterns. Scores were compared to the patient's pre-surgical performance and to a group of healthy older adults.

Results: Results suggest DBS leads to significant, though small declines in executive function (EF) and self-regulation (SR). DBS Patients had significantly worse scores on neuropsychological tests of EF (i.e., phonemic fluency, semantic fluency, and working memory) when compared to their preoperative performance. Similarly, DBS patients had significantly worse scores than controls on measures of EF and verbal memory. With regard to physiological functioning, lower baseline HRV was linked to worse EF but fewer impulsive-compulsive behaviors in DBS patients.

Conclusions: Correlations among measures of theoretically similar constructs (i.e., EF and SR) were modest and variable, challenging the idea that SR in different domains depends on a common resource. Clinically, it is important for health care professionals working with PD to recognize the presence of SR deficits and to be aware of the potential obstacles that might arise from such impairments within a patient's daily life.

Correspondence: Hannah L. Combs, M.S., Psychology, University of Kentucky, 7510 Brompton St. Apt. 598, Houston, TX 77025, United States.E-mail: hannahlanecombs@gmail.com

\section{A.J. DUGGER, A.J. BOHLEN, K.J. MOORE \& H. DAVIS.} Development of Executive Functions: From 5 to 24 Years of Age.

Objective: There is no consensus view of the processes that constitute executive function (EF). Purported EFs include the ability to inhibit and/or switch behavioral responses, hold and manipulate information in conscious awareness (working memory), and engage in goal-directed problem solving. We provide a behavioral assessment of the development of four EFs over an age span that parallels the maturational aging of the frontal lobes.

Participants and Methods: The development of four EFs was examined in individuals in two year increments from 5 to 24 years of age. Inhibition was assessed using a Stroop test with the dependent variable being the difference in time to state what color the words were printed in minus the reading time for the words. The effect of set shifting was assessed using the time to complete Trails B of the Trails Test. Working memory was assessed in a N-back test. The dependent variable was the percentage of correct response on a 100 trial verbal 3-back test. The development of problem solving and planning was evaluated using the Tower of London (TOL) task and the dependent variable was excess moves.

Results: On the Stroop Test assessment of inhibition, as age increased the speed of naming the color words were printed in significantly decreased, $\mathrm{F}(9,1204)=74, p<.001$, with optimal performance achieved by age 15 . For shifting, as age increased the time to complete Trails B significantly decreased, $\mathrm{F}(9,698)=112$, p<.001, with optimal performance achieved by age 15 . For working memory assessment in the N-back task the percent of correct responses increased with age, $\mathrm{F}(9,754)=34.8$, $p<.001$, and optimal performance was achieved by age 17 . The total number of excess moves in the TOL task significantly decreased with age, $F(9,1871)=169.1$, $p<.001$, with optimal performance by age 15 . Conclusions: Increases in four EFs were detectable over 2 year increments during development and switching, inhibition, and working memory significantly correlated with TOL performance. Working memory showed the largest correlation (.40).

Correspondence: Amber J. Dugger, BA, University of Colorado Colorado Springs, Psychology Department, Colorado Springs, CO 80918, United States.E-mail:adugger@uccs.edu

S.W. DUVALL, P. MACLEAN, S. ERICKSON \& J. LOWE. Relationship Between Parent Report of Executive Function and Naturalistic Observational Coding in Preterm and Full Term Preschoolers.

Objective: To compare executive function $(\mathrm{EF})$ in preschoolers born preterm (PT, $<32$ weeks gestation) and full term (FT) using parent report of EF and naturalistic child behaviors.

Participants and Methods: Participants included 61 preschoolers born PT and 40 born FT between the ages of 3 and 4.5 years. Parent report of EF (BRIEF-P) and behavioral coding of EF based on a play interaction between the parent and the child (NICHD Cleanup Defiant Noncompliance) were utilized. Spearman correlations, Analysis of covariance (ANCOVA) and Fischer's r-to-z transformations were used to assess the relationships and significance of the differences between correlation coefficients in the PT and FT samples.

Results: Preschoolers born FT showed greater levels of compliance with parents and preschoolers born PT had higher rates of parent-reported executive dysfunction on the BRIEF-P, both of which remained after correcting for age and FSIQ. The correlations between BRIEF-P GEC and Compliance were significantly different between the PT $(r=1.5)$ and FT groups $(\mathrm{r}=-2.37)$ (discrepancy, $\mathrm{r}=-2.32, \mathrm{p}=.02)$. For the PT group, a negative correlational trend was found $(\mathrm{r}=-.27, \mathrm{p}=.15)$ : as compliance increased, parent reported executive dysfunction decreased. In contrast, for the FT group, the trend was positive $(\mathrm{r}=.20, \mathrm{p}=.21)$ : increased compliance was associated with increased parent reported executive dysfunction.

Conclusions: Preschoolers born PT had poorer EF outcomes than the FT group on observational coding and parent report. Results suggest differing relationships between parent report of EF and observational coding across groups. Our findings that BRIEF-P scores did not align with observational measures of $\mathrm{EF}$ is consistent with previous literature and it has been proposed that parent report and behavioral coding of EF may be more strongly related to general behavioral dysregulation versus EF specific ability. Additionally, compliance may be impacted by parent-child relationship variables.

Correspondence: Susanne W. Duvall, Ph.D., University of Minnesota, Univ New Mexico, 420 Delaware Street SE, MMC 486, Minneapolis, MN 55455, United States. E-mail: swduvall@unm.edu 
M. HALVERSON, M. EULER, M.A. NIERMEYER \& Y. SUCHY. The Effect of Novelty on Motor Control in Healthy Participants.

Objective: The initiation of action during complex motor sequencing strongly predicts executive functioning $(\mathrm{EF})$, and is adversely affected by contextual novelty, primarily among individuals with EF limitations. While simple motor control processes also relate to EF, it is unclear whether (a) these processes are also susceptible to novelty effects, and (b) if low EF predicts a greater vulnerability of these processes to the effects of novelty. This study sought to clarify this issue by examining the effects of novelty on motor control and in relation to EF.

Participants and Methods: Thirty-seven healthy undergraduates completed a joystick-based motor task involving four-movement sequences. Sequences consisted of three joystick movements plus a double-tap button press. Novelty effects were induced by pseudo-randomly changing perceptual aspects of sequence presentation. Motor control was measured based on speed of inter-tap intervals (ITI), and hyperkinetic perseverations (HKP), defined as any sequence containing more than two consecutive taps. An EF composite was calculated based on verbal and design fluency, trail-making, and the Stroop task.

Results: Dividing the sample based on commission of any vs. no HKPs resulted in two approximately equal groups (HKP+, n=19; HKP-, $\mathrm{n}=18)$. Novelty increased median ITI, relative to three familiar conditions, in the group as a whole (all $p \mathrm{~s}<.022$ ), with the HKP+ group showing a particularly large novelty effect $(p=.045)$. Although relations between ITI and EF were in the expected direction (average $r=-.28$, average $p=.096$ ), the effect of HKP group was not significant $(p=.558)$.

Conclusions: Results demonstrated that in addition to impeding motor planning, novelty also affects motor control, as shown by longer ITIs in the full sample, and particularly in the HKP group. Although relations with EF were not significant, the overall results support further investigating the links between motor control, novelty, and EF.

Correspondence: Matthew Euler, Ph.D., Psychology, University of Utah, 380 S 1530 E BEHS 502, Salt Lake City, UT 84109, United States. E-mail:matt.euler@psych.utah.edu

\section{J. SWARTZ \& R. FALLOWS. The Relationship between Interference and Inhibition in a Mixed Clinical Sample.}

Objective: Although the relationship between inhibition and interference has been studied in non-clinical populations to some degree, the relationship in clinical populations is less well studied. This project examined the relationship between color word inhibition and four derived interference scores on the Stroop with proactive and retroactive interference scores on the CVLT-II in a mixed clinical sample.

Participants and Methods: Archival data from an outpatient neuropsychology clinic yielded 63 participants (47.4 years old, SD 16.4; 13.3 years education, SD 3; 47.6\% male; $95.2 \%$ Caucasian) who presented with various neurological/psychiatric diagnoses (most frequent included $37 \%$ psychiatric, 32\% mild neurocognitive disorder, 13\% Dementia \& MCI, and 13\% ADHD) and completed the Stroop (Golden version) and CVLT-II. Stroop scores included Golden's color-word score and interference score. Jensen's interference score, Lansbergen et al.'s ratio interference score, and a relative interference score. Pearson r correlations were used to evaluate the relationship between the five Stroop scores and two CVLT-II interference scores.

Results: A small positive correlation was found between Stroop color word score and CVLT-II proactive interference score $(r=.28, p<.05)$. None of the 4 other Stroop scores were significantly correlated with either of the interference scores.

Conclusions: Overall, there is a small, but positive correlation between color-word reading on the Stroop and proactive interference on the CVLT-II in this mixed clinical sample indicating a possible relationship between prepotent inhibition and proactive interference. Future prospective research examining specific neurological conditions (e.g., ADHD, frontal/temporal lobe epilepsy, FTD/DAT, etc.) will be beneficial in further elucidating the nature of this relationship.
Correspondence: Robert Fallows, PsyD, Neuropsychology, Samaritan Health Services, 400 NW Hickory St., Albany, OR 97321, United States. E-mail:RobertFallows@gmail.com

M.E. FASANO, R. PLOTKIN, A. DITTMAR, D. PREVI \& J. LINTON REESMAN. Concurrent Validity of the Behavior Rating Inventory of Executive Function in Clinically-referred Children who are Deaf or Hard-of-Hearing.

Objective: Empirical examination of the applicability of common neuropsychological measures for deaf or hard of hearing (DHOH) individuals is lacking. The present study examined concurrent validity of the Behavior Rating Inventory of Executive Function (BRIEF) in a sample of clinically-referred DHOH children and adolescents. Inhibit and Working Memory indices were hypothesized to relate to ADHD symptomatology. Participants and Methods: Retrospective review of 119 DHOH children $(63.2 \%$ male, mean age in years $=11.89[\mathrm{SD}=3.71])$ referred for neuropsychological evaluation to an urban specialty clinic for children with hearing loss, parent ratings on the BRIEF and ADHD Rating Scale-IV were examined. General and hearing-related demographic information (e.g., etiology of hearing loss, use of amplification) were reported. Parent ratings on the BRIEF were discretized according to recommended clinical cut-offs. Participants were grouped as "ADHD+" or "ADHD-" based on parent-rated symptom count (i.e., $\geq 6$ on either inattentive or hyperactive/impulsive subscales). BRIEF ratings were compared to ADHD+/ ADHD- grouping using chi-square test of independence.

Results: Total BRIEF scores were significantly related to ADHD grouping. $\mathrm{DHOH}$ children with clinically significant ratings on the BRIEF global executive composite (GEC) were more likely to demonstrate parent ratings consistent with $\mathrm{ADHD}\left(\chi^{2}[3, N=119]=26.13, p<\right.$ .001 , Cramer's $V=.47)$. All indices comprising the GEC were similarly related to ADHD grouping. Clinical elevations on the Inhibit (Cramer's $\mathrm{V}=.47$ ) and Working Memory (Cramer's $\mathrm{V}=.54$ ) indices were related to ADHD grouping within the $\mathrm{DHOH}$ clinical sample.

Conclusions: Current results support the utility of the BRIEF in evaluating executive functioning $(\mathrm{EF})$ in clinically-referred DHOH children and adolescents. Preliminary findings are encouraging for use of this measure with $\mathrm{DHOH}$, and examination of language use of parent and child may assist in delineating factors related to working memory items specifically.

Correspondence: Matthew E. Fasano, M.A., Neuropsychology, Kennedy Krieger Institute, 2300 N. Calvert St, Apt 101, Baltimore, MD 21218, United States.E-mail: fasano@kennedykrieger.org

E.I. FRANCHOW, M.A. NIERMEYER, R. ZIEMNIK, C. BARRON, R. HEYREND, B. BATTILORO \& Y. SUCHY. Naturally-Occurring Expressive Suppression in Older Adulthood: Beyond Executive Functioning.

Objective: Naturally-occurring higher-than-usual regulation of affect (i.e., expressive suppression; ES) is associated with acute depletion of higher-order executive functions (EF), but not lower-order component processes (e.g. processing speed), in young adults (Franchow \& Suchy, 2015). However, considering increasing convergence between EF and component processes in older adulthood (Albinet, Boucard, Bouquet. \& Audiffren, 2012; Ng et al., 2016), the effect of recent, naturally-occurring ES in older adulthood may not be specific to EF

Participants and Methods: 94 adults ( $\mathrm{M}=69$ years old; $65 \%$ female; $\mathrm{M}=15.8$ years of education) scoring within normal limits on the Geriatric Depression Scale completed items assessing burden of ES in general (ES-Gen) and over the past two weeks (ES-2W) as well as an estimate of global cognitive status, the Dementia Rating Scale-2 (Global-Cog). They then completed four subtests from the Delis Kaplan Executive Functioning System, with age-corrected scaled scores reflecting response generation and switching (EF) and basic sequencing and response speed (Component) combined into composite scores. 
Results: EF, Component, and Global-Cog were criterion variables in separate hierarchical regressions; years of education, depressive symptoms, ES-Gen, and ES-2W were entered on separate successive steps. ES-Gen did not contribute significantly in any domain. ES-2W contributed significantly above and beyond previous steps to EF ( $\mathrm{F}_{c h}$ $(1,87)=4.102, p=.046)$ and trended toward significance for Component $\left(\mathrm{F}_{\text {change }}(1,87)=3.614, p=.061\right)$, such that higher ES was associated with poorer performance; unique variance accounted for in $\mathrm{EF}$ and Component by ES-2W was identical $\left(\mathrm{R}^{2}{ }_{\text {change }}=.039\right)$. In contrast, ES-2W did not contribute to Global-Cog.

Conclusions: These results suggest that while the depleting effect of recent ES remains executive in later life, the effect may also include lower-order component processes with increasing age.

Correspondence: Emilie I. Franchow, MS, Psychology, University of Utah, 220 E. 3300 S., Apt. 212, Salt Lake City, UT 84115, United States.E-mail: emilie.franchow@psych.utah.edu

N. HARTLEY, K. ARES \& S.J. HUNTER. Risky Sexual Behaviors in Homeless Youth: The Influence of Executive Functioning and Depression on the Condom Use of Homeless Youth in Chicago.

Objective: Homelessness among youth is a worsening problem in the United States. Homeless youth (HY) are at an elevated risk of developing a mood disorder throughout their lifetime. Additionally, depressed $\mathrm{HY}$ are four times as likely to engage in risky sexual behaviors, such as inconsistent condom use, than are housed youth. This puts the HY population at an increased risk for transmission of HIV and other STIs. HY are also believed to have significant impairments with executive functioning (EF), causing difficulties with regulating impulses and making healthy choices. The purpose of this study is to investigate and how depression and executive dysfunction influence the consistency of condom use among a sample of HY living in Chicago.

Participants and Methods: 149 young adults, aged 18-23, (46\% male and 54\% female; $75 \%$ African American, 5\% Hispanic, 5\% Caucasian, and $15 \%$ other) were recruited from homeless shelters in Chicago. Participant background information was collected including self-reported condom use consistency. Participants were administered a self-report (BRIEF-A) and objective measure (Iowa Gambling Task) of executive functioning. Subjects were also administered a semistructured psychodiagnistic interview (M.I.N.I) to asses for Major Depressive Disorder (MDD).

Results: Binary regression analyses revealed a significant correlation between inconsistent condom use and self-reported deficits in metacognition, the complex process of "thinking about thinking." No significant relationships were found between inconsistent condom use and previous diagnosis of MDD, self-reported deficits in behavioral regulation, or scores on objective EF measures.

Conclusions: Results indicate that homeless youth may engage in risky sexual behaviors due, in part, to deficits in higher-level thinking processes, likely exacerbated by living in unstable environments. Implications point to the need to provide HIV prevention-based interventions aimed at teaching HY systematic and routine problem solving skills while experiencing homelessness.

Correspondence: Nicholas Hartley, Masters (PsyD Candidate), Psychology, Children's Hospital Colorado, 1331 S. Florence St. Apt. \#2025, Denver, CO 80247, United States.E-mail: nhartley@mail. roosevelt.edu

K. HAZLETT ELVERMAN, W.T. MCCUDDY, K. REITER, S. POTTS, A. PORCELLI \& K.A. NIELSON. The Importance of Heart Rate Variability to Executive Functioning Across the Lifespan.

Objective: As the longevity of the population continues to increase, so does the need for understanding, predicting and preventing of cognitive decline. Executive functions, such as inhibitory control (IC), are greatly affected by age and are essential to functional independence. Thayer's neurovisceral integration hypothesis suggests a link between effective autonomic regulation and intact cognition. Therefore, modifiable biomarkers of autonomic functioning might serve as important targets to prevent or address cognitive decline. This project explored the relationship of heart rate variability (HRV) to IC ability.

Participants and Methods: Fifty-nine adults were divided into Young $(\mathrm{M}=23.19, \mathrm{SD}=2.60)$, Middle-Aged $(\mathrm{M}=59.62, \mathrm{SD}=4.44)$, and Older $(\mathrm{M}=72.89, \mathrm{SD}=6.39)$ groups. HRV was measured by electrocardiogram, using overall variability via the SD between heartbeats (SDNN) and low/high frequency power ratio (LHR), a measure of sympathovagal balance, during rest, and during the stop-signal portion of the Parametric Go/No-go/Stop task (PGNGS). Performance was measured by stop signal accuracy (SSA) and reaction time (SSRT).

Results: Young adults had significantly greater baseline SDNN vs. Middle-Aged and Older groups. Greater baseline SDNN was associated with better SSRT across groups $(\mathrm{r}=-.40, \mathrm{p}<.004)$, and greater SDNN and LHR during the task were associated with better overall task performance, including SSA ( $\mathrm{r}=.27$ to .41, $\mathrm{p}<.04)$. Regression showed significant prediction of SSA by LHR $(\mathrm{R} 2=.25, \mathrm{p}<.001)$ after accounting for age and sex.

Conclusions: The results show a general reduction of HRV with advancing age, as expected. Moreover, greater resting HRV, and greater sympathetic upregulation during task completion, was associated with better inhibitory control across the adult age spectrum. Thus, improvements in cardiovascular health and fitness, thereby autonomic regulation and responsiveness, could be key to protecting executive functioning and quality of life into older age.

Correspondence: Kathleen Hazlett Elverman, M.S., Psychology, Marquette University, 1807 N. 57th St., Milwaukee, WI 53208, United States.E-mail:hazlett.ke@gmail.com

J.S. HOLCOMBE, S.L. AITA, S. TAYLOR, J. BEACH, B. SLAGEL, J. HUDSON, S. ROYE, M. CALAMIA \& B.D. HILL. Need for Cognition and its Relation to Self-Reported Executive Dysfunction. Objective: The purpose of the present study was to examine the relation between Need for Cognition, a personality trait of how much a person enjoys effortful mentation, and self-reported executive dysfunction.

Participants and Methods: Participants were healthy college students $(N=130 ; M$ age $=19.45, S D=3.16 ; M$ education $=12.34, S D=0.69$; 73.8\% female; $53.1 \%$ White, 36.9\% African American, 7.7\% Asian). Participants completed the Need for Cognition (NFC) short scale and Barkley Deficits in Executive Function Scale (BDEFS) as part of a larger battery. BDEFS total and subscale scores were used.

Results: Pearson correlations revealed significant negative relations between NFC and BDEFS total and subscales, $r=-.28$ to - -42 ( $p$ values $<.01)$. The BDEFS subscales were entered into a multiple regression to predict NFC. This model explained $20.2 \%$ of the variance in NFC $\left(R^{2}=.202, F(5,124)=6.28, p<.001\right)$. Only the BDEFS Self-Organization and Problem Solving subscales significantly predicted NFC $(\beta=-.35, p=.008)$ with the other BDEFS subscales not significantly predicting NFC.

Conclusions: The personality trait NFC was significantly related to all domains measured by the BDEFS. Greater NFC was associated with less self-reported executive deficits but only self-organization and problem solving significantly predicted NFC. These findings fit into a positive neuropsychology framework where enjoyment of thinking may be linked to robust executive abilities.

Correspondence: Jo S. Holcombe, B.A., Psychology, University of South Alabama, 75 South University Blvd, Mobile, AL 36688-0002, United States.E-mail: jsh1422@jagmail.southalabama.edu

A. ROSA, A.K. HOLLAND, C. BLANCO \& D.W. HARRISON. Assessing Diminishment of Frontal Lobe Function in Trait Aggressive, Violent Prone Men: Examining Changes in Performance on a Cognitive Estimation Task and Grip Strength Measures Using a Dual Task Approach.

Objective: High levels of trait aggression have been associated with decreased performance on tasks measuring executive function (Holler \& 
Kavanough, 2012), which have been associated with diminished regulatory control of the frontal lobes over posterior cerebral regions. High trait aggressive men were predicted to demonstrate poor task performance and evidence heightened sympathetic tone upon exposure to cognitive stress relative to low trait aggressive men.

Participants and Methods: Undergraduates ( $\mathrm{n}=98$ ) completed screening measures including a medical history questionnaire, the Buss Perry Aggression Questionnaire, and a Cognitive Estimation Task (CET). High aggressive $(n=7)$ and low aggressive $(n=8)$ men completed a grip strength task before and after completing a mental subtraction task. Systolic blood pressure (SBP) measures were taken between experimental manipulations.

Results: Aggression was a significant predictor of performance on the CET $(F(1,43)=4.85, p=.03)$. Aggression was also a significant predictor of the number of medical symptoms reported $(F(1,95)=5.36, p=.02)$. Regarding data from laboratory protocol, a main effect for Trial for grip strength across 5 trials was found $(F(4,52)=16.31, p=.01)$, indicating fatigue across 5 trials. A Condition $\mathrm{x}$ Hand interaction was found $(F(1,13)=4.29, p=.05)$, indicating increased grip strength at the left hand in the post stress condition, and a decrease in right hand grip strength in the post stress condition. A main effect was also found for $\operatorname{SBP}(F(1,14)=6.15, p=.02)$ indicating that both high and low aggressive individuals experienced a decrease in SBP after stress.

Conclusions: Results from the regression analyses reveal that high levels of trait aggression may be associated with impaired executive function as well as poor health outcomes. The Condition x Hand interaction provides evidence of increased right parietotemporal activation upon exposure to cognitive stress. The finding of decreased SBP post stress is likely due to the removal of anticipatory stress.

Correspondence: Alissa K. Holland, Ph.D., Psychology, University of South Carolina Lancaster, 476 Hubbard Drive, Lancaster, SC 29721, United States.E-mail: akhollan@mailbox.sc.edu

S.E. JOHN \& B.E. GAVETT. The Unity and Diversity of Neuropsychological Tasks of Executive Functioning: Construct and Ecological Validity of Common Assessment Measures.

Objective: Evidence from both cognitive neuroscience and neuropsychology presents an ill-defined understanding of executive functioning (EF). Some research suggests that subcomponents of EF, such as working memory, are best represented as correlated, but distinct constructs. Other research suggests that subcomponents share a single underlying contributing factor. Computerized measures of EF and total performance scores oversimplify the factor structure of EF and reduce the clinical and ecological generalizability of study findings. Therefore, this study utilized confirmatory factor analysis (CFA) and multiple regression to evaluate the construct validity of nine common paperbased measures of EF and their ability to predict the functional tasks of driving and bill payment.

Participants and Methods: Participants were 192 cognitively heterogeneous community-dwelling older adults between the ages of 54-92 $(M=69.80, S D=8.06)$. They were administered $9 \mathrm{EF}$ tests and 2 tests that approximate functional tasks, NAB Driving Scenes (DS) and Bill Payment (BP). Four hypothesized CFA models were assessed using total scores for each EF measure. Model fit was evaluated using the comparative fit and Tucker-Lewis indices and the root mean square error of approximation.

Results: None of the hypothesized models had adequate fit; the best fitting model was a one-factor model, representing global EF, $\left(\chi^{2}=96.04, \mathrm{df}=27, p<.001 ; \mathrm{CFI}=.84 ; \mathrm{TLI}=.79 ; \mathrm{RMSEA}=.12,90 \%\right.$ CI $[.09, .14], p<.001)$. Regression analyses evaluated each EF test's ability to predict performance on DS and BP, with the Trail Making Test B as the most predictive for both.

Conclusions: Results suggest that total performance scores are poor measures of EF subcomponent functioning that neither map onto distinct subcomponents of EF nor accurately represent a global EF factor. Multiple measures of EF are necessary for interpretation of global
EF ability and single test scores inaccurately estimate specific subcomponents of EF when utilized in isolation.

Correspondence: Samantha E. John, PhD, Neurology, Emory University, 609 Virginia Ave, Apt 2414, Atlanta, GA 30306, United States. E-mail: sejohn@emory.edu

N. JOHNSON, Z. HAWKS \& D. WHITE. Developmental Trajectories of Strategic Processing in Children with Phenylketonuria.

Objective: Phenylketonuria (PKU) is a hereditary disorder characterized by impaired ability to metabolize phenylalanine, resulting in cognitive dysfunction in pre-frontally mediated executive domains. Prior research suggests that strategic processing, one such executive domain, is impaired in children with PKU; however, little is known about the developmental trajectory of strategic processing during childhood and adolescence.

Participants and Methods: The present study evaluated strategic processing in 23 children with early-treated PKU and 44 typically developing controls across three time points using a verbal fluency task. Verbal fluency performance was assessed for clustering and switching in two categories: semantic fluency (animal and food/drink) and phonemic fluency ( $\mathrm{S}$ words and $\mathrm{F}$ words). Whereas clustering was defined as a group of 2 or more words with semantic or phonemic congruence, switching was defined as moving from one word or cluster to an unrelated word or cluster. A composite of mean cluster size and switching was examined to analyze strategic processing.

Results: Results indicated that participants with PKU exhibited worse use of phonemic fluency strategies than controls, and that older baseline age was associated with more efficient semantic and phonemic strategic processing. Of particular interest, there was a significant interaction between group and time point, such that participants with PKU exhibited worse use of semantic fluency strategies over time than controls.

Conclusions: Together, these results suggest that deficits in strategic processing emerge and are exacerbated as children with PKU age. Correspondence: Neco Johnson, Psychology, San Diego State University, 6505 Alvarado Rd, Ste 110, San Diego, CA 92120, United States. E-mail:necoxjohnson@gmail.com

J.E. KARR, G.L. IVERSON \& M.A. GARCIA-BARRERA. Multivariate Assessment of Executive Functions: Frequency of Low Scores on the Delis-Kaplan Executive Function System.

Objective: Neuropsychologists often use multiple standalone measures to evaluate executive functions, without clear guidelines regarding the normal variation that occurs among executive function test scores. The current study quantified the prevalence of low scores among individuals completing a three-test battery from the Delis-Kaplan Executive Function System (D-KEFS).

Participants and Methods: Stratified by education and intelligence, multivariate base rates of low scores were derived for a three-test D-KEFS battery using the adult portion of the normative sample (i.e., ages $16-89 ; N=1,050)$. The battery consisted of the three most commonly administered D-KEFS tests (i.e., Trail-Making, Color-Word Interference, and Verbal Fluency), which together produce seven Total Achievement scores (i.e., the primary indicators of executive function ability).

Results: Low scores occurred commonly among the normative sample, with $62.8 \%$ of the sample having one or more scores at or below the 16th percentile, $36.1 \%$ having one or more scores at or below the 5 th percentile, and $26.8 \%$ having one or more scores at or below the 2 nd percentile. Low scores occurred more frequently among individuals with lower intelligence and less education.

Conclusions: It is common for adults in the general population to obtain one or more low scores when given a brief battery of tests evaluating executive functions. Knowing the frequency of low scores in the general population will improve the accuracy with which clinicians and researchers can identify executive function deficits in clinical 
groups, such as those with traumatic brain injuries or neurodegenerative diseases.

Correspondence: Justin E. Karr, M.Sc., Psychology, University of Victoria, P.O. Box 1700 STN CSC, Victoria, BC VSW 2Y2, Canada. E-mail:jkarr@uvic.ca

M.M. MOHN, K.L. KRICHBAUM, Z.M. SHANKLE \& M.M. KNEPP. Rey-Osterrieth Complex Figure Task Performance and Negative Affect Predict Emotion Regulation and Health Behavior.

Objective: Multiple studies have investigated the relationship between anxiety, health behaviors, and brain function. For example, Nichter and Chassin (2015) found a positive correlation between physiological responses to anxiety and alcohol consumption. Anderson and Shivakumar (2013) found that exercise has been linked to increases in brain-derived neurotropic factor, relating that to decreased behaviors of anxiety and depression. It was hypothesized that decreased performance on the Rey-Osterrieth Figure Task (REYO) would predict higher depression and anxiety scores along with poorer emotion regulation. Individuals with higher worry and/or depression and poor REYO scores would then report more problematic drinking and engage in more negative health behaviors.

Participants and Methods: 114 students at a Midwestern University completed the REYO followed by the modified Beck Depression Inventory (mBDI), Penn State Worry Questionnaire (PSWQ), Emotion Regulation Questionnaire (ERQ), Rutgers's Alcohol Problem Index, and Obligatory Exercise Questtionnaire.

Results: Increased scores on the copy portion of the REYO predicted lower scores of problematic drinking $\left(R^{2}=.114, F(1,111)=14.25\right.$, $p<.001)$, and higher scores on the PSWQ predicted decreased emotion reappraisal on the ERQ $\left(R^{2}=.070, F(1,112)=8.49, p<.005\right)$ and higher depression scores $\left(R^{2}=.146, F(1,112)=19.10, p<.001\right)$. Higher mBDI scores also predicted decreased emotion reappraisal on the ERQ $\left(R^{2}=.185, F(1,112)=25.45, p<.001\right)$ and exercise scores $\left(R^{2}=.036, F(1,112)=4.16, p<.05\right)$.

Conclusions: Higher depression scores were related to lower exercise scores, which concur with the findings of Anderson and Shivakumar (2013). Lower emotional appraisal was significantly associated with higher depression scores, which is supported by findings from Delleman and Fernandes (2015). Future research should further explore how executive function can impact the relationship between negative affect and poor health behaviors.

Correspondence: Michael M. Knepp, PhD, Psychology and Neuroscience, University of Mount Union, 1972 Clark Avenue, Alliance, OH 44601 3993, United States. E-mail: kneppm@mountunion.edu

\section{N. KURNIADI, Z. MESTRE, J. LIANG \& K. BOUTELLE. Specificity of Disinhibition in Overweight and Obese Children.}

Objective: Disinhibition has been linked with overeating in overweight and obese children. However, it remains unclear whether overweight and obese children exhibit disinhibition globally or primarily in response to food stimuli. The current study investigates the differential relationships of global versus food-specific disinhibition and overconsumption in overweight and obese children.

Participants and Methods: One hundred and fourteen overweight and obese, treatment-seeking children aged 8-13 completed baseline assessments. To measure global disinhibition, children were administered the Delis-Kaplan Executive Function System (DKEFS) Color Word Interference Test (CWI) and Stop Signal Task (SST). To measure food-specific disinhibition, children completed a modified version of the Stroop test with calorically-dense food words and non-food words and a modified version of the SST that presented calorie-dense food and non-food pictures. Caloric intake was measured using a free access food laboratory paradigm (i.e. sham taste test).

Results: Response times on food and non-food items were significantly correlated on the Food Stroop task $(r=0.9, p<0.0001)$ and on the SST $(r=0.97, p<0.0001)$, suggesting that participants did not differ between global and food-specific disinhibition tasks. Slower inhibition response time and a higher number of errors in inhibition/switching subtests of the CWI were significantly associated with total calories consumed ( $r=-0.25, p=0.03 ; r=-0.24, p=0.04$, respectively).

Conclusions: Findings suggest that overweight/obese children demonstrate disinhibition in response to both food and non-food stimuli. Global disinhibition was associated with greater food intake. Strenghtening inhibitory control may reduce excessive food intake in overweight and obese children.

Correspondence: Natalie Kurniadi, M.A., Clinical Psychology, California School of Professional Psychology, PO Box 7471, San Diego, CA 92167, United States.E-mail: natalie.kurniadi@alliant.edu

T. LE, E.E. MORAN, K. MCNEAL, A. MASAND, A.M. BRICKMAN \&. M.E. ZIMMERMAN. Executive Function is Associated with Sleep Disruptions in Young Adults with Depression.

Objective: Previous research has established a relationship among sleep, depression, and cognition. Irregularities in sleep are common indicators of depression, and can cause deficits in cognitive functioning. The purpose of this study is to further understand the interrelationship among depression, sleep and cognitive functioning in a non-clinical sample of young adults.

Participants and Methods: This cross-sectional study comprised 82 participants $(74 \%$ female, age in years $=19.9$ (1.3). All participants wore an actigraph for 7-11 days to measure average sleep time and wake after sleep onset (WASO). Participants completed the Beck Depression Inventory (BDI-II) to measure depressive symptoms and completed Trail Making Test Part A (TMTA) and Part B (TMTB) to measure attention and executive function.

Results: Participants with higher depressive symptoms had more WASO $(\mathrm{r}=0.30, \mathrm{p}<0.01)$ and poorer performance on TMTB $(\mathrm{r}=0.26$, $p=0.02)$ and participants with more WASO had poorer performance on TMTB $(\mathrm{r}=0.31, \mathrm{p}<0.01)$. TMTA and total sleep time were not related to BDI-II. A formal test of mediation did not support sleep as a mediator of the relationship between BDI-II and performance on TMTB. Linear regression with the inclusion of an interaction term (overall model $\mathrm{F}(3,69)=9.12, \mathrm{p}<0.01)$ revealed that WASO was only associated with executive function in individuals with clinically significant depressive symptoms $(\mathrm{r}=0.65, \mathrm{p}<0.01)$ and not in those without depressive symptoms $(\mathrm{r}=0.06, \mathrm{p}=0.68)$.

Conclusions: This study further highlights the complex interrelationship among depression, sleep, and cognition and suggests that clinicians should pay close attention to associated disruptions in sleep and cognition in young adults with clinically significant depressive symptoms.

Correspondence: Tiffany Le, Bachelors of Science, Psychology, Fordham University, 550 E. 189 Street, Apt 14, Bronx, NY 10458, United States. E-mail: tle11@fordham.edu

M. MACKIE \& J. FAN. Common Deficits of The Cognitive Control Network in Three Neuropsychiatric Disorders.

Objective: Cognitive control is essential to efficient information processing and behavioral response under conditions of uncertainty. While cognitive control has often been attributed to the frontal lobes, functional neuroimaging studies have revealed the involvement of widespread cortical and subcortical regions (i.e., the cognitive control network, CCN). In neuropsychiatric disorders, there are often reported disruptions to cognitive control functioning and abnormal activity of regions of the CCN. This meta-analysis of functional neuroimaging studies was aimed to provide converging evidence of atypical recruitment of common regions of the CCN across neuropsychiatric disorders. Participants and Methods: We conducted activation likelihood estimation meta-analyses of functional neuroimaging studies of cognitive control in healthy controls and three patient populations: 24 studies of autism spectrum disorder, 46 studies of schizophrenia, and 24 studies of major depressive disorder, in contrast to 59 studies of healthy controls. 
Results: Across the three disorders, atypical patterns of hypoactivity of CCN regions were identified, most notably in anterior cingulate cortex, anterior insula, dorsolateral prefrontal cortex, basal ganglia and thalamus. In addition, a pattern of hyperactivity and failure to deactivate lower-level sensory, default mode, and limbic regions was also observed within each of the three disorders.

Conclusions: These results highlight the common role of the CCN in various neuropsychiatric disorders. In the absence of an adequate level of CCN regulation, external task-irrelevant sensory information and internal thoughts and feelings and may compete with task-relevant stimuli for limited attentional resources, contributing to the clinical presentation of these disorders.

Correspondence: Melissa-Ann Mackie, Psychology, CUNY Graduate Center/Queens College, 1133 Commerce Drive, Apt 519, Decatur, GA 30030, United States. E-mail:mmackie@qc.cuny.edu

T. MAYNARD, E.R. APPLEMAN, A. CRONIN-GOLOMB \& S. NEARGARDER. Objective Measurement of Sleep by Smartphone Application - Comparison with Actigraphy and Relation to Cognition, Mood, and Self-Reported Sleep.

Objective: Sleep plays an integral role in health and cognition; insufficient or fragmented sleep can negatively affect attention and arousal. Multiple technologies have been developed to measure sleep variables such as total sleep time (TST) and sleep efficiency. It remains to be seen whether smartphone applications are comparable to clinical devices that can be used at home, such as actigraphy. The present study aimed to validate the use of one smartphone application in a healthy adult population and explore the relation between self-reported and objective measures of sleep, mood, and cognition.

Participants and Methods: 18 healthy adult participants (mean age $=26.6$ years, std. dev. $=14.4$ years) completed a sleep diary while using a smartphone application to monitor their sleep for one week; a subset of 8 participants also wore a wrist actigraph during the same week. Following data collection, all participants completed self-report questionnaires and assessments of executive functioning and working memory.

Results: There was no significant difference in TST obtained by sleep diaries and the smartphone application. The smartphone application significantly overestimated TST and sleep efficiency when compared to actigraphy. There was no correlation between TST or sleep efficiency and performance on cognitive tasks. Self-reported sleepiness as measured by the Epworth Sleepiness Scale was significantly correlated with anxiety as measured by the Beck Anxiety Inventory and selfreported sleep quality as measured by the Pittsburgh Sleep Quality Index.

Conclusions: While potentially appealing to users, smartphone technology may not provide a sensitive enough metric of sleep in a healthy population; future work is needed to clarify its clinical utility. TST and sleep efficiency do not appear to impact cognition in healthy individuals; however, self-reported sleepiness may be related to additional self-report metrics of mood and sleep.

Correspondence: Taylor Maynard, 90 Burrill Avenue, Room 332, Bridgewater, MA 02324,United States.E-mail:TMAYNARD@student. bridgew.edu

W.T. MCCUDDY, P. PATEL, S. POTTS, K. REITER, K. HAZLETT ELVERMAN, A. PORCELLI \& K.A. NIELSON. The Role of Resting Versus Stress-Induced Autonomic Regulation on Inhibitory Control Performance Across the Lifespan.

Objective: Thayer's neurovisceral integration hypothesis suggests a link between cognitive and autonomic functioning that could be used in predicting future cognitive decline. Yet, how this relationship is affected by both age and stress is not well understood, representing a gap in the literature. This project explores the relationship of heart rate variability (HRV) at rest and during stress on a measure of inhibitory control (IC) across the adult age span.
Participants and Methods: Fifty-nine adults were divided into young Young $(M=23.19, S D=2.60)$, Middle $(M=59.62, S D=4.44)$, and Older $(M=72.89, S D=6.39)$ groups. HRV was measured by electrocardiogram (using SD between heartbeats; SDNN) during three epochs: (i.e., rest, Social Evaluative Cold Pressor Test [SECPT], and a stopsignal [PGNGS] task. The PGNGS task served as a cognitive stressor and provided the primary measure of IC via stop-signal reaction time (SSRT).

Results: Age differences in HRV emerged at rest, $F(2,56)=8.37, p=.001$, at SECPT, $F(2,26)=5.48, p=.010$, and trended at PGNGS, $F(2,45)$ $=3.10, p=.055)$. While rest HRV was positively related to IC across age groups, PGNGS-HRV was related positively to IC in the Young, negatively to IC in the Middle group, and unrelated to IC in the Older group. On examination of the relative impact of age and each HRV variable on IC via linear regression, all but SECPT-HRV were considered independent predictors of inhibitory control (SSRT).

Conclusions: HRV measured at rest and during cognitive stress were differentially related to IC. Greater resting HRV was related to better IC for all age groups. Yet, for middle-aged adults, for whom early detection of cognitive symptoms is key, higher HRV at rest but lower HRV during cognitive demand demonstrated the ideal autonomic pattern for the best cognitive performance. Such an understanding is necessary for research geared toward early detection and development of therapeutic strategies aimed at enhancing and protecting executive functioning into older age. Correspondence: William T. McCuddy, BA, University of Kentucky, 1701 Lin-Wal Ct., Lexington, KY 40505, United States. E-mail: wtmccuddy@ gmail.com

E. MCLEAN, U.D. PERSAUD, M.A. PIEVSKY, K.S. MAZZOLA, L. TIERSKY \& B. FREER. Depression Explains the Relationship Between Cognition and Social Adjustment in Adults with Cognitive Complaints.

Objective: Cognitive decline has been associated with social withdrawal in adults; however, individuals' perceptions of their decline may be misleading and are often weighted by other factors, most notably depression. The present study examined whether objective or subjective measures of working memory (WM) and processing speed (PS) better predict social adjustment.

Participants and Methods: Participants were 110 individuals seeking neuropsychological evaluation for cognitive complaints. Participants completed objective and subjective measures of WM and PS, the MMPI-2 and a measure of social adjustment.

Separate hierarchical linear regression models for each cognitive construct were conducted to predict the Social/Leisure subscale T-score of the Social Adjustment Scale (SAS), after controlling for age and gender. Objective and subjective measures of the PS regression included WAIS-III PSI and the distractibility subscale of the CFQ, while the WM regression included the WAIS-III WMI and forgetfulness subscale from the CFQ. Additional linear regressions were run including the depression subscale from the MMPI-2.

Results: Both regressions revealed that both subjective measures of working memory $(\mathrm{B}=.230, \mathrm{SE}=1.504, \mathrm{p}<.05)$ and processing speed $(\mathrm{B}=.256, \mathrm{SE}=1.509, \mathrm{p}<.01)$ significantly predicted SAS social/leisure subscale. Meanwhile, the linear regression was not significant for objective measures of working memory $(p=n . s$. ) and processing speed $(\mathrm{p}=$ n.s.). However, when depression was included in each model these results were no longer significant $(p>.05)$ and depression remained the sole factor (MMPI-2 Depression subscale both $\mathrm{p}<.001$ ).

Conclusions: Our findings suggest that subjective complaints related to individual's perception of their social relationships; however, perception of social maladjustment was better explained by current depressive symptoms. Thus, it is prudent to consider that complaints of cognition impacting social interactions might largely be due to unacknowledged depressive symptoms. 
Correspondence: Erin McLean, B.A., Psychology, Fairleigh Dickinson University, 317 Old Mill Rd., Spring Lake Heights, NJ 07762, United States.E-mail: eamclean814@gmail.com

E.E. MORAN, K. MCNEAL, T. LE, A. MASAND, A.M. BRICKMAN \& M.E. ZIMMERMAN. Highly Variable Blue Light Exposure is Related to Poor Sleep and Cognition in Young Adults.

Objective: The relationship between sleep deprivation and cognitive dysfunction is well established. Light exposure has a powerful influence on circadian physiology and can greatly impact sleep. The circadian photoreception system is highly sensitive to blue wavelenght light. A ubiquitous source of blue light is electronic devices that contain light-emitting diodes, such as smartphones. As varied pulses of light impact circadian physiology, variability in the light exposure may impact this mechanism. The purpose of this study was to examine the relationship among blue light, sleep, and cognition.

Participants and Methods: A total of 82 participants (74\% female, mean age $=19.9, \mathrm{SD}=1.3$ ) were included in this cross-sectional study. All participants wore an actigraphic monitoring device for a period of 7-11 days to measure sleep and light exposure. Sleep time and blue light exposure in the two hour interval prior to sleep were calculated. The coefficient of variation was calculated for blue light exposure. After completion of the monitoring period, participants underwent assessment of learning and memory and executive functioning using the computer-based CogState battery.

Results: Of the 82 participants, $26(31.7 \%)$ had a coefficient of variation of blue light exposure greater than or equal to 1 , indicating a high degree of variability. Independent samples t tests revealed that those with high variability had significantly shorter sleep time over the study period $(t=3.00, p=0.004)$. Although there was no difference between those with high and low variability in blue light exposure on learning, there was a trend towards significantly more errors on recall $(t=2.37$, $\mathrm{p}=0.058)$ and lower accuracy on the two-back test of working memory $(\mathrm{t}=1.97, \mathrm{p}=0.052)$.

Conclusions: This study demonstrates that high variability in nocturnal blue light exposure is associated with poorer sleep that may negatively affect memory and executive functioning. Future studies should examine relational changes over time between blue light and sleep.

Correspondence: Eileen E. Moran, MSc, Psychology, Fordham University, 600 W. 138th St., Apt 33, New York, NY 10031, United States. E-mail: emoran@fordham.edu

\section{M.A. NIERMEYER, R.E. ZIEMNIK \& Y. SUCHY. Slower Action Planning is Associated with More Obstacle Contacts During an Obstructed Walking Task.}

Objective: We recently proposed that action planning is an aspect of executive functioning (EF; Suchy, 2015), and that action planning allows individuals to quickly reprogram new action trajectories when presented with obstacles in their environment (Niermeyer, Franchow, \& Suchy, 2016). This study examined whether action planning during motor sequencing predicts performance on an obstructed walking task. Participants and Methods: Thirty-two healthy older adults (Mean Age =69.84) completed a computerized sequencing task (Push Turn Taptap task; PTT) designed to assess action planning; an obstructed walking task under both single and dual-task (serial-subtraction) conditions; the Dementia Rating Scale $2^{\text {nd }}$ edition (DRS-2) as an index of general mental status; and selected subtests from the Delis-Kaplan Executive Function System as indices of EF and lower-order component processes (CP; processing speed, graphomotor control ect.).

Results: Hierarchical regressions using the total number of obstacle contacts as the outcome variables were conducted. For the single-task walking condition, CP but not EF nor action planning, accounted for variance in the number of obstacle contacts beyond DRS-2 scores $(p=.004)$. In contrast, for the dual-task walking condition, action planning accounted for variance in the number of obstacle contacts beyond all other variables (DRS-2, CP, and EF scores, $p=.042$ ).
Conclusions: These results support the notion that faster action planning is associated with successful obstacle navigation, at least under executively demanding (i.e., dual task) circumstances. In addition, CP (e.g. processing speed) also relate to obstacle navigation. Among older adults, tripping over an obstacle is a common reported reason for falls (Hill et al., 1999), and dual-task walking performance has been shown to predict falls (Ayers et al., 2014). Taken together with the current results, this suggests $\mathrm{CP}$ and action planning may also predict fall risk. Correspondence: Madison A. Niermeyer, MS, Clinical Psychology Department, University of Utah, Department Of Psychology University Of Utah380 S 1530 E Beh S 502, Salt Lake City, UT 84112, United States.E-mail:madison.niermeyer@psych.utah.edu

\section{K.H. NIXON, A.A. TURK NOLTY, D. BOSCH \& S. AMANO. Can Executive Functioning Bolster Learning During Times of Overwhelming Stress.}

Objective: Experiences of chronic or overwhelming stress lead to cognitive fatigue resulting in a reduction in problem solving and memory efficiency. Individuals who are resilient to stress are able to perform more effectively in the face of stress, presumably due to implementation of strategies implicating more robust executive functions. The objective of this study was to investigate the relationship between executive functioning and learning outcomes in aid workers, a population who are at exposed to traumatic stress during their employment.

Participants and Methods: Twenty-four aid workers (6 women \& 18 men) between the ages of 19 and $60(M=39.0, S D=10.2)$ with an average of 10.4 years $(S D=6.8)$ of experience completed the Rey Complex Figure Test (RCFT) and the California Verbal Learning Test-II (CVLT-II) on two separate days of a training workshop. They also filled out the Behavior Rating Inventory of Executive Functioning (BRIEF-A). Results: The BRIEF-A Behavior Regulation Index correlated negatively with Day 4 RCFT Delayed Recall $(r=-.51, p=.025)$ and a negative trend toward significance for the ratio between Day 4 Delay Recall and Immediate Recall $(r=-.39, p=.096)$. RCFT executive functioning markers also showed a trend toward significance with the ratio of Long Delay Free Recall and Trial 5 on the CVLT-II $(r=.42, p=.054)$.

Conclusions: Based on this study, there appears to be a benefit to enhanced executive functioning on learning outcomes during times of extreme stress that are imperceptible in otherwise sterile conditions. Future studies should investigate how to increase the sensitivity on measures of executive functioning in order the capture aid workers who are at risk to traumatic stress syndromes that otherwise appear adequate on testing in a structured formal setting.

Correspondence: Kyle H. Nixon, M.A., Fuller Graduate School of Psychology, 135 N. Oakland Ave., Pasadena, CA 91182, United States. E-mail: kylenixon@fuller.edu

\section{GANSLER, R. NYENHUIS, E. WASSERMANN, E. HUEY \&} J. GRAFMAN. Measuring patient self-awareness deficit (PSAD). Objective: Patient Self-Awareness Deficit (PSAD) has typically been determined by clinical observation; however, the growth of behavioral rating scales with parallel caregiver and patient forms provides the opportunity to develop psychometric approaches to identifying PSAD. Participants and Methods: Data from 83 patients recruited at the National Institute of Neurologic Disorders and Stroke (NINDS), meeting diagnostic criterion for behavioral variant Frontotemporal Dementia (bvFTD, $n=29$ ), Corticobasal Syndrome (CBS, $n=38$ ), or Primary Progressive Aphasia (PPA, $n=13$ ) were analyzed.

Executive, language, and memory function were measured by the Delis-Kaplan Executive Function System (3 subscale composite), Boston Naming Test, and DRS-2 Memory Scale, respectively. PSAD was operationalized by subtracting the Frontal Systems Behavioral Scale patient after total score from the caregiver after total score, such that higher scores reflected less awareness of the apathy, disinhibition, and executive dysfunction item content. 
Results: PSAD scores were uncorrelated with executive $(r=.06 \mathrm{~ns})$, language $(r=.05, n s)$, or memory function $(r=.01, n s)$.

PSAD scores varied by diagnostic group $(f=6.16, p=.003)$, and were highest in participants suffering from bvFTD.

PSAD scores were predicted by left medial orbital frontal volume $(\beta=-.43)$ after accounting for total intracranial volume.

Conclusions: Analyses support the viability of a psychometric approach to PSAD. A PSAD psychometric was independent of measures of executive, language and memory dysfunction, supportive of divergent validation. PSAD values were highest- as expected- in the diagnostic group with frontal pathology (bvFTD), supportive of criterion validity. Neural correlates of the PSAD psychometric were identified in a brain region associated with social and emotional cognition.

Correspondence: Robert Nyenhuis, Bachelor of Science in Psychology, Psychology, Suffolk University, 201 Prospect Ave. NE, Grand Rapids, MI 49503, United States. E-mail: robnyen@gmail.com

L.C. OBERMEIT, E.E. MORGAN, J.E. IUDICELLO, R.K. HEATON \& I. GRANT. The Cognitive Profile of Sexual Risk-Taking Intentions. Objective: Sexual risk-taking significantly contributes to high rates of STIs/HIV and unplanned pregnancies (e.g., Centers for Disease Control, 2013). Intentions are the most robust and proximal predictors of sexual risk-taking and research has identified multiple predictors of intentions, including attitudes and expectations about the target behavior, perceived susceptibility to consequences, and beliefs about how normative the behavior is (e.g., DeHart \& Birkimer, 2010). However, little is known about the cognitive contributors of intentions to engage in sexual risk. It is important to better understand the predictors of risky sexual intentions in order to improve sexual risk-taking interventions and reduce the consequences associated with such behavior.

Participants and Methods: Participants included 246 adults (ages 18-68) who completed comprehensive neuropsychological and neuromedical assessments. Neuropsychological performances were grouped into 7 cognitive domains (e.g., executive functioning). The associations between demographically corrected domain $\mathrm{T}$ scores and the Sexual Risks Scale Intentions to Try subscale score were evaluated. Due to the robust research showing that past behaviors are among the best predictors of future behaviors, it was predicted that learning and memory would be associated with intentions.

Results: Bivariate correlations showed that executive functioning $(p=.015)$ was significantly associated with intentions to engage in risky sexual behavior and remained significant $(p=.038)$ when accounting for other covariates (age and gender) in a linear regression. Learning $(p=.082)$ and working memory $(p=.080)$ trended toward significance at the bivariate level only.

Conclusions: Higher level cognitive functioning, and possibly learning and working memory, are important for creating behavioral intentions and may be an efficacious target of sexual risk-taking interventions. Correspondence: Lisa C. Obermeit, SDSU/UCSD Joint Doctoral Program in Clinical Psychology, 220 Dickinson Street, Suite B, San Diego, CA 92103, United States. E-mail: lisaobermeit@gmail.com

\section{T.E. OWENS, A. CHAU, V. PAPA \& L.E. MARTIN. Effect of Mood Induction and Trait Anxiety on Delay Discounting Performance in fMRI.}

Objective: The diminished ability to delay immediate rewards to achieve long-term goals is a hallmark of impulsive behavior. Though traditionally characterized by sensitivity to reward and insensitivity to punishment, recent research has shown relationships between impulsive choices and personality traits, such as negative urgency, anxiety, and depression. However, the underlying neural substrates for reward sensitivity, negative affect, and personality traits are relatively unexplored. Participants and Methods: Nonclinical participants $(\mathrm{n}=17)$ engaged in a computerized delav discounting functional magnetic resonance imaging (fMRI) task administered in a 3T Siemens Skyra scanner while undergoing mood induction. Participants made decisions that weighed smaller, short-term monetary rewards versus more valuable, long-term monetary rewards. Negative and neutral mood conditions were induced using corresponding Velten statements paired with mood-congruent music. Behavioral measures of impulsivity were also completed by participants, including the UPPS+P. The Beck Anxiety Inventory Trait, and Center for Epidemiologic Studies Depression Scale (CES-D). Results: Activation in a priori regions of interest (ROI) related to impulsive decision-making showed significant right ventrolateral prefrontal cortex (vlPFC) activation associated with trait anxiety when experiencing negative affect, with no behavioral differences in participants' choices. No other ROIs were significantly related to personality measures. Conclusions: We suggest that the right vIPFC hyperactivation may reflect increased recruitment to maintain performance versus failure to recruit leading to impaired performance, particularly since we did not see a corresponding behavioral change. We believe this increase in activation reflects additional cognitive demand required by those with high trait anxiety to make decisions that are less cognitively taxing for those with lower trait anxiety.

Correspondence: Tyler E. Owens, Ph.D., Psychiatry and Psychology, Mayo Clinic, 670 28th St SE, 6-147, Rochester, MN 55904, United States.E-mail: owens.tyler@mayo.edu

\section{N. PENNINGTON. Frustration Impairs Performance on a Task of Executive Function in Females Only.}

Objective: This investigation aimed to explore the mood-cognition relationship by specifically considering the effects of frustration on executive function. Previous pilot data showed that experimentally induced frustration is associated with poorer executive performance (Pennington, 2016). The present study examined this effect in a much larger sample. Participants and Methods: Frustration's effects on executive function were measured by performance on a modified, more difficult version of the Trail Making Test B. a task thought to heavily employ shifting and working memory abilities. Prior to completing this measure, the experimental group completed a frustration-inducing task. Control participants completed a letter fluency task, representing a neutral mood state. Sixty-six adults $($ Female $=43$, Male $=23)$ with a mean age of 21.47 $(S D=6.73)$ and $13.30(S D=1.64)$ vears of education participated. Participants were randomly assigned to experimental $(\mathrm{N}=33)$ and control $(\mathrm{N}=33)$ conditions.

Results: Independent samples t-tests, two-tailed, confirmed a significantly slower completion time (seconds) in the experimental group $(M=202.24, S D=84.41)$ versus neutral-mood controls $(M=145.36$. $S D=52.69) ; t(64)=3.2836, p=0.0017 ; d=0.808$. Experimental participants also made significantly more errors $(M=2.24, S D=2.28)$ compared to controls $(M=0.88, S D=1.17): t(64)=3.0610$. $p=0.0032 ; d=0.751$. The increased sample size revealed a gender effect: males showed no sionificant differences after frustration, but frustrated females were slower, $t(41)=3.0140, p=0.0044 ; d=0.957$, and made more errors, $t(41)=2.9541, p=0.0052 ; d=0.942$.

Conclusions: These results suggest that frustration may impair executive performance by compromising working memory, shifting, or both. but only in females. The study supports previous evidence that even mild fluctuations in normal, non-clinical moods can markedly impact cognition, and raises implications for validity in neuropsychological assessment and research.

Correspondence: Nicholas Pennington, PSYCHOLOGY, UNIVERSITY OF UTAH, 380 S 1530 E, BEH S 502, SALT LAKE CITY, UT 84112 , United States.E-mail:nicholas.pennington@psych.utah.edu

U.D. PERSAUD, M.A. PIEVSKY, A. Allart, E. MCLEAN, K.S. MAZZOLA, B. FREER \& L. TIERSKY. Cognitive Flexibility and Coping in Adults with Cognitive Complaints.

Objective: Cognitive flexibility is essential for shifting approach to adapt to changing situational demands. It is believed that there are two types of coping strategies one may employ to manage stress: problemfocused coping. in which an individual relies on logical problem-solving. 
and emotion-focused coping, in which the individual manages emotional distress. While cognitive flexibility is related to the use of various strategies to adjust to life stressors, less is known about how coping strategies are employed. The present study examines the relationship between cognitive flexibility and the use of problem- and emotion-focused coping strategies.

Participants and Methods: Participants were 226 individuals seeking neuropsychological evaluation for cognitive complaints at a psychological service center in the northeast US. Participants completed three subtests of the Delis-Kaplan Executive Functioning System assessing cognitive flexibility: the Color-Word Interference Task, Verbal Fluency, and Trail Making Test. Participants also completed the COPE Inventory to assess problem- and emotion-focused coping strategies.

Results: Hierarchical linear regressions were conducted to assess whether neuropsychological measures of cognitive flexibility were related to coping strategies, after controlling for age and gender. Results indicated that performance on the Category Switching condition of Verbal Fluency was negatively associated with the use of emotionfocused coping strategies, $\mathrm{B}=-.02, \mathrm{SE}=.01, \mathrm{r}^{2}=.02, \mathrm{p}<.05$. Tasks of cognitive flexibility were not associated with problem-focused coping strategies.

Conclusions: Our findings suggest that verbal fluency performance is inversely related to the use of emotion-focused coping strategies. This may indicate that the value of developing a broader vocabulary and the ability to flexibly switch between word categories are important to appraising emotional responses in treatment. Our results highlight an important relationship between verbal flexibility and emotion-focused coping.

Correspondence: Usha D. Persaud, PhD, Psychology, Fairleigh Dickinson University, 81 Bessida Street, Bloomfield, NJ 07003, United States.E-mail: upersaud7@gmail.com

B.W. REYNOLDS, M.R. BASSO, L. LAU, A.K. MILLER, D. COMBS \& D. WHITESIDE. Associations of Decision Making and Concept Formation with Risky Choices in Healthy Adults.

Objective: Risky acts (e.g., promiscuity, substance misuse, antisocial behaviors) are a salient problem among young adults. Previous research has evaluated whether personality or cognitive measures of impulsivity predict risk-taking. Nonetheless, few investigations have simultaneously evaluated the relative contribution of personality and neuropsychological aspects of impulsivity and decision-making to risky acts. Towards this end, the current investigation evaluated the relative impact of self-reported and cognitive impulsivity and executive functioning with various aspects of risky behavior.

Participants and Methods: Fifty healthy undergraduate students without neurologic disease or developmental disorder were administered the UPPS-P, a well-validated impulsivity questionnaire (Cyders et al., 2007), the Wisconsin Card Sorting Test (WCST; Grant \& Berg, 1948), and the Iowa Gambling Task (Bechara et al., 1994). Response bias was assessed with the Marlowe-Crowne Social Desirability Scale (MCSDS; Crowne \& Marlowe, 1960) and Reliable Digit Span (RDS; Greiffenstein, et al., 1994). Past two-month risk-taking was also assessed.

Results: Risky acts were classified into four domains with satisfactory internal consistency (alphas >.7): overall risk-taking, promiscuity, antisocial acts, and substance misuse. Risky behaviors were log-transformed to achieve normality. Employing multiple regression, the UPPS-P, IGT, and WCST each emerged as significant predictors of overall risk-taking. Only the WCST accounted for promiscuity and substance misuse. The IGT was the lone predictor of antisocial activity. Significant semi-partial correlations ranged from 2 to .35 .

Conclusions: These findings extend past results indicating that cognitive and personality aspects of impulsivity correlate with risky acts. Extending previous research, these data show that such measures predict promiscuity and antisocial acts in addition to substance misuse. Notably, these occur in a healthy young adult sample, enhancing the ecological validity of the IGT and WCST.
Correspondence: Bradley W. Reynolds, PhD, Psychology, University of Tulsa, 800 S Tucker Road, Lorton Hall 308, Tulsa, OK 74104, United States.E-mail:bradley-reynolds@utulsa.edu

A. RIVERA, L. ABRAMS \& L.J. ALTMANN. Competing conflict leads to bilingual disadvantage: Performance on an explicit and implicit Simon task.

Objective: The purpose of our study was to examine the effects of bilingualism on performance in an implicit and explicit Simon task.

Participants and Methods: Participants included 56 bilingual and 57 monolingual healthy young adults. Participants completed linguistic and nonlinguistic Simon tasks with either implicit vs explicit instructions. Attending to the language/orientation was either implicit (when the task required naming the color) or explicit (when the task required naming the language or orientation). The linguistic stimuli were the color words 'BLUE', 'GREEN', 'AZUL', or 'VERDE' shown in black font, whereas the nonlinguistic stimuli comprised of blue and green rectangles. The directions for the implicit Simon task instructed participants to name the color of the presented stimuli, ignoring the completing conflict of either the language (English or Spanish) or orientation (vertical or horizontal). The directions for the explicit Simon instructed participants to indicate the language or orientation, ignoring the competing conflict of the color of the stimuli. The dependent variables were the Simon Effect (Incongruent RT - Congruent RT) and the Global effect (mean of Congruent and Incongruent RTs).

Results: Results showed that bilinguals were significantly disadvantaged on the Simon effect when the stimuli was in written form (linguistic stimuli) for both implicit (naming the color) and explicit tasks (naming the language/orientation). Bilinguals had a larger Simon effect compared to monolinguals, but there was no significant difference between groups for global effects.

Conclusions: Results suggest a bilingual disadvantage on tasks that require any mode of language processing.

Correspondence: Ana Rivera, PhD, Speech Language and Hearing Sciences, University of Florida, PO Box 117420, Gainesville, FL 32611, United States. E-mail: amrivera@ufl.edu

\section{J. SALVADOR-CRUZ, C.G. ARMENGOL, E.E. SANCHEZ VIELMA \& L.A. LEDESMA TORRES. Executive Function and Reading Comprehension: Performance of Mexican 9-year-olds on a Non-} Linguistic Measure of Inhibition.

Objective: Objective - To explore an understudied aspect of EF: inhibition, on reading comprehension. To examine performance of Spanish-speaking children when using a non-linguistic alternative to the color-word interference test.

Participants and Methods: Participants and Method - Thirty 9 year-old school children (15 boys, 15 girls) with no neurological or psychiatric conditions were individually administered the Reading Comprehension (RC) subtest of PROLEC-RM (Mexican adaptation of a Spanish comprehensive battery of reading ability) and the Five Digit Test (FDT) - a non-linguistic alternative to the Stroop ColorWord Test (SCWT). Independent variables were correct responses for RC and response speed for the FDT interference condition. Given the non-normal distribution of the independent variables, data were analyzed via Spearman's Rho.

Results: $\underline{\text { Results }}$ - Spearman's Rho=.068 was non-significant. No gender differences were observed. Mean correct RC score $=11.431$. Mean FDT interference RT= 75.13.

Conclusions: Discussion - Findings showed no support for inhibitory control as an independent contributor to reading comprehension. Inhibition is an identified component of the triad of executive functions impacting reading, but no consensus regarding its impact on reading comprehension has been reached (Christopher et al., 2013). More sophisticated assessment instruments that capture this complex cognitive process and the impact of socio-cultural variables must be incorporated 
in its clinical and experimental investigation in order to arrive at a more complete model of its neuropsychological underpinnings.

Correspondence: Judith Salvador-Cruz, Ph,D., Mexico City, Mexico. E-mail:salvadcj@gmail.com

O.A. SANTOS \& J. SOBLE. Predicting Performance on a Functional Executive Measure via a Brief Cognitive Screening Test.

Objective: The Montreal Cognitive Assessment (MoCA) is a brief cognitive screening tool with high sensitivity and specificity for detecting mild cognitive impairment (MCI), particularly with visuospatial and executive functioning (EF) impairments. EF also is a strong predictor of everyday functional abilities. The Pillbox Test (PT) is an ecologically-valid functional EF test designed to assess medication management abilities in vivo. This retrospective study investigated whether the MoCA predicted PT performance among Veterans.

Participants and Methods: A mixed clinical sample of 50 Veterans $(94 \%$ men) with a mean age of $64.36 \pm 12.53$ years and a mean education of $13.16 \pm 2.65$ years were administered the MoCA and the PT as part of a comprehensive neuropsychological evaluation. Correlational analyses assessed the associations between MoCA and PT scores and linear regressions examined whether the MoCA accurately predicted PT scores. Results: The MoCA was significantly correlated with some PT scores (-.42 to -.55). Regression analyses revealed the MOCA accounted for $30.6 \%(p>0.0001)$ of variance in the PT total number of pill errors, $23.2 \%$ ( $p>0.001)$ in number of misplacement move errors, $19.3 \%$ $(p>0.001)$ in total time to complete task, and $17.8 \%(p>0.01)$ in passfail rating scores, but did not significantly predict total number of omission errors.

Conclusions: Findings indicated that the MoCA was a significant predictor of four out of five PT scores, including total number of pill/ misplacement errors and task completion time. This study provides preliminary evidence that the MoCA may also be a useful predictor of some functional abilities in addition to functioning as an omnibus measure of cognitive/mental status.

Correspondence: Octavio A. Santos, BA, Psychology, University of Wisconsin-Milwaukee, 2441 E Hartford Ave, Department of Psychology, Garland Hall, Room 224, Milwaukee, WI53211, United States. E-mail: osantossolano@gmail.com

\section{M.E. SELEME, Y. ROMERO-QUINTANA \& L. ROJAS. Executive functioning and academic achievement in a group of elementary school students in Havana.}

Objective: The aim of the present study was to analyze the relation between executive functioning deficits and academic achievement in a group of elementary school students.

Participants and Methods: The participants were typically developing children aged 11 years old ( $\mathrm{n}=23,13$ female). Executive functioning deficits were assessed using the Childhood Executive Functioning Inventory (CHEXI) rated by teachers and performance-based tasks such as the Cancellation Attention Test, TMT part B and WISC-IV Digits subtest. Grading points accumulated (GPA) and grades in language (Spanish) and Math were obtained from school records as measures of school achievement.

Results: Correlation analyses revealed that the two CHEXI factors scores (i.e., working memory and inhibition) were significantly related to academic achievement. Significant and moderate correlations were also found between CHEXI scores and performance-based measures of executive functions, except for Cancellation Attention Test total score. Conclusions: Teacher ratings using the CHEXI are related to both performance-based measures of executive functioning and to academic achievement, which could be taken to mean that the CHEXI could be used to identify executive functions deficits and that this could possibly be used as a screening measure of low academic achievement in elementary school children.
Correspondence: Maria E. Seleme, MSc, Neurophysiology, Playa Community Mental Health Center, 5ta Ave e/38 y 40, Calle 7aA \# 6611, Havana 10300,Cuba.E-mail:esthersel@gmail.com

R.P. SO, C. GAUDET, B.C. KAVANAUGH, F. STEFFENALLEN, R. RANGAMANNAR \& K.A. HOLLER. The utility of neuropsychological assessment in detecting adolescents with a history of prior psychiatric hospitalizations.

Objective: The rate of rehospitalization in youth psychiatric care has significantly increased in recent years, although few studies have investigated the potential role of neurocognitive functions. This study investigated executive function (EF) as a predictor of prior multiple admissions among a sample of adolescents in a psychiatric inpatient program.

Participants and Methods: A retrospective medical chart review was conducted on 67 adolescents (history of prior admission $n=32$ ), who received a neuropsychological evaluation during their most recent hospitalization. Only those EF measures associated with admission status were included in the present study. A series of logistic regression analyses examined the association of $\mathrm{EF}$ to admission status after controlling for demographic variables.

Results: WCST Categories, RCFT Copy Trial, and TMT-B were significantly correlated with the number of hospitalizations and included in subsequent analyses. EF measures $\left(\chi^{2}(3)=15.216, p=.002\right)$ predicted rehospitalization above and beyond non-significant demographic variables $\left(\chi^{2}(3)=5.006, p=.171\right)$, explaining $34 \%$ of the variance in readmission and correctly classifying $68 \%$ of the cases. Examined individually, $\operatorname{RCFT}\left(\chi^{2}(1)=8.916, p=.003\right)$ and $\operatorname{WCST}\left(\chi^{2}(1)=11.954\right.$, $\mathrm{p}<.001)$, but not TMT-B $\left(\chi^{2}(1)=2.719, \mathrm{p}=.099\right)$, predicted rehospitalization with overall model (including demographics) classification rates of $65 \%$ and $73 \%$, respectively.

Conclusions: EF, especially planning/problem solving, may be particularly sensitive to detecting those adolescents with a history of prior psychiatric hospitalizations. If translated to the risk for future rehospitalization, the predictive utility of neuropsychological testing may provide value in treatment planning as well as insurance coverage among inpatient adolescents.

Correspondence: Rachel P. So, M.A., Psychological Sciences, University of Connecticut, 406 Babbidge Road, Unit 1020, Storrs, CT 06269, United States.E-mail: rachel.so@uconn.edu

J. STUDENY, E. WEBER, B.C. KAVANAUGH, K. MCCURDY \& K.A. HOLLER. The Executive and Non-Executive Demands of Constructional Measures within a Children's Psychiatric Inpatient Setting.

Objective: Several studies have identified the importance of non-executive (non-EF) demands on constructional tasks. However, little is known about the impact of executive functions (EF) on constructional abilities. This study examined the association between executive ability and performance on the Rey Complex Figure Test-Copy Condition (RCFT) and Beery-Buktenica Developmental Test of Visual-Motor Integration (VMI) within a children's psychiatric inpatient setting.

Participants and Methods: A chart review was conducted for 91 children (aged 6-12) who received a neuropsychological evaluation during a psychiatric inpatient hospitalization. Attentional/EF dysfunction was defined by a $\mathrm{T}$ score $<1.5 \mathrm{SD}$ below mean on any of 6 attention/EF measures (TMT-B; Stroop C-W; WCST PE; COWAT FAS; CPT-II Variability; WRAML-2 Sentence Memory). Grooved Pegboard (i.e., motor), Matrix Reasoning (i.e., perception), and Block Design (i.e., construction) were used to assess non-EF demands. Multiple regression analyses investigated the role of EF and non-EF demands on RCFT and VMI performance.

Results: Thirty-six percent of participants displayed low performance on the RCFT while $15 \%$ displayed low performance on the VMI. Demographic variables and fine motor skills were not associated with RCFT or VMI. RCFT performance predicted perceptual skills (i.e., Matrix Reasoning; $p=.003 ; \beta=3.44$ ) and attention/EF dysfunction severity 
$(p<.001 ; \beta=-.359)$. VMI performance was predicted by constructional skills (i.e., Block Design; $p<.001 ; \beta=.432$ ), but not attention/EF dysfunction severity $(p=.248 ; \beta=-.111)$.

Conclusions: Results show increased rate of low performance on the RCFT and VMI in children with severe psychiatric disorders. The RCFT was found to contain greater EF demands than the VMI and yields a greater rate of impaired performance in an inpatient child sample as compared to the VMI. Evaluation of children's performance on constructional measures should consider the influence of both constructional and executive functions to ensure proper interpretation.

Correspondence: Jane Studeny, NH, United States. E-mail: jstudeny@ antioch.edu

E. SULLIVAN \& C. NEUMANN. Trauma and Executive Functioning: An Examination of the Relationship between Trauma and Stroop Task Performance using a Large Web-Based Sample.

Objective: Psychological trauma and resulting PTSD symptoms can impact neuropsychological functioning, including higher-order executive functions (EF). The present study sought to examine the relationship between trauma, post-trauma symptoms, and EF using a large web-based sample. Evidence from a pilot study supported hypotheses that level of trauma and PTSD symptoms would relate to poorer Stroop performance.

Participants and Methods: A total of 957 participants (mean age $=$ 34.43 years; $47.9 \%$ female) completed questionnaires followed by two Stroop tasks (traditional and modified emotional), presented through the online platforms Amazon Mechanical Turk (MTurk) and Millisecond Inquisit. Questionnaires included two assessments of trauma: the Trauma History Questionnaire (THQ) and PTSD Checklist (PCL-C). Results: Partial correlations, controlling for age, revealed positive relationships between both number of traumas endorsed $(r=.135 ; \mathrm{r}=.117$; $\mathrm{r}=.175 ; \mathrm{p}<.001)$ and PTSD symptoms $(\mathrm{r}=.194 ; \mathrm{r}=.149 ; \mathrm{r}=.159$; $\mathrm{p}<.001)$ with Stroop response time $(\mathrm{RT})$ on all Stroop conditions (color naming, word reading, and interference). A "likely PTSD" group performed worse on all Stroop conditions than a "non-PTSD" group ( $\mathrm{F}=$ 26.927, $\mathrm{p}<.001 ; \mathrm{F}=4.397, \mathrm{p}<.05 ; \mathrm{F}=8.285, \mathrm{p}<.01)$.

Conclusions: As hypothesized, PTSD symptoms and traumatic experiences were each linked with slower RT across all Stroop conditions. Frequency of trauma experiences appeared to be most directly related to difficulty with the interference condition of the Stroop task. Results suggest that trauma may impact cognition both on simple RT tasks and more complex inhibition tasks. Notably, this relationship may effectively be explored through large web-based samples.

Correspondence: Erin Sullivan, Clinical Psychology, University of North Texas, 2025 Lakepointe Drive, Apt 28D, Lewisville, TX 75057, United States.E-mail: ErinSullivan2@my.unt.edu

S. TAYLOR, S.L. AITA, J. BEACH, J.S. HOLCOMBE, M. ESPENAN, S. ROYE, N. STANLEY, M. CALAMIA \& B.D. HILL. The Relation Between the Mental Clutter Scale and Self-Reported Executive Dysfunction.

Objective: This study evaluated the construct validity of the Mental Clutter Scale (MCS) using an established measure of self-reported executive dysfunction in a healthy sample.

Participants and Methods: 150 healthy participants $(M$ age $=19.67$, $S D=3.75 ; M$ education $=12.35, S D=0.70 ; 74 \%$ female $; 56 \%$ White, $34.7 \%$ African American, 6.7\% Asian) completed the MCS and the Barkley Deficits in Executive Functioning Scale (BDEFS) as part of a larger battery. The MCS is a recent 16 item scale that has been used in pain research. Raw scores from the MCS and BDEFS were used for all analyses.

Results: Pearson correlations for scales and subscales of the MCS and BDEFS yielded correlations ranging from .39 to $.72(p<.001)$. Next, multiple regression was used to predict executive dysfunction measured by the BDEFS using the MCS Cognition and Mental Clarity subscales. MCS subscales accounted for significant variance in the
BDEFS $\left(F(2,143)=80.70, R^{2}=.53, p<.001\right)$. In the regression model, both MCS subscales (Cognition and Mental Clarity) were significant predictors of self-reported executive deficits $(\beta=.30, p=.002 ; \beta=.47$, $p<.001$, respectively).

Conclusions: MCS performance explained a significant proportion of variance in self-reported executive dysfunction. This new short scale of self-reported "mental clutter" was observed to function similarly to the BDEFS in a healthy young adult sample, supporting its convergent validity and possible clinical utility for measuring executive impairment. Correspondence: Sarah Taylor, B.S., 75 South University Blvd, Mobile, AL 36688-0002, United States.E-mail: set1622@jagmail. southalabama.edu

S. NAPOLITANO, N. ABRAHAM, M. BARBOZA, A. KRIVENKO, H. O'HARA, G. OZINGA, M.A. TEAFORD, S.E. TOLFO \& A.M. POREH. The Geriatric Complex Figure: A test for the Assessment of Planning, Visuospatial Ability, and Memory in Older Adult Populations.

Objective: The present study describes the development of a new, simplified complex figure test, the Geriatric Complex Figure (GCF), designed to accommodate elderly individuals. This simplified version, based on theoretical organizational strategy models developed for more complex figures, was developed in order to address the floor effects commonly observed when comparing older participants' results to younger participants' results on other, more complex figure tests.

Participants and Methods: The GCF, alongside four other assessments, was administered to a sample of 126 participants above the age of 60 residing in the North East Ohio region. New technologies for the enhancement of data capture, including a digital pen and computerized scoring software, were utilized to optimize accuracy and efficiency.

Results: This study's findings support that the GCF may be better suited for use in geriatric populations than more complex figures, as score distributions lacked obvious floor effects and were negatively skewed, indicating better performance than expected.

Conclusions: Additionally, the results provide norms and validity data for the GCF and suggest that by using modern computer-based data recording methodologies researchers can reliably discern the multiple cognitive operations that impact participants' performance on this and similar measures.

Correspondence: Sarah E. Tolfo, Master's, Psychology, Cleveland State University, Cleveland State University, Psychology Department, 2121Euclid Avenue, CB 158, Cleveland, OH 44115, United States. E-mail:s.tolfo@vikes.csuohio.edu

\section{J. TRIPP, D.T. PULSIPHER, E.M. KRAPF \& L.D. STANFORD. The Additive Effect of Low Birth Weight and Seizures on Executive Functioning.}

Objective: There is considerable research indicating an elevated risk of executive dysfunction both in individuals born low birth weight (LBW; $\leq 2500$ grams) and in individuals diagnosed with seizures. Additionally, there is a strong correlation between LBW and seizures. The purpose of this study was to investigate whether there is an additive impact of LBW and seizures on executive functioning.

Participants and Methods: This sample consists of 215 individuals (ages 8 to 26 years old; $\mathrm{M}=13.3$ ), referred for neuropsychological evaluation with a history of either $\operatorname{LBW}(\mathrm{n}=82)$, a diagnosis of current seizures $(n=112)$, or $\mathrm{LBW}$ plus a concurrent diagnosis of seizures $(n=21)$ who received portions of the Delis-Kaplan Executive Function System (D-KEFS) as part of a comprehensive neuropsychological battery. A Kruskel-Wallis test was conducted between the three groups and then Mann-Whitney U post hoc tests were conducted.

Results: Trail Making Number-Letter Switching (TM), Design Fluency Switching (DF), and Verbal Fluency Category Switching (VF) were the D-KEFS subtests examined. There was a significant difference found between the three groups on TM $(\mathrm{H}(2)=9.43, \mathrm{p}<0.05)$. A MannWhitney test indicated that performance on TM scores were lower for 
those with LBW plus seizures $(\mathrm{Mdn}=40.64)$ than for those with seizures $(\mathrm{Mdn}=66.76), \mathrm{U}=560.5, \mathrm{p}<0.05$. No significant group differences were found between groups on DF or VF scores.

Conclusions: Although results were only significant for one measure of executive functioning (TM), there was a trend for the LBW plus seizures group to perform more poorly on additional measures (DF and VF). By studying these trends in a larger group or a group of individuals with lower birth weights, more significant results may emerge.

Correspondence: Jennifer Tripp, MA, NeuroDevelopmental Science Center, Akron Children's Hospital, 2649 Maplewood Street, Cuyahoga Falls, OH 44221, United States.E-mail: jtripp@email.jfku.edu

J. WEILHAMMER, E. FIELDS, F. SUMMERSETT-RINGGOLD, J. KAYLEGIAN \& S.J. HUNTER. The relationship between executive behavioral control and emotional distress in urban homeless youth.

Objective: Homeless youth are at higher risk for experiencing psychological distress and cognitive impairments that impact decision making. Yet evidence remains limited about the relationship between homelessness and developing executive behavioral control, which may mediate distress. We examined the association between executive behavioral control, emotional distress symptoms, and their relationship with episodes and length of homelessness in shelter dwelling youth in Chicago. Participants and Methods: 149 homeless youths, 18-25 years of age, predominantly African-American, residing at two homeless shelters in Chicago, completed self-report measures of executive behavioral control (Weinberger Adjustment Inventory), emotional distress (MINI), and an interview regarding homelessness. We examined the relationship between homeless variables (age at first homeless episode, length of time spent in the homeless shelter, number of times homeless, and length of the longest homeless episode), and developing executive functioning and regulation as part of a larger research study.

Results: Bivariate analyses revealed that emotional distress ( $r=.66$, $p<.001)$, homelessness at a young age $(\mathrm{r}=-.17, \mathrm{p}=.04)$, and longer homeless episodes $(r=.23, p=.01)$ were associated with poorer executive control. Hierarchical linear regression indicated that higher scores of emotional distress related to poorer executive control when adjusting for age at first homeless episode and longest homeless episode $(B=.63$, $\mathrm{p}<.001)$.

Conclusions: Results provide a further understanding of the important relationship developmentally between such contextual factors as homelessness, and the development of regulatory capabilities for emotional and behavioral control. These findings represent a further step towards identifying specific domains of EF that can be addressed within supportive interventions for this at-risk population, and guide considerations regarding longitudinal risk and resilience.

Correspondence: Jenalyn Weilhammer, MA, UCM PBN, MC 3077, 5841 S Maryland Ave, Chicago, IL 60637, United States.E-mail:jmw4923@ ego.thechicagoschool.edu

M. WELSH, E. PETERSON, M. JAMESON, M. PEAKE, K. LAFARY, S. MOORE, C. HILL, N. JOHNSON, J. RICHARDSON, M. BAXTER, K. BARTHELEMY, C. WRIGHT, R. CAMP, S. VOGEL \& B. FILIBECK. College Students with a History of Child Maltreatment and Academic Outcomes: The Mediating Role of Executive Functions.

Objective: We examined associations between self-reported history of maltreatment in childhood and academic outcomes in college, as well as the degree to which executive function (EF) mediates this relationship. Of particular interest were both the "cool" and "hot" forms of $\mathrm{EF}$, given evidence of the impact of child maltreatment on tasks that include heightened arousal.

Participants and Methods: Sixty-one male and female college undergraduates self-reported childhood maltreatment history (emotional abuse and neglect, physical abuse and neglect, and sexual abuse) on the Childhood Trauma Questionnaire (CTQ), and were given two EF measures: 1) Go-No-Go (GNG) test that included a Color Condition (cool); Neutral Face Condition (warm); and Emotion Face condition (hot), and 2) Iowa Gambling Task (IGT), a measure of risky decision making that reflects hot EF. Academic outcomes were: 1) GPA (first-semester, cumulative, and semester concurrent with testing), and 2) Student Adaptation to College Questionnaire (SACQ).

Results: Correlational patterns suggested two EF scores as potential mediators. Indirect effects analyses indicated that IGT Block 2 adaptive responding mediated the relationship between CTQ Total score and 1st semester GPA and between CTQ Emotional Abuse and Spring 2016 GPA. Regarding college adaptation, a consistent indirect effect of GNG Neutral Face RT on the relationship between CTQ Emotional Neglect and SACQ total, academic, social, and personal-emotional adaption scores was found.

Conclusions: Our results demonstrate that higher scores on child maltreatment history self-report negatively predict college academic outcomes as assessed by GPA and by self-reported adaptation. In addition, relatively "hot" EF task performance on the IGT and GNG tasks serve as important links between child maltreatment experiences and college achievement and adaptation, suggesting that hot EF skills may be an important focus of intervention efforts to improve academic outcomes for this population.

Correspondence: Marilyn Welsh, PhD, School of Psychological Sciences, University of Northern Colorado, Box 94, University of Northern Colorado, Greeley, CO 80639, United States.E-mail: marilyn.welsh@ unco.edu

R.E. ZIEMNIK, M.A. NIERMEYER \& Y. SUCHY. Predictors of Medication Management: Contrasting Verisimilitude and Veridicality.

Objective: Measuring and developing tests that can accurately predict real-world functioning is one of the foremost goals in neuropsychological research. The ability to take medications as intended is a complex task, with potentially high consequences if error(s) are made. Tests designed to have higher verisimilitude are often presented as the solution to improving ecological validity, though veridicality is of equal importance. We investigated whether the Pillbox Test, which has high face-validity, and the Push-Turn-Taptap (PTT) task, which has been previously found to predict performance on lab-based instrumental activities of daily living (IADLs), may be significant predictors of errors in medication management (MM).

Participants and Methods: Community-dwelling older adults $(n=24$, age 60-79) completed the PTT and the Pillbox Test. To assess MM, participants' pills were counted weekly for eight weeks. Extra and missing pills were documented as MM errors. We generated two variables: MM after one week (MM-1), as a "sample" of real-world MM, and MM after 8 weeks (MM-8) as a more global index of MM.

Results: MM-1 $(r=.46, p=.025)$ and the PTT $(r=-.55, p=.005)$ were significantly correlated with MM-8, but the Pillbox Test was not $(r=-.17, p=.43)$. Linear regression with MM-8 as the criterion variable and MM- 1 and PTT entered as predictors indicated that PTT accounted for $25 \%$ of variance beyond MM-1 ( $p=.005)$, while MM-1 failed to reach significance as an independent predictor $(p=.08)$.

Conclusions: These findings suggest that high verisimilitude is neither necessary nor sufficient for prediction of MM. Tests' veridicality must be empirically established regardless of their verisimilitude before using them as predictors of salient health behaviors outside of a lab-based setting.

Correspondence: Rosemary E. Ziemnik, BS, Psychology Department, University of Utah, 614 Medical Plaza, Salt Lake City, UT 84112, United States. E-mail: rziemnik@gmail.com 


\section{Imaging (Structural)}

\section{M.B. MEMEL \&. L. RYAN. Contributions of Visual Integration and Frontotemporal White Matter Integrity on Associative Memory in Older Adults.}

Objective: Aging is associated with a decline in white matter (WM) integrity across the brain. The medial temporal lobes and prefrontal cortex are involved in associative memory and connected via direct and indirect pathways, including the uncinate, arcuate, and fornix ${ }^{2}$. Uncinate integrity predicts the rapid learning and immediate retrieval of visual associations ${ }^{3}$, but less is known about contributions of other frontotemporal tracts to associative memory. Our goal was to determine whether the integrity of these tracts differentially predicted associative memory for visually integrated and non-integrated object and scene pairs in healthy older adults.

Participants and Methods: Twenty-three cognitively normal older adults studied photographs of objects and scenes. In the Combined condition, an object was positioned naturally in a scene. In the Separated condition, an object was presented on a white background adjacent to and visually distinct from a scene. An associative memory task followed with intact and recombined pairs. Using deterministic tractography, tracts were defined in template space from high angular resolution diffusion-weighted images. FA and radial diffusivity (RD) values were extracted from each tract and entered into separate regressions to predict hits and false alarms.

Results: Whereas greater right uncinate, fornix, and arcuate FA predicted less Separated false alarms, no model predicted Combined false alarms, Hits in both conditions were predicted by the integrity of all 3 tracts, with Combined hits predicted exclusively by RD and Separated hits by both FA and RD values.

Conclusions: WM integrity amongst three frontotemporal tracts differentially predicted hits and false alarms for both visually integrated and non-integrated associative pairs. Arcuate, fornix, and uncinate integrity predicted false alarms with visually non-integrated, but not visually integrated associative pairs. However, frontotemporal WM integrity predicted hits in both conditions.

Correspondence: Molly B. Memel, Master of Arts, Psychology, University of Arizona, 1515 E. Seneca St., Tucson, AZ 85719, United States. E-mail:memel@email.arizona.edu

\section{Language and Speech Functions/Aphasia}

\section{DUTTA. Language Functions in Adults with Epilepsy: A Scoping Review and Data Mining Study.}

Objective: This study involved a scoping review to identify possible gaps in the empirical description of language functioning in epilepsy. With access to social network data, data mining was used to determine if adults with epilepsy are expressing language-related concerns.

Participants and Methods: The review involved exploring online scientific databases to identify pertinent articles. Findings regarding the nature of epilepsy etiologies, patient characteristics, tested language modalities, and language measures used were compiled. Data mining focused on ChaCha, Epilepsy Foundation and Twitter databases to obtain a set of relevant questions.

Results: The search yielded 57 articles. Those with epilepsy types except TLE and older adults were underrepresented. Aphasia tests were usually utilized, indicating only basic language skills were assessed. Although spoken word-retrieval assessment such as naming, word fluency and vocabulary measures was ubiquitous, testing rarely went beyond noun retrieval and characterization of errors was overlooked. Few studies compared the language performances of individuals with epilepsy to age-and educationmatched healthy controls. Only Gauffin et al. (2013) assessed and identified deficits in high-level language skills.

We identified 9200 questions from the ChaCha and 111 posts from the EF data regarding epilepsy-related language problems. Twitter showed no relevant posts. The most frequently used words/phrases included: aphasia, speech, disorder, trouble reading, and speech therapist. Users frequently mentioned problems with word finding, reading, writing, memory, and surgery and medication effects on cognitive-communication skills, following epilepsy onset.

Conclusions: Our findings underscore the need for future specification of the integrity of language in epilepsy, particularly with respect to discourse and higher-level language abilities, to assure not only appropriate counseling and education of those with epilepsy but also their access to speech-language pathology services.

Correspondence: Manaswita Dutta, Ph.D., Speech and Hearing. Sciences, Indiana University, 1750 North Range Road, Apartment C104, Bloomington, IN 47408, United States.E-mail: mdutta@indiana.edu

L. FIELDS, A. BROWN \& K. CROFT CADERAO. Verbal Fluency and Word Retrieval Difficulties in Healthy Older Adults.

Objective: To examine whether age and verbal fluency relate to the likelihood of words identified as very familiar eliciting tip-of-the-tongue (TOT) and, thus, temporarily being unable to be retrieved.

Participants and Methods: Subjects were 30 healthy older adults ages 60 to $93(\mathrm{M}=73.77)$ with 12 to $20(\mathrm{M}=16)$ years of education and without cognitive impairment. Subjects completed a naming-to-definition task, where they indicated whether they know $(\mathrm{K})$ or don't know (DK) the target word, or whether it is very familiar but temporarily cannot be retrieved (TOT). Subjects then completed a brief neuropsychological assessment, including phonemic (Controlled Oral Word Association Test; COWAT) and semantic (Animals) fluency tests. We conducted bivariate correlations and linear regressions to examine the association between age, verbal fluency, and the percentage of TOTs for familiar words [TOT $/$ TOT $+K)=$ Percent TOT $]$.

Results: Percent TOT correlated positively with age $(r=.42, p=.022)$ and negatively with both phonemic $(r=-.47, p=.008)$ and semantic $(r=-.453, p=.012)$ fluency. Percent TOT was not significantly associated with likelihood of eventually retrieving the target word $(p=.76)$. Regressions revealed phonemic fluency $\left(\mathrm{p}=.008 ; \mathrm{r}^{2}=.22\right)$, semantic fluency $\left(\mathrm{p}=.012 ; \mathrm{r}^{2}=.21\right)$, and age $\left(\mathrm{p}=.022 ; \mathrm{r}^{2=} .17\right)$ individually predicted Percent TOT, but age was no longer significant when considered together with phonemic or semantic fluency. Phonemic fluency was the stronger predictor, with lower phonemic fluency scores associated with higher Percent TOT.

Conclusions: Findings suggest that among older adults lower verbal fluency scores are associated with a higher likelihood that familiar words may be temporarily unable to be retrieved (TOT). Although increased age was associated with more retrieval difficulties, age effects were better explained by decreased verbal fluency. Phonemic fluency was the best predictor of whether words identified as very familiar would elicit a TOT in our sample.

Correspondence: Lindy Fields, M.A., Psychology, Southern Methodist University, Psychology Department, P.O. Box 750442, Dallas, TX 75275-0442, United States.E-mail: lloneman@smu.edu

M.W.M. FONG, R. VAN PATTEN \& R. FUCETOLA. Factor Structure of the Boston Diagnostic Aphasia Examination-Third Edition.

Objective: The Boston Diagnostic Aphasia Examination-Third Edition (BDAE-3) is one of the most commonly used test batteries for aphasia, used internationally across inpatient and outpatient settings by multiple disciplines. The BDAE has been substantially revised since its first publication, yet there is no information available about the factor structure of the current version. Therefore, this study aimed to identify the underlying dimensions of the BDAE-3 in patients with acquired aphasia.

Participants and Methods: A total of 335 participants with acquired aphasia mostly due to unilateral left hemisphere stroke were referred to a comprehensive aphasia program for extensive neuropsychological evaluation of language. Forty-one subtests from the four language-related sections of BDAE-3, including auditory comprehension, oral expression, reading, and ideomotor praxis, were selected to assess participants' language. A principal component analysis with Varimax rotation was 
used to examine the underlying dimensions of the selected BDAE-3 subtests.

Results: The principal component analysis revealed a six-factor structure among the selected BDAE-3 subtests, accounting for $77.7 \%$ of the total variance, with the first four factors explaining more than $70 \%$ of the variance. These six factors measured auditory comprehension/ ideomotor praxis, oral expression, reading, syntactic understanding, non-speech oral agility, and basic whole-word recognition.

Conclusions: This study is the first analysis of the underlying dimensional structure of the BDAE-3 in a sizable sample. The factor structure of the BDAE-3 appears to be quite similar to the limited factor analyses of earlier versions. The ideomotor praxis subtests share variance with auditory comprehension subtests. Auditory comprehension, oral expression (including repetition), and reading skills are distinct, primary language domains.

Correspondence: Mandy W.M. Fong, Neurology, Washington University in St. Louis, 4444 Forest Park Avenue, Campus Box 8518, St. Louis, MO 63108.United States.E-mail:wfono@wustl.edu

S. KEY-DELYRIA, A. WEICHSELBAUM \& C. MOSTELLER. Ambiguous Sentence Comprehension and Cognitive Control in Adults after TBI.

Objective: Working memory (WM) and cognitive control (CC) have been identified as crucial to sentence comprehension and are frequently impaired after a traumatic brain injury (TBI). CC appears to be necessary to resolve difficulties in understanding language. Ambiguous sentences in particular seem to involve linguistic processes that closely mirror CC. In order to correctly interpret an ambiguity, the correct meaning must be selected and the incorrect meaning inhibited. Healthy adults with poorer $\mathrm{CC}$ have been shown to process sentences differently than those with better CC. This study asks whether CC and sentence comprehension are linked in individuals with TBI history similar to previous findings in adults with no significant neurological history.

Participants and Methods: 16 individuals with closed-head injury and 26 controls participated. Participants completed cognitive tasks including measures of vocabulary, verbal fluency, WM and CC. Participants also completed a computerized sentence comprehension task involving ambiguous, unambiguous, and control sentences.

Results: Preliminary analyses revealed significant differences between groups on certain cognitive measures, including CC measures. Participants read ambiguous sentences slower than unambiguous sentences and were slower and less accurate in response to yes/no comprehension questions about ambiguous vs. unambiguous sentences. While there were no differences in performance between groups on sentence measures, there were different correlation patterns between sentence measures and cognitive measures in each group.

Conclusions: The lack of group differences on sentence measures is surprising given group differences on measures of CC, the cognitive ability thought to support ambiguous sentence comprehension. However, correlation patterns suggest that groups may be using different cognitive resources to complete the same task. Results are interpreted in light of TBI severity and cognitive performance.

Correspondence: Sarah Key-DeLyria, Ph.D., Speech and Hearing Sciences, Portland State University, PO Box 751 (SPHR), Portland State University, Portland, OR 97207, United States.E-mail: keydel@pdx.edu

T.L. MCAUley, S. MALHI, B. LANSUE \& L. BUCHANAN. Dissociation Between Implicit and Explicit Access: The Failure of Inhibition Theory.

Objective: The Failure of Inhibition Theory (FIT; Buchanan et al., 1999) posits that deep dyslexics (DD) have an intact semantic system but compromised phonological output relating to a failure to inhibit activated lexical neighbours. The purpose of this study was to test this model's predictions that compared to an explicit (oral production) task, performance on a task requiring implicit semantic access would not be affected. Additionally, during oral production, FIT predicts that semantic errors are more likely to be produced for words with denser semantic neighbourhoods.

Participants and Methods: GL is a 33-year-old male with 17 years of formal education who is 8 years post stroke. He completed an implicit semantic task on the computer in which he had to make decisions about iconic relationships between words. A month later, he read the same words during an explicit oral production task.

Results: On the implicit task. GL was faster in correctly responding to abstract $(\mathrm{M}=4441.59, \mathrm{SD}=2303.06)$ than concrete $(\mathrm{M}=6815.04$, $\mathrm{SD}=2996.95)$ relationships, $t(26)=3.11, \mathrm{p}<.05$, as well as iconic $(\mathrm{M}=5030.42, \mathrm{SD}=2234.11)$ than reverse-iconic $(\mathrm{M}=64097.04$, $\mathrm{SD}=3385.73)$ relations, $t(25)=2.17, \mathrm{p}<.05$. On the explicit task, GL's accuracy significantly differed by concreteness, $\chi(1)=15.25, p<.001$, $\phi=.31$, with $87.8 \%$ and $61.3 \%$ correct responses for concrete and abstract words, respectively. Semantic errors had higher semantic neighbourhood densities (SND; M=.40), although analyses were underpowered for supportive findings.

Conclusions: Consistent with FIT, GL demonstrated a dissociation of his performance on an implicit task versus an explicit task. On the implicit task, GL's pattern of performance was comparable to the normal population, whereas on the explicit task, he showed deficits in reading the same words. Common to DD, GL demonstrated a concreteness effect in word reading, and additionally, SND appears to be a valuable semantic factor to consider in future research.

Correspondence: Tara L. McAuley, Bachelor of Science, Psychology, University of Windsor, 10325 Eastcourt Dr., Windsor, ON NSR 1E4, Canada.E-mail:mcauleyt@uwindsor.ca

T.E. OWENS, M. MACHULDA, J. DUFFY, H. CLARK, S. BOLAND \& K. JOSEPHS. Word and Nonword Reading Differences in Primary Progressive Aphasia Variants and Primary Progressive Apraxia of Speech.

Objective: Primary Progressive Aphasia (PPA) subtypes, including agrammatic variant (agPPA), semantic variant (svPPA), and logopenic variant (lvPPA), and Primary Progressive Apraxia of Speech (PPAOS) have differential performance on word-reading tasks, which is hypothesized to be caused by differential breakdown in brain areas needed for language expression. However, there is little information about the clinical utility of word-reading measures in terms of their ability to predict classification of PPA subtypes or group membership. In this study, we evaluated the degree to which word-reading scores could correctly classify patients with well-characterized PPA and PPAOS

Participants and Methods: Participants $(\mathrm{n}=89)$ with confirmed PPA or PPAOS read aloud wordlists with regular phonetic spelling, irregular spelling, and nonwords. Items were scored for correct or incorrect wholeword pronunciation.

Results: Discriminant function analysis showed correct classifications of aphasia variant based solely from irregular word and nonword reading scores for $47.7 \%$ of cases. Classification of svPPA, however, was poor with this model. A second model excluding svPPA and including a measure of aphasia severity improved classification to $73.0 \%$. PPAOS was most consistently classified correctly, with $63.6 \%$ and $87.0 \%$ correct classifications for the first and second analyses respectively.

Conclusions: Irregular and nonword reading scores can make distinctions among PPA subtypes and PPAOS, though comprehensive speech and language evaluations are needed for more reliable clinical diagnosis. The classification utility of these measures improves when distinguishing among fewer groups (e.g., agPPA versus PPAOS) and when including additional information about aphasia severity. Additionally, the consistency of PPAOS classification further supports conceptualization of PPAOS as a distinct entity from aphasia.

Correspondence: Tyler E. Owens, Ph.D., Psychiatry and Psychology, Mayo Clinic, 670 28th St SE, 6-147, Rochester, MN 55904, United States.E-mail: owens.tyler@mayo.edu 
S.B. PILLAY, J. BINDER, L. FERNANDINO, C. HUMPHRIES, D. BOOK \& R. DESAI. Examining the relationship between motor system function and verb processing in aphasia using voxel-based symptom-lesion mapping (VLSM).

Objective: Activation of motor systems in the brain during action verb processing is often cited as evidence for "embodied" concept representation, but this evidence remains controversial. Selective action verb processing deficits in patients with motor neuron disease and Parkinson disease support claims of a causal link between motor system function and action verb comprehension, but the diffuse pathology associated with these degenerative conditions prohibits definite localization of the impaired networks.

Participants and Methods: Participants were 36 right-handed patients with chronic left hemisphere stroke. Participants performed semantic matching tasks with action verbs, abstract verbs, and concrete object nouns. Lesions were mapped using high-resolution MRI. VLSM analyses used action verb accuracy as the dependent variable, with object noun accuracy and abstract verb accuracy as covariates to suppress lesiondeficit correlations associated with non-specific deficits. An additional VLSM examined the lesion correlates of motor deficits in the same patient sample, using MRC strength ratings, averaged across arm, hand, and leg testing.

Results: After controlling for abstract verb and object noun comprehension deficits, action verb deficits were associated with damage to the inferior frontal gyrus, lower precentral gyrus, and mid-insula. Motor deficits overlapped minimally with action verb deficits, mainly involving a focal region near the inferior frontal junction.

Conclusions: Action verb impairments were associated with damage to inferior prefrontal and premotor areas that have been linked with action perception and planning processes. The results suggest that specific knowledge critical for comprehension of action verbs is stored outside primary sensory-motor cortex, in adjacent frontal association cortex linked with higher-level action representation.

Correspondence: Sara B. Pillay, Medical College of Wisconsin, 8701 Watertown Plank Road, Center for Imaging Research, MEB 4670, Milwaukee,WI 53226,United States.E-mail:sara.berentsen@gmail. com

\section{SAADATPOUR, K.M. HEILMAN \& L. DOTY. Primary Progressive} Mixed Transcortical Aphasia, a Case Report.

Objective: Mixed transcortical aphasia is a rare speech disorder characterized by reduced spontaneous speech and decreased comprehension but intact repetiion. This type of aphasia has been seen previously in patients with brain infarction, particularly with ischemia of watershed areas which isolate an intact Wernike's loop (Broca's area, Wernike's area and the arcuate fasciculus) from the rest of the brain. Here we report a 55 year old right-handed female who gradually developed language difficulties over two years. Symptoms began with difficulty finding words and word production. On neuropsychologic testing, she was found to have impaired spontaneous speech, impaired comprehension and naming, with intact repetition. Her MRI showed atrophy in the left frontal, parietal and anterior temporal lobe, without evidence of ischemia.

Participants and Methods: A 55 year old right-handed female who came to our clinic.

Results: Our patient was unable to initiate a conversation. Her comprehension and naming were also impaired. However, her repetition was intact. Her MRI showed atrophy of the left frontal, parietal and anterior temporal lobe. There was no evidence for ischemic injuries.

Conclusions: Several forms of primary progressive aphasia including, logopenic, semantic agrammatic non-fluent and even primary progressive speech abulia have been reported. Mehler (1988) reported a patient with impaired comprehension and intact repetition, however unlike our patient this patient produced phonological paraphasic errors suggesting dysfunction in Wernike's loop. In addition, patients with mixed transcortical aphasia do not speak unless spoken to, and their verbal output is almost entirely limited to echolalia. Thus, this may be the first reported case of a patient with all the signs of a mixed transcortical aphasia due to a neurodegenerative, rather than ischemic injury.

Correspondence: Leila Saadatpour, MD, Neurology, University of Florida, 3230 SW Archer RD, APT\# B110, Gainesville, FL 32608, United States.E-mail: leila.saadatpour@neurology.ufl.edu

\section{Memory Functions}

D.M. ANDERSON, Q. YU, J. ROBERSON, J. PIERCY, A. HEITZER, S. RAZ \& N. OFEN. Systematic Review of the Relationship Between Hippocampal Volume and Memory Performance in Preterm-Born Individuals.

Objective: Preterm birth is associated with alterations in hippocampal structure and deficits in memory; however, the nature of the relationship between hippocampal volume and memory in this at-risk group is not fully understood. We conducted a systematic review of available literature to examine the hypothesis that reduction in hippocampal volume is associated with poorer memory function in individuals born preterm. Participants and Methods: We used PubMed, MedLine, PsychINFO, and Web of Science to conduct a thorough search of English language empirical studies including preterm participants, volumetric data, memory performance data, and sufficient information for effect size calculation. The initial search yielded 280 articles, yet only five met our inclusion criteria. Quantitative integration was based on a random effects model. Effect sizes belonged to the Pearson's $r$ family.

Results: The summary effect size was small-to-medium, but suggested a significant positive relationship $\left(r=0.21,95 \% \mathrm{CI}[0.06,0.35], Z_{\text {test }}=2.28\right.$, $p=.023)$ in accord with expectations. However, only $4 \%$ of the variance in memory performance was explained by hippocampal volume.

Conclusions: Although the overall effect is significant, interpretation must take into account the small number of studies included in this review. The overall effect primarily reflects the relationship between left hippocampal volume and memory. Although this relationship may differ by hemisphere, there was insufficient reporting of effect sizes to investigate possible differences. Despite these concerns for interpretation, the results from this review, compared to the results of a review with control participants (Van Petten, 2004), suggest that the relationship between hippocampal volume and memory may differ between preterm born and full term born individuals. Additional studies of the link between hippocampal volume and memory performance are needed in order to better establish the strength of this relationship, as well as investigate potential differences across hemispheres.

Correspondence: Dana M. Anderson, Clinical Psychology, Wayne State University, 500 W. Willis, Apt. B1, Detroit, MI 48201, United States. E-mail:fj3939@wayne.edu

H.E. BRUNET, J. KRAMER, A. ALIOTO, C. MEAD, D. DELIS \& J. FOLEY. Differences in Learning and Recall Strategies in Older Adults Based on IQ.

Objective: IQ has been shown to be moderately correlated with memory. This study aims to determine how strategies used during learning and recall differ between older adults depending on IQ. We hypothesized that older adults with higher IQs would show greater use of semantic categorization during than individuals with lower IQs.

Participants and Methods: The sample contained 246 healthy older adults (over 65 years) drawn from the California Verbal Learning Test-II (CVLT-II) standardization sample ( $\mathrm{M}=75$ years old, $54 \%$ female, $46 \%$ male, $92 \%$ Caucasian). The majority of participants had 12 years of education (37\%). The Wechsler Abbreviated Scale of Intelligence, Second Edition (WASI-II) was used to measure IQ and the CVLT-II measured learning and memory. ANOVA analyses were used to compare strategies between IQ groups (high-IQ $=>115, n=55$; average-IQ $=114-86, n=177$; and low-IQ $=<85, n=26)$. The groups differed in education level but not in age or gender. 
Results: The IQ groups were compared on mean level of semantic categorization, presented in z-scores. Consistent with our hypothesis, the high-IQ individuals exhibited greater use of semantic categorization during the final learning trial of the CVLT-II $(\mathrm{M}=0.8, \mathrm{SD}=1.6$; $\mathrm{p}<0.001)$ compared to the average $(\mathrm{M}=-0.1, \mathrm{SD}=0.8)$ and low-IQ groups $(\mathrm{M}=-0.5, \mathrm{SD}=0.6)$. The IQ groups also differed in semantic categorization on the delayed recall trial, with the high-IQ group exhibiting greater semantic categorization $(\mathrm{M}=0.8, \mathrm{SD}=1.5)$ than the average-IQ group $(\mathrm{M}=-0.1, \mathrm{SD}=0.8 ; \mathrm{p}<0.001)$ who then display greater categorization than the low-IQ group $(\mathrm{M}=-0.6 ; \mathrm{SD}=0.6 ; \mathrm{p}<0.001)$. Conclusions: As expected, older adults with higher IQs showed greater use of semantic categorization strategies than those with average and below-average IQs. This study suggests that individuals with higher IQs employ greater use of active, strategic approaches to both learning and memory than those with lower IQs, which may explain some of the relationship between IQ and memory.

Correspondence: Hannah E. Brunet, Palo Alto University, $11730 \mathrm{~W}$ Sunset Blvd, Apt 313, Los Angeles, CA 90049, United States. E-mail: hbrunet@paloaltou.edu

A. COOK, E. LOYER, M. CONNELLY, A. RADEMAKER, M. MESULAM, S. WEINTRAUB \& E. ROGALSKI. Is Unusually High Working Memory Performance Associated with SuperAger's Superior Episodic Memory Performance?

Objective: SuperAgers are a unique cohort of adults over age 80 who perform at least as well as average middle-age adults on episodic memory tests and at least average-for-age on tests in other cognitive domains. Age-related working memory decline is hypothesized to contribute to age-related impairment in episodic memory and the transition from Mild Cognitive Impairment to Alzheimer's dementia. The present study examined whether SuperAgers who have superior episodic memory ability also have better working memory than their cognitively average-for-age peers.

Participants and Methods: 60 SuperAgers and 25 cognitively average elderly adults completed the Wechsler Adult Intelligence Scale (WAIS)-III and Rey Auditory Verbal Learning Test (RAVLT). WAIS-III Working Memory Index, Letter-Number Sequencing, and Longest Digit Span backward span were analyzed as tests of working memory. WAIS-III Processing Speed Index was examined to determine if it influenced performance on timed working memory tests. Associations between episodic and working memory were examined with Pearson's correlations. Between-group differences were examined with independent t-tests. Mean \pm SD are reported.

Results: There were no group differences in demographics or estimated premorbid intelligence. Working memory was positively correlated with RAVLT delayed recall across groups $(p ' s<0.05)$. SuperAgers outperformed their cognitively average peers on the WAIS-III Working Memory Index $(121.1 \pm 12.9$ vs. $108.5 \pm 15.1)$, Letter-Number Sequencing raw score (10.5 \pm 2.0 vs. $8.5 \pm 2.1$ ), and longest Digit Span backward span $(5.2 \pm 1.0$ vs. $4.5 \pm 1.2 ; p ’ s<0.01)$. There was no group difference in processing speed.

Conclusions: SuperAgers outperformed their cognitively average-for-age peers on measures of working memory. This difference cannot be attributed to differences in processing speed or demographic factors, including premorbid intelligence. Results suggest that better working memory is associated with better episodic memory even in cognitively average and above-average elderly adults.

Correspondence: Amanda Cook, MA, MS, Cognitive Neurology \& Alzheimer's Disease Center, Northwestern University, 320 E Superior St, Searle 11, Chicago, IL 60611, United States. E-mail: amandacook2017@u.northwestern.edu
L. DE WIT, D.M. O' SHEA, M.E. MCLAREN, S.M. SZYMKOWICZ \& V.M. DOTSON. The Effects of Depressive Symptom Dimensions and Education on Verbal Memory.

Objective: We have previously shown that higher education protects against depression-related verbal learning deficits. Depression is a heterogeneous disorder and comprises different sets of symptom dimensions. Thus, in the current study, we expanded upon our previous results by examining the effects of depressive symptom dimensions on verbal memory and investigating whether education moderates the relationships.

Participants and Methods: Seventy-three community-dwelling adults (aged 18-81 years) completed the Center for Epidemiologic Studies Depression Scale (CES-D) and the Hopkins Verbal Learning Test Revised (HVLT-R). Separate regression models were conducted that included total and delayed recall scores on the HVLT-R as dependent variables, and three core symptom dimensions (depressed affect, lack of well-being, and somatic symptoms) and education as independent variables. Age and sex were included as covariates.

Results: No main effects for the CES-D subscales or education were found. However, we found a lack of wellbeing $\times$ education effect on the HVLT-R total recall $(p=.043)$, and a somatic symptoms $\times$ education effect on HVLT-R delayed recall ( $p=.032)$, such that for the less highly educated individuals, depressive symptoms were associated with worse performance. For the more highly educated, an opposite pattern was found.

Conclusions: We found that education protects against verbal learning and memory impairments associated with lack of wellbeing and somatic symptoms of depression. These findings highlight the need for clinicians to consider both the educational background and symptom profile of depressed patients when evaluating the impact of their depressive symptoms on cognitive functioning.

Correspondence: Liselotte De Wit, M.S., Clinical and Health Psychology, University of Florida, 805 NE 2nd street \#2, Gainesville Fl, FL 32601, United States.E-mail:Ldewit@phhp.ufl.edu

M. OYETEJU, S. JEEVAN, J. CRUMEDY, K. AGBAYANI, J. VOLPI, D. CHIU, Y. ZHANG, G. BRITZ \& M. DULAY. Rates of Memory Loss After Stroke in Areas of the Brain Not Typically Associated with Forgetfulness.

Objective: Previous studies have shown that memory loss occurs after damage to the mesial temporal lobe, frontal lobe and basal ganglia. Less is known about the frequency and magnitude of memory difficulties that may occur after damage to areas of the brain such as the thalamus, pons, and cerebellum. This study examined memory functions after damage to parts of the brain typically and not typically associated with forgetfulness.

Participants and Methods: One-hundred and eight patients (mean age of 62 years-old; $57 \%$ women) underwent neuropsychological assessment an average of 4.5 months after stroke. Groups included individuals with unilateral middle cerebral artery (MCA; $\mathrm{N}=24$ ), cerebellar $(\mathrm{N}=25)$, frontal lobe $(\mathrm{N}=23)$, pons $(\mathrm{N}=17)$, and thalamic $(\mathrm{N}=19)$ stroke. Repeated Battery for the Assessment of Neuropsychological Status subtests represented memory abilities. Patients were excluded with an MMSE score below 25.

Results: As expected, patients who sustained a MCA stroke performed lower than expected with left MCA patients experiencing reduced performance on tests of story memory $(80 \%$ of left MCA CVA patients showed impairment) and right MCA patients experiencing reduced performance for figure recall $(60 \%$ of right MCA patients; overall ANOVA $\mathrm{F}[4,105]=5.82, \mathrm{p}<0.01$; mean performance lower compared to all other groups, post-hoc p<0.01). Frontal lobe and temporal lobe patients showed similar levels of impairment for list learning (post-hoc $\mathrm{p}<0.01$ ). Of note, $50 \%$ of the pons CVA patients showed impaired performance on the list learning task followed by $31 \%$ of cerebellar CVA patients and $20 \%$ for thalamic CVA patients. Side of stroke only showed material specific deficits for MCA patients. 
Conclusions: Results were consistent with previous studies that demonstrated memory difficulties occur after damage to the frontal or temporal lobes. We extend the findings by demonstrating a notable percentage of patients also exhibit memory loss after damage to areas not typically associated with memory functioning.

Correspondence: Mario Dulay, Houston methodist Hospital, 3414 Legends Garden Drive, Spring, TX 77386, United States. E-mail: mdulay@houstonmethodist.org.

E. ESTEVIS, B.M. SCOTT \& A.M. STRUTT. Performance on the Rey Auditory Verbal Learning Test (RAVLT) in neurologically intact Spanish-speaking older adults.

Objective: Latinos represent the fastest and largest ethnic minority group in the United States, with a significant number of this population being primarily Spanish-speaking. The increased risk for developing a neurodegenerative condition and the impact of socio-demographic factors on cognition for this population warrants the implementation of reliable and valid neuropsychological measures accompanied by normative data. Toward this end, the current study examined performance on the Rey Auditory Verbal Learning Test (RAVLT) in a group of neurologically intact Spanish-speaking older adults. Clinical classification accuracy against normative data for English speakers was also assessed.

Participants and Methods: One hundred fifty-nine primarily Spanish-speaking Latinos (age: $\mathrm{M}=61.5, \mathrm{SD}=7.4$; Education: $\mathrm{M}=11.2$, $\mathrm{SD}=4.0$; Years in the US: $\mathrm{M}=20.2, \mathrm{SD}=13.2$ ) with low levels of acculturation, from 13 countries, Cuba, and Puerto Rico were administered the RAVLT as part of a larger norming study.

Results: Age $(\beta=-.27, p=.001)$ and education $(\beta=.29, p<.001)$ were predictors of RAVLT total scores. In comparison to English normative data, the RAVLT was found to have appropriate neurodiagnostic accuracy; five participants were misclassified as impaired. False-positives will be discussed and normative data stratified by age and education will be provided.

Conclusions: The current study revealed that age and education are associated with performance on the Spanish RAVLT. Spanish speakers were found to have scores comparable to their English speaking counterparts and conservative figures resulted in a small number of false positives with appropriate identification of clinical declines. The RAVLT provides neurodiagnostic utility for Spanish speakers with 6 or more years of education.

Correspondence: Eduardo Estevis, Ph.D, Neuropsychology, University of Texas MD Anderson Cancer Center, 1 Hermann Museum Circle \#3106, Houston, TX 77004, United States. E-mail: eestevis@mdanderson.org

O. GAASEDELEN, M.L. MILLER, H. LUU, J.D. BAYLESS, D. MOSER, M.R. BASSO \& D. WHITESIDE. Preliminary Validation of the Subjective Memory Complaint Scale (SMS) for Individuals who have Underwent Electroconvulsive Therapy (ECT).

Objective: Numerous studies show cognitive deficits associated with electroconvulsive therapy (ECT) tend to resolve posttreatment; however many patients continue to have cognitive complaints following treatment (Semkovska \& McLoughlin, 2010). Studies examining the subjective complaints of individuals post-ECT remain sparse, and the present study presents preliminary validation evidence for a new measure, the Subjective Memory Complaint Scale (SMS), which was designed to assess patient perception of memory following ECT.

Participants and Methods: Individuals who underwent a brief neuropsychological assessment, including the RBANS, before receiving ECT within the last 14 years were recruited to return for a follow-up assessment. At follow-up, participants $(N=34)$ were re-administered the initial neuropsychological assessment battery as well as the Beck Depression Inventory-II (BDI-II), Beck Anxiety Inventory (BAI), and the SMS to assess subjective memory complaints of individuals who have undergone ECT.
Results: Findings indicated the SMS demonstrated good internal validity (Cronbach's alpha $=0.73$ ) and had modestly significant correlations with BDI $(r=-0.41 ; p<0.05)$, and RBANS attention domain $(r=0.31$; $\mathrm{p}<0.05)$ and RBANS total score $(\mathrm{r}=0.34 ; \mathrm{p}<0.05)$ post-ECT. Similarly significant correlations were observed between the difference RBANS scores from pre to post-test with the SMS. Correlations with RBANS memory domains were non-significant. A mediation model with BDI scores and RBANS total scale score predicting subjective memory was significant, providing evidence that BDI score mediates the relationship between RBANS total scale and subjective memory.

Conclusions: Results suggest the SMS demonstrates good internal reliability and external validity. Subjective memory complaints appear more related to depressive symptoms than changes in memory as assessed by formal neuropsychological measures. These results are preliminary and thus future research and cross-validation of the SMS is necessary.

Correspondence: Owen Gaasedelen, University of Iowa, 312 Samoa Ct, Iowa City, IA 52246, United States. E-mail: owen-gaasedelen@ uiowa.edu

H. KIM, Y. KANG, K. YU \& B. LEE. Effects of Perceptual and Semantic Encoding on Recall and Recognition Memory in Subjective Cognitive Decline.

Objective: The present study was conducted to examine the effects of perceptual and semantic encoding on memory in Subjective Cognitive Decline (SCD) and to compare them with those in the normal elderly (NE) and amnestic Mild Cognitive Impairment (amMCI).

Participants and Methods: Thirty-three SCD, 35 NE, and 29 amMCI participated in the study. Both perceptual (number of letters) and semantic (manmade/natural) computerized verbal encoding tests were administered. Each test consisted of 12 words and the order of presentation was counterbalanced. After a 20-minute delay, free-recall and recognition tests for the encoded target words were performed. During a 20-minute interval, non-verbal tests that didn't influence on verbal memory process were administered.

Results: On both recall and recognition tests, all three groups did not show any memory differences for perceptually encoded words. For the semantically encoded words, however, the amMCI showed poorer memory than the SCD and NE, although there was no difference between the SCD and NE. At the comparison of the difference of memory performances between perceptual and semantic encoding, the NE showed a greater difference than the amMCI, but the SCD did not show any differences with either NE or amMCI. The interactions between levels of encoding (perceptual/semantic) and types of recall (free recall/recognition) were not found. Although the amMCI showed poorer performance on both recall and recognition tests than the NE and SCD, the SCD did not show any differences in both tests with NE. At the comparison of the difference of the memory performance between the free-recall and recognition, the NE showed a greater difference than the amMCI, but the SCD did not show the difference with either group. Conclusions: These results showed that the SCD already had subtle problems in semantic encoding and memory retrieval but with less degree than amMCI. They suggest that the SCD is an intermediate stage between the $\mathrm{NE}$ and amMCI.

Correspondence: Haeyoon Kim, Hallym University Medical Center, Samsung apartment 101-1204, Dowha-dong, Seoul 121-772, Korea (the Republic of). E-mail: nayuem@hanmail.net

D.R. LABELLE, M. GRUBER \& S.J. BANKS. Curiosity is associated with spatial working memory and right mesial temporal volumes. Objective: Curiosity represents intrinsic motivation for learning, and recent work has suggested that curiosity is associated with co-activation of reward circuitry that modulates hippocampal-dependent learning. Age-related changes in curiosity, and in the relationship between curiosity and limbic/hippocampal circuits, is not yet understood. The current study explores curiosity, neuropsychological function, and structural volumes in an outpatient memory disorders clinic. 
Participants and Methods: Participants included 20 new adult patient referrals presenting to clinic with primary memory complaints. Participants were administered the Curiosity and Exploration Inventory (CEIII) and an abbreviated cognitive assessment including the Montreal Cognitive Assessment, a spatial span task, and validity measures. Ten individuals underwent structural imaging (3T MRI) within 12 weeks of the assessment. Images were processed using Freesurfer 5.3. CEI-II Total, Stretching, and Embracing scores were correlated with assessment results and structural volumes, controlling for age.

Results: CEI-II Total, Stretching and Embracing scores were significantly associated with total volume of the right hippocampus $(r>0.76$, $p<0.03)$, right inferior lateral ventricle $(r<-0.74, p<0.04)$, and right hippocampal fissure $(r>0.83, p<0.01)$. Total and Stretching scores were also associated with right subiculum $(r>0.72, p<0.04)$, and presubiculum $(r>0.80, p<0.02)$ volumes. The Embracing subscale was significantly associated with indices from the spatial span task $(r>0.59, p<0.02)$

Conclusions: Self-reported scores on a curiosity questionnaire were associated with right mesial temporal structural volume and spatial working memory in a sample of older adults, supporting previously reported associations between mnemonic structures and curiosity. The lateralized effect represents a novel finding, and may suggest that, in older adults, curiosity reflects relative preservation of mesial right hemisphere structures and nonverbal cognitive processes.

Correspondence: Denise R. LaBelle, Ph.D., Neurology, Cleveland Clinic - Lou Ruvo Center for Brain Health, 888 W Bonneville Ave, Las Vegas, NV 89101, United States. E-mail: d.r.labelle@gmail.com

A. LaJeunesse, M. PotVIn, V. LABElle, M. CHASles, S. MARCONE, S. JOUBERT \& I. ROULEAU. Semi-Naturalistic Prospective Memory Assessment in Mild Cognitive Impairment. Objective: Prospective memory (PM) is the ability to remember to perform an intention at an appropriate time in the future. PM deficits are common in Mild Cognitive Impairment (MCI) and may affect functional autonomy. The objective of this study was to provide a comprehensive analysis of PM difficulties in MCI using a semi-naturalistic PM task.

Participants and Methods: Eighteen MCI patients and 18 matched healthy controls (HC) between 59 and 85 years of age performed the Ecological Test of Prospective Memory (TEMP), a 20-minute simulated errand task in which participants were required to execute 10 eventbased tasks (EB) and 3 time-based tasks (TB).

Results: Despite the smaller number of actions to be performed, TB condition was more difficult than EB condition for all participants (learning intention, cue/time detection, and action retrieval). Compared to HC, MCI patients were impaired in phases of encoding intention and detecting appropriate target (EB) or time (TB) cues that signal intention execution. Furthermore, MCI patients made more intrusion errors than HC (i.e. identifying a wrong prospective target). MCI patients were also impaired in intention retrieval on TB tasks, but not on EB tasks.

Conclusions: In MCI, difficulties were observed on all aspects of the TEMP with the exception of the retrospective component (i.e. action retrieval) of the EB tasks. Semantic association between target cue and intention might have facilitated EB intention retrieval. A possible explanation for the difficulties on TB tasks may be that it requires divided attention resources to flexibly switch between the PM task and time monitoring, two processes that can be disrupted with aging. Due to its known impact on daily activities (i.e. taking medication), PM impairment should be carefully investigated among elderly.

Correspondence: Ariane Lajeunesse, Psychology, Université du Québec à Montréal, 770 avenue Ampère app.2, Laval, QC H7N 6G7, Canada. E-mail:lajeunesse.ariane@courrier.uqam.ca
C.O. NESTER, A. UZUMCU, C. MALONE, K. TORRES, T. ZINK, S. HOFFSCHIMDT, J. SWEENEY, N. MADIGAN \& M.G. O'CONNOR. Differential Semantic and Episodic Memory for September 11, 2001 in Cognitively Impaired and Healthy Adults.

Objective: The remote memory abilities of healthy and cognitively impaired adults were probed in order to assess the recall of semantic and episodic details from the September 11, 2001 terrorist attacks.

Participants and Methods: An evaluation of the factual knowledge and personal recollections of $9 / 11$ was administered to 51 neurology clinic outpatients (age 50-86 years). Participants included patients with diagnosed cognitive impairment $(n=24$, age $M=71.8)$ and healthy older adults ( $\mathrm{n}=27$, age $\mathrm{M}=66.4$ ). Participants were asked to describe their personal "flashbulb" memories of 9/11; descriptive quality of narratives was scored by five raters on multiple dimensions (people, location. activity, time-course, emotionality, and source). Participants were also probed for additional factual details (semantic memory for 9/11); standardized neuropsychological measures were also administered.

Results: Independent T tests revealed healthy adults were better able to recall factual details for $9 / 11$ than those with cognitive impairment $(\mathrm{t}(49)=2.42 ; \mathrm{p}=019)$. Flashbulb memories trended towards being significantly richer in healthy adults than those who were cognitively impaired $(\mathrm{t}(49)=1.95, \mathrm{p}=.056)$.

Conclusions: The findings of this study are somewhat paradoxical. With cognitive impairment, episodic aspects of memory are recognized to be more vulnerable than that of semantic memory in the degradation of medial temporal structures. However, our patients performed significantly worse in their factual recall for the events of 9/11 than they did in their "flashbulb" memories of that day. Further analyses will focus on the extent to which the degradation of language functions and anterograde memory performance impact capacity for semantic and episodic recall.

Correspondence: Caroline O. Nester, Bachelor of Arts, Cognitive Neurology, Beth Israel Deaconess Medical Center, 1589 Beacon St, \#3, Brookline, MA 02446, United States.E-mail: cnester@bidmc.harvard. edu

D.M. O' SHEA, S.M. SZYMKOWICZ, M.E. MCLAREN, L. DE WIT \&. V.M. DOTSON. Prospective memory strategy use as a predictor of cognitive status in older adults: the role of subjective memory. Objective: In older adults, the spontaneous use of strategies to aid prospective memory (PM) can be considered as a type of adaptive functioning and has been related to global cognitive status. The current study extends previous research by examining the role of subjective memory in this relationship.

Participants and Methods: Prospective memory data was available for 2,345 participants who took part in the 2006 wave of the Health and Retirement Study (age range $=65-90$ years). Using the criteria for cognitive status outlined by Langa and colleagues (2009), two groups were identified: normal $=78 \%$, cognitively impaired $=19 \%(3 \%$ with dementia were excluded). Participants were asked to remind the interviewer to "check something" on their computer in about five minutes (PM task). Whether the participant employed a reminder aid or not was recorded. Subjective memory ratings were based on a five-point scale, with higher scores indicating poorer memory. A binary logistic regression was employed to examine whether or not subjective memory ratings modified the association between PM strategy use and cognitive status. controlling for age, education, sex, race, depression and Instrumental activities of daily living function.

Results: There was no main effect of PM performance on cognitive status. However, the group that did not use a strategy had a $22 \%$ increased odds of being in the impaired group $(\beta=-0.250, p<0.001)$. Furthermore, subjective memory moderated this association, such that those with poor subjective memory and who did not use a strategy had the lowest cognitive scores compared to any other group.

Conclusions: Our findings show that individuals with poor subjective memory who do not spontaneously employ a memory strategy may be at increased odds of having cognitive impairment. The lack of strategy 
use in this group may reflect poor adaptive functioning in the context of cognitive decline. Thus, these individuals may especially benefit from interventions aimed at increasing strategy use to aid prospective memory.

Correspondence: Deirdre M. O' Shea, MS, The Taub Institute, Columbia University,Medical center, 639 NE 6th street, Gainesville, Gainesville, FL32601, United States.E-mail: dmo2123@ufl.edu

\section{A. DACOSTA, B.K. LEBOWITZ, E. ROSEMAN, S. RESCH, T. MYERS \& T. PRESTON. Clinical Evaluation of Prospective Memory in Children: Effect of Distractor Task.}

Objective: To determine whether changing distractor task alters prospective memory performance in clinically referred children and adolescents.

Participants and Methods: Two groups of clinically referred children and adolescents were seen. The first group of 115 participants was given the Prospective Memory Screening (PROMS) while the WIAT III was used as a distractor. The second group of 102 participants was given the same prospective memory measure with mixed distractor tasks. Both groups were given these measures in the context of a full neuropsychological battery.

Results: Findings indicated that PROMS scores did not significantly differ between groups, demonstrating as in past research that distractor task does not have substantial influence on PM performance. It also suggests that the PROMS is a reasonably robust clinical measure of PM. In addition, PROMS results showed modest correlations with executive tasks, but no significant relationship with a measure of declarative memory (CVLT C). PROMS scores were not significantly correlated with most behavioral indices from the CBCL and BRIEF P, probably due to restriction of range issues in the sample.

Conclusions: In two large groups of clinically referred children and adolescents with a variety of problems, results of a clinical prospective memory task were strikingly consistent, despite the use of differing distractor tasks. These findings are similar to those from past work, and demonstrate the potential for the Prospective Memory Screening to be integrated into a neuropsychological battery in a manner that is convenient and ecologically valid.

Correspondence: Thomas Preston, PhD, Neuropsychology Service, Stony Brook University Medical Center, 14 Technology Drive, Suite 12B, East Setauket, NY 11733, United States.E-mail: thomas.preston@ stonybrookmedicine.edu

M. NASSERI, R.K. OLSEN, C.B. MARTIN, J. RABIN, F. GAO, K. HONJO, D.J. PALOMBO, R.N. NEWSOME, S.E. BLACK, J.D. RYAN, M.D. BARENSE \& R. ROSENBAUM. Impaired face discrimination following early mediodorsal thalamic damage.

Objective: The mediodorsal nucleus of the thalamus (MDN) is a diencephalic structure that has been implicated in memory and executive function, but selective unilateral lesions to this structure are seldom reported in humans. The objective of this study is to consider the function of the MDN in the context of the direct input it receives from perirhinal cortex, which is implicated in face and object processing, as this raises the possibility of both mnemonic and perceptual deficits following MDN damage.

Participants and Methods: We present a detailed anatomical and functional characterization of a unique case, $\mathrm{NC}$, who, in relation to an ischemic stroke at 9 days of age, presents with impaired episodic memory. High-resolution MRI images of the anteromedial thalamic nuclei and medial temporal lobe (MTL) were manually segmented with sensitive protocols to localize and quantify structural abnormalities. Recall and recognition memory were assessed with standardized neuropsychological tests and further investigated in NC and demographicallymatched controls using a novel experimental paradigm with closely matched verbal (words) and non-verbal (abstract pictures, faces) conditions. Complex perceptual discrimination was assessed with sensitive oddity tasks that do not place obvious demands on episodic memory.
Results: MRI analyses confirmed significant volume loss within the right MDN and intact MTL, including perirhinal cortex. Performance on recognition memory tests revealed a non-verbal recognition memory deficit limited to face stimuli. Additional probing indicated a distinct perceptual deficit in the complex discrimination of faces presented at different viewpoints, with an additional trend toward impaired discrimination of non-face objects.

Conclusions: Memory and perceptual impairment in NC in relation to a right MDN lesion may reflect a common, impoverished representation due to disrupted connectivity with perirhinal cortex. The findings suggest that organization of function is maintained at the level of the thalamus.

Correspondence: R. Shayna Rosenbaum, PhD, Department of Psychology and Centre for Vision Research, York University, 4700 Keele St., Toronto, ON M3J 1P3, Canada.E-mail: shaynar@yorku.ca

R. ROSENBAUM, D. KWAN, V.M. SMITH, E. CIARAMELLI \& C.F. CRAVER. Not all types of future thinking are affected in episodic amnesia.

Objective: Damage to the hippocampus and its connections can affect both the ability to remember past events and the ability to imagine or "pre-experience" future events. To what extent does impaired episodic prospection affect other types of future-oriented thought and judgment? We studied whether individuals with impaired episodic memory and future imagining display the effects of temporal construal on prospective judgements about the self seen in typical participants - specifically, the tendency to construe the near future in concrete, detailed terms and the distant future in abstract, general terms.

Participants and Methods: Patients with lesions to the hippocampus/ extended hippocampal system or to ventromedial prefrontal cortex and controls were administered two measures sensitive to manipulations of imagined temporal distance: prospective agency and prospective confidence.

Results: Results indicate that individuals with episodic amnesia, like healthy controls, changed their construal of events as a function of temporal distance. Similar to controls, patients gave higher ratings of personal agency and prospective confidence for events and activities framed in the distant future than for those framed in the near future. Conclusions: The results show, surprisingly, that the effects of temporal distance on construal are independent from and so not mediated by episodic prospection. These findings bolster the thesis that, like memory, future thinking is not a unitary cognitive faculty.

amed in the near future.

Correspondence: R. Shayna Rosenbaum, PhD, Department of Psychology and Centre for Vision Research, York University, 4700 Keele St., Toronto, ON M3J 1P3, Canada.E-mail: shaynar@yorku.ca

M. ROSSETTI, R. MARTIN, C.B. DUNN, J. BOOTH \& N. WISDOM. Should Male and Female Normative Data be Separate for Verbal Memory Tests? An In-Depth Look at the CVLT-II versus RVLT.

Objective: Recent research has shown that men and women differ in their rate of decline in memory across the lifespan starting as early as the 40 s. However, the majority of commonly used memory tests for adults do not provide normative data by sex. This has the potential to affect the interpretation of performance on memory tests with increasing age. The purpose of the study is to describe raw score changes and associated variability in the CVLT-II across age groups and sex, as this test provides normative data by sex. As well, this will be explored in the RVLT for which sex-based norms are not available.

Participants and Methods: Mean and standard deviations of delayed recall from each age group for men and women or combined groups from the normative data in the CVLT-II and RVLT manuals were used to calculate the coefficient of variation $(\mathrm{CV}) . \mathrm{CV}$ is a measure of score dispersion that further describes the variability of each standard deviation. It is calculated by dividing the standard deviation by the mean and multiplying by 100 . Mean change in $\mathrm{CV}$ from the youngest to 
oldest normative groups will be compared to examine percent change across time.

Results: As would be expected, mean delayed recall scores decreased across age groups for both tests. Increased variability was noted particularly for CVLT-II scores for men $\left(M_{\text {youngest }}=12, S D=2.5 ; M_{\text {oldest }}=6.5\right.$, $S D=2.75$ ) as they had a CV percentage increase from $20.8 \%$ to $42.3 \%$. In contrast, female delayed recall scores $\left(M_{\text {voungest }}=12.5, S D=2.75\right.$; $\left.M_{\text {oldest }}=9, S D=3\right)$ only showed CV variability of $22 \%$ to $33 \%$. Regarding the RVLT, the CV change over time was $18.8 \%$ to $34.3 \%\left(M_{\text {voungest }}=11.7\right.$, $S D=2.2$ and $M_{\text {oldest }}=7, S D=2.4$ ).

Conclusions: This in-depth look at the variability in normative data highlights the importance of separating norms for men and women given their distinct memory decline patterns. This study demonstrates a possible limitation of the RVLT considering that combined sex norms could contribute to inaccurate data interpretation and potential misdiagnosis.

Correspondence: M. Agustina Rossetti, Ph.D., Michael E. DeBakey VA Medical Center, 1 Hermann Museum Circle Drive, Apt. 3073, Houston, TX 77004, United States.E-mail: agustinarossetti@gmail.com

A. SEKHON, L. KINOSHITA, B. HERDANDEZ, A. NODA, K. DOUDELL, V. ALIPIO-JOCSON \& G. GOODALE. Visual Memory Differences Between Older Veterans with PTSD/OSA and OSA Alone.

Objective: Various cognitive domains have been studied in individuals with either chronic Post Traumatic Stress Disorder (PTSD) or Obstructive Sleep Apnea (OSA). Visual memory has not been often explored in these populations, but when studied, results have been inconsistent. Sleep disturbances, such as OSA are common in older veterans with PTSD. This study explored whether performance on tasks of immediate and delayed visual memory significantly differed between a group of older veterans with a comorbid diagnoses of PTSD/OSA and older veterans with only OSA.

Participants and Methods: Data was analyzed from a longitudinal neuropsychological study of male veterans $(\mathrm{n}=176)$ at the Veteran Affairs Palo Alto Health Care System. As a part of a larger battery, the subjects completed the Benton Visual Retention Test - Revised (BVRT-R) and the Rey O-Complex Figure test. Participants met criteria for OSA (apnea-hypopnea index AHI $\geq 5$ ) and those with PTSD $(\mathrm{n}=106)$ met criteria using the Clinician Administered PTSD Scale. Mean age was 63.4 years (SD: 5.73 ) and educational level 15 years (SD: 2.4) for all participants. Statistical analysis includes t-tests to compare the performance of the two groups on measures of immediate and delayed memory.

Results: A significant difference was found between groups for the BVRT-Memory task $(t=-2.0, \mathrm{p}<.05)$, with the PTSD/OSA group performing lower than the OSA group. In general, individuals with PTSD/OSA performed lower across both visual memory tasks.

Conclusions: Results suggest veterans with a comorbid diagnosis of PTSD/OSA are more likely to perform lower on tasks of visual memory, than compared to individuals with OSA alone. Given these findings it would be beneficial to continue with this research, as further research may help clarify issues in this area. Learning more about these deficits can help inform treatment recommendations in this emerging population.

Correspondence: Amardeep Sekhon, M.S., Clinical Psychology, Palo Alto University, 1791 Arastradero Rd, Palo Alto, CA 94304, United States.E-mail: asekhon@paloaltou.edu

D. STEED, M. HAINES, M. BONNELL, A.M. MYERS-FABIAN \& M.C. HUDSON. Left Ventricular Assist Device Placement Significantly Improves Memory for Patients with Advanced Heart Failure.

Objective: Advanced heart failure (AHF) causes multi-system dysfunction, in addition to cognitive decline. The predominant model speculates cerebral hypoperfusion places patients at risk for cognitive decline. Left
Ventricular Assist Device (LVAD) implant is growing exponentially as a treatment modality for AHF. Despite reports of improvement in multiple body systems following LVAD placement, including cognition, research examining physiological mechanisms impacting longitudinal outcomes of cognition is sparse. We have previously found improved cognition after LVAD implant, including processing speed, verbal recall memory, verbal recognition memory, and visual memory.

Participants and Methods: We followed patients pre- and post-LVAD implantation $(\mathrm{n}=12)$, comparing verbal memory functioning and multiple physiological outcomes across time points (A: 3-6 months; B: 6-12 months) for individuals using linear mixed models, hypothesizing a causal relationship between the trajectory of physiological changes after $\mathrm{LVAD}$ implantation and improvements in verbal memory recall.

Results: Cardiac output was related to improvements in verbal free recall up to12 months post-implant, showing a quadratic relationship with regard to memory, with initial improvement and stabilization over time. B-type Natriuretic Peptide (BNP), a biomarker indicating severity of heart failure, showed an inverse quadratic relationship with verbal memory.

Conclusions: Results indicate as heart failure subsides, marked by increase in cardiac output and decrease in BNP, verbal memory improves initially, and stabilizes by year 1 post-implant. Findings expand the predominant model of improvements in cognition following LVAD placement, highlighting the complexity of the relationship between cardiac functioning and cognitive functioning.

Correspondence: Drake Steed, Psy.D., Rehab Psychology Services, The University of Toledo Medical Center, 3000 Arlington Ave., Toledo, $\mathrm{OH}$ 43614, United States. E-mail: drake.steed@gmail.com

K.S. TALBOT \& U. MÜLLER. Prospective Memory in Childhood: Cognitive and Behavioral Differences Among Subtypes and Experimental Methods.

Objective: The current study aimed to investigate circumstances that influence children's PM skills using a parent-report measure of behavior and cognitive difficulties. Event- (EB) and time-based (TB) PM performances were measured using multiple methods to investigate construct validity. TBPM was further subdivided into general and specific. General TBPM is a memory for action that is carried out within a general time frame (e.g., sometime Monday) whereas specific TBPM is carried out at or after a specific time (e.g., $2 \mathrm{pm}$ ).

Participants and Methods: Thirty-five children (aged 7-13) participated in the two-part study. First, children completed the WASI-II, a lab-based specific TB and EB PM task (MISTY), and two working memory (WM) tasks. Parents also completed parent-report questionnaires of their child's everyday PM and RM failures (PRMQC) and of their behaviors (BASC-2). Next, children completed a general TBPM task with their parents on 6 'target days' at their home.

Results: General TBPM did not significantly relate to the other PM measures. WM was found to significantly relate to the MISTY-PM $[r=.484, \mathrm{p}<.05]$, but not to general TBPM or the PRMQC, though controlling for age resulted in non-significant correlations. Correlational analyses between the PM measures and the BASC-2 indicated that both MISTY and PRMQC scores were significantly related to externalizing problems $\left[r_{\text {MISTY }}=-.441, r_{\text {PRMOQ }}=-.363, p<.05\right]$ and behavior symptoms $\left[r_{\text {Misty }}=-.507, r_{\text {PRMOQ }}=-.482, p<.05\right]$, but not to internalizing symptoms or adaptability. General TBPM was not related to any index measures. Differential relationships between subscale scores on the BASC-2 and PM measures will be discussed.

Conclusions: The current findings suggest poor construct validity among the PM measures used. Additionally, a subset of children's cognitions and behaviors appear to differentially relate to the subtypes of PM. More research is needed to further evaluate the validity of the PM measures and to develop interventions for children with PM difficulties. 
Correspondence: Karley-Dale S. Talbot, B.Sc. Honours in Psychology, Clinical Psychology, UVic, 3800 Finnerty Road, Victoria, BC V8N1M5, Canada.E-mail:kdtalbot@uvic.ca

O. PARAG \& E. VAKIL. Distinct eye movements for different cognitive processes as expressed in the face recognition task.

Objective: Current research literature indicates that eye movements made in the learning and testing phases can predict and affect future recognition processes. Nevertheless, only a partial picture is presented regarding eye movements in the various components of recognition processes: Hits, Correct rejections, Misses and False Alarms (FA).

Participants and Methods: Participants in this study viewed human faces in a yes/no recognition memory paradigm. They were divided into two groups - one group that carried out the testing phase immediately after the learning phase $(n=30)$ and another group with a fifteen minute delay between phases $(n=28)$.

Results: The Immediate group had a lower FA rate than the Delay group, and that no Hit rate differences were observed between the two groups. Thus, based on the Hit and FA rates, the Immediate group was overall more accurate than the Delay group. The two groups also exhibited different patterns of eye movements in the testing phase. In addition, eye movements in the learning phase differed only between the recognition conditions in the learning phase, but not in the testing phase. Conclusions: Different eye movement patterns were observed between Hit and Miss trials in the Immediate group and to a lesser extent in the Delay group only in the learning phase, but not in the testing phase. This pattern of results indicates that although eye movements cannot distinguish between the various components of the recognition process, but rather may predict future recognition.

Correspondence: Eli Vakil, PhD, Psychology, Bar Ilan University, Bar Ilan University Ramat Gan, Ramat Gan 52900, Israel. E-mail: vakile@ mail.biu.ac.il

D. WONG GONZALEZ, L. BUCHANAN \& B. LANSUE. The Effects of Encoding Strategies in Associative Recognition Memory.

Objective: The objective of this study was to examine the effects of elaborative encoding, preexisting word associations, and intention to learn in associative recognition memory.

Participants and Methods: Sixty undergraduate students were asked to learn word pairs under elaborative or non-elaborative conditions. The word pairs were formed by words with a preexisting association (compound word constituents) or words that were semantically unrelated. In order to explore intention to learn and differentiate between intentional and incidental learning, we used the directed forgetting paradigm. In this procedure each word pair was followed by a cue to remember or forget the word pair.

Results: Consistent with previous research, we show that associative recognition was more accurate when participants used an active elaborative strategy during encoding (Craik \& Tulving, 1975). We also show that preexisting semantic associations facilitate associative recognition, as recognition was more accurate for word pairs that were formed by constituents of compound words than by unrelated words. Overall, word pair recognition was more accurate when the word pairs were learned intentionally as opposed to incidentally. However, we also show that word associations can be formed incidentally. Even though recognition accuracy was lower for incidental learning when compared to intentional learning, recognition of incidentally learned word pairs was similarly facilitated by elaborative encoding strategies and preexisting word associations.

Conclusions: These results indicate that associative recognition occurs when word associations are learned both intentional and incidentally. Elaborative encoding and preexisting word associations facilitate associative recognition, and these strategies are useful even when word pairs are learned incidentally.
Correspondence: Daniela Wong Gonzalez, Clinical Neuropsychology, Psychology, University of Windsor, 705-166 University Ave W, Windsor, ON N9A5H9, Canada.E-mail:wonggon@uwindsor.ca

S. YANDALL DEJESUS, H.M. HOLDEN, N.E. DEFORD, H.E. ENDRES, E.J. VAN ETTEN, G. WAGNER, F.V. LOPEZ, C.N. HARTLEY, K. SCROGgINS \& P. GILBERT. Spatial Recognition Memory Across the Adult Lifespan: Evidence for Age-Related Deficits in Spatial Pattern Separation in Middle and Old Age.

Objective: Age-related changes in pattern separation may contribute to spatial memory impairment in older adults. Two recent studies indicate that pattern separation may be less efficient in middle-aged adults on tests involving visual objects or temporal sequences; however, this has not been investigated using a spatial task. We used signal detection theory (SDT) to assess spatial recognition memory across the adult lifespan using a new behavioral test hypothesized to tax pattern separation. Correlations with standardized neuropsychological tests also were examined.

Participants and Methods: Healthy young (YA; $n=39)$, middle-aged (MA; $\mathrm{n}=30)$, and older (OA; $\mathrm{n}=30)$ adults completed our spatial recognition memory test. On each trial, participants remembered the location of a circle on a computer screen. Then, a circle appeared either in the same location or a different location that was separated from the original location by a small or larger separation. Participants indicated whether the circle was in the "same" or a "different" location. Smaller spatial separations on "different" trials were hypothesized to result in greater interference than larger separations, placing greater demands on pattern separation.

Results: On large separation trials, we found that YA significantly outperformed both the MA and OA, while the MA outperformed the OA $(p<.05)$. On small separation trials, the YA outperformed both MA and OA $(p<.05)$; however, there was no significant difference between MA and OA. All groups performed significantly better on large separation trials compared to small separation trials $(p<.05)$. Performance correlated significantly with standardized measures of memory, visuospatial perception, and spatial attention $(p s<.05)$, but not word reading or auditory attention.

Conclusions: Using a new test and SDT, we found that age-related changes in spatial pattern separation may begin in middle age. We also found preliminary evidence for construct validity of our test in healthy adults.

Correspondence: Shannon Yandall DeJesus, Psychology, San Diego State University, 3778 Shasta Street, Unit D, San Diego, CA 92109, United States. E-mail: shannon.nicole.yandall@gmail.com

\section{Visuopatial Functions/Neglect/Agnosia}

L.G. ALTOMARE, E. POLEJAEVA, C.N. SOZDA, K.M. HEILMAN \& W.M. PERLSTEIN. The Influence of Traumatic Brain Injury on the Allocation of Vertical Spatial Attention.

Objective: Research on disordered spatial attention has largely focused on hemispatial neglect in brain-lesioned patients, revealing differential allocation of attention to right versus left egocentric or allocentric hemispace. Less research has examined visuospatial attention to vertical space, but several studies of healthy participants have demonstrated the presence of upward (i.e., vertical) attentional biases. The goal of this study was to learn if chronic moderate-to-severe traumatic brain injury (m/sTBI) survivors exhibit alterations in the alerting, orienting, and/or executive-control attention networks with regard to vertical visuospatial attention as assessed using the Attention Network Task (ANT) which combines trial-wise Posner-type spatial cues and Eriksen flanker targets.

Participants and Methods: 12 chronic m/sTBI patients and 12 demographically-matched neurologically-healthy controls (HCs) completed a 
version of the ANT wherein trials were coded for cue and target locations above and below central visual fixation. Trial-wise reaction time (RT) and accuracy were acquired and subjected to mixed-model analyses of variance and planned contrasts.

Results: Findings support the notion of an upward vertical attentional bias; collapsed across groups, median RTs were significantly faster when targets occurred above than below the central visual fixation $(\mathrm{p}<.0002)$. Error rates were $\sim 2$ times greater for target locations below central fixation $(p<.01)$; however, only HCs' error rates differed as a function of target altitude as reflected in a significant group-related interaction term $(\mathrm{p}<.02)$.

Conclusions: Both groups showed an upward RT bias which was greatest to incongruent targets when compared to neutral and congruent targets. Unlike controls, m/sTBI survivors did not exhibit the anticipated upward error-rate attentional bias. As alteration of spatial attention can be a major cause of disability, present findings suggest that $\mathrm{m} / \mathrm{sTBI}$ survivors exhibit signs of altitudinal neglect that might benefit from treatment.

Correspondence: Lindsey G. Altomare, University of Florida, 4341 Nw 49th St. Unit 103, Gainesville, FL 32606, United States. E-mail: galtomare@ufl.edu

\section{O. BOUKRINA, P. CHEN, N. VATSA \& A. BARRETT. Linguistic} Processing of Single Words in Spatial Neglect.

Objective: This study examined the contribution of orthographic and semantic characteristics of words to the rate of reading errors in individuals with spatial neglect, which is an acquired disorder with an impairment in attending to spatial locations contralateral to the injured cerebral hemisphere.

Participants and Methods: A hundred and ten participants with leftsided spatial neglect after right brain stroke read (or spelled) aloud 1 of 2 matched lists of 36 words, presented at the center of a computer screen one at a time. Presentation was self-paced. Reading errors were scored as neglect (error in the left half of the word) or non-neglect.

Results: To predict neglect or non-neglect errors, we used a stepwise regression with variables including word frequency, orthographic neighborhood (number of words that can be produced by changing a letter in a word of the same length), constrained bigram and trigram counts (number of words with the same 2- and 3-letter combinations in the same position), word length, concreteness, and imageability. The best predictors of neglect errors were word length and trigram count, $\mathrm{F}(2.71)=23.40, \mathrm{p}<.001$, accounting for $64 \%$ of the variance. Longer length and greater number of trigram neighbors produced more neglect errors. The best predictor of non-neglect errors was bigram count. Although concreteness and imageability were correlated with the rate of neglect errors (respectively: $r=-.36, p=.027 ; \mathrm{r}=-.39, \mathrm{p}=.011$ ), they did not account for unique variance.

Conclusions: Spatial neglect affects at least two levels of processing in single-word reading. At perception, longer word length predicts more neglect errors. At a deeper level of processing, the first half of a word is more likely to be read incorrectly when the word has more trigram competitors. We hypothesize that identifying a lexical representation among trigram competitors requires depictive capacity, which may be asymmetrically degraded in spatial neglect. Future studies are required to test the hypothesis.

Correspondence: Olga Boukrina, Ph. D., Stroke Rehabilitation Research, Kessler Foundation, 1199 Pleasant Valley Way, Kessler Foundation Stroke, West Orange, NJ 07052, United States. E-mail: oboukrina@ kesslerfoundation.org.

\section{A.J. GOOD, M.K. HARRIS, A. FALCHOOK, R. WATSON \& K.M. HEILMAN. A 34-Year Follow-Up Study on a Patient with Callosal Disconnection Neglect.}

Objective: In 1984, Watson and Heilman reported a patient with a partial callosal disconnection following an infarction of the anterior portions of the corpus callosum. This woman's performance on line-bisection tasks revealed callosal disconnection neglect. The objective of this research is to reexamine this woman in order to gain information on her recovery.

Participants and Methods: The patient completed visual line-bisection tasks in which horizontal lines were placed in different hemispaces and performed using both right and left hands.

Results: There were no significant main effects for hand or spatial field, as there had been previously. The patient's performance with her left hand did not differ significantly from her right, unlike her performance 34 years ago in which each hand deviated to ipsilateral hemispace, independent of the hemispace of line placement. Additionally, performance did not differ based on hemispace, independent of the hand used, as it had previously.

Conclusions: There were notable differences between this woman's most recent performance on the line bisection and her previous performance 34 years ago. In fact, unlike in the prior testing, this patient's most recent performance resembled the performance of individuals in the previous healthy control group for whom differences in hand and hemispace were not found. This finding is significant in that it suggests this patient with partial callosal disconnection no longer shows the 'callosal disconnection neglect' phenomenon, where each hand revealed ipsilateral deviation. Instead, these results suggest that her hemispatial attentional biases have improved over time and are more closely aligned with the performance of normal control participants.

Correspondence: Allison J. Good, University of Florida, 6304 SW 95th Street, Gainesville, FL 32608, United States.E-mail: agood@ufl.edu

A. MANKOWSKA, M. HARCIAREK, J.B. WILLIAMSON \& K.M. HEILMAN. Right Hemispheric Dominance for the Allocation of Spatially Directed Focal Attention.

Objective: When bisecting horizontal lines, healthy adults deviate their attempted bisections toward the left of center (pseudoneglect). This bias has been attributed to right hemisphere dominance for spatial attention. Based on this right hemisphere attentional dominance hypothesis, it could be posited that healthy individuals might also detect or respond to stimuli presented in left space faster than those presented in right space. The aim of this study was to test this hypothesis.

Participants and Methods: Thirty-two healthy participants (19 woman), aged 20-77, performed a Posner-type visual attention task where to the left and right of the central point two boxes were displayed in which an imperative stimulus could appeared. In each trial this stimulus was preceded by a right or left preparatory cue (box highlighting) that was valid in $80 \%$ of the trials.

Results: Participants responded faster to trials with valid than invalid cues, and this effect was significant for imperative stimuli presented in both left and right hemispace. With valid cues, the reaction times were shorter for the left than right-sided imperative stimuli; however, no similar effect appeared for invalid cues. This left-right asymmetry with valid cues was particularly strong in women, whereas age did not contribute to this effect.

Conclusions: Valid cues can both direct the allocation of spatially focused attention and also prepare the motor networks for action. However, if the left-sided cues with right hemisphere activation prepared the motor systems for action, then even miscues on the left should have reduced reaction times more than miscues on the right, but they did not. Therefore, our results suggest that the reduction of reaction times with valid left cues is related to the allocation of spatial attention. Since this task did not require the allocation for global attention, it appears the right hemisphere is dominant for even allocating focal attention. Correspondence: Aleksandra Mankowska, Master, Institute of Psychology, University of Gdansk, Jana Bazynskiego 8, Gdansk 80-309, Poland.E-mail: aleksandra.wojtowic@gmail.com 
A. SMITH, J.R. SEDGEWICK, B. WEIERS \& L. ELIAS. Native Reading Direction Modulates Lateral Lighting Biases for 3-Dimensional Stimuli.

Objective: Native reading direction has been demonstrated to guide opposing lateral biases in visuospatial attention, which is speculated to influence preferences for visual stimuli. Lateral preferences of lighting for 3-dimensional artworks have been investigated, though only tested in left-to-right reading populations. Our objective was to further explore if native reading direction modulated preferential lighting choices for sculptures.

Participants and Methods: A virtual lighting paradigm was administered via a computer task to native left-to-right (LTR) and right-to-left (RTL) readers. The task consisted of 44 trials of individual sculptures presented on a blank background, to which participants were instructed to manipulate a unidirectional light source to a position they perceived to most aesthetically illuminate the artwork.

Results: Support was found for our primary prediction; lateral lighting choices significantly differed between participants with native LTR and RTL reading systems. However, the directionality of preference was contrary to previous observational study of real-world lighting biases RTL readers demonstrated a leftward bias, whereas no significant bias was exhibited by LTR readers.

Conclusions: Our research highlights how native reading direction can guide perceptual judgments of visual stimuli. With respect to lighting bias, this was the second study to find an opposite preference relative to the cultural group, illustrating that behaviour measured from experimental tasks can conflict with real-world biases.

Correspondence: Jennifer R. Sedgewick, Psychology, Arts and Science, University of Saskatchewan, 9 Campus Drive, Saskatoon, SK S7N 5A5, Canada.E-mail:jrs908@mail.usask.ca

D.N. ZINK, C. BIRD, J.Z. KIRKLAND CALDWELL, J.B. MILLER \& S.J. BANKS. The Relationship Between Parietal Lobe Integrity and Neuropsychological Tests of Visuospatial Function.

Objective: Tests of visuospatial function are often administered in comprehensive neuropsychological evaluations. The neural correlates of these tests, using modern imaging techniques, are not yet clearly understood. They are generally considered assays of parietal function, but also to involve a widespread network of regions. In the current study we investigated the relationship between three commonly used tests of visuospatial function and lobar cortical thickness in each hemisphere. Participants and Methods: Data of 383 patients (mean age $=69.7$, $S D=10.6$; $40 \%$ females; $88.3 \%$ right handed; mean years of education=15.1, $S D=2.7$ ), that underwent a neuropsychological evaluation and MRI scans in an outpatient clinic, were included in the analysis. We examined the relationships between cortical thickness, as assessed with Freesurfer, and performance on three tests: Judgment of Line Orientation (JoLO), Brief Visuospatial Memory Test Revised Copy Trial (BVMT-R-C), and Block Design (BD). Using a series of linear regression models, one for each hemisphere and each test, we assessed which lobe's overall cortical thickness primarily predicted test performance.

Results: In the right hemisphere BD appeared best predicted by cortical thickness in the parietal lobe $\left(R^{2}=.168\right)$, JoLO by the temporal lobe
$\left(R^{2}=.194\right)$, and BVMT-R-C by the parietal lobe $\left(R^{2}=.084\right)$. In the left hemisphere BD appeared best predicted by cortical thickness in the parietal lobe $\left(R^{2}=.159\right.$, ), JoLO by the temporal lobe $\left(R^{2}=.194\right)$, and BVMT-R-C by the parietal lobe $\left(\dot{R}^{2}=.104\right)$.

Conclusions: Constructional tests of visuospatial function appear to correspond with underlying cortical thickness of the parietal lobes, while visuospatial judgement tests appear to correspond to temporal lobe thickness. Notably, BD was more sensitive to right sided parietal cortical thickness. Future research using voxel-wise techniques as well as connectivity techniques, including more diverse samples, may help further understanding of the regions and networks involved in visuospatial tests.

Correspondence: Davor N. Zink, MA, Psychology, University of Nevada Las Vegas, 685 Helmhill Ave., Las Vegas, NV 89123, United States. E-mail:davor13nzp@gmail.com

\section{AM Coffee Break \\ 11:30-11:45 a.m.}

\section{Plenary G (Kaplan Memorial Lecture). Language and the Brain: From Past Studies to Future Aspirations}

\author{
Presenter: Nina F. Dronkers \\ 12:00-1:00 p.m.
}

\section{N.F. DRONKERS. Language and the Brain: From Past Studies to Future Aspirations.}

Past approaches to the study of language and the brain have focused largely on the contributions of Broca's and Wernicke's areas. By using advanced neuroimaging techniques with individuals who have aphasia, we have now learned that language is an extraordinarily complex system that requires an extensive and interactive network of brain regions to sustain it. We have also learned that an intricate system of fiber pathways connect these regions together and has been underestimated in terms of its importance in supporting language. This information has advanced our understanding of how the brain processes language in important ways, while inviting future investigations to embrace novel approaches to the study of brain-behavior relationships.

This lecture is intended to help the listener 1) compare past versus present methods of assessing brain-language relationships, and 2) incorporate localizationist models of language and cognition with a network perspective to better understand the neural mechanisms of language and cognition.

Correspondence: Nina F. Dronkers, PhD, University of California, Davis / VA Northern California Health Care System, 150 Muir Rd (126s), Martinez, CA 94553, United States. E-mail: dronkers@ucdavis.edu

\title{
SATURDAY AFTERNOON, FEBRUARY 4, 2017
}

\author{
Kaplan Lecture Luncheon: A Taste of New Orleans
}

1:00-2:00 p.m. 


\title{
Author Index \\ Forty Fifth Annual Meeting \\ International Neuropsychological Society
}

\author{
February 1-4, 2017 \\ New Orleans, Louisiana, USA
}

\author{
Aanes, S. 97 \\ Aase, D.M. 199, 200, 208 \\ Abate, F. 46 \\ Abbott, K. 271 \\ Abeare, C. 35, 195, 259, 264 \\ Abildskov, T.J. 24 \\ Abraham, N. 39, 43, 316, 317, 328 \\ Abrams, D. 80 \\ Abrams, G. 261, 276, 277 \\ Abrams, L. 326 \\ Acker, M. 181 \\ Acosta, L. 249, 315 \\ Acosta, M.T. 90 \\ Adams, B.S. 5 \\ Adams, S.W. 186 \\ Adamson, L. 80 \\ Adinoff, B. 189 \\ Adler, M.C. 174 \\ Adrover-Roig, D. 105 \\ Agarunov, E. 3 \\ Agbayani, K. 333 \\ Aghjayan, S.L. 216 \\ Aghvinian, M. 53 \\ Aguerrevere, L.E. 198 \\ Ahluwalia, M. 159 \\ Ahmadi, A. 5, 203 \\ Ahn, S.S. 210 \\ Ahrabi-Nejad, C. 67 \\ Aiken, E. 267 \\ Ailes, E. 37 \\ Ailion, A. 25, 155 \\ Aita, S.L. 32, 120, 320, 328 \\ Aitken, M.E. 69, 74, 75 \\ Ajilore, O. 61, 62, 106, 192 \\ Alameddine, L.R. 233 \\ Alarie, C. 265 \\ Albert, M. 245 \\ Albert, P. 299, 305 \\ Aleksonis, H.A. 23, 28 \\ Alexander, A. 83 \\ Alexander, C.J. 144
}

Alexander, D.C. 97

Alexander, J. 271

Alfano, C.A. 302

Alford, U. 52

Alger, J. 148

Ali, S. 273

Alioto, A. 32, 41, 44, 99, 102, 332

Alipio-Jocson, V. 337

Aljabar, P. 97

Alkatan, M. 227

Alkozei, A. 190

Allard, J. 141

Allart, A. 325

Allen, J.B. 296

Allen, M. 310

Allen, M.D. 256

Allen, N. 110

Allen, S. 313

Allison, S. 216

Alluri, P. 87

Almane, D. 11, 305

Almdahl, I. 314

Almeida, D. 209

Alterescu, K. 163

Altmann, L.J. 326

Altomare, L.G. 267, 338

Altonji, K. 25

Alva, J.I. 64

Alvarez, E. 119

Alvarez, G. 66

Alvarez, K. 53

Alverson, W.A. 216

Alves, T. 229

Alzheimer's Disease Neuroimaging Initiative, T. 125

Amador, A. 242

Amano, S. 324

Amaral, J.L. 299

Amari, A. 147

Amariglio, R. 216, 315

Amaro Junior, E. 132, 311
Amaya, S. 261, 272

Ambroziak, A.R. 185

Amedoro, S. 155

Amin, M. 256, 271

Amini, S. 171

Ammons, C. 80

An, E. 193

Anblagan, D. 97

Andersen, R.A. 130

Andersen, T. 18

Anderson, A.W. 23, 27, 28

Anderson, D.M. 332

Anderson, J.R. 281

Anderson, N. 180

Anderson, S. 8, 9, 190, 236, 239, 240

Anderson, V.A. 179, 180, 293, 312

Andrews, G. 43

Ang, T. 118, 119, 228, 265, 307

Angers, K. 200, 204

Anghelescu, D. 158, 159

Anisman, H. 3

Annett, R. 161

Ansaldo, A.I. 105

Ansell, K. 294

Anthony, L. 304

Antonini, T. 66

Aparicio, H.J. 228

Apollonsky, N. 93

Apostolova, L. 224

Apple, A. 315

Appleman, E.R. 233, 323

Arana, V. 143

Arbona, C. 161

Arce Renteria, M. 12, 54

Archambault, W. 66

Arcuri, A. 255

Ardolf, B. 41

Arechiga, A. 110

Arentoft, A. 53

Arentsen, T.J. 48

Ares, K. 320 
Areshenkoff, C.N. 98

Armengol, C.G. 139, 300, 303. 304,326

Armstrong, C.L. 155, 156

Armstrong, G. 43, 176

Arnett, P. 42, 146, 171, 247, 248, 265

Aronova, E. 195

Arredondo, B. 195

Arrington, C. 23, 300, 305

Arruda, F. 143

Asarnow, R. 148, 149

Ashford, J.M. 156

Ashwal. S. 70, 73

Asken, B.M. 248, 266

Assari, S. 200

Asthana, S. 218, 223, 245

Atkinson, T.J. 128, 221

Atri, A. 223, 226

Attix, D. 37

Atwater, K. 77

Au, R. 61, 118, 119, 169, 228, 233. $265,306,307$

Auerbach, S.H. 228

August, S. 210

Aupperle, R. 65, 258

Austiff, M.B. 108

Austin, C.A. 76, 291

Avci, G. 185

Avila, J. 140

Ávila, R. 311

Aviv, O. 182

Ayers, A. 89

Aylward, S. 291

Azar, M. 217, 220, 225, 250

Azmi, H. 272

Babajani-Feremi, A. 214, 215

Babbitt, C. 148

Babi. F. 180

Babicz, M. 122, 128, 133, 249, 315

Babikian, T. 148

Babione, J.M. 199, 200, 208

Babu, P. 200, 204

Babulal, G. 216

Bacevice, A. 24, 71, 72, 179

Backwater, J. 193

Badami, F.S. 133

Baek, J. 104

Baena, A.Y. 217

Bagby, R. 35

Bagger, J.E. 217

Baggett, N. 82

Bagner, D.M. 78, 316

Bai. L. 8

Bailey, B.A. 78, 316

Bailey, C.M. 67

Bailey, K.C. 32
Baird, A. 276

Bajo, S. 168

Baker, J. 197

Baker, L. 24

Bal, T.R. 6

Balcer, L. 207

Baldo, J. 51, 274

Ball, G. 97

Ball, K. 52, 92

Ballantyne, T. 75

Balsis, S. 224

Banerjee, P. 100

Bangen, K.J. 22, 124, 132, 133, 254, 255. 259

Bangert, B. 24, 71, 72, 179

Banks, S.J. 18, 126, 165, 166, 250, 334,340

Banuelos, D. 40, 140, 141

Barba, C. 106, 220

Barbarulo, A. 175

Barboza, M. 39, 43, 316, 317, 328

Barch. D.M. 306

Barense, M.D. 336

Barnett, G. 159

Barr, A. 150

Barr, W. 12, 14, 48

Barrett, A. 339

Barrington, A. 82

Barron, C. 319

Barrow, M. 153

Barry-Tanner, T. 244

Barry, D. 38, 254

Bartfai, A. 19

Barthelemy, K. 329

Barthelemy, O. 238

Barton, M. 81, 82, 85

Bashem, J. 32, 259

Basso, M.R. 37, 40, 44, 134, 199, 200 , 326,334

Bastin, M.E. 97

Batalle, D. 97

Batista, R. 209

Battiloro, B. 319

Bauer, P. 11

Bauer, R.M. 248, 256, 257, 266, 270, $271,306,307$

Baughman, B.C. 67

Baum. K. 73

Baum, K.T. 291

Bava, L. 162

Bavaria, J.E. 181

Baxter, M. 329

Bayless, J.D. 207, 208, 334

Bazan, P.R. 132

Beach, J. 32, 320, 328

Bean, P. 156
Bearden, D.J. 87

Beardmore, B. 221

Beare, R. 312

Beauchamp, M.H. 72, 73, 293. 312,313

Beaudoin, C. 72, 73, 313

Beaulieu, R. 93

Beaver, J. 116, 122

Beck, J. 4, 295

Becker, A. 176

Becker, J. 57

Becker, M. 222

Bedard, A. 281

Bedard, M. 3

Bederson, M. 10, 13, 136, 239

Bedminster, M. 174

Bednarz, H. 80

Beebe, D.W. 136, 286, 291, 296

Beers, S. 215

Beers, S.R. 25, 313

Behrens, S. 116, 118

Behroozi, P. 304

Beiser, A. 169, 228

Bell, S.P. 122, 128, 133, 249, 315

Bellemare, D. 66

Bellerose, J. 313

Belleville, S. 275, 311

Bellinger, D.C. 1

Belser-Ehrlich, J. 102

Beltran, J.L. 54, 58

Bender, H.A. 10, 13, 136, 184, 239

Bender, S. 195

Benjamin, A. 294

Benjamin, E.J. 169

Bennett, E. 142

Bennett, J. 58

Bennett, L. 252

Benoit, A. 137

Benseler, S. 69

Benuzzi, F. 194

Berberian, A. 29

Berezuk, C. 121

Berg, J. 33, 126

Berger, J. 231

Berger, N.I. 80

Bergquist, T.F. 253

Bergström, B. 138, 316

Berl, M. 16, 317

Berman, S. 218

Bermudez, C.I. 8, 9, 236, 239, 240

Bernard, J.A. 203

Bernard, T.J. 75

Bernick, C. 18

Bernier, A. 72, 73, 313

Bernier, R.A. 16, 17, 257, 311

Bernstein, J. 102 
Berroir, P. 105

Berry, D.T.R. 50, 165, 318

Berryhill, S. 190

Bertisch, H. 68

Bertolin, M. 6, 81

Bertrand, E. 217, 250

Bessette, K.L. 201, 207

Best, J. 149, 150

Best, M. 71

Bezdek, M. 18, 190, 194

Bhatikar, P. 238

Bhaumik, A. 29, 30, 125, 137, 252

Biars. J. 159

Biassou, N. 58

Bickford, D. 125

Biedunkiewicz, B. 166, 168, 186

Biegon, A. 230

Biekman, B. 67

Bieliauskas, L.A. 42, 170, 272

Bienkowski, P. 185

Bier, N. 311

Bigler, E.D. 24, 71, 72, 79, 83, 131, 179, 197, 205

Bijanki, K. 245

Bijanki, K.R. 246

Bikson, M. 1, 59

Bilder, R.M. 306, 307

Billings, B. 252

Billings, F.T. 167

Billings, N. 32, 259

Bills, S. 88, 89

Binder, J. 10, 11, 14, 132, 183, 332

Biney, F. 253

Birath, B. 99

Bird, C. 250, 340

Bird, W. 25, 313

Bisconti, S. 18

Bjerke, M. 314

Bjuland, K.J. 97

Black, G. 24

Black, S.E. 121, 336

Blackmon, K. 14

Blackwell, L.S. 70, 78

Blanco-Campal, A. 62

Blanco, C. 140, 320

Blanken, A.E. 62,126

Blazel, H. 223

Blonigen, D. 207

Bloomgarden, G. 79

Bo, J. 84

Boada, R. 75

Boardman, J.P. 96, 97

Bober, C. 173

Bobholz, S.A. 201, 203

Bobova, L. 44

Bocti, C. 275
Bøе, Т. 287

Boege, M. 122

Boelen, D. 312

Boettcher, A. 33

Bogle, J. 295

Bogner, J.E. 122, 128, 133, 249, 315

Bohlen, A.J. 107, 318

Bohsali, A. 256, 271

Boiselle, E.C. 299

Bokhari, H.A. 195

Boland, S. 331

Bolton, J. 15

Bomyea, J. 260

Bondi, M.W. 124, 129, 132, 133, 213. 231, 254, 270

Boneff, K. 293

Bonn-Miller, M. 207

Bonnell, M. 170, 337

Bonner-Jackson, A. 250

Bonner, M.J. 156

Bono, A.D. 13, 163, 195

Book, D. 132, 332

Bookheimer, S. 85, 185

Boone, J.K. 248, 266

Boone, K.B. 255

Booth, J. 336

Boots, E.A. 106

Boress, K. 283

Bornstein, R. 185

Borod, J.C. 113, 163, 195

Bosch, D. 324

Bott, N.T. 32, 99

Bottino, C.M. 132, 311

Bouchard, A. 201

Boukrina, O. 339

Boutelle, K. 322

Boutzoukas, E.M. 317

Bowden, S.C. 280

Bowers, D. 7, 49, 113, 236, 239, 240

Bowler, R.M. 186

Bowman, H. 85

Boxley, L. 163

Boyd, B. 65, 258

Boyer, K. 15

Bradbury, K.R. 81

Braddock, B. 81

Bradley, M. 7, 239

Bradshaw, M.E. 101, 157

Bradson, M.L. 42

Braley, R. 122

Brandt, J. 13, 217

Branum-Martin, L. 303

Bray, S. 82

Brearly, T.W. 209, 251

Brega, A. 162

Breiter, H. 271
Brenner, E. 16, 17, 257, 311

Brenner, L.A. 87, 224

Brent, D. 215

Brescia Morra, V. 175

Bressan, S. 180

Brett, B.L. 67

Brewster, R.C. 184

Briceno, E. 19

Brickell, T.A. 254, 262, 263

Brickman, A.M. 26, 37, 124, 220, 223. 231, 245, 250, 306, 322, 324

Brinkman, T.M. 100, 101

Briskin, S. 6 ?

Britt, J.Y. 33, 38

Britz, G. 177, 191, 333

Brodeur, C. 311

Brontë-Stewart, H. 55

Brook, M. 197

Brooks, B. 69, 72, 294

Brooks, M. 197

Broshek, D. 168

Brothers, S.L. 50

Brown, A. 17, 330

Brown, A.W. 253

Brown, G.G. 44

Brown, K. 37

Brown, K.D. 274

Brown, T. 146, 294

Brown, W.S. 191

Browndyke, J. 180, 181

brownlow, J. 65

Brubakk, A. 97

Brucki, S. 311

Brunet, H.E. 332

Bryant, A.M. 163

Bryant, F. 191

Bryant, K.R. 229

Buchanan, L. 331, 338

Buchanan, T. 150

Buchanan, T.W. 6

Buchard-MacDonald, E. 186

Buckley, R. 105, 216, 315

Budding, D. 263

Bueler, E. 288

Bueso-Izquierdo, N. 196

Buis, B. 300

Bujak, M. 209

Bunt, S.C. 79

Bupp, L.L. 34

Burciaga, J. 261, 276, 277

Burd, I. 309

Burke, A. 206, 215

Burke, J. 252

Burke, T. 62

Burnet, D. 5

Burnett, K. 191 
Burns, A.R. 67

Burns, C.M. 164

Burns, T. 76

Burnsed, J. 309

Burrell, L. 221

Burton, C.Z. 202

Burton, J. 309, 310

Busatto Filho, G. 311

Bush, S. 2

Bussell, C. 108

Bussell, K. 210

Butcher, B. 90

Butler, P. 207

Butterfield, L.C. 49, 113

Butts, A.M. 52

Bylsma, F.W. 51

Byrd, D. 54

Byrkit, D.E. 279

Bytomski, J. 37

Cabaral, M. 146

Cabeen, R. 24

Cáceres, G. 117

Cadden, M. 171

Caesar, E.E. 221

Cahn-Weiner, D. 218

Cai, Q. 245

Cairncross, M. 202

Calabrese, M. 174

Calamia, M. 23, 27, 32, 102, 320, 328

Calhoun, O.C. 29, 30, 125, 126

Calkins, M.E. 135

Calvo, D. 172

Cambronero, F.E. 122, 128, 133 , 249,315

Camp, R. 329

Campbell, L.M. 22, 103, 109, 124, 213

Campbell, M.E. 43, 176, 253

Campbell, R. 90, 92, 94

Campbell, S. 137

Canfield, A. 81

Cannistraci, C.J. 23, 27, 28

Cannon. A. 292

Cannon, B. 218

Caprihan, A. 90

Carbine, K.A. 5, 6

Carlew, A. 41

Carlew, A.R. 172

Carlson, K. 237

Carlson, K.E. 75

Carlsson, C. 218, 223, 245

Carmasin, J.S. 317

Carolin, A. 53

carotenuto, a. 175

Carr, J.J. 122, 133

Carroll. E. 156, 268

Carson, A. 82
Carvalho, J. 317

Casaletto, K.B. 102, 103, 249, 250

Casertano, S. 175

Casey, B. 2

Casey, J.E. 259, 282, 283, 284, 285, 288, 292, 295

Casnar, C. 144, 147

Cass, J. 11

Cassedy, A. 66

Cassidy, A.R. 31

Cassill, C. 110, 282

Castagna, P.J. 23, 27

Castellanos, A. 260

Castelluccio, B. 81

Castillo, G.M. 40, 140, 141

Castillo, T.J. 262

Castor, C. 141

Castro, M. 132

Catroppa, C. 312

Caudle, S. 89

Cavaco, S. 9, 232, 233

Cavallo, F. 280

Cecchi, F. 280

Cella, D. 315

Cerbone, B. 218

Cerny, B.M. 202

Chacko, A. 283

Chacko, R. 191

Chan, A. 177

Chaney, G. 120

Chang, F. 224

Chapieski, M. 156

Chaplin, A.P. 254

Chapman, D. 64

Chapman, S. 176, 217, 219

Chappell, A. 30

Charron, C. 196, 197

Charvet, L.E. 59

Chasles, M. 335

Chatman. T. 191

Chau, A. 325

Chaves, J. 9

Chavez-Valdez, R. 309

Cheema, A. 225

Chen, C.A. 196, 216

Chen, E. 274

Chen, J. 82

Chen, M. 103

Chen, P. 339

Cheng, A. 151

Cheran, G. 120

Cheung, Y. 100, 101

Chevignard, M. 142

Chey, J. 103, 104

Chiaravalloti, N. 172, 173, 254, 256,273
Chibnall, J. 243

Child, A. 155, 300

Childs, K.N. 218

Chiou, K.S. 254, 256, 257

Chiu, D. 333

Cho, I. 82

Choi, A. 105, 167, 171

Christ, S. 88

Christensen Løhaugen, G. 96, 97

Christodouleas, J.P. 161

Christoff, K.A. 156

Christopher, D. 143

Chu, Z.D. 67, 69

Ciabattoni, R. 274, 280

Ciaramelli, E. 336

Ciardullo, J. 239

Cicchetti, D. 193

Cicerone, K.D. 211, 212

Cichon, J.C. 108

Cimino, C.R. 235

Cines, S. 120

Cirino, P.T. 300, 301, 302

Claassen, T. 37

Clancy, S. 147

Clark, A.L. 132, 133, 254, 255, 260,270

Clark, B.E. 156, 268

Clark, H. 331

Clark, L.R. 218

Clark, S.V. 203

Clark, U.S. 17, 19, 54, 191

Clark, V. 30

Clarke, C. 180

Claudino, A. 29

Claus, E.D. 269

Clem, M. 87, 219, 234

Clugston, J.R. 248, 266, 270

Cobia, D.J. 23

Cocchini, G. 176

Cohen, D. 24, 71, 72, 179

Cohen, J. 61, 105, 106

Cohen, M.L. 68

Cohen, N. 207

Cohen, P. 68

Cohen, R. 25, 26, 107, 112, 117

Cole, B. 274

Cole, M. 274

Cole, W.R. 48

Colegrove, D. 271

Coleman, L. 186, 290

Coleman. T. 204

COLIN CANCINO, M. 76

Collier, S. 76

Collins, K. 237

Collins, M.W. 68, 71, 77, 78

Colvin, L.E. 176, 219 
Colvin, M.K. 34, 87, 147

Combs, D. 40, 134, 200, 326

Combs, H.L. 165, 318

Combs, T. 203, 291

Compas, B.E. 23, 27, 28

Conforto, A.B. 132

Conklin, H.M. 100, 156, 159

Connelly, M. 333

conniff, j. 143

Considine, C.M. 10, 35, 160, 264

Contrastano, C.M. 219

CONTRERAS VACA, J. 76

Conturo. T. 24

Cook, A. 333

Cook, D. 274

Cook. I. 99

Cook, N.E. 215

Cook, S.E. 34, 142

Cook, T. 156

Corbetta, M. 178, 211

Cordero, E. 38

Cordova, M. 86

Corello-Soares, V. 71

Corey-Bloom, J. 241

Cornwell, M. 10, 43

Correia, A. 232

Correia, S. 24

Correll, D. 20

Correro, A.N. 35

Corson, A.T. 7

Cosentino, S. 120, 176, 217, 219, 220, $225,237,250,315$

Cosio, D. 217

Costa, S.L. 172

Costabile, B. 168, 170

Costabile, T. 46, 175

Costanza, k. 172

Cote, A. 46

Counsell, S. 96, 97, 98

Courrege, S. 283

Cox, D. 69

Cox, L.E. 156

Cox, S. 97

Cox, S.M. 144

Coxe, S. 189

Cramer, J. 8

Crane, N. 202

Crane, P. 314

Craun. E. 282

Craver, C.F. 336

Crawford, J.L. 201, 203, 210

Crawford, J.R. 293

Crespi, Z. 170

Crewe, M. 30

Cristofalo, E. 310

Crocco, E. 123
Crocetti, D. 289

Crocker, L.D. 65, 258, 260

Croft Caderao, K. 330

Cronin-Golomb, A. 233, 238, 323

Crook, C.L. 122

Crosson, B. 21, 184

Croteau-Chonka, E. 293

Crouse, E. 48

Crowley, S.J. 105, 171, 233

Crumedy, J. 177, 191, 333

Crump, C. 3

Crutcher, J. 23

Crutchley, R. 54

Csernansky, J.G. 23

Cuellar, V.M. 10

Cullen, P. 161

Cullum, C.M. 79

Cullum, M. 16, 63, 124, 167, 189, 219

Culnan, E. 248

Culpepper, W.J. 174

Cummings, J. 126

Cummings, S. 256

Cunningham, W.A. 17

Curiel, R. 123

Curran, B. 274

Cury, M.G. 29

D'Sa, V. 4, 22, 293, 295

da Silva, M.P. 311

DaCosta, A. 336

Dahmen, J. 274

Dalchand, E. 138, 220, 225, 230

Dale, A. 213

Dalley, B.C. Allred. 128, 221

Dalton, K. 313

Daly, M. 34

Danguecan, A.N. 68

Daniel, M. 138. 190, 316

Dapretto, M. 85

Darst, B. 218

Dash. T. 105

Dasher, N.A. 156

Daugherty, J.C. 38, 39, 196

Davatzikos, C. 27, 110

Davey, C. 164

David, D. 293

Davis, G. 180

Davis, H. 107, 318

Davis, J. 227, 228

Davis. K. 122, 301, 302

Davis, L.T. 133

Davis, R. 265

Dawson, E. 163

Day, S. 161

de Haan, E. 98

De Iorio, M. 252

de Jonghe, J.F. 35
De Koninck, J. 137

De La Guarda, S. 8, 9

De Marco, A. 168

De Michele, G. 46

De Rosa, A. 46

De Vivo, D. 145

de Werd, M.M.E. 312

De Wit, L. 333, 335

De-Sollar Hale, A. 13

Deane, K. 291

Deasley, S. 282, 292, 295

Debska-Slizien, A. 166, 186

DeCarli, C. 118, 119, 228, 265

Deck, B.L. 126

Decker, M. 116

DeFeis, B.L. 3, 220, 225

DeFord, N.E. 258, 260, 338

DeJoie, O. 267, 268

Dekhtyar, M. 105

DeKosky, S. 180

Del Bene, V. 12, 54

Delano-Wood, L. 124, 129, 132, 133, $254,255,259,260,270$

DelDonno, S.R. 201, 203

Delgado, D. 261, 263

Delis, D. 108, 332

DeLuca, J. 149, 172, 173, 174 , 175,256

DeMatteo, D. 198

Demers, L. 190

Demery, J. 267

Demeter, G. 29

Demeyere, N. 179

Demro, C. 210

Denckla, M. 290

Denney, D.A. 123

Dennis, E. 148

Dennis, M. 17

Denny, K. 106, 220

Deoni, S. 4, 22, 24, 28, 293, 295

Depp, C.A. 208

DeRight, J. 120, 217

Desai, P. 177

Desai, R. 332

DeSollar, A. 10

Despres, O. 7

Deutsch, S. 30

Dev, S.I. 176, 204, 208

Devine, S. 61, 228

Devinsky, O. 14

DeVito, A.N. 234, 282, 301

Devlin, K.N. 123

Dewey, D. 82, 283

Dewey, R.B. 234

Dezhkam, N. 164

Dhillon, S. 35 
Dhima, K. 16, 167, 219, 234

Dias, D. 9

Diaz Santos, A.L. 123, 229

Diaz-Orueta, U. 61, 62

Dickens, J. 107

Dickerson, B.C. 120

Dickson, D.M. 10

Didehbani, N. 79, 124

Didiano, R. 71

DiFilippo, F. 18

DiGangi, J.A. 199, 200, 208

Dijkerman, C. 98

DiLorenzo, M.G. 177

DiMauro, S. 145

Diminich, E. 207

Dina, S. 23

Dinov, I. 252

Dion, C. 61, 106

Dital, A. 97

Dittmar, A. 319

Divatia, S. 190

Divers, R. 106, 116, 251

Dixon, A. 35

Dobryakova, E. 245, 246

Dodd, A.B. 5, 269

Dodge, H. 137, 252

Dodick, D.W. 295

Dolan, B. 82

Dolan, S.L. 137

Dombrowski, C.V. 196

Dome, G. 144, 157, 158

Dominguez Morales, M. 275

Domokos, F. 191

Donahue, M. 133

Doody, R.S. 216

Dooley, J. 293

Dorociak, K.E. 164

Dotson, V.M. 25, 240, 333, 335

Doty, 1. 332

Doudell, K. 337

Douglass, A. 137

Dowling, M.M. 70

Downey, G. 134

Downing, M. 138

Doyle, K.L. 188

Drane, D.L. 95, 96, 184, 246

Dranikov, A. 239

Dreher, A. 92

Dricot, L. 21

Driscoll, A.E. 262

Driscoll, M. 146

Dronkers, N.F. 1, 340

Drouin, M. 275

Drozdick, L. 308

Duara, R. 108, 111, 112, 119, 229

DuBose, D.N. 248, 266
Duda, B. 17, 191

Duda, J. 237

Duda, T.A. 282, 283, 284, 285, 288

Duff, K. 128, 221

Duffield, T. 84

Duffy, J. 331

Dufour, A. $?$

Dugger, A.J. 107, 318

Dugi, E. 67

Dulay, M. 177, 191, 333

Duncan, B. 69

Duncan, C.J. 11, 12

Duncanson, H. 173

Dunn, C.B. 336

Dunne, K. 180

Duong, P. 304

Dupee, B. 46

Duperrouzel, J. 189

Dupont-Frechette, J. 205

Duran, F.L. 311

Durant, J. 33

Durdle, C. 146

Durgerian, S. 222

Durmusoglu, G. 296

Dutt, S. 102, 103, 240, 249, 250

Dutta, M. 330

Duval, J. 275

Duval, N. 275

Duvall, S.W. 318

Dux, M. 174

Dydak, U. 186

Dykstra, J.B. 11

Eagan, D. 227

Ealey, A. 17, 23

Easter, R.E. 200, 204

Eble, D.J. 265

Echon, R. 20

Eckerstrøm. C. 314

Eckerstrøm, M. 314

Edelman, K. 25, 313

Edelmann, M. 101

Edelstyn, N. 234

Edmonds, E.C. 129, 132, 133

Edwards, A.D. 97

Edwards, K.D. 193

Edwards, M. 24

Eigsti, I. 81

Eikenes, L. 97

Elbin, R. 68, 78, 251

Elias, L. 139, 340

Elkind, M.S.V. 176

Ellemberg, D. 47, 66, 74, 265, 266, 269,270

Elliott, K. 235

Ellis, M. 148, 149

Ellis, R.J. 56
Ellis, S. 234

Ellis, T. 238

Elrod, N. 189

Ely, A. 168

Embry, L. 161

Emery, E. 16

Emmert, N.A. 189, 220

Emrani, S. 123, 124

Endres, H.E. 338

Eng, M.E. 48

Engelman, C. 218

Engelstad, K. 145

England, S. 103

Englert, J. 317

Engström Nordin, L. 19

Epker, J. 33

Eppig, J. 124, 213

Epstein, J. 285

Erdodi, L.A. 196, 197

Erickson, K.I. 99, 105

Erickson, S. 318

Erlanger, D. 46

Erlhoff, S. 32, 44, 99

Erus, G. 27

Eskew, L. 39

Espenan, M. 328

Estepp, J. 93

Estevis, E. 40, 157, 200, 334

Ettenhofer, M. 38, 254

Euler, M. 7, 319

Evangelista, N.D. 132, 255

Evans, J. 186

Evans, M.K. 27

Evensen, K.A. 97

Everett, A. 309

Everett, L. 199, 200, 208

Everhart, D.E. 7

Ewing-Cobbs, L. 69, 74, 75, 136

Eyler, L.T. 176, 204, 208, 213, 260

Faber, J. 69, 74, 75

Fagan, A. 216, 244, 245

Fair, D. 86

Falchook, A. 339

Fallon, E. 120

Fallows, R. 319

Fama, R. 55, 134

Fan, J. 322

Fanning, M. 181

Farias, S. 106, 218, 220

Farrell, E. 12

Farrer, T.J. 34, 37, 142, 197

Fasano, M.E. 319

Faskowitz, J. 148

Fastenau, P. 67

Fatoorechi, S. 255, 267, 272

Fay-McClymont, T.B. 69 
Faytell, M.P. 54, 185

Fazio, R. 43

Feder, A. 283

Federin, L. 304

Federoff, H. 225

Fedio, A.A. 255, 256

Fedio, P. 255, 256

Fedor, A. 177

Fee, R.J. 144, 145, 235, 243

Feenstra, H.E. 251

Fein, D. 80, 81, 82, 85

Feirsen, N. 283

Feld, S. 312

Felix, G. 138, 220, 225, 230

Fellows, R.P. 165

Fennema-Notestine, C. 213

Ferenc, L. 284, 290

Ferland, T. 177

Fernandes, J. 232, 233

Fernandez-Gonzalo, S. 55, 56

Fernandez, A.L. 59

fernandino, 1. 332

Ferreres, A. 59, 60

Festa, E.K. 230

Fiandaca, M. 225

Ficker, L.J. 109

Fields, E. 329

Fields, K.N. 197

Fields, L. 124, 330

Fields, M. 13, 136

Figueiredo, E.G. 132

Figueroa, C.M. 3, 203, 210

Figueroa, P. 191

Filibeck, B. 329

Filla, A. 46

Filoteo, V. 236, 237, 239, 241

Fischer, J.T. 136

Fisher, D. 63

Fisher, M. 155

Fisher, R. 8

Fishman, I. 82

Fitzgerald, D. 256, 270, 271

Fladby, T. 314

Flashman, L. 317

Fletcher-Watson, S. 97

Flores, A. 18

Flowers, A.T. 140, 141

Floyd, T.F. 180, 181

Foldi, N.S. 231

Foley, J. 332

Follett, K. 237

Fong, A.K. 256

Fong, C. 82

Fong, M.W.M. 178, 330

Fonseca, R.P. 209

Foote, K. 236
Forbes, P.W. 299

Ford, A.I. 52, 215

Forman, D. 28

Forte, M. 204

Foss, M.P. 120

Fossatti, D. 168, 170

Foster, S. 268

Foussias, G. 47

Fouty, H. 37

Fowler, C. 245

Fox, E. 103, 250

Fox, J. 197

Fox, M.E. 101

Franchow, E.I. 319

Francis, N. 55

Franco, J. 260

Frando, M. 177

Frank, B.E. 136

Frank, J. 145

Frank, S. 233

Franklin jr, D. 56, 57

Franz, C.E. 213, 259

Frazer, K.M. 134, 138, 220, 225, 230

Freedman-Doan, C. 293

Freer, B. 323, 325

Freier-Randall, K. 70, 73

Freitas, J. 9

French, L.M. 254, 262, 263

Freyer, D.R. 162

Friedrich, T. 139

Frijters, J.C. 300

Frim, D. 169, 267

Frishkoff, G. 21

Froehlich, A. 83

Frost, N. 283, 285

Frost, S. 303

Froudist-Walsh, S. 98

Fucetola, R. 330

Fuchs, L.S. 300

Fujino, H. 251

Fulop, T. 275

Fulton, J.B. 90

Fulton, R. 146

Fungwe, T. 141

Funk, B. 12

Fuster, J. 99, 255, 263, 267, 272

Gaasedelen, O. 199, 207, 208, 334

Gabriel, L.B. 202

Gaillard, W. 16

Gaitley, J. 67

gajjar, a. 156

Gajjar, A. 156, 158, 159

Gale, S.D. 74, 75, 205

Gallo, J. 36, 198

Galusha, J.M. 79

Gamble, D. 141
Gammada, E. 231

Gansler, D. 324

Gao, F. 336

Gao, Y. 82

Garau Rolandi, M. 55, 56

Garcia-Barrera, M.A. 98, 321

Garcia-Egan, P.M. 24

García-León, M. 196

Garcia, A. 107

Garcia, D. 78, 316

García, E. 125

Garcia, J. 92

Garcia, M. 53

Garcia, N.E. 165, 318

Garcia, S. 29, 30, 125, 126, 137

Garner-Roberts, J. 79

Garnett, E.O. 30

Garrity, J.H. 30

Gartner, R. 262

Gatchel, J. 217

Gathright, E. 26

Gau, S. 284

Gaudet, C. 215, 327

Gaudet, C.E. 205, 292

Gauthier. S. 311

Gavett, B.E. 108, 321

Gawron. N. 185

Gaynor, L.S. 256, 257

Gazes, Y. 3

Geck, C.M. 137

Gefen, T. 120

Gencarelli, A. 248

Genik, R.J. 21

Genova, H.M. 173, 174, 254, 256

Gerhart, H. 172

Germeyan, S. 16

Gerner, G.J. 309, 310

Gershon, R. 36

Gerst, E.H. 301

Gerstenecker, A. 174

Gerstle, M. 291

Gettens, K. 118

Geva, R. 96, 97

Gfeller, J. 220

Ghazi Saidi, L. 105, 165, 166

Ghilain, C. 70

Gibbs, M. 301

Gicas, K. 150, 151

Giesbrecht, C. 151

Gifford, K. 122, 123, 124, 128, 133. $226,227,249,315$

Giguère-Rancourt, A. 221

Gilbart, E. 187, 188

Gilbert, B. 275, 311

Gilbert, P. 108, 241, 338

Gill, D. 92 
Gill, J. 263

Gillebert, C. 179

Gilligan, S.K. 305

Gillum, R. 141

Gilmore-Bykovskyi, A. 223

Ginger, M. 268

Ginsberg, T.B. 123, 124

Gioia, A. 88, 144, 157, 158, 160

Gioia, G.A. 67, 76, 79

Giordani, B. 137, 252

Giovannetti, T. 106, 114, 116, 123. 126, 180, 181, 220, 229, 242, 251

Girard, J.M. 299

Giza, C. 148

Glass, J. 100

Glass, L. 10, 11, 88, 93, 183

Gleason, C. 223

Glenn, M.A. 63, 225

Glisky, E.L. 114, 115, 312

Glodé, M. 162

Godbey, E.I. 79

Godfrey, M. 36

Godovich, S.A. 286

Godwin, D. 20

Goff, D. 207

Gogniat, M. 107

Gold, J. 210

Goldberg, J.S. 188, 222

Goldberg, K. 63, 225

Golde, S.D. 195

Goldstein, F.C. 42, 107

Goldstein, M. 293

Goldsworthy, R. 36

Golla, L. 39

Golub, S.A. 49

Gomez, K. 224

Gomez, R. 174

Gonçalves, A. 233

Gonen, C. 53

Gongvatana, A. 56

Gonzales, E.C. 90

Gonzales, M. 61, 110, 125, 218

González, D.A. 39

Gonzalez, I. 92, 108, 111, 112

Gonzalez, J. 225

Gonzalez, K. 140

Gonzalez, R. 57, 189

Good, A.J. 339

Goodale, G. 337

Goodrich-Hunsaker, N.J. 24

Goodspeed, K. 70

Gordon, E. 122, 128, 133, 249

Gordon, W. 259

Gorlyn, M. 206

Gosselin, A. 137

Göthlin, M. 314
Gottuso, A. 242

Goverover, Y. 173, 245, 247

Gracian, E. 14

Gracian, E.I. 108

Grafman, J. 265, 324

Graham, A. 86

Graham, E. 309

Graham, L. 141

Graham, T.D. 76

Grange, D. 88

Granholm, E. 213

Grant, A.C. 13

Grant, I. 56, 188, 325

Gratz, Z. 295

Gravel, J. 72, 73, 313

Graves, B. 284

Graves, J. 174

Graves, L. 108, 241

Green, R. 83

Greenberg, B. 89

Greenberg, D. 17, 23

Greene, J. 65

Greening, S.G. 23, 27

Greenstein, B. 251

Greenstein. J. 199

Greif, S. 78, 270

Greif, S.M. 257

Greif, T. 265

Greig, M. 119

Grenier, S. 311

Grieg, M. 108, 111, 112, 229

Griffin, J.W. 108

Grigsby, J. 162

Grilli, M.D. 249

Grima, N.A. 248

Grimes, K.M. 205

Gross, A. 245

Gross, E.Z. 21, 109, 228

Grossner, E.C. 16, 17, 257, 311

Grote, C. 59, 60

Grove, G. 105

Gruber, M. 334

Grundy, R. 142

Gu, Y. 217, 220, 225

Gualtieri, A. 37

Guay, J. 219

Gubanich, P.J. 136

Guerin, J. 302

Guidotti Breting, L.M. 52

Guillery, N. 285

Guinea, S.F. 117, 125

Guise, B. 38

Gullapalli, R. 27

Gullett, J.M. 256, 271

Gunstad, J. 17, 26, 41, 112, 281

Guo, F. 8
Gur, R.C. 65,135

Gur, R.E. 135

Gurnani, A. 108, 166

Gurney, J.G. 100

Guskiewicz, K.M. 261

Guty, E. 247

Guzman, D. 37

Guzman, V. 184

Guzman, V.A. 26, 54

Haaland, K.Y. 30

Haas, S. 173

Haase-Alasantro, L. 231

Habeck, C.G. 250

Håberg, A.K. 97

Hackett, K. 221

Hagan, M. 19

Hagerty, A. 126

Hagler, D.J. 213

Hahn-Ketter, A. 199

Hahn-Ketter, A.E. 52, 207

Haines, M. 170, 337

Haisley, L.D. 205

Hajnal, J.V. 97

Hakim-Larson, J. 285

Hale, C. 37

Haley, A. 110, 227

Halfacre, M. 163

Hall, L. 93, 158, 159

Hall, S. 156, 268

Hall, T. 84

Hallowell, E.S. 17, 112, 191

Halperin, J. 281, 288, 289

Halstead, T.S. 228

Halverson, M. 319

Hamberger, M.J. 95

Hammeke, T. 169

Hammers, D.B. 128, 221

Hammond, J. 25 ?

Hampstead, B.M. 17, 29, 30, 125, 126 , $132,137,252,311$

Han, D. 31

Hancock, L. 223

Hancock, R. 304

Hanks, R. 32, 259

Hanlon, R. 197

Hannay, H. 136

Hanseeuw, B. 315

Hanson, M. 11

Hanten, G. 67, 69

Happer, K. 171

Harb, G. 65

Harciarek, M. 65, 166, 168, 186, 339

Hardcastle, C. 55, 134

Hardy, D. 99, 261, 263, 267, 272

Hardy, K.K. 88, 89, 90, 144, 157, 158 , $160,161,304$ 
Hardy, L.M. 301

Hardy, S. 88, 89

Harel, B.T. 156

Hargrove, W. 181

Harik, L. 258

Harker, L.A. 15

Harmell, A.L. 221

Harmon, C. 157

Harris, M.K. 339

Harrison Goldman, T. 4, 22, 293, 295

Harrison, C.E. 232, 235

Harrison, D.W. 140, 320

Hart, C. 134

Hart, J. 63, 124

Hartley, C.N. 338

Hartley, N. 320

Hartline, K. 74

Hartman, E. 24

Hartnell, M. 195

Harvey, D. 146, 218, 220

Hasanaj, L. 207

Hasbun, R. 58

Hassan, I. 199, 200, 208

Hassenstab, J. 244

Hathaway, K. 144

Hauger, R.L. 213

Haut, M. 178, 229

Hawks, Z. 88, 321

Hawley, N. 226

Hayes, J. 28, 99

Hayes, S.M. 28, 99

Hays, C.C. 22, 103, 109, 260

Hazlett Elverman, K. 3, 320, 323

He, X. 21

Head, D. 216

Hearps, S. 180, 293

Heaton, I. 67

Heaton, R.K. 44, 56, 57, 188, 325

Heaton, S.C. 78, 316

Heber, E. 281

Heffelfinger, A. 159, 162

Hegde, R. 19

Hegerty, S. 5

Heidebrink, J. 120

Heidel, A. 123

Heideman, E. 87, 291

Heilbrun, K. 198

Heilman, K.M. 65, 166, 168, 186, 332. 338,339

Heindel, W.C. 230

Heinrich, K.P. 160

Heinrichs, R.J. 34, 198

Heinz, A.J. 207

Heitzer, A. 91, 92, 332

Hendershott, T. 55, 235

Henderson, D. 257
Hendriks, M. 37

Henley, J. 77

Henneghan, A.M. 157

Hennessey, L. 48

Hennig, N.M. 193

Henning, J. 269

Hennrick, H. 293

Herdandez, B. 337

Hergert, D.C. 235

Hermann, B. 11, 95, 305

Hernaiz Alonso, C. 105, 167, 171

Herrera-Hamilton, A. 74

Herrera, J. 141

Herringshaw, A.J. 83

Herrold, A. 271

Hershaw, J. 38

Hertzing, W.J. 227

Hessen, E. 314

Hestad, K. 57

Heyanka, D. 273

Heyrend, R. 319

Hidalgo-Ruzzante, N. 196

Hidiroglu, C. 210

Hill-Jarrett, T.G. 112

Hill, B.D. 32, 33, 197, 320, 328

Hill, C. 329

Hill, S. 203

Hillary, F.G. 16, 17, 131, 257, 311

Hilsabeck, R.C. 168

Himali, J. 169, 228, 233

Hinkin, C. 56, 185

Hinrichs, K.H. 200, 204

Hinshaw, S. 287

Hinton, V.J. 144, 145

Hirabayashi, K. 289

Hiralall, A. 171

Hirst, E. 69

Hirst. R. 50

Hizel, L. 105, 167, 171, 233

Ho, J.K. 62, 110, 126

Hodges, E.K. 160

Hoeft, F. 304

Hoelzle, J. 41, 169, 290

Hofer, S.M. 98

Hoffman, J. 221

Hoffman, M. 156

Hoffman, S.N. 258

Hoffschimdt, S. 335

Hohman, T.J. 122, 123, 124, 128, 133. $227,249,315$

Hokkanen, L. 301

Holcombe, J.S. 32, 320, 328

Holden, H.M. 108, 241, 338

Holder, C.M. 214, 215

Holder, N. 79

Holdnack, J.A. 298, 299
Holguin, G. 231

Holiday, K.A. 260

Holland, A. 70, 161

Holland, A.A. 87

Holland, A.K. 140, 320

Holland, C.L. 71

Hollatz, A.L. 75

holler, k.a. 205, 215, 292, 298, 327

Holtzer, R. 102, 103, 114, 117, 183

Holtzman, D. 216

Homaechevarria, A.A. 257

Honer, W.G. 150, 151

Honjo, K. 336

Hoover, B. 90

Hopkins, R. 79

Hoptman, M. 256

Horgas, A. 115

Horn, K. 221

Hortman, K. 25

Horton, D.K. 89, 167

Horwitz, J.E. 166

Hoscheidt, S. 218

Hoskinson, K.R. 17, 23, 27, 28

Hossain, M. 309

Hoth. K. 164

Hovda, D. 99, 255, 261, 263, 267, 272

Hovey, R. 116

Howard, M. 295

Howarth, R.A. 70, 78, 89

Hoyman, L.C. 33, 38

Huang, I. 100

Huang, L. 156

Huber, R.S. 205

Hudon, C. 311

Hudson, J. 320

Hudson, M.C. 170, 337

Hudson, M.M. 100, 101

Huey, E. 120, 220, 237, 250, 324

Huffman, J. 24

Hughes, E.J. 97

Hughes, J. 26

Hughes, S. 89

Huisman, T. 310

Hüll, M. 312

Hulswit, J. 106, 116, 251

Humphries, C. 25, 332

Hundal, J.S. 12

Hunt, I.J. 205

Hunter, B.P. 198

Hunter, J. 67, 69

Hunter, S.J. 55, 134, 320, 329

Huo, Z. 301, 302

Hurd, Y. 288

Hurtubise, J. 196

Hussey, J. 274

Huston-Warren, E.A. 302 
Hwang, E. 161

Hwang, S. 206

Hyland, F. 177

Hynan, L.S. 63, 124

Hysing, M. 287

Iampietro, M. 93

Ibanez-Casas, I. 38, 39

Im-Wang, S. 174

Imbeault, H. 275

Indredavik, M.S. 97

Ingersoll, B. 80

Inman, C. 246

Insel. P. 125

Irish, J. 158

Isaac, L. 39

Isaacson, R.S. 221

Islam, R. 163

Iudicello, J.E. 56, 325

Ivanov, I. 281

Iverson, G.L. 98, 321

Iwamoto, B.K. 70, 73

Izzetoglu, M. 103

Jackson, C.T. 135

Jackson, H. 45

Jackson, L. 85, 303

Jacobson, A.J. 188, 222

Jacobson, L. 297, 298

Jacola, L.M. 100, 158, 159

Jacques, P. 228

Jagid, J.R. 8, 9, 236, 240

Jahedi, A. 82

Jak, A.J. 65, 213, 258, 260

James, A. 21

Jameson, M. 329

Janecek, J.K. 164

Jang, J.Y. 126

Janulewicz-Lloyd, P. 171

Jashar, D. 71

Jasper, B.C. 133

Jay, M. 70

Jeevan, S. 177, 191, 333

Jeffay, E. 47

Jefferson, A.L. 122, 123, 124, 128, 133, 226, 227, 244, 245, 249, 315

Jenkins, L.M. 201, 203

Jennette, K. 187

Jennings, K. 83

Jeremy, E. 213

Jermakowicz, W. 8, 9

Jerram, M. 173

Jiang, Z. 256

Jiménez, D. 125

Jimenez, E. 222

Jin. Y. 148

Jivani, S. 74, 75

Jódar, M. 55, 56
Joelson, S. 22, 293

John, S.E. 321

Johnson, E. 44

Johnson, E.M. 265

Johnson, J. 148

Johnson, K. 216, 217, 315

Johnson, L. 262

Johnson, N. 321, 329

Johnson, S.C. 218, 223, 244, 245

Johnson, S.P. 141

Johnston, M. 310

Jones, A.A. 150

Jones, E. 170

Jones, E.C. 112

Jones, E.F. 294, 298

Jones, J. 11, 56, 240, 305

Jones, J.D. 135

Jones, R. 110, 314

Jones, T. 121

Joo, W. 104

Jorge, R. 65

Jorgenson, M. 285

Josephs, K. 331

Josephson, R. 26

Joubert, S. 335

Juan, R. 198

Judd, T. 59, 60

Julian, L. 174

Julin. P. 19

Junquera, A. 125

Jurick, S.M. 65, 258, 260

K Thiruselvam, I. 178, 229

Kabuba, N. 57

Kadziolka, M. 194

Kaemmerer, T.D. 3

Kahalley, L.S. 155

Kais, L.A. 191

Kaldis, T. 177

Kamali, M. 200

Kamat, R. 245, 246

Kamath, V. 120, 161

Kana, R. 80, 83, 154

Kandasamy, A. 285

Kang, G. 101

Kang, J. 64

Kang, Y. 111, 334

Kangiser, M. 188

Kanner, A. 8, 9

Kanser, R. 259

Kanser, R.J. 32

Kaplan, D. 47

Kaplan, E. 259

Kaplan, L. 231

Kaplan, R. 47

Kaplan, R.F. 118

Kaplan, S. 282
Kapoor, A. 45

Karam, S. 84

Karolis, V. 98

Karpouzian, T. 206

Karr, J.E. 98, 321

Karst, J. 82

Karstens, A. 61, 62, 106, 192

Kasner, S. 181

Kassa, M. 252

Kassel, M.T. 19, 25

Katzel, L. 27

Kaufman, D.A.S. 6, 15, 81

Kaundinya, G. 21

Kaup, A.R. 110, 259

Kaur, A. 171

Kaur, S. 110, 227

Kauzor, K. 40, 140, 141

Kavanaugh, B.C. 205, 215, 292. 298,327

Kavcic, V. 137

Kay, C.D. 120, 222

Kaye, W. 168

Kaylegian, J. 55, 329

Kayser, K. 159

Keating, L. 168

Keatley, E. 259

Keaveney, J. 219

Keedy, S. 134

Keefe, S.M. 161

Keelan, R.E. 32, 259

Kegel, J. 38, 254

Kegeles, L.S. 216

Keilp, J.G. 206, 215

Keith, C. 6

Kelleher, T. 164

Keller, A.V. 65, 258, 260

Keller, J. 102

Kelly, D.F. 261

Kemp, E. 222

Kemp, S. 308

Kenna, M. 293

Kennedy, T. 88, 89, 144, 157, 158, 160

Kenworthy, L. 304

Kepe, V. 18

Kerns, K. 273, 276, 279

Keroack, J. 79

Kerwin, M. 123, 124

Keshavarzian, N. 153, 154

Kesler, S. 157

Kessels, R.P.C. 312

Keutmann, M. 57

Key-DeLyria, S. 264, 331

Kiefel, J. 69

Kiely, T. 223, 226

Killgore, W. 260

Killgore, W.D. 5 
Killgore, W.D.S. 190

Kilshaw, R.E. 98

Kim, H. 104, 334

Kim, J. 103

Kim, K. 275

Kim, M. 187, 206

Kim, N. 21, 261

Kim, R.T. 260, 270

Kim, S. 103

Kim, Y. 111

Kimberly, S. 118, 119

King, T.Z. 25, 101, 137, 155, 184, 203. 277

Kingery, K. 285

Kinoshita, L. 337

Kinsella, G.J. 111

Kipps, C. 4

Kirkland Caldwell, J.Z. 126, 340

Kirkwood, M. 66, 74, 76, 179, 180

Kirlin, K. 11, 12

Kirmse, R. 52

Kirsch, A. 291

Kirton, J.W. 261

Kirwan, B. 205

Kisala, P. 68

Kitchen Andren, K.A. 170, 272

Kitchener, E. 4

Kivisto, L.R. 284, 285, 292, 295

Klarr, J. 91, 92

Klaver, J.M. 312

Klein-Tasman, B. 144, 147

Kling, L.R. 201, 202, 203, 207

Klipfel, K. 89

Knepp, M.M. 322

Knight, R.T. 94

Knight, S. 156

Knopman, D. 164

Kohen. C. 19. 187

Kolcun, J. 8, 9

Kondo, D.G. 205

Kontos, A.P. 68, 71, 77, 78, 251

Koop, J. 159, 162

Kordovski, V.M. 57, 185

Korell, A.M. 20

Kornblith, E. 261, 276, 277

Korthauer, L.E. 11

Koskinen, S. 279

Koss, J.D. 248

Kossman, A. 39

Kotelnikova, Y. 202

Kouo, T. 27

Kovelman, I. 18

Kowalski, K. 71, 72

Koyama, T. 86

Kozlowski, A. 177

Kozlowski, L. 21
Kramer, J. 44, 99, 103, 240, 249. 250,332

Kramer, J.H. 32, 102

Kramer, N. 271

Krapf, E.M. 312, 328

Krasin, M.J. 100

Kravitz, A. 79

Kreisel, C. 224

Kremen, W.S. 212, 213, 259

Krengel, M. 46, 171

Krentzel, A. 70

Kresge, H.A. 133

Krichbaum, K.L. 322

Krijnen, M. 90

Krishnamurthy, L.C. 17, 21, 23

Krishnamurthy, V. 17, 21, 23

Krishnan, K. 253

Kristensen, C.H. 209

Kritikos, L. 249

Krivenko, A. 39, 43, 316, 317, 328

Kriz, D. 84

Krohner, S. 32, 259

Kroll. J. 98

Krone, B. 281

Krull, K.R. 100, 101

Kuhn, T. 56, 185, 192

Kulubekova, S. 64

Kumar, A. 62, 192

Kumar, S. 83

Kurniadi, N. 168, 322

Kurowski, B. 78, 314

Kuttesch, J.F. 23, 27, 28

Kuwabara, H. 141

Kuzniecky, R. 14

Kwak, S. 104

Kwan, D. 336

Kwan, V. 72

Laasonen, M. 301

LaBelle, D.R. 334

Labelle, V. 335

Lace, J. 265

Lacey, W.H. 300

Lacombe, G. 275

Lacritz, L. 16, 234

Lacy, M. 145, 169, 267

LaDuke, C. 198

LaFary, K. 329

Laffer, A. 34, 87

LaFleur, J. 11

Lafo, J.A. 7, 236, 239

Lafosse, J. 142

Lai, E. 237

Lai, J. 177

Lai, J.K. 51, 207

Laidlaw, D. 24

Lainhart, J. 83
Lajeunesse, A. 335

Lajiness-O'Neill, R. 18

Laliberte Durish, C. 72, 294

Lalonde, G. 72, 73

Lam, D. 35

Lam, G. 159

Lamar, M. 61, 62, 106, 123, 124 , 192,211

Lamb, D. 65

Lamb, R. 50

Lampson, E. 87

Landau. S. 230

Landeira-Fernandez, J. 250

Landin-Romero, R. 313

Landry-Roy, C. 73

Landry, A. 84

Landsman, R.A. 297

Lang, A. 65

Lang, D. 150, 151, 152

Lang, M. 143

Langan, L. 10, 184, 239

Lange, N. 83

Lange, R.T. 254, 262, 263

Langenecker, S.A. 19, 200, 201, 202. 203, 204, 207, 259

Langhorst, B. 86

Langlois, F. 275

Lansue, B. 331, 338

Lanzillo, R. 175

LaPoint, M.R. 105

LaPointe, L.L. 4

Lara-Ruiz, J. 40, 261, 263, 267

Larson, M. 5, 6, 205, 267

Last, B.S. 26, 37, 223

Laszlo, S. 80

Latzman, R.D. 78, 203

Lau, L. 40, 134, 326

Launer, L.J. 110

Launes, J. 301

Laurin, D. 221

Lavrador, S. 173

Lawless, S. 217, 220, 225

Lazar, M. 272

Lazosky, A. 202

Lazowski, R.A. 251

Le, T. 322, 324

Leaffer, E.B. 144, 145, 294

Leal, G. 108, 111, 112

LeBlond, E. 73

Lebowitz, B.K. 336

LeCheminant, J. 6

Lechuga, D. 252

Ledesma Torres, L.A. 300, 303, 304,326

Lee-Wilk, T. 174

Lee, A. 223, 226 
Lee, B. 334

Lee, B.G. 18

Lee, C.M. 84

Lee, G.J. 188, 222

Lee, J. 141

Lee, K. 144, 147

Lee, S. 120

Lee, Y. 11, 223

Leese, M. 21

Lefebvre, L. 275

Legarreta, M. 288

Leger, G.C. 33

Leiken, K. 285

Leitner, D. 43, 50, 176, 178, 253

Lemelman, A. 80

LeMonda, B. 48

Lencioni, M. 304

Lengenfelder, J. 173, 174, 254, 273

Lengu, K. 18

Lenihan, J. 70, 73, 159

Leon-Carrion, J. 275

León-Domínguez, U. 275

Leon. K. 92

Leonard, B.E. 38, 39

Leong, S. 141

Leonhart, R. 312

Leow, A. 61, 106, 192

Lépine, J. 66

Leppert, M. 310

Leppo, R.H. 89

Leritz, E. 65, 177

Lerner, A.L.M. 146

Lesnovskaya, A. 29, 125, 126

Lesnovskya, A. 30

Lesser, R. 84

Lessig, S. 236, 237, 239

Letendre, S.L. 56

Leuchter, A. 99

Leung, K. 251

Levenstein, J. 179

Levin, B.E. 236, 239, 240

Levin. H. 67, 131, 149

Levin, H.S. 69, 74, 75, 131, 148

Levine, A. 57

Levitch, C. 261, 286

Levy, S. 105, 112, 171

Li, C. 101

Li. S. 84

Li. T. 19

Li. X. 67.69

Liang, J. 322

Libben, M. 43, 50, 176, 178, 253

Libon. D.J. 61, 123, 124

Lichtenberg. P. 109. 137

Lichtenstein. J. 77

Liebel, S.W. 112, 191
Lieberman, L. 192

Light, S.N. 18, 190, 194

Liguori, A. 46

Lima, E. 74, 110

Limond, J. 142

Limperopoulos, C. 309

Lin, A. 147

Lindbergh, C. 17, 310

Lindsey, H.M. 74, 75

Ling, J. 5

Linnea, K. 77

Linton Reesman, J. 297, 303, 319

Lipinski, D. 251

Lippa, S.M. 254, 262, 263

Lipton, M. 261

Lipton, R. 230, 261

Lisdahl, K. 187

Lisdahl, K.M. 187, 188

Lissek, S. 195

Lithfous, S. 7

Litvan, I. 236, 237, 239

Liu, D. 122, 128, 133, 249, 315

Liu, H. 99, 192

Liu, J. 85

Liu, J.R.M. 74

Liu. S. 224

Liu, W. 100, 101

Liu, Y. 118, 119, 228

Livai, G. 301

Llanes, S. 235

LoBue, C. 63, 124, 219

Locano, E. 260

Locke, D. 11, 12

Loewenstein, D. 108, 111, 112, 119. 123, 229

Loft, S. 54

Lojek, E. 185

Loman. PhD. M. 93

Longoria, J. 286

Lopera, F. 217

Lopes, J. 9

Lopez, F.V. 236, 239, 241, 338

Lopez, L. 159

Lopez, M.G. 167

López, N. 117

Lopez, W. 99, 263, 267

Lordo, D.N. 15

Loring, D. 42, 107, 280

Lortie, J. 74, 270

Losinski, G. 222

Lott, M. 286

Louis, E. 237

Love, C.E. 74

Lowe, D.A. 224

Lowe, J. 92, 318

Lowe, M. 8, 9
Loyer, E. 333

Loyning, A. 264

Lu, L.H. 3

Lu, P.H. 222

Lu, W. 152, 153

Luca, C. 236, 239, 240

Lucchetti, A. 146, 294

Ludwig, M. 177

Lugar, S. 37

Lukács, Á 29

Lumley, M. 259

Lundervold, A. 287

Lundervold, A.J. 287

Lunsford, K. 254

Luo, P. 237

Luong-Tran, C. 304

Lus, G. 175

Luu, H. 207, 208, 334

Luxton, D. 251

Ly, J.J. 231

Lynch, T. 120

Lyon, A. 181

Lyons, M. 213

Lyons, M.J. 213, 259

Mabbott, D.J. 156

MacAllister, W.S. 12, 294

Mace, L.C. 112

Machulda, M. 331

Macina, L.O. 231

Mackala, S.A. 210

Mackay-Brandt, A. 219

Mackie, M. 322

Mackin, S. 125

MacLean, P. 318

MacLeod, M. 69, 74, 75

MacMullen Freeman, L. 90

Macoun, S. 273, 276, 279

MacSween, J. 273, 276, 279

Madercic, S. 67

Madigan, N. 335

Madsen, J.R. 15

Maerlender, A. 77

Maeta, T. 251

Magnuson, S. 12

Mahatan, H.S. 262

Mahdavi, K.D. 232

Mahmood, Z. 56, 185, 192

Mahone, E.M. 284, 289, 290, 294, 296, 297, 298

Mahoney, E.J. 11

Mahoney, J. 102, 103

Mahoney, J.J. 168, 195

Mahoney, M. 142

Maiman, M. 12, 53

Maitland, C.G. 4

Maki, P.M. 57, 201, 230 
Malhas, R. 225

Malhi, S. 331

Malleck, M. 264

Malloy, P. 24

Malone, C. 335

Mancioppi, G. 129

Mancuso, S. 34, 87

Mandelbaum, S. 10, 13, 184, 239

Manderino, L. 41

Mangal, P. 7, 49, 113, 236, 239

Mankowska, A. 168, 186, 339

Manly, J.J. 26, 59, 60, 112, 134, 138 , $220,223,225,230,231,244,245$

Mann, J.J. 206, 215

Manning, C.A. 121, 233, 242

Manning, K.J. 47, 118, 182

Mano, Q. 302

Manoochehri, M. 120

Mansfield, D. 248

Maple, K.E. 188

Mapstone, K. 225

Mapstone, M. 225

Maranhão, M. 29

Marcangelo, M. 145

Marceaux, J. 39

Marcone, S. 335

Marcopulos, B.A. 195

Marcuse, L. 13, 136, 184

Marden, J. 112

Mareci, T. 256, 271

Margolis, A.E. 301, 302

Margolis, S.A. 13

Maricle, D. 286

Marinazzo, D. 6

Marinelli, R.E. 3

Marino, C. 75

Mark, V.W. 281

Marks, W. 237

Marquez, D.X. 106

Marra, D.E. 41, 183, 290

Marreiro, C. 223, 226

Marrone, N. 114

Marsh. A. 22, 298

Marsh, R. 301, 302

Marshall, D. 200, 204

Marshall, P. 290

Marsiske, M. 49, 113, 115

Marson, D. 174

Martin-Willett, R. 122, 128, 133. 249,315

Martin, A. 16

Martin. C.B. 336

Martin. E. 57

Martin, L. 78, 314

Martin, L.E. 325

Martin, M.M. 132, 311
Martin, P. 198

Martin, R. 174, 336

Martindale, S.L. 137, 209, 251

Martinelli, M. 287

Martinez, F. 263, 272

Martinez, M.N. 138, 220, 225, 230

Martins da Silva, A. 9

Martins, C.B. 311

Martinussen, M. 97

Marton, K. 261, 276

Marx, G. 102, 249

Masand, A. 322, 324

Mascarenhas, L.F. 311

Masey, A. 225

Massa, J. 85, 264

Massman, P.J. 54, 58, 101, 216, 218

Massoni, S.G. 304

Mastel, S. 84

Masters, C. 245

Mather, M. 127, 193, 213

Mattes, A. 91, 92

Mattis, P.J. 235, 243

Mattson. S.N. 88

Mattsson. N. 125

Matyi, J. 116, 118

Maxson, C. 277

Maxwell, E.C. 75

Maxwell, K. 242

May, A.C. 135

Maye, J.E. 49, 113

Mayer, A.R. 5, 269

Mayeux, R. 26

Maynard, T. 323

Mazzola, K.S. 323, 325

McArthur, D. 99, 255, 261, 263. 267. 272

McAuley, T.L. 259, 331

McCabe, D.L. 152

McCabe, K.L. 146

McCarthy, J. 276

McCarthy, R. 4

Mccarvel, M. 156, 268

McClean, M. 265

McClintock, K.L. 265

McCrea, M.A. 248

Mccrimmon, A. 82

McCuddy, W.T. 26, 41, 320, 323

McCurdy, K. 215, 298, 327

McDonald, C. 14, 95, 96

McDonald. J. 222

McDonald, K. 177

McDonald, S. 293, 313

McDonnell, M. 188

McFarland, C.P. 277

Mcfarland, J. 156, 268

McFarlane, K. 18
McGee, J. 278

McGinnis, S. 120

McGlade, E. 288

McGlinchey, R. 65, 177

McGrath, M. 219

McGregor, K.M. 21

McInerney, K. 8, 9, 240

McInerney, K.F. 236, 239

McInnis, M. 19, 200, 202, 204

McIntosh. E.C. 64

McKay, D. 41

McKindles, R.J. 3

McKinney, T.L. 7

McKnight, S.E. 146

McKown, C. 193

McLaren, M.E. 25, 333, 335

McLaughlin, R. 12

McLean, E. 323, 325

McLeod, J. 144

McMains, J.T. 191

McManus, D.D. 169

McMillan. T.M. 11

McMorris, C. 283

McNabb, J. 13

McNally, K. 11

McNamara, E. 147

McNeal, K. 322, 324

McNevin, N. 285

McVey, A. 82

McWilliams, K. 153, 154

Mead, C. 41, 102, 332

Means, B.L. 159

Mecca, A.P. 222

Mechanic-Hamilton, D. 45, 123

Medina. L.D. 139

Meffert, B. 207

Mehl, M. 115

Meier, I.B. 37, 124

Meinerding, M.E. 220, 225

Mejia, A. 141

Melhem, N. 215

Melikyan, Z.A. 141

Melo, J.G. 123

Meloy, M. 22, 103, 109, 260

Meltzer, E.P. 113

Meltzer. H. 206

Memel, M.B. 330

Mencl. W. 300

Mendes Estella, N. 29

Mendes, A. 233

Mendez, C. 52

Mendez, M.F. 222

Menon, J. 57

Mercer, E.N. 4, 295

Merritt, V.C. 42,146

Merz, Z. 265 
Messe, S. 181

Mestre, Z. 322

Mesulam, M. 333

Mewborn, C. 107, 310

Meyer, J. 265

Meyers, E. 286

Meza, J. 287

Michalak, H. 222

Michalowski, J. 166, 186

Michalski, J. 161

Michel, S. 140

Michelsson, K. 301

Miciak. J. 301

Mickey, B.J. 203

Midlarsky, E. 113

Miele, A. 28

Mietchen, J.J. 74, 75

Migliorini, R. 135

Mihalov, L. 24, 71, 72, 179

Milberg, W.P. 65, 177

Miley-Akerstedt, A.M.K. 136

Millan, C. 8, 9

Millan, M. 260

Miller, A.K. 40, 134, 326

Miller, A.L. 20

Miller, B. 44, 249

Miller, C. 194

Miller, D. 286

Miller, E. 19

Miller, H. 43, 50, 176, 178, 253

Miller, J.B. 33, 226, 250, 340

Miller, J.S. 242

Miller, L. 107, 178, 229, 310

Miller, M. 207

Miller, M.L. 207, 208, 334

Millman, Z. 210

Milloy, A. 42, 107

Mink, R. 148

Minor, A. 226

Miotto, E.C. 132

Mirabito, G. 18, 190

Miramontes, R. 225

Miskey, H.M. 64, 209

Mistretta, A. 17

Mittal, V.A. 203

Mlodnicka, A. 288

Moberg, P.J. 161

Moccia, M. 175

Modersitzki, E. 6

Moelter, S.T. 63. 112, 225

Moes, E. 173

Moffett, K. 237

Moghekar, A. 245

Mograbi, D.C. 250

Mohapatra, S. 159

Mohlman, J. 234
Mohn, M.M. 322

Molfese, P. 300

Möller, M.C. 19

Molokie, R. 164

Moncata, S. 143

Moore, A. 158

Moore, C. 223, 226

Moore, E. 122, 133

Moore, H. 236, 239

Moore, K. 161

Moore, K.J. 107, 318

Moore, M.J. 179

Moore, R. 236, 237, 239

Moore, R.D. 47, 66, 74, 265, 266 , 269, 270

Moore, S. 329

Moore, T.M. 135

Moran, E.E. 322, 324

Mordecai, K. 174

Moreira, B. 9

Moreira, I. 233

Moreno, C.C. 266

Morgan, A.K. 147

Morgan, E. 134

Morgan, E.E. 54, 188, 325

Morgan, S. 237

Morgello, S. 54

Morin, R. 113

Morissette, S. 137

Mormino, E.C. 216

Morrall, M. 142

Morris, J. 117, 216, 244, 245

Morris, R. 17, 23, 300, 303, 305

Morris, S. 76

Morrison, C. 14

Morrison, L. 94

Morton, A. 3

Mosack, K.E. 108

Moseley, S.A. 114, 115, 312

Moser, D. 207, 208, 334

Moses, J. 48, 51

Moskowitz, T. 32, 41, 44, 99

Mosteller, C. 331

Mosti, C. 266

Mostofsky, S. 287, 289

Moulton, E. 85

Mounsey, T. 13

Mourany, L. 222

Mrakotsky, C. 87

Mueller, S. 125

Mueller, W. 14, 183

Muir, J. 274

Mulhauser, K. 189, 220, 243

Mullen, C. 277

Muller-Oehring, E. 55

Müller, R. 82
Müller, U. 337

Mulrooney, D.A. 100

Munro, C. 57

Muraszko, K. 161

Murdaugh, D. 76, 80, 277

Murphy, H. 239

Murray, A. 164

Murray, E. 163

Murray, N.C. 152

Murray, R. 141

Murre, J. 251

Muths, L.H. 155

Myers-Fabian, A.M. 170, 337

Myers, T. 336

Na, S. 137

Nader, T. 169, 267

Nagorskaya, I. 141

Nair, A. 85

Nair, S. 122, 133

Nakagawa, Y. 86

Nakhla, M. 40, 140, 141

Nakhutina, L. 13

Nakonechny, A. 308

Napolitano, S. 39, 43, 316, 317, 328

Narad, M. 73, 76

Narayana, S. 67, 214, 215

Narkhede, A. 26

Nasrallah, I. 110

Nasreddine, Z.S. 33

Nasseri, M. 336

Natelson Love, M.C. 35

Nation, D.A. 62, 63, 64, 110, 124, 126 , 132,133

Nava, S. 137

Navarro, M. 55

Neal, J.E. 122, 133

Neale, G. 101

Neargarder, S. 323

Nedley, N. 279

Negri, N. 288

Neighbors, C. 54

Nelson, C. 125

Nelson, J.M. 288

Nelson, N.W. 227

Nemeth, D.G. 186, 274, 290

Ness, K.K. 100, 101

Nester, C.O. 335

Nestor, P. 204

Neuhaus, J. 249

Neumann. C. 189, 328

Neumann. S. 30

Neves, S. 232

Newcorn, J. 281

Newell, D. 204

Newman, E. 310

Newman, J.B. 67, 296 
Newsome, M. 149

Newsome, R.N. 336

Ng, R. 193

Ngan, E. 258

Nguyen-Louie, T. 19

Nguyen, C.M. 42

Nguyen, L. 177, 241

Nguyen, T. 41

Nguyen, T.T. 176, 204, 208

Ngwa, J. 141

Ngyuen, L. 241

Niaura, R.S. 191

Niccolai, L. 174

Nichols, J. 70, 73

Nicks, R.C. 198

Nielson, K.A. 3, 26, 222, 320, 323

Niermeyer, M.A. 319, 324, 329

Nishtala, A. 169

Nixon, K.H. 324

Noda, A. 337

Noel, M. 72

Nolan, R. 207

Nolan, V.G. 100

Noll, K.R. 157

Noll, R. 161

Nomura, Y. 189, 288

Nora, D.E. 262

Nordlund, A. 314

Nordstrom, L. 177

Norman, A. 21, 228

Norman, S. 65

Norris, T. 92

Norseth, C. 110

Northington, F. 309, 310

Norton, D. 217, 223

Norton, M. 116

Nosarti, C. 96, 97, 98

Nosovitskaya, M. 141

Nourbakhsh, B. 174

Novakovic-Agopian, T. 261, 276, 277

Novelo, L. 258

Nowinski, C. 36

Ntekim, O. 141

Nukari, J. 279

Nunan-Saah, J. 174

Nunez, C. 224

Nunez, R.A. 192

Nussbaum, N. 10, 12, 184

Nyenhuis, R. 324

Nyman, T. 18

O' Shea, D.M. 25, 333, 335

O'Brien, A.M. 283, 288, 292, 295

O'Connell, M.E. 278

O'Connor, M.G. 335

O'Connor, T.A. 150, 151, 152

O'Desky, I. 295
O'Hara, H. 39, 43, 316, 317, 328

O'Rourke, J. 32

O'Rourke, N. 169

O'Shea, A. 25

O'Suilleabhain, P.E. 234

O'Toole, K. 277

Oberlin, L.E. 99

Obermeit, L.C. 325

Obisesan, T. 141

Odman, S. 288

Ofen, N. 332

Offutt, H. 5

Ogden-Epker, M. 16

Ogedegbe, C. 272

Oghalai, J.S. 89

Ogunlana, O. 141

Oh, C. 4

Ohayon, M. 182

Ohlhauser, L. 50, 176, 253

Ohls, R. 90, 92

Ojemann, J. 95

Okonkwo, D.O. 25, 313

Okun, M.S. 7, 236, 239, 240

Okuneye, V. 134

Olde Rikkert, M.G.M. 312

Oleson, S. 110, 227

Oliveira, A.A. 227

Olivier, T. 274

Olivier, T.W. 147

Olsen, A. 148, 149

Olsen, E. 159, 162

Olsen, R.K. 336

Olson, K. 90, 157

Ong, B. 111

Ono, K. 69, 76

Onyike, C. 120

Oppenheim, J. 159

Oquendo, M.A. 206, 215

Orsillo, S. 173

Osborn, A. 199, 200, 208

Osborn, K.E. 122, 128, 133, 227 , 249,315

Oscarson, B. 161

Oshri, A. 112

Osipowicz, K. 20, 153, 154, 184

Osmon, D.C. 25, 108

Østgård, H.F. 97

Oswald, T.M. 43

Otruba, B. 43

Ott, B. 122

Ott, B.R. 230

Ottino, S. 280

Ouellet, M. 311

Overall, J. 137

Owens, M. 17

Owens, T.E. 325, 331
Oyeteju, M. 177, 191, 333

Ozinga, G. 39, 43, 316, 317, 328

Ozogluoz, H. 85

Pacheco-Colon, I.M. 189

Paciello, F. 46

Padilla, R. 225

Pagen, L. 177

Paguyo, A. 168

Pajkossy, P. 29

Palmer, B. 221

Palmer, S.L. 156

Palombo, D.J. 336

Palomeque, M.E. 8, 9

Panackal, A. 58

Pane, C. 46, 175

Panenka, W. 150, 151, 152

Panfili, D. 153

Panizzon, M.S. 213, 259

Papa, V. 325

Papandonatos, G. 122

Papanicolaou, A. 214

Papp, K.V. 105

Parag, O. 338

Parikh, S.A. 169, 267

Park, H. 13

Park, M. 209

Parker, A.F. 43, 176

Parkhurst, D.K. 11, 12

Parkinson, C. 310

Parks, A. 44

Parks, N. 313

Parra, M.A. 125

Parrini, C. 280

Parsons, M. 159

Parvataneni, H. 105, 167, 171

Pase, M.P. 228

Passarotti, A. 202

Pastorek, N.J. 271

Pataky, R. 97

Patel, A. 74

Patel, J. 200

Patel, P. 323

Patel, S. 278

Patel, S.G. 141

Patel, S.K. 158

Paterson, T.S. 169

Patrick, K. 93

Patrick, K.E. 11

Patt, V.M. 44

Patterson, A. 255

Patwardhan, S.Y. 101

Paul, L. 186

Paul, L.K. 191

Paulraj, S. 174, 274

Paulson, H. 120, 137

Paulus, M.P. 135 
Pawlenko, N.B. 74

Peake, M. 329

Pearson, M.M. 23, 27, 28

Peavy, G.M. 221

Pechlivanidis, H. 138

Pechman, K.R. 122, 133

Pedersen, N.P. 246

Pedraza, O. 44

Peltier, S. 30

Peñate, A. 123

Penedo, F. 315

Penna, S. 237. 269

Penney, D. 265

Pennington, N. 325

Perez Garcia, M. 38, 39, 196

Perez, M. 53

Perkins, S. 161

Perlstein, W.M. 267, 338

Perna, R. 278

Persaud, U.D. 323, 325

Persinger, V.C. 44

Pester, B. 200, 204

Peters, A.T. 19, 86

Peters, B. 91, 92

Peters, J.B. 159

Petersen, J. 153, 154, 172

Petersen, N.J. 271

Peterson, E. 329

Pettigrew, C. 245

Pfefferbaum, A. 55, 134

Phan, K.L. 199, 200, 208

Phelps, A. 141

Phillips, A.M. 283, 288

Phillips, J. 90, 92

Phillips, M. 207

Phillips, S. 24

Piercy, J. 91, 92, 332

Piers, R.J. 61, 169, 228, 265

Pietilä, P. 279

Pievsky, M.A. 323, 325

Pike, K. 111

Pilipenko, V. 314

Pillay, S.B. 132, 332

Pillemer, S. 103, 114, 228

Pilonieta, G. 35

Pineda, D. 59

Pinto, C. 232

Pinto, P. 232

Piper, L.E. 164

Pirogovsky-Turk, E. 236, 237. 239,241

Pitteri, M. 174

Pivonka-Jones, J. 70, 73

Pizzano, B.N. 262

Platt, A. 73

Pleiss, S. 82
Plotkin, R. 303, 319

Pluim, C.F. 237

Plumb, P. 70, 89

Plurad, D. 255, 263, 267, 272

Pluta, A. 185

Poggi, G. 142

Polejaeva, E. 267, 338

Pollock, B. 172

Polsinelli, A. 115, 312

Pommy, J. 90, 92

Ponsford, J.L. 138, 248

Pontifex, M. 80

Ponting, C. 85

Popa, A. 146

Porcelli, A. 26, 320, 323

Poreh, A. 316

Poreh, A.M. 39, 43, 317, 328

Poretti, A. 309, 310

Porges, E. 25, 107, 117

Porto, F.H. 311

Posada-Shea, C. 58

Posecion, L. 174

Possin, K.L. 32, 41, 44, 99

Poston, K. 55, 235

Potter, B. 214. 215

Potter, G. 114

Potts, S. 26, 320, 323

Potvin, M. 335

Poutiainen, E. 279

Powell, M.R. 253

Powell, S. 294

Prescot, A.P. 205

Pressman, P. 240

Presson, N. 25, 313

Preston, T. 336

Previ, D. 319

Price, C.C. 105, 123, 124, 167, 171 , 180, 181, 233, 242

Prigatano, G.P. 123

Prigge, M. 83

Priluck, J.L. 256

Primosch, M. 277

Pritchard, A. 292, 294, 296, 297, 298

Pritchett, A. 13, 189

Proctor-Weber, Z. 273

Procyshyn, R.R. 151

Proescher, E. 199

Prohl, A. 147

Pruitt, P. 203

Ptito, A. 179

Puente, A.E. 38, 39, 130, 141

Puente, A.N. 13

Pugh, E. 222

Pui, C. 100

Pulsipher, D.T. 312, 328

Punyamurthula, S. 82
Putcha, D. 120, 129

Pyne, S. 279

Pyron, M. 110

Quasney, E. 14

Quasney, E.E. 11, 169, 183

Quinn, C. 45

Quinn, D. 30

Quiroz, Y.T. 217

Rabin, J. 336

Rabin, L. 45, 48, 113, 314, 317

Rabinowitz, N. 44

Rabkin, A.N. 312

Rach, A. 295

Rach, A.M. 198

Racsmány, M. 29

Rad, H. 255, 263, 267

Rademaker, A. 333

Raffo, A. 123

Raghavan, M. 183

Rahman-Filipiak, A.A.M. 109, 228

rahman, a. 170

Rahman, S. 82

Raitano Lee, N. 36

Raitano-Lee, N. 53

Raj, S. 73

Rajaram, S. 110

Rajaratnam, S.M.W. 248

Rakhshan, P. 210

Ramalheira, J. 9

Ramig, L. 163

Ramirez Flores, M.J. 76

Ramirez-Coombs, E. 184

Ramirez, F.E. 279

Ramirez, J. 121

Ramsey, L. 178

Randolph, J. 176

Rangamannar, R. 327

Rankin. K. 32

Rankin, K.P. 41, 44, 99

Rao, R. 296

Rao, S. 185, 222

Raphail, A. 175, 182, 248, 279

Rapport, L. 32, 252, 259

Rashid, F. 148

Raskin, S. 267, 268

Rasmussen, K. 221

Ratcliffe, S.J. 181

Ratzon, N.Z. 182

Rauch, A.A. 258

Raz, S. 91, 92, 332

Razani, J. 40, 140, 141

Ready, R. 127, 193

Reale-Caldwell, A. 14, 127

Reckow, J.M. 170

Reda, D. 237

Reddick, W.E. 100, 101 
Reedy, N. 310

Reese, K. 303

Reeves, G. 210

Reeves, V.L. 71

Reife, I. 193

Reilly, J. 107, 116, 206, 271

Reinvang, I. 314

Reis, J. 25 ?

Reiter, K. 26, 320, 323

Remer, J. 4, 293

Renfroe, J. 7

Rensberger, J. 193

Renshaw, P.F. 205

Rentz, D.M. 105, 216, 315

Resch, S. 336

Resch, Z. 14

Rexer, J. 157

Rey, G.J. 8, 9

Reyes, A. 14

Reynolds, B.W. 134, 200, 326

Reynolds, C.M. 11, 12

Reynolds, E. 71, 78

Reynolds, G.O. 127

Reynolds, M. 268

Rezaie, R. 67, 214, 215

Rhines, S. 157

Rhodes, E. 114

Ribot, R. 8. 9

Rice, M. 105, 167

Richard, A.E. 160

Richardson, J. 329

Richardson, R. 264

Ridgel, A. 172

Rieger, R. 90

Rieger, R.E. 92

Riggall, E. 303, 305

Rimol, L.M. 97

Ringman, J. 224

Rinna, K. 293

Riopelle, A. 74

Ris, M. 155

Ritchie, H. 114, 115, 312

Ritchie, K.A. 41, 45, 290

Ritter, A. 226

Rivara, F.P. 68

Rivard, K. 82

Rivera Mindt, M. 54

Rivera, A. 326

Rivera, L. 218

Riviello, J. 15

Rizvi. B. 26

Roach, A. 165

Roalf, D.R. 161

Robbins, J. 178, 229

Robbins, R. 115

Roberson, J. 332
Roberts, A. 303

Roberts, N.A. 11, 12

Robertson, F. 79

Robins, D.L. 80, 81

Robinson, G. 156

Robinson, K. 182

Robinson, T. 107

Robison, L.L. 100, 101

Rochette, A.D. 26

Rodgers, C. 65, 258

Rodriguez, I.G. 229

Rodriguez, M. 52, 108, 111, 112. 119, 229

Roe, C. 216

Rogalski, E. 120, 333

Rogers, S. 115, 232, 235, 241

Rogosch, F. 193

Rohl, B. 237

Rojas, L. 327

Roll, E. 106, 126, 229, 251

Roman, B. 310

Roman, C.A. F. 248

Roman, E. 136, 184

Romero-Quintana, Y. 327

Romero, E. 255, 261

Romero, R. 144

Romski, M. 299

Romyn, A. 17

Rooks, J. 236, 239

Roper, B.L. 48

Roper, C.L. 256

Rosa, A. 140, 320

Rosado-Mueller, A. 114

Rosario, D. 56

Rosario, M. 27

Rosch, K.S. 287, 289

Rose, E. 137

Roseberry, J.E. 10, 160, 203

Roseman, E. 336

Rosen, J. 231

Rosenbaum, R. 95, 336

Rosenzweig, E. 204

Ross, J.M. 189

Ross, R.J. 65

rosselli, m. 143

Rossetti, H.C. 63

Rossetti, M. 336

Rossi, C. 280

Rossi, F. 175

Rossi, M. 158, 159

Rossi, V. 174

Rossion, B. 7

Rotblatt, L.J. 115, 257

Roth, R. 197, 317

Roth, R.M. 196

Rothberg, J. 175
Rothlind, J.C. 237

Rouleau, I. 335

Rowland, J. 20, 64, 137, 209, 251

Rowley, H. 218

Roy, A. 16, 17, 311

Roy, A.A. 268

Roy, A.K. 286

Roye, S. 23, 27, 320, 328

Rubin, L. 57, 230

Rubino, S. 141

Rubio-Gimbert, M. 196

Ruchinskas, R. 167

Ruiz, G. 257, 270

Rum, R.S. 14, 127

Ruparel, K. 135

Ruppert, P. 243

Rushby, J. 313

Russell, K. 158

Russo-Ponsaran, N. 193

Russo, C. 175

Rutlin, J. 88

Ryals, A. 315

Ryan, J.D. 336

Ryan, K. 19, 200, 202, 204

Ryan, L. 330

Ryan, M. 284, 290, 296, 297

Ryan, N.P. 312

Rycroft, S.S. 106, 116, 251

Ryman, S. 5

Saad, L. 45, 123

Saadatpour, L. 332

Sabat, C. 14

Sabaté, J. 110

Sabin, N.D. 100

Sabri, M. 25

Sabsevitz, D. 10, 11, 14, 160, 183

Saccà, F. 46, 175

Sackett, C. 297

Sacktor, N. 57

Sacuiu, S.F. 125

Saczynski, J.S. 169

Sadek, J. 261

Sady, M. 79

Sady, M.D. 76

Safer-Lichtenstein, J. 304

Sagar, S. 196, 197

Saigal, S. 44

Sala Rodó, M. 55, 56

Salama, C.H. 92

SALAS MORALES, G. 76

Salat, D.H. 28

Salazar, R. 238

Salinas, S.L. 269

Salisbury, D.B. 172

Salloum, R. 73

Salloway, S. 24 
Salminen, L.E. 24

Saloner, R. 41, 102, 103, 240, 249, 250

Salorio, C.F. 92

Salvador-Cruz, J. 139, 300, 303. 304,326

Samuels, L.R. 128, 249, 315

Sanborn, V. 129

Sanchez Vielma, E.E. 303, 326

Sanchez-Ramos, J. 235

Sanchez, D.L. 225

Sandel, N. 77, 78

Sanders, C.L. 116

Sanderson-Cimino, M. 65, 124. 213, 258

Sandroff, B.M. 149, 150

Sandry, J. 175

Sanova, A. 5

Santacruz, G. 92

Santorelli, G.D. 127, 193

SANTOS CORDERO, J. 76

Santos, E. 232

Santos, O.A. 52, 327

Sarajuuri, J. 279

Sarapas, C. 192

Sarill, K. 15

Sassoon, S.A. 134

Sathian, K. 17, 252, 311

Satizabal, C. 228

Saunders, E. 200

Saurman, J. 108

Sauve, W. 269

Sawa, A. 201

Sayegh, P. 30

Saykin, A. 317

Scarmeas, N. 135

Scavone, A. 194

Schaefer, L.A. 46

Schaffer, S. 170, 171

Schagen, S.B. 251

Schatz, P. 78

Scheibel, R. 149

Scheibel, R.S. 271

Scheller, A.D. 296

Schiehser, D. 65, 236, 237, 239, 254 . $255,260,270$

Schiffman, J. 210

Schilling, S.L. 269

Schmid, A. 77

Schmid, A.D. 77

Schmidt, A. 67

Schmit, B.D. 3

Schmitter-Edgecombe, M. 50, 116, 119, 122, 128, 165, 220, 274

Schneider, B. 92

Schneider, B.C. 133

Schneider, H. 284, 290, 296, 297
Schneider, H.L. 240

Schneider, J. 55, 134

Schneider, R. 290

Schneider, W. 25, 313

Schoemaker, T. 35

Schoen, C. 117

Schoenberg, M. 14, 127

Schon, K. 27

Schraegle, W. 184

Schraegle, W.A. 12

Schreiber, J.E. 87, 93, 156, 158, 159

Schretlen, D.J. 201, 203, 210

Schreyer-Hoffman, G. 46

Schroeder, M. 315

Schroeder, R.W. 34, 198

Schroth, C. 200, 208

Schrum, A. 294

Schuetze, M. 82

Schulte, T. 55

Schultheis, M. 175

Schultheis, M.T. 182, 248, 274, 278,280

Schultz, A. 217

Schupf, N. 26, 223

Schwab. N.A. 105, 167, 171, 233, 242

Schwam, D. 17

Schwarz, L. 220, 243

Schwarz, N. 177

Scinska-Bienkowska, A. 185

Scorpio, K. 163

Scott, B.M. 7, 236, 239, 334

Scott, J. 65, 135

Scott, M.N. 86

Scott, S.E. 282

Scroggins, K. 338

Seabolt, C. $122,128,133,249,315$

Seagly, K.S. 30

Seaman. B. 269

Sedgewick, J.R. 340

Seehra, S. 225

Seese, S. 304

Segalà, L. 239, 240

Segala, L. 8, 9, 236

Segerstrom, S.C. 165, 318

Segura Villa, A. 300, 304

Seidel, G. 181

Seidenberg, M. 222, 305

Seider, T. 117

Seifan, A. 221

Seke, K. 196, 197

Sekhon, A. 141, 337

Seleme, M.E. 327

Seliger, S.L. 27

Selke, G. 49

Semerjian, C. 169, 267

Semmel, E. 27
Semple, S.I. 97

Semrud-Clikeman, M. 89

Seng, E. 102

Sepeta, L. 16, 317

Serrano, E. 8, 9

Seshadri, S. 228

Sevcik, R. 299, 303, 305

Sever, R. 14, 127

Shaafi Kabiri, N. 46

Shada, K. 86

Shafer, D. 308

Shaff, N.A. 5

Shahid, S. 11, 12

Shair, S. 137

Shaked, D. 27

Shalev, N. 179

Shan, G. 250

Shane, B.R. 260

Shankle, Z.M. 322

Shankman, S. 192

Shapiro, E. 144

Shapiro, R.J. 169

Sharkey, C.M. 160

Sharma, M. 35, 47, 121

Shaughnessy, L. 223, 226

Shaw, M. 59

Shay, N. 214

Sheehan, J.C. 273, 276

Shehorn, J. 114

Sheldon, S. 12

Shenal, B. 251

Shepherd, T. 234

Sheppard, D.P. 185

Sherer, M. 258

Sherman, J.C. 34, 120

Shi, X. 205

Shieh, B. 303

Shields, B.J. 86

Shih, C. 221

Shimony, J. 88

Shipley, E.M. 297

Shipley, T.F. 116

Shirazi, T. 58

Shishido, Y. 78

Shorser-Gentile, L. 87

Shouel, H.L. 138, 220, 225, 230

Shpitser, I. 112

Shuckit, M. 19

Shulman, G. 178

Shum, J. 221

Shungu, D. 145

Shura, R.D. 64, 209, 251

Sicard, V. 47, 74, 265, 266, 270

Sideman, N. 21

Sidney, S. 110

Sidol, C. 285 
Siebenmorgen, M. 93

Siegel, J. 178

Sienkiewicz-Jarosz, H. 185

Signoriello, E. 175

Sikkes, S. 314

Silk, T. 312

Silton, R.L. 191

Silva, G.R. 209

Silva, L. 49

Silverstein, N. 170

Simard, M. 221

Simic, N. 17

Simmons, A. 19

Simon, C.E. 262

Simon, S.S. 132, 311

Simon, T. 146

Simone, A.N. 289

Singer, C. 236, 239, 240

Singh, P. 282, 301

Sinha, P. 167

Siple, P. 32

Sirijuntanan, P. 76

Siugzdaite, R. 6

Skalina, L. 174

Skeel, R. 36, 283

Skerrett, K. 201, 203, 207

Skranes, J. 97

Slagel, B. 320

Slattery, E. 136

Sletten, T. 248

Slifer, K. 147

Sliwinski, M. 261

Sloan, R.P. 3, 37, 306

Slomine, B. 66, 92

Slonim, T. 231

Slotkin, J. 36

Slugs, M. 12

Slyne, K.E. 47

Small, S.A. 37, 306

Smith-Hyle, E. 10, 13, 239

Smith-Paine, J. 78

Smith. A. 134, 139, 340

Smith, C.T. 5

Smith, D. 55

Smith, G.N. 150, 151

Smith, H.A. 5, 6

Smith, J. 222

Smith, M.J. 23

Smith, R. 5, 19, 190

Smith, T. 19

Smith, T.V. 54

Smith, V.M. 336

Snelling, A.C. 262

Snow, J. 58

Snyder, A.R. 257, 270

Snyder, D. 82
So, R.P. 216, 327

Sobanska, M. 185

Soble, J. 32, 327

Sofko, C.A. 128

Soldan, A. 244, 245

Sølsnes, A.E. 97

Song, D. 236, 237, 239

Sorg, S.F. 213, 254, 255, 260, 270

Soto-Añari, M. 117

Sousa, L. 232

Sousa, N. 232

Sozda, C.N. 338

Sparrow, S. 97

Spat, J. 10, 13, 136, 184, 239

Spaulding, W. 201

Spears, A. 120

Sperling, R. 28, 216, 217, 315

Sperling, S.A. 121, 233, 242

Spielman, J. 163

Spielman, L. 259

Spiers, M. 266

Spirou, A. 20

Spitz, G. 248

Spitznagel, M. 26, 172, 281

Split, M. 236, 239

Sprehn, G. 45

Springate, B. 317

Sripada, K.K. 97

Srivastava, D. 100, 101

Srnka, K.D. 305

Stabile, V. 197

Stabler, A.R. 117

Stach, B. 252

Staffaroni, A.M. 48

Stafford, R.J. 163, 195

Stake, K. 229

Stålhammar, J.D. 314

Stancin. T. 66, 74, 76

Stanford, L.D. 312, 328

Stange, J.P. 201

Stanley, N. 328

Stapleton-Kotloski, J. 20

Starkman, M. 19

Starling, A.J. 295

Starsky, A. 169

Statucka, M. 152, 153

Stavinoha, P.L. 70, 87, 161

Steed, D. 170, 337

Steele, N.J. 258

Stefanatos, A.K. 15

Stefanatos, G. 15

Steffen-Allen, F. 216, 327

Stefurak, T. 197

Stegman, R.L. 48

Steinberg, C. 10

Stelmokas, J. 42, 170, 252
Stephan, C. 292, 294, 297, 298

Stephenson, A.J. 7

Stern, M. 237

Stern, Y. 3, 217, 220, 225

Stevens, A. 242

Stevens, E.S. 192

Stewart, B. 277

Stewart, J. 135, 231, 273

Stewart, W. 261

Stierwalt, J.A.G. 4

Stillman, J.N. 256

Stiver, J. 240, 250

Stone, W.L. 81

Storbeck, J.L. 195

Stout, S. 216

Strain, J. 124

Strainge, L. 118

Stratton, J. 197

Stricker, N. 231

Strickland-Hughes, C. 112

Stringer, A.Y. 211, 212, 277

Strober, L. 176, 245, 247

Strong, J. 231

Strutt, A.M. 142, 334

Stuart, J.Z. 34, 142

Stubbs, W.J. 48

Studeny, J. 215, 298, 327

Stuss, D.T. 2

Su, W. 150

Suchy, Y. 7, 319, 324, 329

Sudikoff, E.L. 15

Sufrinko, A. 68, 71, 77, 251

Sufrinko, A.M. 78

Sugarman, M.A. 21

Suhr, J. 163

Suhrie, K.R. 128, 221

Sullan, M. 256, 271

Sullivan, C. 13

Sullivan, E. 189, 328

Sullivan, E.V. 55, 134, 243

Sullivan, J.K. 262

Sullivan, K. 171

Sumida, C. 50, 128

Summers, A. 58

Summersett-Ringgold, F. 329

Sun-Suslow, N. 8, 9, 236, 239, 240

Sunderaraman, P. 220, 225

Sundermann, E.E. 230

Surace, A. 49

Suskauer, S. 66, 92

Sutherland-Owens, A.N. 176, 208

Suwartono, C. 37

Suzuki, J. 10, 239

Svingos, A.M. 78, 257, 316

Swain, M. 156

Swain, M.A. 156 
Swank, P.R. 136

Swanson, S. 10, 11, 14, 183

Swartz, J. 319

Swearer, J. 127

Sweeney, J. 335

Sweeney, K.L. 289, 290

Sweet, L.H. 17, 26, 112, 191

Swenson, R. 61, 123, 124

Swerdloff, R. 261

Swick, C. 18

Swift, A. 209

Swift, R.R. 198

Swift, T.J. 198

Swirsky-Sacchetti, T. 198

Sylvia, A. 156

Sze, A. 317

Szeles, D.M. 224

Szeto, W. 181

Szollosi, Á 29

Szymanska, B. 185

Szymkowicz, S.M. 25, 240, 333, 335

Taber, K. 20

Tadrous-Furnanz, S.K. 158, 290

Taipa, R. 232

Taiwo, Z. 18, 190, 194

Takagi. M. 180

Talbot, K.S. 337

Talon-Croteau, J. 221

Tam, D.M. 48

Tam, H. 285

Tam, J.W. 119

Tan, C.C. 251

Tanaka, H. 110, 227, 304

Tang, I. 193

Tang, R. 49

Tangen, R. 303

Tanner-Woodward, S. 92

Tanner, J.J. 105, 171, 233, 242

Tantiongloc, J. 204

Taormina, I. 157, 160

Tapert, S. 19, 135, 187

Taraben, S. 18

Tarazi, R. 93

Tarkenton, T. 161

Tart-Zelvin, A. 20

Tarumi, T. 110

Tascone, L.d. 311

Tastard, L. 177

Tatarina, O. 217, 220, 225

Taylor, B.P. 241

Taylor, H. 24, 66, 71, 72, 74, 76, 78, $179,180,314$

Taylor, P. 302

Taylor, S. 32, 320, 328

Teaford, M.A. 328

Teboe, C. 137
Teixeira, M.J. 132

Telford, E.J. 97

Tell, E. 54

Tellock, P. 205

Terry, J.G. 122, 133

Terwilliger, V. 271

Tessier, J. 182

Testa, M. 13

Thames, A. 53, 56, 154, 185, 192

Theodore, W. 16

Thesesn, T. 14

Thielke, S. 64

Thomas, A. 188

Thomas, K. 46

Thomas, K.R. 129, 133

Thomas, M.L. 44

Thomas, S. 142

Thompson, A. 73

Thompson, D.M. 262

Thompson, E. 210

Thompson, J. 128, 133, 249, 315

Thompson, L.H. 251

Thompson, L.I. 49

Thompson, P. 148

Thornton, A.E. 69, 150, 151, 152

Thornton, W.J. L. 118, 169

Thursby, M. 122, 128, 133, 249, 315

Thyen, M. 255

Tierney, S.M. 58, 185

Tiersky, L. 323, 325

Timpano Sportiello, M. 304

Timpano Sportiello, M.R. 129, 194, 280

Titus, J.B. 10, 12, 184

Tlustos-Carter, S.J. 74

Tobin, C. 207

Tocchini, S. 194, 304

Tolar, T.D. 300

Tolfo, S.E. 39, 43, 316, 317, 328

Tolliver, A. 297

Tomb, M. 282, 301

Tomfohr-Madsen, L. 71

Tommasini, L. 129

Tomporowski, P. 149, 150

Tonkin, R.J. 98

Toomey, R. 259

Tornes, L. 8, 9

Torres, I. 210

Torres, K. 335

Torres, V.L. 143

Tosun. D. 125

Tourgeman, I. 229

Tournier, J. 97

Tovar Vital, D.S. 139

Towler, S. 21

Towns, S.J. 52
Tracy, J.I. 21, 153, 183, 184

Trahan, D.E. 199

Traino, K.A. 58

Tran, B. 241

Tran, M. 260

Tran, S. 21

Travers, L.V. 147

Treadwell, K. 196

Treble-Barna, A. 78, 314

Tremont, G. 122, 127, 129, 228

Trenova, L. 65

Trenova, L.S. 258

Triebel, K. 174

Trifilio, E. 49, 113, 236

Tripp, J. 328

Trittschuh, E. 64

Troyanskaya, M. 271

Truong, D.Q. 59

Tsang, T. 85

Tsapanou, A. 135

Tschanz, J. 116, 118

Tseng, C. 98

Tso, I.F. 202

Tuchscherer, V. 86

Tucker, B.J. 256

Tucker, D. 282

Tulsky, D. 68

Tupper, D. 164

Turk Nolty, A.A. 158, 193, 324

Turkstra, L. 293

Turley-Ames, K.J. 20

Turner, B. 282

Turner, J. 141

Turner, J.A. 5, 184, 203, 305

Turski, P. 218

Tusor, N. 97

Tuulio-Henriksson, A. 301

Twaite, J.T. 163, 195

Twamley, E.W. 65, 258, 260

Tyner, C. 174

Ukueberuwa, D.M. 170, 272

Umucu, E. 223

Upshaw, J. 43, 50, 176

Uy, P. 43

Uzumcu, A. 335

Vaccariello, E. ?

Vaillancourt, A. 191

Vakil, E. 182, 338

Valálik, I. 29

van der Heijden, P. 37

van Dijk, M. 37

Van Dyk, K. 231

Van Eman, G. 25, 313

Van Etten, E.J. 241, 338

Van Meter, A. 19

van Meurs, B. 195 
Van Patten, R. 6, 189, 265, 330

VandeBunte, A.M. 241

Vannini, P. 315

Vannucci, M. 44

Vargha-Khadem, F. 130

Vargo, T.L. 53

Varvaris, M. 201

Vasan, R.S. 228

Vasquez, A.C. 67

Vasquez, D. 173

Vasserman, M. 285

Vatsa, N. 339

Vaughan Van Hecke, A. 82

Vaughan, C.G. 76, 79

Vaughn, D.W. 50

Vaurio, L. 12

Vederman, A. 19

Vega, C. 15, 14 ?

Velez Lopez, A. 27

Velez Uribe, I. 143

Velez-Ruiz, N. 8, 9

Velez-Uribe, I. 143

Venezia, R. 170, 171

Venkatesan, U.M. 16, 230

Verbalis, A.D. 304

Verduzco, M. 54, 57, 58

Verfaellie, M. 28, 99, 249

Verhaak, A. 161

Vermeulen, I.E. 251

Vermilion, B. 32, 252, 259

Vernon, E.K. 118

Vespa, P. 99, 255, 261, 263, 267, 272

Vickers, K.L. 161, 182, 274, 278, 280

Vila-Castelar, C. 231

Vila-Chã, N. 233

Vila-Rodriguez, F. 150

Villalobos, J. 188

Villalon-Reina, J. 148

Villegas, A. 53

Vinck, K. 143

Vinette, S. 82

Vingerhoets, G. 6

Viqar, F. 136

Virta, M. 301

Viscogliosi, C. 311

Vlasova, R. 148

Vo, T.T. 50, 128, 274

Vock, D.M. 227

Voelbel, G. 272

Voelker, S. 285

Vogel, S. 250, 329

Vogt, E. 41, 290

Vogt, G.S. 271

Voigt-Radloff, S. 312

Vojtila, L. 205

Volkovinskaia, A. 283
Volpi, J. 333

Voorheis, D. 20

Voss, J.L. 315

Vossel, K. 240

Vossler, H. 267, 268

Votruba, K. 156

Waber, D.P. 15, 87, 299

Waclawik, K. 150, 151, 152

Wade, S.L. 66, 73, 74, 76, 78, 314

Wadsworth, H. 16, 167, 219, 234

Wagley, N. 18

Wagner, G. 231, 338

Wagner, L.I. 315

Wagner, M.T. 224

Waite, E. 261

Waiwood, A. 99

Wakefield, D. 118

Wakschlag, N. 261

Walder, A. 271

Waldstein, S.R. 27

Wali, L. 97

Walker, A. 224, 255, 267, 272

Walker, K.A. 168, 170, 171

Walker, R. 189

Walkowiak, J. 84

Wallace, E.R. 50

Walley, J. 79

Wallin, A. 314

Walljasper, L. 128, 133, 249, 315

Walls, B.D. 50

Walsh, K.S. 144, 157, 158, 160, 161

Walsh, R. 165, 166

Walsh, V. 209

Walters, R. 199

Waltz, J. 210

Walzak, L.C. 118

Wan, C. 147

Wang, C. 261

Wang, J. 68

Wang, L. 23, 315

Wang, M. 245

Wang, N.Y. 150, 151, 152

Wang, T.J. 122, 133

Wang, W. 93

Wang, Y. 8, 141

Wank, A.A. 249

Warfield, S. 147

Warner, E. 105, 171

Warren, Z.E. 81

Washington, D. 223

Wasserman, V.J. 118, 119

Wassermann, E. 324

Watchmaker, J. 133

Watson, C.W. 231

Watson, E. ?

Watson, J. 50
Watson, R. 339

Watt, J.C. 105

Waubant, E. 174

Weakley, A. 119, 220

Weaver, F. 237

Weber, E. 215, 273, 298, 327

Weber, M. 190

Wefel, J.S. 101, 157, 161

Wegele, A. 94

Wehde, A.T. 253

Weichselbaum, A. 331

Weiers, B. 340

Weilhammer, J. 329

Weiner, M. 125

Weinstock, J. 189

Weintraub, S. 120, 333

Weisenbach, S. 19, 201

Weissberger, G. 231

Weizenbaum, E. 238

Weldon, A. 19, 203

Welkowitz, L. 83

Welsh, M. 329

Werhane, M.L. 132, 133, 254 . 255,270

Werry, A.E. 138, 190, 316

Wertheimer, J. 242

Wertz, C.J. 5

Wesonga, E.M. 305

West, R. 235

Westhafer, J. 41

Westmacott, R. 68, 177

Wethe, J.V. 295

Weuve, J. 112

Wheeler, J.M. 51

Whipple, E. 182

Whitaker, A.M. 162

White, D. 88, 305, 321

White, J. 167

White, L. 93, 158

White, M. 92

White, W. 118

Whiteneck, G. 258

Whiteside, D. 40, 44, 52, 134, 164 , 199, 200, 207, 208, 326, 334

Whitman, R.D. 32

Whitney, N. 28

Whyte, R. 188

Wickham, R. 48

Wicklund, A.H. 268

Wieben, O. 218

Wiener, J.R. 43, 229

Wier, R. 17, 23, 28

Wierenga, C.E. 22, 103, 109, 168

Wiggins, M.E. 242

Wiggs, E. 16

Wilde, E.A. 67, 69, 74, 75, 148, 149 
Wiley, J. 3

Wilkinson-Smith, A. 89

Willar, K. 82

Willi, T. 150

Williams, J. 153, 154

Williams, K. 87

Williams, T. 68, 177

Williams, V.J. 28, 99

Williamson, E. 273

Williamson, J.B. 65, 166, 168, 186, 339

Williamson, P. 58

Williamson. T. 185

Willie, J.T. 246

Willis, S. 220

Wilmoth, K. 79, 124

Wilson, B. 146

Wilson, B.A. 211, 212

Wilson, C. 210

Wilson, C.L. 101

Wilson, J. 30

Wilson, K. 30

Wilson, R.S. 63, 225

Wing, B.H. 256

Winslett, M. 80

Winter, R. 303. 305

Winter, S. 182

Wisdom. N. 336

Wise, S. 88, 89

Withrow, S. 51, 162

Wolak, T. 185

Wolday, S. 141

Wolf, D.H. 135

Wolf, S. 224

Wolfson, L. 47, 118

Wolk, D.A. 45, 112, 123

Wollam, M.E. 105

Womack, K. 124

Wong Gonzalez, D. 338

Wong, C.G. 252

Wong, L. 146

Wong, M.M. 282

Woo, E. 99, 224, 255, 263, 267, 272

Woodard, J.L. 21, 222, 228

Woodburn, M.A. 22

Woods, A.J. 25, 59, 107, 117

Woods, R. 3

Woods, S.P. 54, 57, 58, 185, 301

Woon. F. 63
Wooten, K. 186, 290

Worthington, G.M.G. 121

Wright, B. 159

Wright, C. 329

Wright, I. 22, 298

Wright, K.L. 79

Wright, M.J. 99, 255, 261, 263, 267, 272

Wright, M.M. 262

Wright, N.E. 187

Wu, L. 120

Wu, S. 272

Wylie, G. 272

Wyman-Chick, K.A. 121, 233, 242

Wynn, M. 240, 250

Xavier, A.B. 132

Xia, F. 110

Xu, C. 238

Xu, X. 17, 20, 191

Xu, Y. 110

Yaffe, B. 235, 243

Yaffe, K. 110, 259

Yakobson, M. 168

Yalem, L. 305

Yamaguchi, T. 251

Yandall DeJesus, S. 338

Yang, B. 108

Yang, J. 85

Yang, S. 192

Yanos, B.R. 262

Yarmolovky, J. 97

Yassay, L.J. 252

Yatham, L.N. 210

Yeates, K.O. 17, 24, 66, 68, 69, 71, 72. 74, 78, 179, 294, 312, 314

Yee, M.K. 171

Yeh, T. 255, 272

Yeo, R. 90, 92, 94, 269

Yeung, L. 37

Yew, B. 63

Yoash-Gantz, R.E. 64

Yochim, B. 51

Yoo, J. 13, 136

Yoon, H. 103

York, M. 237

Yoshida, H. 301

Yots, J. 264

You, X. 16
Youm, Y. 104

Young, K.J. 196

Yozawitz, L. 43

Yu, F. 227

Yu, K. 334

Yu, Q. 332

Yuan, J. 183

Yund, B. 144, 147

Yurgelun-Todd, D. 205, 288

Zabel, T.A. 292, 294, 298

Zachery, A. 16

Zaheed. A.B. 51

Zahodne, L.B. 112, 138, 220, 225. 230,231

Zahr, N. 134

Zakzanis, K.K. 35, 47, 121, 205

Zamzow, J. 182, 248

Zane, K.L. 189, 243

Zang, H. 78

Zartman, A. 41

Zarzour, R. 37

Zeal, J. 159, 162

Zeiner, H.K. 33, 38, 48

Zemon, V. 207

Zetterberg, H. 249, 315

Zhan, L. 61, 106

Zhang, A. 192

Zhang, H. 97, 100, 156

Zhang, J. 309

Zhang, N. 78

Zhang, Y. 191, 333

Zhao, W. 82

Zhu, C. 220

Zhu, D. 235

Ziemnik, R. 319

Ziemnik, R.E. 324, 329

Zimmerman, M.E. 261, 322, 324

Zink, D.N. 340

Zink. T. 335

Zlatar, Z.Z. 22, 103, 109, 132

Zobel, H. 15

Zolliecoffer, C.J. 203, 210

Zonderman, A.B. 27

Zougari, A. 196, 197

Zubieta, J. 19, 203

Zuccato, B.G. 195, 259

Zumberge, N. 24, 71, 72, 179

Zuppichini, M.D. 175 


\title{
Keyword Index \\ Forty Fifth Annual Meeting \\ International Neuropsychological Society
}

\author{
February 1-4, 2017 \\ New Orleans, Louisiana, USA
}

academic achievement 28, 40, 52, 53, 87, 88, 90, 139, 143, $150,159,160,161,177,197,276,280,284,287,290$. 300, 302, 303, 304, 305, 327, 329

acculturation 60, 108, 138, 140, 141, 142

activities of daily living / adaptive functioning 15, 39, 43, 46,

$75,84,85,87,89,93,103,119,121,122,125,128$.

$129,144,157,163,165,173,182,218,220,221,226$,

230, 239, 241, 242, 252, 272, 277, 284, 292, 298,

312,329

adolescence $2,49,70,73,75,77,79,81,135,136,187,188$, $189,197,210,251,283,287,288,329$

affective processing disorders 7, 49, 84, 113, 195, 201, 208,260

affective processing, normal 5, 18, 19, 111, 140, 191, 192 ,

193, 194, 195, 246, 320, 321

aggression 198, 256, 321

aging disorders 4, 31, 39, 45, 46, 61, 62, 64, 108, 109, 110 , $113,114,119,126,127,128,129,133,136,150,155$, $185,213,218,220,221,222,225,228,235,247,252$, $275,279,311,312,315$

aging, normal 17, 21, 22, 23, 24, 25, 26, 29, 46, 48, 50, 62, $63,64,99,102,103,104,105,106,107,108,109,110$, 111, 112, 113, 114, 115, 116, 117, 118, 123, 133, 138, $139,141,150,181,183,190,193,214,216,250,259$, 298, 306, 307, 311, 312, 316, 318, 320, 321, 323, 324, 330, 333, 336, 337, 338

agrammatism 264

alcohol 19, 134, 187, 188, 189, 207, 243, 270, 322

amyotrophic lateral sclerosis 165

anomia 51, 142

anosognosia 203, 236, 250, 272, 325

anterior cingulate 194, 246

antiretroviral treatment 55, 56

anxiety $23,71,126,127,182,192,195,196,201,206,208$,

229, 232, 234, 261, 271, 274, 279, 283, 301, 318, 322,325

apathy 57, 126, 222, 223, 224, 239, 246

apnea 137, 282, 337

apolipoprotein E 3, 122, 124, 128, 133, 222, 227, 228

apraxia $31,237,331$

arousal 214 assessment 12, 32, 34, 36, 37, 38, 39, 40, 42, 43, 44, 48, 49 , $50,51,52,67,73,85,108,116,123,125,130,142$, $144,173,178,196,197,198,219,227,246,247,251$, $254,255,258,261,285,291,292,293,294,295,296$, 298, 299, 301, 306, 307, 308, 317, 318, 322, 327. 328,340

asymmetry $140,217,340$

ataxia $46,98,147$

attention 5, 7, 21, 44, 50, 74, 87, 97, 100, 101, 115, 138 , 145, 148, 160, 166, 167, 169, 171, 173, 186, 192, 208 , 231, 255, 273, 275, 276, 282, 283, 286, 287, 288, 291, 296, 300, 302, 306, 339

attention deficit hyperactivity disorder 13, 35, 48, 51, 86, 89 , 93, 282, 283, 284, 285, 286, 287, 288, 289, 290, 291.

292, 295, 296, 297, 299, 304, 305

autism spectrum disorders 52, 80, 81, 82, 83, 84, 85, 86, 87, $144,154,299,304$

autoimmune disorders 69, 70, 88, 89, 164, 166

awareness 75, 123, 127, 176, 194, 210, 219, 250, 258, 317

basal ganglia 289

bilingualism 105, 120, 140, 143, 301, 326

bipolar disorder 58, 177, 200, 202, 203, 204, 205, 207. 208,210

bone marrow transplant 157

brain damage 309

brain development 22, 28, 145, 149, 201, 304, 309

brain disorder 120, 145, 166

brain function $6,19,90,112,181,182,185,214$

brain injury 18, 20, 39, 66, 68, 71, 74, 80, 130, 171, 182 , $248,258,261,267,270,271,273,278,309,310$

brain plasticity $28,30,99,184,195,278,279$

brain structure $63,103,110,151,201,250,267,271$

brain tumor 23, 27, 28, 73, 92, 101, 143, 155, 156, 157, 159 , $160,161,163,195$

breast cancer 158,316

cancer 100, 101, 102, 113, 145, 156, 157, 158, 159, 160 , 161,162

cannabis 50, 56, 135, 187, 188, 189

cardiovascular disease 43, 100, 111, 116, 122, 165, 170, 181, 211, 219, 229, 337

caregiver stress/burden 13,121 
cerebellum 147, 155, 162, 170, 267, 334

cerebral blood flow 23, 103, 105, 132, 133, 219, 254

cerebrevascular accident/stroke 69, 132, 177, 178, 191, 265 ,

334,336

cerebrovascular disease 63, 66, 102, 133, 178

chemotherapy 100, 162

child brain injury 17, 66, 67, 69, 70, 71, 72, 73, 74, 76, 77,

78, 79, 136, 148, 149, 312, 317

child development disorders 15, 52, 53, 84, 85, 88, 90, 145,

146, 187, 189, 279, 283, 284, 293, 295, 305, 336

child development, normal 4, 18, 22, 23, 107, 150, 193, 295 ,

318,338

childhood maltreatment 143, 204, 329

chronic pain $33,72,87$

chronic stress 194, 324

clinical trials 36, 102, 173, 221, 223, 225, 227, 231, 235.

270,281

cocaine 54, 57, 134, 151, 190

cognitive 12, 23, 24, 55, 56, 59, 63, 80, 107, 114, 115, 136 ,

$151,158,167,175,189,198,220,221,223,227,237$.

246, 285, 288, 293, 301, 310, 325, 338

cognitive control 5, 6, 14, 47, 54, 100, 140, 192, 204, 206,

207, 216, 222, 267, 270, 275, 281, 320, 323, 326

cognitive course 118, 151, 200

cognitive functioning $14,19,27,30,39,40,43,50,53,55$,

$57,58,65,70,74,76,87,88,90,94,97,102,104,106$.

$109,110,113,114,115,116,117,118,119,120,122$,

$128,134,135,136,143,145,147,148,150,156,159$,

$160,162,164,168,169,170,172,173,174,178,185$.

188, 190, 196, 200, 202, 204, 206, 207, 221, 224, 225,

230, 232, 235, 239, 240, 241, 242, 247, 248, 249, 257 ,

$259,260,262,272,275,302,306,312,316,319$,

323,324

cognitive neuroscience 3, 4, 5, 7, 17, 23, 97, 184, 191, 193 ,

$206,213,214,249,257$

cognitive processing $3,5,61,62,87,94,126,146,159,200$,

210, 243, 267, 288, 295

cognitive rehabilitation $1,30,64,65,70,78,84,152,153$,

173, 174, 182, 212, 221, 234, 256, 274, 275, 276, 277 , $278,279,280,311,312$

cognitive reserve $26,99,105,106,110,117,139,172,173$, 181, 182, 183, 214

cognitive screening 33, 34, 42, 88, 128, 167, 181, 229 ,

236,327

computerized neuropsychological testing $33,39,41,45,47$, $61,74,78,88,107,138,156,171,221,251,252,265$, 270,274

concussion $37,45,65,67,68,69,70,71,72,74,76,77,78$, 79, 124, 136, 152, 153, 247, 248, 258, 259, 264, 265.

266, 267, 269, 270, 271, 272, 273, 313

congential disorders 86,94

corpus callosum 191, 339

cross-cultural issues 2, 9, 19, 39, 55, 112, 137, 139, 141, 142 , 143, 155, 185, 220, 224, 225, 226, 230, 231, 308, 309,334 decision-making 3, 8, 109, 195, 287, 289, 325, 326

deep brain stimulation 30, 236, 238, 240, 318

delirium 167, 171

dementia with Lewy bodies 44, 231

dementia, Alzheimer's disease 24, 34, 36, 42, 51, 52, 62, 63, $64,104,110,112,113,123,126,132,139,166,199$, $213,216,217,218,219,220,222,223,224,225,226$, $227,228,229,231,232,235,243,244,245,249,250$, $278,279,306,312,314,315,335$

dementia, other cortical 4, 46, 120, 121, 237, 278

demential, subcortical 52

demographic effects on test performance 16, 93, 119, 142 , 196, 225,264

depression 3, 13, 14, 20, 25, 35, 111, 113, 125, 157, 158 , 161, 163, 168, 169, 170, 176, 184, 187, 190, 191, 192. 193, 201, 202, 204, 206, 207, 208, 209, 215, 216, 224, $228,233,236,239,240,241,242,246,247,259,261$. $265,269,274,277,279,288,320,322,324,333,334$

diabetes 64

disinhibition 65, 189, 261, 322, 325

driving 139, 157, 175, 182, 216, 230, 275, 278

dysgraphia 289

dyslexia 18, 179, 304, 305

eating disorders 29, 168, 322

ecological validity $53,321,329,335$

effort 32, 34, 40, 41, 43, 58, 69, 196, 197, 198, 199, 255. 290, 294, 296, 298

electroencephalography 6, 7, 8, 82, 96, 97, 167, 216, 266, 285

endocrine disorders 20

ethnicity 140, 141

event related potentials $3,5,8,81,149,166,239$

everyday functioning 45, 53, 54, 106, 116, 122, 129, 133, $152,169,171,176,182,186,192,202,224,230,252$, $256,258,261,273,274,275,276,280,304,321,327$ executive abilities, abnormal 13, 14, 25, 29, 33, 37, 45, 49. $52,53,57,68,69,73,75,76,78,86,90,100,128,129$, 137, 144, 145, 147, 155, 158, 159, 161, 164, 165, 174, 177, 184, 190, 191, 201, 202, 205, 209, 219, 238, 269, 270, 273, 276, 277, 278, 281, 284, 292, 295, 296, 297. $314,316,318,319,320,321,322,323,327,328,329$ executive abilities, normal $7,25,27,40,51,55,56,82,87$. 93, 99, 103, 106, 115, 117, 118, 134, 135, 140, 141. 158, 194, 202, 267, 274, 280, 291, 301, 302, 304, 317. $318,319,320,321,322,323,324,325,326,327,329$

face processing $7,33,80,98,103,120,183,286,338$

facial affect $33,82,163,173,174,175,260$

fatigue $19,55,153$

fetal alcohol syndrome 88, 303

fluency $140,242,304,317$

forensic neuropsychology 43, 196, 197, 198

frontal lobes $3,27,30,99,160,167,183,269,273,322,336$ generalization 195

genetic neuropsychology 24, 36, 78, 87, 101, 144, 145, 146, $147,216,255,314$ 
geriatric depression 114, 219

head injury, closed 131, 153, 258, 272

hippocampus 24, 28, 106, 108, 126, 139, 226, 249, 250, 251, $316,332,335,336$

HIV/AIDS 54, 55, 56, 57, 58, 135, 151, 185, 246, 320

hormones $92,162,271$

Huntington's disease 236, 241

hydrocephalus 168

hypertension $110,116,239$

hypoxia $182,309,310$

Imaging (Functional) 6, 16, 17, 18, 20, 21, 107, 131, 149 , $201,214,217,305,311,316$

information processing speed 32, 102, 105, 158, 177 , 208, 301

inhibitory control 6, 37, 105, 168, 187, 192, 281, 317 , 320,323

intellecutal functioning 15, 35, 39, 69, 85, 89, 146, 287, 293, $298,317,333$

intelligence 26, 38, 48, 86, 91, 197, 294, 302

language $6,10,16,21,30,49,51,80,91,107,142,316$. $330,331,339,340$

language: aphasia 2, 68, 120, 132, 264, 331, 332

language: development 10, 80, 83, 86, 91, 92, 285, 299,310

language: second/foreign 143

laterality 14, 16, 139, 214, 237, 339, 340

learning 4, 43, 58, 84, 99, 106, 109, 173, 176, 187, 190, 232, $241,303,319,324,325,333,338$

learning disabilities 18, 187, 266, 295, 299, 301, 303, 305

leukemia 101, 162

low birth weight 329

magnetic resonance spectroscopy 205, 216, 227

magnetoencephalography 20, 214

malingering $32,35,38,48,51,196,197,198,199,255$, 283, 297

mathematics ability $300,301,302$

medical illness 150, 164, 166, 167, 168, 169, 170, 171. 181. 186

memory complaints 13, 38, 43, 95, 123, 161, 164, 198, 199 , $216,224,226,229,280,307,314,315,334$

memory disorders $4,10,15,22,35,36,55,56,58,62,64$, $65,99,120,126,129,130,132,170,176,178,184$, $216,217,225,232,235,249,250,251,253,264,267$. $274,332,334,336,337$

memory training $17,275,278,311,312$

memory, false 6

memory, implicit 4, 51, 74, 183, 283, 303

memory, normal 11, 22, 26, 28, 37, 42, 46, 50, 99, 105, 108,

$115,132,141,153,154,193,250,316,328,330,333$,

$334,335,337,338$

memory, prospective $13,30,54,111,167,268,276,335$, 336,338

meningitis 58

mental retardation 53

metacognition 23, 210, 224, 254, 320 metamemory 210, 219, 250, 276, 336

methamphetamine 189

mild cognitive impairment 29, 34, 36, 42, 45, 111, 112, 113 , 114, 116, 120, 121, 122, 123, 124, 125, 126, 127, 128 , 129, 138, 166, 213, 218, 221, 228, 229, 230, 235, 237 . $249,252,280,311,312,314,317,334,335$

mild traumatic brain injury 19, 38, 41, 65, 68, 71, 72, 77. $146,153,169,179,180,199,251,253,254,255,256$. $261,262,268,269,272,294,313$

minority issues 231

motivation 47, 189, 197, 246, 287, 288

motor function 22, 55, 84, 91, 103, 117, 134, 169, 186, 243 . $265,283,284,290,319,324,332$

motor speed 46, 209, 234

multiple sclerosis 150, 172, 173, 174, 175, 176, 247, 248, 249, 278

muscular dystrophy 94, 145

naming 71, 137, 218, 330, 332

neglect 50, 179, 339

neural circuitry 18,98

neurocognition 18, 56, 57, 98, 112, 121, 152, 202, 203, 206 , $210,213,217,325$

neurofibromatosis 90, 93, 144, 148, 155

neuroimaging, functional 5, 17, 19, 20, 21, 22, 83, 96, 100 , 103, 104, 111, 135, 149, 153, 154, 166, 183, 204, 206. 214, 215, 243, 256, 261, 304, 323, 325

neuroimaging, functional connectivity 18, 21, 22, 83, 86, 98, 132, 148, 181, 184, 203, 301, 302

neuroimaging, structural 11, 23, 25, 26, 27, 28, 29, 30, $32,42,67,69,76,80,83,91,92,96,98,104,107$. 108, 112, 124, 125, 132, 151, 155, 166, 171, 178 , 185, 186, 188, 193, 204, 222, 223, 228, 231, 242 , $249,252,257,260,265,270,272,300,309,310$, $312,314,335$

neuroimaging, structural connectivity $24,25,26,27,62,74$, 82, 89, 97, 101, 149, 151, 184, 192, 234, 246, 257. 313,330

neuropsychological assessment 10, 11, 15, 27, 29, 32, 35, 36 . $38,39,43,44,45,46,48,49,50,53,61,62,74,84$. 107, 110, 112, 115, 116, 119, 120, 127, 128, 131, 137, $141,143,145,147,152,164,165,166,168,175,178$, 179, 180, 182, 185, 187, 196, 202, 203, 205, 207, 210, 218, 223, 226, 231, 232, 233, 237, 241, 243, 252, 253, 258, 275, 276, 283, 285, 289, 291, 294, 296, 297, 301. $316,322,325,326$

neuropsychological outcome 12, 14, 54, 76, 84, 86, 92, 131, $133,141,157,165,171,172,177,186,188,209,221$, $228,233,238,262,263,269,278,284,314$

neurotoxicity 161, 186

neurotransmitter systems 202

nonverbal learning disability 53, 146

normative data 44, 46, 134, 142, 264, 295, 334, 337

obsessive-compulsive disorder 88, 206

outcome 14, 68, 72, 92, 138, 156, 159, 178, 181, 215, 219. $254,269,299$ 
parietal lobes $98,101,340$

Parkinson's disease 7, 30, 55, 150, 163, 164, 165, 166, 232 , 233, 234, 235, 236, 237, 238, 239, 240, 241, 242 , 243,318

pediatric neuropsychology 1, 4, 13, 16, 27, 28, 31, 67, 69, 70, $72,75,76,82,85,87,89,91,93,144,145,147,155$, $156,158,160,161,162,163,174,180,193,205,215$, $239,273,274,277,286,290,292,293,294,295,296$, $297,298,303,313,317,319,328,329$

perinatal factors 302

perseveration 319

personality 7, 8, 115, 134, 143, 204, 217, 219

phenylketonuria 89, 321

phonology 302

planning 87, 274, 327, 328

positron emission tomography 18, 126, 230, 315

post traumatic stress disorder 20, 30, 33, 35, 38, 40, 64, 65 , 66, 137, 182, 194, 200, 207, 208, 209, 255, 256, 258, $261,263,337$

praxis 6

prematurity $91,92,318,332$

premorbid functioning 35, 39, 142, 181, 198

psychometric constructs 33, 37, 40, 41, 42, 44, 54, 99, 219 . $236,258,286,294,320,322,328,331$

psychopathy 205, 292, 293

psychopharmacology 206, 281, 291

psychophysics 230

psychosis 6, 151, 202, 203, 205, 209, 210, 220

pulmonary disorders 100

quality of life $9,11,16,52,55,57,69,73,102,119,121$,

$164,171,176,208,217,247,253,263,283$

radiotherapy 100,159

reaction time $105,285,328$

reading disorders $80,299,300,301,302,331,339$

reading, normal 23, 30, 300, 301, 302

schizophrenia 24, 47, 137, 201, 202, 203, 204, 205, 206 , 208,210

seizure disorders / epilepsy 10, 11, 12, 13, 14, 15, 16, 21, 22, $90,95,96,147,153,183,306,329,330$

seizure disorders / epilepsy, surgical treatment 8, 9, 14, 15, $16,95,96,183,185$

seizures, psychogenic 12

self-monitoring $85,254,325$

self-report 12, 33, 58, 76, 114, 123, 156, 157, 191, 193, 237. $272,315,320,323,324,328$

semantic processing 4, 132, 230, 331, 332

sensory integration 230,300 sickle cell disease 88, 89, 93, 94, 164, 165

sleep 8, 10, 71, 72, 79, 103, 118, 136, 137, 148, 153, 177 . 223, 237, 240, 248, 256, 260, 271, 281, 286, 287, 296, $318,322,323,324$

sleep disorders 8, 118, 147, 248, 286

social cognition 3, 10, 17, 18, 81, 82, 83, 86, 97, 98, 120 ,

148, 149, 191, 193, 194, 195, 230, 271, 293, 313

sports-related neuropsychology 41, 42, 45, 67, 77, 78, 79, $124,146,248,251,253,259,261,262,265,266,267$. $269,271,296$

stimulants 281

strategic processing 20, 321

stroke recovery $45,46,50,70,75,150,176,177,178,211$, $253,256,265,274$

substance abuse 57, 135, 150, 152, 187, 188, 189, 191

target detection 7

temporal lobes $8,9,12,14,15,184,254$

test $33,36,81,176,253,293$

test development $35,37,47,51,78,122,190,229,251$, 314,334

test reliability 37, 48, 50, 127, 138, 251, 294, 296

test theory 53

test validity $32,33,35,38,41,42,44,47,48,50,74,89$, 127, 199, 232, 235, 251, 252, 258, 282, 292, 295, 319, 331,338

thalamus $25,99,178,336$

theory of mind 118, 173, 191, 193, 312

traumatic brain injury $16,25,32,35,63,66,67,71,74,75$, $78,79,99,131,136,137,138,148,149,152,195,196$, 198, 246, 248, 253, 254, 255, 256, 257, 258, 259, 260, $261,262,263,264,265,266,267,269,270,272,273$, $276,313,314,331,339$

treatment outcome 14, 30, 65, 66, 79, 81, 94, 143, 147, 158, 163, 168, 171, 207, 215, 253, 275, 280, 312, 337

Turner syndrome 147

verbal abilities 43, 51, 82, 142, 162, 209, 230, 233, 238

visual imagery 84

visuoconstruction 101, 328

visuospatial 12, 49, 83, 99, 117, 121, 146, 164, 169, 171, $186,208,280,328,337,338,339,340$

vocation 52

William's syndrome 146

working memory 5, 20, 47, 109, 124, 126, 138, 200, 203, $204,208,216,274,282,283,284,290,291,305,324$, $331,333,335$

writing 289,317 